\title{
$D R-1862$
}

CONF-690401

proceedings of

conference on

\section{THE EFFECTIVE USE \\ OF COMPUTERS}

\section{IN THE NUCLEAR INDUSTRY}

April 21-23, 1969

Knoxville, Tennessee

sponsored by

The Mathematics and Computation Division of The American Nuclear Society

and

The Oak Pidge National Laboratory

and

The University of Tennessee

U.S. ATOMIC ENERGY COMMISSION

DIVISION OF TECHNICAL INFORMATION 


\section{DISCLAIMER}

This report was prepared as an account of work sponsored by an agency of the United States Government. Neither the United States Government nor any agency Thereof, nor any of their employees, makes any warranty, express or implied, or assumes any legal liability or responsibility for the accuracy, completeness, or usefulness of any information, apparatus, product, or process disclosed, or represents that its use would not infringe privately owned rights. Reference herein to any specific commercial product, process, or service by trade name, trademark, manufacturer, or otherwise does not necessarily constitute or imply its endorsement, recommendation, or favoring by the United States Government or any agency thereof. The views and opinions of authors expressed herein do not necessarily state or reflect those of the United States Government or any agency thereof. 


\section{DISCLAIMER}

Portions of this document may be illegible in electronic image products. Images are produced from the best available original document. 


\section{LEGAL NOTICE}

This report was prepared as an account of Government sponsored work. Neither the United States, nor the Commission, nor any person acting on behalf of the Commission:

A. Makes any warranty or representation, expressed or implied, with respect to the accuracy, completeness, or usefulness of the information contained in this report, or that the use of any information, apparatus, method, or process disclosed in this report may not infringe privately owned rights; or

B. Assumes any liabilities with respect to the use of, or for damages resulting from the use of any information, apparatus, method, or process disclosed in this report.

As used in the above, "person acting on behalf of the Commission" includes any employee or contractor of the Commission, or employee of such contractor, to the extent that such employee or contractor of the Commission, or employee of such contractor prepares, disseminates, or provides access to, any information pursuant to his employment or contract with the Commission, or his employment with such contractor.

This report has been reproduced directly from the best available copy.

Printed in USA. Price $\$ 3.00$. Available from the Clearinghouse for Federal Scientific and Technical Information, National Bureau of Standards, U. S. Department of Commerce, Springfield, Virginia 22151. 
TID-4500 (53rd ed.)

\title{
Proceedings of Conference
}

on

\section{THE EFFECTIVE USE OF COMPUTERS IN NUCLEAR INDUSTRY}

Knoxville, Tennessee

April 21-23, 1969

Papers presented at the third biennial topical meeting of the Mathematics and Computation Division of the American Nuclear Society

\section{Betty F. Maskewitz}

General Chairman

R. Ehrlich

Chairman, Technical Program Committee

D. R. Vondy

Chairman, Publications Committee

\author{
Cosponsored by
}

OAK RIDGE NATIONAL LABORATORY

Operated by Union Carbide Corporation

for the U.S. Atomic Energy Commission

and

THE UNIVERSITY OF TENNESSEE

This report was prepared as an account of work

sponsored by the United States Government. Neither

the United States nor the United States Atomic Energy

Commission, nor any of their employees, nor any of

their contractors, subcontractors, or their employees.

makes any warranty, express or implied, or assumes any

legal liability or responsibility for the accuracy, com-

pleteness or usefulness of any information, apparatus,

product or process disclosed, or represents that its use

would not infringe privately owned rights. 
The Mathematics and Computation Division of the American Nuclear Society initiated in 1965 the first of what was hoped to be a continuing series of topical meetings to be held biennially, the topic to be selected for its current interest and need for exploration. The first was held at Argonne National Laboratory as a Conference on the Application of Computing Methods to Reactor Problems and resulted in proceedings reported as ANL-7050. In 1967 the meeting was convened in Mexico $\mathrm{C}$.ty. The proceedings were published in three volumes as report number CNM-R-2 as the International Conference on the Utilization of Research Reactors and Reactor Mathematics and Computation.

The Conference on the Effective Use of Computers in the Nuclear Industry was proposed and planned by the ANS M \& C Division with the Oak Ridge National Laboratory making all local arrangements and the University of Tennessee supplying the facilities.

R. Ehrlich of the Knolls Atomic Power Laboratory served as Chairman of the Technical Program and was assisted by G. E. Whitesides, Union Carbide Nuclear Division; K. F. Hansen, Massachusetts Institute of Technology; H. C. Honeck, Savannab River Laboratory; Margaret K. Butler and C. N. Kelber, Argonne National Laboratory; R. Vichnevetsky, Electronic Associates, Inc.; R. J. Breen, Bettis Atomic Power Laboratory; P. F. Pasqua, University of Tennessee; K. D. Lathrop, Los Alamos Scientific Laboratory; F. H. Clark and D. R. Vondy, Oak Ridge National Laboratory; R. A. Blaine, International Business Machines; and R. R. Coveyou, International Atomic Fnergy Agency.

The Conference papers are being published as the result of a continuing policy of the American Nuclear Society Mathematics and Computation Division to make available, by every possible means, information which is likely to be of value to its members. Special appreciation is expressed to the individual authors and their employers who devoted much time and effort toward making the conference a success and this publication possible. It is hoped that the meeting and the information in this collection of papers will advance the state of the art in the effective use of computers in the nuclear industry.

Deserving of special recognition are the USAEC Division of Technical Information Extension, who agreed to publish and distribute these papers and the Conference Publications Committee whose members are D. R. Vondy, Chairman; F. E. Marsh, Jr., UwAEC-DTIE; C. E. Robertson, Jr., GeneraI Electric Company; H. C. Roland, University of Tennessee; Ann W. Savolainen, Oak Ridge National Laboratory; Phyllis l.. Walker, Argonne National Laboratory; and G. E. Whitesides.

The advice and counsel of $K$. F. Hansen, ANS M \& C Division Chairman, and of O. J. Du Terple, R. R. Quinn, and other members of the American Nuclear Society stafi is much appreciated. Special thanks are due to the local arrangements staff - to C. E. Hormand, C. T. Long, Jane Gurney, and Vivian Jacobs.

Betty F. Maskewitz, ORNL Conference Genera1 Chairman Mathematics and Computation Division American Nuclear Society 


\section{BLANK}


CONTENTS

\section{SESSION I}

KINETICS

Chairman: W. M. StaceJ, Knolls Atomic Power Laboratory

REVIEW OF COMPUTATIONAL METHODS FOR SPACE-TTME KINETICS

A. F. Henry, MIT .

TART - AN LMEBR TRANSIENT ANALYSIS PROJECI

M. G. Stevenson, B. E. Bingham, B\&W ..................... 16

COUPLING OF DYMANICS CALCUIATIONS IN THE FREADM CODE

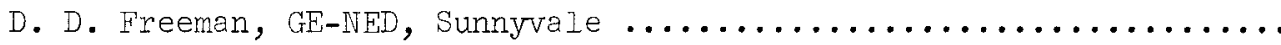

FAST REACTOR ACCIDENT STUDY CODE, SASIA

G. J. Fischer, J. C. Carter, T. J. Heames, D. R. MacFarlane,

IN. A. MoNeal, C. K. Sanathanan, W. T. Sha, C. K. Youngdahl, ANI ....

ON THE SOLUTION OF THE TIME-DEPENDENT GROUP DIFFUSION EQUATIONS

BY AN IMPLICIT TIME DIFFEREINCED ITERATIVE NETHOD

J. B. Yasinsky, L. A. Hageman, W-BAPL

NUMERICAL SOLUTION OF THE TWO-DIMENSIONAL TIME-DEPENDENT

MULTIGROUP EQUATIONS

W. T. McCormick, Jr., K. F. Hansen, MIT $\ldots \ldots \ldots \ldots \ldots \ldots \ldots \ldots \ldots$

NUCLEAR REACTOR SPATIAL KINETICS CALCULATIONS

G. Buffoni, A. Galati, P. Loizzo, S. Lopex, F. Norelli,

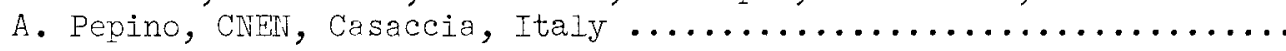

\section{LUATCHEON SENSSION}

Presiding: H. C. Honeck, Savannah River Laboratory

SOME THOUGHIS ON AEC FUNDED CODING

W. H. Hannum, J. W. Lewellen, USAEC

\section{SISSION II \\ DATA PROCESS ING \\ Chairman: R. A. Blaine, International Business Machines}

LEIRD GLNERATION CONPUTER USED TO CONTROL FUEL ELEMENT ASNEMBLY

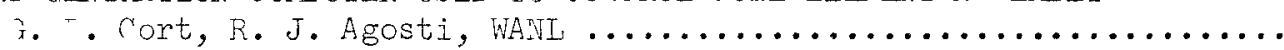

W

CROW SECIIOR DATA AND THE CONTROL OF NEUTRON DATA ACQUISITION

FACILITIES AROUTD A VAN DE GFAAFF AND AIN GLECTRON LJINEAR AC-

CELIERATOR

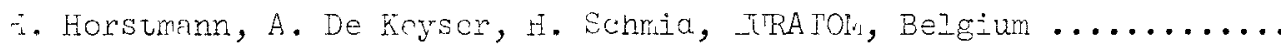

DECIGN OF A INUCLEAR DATA ACQUISITIOH "YSTL"

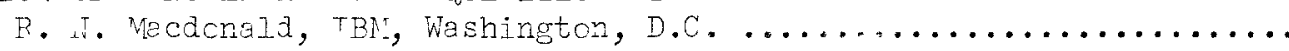

APPLICATION OF AN INAGE PROCESOIING SYSTEM TU FRAGMENIA IION SIUDIES

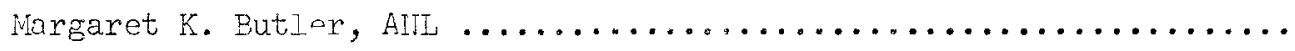


Session II (cont.)

NOISYI - A PROGRAM FOR CAICULATION OF SPACE DEPENDFHNT AUTO AND

CKOÉS SPECTRAL DEISIMTES IN REACTORS

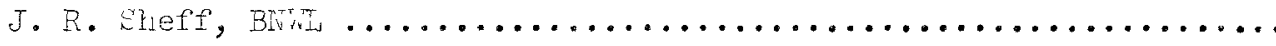

SE'SSION III

MULIICROUP CALCULATICNC

Chairman: R. C. Dahlberg, Gulf General Atomic

THE APDLICA IOR, OF THE BLOCK INVERSION TECHNIQUE TO MULTI-

DINENISIONAL REACTOR CALCULATIONS

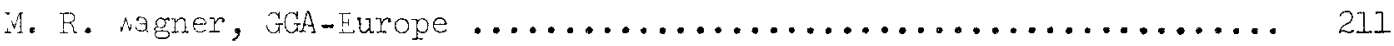

REDUCPIO OF NACHINE IT IN DISCRETE ORDINATES CALCUTAPIONÖ

Q. ᄂ. Sulivan, L. K. Albers, NASA-IRC, D. Yu, Univ. of OKIa ..... 234

A IWO-LIIEWISEOML IEALVPORT THEORY PROGRAM IN A MODUTAR SVSTEM

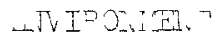

J. P. F. iedman, B. H. Creiwford, CE-KAPL

OPTIMIZATIOIF OF THE DOPPLER EFEECT IN A FAST MATERIAL TEST

PFACTOE BY PROGRAMMTIT TECHNIQUES

A. L...ni, $\because$ Sa Ivatores, G. Sena, CHErr, Casaccia, ItaIy .........

256

\section{SESSIOI IV}

REACIOK DESIGIS SIS.E:

thairman: C. J. Pfeifer, Bettis Atomic Power Laboratory

VARIA TIOTAL METHODS AID NETTRON FLUX SYINTHESIS

I. I. Wachspress, GE-KAPL

CARONTE - THE EURATOM SYSTEM FOR AUTOMATIC CONTROL OF LINKED

CAICUIA TIONIS

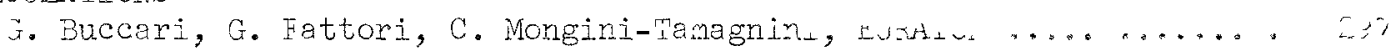

IHE CODNUC SYSTEI - A MODULAR SYSTTM OF SUBROUTIIES FOR

REACTOR CAICULATION

J. P. Bayard, R. Boudet, CEN, Saclay, France

HITACHI NUCLEAR CODES CONTROL SYSTEM, NCCS

. Watanabe, K. Arai, T. Noda, H:tachi, Ltd. .............. 313

JOSHJA - A RTACTOR PHYSICS COMPITATIONAI SYSTEM

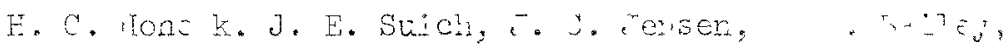

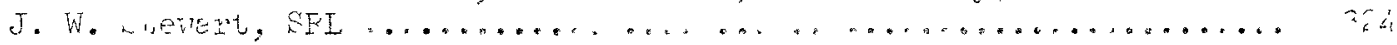

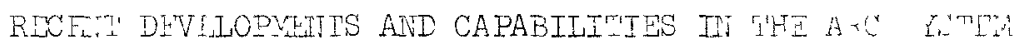

L. C. Just, P. Walker, B. J. Toppel, $A$. $\ldots \ldots \ldots \ldots \ldots \ldots \ldots \ldots$

THREE DIMEINSIOIAI BWR SIMULATION

R. L. Crowther, W. P. Petrick, A. Weitzberg, GE-NED, San Jose ..... 344

SESSION $V$

HYBRID COMPUTATIONS

Chairman: S. H. Jury, University of Tennessee

HYBRID SOLUTION OF PARIIAL DIFEERENTIAL EQUATIOIS IN NUCLEAR

PLANTS SIMULAIION: A REVIEW

R. Vichnevetsky, EAI 
Session $V$ (cont.)

THE USE OF HYBRID COMPUTERS IN THE ITALIAN CNEN NUCLEAR PROGRAMME

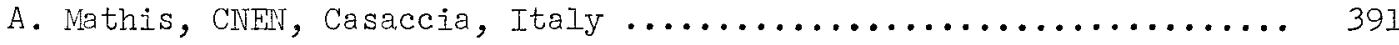

HYBRID SIMULATION IN FFTT SYSTEM CONCEPTUAL DESIGN

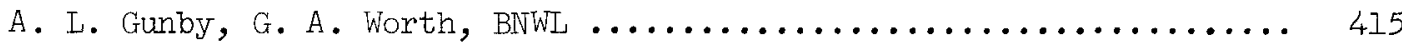

\section{SESSION VI \\ COMPUTATIONAL TECHNIQUES \\ Chairman: K. D. Lathrop, Los Alamos Scientific Laboratory}

THE EFFECTIVE USE OF THE COMPUTER STORAGE HIERARCHY

T. I. Jordan, W. J. Worlton, LASL

COMPUTER INDEPENDENCE OF LARGE REACTOR PHYSICS CODES WITH

REFERENCE TO WELI BALAINCED COMPUTER CONFIGURATIONS

R. Froehlich, GGA

451

DATA MANAGEMENT FOR MULIACCESS COMPUTATIONAL SYSTEMS

J. E. Suich, J. C. Jensen, H. C. Honeck, SRL .............. 471

A BURNUP SYSTEM CONTROL AND CALCULATION METHOD

E. R. Volk, J. S. Gubkin, D. B. Wehmeyer, C. S. Yeh, APDA ........ 482

NUCLEAR MODEL PROGRAMMING FOR DATA GENERATION

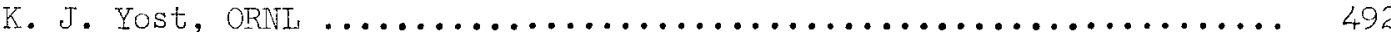

SESSION VII

ENGINEERING ANALYSIS

Chairman: J. E. Olhoeft, Westinghouse Corporation, Atomic Power Division

ON COMPUTATIONAL METHODS IN SOLID MECHANICS AND STRESS ANALYSIS

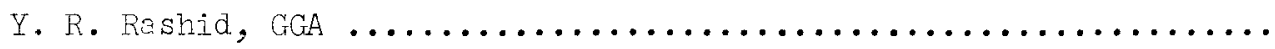

AUIOMATED THERMAI ANALYSIS OF NUCLEAR REACTOR COMPONENTS

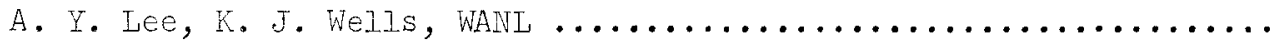

NUMERICAL ANAIYSIS OF TWO-DIMEINSIONAL, DYNAMIC, THERMAL

S RRE,BSLS IIN A HOLLOW CYLINDER

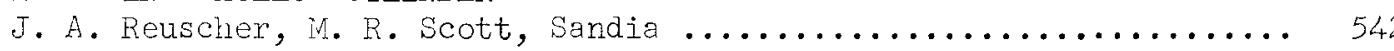

AN EXPLICIT UNIIMI JWD STABILITY APPROACH IO THE TRANSIENT

CONDUC I IOIT-COIJVECTIOIJ EQUATIONS

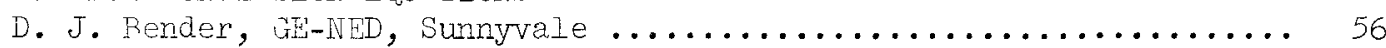

TRACK II, A COMPUTER PROGRAM FOR TRANSIENT THERMAL ANALYSIS OF

CONPLEX IUCLEAR REACTOR SYSTEMS WITH MULTIPLE PARALLEL AND

SERIE'S TLW PATHA

M. D. Woods, I. D. Hamlin, WAIIL

ROECT - A COMPUTER PROGRAM TO SOLVE THE BOWING PROBLEM IN

KOI-TYPE FUEL ELENEITTS

J. J. Urbaniak, W-WAPI

SESSION VIII

MONTE CARIO

Cnairnan: F. H. Clark, Oak Ridge National Laboratory

A NEW I.ON-IULLITROUP ADJOINT MONTE CARIO IECHNIQUE

I. B. Isvitt, AI, J. Spanier, NAR 
Session i III $($ cont. $)$

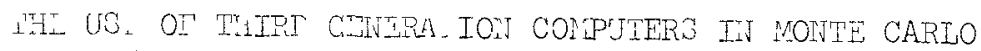

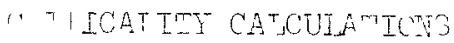

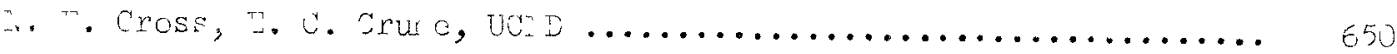

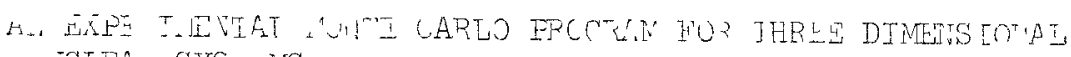

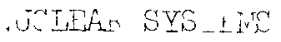

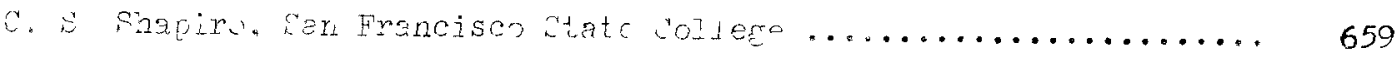

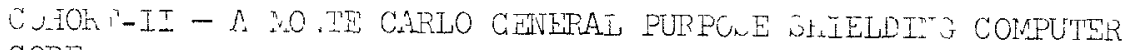
$\mathrm{CODE}$

I. in $\mathrm{PCr}^{2}$, I. r. C.emons, Jr., JJARA-LRC 
The Technical Program Committee arranged the meeting to encourage participation by all of the members of the Mathematics and Computation Division of the American Nuclear Society. Invited talks were severely limited to allow for a maximum of contributed papers. The response was excellent and the committee had a difficult task in selecting papers for presentation from the 78 contributed abstracts. Although no attempt was made (in this third meeting of the series started in 1965) to arrange international sponsorship, the committee was delighted at the large number of papers contributed by authors from different countries.

Informal exchange of information was encouraged at the meeting by several round-table discussions. Round-table discussions were arranged prior to the meeting and others could be added if desired by the participants. The prearranged around-table discussions on topics appropriate to the conference were monitored by J. E. Suich (SRL), W. R. CadwelI (W-BAPL), G. L. Gyorey (GE-APD), H. J. Kopp (GE-KAPI), and R. A. Blaine (IBM) and were summarized at the meeting. To avoid delay in publication the discussions following the presentation of papers and the summaries of the round-table sessions are not included herein. Summaries of the round-table discussions will be made available in an M \& C Division newsietter. The Committee hopes that this format will be an effective means of achieving the goal implied by the Conference title. 
SESSIOII I

KINETICS

Chairman: W. M. Stacey Knolls Atomic Power Laboratory 
BLANK 
REVIEN OF COMPUTATIONAL METHODS FOR SPACE-TIME KINETICS

\author{
A. F. Henry \\ Massachusetts Institute of Technology \\ Cambridge, Massachusetts 02139
}

\title{
I. INTRODUCDION
}

The description of the space and time dependence of neutrons in a reactor is made complicated by several factors. First of all nuclear cross sections provide only the probability that any particular reaction will take place. Thus it is not possible - even given the positions and momenta of all the neutrons in a reactor - to predict with certainty their future behavior. Secondly, even if one knew with certainty the outcome of any given interaction bet ween a neutron and a nucleus, calculation of the detailed time behavior of the population requires that a rreat variety of interactions occurring at all points in the reactor, with neutrons travelins in all directions and having all energies in tne rance below about $15 \mathrm{Mev}$ be accounted for. Moreover, the time for these interactions to occur varies from $10^{-13}$ seconds to minutes. Thus even deterministic computauions appear to be almost impossibly formidable. Finally, fission reactions cause heating which can result in changes in core geometry, density of whe constituent materials or themal motion (temperatures) of these materials. These effects in turn change the local nuclear reaction rates and hence influence tne subsequent kinetic benavior of the reactor. Thus it appears necessary to compute reactor heating and flow effects simultaneously with neutron effects.

Despite unese difficulties people design and build reactors on the basis of unen previctou rinetic behavior. The attempt is to design on the conservative siae of predicted performance, and much advantage is taken of the experience gined from testing previously built reactors or from analysis of the sustematic experimental transient studies performed in, for example, the Spert Propram. Nevertheless predicted performance and subsequent design decisions are male on the basis of theoretical computations of reactor transient.. I shall attempt in this review to point out the physical features whic rak it prsitlo to describe neutron transients using a fairly simple ma unerical movel, to indicate how the resultant equations have been attacked to date whe o aiscuss the more refined methods which are now being introduced and whind are likely to form the basis of future design methods.

\section{IHE FEW-GROUP DIFFUSION THEORY MODEL}

For o larke class of reactor transients the number of neutrons involved it in excciso of $\mathrm{i}$ million. Under these circumstances it is usual to assume that fluct lation effects may be ignored. Thus for a large class of transients the first problem mentioned above does not arise, and the kinetic behavior of the populatior may pe described by a set of deterministic equations, namely the time aependent transport equation along with associated equations needed 
to describe fluid flow (including fuel, if it is in fluid form), structural and temperature chances within the core. (In general these latter equations will be coupled to those describing the behavior of the rest of the plant.) We shall consider orily the neutron equations in the present discussion and shall in addition limit this discussion to the deterministic case for ctationary Iuel.

It has "een found satisfactory in many practical situations to describe the stalic neutron aistribution in a reactor by an energy group diffusion theory model (generally making use of "effective" diffusion theory parameters to compensate for the inadequacy of straightforward diffusion theory). This simpilfication partially gets around the second difficuity mentioned above. Accoraingly we shall present the various approaches to analyzing neutron kinetic phononena in terms of group diffusion theory. Moreover, for purposes of simplic:i, we shall illustrate the methods using a two energy group model. Extorsion $\partial^{\text {f }}$ the ideas to models involving more energy groups or embodying approximalions to the transport equation more accurate than diffusion theory is straict, forwary (altnough the associated sets of equations may become forbin ix r o lo $\left.r_{\mathrm{e}} \mathrm{e}\right)$.

One point related to selection of an adequate theoretical model should be -roscer: There is no guarantee that the time dependent extension of a model nic, $\cdots, \cdots,-1$ atic benavior with acceptable accuracy will provide an ‥ ,...iption of kinetic effects. This point becomes increasingly

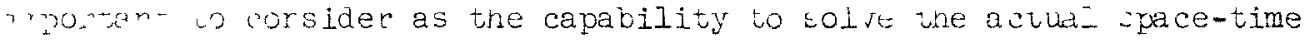
"xtension of the static equations is attained.

The two rroup diffusion theory model is based on the aivision of all neutrons irto two classes depending on whether their energy is greater or less thar that correspondine to some arbitrary cut point generally taken to be acout $1 \mathrm{ev}$. It is assumed that within given rexions of the reactor (Eenerally corresponding to given compositions) the distributions in energy of neutrons belonging to the two groups are independent of position. The total concentrajions of neutrons a position $r$ and time $u$ lc symcolized $h_{y} \Phi_{1}(\underline{r}, t) / v_{1}$ and $\varphi_{2}(\underline{r}, t) / v_{2}, \varphi_{1}$ and $\varphi_{2}$ being the group I'luxes, $v_{1}$ and $v_{2}$ beint the average speed of neutrons in the two groups and subscript (1) rcferming to the higher energy group.

Total reaction rates at any location and time are given by the product of fluxes Ly appropritte macroscopic cross sections $\Sigma_{n}(\underline{r}, t)$ where subscript $n$ $(n=1,2)$ refers to the energy group. The $\Sigma_{n}(r, t)$ themselves are sums over isotopes, $j$, or tae products of number densities, $\mathbb{N}^{j}(\underline{r}, t)$, of material con-

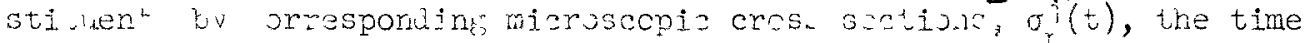
dependence of urese zatur arisir, from tic luct that the are averages over the thermal molion of the nulei makin up the ractor and over the assumed energy distritution of the neutrons in the various regions of tne core. Thus feedback effects chance the $\Sigma_{n}$ by virtue of altering the values of the group averaged microscopic cross sections as well as the material concentrations, $\mathbb{N}^{j}$. The term $\Sigma_{2 l}(r, t)$ will indicate a cross section for transferring neutrons by enercy degradation from group (l) to group (2). The term $\nu^{j} \sum_{f}(\underline{r}, t)$ will indicate the cross section for the production of neutrons due to fissions of isotope $j$ in group $n$. ( $\Sigma_{f n}^{j}$ is the fission cross section for isotope $j$, and $\nu j$ is the corresponding total number of neutrons produced by that fission.) $\mathrm{A}$ fraction $\beta(j)$ of the $v j$ neutrons produced in fission in group $n$ appears only after a $\beta$-decay of one of the fission fragments. There are a number of these "precursor "roups"; the fraction of delayed reutrons appearing from the $i^{\text {th }}$ such Eroup will be designated by $\beta_{i}(j)\left(\beta(j)=\sum_{i} \beta(j)\right)$. The concentration of 
the $i^{\text {th }}$ precursor group will be symbolized by $c_{i}(\underline{r}, t)$, and $\lambda_{i}$ will indicate the probability per second that it will decay.

In the aiffusion theory approximation tne leakage rate of group-n neutrons out of a init volume is fiven by $-V \cdot D_{n}(\underline{r}, t) \vee \varphi_{n}(\underline{r}, t)$, where $D_{n}(\underline{r}, t)$ is the diffusion constant for group $n$. It depends on the number densities, temperatures and nuclear cross sections of the maierials at point $\underline{r}$.

As a consequerce of the various reaction rates and processes described above the rate of change of the neutron population according to the two group model becomes

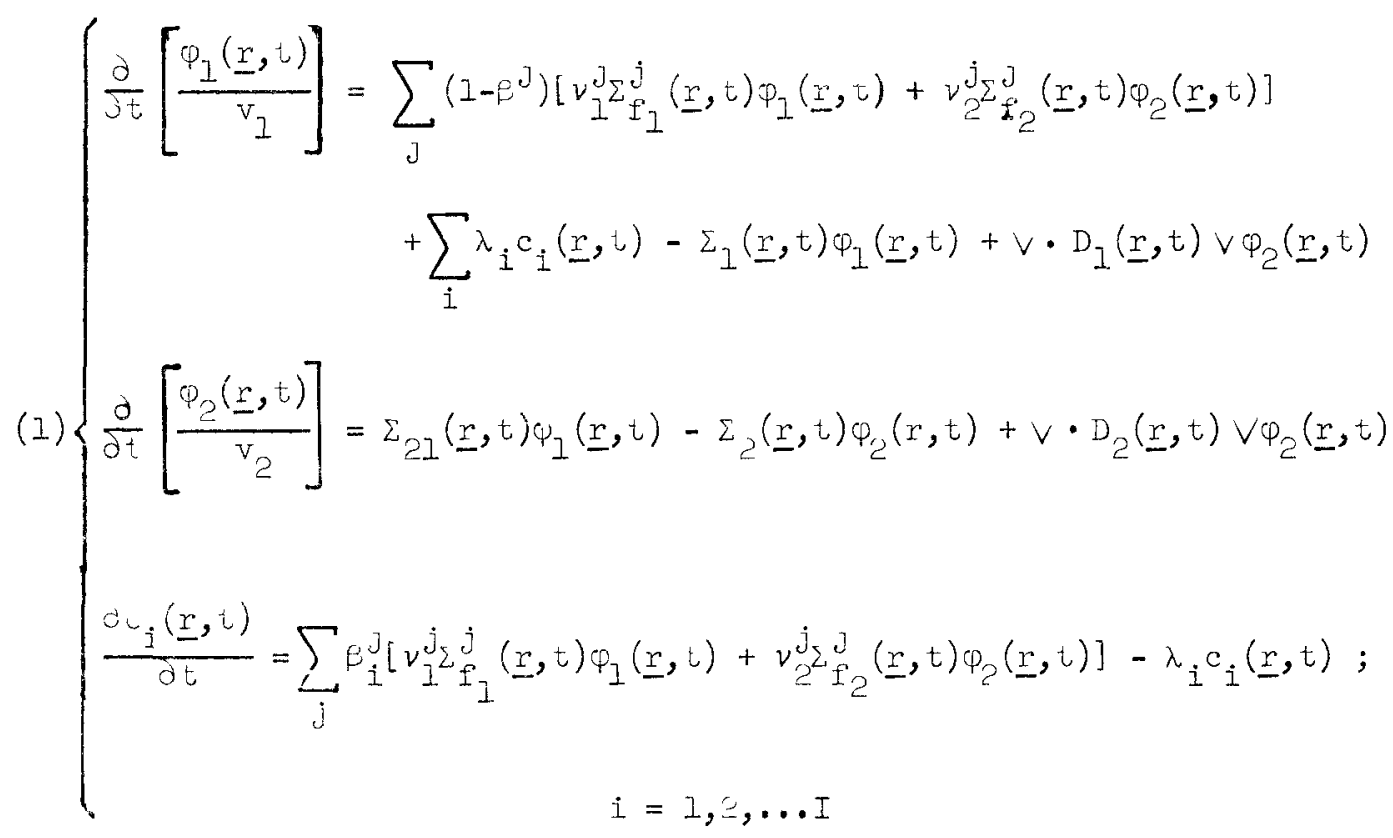

wilero a luve assumon that neutrons from fission appes only in group 1.

Wquitione (1) are tne two group tine dependent dî́iusion equations inprop it te to wh wave of stationary fucl axd no external source of neutrons. Since tha iruimted tinc dependence of the crows sections depends in ceneral

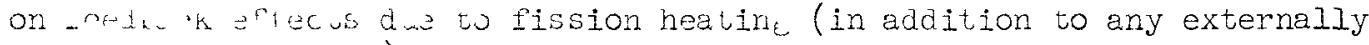

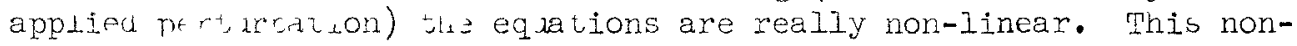
Linesrit, is the uhro difficulty mentioned in the first paragraph. It would

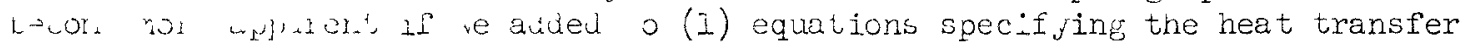
post to "if ull reacur. The diricuity it presents is circurvented in practl-w by colvir, the reutron and feedback equations in tandem. Thus with tu, fis on m te nor $r^{+}$come initial time, t, material temperatures and

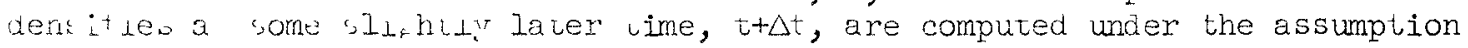
that the Iission rate stays constant auring the time interval $\Delta t$. The new temperatures 3i. denoities are then used to estimate the behavior of the niffuw on theory paramevors durins $\Delta t$, and Eqs (1) are solved during the intervi a. cet of Innfr equition witn time depenuent coefficients. The

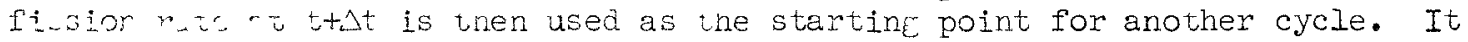
Is af colrwe pn sible to iterate this process. However changes in material prope tien ac eloralyy so much slower than chances in neutron levels that 
this is not a profitable numerical strategy.

Methods for colrin: Egs (1) thus form a very imoortant part of the overall procedure for rreaicting the transient behavior of reactors. As a preluae to ousining suca metnods it is convenient to rerrite these equations un nole corpad natrix form. The ro ult is

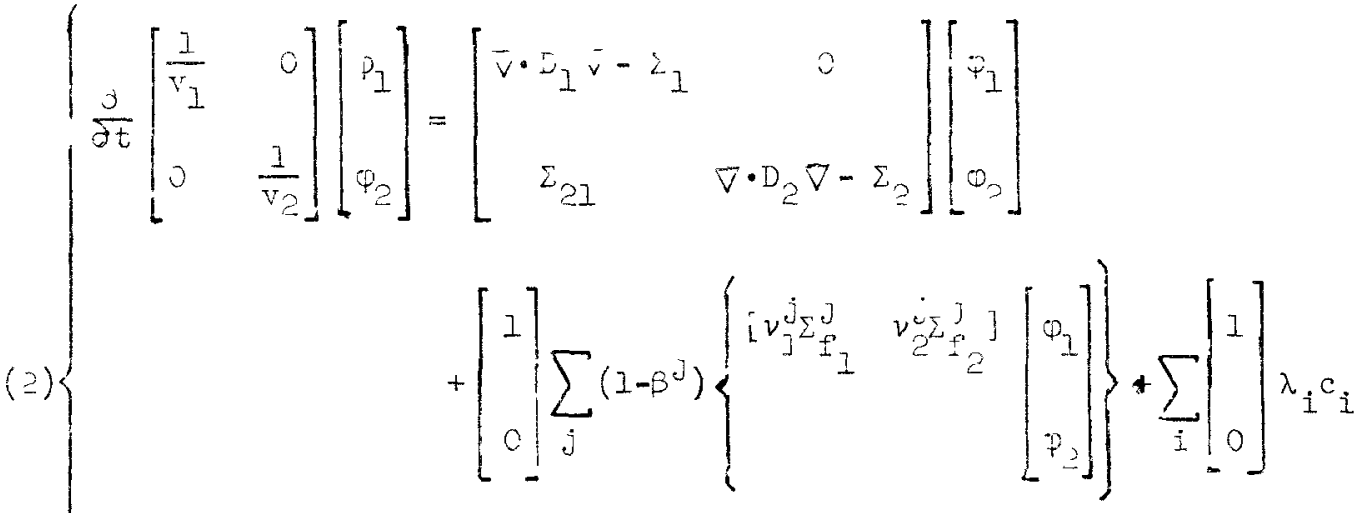

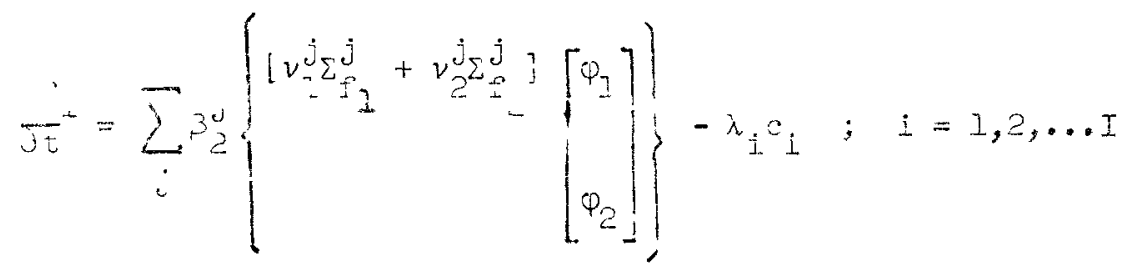

or

(3)

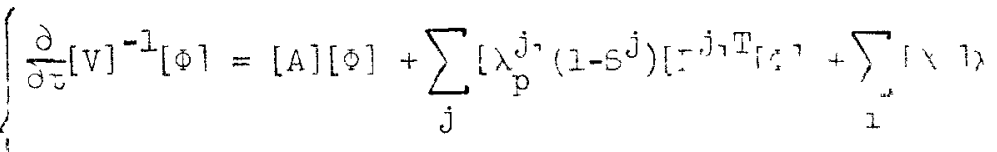

$$
\begin{aligned}
& \frac{\partial c_{i}}{\partial \tau}=\sum_{j} \beta_{i}^{j}\left[F^{j}\right\}[\varphi]-\lambda_{i} c_{i} \quad ; \quad i=1,2, \ldots I
\end{aligned}
$$

where superscript $T$ indicates a transpose (so tnat $\left[F^{j}\right]^{T}$ is the row vector, row $\left.\left\{v_{1}^{j}\right\}, v_{L^{2}}^{j} j\right\}$ ana other vems in Eq (j) ur lefined $k y$ comparison

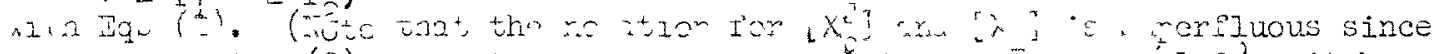

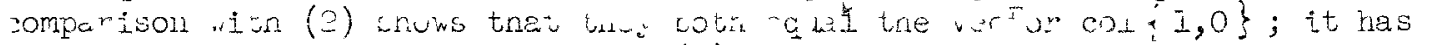
been introaued wince, ith it, Eqs ( ) inen nave th: renerai nultigroup form.)

Equation (3) is to be solved subject to the bourdiry conditions that [ $\Phi]$ be everywhere continuous and non-negative inside the reactor and vanish on the outer boundary and that the components perpendicular to any internal surface of the vectors $D_{i} V \varphi_{i}$ be continuous across that surface. In the sections below we shall indicate the various approaches which have been used to obtain approximate solutions to (S) obeying these conditions. 


\section{METHODS FOR SOLVING THE SPACE-TIME EQUATIONS}

In virtually all attacks on (3) involving use of a digital computer the spatial derivative operators in $[A]$ and the time derivative operators are replaced by finite difference counterparts. Thus ( 3 ) is approximated by a set of inhomogeneous algebraic equations the solution of which are the values of $[\Phi]$ and the $c_{i}$ at each spatial mesh point at given time steps. One wishes to find an acceptably accurate approximalion to this solution in a minimum computing time.

\section{A. Finite Differencing in the Space and Time Variables}

There is no systematic way of evaluating the accuracy of an approximate method for solving the difference equation counterpart of (3) other than to compare results with a solution which is essentially exact. For this reason efficient methods for a complete space-time finite difference solution of (3) are being developed. For one dimensional cases the problem is well in hand. For example the programs GAKIN ${ }^{1}$ and WIGL ${ }^{2}$ (which differ primarily in their treatment of the time differencing) provide finite difference solutions to one dimensional diffusion theory problems. The fact that solving the spatial part of the problem requires no iteration is what makes this one dimensional case so tractable. The time to compute a spatial shape for a fixed number of spatial mesh points is independent of the nature of the problem or the size of the time step. What does matter is that the time differencing be carried out in an efficient manner so that large time steps can be used without impairing accuracy. The chief difference between GAKIN and WIGL is in their approach to this part of the problem.

Obtaining a complete space-time difference solution in two dimensions is a much more difficult matter. Here the strategy for solving the spatial part of the protlem interacts with that for solving the temporal part. Running times become quite problem dependent. Papers (4) and (5) in the present session deal explicitly with methods for determining complete space-time difference solutions for the two dimensional case, and I shall say no more about the strategies being employed. I do want to make the point, however, that I feel exact solutions of the difference equations should be used primarily as numerical standards for testing the much faster approximate methods to be discussed below. Exact two dimensional solutions are costly, particularly if the number of mesh points involved exceeds around a thousand. Moreover, although some of the ideas for doing two dimensional problems appear to be extendible to the three dimensional case, it is quite likely that a 1000 point three dimensional problem would cost even more than a 1000 point two dimensional one. Thus if any amount of detail is desired, one is restricted in this method by economic considerations to study of only a few cases. Moreover, even if a crude spatial mesh can represent the reactor satisfactorily, approximation schemes should still be used since they can almost always be made cneaper and since for a crude spatial mesh their accuracy is generally quite acceptable.

\section{B. Other Approximation Methods}

Almost universally, approximate methods for solving Eq $(S)$ are based on some assumed knowledge of the spatial flux shapes. We shall group the various schemes used into two categories dependent on whether a priori knowledge is assumed about flux shapes over the entire core or over only portions of the core. (It can be seen that the first category is really a special case of the second. However it is convenient to treat them as distinct.) The first category we shall refer to as time synthesis, the second, as space-time synthesis. It is 
possible to derive general equations for which any approximation is a special case. We shall do that only for the first (time synthesis) category and shall discuss approximations belonging to the second category on an individual basis.

\section{i. Time Synthesis Methods}

For time synthesis the most general assumption which can be made about the flux is that over a certain interval of time, $\Delta t$, (either predetermined or computed in the course of running the problem) the flux, $\varphi_{n}(\underline{r}, t)$ for group $\mathrm{n}$ can be represented as

('t) $\varphi_{n}(r, t)=\sum_{k=1}^{K} \psi_{n k}(r) T_{n k}(t) \quad t C \Delta t$

so that

(5) $\Phi(\underline{r}, t) \simeq\left(\begin{array}{l}\varphi_{1}(\underline{r}, t) \\ \varphi_{2}(\underline{r}, t)\end{array}\right)=\sum_{k=1}^{K}\left(\begin{array}{cc}\psi_{I k}(\underline{r}) & 0 \\ 0 & \psi_{2 k}(\underline{r})\end{array}\right)\left(\begin{array}{l}T_{I k}(t) \\ T_{2 k}(t)\end{array}\right)=\sum_{k=1}^{K}\left[\Psi_{k}\right]\left[T_{k}\right]$

where tre $\Psi$ are predetermined spatial "trial functions" obeying the same consinutur and spatial boundary conditions as the $p_{n}$, and the $T_{n k}$ are "mixing coefficiens" which are to be determined by requiring (4) to be a solution of (3) in a weithted irtegral sense.

We obtain equations for the colum vectors $\left[\mathrm{T}_{k}\right]$ by inserting (5) into (3), waltiplying successively by a series of probetcrminc inght functions $\left[W_{p}(r)\right]\left(\left[W_{p}(r)\right]=\operatorname{diag}\left\{W_{I p}(\underline{r}), W_{2 p}(\underline{r}) ; ; p=I, 2, \ldots K\right)\right.$ and integrating over the volume of the reactor. The result is

(6) $\left\{\int_{V}\left[w_{p}\right][V]^{-1} \sum_{i=1}^{K}\left[\Psi_{k}\right] d v\right\} \frac{\partial}{\partial t}\left[T_{k}\right]=\left\{\int_{V}\left[w_{p}\right]\left[A+\sum_{j}\left(1-\beta^{j}\right) x_{p}^{j_{F}} j^{T}\right] \sum_{k=1}^{K}\left[\Psi_{k}\right] d v\right\}\left[T_{k}\right]$

$$
+\sum_{i} \lambda_{i} \int_{V}\left[w_{p}\right]\left[x_{i}\right] c_{i}(\underline{r}, t) d V ; p=1,2, \ldots k .
$$

For the second of Eqs (3) we multiply by the column matrix

$\left[w_{p}\right]\left[x_{i}\right]$ and again integrate over volume to obtain 
(7) $\frac{\partial}{\partial t} \int_{V}\left[w_{p}\right]\left[x_{i}\right] c_{i}(\underline{r}, t) d V=\left\{\int_{V} \sum_{j} \beta_{i}^{j}\left[w_{p}\right]\left[x_{i}\right]\left[F^{j}\right]^{T} \sum_{k=1}^{K}\left[\psi_{k}\right] d V\right\}\left[T_{k}\right]$

$$
-\lambda_{i} \int_{V}\left[w_{p}\right]\left[x_{i}\right] c_{i}(\underline{r}, t) d V ; p=1,2, \ldots K
$$

Equations (6) and (7) relate the $K$, two-element column vectors $\left[T_{k}\right]$. They depend on time alone, all spatial dependence having been integrated out. (Generally the $\left[\Psi_{k}\right]$ and $\left[W_{\mathrm{p}}\right]$ will be finite difference solutions. The indicated integrals over volume are then to be interpreted as sums over mesh points.)

The structure of these equations can be somewhat more easily seen if we define $K x K$ matrices $[F],[\rho],[\Lambda]$ and $[\bar{\beta}]$ the elements, $[F]_{p k},[\rho]_{p k}$ etc. of which are the following $2 \times 2$ matrices

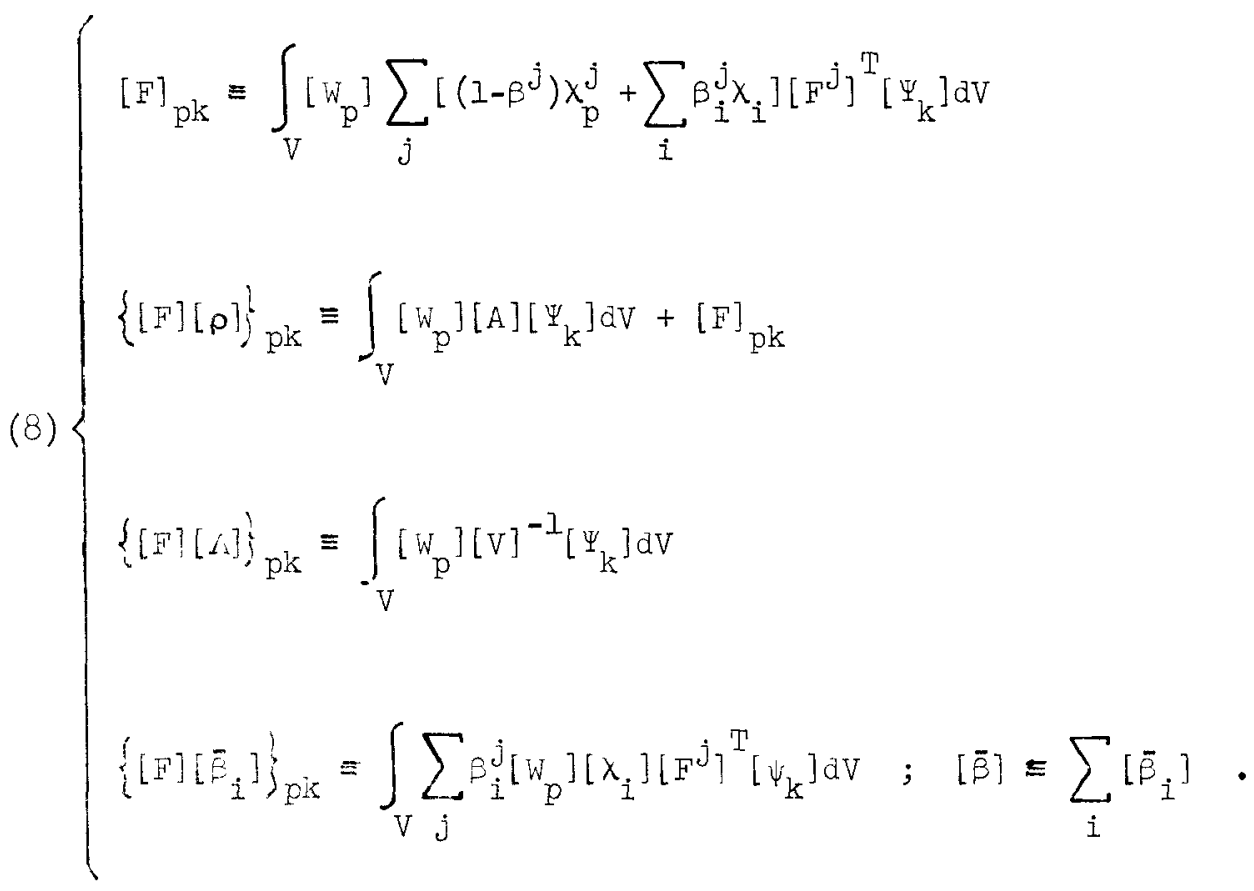

If finally we define [T] to be the $K$ elenent column vector having for its $k^{\text {th }}$ element the two element column vector $\left[T_{k}\right]$ and $[F][\Lambda]\left[C_{i}\right]$ to be the $K$ element column vector having for its $k^{\text {th }}$ element the two element column, $\int_{V}\left[w_{p}\right]\left[x_{i}\right] c_{i}(\underline{r}, t) d V$, Eqs $(6)$ and $(7)$ may be written 
(9) $\left\{\begin{array}{l}{[F][\Lambda] \frac{\partial}{\partial t}[T]=[F][\rho][T]-[F][\bar{\beta}][T]+\sum_{i} \lambda_{i}[F][\Lambda]\left[C_{i}\right]} \\ \frac{\partial}{\partial t}[F][\Lambda]\left[C_{i}\right]=[F]\left[\bar{\beta}_{i}\right][T]-\lambda_{i}[F][\Lambda]\left[C_{i}\right]\end{array}\right.$

or

(10) $\left\{\begin{array}{l}\frac{\partial}{\partial t}[\mathrm{~T}]=[\Lambda]^{-1}[\rho-\bar{\beta}][T]+\sum_{i} \lambda_{i}\left[C_{i}\right] \\ \frac{\partial}{\partial t}\left[C_{i}\right]=[\Lambda]^{-1}\left[\bar{\beta}_{i}\right][T]-\lambda_{i}\left[C_{i}\right]\end{array}\right.$

Equations (10) will be recognized as a generalization of the point kinetics equations. Because of the very large range of time constants involve: solving them efficiently on a digital machine is a difficult problem. For the artual point case (see e) below) a number of strategies have been swescici. Comprehensive lists of pertinent references were given by cohen ${ }^{3}$ in 1358 and Vigel in 1967 . They fall roughly irto four categories: Taylor senics expansion methods ${ }^{4}, 5$, finite differencing 7 , direct integration coproximating the behavior of the dependent variable in the integrand $5,8,9$, and direct integration approximating the resulting quotient of exponential matrices by quotients of rational functionslo. There is not general agreement as to which method is best, and I am unaware of any extension of these methods to the more general situations encompassed by (10).

In describing the various particuler approximetion metnods in the time syntnesis category it will be helpful to fix attention on Eq (4) while discussing the trial functions and underlying physical notions and on Eq (10) while discussins the mathematical nature of the approximation equations. In the brief summaries which follow I shall describe only approximations which have actually been investigated. Other combinations of the ideas will be seen to be possible. There may be some disagreement about the names I have chosen for the various methods.

\section{a) General Time Synthesis ${ }^{\text {II }}$}

The trial functions $\psi_{n k}(\underline{r})$ are taken from static computations (obiained by artificial adjustment of the $v_{j}^{j}$ and $v_{\partial}^{j}$ ) for the core in a number of conditions bracketing or typical of the conditions actually expected during the transient in question. Thus the trial functions are fundamental modes of a number of different operators characterizing different states of the system. These are expensive to find especially in two and three dimensions. Accordingly the weight functions, $W_{n p}(\underline{r})$, are usually taken to be the same as the trial functions, and neither set is altered during the course of the calculation. The most general form of $\mathrm{Eq}$ (10) results. Negative fluxes can turn up with this method (as well as with the next two). In practice this has not turned out to be a serious problem. 


\section{b) Analysis using Modal Flux Shapes ${ }^{12}$}

The $\psi_{n k}(\underline{r})$ are taken to be shapes associated with static solutions for one particular condition of the reactor. Thus they are a number of different modes of a single operator. They are extremely difficult to find in unsymmetrical tno and tnree dimensional cases. Also, since they are computed for core conditions appropriate to only one stage of the transient it may be necessary to use a large number of modes. (Consider for instance the problem of a moving control roa.) The $W_{n p}(\underline{r})$ may be taken from the modes of the corresponding adjoint equation. But this doubles the work of finding the functions and appeass likely to result in no overall gain.

$$
\text { Ielmholtz Modes }{ }^{15}
$$

The $\psi_{n k}$ and $J_{n p}$ are taken to be solutions to the diffusion equation appropriate to a bare nomogeneous reactor having the same outer surface as the reactor in question. Thus the $y$ are known functions, and no static problems need be run to obtain them. However the number of modes, $K$, required for an accurate description of the flux shape can be very large. (Concider for instance trying to describe a cross shaped control rod slightly off center and being withdrawn.)

\section{c) The Collapsed Group Method ${ }^{\text {II }}$}

For the approximation metnods described so far the fast and slow fluxes have been permitted to vary independently. That is, the components $\psi_{1 k}(\underline{r})$ anu $\psi_{2 k}(\underline{r})$ of a given trial function have separate time dependent coefficient $T_{1 k}(i)$ and $m(t)$. Physically this permits the fast to slow flux ratio $\left(T_{2 k} / T_{2 k}\right)$ for a given trial function to change during the course of a transienc and thus provides a greater capability for the components of $\Phi(\underline{r}, t)$ (Eq 5) to account for such a change. It is found however that the ratio $\varphi_{-}(\underline{r}, t) / \nu_{,}(\underline{r}, t)$ chanoes little during a great many transients, particularly thos associatea ith tnermal reactors. Thus a reauction in the complexity of

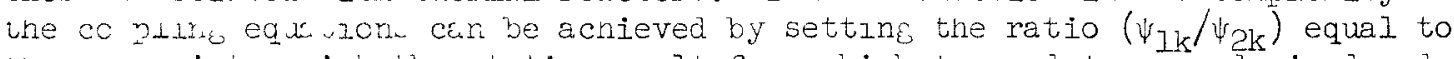
that ar,ccizte, ith the static result from which $\psi_{1 k}$ and $\psi_{2 k}$ are derived and replazirs ( $)$,

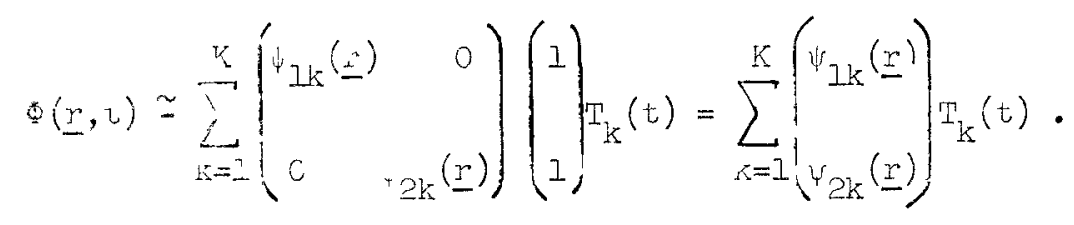

To ortajn he a w wions which determine the scalars $T_{f}(t)$ we then multiply the lir of $f(-1) \times W_{1 p}(\underline{r})$, ine secona by $N_{2 p}(\underline{r})$ and add the two results. lautu aatinull, ni, corresponds to replacing the dicsonal matrix $\left[w_{p}(\underline{r})\right]$ in (6) and $(7) \mathrm{b}$ the row matrix $[1,1]\left[w_{\mathrm{p}}(\underline{r})\right]$. Thus the elements $(8)$ of the matrices

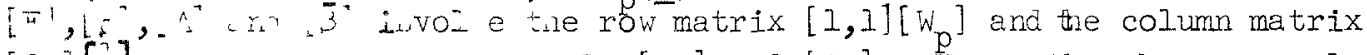

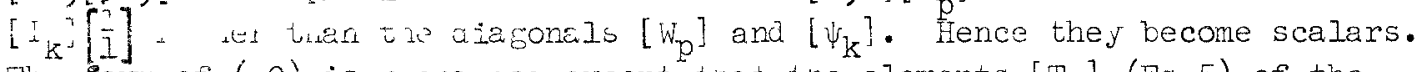

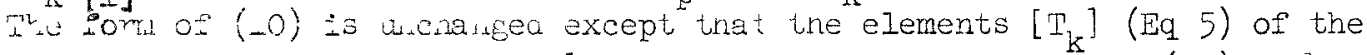
colume vector, [ $\left[\right.$ and the $\left[C_{i}\right]$ are now the scalars, $T_{k}$ of $E q(I I)$ and

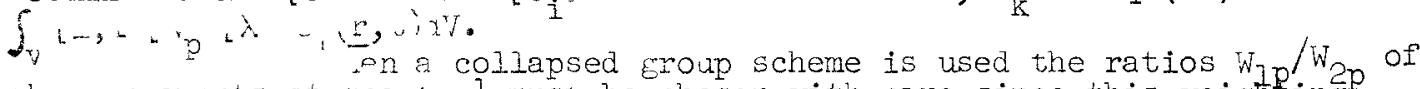
the omoncrits of tre $1, p$ l must be chosen with care since this weighting affe. ust ..tudu balance and hence the rate of change of the overall neutron 
population. There is what Stanley Kaplan calls a folk theorem in reactor theory to the effecu that selecting tne $\left[\mathrm{w}_{\mathrm{p}}\right]$ to be the solutions of the static adioini equitions asea to find the $\left[\Psi_{k}\right]$ leads to the most accurate results. phere is come empicical viaence to support this velief. It Fowever whe use of adjoint ,eighting has also led to cerious numerical difficulties. 15 The present fealing is that to use weight functions havin a component ratio (WIp/wep approximating that of the adjoint flux vector is probably the best conbination of economy and accuracy. I am una hare of any evidence - at least for tnermal raactors - that use of tne actual shapes ot the aajoint fluxes for the $w_{l p}$ and $w_{2 p}$ leads to more accurate results thur use of the shapes $w_{1 p}$ and $\psi_{2 p}$ of the trial functions themselves.

\section{d) Adiabatic Approximations}

In usine the approximation methods described to this point it is usuall, the best strategy to retair the same trial functions throughout the entire course of the transient. Thus the $\Delta t$ introduced with Eq (4) is taken to be the entire time stan of interest.

There is however another way of attacking the problem ${ }^{10}$, and that is to break up the overall time interval into small time steps using only ore rial function $\left[\Psi\left(\underline{r}, t_{n}\right)\right]$ (of he collapsed type) to approximate $\phi(\underline{r}, t)$

$r-1) \cdot a_{y}$-iven time. That trial function is then changed after each time cor. " $n$ an estimate of the ne' srape of the flux at that time. A

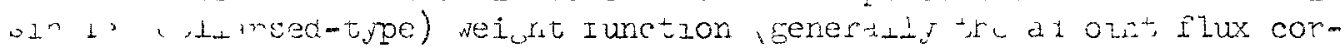
resmonath to tre initial or asymptotic conaicion) if ds ce at 21 .

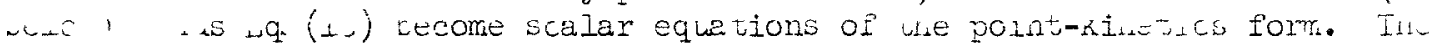
squence of trial functions is normalized so that

$$
\int_{V}[W(\underline{r})]^{T}[V]^{-I}\left[\Psi\left(\underline{r}, t_{n}\right)\right] d V=I .
$$

Tn is, frem $(3)$

$$
\int[W(\underline{w}, \tau)]^{T}[V]^{-I}[\Phi(\underline{r}, t)] d V=P(t)
$$

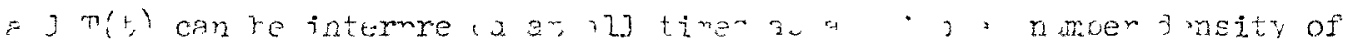

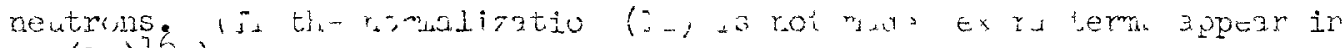
E: $(20)^{15}$.)

The difriculty with the adiapatic method is tnat a new trial function must be computed after every time step. In the aaiabatic method gets its name from the fact that static shapes characteristic of the instantaneous properties of the perturbed reactor made critical by an artificial variation of the $\nu_{n}^{j}$ were suggested originally for the $\left[\Psi\left(\underline{r}, t_{n}\right)\right]$. For specialized cases the problem of continually computing new trial functions can be circumvented by deriving "reactivity coefficients" before the transient calcula ior is commenced. If However it is necessary to know a good deal beforehand about the characteristics of the transient if this method is to be succesofiz. 
Ott and Meneley ${ }^{18}$ have extended the adiabatic approach by suggesting a way to compute the $\left[\Psi\left(\underline{r}, t_{n}\right)\right]$ which reflects more correctly the effects of changing delayed neutron spatial shapes. They also point out that the time steps in which a given $\left[\Psi\left(r, t_{n}\right)\right]$ can be used can be much larger than the time steps required for an accurate finite difference solution of Eq (10). Paper No. 6 in the present session describes what appears to be a similar extension.

\section{e) Point Kinetics}

The most elementary form of time synthesis is obtained when a single collapsed-type trial function and weight function (generally the corresponding adjoint flux) are used throughout the whole course of the transient. This approximation is called point-kinetics. It is still the most widely used method for analyzing reactor transients. Just as in the adiabatic case Eqs (10) reduce to scalar form for point kinetics. Moreover, because of the fixed nature of the trial and weight functions, the reactivity, $\rho$, (Eq 8) becomes a linear functional of the perturbation in the properties of the core. Thus contributions to $\rho$ arising from perturbations in temperatures and densities in various sub-regions of the reactor can be summed. (Use of local temperature and density coefficients is generally made in carrying out this procedure.) The parameters $\Lambda$ and $\bar{\beta}$ are also linear functionals of the perturbation, but changes in them are fractionally so small that they can be ignored. (Such changes are aIso commonly neglected in the adiabatic method.) Thus the behavior of $T(t)$ in the point kinetics model is determined entirely by the behavior of $\rho$.

The point kinetics equations are sufficiently simple that they may for many studies be solved on an analog computer simultaneously with the equations describing the thermal part of the plant. If instead, such a problem is done digitally, it is important to work out a computational strategy which takes advantage of the different time scales of the processes involved. Papers (2) and (5) in this session describe programs which illustrate such overall strategies. Paper No. I reports on an overall plant description where a onedimensional space-time finite difference calculation is also available for computation of the neutron behavior during the transient.

\section{Space-Time Synthesis}

All of the methods described in section (B.I) suffer from the difficulty inat for numerous cases of practical interest a great many trial functions ausi be used - and in the general case these are three dimensional shapes. The reason that many are needed is twofold. First, one may not have too food an iJea zeforehand of the shape changes to be expected and hence must provide a space of trial functions sufficiently general to include shapes associated with unforeseen neutron behavior. Secondly, the nature of the transient iuselt, even if its general character is predictable, may require that a large number of trial functions be employed. (For example, consider a transient induced by withdrawal of a single control rod.)

The various space-time kinetics methods represent schemes for gettine around these aifficulties by elfminating some of the inflexibility imposed $k_{y}$ the wse of rigid three dimensional trial functions. The basic idea is to replase the trial function expansion (4) by the more flexible approxima izion 
(14) $\quad \varphi_{-}(x, t) \simeq \sum_{k=1}^{K} \psi_{n k_{i j}}(x, y) \mathrm{T}_{n k_{i j}}(z, t) ; t \subset \Delta t ; x, y \subset \mathrm{R}_{j} ; z \subset \Delta z j$

where the $R_{i}$ are refions resulting from a partitioning of the $X Y$ plane into sub-regions, and the $\forall$, result from a similar partitioning of the $Z$ axis. (Ihe $\psi_{\mathrm{nk}}$; can equaliy well be furetions of racius and angle $(r, \theta)$ or of coordinatesuppropriate to hexagonal geometry.)

The trial functions $\Psi_{n k j}$ may be taken as portions of overall static (XY) flux shapes appropriate to various axial locations and reactor conditions exnected during the transient 9 or may be taken as shapes resulting from varicus tw uimensional inhomogeneous source calculations. 20 Corresponding weight, funct ions $W_{\text {rip }}(x, y)$ are needed, and, as in the point case, these may be taken to $b^{2}$ the trialufunctions themselves (the Galerkin Method) or to be the resuirs ti corrcopondirg static ajoint computations. Trial and reigh function, wai le ejther general or of the collapsed type.

Equations for the coupling coefficients $T_{n k}$, are best found by use cf a variat:onil principle which admits of discontinuous idrial functions. 21

- + + qutions are time dependent coupled differential equations in the - $\quad r^{-}$the are more aifficult to solve than the corresponding point

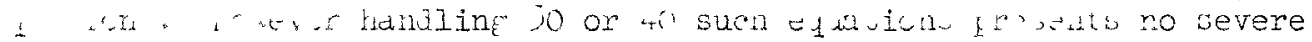

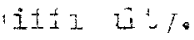

Ho fer as I am awa, the major development efforts in the area of space-ime yntresiz have been at the KAPL and Bettis labonatories. In the Eet is in the rograms developed nave involved only a single radial region, $F_{i}$ anconpasoin's the whole radial plane. Thus at least two full core (XY) calculations must he run to provide the flexibility needed to describe a flux

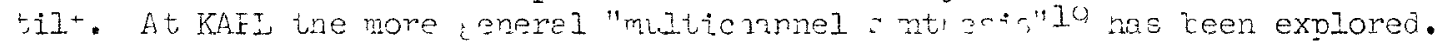

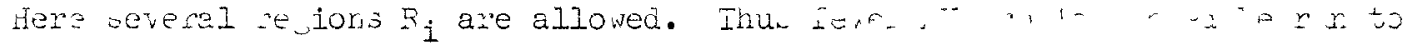
uta: $\mathrm{r}$ tzic functions. The radial discontinuities waich resunt from uris ippror k. a ea. co be small. 22

IV. CuNLIUIDT

It is ry opinion that the present review snows that a wide range of tractable approximation methods exist for describing spacc-time neutron veharior. They can all be viewed as the consequences of certain selections

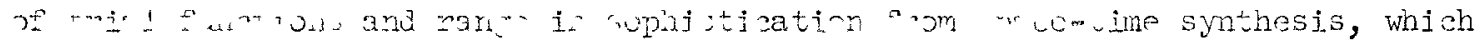

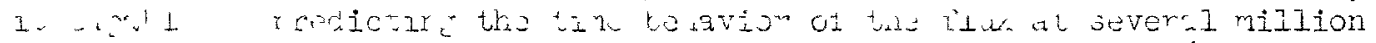

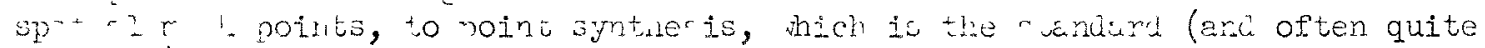
adequal bor.hor, of reactor knnetics. Furthermore, jetailed exact solutions of tire spaze-ime difference equations are toda $J$ positile for mall sized proulems, and these have been used to validate the accurcy of the various approximation methods.

The major effort for the next few years should I feel be one of gaining experience in the accuracy and adequacy of the many methods available and of developing further the associated numerical solution techniques and overall complter wravgig for hanaling large scale kinetics problems. Thus I think that fron a broad point of view matters are well in hand. However much work remilz in, ior.t. I cxpect a few surprises ill turn up as we proceed. 
1. K. F. Hansen and S. R. Johnson, GAKIN - A One Dimensional Multigroup Kinetics Code, GA-7543 (1967).

2. A. V. Vota, N. J. Curlee and A. F. Henry, WIGI3 - A Program for the SteadyState and Transient Solution of the One-Dimensional, Two-Group, Space-Time Diffusion Equations Accounting for Temperature, Xenon, and Control Feedback, WAPD-TM-788 (1969).

5. E. Richard Cohen, "Some Topics in Reactor Kinetics," 1958 Geneva Conference Paper A/Conf/5/P/629.

4. J. C. Vigel, Nucl. Sci. and Eng. 29, 392 (1967).

5. J. Mennle and T. Auerbach, Nucl. Sci. and Eng. 28, 159 (1967).

6. H. D. Brown, Nucl. Sci. and Ene. 2, 687 (1957).

7. W. R. Cadwell, A. F. Henry, A. J. Vigilotti, WIGIE - A Program for the Solution of the Two-Group Space-Time Diffusion Equations in Slab Geometry, WAPD-TM-4I6 (1964).

8. J. J. Kaganove, Numerical Solution of the One Group Space Independent Reactor Kinetics Equations for Neutron Density Given the Excess Reactivity,

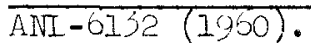

9. K. F. Hansen, B. V. Koen and W. W. Little, Nucl. Sci. and Eng. 22, 51 (1965).

10. T. A. Porsching, Nucl. Sci. and Eng. 25, 183 (1966).

11. S. Kaplan, O. J. Marlowe, J. Bewick, Nucl. Sci. and Eng. 18, I63 (1964).

12. L. R. Foulke and E. P. Gyftopoulos, Nucl. Sci. and Ene. 30, 419 (1967).

15. H. L. Garabedian, C. B. Leffert, Nucl. Sci. and Eng. 6, 26 (1959).

14. J. A. Bewick and S. Kaplan, A Test of the Time Synthesis Approach for the Solution of Reactor Kinetics Problems, WAPD-TM-64I (1966).

15. J. E. Yasinsky and S. Kaplan, Nucl. Sci. and EnE. 31, 337 (1968),

16. A. F. Herry, ruel. Sei. and Eng. 3, 22 (1958).

17. N. T. Surlee, Nucl. Sci. and Eng. 6, I (1959).

18. K. O. Ot and D. A. Meneley, Accuracy of the Quasistatic Treatment of Spatial Roactor Kinetics, Proceedings of the Brookhaven Conference on Indust. ial Needs and Academic Research in Reactor Kinetics, p.192, April 1968.

19. E. L. Nachspress, R. D. Eurgess and S. Baron, Nucl. Sci. and Eng. 12, 381 $(1907)$.

20. D. 2. To he hers and C. T. Shen, Wued. Sci. and Ene. 13, 141 (1962).

21. J. B. Yasinsky and S. Kaplan, Nucl. Sci. and Eng. 28, 426 (1967).

22. E. I. Wachspress, Nucl. Sci. and Eng. 26, 373 (1966). 


\section{TART - AN LMFBR TRANSIENT ANALYSIS PROJECT}

by

M. G. Stevenson and B. E. Bingham

Babcock and Wilcox

\section{Introduction}

The design and safety analysis of a large LMFBR requires detailed computational models and programs to describe the transient response of the reactor $c:=z^{\circ}$ olart to a variety of conditions. The purpose of the Babcock \& Wilcox

..... Than - Reactor Trasients) projoct is to develop digital confuter methods which will provide a means to analyze the dymamic characterictirs of +3 rge LMFBRs as accurately as necessary at a minimum computational cost.

Both point kinerics and one-dimension multigroup diffusion neutronics models are available as separate versions of the TART program. The point or

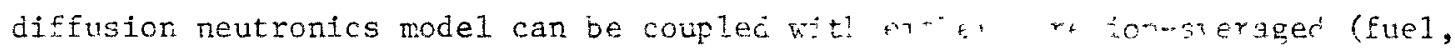
clad, and coolant nodes) or detailed (two-dimension multinode) thermalhydraulics solution fer an average fuel pin in each thermal-hydraulics region. A primary coolant model corresponding to the pool primary system used in the Babcock and Wilcox 1000 MWe LMFBR reference design ${ }^{1}$ may be included with either chermal-hydraulics solution, and the actions of the reactor control and safety svatir. can be simulated at the user's oftion. The time steps used in the thermi- nglraulics solution was be an integral number of neutronics solution tine steps with each selected according to preset error criteria. A block flow diagram of the TART program is shown in Figure 1 .

\section{Neutronics Solutions}

The neutronics solutions in TART are based on the exponential extrapolation methods developed under the supervision of $\mathrm{k}$. Hansen. Either the point $^{2}$ or diffusion ${ }^{3}$ method yields a comparatively fast solution and is numerically stable for any time step size. Up to six neutron energy groups are allowed in the diffusion version of TART, and six delayed neutron groups may be used in either version. 
Figure 1. TART Flow Chart

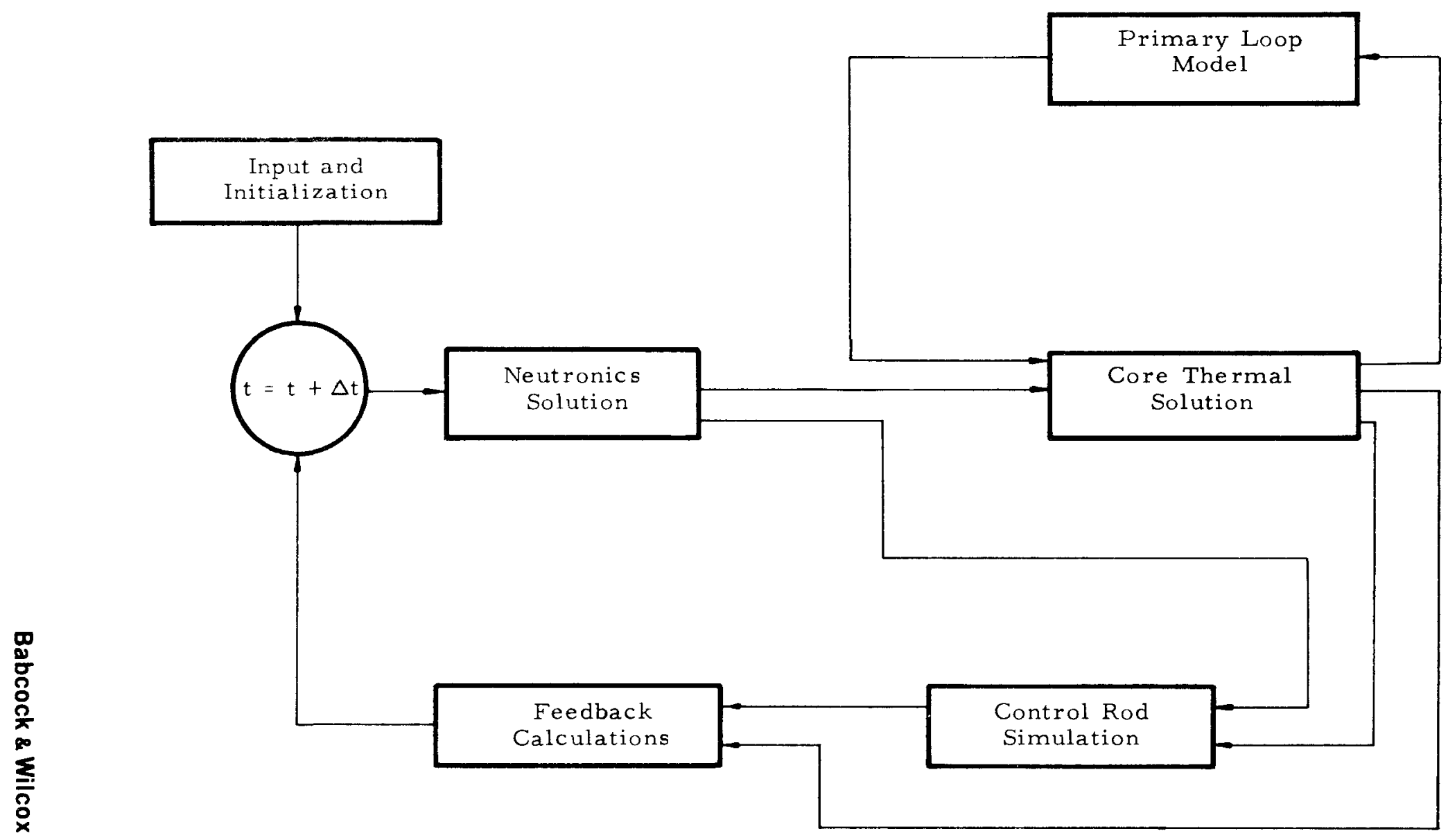


Both methods are based on approximating the time-dependent behavior of the neutron density (or group fluxes) and the precursor concentrations by an exponential over each time step. The set of ordinary differential equations corresponding to the point model or to the spatial-differenced diffusion equations is then integrated over the time step using this approximation. The resulting set of algebraic difference equations can be solved explicitly in the point kinetics case and can be solved through the inversion of tridiagonal matrices in the multigroup diffusion case. It should be emphasized that because the matrices to be inverted remain tridiagonal regardless of the number of neutron energy groups, the method is not penalized excessively as the number of groups is increased.

To summarize the basis of the neutronics method for the one-dimension diffusion case as given by Andrews and Hansen in reference (3), the set of ordinary differential equations resulting from a conventional spatialdifferencing is written as

$$
\frac{3}{d i}-i v
$$

whene th. vector $\psi$ contains the multigroup flux and precursor concentrations at each mesh point. The matrix $A$ is factored as

$$
A=\Gamma+L+U+H
$$

where $L$ and $U$ are strictly block lover and upp- :-iar $u^{-2} \cdots$, rospectively, and $\Gamma$ and $H$ are block tridiagonal. For the flux variables the submatrices $\mathrm{H}_{\mathrm{g}}$ and $\Gamma_{\mathrm{g}}$ are defined by

$$
H_{g}=v_{g} \frac{\delta D \delta}{h_{x}^{2}}
$$

and

$$
\Gamma_{\varepsilon}=v_{g}\left(x_{g} \nu \Sigma_{g}^{f}-\Sigma_{g}^{t}\right)
$$

where $\frac{\delta D \delta}{h_{x}^{2}}$ represents the difference form of the diffusion term.

It should be noted that all submatrices are diagonal except the $\mathrm{H}_{\mathrm{g}}$ which are tridiagonal.

If equation ( $I$ ) is then written as

$$
\frac{d \psi}{d t}-\Gamma \psi=(L+U+H) \psi
$$


and integrated over the time interval $h=t_{j+1}-t_{j}$, assuming that the matrix elements are constant over $h$, then the result can be written as

$$
\begin{aligned}
\psi_{j+1}= & \exp (\Gamma h) \psi_{j}+\int_{0}^{h} \mathrm{~d} \xi \exp [\Gamma(h-\xi)](L+U) \psi\left(t_{j}+\xi\right) \\
& +\int_{0}^{h} \mathrm{~d} \xi \exp [\Gamma(h-\xi)] H \psi\left(t_{j}+\xi\right)
\end{aligned}
$$

If $\psi\left(t_{j}+\xi\right)$ in the first integral is replaced by

$$
\psi\left(t_{j}+\xi\right)=\exp (\omega \xi) \psi_{j}
$$

and in the second integral by

$$
\psi\left(t_{j}+\xi\right)=\exp [-\omega(h-\xi)] \psi_{j+1}
$$

where $\omega$ is an extrapolation parameter, then the integrals can be evaluated. The flux vector at time $t_{j+1}$ is then given by the solution to

$$
\begin{gathered}
{\left[I-(\omega I-\Gamma)^{-1}\{I-\exp [(\Gamma-\omega I) h]\} H\right] \psi_{j+1}=[\exp (\Gamma h)} \\
\left.+(\omega I-\Gamma)^{-1}\{\exp (\omega h I)+\exp (\Gamma h)\}(L+U)\right] \psi_{j}
\end{gathered}
$$

It should be noted that the matrix on the left side of (9) is block tridiagonal and may be easily inverted. The resulting solution method can be shown to be numerically stable and to yield the asymptotic solution for the case of a step change in the properties of the system. The extrapolation parameter $\omega$ as used in the TART program is calculated at time $t_{j}$ by

$$
\omega_{j}=\left[\ln \left(\psi_{j}^{i} / \psi_{j-1}^{i}\right)\right] / h_{j-1}
$$

where the superscript $i$ represents a particular variable in the vector $\psi$.

\section{Thermal-hydraulics Models}

Each of the two thermal-hydraulics models used in TART is based on a thermal analysis of an average fuel pin in each thermal-hydraulics region. The first of these models, the "region-averaged" model, considers a single fuel node, clad node, and coolant node in each region. The equations describing the average temperatures corresponding to these nodes are for each region 


$$
\begin{aligned}
& (\overline{\rho C V})_{f} \frac{\bar{d}_{f}}{d t}=-(\overline{U A})_{f}\left(\bar{T}_{f}-\bar{T}_{c I}\right)+r_{f}^{q} \\
& \overrightarrow{(\overline{\rho C V})}=1 \frac{d \bar{T}_{c}}{d t}=(\overline{\mathrm{UA}})_{f}\left(\overline{\mathrm{T}}_{f}-\overline{\mathrm{T}}_{c 1}\right)-(\overline{\mathrm{UA}})_{c}\left(\overline{\mathrm{T}}_{c 1}-\widehat{\mathrm{T}}_{c}\right)+r_{c 1} q \\
& \overline{(\overline{\rho C V})_{c}} \frac{\overline{d \bar{T}} c}{d t}=(\overline{\mathrm{UA}})_{c}\left(\overline{\mathrm{T}}_{c 1}-\overline{\mathrm{T}}_{c}\right)+W\left[\left(c_{c}^{\text {in }}+c_{c}^{\text {out }}\right) \mathrm{T}_{\text {in }}\right. \\
& \left.-2 c_{c}^{\text {out }} \overline{\mathrm{T}}_{c}\right]+\mathrm{r}_{c} \mathrm{q}
\end{aligned}
$$

where

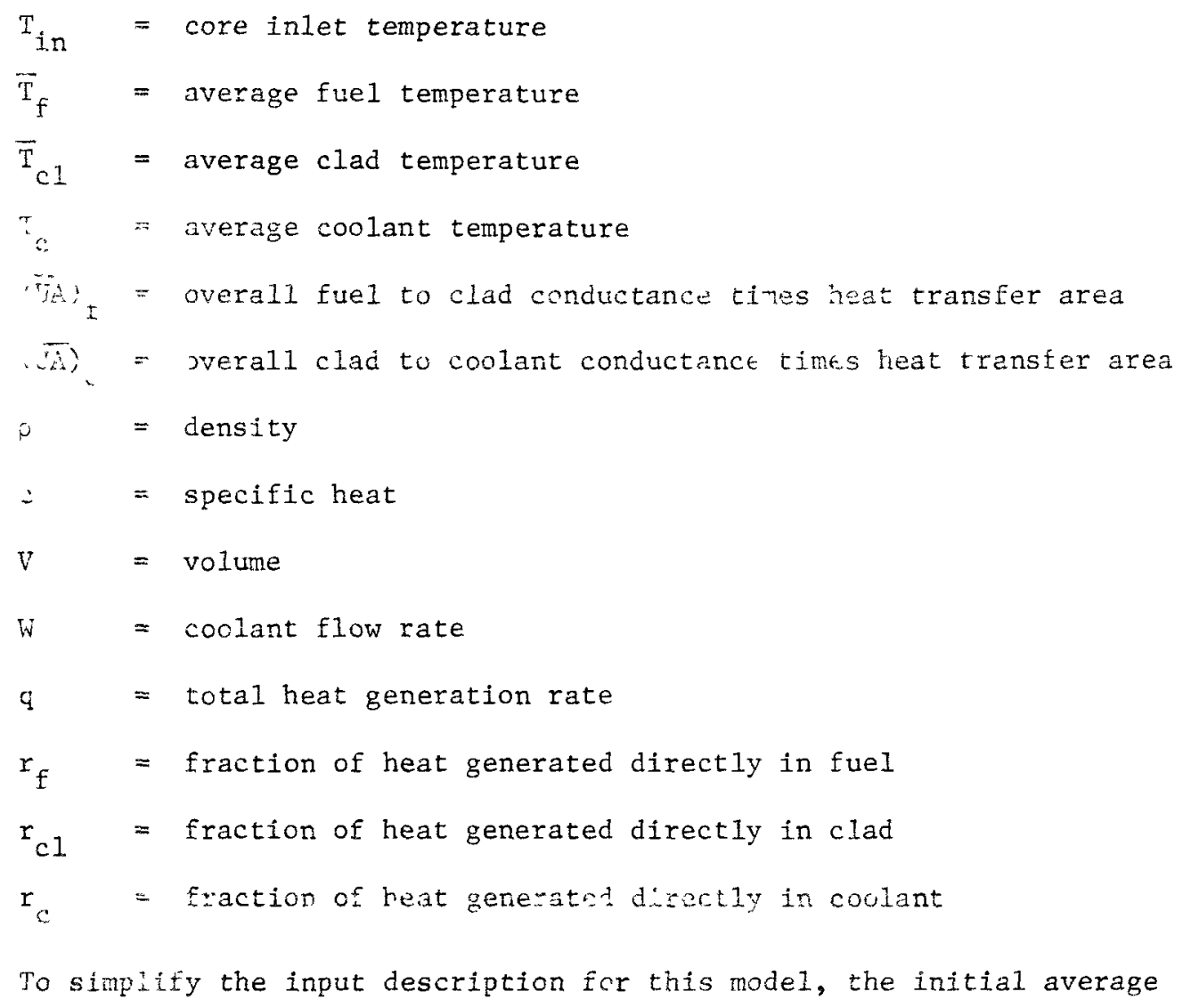
fuel, clad, and coolant temperatures are input for each thermal-hydraulics region, and the overall conductances and initial flow in each region are then calculated using these temperatures. These initial temperatures can be obtained from detailed steady-state calculations. Coolant properties are expressed in polynomial form and evaluated in each region during a transient, but input fuel and clad properties are assumed to remain constant throughout a transient. 
The detailed thermal model used in TART is based on the heat conduction equation in the fuel and clad, i.e.

$$
\rho c \frac{\partial T}{\partial t}=\nabla \cdot k \nabla T+q_{1}
$$

where $k$ is the thermal conductivity and $q_{1}$ is the volumetric heat generation rate. If axial heat conduction is neglected and azimuthal symmetry is assumed, this equation becomes in cylindrical geometry

$$
\rho c \frac{\partial T}{\partial t}=\frac{1}{r}\left[\frac{\partial}{\partial r}\left(r k \frac{\partial T}{\partial r}\right)\right]+q_{1}(r, z, t)
$$

Spatial difference equations are obtained from (15) by using Simpson's rule integration over a radial mesh interval. The thermal conductivity is evaluated at the midpoint between mesh points, and the heat generation rate, density, and specific heat are evaluated at the mesh point. The ordinary differential equation resulting from the spatial integration over the mesh interval about an interior mesh point $i$ is then

$$
\begin{aligned}
& \frac{1}{12}\left(1-\frac{\Delta r}{2 r_{i}}\right)(\rho c)_{i-1} \frac{\mathrm{dT}_{i-1}}{\mathrm{dt}}+\frac{5}{6}(\rho \mathrm{c})_{i} \frac{\mathrm{dT}_{i}}{\mathrm{dt}}+\frac{1}{12}\left(1+\frac{\Delta \mathrm{r}}{2 \mathrm{r}_{i}}\right)(\rho \mathrm{c})_{i+1} \frac{\mathrm{dT}_{i+1}}{\mathrm{dt}} \\
& =-\frac{\mathrm{k}_{i-1 / 2}}{(\Delta \mathrm{r})^{2}}\left(1-\frac{\Delta \mathrm{r}}{2 \mathrm{r}}\right)\left(\mathrm{T}_{i}-\mathrm{T}_{i-1}\right)+\frac{\mathrm{k}_{i+1 / 2}}{(\Delta \mathrm{r})^{2}}\left(1+\frac{\Delta \mathrm{r}}{2 \mathrm{r}}\right)\left(\mathrm{T}_{i+1}-\mathrm{T}_{i}\right) \\
& \quad+\mathrm{q}_{i}(\mathrm{t})
\end{aligned}
$$

The heat convection equation describing the temperature of the coolant is approximated by

$$
\rho c \frac{\partial T_{c}(z, t)}{\partial t}=-W \frac{\partial\left[c T_{c}(z, t)\right]}{\partial z}+q_{2}+q_{3}
$$

where $\mathrm{q}_{2}$ represents the heat source input to the axial segment from the clad and $\mathrm{q}_{3}$ represents the heat generated directly in the coolant. The coolant temperature is assumed to vary linearly within each axial segment and the resulting spatial difference equation for axial segment $m$ is

$$
\begin{aligned}
(\rho c)_{m} \frac{\mathrm{dT}}{\mathrm{dt}}= & -\mathrm{W}\left[2 c_{m+1 / 2} \mathrm{~T}_{\mathrm{m}}-\left(c_{m+1 / 2}+c_{m-1 / 2}\right) \mathrm{T}_{\mathrm{m}-1 / 2}\right] \\
& +\mathrm{q}_{2}+\mathrm{q}_{3}
\end{aligned}
$$


The sets of coupled ordinary differential equations resulting from the spatial differencing are integrated over a time step using trapezoidal integration, and the resulting algebraic equations are solved for each axial segrent $x y$ the inversion of a tridiagonal matrix. This solution technique is numerically stable for any time step size and has a low truncation error. ${ }^{4}$

Functicnal expressions are huilt into the program for the fuel density, specific heat, and thermal conductivity, for the coolant density and specific heat, and for the coolant-clad heat transfer coefficient. Injut information is used fcr the central void radius and for the fuel-clad gap size and ccnductare. Difeerent pin sizes nay be used th correspond to actual pin dimensions in the core and radial blanket. The initial steady-state solution is obtained by first inputting either the initial flow rate or initial coolant temperatuce rise in each themal-hydraulics region, and then calculating the temperatures in each region by an iterative technique.

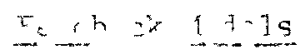

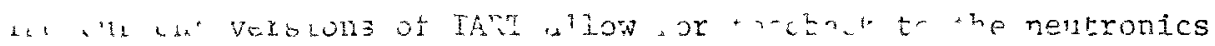

Jar.meter due to fuel temerature, coolant densits ard control rod bosition clinf s, he reautivity feedback for the point kinetics rodel is ssoume, fr be ot tot form

$$
\begin{aligned}
\delta p(t)= & \sum_{n}\left\{\alpha W_{n}^{T} \ln \left[\bar{T}_{n}^{F}(t) / \bar{T}_{n}^{f}(0)\right]\right. \\
& +W_{n}^{F} \underset{m}{F} \alpha_{m}^{p}\left[\left(P_{n m}(t)-P_{n m}(0)\right) / P_{n i n}(0)\right] \\
& \left.+a_{n}^{R} f(R)\left[R_{n}(t)-R_{n}(0)\right]\right\}
\end{aligned}
$$

$\overline{\mathrm{T}}_{\mathrm{n}}^{\mathrm{f}}$ is the weighted average fuel temperature in region $\mathrm{n}, \mathrm{P}$. is the coolant

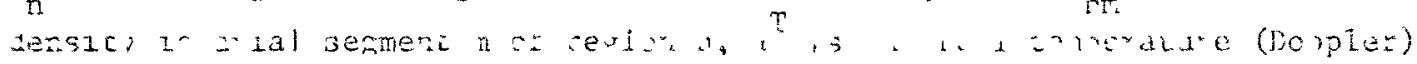
reactivity coefficient, $\alpha_{\text {in }}^{p}$ is the volare desito res trity cuerilcient in axial segment $m$, and $w_{n}^{T}$ and $w_{n}^{2}$ are radial vexghting coetficiencs. $R_{n}(t)$ represents the relative rod position (from $0 \ldots$ fin 1 ..t to 1 at full in), and $a_{n}^{R}$ represents the total reactivity difference between rods in and rods out in region $n$. The optional function $f(R)$ provides a means to approximate the usual S-shaped rod reactivity curve with an input table.

The feedback model for the space-dependent diffusion equation solution takog to from 


$$
\begin{aligned}
\delta \Sigma_{n}(t)= & \frac{\partial \Sigma_{n}}{\partial \bar{T}_{n}^{f}}\left[\bar{T}_{n}^{f}(t)-\bar{T}_{n}^{f}(0)\right]+\frac{\partial \Sigma_{n}}{\partial \vec{P}_{n}^{c}}\left[\bar{P}_{n}^{c}(t)-\bar{P}_{n}^{c}(0)\right] \\
& +\Delta \Sigma_{n} f(R)\left[R_{n}(t)-R_{n}(0)\right]
\end{aligned}
$$

for each of the group cross sections and

$$
\begin{aligned}
\delta\left[1 / D_{n}(t)\right]= & \frac{\partial\left(1 / D_{n}\right)}{\partial \bar{P}_{n}^{c}}\left[\bar{P}_{n}^{c}(t)-\widehat{P}_{n}^{c}(0)\right] \\
& +\Delta\left(1 / D_{n}\right) f(R)\left[R_{n}(t)-R_{n}(u)\right]
\end{aligned}
$$

for the group diffusion coefficients.

Primary Coolant Loop and Flow Coastdown Models

The primary coolant loop thermal model included in TART considers nodes in the core outlet plenum, in each IHX (Intermediate Heat Exchanger) prinary side, tube wall, and secondary side, and in the pot. The coolant flow oti of the core is assumed to mix and then to flow into the outlet plenum. The bulk reactor outlet temperature can then be determined by the energy balance

$$
T_{\text {out }}(t)=\frac{\sum_{n} c\left(T_{\text {out }}^{n}\right) T_{\text {out }}^{n}}{W_{T} c\left(T_{\text {out }}\right)}
$$

where $w_{n}$ is the coolint flow rate in thermai-hylraulic region $n$, $T^{n}$ out is the outlet temperature in region $n, c$ is the specific heat. $w_{T}$ is the totai coolant flow rate, and $T_{\text {out }}$ is the bulk outlet temperature.

The average temperature in the core outlet plenum is given bv

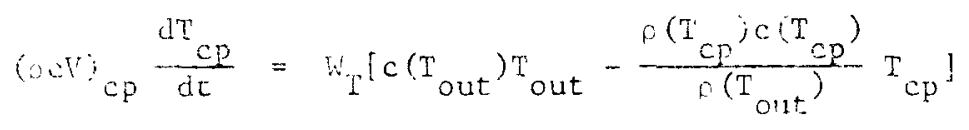

The primary coolant temperature is assumed to vary lineariy in the

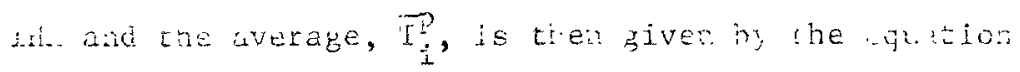

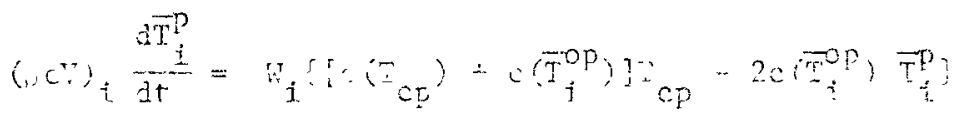

$$
\begin{aligned}
& -(\mathrm{UA}){ }_{i}^{\mathrm{F}}\left(\overrightarrow{\mathrm{T}_{i}^{\mathrm{p}}}-\overrightarrow{\mathrm{T}_{i}}\right)
\end{aligned}
$$


where $W_{i}$ is the coolant flow rate through each $I H X$, where $\vec{T}_{i}^{w}$ is the average tube wall temperature, $\overline{\mathrm{T}}_{i}^{O P}$ is the IHX primary coolant outlet temperature, and (UA) ${ }_{i}^{p}$ is the overall conductance from the primary coolant to the center of the tube wall.

The average tube wall temperature is given by

$$
(\rho \subset)_{\mathrm{W}} \frac{\mathrm{dT}_{i}^{\mathrm{W}}}{\mathrm{dt}}=(\mathrm{UA})_{i}^{\mathrm{P}}\left(\overline{\mathrm{T}}_{i}^{\mathrm{P}}-\overrightarrow{\mathrm{T}}_{i}^{\mathrm{W}}\right)-(\mathrm{UA})_{\dot{i}}^{\mathrm{S}}\left(\overline{\mathrm{T}}_{i}^{\mathrm{W}}-\overrightarrow{\mathrm{T}}_{i}^{\mathrm{S}}\right)
$$

where (UA) ${ }_{i}^{S}$ is the overall conductance from the center of the tube wall to the secondary coolant and $\overline{T_{i}}$ is the average secondary coolant temperature. $\overline{\mathrm{T}}_{i}^{\mathrm{S}}$ is given as a fixed input quantity or as an input time table.

The average temperature of the coolant in the pot (the core inlet temperature) is calculated from

$$
(\rho \mathrm{cV})_{p o t} \frac{\mathrm{dT}_{i n}}{\mathrm{dt}}=\sum_{i} \mathrm{~W}_{i} \mathrm{c}\left(\overline{\mathrm{T}}_{i}^{\mathrm{Op}}\right) \overline{\mathrm{T}}_{i}^{\mathrm{op}}-\rho\left(\mathrm{T}_{i n}\right) c\left(\mathrm{~T}_{i n}\right) \mathrm{T}_{i n} \sum \frac{\mathrm{W}_{i}}{c\left(\overline{\mathrm{T}}_{i}^{\mathrm{Op}}\right)}
$$

TART contains an option which allows the solution of the primary loop flow coastdown equations for the case of power loss to one or more primary pumps. A simplified primary loop flow model results in equations giving the rotational speed of each pump, the flow through each pump, the pressure rise across each pump, the gravity head across the IHXes, and the flow through each IHX. Time-dependent core flow rates may also be specified by the use of an input time table.

\section{Control System}

The control system incorporated into TART corresponds roughly to that in B\&W'S 1000 MWe LMFBR reference design. Both safety and shim-regulating control rods are included, and a rod of either type is allowed in any therma1hydraulics region. Two rod speeds are permitted, and a delay time is allowed between the scram command from a monitor and the beginning of rod insertion.

In general, the control system logic follows the pattern

(1) Monitor all necessary system variables.

(2) Determine correct mode of action according to preset conditions on the system variables.

(3) Follow this mode of action until all conditions for action are satisfied or until all rods are completely inserted. 
The monitors in the control and safety simulation include reactor power, instantaneous period, bulk outlet temperature, outlet temperature in each thermal-hydraulics region, and the power to flow ratio. The modes of action are normal control, fast setback, and scram, and the limit conditions for each mode are set by user input.

\section{Application}

Both the point kinetics and one-dimension diffusion TART programs have been used to calculate transients due to flow coastdown and ramp reactivity insertions. The reactor model used corresponds to the Babcock and Wilcox 1000 MWe LMFBR reference design. In these analyses the reactor was divided into six radial zones (three core zones, two blanket zones, and a reflector). Six neutron energy groups were used in the space-dependent diffusion calculations. The changes in the six-group neutronic parameters due to changes in fuel temperature and coolant density were calculated using 18-group $B \& W$ modified SIZZLE ${ }^{5}$ criticality calculations for different fuel temperatures and sodium densities and then collapsing to six groups to evaluate the changes in neutronic parameters. The reactivity coefficients for the point model were then calculated by the PERT code ${ }^{6}$ using the same six-group changes. The identical thermal model was used in the point kinetics and diffusion calculations, with six thermal-hydraulics regions corresponding to the six core zones being used. The actual core and blanket pin sizes were used, and 9 axial segments were used in each region with each segment consisting of a radial mesh of is nodes.

The point kinetics version was compared with the FORE-II code ${ }^{7}$ for the flow coastdown problem with the results agreeing within a few percent. The point TART took approxinately 15 seconds of central processor time on the CDC 6600, while for the same problem the FORE-II code required about 150 seconds.

A \$15isecond reactivity insertion was used to initiate a fast transient calculated rith ro foint TART. An equivalent reactivity ramp insertion was used with the space-cependent TART for two comparison cases. The first of the latter two cases was initiated by a spatially-uniform linear increase in the fission cross sections, while the second was initiated by a linear increase in the fission cross sections of the central core zone only. The normalized tire-dependent power for these three cases is shown in Figure 2. The rather significant difference between the point kinetics and 6-group diffusior results can be attributed primarily to the effective increase in reactivity (greater than $\$ 15 / \mathrm{sec}$ ) in the diffusion cases. 
Figure 2. Normalized Power ( $\$ 15 / \mathrm{sec}$ ramp)

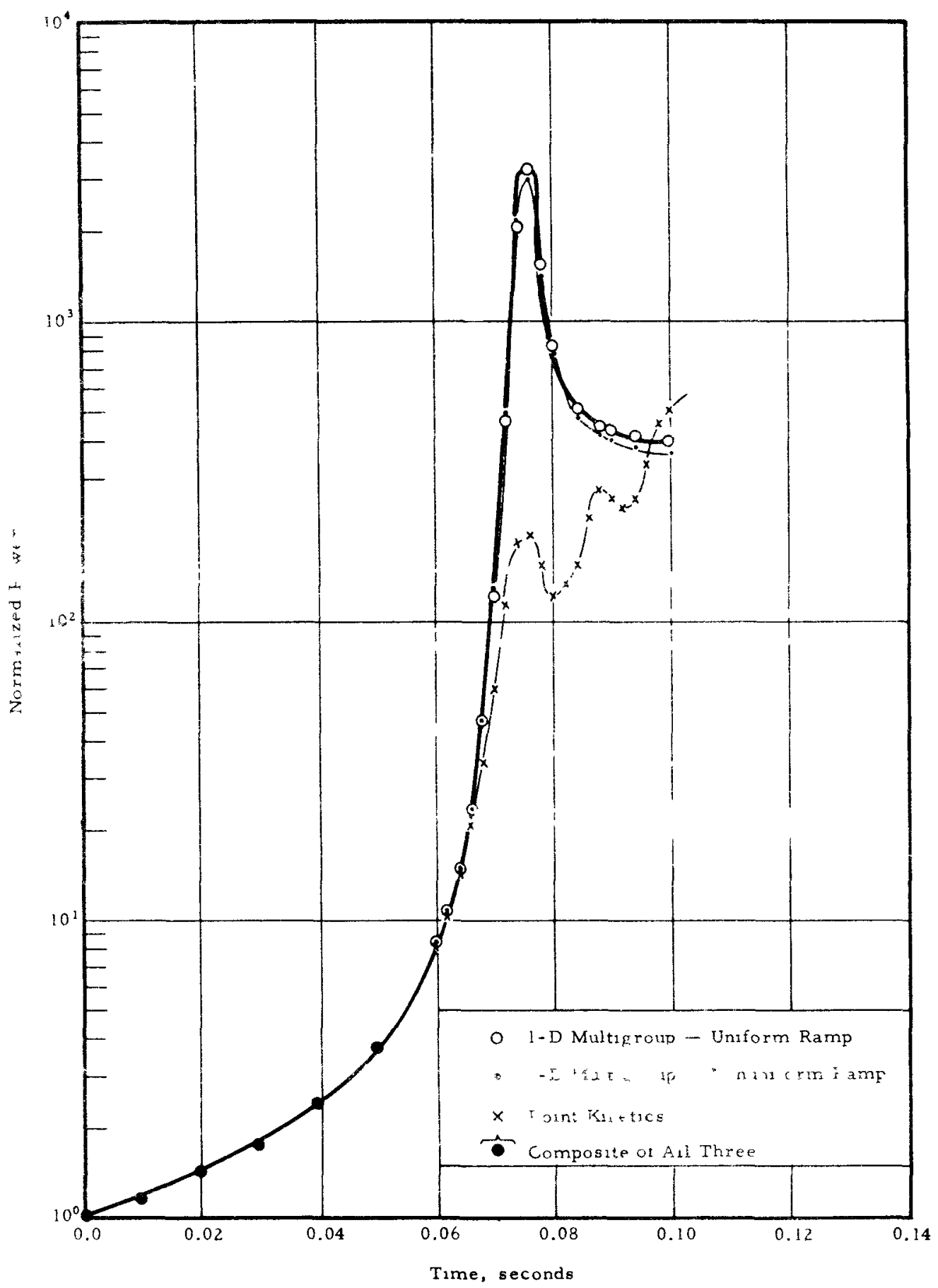

Babcock \& Wilcox 
To indicate the magnitude of the spectral and spatial changes occurring during the transient, Figure 3 shows the normalized amplitudes of groups 1 and 6 at the center of the core and of group. 1 at the center of the radial blanket for the transient due to the nonuniform perturbation. As might be expected, the spatial shift, although small, is greater in the first part of the transient while the spectral shift is greater in the latter part since the fuel temperature changes cause spectrally nonuniform changes in cross sections.

The point kinetics calculation required slightly over 3 minutes of CDC 6600 central processor time, taking about 1900 neutronics time steps and 700 thermal time steps. The diffusion calculations required about 10 minutes of central processor time, taking 2000 neutronics time steps and about 800 thermal time steps.

Sumnary

The TART programs form a flexible and comprehensive system for use in LMFBR transient and safety analysis studies and are relatively economical to use. The modular design of the TART system simplifies further extensions such as those involving two-dimensional neutronics methods, sodium boiling models, and thermal stress models. The availability of the different neutronics and thermal-hydraulic models and options in TART allows the reactor designer or safety analyst to obtain a calculational accuracy which is consistent with the nature of a particular problem and with the uncertainties which may be involved either in the statement of the problem or in the input parameters. In addition, the use of either point or multigroup diffusion kinetics and either region-averaged or detailed thermal-hydraulics calculations within a single comprehensive system permits a consistent analysis of the range of applicability for the simpler models. 
Eigure 3. Flux Amplitude Factors (nonuniform ramp)

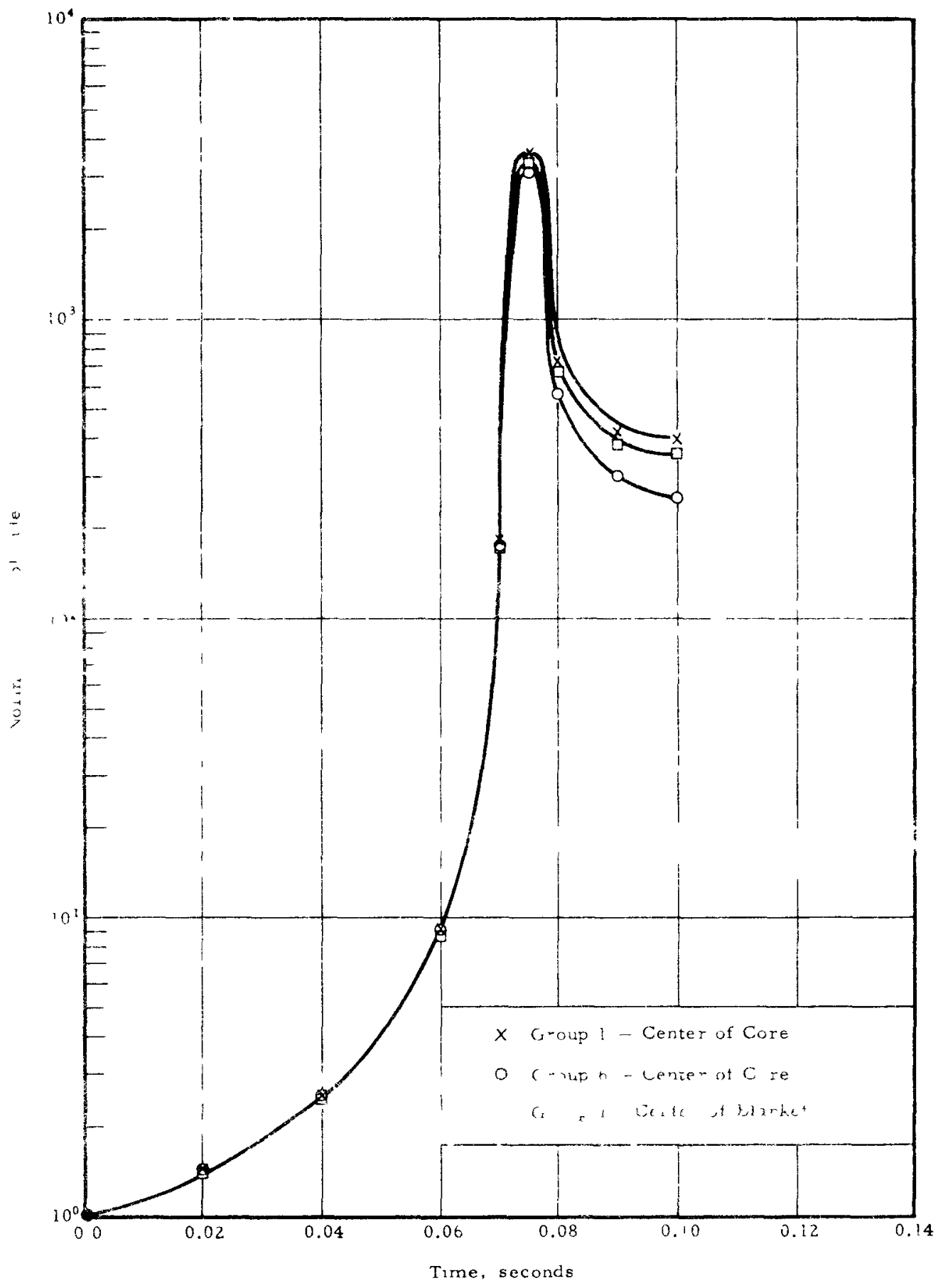

Babcock \& Wilcox 
1. 1000 MWe LMFBR Follow-On Study, Babcock \& Wilcox Co., Nuclear Power Generation Department, BAW-1328 (1968).

2. K. F. Hansen, B. V. Koen, and W. W. Little, "Stable Numerica1. Solurions of the Reactor Kinetics Equations." Nucl. Sci. Engr., 22, 51-59 (1965).

3. J. F. Andrews, II and K. F. Hansen, "Numerical Solution of the TimeDependent Multigroup Diffusion Equations," Nuc1. Sci. Engr., 31, 304-313 (1968).

4. G. Birkhoff and T. F. Kimes, CHIC Programs for Thermal Transients, WAPD-TM-245 (1962).

5. R. J. Neuhold, D. H. Roy, and N. C. Thomas, A Computer Package for Fast Reactor Design Calculations, BAW-TM-187 (1967).

6. H. P. Flatt, PERT: A Perturbation Theory Code, NAA Code Report (1961).

7. J. N. Fox, B. E. Lawler, and H. R. Butz, FORE-II, A Computational Prngram for the Analysis of Steady-State and Transient Reactor Performance, GEAP-5273 (1966). 


\title{
COUPLING OF DYNAMICS CALCULATIONS IN THE FREADM CODE*
}

\author{
D. D. Freeman
}

General Electric Company

Nuclear Energy Division.

Breeder Reactor Development Operation

A paper to be presented at the ANS Topical Meeting on Effective Use of Computers in the Nuclear Industry, April 21-23, 1969.

* Work performed for the USAEC under contract AT(04-3)-189, PA-10, Fast Ceramic Reactor Development Program. 


\section{SUMMARY AND CONCLUSIONS}

The FREADM code is being developed to provide sensitivity information pertinant to fast reactor safety analysis. Simultaneous monitoring of temperatures in several core locations is required to follow the course of an accident as it proceeds from its initiation to its conclusion.

The time step control developed for the code reduces the number of relatively costly heat transfer time steps to one-half to one-tenth the number of nuclear kinetics time steps for a range of transients of interest with little or no sacrifice in accuracy of the integrated power. The cost of performing the many reactor dynamics calculations required in sensitivity studies is expected to be substantially reduced through the use of the time step control. 


\section{INTRODUCTION}

Self-destructive nuclear transients are of great interest in the safety design of fast reactors. Chief concerns are with:

1. Dosigning to eliminate or minimize the effects of their occurance.

2. [nstrumentation to detect and check their initiation.

3. Containment to protect the environment.

Loc:. voluing and fuel compaction have been identjfied as potential sources cf large reactivity insertions in sodium-cooled $f$ ast power reactors? High rates of reactivity insertion have been postulated which lead to explusive lisassembly of the core? A prompt negative Doppler coefficient of reictint has been shown to substantialiy reduce the magnitud of the energy release for assumed rates of reactivity insertion and core configurations?

rovicus analysis has not considered in detal 1 the interaction of the valiols neanañsmo for reactivity feedback prior to disassembly. Information

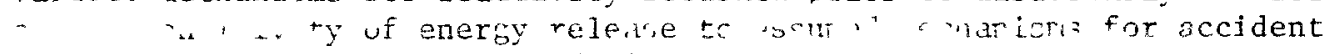
$-21^{+}+\omega^{2}$ lid propagation is desired to:

1. Jevelop safety related design criteria.

2. Eliminate some types of initiating events as leading to alsassembly.

3. Direct safety related experimental studies.

A rew Fast Reactor Excursion and Accident Dynamics Model, the FREADM code, is being developed to include models for nuclear kinetics, heat transfer, coolant flow and voiding, fuel redistribution, failure propagation and programmed reactivity insertion.

A unique feature of the FREADM code is the method used to couple the varzous dynamics models. Features of the method are: 
1. Each process is computed using a time step determined by the accuracy requirements for that process.

2. Calculations are straight ahead, i.e. once a successful time step is taken for a given process no readjustment is required.

3. Coincidence in time of the various calculations is assured by controlling the time step within the neutron kinetics calculation.

This paper summarizes the FREADM calculational model and presents results which demonstrate the effectiveness of the time step control. 


\section{CALCULATIONAL MODEL}

Accidents for which the FREADM code is designed may be characterized as initiating and propagating uniformly within annular or cylindrical core regions. Conceptually, the core is divided into a number of annular regions, each region consisting of one or more bundle types. Variables between bundle types include material properties, flow parameters, power peaking factors an 1 failure mechanisms.

Detailed temperature distributions are computed numerically for a single pin in each bundle type. Fuel failure and/or sodium voiding may then be triggered within a fuel bundle type by monitoring the temperature distributions for present trigger levels.

Initial values for temperature and flow variables for each bundle type are computed within the code assuming steady state conditions. Nuclear kinetics parameters are normalized to an initial steady state relative neutron density of 1.0. Transients may be initiated by perturbations in coolant flow and/or inlet temperature to one or more bundle types, or by programmed reactivity insertions.

" $x^{n} \geq$ ical methods used in the various dynamics models are based largely on previously used techniques. Integration of the nuclear kinetics equations fnlicr. garinily the method used in the FOR -." crrot. The implicit method is $45 t^{4}$ icx computing radial heat transfer with crout's method for reducing the coefricient matrix. Sodium flow and voiding calculations are an extension $0^{-}$ariler work by Meinhardt and others,"7 The reactivity effect of local voiding is computed using parabolic fits to static flux calculations.

Models for fuel failure and failure propagation developed for the code are simple to use and flexible enough to include a broad range of failure types. Four modes of fuel failure are available, ranging from complete collapse of one bundle section upon mother $t=f a t: m, f=s, c, l e$ axial node with interaction with flow, gravity and/or voiding aynawlcs. A key idea of the failure modes is that once failure is triggered all subsequent motion is determined either from user input or known conditions within the bundle.

Temperature dependent reactivity feedback is computed using local coefficients with fuel temperatures averaged over each axial fuel node. Doppler coefficients for bundle type are dependent on the condition of voiding within that bundle type. The temperature is computed assuming temperature-dependent fuel properties with constant vajues ir clad and coolant pr sperties and gap and fim coefficirnts y rur to thei fuilure or voiding Heat of fusion is included in the ralcu ation. 


\section{TRANSIENT LOGIC}

The transient logic has been arranged to minimize the number of heat transfer and voiding calculations required. Since these calculations are the most detailed, minimizing their number should tend towards minimum computation costs. This approach is motivated by the following considerations:

1. Sodium voiding is essentially independent of the neutron kinetics, and for cases of high superheat proceeds somewhat independently of heat transfer?

2. The detailed temperature distributions are not required for prompt reactivity feedback and need not be computed as often as reactivity requirements might dictate.

3. Point kinetics calculations are relatively inexpensive per time step.

The logic used is shown in Figure 1 .

The calculational procedure is as follows:

1. Compute coupled flow and sodium voiding for one time step. This may determine the maximum allowable neutronics time step.

2. Adjust reactivity for any new sodium voiding.

3. Compute relative power density (point kinetics). The time step is controlled by void time step, fractional power change, estimated fuel temperature and/or estimated reactivity change. Integrated power is used to estimate temperature changes required for feedback calculations. The power integrated since the last heat transfer calculation is used to determine whether a new heat transfer calculation is required.

4. Perform heat transfer calculations for those bundle types for which a calculation is required. 


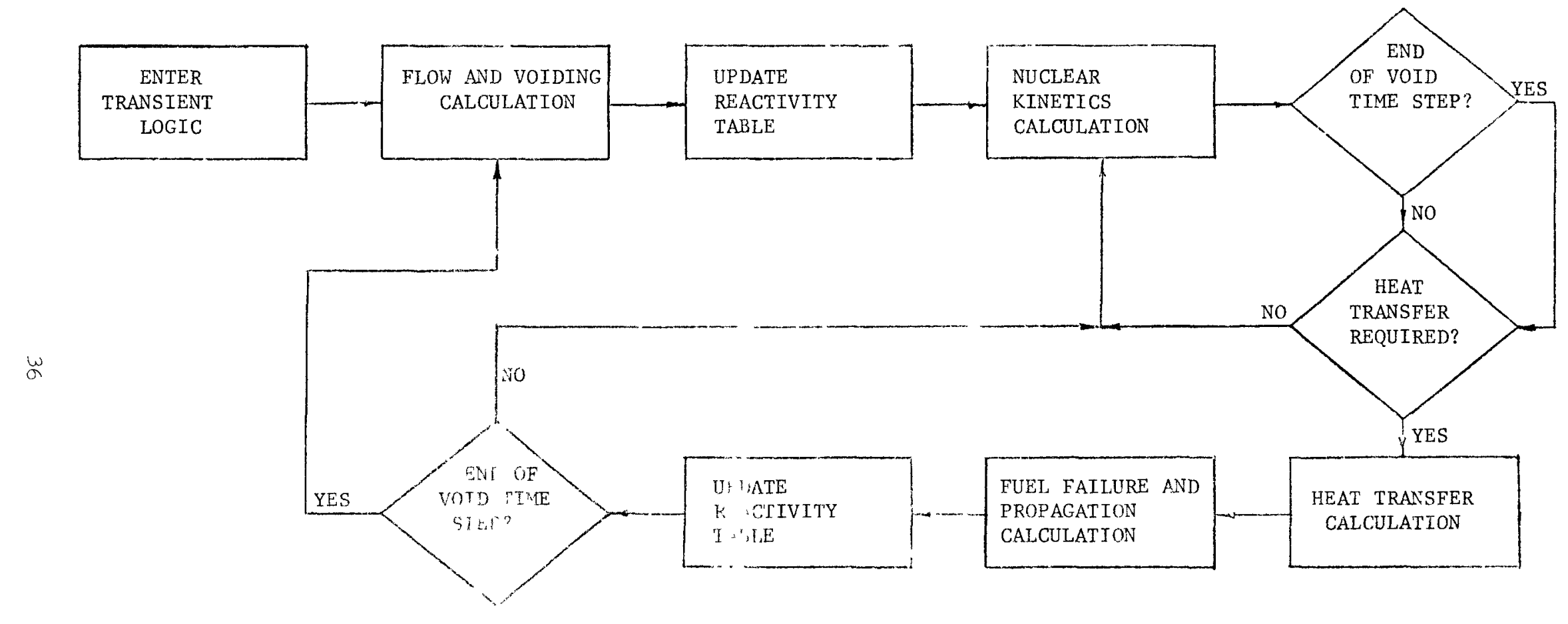

TRANSIENT CH UULATIUN LOGIC FOR FREADM

FIGURE 1 
5. Check temperatures for initiation of fuel redistribution and set appropriate flags.

6. Perform indicated fuel redistribution calculations.

7. Adjust reactivity and check elapsed time since last void calculation to determine whether the end of the void time step has been reached. If not, continue neutronics calculations. Otherwise return to void and flow calculation.

The time step control is within the neutronics calculation. Maximum values of reactivity change, average fuel temperature change by axial node and relative neutron density change per time step are input. The maximum starting time step is determined either by the void time step, or by reactivity considerations. A detailed heat transfer calculation is called for whenever the estimated average fuel temperature change in a node exceeds a value input by the user, or whenever the number of kinetics steps taken since the previous heat transfer calculation reaches a number specified by the user. 
Time step linitations due to accuracy requirements for prompt feedback in the nuclear kinetics are much more restrictive than time step limitations due to accuracy requirements for heat transfer calculations for a large number of cases. A method was developed for computing temperature dependent prompt feedback between detailed calculations of the temperature profile. The method treats only the average fuel temperature of each axial node. Clad and coolant temperatures are eliminated as variables, but changes in the rate of heat transfer from the fuel are accounted for.

The average fuel temperature for each axial fuel node is estimated between heat transfer calculations as follows:

1. Compute spatial averages for the fuel properties conductivity, specific heat and thermal diffusivity.

2. Integration of the heat balance equation with constant coefficients over the node gives:

$$
\left(\overline{\nabla^{2} \mathrm{~T}}\right)=\frac{1}{\alpha} \frac{\partial \overline{\mathrm{T}}}{\partial \mathrm{t}}-\frac{\overline{\mathrm{Q}}}{\mathrm{k}}
$$

where:

$\alpha$ and $k$ axe average values of thermal diffusivity and conductivity,

$\vec{T}$ is the average fuel temperature for the axial node,

$\overline{0}$ is the average power density in the fuel,

$\left(\overline{\nabla^{2} T}\right)$ is the average value of the Iaplacian in the fuel

Estimate the Laplacian assuming $\frac{\partial T}{\partial t}=\frac{\delta T}{\delta t}$ for the last time step, and using $\bar{Q}$ at the end of the last stêp.

$$
\left(\overline{\nabla^{2} \mathrm{~T}}\right) \approx \frac{1}{\alpha} \frac{\delta \overline{\mathrm{T}}}{\delta \mathrm{t}}-\frac{\overline{\mathrm{Q}}}{\mathrm{k}}
$$


3. Integrate the heat balance over one time step assuming that material properties and $\left(\overline{\nabla^{2} \mathrm{~T}}\right)$ remain constant over the time step at their values at the beginning of the time step giving,

$$
\delta \bar{T}=\alpha\left[\left(\overline{\nabla^{2} T}\right) \delta t+\frac{1}{k} \int_{t_{1}}^{t_{2}} \bar{Q}(\tau) d \tau\right]
$$

The integral quantity is proportional to the integrated power over the time step given by the point kinetics model. The time derivative and the fuel temperature are renormalized each time a detailed heat transfer calculation is performed.

Note that the change in fuel average temperature has been computed independently of instantaneous clad or coolant temperatures. Starting valies of the temperature and Laplacian are easily obtained from steady state considerations.

Heat transfer calculations for a single axial fuel node were coupled to the point kinetics model to test the time step control and demonstrate its effectiveness in reducing computation costs. Three series of cases were run with combinations of reactivity input, Doppler feedback and time step controls as shown in Table 1. Also shown in Table 1 is the ratio of nuclear kinatics time steps to heat transfer time steps actually takell for each case.

Time step controls which were input included:

El - The maximum allowable fractional change in power over a kinetics time step.

E2 - The maximum allowable change in average fuel temperature over a kinetic tine step.

E3 - The maximum allowable change in reactivity over a kinetics time step.

TMAX - The minimum change in average fuel temperawure between calculations of the radial temperature profile.

JiKS - The maximum number of nuclear kinetics time steps per heat transfer calculation.

Values of $F 1, F 2$, and $\mathrm{E} 3$ were $2.0,50.0$, and 0.1 , respectively, for all cases. In in thum kinerics time orep taken was 0.01 sec. The time step was halvic incnever criterua set by vaiues of $E 1, \mathrm{E} 2$, or E3 were not satinfied and the salculation repeated. The time scep was reset to 0.01 at the beginning of each timester to simulate ar upper limit imposed by vojdine ne nther reativitu-tire intervals. 
STMMARY OF TEST CASE PARAMETERS

Series 1 - Step Bf sctivity Insertion, No Feedback

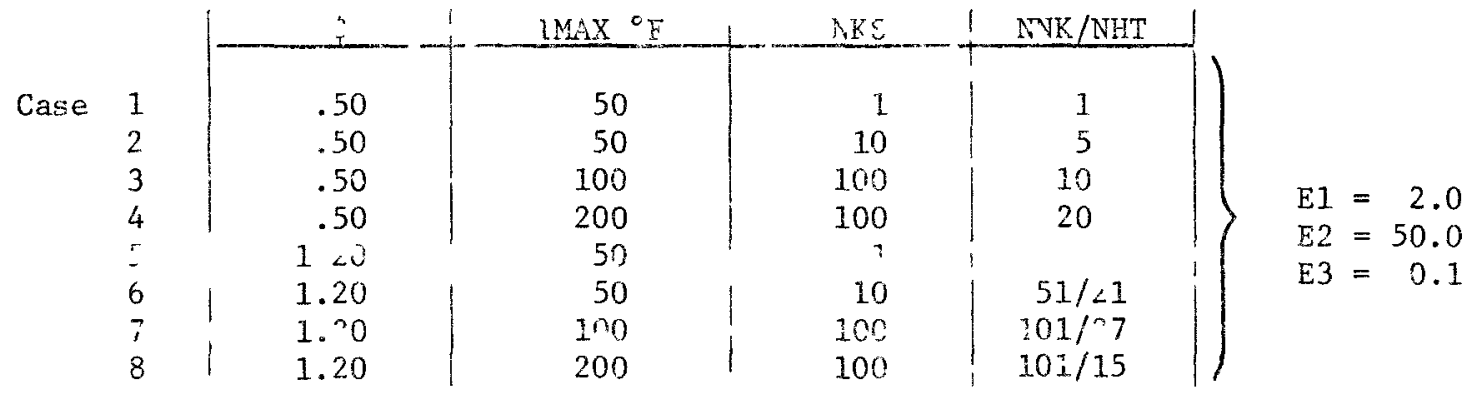

Ser土es 2 - Stap Reactivity Insertion, $T \mathrm{dk} / \mathrm{dT}=-.006$

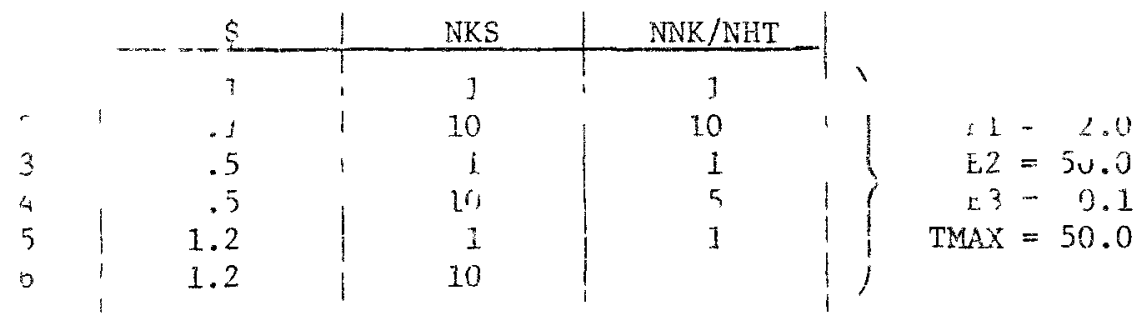

Sexies 3 - Ramp Reactivity Incertion of $\$ 320 \mathrm{~T}$ dk! $2 \mathrm{~T}=-306$

\begin{tabular}{|c|c|c|c|c|c|}
\hline & S/sec & $I_{M A X}^{\circ} \mathrm{F}$ & NKS & 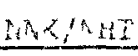 & \\
\hline Case & 1 & 50 & 1 & 1 & \\
\hline 2 & 1 & 50 & 10 & & \\
\hline 3 & 1 & 10000 & 100 & 100 & $E 1=2.0$ \\
\hline 4 & 10 & 50 & 1 & 1 & $E 2=50.0$ \\
\hline 5 & 10 & 50 & 10 & & $\mathrm{E} 3=0.1$ \\
\hline 6 & 10 & 10000 & 100 & 100 & \\
\hline
\end{tabular}

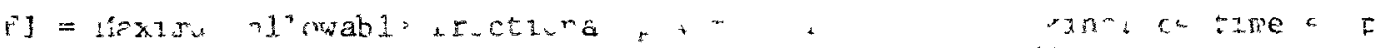

$\mathrm{E} 2$ = Maximum aliowable nudal fue: temptra ure sncrease ( ${ }^{\circ} \mathrm{F}$ )

$\mathrm{E} 3$ = Maximum allowable reactivity change ( $\$$ )

TMAX = Minlnuir temperature change beiween heat Esansfe. cdiculations NKS $=$ Maximum number of kinetics steps per heat trarsfur step

$\mathrm{NNK} / \mathrm{NHT}=$ Average ratio of kinetics steps per heat transfer step 
A11 cases were initiated from steady state with a constant inlet coolant temperature of $700^{\circ} \mathrm{F}$ and a linear power of $11 \mathrm{kw} / \mathrm{ft}$ in a $0.25-$ inch o.D. mixed oxide fuel pin. Inlet coolant velocity was $10 \mathrm{ft} / \mathrm{sec}$ with a fuel pin pitch-to-diameter ratio of 1.20 . The length of the node was 3 inches. Hence, starting temperatures are characteristic of a sodium-cooled fast reactor at the core inlet.

The first series of cases is for step insertion of reactivity with no feedback. Step insertions of $\$ .50$ and $\$ 1.20$ were made with variations in TMAX and NKS as shown in Table 1 . The results of Series 1 are shown in Figures 2-5. The accuracy of the heat transfer model with large temperature changes $\left(>200^{\circ} \mathrm{F}\right)$ is shown in Figures 2 and 3 for sub-prompt critical $(\$ .50)$ and super-prompt critical $(\$ 1.20)$ excursions. The average fuel temperature for cases in which heat transfer calculations were performed after 50,100 and $200^{\circ} \mathrm{F}$ temperature changes (TMAX $\left.=50,100, \& 200\right)$ are compared with a case in which heat transfer calculations are performed each kinetics time step (NKS $=1$ ).

Values of average fuel temperature computed (by the approximation) just prior to a new heat transfer calculation are compared for TMAX of 50, 100, and 200 with values obtained with NKS of 1 in Figures 4 and 5 . The ability of the average temperature approximation to follow slow and fast transients is shown in these curves.

Series 2 is a set of problems with step insertions of reactivity and a Doppler coefficient of reactivity $(\mathrm{T} \mathrm{dk} / \mathrm{dT})$ of -.006 . Two sub-prompt critical reactivity steps $(\$ 0.1, \$ 0.5)$ and one super-prompt critical reactivity step $(\$ 1.20)$ were considered. Comparisons were made between cases with values of NKS of 1 and 10 for each step insertion considered. Figures 6,7 and 8 show the transient power and temperature plotted as lines for NKS $=1$ and as points for NKS $=10$. The value of TMAX is 50 for all cases. The agreement in power and average fuel temperature between cases with NKS $=1$ and NKS $=10$ demonstrates that prompt reactivity feedback effects are adequately handled lising the approximation for a wide range of transient conditions.

Ramp rates of roactivity insertion were considered in Series 3 . A Doppler coefficient $(\mathrm{T} \mathrm{dk} / \mathrm{dT}$ ) of -.006 was assumed with insertions of $\$ 1.20$ at $\$ 1 / \mathrm{sec}$ and $\$ 13 / \mathrm{sec}$. Figures 9 and 10 show the transient power and average fuel temperature as a line for NKS $=1$ and as points for NKS $=10$. The value of TMAX was set at $50^{\prime} \mathrm{F}$ in these calculations. Again, prompt reactivity feedback effects are adequately handled without recomputing the temperature distribution each kinetics time step. 
FISIRE 2

TEMPERATURE VS. TIME

$50 \mathrm{C}$ STEF, NO FEEDBACK

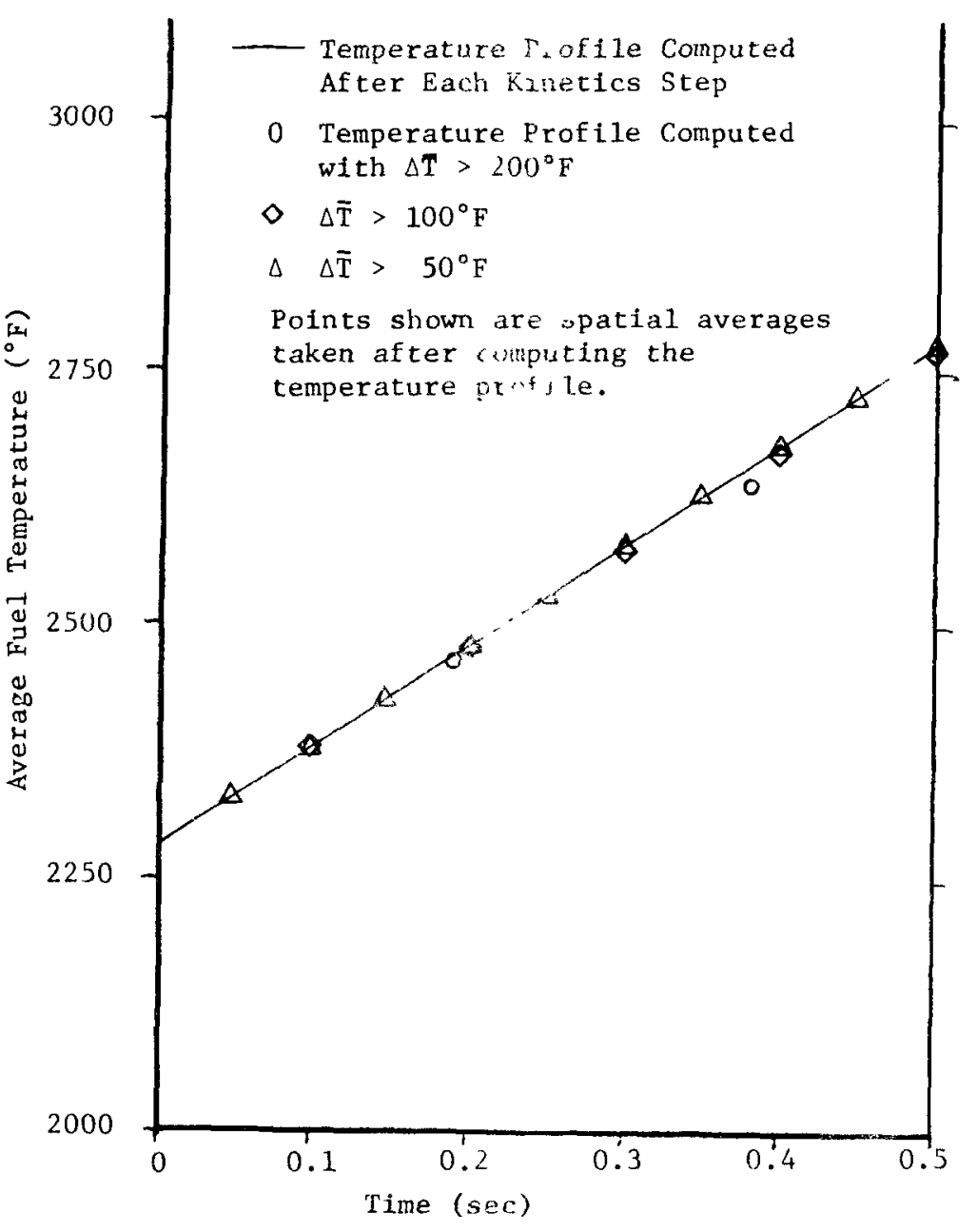

FIGURE 3

TEMPERATURE VS. TIME

$\$ 1.20$ STEP, NO FEEDBACK

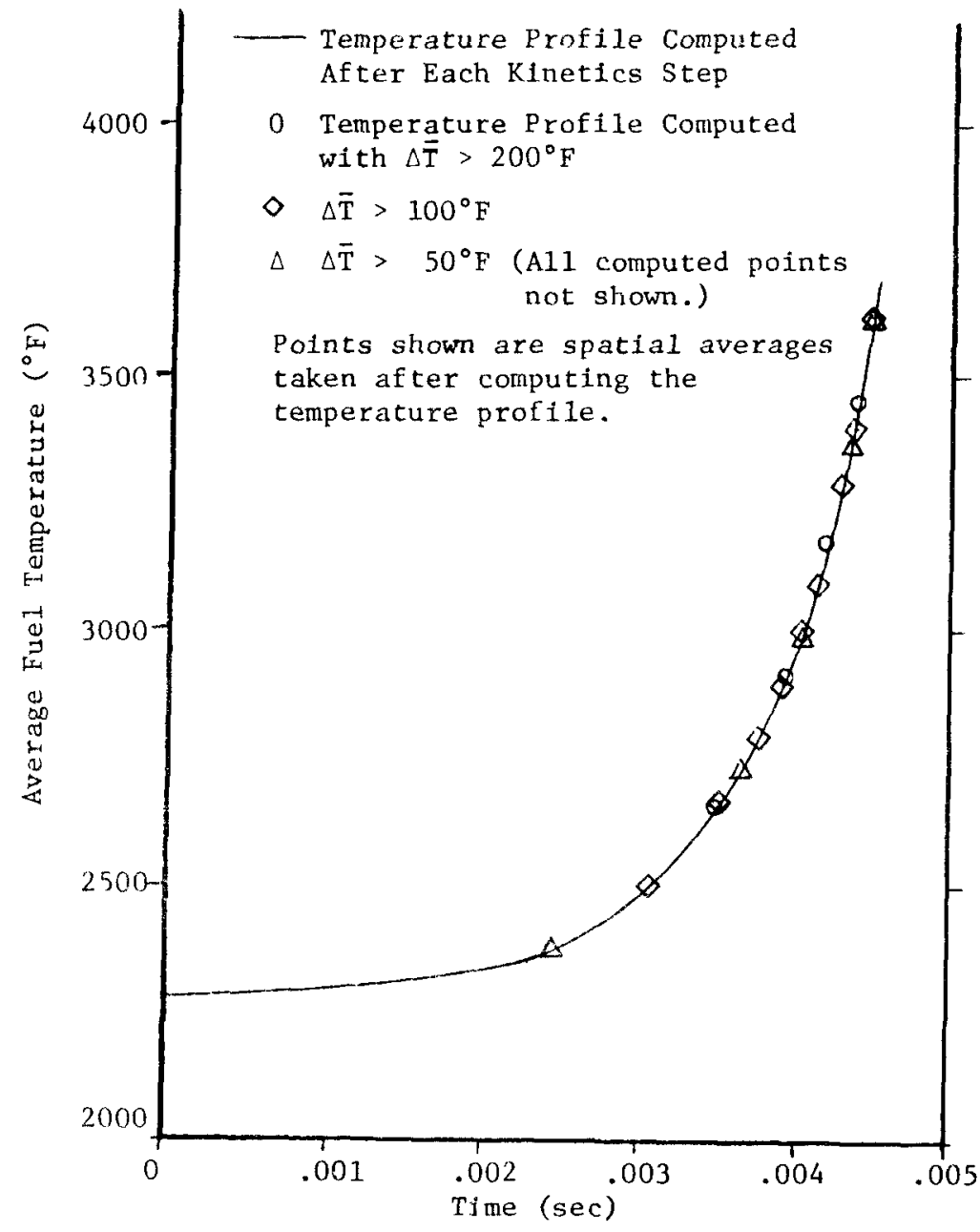


FIGURE 4

ESTIMATED TEMPERATURE VS. TIME

\section{OC STEP, NO FEEDBACK}

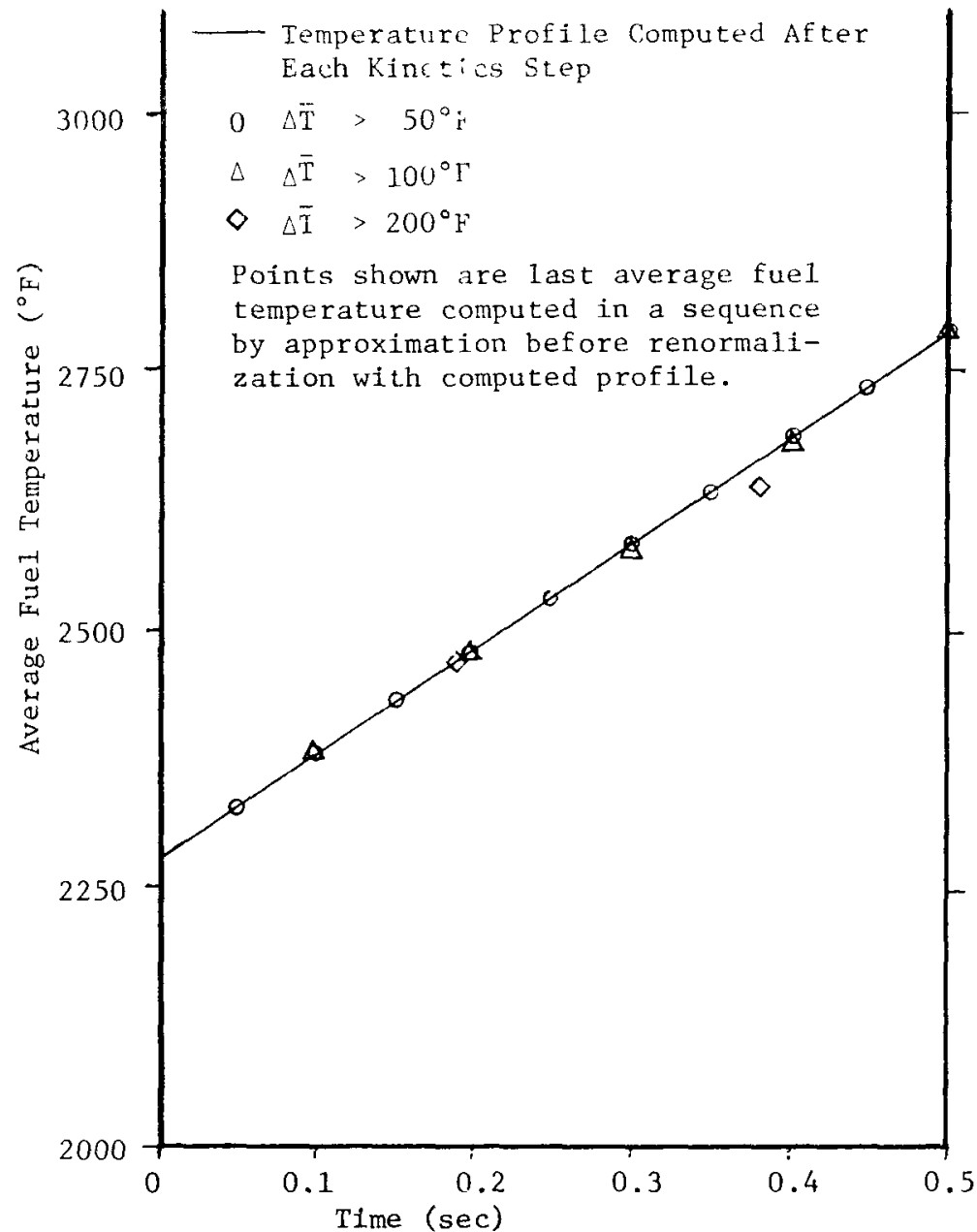

FIGURE 5

ESTIMATED TEMPERATURE VS. TIME

\section{$\$ 1.20$ STEP, NO FEEDBACK}

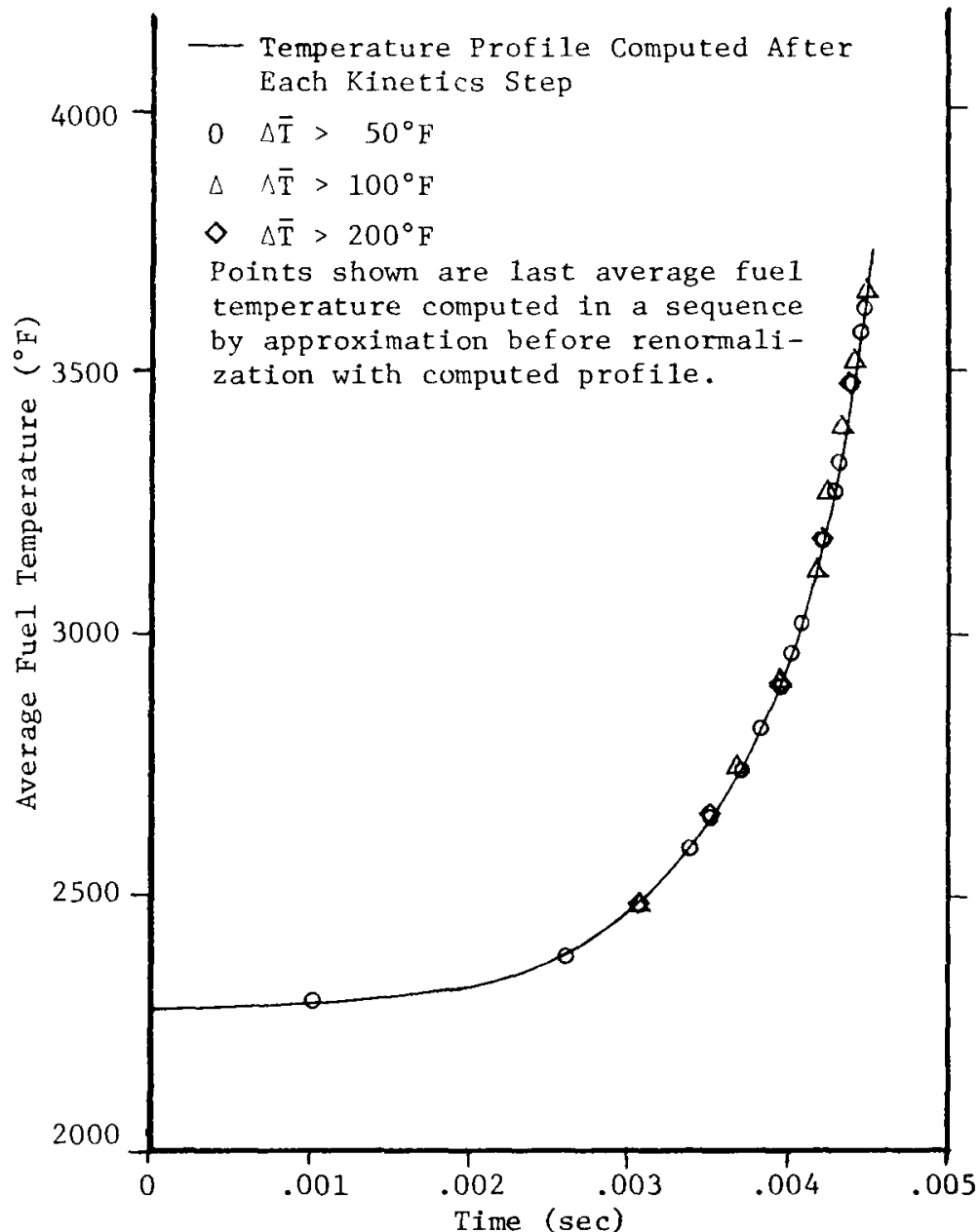


FIGURE 6

RELATIVE POWER AND TEMPERATURE

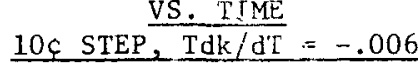

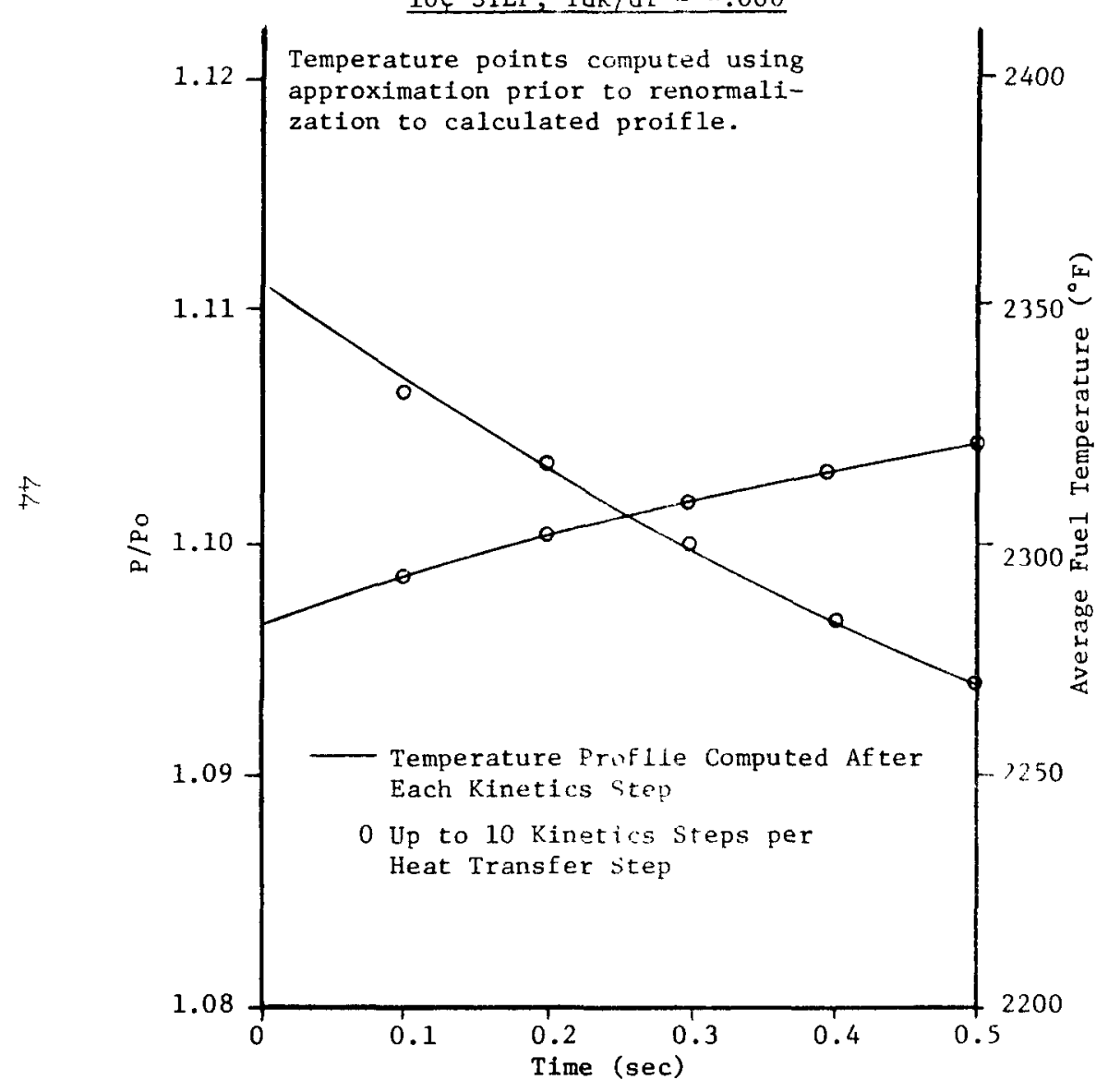

FIGURE 7

RELATIVE POWER AND TEMPERATURE

$50 C \operatorname{STEP}, \frac{V \text {. TIME }}{\mathrm{Td} \mathrm{dT}}=-.006$

Temperature points computed using approximation prior to renormalization to calculated profile.

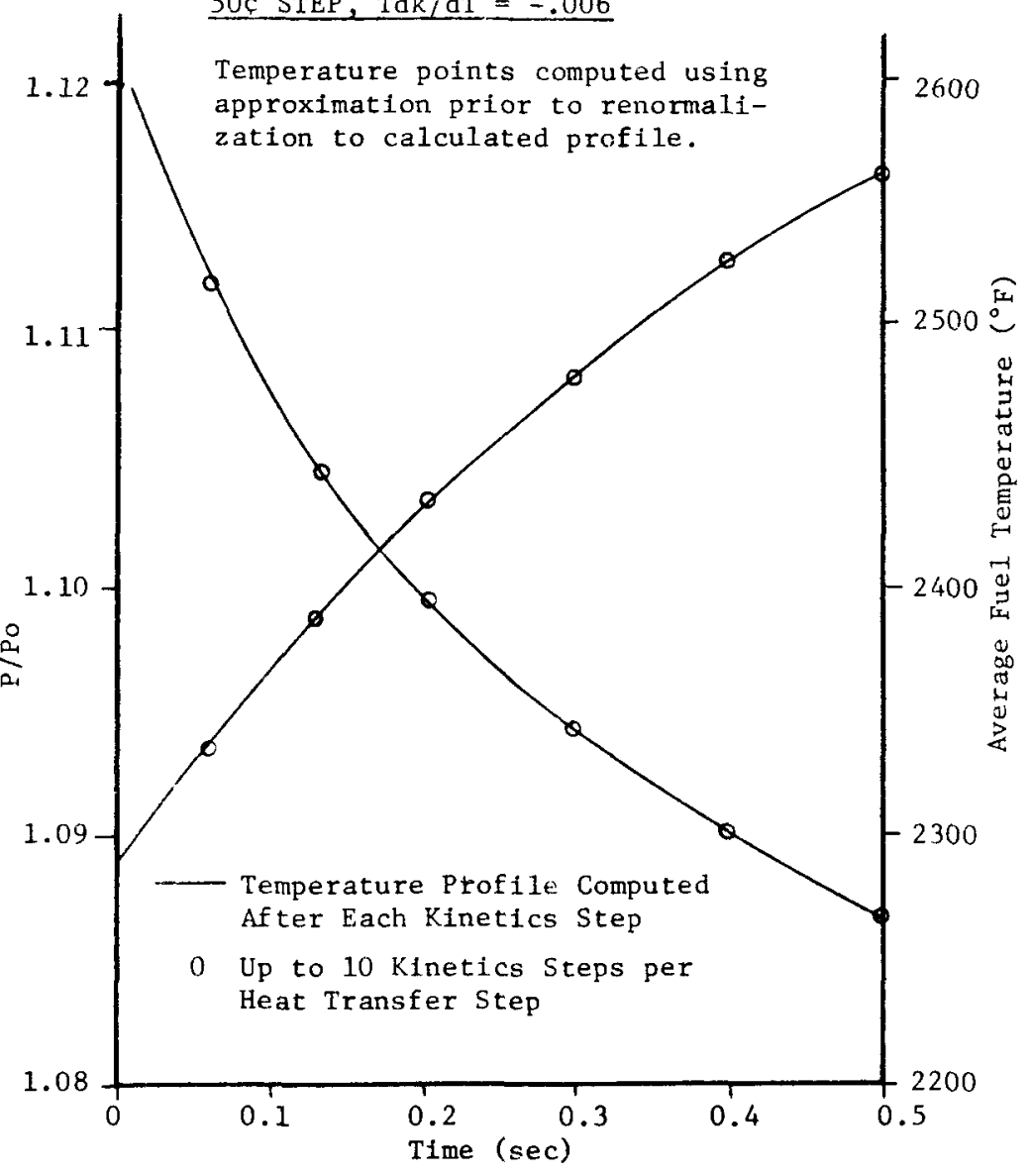


FIGURE 8

RELATIVE POWER AND TEMPERATURE

SS. TIIA

$\$ 1.20 \mathrm{STEP}, \mathrm{T} \mathrm{dk} / \mathrm{dT}=-.006$

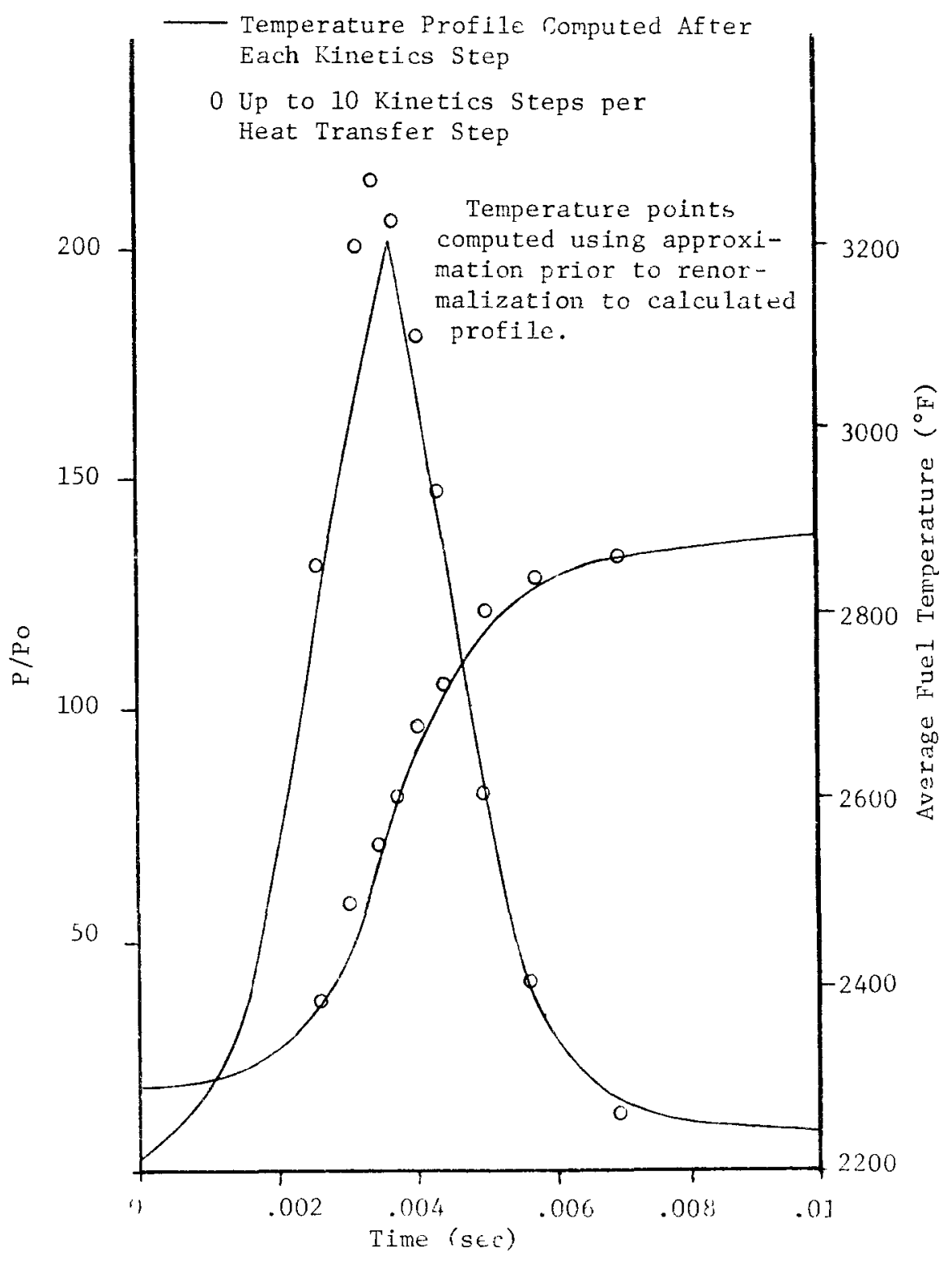


EIGURE 9

RELATIVE POWER AND TEMPERATURE
FIGURE 10

RELATIVE POWER AND TEMPERATURE

$$
\text { VS. TIME }
$$

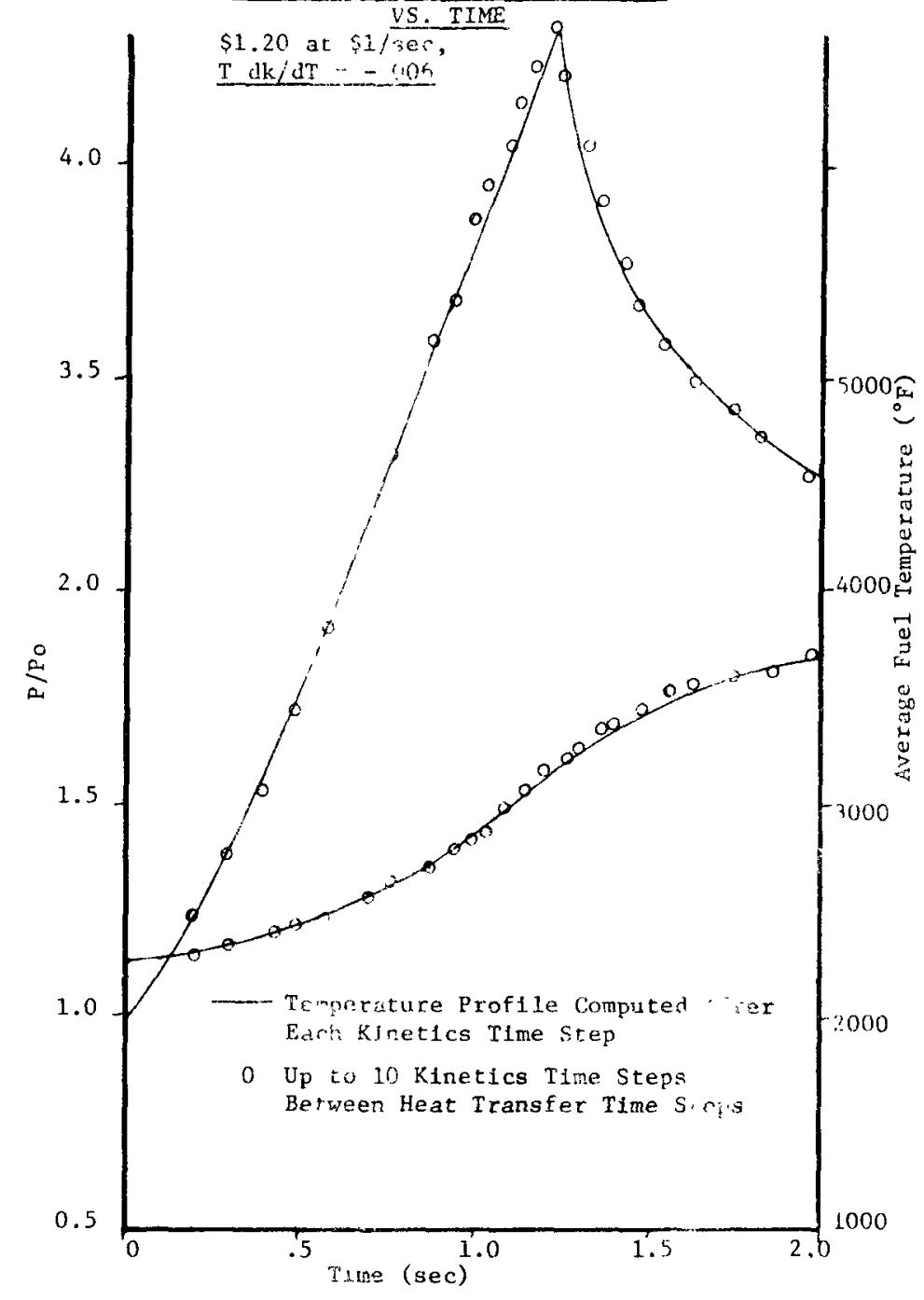

$\$ 1.20$ at $\$ 10 / \mathrm{sec}, \mathrm{T} \mathrm{dk} / \mathrm{dT}=-.006$

- Temperature Prof: le Computed After Each Kinetics Tine Step

0 Up to 10 Kinetics Time Steps Between Heat Transfer Time Steps

20
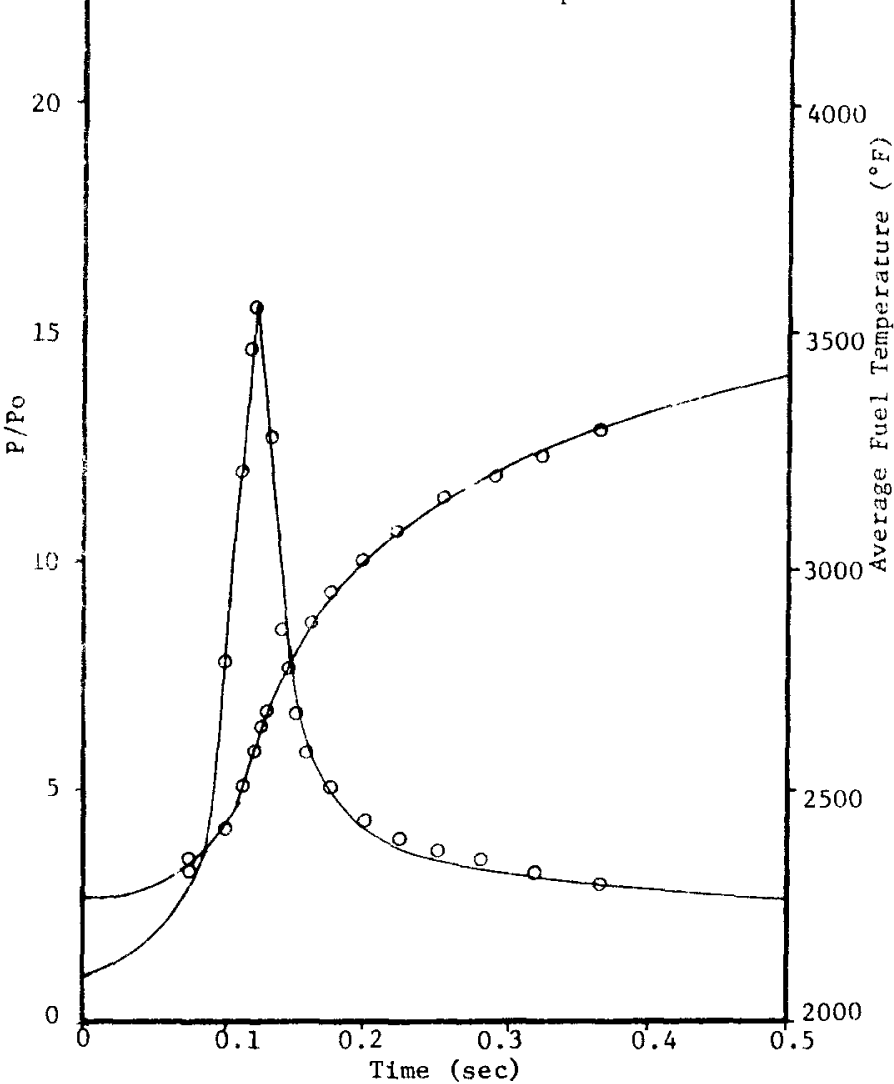
Scoping of transients prior to performing detailed transient calculations may also be done using the average fuel temperature approximation as shown in Figures 11 and 12. In these figures transient power and average fuel temperature are compared for ramp rates of $\$ 1 / \mathrm{sec}$ and $\$ 10 / \mathrm{sec}$ with NKS $=1$ in one case (detailed temperature profile each kinetics step) and NKS $=200$, TMAX $=10000$ (no detailed temperature profile after steady state) for another case. The power and fuel temperature traces obtained with the simple model may be used to set time step controls in a detailed calculation.

Substantial cost savings are possible if the cost of radial heat transfer calculations is a significant portion of the total transient calculation cost. From the one node model one radial heat transfer time step for one axial node with ten rádial fuel segments takes $\sim .007 \mathrm{sec}$ of GE-635 processor time. The potential savings in processor time was estimated for large problems using the results of the one node calculations. Processor time used in the transient calculations was separated into time used for heat transfer and that used for transient power calculations. A 1arge heat transfer problem, say 12 bundle types and 7 axial nodes (total of 84 axial nodes) was assumed to take about the same amount of processor time per node for heat transfer, and the same total processor time for transient power calculations as the corresponding one node calculation. Results are summarized in Table 2 .

Processor times shown in Table 2 are estimates for power transients identical to the single node calculations assuming a total of 84 axial fuel nodes (12 bundle types, 7 axial nodes). The number of kinetics steps and the number of heat transfer calculations required for the various cases considered is shown for the single node problems which were run. The temperature change for each case is compared with the temperature change for similar power transients with heat transfer computed each kinetics time step (NKS $=1$ ). The ratio of fuel temperature change with control of the heat transfer time step to temperature change with no control is shown as a measure of the accuracy obtainable with the heat transfer time step control. For the cases studied, no significant sacrifice in accuracy was made when the number of radial heat transfer calculations was reduced.

The total processor time required for transient calculations was also compared with the tine required when radial heat transfer calculations were performed after each kinetics step for each case. The ratio of total processor time with control of the heat transfer time step to total processor time with no control of the heat transfer time step is given in Table 2 as the cost ratio. This ratio ranges from one-tenth to one-half for the cases considered. Since calculation of transient conditions will be a major portion of the total computer cost in sensitivity studies, significant cost savings should be realized with the time step control in the FREADM code. 

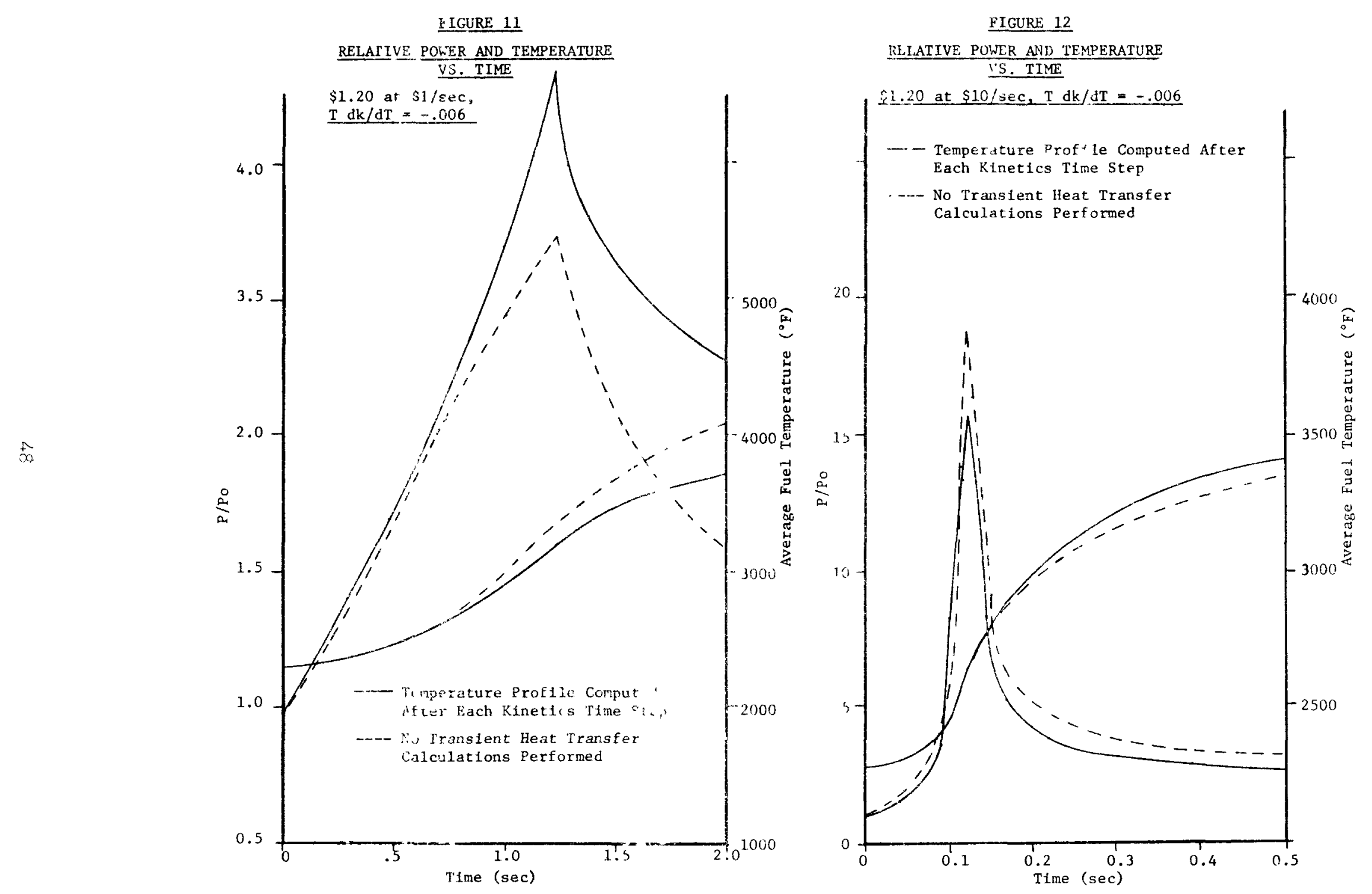
TABLE 2

SLMMARY OE PROCESSOR TIME ESTIMATES

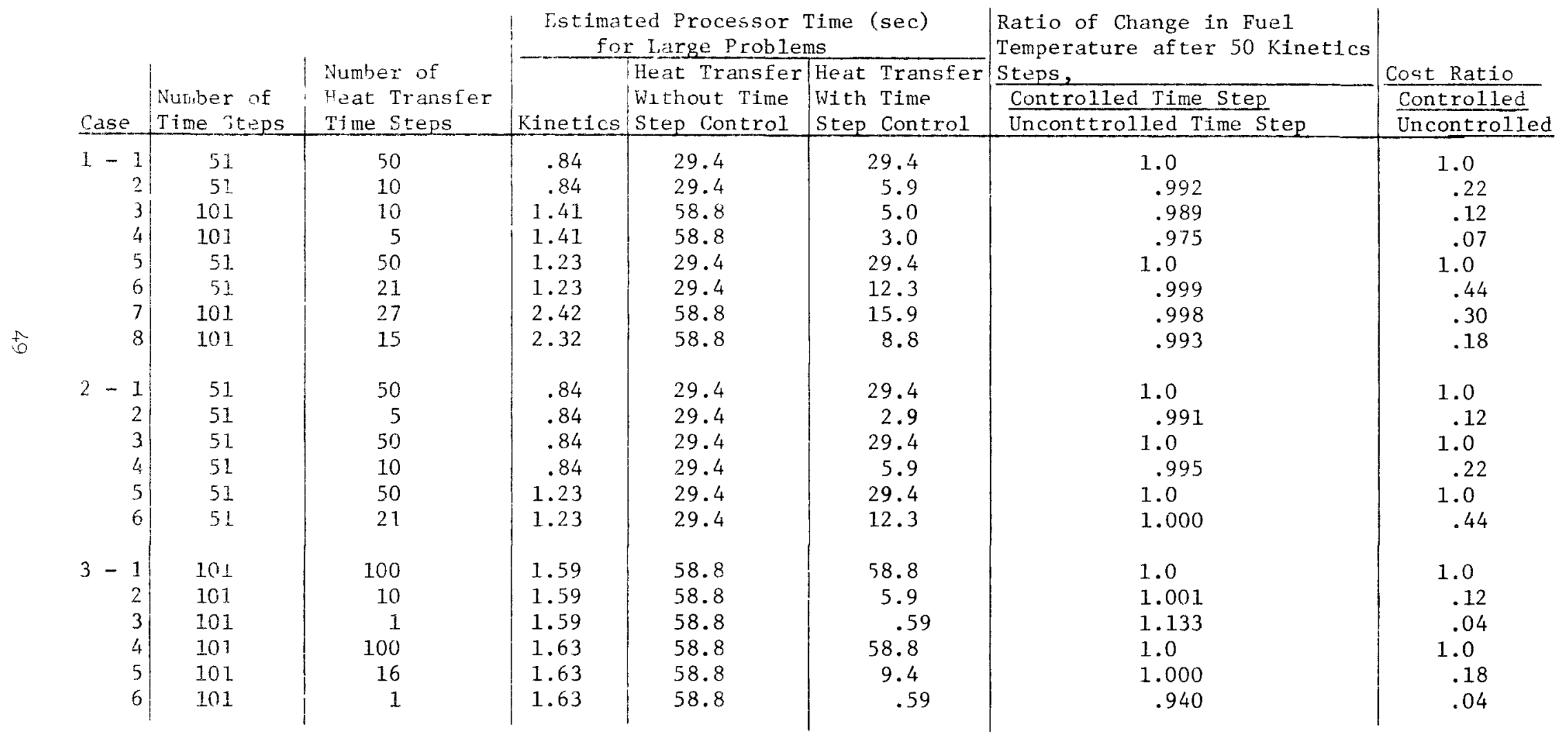


1. P. Greebler, Recent Improvements in Calculations of Doppler and Sodium Reactivity Effects for Large Fast Reactors, GEAP-4410, (Nov. 13, 1963).

2. K.P. Cohen and G.L. O'Nei11, "Safety and Economic Characteristics 1000 MWe Reactor," Advances in Nuclear Science and Technology - Vo1.4.

3. R.A. Meyer and B. Wolfe, "Fast Reactor Me1tdown Accidents," Advances in Nuclear Science and Technology - Vol. 4, p. 197; Academic Press (1968) p. 67; Academic Press (1968).

4. J.N. Fox, et a1, FORE-II - A Computational Program for the Analysis of Steady State and Transient Reactor Performance, GEAP-5273 (Sept. 1966)

5. Hildebrand, Methods of Applied Mathematics, 2nd Edition, p. 339; Prentice-Hall (1965).

6. H.K. Fatske, "Liquid Metal Boiling in Relation to LMFBR Safety Design," Paper Presented to 10th National Heat Transfer Conference, Dhiladelfiria, Pennsylvania (Aug. 1968).

i. iN.G. Meinhardt, "Calculating Vapor Growth in a Superheated Liquid Metal Within a Heated Channel," Paper Presented to ANS Winter Meeting, pittsburgh, Pennsylvania (Nov. 1966). 


\title{
FAST REACTOR ACCIDENT STUDY CODE, SASIA
}

\author{
G. J. Fischer, J. C. Carter, T. J. Heames, D. R. MacFarlane, \\ N. A. MoNeal, C. K. Sanathanan, W. T. Sha, C. K. Youngdahl
}

Argonne National Laboratory Angonne, Illinois

The SASIA code represents the first major step within the Safety Analysis Section of Argonne National Laboratory in the development of a new generation of computer codes directed toward more accurate and credible computation of fast breeder reactor accident histories. The code has been kept relatively simple in many of its aspects in onder to achieve relatively fast running time and to be compatible in sophistication with the level of understanding of the phenomenology and of the present technical uncertainties of fast reactor mechanisms. Nevertheless it is considerably more advanced in its sophistication than are many previous codes. Because of the modular structure of the code new or alternate versions of any aspect of safety analysis can be adapted into SASIA with a maximum of ease. The code can thus be used to evaluate experimental research needs and to plan effective experiments, and it can quickly incorporate the consequences of these experiments. Running time for the code is relatively short. About 5 minutes of IBM 360 50/75 time has been sufficient to calculate most cases tried to the present. Future developments will improve computing efficiency anc add important new tecnnical features. The code is written entirely ir Fortran IV. It should be available through the Argonne Code Center in CDC 6600 and IBM 360 versions at the date of this conference. Major modules of the code also function as ARC system modules.

The SASIA code can, in one continuous computation at the computer, initialize the thermo-hydraulic operating conditions to the user's specifications in onler' to defint the pre-accicient steady operating state, compute the history of reactor response to aciidents, such as loss-of-flow, and calculate the physical history of any disassembly which might become a consequence of the sequence of computer events. Besides the advantage of corvenience to the user, severe accidents computed with SASIA for "Design Basis Accidents" portions of saiety eraluarion sturies will use iritial condicions of the disassembly accident which ar computel as direct consequences of the previous history of the accidert sequence. They need no ionger be hypothetical, and often exceptionall $v$ pessimistic.

Because sodium voiding represents an important source of rapid reactivity insertion, vciling dynamics is calculated with cane. The methods reported by MacFar Lane [1] have been improved for range of applicability and computation time reduction. Ar altemate voiding module [c] which represents an improvement on the simpier BLOW code [3] has been written and tested, and will be mare avajiar Ie in SAJIA as an alternate sption. Fuel deformation mechanics durire accitent conditione, and treatment of feedback mechanics are other facets of SASIA zoncentration to be aescribed below. The explosion routine was 
adapted from the APDA code MARS, [4] with addition of several desirable improvements [5].

i- poszible limitation of SASIA, for some areas of accident analysis, is that point kinetics is used in the code. Because of the nature of the spatial deperderice of reactivity-changing mechanisms during several phases of fast reactor accidents, two-dimensional time-dependent neutronics calculational capability could be irmortant. Computation time for any problem, however, would increase sigrificantly if such a routine were available and used. Such a routine is being developed at ANL but it will be used in a more sophisticated code, SASIB, which will incorporate several other features of consistent sophistication. The VENUS severe disassembly accident code [6] contains a routine for two-dimerisional hydrodynamics computation which is an early-developed component of the SASIB code package.

Another current limitation of SASIA is that except for a relatively simple approximation for non-coherence of phenomena the accident analysis is based on what is called a single-channel approximation. The accident dynamics of the full reactor is related to themal-hydraulic events occurring in a single fuel pin with its associated cladding, coolant, and structural components. Noncoherence of voiding is approximated by allowing the code user to specify the further increments of nuclear heating required to initiate boiling in up to three cther chanrels. The pattern of reactivity change with time for these arameis, nowever, is oniy that of the first channel except for the computed in: telity.

This rostricticn will be relieved relatively soon through logic and input changes wrich will allow explicit multiple channel calculations.

The code is written in modular form. A MAIN program calls four driver programs. These handle the areas of 1) input-output, with diagnostics on user difficulties, 2) steady state initialization, to define the cone state before the initiation of the accident, 3) the transient state driver, for the largest part of the code, and 4) the driver for the explosion routines. Eighteen subroutines are used. These are described in detail in the write-up provided with the code, so that only the more important features of the subroutines of greatest interest to safety analysts will be described here.

The transient state heat transfer routine employs an extrapolated CrankNicholson computing scheme similar to that proposed by M. Lees [7]. Large time steps can be taken by this implicit scheme. Non-linearities caused by wide variations in heat conductivity of oxide fuel are handled easily. Moving mesh coondinaters adapt to dimensional changes due to heating and changes of phase of the fuel, and account for closing of possible gas bond spaces.

The fue mechanics routine computes the stresses and deformations occurring during the power transients, changes of phase, closing of the center hole if one exists, as for an oxide fuel, as melting procedes, and elastic as well as plastic deformation of the cladding is calculated.

The moment of possible rupture of the fuel cladding can be very badly mis-calculated if the possibility of relief of intermal pressures due to the displacement of the highly fluid molten $\mathrm{UO}_{2}$ is not taken into account. The FUEIDYN subroutine attempts to do this. While it is very simple in its present formulation, its physical foundation is sound. Studies using the routine have shown very ample reason for further development. This routine contains user-supplied tables of the terperature-dependent strength characteristics of the steel of the cladding, which FUEIDYN uses to compute the core-height 
dependence of the position of rupture. Experimental calculations indicate that rupture almost always occurs well above the midplane of the core. This information has important neutronic significance. Since cladding rupture could lead to fuel-coolant interaction and very rapid expulsion and reactivity change dynamics, FUELDYN will supply important input to future fuel-coolant interaction routine.

Sodium voiding is calculated explicitly during the accident. The rate of voiding is responsive to changes in heat transfer rates during the accident, much of which can be a result of reactivity changes induced by sodium voiding which has developed to that moment. Voiding can occur at any position along the lengths of the fuel. It then grows in both directions according to a mathematical model which allows two phase flow. Developments on this model [1] have allowed an extension of the voidirg history and accelerated computing speed. Reactivity changes associated with the sodium voiding employ an interpolation on a table look-up, where the user supplies the data to the table. The user is then free to and responsible for choice of neutronic cross sections and voiding calculation methods. Similarly, the Doppler effect feedback employs a table look-up procedure where the table wants data on the axial dependence of the Doppler effect and the change in magnitude of the Doppler effect with extent of local voiding of sodium.

It is important to recognize that change in density of sodium as it heats and before it reaches the boiling state can have a major effect on the reactor neutronic performance. SASIA accounts for this effect as well.

Other feedback calculations of SASIA are due to axial expansion of the fuel and radial expansion of the structure. Because of the well-known difficulties of characterization of oxide fuels for axial expansion, the code user must be careful of the meaning he wishes to assign to the axial expansion effect. The radial expansion feedback treatment represents a first-order approach to allowing the user to treat his radial expansion or bowing problems. This feedback depends upon the heat generation rate (by conduction) in the structure, as a function of core height. The feedback can be positive or negative, it can have a threshold for starting, as that required to take up clearances, and it can be turned off, as when clearances are entirely removed. The shutdown response of control and safety rods, if tripped, is of counse available.

The two-dimensional $(R-Z)$ explosion routine of SASIA was adopted from the APDA code MARS [4]. The interest and cooperation of A. Klickman of APDA was greatly appreciated. This routine once again employs the Bethe-Tait formalism and includes Dopples cffect feedback. ANL changes to MARS include a second order differentiäl equation routine not made available by APDA, a more effective kinetics routine, and an addition to account for boundary motions upon reactivity [8]. 
1. D. R. MacFarlane, N. A. MoNeal, D. Meneley, C. K. Sanathanan, Theoretical Studies of the Response of Fast Reactors During Sodium Bojling Accidents, Proc. of Int. Conf. on Safety of Fast Reactors, Aix-en-Provence, France, (1967).

2. A. W. Cronenberg IIt, SIug Expulsion of Boiling Sodium from a Reactor Coolant Channel, M. S. Thesis, Northwestern University, I1l., (1969).

3. W. Peppler, et.al., Sodium Boiling and Fast Reactor Safety, Proc. of Int. Conf. on Safety of Fast Reactors, Aix-en-Frovence, France (1967).

4. N. Hirakawa, MARS, A Two-Dimensional Excursion Code, APDA-198, June 1967.

5. W. T. Sha, MARS/SAS, A Two-Dimensional Weak Explosion Program, to be publisned.

6. W. T. Sha and T. H. Hughes, VENUS, A Two-Dimensional Coupled Neutronics Hydrodynamics Reactor Excursion Computer Program, to be published.

7. M. Jees, An Extrapolated Crank-Nicholson Difference Scheme for Cuasilinear Parabolic Equations, Proc. Symposium on Non-Linear Partial uifierental Lquations, Ed., W. F. Ames (Acadenic Press, to be printed).

5. W. T. Sha and R. B. Iicholson, Maximum Accident of Zoned Fast Reactors ANS Transactions, Vol. 11, No. 2, 1968. 
ON THE SOLUTION OF THE TIME-DEPENDENT GROUP DIFFUSION EQUATIONS

BY AN IMPLICIT TIME DIFFERENCED ITERATIVE METHOD

J. B. Yasinsky and L. A. Hageman

Westinghouse Electric Corporation

Bettis Atomic Power Laboratory

I. INIRODUCTION

In recent years much emphasis has been devoted to the problem of solving multi-dimensional reactor kinetics problems. Some of this emphasis has been focused on the two-dimensional problem; the goal being to obtain a dependable, economic, easy-to-use method which would serve as the basis for computer programs capable of analyzing a restricted class of design and accident problems. Such a program would also provide a standard of comparison for the efficient development of more elaborate three-dimensional methods (such as space-time synthesis (Ref I)). To date much of the work aimed at such a two-dimensional computer program has been of the method comparison type (Refs $2,3,4$ ) where only estimates of the capabilities of several methods are discussed.

This paper concerns the two-dimensional space-time differencing method used in the TWIGL (Refs 5, 6) program. The relevant difference equations and solution techriques are described and some numerical evidence relating to the accuracy, running time and efficient use of this program is presented.

II. THE SPACE-TTME DIFFUSION EQUATIONS

The two-group diffusion and delayed precursor equations solved by TWIGL are

$$
\begin{aligned}
\nabla \cdot D_{1}(\underline{r}, t) \nabla \Phi_{1}(\underline{r}, t) \cdots \Sigma_{1}(\underline{r}, t) \varphi_{1}(\underline{r}, t) & +(1-\beta)\left[v \Sigma_{\underline{f}_{1}}(\underline{r}, t) \varphi_{1}(\underline{r}, t)\right. \\
& \left.+v \Sigma_{\underline{f}_{2}}(\underline{r}, t) \varphi_{2}(\underline{r}, t)\right]+\sum_{i=1}^{I} \lambda_{i} C_{i}(\underline{r} ; t)=\frac{1}{v_{1}} \frac{\partial}{\partial t} \varphi_{1}(\underline{r}, t)
\end{aligned}
$$




$$
\begin{aligned}
& \nabla \cdot D_{2}(\underline{r}, t) \nabla \varphi_{2}(\underline{r}, t)-\Sigma_{2}(\underline{r}, t) \varphi_{2}(\underline{r}, t)+\Sigma_{r_{1}}(\underline{r}, t) \varphi_{1}(\underline{r}, t)=\frac{\underline{I}}{v_{2}} \frac{\partial}{\partial t} \varphi_{2}(\underline{r}, t) \\
& \beta_{1}\left[v \Sigma_{f_{1}}(\underline{r}, t) \varphi_{1}(\underline{r}, t)+v \Sigma_{f_{2}}(\underline{r}, t) \varphi_{2}(\underline{r}, t)\right]-\lambda_{1} C_{i}(\underline{r}, t)=\frac{\partial}{\partial t} c_{i}(\underline{r}, t) ; 1=1, \ldots, I ;
\end{aligned}
$$

where $\underline{r}$ represents $\mathrm{x}, \mathrm{z}$ (rectangular geometry) or $\mathrm{r}, \mathrm{z}$ (cylindrical geometry). These equations are to be solved subject to zero flux boundary conditions and equilibrium inftial conditions. The $D_{g}, \Sigma_{g}, v \Sigma_{I_{g}}$ and $\Sigma_{r_{I}}$ are functions of time through specified linear changes and through both coolant and fuel temperature feedback.

The spatial dependence of $\Phi_{1}, \Phi_{2}$ and the $C_{i}$ will be defined relative to a mesn grid with $N$ and $M$ solution points, respectively, in the $x$ (or $r$ ) and $z$ directions. Using the integration method (Ref 7), the spatially discretized form of (2.3) wan be written as

$$
\begin{gathered}
-H_{I}(t) \Phi_{1}(t)+B_{2}(t) \Phi_{2}(t)+\sum_{i=1}^{I} \lambda_{i} \underline{C}_{i}(t)=\Omega_{1} \dot{\Phi}_{I}(t) \\
-H_{2}(t) \varphi_{2}(t)+B_{1}(t) \Phi_{I}(t)=\Omega_{2} \dot{\Phi}_{2}(t) \\
K_{I_{I}}(t) \Phi_{I}(t)+K_{i_{2}}(t) \Phi_{2}(t)-\lambda_{i} \underline{C}_{i}(t)=\dot{\underline{C}}_{i}(t) ; i=1, \ldots, I,
\end{gathered}
$$

where $\underline{\varphi}_{1}, \underline{\varphi}_{2}$ and the $\underline{C}_{i}$ are column vectors with NM elements, $\Omega_{1}$ and $\Omega_{2}$ are NMXNM diagonal matrices representing the $1 / v_{1}$ and $1 / v_{2}$ terms. $B_{1}, B_{2}, K_{i_{1}}$, and $K_{i_{2}}$ are NMXIM diagonal matrices resulting from the spatial discretization of the $\Sigma_{r_{I}}$, $(I-\beta) v \Sigma_{f_{2}}, \beta_{i} v \Sigma_{f_{1}}$ and $\beta_{i} v \Sigma_{f_{2}}$ terms, respectively. $H_{1}$ and $H_{2}$ are NMxNM matrices with at most five non-zero elements in any row. These matrices are the discrete representations of the $\left[V \cdot D_{1} V-\Sigma_{1}+(I-\beta) v \Sigma_{f_{1}}\right]$ and $\left[\nabla \cdot D_{2} \nabla-\Sigma_{2}\right]$ terms.

We seek to approximate the solution to (2.2) at a sequence of time points $t_{0}, t_{1}, \ldots, t_{j}, \ldots$, which are separated by the time intervals $\Delta t_{j} \equiv t_{j+1}-t_{j}$. Let 
superscript $j$ denote a quantity evaluated at time $t_{j}$. If we integrate Eqs (2.2) from $t_{j}$ to $t_{j+l}$, using the approximation

$$
\int_{t_{j}}^{t} j+1
$$

for all terms involving the fluxes, and

$$
\int_{t_{j}}^{t} \underline{c}_{i}(t) d t \cong \frac{\Delta t}{2}\left[\underline{c}_{i}^{j+1}+\underline{c}_{i}^{j}\right],
$$

then we obtain

$$
\begin{aligned}
& {\left[\Omega_{1}+\theta \Delta t_{j} H_{1}^{j+1}\right] \varphi_{1}^{j+1}-\theta \Delta t_{j} B_{2}^{j+1} \varphi_{2}^{j+1}=\left[\Omega_{1}-(1-\theta) \Delta t_{j} H_{1}^{j}\right] \varphi_{1}^{j}} \\
& (1-\theta) \Delta t_{j} B_{2}^{j} \Phi_{2}^{j}+\sum_{i=1}^{I} \frac{\lambda_{i}}{2} \Delta t_{j}\left[\underline{C}_{i}^{j+1}+\underline{C}_{i}^{j}\right] \\
& {\left[\Omega_{2}+\theta \Delta t_{j} H_{2}^{j+1}\right] \varphi_{2}^{j+1}-\theta \Delta t_{j} B_{1}^{j+1} \varphi_{1}^{j+1}=\left[\Omega_{2}-(1-\theta) \Delta t_{j} H_{2}^{j}\right] \varphi_{2}^{j}+(1-\theta) \Delta t_{j} B_{1}^{j} \varphi_{1}^{j}}
\end{aligned}
$$

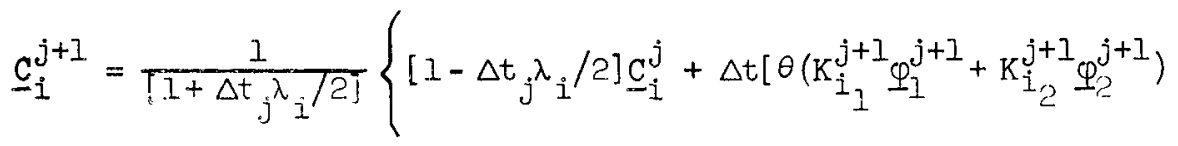

$$
\begin{aligned}
& \left.\left.+(1-\theta)\left(K_{i_{1}}^{j} \varphi_{1}^{j}+K_{i_{2}}^{j} \varphi_{2}^{j}\right)\right]\right\} \text {. }
\end{aligned}
$$

Here $\theta$ is a number between $O$ and $I$ which is specified by the user. If we use Eq $(2.3 c)$ to eliminate $\underline{C}_{i}^{j+1}$ in Eq $(2.3 \mathrm{a})$, then we are left with the following inhomo renerus spatial d fference equations which must be solved at each time step.

$$
\begin{aligned}
& A_{1}^{j+1} \varphi_{1}^{j+1}-\tilde{B}_{2}^{j+1} \varphi_{2}^{j+1}=S_{1}^{j} \\
& A_{2}^{j+1} \varphi_{2}^{j+1}-\tilde{B}_{1}^{j+1} \Phi_{1}^{j+1}=S_{2}^{j}
\end{aligned}
$$


In Eq (2.4), $\underline{S}_{1}^{j}$ and $\underline{S}_{2}^{j}$ are NM element column vectors which contain all the terms involving the known fluxes $\Phi_{1}^{j}, \Phi_{2}^{j}$ and the known precursor concentrations $\underline{c}_{i}^{j} ; 1=1, \ldots$, .

III. SOLUTION OF THE SPATIAI DIFFERENCE EQUATIONS

The TWIGL program uses the cyclic Chebyshev polynomial method (Refs $8,9,10$ ) to solve the system of Eqs (2.4). Given an initial guess vector $\Phi_{2}(0)$, the cyclic Chebyshev method generates successive estimates for the solution vectors $\Phi_{1}$ and $\Phi_{2}$ (for convenience we have dropped the time index $j$ ) by the process

$\left.\begin{array}{l}\Phi_{1}(k+1)=\alpha_{k+1}\left[\underline{S}_{1}(k+1)-\varphi_{1}(k)\right]+\varphi_{1}(k), \text { where } A_{1} \underline{S}_{1}(k+1)=\tilde{B}_{2} \Phi_{2}(k)+\underline{S}_{1} \\ \Phi_{2}(k+1)=\gamma_{1+1}\left[\underline{S}_{2}(k+1)-\varphi_{2}(k)\right]+\Phi_{2}(k) \text {, where } A_{2} \underline{s}_{2}(k+1)=\tilde{B}_{1} \Phi_{1}(k+1)+\underline{S}_{2}\end{array}\right\}$

where $k$ is the Chebysher iteration index and $\alpha_{k+1}, \gamma_{k+1}$ are the Chebyshev scceleration parameters. If $\alpha_{k+1}=\gamma_{k+1}=1,(3.1)$ is the Gauss-Seidel method if iteration.

In order to carry out the iterative process (3.1), group equations of the form

$$
A_{g} \underline{s}_{g}=\underline{h}_{g}, g=1,2,
$$

must be solved for $\Sigma_{g^{\prime}}$. The solutions $\underline{s}_{g}$ to (3.2) are approximated by the oneIine successive overrelaxation iteration method (Ref 8 ). The iterations used to obtan theit dpproximations are cailed inner iterations whic the iterations of (3.1) are calied outer iterations. A fixed number, $\mathrm{m}_{g}$, of inner iterations is performed in group $g$ for every outer iteration. The program tries to choose $\mathrm{m}_{\mathrm{g}}$ such that the inner iteration error vector after performing $\mathrm{m}_{\mathrm{g}}$ iterations is about 0.05 of the inftial error vector. This prediction is made in what is called the "omega routine", which is carried out prior to the start of the outer iterations. 
The overrelaxation factor, $\omega_{g}$, required by the overrelaxation method is also determined in the omega routine. The $\mathrm{m}_{\mathrm{g}}$ and $\omega_{\mathrm{g}}$ computed for the first time step are used for succeeding time steps. If the norm of the error vector after $\mathrm{m}_{\mathrm{g}}$ inner iterations is greater than 0.2 that of the initial error vector, the $\omega_{g}$ and $\mathrm{m}_{\mathrm{g}}$ constants are no longer considered adequate and are recomputed for the next time step.

The optimum Chebyshev parameters $\alpha_{k}$ and $\gamma_{k}$ for the outer iterations are functions of the eigenvalue domain of the Jacobi iteration matrix

$$
J=\left[\begin{array}{cc}
0 & A_{1}^{-I} \tilde{B}_{2} \\
A_{2}^{-I \tilde{B}_{1}} & 0
\end{array}\right] \text {. }
$$

For reasonable values of $\Delta t$, the elgenvalues of $J$ are bounded by the elipse $\mathrm{x}^{2} / \sigma^{2}+\mathrm{y}^{2} / \epsilon^{2}=1$, where $\epsilon^{2}<\sigma^{2}$ and $\sigma$ is the spectral radius ${ }^{*}$ of the matrix $\mathrm{J}$. For this case, the acceleration parameters $\alpha_{k}$ and $\gamma_{k}$ are given (Refs 9,10) by $\alpha_{1}=1, \gamma_{1}=2 /\left(2-p^{2}\right)$ and for $k \geq 1$

$$
\alpha_{k+1}=\frac{1}{1-\frac{\rho^{2}}{4} \gamma_{k}}, \quad \gamma_{k+1}=\frac{1}{1-\frac{\rho^{2}}{4} \alpha_{k+1}} \text {, }
$$

where $\rho^{2}=\sigma^{2}-\epsilon^{2}$ A value of $\sigma$ less than unity is required for convergence and for a fixed, the rate of convergence increases as o decreases. It is thought by some (RESS 10,1I) that the eigenvalues of $\mathrm{J}$ are real but that the inner iterations introduce complex eigenvalues whose moduli depend on the degree of convergene of the znner iteration. In the TwIGL program it is assumed that an

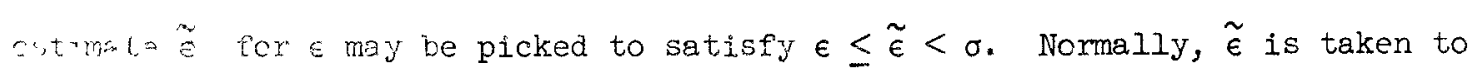

\footnotetext{
* $T \hat{1} \lambda_{j} \ldots, \lambda_{n}$ are the eigenvalues of the $n \times n$ matrix $U$, then the spectral radius, $\sigma(U) ;$ of $U$ is $\sigma(U)=\underset{i}{\max }\left|\lambda_{1}\right|$.
} 
be 0.1. An accurate estimate for $\sigma$ is now needed for the efficlent use of the Chebyshev method.

In the TWIGL program, numerical estimates for o are sbtained by observing the decay rate $t_{0}$ the res-ud 1 vector $\underline{y}(k+1)=\underline{s}_{2}(k+1)-\underline{P}_{2}(k)$, where $\underline{s}_{2}(k+1)$ and $\Phi_{L}(k)$ are gives. $r y(k .1)$. The rosudual vector quctient is defined as

$$
Q(k+1)=\frac{\|y(k+1)\|}{\|y(k)\|},
$$

where $\| \underline{x} \mid !$ is the $l_{2}$ norm of the rector $x$; $1 . e .$, if $x_{j}$ are the elements of $x$, then $\|x\|-\left[\sum_{1=1}^{n}\left|x_{1}\right|^{2}\right]^{1 / 2}$. Since $\lim _{x \rightarrow \infty} Q(k)=\sigma^{2}$ for the causs-seidel method (Ref 9 ), an initial estimate for $\sigma^{2}$ nay be obtained by doing a few Gauss-seldel iterations before starting the Chebyshev process. Once the Chebyshev process has started, new cottrates fmo a san be obtained by compartng the convergence rate actually being obtalne inth the thecreticel convergence rate one would oftain if the estimate being sec for $\sigma$ were correct. In order to obtain a new estimate for $\sigma$, whever, cne needs (Ref 9, App. B) to stop the Chebyshev process, do one Gauss-Seidel iteration, compute a new estimate for $\sigma$ and then start the Chebyshev process over again,

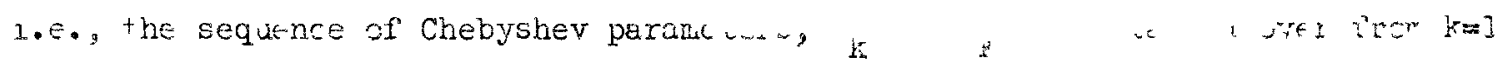
using this rew estumate for $\sigma$. The strategy for estimating $\sigma$ is discussed in more detasi in Ket $y$, App. B.

The estimate for $\sigma$ ottanne from the outer iterations for the first time step is used for succeeding time steps. However, if the ratio of "actual convergence

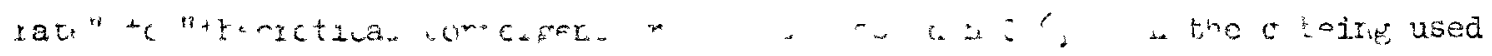
is no longer conodered adequte and $\checkmark$ is lamestima veu for the next time step. The outer sterations (3.1) axe considerea wervercu wan

$$
\left[\frac{1}{1-Q(k+1)}\right]\left[\frac{\|\underline{y}(k+1)\|}{\left\|\Phi_{2}(k)\right\|}\right] \leq \delta,
$$


where $\delta$ is an input quantity. The left side of (J.ל) is an approximation (Ref y) to the $h_{2}$ norm of the error vector, $\underline{\varepsilon}(k)=\underline{\varphi}_{2}(k)-\underline{\varphi}_{2}$, normalized by $\left\|\underline{\varphi}_{2}\right\|$. The proeram will not allow convergence in less than five outer iterations.

IV. NUMERICAI STUDY

In the preceding section we saw that TWIGL solves the group diffusion difference equations at each time step by an iterative process. The number of ouver iterations requirea to convere this process al each time step depends not only on the converence parameter, $\delta$, but also on the value of the spectral rauius, $\sigma$, of the Jacobi masix (and Low well we estimate it). The Jacobi matrix itself is a function of the time step $\Delta t$, the time differencing parameter $\theta$ and tne state of the reaccor (sulcritical, supercritical). Hence tne nunker of outer ilera uions required for conversence at each time step (thus tne problen runing time) is a function of 8 , $\Delta \tau, \theta$ ard the state of hne reactor. The accuracy of the solution is also dependenu on these quantiuies.

In uhis section we discuss some numerical stuaies wnich are cined at examining the accuracy and runin, time characteristics of ine TWIGL solution mothod. PA reactor consiaered for nost of these problems (typical of a Prk seed-blanket reactor) is described in Fic. 1. Zero flux boundary conditions are applied on the extesnal surfaces. Tro basic proklems are considered relative to tnis reactor, one delajed supus crivical and the other prompt supercrivical. 'The material perturivalions and cine aurations of interesu for chese prollems are given in Table I.

The initial question we wart to answer is: What value of the spa cial converience paramcter, 8, must ke ased to achieve a desired accuracy in tne spatial Loluion at eacn time step? Usine the TwIGL program, with $\Delta t=0.005$ sec and $\theta=I$, we colved the delajed supercritical protiem for 50 time steps (wo $t=0.25$ sec), usine, first $\delta=10^{-2}$, then $\delta=5 \times 10^{-3}, 1 \times 10^{-3}, 5 \times 10^{-4}, 1 \times 10^{-4}$ and finally $\delta=5 \times 10^{-5}$. Comparisons of thermal flux values at $t=0.25 \mathrm{sec}$ were used to 


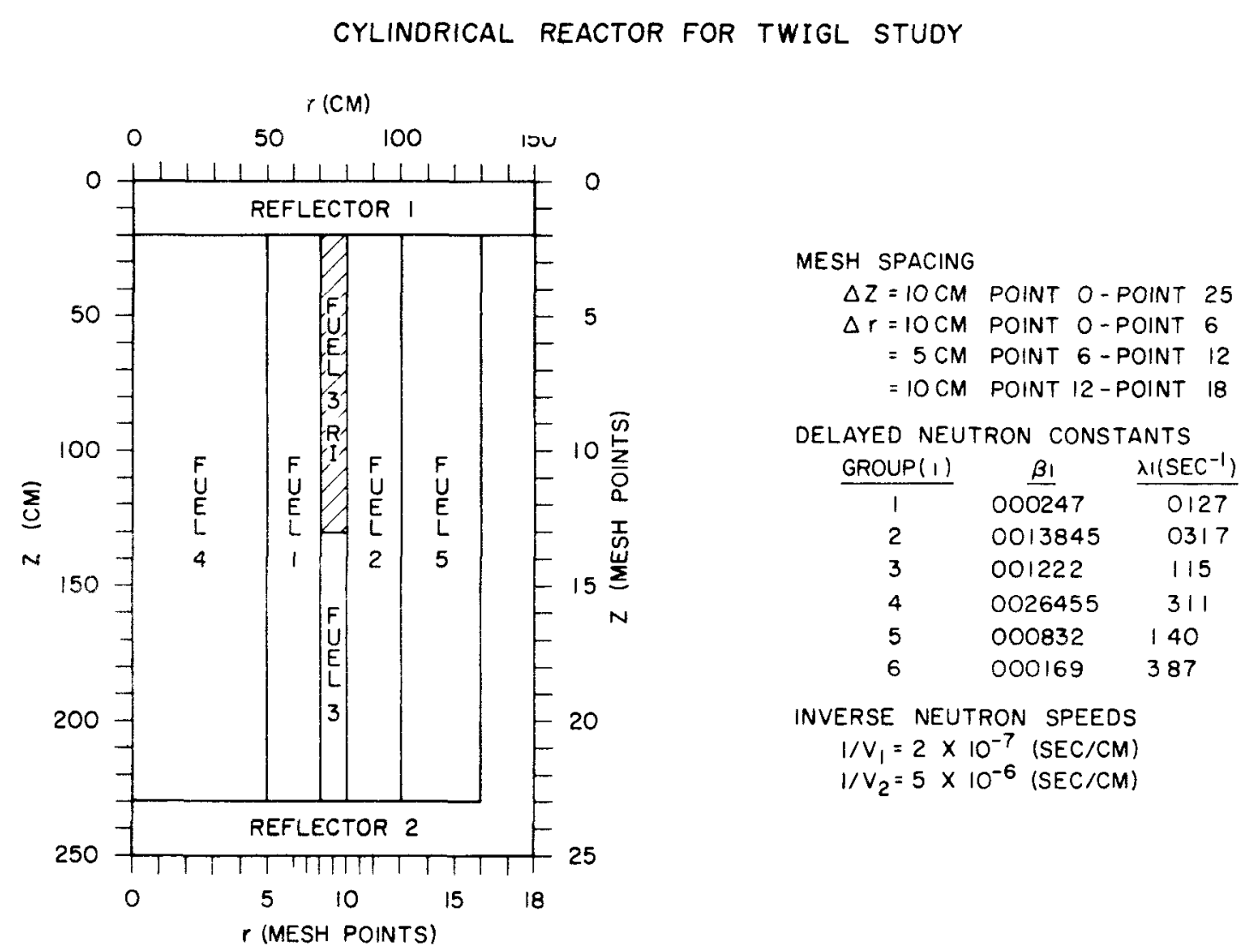

CRITICAL MATERIAL PARAMATERS

\begin{tabular}{|c|c|c|c|c|c|c|c|c|}
\hline & FUEL I & FUEL 2 & FUEL $3(R I)$ & FUEL 3 & FUEL 4 & FUEL 5 & REFL I & REFL 2 \\
\hline$D_{1}$ & 1367 & 1404 & 1377 & 1377 & 1291 & 1297 & 1412 & 1625 \\
\hline$\Sigma_{a_{1}}$ & 01099 & 00661 & 01061 & 006551 & 008494 & .0084 & 001483 & 000365 \\
\hline$\Sigma_{r_{1}}$ & 01309 & 01582 & 01309 & 01586 & 01116 & 01114 & 005923 & 03797 \\
\hline$\nu \Sigma_{f_{1}}$ & 0060575 & 0055995 & 0051279 & 0051279 & 0022705 & 0022739 & 0 & 0 \\
\hline$D_{2}$ & 3825 & 3778 & .3384 & 3384 & 4565 & 4572 & 7162 & 2750 \\
\hline$\Sigma_{\alpha_{2}}$ & $|5| 1$ & 09493 & 1339 & 09039 & 04975 & 04727 & 006549 & 01083 \\
\hline$\nu \Sigma_{f_{2}}$ & 164965 & 125357 & 145203 & 145203 & 049362 & 046233 & 0 & 0 \\
\hline
\end{tabular}

Fig. 1 


\section{TABLE I}

\section{DELAYED SUPERCRITICAL PROBIEM}

Simulatjon of $60 \mathrm{~cm}$ Partial Rod Withdrawal in Fuel 3 - R.I. Region Material Perturbations

$$
\begin{aligned}
& \Delta \Sigma_{1}=-\left(1.48 \times 10^{-3} / 0.2\right) t \\
& \left.\Delta s_{r_{1}}=\left(1.11 \times 10^{-3} / 0.2\right) t\right\} 0 \leq t \leq 0.2 \mathrm{sec} \\
& \Delta \Sigma_{2}=-\left(2.39 \times 10^{-2} / 0.2\right) \mathrm{t} \\
& \text { Constant values for } 0.2 \leq t \leq 0.5 \mathrm{sec} \\
& \text { Asymptolic Reactivity } \simeq 0.0032
\end{aligned}
$$

\section{PROMPT SUPERCRITICAL PROBLEM}

Simulation of Total Rod Withdrawal in Fuel 3 - R.I. Region Material Perturbations

$$
\begin{aligned}
& \Delta \Sigma_{2}=-\left(1.29 \times 10^{-3} / 0.002\right) t \\
& \left.\Delta \Sigma_{1}=\left(2.77 \times 10^{-3} / 0.002\right) t\right\} 0 \leq t \leq 0.002 \mathrm{sec} \\
& \Delta \Sigma_{2}=\left(4.35 \times 10^{-2} / 0.002\right) t \\
& \text { Constant values for } 0.002 \leq t \leq 0.02 \mathrm{sec} \\
& \text { Asymptotic Reactivity } \approx 0.017
\end{aligned}
$$


imply an error versus $\delta$ relation. Figure 2 shows some of these results for the two space points that exhibited the maximum errors; namely, the point of peak thermal flux, and the center of the perturbed region. All such comparisons indicate that a $S=10^{-3}$ is adequate for the spatial accuracy to better than one percent. Also included in Fig. 2 are the values of the problem running times. From this point on all problems used $\delta=10^{-3}$.

By decreasing the value of $\Delta t$ or $\theta$ belng used in a TWIGL problem we reduce the spectral radius of the Jacobl matrix and thus increase the rate of convergence of the iterat1ve procedure. Figure 3 shows the number of outer 1terations required at each time step for four solutions of the delayed supercritical problem. Comparling the right side results with those on the left shows that using $\theta=1 / 2$ Irstesi of $\theta=1$ can substantially increase the rate of convergence, especlally Wille the material properties of the reactor are changing with time $(0 \leq t \leq 0.2$ sec) Varving $A$ can also affect the accuracy of the time differencing approximation. Normally one should restrict his choice of $\theta$ to be between 0.5 and 1 , since for $\theta<0.5$ the time differencing approximation can become unstable for some values of $\Delta t$. For sufficiently malz values of $\Delta t$, a solution with $\theta=0.5$ is more accurate than cne wath $\theta=1$. However, for larger values of $\Delta t$ a solution with $\theta<1$ may be undesirable die to an oscillatory type solution behavior which cannot occur for $\theta=1$. Comparing the top and bottom results of Fig. 3 we see how a decrease in $\Delta t$ can increase the rate of convergence of the TWIGL solution. The total run times are

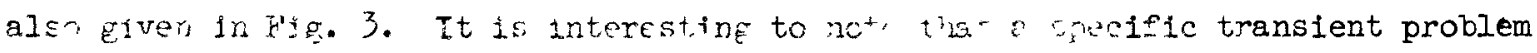
can be solvad faster using 200 time steps than it csn ueing 50 time steps (e.g. upper left and lower right results in Fig. 3). We also note from this figure that once the reactor properties stop changing, and the flux shape tends towards its asymptotic state, the number of iterations required at each time step approaches a constant value. 


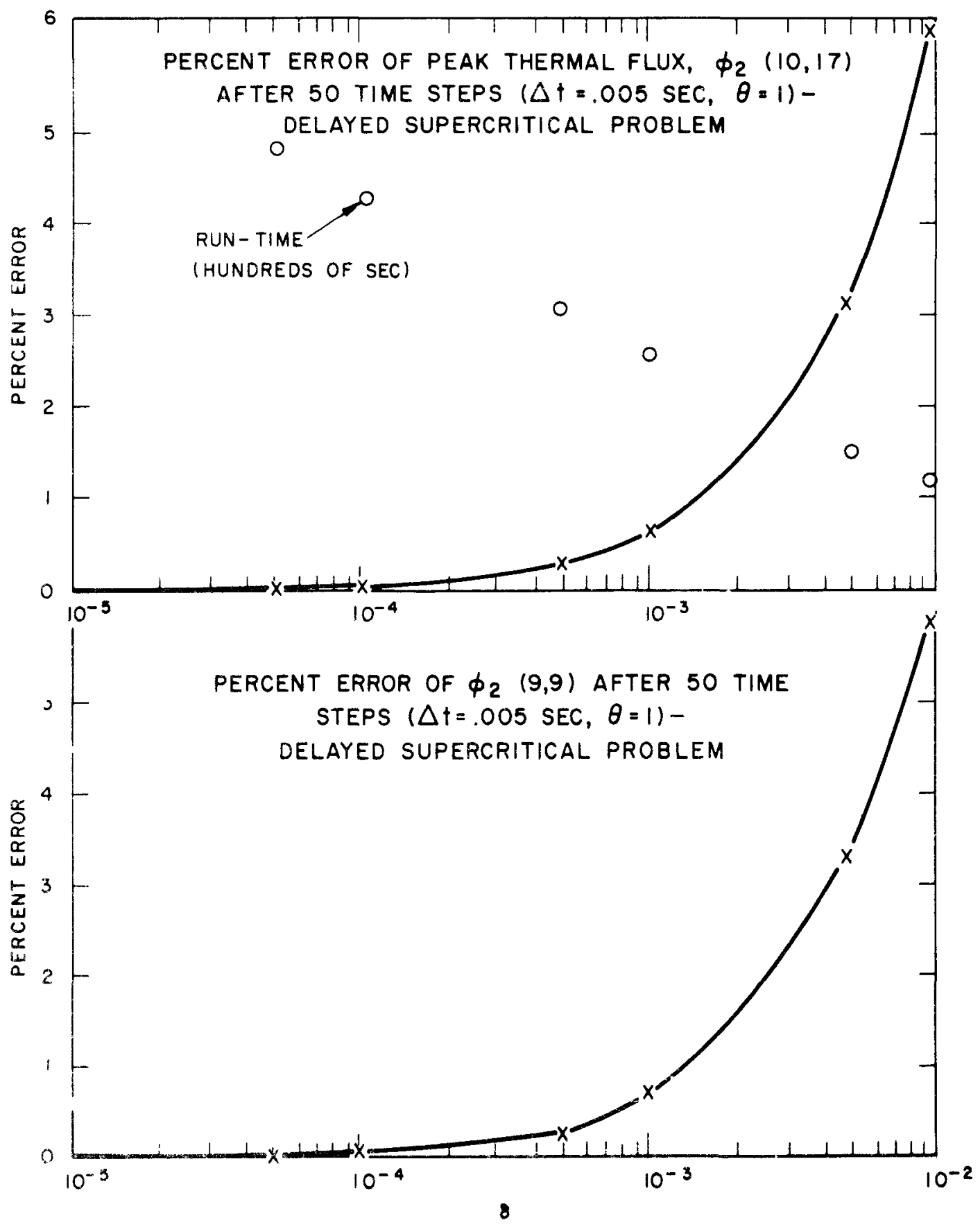

Fig. : 
OUTER ITERATIONS VS TIME STEP FOR DELAYED SUPER CRITICAL PROBLEM

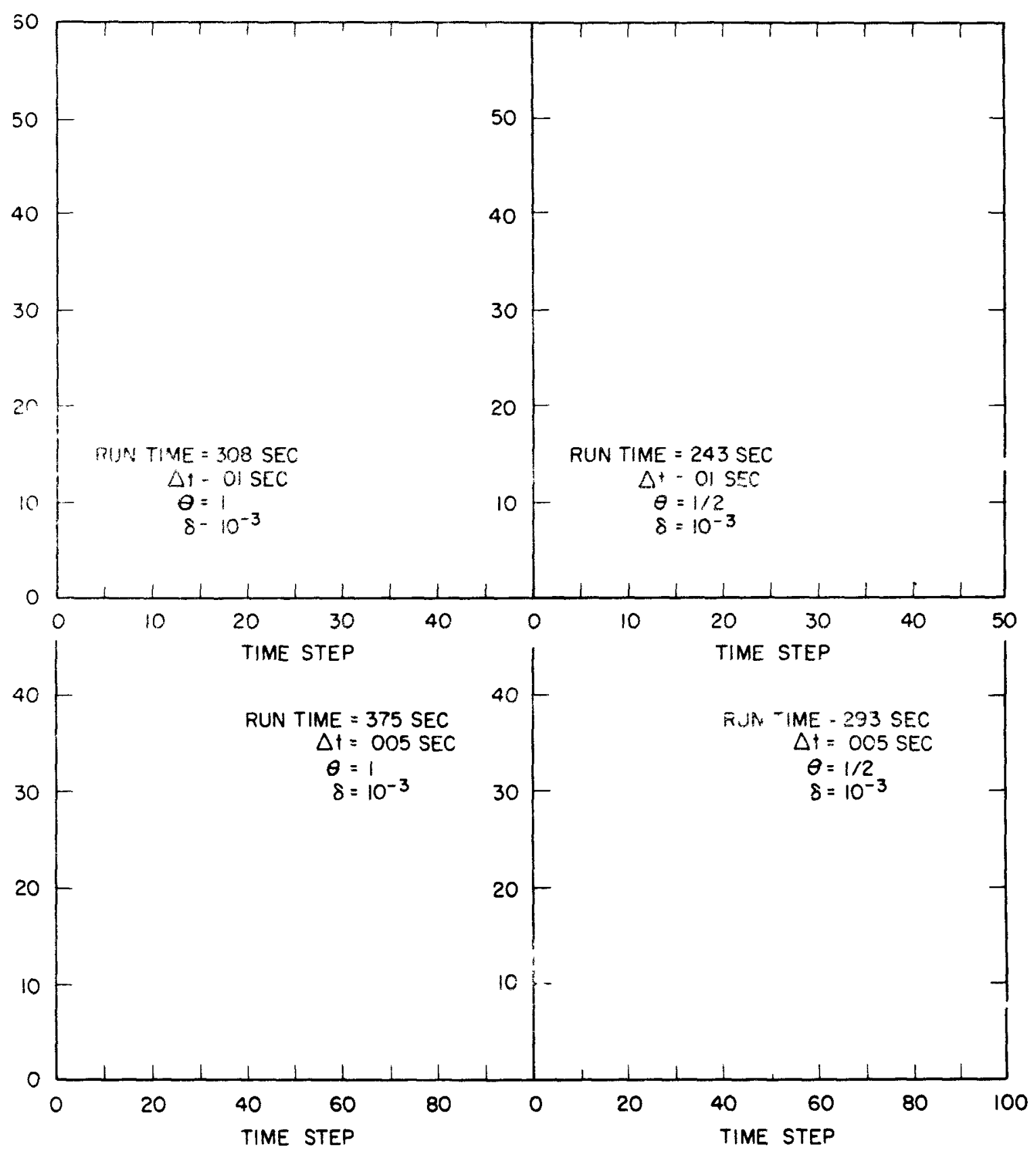

Fig. 3 
Earlier we mentioned that our estimate for the spectral radius, $\sigma$, of the Jacobi matrix is not recomputed each time step. In practice $\sigma$ is normally recomputed only a few times during a problem. Referring again to Fig. 3 , we cite two examples. For the case with $\Delta t=0.01$ sec and $\theta=1$, $\sigma$ was computed only at time steps 1 and 20. For the case $\Delta t=0.005 \mathrm{sec}$ and $\theta=1$, $\sigma$ was computed at time steps $1,3,4,5$ and 30 .

Next we examine the question of accuracy of the time differencing scheme for the delayed supercritical problem. For "model problem" conditions, and "sufficiently EmalI" values of $\Delta t$, one can prove that the use of one value of $\theta$ is better than another. Hovever, for realistic reactor geometries and practical sized time steps our only recourse is to examine the question of accuracy by comparing numerical solutions. For such comparisons, an "exact" solution is obtained by solving the same problem with smaller and smaller values of $\Delta t$, until the fluxes stop changing (within the error band implied by the value of $\delta$ used) with changing $\Delta t .8=10^{-4}$ was used in determining the "exact" time differenced solution.

The delayed supercritical problem was then solved using $\Delta t=0.0025,0.01$ and $0.1 \sec$ for $\theta=1$ and 0.5

Tabie II shows the time variation of the thermal flux at mesh points (rxio, $z=17)$ and $(r=9, z=9)$ forthe case of $\theta=1$ and $\theta=0.5$. The problem running times are also fiven. Whle examinung the results given in this table we must recall that the use if $\delta=10^{\circ 3}$ implies an uncertainty of the order of one percent or less. This azciuntr far the fact that some of the solutions for $\Delta t=0.01$ are closer to the "exact sciuticn" than are the $\Delta t=0.0025$ results. These comparisons indicate trat Fr $c^{ \pm}=0.01$ sec the solutions with $\theta=1$ on $\theta=1 /$ ? are of quite acceptable accuracy. However, whth $\theta=1 / 2$ we risk the oscillatory behavior that is displayed in the error of the $\theta=I / 2$ sclution for $\Delta t=0.01$. The $\theta=I$ solution with $\Delta t=0.2$ is in error by at most orly about $5 \%$. 
THERMAI FLUX COMPARISONS FOR DELAYED SUPERCRITICAL PROBLEM WITH $\delta=10^{-3}$

$$
\Phi_{2}(10,17)
$$

\begin{tabular}{|c|c|c|c|c|c|c|c|}
\hline \multirow[b]{2}{*}{ Time } & \multicolumn{3}{|c|}{$\theta=1$} & \multirow[b]{2}{*}{ Exact } & \multicolumn{3}{|c|}{$\theta=1 / 2$} \\
\hline & $\Delta t=0.1$ & $\Delta t=0.01$ & $\Delta t=0.0025$ & & $\Delta t=0.0025$ & $\Delta t=0.01$ & $\Delta t=0.1$ \\
\hline 0 & 30.2 & 30.2 & 30.2 & 30.2 & 30.2 & 30.2 & 30.2 \\
\hline 0.05 & & 32.3 & 32.2 & 32.2 & 32.2 & 32.3 & \\
\hline 0.10 & 36.4 & 36.0 & 35.9 & 36.0 & 35.9 & 36.0 & 35.8 \\
\hline 0.1 .5 & & 42.2 & 42.0 & 42.3 & 42.0 & 42.2 & \\
\hline 0.20 & 5.5 & 54.8 & 54.4 & 54.6 & 54.2 & 54.5 & 56.1 \\
\hline 0.25 & & 60.9 & 60.7 & 61.3 & 61.0 & 61.2 & \\
\hline 0.50 & $6 a \cdot 7$ & 02.6 & 62.4 & 62.9 & 62.7 & 62.7 & 65.4 \\
\hline 0.35 & & 63.9 & 63.7 & 64.1 & 64.0 & 64.0 & \\
\hline 0.40 & 65.5 & 65.2 & 64.9 & 65.1 & 65.2 & 6.5 .2 & 64.7 \\
\hline 0.45 & & 66.3 & 66.1 & 66.4 & 66.4 & 66.4 & \\
\hline 0.50 & 67.9 & 67.5 & 67.2 & 67.6 & 67.6 & 67.6 & 68.5 \\
\hline
\end{tabular}

$\begin{array}{cccccccc}0 & 2.17 & 2.11 & 2.11 & 2.11 & 2.11 & 2.11 & 2.11 \\ 0.05 & & 2.75 & 2.74 & 2.74 & 2.74 & 2.75 & \\ 0.10 & 3.87 & 3.82 & 3.81 & 3.83 & 3.81 & 3.82 & 3.80 \\ 0.15 & & 5.76 & 5.72 & 5.76 & 5.73 & 5.75 & 10.2 \\ 0.20 & 10.5 & 9.97 & 9.87 & 9.93 & 9.86 & 9.91 & 11.2 \\ 0.25 & & 11.2 & 11.1 & 11.2 & 11.2 & 11.2 & 12.0 \\ 0.30 & 11.5 & 11.5 & 11.4 & 11.5 & 11.5 & 11.5 & \\ 0.35 & & 11.7 & 11.7 & 11.7 & 11.7 & 11.7 & 11.8 \\ 0.40 & 12.0 & 11.9 & 11.9 & 11.9 & 11.9 & 12.0 & 12.9 \\ 0.45 & & 12.2 & 12.1 & 12.2 & 12.2 & 12.2 & 12.6 \\ 0.50 & 12.5 & 12.4 & 12.3 & 12.4 & 12.4 & 12.4 & \end{array}$

$\underset{\text { Time }}{\text { Run }} \quad 115 \mathrm{sec} \quad 308 \mathrm{sec} \quad 504 \mathrm{sec} \quad 463 \mathrm{sec} \quad 243 \mathrm{sec} \quad 103 \mathrm{sec}$


Figure 4 displays graphically the problem run time versus $\Delta t$ for both the $\theta=I$ and $\theta=I / 2$ solutions of the delayed supercritical problem.

In section III we pointed out that if the Chebyshev acceleration parameters $\alpha_{k}$ and $\gamma_{k}$ are both set equal to unity for all $k$, then the TWIGL program uses the Gauss-Seidel iteration method. Figure 5 shows the number of outer iterations required (at each of the first 15 time steps) for the solution of the delayed supercritical problem by both the cyclic Chebyshev method (TWIGL) and the GaussSeidel method (TWIGL with $\alpha_{k}=\gamma_{k}=1$ ). Figure 6 shows similar results for another problem. These comparisons show that the cyclic Chebyshev method can be of the order of ten times faster than the Gauss-seldel method for solving the neutron group diffusion equations.

Comparisons were made of accuracy and running time for the prompt supercritical problem described in Table I. For a highly supercritical problem such as this a relatively small value of $\Delta t$ is required to insure an accurate time differenced soiution. However, the use of a small $\Delta t$ increases the rate of convergence at each time step and it is found that in such cases the number of outer iterations per time step is ustaily constant. In addition we find that the $\theta=1 / 2$ solutions are significantiy more accurate than the $\theta=1$ solutions - this being expected for the smaller time steps involved. Table III summarizes some of the prompt supercritical probiem results. The flux error given in this table is the error in the peak thermal flux at $t=0.02 \mathrm{sec}$, the transient termination time. We see that a time step of $10^{-3} \mathrm{sec}$ is quite adequate when $\theta=1 / 2$ is used, while this same time step results in almost a $75 \%$ flux error with $\theta=1$.

The TWIGL program considers the effects of changes in both the coolant temperature and the fuel temperature. The neutron equations couple with the temperature equations in a non-linear fashion; however, we have been able to discuss the solution of the neutron equations independent of the temperature 
TOTAL PROBLEM RUN TIME FOR DELAYED

SUPERCRITICAL PROBLEM $(0 \leq t \leq .5$ SEC)

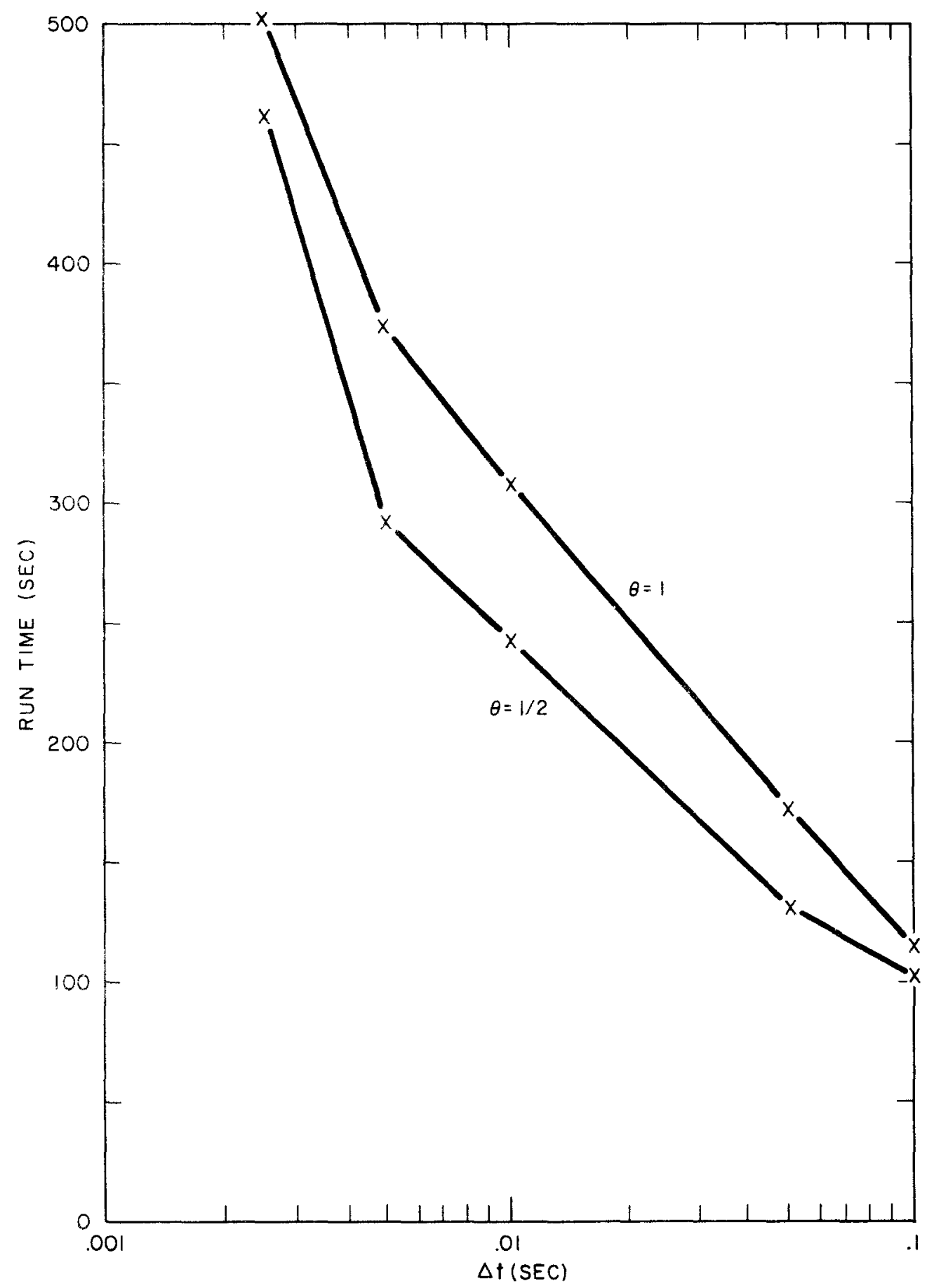

Fig. 4 


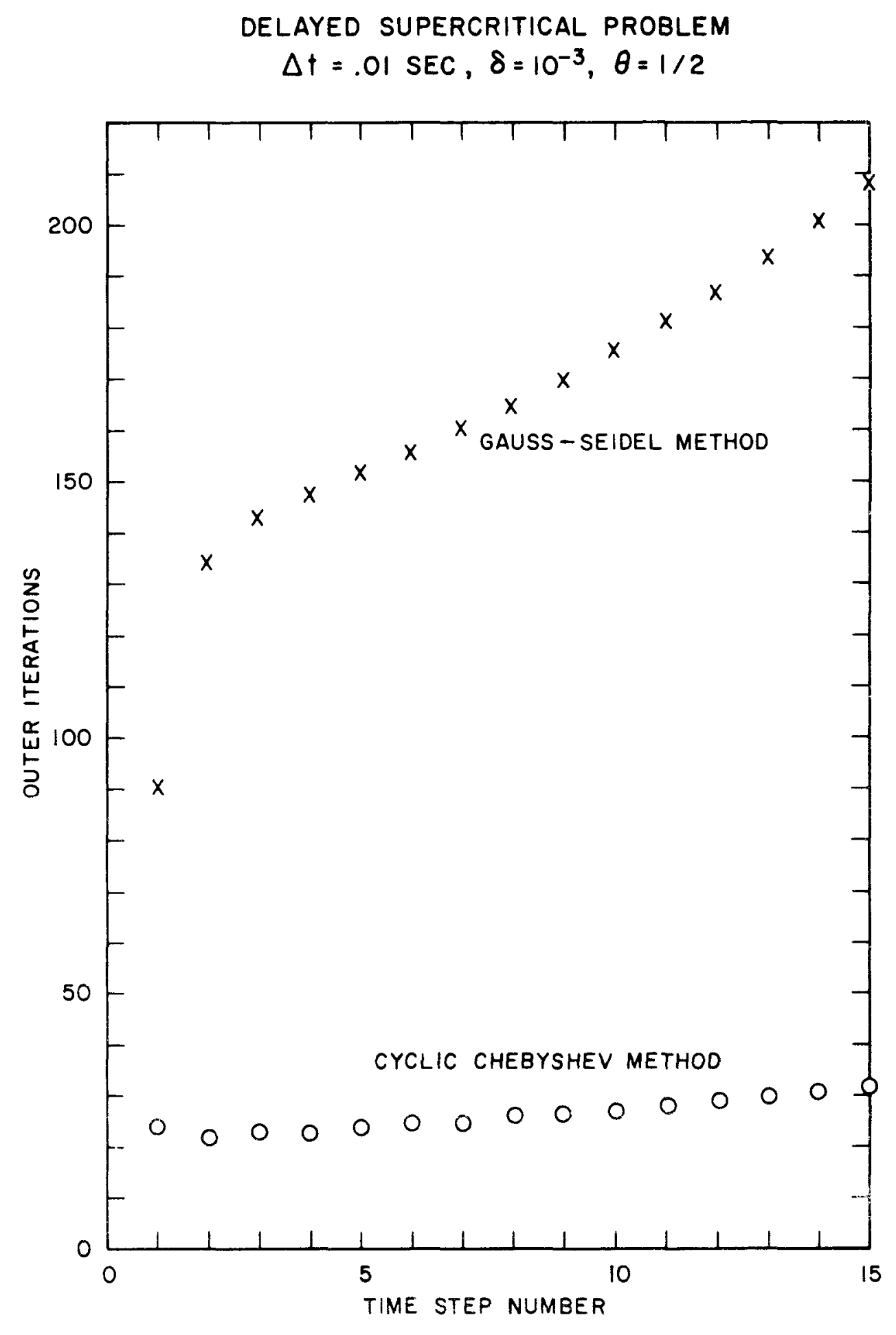

FiE. 5 


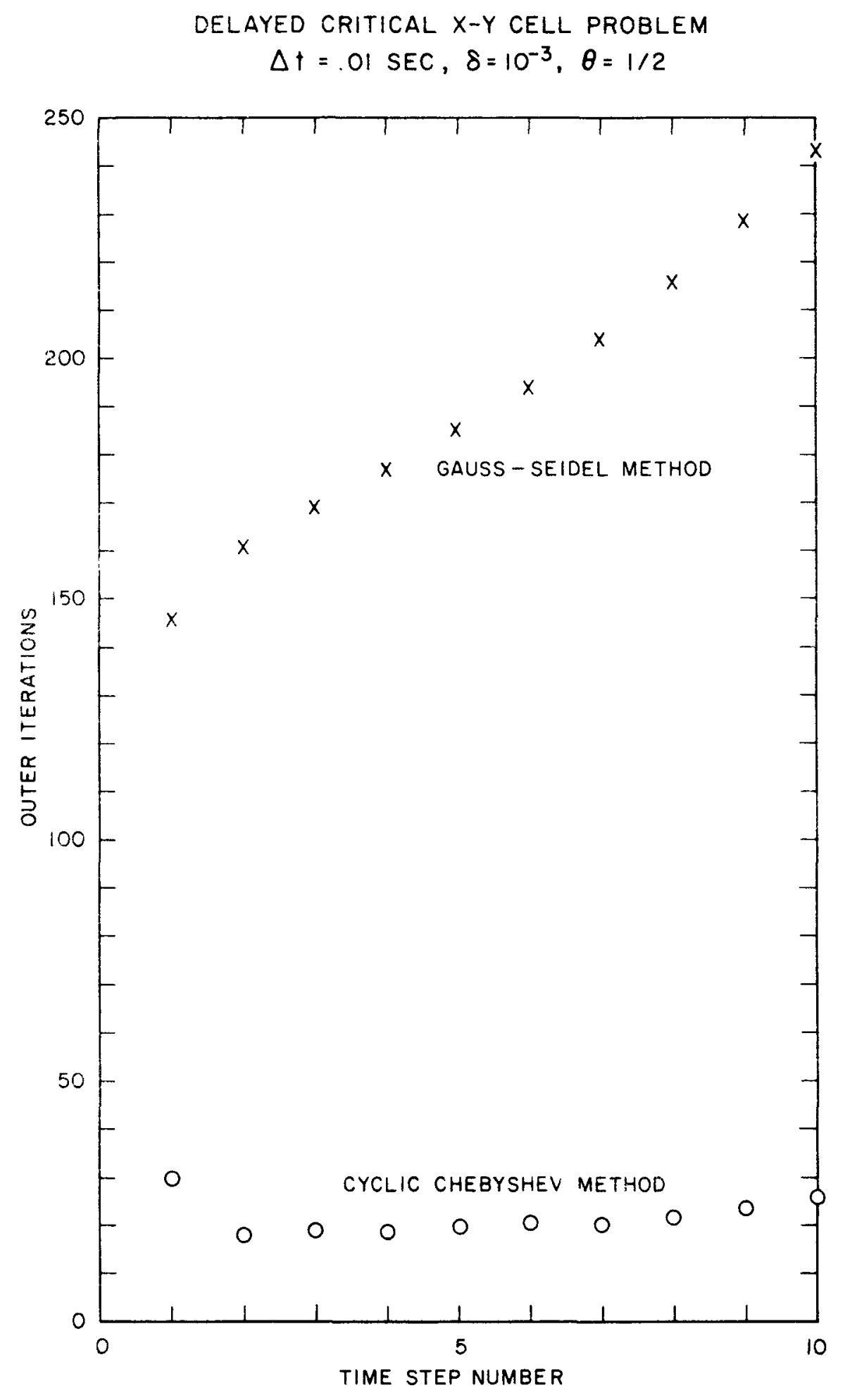

Fig. 6 


\section{TABIE III}

PROMPT SUPERCRITICAL PROBIFM RESULTS

$$
\theta=1 / 2 \text { Problems }
$$

\begin{tabular}{|c|c|c|c|}
\hline Time Step (sec) & Flux Error & Outer Iterations/ $\Delta t$ & Problem Run-Time (sec) \\
\hline $1 \times 10^{-4}$ & $0.00 \%$ & 8 & 401 \\
\hline $2 \times 10^{-4}$ & $0.01 \%$ & 8 & 204 \\
\hline $4 \times 10^{-4}$ & $0.88 \%$ & 1.1 & 132 \\
\hline $1 \times 10^{-3}$ & $2.92 \%$ & 15 & 72 \\
\hline \multicolumn{4}{|c|}{$\theta=1$ Problems } \\
\hline Time Step (sec) & Flux Error & Outer Iterations $/ \Delta t$ & Problem Run-Time (sec) \\
\hline $1 \times 10^{-214}$ & $4.53 \%$ & 8 & 403 \\
\hline $2 \times 10^{-14}$ & $4.8 \times 6$ & 9 & 230 \\
\hline $4 \times 10^{-44}$ & $39.3 \%$ & 13 & 151 \\
\hline$\therefore \times 2=-3$ & $73.8 \%$ & 23 & 103 \\
\hline
\end{tabular}


equations since TWIGL solves these equations in a linear fashion. That is, temperatures are assumed constant over the flux solution time step, and then the flux is assumed constant while the new temperatures are calculated. TWIGL solves for average coolant and fuel temperatures over specified "thermal-hydraulic regions".

For all of the problems discussed in this section the number of inner iterations, per outer iteration, $\mathrm{m}_{\mathrm{g}}$, has been three for both energy groups. For most TWIGL probiems examined to date it has been found that $m_{8}=3$ or 4 is sufficient to reduce the inner iteration error vector to at least 0.05 of its initial value.

Based on empirical results from a CDC-6600 computer, the running time of a

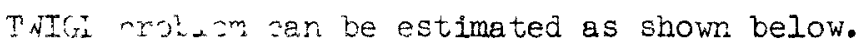

$$
\text { Tun mand }\left(\operatorname{soc}^{1} \sim\left(3 \times 10^{-4}\right) \times \mathbb{N} \times \mathrm{M} \times \text { OI } \times \mathrm{II}+\left(6 \times 10^{-5}\right) \times \mathrm{K} \times \mathrm{mS}\right. \text {, }
$$

where $\mathbb{N}$ and $M$ are the number of fiux solution points in the $x$ (or $r$ ) and $z$ directions, $K$ is the number of thermal hrdraulic resicns specified, TS is the number of trme sters, II is the number of inner iterations per outer iteratior and $C I$ is the tota number of outer iterations required. If the same number of outer iteratius is required at each time step, then OI is just TS times the number of outers per time step. Limitations in TWIGI are $\mathrm{N} \leq 35, \mathrm{M} \leq 35$ and $\mathrm{K} \leq 100$. For example, If $N=M=35$ (-2925 snlution roints) and $K=100$, and 2000 total outer iteratirs tre required for a transient, soiution witr. thres inders per outer, then the problem run time would be

$$
\text { Run Time } \sim[1470+0.6(\text { TS })] \sec .
$$

If 10 outers were used per time step, then we would have used 200 time steps, hence the run-lame would ba about 26 minutes. 


\section{REFERENCES}

1. J. B. Yasinsky, "Combined Space-Time Synthesis with Axially Discontinuous Trial Functions," WAPD-TM-736 (October 1967).

2. K. F. Hansen, "A Comparative Review of Two-Dimensional Kinetics Methods," GA-8169 (August 1967).

3. J. C. Peak, "Reactor Kinetic Considerations in High Temperature Gas Cooled Reactors," Proc. of the Brookhaven Conf. on Indust. Needs and Acad. Research in Reactor Kinetics (Apri? 1968).

4. N. E. Carter, "Review of Physics Methods Used for Computing the Kinetic Behavior of Large Power Reactors," BNWL-738 (January 1968).

5. J. 3. Yasinsky, M. Natelson, I. A. Hageman, "TWIGI-A Prokram to Solve the Two-Dimensional, Two-Group Space-Time Neutron Diffusion Equations with Temperatire Feedback," WAPD-TM-743 (Februarg 1968).

6. J. B. Yasinsky, M. Natelson, L. A. Hageman, Nucl. Sc1. and Eng. 33, 355 (1968).

7. I. A. Hageman, "Numerical Methods and Techniques Used in the Two-Dimensiona? Neutron Diffusion Program PDQ-5," WAPD-TN-364 (February 1963).

8. R. S. Varga, Matrix Iterative Analysig, Prent1ce-Hall, Englewocd Cliffs, N.J. $(=962)$.

9. I. A. Hageman, "The Chebyshev Polynomial Method of Iteration," WAPD-TM-537 $(1967)$.

10. E. L. Wachsprese, Iterative Solution of Elliptic Systems, Prentice-HaII, Englewood Cliffs, N.J. (1966).

11. L. A. Hageman ana C. J. Ffeifer, "The Utilization of the Neutron Diffusion Frogram EDQ $=5$," WAPD TM-395 (1965). 


\title{
NUMERICAL SOLUTION OF THE TWO-DIMENSIONAL TIME-DEPENDENT MULTIGROUP EQUATIONS
}

\author{
W. T. McCormick, Jr. \\ K. F. Hansen \\ Department of Nuclear Engineering \\ Massachusetts Institute of Technology
}

\begin{abstract}
A numerical algorithm for the solution of the two-dimensional time-dependent multigroup neutron diffusion equations is presented. The method assumes that the variation in the neutron flux at each mesh point can be represented as an exponential function of time over each integration time step. Additionaliy, the assumption is made that the transverse leakage in one spatial direction can be approximated by a pointwise transverse buckling over one time step. These assumptions together with an appropriate factoring and integration of the matrix form of the semi-discrete multigroup equations produce a mathematically consistent approximation and an uncorditionaliy stable algorithm. It is also shown, for the special case of a constant step change in reactor properties, that the asymptotic numerical solution is proportional to the asymptotic eigensolution of the semi-discrete multigroup equations. The experimentally observed truncation error is discussed and several numerical experiments are presented which illustrate the accuracy and utility of the method.
\end{abstract}




\section{INTRODUCTION}

The numerical solution of the two-dimensional time-dependent multigroup equations has been obtained by several methods. ${ }^{1,2}$ At present, however, the methods known to the authors are either limited to two energy groups or are restrictive in the approximations made to obtain solutions in reasonable computing time.

Additionally it has recently been shown ${ }^{3}$ that few-group kinetics can, in certain instances, lead to considerable error in computing the time-dependent behavior of the neutron flux. The purpose of this paper is to report on the developments of a multigroup, two-dimensional, fine-mesh method, which is an extension of the GAKIN algorithm ${ }^{4}$ to higher spatial dimensions.

Initially the time-dependent semi-discrete multigroup equations will be formulated in matrix notation and the difficulties in obtaining a solution will be briefly discussed. In section II the proposed numerical algorithm will be derived and shown to be a mathematically consistent approximation. Additionally, the method will be shown to be numerically stable and to possess a desirable asymptotic behavior. In section III several refinements to the basic algorithm are presented as well as a discussion of the selection of the free parameters. Section IV contains a number of numerical experiments to illustrate the general utility and capabilities of the method.

The time-dependent diffusion equations may be written for each of the $G$ neutron energy groups in the form

$$
\begin{aligned}
\frac{I}{v_{g}} \frac{\partial \phi_{g}}{\partial t}= & \underline{\nabla} \cdot D_{g} \nabla \phi_{g}-\sigma_{g} \phi_{g}+\sum_{g^{\prime}=1}^{G} t_{g g^{\prime}} \phi_{g^{\prime}} \\
& +\sum_{i=1}^{I} f_{g i} \lambda_{i} c_{i}+S_{g}, \quad(I \leqslant g \leqslant G)
\end{aligned}
$$


with

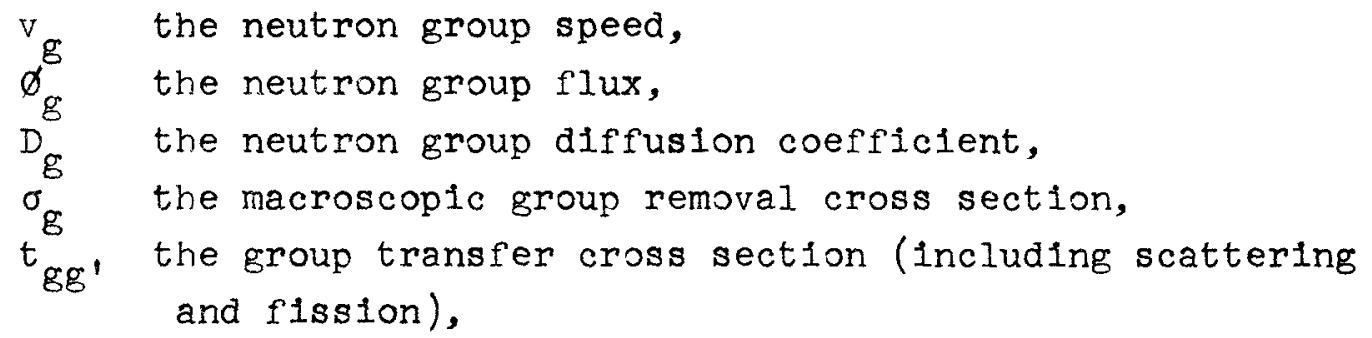

$f_{g 1}$ the delayed neutron energy spectrum from decay of precursor,

$\lambda_{1}$ the precursor decay constant,

$C_{1}$ the precursor concentration,

$\mathrm{S}_{\mathrm{g}}$ the neutron group external source,

The time-dependent equations for each of the I delayed neutron precursor groups may be written

$$
\frac{d C_{1}}{d t}=\sum_{g^{\prime}=1}^{G}\left(\nu \sigma_{f^{\beta}}\right)_{g^{\prime}} \emptyset_{g^{\prime}}-\lambda_{1} c_{1},
$$

with $\left(\nu \sigma_{f} \beta_{1}\right)_{g}$, the yield into delayed group $i$ from fission in neutron energy group $\mathrm{g}$. All of the quantities appearing in Eq. (I) and $\mathrm{Eq}$. (2) except $\lambda_{i}, \beta_{1}$, and $\nu$ may be functions of both space and time.

Defining a $(G+I)$ dimensional vector $\Phi$, of group fluxes and precursor concentrations, the set of equations (1) and (2) may be written collectively in matrix form as

$$
\frac{\mathrm{d} \Phi}{\mathrm{d} t}=\theta \Phi+\underline{\mathrm{R}},
$$

where $\underline{R}$ is the source vector and $\theta$ is a $(G+I)$ by $(G+I)$ dimensional operator which includes all the production, scattering, removal and leakage terms. The above equation can now be further reduced to the so-called semi-discrete form by making the spatial behavior 
discrete on a mesh while leaving the time behavior continuous.

It will be assumed for the present discussion that the reactor is a two-dimensional rectangular slab in $x-y$ geometry, although in general the algorithm is by no means restricted to this geometry. The spatial derivatives in the $x$ any $y$ directions are approximated by standard finite difference relations. The $\underline{\nabla} \cdot \mathrm{D}_{\mathrm{g}} \underline{\nabla}$ operator at mesh point $\mathrm{x}_{\ell}, \mathrm{y}_{\mathrm{K}}(1 \leq \mathrm{K} \leq \mathrm{K}, 1 \leqslant \ell \leqslant \mathrm{L})$, is replaced by the second central difference operator

$$
\frac{\delta D_{g} \delta}{h_{x}{ }^{2}}+\frac{\delta D_{g} \delta}{h_{y}^{2}},
$$

where $h_{x}$ and $h_{y}$ are the mesh spacings in the $x$ and $y$ directions respectively. This particular difference approximation in space is well known to be consistent and accurate to order $h_{x}{ }^{2}$ and $h_{y}{ }^{2}$. $A(G+I) K L$ dimensional discrete solution vector $\psi$ of group fluxes and precursors are defined, and the spatially discrete form of Eq. (3) becomes

$$
\frac{d \underline{\psi}}{d t}=A \underline{\psi}+\underline{S}
$$

with $A$ a $(G+I) K L$ by $(G+I) K L$ dimensional matrix and $S$ a $(G+I) K L$ dimensional source vector. The matrix $A$ may be written as

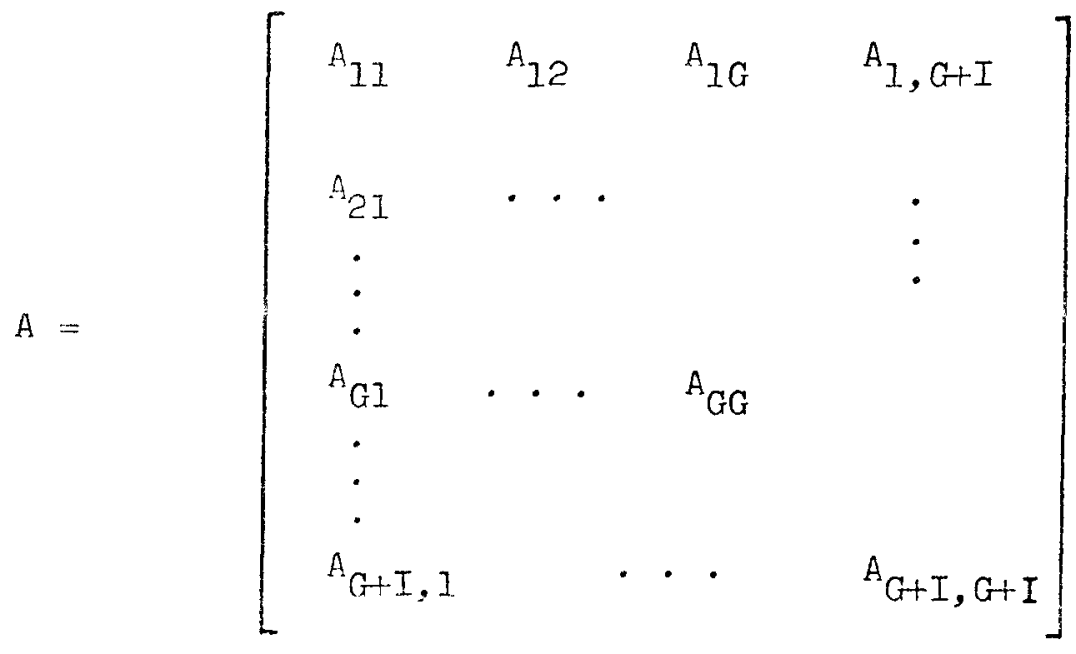


where each $\mathrm{A}_{\mathrm{gg}}$ is a $\mathrm{KL}$ by $\mathrm{KL}$ dimensional square submatrix. The special form and constituent elements of these submatrices are described below.

$$
A_{g g}=v_{g}\left[\frac{\delta D_{g} \delta}{h_{x}^{2}}+\frac{\delta D_{g} \delta}{h_{y}^{2}}+t_{g g}-\sigma_{g g}\right] \quad(1 \leqslant g \leqslant G),
$$

with block tridiagonal form.

$$
A_{g g^{\prime}}=v_{g^{t}} g_{g^{\prime}}, g \neq g^{\prime}, \quad\left(1 \leqslant g, g^{\prime} \leqslant G\right),
$$

with diagonal form.

$$
A_{g, G+i}=v_{g} f_{g i} \lambda_{1}, \quad(1 \leqslant i \leqslant I, I \leqslant g \leqslant G),
$$

W: diagonal form.

$$
A_{G+1, g}=\left(\nu \sigma_{f}^{\beta}{ }_{i}\right)_{g}, \quad(I \leq 1 \leq I, I \leq g \leq G),
$$

with diagonal form.

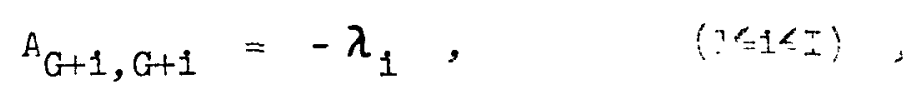

with diagonal form.

$$
A_{G+1, G+I^{\prime}}=0, \quad\left(1 \leq 1,1^{\prime} \leq I\right)
$$

with null form.

From the definitions of the elements of 4 , it is observed that, In general, $A$ is an irreducible matrix with all off-diagonal elements non-negative. Thus, $A$ is an essentially positive matrix ${ }^{5}$ and can be shown to have a largest eigenvalue whose corresponding

80 
eigenvector has positive components. It can also be shown for the case where $A$ and $\underline{S}$ are constant with time that $\mathrm{Eq}$. (4) possesses the analytic solution

$$
\underline{\psi}(t)=\exp (t A) \underline{\Psi}(0)+A^{-1}[\exp (t A)-I] \underline{S},
$$

where $\exp (t A)$ is defined as

$$
\exp (t A)=I+t A+\frac{t^{2}}{2 !} A^{2}+\ldots
$$

The evaluation of the above exponential to a sufficient number of terms is prohibitively expensive; therefore, approximate numerical methods are required. Defining $\psi^{j}=\Psi\left(t_{j}\right)$ and a time interval $h=t_{j+1}-t_{j}$, the analytic solution given in Eq. (6) over a time step is simply

$$
\Psi^{J+I}=\exp (h A) \Psi^{J}+A^{-I}[\exp (h A)-I] \underline{S}^{J}
$$

The solution to equation (4) may be approximately obtained by approximating the series given in Eq. (7). One example is formed by simply truncating the series and is called the explicit method,

$$
\underline{\Psi}^{J+1}=(I+h A) \underline{\Psi}^{J}+h \underline{S}^{J}
$$

Another approximation is the implicit method given by

$$
\Psi^{\mathrm{J}+1}=(I-\mathrm{hA})^{-1} \underline{\psi}^{J}+\mathrm{h} \underline{S}^{J}
$$

It has been shown ${ }^{6}$ that the explicit method encounters conditional stability requirements due to the large negative elgenvalues of $A$. This results in necessarily taking very small time steps to obtain any solution at all, and renders the method impractical. On the other hand, although the implicit method may easily be shown to be unconditionally stable it has the 
computational disadvantage of requiring the inversion of a very large matrix. Additionally, if the matrix properties change with time, then an inversion at each time step is needed, and the method becomes computationally unfeasible. It is evident that there is a tradeoff between numerical stability and computational advantage per step, and thus a semi-implicit method is now presented for solution of the multigroup equations.

\section{THE PROPOSED ALGORITHM}

Thus far the original multigroup differential equations have been reduced to a semi-discrete matrix equation which is written in the form

$$
\frac{\mathrm{d} \underline{\psi}}{\mathrm{dt}}=\mathrm{A} \underline{\psi}+\underline{S}
$$

with $\Psi$ the solution vector. The matrix A can now be split into 5 matrices of the same order and Eq. (11) becomes

$$
\frac{d \underline{\Psi}}{d t}=(I+U+H+V+r) \underline{L}+\underline{S}
$$

with I a strictly block lower triangular matrix given as

$I=\left[\begin{array}{lll}0 & & 0 \\ A_{21} & 0 & \\ A_{31} & A_{32} & \\ A_{G+I, I} & \cdots & A_{G+I, G+I-I}\end{array}\right]$,

U a strictly block upper-triangular matrix given as 


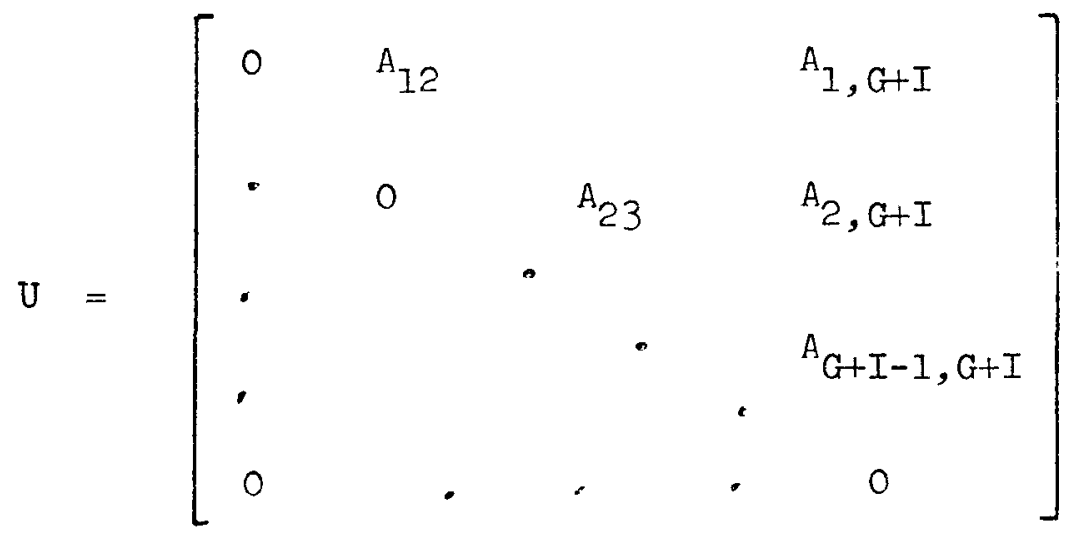

and $\Gamma, \mathrm{H}$ and $\mathrm{V}$ block diagonal matrices given as
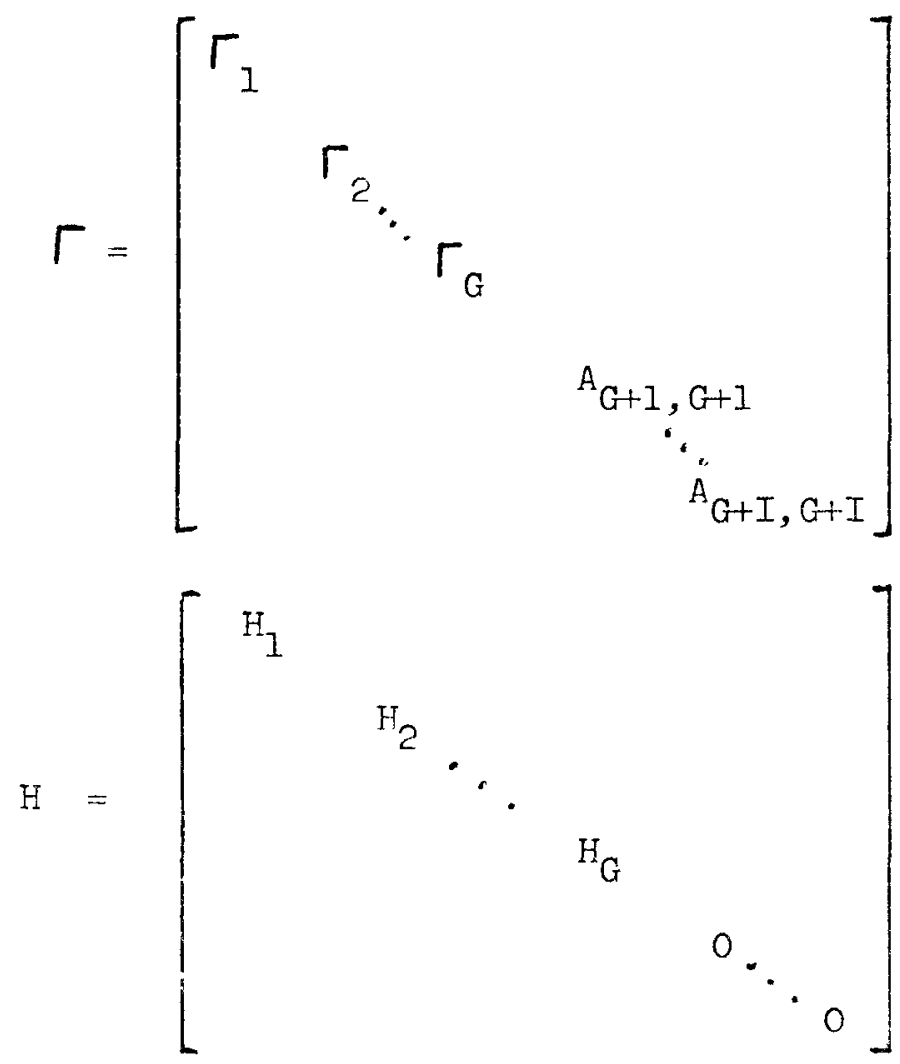


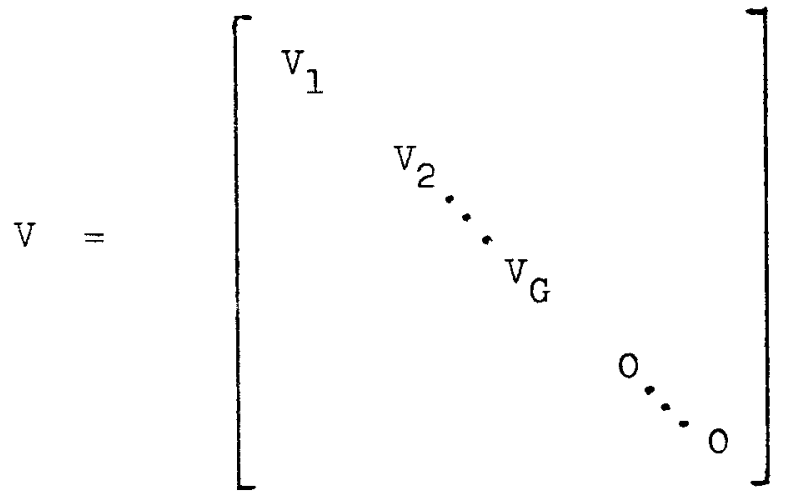

The KL by KL dimensional submatrices $\Gamma_{g}, H_{g}$ and $V_{g}$ are defined as

$$
\begin{array}{ll}
H_{g}=v_{g} \cdot \frac{\delta D_{g} \delta}{h_{x}^{2}} & (1 \leqslant g \leqslant G), \\
v_{g}=v_{g} \cdot \frac{\delta D_{g} \delta}{h_{y}^{2}} & (1 \leqslant g \leqslant G), \\
r_{g}=v_{g}\left[t_{g g}-\sigma_{g}\right], & (1 \leqslant g \leqslant G),
\end{array}
$$

and therefore

$$
A_{g g}=H_{g}+\Gamma_{g}+V_{g} .
$$

All the above submatrices are diagonal except the $\mathrm{H}_{\mathrm{g}} \mathrm{s}$ and the $V_{g ' s}$ which both contain 3 stripes. The integration of $\mathrm{Eq}$. (12) may be accomplished by making a simple anproxtmation which ylelds a stable numerical algorlthm.

The diagonal matrix $Q$ is defined such that over one time step

$$
\mathrm{Q} \underline{\psi}=\mathrm{V} \underline{\Psi} \text {. }
$$


This approximation corresponds to replacing the transverse leakage in the vertical direction by an effective pointwise buckling.

Eq. (12) may then be written as

$$
\frac{d \underline{\psi}}{d t}-(\Gamma+Q) \underline{\psi}=(I+U+H) \underline{\psi}+\underline{S}
$$

Letting $G=\Gamma+Q$ and assuming all the above matrix elements are constant over the time interval $h=t_{j+1}-t_{j}, E q$. (14) may be integrated to obtain

$$
\begin{aligned}
\Psi^{j+1} & =\exp (G h) \underline{\Psi}^{j}+\int_{0}^{h} d \xi \exp [G(h-\xi)](I+U+H) \underline{\psi}\left(t_{j}+\xi\right) \\
& +G^{-1}[\exp (G h)-I] \underline{S}^{j} .
\end{aligned}
$$

To proceed, some assumption must be made concerning the behavior of $\Psi\left(t_{j}+\xi\right)$ over the time interval $h$. For the moment, let $1 t$ be assumed that the reactor is bare, homogeneous and uniformly perturbed. This assumption is really not necessary but is only used here so that the following derivation may be easily written in matrix form. It is now reasonable to suggest that over the time interval the flux will vary exponentially with time, that is,

$$
\Psi\left(t_{j}+\xi\right)=\exp (\omega \xi) \Psi^{J}
$$

and

$$
\underline{\psi}\left(t_{j}+\xi\right)=\exp [-\omega(h-\xi)] \Psi^{j+1},
$$

with $\omega$ a scalar quantity that is chosen at each time interval to reduce the truncation error. The selection of this parameter ard its generalization to a matrix will be discussed in section III. Integration of $\mathrm{Fq}$. (15) nay now be performed using FC. $(16 a)$ for $\Psi\left(t_{j}+\xi\right)$ in the cases where it multiplies the matrices 
$I$ and $U$ and using Eq. ( $16 \mathrm{~b}$ ) in the case where it multiplies the matrix $H$. This has the result of making the algorithm implicit in the $H$ operator and gives the form

$$
\begin{gathered}
{\left[I-(\omega I-G)^{-1}\{I-\exp [(G-\omega I) h]\} H\right] \psi^{j+I}} \\
=\left[\exp (G h)+(\omega I-G)^{-1}\{\exp (\omega h I)-\exp (G h)\}(I+U)\right] \Psi^{j} \\
+G^{-1}[\exp (G h-I)] \underline{S}^{j},
\end{gathered}
$$

or more simply,

$$
\mathrm{F}_{1} \underline{\psi}^{j+1}=\mathrm{F}_{2} \underline{\psi}^{j}+\mathrm{F}_{3} \underline{S}^{j},
$$

whare the definitions of $F_{1}, F_{2}$ and $F_{3}$ are evident from $E$. (17). To metrix $E_{\perp}$ is tridiagonal and may he partitioned in the f orm

$F_{I}=\left[\begin{array}{ccccc}I_{I} & & & & \\ & E_{2} & & 1 \\ & & E_{G} & 0 \\ \cdots & \cdots & & I\end{array}\right]$

with each matrix $E_{G} \quad a \mathrm{KL}$ by $\mathrm{KL}$ dimensional tridiagonal matrix. The unit matrix has dimensions IKL by IKL and represents the precursor concentration equations. Each matrix $E_{g}$ may easily be shown to be diagonally dominant with positive diagonal and non-postive off-dlagonal elemerts and thus possesses a positive inverse.

It may also be easily shown that the entire matrix $F_{1}$ has a non-negative inverse. The algorithm may now be written in the form

$$
\underline{\Psi}^{j+1}=\mathrm{B}_{\mathrm{v}} \underline{\Psi}^{j}+\mathrm{P}_{\mathrm{v}} \underline{S}^{j},
$$


where $B_{v}$ is a non-negative matrix since both $F_{I}^{-1}$ and $F_{2}$ are non-negative. The matrix $B_{V}$ is also readily seen to be irreducible and since it has positive diagonal entries it consequently is also primitive. 8 From these properties of the matrix $B_{v}$ the algorithm is numerically uncondftionally stable in the sense that for all reactor properties and integration time step sizes, the solution vector will always be non-negative and can therefore never oscillate in sign. Additionally, by the theorem of Perron and Frobenius ${ }^{9}, B_{v}$ possesses a positive real simple elgenvalue $\rho_{o}$, equal to its spectral radius, and a corresponding positive ejgenvector. For the case of a step change in reactor properties, sussessive operation of $B_{v}$ on a solution vector repeatedly reduces all components of the solution along the directions of the eigenvectors corresponding to the smaller eigenvalues until the single eigensolotion remains. That is, the asymptotic solution is exactly proportional to the eigenvector corresponding to the largest eigenvalue of $B_{v}$.

The subscript $v$ appearing on the advancement matrix $B_{V}$ Indicates the transverse pointwise buckling approximation was in the vertical direction. However, a derivation entirely equivalent to that which led to $\mathrm{Eq}$. (19) may be performed to obtain an advancement matrix $B_{H}$ and a $\mathrm{P}_{\mathrm{H}}$ using an approximation of the transverse leakage in the horizontal direction. These matrices may be used to compute the solution vector at the next time step, that is,

$$
\Psi^{J+2}=B_{H} \Psi^{j+1}+P_{H} S^{j+1}
$$

Equatiois (19) and (20) taken as a continucus alternation scheme when combined with the selection of the free parameter $\omega$ are 
used to minimize the truncation error of the method. So far in the preceeding discussion nothing has been said about which values of the flux over the time step $h$ are used in the calculation of the transverse buckling in the matrix $Q$. As a first approximation $1 t$ will be assumed that $Q$ during the interval of time from $j$ to $j+l$ will be just those values computed using the flux at time $f$, that 15 , the transverse buckling will be constant over the step $h$ and equal to that value at the beginning of the interval. Using this method of calculating Q, it will now be shown that the algoritrms of elther equation (19) or (20) are mathematically consistent approximations; that is, they agree with the exact solution $\Psi^{j+1}=e^{A h} \Psi^{j}$ through at least the order $h$ term in the expansion of $e^{A h}$. This will be accomplished by simply letting the advancement matrix $B_{V}$ operate on $\Psi^{j}$. Using the form of $\mathrm{Eg}$. (17) without sources

$$
\begin{gathered}
B_{\underline{I}} \underline{\Psi}^{j}=\left[I-(\omega I-G)^{-I}\left(I-e^{(G-U I) h}\right) H\right]-I . \\
{\left[e^{G h}+(\omega I-G)^{-1}\left(e^{w I h}-e^{G h}\right)(I+U)\right] \Psi^{J}}
\end{gathered}
$$

Expanding and retaining terms through order $h^{2}$ gives

$$
\begin{aligned}
& B \Psi^{j}=\left[I+h\{H+G+I+U\}+h^{2}\left\{-\frac{1}{2} w H+\frac{1}{2} G H+H G\right.\right. \\
& \left.\left.+\frac{G^{2}}{2}+\frac{W}{2}(I+U)+\frac{G}{2}(I+U)+H(I+U)\right\}+\ldots\right] \Psi^{j} .
\end{aligned}
$$


The intermittent expansion of inverse matrices are valid since consistency is show in the limit as $\mathrm{h}$ is taken arbitrarily small. Now, recalling that $\mathrm{V} \underline{\psi}^{\mathrm{J}}=\mathrm{Q} \underline{\Psi}^{\mathrm{J}}$ and $\mathrm{G} \underline{\psi}^{\mathrm{J}}=\mathrm{V} \underline{\psi}^{\mathrm{J}}+\Gamma \underline{\Psi}^{\mathrm{J}}$, the above equations may be written

$$
\mathrm{B} \underline{\Psi}^{j}=\left\{I+h(\mathrm{H}+\mathrm{V}+\Gamma+I+\mathrm{U})+O\left(h^{2}\right)\right\} \Psi^{j}
$$

or

$$
B \Psi^{j}=\left\{I+h A+o\left(h^{2}\right)\right\} \Psi^{j} .
$$

It is now quite apparent that the expanded form of the algorithm in Eg. (21) does in fact agree with the exact solution $e^{A h} \frac{\psi^{j}}{2}$ through terms of order $h$ and has truncation error of order $\overline{h^{2}}$. It may also be shown that the agreement with the exact solution in the source term is also through order $h$, and the truncation error is in the $h^{2}$ term.

Now the asymptotic properties of the algorithm will be examined for the case of a step change in reactor properties excluding external sources. The governing matrix equation is again

$$
\frac{d}{d t}=A \Psi
$$

where $A$ is essentially positive and thus has a largest real eigenvalue $\rho_{0}$. Since $A$ is not a function of time, the analytic solution may be expanded in terms of the eigenvectors $\underline{u}_{n}$ as

$$
\Psi(\text { analytic })=\sum_{n} a_{n} e^{\omega_{n}^{t}} \underline{u}_{n}
$$

$$
n=0,1 \ldots(G+I) K I-I
$$

where $u_{n}^{\prime}$ are the corresponding eigenvalues. It. is clear that asymptotically the persisting solution is $a_{0} e^{w_{0} t_{u_{0}}}$ where $u_{0}^{\prime}$ is the largest eigenvalue of $A$. To examine the asymptotic 
properties of the numerical algorithm, the effect of operating on $\underline{u}_{0}$ must be determined. So,

$$
\begin{aligned}
B \underline{u}_{0}= & {\left[I-(\omega I-G)^{-1}\{I-\exp [(G-\omega I) h]\} H\right]^{-I} \cdot } \\
& {\left[\exp (G h)+(\omega I-G)^{-1}\{\exp (\omega I h)-\exp (G h)\}(I+U)\right] \underline{u}_{0} }
\end{aligned}
$$

Since

$$
A \underline{u}_{0}=w_{0} \underline{u}_{0}
$$

or

$$
(I+U) \underline{u}_{0}=\left(U_{0} I-G-H\right) \underline{u}_{0} .
$$

Substituting Eq. (25) into Eq. (24) and reducing ylelds

$$
B \underline{u}_{0}=\exp \left(\omega_{0}^{h I}\right) \underline{u}_{0} \text {. }
$$

So it is founo that exp $\left(\omega_{0}^{\prime} h I\right)$ is an eigenvaiue of $B$ and $u_{0}$ is its corresponding eigenvector. Thus the numerical asymptotic solution is proportional to the analytic asymptotic solution if $\exp \left(u_{0} h\right)$ is the largest eigenvalue of $B$. This can easily be verifled in a manner equivalent to that for the one dimensional case. 10

Besides being consistent, stable, and raving desirable asymptotic properties, the algorithm has considerable calculational attractiveness, since computing either $B_{\mathrm{H}}$ or $\mathrm{B}_{\mathrm{V}}$ requires only an inversion of a tridiagonal matrix which is linearly related to the number of unknowns in the solution vector.

\section{MODIFICATION TO THE BASIC ALGORITHM}

Before describing the tecrnique for selfocirig the free parameter $w$, several modifications of the basic method will be presented.

Since all the energy coupling between neutron energy groups is handled explicitly, the inversion of the matrix $F_{1}$ in Eq. (18) is really accomplished by inverting independently the $G$ tridiagonal matrices that constitute $F_{1}$. Thus, in computing the 
fluxes at each time step the group fluxes are calculated successively one after another from highest energy to lowest, always using the old values of the flux on the RHS of $\mathrm{Eq}$. (18). Another procedure might be to use the most current values of the flux in the previously computed higher energy groups when computing the flux in a lower group. A third procedure which might he expected to be more accurate than e1ther, would be to use some welghted average of the new and old values. In this case the matrix $A$ is split in the form

$$
A=G+[(1-\alpha) I+U]+[d L+H],
$$

with $\alpha$ a constant weighing factor. Eq. (19) would then be

$$
\begin{aligned}
& {\left[I-(\omega I-G)^{-I}\{I-\exp [(G-\omega I) h]\} H\right] \underline{\psi}^{j+1} } \\
&= {\left[\exp (G h)+(\omega I-G)^{-1}\{\exp (\omega h I)-\exp (G h)\} .\right.} \\
&\{(I-\alpha) I+U\}] \Psi^{j}+[\exp (-\omega h I)] \propto L \underline{\psi}^{j+I} \\
&+G^{-1}[\exp (G h)-I] \underline{S}^{j} .
\end{aligned}
$$

The modified algorithm given in $\mathrm{Eq}$. (27) retains all the previously mentioned properties of the original algorithm and has been observed to give smaller truncation error, especially in large step change proilems. $\quad \alpha=\frac{1}{2}$ seemed to be a near optimal weighting.

The use of a single free parameter $w$ could obviously be restrictive since the flux in different regions of a system may respond at different rates to a localized perturbation. The second modification 18 to extend the method to use a alagonal matrix $\Omega$, of pointwise free parameters. For the purposes of integrating Eq. (15) the fluxes are now assumed to berave as

$$
\Psi\left(t_{j}+\xi\right)=\exp (\xi \Omega) \Psi^{j}
$$


and

$$
\Psi\left(t_{j}+\xi\right)=\exp [-(h-\xi) \Omega] \psi^{j+1} .
$$

Since exp $(\Omega \xi)$ is no longer a constant diagonal matrix, 1.e. a scalar, it does not commute with $H, I$ or $U$ and thus Eq. (15) must be integrated term by term. The form of the general algorithm is now given as

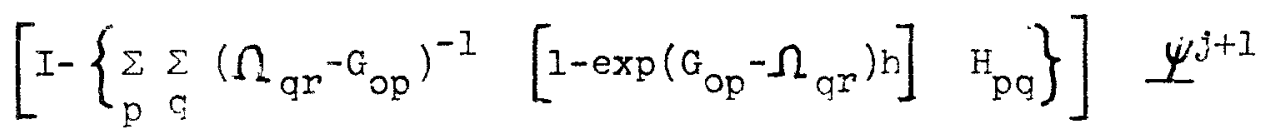

$$
\begin{aligned}
& =\left[\exp (G h) \psi^{j}+\left\{\sum_{p} \sum_{q}\left(\Omega_{q r}-G_{o p}\right)^{-1} .\right.\right. \\
& \left.\left.\left[\exp \left(\Omega_{a n} h\right)-\exp \left(G_{o p} h\right)\right] \cdot\left[(1-\alpha) I_{p a}+U_{p q}\right]\right\}\right] \psi^{j}
\end{aligned}
$$

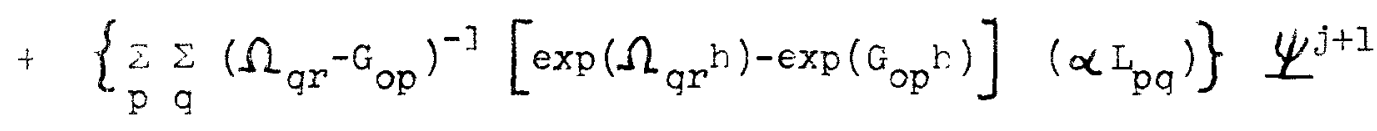

$$
\begin{aligned}
& +\mathrm{G}^{-1}[\exp (\mathrm{Gr})-I] \mathrm{S}^{j},
\end{aligned}
$$

where the braces indicate a matrix whose $o, r^{\text {th }}$ element is given by tre indicated summations. The final form of the algorithm may be written as

$$
\dot{\psi}^{j+1}=B^{\prime}(\Omega) \underline{\varphi}^{j}+\mathrm{Y} \underline{\Sigma}^{j}
$$

where $f^{\prime}(2)$ has all of the previously mentisned matrix properties as $B_{H}$. This form of the method would allow the use of a different $\Omega_{i}$ at each space point and in each energy group. It has been shown ${ }^{11}$ that for thermal reactors only the spatial frequencies in the thermal controlling group are necessary for good accuracy. However, in a fast reactor where the importance of the energy groups are more equal it may often be necessary to use group 
dependent as well as space dependent frequencies in the very early part of a transient. The results quoted here are principally for thermal reactors, hence the spatially dependent frequencies in the thermal group are also used for the other energy groups.

A final modification of the algorithm involves the calculation of the matrix $Q$ of the transverse pointwise bucklings. It would seem Iikely that the best values of the pointwise bucklings would be those computed using some weighted average of the bucklings at the beginning and at the end of the time interval $h$, that is,

$$
Q=W Q^{j}+(1-W) Q^{j+1},
$$

with

$$
\mathrm{Q}^{j} \Psi^{j}=\mathrm{V} \underline{\Psi}^{j}
$$

and

$$
Q^{j+1} \underline{\Psi}^{j+1}=V \underline{\psi}^{j+1}
$$

If the reactor flux shape does not change, as is the case in an asymptotic reactor, or in a bare homogeneous uniformly perturbed reactor, then $Q^{j}=Q^{j+I}$. At the beginning of the time step, however, $\psi^{j+1}$ is not known but can be approximated by $e^{\Omega h} \psi^{j}$, so that

$$
2^{j+1} e^{\Omega h} \psi^{j} \approx V e^{\Omega h} \psi^{j} .
$$

Thus a better value of $Q$ may be evaluated f'rom Eq. (30), at least approximateiy.

'To determine the free parameters initially, some estimate of each $\Omega_{i}^{j}$ is chosen. These estimates are then used in Eq. (29) to carry out a time step. A check is then made to determine if the thermal flux at particular test points did in fact change exponentially with time as assumed. That is, if the expression

$$
\left|\exp \Omega_{r, s}^{j}-\Psi_{r, s}^{j+1} / \Psi^{j} r, s\right|<\text { tolerance }
$$


is satisfied for all test points $(r, s)$. Then new values of the free parameters are calculated from

$$
\Omega_{r, s}^{j+1}=\frac{1}{h} \ln \left(\underline{\psi}_{r, s}^{j+1} / \underline{\psi}_{r, s}^{j}\right),
$$

and these values are used in Eq. (28) for the next time step. If relation (3I) is not satisfied for all test points, new values of the $\Omega_{i}{ }^{\prime}$ s are calculated at every point from Eq. (32) and the calculation of the time step is repeated. This method of determing the free parameter converges rapidly in most cases, and in many instances iteration is not necessary for every time step.

\section{NTMERICAT RESUITS}

A variety of numerical experiments have been carried out to test the accuracy and the speed of the method. The observed $t$ : crisation error is primarily controlled by the allowed flux chanra per time step and the magnitude of the tolerance given in - . (31). The flux change per step is given approximately by Fie pisduct oi the largest frequency, $\Omega$ max, and the time interval, h. For best results it was found that the percentage change, $\Omega \max h$, should not exceed about $1 \%$ except when the system is approaching an asymptotic behavior. With this percentage change, and a tolerance about 100 times smaller $1 . e .10^{-4}$, most transients could be analyzed with an accuracy of about $2 \%$ or less over a flux change of a factor of 10 . For greater accuracy one would merely reduce the time step, hence the percentage growth, and correspondingly reduce the tolerance.

The efficiency of the method is defined as the number of acconter stops divided by the number of attempted steps, where the accectance of a step is determined by whether relation (31) is satisfied. Dursing the very early parts of transients when the frequencies change rapidly with time, the efficiency is often as Iow as 20 to $30 \%$. However, as a transient progresses the efficiency rises rapidly until the overall efflciency for a transient to asymptotic may be $60 \%$ or more. It was found that best results could be obtained by taking less than $1 \%$ change over 
the very early part of the transient. Thus, as the efficiency increased, the time step $h$ could also be increased and when the system became asymptotic as large a time step as desired could be taken.

In reporting the number of calculational steps for the following results, the total number of attempted steps have been recorded, and the thermal neutron flux at the center point in the reactor is given. It was found that the error in the other neutron energy groups was very nearly the same as the error in the group reported.

The results for the bare homogeneous, uniformly perturbed reactors were compared with an analytic solution obtained by finding the elgenvalues and eigenvectors of the matrix $A$ and expanding the initial conditions as given in Eq. (23). Table I gives the results for four Two-Neutron Group and One-Delayed Group problems. There are two delayed critical problems of approximately $40 \notin$ and $80 \notin$, a subcritical problem of $-50 \not$, and a prompt critical one of about $\$ 1.20$. In all of these problems there was a mesh spacing of $15 \mathrm{~cm}$. and there were 11 mesh points in each direction. Also shown are the tolerances used and the actual total computer time required on the IBM $360 / 65$, including the printing of the results. Table II shows the results for a 2-Group, 6-Delayed Group problem with about +80e reactivity and also a 4 neutron group, 1 delayed-group problem with about $+50 \not$ reactivity. The 4 group problem is a fast reactor experiment as is evident from the very rapid prompt jump. In the two-group problem the thermal flux is reported and in the four-group problem the third highest energy group flux is given. Again the flux values are taken at the geometrical center of the reactors. To obtain a correlation between the temporal truncation error and the allowed maximum percentage growth, $\Omega_{\max } h$, the two-group onedelayed Eroup +80 reactivity case was run for several cholces of the $\Omega_{\max }{ }^{h}$ and corresponding tolerances. 


\section{TABLE I}

NUMERICAL RESULTS FOR TWO-GROUP, ONE-DELAYED CASE

\begin{tabular}{|c|c|c|c|c|c|c|c|}
\hline \multicolumn{2}{|c|}{$\rho=+40 d$} & \multicolumn{2}{|c|}{$\mathrm{EPI}=.2 \times 10^{-4}$} & \multicolumn{2}{|c|}{$\rho=+80 \notin$} & \multicolumn{2}{|c|}{$\mathrm{EPI}=.4 \times 10^{-4}$} \\
\hline Time (sec) & Steps & Flux & \% Error & Time (sec) & Steps & Flux & \% Error \\
\hline .000 & 0 & .382 & 0.0 & .000 & 0 & .382 & 0.0 \\
\hline .050 & 83 & .444 & 0.2 & .040 & 80 & .503 & 0.2 \\
\hline .214 & 230 & .555 & 0.4 & .100 & 135 & .672 & 0.6 \\
\hline .963 & 402 & .644 & 0.8 & .207 & 222 & .935 & 1.1 \\
\hline 2.74 & 462 & .692 & 0.1 & .444 & 331 & 1.403 & 1.8 \\
\hline \multicolumn{4}{|c|}{ running time $=220 \mathrm{sec}$} & \multicolumn{4}{|c|}{ running time $=164 \mathrm{sec}$} \\
\hline \multicolumn{2}{|c|}{$\rho=-50 \not$} & \multicolumn{2}{|c|}{$E P 1=.2 \times 10^{-4}$} & \multicolumn{2}{|c|}{$p=\$ 1.20$} & \multicolumn{2}{|c|}{$E P I=.4 \times 10^{-4}$} \\
\hline Time (sec) & Steps & Flux & \% Error & Time (sec) & Steps & Flux & \% Error \\
\hline .000 & 0 & .3823 & 0.0 & .000 & 0 & .382 & 0.0 \\
\hline .020 & 46 & .3487 & 0.1 & .027 & 57 & .516 & 0.2 \\
\hline .060 & 126 & .3050 & 0.2 & .074 & 135 & .777 & 0.3 \\
\hline .130 & 221 & .2700 & 0.3 & .154 & 208 & 1.302 & 1.0 \\
\hline .324 & 323 & .2509 & 0.3 & .381 & 337 & 3.684 & 2.2 \\
\hline \multicolumn{4}{|c|}{ munning time $=152 \mathrm{sec}$} & \multicolumn{4}{|c|}{ running time $=153 \mathrm{sec}$} \\
\hline
\end{tabular}


TABLE II

NUMERICAL RESUITS FOR TWO-GROUP AND FOUR-GROUP CASES

\begin{tabular}{|c|c|c|c|c|c|c|c|c|}
\hline \multicolumn{4}{|c|}{$G=2, I=6, \rho=+80 \notin E P I=.4 \times 10^{-4}$} & \multicolumn{5}{|c|}{$G=4, I=1, \rho=+50 \notin E P 1=.4 \times 10^{-4}$} \\
\hline Time (sec) & Steps & Flux & $\%$ Error & Time (sec) & Steps & FIux & $\%$ & Error \\
\hline .000 & 0 & .3823 & 0.0 & .0000 & 0 & 1.006 & & 0.0 \\
\hline .040 & 71 & .503 & 0.3 & .0003 & 120 & 1.412 & & 0.6 \\
\hline .123 & 155 & .733 & 1.0 & .0006 & 170 & 1.738 & & 0.6 \\
\hline .325 & 270 & 1.227 & 1.3 & .00096 & 241 & 2.013 & & 1.0 \\
\hline 1.037 & 418 & 2.974 & 3.0 & .00193 & 341 & 2.421 & & 2.0 \\
\hline \multicolumn{4}{|c|}{ running time $=274 \mathrm{sec}$} & \multicolumn{5}{|c|}{ running time $=307 \mathrm{sec}$} \\
\hline
\end{tabular}

Figure (1) shows that roughly a linear relationship exists and the truncation error is quite predictable and reasonably good.

FIGURE 1

Percentage error at $0.2 \mathrm{sec}$

for $+80 d$ reactivity

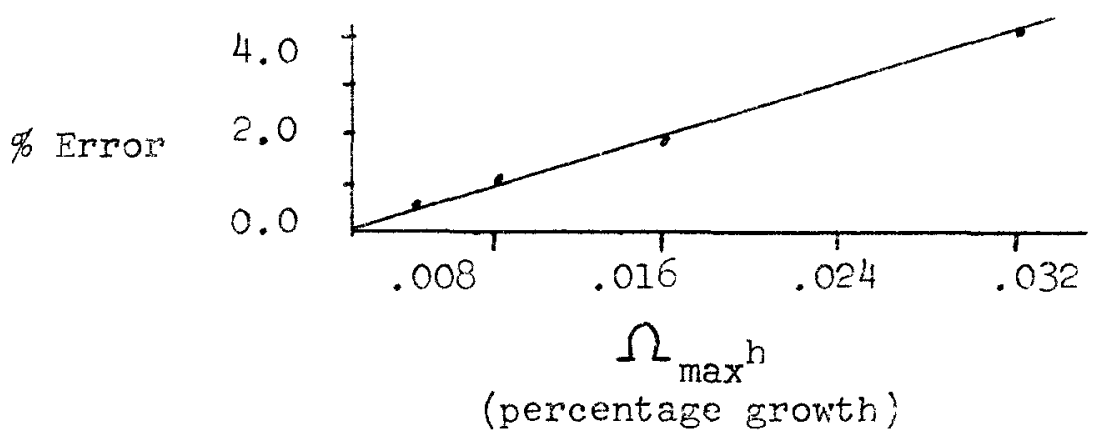


A sample result for a non-homogeneous, non-uniformly perturbed reactor is also glven. In this case the spatial variation of the flux changes as time progresses. Figure 2 illustrates the geometry of the system and shows 441 spatial mesh points in a square array with a mesh spacing of $8 \mathrm{~cm}$. In each direction. Additionally, there were three different material compositions which are numerically denoted in their respective regions in Figure 2.

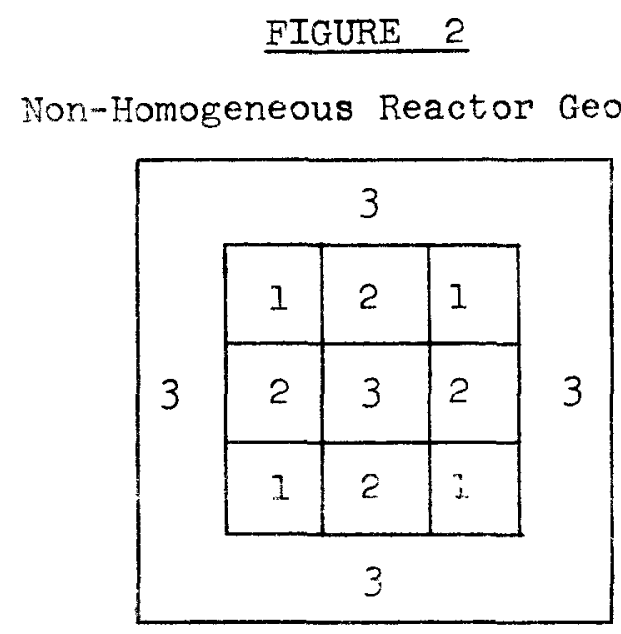

T'wo neutron energy groups and one delayed group were used in the calculation. The reactor was driven by a linear decrease with time in the thermal capture cross section in the regions containing material composition 1 as illustrated in Figure $a$. The total reactivity inserted was approximately $+50 \notin$ during a ramp lasting 0.2 seconds. Following the ramp the capture cross section assumed the value at $0.2 \mathrm{sec}$. for the remainder of the transient. The thermal flux is reported out to 0.45 seconds of reactor time and is compared to a TWIGIE ${ }^{1}$ solution. The results are given in Table III where the flux values at the center point of the reactor are given, and the percent errors quoted are the pcrcent difference between the proposed method and TWIGIE. Throughout the entire transient the thermal flux values of the two methods agreed to within $1.7 \%$ at every point in the reactor. 
TABLE III

\begin{tabular}{lccc}
$G=2$ & & $\rho=+50 \notin$ & EPI $=.8 \times 10^{-4}$ \\
\hline Time & Steps & Flux & $\%$ Error \\
\hline 0.0 & 0 & 16.75 & 0.0 \\
0.05 & 93 & 18.80 & 0.2 \\
0.10 & 119 & 21.73 & 0.1 \\
0.15 & 172 & 26.03 & 0.3 \\
0.20 & 193 & 32.49 & 0.3 \\
0.30 & 300 & 34.83 & 1.7 \\
0.45 & 313 & 35.38 & 1.7 \\
\hline
\end{tabular}

running time $=1097 \mathrm{sec}$

\section{CONCLUSIONS}

The algorithm presented has been shown to be consistent, stable, and to possess desirable asymptotic behavior. Furthermore, the numerlcal results indicate the proposed method is both accurate and rapid in operation. Since the inversion of the matrix $F_{I}$ is linearly proportional to the total number of unknowns (i.e. spatial mesh points times the number of neutrons energy groups), the computation time experimentally has been found to vary linearly with the product of the number of mesh points and energy groups. This has an obvious benefit when more than two energy groups are desired in a calculation. Furthermore, flve additional delayed groups may be included for about the cost of one additional neutron energy group. Another advantage of the method is that the computation time does not depend on the number of material regions used.

An empirical formula for the calculation time is .002N* $(G+.2 I)$ seconds/time step on an IBM 360/65, where $N$ is the total number of mesh points, $G$ is the total number of neutron energy groups, and $I$ is the total number of delayed groups. Depending upon the transient, from 100 to 500 time steps would be required 
to yield a solution accurate to within a few percent.

Finaliy, it is to be noted that all the properties of the algorithm, 1.e. stability, consistency etc., may be shown to be equally valid in the direct three-dimensional extension of the algorithm.

\section{ACKNOWLEDGEMENTS}

This work was sponsored by the United States Atomic Energy commission under USAEC Contract AT(30-1)-3903. The providing of TWIGLE results for comparison by Dr. John B. Yasinsky of Bettis Atomic Power Laboratory is gratefully acknowledged.

\section{REFERENCES}

1. J. B. Yasinsky, M. Natelson and I.A. Hageman, "TWIGLE - A Program to Solve the Two-Dimensional, Two-Group, SpaceTime Neutron Diffusion Equations with Temperature Feedback", WAPD-TM-743, Bettis Atomic Power Laboratories (February 1968).

2. T. W. Riese, "VARI-QUIR-A Two-Dimensional Time Dependent Multigroup Diffusion Code", WANL-TNR-133, Westinghouse Astronuclear Laboratory (1963).

3. J.T. Hitchcock, "Problems in Using the Unperturbed Spectrum for Elew Group Reactor Kinetics", SM Thesis, Department of Nuclear Engineering, MIT (June 1969).

4. K.F. Hansen and S.R. Johnson, "GAKIN, A Program for the Solution of the One-Dimensional, Multigroup, Space-time Dependent Diffusion Equations", USAEC Report GA-7543, General Atomic Division, General Dynamics Corporation (1967).

5. R.S. Varga, Matrix Iterative Analysis, Chapter 8, p. 257, Prentice Hall, Englewood Cliffs, New Jersey (1962).

6. K.F. Hansen, "A Comparative Review of Two-Dimensional Kinetics Methods", USAEC Report GA-8169, General Atomic Division, Gereral Dynamics Corporation (1967).

7. R.S. Varga, Matrix Iterative Analysis, Chapter 3, p. 85, Prentice Hall, Englewood Cliffs, New Jersey (1962).

8. Ibia, Chapter 2, p. 41.

9. Ib Id, Chapter 2, p. 30 .

10. J. Barclay Andrews, II, and K. F. Hansen, "Numerical Solution of the Time-Dependent Multigroup-Diffusion Equations', Nucl. Sci. Engr., 31, 304-313 (1968). 
11. W.M. Benjamin, "A Numerical Method for Solution of SpaceTime Kinetics for Fast Reactors", SM Thesis, Department of Nuclear Engineering, MIT (January 1969). 


\title{
NUOIEAR REACTOR SPATIAL KINETICS CAICULATIONS
}

\author{
by G. Biffoni, A. Galati, P. Loizzo, 'S. Lopez, \\ F. voreizi, A. Pepino \\ C.N.E.N. - Casaccia (Rome) - ITALY
}

\section{1. - INTRRDIOTION}

Porrinkarie efforts have lately been devoted to the solution of the proleme inherent to Space-Time Kinetics. The introduction on the riarket of the new large power reactors and the commercial necessity of reducing the design safety-margins involve new y $r$. fon tie reactor designer which cannot be solved with the $\cdots \cdots-\cdots-1 j m a n s i o n$ modela.

$\therefore$... sin Frobiem is very difficult and expensive, most of the research activity is oriented toward the discovery of simpified spatial neutronics methots, valid for a large range of reactor transients.

One uf these methods is the metastatic model which is based on the sisumption that the spatial flux shape reaches a dynamic equilibrium a few neutron lives after any perturbation.

Two codes based on this methol have boen written: the KINEX code solves the one dimension linear kinetics problem; the ALADINO code studiea a boiling water reactor channel and takes into account the feefrack due to the fuel temperature and void fraction distribution. Both codes use a two energy group model with six delayed groups in one (axial) dimension.

\section{2. - DESCRTPTION AND USE OF THE CODES}

The FOBTRAN code ALADINo calculates the dynamic response of a water-moicd reactor to a reactirity input generated by control rod novements.

The first step consists in a steady-state calculation, including the neutron power distribution as well as the consistent thermokydraulic characteristics. Subsequently, some reactivity agent intervenes and the power transient initiates.

In this edition of the code the reactivity agent may be constituted by the movement of one or many control rods (represented as poison cross sections) being oscillated at any frequency or extracted step- or rampwise.

The transient problem is linearized as a sequence of neutronic and thermohydraulic calculations according to the following soheme: 
- the neutron flux consistent with the reactivity agent is evaluated after a time $\Delta t$ by means of tho metastatic method;

- the fuel temperature and heat flux are calculated according to the power generated during the time-interval and are obtained by the Hankel transform method;

- starting from the heat flux, the new boizing boundary and the void fraction are calculated by integrai... tire unal equations, expressing mass and energy conservatu, aiong the chartteristic curves.

For the next time step, the code generates at this point new nuclear constants, including external reactivity effects as well as feedback ones.

The upper duration limit of the time step is dictated by the feedback actions rather than by neutronics.

In the next edition of the code a more sophisticated thermohydraulic model will be included in order to consider new classes of accidents such as cold water adition, coolant flow loss, pressure effects, paraliel channels, etc.

Up to fifty meshes are allowed for the axial description of the reactor.

A nuclear constant table is supplied as an input; the nuclear constants in each mesh are caiculated by a second order, forward or backward newtonian interpolation for mula.

In this table two group constants are given as a function of the water density and Doppler fuel temperature. The constants for all the calculations described in this paper were supplied by the RIBOT code?

The KINEX code has the same main features as the ALADINO, but it solves the linear neutron kinetics only. By means of the KINEX code faster transients can also be calculated with the inclusion of the faster decaying negative eigenvalue (Rossi- $\alpha$ ).

It was employed in order to test the method and its validity limits and to calculate high-frequency zero-power transfer functions.

\section{3. - WEUTRON KATETICS: THE METASTATIC METHCN}

$T$ we reflare $\partial / \partial t$ by $\omega$ in the time-dependent reactor equations and Eliminate the resulting algebraic equations for the concentrations of the neutron precursors, we may write time-indepen-

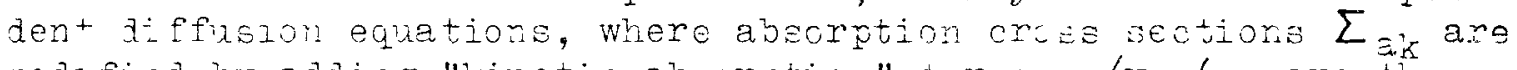
modefied by adaing "kinetic absorption" terms $\omega / v_{k}$ ( $v_{k}$ are the neutron velocities):

$$
\begin{aligned}
& -D_{1} \nabla^{2} \Psi_{1}+\left(\Sigma_{21}+\Sigma_{r}+\omega / v_{1}\right) \psi_{1}=\frac{1}{\mu}\left(\nu \Sigma_{E 1} \Psi_{1}+\nu \Sigma_{E 2} \Psi_{2}\right), \\
& -D_{2} \nabla^{2} \Psi_{2}+\left(\Sigma_{22}+\omega / v_{2}\right) \Psi_{2}=\Sigma_{r} \Psi_{1}, \\
& \frac{1}{\mu}=1-\sum_{i=1}^{6} \frac{\omega \beta_{i}}{\lambda_{i}+\omega}
\end{aligned}
$$


If the algebraically dominant eigenvalue, say $\omega_{\boldsymbol{1}}$, is considerej, we can give an asymptotic representation of the flux. This consists of a flux srape evolving exponentially, i.e.:

$$
\phi_{k}(z, t)=P_{1} \psi_{k}(z) e^{\omega_{1} t}+q_{1 k}(z, t) \quad, 0^{+} \leqslant t \leqslant \Delta t^{-}
$$

Here $k$ is the "sroup" injex, $a_{1 *}(z, t)$ is a fast vanishing function which represents the deviation from the asymptotic flux due to the rapidly decaying initial transients, and $P_{I}$ is a normalization factor accounting for the initial conditions:

$$
P_{1}=\frac{\int d z\left\{\psi_{1}^{*} v_{1}^{-1} \phi_{o 1}+\psi_{2}^{*} v_{2}^{-1} \phi_{o z}+\sum \frac{\lambda_{i}}{\lambda_{i}+\omega_{1}} \psi_{1}^{*} c_{o i}\right\}}{\int d z\left\{\psi_{1}^{*} v_{1}^{-1} \psi_{1}+\psi_{2}^{*} v_{2}^{-1} \psi_{2}+\sum \frac{\lambda_{i} \beta_{i}}{\lambda_{i}+\omega_{1}}\left(\nu \sum_{g \psi_{1}}+\nu \sum_{\mathrm{I2} 2_{2}}\right)\right\}},
$$

wre! $\psi_{k}$ and $\psi_{k}^{\#}$ are the usual direct and adjoint flux shapes, :i the conzentration shapes of the delayed neutron precursor,

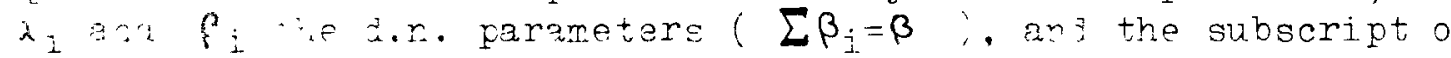
refers to the "initial" situation in the time interval being connijered. "re rypothesis that $q_{1 k}(z, t)$ is a rapidly vanishing function originates from the results obtained by some authors ${ }^{1}, 3$ reiated to the fact that the flux equilibrium is reached in a very short time iof the order of some prompt neutron lifetimes) after the reactivity insertion.

Let us suppose that, withoid loss of gererality, two delayed

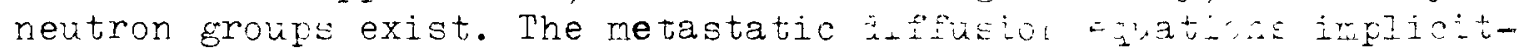
Iy define an infinity of functions correlating $\omega$ and $\mu$. Tre ons corresponaing to all-positive fluxes, say $\omega=f(\mu)$, and the third equation (I), say $\omega=g(\mu)$, will define the first $3(\circ r n+I$ where $n$ is the number of d.n. groups) eigenvalues of the metastatic equations.

The highest one, $\omega_{\mathbf{1}}$, is the inverse asymptotic period; the second eigenvalue has a value between $-\lambda_{1}$ and $-\lambda_{2}$ (or, in general, the next $n-1$ have a value between $-\lambda_{1}=1$. $-\lambda_{n}$ ); the last one correaponis to the Rossi- $\alpha$.

To the first eigenvalue corresponds ar eigenfunction different from the static one; the difference is due to the kinetic absorption terms $\omega_{1} / v_{\mathbf{x}}$. If the excess of reactivity is small, the first $n$ eigenfunctions are not much different from the statically evaluated (i.e. without the kinetic absorption terms) flux distributions.

The one corresponding to $\alpha$ will be, in general, different from the first one.

As could easily be demonstrated, two cases exist and both allow a convergent calculation technique for any $\omega$. In Fig. $I$ and 2 the qualitative trends of the functions $f(\mu)$ and $g(\mu)$ are graphed (dotted and continuous lines, respectively). $f(\mu)$ is a monotonously decreasing function, $\mathrm{g}(\mu)$ has a pole corresponding 

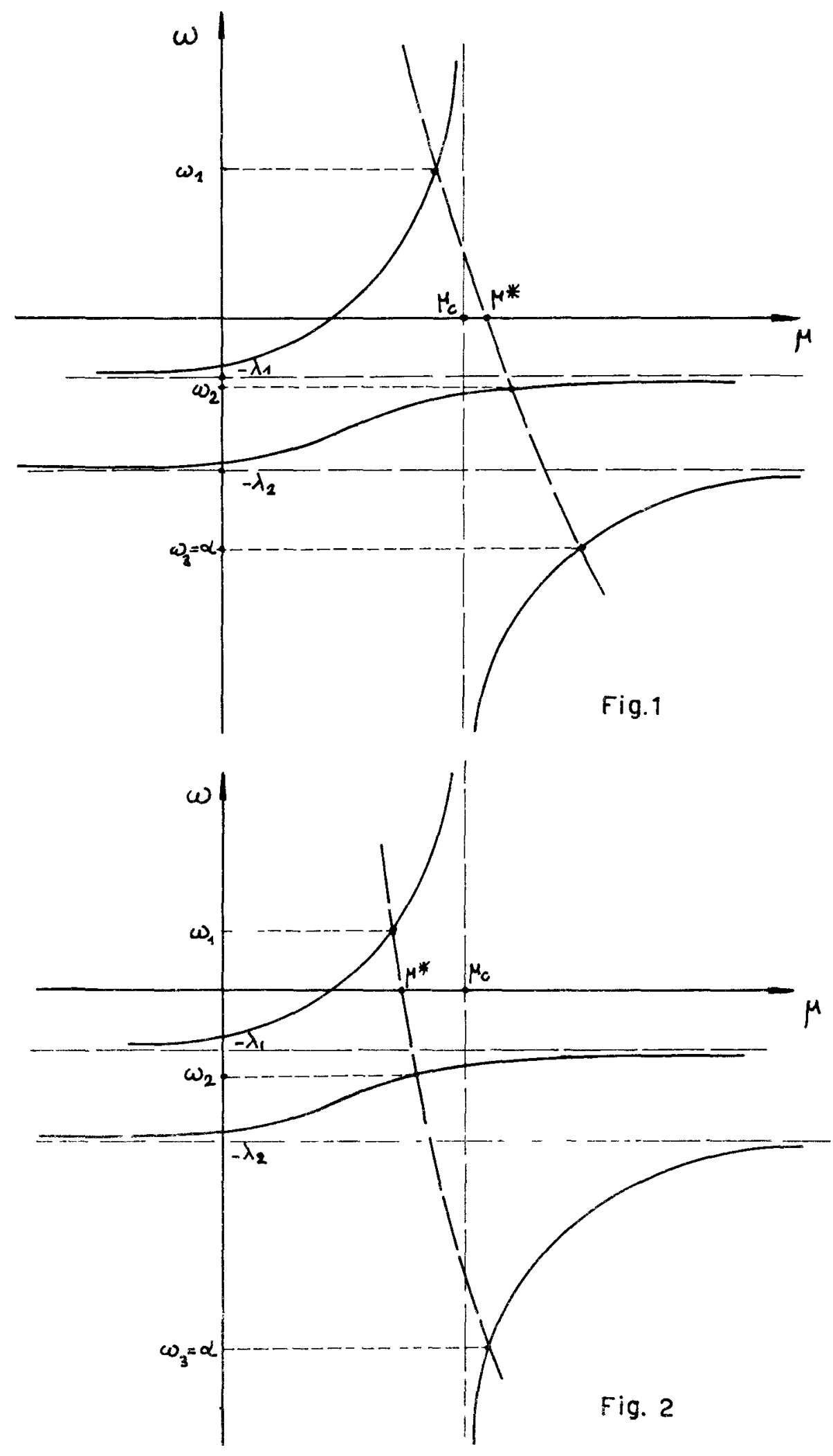
to the abscissa $\mu=\mu_{c}=(1-\beta)^{-1}$. In the case of Fig. $1, f(\mu)$ vanishes at $\mu^{*}>\mu_{c}$, in the one of Fig. 2 at $\mu^{*}<\mu_{c}$.

The intersection corresponding to the searched value $\omega_{I}$ can be reached, starting from a guess value $\mu_{0}$ or $\omega_{0}$, byaconvergent "clockwice" iteratine scheme in the first case, or by a "counterclockwise" scheme in the second case. In the first case, a superpromptcritical situation exists and the eigenvalue $\omega$ must be searched in the metastatic diffusion equations; in the second case, the usual eigenvalue $\mu$ is searched. Thus, the problem is easily solvea by means of the ordinary static calculation methods, such as the well-known "power" method.

If the reactivity is so small ( $K_{\text {eff }}<1+\beta / 5$, say) that the first time constant $\omega_{1}$ is not much greater than the others, we cannot neglect the representation of the eigenfunctions corresponding to the successive $n-l$ values of $\omega_{j}$. We assume in this case that tre $F^{-}$genfunctions associated with the other eigenvalues have the same shape as the first eigenfunction . The normalization factors are defined, accordingly: so

$$
\begin{aligned}
& \phi_{i k}=P_{i} \Psi_{k}(z) e^{\omega_{i} t}, i=1,2, \ldots, 6 \\
& \text { The "totaI" flux is represented by: } \\
& \phi_{k}(z, t)=\sum_{i} \phi_{i k}+q_{k}(z, t)
\end{aligned}
$$

where $q_{k}(z, t) \quad$ is a "short transient" rapidIy vanishing function, so that by neglecting $q_{k}(z, t)$ we commit only an insignificant error, when the reactivity insertion mots is roge than

If the same flux shapes are used for all the first n figenvalues, tre velocity dependent terms must be dropped in $\mathrm{Eq}$. (3). On the contrary, if small reactivities are inserted at high frequercy, prompt neutron variations become quite important, so that the calculation of the Rossi- $\alpha$ becomes essential. This procedure is fol]owed in the KINEX code.

Finally, if very large reactivities are inserted, the first exporential term becomes prefcraerant end no proinems arise.

Galati nade a comparison I petweor the metastatic, the adiabatic, the sythesis and the "exact" methods by calculating the "bench mark" problems proposed by Yasingky and Henry 4. The metastatic method gave more accurate results than any other approximation method, both in superpromptcritical and in subpromptcritical cases.

In superpromptcritical excursions the metastatic method differs from the adiabatic one

a) because of the kinetic absorption term, and,

b) because the former utilizes the instantaneous adjoint fluxes for the definition of the normalization level.

\# Note that the Rossi- $\alpha$ and its flux shape are not considered here. 
The effects of (a) and (b) have opposite signs and (a) is quantitatively half the size of (b). With the trial functions used by Yasinsky and Henry, only the effect of (a) is present in the synthesis method.

In subpromptcritical excursions the kinetic absorption term is negligible and only the consideration of adjoint fluxes determines the good success of the method.

\section{4.- ZERO-PONER TRANSFER FUNCTIONS}

The results of the KINEX and ALADINO codes were compared with experimentally measured zero-power reactor transfer functions.

Starting from the experimental test on the EBNR-Pu 5 , we performed zero-power calculations referring to a frequency ranging from 0 to 50 radians/sec.

The calculations showed that for small frequencies ( $1+2 \mathrm{cps}$ ) we may neglect the Rossi- $\alpha$. However, if high frequencies are considered, the Rossi- $\alpha$ cannot be neglected. The calculations and the experiments agree very well in all the frequency range (see Table 1):

TABLE 1

ZERO-POWER TRANSFER FUNCTION CALCULATED BY MEANS OF THE ALADINO CODE

\begin{tabular}{|c|c|c|c|c|}
\hline $\begin{array}{l}\text { frequency } \\
\Omega(\text { rad } / \text { sec }\end{array}$ & $\begin{array}{l}\text { Calc.gain } \\
(\mathrm{d} b)\end{array}$ & $\begin{array}{c}\text { Exp. gain } \\
(d b)\end{array}$ & Calc.phase & Exp.phase \\
\hline .18 & 4.4 & 5.1 & $-32^{\circ}$ & $-34: 1$ \\
\hline .551 & 2.3 & 2.1 & $-21^{\circ}$ & $-21: 3$ \\
\hline 3.176 & .3 & .3 & $-5^{\circ}$ & -8.7 \\
\hline \multirow{2}{*}{13.124} & $0^{*}$ & \multirow{2}{*}{-.1} & $-80^{*}$ & \multirow{2}{*}{$-7: 9$} \\
\hline & 0 & & $-2^{0}$ & \\
\hline \multirow{2}{*}{53.554} & $-1.0^{*}$ & \multirow{2}{*}{-.8} & $-23^{\circ} *$ & \multirow{2}{*}{$-23^{\circ}$} \\
\hline & 0 & & $0^{\circ}$ & \\
\hline
\end{tabular}

(*) Calculated with the inclusion of the Rossi- $\alpha$ term (KINEX).

Spatral effects on the transfer function can be noticed only wher $\Omega \gg \alpha$, and thus the neutron waves become evident.

5. - THERTOEIYDRAJTICS

The main thermohydraulic problems are solved as follows:

a) Euel_- Temperature_and_Heat Flux

Me suppose that the coolant temperature is constant and that the fuel corducibil工t $\Gamma$ is not temperatire-dependent.

We consequently solve the problem by means of a Hankel transform 6. Fuel temperature is calculated as a function of radial variahle $r$, hei ght $z$, and time $t$. The central and boundary fuel temperatures are then evaluated and, hence, the Dyppler effect. 
The heat source is expressed by:

$$
S(z, r, t)=A(z, t)\left\{a+b J_{0}(\gamma r)\right\}
$$

where $A(z, t)$ is proportional to the neutron power.

The solution is represented by a fast convergent infinite series. Ten terms are sufficient to correctly describe the fuel temperature in an oxide fuel rod. The heat flux in the water, $\mathrm{H}(\mathrm{z}, \mathrm{t})$, is calculated from the formula:

$$
H(z, t)=-\Gamma\left(\frac{\partial T}{\partial r}\right)
$$

rod boundary

b) Coolant Temperature $=$ Boiling Boundary

The coolant temperature is assumed to be constant and equal to saturation temperature in the two-phase volume. In the subcooled zone the temperature is a monotonjcally increasing function of $\mathrm{z}$. In the present version of the AIADINO the subcooled boiling is not considered.

If in the heat diffusion equation we neglect the axial conducticr witi respect to the convection, we have:

$$
\frac{\partial T}{\partial t}+u \frac{\partial T}{\partial z}=Q(z, t)
$$

where $u$ is the (constant) coolant inlet velocity and $Q$ the heat source in the water, which is proportional to the heat flux. Using the initial conditions as well as the boundary condition ( I irlet = constant) Eq. (4) can be analytically solved and the boiling boundary calculated.

\section{c) Voía_Fractions_Calculationg}

The problem regarding the motion of a two-phase fluid mixture in $a$ pipe with a fixed space and time variation of enthalpy requires the integration of a non-linear, partial differential equation system.

Several kinds of difficulties are connected with the integration of these equations:

- ti.a phace ajstribution, over in th" "ans of a cingle rarrow pipe, camot te considered a function of the axlal dimension only;

- the mass, energy, and momentum conservation equations, coupled with a state equation, define the motion; in general, little can be said about the microscopic nature of the friction forces acting on the fluid elements;

- furthermore, no mathematical instmument has as yet been devised which gives a reliable description of the behavior of the pressure waves.

Many approximations have been made to solve some particular aspects of the problem. The approximations used in the ALADINO are the following:

- experimental correlations between the average axial steam and water velocities permit us to average the effect of the di- 
mension $r$.

The Bankoff slip ratio correlation ${ }^{7}$ is used in the static as well as in the dynamic conditions:

$$
s=(1-\alpha)(c-\alpha)^{-1}=u_{v} u^{-1}
$$

where

$\alpha=\alpha(z, t)$ is the "void fraction";

$c$ is a pressure depending parameter;

$u_{v}=u_{V}(z, t)$ is the steam velocity;

$u_{H}=u_{H}(z, t)$ is the liquid coolant velocity;

- by assuming that both phases are uncompressible ( $\mathbf{u}_{\mathrm{H}}$ and $\mathbf{u}_{\mathrm{v}}$ independent from fluid temperature and pressure), the sound velocity is automatically set to infinity. It is very difficult to evaluate the consequences of this assumption, especially in the large power reactors of the present generation;

- in the actual version of the ALADINO, friction, buoyancy, and inertial forces are neglected with respect to the pump driving forces. In this way, the problem is reduced to a purely kinematic one; the inlet velocity is a given time function. This assumption, which is valid for forced circulation channels, is being eliminated. tions:

Under these hypotheses we can write the conservation equa-

$$
\begin{array}{ll}
\left(e_{v}-e_{H}\right) \frac{\partial \alpha}{\partial t}+e_{H} \frac{\partial}{\partial z}\left\{u_{H}(1-\alpha)\right\}+e_{v} \frac{\partial}{\partial z}\left\{u_{v} \alpha\right\}=0, & \text { (mass equation) } \\
\frac{\partial \alpha}{\partial t}-\frac{\partial}{\partial z}\left\{u_{H}(1-\alpha)\right\}=f(z, t), & \text { (energy equation) }
\end{array}
$$

where $e_{V}$ is the steam density;

$\rho_{H}$ is the liquid density;

$f(z, t)$ is proportional to the heat flux.

By substituting the Bankoff correlation in the conservation equations, we easily find:

$$
u_{v}(z, t)=\frac{u_{H}^{\text {inlet }}}{c}+\frac{I}{c(r-I)} \int_{\zeta(t)}^{z} f(z ; t) d z^{\prime}
$$

where

$$
\begin{aligned}
& -r=\frac{e_{\mathrm{H}}}{e_{H}-e_{\mathrm{V}}} \\
& -\zeta=\varphi(t) \text { is the (time varying) boiling boundary. } \\
& \text { The energy equation becomes: }
\end{aligned}
$$

$$
\frac{\partial \psi}{\partial t}+u_{v}(z, t) \frac{\partial \psi}{\partial z}=\psi f(z, t)
$$

and $\psi=\operatorname{cr}-\alpha$.

The last problem is solved by the characteristics method. starting from a time interval $\Delta t$ and the heat flux at the be- 
ginnıng and at the end of $\Delta t$, the ALADINO selects an internal $\Delta t$ ' such that the corresponding space intervals $\Delta z_{i}^{\prime}$ on the characteristic curves are smaller than the space mesh amplitudes $\Delta z_{i}$

Knowing the solution on the points $z_{i}^{*}$ along the characteristic lines, $\psi$ is then calculated on the points $z_{i}$ of the original discretization, by a suitable interpolation formula.

In the AIADINO use is made of an interpolation for mula of the type $\alpha=I /(a+b I)$ where $I \propto \int H(z, t) d z$.

The usual explicit difference methods, such as the "LaxWendroff" technique 8 would correspond to different interpolation formulae (linear or parabolic in $\mathrm{z}$ ). The rational two points interpoiation formula was used in the AIADINO because the Iinear one showed some numerical instabilities.

\section{6. - RESTITS OF HIGH POWER CALCULATIONS}

A series of calculations was carried out with two purposes:

a) to test the basic physical and mathematical assumptions made in the code by comparing the results with the experiments;

b) to focus the effects due to the spatial behavior of the core durirg a transient.

The reference reactor was tine EB:R.

As part of the plutonium experiment in the EBWR 9 a series of transfer function measurements was made by oscillating a control rod in the reactor at different power levels ${ }^{5}$. The experimental results are shown in Fig. 3.

Several types of fuel elements were present in the reactor during the experiments:

a) 36 mixed oxides bundies ( $1.5 \% \mathrm{PuO}_{2}$ in fepleted $\mathrm{UO}_{2}$ );

b) $525.7 \%$ enriched $\mathrm{JO}_{2}$ bundles witn $\mathrm{Iu}$ and $\mathrm{Sm}$ as burnabie poisons;

c) 8 spike bundles ( $93 \%$ enriched $\mathrm{UO}_{2}$ in $\mathrm{Al}_{2} \mathrm{O}_{3}$ );

d) 5 I natural $\mathrm{UO}_{2}$ bundles.

The M.O. and the enr. $\mathrm{UO}_{2}$ bundles generate more than $80 \%$ of the power and are responsible for the dynamic behavior of the reactor.

The average fraction of delayed neutrons calculated to be $.534 \%$.

The over-dil water density effect on reactivity does not differ considerably in both zones, being mainly a leakage effect due to change in $\mathbb{H}_{1}^{2}$ with density.

Therefore, the reactor channel was supposed to be constituted by the mixed oxides. The radial buckling was $16 \mathrm{~m}^{-2}$ (reactor radius of $60 \mathrm{~cm}$ corresponding to zones $a+b+c)$.

As a first step the stability of the numerical solutions was checked. The reactor was initialized at 40.5 nW (here and in the following, by "initialize" we intend making voids consistent with power density distribution at a given power level; after convergence the reactor $k_{\text {eff }}$ is set equal to 1 ).

After the initialization the central rods were not moved and the reactor was allowed to evolve freely. The water density 


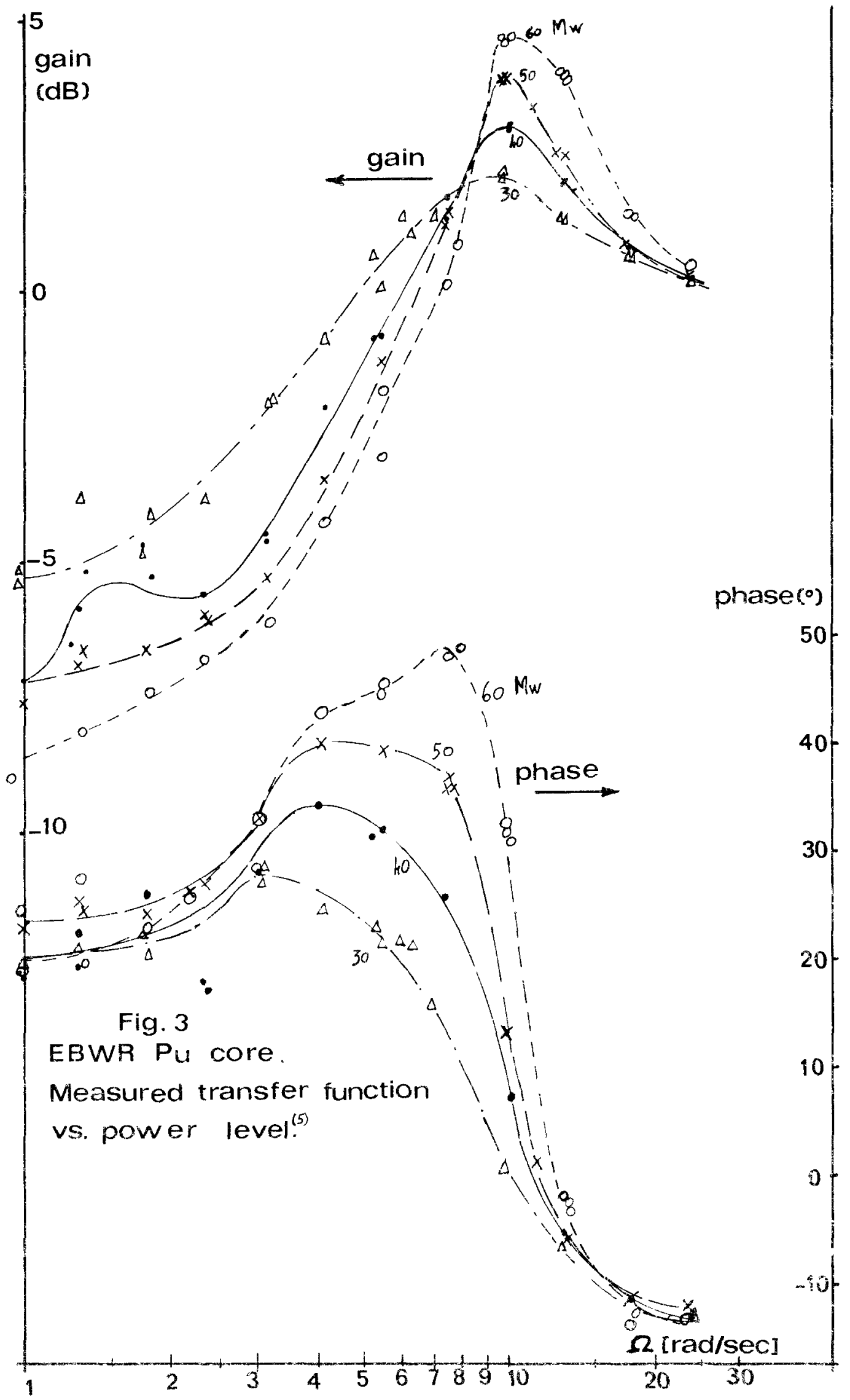


distribution slightly changed, because of the discretization of the time variable in the void equation.

This change in void distribution caused a reduction of the reactivity of $10^{-6}$ and, after a very small transient, the power stabilized at $40.499 \mathrm{MW}$. The transfer functions were then calculated as a function of the reactor power. The results and the comparison with the experiments are given in Table 2:

TABLE 2

HIGH POWER TRANSFER FUNCTION CALCULATIONS AND EXPERIMENTS

\begin{tabular}{|c|c|c|c|c|c|}
\hline$\Omega(\mathrm{rad} / \mathrm{sec})$ & $\begin{array}{c}\text { Power } \\
\text { MW }\end{array}$ & $\begin{array}{c}\text { Calc.gain } \\
\text { db }\end{array}$ & $\begin{array}{c}\text { Exp.gain } \\
\text { db }\end{array}$ & Calc.phase & Exp.phase \\
\hline \multirow{3}{*}{3.176} & 40.5 & -1.6 & -4.5 & $31^{\circ}$ & $28^{\circ}$ \\
\cline { 2 - 6 } & 57.0 & -1.9 & -6.1 & $33^{\circ}$ & $33^{\circ}$ \\
\hline \multirow{2}{*}{5.494} & 40.5 & 1.4 & -0.8 & $25^{\circ}$ & $31^{\circ}$ \\
\hline \multirow{2}{*}{12.0} & 40.5 & 2.2 & 3.0 & $7^{\circ}$ & $7^{\circ}$ \\
\cline { 2 - 6 } & 57.0 & 2.4 & 4.7 & $12^{\circ}$ & $32^{\circ}$ \\
\hline 13.124 & 40.5 & 1.4 & 2.0 & $-7^{\circ}$ & $-8^{\circ}$ \\
\hline
\end{tabular}

From the results of Table 2 it is clear that the calculated gain trenls are much flatter thar the expermor tal ralues and also that they do not change with the power to such an extent as the experiments indicate. If we assume as reference points $\Omega=3.176 \mathrm{rad} / \mathrm{sec}$ and $\Omega=10.0 \mathrm{rad} / \mathrm{sec}$, the gain variation at 40.5 MW is $3.8 \mathrm{db}$ (exp. $7.5 \mathrm{db})$, and at $57 \mathrm{MW} 4.3 \mathrm{db}(\exp .10 .8 \mathrm{db})$.

Obviously, a decrease of the void coefficient may increase the agreement between calculations and experiments. However, it was found from a theoretical experimental comparison of the static resilts that the void coefficient was already pxceedingly negative.

It is very likely that the reasons for the disagreement are to be found in the pressure variations. If the momentum conservation equation is introduced at low frequency, the pressure drop in the boiling channel will increase as the heat flux increases.

Since the total pressure loss in the circuit is constant, the inlet pressure will decrease with a time constant which depends on the single phase channel length and on the single phase pressure drop. Therefore, the water inlet flow will decrease; this will result in an increased void fraction and in an apparent increase of the (negative) void effect.

The above effect will be increasingly pronounced at higher power levels and is particularly sensitive in the EBWR because of the natural circulation. 
The pressure effect is being introduced in the ALADINo code.

To test quantitatively the magnitude of the apparent (negative) increase of the void effect, a calculation was made after having artificially changed the void coefficient. This was $\frac{\mathrm{dk}}{\mathrm{de}}=(.342-.333 \mathrm{~g}) \mathrm{cm} / \mathrm{g}$ for the calculations of Table 2. Altering it to $\frac{\mathrm{dk}}{\mathrm{de}}=(.972-1.078 \mathrm{e}) \mathrm{cm}^{3} \mathrm{~g}$ the gain variation from $\Omega=3.176$ to $\Omega=$ $=10.0 \mathrm{rad} / \mathrm{sec}$ was $7.1 \mathrm{db}$.

A second set of calculations was made in order to focus the effect due to the spatial behavior. For this purpose two accidents were simulated in the same reactor by means of a step extraction of a control rod worth about .5 $\beta$ (transient No. I) or $\beta$ (transient No. 2). The pressure effect was not taken into account. This corresponas roughly to the assumption that the inlet water flow is maintained constant by an oversized pump in series with a very narrow nozzle.

The initial reactor power was $40.5 \mathrm{NW}$ in both cases; the maximum heat flux (at $28.4 \mathrm{~cm}$ from the core bottom) was 48.89 $\mathrm{W} / \mathrm{cm}^{2}$.

The first transient was generated by a sudden rod extraction from the core top $(2.43 \mathrm{mk})$. The power reached a peak of $105.7 \mathrm{MW}$ after. I sec and the heat flux a peak of $54.1 \mathrm{~W} / \mathrm{cm}^{2}$ after . $15 \mathrm{sec}$. After 4 sec the power was slowly increasing at a value of $44.88 \mathrm{MW}$ and the heat flux had a value of $51.56 \mathrm{~W} / \mathrm{cm}^{2}$. The final power distribution was flatter than the initial one.

The spatial effects were already evident: in fact, from the initial to the final situation the total power as well as the average heat flux increased by $11 \%$, while the maximum heat flux increased by $5.5 \%$. At the peak, the average heat flux increased by $13.5 \%$, while the maximum heat flux increased by $10.5 \%$. The spatial effects were even more evident in transient No. 2 $(\delta \mathrm{k}=5.22 \mathrm{mk})$. Here the power had a maximum $(1,150 \mathrm{MW})$ at $t=\mathrm{sec}^{-1}$. 0
$=. I \mathrm{sec}$ and made a series of damped oscillations wi th $\Omega \approx 10$ At $t=4$ sec the neutron power was still oscillating at a value of $50.40 \mathrm{MW}$. The maximum heat flux had a peak $\left(86.69 \mathrm{~W} / \mathrm{cm}^{2}\right)$ at $t=. I$ sec and oscillated then with the same frequency and with a phase delay of $.11 \mathrm{sec}\left(-63^{\circ}\right)$; at $t=4$ sec the maximum heat flux had a value of $53.13 \mathrm{~W} / \mathrm{cm}^{2}$.

The water density had a minimum: at $t=.2$ sec when $z=28.4 \mathrm{~cm}$, at $t=.4$ sec at the channel exit $(z=123.2 \mathrm{~cm})$. After the minimum the water jensity at $28.4 \mathrm{~cm}$ oscillated with a phase delay of .2 sec $\left(-i 20^{\circ}\right)$ with respect to the neutron power.

The exit water density did not oscillate after the minimum. In this case, the total power increased between the final and the initial situation by $24.5 \%$, the average heat flux increased by $17.5 \%$ while the raximum heat flux increased by $8.5 \%$ only.

At the peak ( $t=.1$ sec), the average heat flux increased by a factor 1.90, while the maximum heat flux increased by a factor 1.79.

This comfarlsor, of course, focuses the differences between 
average heat $f l u x$ and maximum heat flux tue to a power shape change during the transient. Evidently, the major differences between zero dimension kinetics and spatial kinetics as pointed out by Yasinsky and Henry are still valid.

The time required for an ALADINO calculation is of the same order of magnitude as for an adiabatic model (on IBN 7094, about 3 sec per time step on a 30 mesh problem, including initialization; transient No. 2 required a time step of $.05 \mathrm{sec}$ and a total of 4 minutes for the 4 second transient).

From these results it is easy to realize the possible application of the method to two or three dimension problems. 


\section{REFERENCES}

1. A. Galati, "The Metastatic Method in Nuclear Reactor Core Kinetics Calculations" (to be published in Nucl. Sci.Eng.)

2. P. Loizzo, RIBOT: A Physical Model for the Light-Water Lattice Calculations, BNWL-735 (1968).

3. A. Foderaro and H.I. Garabedian, "Two Group Reactor Kinetics", Nucl. Sci. Eng., 14, 22-29 (1962).

4. J.E. Yasinsky and A.F. Henry, "Some Numerical Experiments Concerning Space-Time Reactor Kinetics Behavior", Nucl. Sci. Eng., 22, I7I-181 (1965).

5. T.P. Mulcaney, C.W. Michels and G. I. Yingling, personal communication (1957).

6. G. Buffoni and A. Galati, Evaluation of the Temperature Distribution in the Fuel under Transient Conditions of Power, CNEN RT/FI(65)52,(1965).

7. S.G. Bankoff, "A Variable Density Single-Fluid Model for TwoPhase Flow with Particular Reference to Steam-Water Flow", Trans. ASME, Ser. C, J. Heat Transfer, 82, 265-272 (1960).

8. G. Birkoff and R.E. Lynch in Numerical Solution of Partial Differential Equations, J.H. Bramble, Editor, pp 289-315,

(Academic Press, New York, 1966).

9. R.C. Ijickala, U.P. Jenquin and W.A. Reardon, The $\mathrm{PuO}_{2}-\mathrm{UO}_{2}$ Experiment in the EBWR, BNWI-126 (I965).

B.J. Toppel and R. Avery, Some Physics Aspects of the Plutonium Experiment in the EBWR, ANI-7019 (1965). 
BLANK 
LUNCHEON SESSION

Presiding: H. C. Honeck

Savannah River Laboratory 
BLANK 
SOME THOUGHTS ON AEC FUNDED CODING

\author{
W. H. Hannum \\ J. W. Lewellen \\ Reactor Physics Branch \\ Division of Reactor Development \\ and Technology \\ U. S. Atomic Energy Commission \\ Washington, D. C. 20545
}

There is a clear and unmistakable need within the nuclear industry for improved computational capability. This need arises from various causes, most of which are easily recognized. For example, the increase in the quality and quantity of available basic data (e.g., cross sections) permits the use of substantially more detail in calculations; the natural desire to utilize nuclear power as efficiently as possible drives us to design and operate plants with greater precision; a broader range of neutron energies must be considered for fast reactors than was required for thermal reactors; more complex configurations including zoned configurations, spoiled geometries, etc., are considered. These factors will drive us toward more and more complex computations. Simultaneously, our backlog of familiarity grows; the degree of commercial application, rather than research orientation, increases; replication of designs is apparent in some areas. These factors suggest that the computational burden should be eased. A third influence is clearly the availability of new machines, improved in speed, size, or precision.

In this environment, we must ask (1) what capability exists, (2) what further capability is required, and (3) what is a realistic way of obtaining that further capability which is required.

Before addressing these questions, it may be worth making a few general comments. The calculational capability to which these comments are addressed is that which goes from a reasonable compilation of cross sections (e.g., ENDF/B ${ }^{2}$ ) and ends up with data in a reasonable form for use in engineering (or other) designs (e.g., power distributions, fuel management evaluations, transient performance). Our comments are limited to those where it appears that significant AEC participation is required. That is, we will be considering only those areas which are independent of large scale proprietary interest. The running times of codes are of interest only to the extent that they must be short enough to permit doing those calculations which must be done.

Within these limits, there exist requirements for multiple capability. That is, the designer wants to be able to do a "quick-and-dirty" calculation which will be "good enough." He also wants to be able to confirm the adequacy of his calculational approximation by comparison with more sophisticated calculations (e.g., 
synthesis against explicit multidimensional representations). He wants to have unambiguous interpretations of critical experiments, but that requires very specialized and detailed analyses (e.g., heterogeneity). The requirements on these various paths are frequently contradictory.

\section{Current Situation}

It is possible to compile a reasonable catalogue of available calculational codes in various ways, but it is clear that no such catalogue will properly describe the codes available to everyone. The following listing is meant to be illustrative rather than complete.

\section{Constants Preparation Codes}

There are at present the ELMOE, GAF-GAR, FCC-IV, and $\mathrm{MC}^{2}$ codes available ${ }^{3,4,5,6}$ These codes appear to provide a reasonable capability for computing constants without including spatial dependence. However, techniques to improve the handling of resonance and heterogeneity effects need further attention.

Codes which incorporate spatial spectrum dependence are also needed. The work with the XSDRN ${ }^{7}$ code at ORNL is one significant effort now underway in this area. Investigative calculations of the ELMOE-SNARG 8 type are in progress at ANL.

\section{One -Dimensional Diffusion Codes}

A variety of codes exist, with effectively each organization having their own code. A conservative list shows fourteen 1-D diffusion codes of varying characteristics in the ANL code library, several with multiple versions. This capability appears adequate, and we do not foresee further AEC support for this category of coding.

\section{One-Dimensional Transport Codes}

An adequate interim capability, represented by the ANISN 10 DTF-IV, ${ }^{11}$ and SNARG $^{12}$ codes, exists now. Continued attention will be given to experience with these codes in order to evaluate their adequacy (efficiency) for long-term application.

\section{Two-Dimensional Diffusion Codes}

Several promising codes exist. However, independent efforts in the past have left the overall capability in a fragmented stafe. We are now trying to evaluate the relative efficiency of the CITATION, ${ }^{3}$ PDQ $-7,{ }^{14}$ SCRAMBLE, ${ }^{15}$ and $2 \mathrm{DB}{ }^{16}$ codes. New coding is not foreseen pending completion of this evaluation.

\section{Two-Dimensional Transport Codes}

The current codes are DOT 17 and 2DF. ${ }^{18}$ We consider the situation here as comparable to that for one-dimensional transport codes; further development will be predicated upon the outcome of experience gained with DOT and 2DF. 


\section{Three-Dimensional Diffusion Codes}

The only current explicit 3D codes are PDQ -7 , which is of limited availability and applicability to fast reactor problems, and CITATION, which is still under development and relies on extended core storage. At least a backup level of capability is desired so that explicit three dimensional effects can be investigated when needed and synthesized solutions can be verified.

\section{Monte Carlo Codes}

The AMC ${ }^{19}$ and MORSE ${ }^{20}$ codes are available now, and the ORNL series of codes and the VIM work is a continuation of the line of effort which resulted in the HWOCRSAFE code. ${ }^{21}$ Application of Monte Carlo to three-dimensional and heterogeneity problems is anticipated. Its suitability for providing "reference" or "standard" calculations for comparisons with the results of other, less general methods will also be evaluated.

\section{Synthesis - Statics}

Little current capability exists beyond PDQ-7 and BISYN. 22 The LMFBR Program Plan includes work on synthesis methods for LMFBR's, including continuous and discontinuous trail functions, leading to one or more 2D and 3D codes.

\section{Dynamics Codes}

The FORE II code 23 is the most representative code based on point kinetics now in use. Currently aygilable space dependent dynamics codes are GAKIN, ${ }^{24}$ NOMURK, ${ }^{25}$ QX $-1,26$ TWIGLE, ${ }^{27}$ and WIGLE 2.28 The need for more dynamics codes is clear, but the identification of what particular codes are required is not. Developments in this area must include some form of multigroup, two-dimensional capability to enable stability and response studies to be made of typical fast reactor designs.

\section{Burnup and Fuel Management}

The 1-D FARED ${ }^{29}$ code is being developed at $\mathrm{B}$ \&W and the $\operatorname{ARC}^{30}$ modules $(1,2$ or 3D) are planned. Adequate capability in this area does not exist at present.

\section{Shielding Codes}

Basically, the capability consists of codes listed above and adaptations thereof, such as ANISN, DOT, and $05 R ?^{31}$

This listing must be qualified in several ways. As stated it is illustrative; not all codes listed are available to, or desirable for, all users; not all codes listed have a satisfactory capability for some applications; many codes for particular applications have been omitted. Two observations are clear, however. In some areas there is substantial overlap (if not duplication) among codes, e.g., 1- and 2-D diffusion theory codes. Secondly, there are several gaping 
holes--the most apparent are in the kinetics, 3D, and synthesis areas, where codes are large. It is not apparent from the list, but there are other holes, in that codes must in many instances be responsive to particular requirements such as speed of operation for survey work and particular usage features (interfacing).

This inventory of codes, in spite of the devious holes, overlaps, and other problems does represent a massive and valuable investment which is available to the nuclear community ${ }_{32}$ By virtue of the highly successful operation of the ANL code center and RSIC, 32 this asset is readily and conveniently available to the nuclear community, both within the U.S. and in a fair exchange, with the capability developed by our European colleagues. By a modest evaluation, we note that the cost avoidance associated with the operation of our two code centers (that is, the expenditure which would be required to generate independently each code distributed by these centers) is approximately equal to the total AEC budget for all civilian reactor programs. Our only regret is that these are virtual, and not real dollars.

\section{U1timate Objectives}

It is clear that a program that would complete the catalog of codes described above to a fully satisfactory level for both designer and analyst would require a massive effort. It is not clear that such an effort would in fact be convergent nor that the result of any finite effort along these lines would be satisfactory.

It may be of interest to consider briefly an idealized situation. If all coding were done in a common language and this language was optimum for all hardware in use, there would be no problem in doing machine independent coding. If a11 coding were done to a common user/machine interface, the user adaptability would be greater. If the output of all codes were directly suitable as input to subsequent codes, the users' job would be greatly simplified. If optimum and generally applicable methods were available for all phases of a calculation, we could use those methods now.

None of these conditions however is real, and we must talk in terms of an objective which is consistent with reality. It can be safely assumed during the time span of concern that there will be various hardware capability. User oriented software will not be fully optimum. Existing codes cannot be disregarded, and we must rely on efforts other than those directed specifically at reactor applications .

Within these constraints, it is still feasible to talk of an orderly, cooperative effort, ending in a library of codes which satisfies the broad needs of the industry. Two aspects must be considered--the effectiveness and efficiency of the coding effort, and the efficiency of the resulting product. There are groups who have proposed that a code system such as ARC is the best way to meet these requirements. Such a system would be fully open ended, would include multiple paths linking a flexible set of interfaces, and would have an operating system which can generally and flexibly link modules into preselected paths. From a coding point of view, various participating groups would contribute modules, and these would be immediately incorporated into the master system. Intercomparison among methods is straightforward. Coupling between code segments would be without 
manual intervention. Common input could be prepared for a broad range of calculations. Many coding duplications are avoided.

In addition to the ARC system, we find the JOSHUA33 system, DATATRAN, 34 CITATION, the British SCRAMBLE, the FARED code, and other efforts which include code system aspects. The program for this conference in fact shows more discussion of code systems than of new specific codes. With this sort of evidence to support the benefits of some form of system, it does not seem necessary to debate further the specific benefits which are potentially available from a code system.

It should be noted, however, that this form of coding is not without disadvantages. Perhaps the most basic potential problem is that each user group may find it necessary to develop their own system. We have then lost not only the potential for reduced duplication, but have in fact added another layer. Two other major problems arise. These code systems tend, if they are to realize any substantial part of their capability, to become massive and complex. In line with Parkinson's law, they tend to expand to fill the available capacity. Thus, they tend to become more specific to a specific installation than individual stand-alone codes ever were. Secondly, and perhaps more basically, the concept of a system relies in varying degree on the capability to manage large amounts of data at interfaces. Code systems also by virtue of the generality of data handling tend to suffer a loss in efficiency relative to stand-alone codes or specialized code systems. Since for the current generation of machines, data management appears to be the limiting factor in calculational efficiency, a code system becomes dependent on not only hardware configuration, but even on basic data handling capability and procedures of the machine. This currently differs significantly among the various machines. Thus, we can readily expect that current code systems to be machine and installation dependent, and in some instances even operating mode (multi vs batch processing mode) dependent.

In this environment, can the AEC justify the support for development of a code system? Can we justify support for twelve systems?

\section{Current Targets}

It can be observed that a significant improvement has been achieved over the past few years. One of the major contributing factors has been the ANS committees which have developed ANS $10^{35}$ and drafted ANS 10-2, "Recommended Programing Practices to Facilitate the Interchange of Digital Computer Programs." It is our intention to encourage by such means as are available to us the use of these as minimum efforts at good coding practice and documentation.

While this is highly useful progress, it appears that there is room for significant further levels of cooperation. In view of the preceding discussion, it does not appear justified to develop a code system for a single group for the sake of a system. But it does appear that there is a lesson that can be learned here which can lead to an improvement in the coordination of code development. The objective here is to develop a situation in which all the active coding groups receiving direct support from RDT can cooperate in the following senses: 
Work done at one installation will be readily and rapidly available to all other groups.

Effort can be concentrated on specific areas without unnecessary redundancy of coding.

Intercomparison and selection of various methods or approximations are feasible without extensive complication.

Sufficient flexibility will remain to allow imagination, improvement, and adaptation to local environments.

None of these characteristics require reference to a system. Rather, they require only a degree of commonality. In particular, if there were a universal set of interfaces (as used here, an interface is taken to mean a specified collection of data sets required by or produced by a calculational algorithm and available for use external to that algorithm), it would then appear to be feasible to meet all of the above objectives. To this end, a working group has been assembled to develop on an urgent basis that set of interfaces which can be generally accepted. This group met for the first time in March, and is now actively engaged in this effort. While it is not possible (or appropriate) to predict what the outcome of this effort will be, it is hoped that we can obtain some substantial level of agreement. The group has been given a charter to work for approximately two months, with firm recommendations due by the end of May. They have been asked to consider several general areas such as:

1. The potential gains available from standardizing interfaces.

2. The tradeoffs between flexibility and rigidity of interfaces.

3. Interactions of interfaces with hardware and software currently available and the implication of future hardware and software developments.

More specifically, the group has been asked to:

1. Specify a set of interfaces.

2. Consider the location of standard interfaces within calculational sequences.

3. Specify means of accommodating the required level of flexibility, if required.

Presuming, they are successful in accomplishing these objectives, the group will be asked in formulating their final recommendations to:

1. Consider the potential for expansion of the scope of cooperation in the use of standard interfaces.

2. Discuss the degree of permanence which might be implied by current adoption of fixed interfaces. 
3. Specify any restraints which preclude immediate implementation of their recommendations.

The working group is being coordinated by the Liquid Metal Fast Breeder Reactor (LMFBR) Program Office located at Argonne National Laboratory, with Dr. Dan Butler of that office acting as group leader. The investigations of the group are being carried out, insofar as needed with the work of internal subcommitees. Because the group will be dissolved after three meetings, it is not formally organized beyond this point.

If we may be permitted a few minutes for some speculation, we would like to speculate as to what could result from an immediate implementation of fixed interfaces. The first and safest prediction is that there would be a multitude of cries of anguizh, justifiably pointing out the incompatibility of the new interfaces with those now in use. (Any interface adopted by the working group is likely to correspond to at most one existing interface.) For perhaps a year, there would be an almost complete lack of progress by those who cooperated with the standards. A great deal of time would be "wasted" in conversion of existing codes to standard interfaces; many codes will be abandoned in that they could not reasonably be made to conform; there would be extended and occasionally heated discussions as to the conversion of codes which overlap in function; each code converted will suffer a measurable loss in efficiency; etc.

If we may be permitted a further degree of speculative foresight, we can look to see what will be left in the debris at the end of this period. There will be a capability which can be implemented at any interested installation with only a modest effort. The codes which remain will be only a fraction of those currently existing, but no one will have lost capability, and various places will discover that they have in fact gained capability. The arguments as to the relative benefits of various codes will be quietly forgotten either because of implicit or explicit acknowledgment as to what is the best method. That is, the easily predictable wasted year may in fact result in a thinning of the ranks of codes, ending with a real and positive gain in capability by all concerned. The real gain of course would be from that point on, in which the criteria listed at the beginning of this section would be largely satisfied.

It should be noted that these gains are speculative. It is not really apparent that they would be obtained, and we have thus honestly asked the working group to evaluate this as a potential approach. It can only be noted that if their response is that this is not the way to improve the coordination among coding efforts, we will have to find some better way.

One final factor should be considered with regard to standardization of interfaces. It is a truism that any standardization removes flexibility. We must be sure in this operation that we do not remove the user's flexibility, while we seek better coordination among coding efforts. There is no stated or implied intent at this point to suggest a best way to use the resulting product.

A code system will be neither required nor precluded. Any user group will be expected to implement those capabilities which are of use. It can be expected that the national laboratories would emphasize more detailed calculational routes than those desired by industrial contractors. In addition it is expected that industrial contractors would, on their own initiative, modify the basic capability 
in terms of proprietary information, improved efficiency, or otherwise. More detailed analytic methods would always be readily available either for local use, or through national laboratories, to justify or verify more design oriented methods.

For those of you who are not directly involved in the working group charged with developing interfaces (if and as feasible), we earnestly solicit your comments, your constructive criticism, and your cooperation. Within the U.S. reactor community, we have tried to include a broad cross section of representation, but undoubtably we have missed some interested people. For our colleagues from outside the U. S., we will be seeking to broaden the scope of cooperation when and if we find a gain' from this or some other such course of action. We solicit your interest and comments as well, and we will try to keep you informed of our plans and progress.

IV. Summary

A casual review of the calculational capabilities available to the reactor community shows a broad and impressive range of codes. There are, however, both substantial holes in this capability and significant duplications in both past and current efforts. Since the job remaining is large and the effort which can be put on this is limited, it is desirable that we seek ways of concentrate and coordinate our efforts. Many ideas and proposals have been made on various levels with varying degree of demonstrable success. These included the undeniable success of the code centers, to the very promising early benefits from ANS-10 and 11, the yet unfulfilled benefits of systems such as ARC, and some less successful efforts which are mercifully forgotten.

It is our judgement that there is currently room for significant and potentially dramatic improvement in coordination. The approach which has been most clearly posed for us is in the area of establishing standard code interfaces. So as to avoid having this as purely a topic of academic discussion, and to permit early implementation, an ad hoc working group has been assembled and is being coordinated by the Argonne Program Office, to urgently select and define, if possible, standard code interfaces. If so advised by this group, the ACRP, and other interested parties, we will seek the early implementation of such interfaces. 


\section{References}

1. Liquid Metal Fast Breeder Reactor Program Plan, Volume 9, Physics, WASH-1109 (1968).

2. H. C. Honeck, ENDF/B, Specification for an Evaluated Nuclear Data File for Reactor Applications, BNL-50066(T-467).

3. A. L. Rago and H. H. Humme1, ELMOE: An IBM-704 Program Treating Elastic Scattering Resources in Fast Reactors, ANL-6805 (1964).

4. D. R. Mathews and R. J. Archibald, GAF, A Computer Program for Calculation of Neutron Spectra and Average Cross Sections in the High Energy Region, USAEC Report GA-7169 (1967) and

C. A. Stevens and R. J. Archibald, GAR, A Computer Program for Evaluating Leakage Dependent Resonance Absorption, General Atomics Report GA-6952 (1966).

5. W. W. Little, Jr. and R. W. Hardie, FCC-IV, A Revised Version of the FCC Fundamental Mode Fast Reactor Code, BNWL-450 (1967).

6. D. M. O'Shea, B. J. Toppe1, and A. L. Rago, $\mathrm{MC}^{2}$, A Code to Calculate Multigroup Cross Sections, ANL-7318 (1967).

7. N. M. Green and C. W. Craven, XSDRN, A Discrete Ordinates Spectral Analysis Code, USAEC Report ORNL-TM-2500 (In preparation).

8. D. Meneghetti, A. L. Rago, and K. E. Phillips, Resume of Articles Presented at the International Conference on the Utilization of Research Reactors and Reactor Mathematics and Computation, Sponsored by the Comision Nacional de Energia Nuclear de Mexico, Mexico City, May 2-4, 1967, page 177.

9. M. K. Butler, et al, Argonne Code Center: Compilation of Program Abstracts, ANL-7411.

10. W. W. Engle, Jr., A Users Manual for ANISN, A One-Dimensional Discrete Ordinates Transport Code with Anisotropic Scattering, K-1693 (March 30, 1967).

11. K. D. Lathrop, DTF-IV, A FORTRAN IV Program for Solving the Multigroup Transport Equation with Anisotropic Scattering, LA-3373 (July 15, 1965).

12. G. J. Duffy, H. Greenspan, S. P. Sparck, J. V. Zapatka and M. K. Butler, SNARG-1D, A One-Dimensional, Discrete-Ordinate, Transport Theory Program for the CDC-3600, ANL-7221 (June, 1966).

13. T. B. Fowler and D. R. Vondy, Nuclear Reactor Depletion and Dymamics Code, CITATION, USAEC Report ORNL-TM-2496 (In preparation).

14. W. R. Cadwe11, PDQ-7 Reference Manual, WAPD-TM-678 (January, 1967) .

15. SCRAMBLE User's Manua1, Computer Group, AEE, Winfrith (unpublished) . 
16. W. W. Little, Jr. and R. W. Hardie, 2DB, A Two-Dimensional Diffusion-Burnup Code for Fast Reactor Analysis, BNWL-640 (January, 1968) Addendum and Errata (April 12, 1968).

W. W. Litt1e, Jr. and R. W. Hardie, 2DB Users' Manual, BNWL-831 (July, 1968).

17. F. R. Mynott, A Users' Manua1 for DOT, USAEC Report K-1694 (to be published).

18. 2DF, A Two-Dimensional Transport Code From the Los Alamos Scientific Laboratory (Unpublished).

19. L. B. Miller, Monte Carlo Analysis of Reactivity Coefficients in Fast Reactors: General Theory and Applications, ANL-7307 (1967).

20. C. L. E1lis, D. B. MacMillan, 05R User's Manual, KAPL-M-6741 (1967).

21. L. B. Levitt, Applications on Monte Carlo to Problems in Reactor Design and Experimental Analysis, ANS Transactions, Vol. II, No. 1 (June, 1968).

22. P. Greebler, M. D. Kelley, R. A. Davis, C. A. Keck, and W. A. Duncan, BISYN-A Two-Dimensional Synthesis Program, GEAP-4922, (July 15, 1965)

23. J. N. Fox, B. E. Lawler, and H. R. Butz, FORE-II, A Computational Program for the Analysis of Steady State and Transient Reactor Performance, GEAP-5273.

24. K. F. Hansen and S. R. Johnson, GAKIN, A Program for the Solution of the One-Dimensional, Multigroup, Space-Time Dependent Diffusion Equations, $\overline{\mathrm{GA}} 7543$.

25. J. Chernick, et al, Reactor Kinetics Research at Brookhaven National Laboratory, BNL-50117 (T497).

26. D. A. Meneley, K. Ott, and E. S. Wiener, Space-Time Kinetics, The QX-1 Code, ANL-7310 (1967).

27. J. B. Yasinsky, N. Natelson and L. A. Hagemen, TWIGLE, A Program to Solve the Two-Dimensional, Two-Group Space-Time Neutron Diffusion Equation with Temperature Feedback, WAPD TM-743.

28. A. F. Henry and A, V. Vota, WIGL2--A Program for the Solution of the OneDimensional, Two-Group, Space-Time Diffusion Equations Accounting for Temperature, XENON, and Control Feedback, WAPD-TM-532 (October, 1965) and Addendum 1 (January, 1967).

29. Fast Breeder Reactor Static Physics Methods Development and Analysis Project, (Quarterly Technical Reports), BAW-3867-1 to BAW-3867-6.

30. B. J. Toppe1, The Argonne Reactor Computation (ARC) System, ANL-7332 (1967), 
31. D. C. Irving, et al, 05R, A General Purpose Monte Carlo Neutron Transport Code, ORNL-3622 (1965).

32. Abstracts of Digital Computer Code Packages Assembled by the Radiation Shielding Information Center, Oak Ridge National Laboratory document ORNL-RSIC-13.

33. J. E. Suich, J, C. Jensen and H, C. Honeck, JOSHUA--An Operating System for Reactor Physics Computations (this conference).

34. H. J. Kopp and P. S. Selengut, DATATRAN--A Data-Handling Computer Language for a Large Modular Reactor Design System, Resume of Articles presented at the International Conference on the Utilization of Research Reactors and Reactor Mathematics and Computation, sponsored by the Comision Nacional de Energia Nuclear de Mexico, Mexico City (1967).

35. ANS Standards--A Code of Good Practices for the Documentation of Digita1 Computer Programs, ANS-STD.2 (1967). 
BLANK 
SESSION II

DATA PROCESSING

Chairman: R. A. Blaine

Intermational Business Machines 
BLANK 
THIRD GENERATION COMPUTER USED TO CONTROL FUEL ELEMENT ASSEMBLY

G. E. Cort and R. J. Agosti

March 1969

Westinghouse Electric Corporation

Astronuclear Laboratory

Pittsburgh, Pennsylvania 


\title{
THIRD GENERATION COMPUTER USED TO CONTROL FUEL ELEMENT ASSEMBLY
}

\begin{abstract}
This paper presents an example of the use of third generation computers to control the assembly of fuel elements in a nuclear reactor. Through a combination of engineering calculations and a business type data handling system to provide checking and quality contrcl monitoring, an individual set of assembly instructions is produced for each unique fuel element. The result is an optimized thermal performance for each solid graphite enriched uranium fuel element which operates within narrow limits of the average core temperature. Many fuel elements with up to a hundred different detail drawing configurations are used in each reactor. Approximately fifty different input quantities are needed to Irscit. ach elmment. These data are stored on four different magnetic tapes with public PEAD access, but WRITE access restricted to four different functional groups responsible for processing and updating the information on its own tape. The program uses these four controlled access tapes as input for the engineering calculations, for verifying that all elements were properly inspected and released, and for monitoring the assembled reactor against the planned design configuration.
\end{abstract}

\section{DESCRIPTION OF REACTOR}

The active core of the NERVA nuclear reactor consists of solid graphite enriched uranium fuel elements, each containing many individual flow channels. Many fuel elements are clustered together to form the cylindrical active core volume. Hydrogen propellant is heated in these axial flow channels from cryogenic temperatures at the inlet to well over $4000^{\circ} \mathrm{R}$ at the exit. To protect the graphite from chemical attack by the hot flowing hydrogen, the channels are coated in a vapor deposition process with refractory metal carbides. Orifice flow meters are installed at the inlet end of the fuel elements and are sized according to the expected heat input and hydraulic diameter. The significance of precise temperature control can be appreciated by considering that the engine's performance increases rapidly with the average propellant exit temperature while the graphite corrosion rate increases exponentially with temperature.

\section{PROGRAM PURPOSE AND LIMITATIONS}

To build the reactor rapidly, installing the correct size orifice meters in the correct element and assembling the element into the correct cluster without error requires excellent communication between engineering and reactor assembly personnel. The orifice meter sizes are calculated by the computer program under discussion and results are displayed in a compact, readable instruction printout used directly to assemble the core. Over fifty separate pieces of information are needed as input to describe each fuel element and its core location. One use of these data is to calculate the required orifice meter sizes for a 
uniform exit gas temperature from each element. The instruction printout not only specifies these orifice meters, but also assigns the fuel element to its unique core location. The computer checks the fuel element characteristics such as uranium loading and hudraulic diameter to assure they are within specification tolerances. In addition, it verifies that the fuel element was properly inspected and the assigned reactor core location is consisfent with its detail drawing identification.

Figure 1 is a sample printout of these assembly instructions for a part of one cluster. Two fuel elements are on the page with the required orifice sizes designated. The data blocks along the right-hand margin are to be filled in by the technicians and/or inspectors as a check that assembly operations are completed. Note that the orifice meters are colorcoded by the use of a plastic cap and photos are taken as a permanent record. The identifying five digit element serial number printed on Figure 1 is engraved on the graphite fuel element at the earliest practical stage of production. The four character core location code, printed beside the serial number, assigns the fuel element to its unique core location and standard orientation. This printout, which carire; the status of a drawing, is used as primary assembly instructions by the reactor assembly technicians to build the active core volume from the inventory of fuel elements. Other assembly drawings are, of course, required for building the clusters, and for assembling the clusters into the completed core. The designation of unfueled, instrumented elements and nuclear shimming requirements is also carried on this printout.

The orifice meter calculations and the quality control checking are carried out in one job submittal, so that reactor assembly can proceed without delay. To complete the core, numerous computer runs over a time interval of weeks are necessary to accommodate new production, replace elements damaged in shipping and handling, and adjust reactivity shimming. The magnitude of the quality control function is illustrated by the fact that many fuel elements with up to a hundred different defail drawing identifications are produced per core. Each element is inspected many times during production and assembly for conformance to close external dimensional tolerances, thickness and corrosion resistance of carbide coating, uranium content, hydraulic channel diameter, etc. Because of the high reliability requirements common to aerospace projects and vulnerability of the graphite fuel elements to damage, rejection rates are relatively high. This places even greater importance on the quality control monitoring feature of this computer program.

Although the program has been specifically designed for the NERVA reactors, it is versatile enough to be used with any solid core reactor built up to discrete fuel elements containing parallel flow channels. It probably could be adapted to a liquid-cooled parallel rod bundle core as well. Its primary utility is in cases where a large amount of basic nuclear power prediction and fuel inspection data must be quickly brought together to define a set of optimized assembly instructions for the reactor core.

\section{PROGRAM ORGANIZATION}

Four controlled access tapes under the third generation computer's permanent file manager system are used as the primary input to the program. Some of these tapes are also used in other phases of the operation, such as inventory and criticality control. Each tape is the responsibility of a different functional group within the laboratory. The responsible group has the only WRITE access to its tape (by specifying a series of four code words, for example). Users of the tapes have READ access only. The tapes, described in Figure 2, are updated as often as necessary by the responsible groups so that the latest information is 
NRX-A6 REACTOR, CONFIGURATION 123C, JPTATE 12,4/25/67

CLUSTER 43

1. CORE LOCATION 0734

ORIFICE METER SIZE :

USE 3 , SIZE C , DIAMETER 0.145 INCHES, COLOR CODE BROWN

2. CORE LOCATION 0735

ELEMENT SERIAL NUMBER 64318

ORIFICE METER SIZE :

USE 1 , SIZE B , DIAMETER 0.175 INCHES, COLOR CODE YELLOW

CLUSTER 43 CONTINUED ON NEXT PAGE:
CHECKLIST DATA BLOCKS

ACCOUNTABILITY

OPERATOR

INSPECTOR

PHOTO TAKEN

PHOTO VERIFIED

LEAK CHECK

VISUAL INSPECTION

ACCOUNTABILITY

OPERATOR

INSPECTOR

PHOTO TAKEN

PHOTO VERIFIED

LEAK CHECK

VISUAL INSPECTION

Figure 1. Sample Assembly Instruction Printout 


\section{DESCRIPTION}

TAPE 1

ELEMENT INSPECTION

DATA
RESPONSIBILITY

(WRITE ACCESS)

THERMAL AND HYDRAULIC

ENGINEERING GROUP

(USING INFORMATION

FURNISHED BY FUEL

SUPPLIERS)

NUCLEAR ENGINEERING

GROUP

NUCLEAR PARAMETERS

TAPE 3

CERTIFIED ELEMENT

LIST

QUALITY CONTROL GROUP

TAPE 4

CORE CONFIGURATION ENGINEERING ADMINISTRATION GROUP

LIST OF ALL CORE LOCA-

TIONS WITH NORMALIZED

POWER DISTRIBUTIONS.

CORE LOCATION, LOCAL RADIAL POWER

(NORMALIZED), NOMINAL URANIUM

LOADING USED TO OBTAIN POWER

DISTRIBUTION, AXIAL POWER DISTRIBUTION

LIST OF ALL INSPECTED FUEL ELEMENTS WHICH MEET SPECIFICATIONS

ELEMENTS LISTED BY AL NUMBER WITH

LOCATION (IF ANY) AND

MEASURED PARAMETERS.

PROCESS INFORMATION (MATERIALS USED, EXPERIMENTAL PROCESS, SUPPLIER, ETC.), ASSIGNED CORE LOCATION.

LIST OF ALL CORE LOCATIONS WITH REQUIRED

ELEMENT PART NUMBER
SERIAL NUMBER, DETAIL DRAWING IDENTIFICATION, DESCRIPTIVE INFORMATION

CORE LOCATION, REQUIRED DETAIL DRAWING IDENTIFICATION FOR FUEL ELEMENT TO BE USED IN THIS LOCATION, DESCRIPTIVE INFORMATION 
always used for each job submittal. This is important for the NERVA application since new production elements are normally being fed into the system, while others may be deleted for damage in shipment or handling.

Tape 1, the responsibility of the thermal and hydraulic engineering group, consists of two binary coded decimal records per element. These tape records contain the element's unique serial number, its assigned core location, and measured characteristics important for sizing orifice meters. The data for spare elements, containing blanks in the core location field, can also be stored on this tape. The fuel elements are assigned to core locations, by filling in the appropriate core location code in this field. Tape 2, the responsibility of the nuclear analysis group, consists of two binary coded decimal tape records for each core location, and includes a complete description of the nominal nuclear characteristics. Each fuel element location is described by the core location code, normalized nuclear power generated at that location, and the nominal uranium loading used to calculate the power distribution. This tape is prepared based on nuclear analysis and experiments carried out before the start of core assembly.

After sorting Tape 1 according to the core location code (see Figure 3 for the program sequence), the program matches these locations against corresponding locations on Tape 2. Tine program then checks input data for validity (such as the measured loading on Trre ! : inc within tolerance of the nominal loading on Tape 2). It prints an error message if required, or if no errors are found, proceeds to the subroutine to calculate orifice meter sires as indicated by Figure 3. To save computer time that would normally be consumed in tape nandling, the information taken from the tapes is stored in memory. This requires the use of a $64 \mathrm{~K}$ memory plus storage of three variables simultaneously in one memory location by appropriate programming.

The parametric thermal and hydraulic reactor data, orifice meter characteristics, and control indicators for the orificing are read in from data cards. Required meter sizes are calculated after the measured uranium loading for the fuel element has been used to adjust the nominal local power prediction given on Tape 2. Based on the corrected local nuclear power, the element's measured hydraulic diameter and the parametric reactor data, the required orifice meter size is determined. The nearest available size is selected from the inventory of discrete sizes available and the as-built exit gas temperature is calculated for the selected size. The measured reactor exit gas temperatures from the most recent NERVA test exhibit very little random scatter due to the combination of uncertainties in nuclear power prediction and fuel element measurement, reactor design point prediction, and selection of orifice meter sizes.

The output from the orifice meter size calculations is the complete cluster definition shown in Figure 1 which goes directly to reactor assembly personnel. In addition, a printout of auxiliary engineering information including the element's power generation, flow rate and exit gas temperature is produced. Also, the assigned core locations and fuel element serial numbers are saved to monitor the assembled core. This list is designated "Tape 5 " in Figure 3, but is normally on a disk file for immediate use.

After completing the assembly instruction printouts for the fuel clusters, the computer monitors the as-built core for quality control purposes. This checking option is a separate program, (designated "CHECK" in Figure 3) called by control cards using the internal file management system. It compares the list of serial numbers and core locations on "Tape 5" against the Certified Element List (Tape 3) and the Core Configuration (Tape 4) as described in Figure 2. Tape 3 is the responsibility of the quality control group whose 


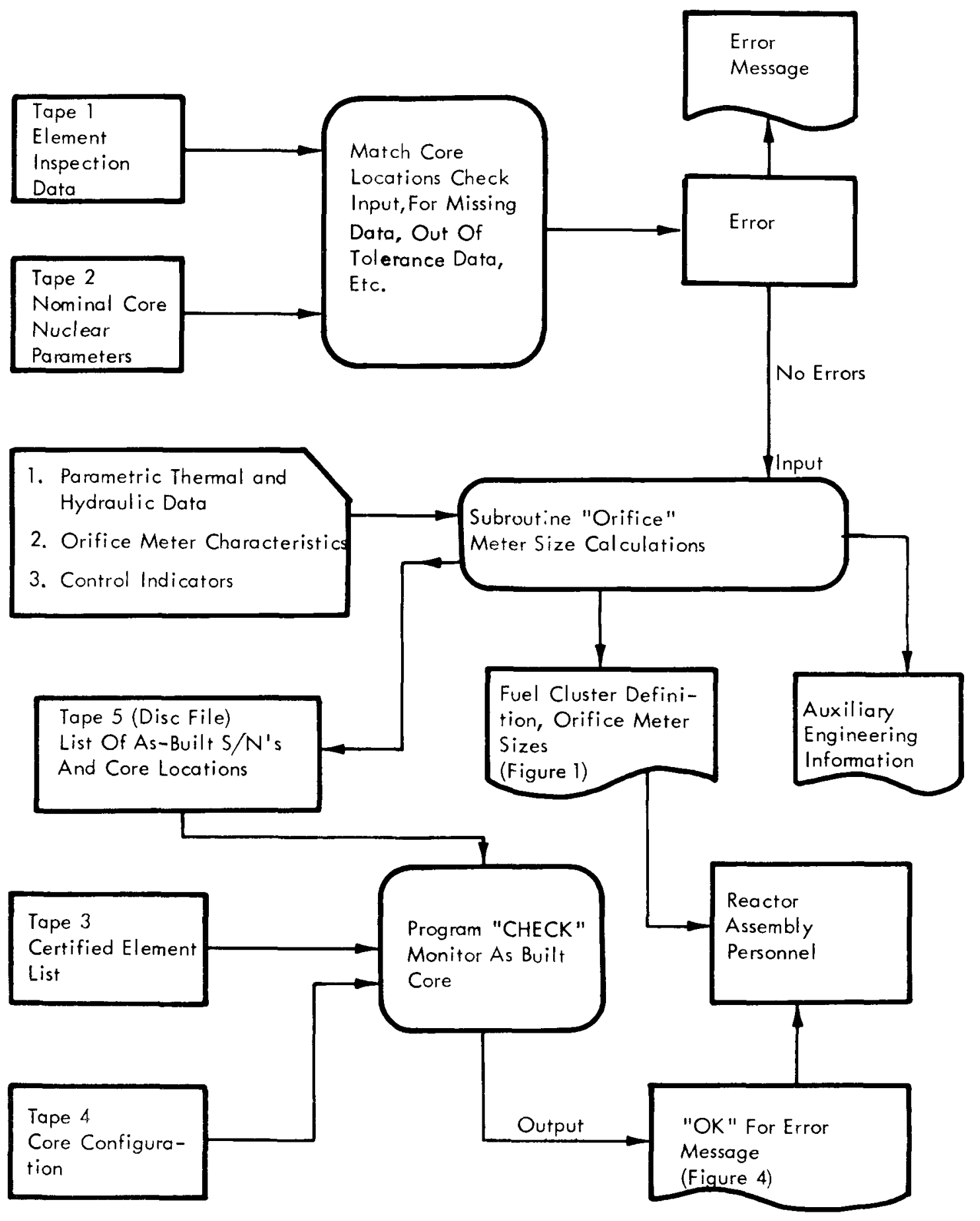

Figure 3. Program Sequence for Fuel Element Orificing and Assembly Code 
responsibility is to verify that the elements have successfully passed through the detailed inspection, non-destructive testing, and other certification procedures as required by drawings and specifications. There is one binary coded decimal tape record per element containing the serial number and part number (detail drawing identification) to which the element was inspected and released. Auxiliary descriptive information is provided on this tape to assist in inventory control.

Tape 4 is the responsibility of the engineering administrative group which controls the reactor drawings and the formal change order system. The required fuel element assembly configuration is stored on this tape with each record containing the reactor core location and the required element part number for that location. Changes to this tape are frequently made while the reactor is being put together and all changes are controlled by the responsible group with appropriate signatures to drawings, followed by a tape update. The identification of Tapes 3 and 4 remains constant for each reactor, so that users need only request the tapes using the appropriate code words to obtain the latest information. Each tape is identified by a title, latest update sequence, and calendar date as the first record. The printout of the check program contains these identifications plus the title and update information printed on the fuel cluster definition (Figure 1) furnished to the assembly personinel. The sample printout in Figure 4 includes a summary of the elements checked and lists any error messages. In this example, ten new elements were added to the core and three enors were found. The first error indicates the element assigned to location 0735 is not on the Certified Element List. This might occur, for example, because the element (serial number 64318) had been damaged and deleted from the most recent update revision of Tape 3. The second error shows that the required detail drawing identification for location 1403 given by the Core Configuration tape (Tape 4) does not agree with that of the actual element given by the List of Certified Elements (Tape 3). This could be caused, for example, by a keypunch error in preparing either tape. The third error, affecting core location 0042, is similar to the second. In this case, the part number letter revision has not been updated on Tape 3 from $L$ to $M$ as required by the core configuration tape (Tape 4). This might occur, for example, due to oversight, or it may be that the element, when re-inspected, did not meet the new drawing requirements. Typically, one would not expect this many errors in one run containing only ten elements. The errors would normally occur because of changes in the element's status on the quality control cerfified list (Tape 3 ) or, more rarely, changes in the desired core configuration. These changes are incorporated into Tapes 1 or 2 (which will define the as-built core) before the jab is executed, and the checking program provides a back-up to guarantee that desired changes have been made.

After the errors indicated in Figure 4 have been corrected by assigning new elements in the as-built reactor, a re-run of the orifice calculations and checking option will produce a printout showing zero errors. It is then forwarded to the assembly personnel verifying that the new assembly instructions (Figure 1) have been checked and that the ten elements are to be orificed, clustered, and added to the reactor in the locations shown.

Rapid, error-free communication of the many thousands of reactor assembly details is vital to this phase of the NERVA reactor construction.

\section{ACKNOWLEDGEMENT}

The Nuclear Engine for Rocket Vehicle Application Program (NERVA) is administered by the Space Nuclear Propulsion Office, a joint office of the U. S. Atomic Energy Commission and the National Aeronautics and Space Administration. Aerojet-General 
TAPE IDENTIFICATION

TAPE 3 CERTIFIED ELEMENT LIST $471 B 233$ REVISION 14. 4/22/67

TAPE 4 CORE CONFIGURATION NRX-A6 REVISION 3. 4/10/67

TAPE 5 AS-BUILT ELEMENT LIST 123C UPDATE 12. 4/25/67

CHECK OPTION EXECUTED $4 / 25 / 67$

10 ELEMENTS ADDED TO AS-BUILT REACTOR

10 ELEMENTS CHECKED

3 ERRORS FOUND

ERROR SUMMARY FOLLOWS -

AS-BUILT CORE TAPE 5

NO. SERIAL NUMBER

CORE LOCATION

1

2

3

64318

0735

82141

1403

0042

04302

CERTIFIED ELEMENT LIST

TAPE 3

SERIAL NUMBER

PART NUMBER

MISSING

82141

04302 $-\cdot-$

$104-M-110$

$721-L-453$
CORE CONFIGURATION TAPE 4

\begin{tabular}{cc} 
CORE LOCATION & PART NUMBER \\
\hline 0735 & $721-M-453$ \\
1403 & $114-M-110$ \\
0042 & $721-M-453$
\end{tabular}

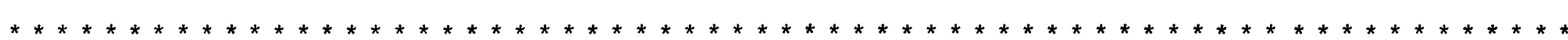

Figure 4. "CHECK" Option Sample Printout 
Corporation, as prime contractor for the engine system, and Westinghouse Electric Corporation, as subcontractor for the nuclear subsystem, are developing a nuclear propulsion system for space applications. 
Use of a Process Control Computer System in Analysis of Neutron Cross Section Data and the Control of Neutron Data Acquisition Facilities around a Van de Graaff and an Electron Linear Accelerator

by

H. Horstmann, A. De Keyser, and H. Schmid

Central Bureau for Nuclear Measurements

EURATOM, Geel, Belgium

\section{GENERAL DESCRIPTION OF THE DATA PROCESSING SYSTEM}

The data processing system of the Central Bureau for Nuclear Measurements (CBNM) has been installed for the purpose of data acquisition control and scientific data analysis. The source of most of the data to be handled and analysed are neutron cross section measurements at a $90 \mathrm{MeV}$ electron linear accelerator and a $3 \mathrm{MeV}$ Van de Graaff.

The data processing system is based on an IBM 1800 computer which is linked to the experimental equipment around the accelerators by special interface units. According to various experimental conditions the interface units request computer interrupts on different priority levels in order to start data transfers to the computer. Such data transfers are completely controlled by computer commands for the corresponding interface units. Several validity checks are made on the transferred data before they are reduced and stored on disk to be used later on for numerical analysis with respect to interesting physical parameters.

The data analysis is performed on-line or off-line depending on the complexity of the method to be applied. Simple operations like display, background corrections etc. can be initiated on-line by the operator of a data acquisition station by pressing push-buttons and adjusting data entry switches. More complicated analysis problems are solved off-line on the IBM 1800 (Time Sharing Execulive System) or the IBM 360/65 computer of EURATOM Ispra/ Italy, about 650 miles away from the CBNM. Data and programs are transmitted to the IBM $360 / 65$ by a tele-processing system operating with two magnetic tape transmission terminals IBM 7702. Fig. I shows a block diagram of the complete data processing system including future extensions. 
LINEAR ACCELERATOR

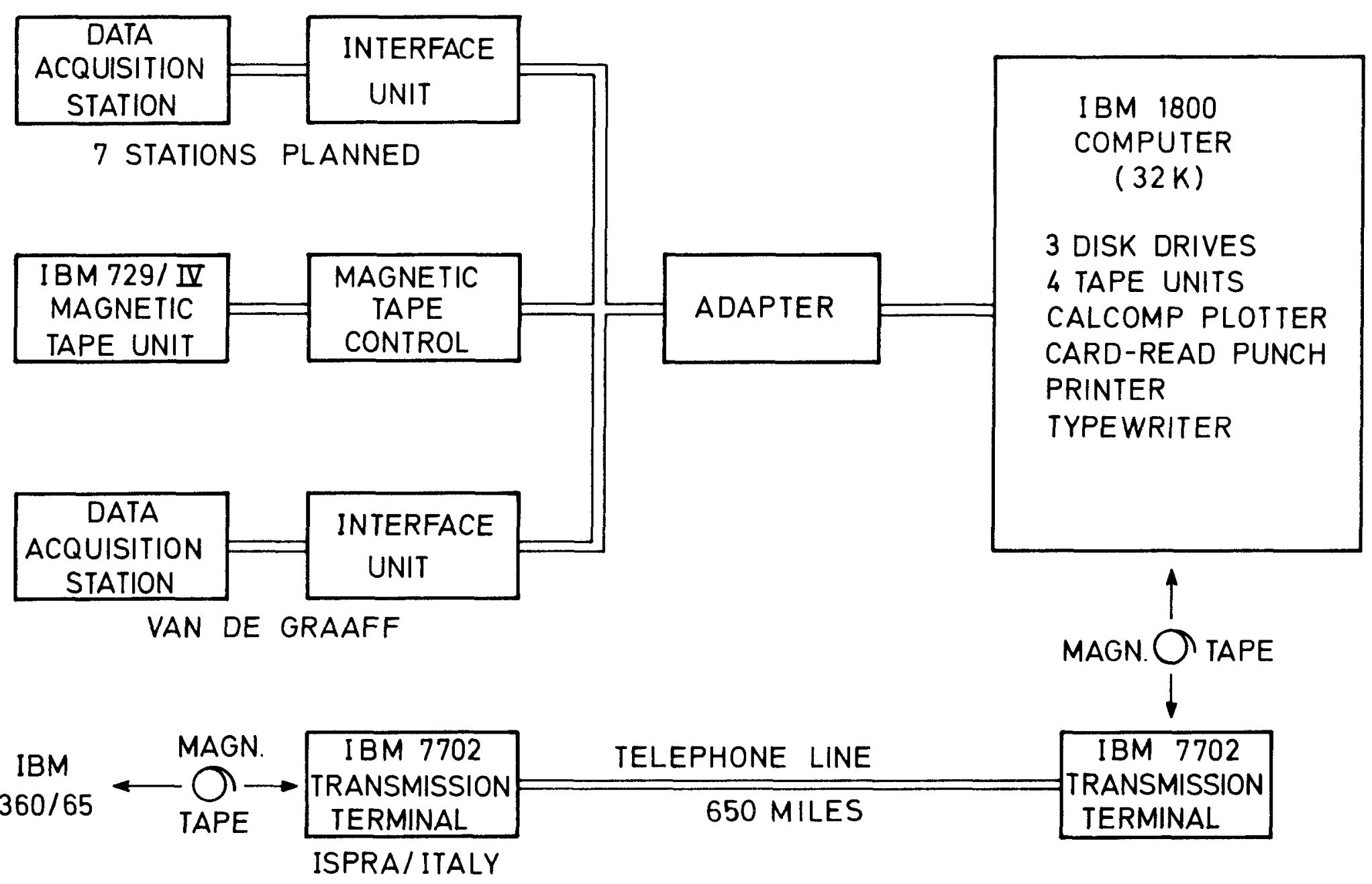

Fig.1 Data processing at CBNM accelerators 


\section{MEASUREMENT PROGRAM FOR THE ACCELERATORS}

Most of the data to be processed are obtained by neutron time-of-flight techniques. Experimental groups for the measurement of the following neutron data have to be serviced.

Linear Accelerator:

- Total cross sections

- Fission cross sections

- Capture cross sections

- Scattering cross sections

- Capture $\gamma$-ray spectra

Van de Graaff:

- Cross sections for neutron induced threshold reactions

- Differential elastic and inelastic scattering cross sections

3. HARDWARE COMPONENTS OF THE AUTOMATIC DATA ACQUISITION

SYSTEM

The data acquisition system consists of the following hardware components: IBM 1800 computer, data acquisition stations, interface units, signal level adapter, IBM 729/IV magnetic tape unit. Fig. 2 shows a block diagram of the system.

The IBM 1800 computer system has the following configuration:

1802 processor-controller, 32768 words, 2 usec cycle time

24 interrupt levels

8 data channels

digital input, digital output, process interrupts

$2310 /$ A3 disk storage

4 magnetic tape units 2401 , model l

$1443 / 2$ printer

$1442 / 7$ card read punch

1816 printer-keyboard

CALCOMP plotter; model 506.

There are two types of data acquisition stations around the accelerators: integrating and non-integrating devices. The integrating devices are fast time-of-flight multi-channel analysers 1 ) (4096 channels, $10^{6}$ counts per channe1). The non-integrating devices are 24 track magnetic tape units on which nuclear events are recorded without any sorting operation ${ }^{2}$ ).

Both types of data acquisition stations are connected to the computer by special interface units which request computer interrupts either automatically according to preset experimental conditions or by operator interventions. Having accepted an interrupt the computer issues commands through digital output which cause the corresponding interface unit to direct the data transfer between the data acquisition station and the computer. In this way the computer takes over complete control of the data transfer.

The IBM $729 /$ IV magnetic tape unit is used as a back-up device for data storage in case of a computer defect or lack of disk storage. 


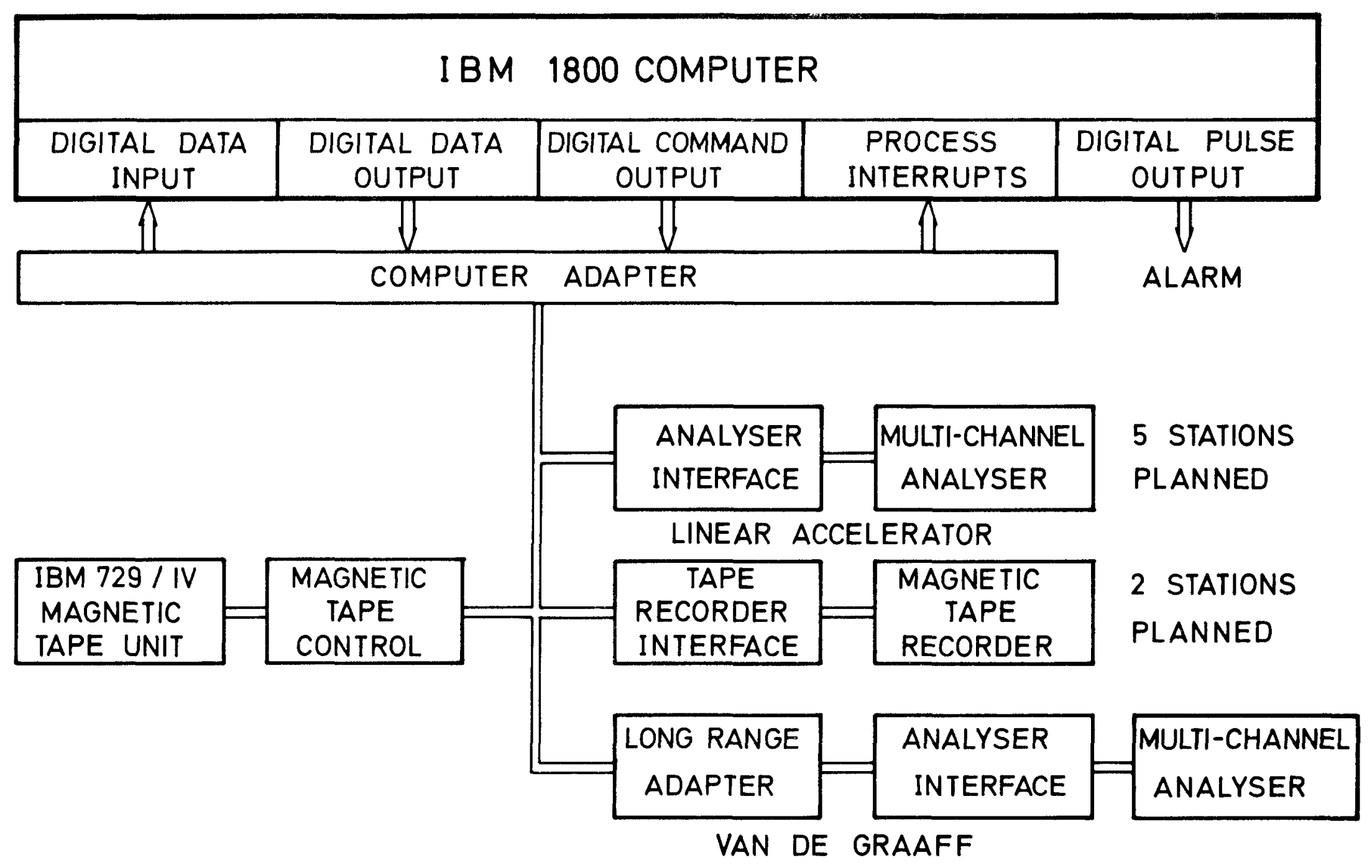

Fig. 2 Automatic data acquisition system of the CBNM 
At the linear accelerator one data acquisition station with a small on-line computer operating as satellite of the IBM 1800 is being planned. This station will be used for experiments with complicated data reduction problems (e.g. multi-parameter experiments).

\section{FORMAT SPECIFICATIONS FOR EXPERIMENTAL DATA AND RELATED}

\section{CONTROL INFORMATION}

In the core memory of a multi-channel analyser the data are stored in BCD format, i.e. 6 decimal digits per channel. The data are transferred from the analyser to the computer in $\mathrm{BCD}$ and converted to binary by a computer program.

The general format of these data read on digital input is shown in Fig. 3a. The 24 bits of the 6 decimal digits of one analyser channel are split into two groups of 12 bits for two computer words. The remaining 4 bits in each 16 bit word are used for control information as follows:

Bit position 0 is a flag bit specifying special data words.

Bit position 1 contains the odd parity of the 16 bit word.

Bit position 2 and 3 specify the first or second group of 3 decimal digits of one analyser channel. By reserving 2 bits for this identification provision has been made for the transfer of information containing up to 64 bits (4 computer words). This is useful for multi-parameter experiments.

The analyser memory of 4096 channels (analyser words) is read out in 16 separately addressed blocks of 256 channels each. This group of 16 blocks is preceded by 4 control words ( 32 bits each) and up to 9 scaler words ( 32 bits each) identifying the spectrum of 4096 channels. Control and scaler words are supplied by the interface unit and have the same format as the analyser words (cf. Fig. 3a).

The 4 control words contain information specifying the type of experimental data and the subsequent treatment by the computer. The information in the control words is related to the experimental equipment, the multi-channel analyser, and digit switches at the interface unit.

Control word 1 contains the address of the data acquisition station, the identification number of the spectrum to be handled and an indicator specifying whether the memory of the multi-channel analyser has to be cleared or not after the data have been transferred to the computer.

Control word 2 mainly contains information about the experimental conditions under which the data have been collected and how the data have to be treated by the computer.

Control word 3 contains information about the utilization of the analyser memory: The total number of analyser blocks with useful data and the number of the first block of these data.

Control word 4 contains a preset count or time specifying the duration of one experimental run.

The scaler words contain additional information characterizing the experimental data, i.e. monitor counts, total count of the spectrum, the daytime 


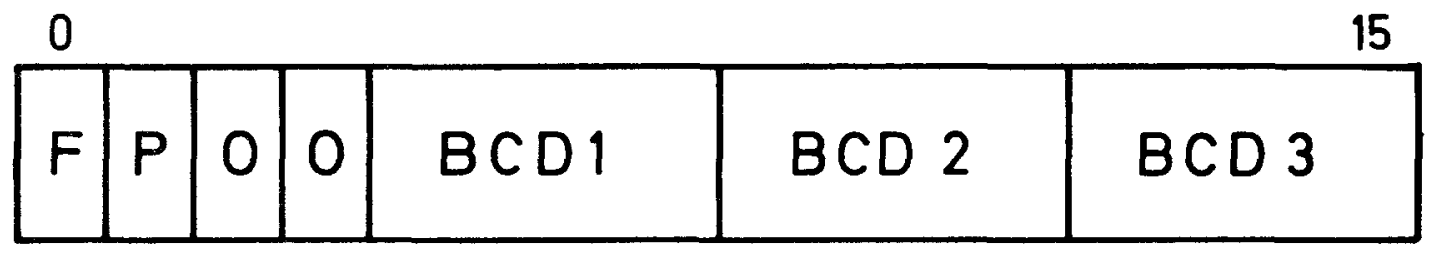

FIRST WORD

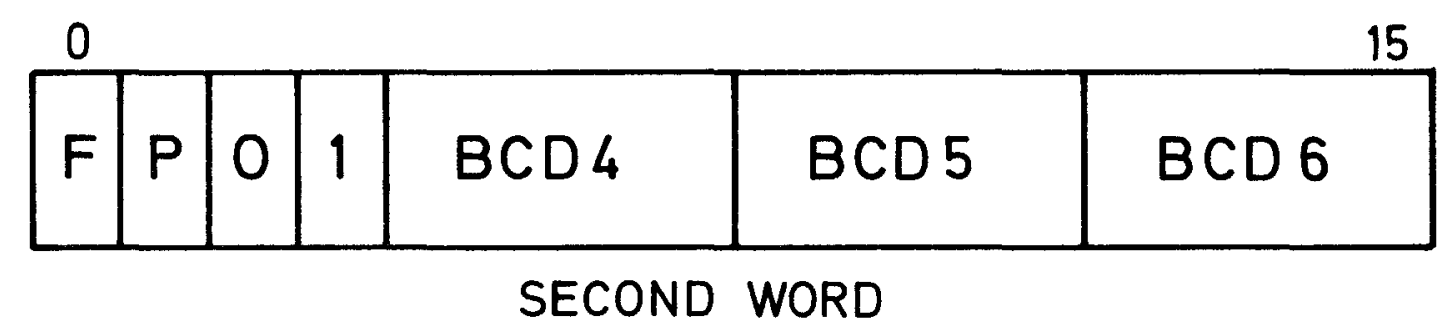

Fig.3a General data format for digital input

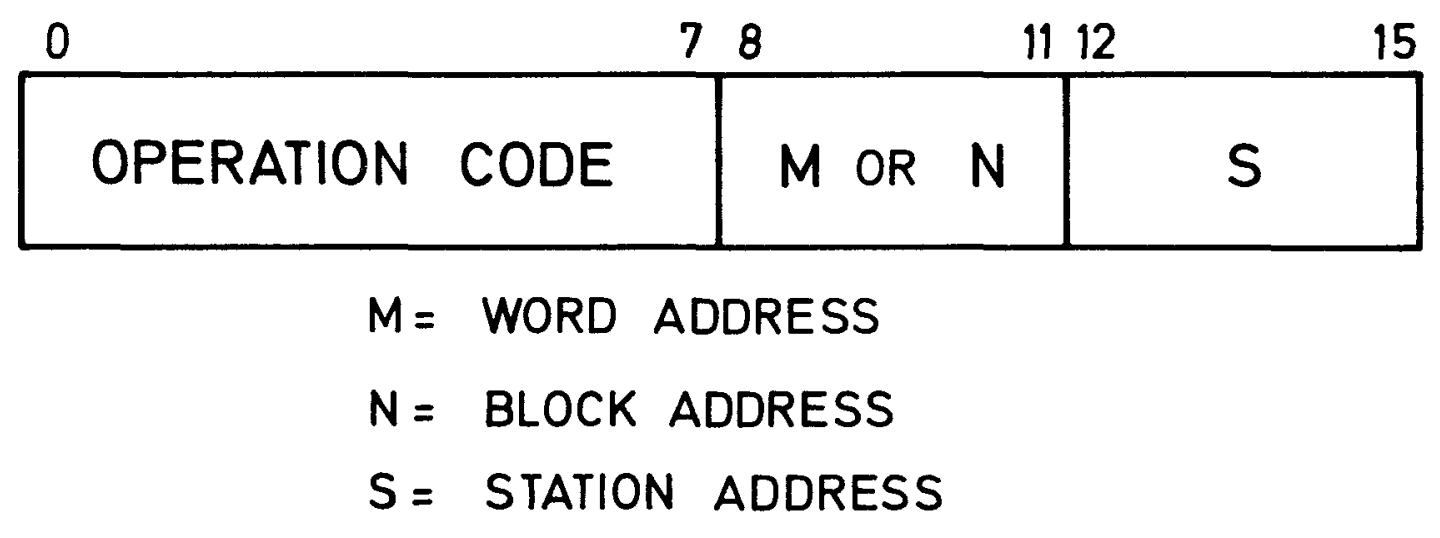

Fig.3b Format of control command for interface unit 
of the experiment, etc.

\section{FUNCTIONAL CHARACTERISTICS OF THE ANALYSER COMPUTER}

\section{INTERFACE UNIT}

In the following the function of the interface unit for multi-channel analysers 3,4$)$ will be discussed in some detail. This unit contains storage for 4 control words, nine counting registers (scalers), instruction and address decoders, and control circuitry for the supervision of the data accumulation in the analyser and the subsequent transfer to the computer (cf. Fig. 4). The computer controls this transfer by special commands on digital output for the interface unit. The format of the control commands is shown in Fig. 3b.

Bits 0-7: Operation code

Bits 8-11: Word address M (scaler or control word) or block address $\mathrm{N}$ Bits 12-16: Address S of the data acquisition station

The function of the interface unit may be understood by a discussion of the instruction set by which it is controlled. The bit configuration of the instructions is given in hexadecimal representation.

140S PRINT ALPHAMERIC

If an error condition during the data transfer or the subsequent analysis is met the computer informs the interface unit of the corresponding station $S$ that an error message is to be printed on the interface typewriter. The message itself is transferred in data channel operation to the typewriter.

24MS READ WORD M

The computer wants to read an interface word (scaler or control word) with address $M$.

2AMS WRITE WORD M

The computer wants to write a double word into the interface scaler (counting register) with address $M$.

20NS WRITE BLOCK ADDRESS $N(N=1, \ldots, 16)$

The computer writes a block address into the address register of the multi-channel analyser.

$340 \mathrm{~S}$ READ BLOCK

The computer wants to read (in data channel operation) the analyser data block previously addressed by the instruction 20 NS.

3AOS WRITE BLOCK

The computer wants to write disk data into the analyser block previously addressed by the instruction $20 \mathrm{NS}$.

300S RESE $\Gamma$ BLOCK

The analyser data block previously addressed by the instruction 20 NS is reset to zero. 


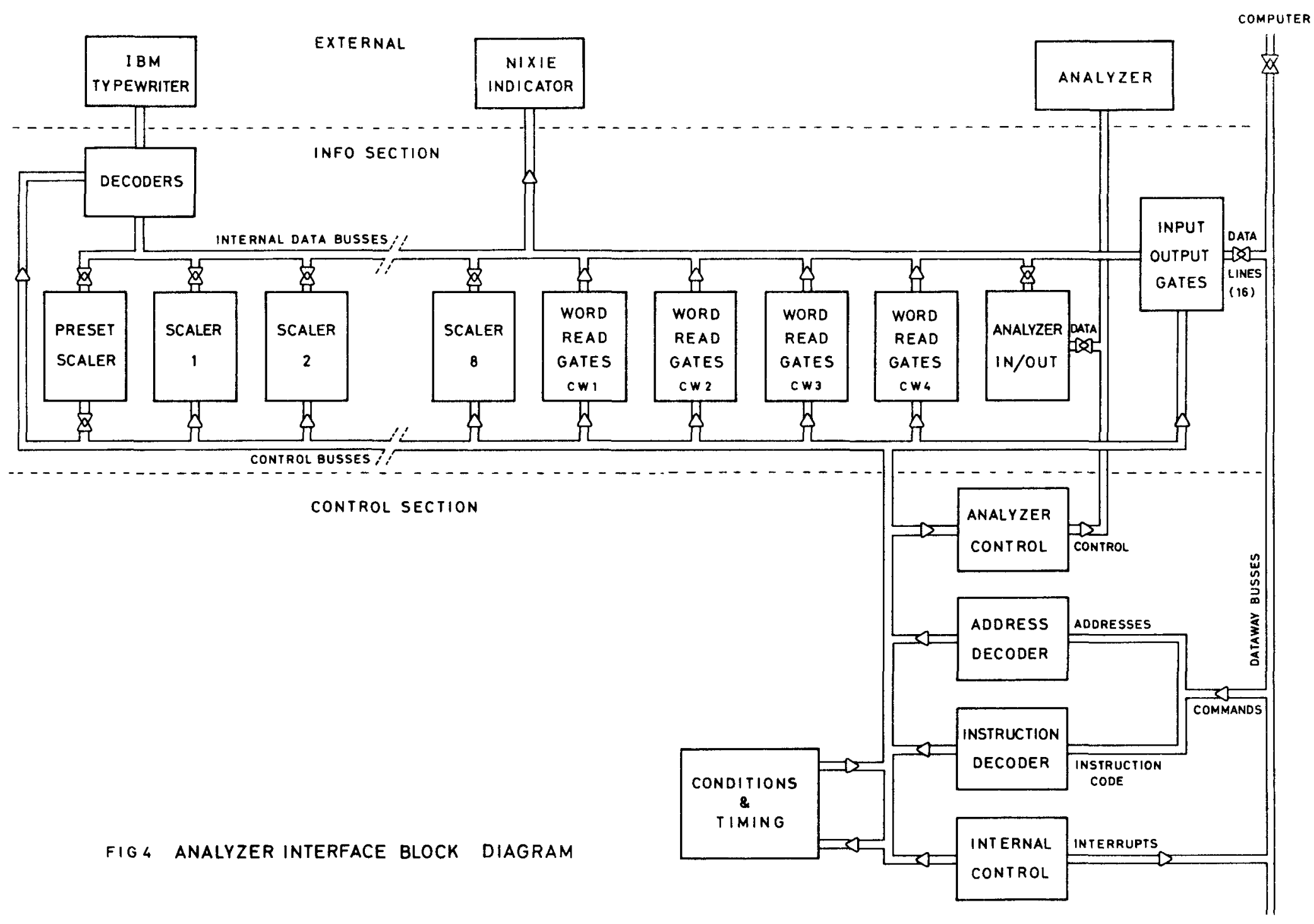


The computer wants to read the 4 control words and the 9 scalers in data channel operation.

COOS PRINT WORDS

The 4 control words and the 9 scalers are printed on the interface typewriter.

DOOS RESET SCALERS

All scalers in the interface unit are reset to zero.

EOOS END OF OPERATION

The computer informs the interface unit that the interrupt has been serviced.

F800 ENABLE ALL INTERRUPTS

All data acquisition stations are enabled to request computer interrupts.

F400 DISABLE ALL INTERRUPTS

All data acquisition stations are disabled to request computer interrupts.

F20S ENABLE STATION S

Only the data acquisition station $S$ is enabled to request computer interrupts.

FO0S DISABLE STATION S

Data acquisition station $S$ is disabled to request computer inter rupts.

The interface unit can also be used by the operator of the data acquisition station to initiate immediate and delayed computer operations by pressing push-buttons and adjusting data entry switches.

Immediate operations require only short execution times and do not use the line printer of the IBM 1800 . The following immediate operations can be performed.

- A spectrum stored on disk can be retransmitted to the data acquisition station in order to be examined on a display unit.

- Up to 10 spectra can be summed up and the resulting spectrum stored on disk.

- The count rates between channel limits can be integrated and the results printed on the interface typewriter.

- A spectrum can be partly or in total multiplied by a normalization factor. The resulting spectrum is stored on disk.

- Spectra stored on disk which are no longer useful can be deleted.

- The identification numbers of all disk spectra with the same station address can be listed on the interface typewriter.

For delayed operations the computer stores the incoming requests on disk. At suitable time intervals the computer operator starts the execution of the corresponding programs. This method has been chosen for programs with long execution times and/or large amounts of printed or punched output. 
When the data accumulation in a multi-channel analyser has been terminated the interface unit requests a computer interrupt which is serviced by an interrupt core load.

First of all the 4 control words and up to 9 scalers are read on digital input by direct program control. The computer requests each interface word by sending the appropriate command on digital output to the interface unit. Reading is stopped when a flag bit is detected on a scaler word. The program checks each control or scaler word for parity errors or invalid digits. In case an error condition is met the word under consideration is read again. If after a certain number of reading the error still persists error messages are printed on the interface typewriter and on the 1053 printer, an audible alarm is turned on, and the interface unit is disabled to send further interrupt re quests to the computer.

If the control and scaler words are correctly read they are decoded in order to provide the program with information about the type of data to be handled, i. e. the total number of data blocks in the spectrum (maximum 16 blocks or 4096 channels), the number of the first block to be addressed, the identification number of the spectrum for disk storage or the identification number of a disk spectrum to which the present one has to be added, etc.

Next the data blocks are read in data channel operation with external synchronization at a word rate $(32 \mathrm{bits})$ of $10 \mathrm{kc}$. Each block is separately addressed by an interface command. A read-back check on the address makes sure that the correct address has been written into the address register of the analyser. In this case the data channel operation is initiated. The computer checks if the operation is terminated correctly. If this is not the case a retry-method is applied which will lead to a correct transfer of the data block or to an error condition.

When all blocks of the spectrum are correctly read the total spectrum is converted from BCD to binary and stored on disk. An extended version of the program allows for on-line data reduction by summing up spectra of the same type and saving only the sums on disk. This method is especially used for the measurement of total cross sections (cf. 2).

When the spectrum has been transferred to the disk storage the command PRINT WORDS causes the interface unit to print its control and scaler words on the interface typewriter. Finally the command END OF OPERATION informs the interface unit that the interrupt has been serviced by the computer.

For a more detailed description of the on-line programs for digital data input and reduction the reader is referred to reference ${ }^{5}$.

\section{DISK ORGANIZATION FOR ON-LINE DATA STORAGE}

In order to store analyser data in a most efficient way on disk a somewhat intricate organization concerning the identification tables and data zones has been established. In this paper only the principles of this organization can be outlined, more details will be published elsewhere 6).

The experimental data are read by the computer as spectra of up to 16 blocks (4096 channe1s). The maximum count rate which can be stored in one disk 
word (16 bits) is 65535 . A channel with a count rate higher than 65535 , a so-called overflow channel, requires two disk words for storage. Normally only a small number of the 4096 channels in a spectrum are overflow channels. Therefore the spectra are classified and stored in the following way.

Spectra of 16 blocks (4096 channels):

If the spectrum has more than 2048 overflow channels two disk words are used for each channel. If the spectrum has less than 2048 overflow channels the 16 least significant bits of each of the 4096 channels are stored in 4096 disk words. For each overflow channel two additional words are reserved, the first one for the channel number and the second one for the overflow count (16 most significant bits).

Spectra with less than 16 blocks:

1 channel $=1$ word if no overflow channels in the spectrum

1 channel $=2$ words if overflow channels in the spectrum.

In the measurement program spectra with less than 16 blocks are not so numerous as those with 16 blocks.

When the spectra stored on disk are read back into the core memory they are always restored in a format with two words per channel.

\section{SURVEY OF ON-LINE AND OFF-LINE DATA PROCESSING}

On-Line Programs

For the acquisition and reduction of neutron data measured at the accelerators a set of programs for the Time-Sharing Executive System of the IBM 1800 computer is being used. These programs service the automatic and operator interrupt requests from the interface units, e.g. for data transfers to the computer, display of disk data at the data acquisition stations, and data normalization and reduction. Requests for programs with long execution times and/or large amounts of printed or punched output are recorded to be serviced when computer time is available. All on-line programs are written in ASSEMBLER language.

Off-Line Programs

Complicated data reduction and the analysis for interesting physical parameters are performed in time-sharing with the on-line work on the IBM 1800 computer or by tele-processing on the IBM $360 / 65$ of EURATOM Ispra/Italy. Programs for single-level and multi-level analysis of neutron resonances, multiple scattering corrections for elastic and inelastic differential neutron cross section data, self-absorption corrections, etc. are continually used.

The efficiency of the data analysis is considerably increased by the operation of a CALCOMP plotter (model 506) with tape and disk buffering in multi-programming mode 7 ). 
1) A. De Keyser, in Automatic Acquisition and Reduction of Nuclear Data, K. H. Beckurts, W. Gläser G. Krüger, Editors, pp. 79-82, (Gesell schaft für Kernforschung m. b. H., Karlsruhe, 1964).

2) A. B. Idzerda, in Automatic Acquisition and Reduction of Nuclear Data, K. H. Beckurts, W. Gläser, G. Krüger, Editors, pp. 104-109, (Gesellschaft für Kernforschung m. b. H., Karlsruhe, 1964).

3) A. De Keyser, S. de Jonge, T. van der Veen, and P. ter Meer, Analyser Computer Interface, paper presented at the International Symposium on Nuclear Electronics, Versailles (Sept. 1968).

4) A. De Keyser, S. de Jonge, T. van der Veen, and P. ter Meer, EUR report to be published.

5) H. Horstmann, EUR report to be published.

6) H. Schmid, H. Claessens, H. Horstmann, EUR report to be published.

7) H. Schmid, An IBM 1800 Program Package for On-Line and Off-Line Operation of a CALCOMP Digital Incremental Plotter, EUR 4225e (1969). 
DESIGN OF A NUCLEAR DATA ACQUISITION SYSTEM

Robert N. Macdonald

IBM Corporation

\section{ABSTRACT}

A multi-user nuclear data acquisition system has been designed for the IBM 1800 computer. The system is based on a control unit which allows most standard nuclear instrumentation, such as analog to digital converters or scalars, to be plugged directly to the interface with no modifications. By means of a diode pin matrix plugboard, the control unit can be reprogrammed to handle eight different configurations of up to fifteen components while the system is on-line. This can be accomplished without dismupting the experiments in progress. Data transfer takes place via a high speed data channel operating in the cycle steal mode. This minimizes data collection time and permits a higher burst data rate.

The programming system (PALS) used is highly interactive, allowing multi-tasking on each of two priority levels, plus a background job. System resources, such as core storage and disk space, are allocated dynamically as they are requested by the user and returned to the system when a user's program exits. If desired, user programs may be written in a special application-oriented language which relieves the experimenter of the necessity of knowing assembler language. Full use of all system facilities is provided at remote locations, thus, permitting operation of the computer from the experiment area rather than the computer room. 


\title{
DESIGN OF A NUCLEAR DATA ACQUISITION SYSTEM
}

\author{
INTRODUCTION
}

Small binary computers have been used for several years in nuclear data acquisition applications, such as pulse height analysis or reactor physics experiments. 1, 2, 3. In general, these machines are dedicated devices serving one experiment at a time. Their function is to collect and store the data in machine-readable format for subsequent off-line analysis. The analysis is then done either on a large central computer or on the data acquisition computer with typical turn around times measured in hours or days. This time delay is sufficiently long and reactor or accelerator time sufficiently limited that the tendency is to run a complete set of experiments before analyzing the results. Often this means that the experimenter learns too late that he has taken insufficient data or that he has thoroughly surveyea a set of operating conditions which holds little interest.

To prevent this, the computer should be used as a true "on-line" system and do at least part of the data reduction while the experiment is in progress. It should present the experimenter with meaningful summary of the data being collected and through suitable interaction, allow him to eliminate the unproductive runs and to shorten the total experiment time.

While the ability to go on-line and perform calculations in real time is desirable, sufficient file handling and arithmetic capabilities to approach this goal are beyond the scope of a typical small laboratory computer. Interactive situations which enable the experimenter to change the order of the data collection and analysis tasks at will implies a series of programs which can be set into execution in an unpredictable sequence. Core storage will, in general, not be large enough to contain all possible programs simultaneously so a random access storage device, such as disks or a drum will be required. If the experiment requires the comparison of calculated data to established standards then magnetic tapes will be needed to hold the bulk data. CRT displays are needed for presentation of graphical data to the experimenter and both digital plotters and line printers are useful for providing hard copy output. Finally, an operating system is required to handle the transition from one task to another and to efficiently operate the varied input and output devices which are in use by the system. It is these I/O devices which bring the cost of true real time system to an order of magnitude greater than that of a small laboratory computer and places it far outside the budget of a single experimenter.

If a medium sized computer with the necessary I/O devices could be shared among several users, the system could be viewed as a capital equipment purchase requiring no greater investment than acquiring separate 
small computers for each staff member. Unfortunately, time sharing a larger computer among multiple users has, to date, not been practical. First, computer manufacturers have not provided a time shared operating system for the laboratory environment, and, secondly, the interdependence of the user's program and the computer-experiment interface has caused unacceptable interference at the hardware level between the various users.

Within the last year there have been two developments which help avoid these problems and may make time shared systems truly practical. These are an interface control unit ${ }^{4}$ which was designed during a joint project between IBM. Research, and Yale University and, secondly, a new programming system ${ }^{5}$ which was written for the IBM 1800 computer by IBM in Palo Alto, California. A system based on these two concepts is now being placed in operation at our facilities in Washington, D. C., and its functional characteristics are described below.

\section{INSTRUMENT INTERFACE}

In a data acquisition system multiple instruments must, in some way, share a single input channel. As each instrument is ready to transfer data it must be switched onto the data buss for the period of time that it is active. Since the data transfer from independent experiments operating at different time bases is completely impossible to schedule in advance, a priority interrupt structure is needed to switch to each instrument on demand. This dependence on the interrupts causes a significant fraction of the computer's time to be used up in non-productive overhead and often results in an instrument being "locked out" at the time it needs to transfer data. The alternate approach of providing a separate channel for each ADC or scaler is simply too expensive.

To avoid these difficulties the following ground rules were established to guide the interface design:

1) Data transfer should be on a demand/response basis under channel control requiring one machine cycle per word transferred.

2) Interrupts should be reserved for starting or stopping programs or for signaling the end of a block of data.

3) The interface should provide connection points for standard nuclear instrument modules, such as ADC's or scalers.

4) It should be possible to change the number and type of instruments in use while the system is on-line.

5) Multiple users should be able to share the interface simultaneously.

6) The system should be operated from the experiment area rather than the computer room. 
7) Any special programming for the interface should be independent of the quantity or nature of the data transmitted.

The interface to the IBM 360/44 at Yale University's Nuclear Structure Laboratory, although designed for data acquisition from a tandem Van de Graff accelerator, is sufficiently close to the desired specifications that it was decided to implement as much of this design as possible. In function, the unit described herein is very similar to that at Yale, although the hardware implementation is completely different.

The Nuclear Interface Control Unit is shown in Figure 1. This unit provides the capability of attaching up to fifteen nuclear instruments to a high speed data channel operating in the cycle steal mode. Via the matrix pinboard, the unit is programmable such that any of eight request pulses can signal for a data readout from selected instruments. The unit requires 12 inches of panel space in a 19-inch rack and can be installed at a location remote from the computer. A simplified logic diagram is shown in Figure 2.

Inputs to the system are eight Event lines at the left of the diagram ard fifteen Component Connectors at the bottom. The Component Connectors conriat of 16 vata bits, two control lines (Select and Reset) and a common line. These connectors provide the input points for ADC, scalar-timers, or registers for holding any bit pattern. The only restriction is that the instruments have outputs which can be gated and reset by the two control lines. The electrical specifications are that a logical one is between 0 and +1.0 volts and a logical zero is between +1.7 and +12 volts. The control unit is electrically isolated from the computer and these levels may be reversed by tine interchange of two wires at the computer input terminal.

The Event Iines are simple timing or transfer request lines which are activated by grounding the input. The Event Signal may be produced by a scalar overflow, a conversion complete pulse from an ADC, or pulse from a coincidence circuit. When an event occurs, it is routed through the inhibit gates to the Event Iatches. If the circuits are free, it will immediately be sent through the priority or sequential scan tie-breaking logic and into the diode pinboard at the center of the diagram, otherwise it will be held pending until it can be processed. Wrile the event is pending or in process, the corresponding Event Pending Output line goes to zero and thus can be used to gate an external timer for measuring system live time.

The diode pinboard is the device which programs the control unit and consists of three sections, an 8 X 16 Component Select Matrix, an 8 X 1 Interrupt Miatrix, and an 8 X 8 Event Inhibit Matrix. Pins placed in the appropriate columns along the Event Row will cause the corresponding Component Select Latches to be set whenever an event occurs. Note that each event can call for transfer of from one to sixteen data words and that components may be shared between events. If, for example, Events 2 and 3 share the same ADC, then the two events are mutually exclusive and should not be allowed to occur simultaneously. This can be done via the Event Inhibit Niatrix. If Event 3 is inhibited by Event 2, then the input gate to 3 is closed while 2 is pending or in process. 


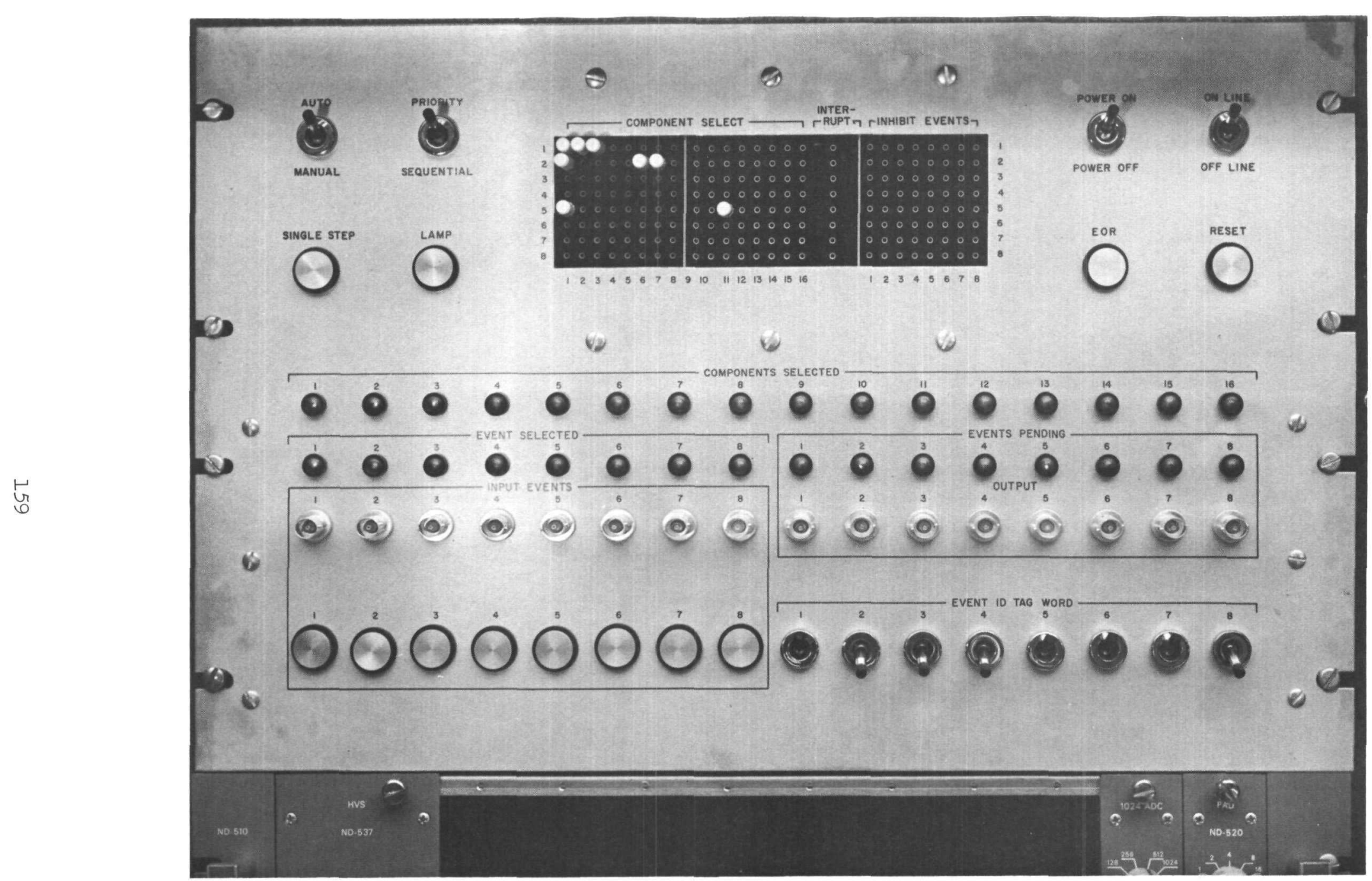

Figure 1. Nuclear Interface Control Unit 
EVENT INHIBIT LINES

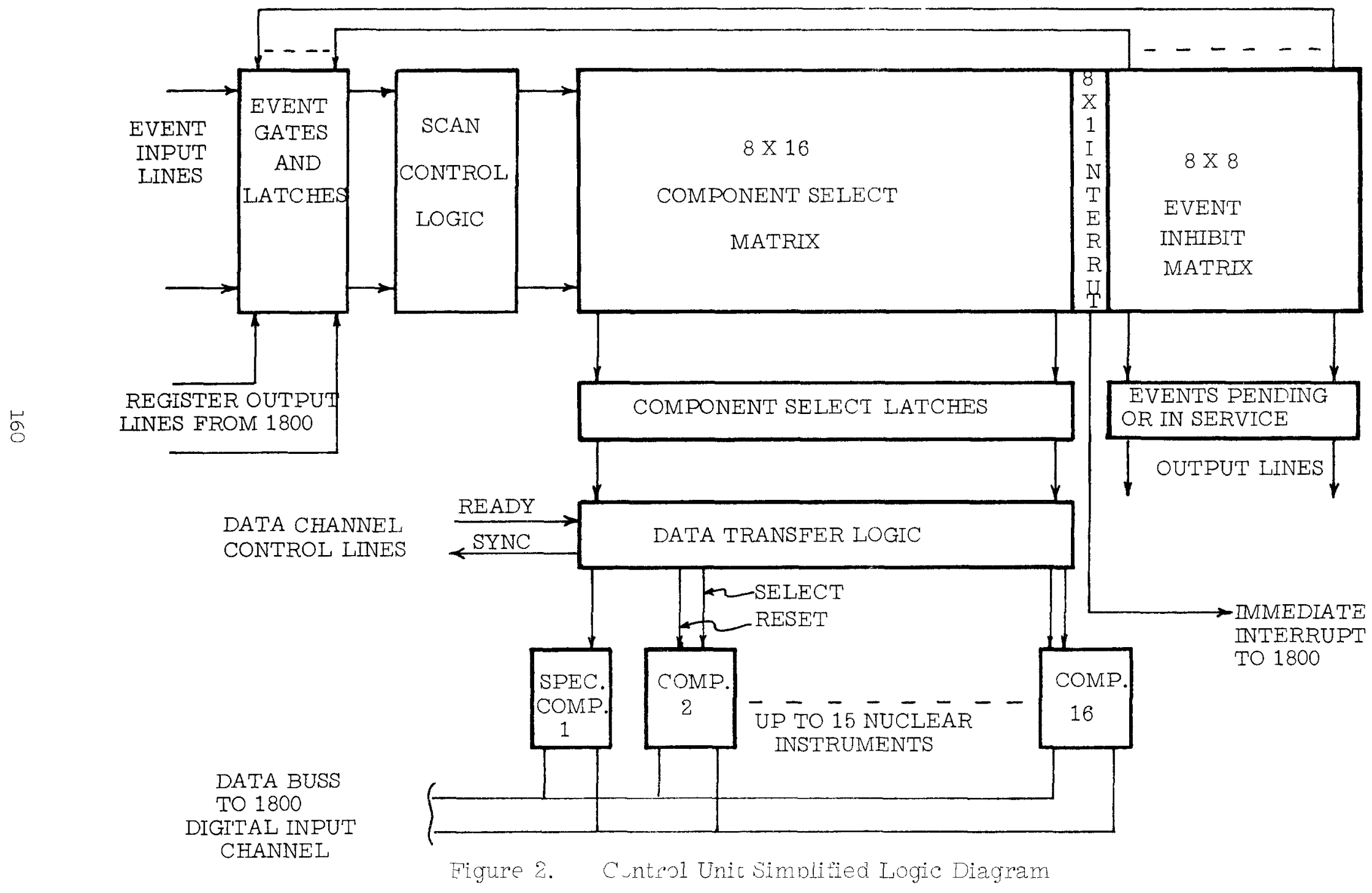


The Intermpt Matrix provides facility for sending an immediate interrupt to the CPU whenever a particularly interesting event occurs. In the case of a rare event it might signal for a special program to be loaded from disk. Note that it is not used for data transfer.

Once an event has been selected for processing and the Component Latches have been set, the data transfer logic sexially signals each component to place its output on the data buss and sends a Sync signal to the 1800 data channel. When the channel responds by dropping the Ready line, a reset pulse is sent to the component and the cycle repeats as long as any components or events are pending. To provide noise rejection the transfer time is currently set for $10 \mu \mathrm{s}$ per word although $4 \mu \mathrm{s}$ is possible. Of this, only $2 \mu s$ are required for actual transfer to core.

Since the eight events may belong to different experiments, some provision must be made for identifying the data in core. This can be done by tagging each word externally or by placing a pin in the first column. This corresponds to a special component which transfers an identification word prior to the data. The ID word is composed of eight bits from toggle switches on the front panel and eight bits from the event scan logic corresponding with the event which is in process. The transmitted data thus forms a table in core such that each block of data is separated by an ID word. Bit 0 is normally left on thus generating a negative ID which simplifies program recognition of each block.

At times it may be desirable to selectively inhibit one or more events while allowing the others to proceed normally. This is accomplished by controlling the Event Gates with eight register output points on the 1800. Thus, any experiment can effectively shut itself off without disturbing any other.

Other functions available are a switch to take the control unit off-line for manual check of the operating sequence, a single step button to transfer one word, and a rnanual interrupt which can be used to initialize or terminate the channel operation.

\section{PROGRAMMING SYSTEM}

The programming system used (PALS) is a multi-tasking operating system which allows program execution on either of two priority levels. The high level or foreground is used for real time data acquisition programs and is allowed a $5 \mathrm{~ms}$ time slice. The second level or middleground is used for on-line data analysis and is allocated $100 \mathrm{~ms}$ time slices. A background level is also available for time independent programs which are run to completion. Programs may be written either in a high level macro language which provides the capability of setting up special functions such as pulse height analyzer with one statement or in assembly language. A knowledge of assembly language will not be necessary to use the system.

All programs are saved on the disk in a relocatable format. They are loaded on reauest and may be scattered into any available 512-word page of core. All system resources, such as core pages and disk space, are dynamically allocated by the system and are reclaimed by the system when a program terminatos. The disk is organized as a large number of logical tapes 
which are created as needed. Thus, it is not necessary to know in advance how many words will be written to the tape as additional space is made available automatically. All requests to use data processing $I / O$ devices such as the line printer are automatically spooled if the device is busy.

The operation of this system could proceed in the following manner. To wire the system the experimenter will plug the instruments to be used into any available component position. Any event line can be used to trigger the transfer. As soon as pins are placed in the board and the event line attached, data collection is ready to begin. An interrupt push button will cause his program to be loaded in foreground mode into any available core pages. If no other program is using the interface, a data collection routine is loaded which starts the data transfer to core. If the interface is in use by another experiment, no load occurs but the linkage is changed to reflect the new program in the system.

At this time neither the user's program nor the data collection program is in execution although both are still in core. They have simply relinquished their use of the CPU until a buffer has been filled. The input channel is responsible for the actual data transfer, thus leaving the CPU free to work on any othe: program which needs service. If no otrer tasks are pending, the zy ${ }^{\vee}=m$ jinl go into a wait state.

Wren a buffer is full, an end of table intermupt initiates the unloading routine. The first step is to switch the incoming data to be read into an altemate buffer and then begin searching the table for ID words. When an ID is found, control is transferred to the proper user by executing an indirect branch through the event linkage table which was previously established. The event routine performs whatever task was assigned and then returns to the unpacking program.

At any time the experimenter may check his data by requesting $\in$ ither a plot or alpha-numeric display to be drawn on a storage oscilloscope. The display program is loaded from the disk, runs in the middleground, and thus takes no time from the real time program. When sufficient data has been accumulated, a second press of the interrupt button will remove the program from active status then load and pass control to an analysis program. This can be a middleground program which reduces the data and produces a printed report or a graphic display. When the analysis program is complete it may restart the data acquisition phase or exit, thus releasing its core pages to tine system.

If it is desired to record data on disk or magnetic tape for future offline analysis one simply pushes an interrupt button marked RECORD. Raw data from the input buffers is then dumped to tape until the process is terminated by a second push of the RECORD button. The record function can run concurrently with real time tasks or can be operated independently with no user's program in the system. Since the data being saved is exactly the same as that coming from the interface, a real time program can be written to accept data either on-line from the interface or off-line from the tape.

Note that all operations have been performed at a remote (from the computer) location without any restrictions or interference from the other 
tasks using the system. Furthermore, there is no restriction on the number of tasks which can be set into operation. Thus, if only one experiment is active, it has the entire capability of the computer at its disposal. As more experiments are brought on-line the system resources will be shared as requested.

\section{SYSTEM AVAILABILITY AND THRUPUT}

Two quantities which must be determined for any computer system are the fraction of the total time that the computer is available for reading data and secondly, the system thruput or average data rate. For time-shared systems these numbers are of vital importance and yet are virturally impossible to calculate. They must be determined by direct measurement. It will be useful, however, to make some estimates for the PALS system and compare them with computed times for two sample experiments which are described later.

First, consider the Interface Control Unit. Since this device operates on a data channel under control of the experiment, conventional lock-out of a fast instrument by a slow one will not be experienced. Data will be sent to the computer at $10 \mu$ s per word regardless of which program is in execution. The only delays experienced will be when one event must wait for a previous event to be cleared before the second is serviced. These delays can be easily calculated or measured by a live time meter on the Event Pending Output.

While a burst rate of 100,000 words per second is attainable, data transfer must stop when the core buffers are full. The fastest output device available is the magnetic tape at 45,000 words per second. Assuming a factor of $25 \%$ for system overhead the effective thruput rate in the record only mode becomes 34,000 words per second.

When operating in the real time mode, additional overhead is necessary. The input buffer must be scanned for ID words at $16 \mu \mathrm{s} /$ word and when one is found, an additional $19 \mu \mathrm{s}$ is needed to identify it and branch to the correct servicing routine. Assuming 4 words per event and an event servicing routine of 40 instructions averaging $5 \mu$ s per instruction, the maximum data rate becomes 17,000 words/sec. The system overhead factor associated with time slicing and buffer chaining may further diminish this to an effective rate of 13,000 words/sec.

Display and analysis programs which run in the middleground are of lower priority than the real time tasks and thus will not take any time away from the data acquisition process. The time required to set them up in the job queue has already been included in the system overhead. The problem is that when data are being collected rapidy the middleground routines may not run at all. They will, however, be held pending until the data rate slows and the input buffers have been unloaded. This shows the importance of preventing transfer of unnecessary data.

Clearly the advantage of the system described is not speed. If pure speed is required then fixed wire devices or dedicated computers should be 
used. (As a dedicated device the 1800 is capable of thruput rates of 150,0 , 0 words/sec. and single parameter pulse height analysis at 300,000 counts/sec.) The advantage of such a system is, rather, its great flexibility. The capacity for simultaneously handling data from unrelated experiments, of queueing multiple tasks at different priority levels, and providing an interactive environment for users at remote locations are the essential ingredients of a timeshared system.

These features make it very easy to use the computer. When combined with the characteristic that the system makes no attempt to restrict either the number of tasks in execution or the data rate, a situation will invariably develop in which the machine is overloaded. This will be immediately apparent to the experimenter, however, because his live time meter on the interface will drop to an unacceptable level. Furthermore, the solution is easy. Simply disconnect one or more of the experiments until the CPU has time to clear out the work pending.

Examples illustrating two typical experiments which may run concurrently are given in the following sections.

\section{PULSE HEIGHT ANALYSIS EXPERIMENT}

Assume that a spectrum to be studied has major peaks in areas which are widely spearated in energy with the intervening area of little interest. It is possible to define a non-linear analyzer such that the intermediate energies can be compressed into a few channels while the peaks are kept at maximum resolution. This is illustrated in Figure 3. Only two statements are required to set up the analyzer. The ANAI macro reserves the specified number of bins and assigns them a name. The PHA macro does the actual work of checking the upper and lower limits (U and I, factors), compressing the data by powers of 2 (M factor), adding the offset (O factor), and incrementing core storage.

Since the maximum positive count in a 16-bit machine is 32767 , some of the channels will overflow during the experiment. The PHA macro handles this by recording on disk the fact that a particular channel has overflowed and then resets the channel to zero. This overflow area which is referenced by the same name as the analyzer can be checked by an analysis program to determine the number of times a channel has overflowed.

A program to handle this experiment is shown in Figure 4 with the definitions of each macro in Table 1 . The entry point and name of the program is PROGA. It can be called into execution by process interrupt 20 or by command from a keyboard printer. The program sets up the control unit to read data and branch to E5 when an ID word corresponding to Event 5 is found. Process interrupt 19 is assigned to a plot routine and 20 is reassigned to cancel the data acquisition phase and start the analysis phase. Since there is no processing to do PROGA then relinquishes control.

Data collection is now proceeding automatically and the experimenter need do nothing until the display is desired. At that time pushing process interrupt button 19 causes the program to be re-entered at PLOT. The display programs are loaded into core, executed in the middleground, and then 


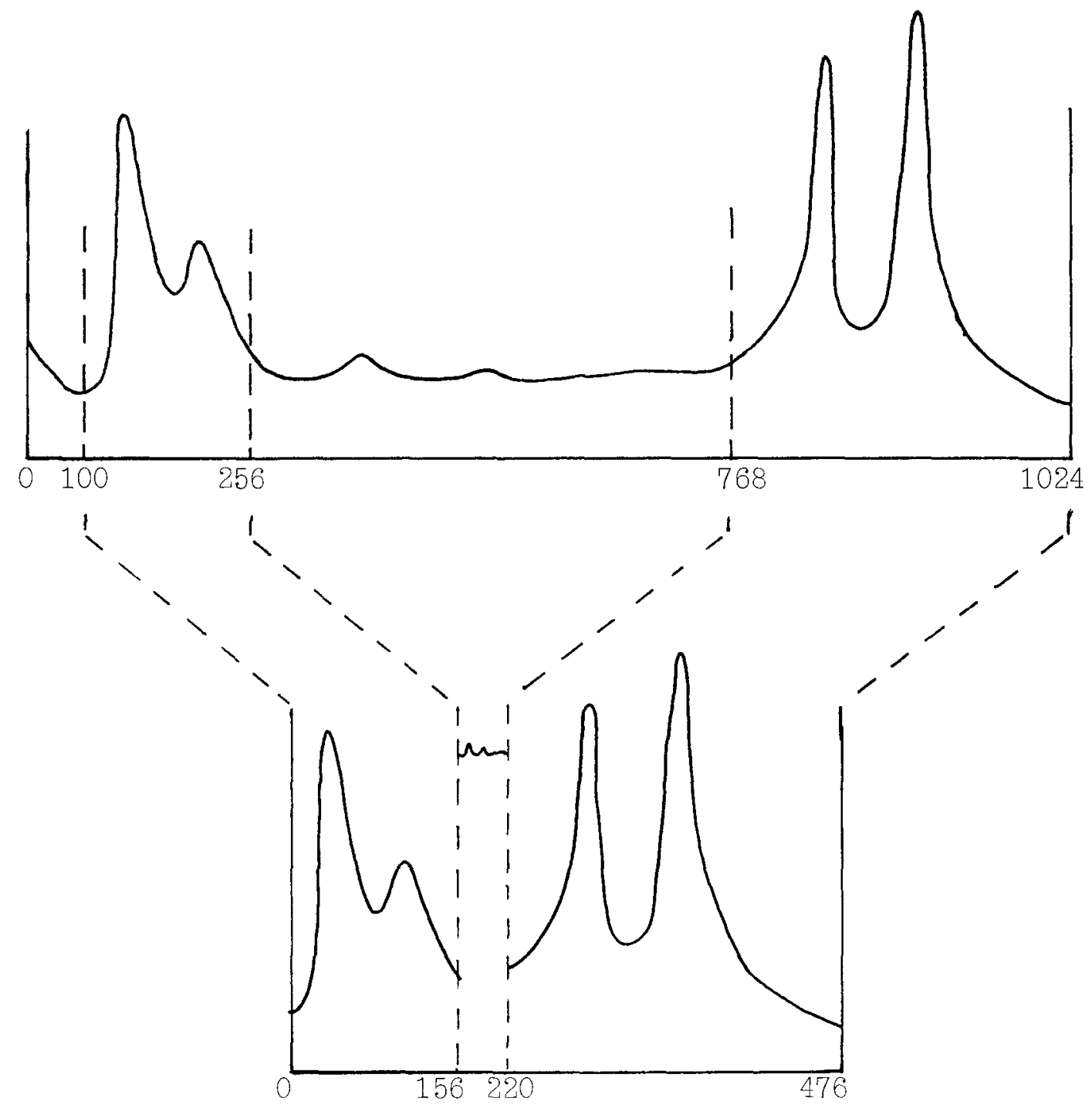

PHA NAME L1, U1, M1, 01, L2, U2, M2, 02, L3, U3, M3, O3

ANAL NAME, BINS

Figure 3. Non-linear Multichannel Analyzer 


\section{INIT}

\begin{tabular}{|c|c|c|}
\hline PROGA & SPCU & $0, \curvearrowright, \cap, 0, \pm 5,0,0,0$ \\
\hline & ASPI & PI2O, CAIC \\
\hline API & ASPI & PI19, PLOT \\
\hline & RELQ & \\
\hline PLOT & ERAS & J, C3 \\
\hline & PLTP & $J, X O, Y O, N P$ \\
\hline & $\mathrm{GO} 2$ & API \\
\hline CALC & GATE & 5,0 \\
\hline & CALS & PROG B, 2, TVEC \\
\hline & TERM & \\
\hline E5 & EVRT & 1 \\
\hline & PHA & $\begin{array}{l}\mathrm{SPEC}, 100,256,0,0,257,768,2, \\
157,769,1024,0,769\end{array}$ \\
\hline & FIN & E5 \\
\hline & ANAL & SPEC, 476 \\
\hline & END & PROG A \\
\hline
\end{tabular}

Figure 4. PROGRAM FOR PULSE HEIGHT ANALYSIS 
released from core. When the plot is complete the program loops back through the process interrupt assignment and again relinquishes control. This sequence may be repeated as often as a plot is desired.

Table 1. Macro Definitions

Macro Name

ANAL

ASPI

CALS

CNTR

ERAS

EVRT

FIN

GATE

GO2

INIT

PHA

PLTP

REIQ

SPCU

TERM
Use

set up a multichannel analyzer

assign a process interrupt to a program entry point

loan and execute the named program

set up a program counter

erase a storage scope

define the start of an event-handling routine

define the end of an event-handling routine

open or close the event input gates

branch to the named location

initialize a user's program

perform pulse height analysis

plot points on a storage scope

remove a program from the active queue

set up the linkage to event-handling routines and start the interface control unit

restore standard linkages for the control unit and remove this program from core

To stop the data collection phase, process interrupt 20 closes the event gate, calls an analysis program (PROG B) and exits from core. On exiting, all linkages and process interrupts are restored to their original values so that the entire process may be repeated if desired. at $\mathrm{E} 5$.

The timing for this program may be computed from the event routine 


\begin{tabular}{|c|c|c|}
\hline Find ID & anch to $\mathrm{E} 5$ & $35 \mu$ \\
\hline EVRT & & 18 \\
\hline PHA & & 95 \\
\hline FIN & & 12 \\
\hline & TOTAL & $160 \mu$ \\
\hline
\end{tabular}

Assuming $20 \%$ overhead, a sustained rate of 5000 events per second is possible.

If only one experiment were operating, the ID words would not be needed and the PHA macro could operate directly on the input buffer. Under these conditions the time for PHA would be $70 \mu \mathrm{s}$ and a data rate of 11,500 words/second could be attained. If no limit checking is desired the rate further increases to 34,000 words/second.

Exchanging the multi-user input routines for a single user routine can be done on demand from the experiment interface. Approximately two seconds are required to load the new routine from disk. The exchange in no way affects any program which is being run in the middleground or background.

\section{NOISE ANALYSIS EXPERIMENT}

A technique for analyzing noise in fast reactors by polarity correlation has recently been described by Cohn. ${ }^{6}$ This method allows calculation of the cross correlation function between two signals while recording only whether each signal is positive or negative at the time of sampling. Since this method involves only the manipulation of ones and zeros it is ideally suited for use on a fixed point computer.

The input instrumentation consists of a Schmitt trigger which provides data pulses to two pair of shift registers (serial to parallel converters). Each pair contains a sixteen bit register for each input signal. Data fills first one pair then the other so that data collection will be uninterrupted while the registers are being transferred to the computer.

These four registers may be plugged into the interface at any available component location. Since they will probably be driven near the maximum transfer rate the transfer signal from bank one should be assigned to Event 1 and that from bank two to Event 2. The pinboard is set up as shown in Figure 6. To insure that the data will be handled as fast as possible the scan control switch should be set to the priority mode. This causes Event 1 or 2 to be serviced before the pulse height analysis experiment using Event 5.

To compute the cross correlation function approximately 800 sample points or 50 sixteen-bit words for each signal will be used. At the high data rates used more than sufficient data will already be in the buffers by the time the event servicing routine has been notified that the first word is ready. Under these conditions the event routine has only to pick up the required quantity 


$\begin{array}{lll} & \text { INIT } & \\ \text { NOISE } & \text { SPCU } & \text { E1, } E 1,0,0,0,0,0 \\ & \text { RELQ } & \\ \text { FULL } & \text { GATE } & 1,0 \\ & \text { GATE } & 2,0 \\ & \text { CALS } & \text { XCORR, 2, TVEC } \\ & \text { CNTR } & \text { STOP, 10 } \\ & \text { GATE } & 1,1 \\ & \text { RELQ } & \\ \text { STOP } & \text { TERM } & \\ \text { E1 } & \text { EVRT } & 2 \\ & \text { FILL } & \text { TABA, 100, 2, FULL } \\ & \text { FIN } & \text { E1 } \\ & \text { END } & \text { NOISE }\end{array}$

Figure 5. NOISE ANALYSIS PROGRAM 


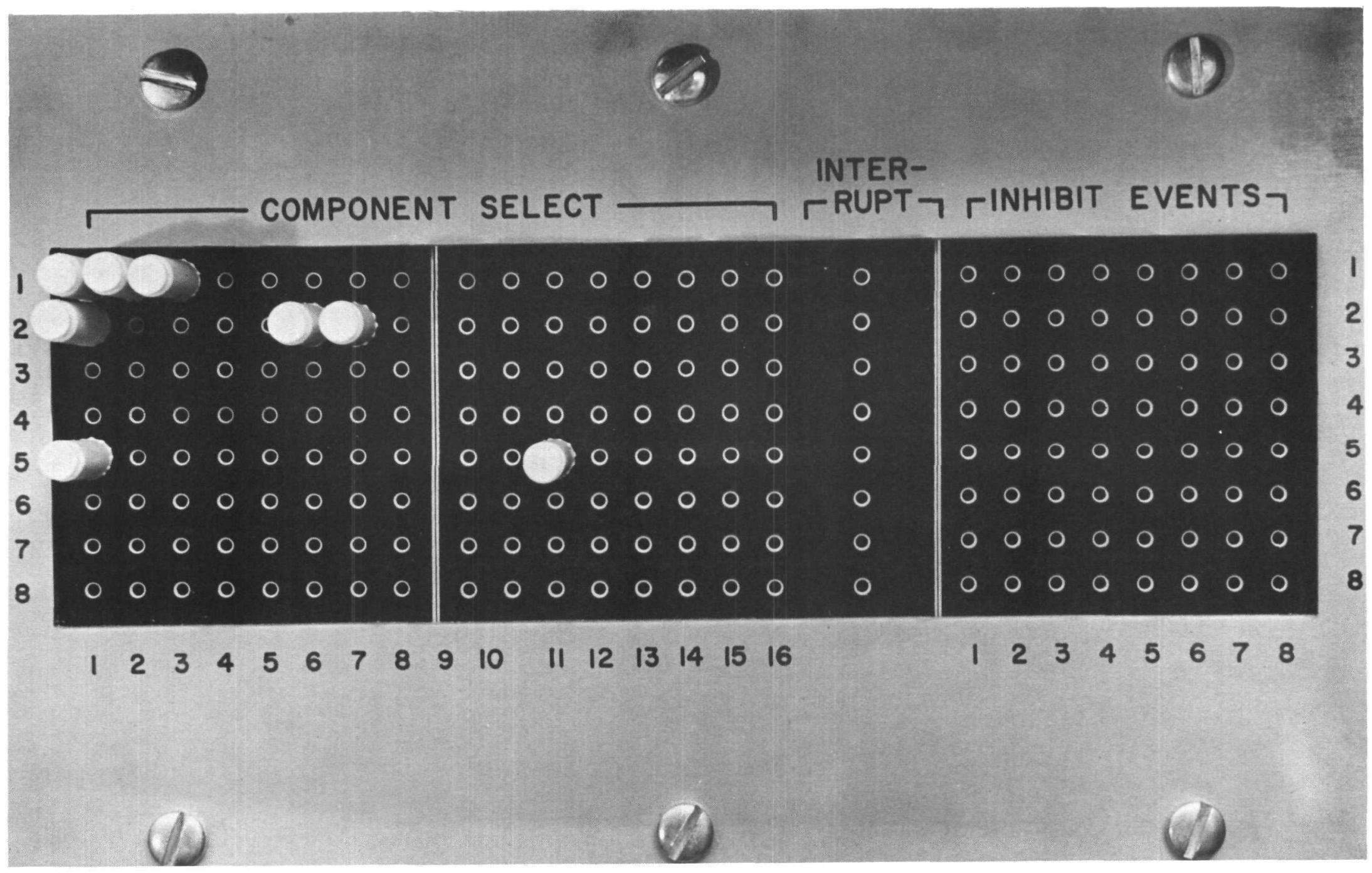

Figure 6. Pinboard Set-Up for PHA and Noise Analysis Experiments 
of data and move it to a work space for the analysis routine. A program to handle this experiment is shown in Figure 5.

The NOISE program can be loaded and set into execution by a process interrupt. On entering the program finds the control unit already in use by the pulse height analysis program, modifies the linkage so that both Event 1 and 2 will be serviced by the routine at $E 1$, and immediately relinquishes control. No processing can be done until the FILL routine in $\mathrm{E} 1$ has loaded. the work area and returns to the main program at FULI. Event Gates 1 and 2 are then closed to prevent unnecessary data transfer and the cross correlation program is started in the middleground. While this calculation is being done FILL clears any unused data from the input buffers so that the pulse height analysis program may continue. On return from the analysis, the input gates are opened so that a new set of data may be obtained. The process repeats ten times before being terminated.

Each cross correlation function calculated required $15 \mathrm{~ms}$ of real time operation to unload and clear the input buffers and $600 \mathrm{~ms}$ of middleground computation. Thus, while the burst rates are high, less than $2.5 \%$ of the total time is actually required for gathering the data. This intermittent use of the real time level allows the pulse height analysis program to proceed virtually unhindered.

\section{CONCLUSION}

The data acquisition system described has all the ingredients of a practical multi-user system. These are a hardware interface which accepts standard nuclear instrumentation as plug-in components, a medium size computer with large file capabilities and a programming system which allows multiple, real time and background programs to be in execution concurrently.

Fxperience gained thus far indicates that such a system will be most useful for those experiments which require relatively large amounts of file manipulation and interaction with the experimenter in real time. A realistic aggregate data rate of 5000 words per second or a burst rate of 100,000 words per second is permissible while still providing real time computation. This rate is independent of the number of programs sharing the system. Automatic adjustments in the operating system accommodate any number of users and experiments may be brought on-line or removed freely without disrupting any work in progress.

For those experiments which require a large fraction of the computer's resources, the system must be temporarily dedicated to a single real time user. This is done from the experimental area by loading a specialized input routine to handle data from the control unit and does not interfere with any middleground or background programs which may be in progress. In this mode data rates of 20,000 words/sec. may be realized while still maintaining the capability of real time computation.

This type of system should not be considered as a replacement for multichannel analyzers or other special purpose devices. It is rather a general purpose instrument which can operate in conjunction with fixed wire 
devices to provide the experimenter with meaningful data reduction in time for it to be useful to him in controlling the course of his experiment.

\section{ACKNOWLEDGEMENTS}

I wish to express my appreciation to Dr. Joel Bimbaum of IBM Research in Yorktown Heights, New York, and Dr. Charles Sederholm of the IBM Scientific Center in Palo Alto, California, for allowing me to borrow so many of their ideas, and also to Diana Block, Chris Jenny and Donna Nelson with IBM, Palo Alto, for being so very patient and helpful in making the programming system operational.

\section{REFERENCES}

1. IBM Journal of Research and Development, (13), (January, 1969).

2. Proceedings of the Conference on Automatic Reduction and Acquisition of Tuclear Data, Karlsruhe (1964).

3. Use cf Computers in Analysis of Experimental Data and the Control of Nuclear Facilities, AEC Symposium Series \#10, (May, 1967)

4. H. L. Gelernter et al., "An Advanced Computer-Based Nuclear Physics Data Acquisition System, "Nuclear Instruments and Methods, (54), 77-90, (1967).

5. C. H. Sederholm, "1800 Palo Alto Laboratory System," IBM Program Information Department, Hawthorne, New York, PID 1800-05.1.002, IBM Corporation, (1968).

6. C. E. Cohn, "Reactor Noise Studies with an On-Line Digital Computer," Seminar on Reactor Noise Analysis, Kyoto, Japan, (September, 1968). 


\title{
APPLICATION OF AN IMAGE PROCESSING SYSTEM TO FRAGMENTATION STUDIES*
}

\author{
Margaret K. Butler \\ Argonne National Laboratory
}

\begin{abstract}
Over the past six years image processing at Argonne has become a way of life. Since the CHLOE film measuring system became operational early in I963 it has found application to such diverse problems as chromosome classification, $1,2,3$ spark chamber track processing, 4 and fingerprint identification. ${ }^{\text {. }}$ Recently the CHLOE hardware and the image processing software system developed for biological image processing have been put to work in an attempt to facilitate chemical engineering studies of physical and chemical interactions between reactor structural and fuel materials and reactor coolants. Fragmentation experiments are a basic part of these studies. In these experiments single drops of materials such as molten tin, lead, bismuth, and uranium dioxide are dropped into reactor coolants such as water and sodium. The degree of fragmentation that ensues is a function of the drop height (entrance velocity) and/or drop size, and the temperatures of the molten metal and the quenching medium. Measures of this fragmentation (e.g., the count of the number of particles, their individual surface areas, and the cumulative particle size distribution) are made and the information gained is then used in reactor safety heat transfer calculations.
\end{abstract}

In the experiments considered to date a piece of tin of approximately 0.4 grams in weight and with a spherical surface area of $0.6 \mathrm{~cm}^{2}$ has been used. It is heated to a specific temperature in the $250^{\circ}$ to $600^{\circ} \mathrm{C}$ range in a quartz dropper positioned in a small electric resistance furnace. A single drop is visually observed to fall from a height of one to four feet into room temperature water when a slight overpressure is applied in the dropper. These experiments have been conducted both in air and in an inert atmosphere (argon). Five separate runs are made of each experiment. When a run is completed the metal fragments, varying in size from a few microns to a centimeter, are collected and measured.

Sieving, the most common method of particle size measurement, 6,7,8 involves the use of a number of open containers each with uniform regularlyspaced openings in the wire-cloth bottom. These pans are stacked in order of increasing aperture size so that when the sample is placed on top and shaken through the stack it is divided into fractions on the individual sieves. These fractions are then weighed to obtain the particle size distribution. Because of the difficulty of producing wire cloth with uniform openings of small size sieve analysis is generally restricted to particles larger than $50 \mathrm{mi-}$ crons. Newer micromesh sieves of electroformed nickel have extended the range of applicability downward and successful sieving of 20 micron particles has been reported. In these fragmentation experiments the quantity of material involved is so small (less than one-half gram) that sieving is impractical.

A second technique employed in particle size analysis is microscopy. Using an optical microscope the images of particles can be measured and

*Work performed under the auspices of the United States Atomic Energy Commission. 
classified, by size, either by direct visual examination, by projection on a screen, or from recorded photomicrographs. In this technique use of the electron microscope permits study of particles down to the .001 to 5 micron size range. A major limitation of microscopy is the time and effort necessary to ensure that a representative and sufficient sample is selected and that accurate size measurements are recorded. In an attempt to remove these limitations, automatic and semiautomatic scanning devices have been introduced to permit measurement of a large number of particles in a reasonable time and to eliminate the bias inherent in the tedious human measurement procedures. Consequently, it was only natural that chemical engineers at Argonne faced with the measurement of fragmentation residue considered the possibility of using CHLOE.

At the completion of a run when the metal fragments have been collected they are carefully arranged so that the individual particles are separated and then photographed with a Nikon camera onto a $35 \mathrm{~mm}$ film strip together with a photograph of a standard of known area. The standard used is a flat, circular washer with an outside diameter of $1.44 \mathrm{~cm}$. A schematic diagram of the photographic setup devised to produce the film strips is shown in Figure l. Plus-X film is used with a high-contrast development process.
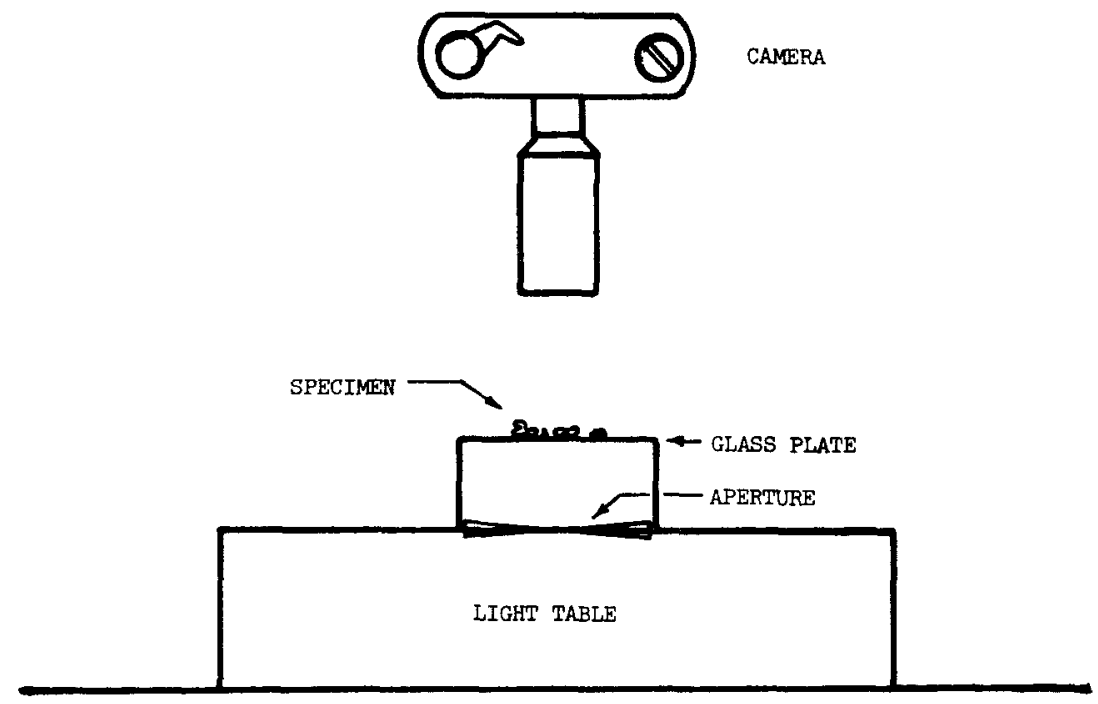

Figure 1. Schematic Diagram of Photographic Setup

These film strips serve as the input to the CHLOE system where the measurement function and the first part of the particle size analysis are carried out. Major components of the CHLOE system, designed and developed in the Applied Mathematics Division at Argonne, include an ASI-210 digital computer, an optical scanner and a visual display unit. ${ }^{9}, 10$ The commerically-built computer has an 8192 21-bit word core memory with a 2 us cycle time, 64 external device 
interrupt lines, and two buffered input/output channels which can simultam neously transfer data at a rate of 30,000 words/second. It is equipped with a console typewriter, paper-tape reader and punch, and a single magnetic tape unit. The cathode-ray tube (CRT) scanner operates by projecting a spot of light onto the film and viewing the light transmitted through the film with a photomultiplier. The CRT light spot is directed by a pair of $\mathrm{x}, \mathrm{y}$ counting registers to scan a selected rectangular area of the 1.25 by 1.25 inch aperture. The spot appears in one position of the 4096 by 4096 raster for one microsecond, is blanked out for three microseconds, and appears in the succeeding location the following microsecond. Thus, it is possible to scan the complete raster, if desired, in roughly one minute. When the photomultiplier registers a specified change in the transmitted light from one spot to the next the contents of the counting registers, i.e., the $x, y$ coordinates of the spot, are transmitted to a prescribed buffer area in the computer memory, together with the observed density. This density is recorded at eight levels, representing the eight discriminator circuits included in the photomultiplier design. A block diagram of the scanner system is shown in Figure 2. Other features of the equipment are the ability to control the CRT beam current and the film transport mechanism, and to set the scanner to operate in a horizontal (left to right) or vertical (bottom to top) mode and on black or white background film. The computer directs the operation of the scanner and its associated equipment as it does its own peripheral equipment through the use of an external device control word (EDCW) assigned that operation. Consequently, these operations are programmable, realizing one of the major design objectives of the system--a flexible means of digitizing photographic information regardless of its experimental origin.

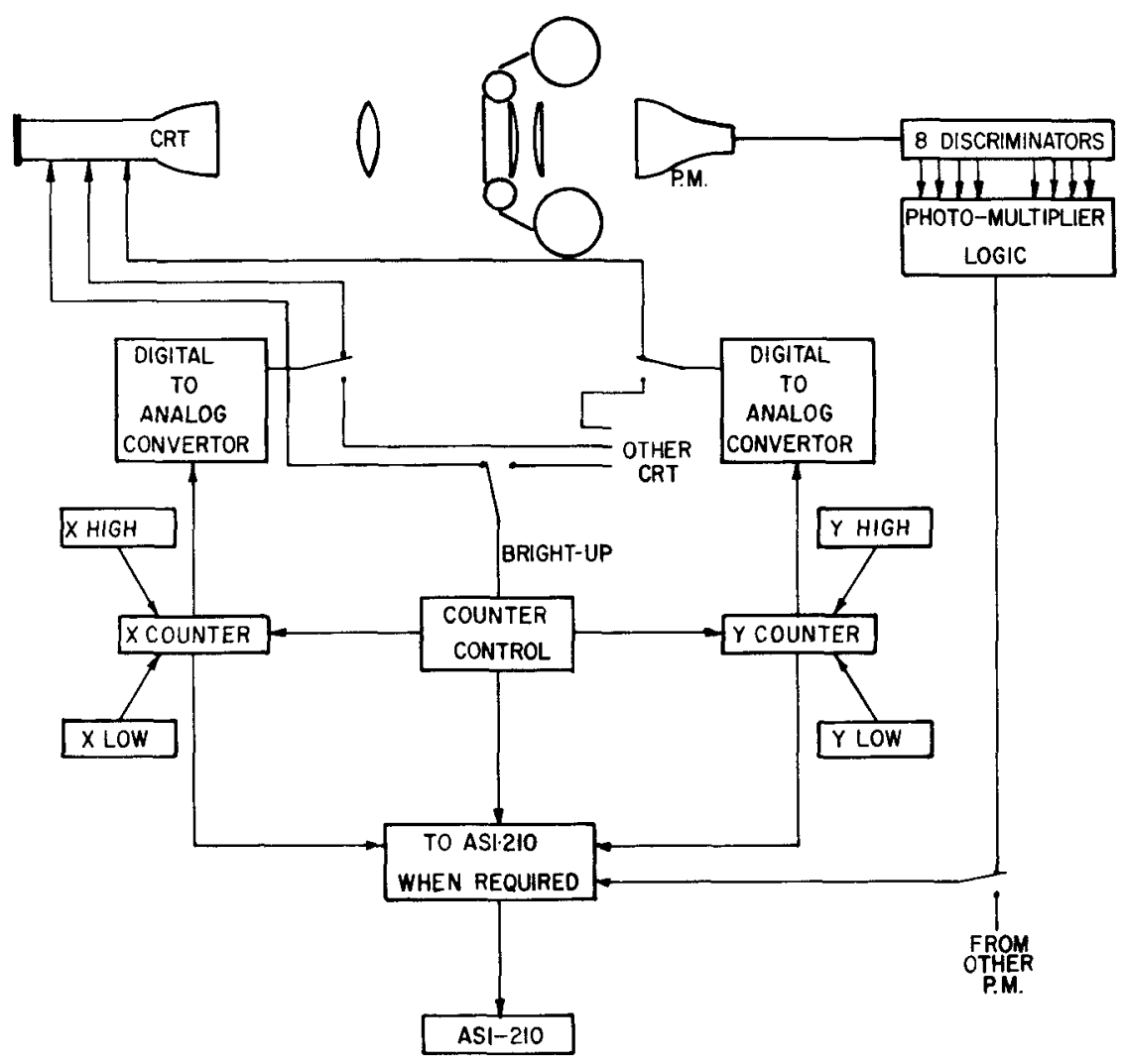

Figure 2. Schematic Diagram of the CRT Scanner 
This same chilosophy has been carried over in the development of the software systems for CHLOE. Two general-purpose systems have evolved. One was primarily designed to handle the large volume of film emanating from spark-chamber experiments in bigh-energy physics, a need which dictated the construction of CHuT. II The cther was originally created in cooperation with the Biological and Medical Research Division for the autonatic analysis of chromosome dat'a. A schematic diagram of this biological image-processing system as applied to the fragmentation resilue problem is shown in Figure 3 . The KARYOGRAM program for the CHLCE system accepts as problem parameters the setting of the film ccndition (i.e., black-on-white or white-on-black), the boundaries $c I$ the scanning area, the scan frequency or raster size, an indication of the sprocket hole/frame ratio of the film, and arguments for the density computer ilgorithm used to determine the CRT beam current and discriminator level settings and for the measurement and shape construction routines.

APPLIED MATHEMATICS IMAGE PROCESSING SYSTEM

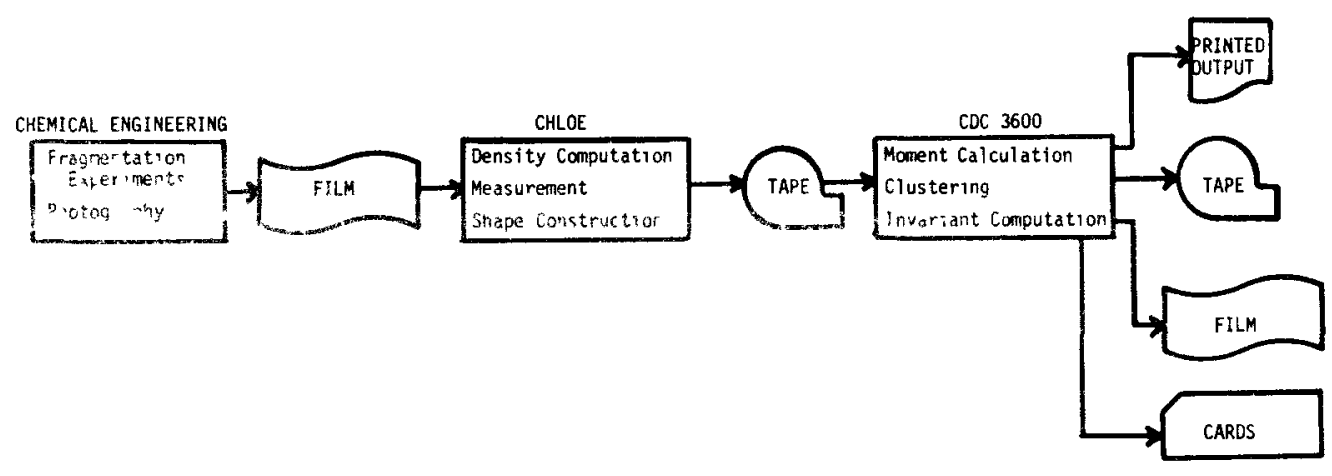

Figure 3. Application of an Image Processing

System to Fragmentaticr St liki-s

When the reel of film to be measured has beeri placed in the scanner unit and the KARYOGRAM progrem is iritially read into the ASI-210 computer it requests the operat on to load the problem parameter tape and to type in identification information for the film sequence and the magnetic tape which will be used for recording the output information. A coarse scan is then initiated over the prescribed area and repeated giving the operator the opportunity to select and/or adjust the frame for proper registration in the aperture of the scanner. This is accomplished by observing the monitor display and typing the appropriate character to move the film forward $(+)$ or backward $(-)$ in $\frac{1}{4}$ sprocket-hole steps, or to advance the film one frame $(R)$. Once the desired frame is seen to be in position for measurement, striking the typewriter space bar causes the program to proceed to the density computation. The "density computer" algorithm in the present program is tne result of a series of program modifications and engineering changes incorporated as a result of our experience to date. ${ }^{3}$ Its objective is to determine the beam current value and discriminator level best suited to digitize the information contained in a particular frame image.

To describe this algorithm it is necessary to first explain the action of the discriminators in the CHLOE photomultiplier circuit. The eight levels are set to register evenly-spaced intervals of light in density ranging from completely black film (level 0) to clear film (level 8). Whenever a particular level is triggered, all lower levels will have registered as well. When a scan operation is started and at the beginning of every scan line the density of the previously-viewed point (a special register) is automatically set to the 
background density of the film. As the scan proceeds the density is measured at each point by the "active" discriminator levels and compared with that measured at the previous point and remembered by the special register. When the density is different the coordinates and density of the next point are sent to the computer memory via the second input/output channel. If the density has not changed the counters advance and the next point is examined. When the last point of a line is examined its density is forced to the background density of the film. Figure 4 shows CHLOE examining fragmentation residue.

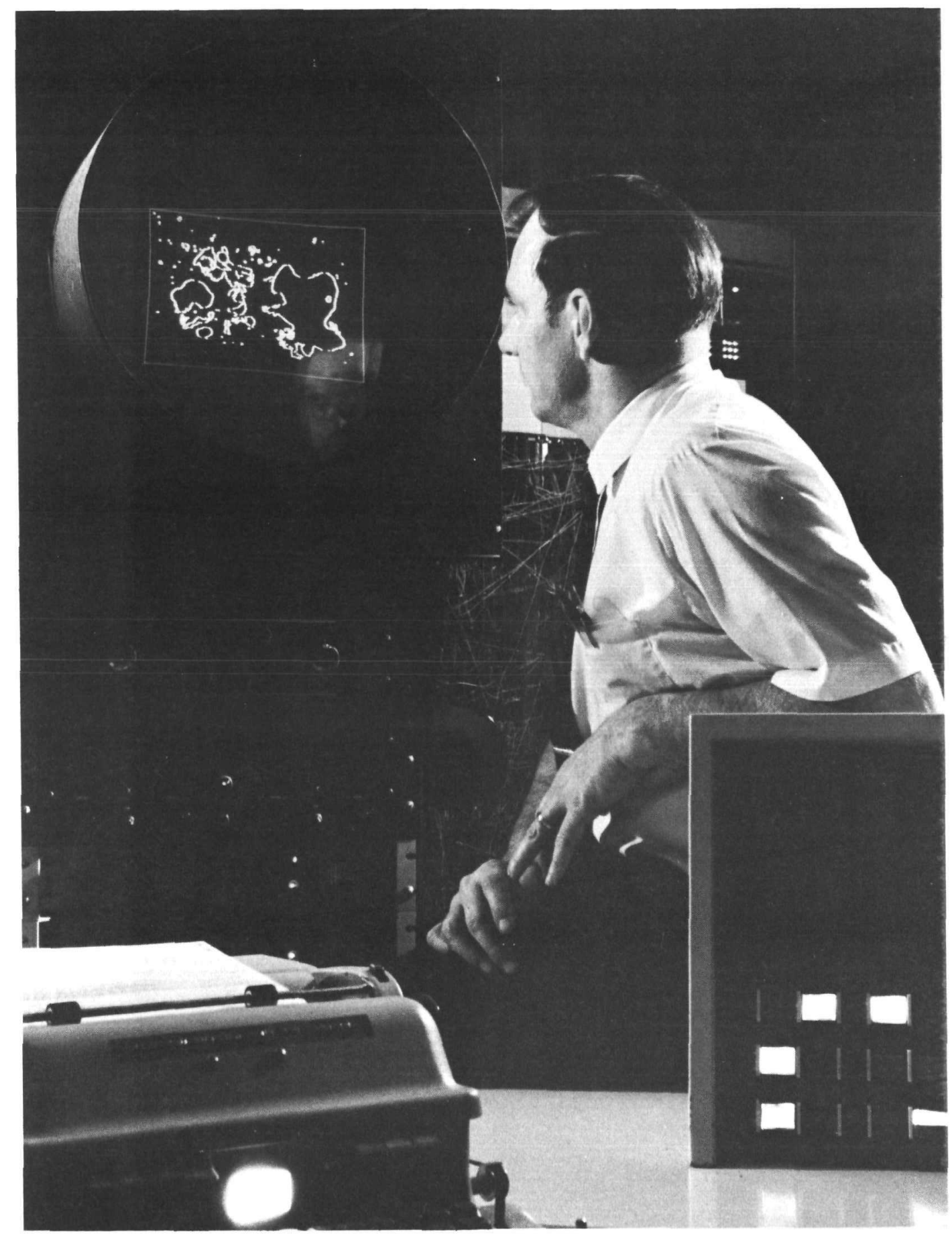

Figure 4. CHLOE Visual Display Unit View of Fragmentation Residue 
It was observed that for film of good contrast quality a plot of the number of digitized coordinates against discriminator level showed a definite plateau. At one end of the discriminator range the number of coordinates decreases, while at tre other erd the number increases as the machine begins to digitize noise from the CRm phosphor grain and electrical noise from other system components. The algorithm used generates a CRT beam current in a quasirandom fashion and then performs a cuarse (128 by 64 ) scan with all eight discriminator levels activated. These are activated by setting the appropriate bits in the piotomuliplier masi of the Pnctomlitiplier Function Register (EDCW 62). From the coordinatc and density nformaticn obtained from this single scan a curve of segment courts fur each level is derived. This calculated curve is then tested against criteria kased cn noput parameters to see if the required number of levels meet the raximum and minımum segment count requirements. If so, the beam count is accepted, the discriminator level is set to the central value of the calculated plateen and the program proceeds. If not, the CRT beam current is varied and a second scan is made. If, after a prescribed number of trials, success has not been achieved, the frame is rejected as having too low a contrast quality and the next frame is examined.

Once the density computer algorithm has successfully set the CRT beam current and discriminator level the program proceeds to what is called the preliminary scan. This is a short period during which the scanning unit is directed to scan the prescribed area sixteen times using a 128 by 128 raster. This gives the operator a chance to intempot and realign the film if necessary or

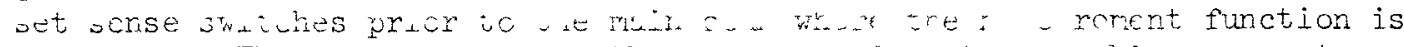
pertormed. The raster sıze for the man scar is set $4 y$ problem parameters.

Since the hardware transmits coordinate and Jensity infurmation only when there is a density change of a specified magnitude, only the edge coordinates of the images are recorded in the buffer area. With the horizontal scan mode in operation these are entered as two words for the left-edge coordinate with $\mathrm{x}$-coordinate and density in the first word and $\mathrm{y}$-coordinate and density in the second. Then two more words with the right-edge information are transmitted in the same format. The KARYOGRAN program regards trege four-word units as "Iine segments." Scanning proceeds as shown in Figure 5. The coordinates and density values transmitted for this illustration would be as shown in Table I considering the film to have a black background with the area external to the clcsed shape "black" and the active discriminator level as number four.

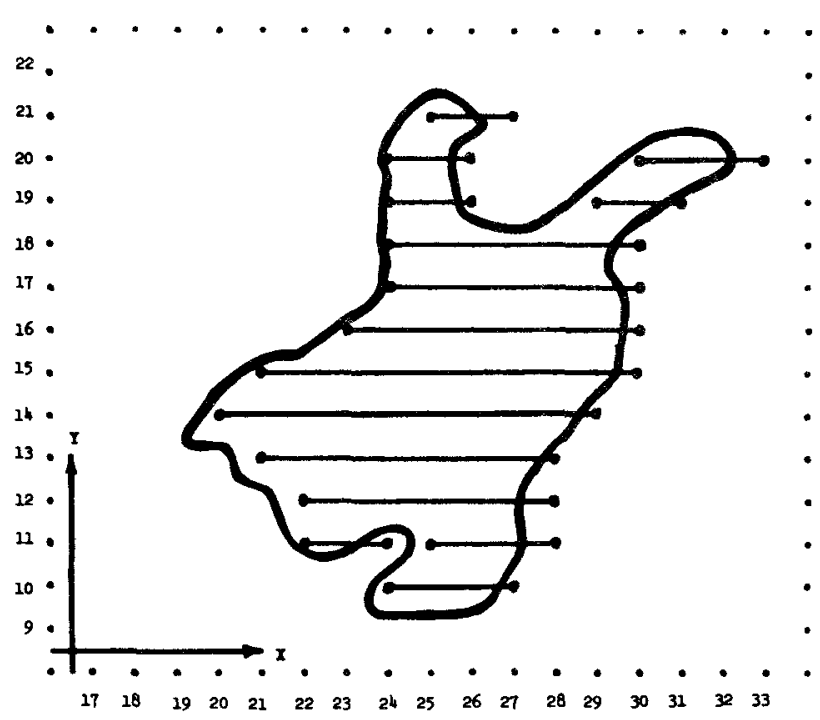

Figure 5. Operation of Scanner 
TABLE I, Data Transmitted to Buffer Area as a Result of Scanner Operation Shown in Figure 5

\begin{tabular}{c|c|c|c|c}
\hline $\begin{array}{c}\text { Line Segment } \\
\text { Number }\end{array}$ & \multicolumn{2}{|c|}{ At Left-Edge of Shape } & \multicolumn{2}{c}{ At Right-Edge of Shape } \\
\cline { 2 - 5 } & First Word & Second Word & First Word & Second Word \\
\hline 1 & 0014010 & 0005010 & 0015400 & 0005000 \\
2 & 0013010 & 0005410 & 0014000 & 0005400 \\
3 & 0014410 & 0005410 & 0016000 & 0005400 \\
4 & 0013010 & 0006010 & 0016000 & 0006000 \\
5 & 0012410 & 0006410 & 0016000 & 0006400 \\
6 & 0012010 & 0007010 & 0016400 & 0007000 \\
7 & 0012410 & 0007410 & 0017000 & 0007400 \\
8 & 0013410 & 0010010 & 0017000 & 0010000 \\
9 & 0014010 & 0010410 & 0017000 & 0010400 \\
10 & 0014010 & 0011010 & 0017000 & 0011000 \\
11 & 0014010 & 0011410 & 0015000 & 0011400 \\
12 & 0016410 & 0011410 & 0017400 & 0011400 \\
13 & 0014010 & 0012010 & 0015000 & 0012000 \\
14 & 0017010 & 0012010 & 0020400 & 0012000 \\
15 & 0014410 & 0012410 & 0015400 & 0012400 \\
\hline
\end{tabular}

After a buffer area has been filled with line-segment information the shape construction routine examines the horizontal position of each segment in that buffer relative to those on the $\mathrm{N} y$-levels below it, where $\mathrm{N}$ is a problem parameter. Segments whose projections onto the $\mathrm{x}$-axis have a non-zero intersection are forced to acquire the same shape identification number indicating they belong to the same connected shape. An overlap parameter, $\varepsilon$, supplied on the problem input tape is incorporated in making this comparison. Since the line segments are horizontal the right-edge $\mathrm{y}$-coordinate and density word is superfluous and these memory locations are used for shape labeling. Line segments which the routine is unable to associate with a shape are labeled with a negative segment accession number. The CHLOE program uses a circular buffer scheme which overlaps accepting edge-coordinate information from the scanner (I/O channel 2), examining and linking these line segments to construct possible shapes, and writing this labeled information onto magnetic tape (I/O channel 1) for use as input to the second or CDC-3600 phase of the system. Consequently, due to the finite length of the buffer areas, it is necessary to maintain throughout the processing of each frame a table of shape number correspondences which can be used later by the CDC-3600 program, METAPHAZ, to complete the shape construction process.

As the coordinate tape is read into the larger machine the program computes the first ten absolute xy moments (through the third order) of each accepted shape; at the same time, if requested, the reconstituted frame will be plotted by the DD80A film recorder and stored on the disk for later plotting as necessary. Next, METAPHAZ finishes the shape construction operation and attempts to form initial clusters of shapes for possible assembly into fragments. In processing an image, shapes may often be viewed by the scanner as several disconnected pieces; the clustering algorithm attempts to join these pieces. This is done by approximating each shape by an ellipse and putting nearby shapes into the same initial cluster. Up to six elements can be associated 
into a single cluster. At this point the invariant computation is performed. The shapes are classified by moment invariants as described by $\mathrm{Hu}{ }^{12}$ the particular invariants used here are Euclidean moment invariants since they are chosen to be invariant under the extended Euclidean group, that is, the group in the plane consisting of translations and proper and improper rotations. The first seven invariants are formed from the ten absolute xy moments. After an initial cluster is formed the program tests all possible subclusters by computing their moment invariants and accepts the largest clusters which meet input requirements. Successful clusters, or fragments, are then numbered and printed in an edited output format in the form of nine identifying quantities, the coordinates of the centroid and the seven invariants. The first moment invariant represents the computed area of the fragment. In addition, system options provide DD80A film recorder output of the reconstituted picture showing each of the identified particles or fragments, labeled two per frame, and cumulative fragment library tapes and/or cards for additional processing as appropriate to further study of the application. In the chromosome application this material is utilized for decision plane calculations. ${ }^{3}$

So far the application of this image-processing system to fragmentation studies has been concerned primarily with the optimization of the two initial stages of the process: the photographic techniques and the data acquisition from the film strips. Fragmentation residue covers a wide size range and the preparation of the particles for photographing is a tedious operation. The individual particles must be adequately separated and the lighting must be adjusted to ensure uniformly high contrast over the frame. The smaller fragments or a particular area in early experiments tended to have a markedly lower contrast than the remainder. This is hard to ascertain at the time the film is being prepared but is painfully obvious to CHLOE and often apparent upon examination of the print. In addition, the irregularly-shaped three-dimensional quality of the larger fragments lends itself to a shadowing effect which is readily apparent in Figure 6 . Over the past year experimentation in running film strips prepared with the specimens at different elevations over the light table, using different size apertures, and a range of camera positions has resulted in a steady improvement in the quality and uniformity of the input medium.
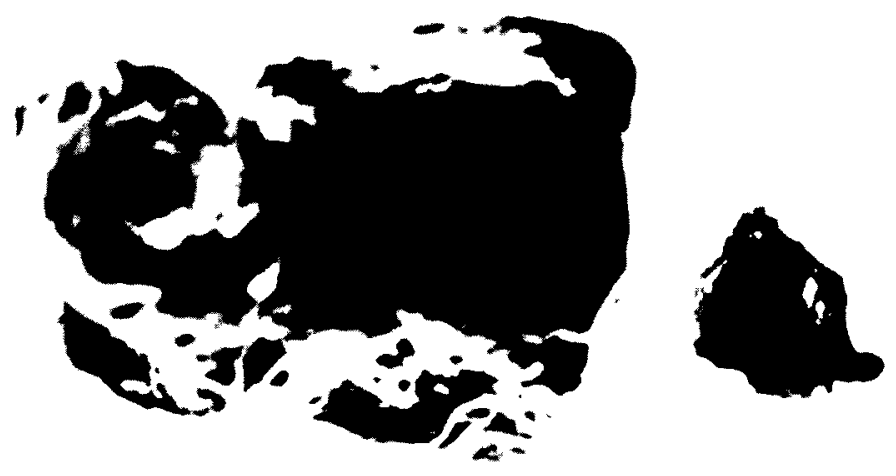

Figure 6. "Shadowing" Effect 
The second stage refers to the optimization of operating procedures and parameter values so that the digital information supplied to the computer truly represents the information one wants to extract from the photograph. Obviously, this optimization is heavily dependent upon the success achieved in the first stage. For example, with the presence of the shadowing effect the scanner "sees" the fragment as a large number of disconnected pieces. The parameters of the computer programs must then be adjusted to force the shape construction and clustering routines to merge these into a single acceptable cluster. In addition, efforts have been made to optimize the parameters involved in the photomultiplier level and beam setting procedures and to determine optimal values of other problem parameters such as the raster size, minimum shape area, and segment overlap length. In raster-size experiments two frames were examined extensively, Frame PTSZ 15-9 (Figure 7) and Frame PTSZ 15-3 (Figure 8). The fragments shown on each are identified by number and some of the results are presented in Tables II and III. At the time this experiment was conducted the shadowing effect was primarily responsible for the large shape counts shown. It has been reduced significantly. However, it can be seen that while use of the 4096 by 4096 raster (SDX,SDY $=12$, representing the power of 2) may or may not measure the smaller particles, measurement of these particles is accompanied by extraneous noise measurements. Later, an experiment comparing the 2048 by 2048 raster scan with the 1024 by 1024 scan for a complete run of 36 frames showed no indication of increased recognition of the smaller particles.

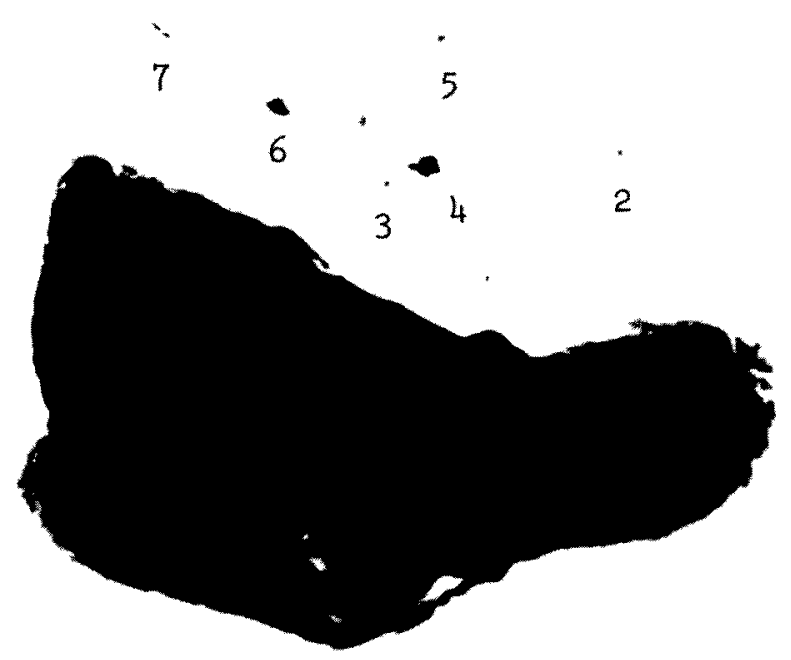

1

Figure 7. Frame PTSZ 15-9 
TABLE II. Study of Areas, Frame PTSZ 15-9

\begin{tabular}{|c|c|c|c|c|c|c|c|c|c|}
\hline $\begin{array}{cc} & \text { SDX, SDY } \\
\text { PM/BEAM } \\
\text { Shape } \\
\text { CIDEX } \\
\text { ID } \\
\end{array}$ & $\begin{array}{l}\text { DNERGE }=.00 \\
11 \\
4 / 944 \\
7 \\
6 \\
\end{array}$ & $\begin{array}{l}39 \\
4 / 920 \\
6 \\
7 \\
\end{array}$ & \begin{tabular}{|l|} 
Sequence 113 \\
12 \\
$6 / 1008$ \\
4 \\
12 \\
\end{tabular} & $\begin{array}{l}31 \\
10 \\
3 / 912 \\
5 \\
5 \\
\end{array}$ & $\begin{array}{l}10 \\
6 / 1000 \\
4 \\
3 \\
\end{array}$ & $\begin{array}{l}10 \\
4 / 936 \\
7 \\
3 \\
\end{array}$ & \begin{tabular}{|l|} 
DNERGE $=.01$ \\
11 \\
$4 / 944$ \\
7 \\
5 \\
\end{tabular} & 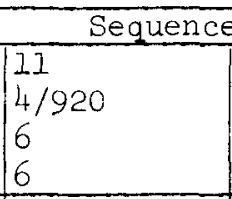 & $\begin{array}{l}1278 \\
12 \\
6 / 1008 \\
4 \\
10 \\
\end{array}$ \\
\hline 1 & $\begin{array}{l}.000001431 \\
.00003743 \\
.03398 \\
.00004363 \\
.034062 \\
\end{array}$ & $\begin{array}{l}.000002623 \\
.03308 \\
.0000004768 \\
.00002789 \\
.0000174 \\
.033128\end{array}$ & $\begin{array}{l}.00004959 \\
.000002086 \\
.0000001192 \\
.0000003576 \\
.000001132 \\
.0000008941 \\
.00004303 \\
.000004888 \\
.03411 \\
.034212\end{array}$ & $\begin{array}{l}.000002861 \\
.00003338 \\
.03368 \\
.033716\end{array}$ & .03411 & .03394 & $\begin{array}{l}.000001431 \\
.03402 \\
.00004363 \\
.034065\end{array}$ & $\begin{array}{l}.000002623 \\
.0000004768 \\
.03311 \\
.00001740 \\
.033130 \\
\end{array}$ & $\begin{array}{l}.00004959 \\
.000002086 \\
.0000008941 \\
.00000149 \\
.00004315 \\
.000004888 \\
.03411 \\
.034212\end{array}$ \\
\hline $\begin{array}{l}2 \\
3\end{array}$ & & & & & & & & & \\
\hline $\begin{array}{l}4 \\
5\end{array}$ & .00003815 & .00002289 & .00003868 & .00003338 & .00003624 & .00003338 & .00003815 & .00002289 & .00003868 \\
\hline $\begin{array}{l}6 \\
7 \\
8\end{array}$ & .00002098 & .000009298 & $\begin{array}{l}.00002509 \\
+ \text { noise }\end{array}$ & .00001240 & .00002098 & .00001717 & .00002098 & .000009298 & $\begin{array}{l}.00002509 \\
+ \text { noise }\end{array}$ \\
\hline Spread Area & .03412 & .03316 & .03427 & .03376 & .03416 & .03399 & .03412 & .03316 & .03427 \\
\hline
\end{tabular}



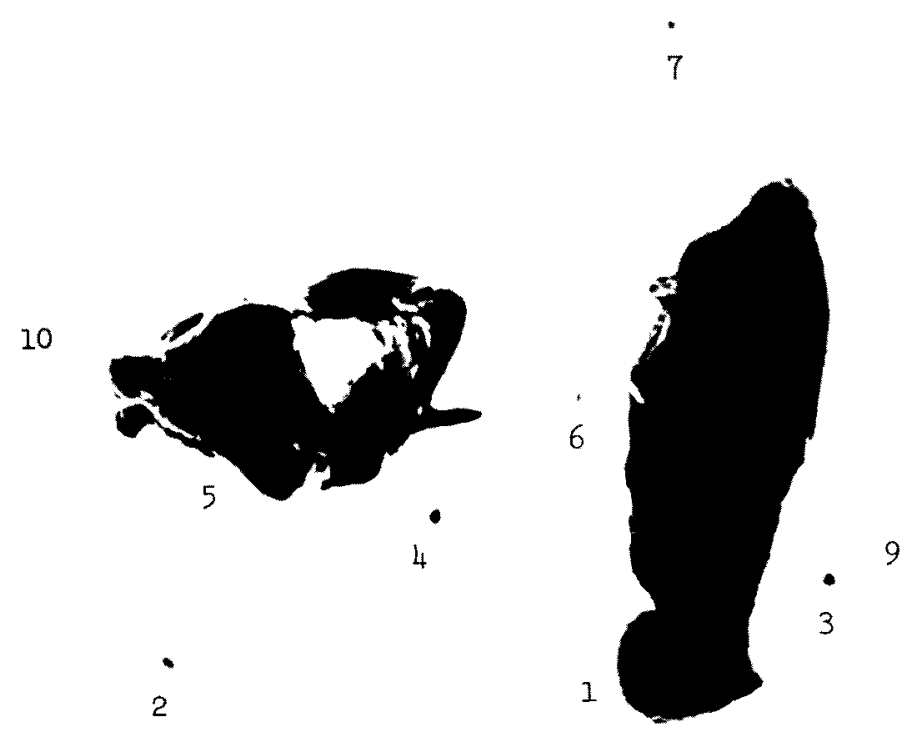

Figure 8. Frame PTSZ 15-3

TABLE III. Study of Areas, Frame PTSZ 15-3

\begin{tabular}{|c|c|c|c|c|c|c|c|}
\hline \multirow[b]{2}{*}{$\begin{array}{c}\text { SDX,SDY } \\
\text { PM/BEAM } \\
\text { Shape } \\
\text { ID } \\
\text { CIDEX } \\
\end{array}$} & \multicolumn{4}{|c|}{ DMERGE $=.0039$ Sequence 1104} & \multicolumn{3}{|c|}{ DMERGE $=.01$ Sequence 1204} \\
\hline & $\begin{array}{l}11 \\
4 / 920 \\
6 \\
20 \\
\end{array}$ & $\begin{array}{l}11 \\
4 / 920 \\
6 \\
22 \\
\end{array}$ & \begin{tabular}{|l|}
12 \\
$4 / 920$ \\
7 \\
22 \\
\end{tabular} & $\begin{array}{l}12 \\
4 / 920 \\
6 \\
35 \\
\end{array}$ & $\begin{array}{l}11 \\
4 / 920 \\
6 \\
17 \\
\end{array}$ & $\begin{array}{l}11 \\
4 / 920 \\
6 \\
16 \\
\end{array}$ & $\begin{array}{l}12 \\
4 / 920 \\
7 \\
21 \\
\end{array}$ \\
\hline 1 & .013692 & .013718 & .014852 & .013717 & .013692 & .013718 & .014854 \\
\hline 2 & + & & $1.335^{-5}$ & & & & $1.335^{-5}$ \\
\hline 3 & $4.053^{-6}$ & $4.53^{-6}$ & $1.842^{-5}$ & $6.199^{-6}$ & $4.053^{-6}$ & $4.53^{-6}$ & $1.842^{-5}$ \\
\hline 4 & $5.722^{-6}$ & $5.96^{-6}$ & $1.919^{-5}$ & $7.153^{-6}$ & $5.722^{-6}$ & $5.96^{-6}$ & $1.919^{-5}$ \\
\hline 5 & .007088 & .007084 & .008684 & .007081 & .007088 & & .008684 \\
\hline 6 & & & $1.490^{-6}$ & & & & $1.490^{-6}$ \\
\hline 7 & & & $3.934^{-6}$ & & & & $3.934^{-6}$ \\
\hline 8 & $1.431^{-6}$ & $1.192^{-6}$ & $9.358^{-6}$ & $1.431^{-6}$ & $1.431^{-6}$ & $1.192^{-6}$ & $9 \cdot 358^{-6}$ \\
\hline 9 & & & $2.980^{-7}$ & & & & $2.980^{-7}$ \\
\hline 10 & & & $\begin{array}{r}3.576^{-7} \\
+ \text { noise } \\
\end{array}$ & & & & $\begin{array}{l}3.576^{-7} \\
+\quad \text { noise } \\
\end{array}$ \\
\hline Ppread Area & .02079 & .02082 & .02362 & .02082 & .02079 & .02082 & .02362 \\
\hline
\end{tabular}


An investigation of the optimum minimum area cutoff revealed that noise and smaller fragments were equally likely to be measured when this parameter was reduced to about $3 \times 10^{-6}$. The 1.25 by 1.25 inch scanner aperture is considered to have unit area. Manual selection and refinement of the discriminator level and beam eurrent settings was tested and the results compared with those achieved by the density computer algorithm. The reproducibility of these results has also been investigated. Table IV lists the true shape counts and those determined by both the refined manual and automatic procedures for the most recent film strip sample. The second run figures indicate a measure of the reproducibility of the system. Note that the "automatic" runs were made almost a week apart. The SURFA-2 film strip contains many fragments of a size below the minimum size acceptable for CHLOE digitization, accounting for the differences in the number of shapes on the film and the number counted by CHLOE. To include these in the analysis it would be necessary to magnify the smaller particles by a larger factor prior to photographing. In this application the engineers nave chosen instead to restrict the analysis to particles "visible" to CHLOE. It appears that the parameters now in use with the KARYOGRAM program can, given a photograph of good contrast quality, extract the digitized information desired.

TABLE IV. Comparison of Shape Count and Repeatibility, with Manual and Automatic Discriminator Level and Beam Current Setting

Tape $039652 / 11 / 69$

Tape $10884 \quad 2 / 17 / 69$

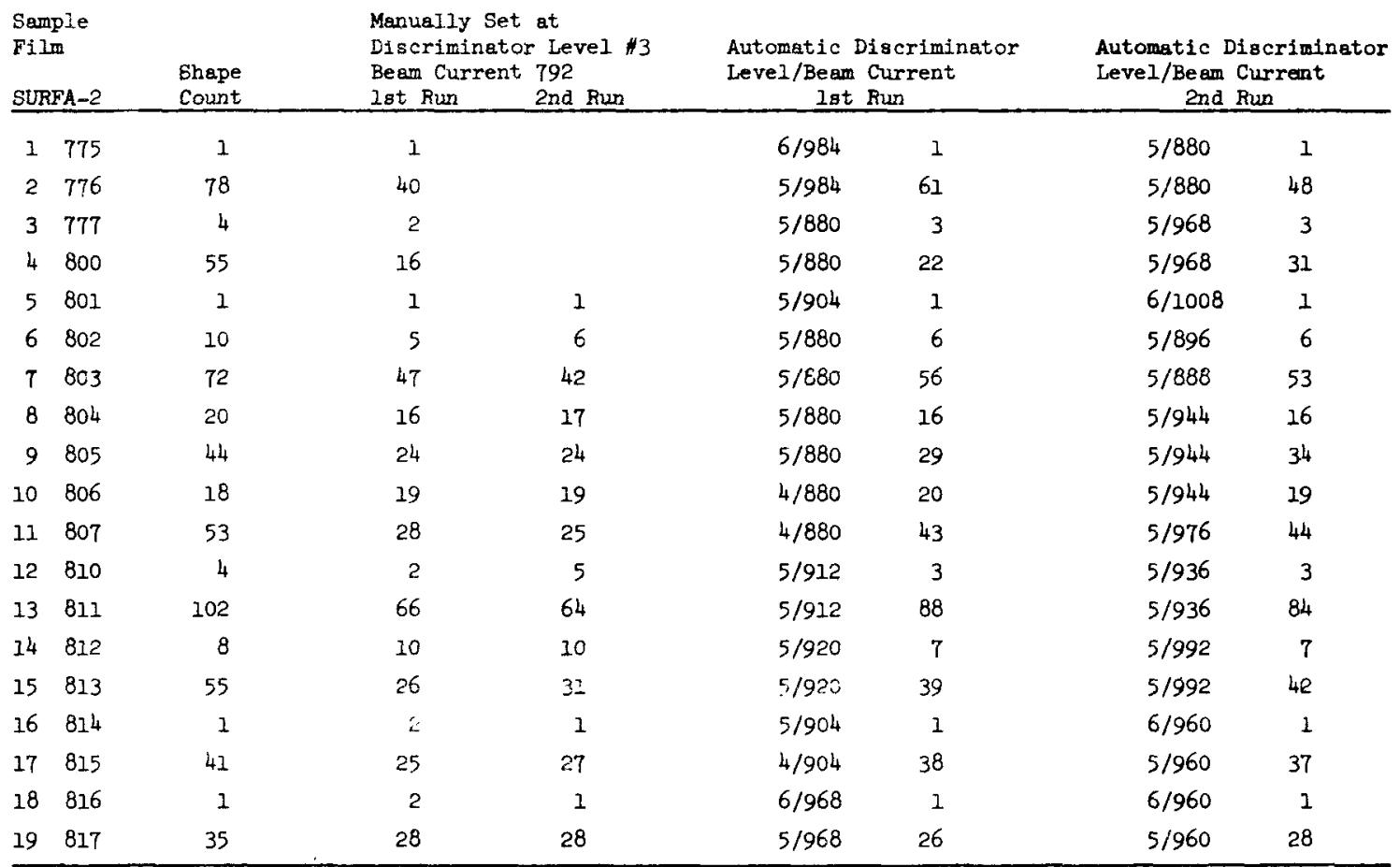

It is necessary still to optimize the two later stages in the process: the recognition of fragments and the determination of the particle size distribution. In the chromosome application decision surfaces in the 7-dimensional measurement space are used to separate chromosome "points" from non-chromosome ones (e.g., nucleii, noise). These decision surfaces are computed from the analysis of sample data and fed back into the system as program parameters. No use of this part of the image-processing system has been made in the fragmentation residue analysis. 
A selection of photographs indicative of the samples obtained in these fragmentation experiments together with the corresponding reconstituted piccures is shown in Figures 9-1l.
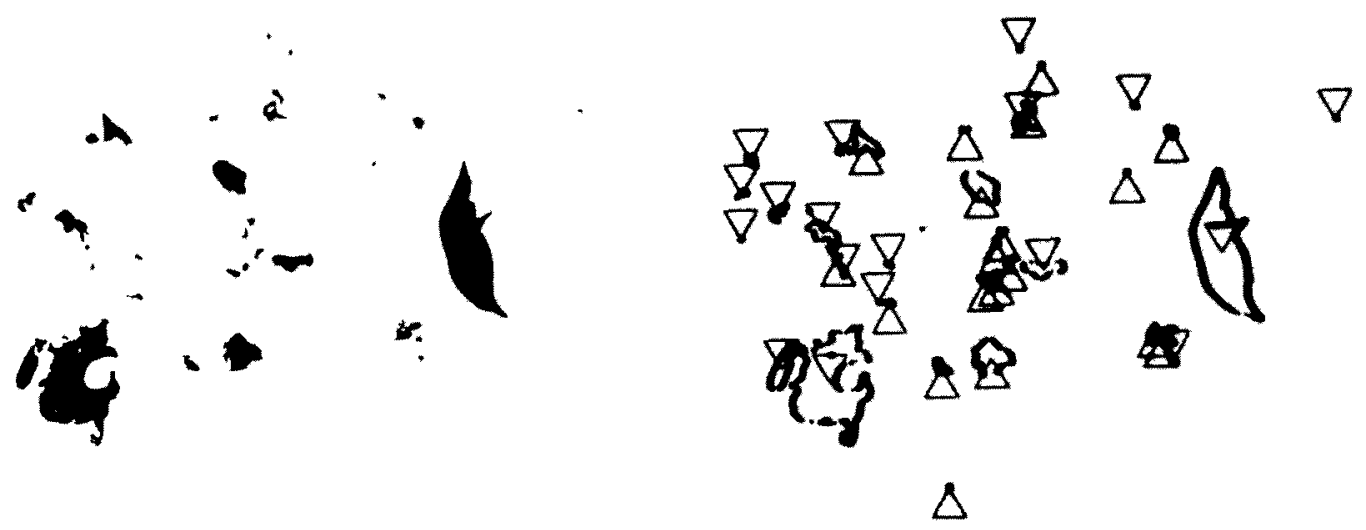

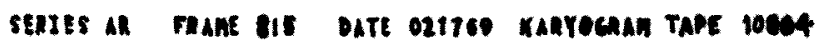

Figure 9. SURFA-2 Film, Frame 17
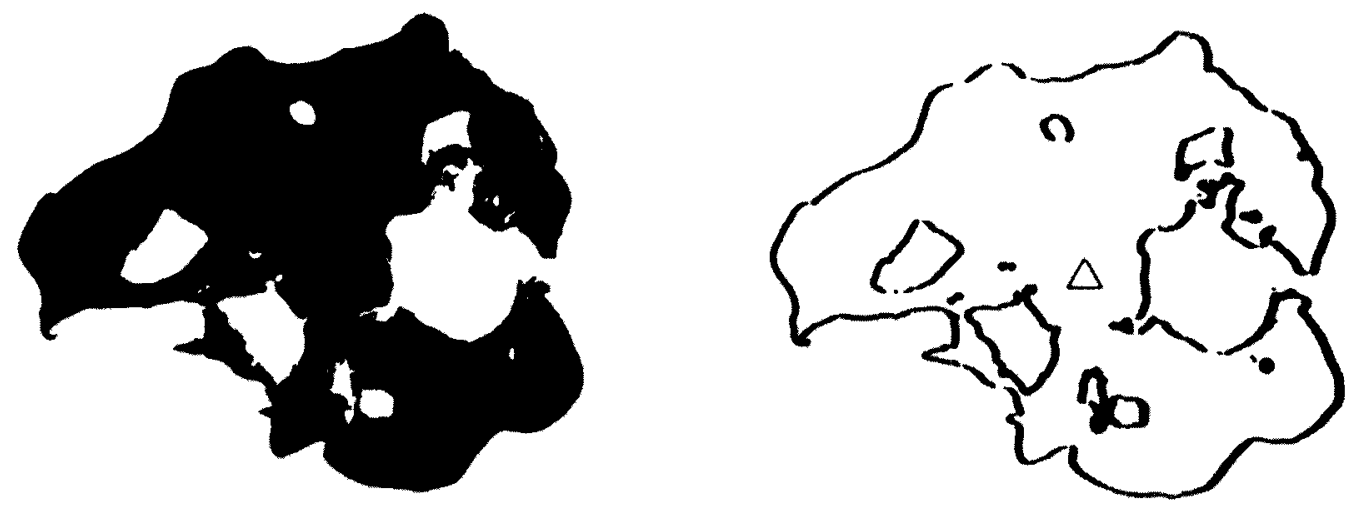

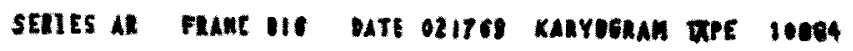

Figure 10. SURFA-2 Film, Frame 18

185 


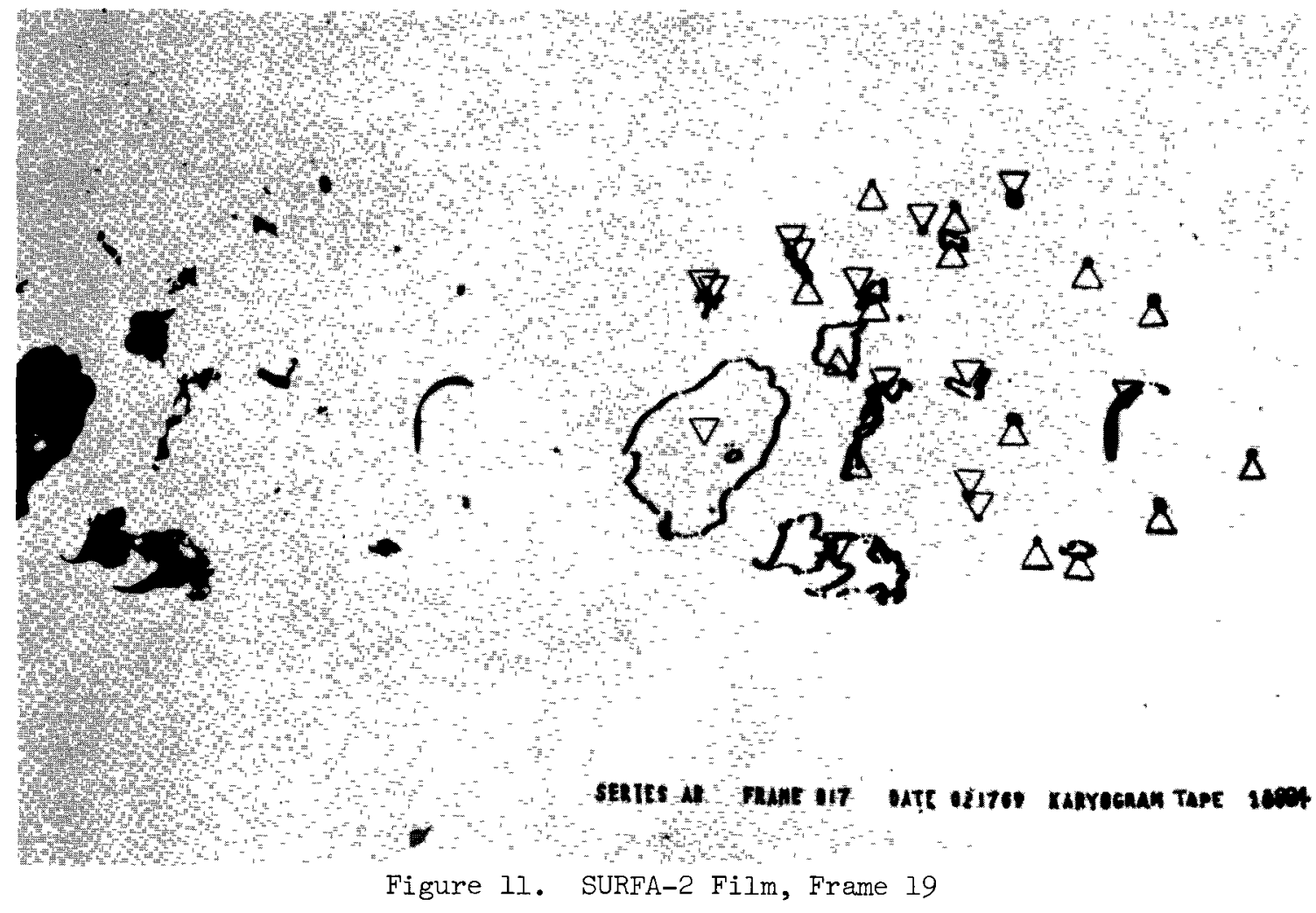

In our work to date the chemical engineers have taken the printed output (Figure 12) together with the pictorial output (Figures 13-15) and using a film strip projector screened the system results to ensure that no particle of interest has been skipped and that no noise has been included. If the system has failed to merge pieces of a fragment this is noted and the appropriate area correction made. Using this information together with the shape tape they have devised auxiliary computer programs to compute the particle size distribution and related computations of interest for the run including such things as initial drop diameter, Sauter mean diameter, average particle thickness, total two-dimensional surface area, and Weber number. The cumulative particle size distribution is then plotted on log probability paper. The distribution function most frequently associated with particle size analysis is the lognormal distribution ${ }^{14}$ which can be obtained from the normal distribution by the transformation $z=\gamma+\delta \log x$. A straight-line approximation is drawn through the plotted points and the mean and the standard deviation can be readily determined from the graph. The mean is read off at the 50 percent probability level and the standard deviation is calculated as the ratio of either the 84.13 percent to the 50 percent probability value or the 50 percent to the 15.87 percent value. A useful property of the lognormal graph is that the transformation from the cumulative particle size distribution to a surface area distribution or a particle volume distribution can be readily accomplished. The distributions will be parallel lines displaced vertically by $2.3026 \delta \sigma_{z}^{2}$ where $\delta=2$ for the surface distribution and 3 for the volume distribution.

Future efforts will be devoted to further refinements of the first two stages of the process with major attention directed to optimizing these last stages of fragment recognition and the development of a particle analysis system for production use. 


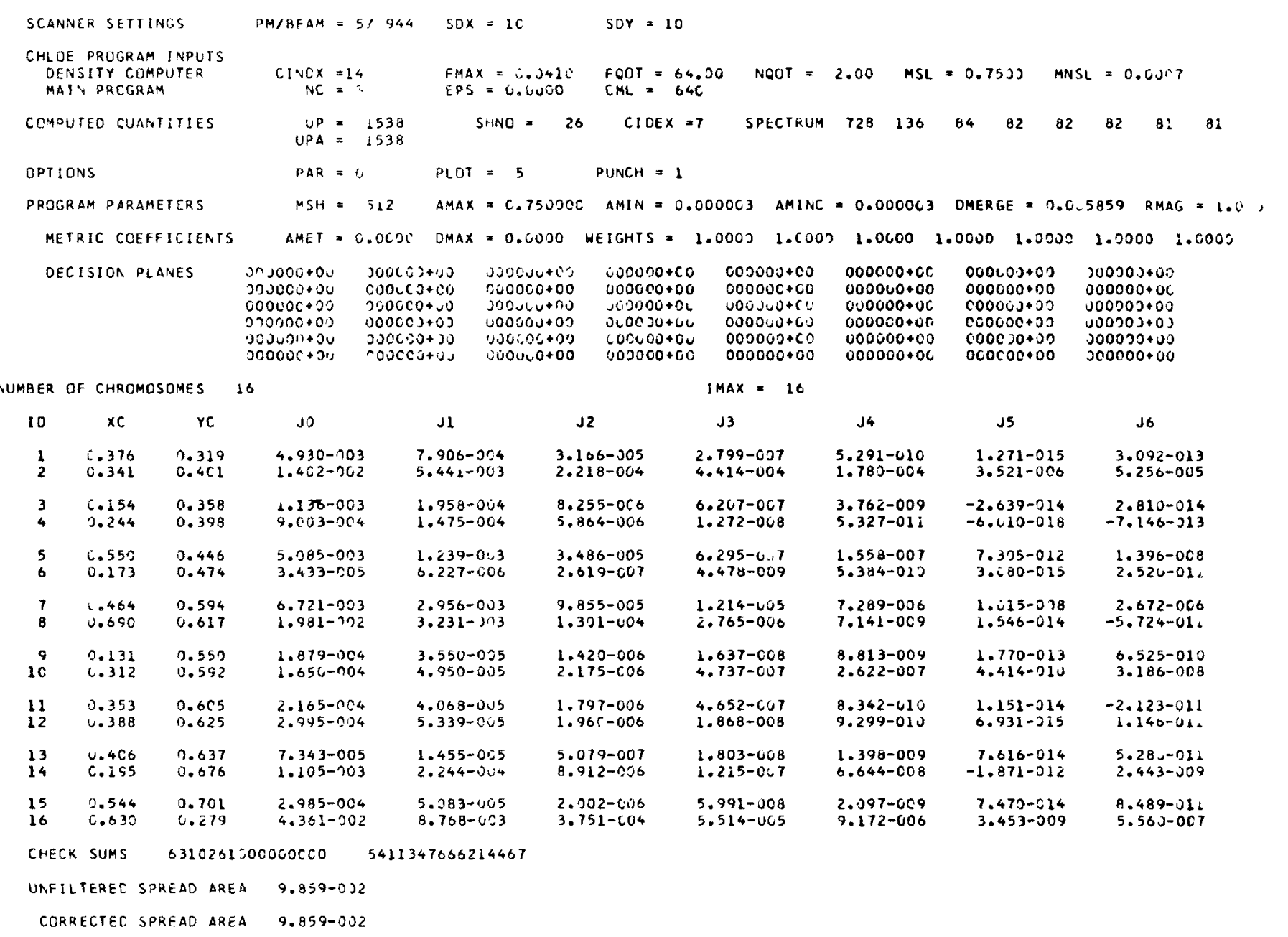




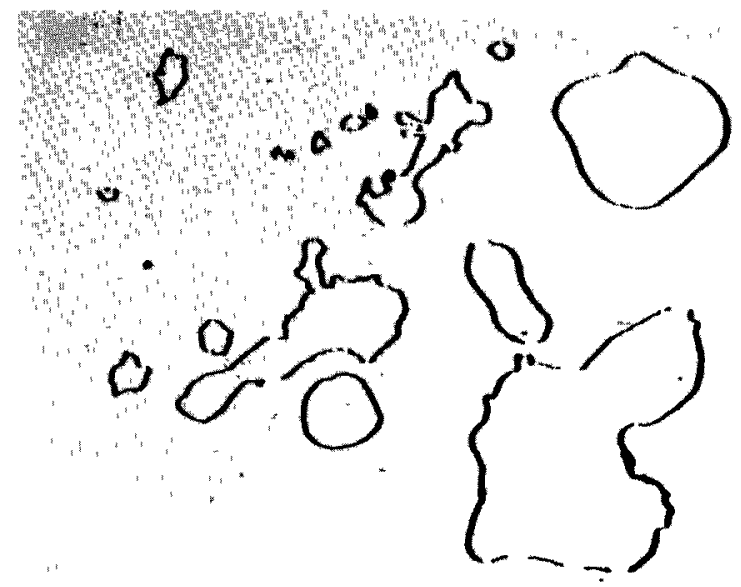

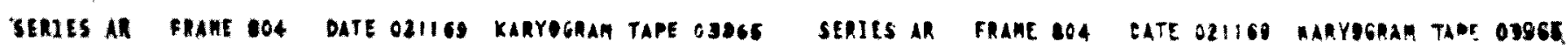

$\underset{\substack{\infty \\ \infty}}{\infty}$
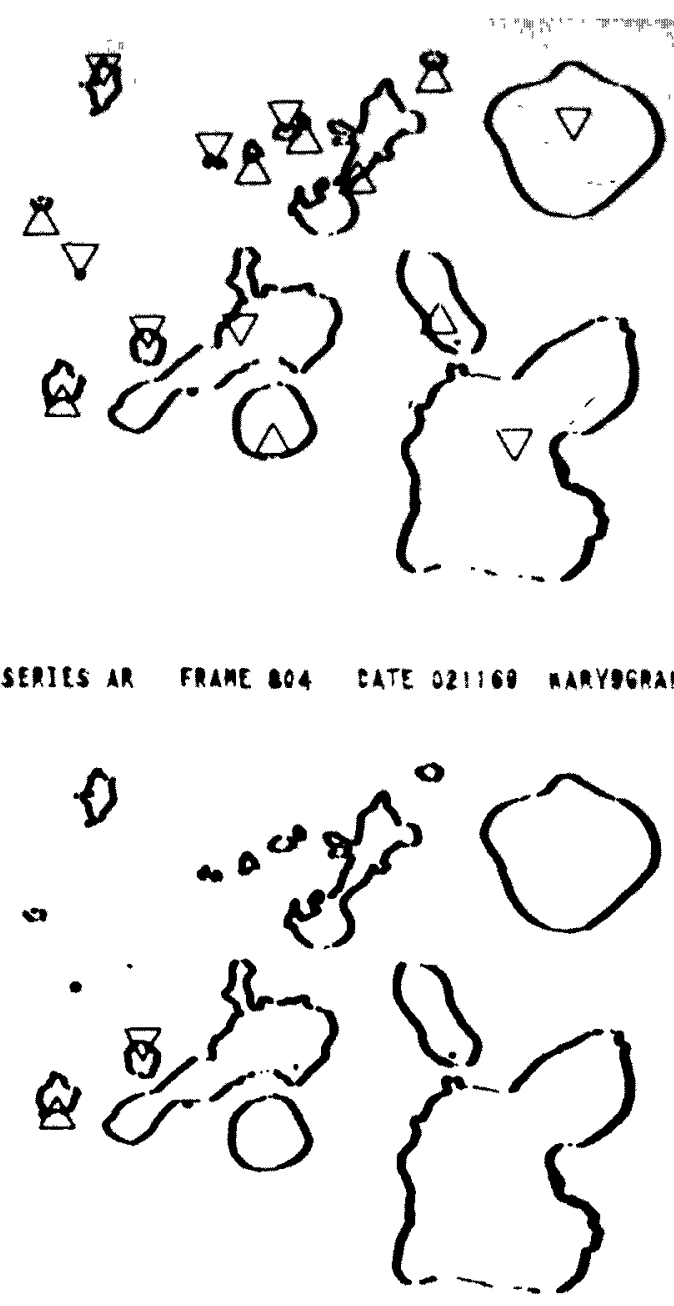

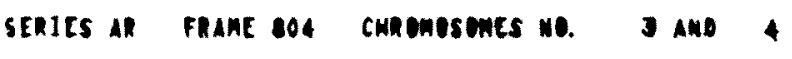

Figure 13. Pictorial Output for SURFA-2 Film AR 804, lIst Four Frames 


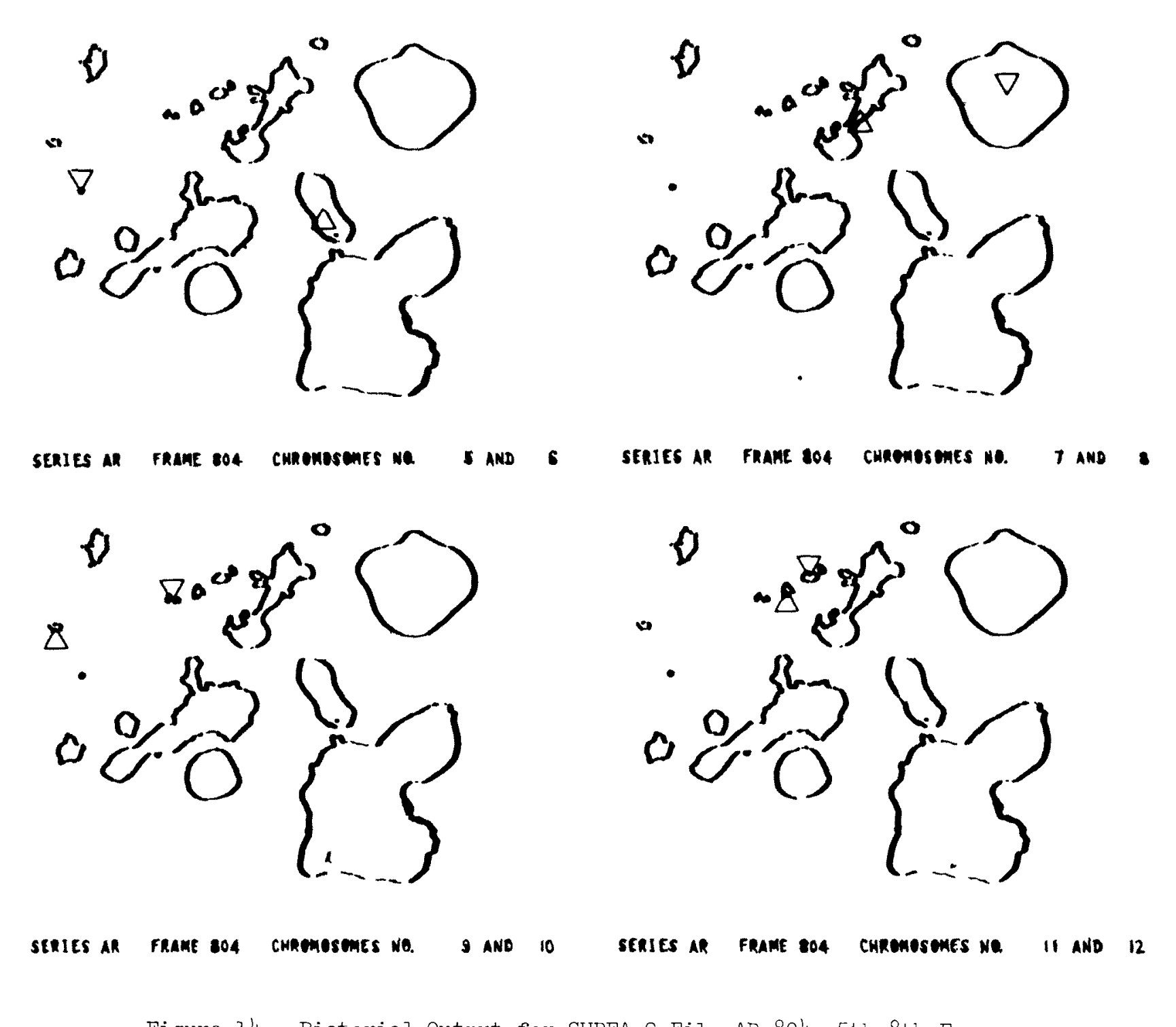




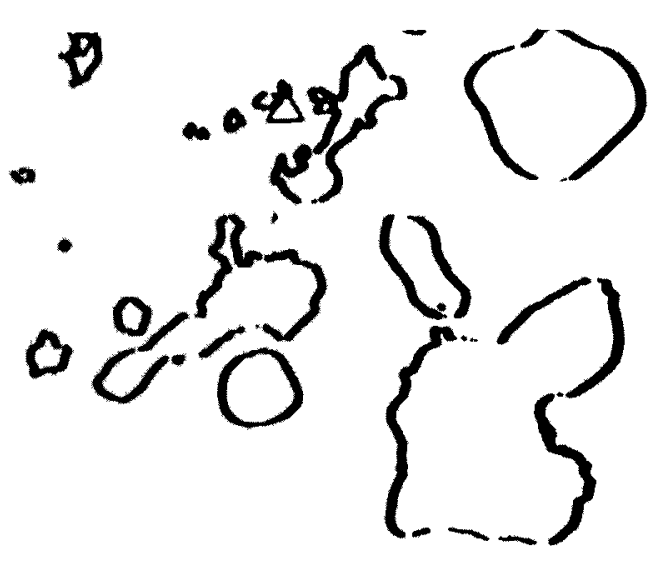

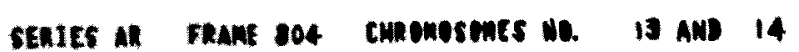

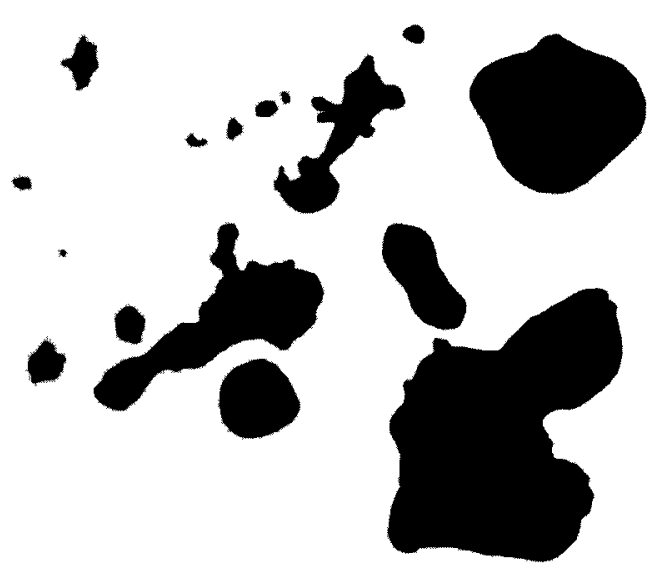

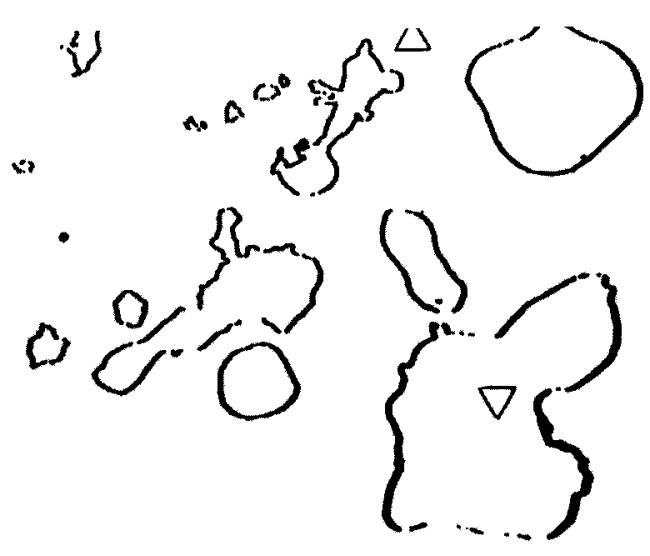

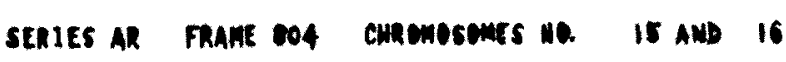

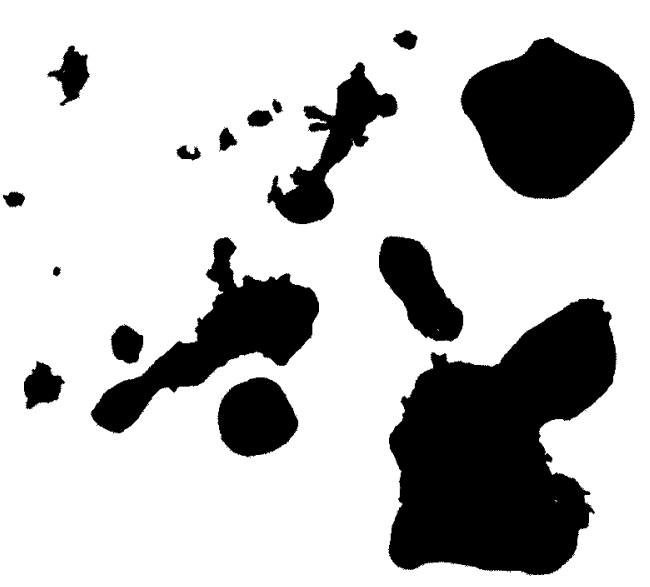

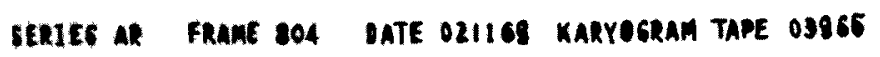

Figure 15. Pictorial Output for SURFA-2 Film AR 804, 9th and 10th Frames Fill Option, and Original Frame 
It is a pleasure to acknowledge the contributions of many persons to this endeavor. D. Armstrong and W. Gunther are the chemical engineers who brought the problem to our attention and plan to make use of the results. They perform the fragmentation experiments and have developed and optimized the photographic procedures and written the auxiliary computer programs to complete the particle size analysis. J.W. Butler, one of the designers of CHLOE and the mathematician primarily responsible for the development of the image-processing system, has contributed advice and assistance in its application to this problem. Use of the CHLOE hardware has been enhanced by the presence of $B$. Kroupa and

$\mathrm{R}$. Wehman who manage to keep an "old" machine not only operable but often in an improved manner. Sue Katilavas handled the routine production for the Chemical Engineering Division runs and Doris Haight prepared the tables and typed the manuscript. 
References:

1. J. W. Butler, Margaret K. Butler, and Agnes Stroud, "Automatic Classification of Chromosomes" in Data Acquisition in Biology and Medicine, Vol. 3, K. Enslein, Editor, pp. 261-275 (Pergamon Press, London, 1964).

2. J.W. Butler, Margaret K. Butler, and Agnes Stroud, "Automatic Classification of Chromosomes II" in Data Acquisition in Biology and Medicine, Vol. 4, K. Enslein, Editor, pp. 47-57 (Pergamon Press, London, 1965).

3. J. W. Butler, Margaret K. Butler, and Agnes Stroud, "Automatic Classification of Chromosomes III" in Data Acquisition and Processing in Biology and Medicine, Vol. 5, K. Enslein, Editor, pp. 21-38 (Pergamon Press, London, 1968).

4. Robert Clark and W. F. Miller, "Computer-based Data Analysis Systems" in Methods in Computational Physics, Vol. 5, B. Alder, et al., Editors, pp. 47-98 (Academic Press, New York, 1966).

5. C. B. Shelman, "Machine Classification of Fingerprints" in First National Symposium on Law Enforcement, Science, and Technology, Vol. I, pp. 467-477 (Thompson Book Company, New York, 1967).

6. Terence Allen, Particle Size Measurement (Chapman and Hall, London, 1968).

7. R. R. Irani and Clayton F. Callis, Particle Size: Measurement, Interpretation, and Application (John Wiley and Sons, New York, 1963).

8. G. Herdan, Small Particle Statistics, 2nà ed. (Butterworth and Company, London, 1960).

9. D. Hodges, "Photographic Scanning Systems in the Applied Mathematics Division" in Proceedings of a Conference on the Use of Computers in Analysis of Experimental Data and the Control of Nuclear Facilities, AEC Symposium Series 10, CONF-660527, pp. 177-185 (U. S. Government Printing Office, Washington, 1967).

10. Donald Hodges, CHLOE, An Automatic Film Scanning Equipment, Hardware Reference Manual, ANL-AMD Technical Memorandum No. 61 (November 1963).

11. Donald Hodges, The LIBERATOR, ANL-AMD mechnical Memorandum No. 127 (July 1966).

12. Ming-Kuei Hu, "Visual Pattern Recognition by Moment Invariants" in IRE Trans., IT-pp. 179-187 (1962).

13. J. W. Butler, Margaret K. Butler, and Barbara Marczynska, "Automatic Analysis of 835 Marmoset Spreads," to be published in Annals of the New York Academy of Science (1967).

14. M. G. Kendall and A. Stuart, The Advanced Theory of Statistics, Vol. I, pp. 168-170 (Charles Griffin, London, 1963). 
NOISYI - A PROGRAM FOR CALCULATIUN OF SPACE DEPENDENT AUTO AND CROSS SPECTRAL DENSITIES IN REACTORS (a)

James R. Sheff ${ }^{(b)}$

FFTF Reactor Physics and Operations

Battelle Memorial Institute

Pacific Northwest Laboratory

Richland, Washington 99352

March 11, 1969

Paper for presentation at the Conference on the Effective Use of Computers in the fruclear Industry, Knoxville, Tennessee, April Ll-k3. 1969.

(a) This paper is based on work performed in part under United States

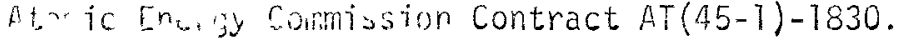

(b) Seniar Restirch Engineer, FFTF Project, Pacific Northwest Laboratory, operared by Eattolle Memorial Institute for the United States Atomic Eneryy Commission, Richland, Washington. 


\section{INTRODUCTION}

A system for trit caloulation of space dependent auto and cross spectral densities in bare cubical systems has been under development for several years. For several reasons it seems at this time particularly appropriate to describe its capabilities and make it generally available. Firstiy, only recently new techniques of measurement have successfully been employed revealing pure space dependent effects which are both useful in themselves and of such nature as to at least dictate correction of the experimental data via space dependent calculations before data reduction via space independent model forms. Secondly, these pure space dependent effects occur in relatively small systems and, hence, will be rather widely observable. Finally, some of the recent measurements are perhaps not casily explainable to a good approximation by either calculations with this system or with the other known systems. By making this system generally available it can perhaps provide an advanced basis for the necessary extensions.

The caiculated auto and cross spectral densities are evaluated from anaiytica? expressions for nonperturbing point or cubical neutron detectors located arbitrarily in a cubical reactor. These analytical expressions inich are desmibed in part two of this paper are based on one energy group uiffusid wiesiry 1 ciuding an arbitrary number of delayed neutron groups.

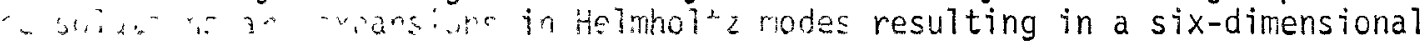
sel its, which we diat uf ts size presents a sizabie summation task the solution of which is discussed in part four.

The program currently operates on a UNIVAC 1108 and is essencially written in FORTKAN IV, but inciuded are several unique features of UNIVAC FORTRAN $V$. Extensive amouncs of the output results are capable of being graphed by the printer using printer-constructed-graphs as part of the output. Key parts of the output are also stored on tape as individually named unique absolute-data-elsments which can a directly reprocessed in

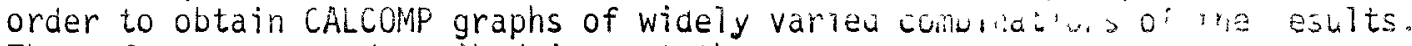
These features are described in part three.

One and two temperature coefficient feedback effects are included as an available option in a fundamental mode or space independent sense. This was done in a marmer which will allow for relatively simple extension to more coefficients or to space dependent temperature effects in a fundamenta? mode sense.

\section{THEORET:SAL WUDEL}

The calculations performed by NOISYI are the auto and cross spectral density functions of the neutron density and detector count rates in bare cubical reactors. The reactors neutron density is described by one energy group diffusion theory and it has a fundamental space mode source distribution when subcritical. The spectral densities may be calculated at either localized points or for finite nonperturbing regions (detectors) which fully overlap for the auto spectra case and do not overlap for the cross spectra case.

The theoretical development is published elsewhere $(1-4)$ and thus, will only be sketched briefly here. The auto and cross spectral density can be written as follows: 


$$
\begin{aligned}
& \Phi_{z_{1} z_{2}}\left(\bar{r}_{1}, \bar{r}_{2}, \omega\right)=C_{0} \frac{(2 / \pi)^{3}}{a b d} \Delta V\left\{\left(\sum_{\frac{g}{g}} G-R \frac{2}{g}\left(\bar{r}_{1}\right) \frac{\left(\delta k_{\bar{T}}+k_{\bar{\eta}} \beta\right) / \ell \bar{T}}{\left(\delta \bar{k}_{\bar{g}}+k_{g} \beta\right) / \ell \bar{g}}\right.\right. \\
& \left.+2 \sum_{g h} G_{\overline{g h}} R_{\bar{g}}\left(\bar{r}_{\eta}\right) R_{\bar{h}}\left(\bar{r}_{1}\right) \frac{\left(\delta k_{\bar{\eta}}+k_{\bar{\eta}} \beta\right) / l_{\bar{T}}}{\left(\delta k_{\overline{g h}}+k_{\overline{g h}} \beta\right) / \ell \overline{g h}}\right) \delta_{r_{\bar{T}}, r_{\overline{2}}} \\
& +\varepsilon^{\prime}\left(\frac{k \bar{j}}{\ell_{\overline{1}}}\right)^{2}\left|1-i \omega \sum_{j=1}^{N} \frac{\beta_{j}}{i \omega+\lambda j}\right|^{2}\left(\frac{\overline{\nu^{2}-\bar{v}}}{\bar{\nu}^{2}}\right) \\
& \left.\left(\sum_{g} \frac{G_{g} R_{\bar{g}}\left(\bar{r}_{7}\right) R_{\bar{g}}\left(\bar{r}_{2}\right)}{\left[\alpha \frac{2}{g}+\left(\gamma_{\bar{g}} \omega\right)^{2}\right]}+\sum_{\substack{g h \\
g \neq h}} \frac{G_{g h} R_{\bar{g}}\left(\bar{r}_{\eta}\right) R_{\bar{h}}\left(\bar{r}_{2}\right)}{\left[\alpha_{\bar{g}}^{\alpha} \bar{h}+\left(\gamma_{\bar{g}} \omega\right)^{2}+i \omega \frac{L^{2}}{\ell}\left(B \frac{2}{g}-B_{\bar{h}}^{2}\right)\right]}\right)\right\}
\end{aligned}
$$

The result has been arranged to emphasize which terms are real and which terms are complex, and the effect of delayed neutrons has been included
The detailed definitions of the quantities in $\mathrm{Eq} .1$ have been given $(1-4)$. Briefly, the $G^{\prime} s$ are constants, $R_{g}^{-}\left(\bar{r}_{7}\right)$ and $R_{\bar{h}}\left(\bar{r}_{2}\right)$ are three-dimensional space eigenfunctions, $\bar{g}$ and $\bar{\hbar}$ are triple summation indexes, and $\varepsilon^{\prime}$ represents the counts per unit detector volume per fission per unit reactor volume at the detector locations. The quantity $\alpha \bar{g}$ is approximately $\left(\delta k_{\bar{g}}+k_{\bar{g}} \beta\right) / l_{\bar{g}}$ for large $\omega ; \gamma \bar{g}$ is approximately unity for large $\omega$. The quantities $\alpha \bar{g}(\omega)$ and $\gamma_{\bar{g}}(\omega)$, aside from their dependence upon higher order bucklings, are the usual quantities found in the spectral density, including delayed-neutron effects, and are the real and the imaginary part of the inhour equation where the roots have been taken as $i \omega$. Along with the previously noted assumption of one-energy-group diffusion theory, the assumption of an extraneous neutron source distribution is necessary for the subcritical case. A most interesting and realistic form to choose for the source distribution is the critical fundamental mode. The generalization to the point source distribution can be easily made if desired.

The detector efficiency is given by

$$
\varepsilon^{\prime}=\frac{\Sigma_{d}}{\Sigma_{f}}=\frac{v \Sigma_{d} n}{v \Sigma_{f} n}
$$

and is the ratio of detector counts per unit volume to fissions per unit volume. The definition most frequently used in the space-independent theory is the ratio of total counts in the detector to total fissions in the reactor.

One interesting fact about this result obtained by numerical calculations is that, when the space-independent form, or the leading term of the series, dominates, it is not primarily because of rapid convergence but because of a high degree of cancellation among the higher order terms. As a result, in a bare cube the individual breaks, in general, for higher order terms will not be observed as such since the deviation from the fundamental term is a complex sum of many terms. 
The definition of the eigenfunctions, $R_{\bar{g}}\left(\bar{r}_{\eta}\right)$, generalized to include the case of a finite detector of edge a' will be given as it has not appeared previously.

$$
\begin{aligned}
R_{g}(\bar{r})= & \left.\sin \frac{[g}{a}\left(x-\frac{a}{2}\right)\right] \sin \left[\frac{n \pi}{b}\left(y-\frac{b}{2}\right)\right] \sin \left[\frac{p \pi}{d}\left(z-\frac{d}{2}\right)\right] \\
& x\left\{\frac{\sin \left[\frac{g r}{a} \frac{a^{\prime}}{c}\right] \sin \left[\frac{n \pi}{b} \frac{a^{\prime}}{2}\right] \sin \left[\frac{p \pi}{d} \frac{a^{\prime}}{2}\right]}{\frac{1}{8}\left(\frac{g \pi}{a}\right)\left(\frac{n \pi}{b}\right)\left(\frac{p \pi}{d}\right) \Delta V}\right\}
\end{aligned}
$$

The quantity within the brackets represents the finite detector contribution and is identicali! equal to one for the case of $a^{\prime}=0$.

\section{FROGRAM FEATURES INDEPENDENT OF NOISYT ITSELF}

The basir framework into which NOISYi is installed is essentially a

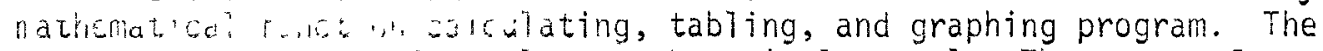

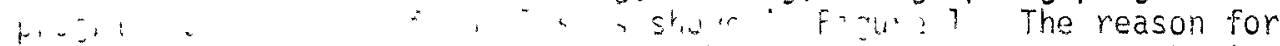
froce-uig $r$ is coner wes to develor a read to go system having convenuont features ire, which desired function evaluations could be inserted To tris wey of large extensive production systems, this is probabiy not ton important a feature, but to the scientist or engineer user wrio has raayy varied and frequently short lifetime calculations, it is of the greates $L$ benefit. I am sure everyone is well acquainted with the "quickie" progran which prints out several pages of completely unidentified output which is totally useless to everyone except perhaps the writer. A week after tue program was last 11:ed, it is usually even difficult for the writer to wade through such resulics.

The first subroutine or the "main" program has control over reading of the general input quantities, control of the calling sequence of most other subroutines, and does the basic housekeeping chores of the program. This includes decisions about repeating calculation sequences for more data sets with or witnout mode changes and shutdown. Included are calls to routines which recover from and generate shutdown procedures after "end of file' or "error' status modes are encountered by the system. These chores include bie setting if of the 315,23 wo produce an in-house or inmgroup accounting ad showing operator lume, run number, time spent, pages output, cards output, and to what accourt nunder it was charged. The error status routine currently recovers providing this summary in all cases except when of maximum time and page expectations are exceeded.

In keeping with the concept of general usage function evaluation, a fivedimensional matrix of input data is generated via specification of initial values, increments and the numbers of points to be calculated. One dimension, the one associated with the frequency in the spectral density calculations, has a much more extensive system for specification of the input array. Least often used options are direct reading of an input data set and various linear incrementing schemes. The most used alternative is a logarithmic data generating scheme. As input, one specifies a starting point and up to three pairs of integers for the points per decade and the number of decades desired. The spacing of the points in a given decade can be specified as either uniform or "almost" uniform. The first is ideal for plotting 


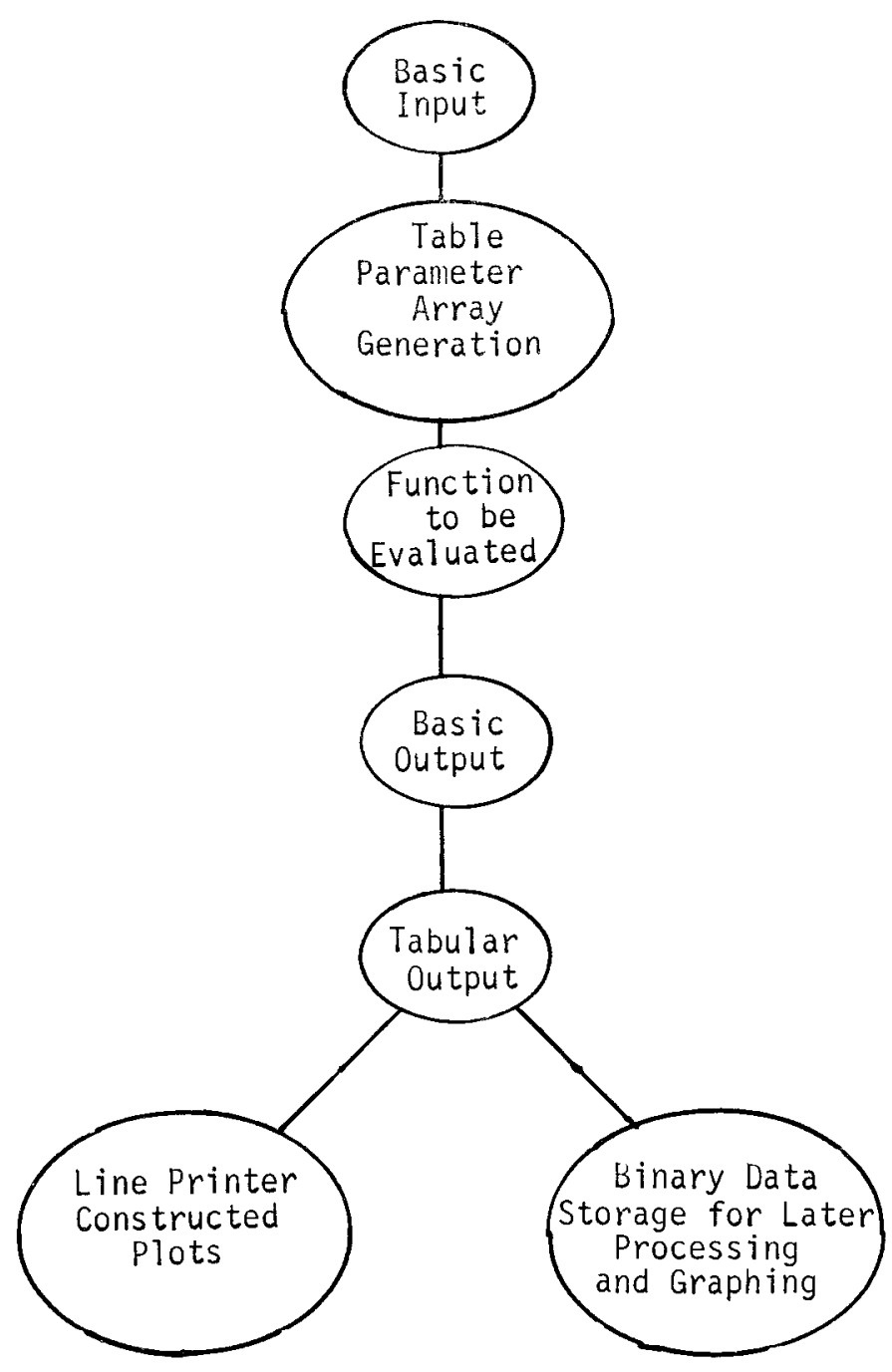

Figure 1. Basic Program Layout.

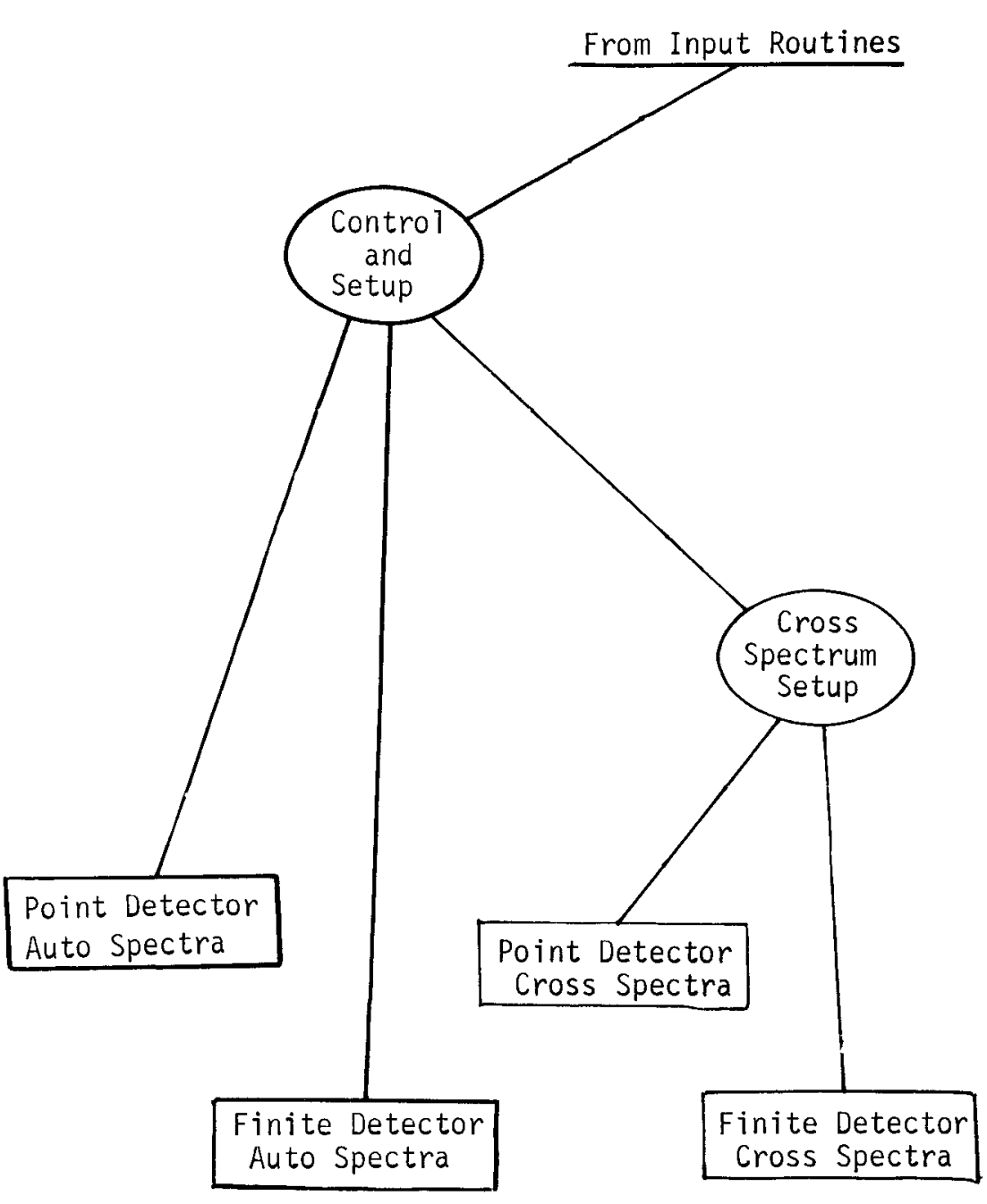

Figure 2. Progrom Flow in the Calculation Section Showing the Basic Paths. 
log information on linear paper and the second via picking near by integer values allows simple aimost uniform plotting on typical log graph papers. The uniform syster is ise one most generally utilized and also is best for mechanized graph arawing machines.

The basic ultudt routine is rather conventional and is tailored to the calculation being done. In the way of special features, however, it does check the specitied iel. truil detectcr location to see if it either is or is near to a reasonable fraction of the reactors size. If such a reasonable fraction is found, it is given as output along with its decimal equivalent to eight p?aces. This is a useful check upon the input which is frequently specified as a fraction and has been known to have beer entered incorrectly.

The tabular output routine is built around use of fifteen three dimensional arrays which are available for various quantities that are desired as output. Via setting internally of an integer associated with each array, the output may include also the logarithim of the array and/or a printer constructed piot of one or the other. The fifteen arrays and considerable of the other wutput is stored as a named absolute deck (binary) along with the program and other elements on tape.

\section{$\because+$ ATUPES OF THE NOISYI CALCULATIONS}

17. actual ariulation subroutines are spitit into reur bimi? or or

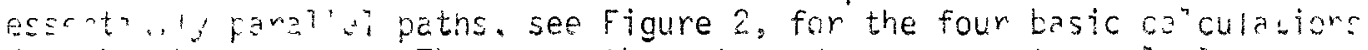
done $t_{y}=1$ piogram. These are the auto and cross spectra caicilations each for woth point and finite detectors. These four paths are assigned to mutual core ocations or "overlayed" via the UNIVAC Memory Ailocator Processor (MAP).

The principle task performed by each of these paths is to simply

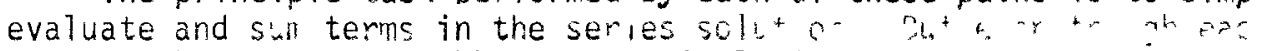
individual term is actually rather simple in form, the series $:$.ixdimensional corresponding to the two detector points $\bar{r}_{1}$ and $\bar{r}_{2}$ (this is still true whon $\bar{r}_{l}=\bar{r}_{2}$ ), and this leads to a sizable term summation problem even when convergence would normally be considered good. For example, for the relatively nominal sing?e dimensional series requiring ten terms for adequate convergence, the equivalent six-dimensional series might require on the order of $10^{6}$ terms.

It is posstile. of course to set un a sirnle gant six-dimensional

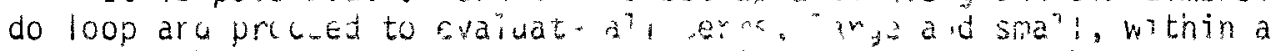
cubical volume in the six space, but tois is vary vasteful in terms of machine utilization and actually makes such calculations out of reach in terms of the funds available. Such an approach can be supplemented via checks of convergence, but because the region of raximum term size lies along and near to the six dimensional diagonal, the checks become quite complex and time consuming. Also, in view of the absence at the time of the programs inception of any information about the relative effects of various types of terms and their convergence in various situations, it seemed desirable to seek an alternate calculational scheme.

The final basis for a workable system was to structure the program so that one would concentrate the summing first close to and along the diagonal in the six space. This was accomplished by first summing over the three-dimensional set of terms obtained by making equal the three pairs of indexes for the two points. Thus, for $\bar{r}_{1}$ and $r_{2}$ one has the vector indexes $\bar{g}(g, m, p)$ and $\bar{h}(h, n, q)$ in which one requires $g=h, m=n$, 
and $p=q$. It should also be noted that all terms involving an even and an odd eigenfunction go to zero, so for convenience, the series is split along even and odd terms (sines and cosines). Out of the 64 combinations of a given set of even and adjacent odd indexes, only 8 are non-zero. Thus, when a specific term is mentioned, one is really talking about 8 . When 106 terms are referred to, one means the $8\left(10^{6}\right)$ non-zero terms out of $(20)^{6}=64\left(10^{6}\right)$ terms possible.

It turns out that summations over this single set of diagonal terms usually yields an answer which very closely approximates the full summation. Thus, for all detector positions near the core center, the summation of $10^{3}$ terms yields approximately the same result as would be obtained by blind summation of $10^{6}$ terms or a $10^{3}$ reduction in effort results without need of any convergence checks.

For the extreme limiting case of a detector at $3 \mathrm{~s} / 8$ on the diagonal, this comparison between the "exact" answer and the approximation using only the terms diagonal in three dimensions or the "fully diagonal terms" (DUU) is shown in Figure 3.

Inspection of the low frequency end of Figure 3 shows that the curves do differ in shape even in the plateau regions, but the difference does not become significant until about $\omega=100$. For $\omega>100$, the "exact" curve falls below the approximation, and approaches the infinite medium point detector shape, $c / \omega] / 2$, at $\omega$ s $10^{4}$. Note also that the slope of the "exact" result in the normal "space independent" region, however, is $c \% / \omega$ rather than $c^{*} / \omega^{2}$. The dashed curves will be discussed below.

The observation that in many cases the DDD terms are an adequate approximation has been freely utilized in the calculationally mych more difficult problem of the spectrum in three region problems $(5)$. In that case, the fully diagonal terms are the only ones which have been programmed at this time.

To improve upon the results obtainable via summation of the fully diagonal terms, one must then search for the next larger set of terms and sum these. The obvious next step then is to gradually move away from the six space diagonal in other directions. It is beneficial to draw a comparison to the cube in three spaces. The summation of the fully diagonal terms has included all terms on the principle diagonal or by analogy along the diagonal running between opposite corners of a cube. These are the terms for which all of the six (3 pairs) indexes are equal. Also included have been terms along the minor diagonals such as the terms along the diagonal between opposite corners on the face of a cube. These correspond to the case where say 2 or 4 ( 1 or 2 pairs) of indexes are equal to each other but not to the other 2 ( 1 pair) indexes.

At this point, one must move to even lower order diagonals which would correspond in the cube to terms along an edge. The analogy has, of course, started to break down at this point as these terms really correspond best to the fully non-diagonal terms. In any case, the next set of terms should be diagonal by pairs in four dimensions and nondiagonal in the other two. The requirement upon the vector indexes $\vec{g}(g, m, p)$ and $\bar{h}(h, n, q)$ thus becomes $g=h, m=n$, and $p \neq q$ and the other two possibie rotations of this. In the special case of auto spectra, further simplification results because of symmetry between the case when $p>q$ and $q>p$. Whether symmetry exists or not, however, one now must in some manner 


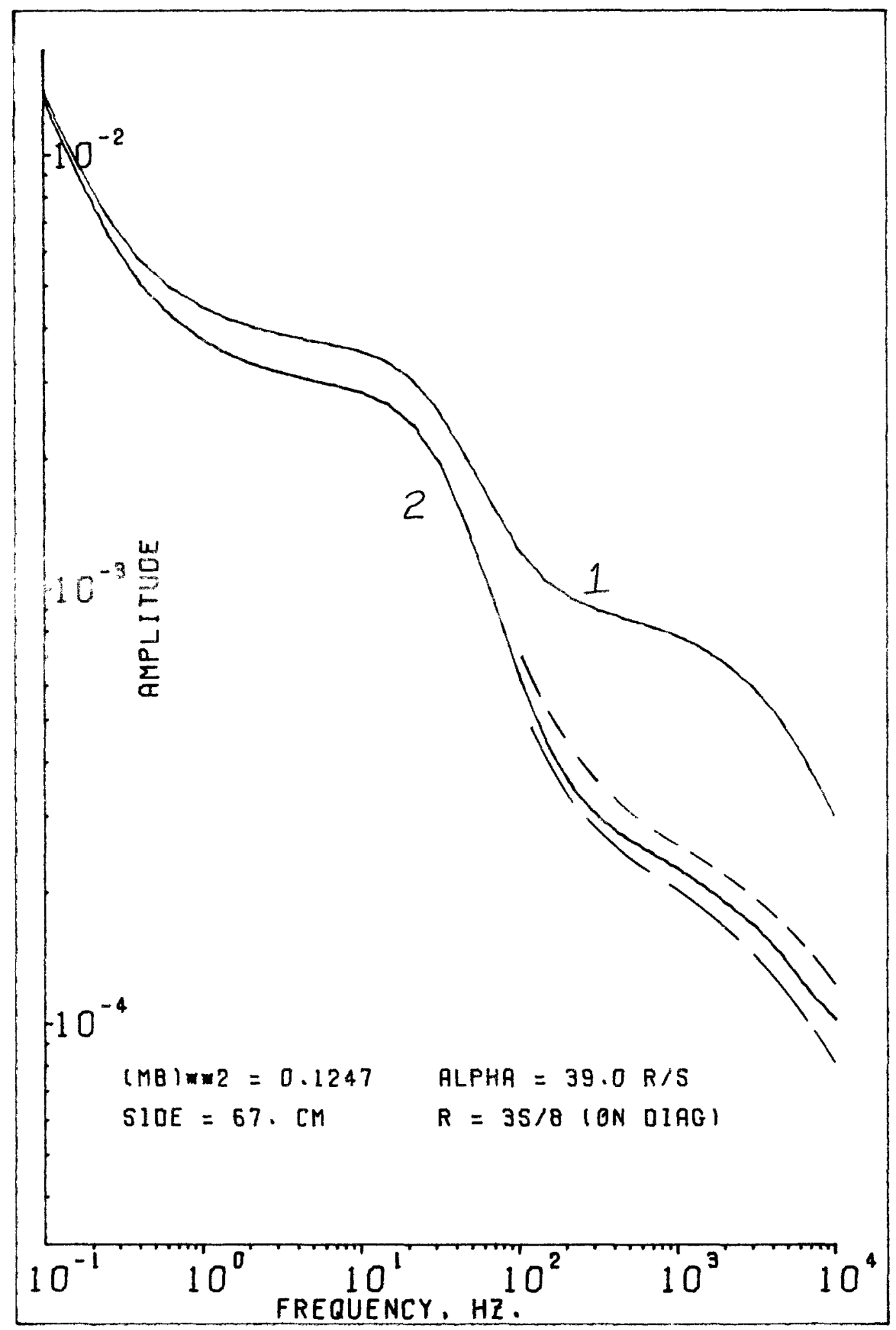

Figure 3. Comparison of the Auto Spectral Density Function (Curve 2) and the "Fuily Diagonal" Term Approximation (Cume 1). 
sum over these three four-dimensional series in which one index is restricted to values less than another. The programming is most simple if one sacrifices memory by neither attempting to utilize in tandem the already set up fully diagonal term logic or the tandem usage of a single new four-fold do loop. Thus, one is lead to three essential identical loops which differ only in the manner in which the indexes are arranged. This fact is relevant in that they are in usage in many places machines of capacity quite inadequate compared to a UNIVAC 1108, and hence, via expenditure of some programming effort the programs storage requirements could be reduced to a significant degree.

These additional terms, the "diagonal, diagonal, non-diagonal terms" (DDND), are less significant contributors to the result, and thus, the program makes provision for the user to sum these terms to a different level than that picked for the diagonal terms. It has been found through experience that the level should be picked at about $2 / 3$ that of the fully diagonal terms. The actual value is in general not critical as the do loops, also, at the option of the user, incorporates a fairly high level of convergence checking and, hence, compensates quite well for a poor choice in this index.

The curves labeled 1 and 2 in Figure 4 are the DDD and DDND terms for the example given above in Figure 3 . The sign of the DDND terms is negative and, hence, inclusion of only this additional set of terms would make the result negative for $\omega>200$. The level of summation for the DDND terms was 4 for low frequencies and 6 for high frequencies. The change can be noted by the discontinuity in the curve near the break frequency.

The general attack upon the problem has been sufficiently well described now as to make a quantitative formulation of it useful in order to show clearly where one must next proceed in order to provide the capability of complete summation for at least check purposes. For a six-dimensional series, summed to a level of $N$, there are $N^{6}$ terms. Out of the $N^{2}$ in one pair of components, I have picked the $N$ terms which have equal indexes. This leaves $N(N-1)=N^{2}-N$ remaining and provides the clue to how one should express the $N^{6}$ terms. One has that

$$
\begin{aligned}
N^{6} & =\{N[1+(N-1)]\}^{3} \\
& =N^{3}+3 N^{3}(N-1)+3 N^{3}(N-1)^{2}+N^{3}(N-1)^{3}
\end{aligned}
$$

Where the first term, $\mathrm{N}^{3}$, is the number of fully diagonal terms and the second term, $3 N^{3}(N-1)$, is the three sets of DDND terms. The third term is three sets of what will be referred to as "diagonal-nondiagonal-nondiagonal terms", DNDND, and the final term, $N^{3}(N-T)^{3}$, is the set of "full nondiagonal terms", NU.

These last two sets of terms are also programed with control of the level of the number of terms left to the user in the sense that the DNDND is set at the same level as the DDND and the ND is adjustable directly through input.

The relative magnitudes of these four contributions are shown for the example case in Figure 4. The level of summation was 10 for the DDD terms, and 6 for the DDNO and DNDND terms and 5 for the ND terms. For $\omega<\beta / \ell$, the 


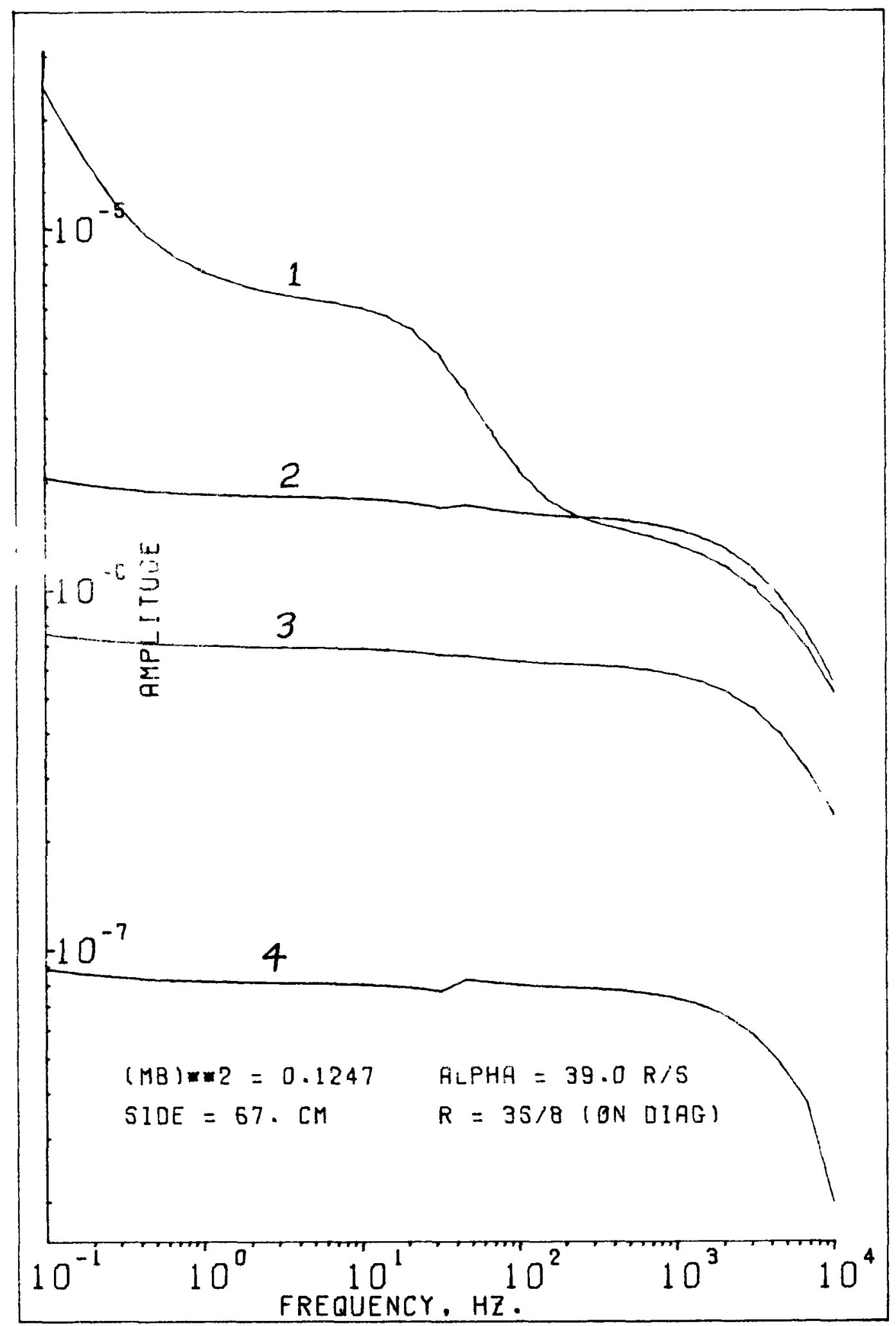

Figure 4. Contributions to the Auto Spectral Dersity. 1 is DED, $\therefore$ is minus DLWD, 3 is DWDND, and 4 is minus ID terms. 
level is reduced by 2 for all terms other than the DDD ones. The program was running with the convergence checking mode operating for these calculations, but because of the optimum choice of the level of summation, the results differ significantly enough to be shown only when combined into the final results. The dashed curve above the solid line shown in Figure 3 is the same case without the convergence mode operating. For comparison, if the DDND, the DNDND, and the ND terms are summed to a level of 10 terms with convergence checking the lower dashed curve is obtained.

The fully non-diagonal terms are almost never of significant consequences for the cases observed, so that by this choice of attack one is able to el iminate from the calculation a very large fraction of the terms without necessity of any convergence checks. In quantitative terms, the fraction eliminated is

$$
\begin{aligned}
\frac{N^{3}(N-1)^{3}}{N^{6}} & =\frac{(N-1)^{3}}{N^{3}} \\
& =1-3 / N+3 / N^{2}-\frac{1}{N^{3}}
\end{aligned}
$$

or for the case of $N=10$, seven tenths of the terms can be easily eliminated.

As stated above, in the process of programming for the UNIVAC 1708 , it was expediate to sacrifice machine capacity through almost redundancy in these latter summations rather than expend programming time.

The relative calculational effort required can be bracketed between $\frac{1}{N^{3}}$
the fully diagonal terms only and ny $3 / \mathrm{N}$ for exclusion of only the for the fully diagonal terms only and
fuily nondiagonal terms where $N>3$.

\section{OPERATION MODE AND DELETE CONTROL}

The desire for extensive displays of debugging information and benchmarks in a program usually poses the problem of how this can be accomplished without overloading the machines capacity and/or greatly slowing down operating speed with the many checks to see if printing is desired. Similarly one frequently encounters a need for being able to calculate using more than one operational mode or strategy. This was the case with NOISYI, and an ideal solution seems to exist with the combination of two operations availabie in UNIVAC FORTRAN-V.

In the UNIVAC system, the FORTRAN language programs (symbolics), the machine language programs (relocatables) and the coupled groups of machine language subroutines forming a full program (absolutes) are a 11 named and stored on tape. The symbolics can be altered via a system allowing cards to be removed and/or inserted. Cards common to many symbolics may be named and stored separately once and then called automatically into a particular symbolic when compilation is taking place. This is the INCLUDE option.

Also available is a so called DELETE option which provides a system for telling the compiler to either ignore or not ignore groups of cards at compile time. DELETE is a poor title, however, because although the chosen 
cards are deleted frum the compilation, they are not deleted from the symbolic deck. Thus a better word is "Ignored" and perhaps this option should have been so : alled. The key that allows the compiler to decide whether the referenced group of cards should be ignored or not is the value of an integer as specified by a PARAMETER definition card within the symbolic dech. Tris FARAMETER card can be most simply changed and easily routed to all desired places via the INCLUDE option.

Consider, as an example of this usage, the problem of converaence checking in the many series involved in this program. As will be noted below, the convergence in the presence of the trigonometric terms is not uniform, but oscillates about zero where the absolute maximum value is converging. Thus, the convergence checking is not always desirable and certainly should be checked in cersain cases. One is also concerned about the time spent in checking versus the time required for excessive calculations without checking. Hence, one would like to have two versions of the program, one with checking and one without. Normally such a situation result.s in at least frustration if nothing more serious in the attempt to keep tne two versions identical with respect to improvements and revisions.

In. lenerting tris system to this case of a need for several operating versions, then prusets as follows. At the end of each do loop in which

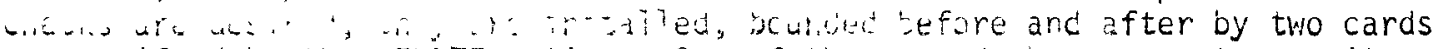
as spes: fied by the JELITE uption. One of these cards has present upon it a control integer wiose value tells the compiler whether or not to ignore thise cards when camiling. This control integer is set within the symbolic deck by compiler request from a group of similar symbolic cards stored together in a named element called a Procedures Definition Table.

The production of the two desired versions proceeds directly then by naming one set of "relocatables" and its "absolute" one name and the other set another. Both, however, were producer frot the same sft nf "symbolic" decks. At compile time, in one case the single relevarit card in: the Procedures Uefinition Table was used as it was, and in the other case it was changed immediately before compilation to the alternate value. That is the integer parameter is changed in value from zero to one or the reverse.

The system has also been used in the program to include options for calculating fewer terns at frequencies below the break than above and to allow one to printout basi information for each term in the series.

The debugging of the prograli has seen under this type of control for some time now in an "instäl what is necessary at the time" manner. Hence, many debugging problems are now being answered by merely rerunning the problem containing the difficulties after recompiling the relevant subroutines with the debugging parameter card set so as to include in the compilation such debugging cards as are available. 


\section{SERIES CONVERGENCE AND THE EXISTENCE OF HIGHER ORDER BREAKS}

The series convergence can be easily determined by inspection of Eq. (1). Consider first the diagonal terms which appear as the first summation of the last line of Eq. (1). $G_{\bar{q}}$ starts at $(4 / 3)^{3}$ and asymptotically approaches one, and the eigenfunctions in general oscillate between minus and plus one. Thus, one must depend upon the denominator for convergence. Clearly for all terms with $\gamma g^{\omega}{ }^{\prime} \alpha_{\bar{g}}$, the series will not converge; thus one must always sum up to terms where $\alpha_{g}$ is sufficiently large that the series will converge.

Inspection of the various nondiagonal terms, the second summation in the last line of Eq. (1), reveals exactly the same situation except that convergence is also aided by the coefficient $G \overline{g h}$ which goes to zero as the disparity between $\bar{g}$ and $\bar{h}$ increases.

The lowest order terms are shown in Figure 5. Curve \#1 is the fundamental mode or space independent term. and has three pairs of cosines in the eigenfunctions. Curve 2 is the next higher mode, and because of symmetry, there are three such terms each containing two pairs of cosines and a pair of sines. Curve 3 has two pairs of sines and one pair of cosines and again represents three terms. Curve 4 has three pairs of sines. The discontinuity at the left hand edge of the lines indicates the asymptotic level neglecting delayed neutrons.

Note how closely all of the higher order break frequencies are bunched together as compared to the separation of the fundamental and the first higher order break. The breaks become progressively more compressed at a rapid rate which essentially rules out the possibility of discrete separate breaks appearing in the final sum. In fact, there are about 512 terms having break frequencies less than $10^{4}$ for the case considered. 


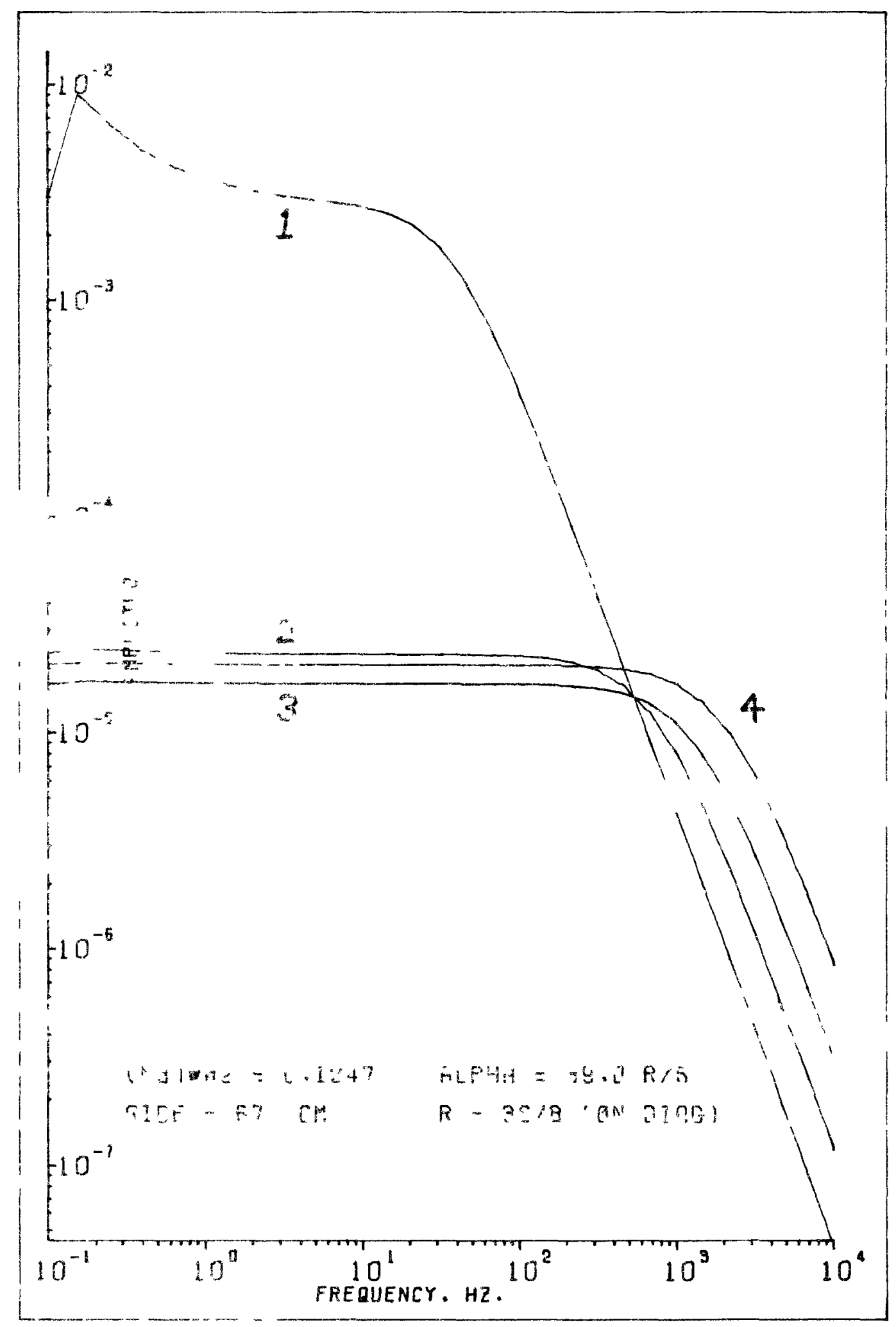

$F \cdot P=I 2+e^{+}$pruer enms in the wto Spectral Density.

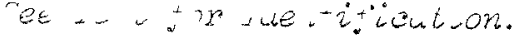




\section{SUMMARY AND CONCLUSIONS}

The features of the NOISYl program for calculation of space dependent auto and cross spectral density functions of the noise in a nuclear reactor has been described. It was shown that because of the long period over which this system has evolved, an extensive program has resulted which has many features advantageous to potential users of such calculations. Secondly, because of its comparisons with special case calculations using a three region program under development, it has stood and passed the test of line by 1 ine comparison with an independent system. Thus, it can be assumed to be functioning correctly providing error free answers. For these reasons, i.e. convenience of use and error free accuracy proven by test, one concludes that NOISYl provides a logical basis for doing experimental test planning and result analysis calculations.

Furthermore, for those many situations in which NOISY] is still inadequate, it should provide an advanced basis from which to work toward a better more complete solution of the space and energy dependent noise problem. Indeed, because of the programs basic structure, it can be a beneficial basis for any desired calculation, especially those which are expected to be short lived.

Several unique uses of some of the features available on at least Univac machines were described as applied to NOISYl, and it is felt that they are of general benefit to the goal of convenient trouble free programs.

\section{REFERENCES}

T. J. R. Sheff and R. W. Albrecht, "Space Dependence of the ReactorNoise Spectral-Density Function", Neutron Dynamics and Control, pp. 213, 322, AEC Symposium Series, No. 7 (CONF-650413), 1966.

2. J. R. Sheff, The Cross Correlation of the Neutron Density Fluctuation at Two Points in a Nuclear Reactor, Ph.D. Thesis, University of Washington, 1965 (University Microfilms, Inc., Abstract No. 65-8530).

3. J. R. Sheff and R. W. Albrecht, "The Space Dependence of Reactor Noise, I-Theory and II-Calculations", Nucl. Sci. Eng., 24: 264 (March 1966) and 26: 207 (October 1966).

4. J. R. Sheff, "Space Dependent Cross Spectral Density in a Bare Cube", Neutron Noise, Waves, and Pulse Propagation, pp. 623-648, AEC Symposium Series, Wo. 9, (CONF 660206) 1967.

5. J. R. Sheff, "Exact Treatment of Neutron Fluctuations in Reflected Systems", ANS Trans., II, No. 1, pp. 233 (1968). 
BLANK 
SESSION III

MULTIGROUP CALCULATIONS

Chairman: R. G. Dahlberg Gulf General Atomic 
BLANK 
THE APPLICATION OF THE BLOCK INVERSION TECHNIQUE

TO MULTI-DIMENSIONAL REACTOR CALCULATIONS

\author{
M. R. Wagner \\ Gulf General Atomic Europe \\ Zurich/Switzerland
}

\title{
1. Introduction
}

During the past years a number of authors have developed non-iterative methods of solution for the inhomogeneous onedimensional multigroup diffusion theory equation. The method used most widely is a direct generalization of the "forward elimination-backward substitution" method for tri-diagonal matrices. For multigroup diffusion problems with a full scattering matrix the factorization technique can be generalized to the solution of the resulting linear equations with block-tridiagonal coefficient matrices 1,2 .

Another method to solve one-dimensional multigroup boundary value problems was proposed by Lucey and Hansen 3 . This method, the reconditioning technique, treats the multigroup equations as an initial value problem. Making use of the Iinearity of the equation the solution vector is expanded in terms of a complete and linear independent set of homogeneous solutions and one inhomogeneous solution, which are calculated in an unstable march-out process. The instability, however, can be controlled by periodically applying so-called reconditioning transformations to restore a good linear independence of the homogeneous solutions. The error growth can further be reduced by an error-compensating method, which will be discussed later in this paper in a similar context. It was shown by Froehlich 2 that the conventional forward elimination-backward substitution method may be interpreted as a special case of the reconditioning method. Froehlich also made a careful comparison of the relative merits of both methods.

Based on the experience and the reliable results obtained with this technique the question arose, whether similar methods could be used for multi-dimensional reactor problems. Although, in principle, the reconditioning method can immediately be applied for inhomogeneous $2-D$ one-group problems, its use is only indicated for elongated meshes. One possibility would be to di- 
vide the spatial mesh into a number of stripes for each of which the reconditioning method could be used. It seems, however, more consistent to subdivide the mesh into a number of subgroups of mesh points ("ajffu=jor blocks") with dimensions of the same order in both directions. If the block length is chosen properly one can even consider to do a direct inversion for each block without the use of reconditioning transformations.

This techr:cue of block inversion 4 for a given set of flux values at the block boundaries is discussed in section 2 . The block inversion is still based on an initial value treatment by expanding the flux at the inner mesh points in terms of a complete set of homogeneous solutions and a particular solution which satisfies tho given boundary conditions. An alternating iterative technigue is used to solve the inhomogeneous problem for the full mesh. For homogeneous eigenvalue problems the overall convergence is further improved by applying two independent acceleration inethods: a coarse mesh rebalancing technique 1,5 and a special asymptotic source and flux extrapolation method.

For practical reasons the block inversion method was first appl... th a two-dimensional uniform triangular mesh with a hexa-

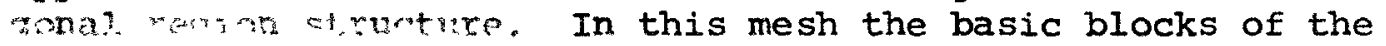
bufty. Aue ch ale cluwters or "patches" of seven hexagonal elements. As a resuit of this development a two-dimensional diffu=Lon-depletion program GAUGE 6 was written which is described in some detail in section 4 .

The results obtained with GAUGE were encouraging enough to attack the same problem in three dimensions. The code GATT, 8 a three-dimensional static neutron diffusion theory program, also based on the block inversion methon, was written for a hexagonal $z$ mesh and will be discussed in section 5 .

Finally, a three-dimensional low order discrete ordinate transport code $3 \mathrm{Dr}, 9$ which was developed recently as a prototype program, is described briefly in section 6. The technique of solution, although not a direct analogon to the block inversion, has some similarity which may be of some interest in this connection.

\section{The Block Inversion Method}

In the applications discussed later the conventional fission source iteration will be used to solve multi-dimensional diffusion theory eigenvalue problems. In this section we will discuss only the inner iteration scheme which requires to solve repeatedly the inhomogeneous multi-dimensional difference equations for $G$ groups, $g \leq G$,

$$
A_{g} \varnothing_{g}=Q_{g}
$$

for varying source terms $Q_{g} \cdot A_{g}$ is a matrix of the order of 
the total number of mesh points $N$. The subdivision of the mesh into $M$ blocks corresponds to a reordering, such that the matrix $A=A g$ of $\mathrm{Eq}$. (1) is partitioned into $M$ submatrices $A_{m}$ with no direct coupling to all other $A_{m^{\prime}}, m^{\prime} \neq m \leq M$, plus a submatrix $A_{M+1}$ with direct coupling to all other $A_{m}$.

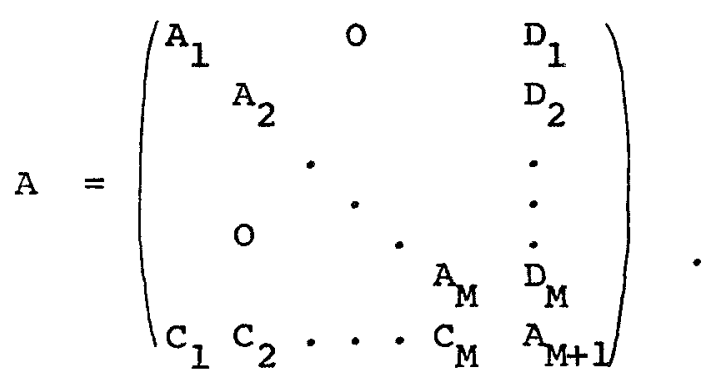

The submatrices $A_{m}, m \leq M$, of order $N_{m}$ comprise the inner mesh points of block $m$ whereas the "boundary block" $A_{M+1}$ includes the mutual coupling of all remaining points at the block boundaries.

The problem of solving Eq. (1) is decomposed into 1) the problem of solving for the fluxes in the interior of the diffusion blocks for a given set of boundary values; and 2) the problem of iteratively improving the boundary fluxes, taking into account the interaction with the adjoining blocks.

The problem 1) is solved by the block inversion method. The method will be described for a simple rectangular mesh block, Fig. 1 . However, the same principles can also be applied to other types of geometry.

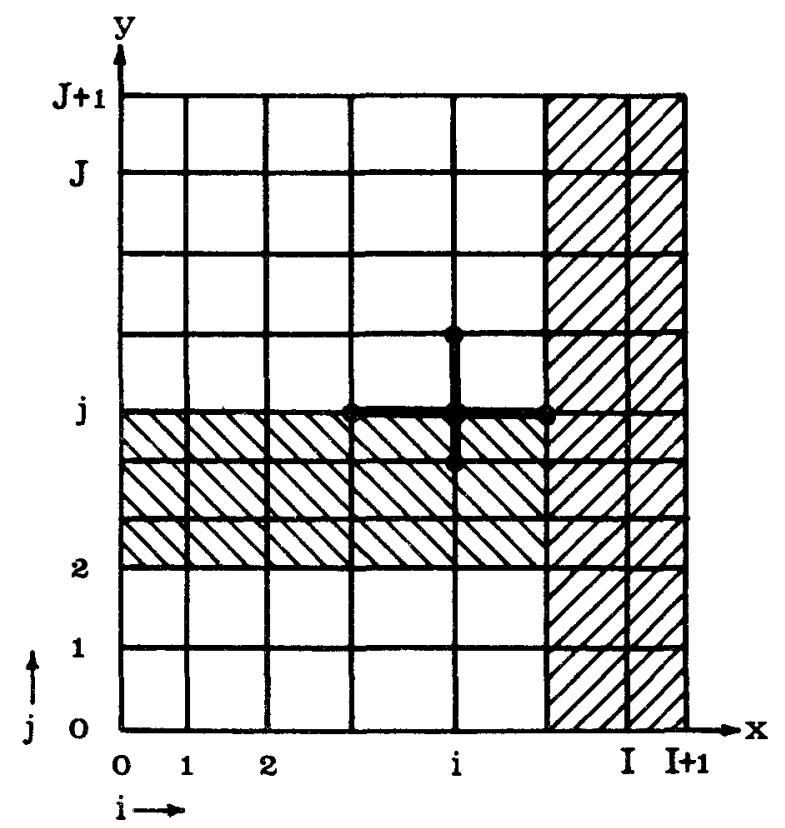

Fig. 1

Two-Dimensional Rectangular Mesh Block with I Inner Rows and $J$ Inner Lines 
The discrete equations for the neutron flux $\Phi(1, j)$ at mesh point $i, j$ is given by a five-point difference expression in the form

$$
\begin{aligned}
& a_{0}(i, j) \Phi(1, j)-a_{1}(i, j) \Phi(i-1, j)-a_{2}(i, j) \Phi(i, j-1)(3) \\
&-a_{3}(i, j) \Phi(i+1, j)-a_{4}(i, j) \Phi(i, j+1)=Q(i, j) \\
& \text { with } i=1, \ldots, I ; j=1, \ldots, J
\end{aligned}
$$

The problem is to solve simultaneously the system, Eq. (3), for a given volume source $Q(i, j)$ and for a fixed set of boundary values $\Psi$

$$
\begin{aligned}
& \Phi(0, j)=\Psi_{L}(j) \quad ; \quad \Phi(I+1, j)=\Psi_{R}(j) \\
& \Phi(i, 0)=\Psi_{B}(i) \quad ; \quad \Phi(i, J+1)=\Psi_{T}(i) .
\end{aligned}
$$

Due to the block-tri-diagonal form of the coefficient matrix $A_{\mathrm{m}}$

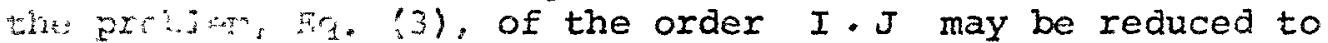
$\cdots$ r. $\because \cdots \cdots$, ut us esume that the correct solution would he wan as in vector $\alpha$ at the first inner line

$$
\alpha(i)=\Phi(i, 1) \text {, }
$$

then Eq. (3) may be considered as a recursive relation which allows to calculate the inner fluxes $\Phi(i, j+1), i=1, \ldots, I$, in line $j+1$ from the fluxes in two successive lines $j$ and $j-1$.

Introducing $\alpha$ as an unknown vector which will be determined later, one may expand the solution of $\mathrm{Eq}$. (3) for each point $i, j$ in terms of a complete set of $I$ linear independent homogeneous solutions $U\left(i, j ; i^{\prime}\right), i^{\prime}=1, \ldots, I$, and a particular solution of Eq. (3)

$$
\Phi(i, j)=\sum_{1^{\prime}=1}^{I} U\left(i, j ; i^{\prime}\right) \alpha\left(i^{\prime}\right)+W(i, j) .
$$

Each homugeneous solution $U$ satisfies zero boundary conditions at the left and right boundaries

$$
\begin{aligned}
& U\left(0, j ; i^{\prime}\right)=0 \quad j=1, \ldots . J \\
& U\left(I+I, j ; i^{\prime}\right)=0
\end{aligned}
$$

and at the bottom line $j=0$

$$
U\left(i, 0 ; i^{\prime}\right)=0 \quad i=1, \ldots, I
$$


whereas the inhomogeneous solution $W$ takes on the given boundary values

$$
\begin{aligned}
& W(0, j)=\Psi_{L}(j) \\
& W(I+1, j)=\Psi_{R}(j) \\
& W(i, 0)=\Psi_{B}(i) .
\end{aligned}
$$

Since $\alpha$ represents the flux at line $j=1$ one has

$$
\begin{aligned}
U\left(1,1 ; i^{\prime}\right) & =\delta_{1 i} \\
W(i, 1) & =0 .
\end{aligned}
$$

With the boundary conditions, Eqs. $(7,8)$ and the initial values, Eq. (9), the complete set $U$ and the inhomogeneous solution $W$ is calculated in a step-by-step process, proceeding from line $j=2$ to $j=J+1$. Note, that for the calculation of $U$ the homogeneous form of Eq. (3) is used. The vector $\alpha$ can then be determined from the requirement that the linear combination Eq. (6) must fulfil the remaining boundary condition at the top line $j=J+1$

$$
\sum_{i=1}^{I} U\left(i, J+1 ; i^{\prime}\right) \alpha\left(i^{\prime}\right)+W(i, J+1)=\Psi_{T}(i),
$$

or in matrix notation

$$
\mathrm{U}_{\mathrm{T}} \alpha=\Psi_{\mathrm{T}}-\mathrm{W}_{\mathrm{T}}
$$

Having obtained the flux vector $\alpha$ at line $j=1$

$$
\alpha=U_{T}^{-1}\left(\Psi_{T}-W_{T}\right)
$$

one can now start again to calculate the full field $\Phi(i, j)$ of inner fluxes recursively from Eq. (3) in a second sweep upwards. If it were not for round-off errors the flux vector $\Phi$ 우 obtained in this march-out would be equal to the given boundary vector $\mathbb{T}_{T}$.

The question of numerical stability will be discussed in the next section. Here we note, that, during the fission source iteration, the block inversion must be done repeatedly for varying volume sources $Q$ and boundary values $\Psi$. The matrix $U_{T}$, as a homogeneous solution, depends only on the coefficients $a_{k}(i, j)$ in Eq. (3) and not on $Q$ or $\Psi$. Hence $U_{T}$, or rather the inverse $\mathrm{U}_{\mathrm{T}}^{-1}$, can becalculated once and for alf. Often the matrix $A_{m}$ also shows symmetries which may be reflected in symmetry properties of $\mathrm{U}_{\mathrm{T}}^{-1}$. A further implication is the fact, that there is no need to store the field of inner fluxes once they have been calculated and used to form the downscatter sources and related quantities. 


\section{Numerical Stability of the Block Inversion}

It is well known that Eq. (3), treated as an initial value problem, is inherentiy unstable. Therefore, the block inversion, when used without ipecial precautions will lead to computational difficulties that arise from the finite computer word length. This is due to the fact that with increasing number of steps $J$ in $y$ direction the $i^{\prime}$ vector components of the homogeneous solution $U\left(i, J, i^{\prime}\right)$ become increasingly proportional to each other. The matrix $U_{T}$ in EG. (11) will become ill-conditioned and the inversion of $U_{T}$ results in a considerable loss of significant Eigures.

Although reconditioning transformations may be used to stabilize the march-out, another simple approach was preferred. The method, described below, is a rapidly converging iterative errorcompensation technique that allows to stabilize the solution, at least for blocks with a limited number $J$ of steps.

To simplify the notation we will denote the solution vectors at line $j$ by $\Phi_{j}, w_{j}$. Similarly $U_{j}$ is the matrix of homogonocis solutions at line $j$. Let further be $U_{T}^{*}-1$ the matrix

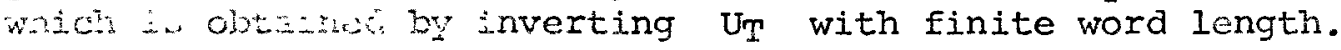

Bavaves of the ill-conditioned matrix $U_{T}$ the solution $\alpha^{\circ}$ of Eq. (1.) vill be inaccurate. Let $\alpha$ denote the correct solution

$$
\alpha=\alpha^{0}+\delta \alpha,
$$

where $\delta \alpha$ is an unknown error vector that can be determined approximately from the following consideration. At line $j \Phi_{j}$ is represented by

$$
\begin{aligned}
\Phi_{j} & =U_{j}\left(\alpha^{\circ}+\delta \alpha\right)+W_{j} \\
& =\Phi_{j}^{\circ}+U_{j} \delta \alpha
\end{aligned}
$$

so that the error Eunction

$$
\delta \sigma_{j}=\Phi_{j}-\Phi_{j}^{O}=\mathbb{T}_{j} \delta \alpha
$$

is proportional to the homogeneous solution. Since $\delta \mathrm{DT}_{\mathrm{T}}$ for the top boundary is known, $\delta \alpha$ may be chosen such that

$$
\mathrm{U}_{\mathrm{T}} \delta \alpha=\delta \Phi_{\mathrm{T}}=\Psi_{\mathrm{T}}-\Phi_{\mathrm{T}}^{\circ}
$$

with the approximate solution

$$
\delta^{1} \alpha=U_{T}^{*-1}\left(\Psi_{T}-\Phi_{T}^{\circ}\right) .
$$


The first order correction $\delta^{1} \Phi_{j}$ can then be determined in an additional sweep, again using the homogeneous portion of Eq. (3), with the initial values

$$
\begin{aligned}
& \delta^{1} \Phi_{0}=0 \\
& \delta_{\Phi_{1}}^{1}=\delta^{1} \alpha .
\end{aligned}
$$

so that the corrected solution

$$
\Phi_{j}^{1}=\Phi_{j}^{0}+\delta^{1} \Phi_{j}
$$

will fulfil the boundary conditions at $j=J+1$ to a much better degree. Clearly this procedure is iterative: calculate $\delta^{\text {n }} \alpha$ from

$$
\delta_{\alpha}^{n}=U_{T}^{*-1}\left(\Psi_{T}-\Phi_{T}^{n-1}\right), n=1,2, \ldots
$$

to obtain in the $n$-th error sweep with initial conditions

$$
\begin{aligned}
& \delta_{\Phi_{0}}^{n_{1}}=0 \\
& \delta_{\Phi_{1}}^{n_{1}}=\delta_{\alpha}^{n_{\alpha}}
\end{aligned}
$$

the corrected solution for the inner fluxes

$$
\Phi_{j}^{n}=\Phi_{j}^{n-1}+\delta^{n} \Phi_{j} .
$$

The convergence of this error compensation method is extremely good as long as

$$
\left\|I-U_{T} \cdot U_{T}^{*-1}\right\| \ll 1 .
$$

The iteracisn is terminated as soon as

$$
\left|\Psi_{T}-\Phi_{T}^{n}\right|<\Psi_{T} \text { for } n \leq n_{0}
$$

where no is a limit for the number of exror sweeps. In the code GATI' no $=2$, so that the calculation stops and prints an error message if more than two additional sweeps should become necessary. This is usually also a good input check indicating that sorwic ticrscopic cross sections have beccme exceedingly large.

$$
\text { It slould be mentioned that, even if condition (23) is }
$$
satisflad. it is necessary to check whether the difference equations (3) at the last inner mesh line $J$ is fulfilled withir the same criterion $t$, since round-off errors in adding $\delta \mathrm{n} \mathrm{j}$. Eq. (21), are not corrected by this procedure. 
It is olvious, that the use of higher arcuracy computation, for instance double precision floating point arithmetic, will incrcase the limiting block length or reduce the number of error iterations for a molock size. Note, however, that error sweeps often besome necen:ary only for certain energy groups with a large romoval cross sastion. In the applications a computer with 36 bits word lengtin 2 : been ased (UnIVAC 1108) and double precision arithmetic was verd only for the inversion of the matrix UT.

\section{GAUGE, a Two-Dinensional Diffusion-Depletion Program for} a Uniform Triangular Mesh

The first appitication of the block inversion method was made in the code GAUGE ${ }^{6}$. This code was developed specifically to calculinte the tro omensional flux and power distribution and the burnup hehayjor of large power reactors with hexagonal core confuguration. The man objective was to make available to the nuclear rngineer a dasign tool that takes into account important two-dimens onal $\in f f r_{0}$ t, and which, on the other hand, is efficient enough to calculate on a survey basis complete, detailed reactor life histories with modest cost.

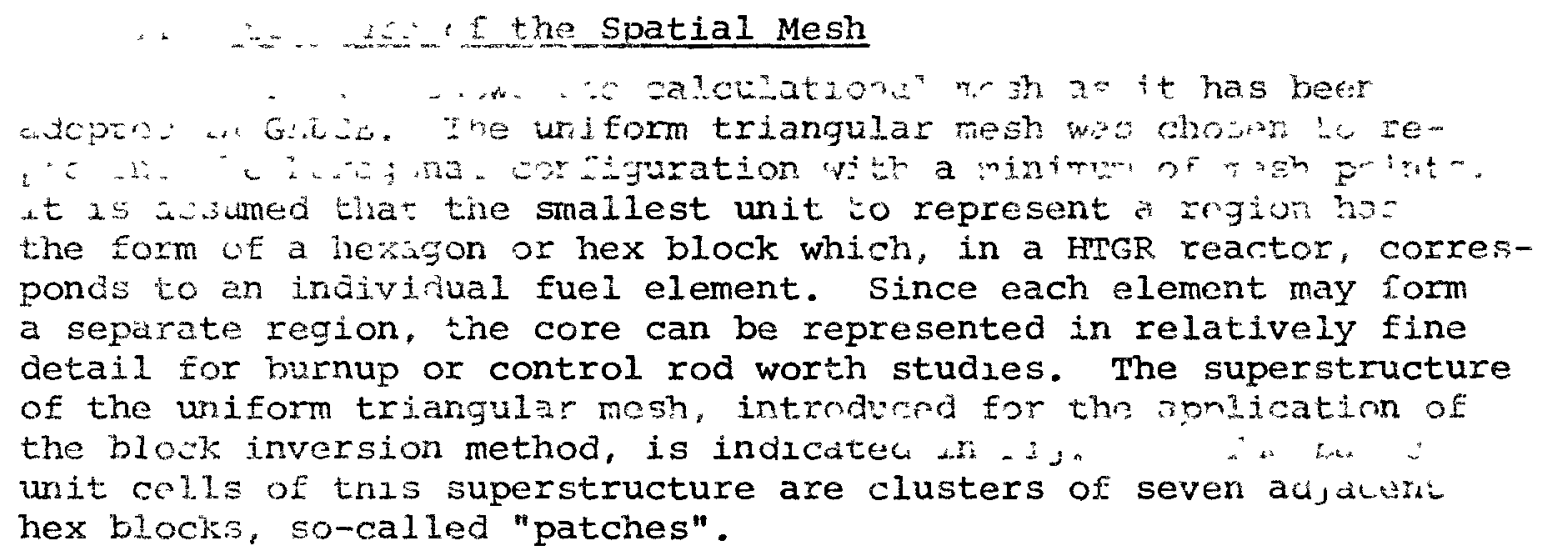
ponds to an indivirual fuel element. Since each element may form a separate region, the core can be represented in relatively fine detail for burnup or control rod worth studies. The superstructure of the uniform triangular mosh, introdered for tho rongication of

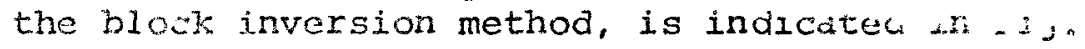
unit cells of tnis superstructure are clusters of seven aujacsin hex blocks, so-called "patches".

To achieve the goal of a fast $2-D$ reactor survey and design code some concessions have been made with regard to the accuracy with which the outer reflector boundary can be treated. It was assumed that the neutron flux for all groups vanishes at the outer bondury of the calculational mesh. Als, as Fig, 2 shows, it is diffurist to saresent a arcular outer reflector boundary. In the GAUGE model the o.ster boundary na a roughly hexagonal shape, which leads to an azinuthal variation of the reflector thickness. However, one can vary the density of the moderator in the outermost reflector elements such that the effective thickness is nearly constant in radial direction. Comparisons with other, more refined models have shown that this approximation leads to a small error, which can be tolerated for most practical applications.

For the uniform 60-degree triangular mesh selected in GAUGE the spatial difference equation at a corner point between 


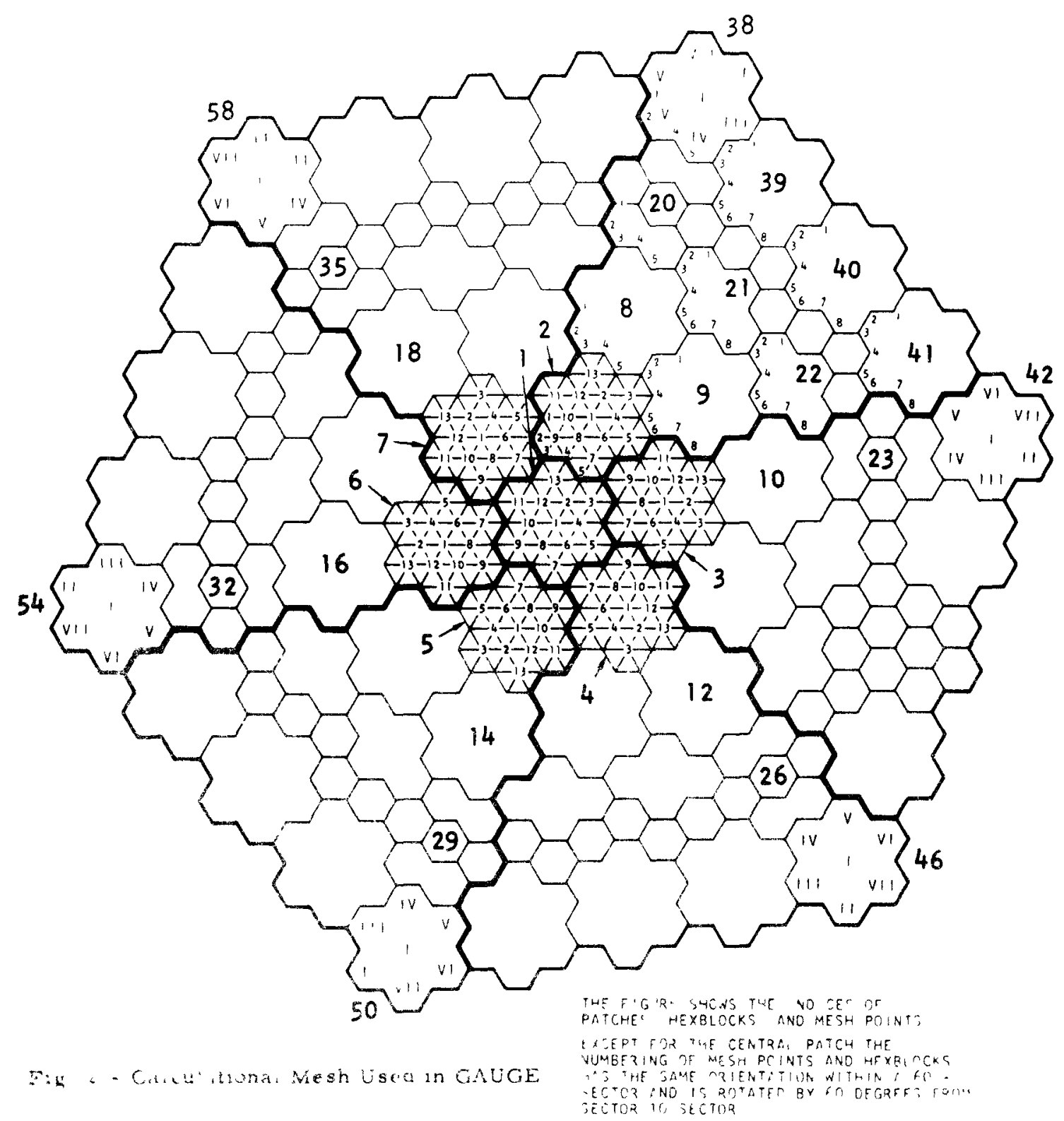


three different hex blocks I, II, and III has the following form

$$
\begin{aligned}
& \left(6 \bar{D}+\frac{3}{2} h^{2} \bar{\Sigma}\right) \Phi_{0}-D_{I} \Phi_{I}-D_{I I} \Phi_{3}-D_{I I I} \Phi_{5} \\
& -\frac{D_{I}+D_{I I}}{2} \Phi_{2}-\frac{D_{I I}+D_{I I I}}{2} \Phi_{4}-\frac{D_{I I I}+D_{I}}{2} \Phi_{6}=\frac{3}{2} h^{2} Q_{0} .
\end{aligned}
$$

where $h$ is the mesh spacing and

$$
\begin{aligned}
& \bar{D}=\frac{1}{3}\left(D_{I}+D_{I I}+D_{I I I}\right) \\
& \bar{\Sigma}=\frac{1}{3}\left(\Sigma_{I}+\Sigma_{I I}+\Sigma_{I I I}\right) .
\end{aligned}
$$

\subsection{Block Inversion in GAUGE}

A block in the sense of the block inversion method is the hesagcad patch, for which the numbering of inner fluxes *, $i=1, \ldots, \ldots$ be mandary fluxes $w ;, j=1, \ldots, 18$, and hex biocks $z=1, \ldots, y$ is shown in Fig. 3 . In this case the block inversion is used to solve 13 difference equations of type (24) for the interior fiuxes for a given set of volume sources $Q_{i}$ and boundary values $\Psi_{j}$. It is felt unnecessary to write down the seven-point difference expressions in extenso; they may be found in reference 6 . It may suffice to say that, in this simple application, the fluxes $\Phi_{i}$ are expressed as a linear combination

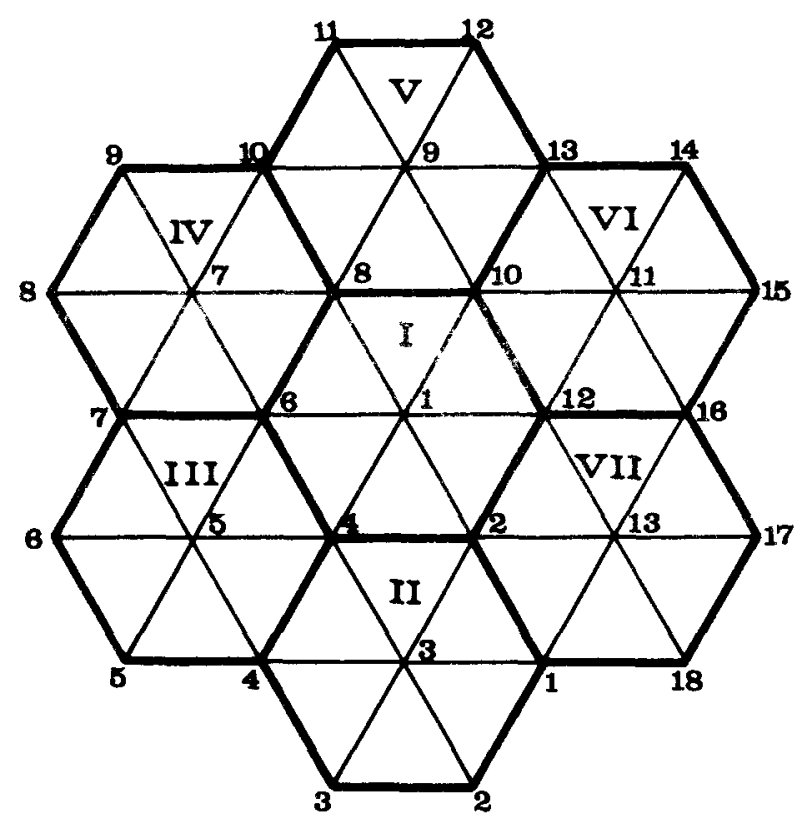

Fig. 3

Triangular Mesh for a Hexagonal Patch 


$$
\Phi_{i}=\sum_{j=1}^{3} U_{i j} \alpha_{j}+w_{i}
$$

in terms of the parameters $\alpha_{j}$

$$
\alpha_{1}=\Phi_{2}, \quad \alpha_{2}=\Phi_{3}, \quad \alpha_{3}=\Phi_{13}
$$

which correspond to the inner fluxes at the "first inner line" with points 2,3 and 13 , so that $U_{i j}$ and $w_{i}$ start with the initial values

$$
\begin{aligned}
& \mathrm{U}_{2,1}=1, \mathrm{U}_{3,1}=\mathrm{U}_{13,1}=\mathrm{w}_{2}=0 \\
& \mathrm{U}_{3,2}=1, \mathrm{U}_{2,2}=\mathrm{U}_{13,2}=\mathrm{w}_{3}=0 \\
& \mathrm{U}_{13,3}=1, \mathrm{U}_{2,3}=\mathrm{U}_{3,3}=\mathrm{w}_{13}=0
\end{aligned}
$$

in a sweep across the inner mesh of the patch ending at points 7,8 and 9 . The parameters $\alpha_{j}$ are determined from the requirement that the proper linear combination (25) must fulfil the difference equations (24) at the points 7.8 and 9 . One obtains a linear system of equations of the order three

$$
\mathrm{M} \alpha=\mathrm{N},
$$

where dgain the matrix $M$ of the homogeneous solutions depends only on the material properties $\Sigma_{l}, \mathrm{D}_{\ell}$ of the patch. Therefore, in inverse $\mathrm{M}_{\mathrm{k}, \mathrm{g}}^{-1}$ is calculated once for all and stored in high speed memory for all patches $k$ and groups $g$. With is available, two sweeps through the inner mesh of the patch plus a matrix-vector multiplication are required to perform a block inverwion Fi. the given set of $Q_{i}$ and $\Psi_{j}$. In this degenerated case error iterations are seldom required, although they are included in GAUGE to cnsure absolute stability.

The inner iterations for the full mesh, Fig. 2 , start with a suitable guess for the boundary fiuxes of group $g$. Then block inversions are performed for all patches. The next phase

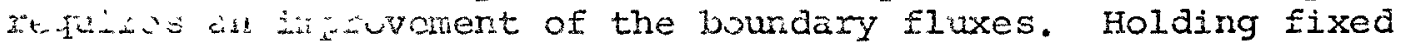
Lise susw nuxtm, a one-dinensionat calculation for the boundary ruses ia lere for the sequence of 5 or 8 points at the bourdar of ead pacill, procecding in a spiral sweep inwards. With the improved set this alternating scheme may be continued until the boundary fluxes are converged. However, due to the rapid exror zeduction, it turns out to be the optimal strategy to aizow miy on inner ateration per outer source iteration.

\subsection{Accejeration of Fission Source Iterations}

From the above remarks follows that the overall convergence of the 2-D diffusion calculation in GAUGE depends mainly on the convergence rate of the outer iteration. Two independent 
acceleration techniques have been adopted or modified to speed up the convergence of the conventional fission source iteration scheme. Their combined use reduces the total running time by almost an order of magnitude, Fig. 4.

As soon as an asymptotic convergence state has been reached a fission source extrapolation is applied to damp out the next-to-lowest eigenmode. The method, a modification of a similar technique described in reference 7 , is based on the fact that, asymptotically, the fission source vector iterates $\Psi(\mathrm{n})$ behave like

$$
\Psi^{(n)}=\Psi^{(\infty)}+R \sigma^{n}
$$

where $\sigma$ is a constant, independent of $n, g$ and the spatial variables. With

$$
\Psi^{(n)}-\Psi^{(n-1)}=R \sigma^{n-1}(\sigma-1)
$$

this leads to the extrapolation formula

$$
W^{*}=\psi^{(n)}+\frac{\sigma}{1-\sigma}\left(\psi^{(n)}-\Psi^{(n-1)}\right),
$$

where $v^{*}$ is the extrapolated fission source and $\sigma$ is given by

$$
\begin{aligned}
\sigma & =\frac{\bar{\epsilon}_{n}}{\bar{\epsilon}_{n-1}}\left(1+\bar{\epsilon}_{n-1}\right) \\
\bar{\epsilon}_{n} & =\frac{1}{N} \sum_{i} \frac{\left|\Psi_{i}^{(n)}-\Psi_{i}^{(n-1)}\right|}{\Psi_{i}^{(n-1)}} .
\end{aligned}
$$

For the success of this extrapolation scheme it is obviously important to use suitable criteria which allow the program to decide whether an asymptotic behavior has been reached. The detailed description of these criteria is given in reference $\dot{6}$ The essential point is to make certain that the pointwise deviations from the integral quantity $\sigma$ are sufficiently small over the whole mesh. Another sensitive indication for the asymptotic behavior is the fact, that the maximum relative change in the predicted extrapolation factors $\theta(n)$ is less than, say, 10 percent during the last two iterations

$$
\begin{gathered}
\delta^{(n)}=\frac{\mid \theta^{(n)}-\theta^{(n-1) \mid}}{\theta^{(n-1)}}, \\
\operatorname{Max}\left(\delta^{(n)}, \delta^{(n-1)}\right) \leq 0.1 .
\end{gathered}
$$


The extrapolation factors $\theta$ may become large, often factors of the order 100 are observed. In Fig. 4, curve B, this results in a sudden increase of $\operatorname{Max}\left(\delta \Psi_{i} / \Psi_{i}\right)$ followed by a steep drop until a new asymptotic behavior is reached.

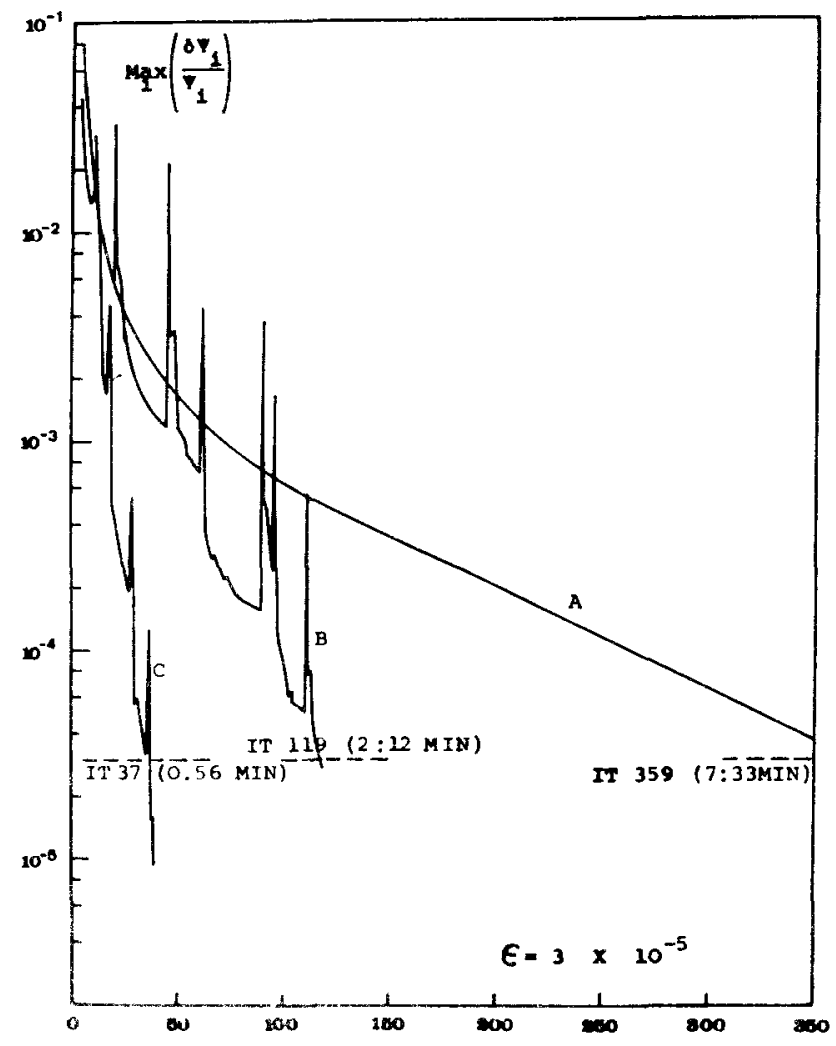

Fig. 4

Convergence Behavior of GAUGE

4 Group Problem with

61 Patches

A: No Acceleration

B: Asymptotic Source Extrapolation

C: Source Extrapolation plus Rebalancing

Experience from other two-dimensional neutron diffusion codes, e.g. 7 has shown that the use of a coarse mesh rebalancing technique 1,5 can lead to considerably improved convergence rates. The principle idea is to switch temporarily to a coarse energy-space mesh, accepting for a moment the current fine flux distribution within each coarse mesh cell, and to multiply each flux value in a coarse cell with a non-negative number such that the overall neutron balance for each cell is fulfilled. If, as in GAUGE, the same superstructure that is used for the block inversion is also utilized for the rebalancing coarse mesh, one can expect a very good overall convergence rate.

The rebalancing equations may be derived from a variaiional principle 5 . Let $s_{k}$ be $k$ disjunctive subsets of the mesh. In this particular application, $s_{k}$ comprises all energyspace mesh points of patch $k$, including the group of 5 or 8 bound- 
ary points assigned to that patch, Fig. 2 . Then we define

$$
\begin{aligned}
& \varphi_{k,-5}=1,(n) \quad \text { for a.1 } i, g \in S_{k} \\
& \varphi_{k, i, j}=0 \quad \text { for all other } i
\end{aligned}
$$

and choose as trial function

$$
\varphi_{i, g}=\sum_{k=1}^{K} a_{k} \varphi_{k, i, g} .
$$

with group independent coefficients $a_{k}$. Inserting the trial function (34) in the fine mesh equations one obtains contracted one-group ecruations

$$
A^{\prime} \mathrm{a}=\frac{1}{\lambda^{\prime}} \mathrm{p}^{\prime} \mathrm{a}
$$

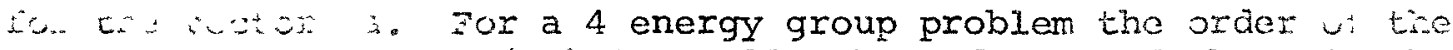

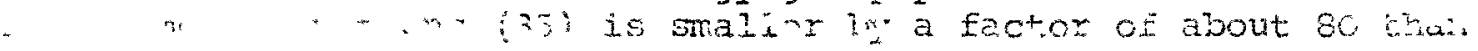
in correspcuring fine-mesh problem. Therefore, the it tat $\mathrm{v}$.

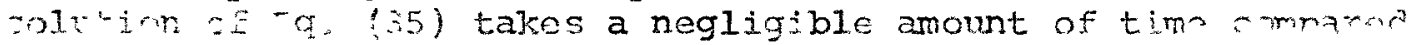
ro ansile ute: iteration sweep through the fine merh. ma computations requirod to calculate the coeffjcients of the contracted matrices $A^{\prime}$, $p^{\prime}$ can be reduced if explicit use is made of the fact that a direct block inversion is done for each patch, so that the residuals of the difference equations (24) are exactly zero For the inner mesh points. The time reguired for the compl te coarse mesh rebalancing calculation iricluc..rg $z^{-}$it.. : is the innproved solution Eq. (34), takes not more than abulu \& pu.:cent of a single outer iteration. On the other hand, the asympto-tic convergence rate is increased by more than an order of magnitude, curve C of Fig. 4 .

\section{4 other Eeatures of GAUGE}

The code GAUGE was developed as a diffucion-depletion

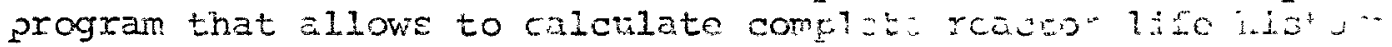
with partial. refueling at a number of xeload time poirts. $\therefore$ each discrete time point a control rod searct ray be performat $t$. maintain criticality at all times, allowing the adjustrent of a number of control rod banks according to a prestribed rod stouencing scheme.

The depletion scheme of all burnable nuclides is specifled by the user at execution time. A general and flexible burnup routine system 6 was developed which allows an optimal choice of either a difference approximation or an analytical solution of the depletion equations. The code decides internally, for each time step and every nuclide, which approximation is used. As the most gereral case, a full triangular depletion matrix is assumed. 
GAUGE allows 1519 hex blocks with 4405 mesh points, 4 energy groups, 280 burnup regions, 40 burnable nuclides and 80 microscopic cross section blocks. A 4-group problem with 61 patches, 1201 nesh points, 35 nuclides, 170 regions, no control search, recuires 1 min. per long time step $(5$ small time steps between diffusion calculations). The same problem with criticality search requires 2 min. (UNIVAC 1108).

5. GATT, a Three-Dimensional stalic Diffusion Code for a Hexagonal-z Mesh

Based on the experience gained in the development of GAUGE and on the conviction that the block inversion will show particular advantages also in three-dimensional problems, a static 3-D diffusion code GATT was developed. GATT is, particularly with respect to geometry, a direct analogue to GAUGE. The same calculational mesh, Fig. 2, for the horlzontal planes has been adopted ard a three-dimensional prismatic patch, Fig. 5, is used as the basic unit block of the mesh superstructure. Very similar to the actual configuration of a HTGR core the reactor may consist of a number of horizontal layers of patches and it is assumed that each layer or "slice" is homogeneous in axial direction. Control rods are represented by an absorber which is smeared over the central hex block of a patch. A rod can be inserted into a position that coincides with the boundary between two slices.

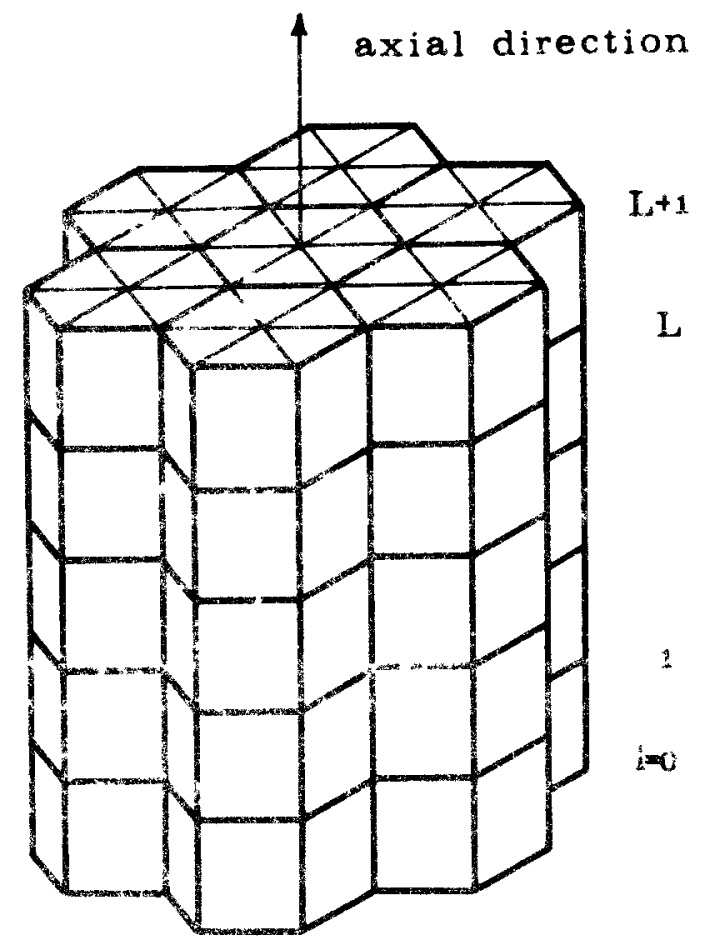

Fig. 5 Three-Dimensional. Block ("Patch")

In ach axial region, the axial mesh spacing $h_{z}$ is assumea to be conctant. However, $h_{z}$ mar change from region to region $T$ the case that the central point of the difference operator 1 ; iocated at a corner between three different hex blocks 
and between two adjacent axial regions with spacings $h_{z}^{+}$and $h_{\bar{z}}$ the following nine-point difference expression is used

$$
\begin{gathered}
\left\{2\left(\overline{\mathrm{D}}_{1}+\overline{\mathrm{D}}_{2}+\overline{\mathrm{D}}_{3}\right)+\frac{3}{2} \mathrm{~h}_{\mathrm{x}}^{2}\left[\frac{\bar{\Sigma}_{1}+\bar{\Sigma}_{2}+\bar{\Sigma}_{3}}{3}+\frac{\overline{\mathrm{D}^{-}}}{\left(\mathrm{h}_{\mathrm{z}}^{-}\right)^{2}}+\gamma \frac{\overline{\mathrm{D}^{+}}}{\left(\mathrm{h}_{\mathrm{z}}^{+}\right)^{2}}\right]\right\} \Phi_{0}^{\circ} \\
-\overline{\mathrm{D}}_{1} \Phi_{1}^{\circ}-\overline{\mathrm{D}}_{2} \Phi_{3}^{\circ}-\overline{\mathrm{D}}_{3} \Phi_{5}^{\circ}-\frac{\overline{\mathrm{D}}_{1}+\overline{\mathrm{D}}_{2}}{2} \Phi_{2}^{\circ}-\frac{\overline{\mathrm{D}}_{2}+\overline{\mathrm{D}}_{3}}{2} \Phi_{4}^{\circ}-\frac{\overline{\mathrm{D}}_{3}+\overline{\mathrm{D}}_{1} \Phi_{6}^{\circ}}{2} \\
-\frac{3}{2} \mathrm{~h}_{\mathrm{x}}^{2}\left[\frac{\overline{\mathrm{D}^{-}}}{\left(\mathrm{h}_{\mathrm{z}}^{-}\right)^{2}} \Phi_{0}^{-}+\gamma \frac{\overline{\mathrm{D}^{+}}}{\left(\mathrm{h}_{\mathrm{z}}^{+}\right)^{2}} \Phi_{0}^{+}\right]=\frac{3}{2} \mathrm{~h}_{\mathrm{x}_{0}^{2}}^{\circ},
\end{gathered}
$$

where

$$
\begin{aligned}
& \bar{D}_{i}=\frac{D_{i}^{-}+\gamma D_{i}^{+}}{2} \\
& \bar{\Sigma}_{i}=\frac{\Sigma_{i}^{-}+\gamma \Sigma_{i}^{+}}{2}
\end{aligned} \quad i=1,2,3
$$

and

$$
\begin{gathered}
\overline{D^{+}}=\frac{1}{3}\left(D_{1}^{+}+D_{2}^{+}+D_{3}^{+}\right) \\
\overline{D^{-}}=\frac{1}{3}\left(D_{1}^{-}+D_{2}^{-}+D_{3}^{-}\right) \\
\gamma=\frac{h_{z}^{+}}{h_{z}^{-}} .
\end{gathered}
$$

The static eigenvalue problem for this hexagonal-z mesh is solved making use of the block-iterative technique described in the previous sections. Since the prismatic patch in Fig. 5 is considered as the unit cell of the coarse mesh the block inversion technique can be used efficiently to calculate the flux within such a block, holding fixed the flux values along the boundaries between the blocks. Each patch contains $13 . \mathrm{L}$ inner mesh points, where $I$ is the number of inner mesh planes in a particular slice, $I_{\max }=9$. Accordingly, the flux at point $i, l$ is represented by

$$
\Phi_{i}^{l}=\sum_{j=1}^{13} \mathrm{v}_{i j}^{l} \alpha_{j}+w_{i}^{l}
$$

where $i=1, \ldots, 13 ; \quad l=0,1, \ldots, I+1$

The explicit form of the recursiye difference equations for the set of homogeneous solutions $U_{i j}$ and for the inhomogeneous 
solution $w_{i}^{l}$ can easily be derived from Eq. (36) and may be found in reference 8 . The initial conditions for $U$ and $W$

$$
\begin{aligned}
& U_{i j}^{0}=0 ; \quad U_{i j}^{1}=\delta_{i j} \quad i, j=1, \ldots, 13 \\
& w_{i}^{0}=\Psi_{B, i} ; \quad w_{i}^{1}=0
\end{aligned}
$$

are chosen such that $\alpha$ represents the flux vector at the first inner mesh plane $\ell=1$. Again $\alpha$ is obtained from the matrix

$$
\mathrm{U}_{\mathrm{T}} \alpha=\Psi_{\mathrm{T}}-\mathrm{W}_{\mathrm{T}}
$$

where the subscript $T$ refers to the top mesh plane $l=I+1$. The matrix $U_{T}$ of order 13, or rather its inverse $U_{\mathrm{T}}^{-1}$, is calculated for each patch and energy group in the initial phase of the calculation. In the first version of GATT an additional assumption has been made with respect to the symmetry structure of the patch. It was assumed that each patch is composed of only two regions, the central hex block with macroscopic constants $\Sigma_{0}$, $D_{0}$ and a ring of six surrounding hex blocks with constants $\Sigma_{1}$, $\mathrm{D}_{1}$. It is easy to show that the resulting 60-degree symmetry leads to a highly symmetric matrix $U_{\mathrm{T}}^{-1}$ with only 19 different elements. An extended version of GATT is presently being developed which allows a more general region structure for each patch, both axial and radial.

The iteration strategy of the present GATT version follows closely the scheme used in GAUGE. The large maximal problem size of more than $400 \cdot 000$ group mesh points, however, requires a subdivision of the total problem size into smaller portions that can be held simultaneously in the fast computer memory. It was decided to use as the core problem size one horizontal layer of patches for one energy group, with a maximum of 61 patches per slice. First a block inversion is done for all patches in the slice, followed by one sweep to improve the boundary fluxes. During this time an overlapping transfer of data for the previous and for the next slice is possible with a minimum of waiting time. This operation is continued until a full axial sweep through all layers is completed. For each outer iteration two such axial iteration sweops are performed in alternating directions.

In the present GATT version only one of the two acceleration procedures is applied to speed up the outer fission source iteration. The use of the asymptotic source and flux extrapolation, as described in section 4.3 , results in a net saving of about a factor of three, relative to the unaccelerated source iteration. It $i s$ expected, that the inclusion of the coarse mesh rebalancing in the extended version of GATT will improve the convergence rate by arother factor of this order. 
GATT is written in FORTRAN IV and requires a $65 \mathrm{k}$ central memory and 10 files of drum storage with a total of $500 \cdot 000$ words capacity. As an example, a four-group problem with 42'000 mesh points representing a $330 \mathrm{MW}(\mathrm{e})$ HTGR reactor with four fully and three partially inserted control rods can be run to tight convergence in 80 minutes on a UNIVAC 1108 computer.

\section{3DT, a Specialized Three-Dimensional Transport Code}

Finally, in this section a simple approach for the numerical solution of a certain class of 3-D neutron transport problems is described briefly 9 . The method was developed in an attempt to analyze various configurations of the thermionic critical experiment (TCE) of Gulf General Atomic 10, but may be of interest for similar applications. The geometry of this critical assembly is a rectangular parallelepiped with a region structure that allows the use of a uniform spatial mesh with constant spacings $h_{\mathrm{x}}, h_{\mathrm{y}}$ and $h_{\mathrm{z}}$ in each direction. A unit rectangular mesh cell is shown in Fig. 6 .

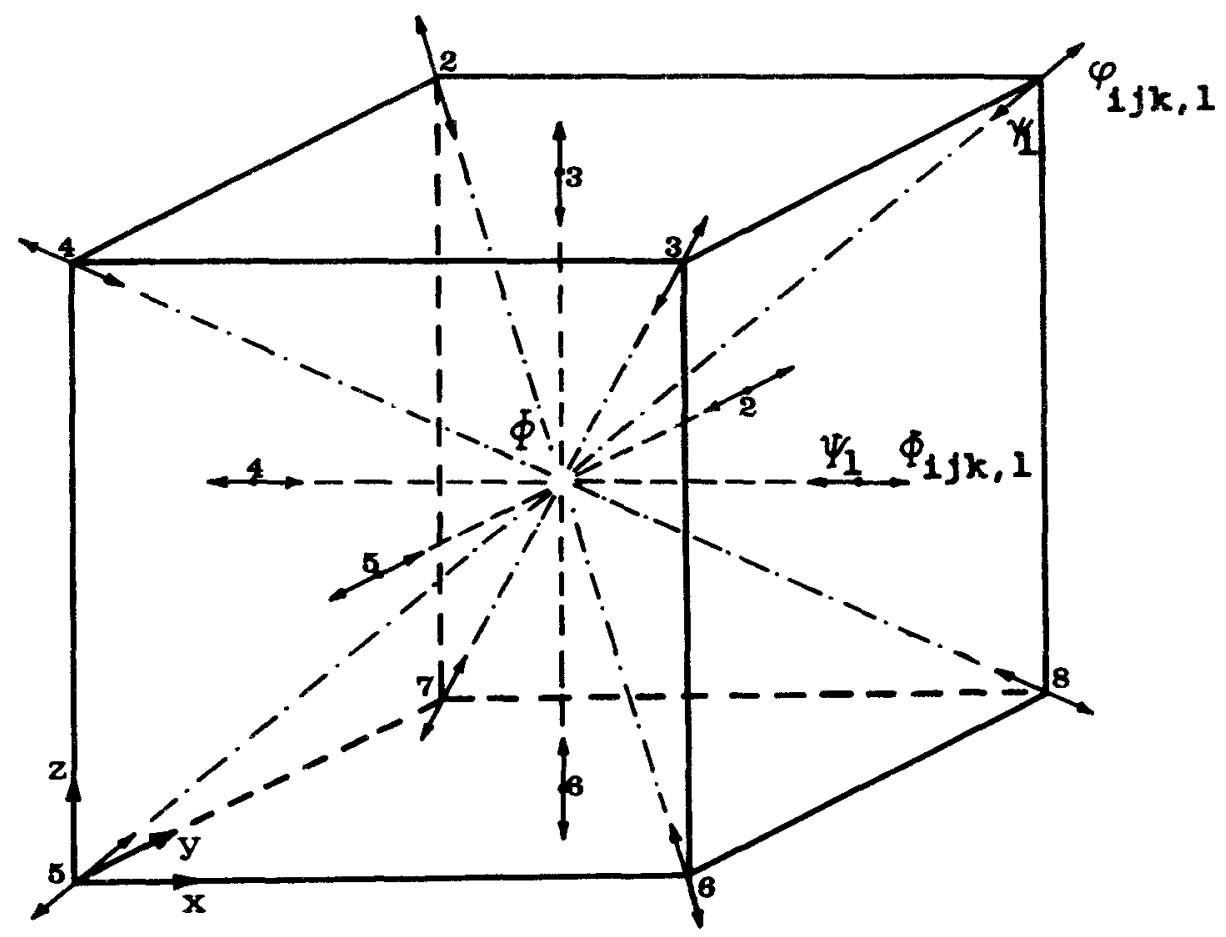

Fig. 6

The 14 Angular Flux Components of a Unit Mesh Cell 
The angular flux distribution in cell $i, j, k$ is approximated by a low-order discrete ordinate model. It is assumed that the neutron flux can be described in terms of 14 angular flux components

$$
\begin{array}{ll}
\Phi_{i j k, \ell} & \ell=1, \ldots, 6 \\
\varphi_{i j k, m} & m=1, \ldots, 8,
\end{array}
$$

where the first 6 components point in the directions of the main axes and the remaining 8 components along the space diagonals of the cell in outgoing directions. To each cell belongs a set of ingoing components $\Psi_{\ell}, \psi_{\mathrm{m}}$ which are stored as outgoing components of 14 neighbor cells.

Assuming that the group sources $Q_{i j k}^{0}, \vec{Q}_{i j k}^{l}$ and the Po and Pl scattering sources are constant within each cell and omitting spatial subscripts $i, j, k$ the discrete ordinate equations which describe the particle motion in the normal directions may then be written

$$
\begin{aligned}
& \Phi_{\ell}=\Gamma_{\ell}{ }_{\ell+3}+\Delta_{\ell}\left[\sigma_{\mathrm{s}}^{0} \Phi+Q^{0}+\left(\overrightarrow{\mathrm{n}}_{\ell}, \sigma_{\mathrm{s}}^{1} \vec{J}+\vec{Q}^{I}\right)\right] \\
& { }_{\ell+3}=\Gamma_{\ell}{ }^{\Psi} \ell+\Delta_{\ell}\left[\sigma_{\mathrm{s}}^{0} \Phi+Q^{0}-\left(\overrightarrow{\mathrm{n}}_{\ell}, \sigma_{\mathrm{s}}^{1} \vec{J}+\vec{Q}^{I}\right)\right],
\end{aligned}
$$

with $l=1,2,3$ and

$$
\begin{gathered}
\Gamma_{\ell}=\exp \left\{-c_{t} h_{\ell}\right\} \\
\Delta_{\ell}=\frac{1-\Gamma_{\ell}}{\sigma_{t}} .
\end{gathered}
$$

Similar ray equations are obtained for the diagonal components, $m=1, \ldots, 4$,

$$
\begin{aligned}
& \varphi_{m}=\gamma \psi_{m+4}+\delta\left[\sigma_{s}^{\circ} \Phi+Q^{\circ}+\left(\vec{d}_{m}, \sigma_{s}^{l} \vec{J}+\vec{Q}^{1}\right)\right] \\
& \varphi_{\mathrm{m}+4}=\gamma \psi_{\mathrm{m}}+\delta\left[\sigma_{\mathrm{s}}^{0} \Phi+Q^{0}-\left(\overrightarrow{\mathrm{d}}_{\mathrm{m}}, \sigma_{\mathrm{s}}^{1} \vec{J}+\vec{Q}^{1}\right)\right], \\
& \gamma=\exp \left\{-\sigma_{t} h_{D}\right\} \\
& \delta=\frac{1-y}{\sigma_{t}} \\
& h_{D}=\sqrt{h_{1}^{2}+h_{2}^{2}+h_{3}^{2}} \text {. }
\end{aligned}
$$


$\vec{n}_{\ell}$ are unit vectors in the three coordinate directions, $\vec{d}_{m}$ are unit vectors in the direction of the space diagonals (with positive $z$-components). Simple expressions can be used to approximate the neutron current vector $J$ in terms of the discrete components, for instance

$$
\begin{gathered}
J_{x}=w_{1}\left(\Phi_{1}-\Psi_{1}\right)-w_{4}\left(\Phi_{4}-\Psi_{4}\right) \\
+w \frac{h_{x}}{h_{D}}\left[\left(\varphi_{1}-\psi_{1}\right)-\left(\varphi_{2}-\psi_{2}\right)+\left(\varphi_{3}-\psi_{3}\right)-\left(\varphi_{4}-\psi_{4}\right)\right. \\
\left.-\left(\varphi_{5}-\psi_{5}\right)+\left(\varphi_{6}-\psi_{6}\right)-\left(\varphi_{7}-\psi_{7}\right)+\left(\varphi_{8}-\psi_{8}\right)\right] .
\end{gathered}
$$

The discrete ordinate model associates with each discrete direction an angular range or quadrature weight $\mathrm{w}_{l}$ or $\mathrm{w}_{\mathrm{m}}$. For symmetry reasons $w_{m}=w$ for all $\mathrm{m}$. The weights fulfil the condition

$$
\sum_{l=1}^{6} w_{l}+8 w=1
$$

The set of normal weights $w_{l}$ may be derived from the requirement that the average cosine with respect to the three coordinate directions must be $1 / 3$.

With

$$
\frac{8 w}{\sum_{l} w_{l}}=\beta
$$

one obtains

$$
\begin{gathered}
\mathrm{w}_{\ell}=\frac{1}{6}\left(1-\frac{3 \beta}{\beta+1} \frac{\mathrm{h}_{\ell}^{2}}{\mathrm{~h}_{\mathrm{D}}^{2}}\right), \quad \ell=1,2,3 \\
\mathrm{w}_{\ell+3}=\mathrm{w}_{\ell} .
\end{gathered}
$$

The average scalar flux $\Phi$ in a cell is approximated by

$$
\Phi=\sum \mathrm{w}_{l} \bar{\Phi}_{l}+\mathrm{w} \sum_{\mathrm{m}} \bar{\varphi}_{\mathrm{m}}
$$


where $\bar{\Phi}_{\ell}, \bar{\varphi}_{\mathrm{m}}$ are average values of $\Phi_{\ell}, \varphi_{\mathrm{m}}$ integrated on the rays $\ell, \mathrm{m}$, so that, e.g.

$$
\begin{aligned}
& \sum_{\ell} w_{\ell} \bar{\Phi}_{\ell}=\sum_{\ell} \frac{1}{h_{\ell}} \int_{0}^{h_{\ell}} \Phi_{\ell} d s_{\ell} \\
& =\sum_{l} w_{l}\left[\frac{\Delta_{l}}{\mathrm{~h}_{\ell}} \Psi_{l}+\frac{\Delta_{l}}{\sigma_{t}}\left(\sigma_{s}^{0} \Phi+Q^{0}\right)\right]
\end{aligned}
$$

and similar for $\sum_{\mathrm{m}} \bar{\varphi}_{\mathrm{m}}$. Inserting this into Eq. (50) and using the ray equations (42) and (44) one obtains a neutron balance

$$
\left(\sigma_{t}-\sigma_{\mathrm{s}}^{\circ}\right) \Phi=Q^{0}-\left\{\sum_{l=1}^{6} \frac{\mathrm{w}_{l}}{\mathrm{~h}_{\ell}}\left(\Phi_{\ell}-\Psi_{l}\right)+\frac{\mathrm{w}}{\mathrm{h}_{\mathrm{D}}} \sum_{\mathrm{m}=1}^{8}\left(\varphi_{\mathrm{m}}-\psi_{\mathrm{m}}\right)\right\} .
$$

During the inner spatial iteration a procedure is used which is related to the block inversion method. While passing through cell $i, j, k$ the current set of ingoing fluxes $\Psi_{l}, \psi_{m}$ for all directions is held constant momentarily and the set of outgoing fluxes $\Phi_{l}, \varphi_{\mathrm{m}}$ is calculated from the ray equations such that the neutron balance is fulfilled for that cell. A consistent value for $\Phi$ is obtained from Eq. (52) by eliminating the outcomponents with the use of Eqs. (42), (44)

$$
\Phi=\frac{a^{0}+\sum_{l} \frac{W_{\ell}}{h_{\ell}}\left(1-\Gamma_{\ell}\right) \Psi_{\ell}+\frac{w}{h_{D}}(1-\gamma) \sum_{m} \Psi_{m}}{\sigma t-a \sigma_{s}^{\circ}},
$$

where

$$
a=I-\left(\sum_{\ell} \frac{w_{\ell}}{h_{\ell}} \Delta_{\ell}+8 \frac{w}{h_{D}} \delta\right) .
$$

With this value for $\Phi$ the ray equations may be solved. If $\mathrm{Pl}$ scattering is present $\sigma_{s}^{1}=0$. Egs, (42), (44) represent a coupled innear system of equations. Instead of solving this system explicitly for each inner iteration $n$ it is more expeditious to account for the linear anisotropic scattering terms in an iterative way be using the neutron current vector $\vec{f}(n-1)$ from the previous inner iteration. 
Based on this model a prototype computer code 3DT was written. The code, in its present form, is core-contained and, therefore, allows only a relatively small problem size, namely, 2 energy groups and 1120 mesh cells. An eigenvalue calculation for the TCE assembly with 2 groups, 1120 cells and $\mathrm{Pl}$ scattering requires 1.1 minutes on the UNIVAC 1108 computer. 3DT was used to calculate individual fuel block reactivity worths for the TCE assembly. The comparison 11 with experiment and with a 3-D synthesis perturbation method is in general good. In all cases but one, the predicted and measured reactivity worths agree within the margin of error. 


\section{References}

1. Wachspress, E.I., Iterative Solution of Elliptic Systems, Prentice-Hall, Inc., Englewood Cliffs, 1966.

2. Froehlich, R., on Stable Methods of Matrix Factorization, Parts 1 and 2, GA-7164 (1966).

3. Lucey, J.W., and K.F. Hansen, A Stable Method of Matrix Factorization, Trans. Am. Nucl. Soc. 7, 259 (1965).

4. Kraetsch, H., and M. Wagner, The Block Inversion Method, Trans. Am. Nucl. Soc. 10, 173 (1967).

5. Froehlich, R., A Theoretical Foundation for Coarse Mesh Variational Techniques, GA-7870 (1967).

6. Wagner, M.R., GAUGE - A Two-Dimensional Few Group Neutron Diffusion-Depletion Program for a Uniform Triangular Mesh, GA-8307 (1968).

7. Dorsey, J.P., and R. Froehlich, GAMBLE-5, A Program for the Solution of the Multigroup Neutron-Diffusion Equations in Two Dimensions, GA-8188 (1967).

8. Kraetsch, H. and M.R. Wagner, GATT - A Three-Dimensional Few-Group Neutron Diffusion Theory Program for a Hexagonal-z Mesh, GA-8547 (1968).

9. Wagner, M.R., A Numerical Method for the Solution of Three-Dimensional Neutron Transport Problems, Trans. Am. Nucl. Soc. 11, 534 (1968).

10. Cohen, S., et al, Thermionic Critical Experiment Description and Safety Analysis, GA-8091 (1967).

11. Cohen, S. and D. Sargis, Personal Communication, Jan. 1969. 
REDUCTIUN OI MRCHINE TIME IN DISCRETE ORDINATES CALCULATIONS

\author{
hy Zi_rt $\Xi$. Elllivan*, Duhsoong Yu**, and Iynn $Y$. Albers* \\ Lewis Research Center \\ Notional feronautics and Space Administration \\ Cleveland, Ohio

\section{INTRODUCTION}

Recent grvences in computer design make it possible to obtain numerical solutions to problems previously too large to process. However, the direct apblication of existing methods to these larger problems can require prohibitive1. 'fr.. ype ytures of machine time. One class of problem of which this is

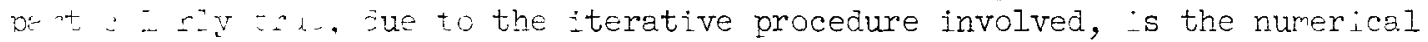

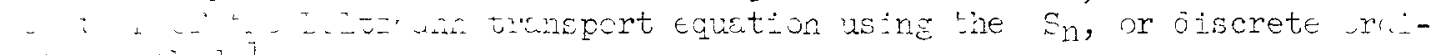
Miter tinor. I

$$
\begin{aligned}
& \text { rine wolut ... of the stat.onary transport equation } \\
& \qquad \hat{\overrightarrow{3}} \cdot \mathrm{W}(\mathrm{t}, \overrightarrow{0}, \hat{\imath})+\sigma_{t}(E, \vec{\rho}) \psi(E, \vec{\rho}, \hat{\Omega})=S(E, \vec{p}, \hat{\imath})
\end{aligned}
$$

entais the determination of a particle flux distribution which is a function uf crergy, $F$, position, $\vec{\rho}$, and direction, $\hat{\Omega}$, in terms of the total cross section, $\sigma_{t}$, and the scurce $S$. In the $S_{n}$ mecnod, this is accomplished by establishing a cuaorature on the domain of each of these indenenient va" ables and, using an nitial approximate distribution, iterating on eacn quacrature until a specifiea degree of convergence is attained. Several techniques ior improving the efficiency of the method by using various scaling and acceleration 3 procecures to force more rapid convergence on the flux distribution have been successfully applied. Alternate techniques for directly reducing the number of numerical calculations involved by varying the manner in which the quadratures are applied seem to have been less thoroughly investigated. Thus, existing applications of the $S_{n}$ method are basically similar4 in that they require a constant order of duadrature for each of the independent variables.

In obtaining a solution to the transport equation for a problem in which no prior information concerning the flux distribution is available, it is customary to use as a first approximation a distribution which is constant in the independent variables. The major portion of the numerical calculation is ther devoted to obtaining successively more accurate approximations to the flux distribution. Since the time required to perform an iteration depends strongly on quadrature order and since the accuracy of the early iterations is low, use of the high quadrature orders required for the final solution does not appear warranted in the preliminary stages of the calculation. A more optimum procedure might be to vary the quadratures as a function of the progress of the calculation so as to more rapidly obtain a reasonably accurate preliminary flux distribution.

\footnotetext{
* Iewis Research Center.

* University of Oklahoma.
} 
Although the flux distribution is a function of energy, position, and direction, the energy quadrature is effectively removed from the actual discrete ordinates calculation due to the group averaging methods normally used. The two remaining independent variables, direction and position, will be discussed separately.

The central feature of the $S_{n}$ technique is the representation of the continuous angular distribution of the particle flux by a number of discrete intervals. If the direction unit vector, $\hat{\Omega}$, is defined by the angles, $\mu, \eta$, and $\xi$, it may be written, in rectangular coordinates, as

$$
\hat{\Omega}_{m}=\cos \mu \hat{i}+\cos \eta \hat{j}+\cos \xi \hat{k}
$$

where $m$ denotes the total angular dependence. The direction $\hat{\Omega}_{\mathrm{m}}$ represents a point on the surface on a unit sphere and has an associated surface area, $\mathrm{w}_{\mathrm{m}}$, which indicates the weight assigned to that direction. The range of integration over the unit vector is divided into a number of intervals, $M$, corresponding to the order of the quadrature, and the angular flux is assumed to be constant within each interval. The integral over the angle may now be replaced with a summation over the weighted intervals so that the group scalar flux $\varphi_{\mathrm{g}}$ is given by,

$$
\varphi_{g}(\vec{\rho})=\frac{I}{4 \pi} \int_{\hat{\Omega}} \psi_{g}, \bar{i}, \bar{k}, \bar{m}(\vec{\rho}, \hat{\Omega}) \mathrm{d}^{2} \hat{\Omega}=\sum_{m=1}^{M} w_{m} \bar{\psi}_{g}, \bar{i}, \bar{k}, \bar{m}
$$

where $\psi_{g}, \bar{i}, \bar{k}, \bar{m}$ represents the average flux in an interval for a given direc-
tion.

Since the calculation time increases rapidly with the number of discrete intervals employed, limiting this number of directions in the initial stages of the calculation should be a valid procedure. Historically, several variations in the standard $S_{n}$ procedure have attempted to achieve this end by first obtaining a preliminary flux distribution for use in the main calculation. Thus, some $S_{n}$ codes have contained an option for first performing a diffusion theory solution, 5 for first performing one outer iteration using an $S_{2}$ quadrature, ${ }^{G}$ or for reducing the number of inner iterations in the initial outer iterations. ${ }^{7}$ There seems to be little or no published information on the effect of these procedures on accuracy and running time.

In the standara $S_{n}$ treatement, the spatial dependence of the particle flux is approximated by superimposing a finite mesh on the configuration to be analyzed. While the mesh spacing in any one direction is arbitrary, no variation is permitted in that mesh in an orthogonal direction. For example, in rectangular coordirates, the mesh set un alcng the $x$ coordinate must be retained for all values of the $y$ coordinate.

In the discrete ordinates method, determination of the value of the central average angulitr iluxes, $\bar{\psi}$, involves the use of a physical model to relate the angular fluxes at the mesh cell bourdaries with the central flux. In the commonly used "diamond" rodel

$$
\begin{aligned}
\bar{\psi}_{g, \bar{i}, \bar{k}, \bar{m}} & =\frac{1}{2}\left[\psi_{g}, i+1, \bar{k}, \bar{m}+\psi_{g, i, \bar{k}, \bar{m}}\right] \\
& =\frac{1}{2}\left[\psi_{g}, \bar{i}, \underline{k}+1, \bar{m}+\psi_{g}, \bar{i}, k,-\bar{m}\right] \\
& =\frac{1}{2}\left[\psi_{g}, \bar{i}, \bar{k}, m+\psi_{g}, \bar{i}, \bar{k}, m-I\right]
\end{aligned}
$$


where $i$ and $k$ represent mesh boundaries in orthogonal directions and $m$ again denotes the angular dependence. Since all these quantities must be calculated repeatedly in each interval, for each quadrature direction, as indicated in equation (3), it is obvious that any method of eliminating unnecessary spatial mesh intervals would siso reduce calculation time.

The objective of this study was to determine the general feasibility of reducing computer time in discrete ordinates calculations. In particular, several modifications to the standard $S_{n}$ procedure, which relax the constant quadrature requirements, were investigated and their effect on accuracy and calculation time observed.

\section{ANALIYSIS AND RESUITS}

For this study, the modifications made to present applications of the dis crete ordinates method are restricted to relaxation of the constant quadrature requirements. The primary objective in allowing quadratures to vary during the calculation is to more rapidly establish improved scalar flux distributions. This is accomplished by performing preliminary calculations with lower order quadratures, and a concomitant smaller number of angular fluxes, and using the resulting scalar fluxes to enter the final calculation.

Removal of the energy dependence from equation (1) results in a set of integro-:. fierential equations coupled by a source term involving only the sialar fiues. In the normal flow of the discrete ordinates calculation there ale two major iterative procedures. The first, or inner iteration, consists of a determination of the angular fluxes for all discrete directions over the entire spatial mesh. This results, as indicated in equation (3), in a revisea scalar flux distribution. The second, or outer iteration, involves calculation of the source terms coupling each equation and involves only these scalar fluxes. Since this outer iteration is essentially independent of the angular fluxes, the completion of each such iteration affords a convenient point at which to vary angular quadratures.

While it is also possible to vary the spatial mesh on termination of an outer iteration, modifications to the angular and spatial quadratures are not analogous. Permissible variations in the spatial quadrature should comprise not only alterations in the order of the uniform mesh but, more importantly, the elimination or combination of noncritical mesh intervals. This latter consideration requires that some assumptions be made regarding the behavior of the individual angular fluxes.

Due to the dissimilar procedures involved, the modifications to the angular and spatial quadratures will be treated separately. All modifications were made utilizing an existing two dimensional $S_{n}$ program, ${ }^{3}$ TDSN. TDSN is a variable storage allocation code which is similar in flow diagram to other current discrete ordinates codes.

The configuration used for testing these modifications is shown in figure 1. It is a rectangular coordinate representation of the transverse section of a cylindrical reactor. The reactor is fueled with $\mathrm{U}^{23} \mathrm{~N}_{\mathrm{N}}$ and uses $\mathrm{Li}^{7}$ as a coolant. Control is effected by the rotation of two noncentral drums containing $\mathrm{B}_{4} \mathrm{C}$ and the reflector is $\mathrm{BeO}$.

Eight group cross sections, seven fast and one thermal, generated from the GAM-II 8 and GATHER-II 9 library are used. While this configuration was run to verify the accuracy of all the modifications, the angular quadrature results are based primarily on a one dimensional representation of this core using two dimensional quadrature sets. 
TEST CASE CONFIGURATION

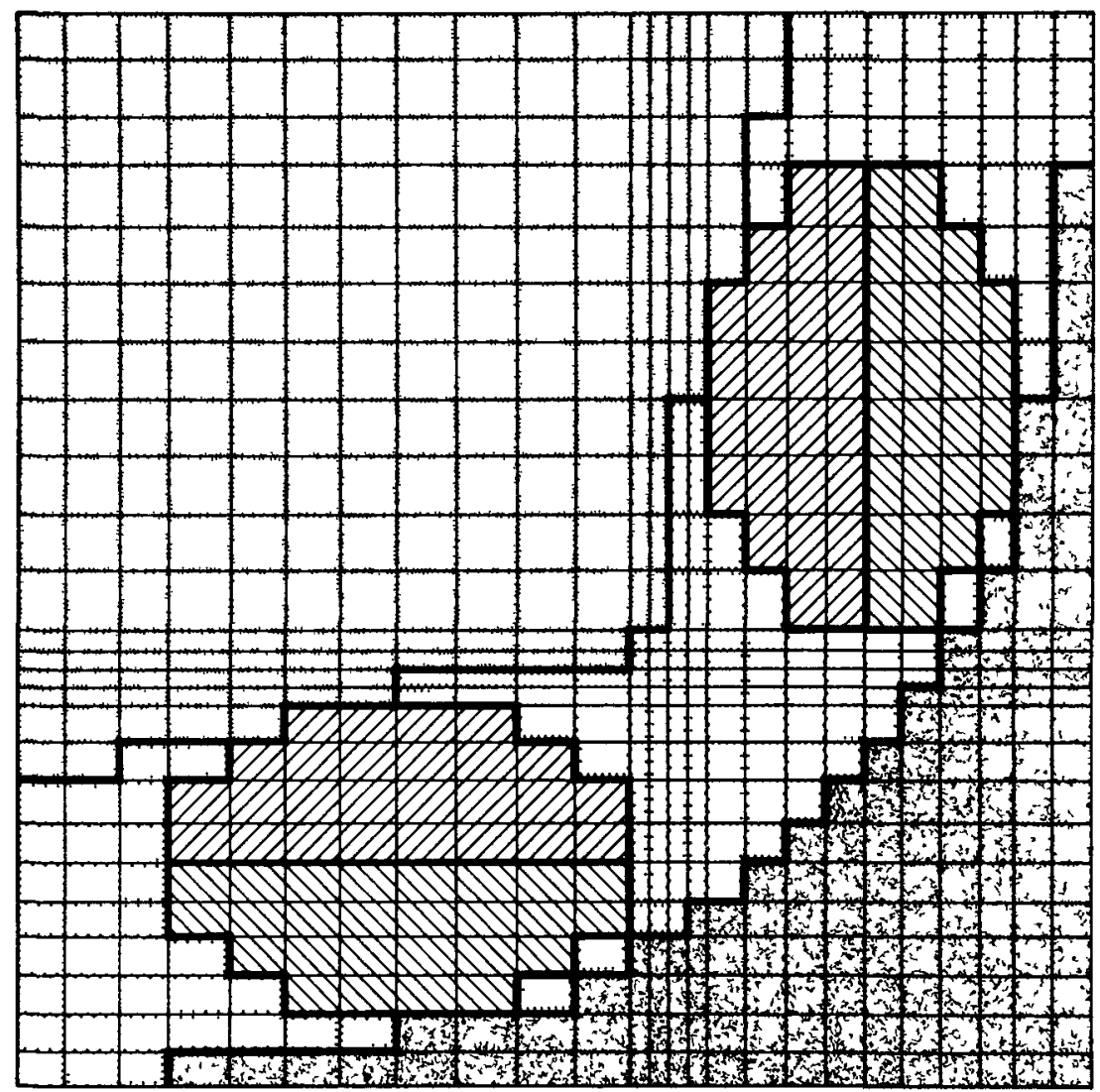

$\square$ CORE

$\square$ REFLECTOR

ZAs вео
DIV $\mathrm{B}_{4} \mathrm{C}$

EXTERIOR INTERVALS

CD-10313-22

CS- 49284 


\section{ANGUTAR QUADRATURE MODIFICATIONS}

Analys is

Consideration of the flux values established during the early iterations of a higher order angular quadrature calculation indicates that a large number of unnecessary numerical steps are performed in establishing an accurate preliminary scalar flux distribution. This is caused, primarily, by the use of a larger number of discrete directions than required to obtain scalar flux approximations which are varying rapidly. The use of any of the previously described methods $5,6,7$ should be a valid, but perhaps inefficient, procedure. The basic question of the optimum manner in which to approach the final solution requires a more general modification of the standard $S_{n}$ coding. options should be included which would allow studies to be made of the eifect on convergence of using any progression of quadratures in conjunction with arbitrary termination criteria. In addition, since most reactor configurations involve energy dependent cross sections in which the optimum quadrature order would not be identical for all energy groups, it would be of interest to observe the effect of permitting the quadrature order to be a function of the energy.

Since the lower order quadratures cannot be expected to yield scalar flux distributions with an accuracy equivalent to that of higher order quadratures, there is little point in solving the lower order calculation to the same degree of convergence required for the final calculation. The major benefit in the final calculation should be derived from the use of a trial scalar flux which minimizes the number of inner iterations required for convergence on the final quadrature and not from fully converged low order scalar fluxes. An important question in this regard is that of determining the optimum point at which to effect the quadrature variations.

In order to study the effect on solution time of the modifications discussed, the computer program previously referenced ${ }^{3}$ has been altered to perform the following sequences of operation:

(1) Any number of preliminary lower order quadrature sets may be used.

(2) The use of a given quadrature set may be terminated either by completion of a given number of outer iterations or by attaining to some specified degree of convergence.

(3) When a given number of outer iterations is specified, a convergence criterion may be used which will terminate the calculation on the set if attained before all the outers are performed.

(4) The final quadrature order may be made a function of the energy group. This feature may be used with or without the preliminary lower order calculation.

Sequences (2) and (3) imply that more than one degree of convergence may be used on the same quadrature set. This, in effect, serves to limit the number of inner iterations in the initial phases of the calculation. The multiple sets of direction numbers need not be read in as the program generates moment modified sets ${ }^{10}$ as required. In addition, all the options available in the original code, including convergence tests and acceleration procedures, are retained. None of the modifications made which involve varying the angular quadrature order during the course of the calculation should affect the accuracy of the final results. Convergence on the final values of the angular fluxes, eigenvalues, and other quantities must not depend on the manner in which the calculation is begun. This is also true, within the framework of the quadrature orders used, for the modification in which the quadrature is made a function of 
the energy. The main problem here is a foreknowledge of the quadrature required to accurately represent a given energy group.

\section{RESUTTS}

The most important consideration concerning the use of preliminary low order quadratures is that of determining the optimum manner in which to approach final convergence. Results from the one dimensional cases collected in table 1 indicate that this optimum method depends on the order of the final quadrature desired.

\section{TABLE 1. - QUADRATURE MODIFTCATIONS}

1. Base Case is $S_{8}$ with convergence criterion of $10^{-4}$ requiring 2.53 minutes for final convergence.

$\begin{array}{lcccc}\text { Case } & \begin{array}{c}\text { Preliminary } \\ \text { quadratures }\end{array} & \begin{array}{c}\text { Quadrature } \\ \text { switch } \\ \text { criterion* }\end{array} & \begin{array}{c}\text { Running } \\ \text { time } \\ \text { (min) }\end{array} & \text { Eigenvalue } \\ \text { a. } & \mathrm{S}_{2} & 20 & 1.90 & 1.6051 \\ \text { b. } & \mathrm{S}_{2} & 30 & 1.84 & 1.6051 \\ \text { c. } & \mathrm{S}_{2} & 10^{-2} & 1.63 & 1.6051 \\ \text { d. } & \mathrm{S}_{2} & 10^{-3} & 1.77 & 1.6051 \\ \text { e. } & \mathrm{S}_{2} & 10^{-4} & 2.0 & -.-1 \\ \text { f. } & \mathrm{S}_{2}, \mathrm{~S}_{8} & 20,10^{-2} & 1.52 & 1.6051 \\ \text { g. } & \mathrm{S}_{2}, \mathrm{~S}_{8} & 20,10^{-3} & 1.21 & 1.6051 \\ \text { h. } & \mathrm{S}_{2}, \mathrm{~S}_{4} & 20,10^{-3} & 1.70 & 1.6051\end{array}$

2. Base Case is as above but with $S_{16}$ final quadrature, requiring 9.33 minutes for final convergence.

$\begin{array}{ccccc}\text { Case } & \begin{array}{c}\text { Preliminary } \\ \text { quadratures }\end{array} & \begin{array}{c}\text { Quadrature } \\ \text { switch } \\ \text { criterion }\end{array} & \begin{array}{c}\text { Running } \\ \text { time } \\ \text { (min) }\end{array} & \text { Eigenvalue } \\ & & 20,40,10^{-3} & 3.98 & 1.6052 \\ \text { a. } & S_{2}, S_{4}, S_{8} & 40,10^{-3} & 4.80 & 1.6052 \\ \text { b. } & S_{2}, S_{16} & 10^{-4}, 10^{-4}, 10^{-4} & 5.50 & 1.6052\end{array}$

${ }^{*}$ no represents the number of outers run on the indicated quadrature set.

For the test case used, with final quadratures of $S_{8}$ or lower order, the maximum time reduction is obtained when only a single preliminary $\mathrm{S}_{2}$ calculation is performed. For problems involving quadratures of higher order than $\mathrm{S}_{8}$, the optimum approach is to use more than one preliminary quadrature. This is best illustrated, for the $S_{8}$ or lower quadratures, by case Ih which uses both an $S_{2}$ and $S_{1}$ preliminary calculation and whose running time to final convergence exceeded that of case $I g$ which had only an $S_{2}$ start. For an $S_{16}$ final quadrature, however, case $2 \mathrm{~b}$, which used only an $S_{2}$ preliminary calculation, required considerably longer to converge than case $2 \mathrm{a}$, which used successively higher quadratures to approach final convergence.

For those problems in which the use of only a single preliminary quadrature is most efficient, a number of trial cases ( $l a$ to $l g$ ) were run in order to determine when to shift from the preliminary to the final quadrature. As discussed previously, full convergence on the preliminary quadrature is probably not desirable. This is borne out by case le which converged the $S_{2}$ start to the 
final $\left(10^{-4}\right)$ criterion and required more running time than any of the other methods applied. These cases also indicate that final convergence is best approached by first performing several outer iterations and then converging to some intermediate criterion on the final quadrature. The use of this intermediate criterion essentially acts to limit the number of inner iterations per outer :teration during that portion of the calculation when the flux distribution is changing rasidis. The same effect could be achieved by directiy controlling the number of inner iterations per outer iteration but this procedure affords no way of insuring that the flux in each energy group is equally well converged. Case lE, which used this approach, yielded the shortest running time for the problem.

For those problems in which multiple quadratures are used to approach final convergence, it is more difficult to specify an optimum approach. The calculation of all of the large number of permutations afforded by allowing both a given number of outer iterations and an intermediate convergence for each quadrature criterion would require an inordinate amount of machine time. Again, however, case $2 \mathrm{c}$ demonstrates that running to full convergence on each preliminary quadrature is less efficient than use of other available options. Consideration of the results of the cases run shows that the shortest running time for the $S_{16}$ solution was achieved by performing a limited number of outer terations on both an $S_{2}$ and an $S_{4}$ preliminary calculation followed by inter$r \rightarrow a^{\prime}$ te convergerce on an $S_{8}$ quadrature and, finally, by final convergence on the, , sclution. Case 20 , which followed this pattern, reduced the tine re-

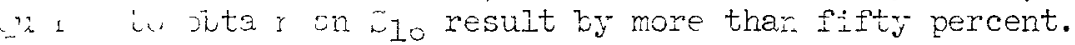

The program modification which allows the quadrature order to be specified by snergy group was also tested on the same base case but with the quarirature sets for the ght energy groups arbitrarily chosen as $2 S_{8}, 4 S_{4}$, and $2 S_{2}$ starting with the highest energy group. For this test case the running time was reduced by about sjxty percent. The low order quadrature start may also we use in conjunction with this modification to further reduce the running time. The value of this modification is questionable due to the difficulty involved in specifying appropriate quadrature orders.

\section{SPATIAL QUADRATURE MODIFICATIONS}

Analysis

Present applications of the discrete ordinates methou require that a uniform spatial mesh be employea. This means that the interval spacing used in one direction must be retained over the whole configuration. For example, the use of rectangular coordinates to represent the cylindrical boundary shown in figure 1 requires retention of the extraneous intervals lying outside the configuration. The typical reactor problem also contains larger homogeneors regions which should not require the same detailed spatial treatment as is necessary near material interfaces or boundaries. Since the angular flux in each discrete direction must be repetitively computed for each mesh interval, recuction or elimination of unnecessary intervals would improve the efficiency of the calculation. Another desirable option would be to obtain an initial improved flux estimate by first solving a less detailed problem.

Although consideration of the constant spatial quadrature requirement indicates many possible modifications, those accomplished for this study were restricted to the following:

(1) First, an option for specifying the number of mesh intervals in the configuration in one direction as a function of the other direction is added. In effect, this allows the normal mesh spacing to be retained but permits the truncation of individual rows in the configuration. The boundary conditions 
are then applied to the initial and final remaining intervals in each row.

(2) Second, an option for varying the mesh interval spacing in the interior regions for both directions is developed. In effect, this allows several intervals to be combined into one and permits the angular fluxes to be calculated only once, in each inner iteration, for this larger interval rather than once for each original separate interval.

(3) Finally, if solution accuracy equivalent to that of the original $S_{n}$ mesh is required, a provision is made for expanding the scalar flux distribution developed in part (2) and performing final iterations on the original mesh configuration.

Due to the numerous ways in which alterations to the spatial mesh portion of the standard $S_{n}$ flow may be accomplished, no spatial modification may be regarded as unique since the same end effect, the reduction of the number of intervals to be treated in the calculation, may be achieved using many different approaches. In general, the modifications described here have been restricted to Cartesian coordinates and an attempt made to keep the additional input as simple as possible.

\section{Results}

The first modification tested was that in which the number of intervals in each row is allowed to vary. This option is primarily useful where it is necessary to use rectangular coordinates to analyze a cylindrical core such as shown in figure 2. Any combination of the normal boundary conditions is permitted so that the quarter, half, or full configuration may be run. In the standard $S_{n}$ treatment, it is necessary to run the complete rectangular mesh with some artificial material in the extraneous intervals. It is possible to compare the accuracy of this modification with that of the standard treatment by inserting a "perfect absorber" into these intervals. Comparison of the eigenvalues from such calculations shows agreement, at the end of each outer iteration, to better than the accuracy required by the convergence criterion used. The time reduction depends, of course, on the complexity of the original case and on the number of intervals deleted. For the configuration of figure 2, with the exterior intervals removed from the computation, the time re. duction is approximately 25 percent.

As a test of the leakage calculation, a simple case was run using cylindrical coordinates in the original code to obtain the eigenvalue and leakage for a 10 centimeter radius cylinder with no internal details. This run was then compared with the interval deletion modification results using a ton interval approximation of the cylindrical boundary. The comparison yields

$\begin{array}{lcl} & \text { Eigenvalue } & \begin{array}{l}\text { Leakage } \\ \text { source } \\ \text { neutron }\end{array} \\ \text { Original TDSN } & 0.9623 & 0.0377 \\ \text { Interval deletion } & 0.9617 & 0.0383\end{array}$

which is quite accurate since only ten steps were used to approximate the boundary.

The second modification, in which interior mesh intervals within homogeneous regions of the core are combined, is the most complex in terms of alternations made to the standard flow. Since the method of implementing any 
INTERVAL TRUNCATION MODIFICATION

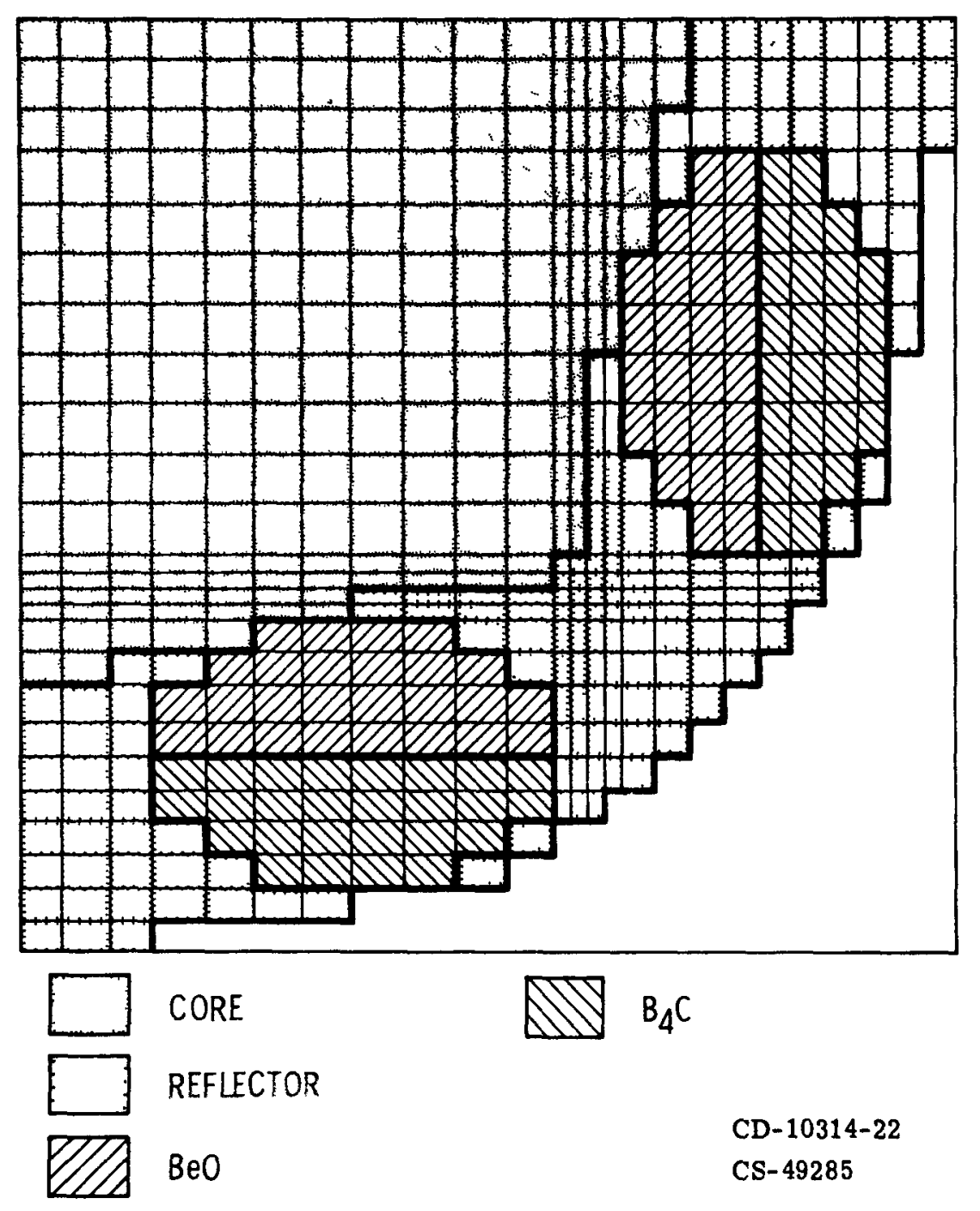

- Figure 2 
such scheme is quite arbitrary, a brief description of the procedure followed will be given.

The normal spatial mesh is first defined. Two interior coordinate boundaries are then specified. Within these boundaries, the interval reduction, or combination, may be applied. Each original region is comprised of a given number of intervals, bounded by mesh lines. The interval reduction is accomplished by choosing the number of mesh lines, in each direction, to be eliminated. Thus, sets of from one to the total number of interval lines may be removed. As an example, if sets of two are removed, two interval boundaries are deleted, the next is retained, the next two removed and the process continued until the outer region boundary is reached. The elimination is performed independently in each coordinate direction with the only restriction being that the last remaining interval boundaries must coincide with the original region boundaries. Figure 3 indicates the results of applying this process to the test case. Since the process results in dissimilarly sized adjacent intervals, some assumption is necessary concerning the behavior of the angular fluxes across such boundaries. No attempt has been made in this study to develop interpolation formulae for such cases and simple flux averages have been used to extrapolate the angular fluxes.

As an example of the use of this modification, the configuration shown in figure 3 was run with the intervals combined as indicated. This problem should represent an extreme test of the modification since some intervals have been combined in regions, such as near the control drums and material interfaces, where the angular fluxes are expected to vary rapidly. Two test cases were run and compared with the results of the original version of the program in order to compare both eigenvalues and reactivities. The second case is also as in figure 3 but with the control drums rotated $90^{\circ}$ clockwise. Results for the first case are

$\begin{array}{lcc} & \begin{array}{c}\text { Time } \\ \text { (min) }\end{array} & \text { Eigenvalue } \\ & 17.22 & 1.285 \\ \text { Original TDSN } & 11.96 & 1.280\end{array}$

and the eigenvalues differ by 0.39 percent. The running time is reduced by about 35 percent.

For the $90^{\circ}$ control arum rotation, the results are

$\begin{array}{lcc} & \begin{array}{c}\text { Time } \\ (\mathrm{min})\end{array} & \text { Eigenvalue } \\ & & \\ \text { Criginal TDSN } & 22.88 & 1.256 \\ \text { Interval reduction } & \mathbf{1 5 . 4 4} & 1.252\end{array}$

and the eigenvalues differ by 0.32 percent with a time reduction of about 33 percent. A comparison of the control reactivities shows that the original version yields 2.28 percent $\delta \mathrm{k} / \mathrm{-k}$ and the interval reduction scheme 2.21 percent $\delta \mathrm{k} /-\bar{k}$. This is good agreement in view of the fact that intervals have been deleted in regions where the flux does not conform to an assumption of relative constancy. 
INTERVAL COMBINATION MODIFICATION

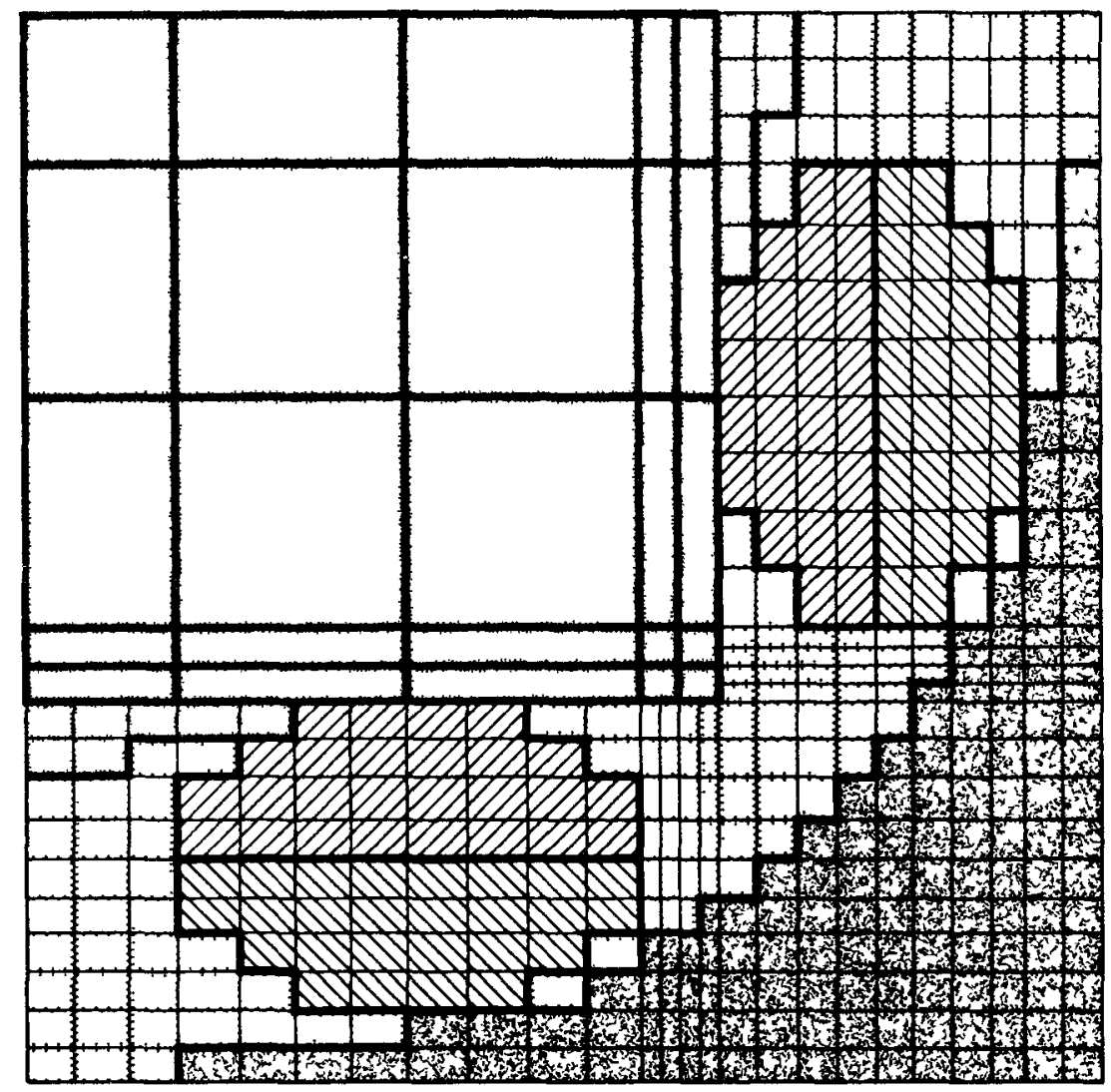

$\square$ CORE

$\square$ REFLECTOR

ED Beo
AII $\mathrm{B}_{4} \mathrm{C}$

EXTERIOR INTERVALS

CD-10315-22

CS- 49286 
The most general application of this modification is to generate a preliminary scalar flux guess for use in a final calculation over the original spatial mesh. To demonstrate this, a configuration similar to that shown in figure 3 was run, using a coarse spatial mesh with less detail in the control drum region, for three outer iterations of the reduced mesh. The scalar fluxes resulting from this calculation were then expanded to fit the original mesh, and the final iterations converged on this mesh. A comparison of the times involved yields

\begin{tabular}{|c|c|c|}
\hline & $\begin{array}{l}\text { Time } \\
(\min )\end{array}$ & Eigenvalue \\
\hline Original TDSN & 13.32 & 1.145 \\
\hline$\left[\begin{array}{l}\text { Coarse mesh } \\
\text { Original mesh }\end{array}\right.$ & $\begin{array}{r}4.192 \\
+3.830 \\
=8.022\end{array}$ & $\left.\begin{array}{l}1.135 \\
1.145\end{array}\right]$ \\
\hline
\end{tabular}

for a time reduction of approximately 40 percent.

\section{CONCLUSIONS}

Some general conclusions regarding the utility of modifications of the type accomplished for this study may be drawn as a result of the test cases run. These conclusions depend, to some extent, on the accuracy required for the final calculation.

For repetitive survey calculations, in which high accuracy' is not requirea, approximate solutions themselves greatly reduce machine running times and are more accurate than the results yielded by merely solving a less detailed straight $S_{n}$ calculation. For the modification using a less detailed angular representation in the preliminary calculations, an approximate solution may be obtained by permitting the quadrature order to be a function of the energy group. While the question of the quadrature order required to adequately Jescribe a given energy group may be difficult, test case results have not varied greatly with reductions in quadrature order. Moreover, the energy dependent quadrature solutions, particularly when combined with a low order preliminary calculation, afforỏ a means of rapidly obtaining approximate answers. For the modification in which interval boundaries are omitted, an approximate solution is obtained by combining less important mesh intervals. The question of which intervals to combine is much less difficult than that of predetermining angular quadrature orders. Since the configuration to be analyzed is known and the spatial angular flux averages postulate a slowly varying angular flux, intervals should be combined only in regions which are homogeneous and removed from material interfaces. The test case results, in which the interval reduction scheme was applied to regions which do not rigorously satisfy the postulate, indicate that this limitation is not severe.

For problem solutions in which accuracy identical to that yielded by the standard $S_{n}$ method application is required, several conclusions regarding the optimum approach to final convergence may be drawn.

1. Allowing full convergence on each angular quadrature set significantly increases running time.

2. For problems involving low order angular quadratures, the most efficient approach to final convergence is through the use of a single preliminary $\mathrm{S}_{2}$ quadrature followed by intermediate convergence on the final quadrature 
prior to the final calculation.

3. For problems using higher order angular quadratures, the greatest time reduction is observed then multiple quadrature sets are used to approach final convergence.

4. For problems in which a less detailed spatial quadrature is used to establish a preliminary flux distribution, the optimum time to final convergence is observed when only a coarse mesh is used to represent the spatial and material configuration in the preliminary calculation.

It may be concluded that modifications of the type outlined are capable of significantly reducing machine execution times without impairing solution accuracy.

\section{REFERENCES}

1. B. G. CARLion, "Solution of the Transport Equation by $S_{n}$ Approximations," IA-I891, Los Alamos Scientific Lab. (Tebruary, 1955).

2. K. D. IATHROD, "DTF-IV - A Fortran-IV Program for Solving the Multigroup Transport Equation with Anisotropic Scattering," LA-3373, Los Alamos Scientific Lab. (July, 1965).

3. $\therefore$. P. PIRBER, "A Fortran IV Two-Dimensional Discrete Angular Segmentation Transport Program," NVASA TN D-3573 (August, 1966).

4. I. F. MINATT, "DOT, A Two-Dimensional Discrete Ordinates Transport Code," K-1694, Union Carbide Corp. (1967).

E. B. CARISON, W. J. WORLION, W. GRUBER, and M. SHAPIRO, "DTF Users Manual," Phys/Math-3321, United Nuclear Corp. (November, 1963).

6. B. CARLSON, C. LEE, and J. WORLTON, "The DSN and TDC Neutron Transport Codes," IAMS-2346, Los Alamos Scientific Lab. (October, 1959).

7. J. H. ALEXANDER, G. W. HINMAN, and J. P. TRIPLETT, "GAPLSN - A Modified DSN Program for the Solution of the One-Dimensional Anisotropic Transport Equation," GA-4972, General Dynamics Corp. (March, 1964).

8. G. D. JOANOU and J. S. DUDEK, "GAM-II. A B B Code for the Calculation of Fast-Neutron Spectra and Associated Multigroup Constants," GA-4265, General Dynamics Corp. (September, 1963).

9. G. D. JOANOU, C. V. SMITH, and H. A. VIEWEG, "GATHER-II - An IBM-7090 Fortran-II Program for the Computation of Thermal-Neutron Spectra and Associated Multigroup Cross Sections," GA-4132, General Dynamics Corp. (July, 1963).

10. B. G. CARLSON and G. I. BELI, "Solution of the Transport Equation by the $S_{n}$ Method," Proceedings of the Second International Conference on the Peaceful Uses of Atomic Energy, Vol. 16, United Nations, 1958, p. 535. 
A TWO-DIMENSIONAL TRANSPORT THEORY PROGRAM IN A MODULAR SYSTEM ENVIRONMENT

Jack P. Friedman and Bruce W. Crawford

$$
\text { April 21, } 1969
$$

General Electric Company KNOLLS ATOMIC POWER LABORATORY 


\author{
A TWO-DIMENSTONAL TRANSPORT THEORY \\ PROGRAM IN A MODULAR SYSTEM ENVIRONMENT
}

\title{
INTRODUCT ION
}

IT is a general purpose two-dimensional, multigroup, multiregion, reactor physics code capable of solving several approximations of the neutron transport equation. A modular system environment for IT's development has contributed greatly to the incorporation of a large variety of approximations for rectangular, hexagonal, and azimuthally-symmetric geometrical configurations in both the energy and spatial domain. Complementary to its use for obtaining accurate transport theory scalar flux solutions, IT is a research tool for examining the accuracy of various approximations of the transport equations.

\section{MODULAR SYSTEM CONCEPTS}

A modular system contains an accumulated bank of computer programs, called "modules", which may be tapped for automated execution in arbitrary sequences. Data files serve as the basic communication link between modules. Modules should be as independent of one another as possible in order to maximize the number of ways in which modules may be combined for specific applications. The following basic advantages of a modular system have been exploited by the IT code:

1. Available central memory space has been expanded considerably since modules do not coexist in central memory simultaneously. As a consequence, problems having the order of $10^{4}$ mesh points, 200 regions, and 60 energy groups can be solved by IT in tractable amounts of computer time.

2. Programing effort for IT has been considerably reduced by the utilization of previously existing modules whenever possible.

3. Considerable flexibility in capabilities has resulted from the synthesis of a large variety of module execution sequences.

4. Generalization (and alteration) of IT has been implemented with relative simplicity by modifying module execution sequences, modifying specific modules, adding new modules to the system, and generating/utilizing new data files as required.

5. Debugging has been simplified via localization of errors to specific modules.

There are two essential software components of a modular system: a module execution monitor and a data manipulation language. The execution monitor used by IT analyzes a "path" which is a string of the form: $R_{1}, R_{2}, \ldots R_{n}$ supplemented by a test parameter $T . R_{m}(m=1,2, \ldots)$ stands for 1) a list of module names, 2) a set of control variables, or 3) a combination of module names and control variables. $T$ has the value of 0 or $\neq 0$. Three 
types of control variables are included: unconditional branching from one portion of the path to another (e.g., branching from $R_{m}$ to $R_{m+5}$ ); conditional branching from one portion of the path to another (e.g., branching from $R_{m}$ to $R_{m+3}$ if $T \neq 0$ as opposed to executing $R_{m+1}$ after $R_{m}$ if $T=0$ ); iterative looping within a path subset (e.g., executing $R_{m}, R_{m+1}, \ldots R_{m+4}$ eighty times). IT takes advantage of the flexibility afforded by the preceding logic by dynamically generating its path in accordance with the requirements of specific IT applications. Note that the execution monitor is implemented in less than 100 lines of FORTRAN coding.

A data manipulation language for generating, accessing, and altering data files is essential for communication between modules. Such a language should be both efficient and easy to use, a combination which is difficult to achieve in practice and which requires considerable coding effort. To be efficient, the coding effort must take every advantage of its background hardware configuration and will therefore vary from installation to installation. Once a good language is developed at a particular installation, however, it can become a basic component of that installation's modular system, thereby servicing the data manipulation requirements of the participating modules.

\section{FORMAL BACKGROUND}

IT's program capabilities result primarily from its modularized implementation of two basic concepts: (1) a decomposition of multigroup (or few group) problems into a cyclical sequence of one-group calculations and (2) the use of an iterative series method for solving each such one-group problem.

For a problem having $G$ energy groups, identified by group numbers $g=1,2, \ldots, g$ in order of decreasing energy, the group-g transport equation for the scalar flux $\Phi^{g}$ is

$$
\Phi^{\mathrm{g}}(\underline{\mathrm{r}})=\mathrm{T}\left[\mathrm{C}^{\mathrm{g}} \Phi^{\mathrm{g}}+\mathrm{S}^{\mathrm{g}} / \Sigma_{\mathrm{T}}^{\mathrm{g}}\right]
$$

where $T$ is the two-dimensional transport theory operator

$$
\mathrm{T}=(2 \pi)^{-1} \int_{0}^{2 \pi} \mathrm{d} \phi \int_{0}^{s_{\mathrm{b}}} \mathrm{ds} \mathrm{Ki}_{1}(\mathrm{~s})
$$

and $C^{g}$ and $\Sigma_{T}^{\mathcal{E}}$ are the scattering/total and total cross sections respectively. The angular variahle $\phi$ defines rays emanating from $\underline{r}$ and $s$ is optical distance measured from $\underline{r}$ in the $\phi$-direction. The quantity in square brackets appearing in (1) is evaluated in the direction at an optical distance $s$ from $\underline{r}$. The s-integration ends at a physical boundary, denoted by $s_{b}$, or at a specified maximum optical distance.

$\Phi^{g}$ is coupled to scalar fluxes of other energy groups via the sourca tern. $S^{\mathscr{E}}$ which is a linear combination of group $\Phi^{g^{\prime}}\left(g^{\prime}=1,2, \ldots, G\right)$, resulting from isotropic scattering of neutrons from groups $g^{\prime}$ to group $g$ and contributions of fission neutrons originating from group $g^{\prime}$ and re-entering in group $g_{0}$. The Elssion component of $s^{g}$ may be replaced by a flux-independent source $S_{0}^{g}$ in which case an eigenvalue problem is replaced by a "fixed source" problem. This coupling between group fluxes is implemented in the form of source-updating procedures outside of the modular sequence for solving group $g$. Hence, the algorithr for solving (1) for group $g$ treats $s^{g}$ as a flux-independent quantity. 
Equation (1) is solved successively starting with group 1 and ending with group G. After the group-g equation is solved, scattering source contributions from group $g$ to other groups $g^{\prime} \neq g$ are updated. Fission sources (for eigenvalue problems) are updated after the solution to group $G$ is obtained. Aside from the special case of a fixed source problem with no upscattering, it is necessary to repeat the cycle through groups $1,2, \ldots, 6$ several times until s converges. Each such cycle from group 1 through group $G$ is called an "outer cycle".

The iterative series method calculates $\Phi^{g}$ as a convergent series of solutions to easier-to-solve problems: 1

$$
\Phi^{g}=\phi_{01}^{g}+\left(s_{02}^{g}+\phi_{02}^{g}\right)+\left(s_{03}^{g}+\phi_{03}^{g}\right)+\ldots
$$

where ${ }_{0 \mathrm{~m}}^{\mathrm{g}}(\mathrm{m}=1,2, \ldots)$ satisfy diffusion-type differential equations and $s_{0 m}^{g}(m=2,3, \ldots)$ are two-fold quadrature evaluations. Specifically,

$$
\begin{aligned}
& { }_{D}^{D}{ }_{01}^{g}=c^{g}{ }_{01}^{g}+s^{g} / \Sigma_{T}^{g} \\
& s_{02}^{g}=T\left(c^{g}{ }_{01}^{g}+s^{g} / \Sigma_{T}^{g}\right)-\phi_{01}^{g} \\
& D \phi_{0 m}^{g}=C^{g}\left(\phi_{0 m}^{g}+s_{0 m}^{g}\right) ; m=2,3, \ldots \\
& s_{0 m}^{g}=T\left(c^{g_{\phi} g}{ }_{0, m-1}+c^{g} S_{0, m-1}^{g}\right)-\phi_{0, m-1}^{g} ; m=3,4, \ldots
\end{aligned}
$$

Note that $\phi_{01}^{g}$ satisfies (1) under the symbolic replacement of $\mathrm{T}$ by the diffusion theory operator $\mathrm{D}^{-1}$ and its inversion to differential form.

Each successively parenthesized sum in (3) represents an additional order of accuracy. $\Phi^{g}=\phi_{01}^{g}$, the zeroth order solution of (1), represents diffusion theory. An Nth order truncation of the series ends with the $\phi_{0, N+1}^{g}$ term and contains a total of $2 \mathrm{~N}+1$ terms: $\mathrm{N}+1$ diffusion equation solutions and $N$ quadrature evaluations.

The iterative series method may be thought of as an accelerated Neumann series solution technique. If the diffusion theory terms in the iterative series are set equal to zero, the solution reduces identically to the Neumann series solution. It is also possible to interpret the diffusion theory terms as playing a role analogous to "importance sampling" in Monte Carlo solution methods.

Experience indicates that the iterative series method is often rapidly convergent, yielding accuracy to within several percent for an $\mathrm{N}=1$ truncation for a wide variety of problems. Pointwise-independent quadrature evaluations permit very accurate transport theory solutions along user-specified profile lines without requiring comparable accuracy elsewhere in the problem. It is also possible to choose a "fine mesh" in $\underline{r}$ for the $\phi_{01}^{g}$ calculation and a subset of this (known as a "coarse mesh") for the other terms (i.e., $s_{02}^{g}, \phi_{02}^{g}$, etc.). These features have the effect of considerable computational efficiency. 
Stochastic methods, applied to the -integrations for the $\mathrm{s}_{0 \mathrm{~m}}^{\mathrm{g}}$ evaluations, eliminate the spatial flux peaking of "ray effects" which are characteristic of discrete ordinates $S_{n}$ solutions for certain types of problems. ${ }^{2}$ Directional

biasing (which can cause ray effects) is avoided in IT by picking a random set of $\phi^{\prime} s$ independently at each point $\underline{x}$ where $S_{0 m}(\underline{r})$ is evaluated. A stochastic treatment of the variable $s$ eliminates additional numerical bias.

\section{IMPLEMENTATION}

A flow diagram for IT is shown in Figure 1. Note the following:

A. The module packages for initial processing of boundary conditions, geometry, and cross sections are independent of one another and couple only loosely with other modules used by IT. Capabilities in any of these areas may, therefore, be expanded by confining programing effort to highly localized portions of IT.

B. All of the indicated module packages, with the exception of the $\mathrm{s}_{0 \mathrm{~m}}^{\mathrm{g}}$ calculations and interface modules (omitted for simplicity), are basic components of the servicing modular system which existed prior to the IT programming effort. Most of the IT progranming has therefore been concerned with the interface modules. This illustrates the enormous potential of programing utilization which a well-organized modular system can provide.

C. Four basic solution methods are available to IT:

1. Diffusion theory results if the iterative series is truncated at zeroth order (i.e., $\Phi^{g}=\phi_{01}^{g}$ ). The modules which calculate $\mathrm{s}_{02}^{\mathrm{g}}$, $\phi_{02}^{\mathrm{g}}$, etc., are thus bypassed.

2. Simplified $P_{n}$ (for odd positive integers $n$ ) solutions satisfy $(n+1) / 2$ coupled diffusion-type differential equations per energy group and may therefore be obtained via IT under the diffusion theory option for $G(n+1) / 2$ groups. Simplified $P_{n}$ theory requires that a certain linear transformation of the cross sections be performed. This is convenientiy accomplished in the "CROSS SECTIONS" initial processing package.

3. Nth order iterative series transport theory is obtained by truncating beyond the $2 \mathrm{~N}+1^{\text {th }}$ term in (3) as discussed previously.

4. An Nith order Neumann series solution to (1) obtained by replacing the diffusion theory module by one which sets the ${ }^{\circ} \mathrm{Om}_{\mathrm{m}}\left(\mathrm{m}_{\mathrm{l}}=1,2, \ldots\right)$ terms identically to zero.

D. Since inoaule execution sequences need not be the same for each energy group, if ids the capability of utilizing group-dependent solution methods. One may elcct, for example, to solve groups $1,2, \ldots, J$ with diffusion theory and groups $. T+1, J+2, \ldots, G$ with any $N$ th order iterative series transport theory. Many group-dependent combinations of the four basic solution methods are

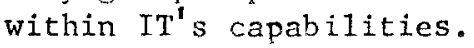

E. Group depentent diffusion theory modules may be utilized since the automated replzienent of one module by another is trivial to accomplish. This can be especiaily valuable when the efficiency of various diffusion theory solvers is sensitive to group-dependent variables (such as optical spacing between mesh points). 


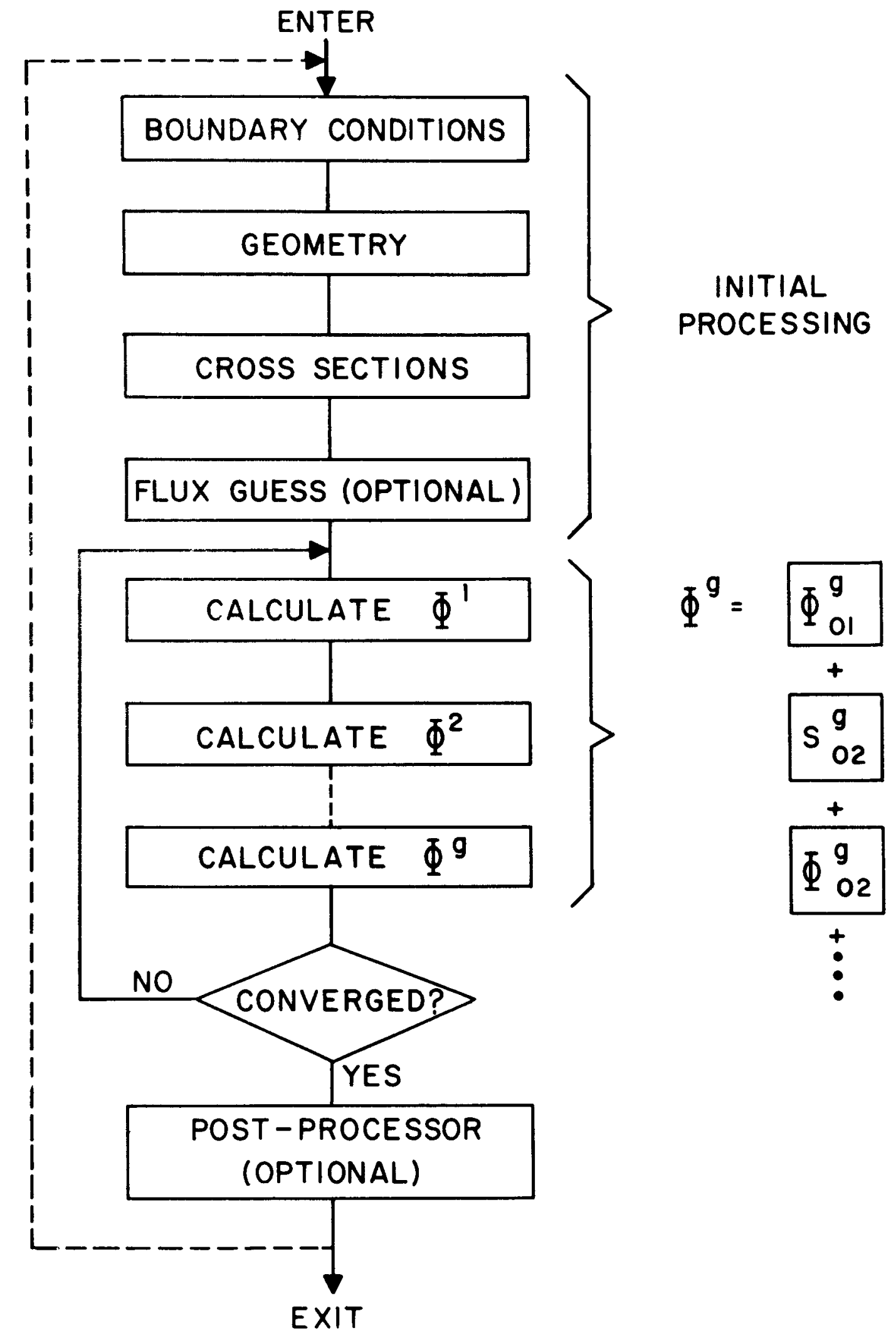

Figure 1. Simplified description of IT. Each box represents a module or sequence of modules. The dashed line indicates the possibility of iterative looping through IT. 
F. Since the servicing modular system need supply on $1 y$ one-group diffusion theory modules, any necessity of having a variety of multigroup (or few-group) diffusion theory programs designed for specific applications is eliminated. This simplification in accessory software requirements prevents restrictive constraints on IT's capabilities.

G. The "POST-PROCESSOR" box in Figure 1 may be used for inserting modules which do auxiliary calculations such as the contour plotting of the group fluxes. An alternative usage is for interfacing successive IT calculations as is implied by the dashed line. For example,

1. Multigroup, diffusion theory spectra may be obtained via IT. Such spectra may be utilized for cross section generation in a second pass through IT for doing a few-group Nth order transport theory calculation.

2. Few-group $\mathrm{N}^{\text {th }}$ order transport theory solutions may be obtained with the accompaniment of self-consistent multigroup diffusion theory spectra. This is done by iterative repetition of the previous procedure (1.) such that the source to each multigroup (diffusion theory) problem is derived from the power distribution of the preceding few group (transport theory) problem. The procedure is terminated when the power distribution converges.

H. The outer cycle looping procedure may be accelerated via the "FLUX GUESS" package in Figure 1. An automated procedure for obtaining a flux guess is to include within this package a module which does a multigroup (or few group) diffusion theory calculation. Another option is to input a flux guess directly.

I. Other features available to IT may be found in Appendix A.

\section{CONCLUSION}

Multi-method features, as well as other major capabilities result from the integration of the iterative series approach with the modular system framework. Since the code is effectively open-ended with respect to both solution methods and procedural synthesis, one can easily design his use of IT to satisfy his needs. For the same reasons, IT can be extremely valuable as a research tool for studying the validity of various solution methods and procedures in given application areas.

APPENDIX A

Specifications for IT

A. Problem Types:

1) Fixed Source

2) Eigenvalue

B. Solution Methods:

1) Diffusion theory

2) Simplified $P_{n}$

3) Iterative series transport theory

4) Neumann series transport theory

5) Mixture of preceding methods

C. Energy Treatments:

1) Multigroup

2) Few group 
D. Spectra for Few Group Cross Sections:

1) Infinite medium

2) Multigroup diffusion theory

3) Simplified $P_{n}$

E. Neutron Scattering

1) Energy dependence - downscattering and upscattering between all energy groups

2) Angular dependence

a) Between groups - isotropic

b) Within groups - isotropic plus forward component

F. Geometries (two-dimensiona1):

1) Rectangular

2) Hexagona1

3) Azimuthally - symmetric*

G. Boundary Conditions (external and interna1*):

1) Vacuum ${ }^{* *}$

2) Reflecting

3) Periodic

4) Current/flux ratios for each energy group*

5) Constants $a^{g}, b^{g}, c^{g}$ satisfying $a^{g} \Phi^{g}+b^{g} D^{g} \nabla \Phi^{g}=c^{g}$ for each energy group $\mathrm{g} . *$

H. Maximum Size Problem:

1) No. of regions $\leqslant 3000$ for diffusion theory/simplified $P_{n}$

$\leqslant 500$ for transport theory

2) No. of mesh lines in each coordinate direction $\leqslant 3000$

3) No. of energy groups $\leqslant 600$

4) No. of regions $x$ no. of groups $\leqslant 12000$

I. Edits:

1) Region - integrated fluxes (and other quantities)

2) Pointwise group fluxes

3) Pointwise and region-integrated contributions from each term of the iterative series

4) Group-summed flux edits (pointwise and region-integrated)

5) Power edits (pointwise and region integrated)

6) Picture of problem

a) mesh-oriented picture

b) dimension-oriented picture

7) Perspective and contour plots of group fluxes

8) Edit-delete options

\footnotetext{
$*_{\text {Not }}$ available in transport theory

${ }^{* *}$ Not available in simplified $P_{n}$
} 
J. Miscellaneous Options:

1) Group-dependent solution methods

2) Group-dependent diffusion theory modules

3) "FLUX GUESS" package (see Figure 1 and text) for accelerating outer cycle convergence rate

4) Processing of energy groups in reverse order for adjoint-flux calculations

5) Two spatial meshes:

a) Fine mesh for zeroth-order (diffusion theory) term in iterative series

b) Coarse mesh (subset of fine mesh) for higher order (transport theory) terms in iterative series

6) Especially-accurate flux calculations along user-selected mesh lines

7) Flux renormalization to assure neutron conservation in cell problems

8) Mechanism for interrupting an IT calculation at an intermediate stage and restarting the problem at a later time

K. Program Arrangement:

Exists in a modular system environment and exploits modular system features to: (1) utilize central memory space efficiently; (2) achieve general capabilities; (3) simplify programming, debugging, and generalizing of the code.

\section{REFERENCES}

1. B. W. Crawford and J. P. Friedman, "MULTIGROUP TWO-DIMENSIONAL NEUTRON TRANSPORT", Trans. Am. Nucl. Soc. 10, 1, 172 (1967).

2. K. D. Lathrop, "RAY EFFECTS IN DISCRETE ORDINATES EQUATIONS", Nuc1. Sci. Eng., 32, 357-369 (1968). 
OPTIMIZATION OF THE DOPPLER EFFECT IN A FAST MATERIAL TEST REACTOR BY PROGRAMMING TECHNIQUES

A. Gandini (CNEN), M. Salvatores (CNEN) and G. Sena (CNEN).

ABSTRACT.- A method is described that adopts the generalized perturbation theory and programming techniques for optimizing reactor integral parameters, linear or bilinear with the real and adjoint neutron densities, after assumtion of given constraints. An application of these methods to the optimization of the berillium distribution with respect to the Doppler effect in a fast ceramic test reactor is shown.

1. - IIJTRODUCTION

The availability of fast computers of increasingly higher performance on one side and on the other the development of generalized perturbation methods of first and higher order, suggests an extensive use of programming techniques for power reactor design.

The specific problem considered deals with the need of ensuring an intrinsic safety mechanism in a 500 litre ceramic fast reactor fuelled with $U-235$ enriched uranium and cooled with sodium. For this purpose it is deemed convenient to find the optimal distribution of a given quantity of berillium oxide in the four subregions into which the driver core has been subdivided, so that the Doppler reactivity effect beoomes enhanced. This is consequent to the increase of the neutron flux at low energies where the U-238 capture resonances are present. Therefore, a prompt negative feedback is ensured in case of an accidental excursion leading to a temperature increase. In the present case a temperature increase $\Delta \mathrm{T}$ of $1200^{\circ} \mathrm{C}$, starting from operating conditions, has been assumed.

By means of the method developed for this specific purpose it is possible to calculate this berillium distribution, certain limiting conditions, or constraints, being given. Once these constraints and the quantity to be optimized are set, the basic procedure consists in the parametrization of the problem in terms of linear and/or quadratic (or higher order) expressions. This is appropriately allowed for by adopting the generalized perturbation techniques (see Appendix), relating changes of the reactor nuclear parameters to changes of functionals of the real and/or adjoint neutron densities, such as the Doppler reactivity effect. 


\section{2. - DESCRIPTION AND SPECIFICATIONS OF THE SYSTEM}

As has been said above, the optimization study was carried out on a 500 litre ceramic fast reactor fuelled with enriched uranium oxide and cooled with sodium. Its core, formed by test and driver regions, is a cylinder with a height/diameter ratio of 1.I. The inner and outer radia of the four subregions of the driver core, which surrounds the test region, and those of the outer reflector and shield, are shown in TABLE 1 together with the regional compositions of the reference system. In order to enhance the Doppler effect, an amount of berillium oxide corresponding to $5 \%$ of the volume has been evenly distributed in the

\section{TABLE 1 - DIMENSIONS AND COMPOSITIONS}

\begin{tabular}{|c|c|c|c|c|c|c|c|}
\hline Region & Subregion & $\begin{array}{l}\text { Outer } \\
\text { Radius } \\
\text { (cm) }\end{array}$ & $\mathcal{F}-\mathrm{Na}$ & $V_{-S S}$ & $\mathcal{F}-\mathrm{UO}_{2}$ & $\mathrm{~F}-\mathrm{PuO}_{2}$ & $\sqrt{F}-\mathrm{BeO}$ \\
\hline Test & $\begin{array}{l}\text { Diluent } \\
\text { Test fuel } \\
\text { Diluent }\end{array}$ & $\begin{array}{r}5.72 \\
9.53 \\
15.25\end{array}$ & $\begin{array}{l}0.60 \\
0.596 \\
0.60\end{array}$ & $\begin{array}{l}0.40 \\
0.125 \\
0.40\end{array}$ & $0 . \overline{2} 37$ & 0.042 & - \\
\hline Driver & $\begin{array}{l}\text { Subr. } \frac{1}{2} \\
\text { Subr. } \\
\text { Subr. } 3 \\
\text { Subr. } 4\end{array}$ & $\begin{array}{l}21.73 \\
28.21 \\
34.70 \\
41.18\end{array}$ & $\begin{array}{l}0.423 \\
0.423 \\
0.423 \\
0.423\end{array}$ & $\begin{array}{l}0.198 \\
0.198 \\
0.198 \\
0.198\end{array}$ & $\begin{array}{l}0.329 \\
0.329 \\
0.329 \\
0.329\end{array}$ & - & $\begin{array}{l}0.05 \\
0.05 \\
0.05 \\
0.05\end{array}$ \\
\hline $\begin{array}{l}\text { Control } \\
\text { (rods out }\end{array}$ & - & 43.09 & 0.75 & 0.25 & - & - & - \\
\hline & & & & & & & $\sqrt{F-N i}$ \\
\hline Reflect. & - & 82.75 & 0.182 & 0.25 & - & - & 0.568 \\
\hline & & & & & & & $\sqrt{ }-B_{4} C$ \\
\hline Shield & - & 98.85 & 0.182 & 0.152 & - & - & 0.666 \\
\hline
\end{tabular}

driver. The optimization consists in redistributing this quantity or in distributing assumed larger amounts of berillium, so that the negative Doppler effect reaches a maximum in absolute value. These distributions must satisfy a number of constraints derived from physical and technological specifications:

1 - the sum of the berillium oxide volume fraction and that of the fuel in each subregion into which the core is subdivided must be kept constant;

2 - the reactor must be maintained critical by correspondingly changing its U-235 enrichment which must be the same in all subregions;

3 - the power of the test region is fixed;

4 - the power in each fuel pin must not be higher than a safety limit;

5 - the composition of the test region is fixed;

6 - the neutron spectrum in the test region must satisfy a given hardness condition. 


\section{3. - THZ OFTIEIZATION PROBITM}

The model assumed for the calculations is represented by a fast driver core subdivided into $N$ subregions (four, in our case) corresponding to the rows of the fuel subassemblies. Then we assime that in each driver subregion $\mathrm{n}$, the material replacements are relevant to $\mathrm{M}$ materials (in our case: $\mathrm{U}^{23} \mathrm{O}_{2}, \mathrm{U}^{238} \mathrm{O}_{2}, \mathrm{BeO}$ ).

Let us now represent the Doppler reactivity effect relative to a core temperature change $\Delta \mathrm{T}$ by the functional (using a multienergy group vector notation):

$$
\rho_{D E}=\frac{\int_{\text {Syst }} \Phi^{\Phi^{*} \Delta \rho \Phi d \underline{r}}}{\int_{\text {Syst }} \Phi^{*} \mathcal{F}_{\Phi} d \underline{r}}
$$

where $\Delta \rho$ represents the perturbation of the Boltzman operator $\rho$ related to the temperature change $\Delta T$ and $\mathcal{F}$ represents the fission source matrix. The vector functions $\Phi$ and $\Phi^{*}$ represent the mutigroup real and adjoint neutron fluxes.

The optimization of $\rho_{\mathrm{DE}}$ corresponds to the maximization of the fractional quantity:

$$
\delta_{S_{D E} / \rho_{D E}}=\sum_{n=1}^{N} \sum_{m=1}^{N} a_{n, m} x_{n, m}
$$

where $x_{n, m}$ represents (in weight) the amount of material $m$ introduced ninto or subtracted from region $\mathrm{n}$. The coefficients $a_{n}$ are sensitivity coefficients calculable with generalized perturibation formulae. The coefficients $a_{n, m}$ relative to the materials involved in the operator $\Delta \rho$ of $\mathrm{Eq}$. (I) are the sum of two contributions: one contribution is related to an indirect effect (spectral effect), the other one is related to a direct effect (material density change).

The constraints mentioned in the previous section may be translated into parametric relations as shown below.

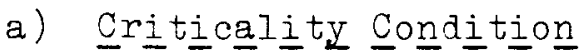

It is necessary that the criticality of the system is not affected by the optimization. We then consider the ratio:

$$
\rho_{\text {Mat }}=\frac{\int_{\text {syst }}{\underline{\phi^{*}}}^{*} \phi d \underline{r}}{\int_{\text {syst }} \phi^{*} \mathcal{F}_{\phi d \underline{r}}}
$$

where $\beta$ is the Boltzmann operator related to the material distribution in the reactor. Recalling that $\rho \Phi=0$ for a critical system, $\rho_{\mathrm{Mat}}=0$. The criticality condition becomes: 


$$
\delta_{\text {Mat }}=\sum_{n=1}^{N} \sum_{m=1}^{N} b_{n, m} x_{n, m}
$$

where the coefficients $b_{n}, m$ may be calculated using the conventional perturbation techniques.

b) Constant_Enrichment Condition

The enrichment must be constant in all the driver subregions. If $m_{1}$ is the fissile.material index and $m_{2}$ that of the fertile one, this condition may be represented by the quadratic equations:

$$
\frac{\bar{x}_{N, m_{1}}+x_{N, m_{1}}}{\bar{x}_{N, m_{2}}+x_{N, m_{2}}}=\frac{\bar{x}_{n, m_{1}}+x_{n, m_{1}}}{\bar{x}_{n, m_{2}}+x_{n, m_{2}}} \quad[n=1,2, \ldots,(N-1)]
$$

where $\bar{x}_{n, m}$ represents the amount of material $m$ in region $n$ before the optimization.

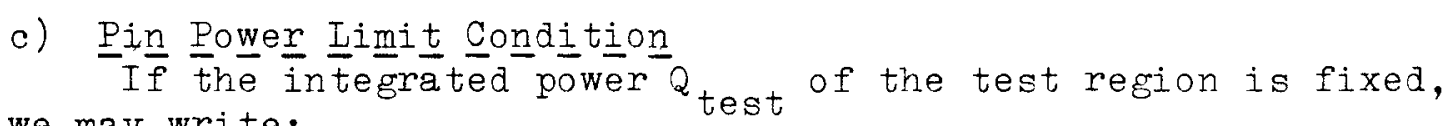
we may write:

$$
Q_{\text {test }}=q \int_{\text {test }} \underline{\Phi}^{\prime} \underline{\Sigma}_{f}^{\text {test }} d \underline{r}=K_{\text {test }}
$$

where $K_{\text {test }}$ is a given positive constant, $q$ represents the energy per fission, and $\Phi^{\prime}$ the neutron flux perturbed by the material redistribution due to the optimization process. On the other hand, in each subregion $n$ the power per fuel pin $Q_{n}$ must be Iimited for safety reasons. Therefore, it must be:

$$
\begin{aligned}
& Q_{n}=q \sum_{i=1}^{2} \int_{(n)} \Phi^{\prime} \gamma_{n}\left(\bar{x}_{n, m_{i}}+x_{n, m_{i}}\right) \underline{\sigma}_{f}^{m_{i}} d \underline{r} . \\
& =\frac{\bar{x}_{n, m_{1}}+\bar{x}_{n, m_{2}}}{\alpha_{\mathrm{n}}\left[\left(\bar{x}_{n, m_{1}}+x_{n, m_{1}}\right)+\left(\bar{x}_{n, m_{2}}+x_{n, m_{2}}{ }_{4}\right]\right.} \leqslant k_{0}
\end{aligned}
$$

where $K_{0}$ is another given positive constant, $\gamma_{n}$ is a coefficient which translates the weight $(\bar{x}+x)$ relative to the fissile and fertile materials into volume fractions and $\alpha_{n}$ represents the number of fuel pins in the $n$-th region in the system before the optimization. Therefore, the fractional coefficient in represents the inverse of the number of the fuel pins after the optimization.

Our condition may now be written:

$$
\frac{Q_{n}}{Q_{\text {test }}} \leqslant \frac{K_{0}}{K_{\text {test }}}=\bar{K} \quad(n=1,2, \ldots, N)
$$


Recalling expressions (6) and (7) and that the quantities $\left(\bar{x}_{n, m}+x_{n, m}\right)$ are assumed to be always positive,

condition (8) becomes:

$$
\begin{aligned}
& \sum_{i=1}^{2}\left(\bar{x}_{n, m_{i}}+x_{n, m_{i}}\right) \rho_{n, i}^{\prime}-\frac{\bar{k} \alpha_{n}}{\gamma_{n}} . \\
& \frac{\left(\bar{x}_{n, m_{1}}+x_{n, m_{1}}\right)+\left(\bar{x}_{n, m_{2}}+x_{n, m_{2}}\right)}{\left(\bar{x}_{n, m_{1}}+\bar{x}_{n, m_{2}}\right)} \leqslant 0
\end{aligned}
$$

where

$$
\rho_{n, i}^{\prime}=\frac{\int_{(n) \Phi^{\prime} \underline{\sigma}_{f}^{m_{i}} d \underline{r}}}{\int_{\text {test } \Phi^{\prime} \underline{\sum}_{f}^{\text {test }} d \underline{r}}} \quad(i=1,2)
$$

The difference $\delta \rho_{n, i}$ between the perturbed values $\rho$ ' $n$, i and the unperturbed ones $p n, i$ may also be written in terms of the generalized perturbation techniques. Iimiting the approximation to first order, we may write the following fractional variations:

$$
\frac{\delta p_{n, i}}{\rho_{n, i}}=\sum_{u=1}^{N} \sum_{m=1}^{M} c_{u, m}^{(n, i)} x_{u, m} \quad(i=1,2)
$$

where $c_{u, m}^{(n, i)}$ are calculable coefficients relative only to indirect ( $i^{\mathrm{m}}$ e. spectral) effects on functionals $\rho_{n}, i$. Substituting in (9) $\rho_{n, i}^{\prime}$ with $\rho_{n, i^{+}} \delta \rho_{n, i}$ and recalling expressions (II) for $\delta \rho_{n, i}$, we obtain:

$$
\begin{aligned}
& \sum_{i=1}^{2} \rho_{n, i}\left(\bar{x}_{n, m_{i}}+x_{n, m_{i}}\right)\left(1+\sum_{u=1}^{N} \sum_{m=1}^{M} c_{u, m}^{(n, i)} x_{u, m}\right)- \\
& -\frac{\bar{k} \alpha_{n}}{r_{n}} \frac{\left(\bar{x}_{n, m_{1}}+x_{n, m_{1}}\right)+\left(\bar{x}_{n, m_{2}}+x_{n, m_{2}}\right)}{\left(\bar{x}_{n, m_{1}}+\bar{x}_{n, m_{2}}\right)} \leq 0
\end{aligned}
$$

which represents ${ }_{\mathrm{n}}^{\mathrm{a}}$ quadratic condition. Neglecting the quadratic terms $\left(x_{n, m_{i}} c_{u, m} x_{u, m}\right)$, this condition may be synthetically written:

$$
\sum_{u=1}^{N} \sum_{m=1}^{M} d_{u, m}^{(n)} x_{u, m} \leqslant 0 \quad(n=1,2, \ldots, N)
$$

d) Constant_Fuel and Berillium Volume_Condition

The berilitium replaces equal fuel volume. This is translated into the condition:

$$
\sum_{m=1}^{M} r_{n, m} x_{n, m}=0 \quad(n=1,2, \ldots, N)
$$


where $y_{n, m}$ is a coefficient which reduces $x_{n, m}$ into volume fractions.

e) Fixed Berillium Amount Condition

Once the amount $W$ of berilinm (labelled with index $\mathrm{m}_{3}$ ) to

be distributed into the system is fixed, it must be:

$$
\sum_{n=1}^{N}\left(\bar{x}_{n, m_{3}}+x_{n, m_{3}}\right)=w
$$

f) Spectrum_Haraness Condition

The spectrum of the test region must satisfy a given hardness condition. Having defined the ratio:

$$
\rho_{h}=\frac{\int \operatorname{tes} \bar{t} \int_{1}^{\infty} \phi(E) d E}{\int \operatorname{tes} \bar{t} \int_{0}^{\infty} \phi(E) d E}
$$

the following inequality has been imposed:

$$
\delta \rho_{h} / \rho_{h} \geqslant-0.03
$$

where $\delta \rho_{h}$ represents the change of $\rho_{h}$ consequent to the perturbation of the flux spectrum due to the material redistribution in the optimization process. Employing once more the generalized perturbation techniques, it may be written:

$$
\sum_{n=1}^{N} \sum_{m=1}^{11} e_{n, m} x_{n, m} \geqslant-0.03
$$

where $e_{n, m}$ are calculable coefficients.

The total number of conditions is $3 \mathrm{~N}+2$ represented by $2 \mathrm{~N}+1$ equations and $\mathrm{N}+1$ inequalities. A check to see whether there are solutions can be made by the drastic reduction to one subregion only. We see, as expected, that the equations are reduced to three, allowing only one solution, (three being also the number of the interchangeable materials), if this is permitted by the inequalities.

\section{4. - CALCULATIONAI METHODS ADOPTED}

The calculation of the real and adjoint neutron fluxes and of the importance functions have been performed in infinite cylindrical geometry adopting the multigroup diffusion approximation and the 26 group cross section library by Abagjan et al. Allowance for the axial finiteness has been made by introducing into the calculation a transverse leakage term. Use of the TAIn Code ${ }^{5}$ for the real and adjoint flux calculations has been maje, while the importance functions and the generalized perturbation calculations ${ }^{\text {have }}$ been performed with the CIAP-ID and the GLOBPERT-ID Codes ${ }^{2}$, respectively.

For the solution of the optimization problem two different non-linear programming algorithms have been adopted. The first 
algorithm consists in the iterative application of the simplex method and will be referred to as the MAP method (Method of Approximation Programming). The second algorithm used consists in the so called SLUMT method ${ }^{9}$ (Slacked Unconstrained Minimization Technique). It reduces the problem of minimizing (or maximizing) a function, subject to given constraints, to that of minimizing (or maximizing) an unconstrained function.

\section{5. - DISCUSSION OF THE RESULTS}

The calculations have been performed for various amounts of berillixm oxide to be distributed in the driver, i.e. for different average volume fractions. The results obtained using the independent MAP and SIUMT methods agree up to the fourth significant figure, which proves the reliability of the calculations.

In TABIE 2 the optimal distribution of berillium and fuel materials are shown for each average volume fraction of berillium oxide. To be noticed the tendency of the berillium distribution to spread from inner subregions toward outer ones with increasing average volume fractions of the berillium.

THEIE 2 - CETTAAL BERILIIUM AND FUEL MATERIAL DISTRIBUTIONS (IN TOLLME FRACTIONS) BY THE SLUMT AND MAP METHODS

\begin{tabular}{|c|c|c|c|c|c|c|c|c|c|}
\hline \multirow{2}{*}{$\frac{\dot{y}}{3}$} & \multicolumn{3}{|c|}{$\bar{F}-\mathrm{BeO}=0.05$} & \multicolumn{3}{|c|}{$\overline{\sqrt{F}}-\mathrm{BeO}=0.10$} & \multicolumn{3}{|c|}{$\bar{F}-\mathrm{BeO}=0.15$} \\
\hline & $\mathrm{BeO}$ & $\mathrm{U}^{235} \mathrm{O}_{2}$ & $\mathrm{U}^{238} \mathrm{O}_{2}$ & $\mathrm{BeO}$ & $\mathrm{U}^{235} \mathrm{O}_{2}$ & $\mathrm{U}^{238} \mathrm{O}_{2}$ & $\mathrm{BeO}$ & $\mathrm{U}^{235} \mathrm{O}_{2}$ & $\mathrm{U}^{238} \mathrm{O}_{2}$ \\
\hline 1 & 0.295 & 0.028 & 0.056 & 0.152 & 0.085 & 0.142 & 0.092 & 0.114 & 0.173 \\
\hline 2 & 0.008 & 0.124 & 0.247 & 0.242 & 0.051 & 0.086 & 0.158 & 0.088 & 0.133 \\
\hline 3 & 0. & 0.127 & 0.253 & 0.077 & 0.113 & 0.190 & 0.225 & 0.061 & 0.093 \\
\hline 4 & 0. & 0.127 & 0.253 & 0. & 0.142 & 0.237 & 0.111 & 0.107 & 0.161 \\
\hline
\end{tabular}

Since in the optimization process different volume fraction variations occur in each subregion, the self-shielding factors of the Abagjan library also change, which is not accounted for in the optimization process. In order to check the influence of this effect and in general the validity of the generalized perturbation techniques adopted, direct calculations of the Doppler effect with corrected and uncorrected cross sections have been performed. The results of the optimization procedure and those obtained by direct calculations are shown in TABIE 3 together with the Doppler effect value of the reference case. As may be seen, the agreement between the various sets of data is rather good. The changes of the self-shielding factors in the Abagjan schematization seem to play a minor role. The major deviations seem attributable to the linearization of the generalized perturbation expressions, since, as expected, they tend to increase with increasing amounts of added berillium. 
TABLE 3 - VALUES OF PDE $\left(\Delta \mathrm{K} / \mathrm{KxIO} \mathrm{O}^{3}\right)^{\mathrm{a}}$

\begin{tabular}{|l||c|c|c|}
\hline \multicolumn{1}{|c||}{$\bar{F}-$ Be0 } & $\begin{array}{c}\text { SLUMT } \\
\text { \& MAP }\end{array}$ & $\begin{array}{c}\text { Uncorrected } \\
\sigma^{\prime} \mathrm{s}\end{array}$ & $\begin{array}{c}\text { Corrected } \\
\sigma^{\prime} \mathrm{s}\end{array}$ \\
\hline $0.05(\operatorname{Ref})$. & - & $-3.297(+0.55)$ & $-3.097 \mathrm{~b}$ \\
0.05 & -3.279 & $-3.275(-0.12)$ \\
0.10 & -3.803 & $-3.750(-1.38)$ & $-3.724(-2.08)$ \\
0.15 & -4.043 & $-3.895(-3.65)$ & $-3.855(-4.90)$ \\
\hline
\end{tabular}

a - The figures in parentheses represent the percentage deviations from the SLUMT and MAP results.

b - Doppler effect in absence of Be: $-0.00256 \Delta \mathrm{K} / \mathrm{K}$.

In TABIE 4 power volume density distributions, normalized to an average power density of unity in the test fuel subregion are shown as resulting from the data of the optimization process itself and from direct calculations, with cross sections corrected for self-shielding. Also here the agreement between the two sets of results is rather good, which assesses the reliability of the optimization procedure adopted. As may be seen, the average power density per unit volume, normalized to unity in the test fuel subregion, is reduced in the driver by the optimization of the berillium distribution. This may be due to different factors.

\section{TABLE 4 - POWER VOLUME DENSITY DISTRIBUTIONS}

\begin{tabular}{|c|c|c|c|c|c|c|c|}
\hline $\bar{F}-B e O$ & $0.05(\operatorname{Ref})$. & & & & & & .15 \\
\hline Subreg: & direct & $\begin{array}{l}\text { SLUMT } \\
\& \text { IMAP } \\
\end{array}$ & direct & $\begin{array}{l}\text { SLUMT } \\
\& \text { MAP }\end{array}$ & direct & $\begin{array}{l}\text { SLUMT } \\
\text { \& MAP } \\
\end{array}$ & direct \\
\hline $\begin{array}{l}1 \\
2 \\
3 \\
4\end{array}$ & $\begin{array}{l}0.936 \\
0.855 \\
0.762 \\
0.693\end{array}$ & $\begin{array}{l}0.310 \\
0.896 \\
0.746 \\
0.666\end{array}$ & $\begin{array}{l}0.295 \\
0.899 \\
0.744 \\
0.669\end{array}$ & $\begin{array}{l}0.827 \\
0.500 \\
0.790 \\
0.832\end{array}$ & $\begin{array}{l}0.851 \\
0.482 \\
0.802 \\
0.822\end{array}$ & $\begin{array}{l}1.029 \\
0.798 \\
0.558 \\
0.756\end{array}$ & $\begin{array}{l}1.065 \\
0.812 \\
0.527 \\
0.755\end{array}$ \\
\hline average & 0.825 & - & 0.681 & - & 0.746 & - & 0.755 \\
\hline
\end{tabular}

As far as the 5\% and 10\% vol average berillium distributions are concerned, the resulting configuration appears to be a sort of flux trap in the test region, since most of the berillium is concentrated in the inner subregions of the ariver. As for the $15 \%$ Vol distribution, the decrease of the critical mass, consequent to the leakage reduction, seems to play the major role.

In TABLE 5 the distributions of the U-235 critical masses for the various cases considered are shown. We notice that in the case of 5\% vol average optimal berillium distribution, the critical mass insreases, since its concentration within the inner subregion of the driver, as compared to the even distribution cf the reference case, reduces its benefit on the neutron leakage. Since this tendency reverses for higher berillium average concentrations, the critical masses correspondingly tend to become increasingly reduced. 


\begin{tabular}{|c|c|c|c|c|}
\hline Subreg. & \multicolumn{1}{|c|}{$\begin{array}{c}0.05 \\
(\text { Ref.) }\end{array}$} & 0.05 & 0.10 & 0.15 \\
\hline 1 & 58.41 & 15.75 & 47.33 & 63.86 \\
2 & 78.88 & 93.37 & 38.66 & 66.35 \\
3 & 99.36 & 120.11 & 107.31 & 58.44 \\
4 & 119.84 & 145.10 & 162.71 & 122.50 \\
\hline Eotal & 356.50 & 374.33 & 356.01 & 311.15 \\
\hline
\end{tabular}

In a previous analysis ${ }^{10}$ of the same problem presented in this study, linear programming techniques were adopted which corresponds to the linearization of condition $b$ ) of section 3 . As may be seen from TABLES 6 and 7 , the resulting distributions and consequent parametric changes did not turn out to be substantially different from those obtained here, although the constant enrichment condition is only fairly satisfied. This means that for

TABIE 6 - OPTIMAL BERILIIUM DISTRIBUTION (IN VOLUME FRACTIONS) BY LINEAR PROGRAMMING TECHNIQUES

\begin{tabular}{|c|c|c|c|c|}
\hline Subreg. & 1 & 2 & 3 & 4 \\
\hline$\vec{F}$-BeO & & & & \\
\hline 0.05 & 0.305 & 0.210 & 0. & 0. \\
0.10 & 0.230 & 0.281 & 0.0 & 0. \\
0.15 & 0.061 & 0.219 & 0.328 & 0. \\
\hline
\end{tabular}

TABLE 7 - VALUES OF PED $\left(\triangle \mathrm{K} / \mathrm{KXI0^{3 }}\right)$ AND CRITICAL MASSES RESULTING FROM THE OPTIMIZATION BY IINEAR PROGRAMUING TECHNIQUES

\begin{tabular}{|c|c|c|c|}
\hline$\vec{V}-B e O$ & 0.05 & 0.10 & 0.15 \\
\hline PED & -3.250 & -3.842 & -4.013 \\
\hline $\begin{array}{c}\text { critical } \\
\text { mass(Kg) }\end{array}$ & 370.99 & 357.47 & 314.45 \\
\hline
\end{tabular}

a number of reactor problems inherent to project survey analysis, advantage may be taken of the faster and well established linear programming techniques with a fair degree of confidence.

\section{6. - CONCLUSION}

From the accurate results obtained in this optimization analysis it seems that the generalized perturbation techniques of first and higher order may be effectively used in connection with the most sophisticated programming techniques developed up to now. The confluence of these two different fields of activity should offer the reactor designer a powerful tool for system analysis and optimization in a large number of cases of interest relevant to control, economics, safety. With regard to the improvement of the optimization method proposed in the present 
study, a further investigation of the programming techniques themselves, and of the numerical analysis involved, should be made so that an increasing amount of information available may be consistently exploited in the optimization process.

\section{APPENDIX}

Let us consider a reactor system in the time interval ( $\left.t_{0}, t_{F}\right)$ and let us define at $t_{F}$ a functional $Q$ linear with the neutron density. Using a multigroup vector notation (in diffusion approximation) we may then write:

$$
\left.Q=\int \underline{h}+\underline{r}\right) \underline{n}\left(\underline{r}, t_{F}\right) d \underline{r}
$$

where the integral over $\underline{r}$ is intended over the whole system and $\underline{n}\left(\underline{r}, t_{F}\right)$ represents the neutron density vector which, together with the precursor density vector $\mathrm{R}$, satisfies the integro-differential equation:

$$
\frac{\partial}{\partial t}\left|\begin{array}{l}
\underline{n} \\
\underline{\underline{ }}
\end{array}\right|=M\left|\begin{array}{l}
\underline{\mathrm{n}} \\
\underline{\mathrm{p}}
\end{array}\right|
$$

Mrepresents the Boltzmann integro-differential operator extended also to the precursor density. Having set

$$
\underline{c}=\left|\begin{array}{c}
\underline{\mathrm{n}} \\
\mathrm{g}
\end{array}\right|
$$

if a perturbation $\delta \mathcal{M}$ (and $\delta \underline{h}^{+}$) is introduced into the system, the following generalized perturbation expression may be derived ${ }^{2}$ :

$$
\delta Q=\int \delta \underline{\underline{h}}+\underline{\underline{r}} \underline{\underline{\underline{r}}}\left(\underline{\underline{r}}, t_{F}\right) d \underline{\underline{r}}+\int_{t_{0}}^{t_{F}} d t \int \underline{\underline{c}}^{*} \delta \mu \underline{\underline{c}^{\prime}} d \underline{\underline{r}}
$$

where $\underline{c}^{\prime}$ represents the perturbed neutron and precursor density and where $a^{\text {is }}$ the importance function satisfying the adjoint differentiāl equation:

$$
-\frac{\partial c^{*}}{\partial t}=\mu^{*} \underline{c}^{*}
$$

and the "ifinaI" condition at $t_{F}$ :

$$
\begin{gathered}
\underline{\underline{C}}^{*}\left(\underline{\underline{r}}, t_{F}\right)=\left|\underline{\underline{h}}_{0}^{+}(\underline{\underline{r}})\right| \\
\text { If a functional } Q^{*} \text { Iinear with the importance function is de- } \\
\text { fined at } t_{0}, \text { as given by the expression: } \\
\text { the following perturbation expression may also be written: }
\end{gathered}
$$

$$
6 \underline{Q}^{*}=\int \underline{\underline{n}}^{*}\left(\underline{\underline{r}}, t_{0}\right) \delta \underline{\underline{h}}\left(\underline{\underline{r})} d \underline{\underline{r}}+\int_{t_{0}}^{t_{F}} d t \underline{c}^{*} \delta \mathcal{M} \underline{\underline{c}} \underline{\underline{r}}\right.
$$

where $\underline{e}^{\prime}$ is the perturbed importance density function. Now let us suppose that the system is critical and that 
$t_{0} \ll t_{F}$. If ratios $R$ and $R^{*}$ are considered as given by the expressions:

$$
R=\frac{Q_{1}}{Q_{2}}=\frac{\int \underline{h}_{1}^{+}(\underline{r}) \underline{n}(\underline{r}) d \underline{r}}{\int \underline{h}_{2}^{+}(\underline{r}) \underline{n}(\underline{r}) d \underline{r}} \quad R^{*}=\frac{Q_{1}^{*}}{Q_{2}^{*}}=\frac{\int \underline{n}^{*}(\underline{r}) \underline{h}_{1}(\underline{r}) d \underline{r}}{\int \underline{\underline{n}}(\underline{r}) \underline{h}_{2}(\underline{r}) d \underline{r}}
$$

where, since $t_{0}<t_{F}, \underline{n}(\underline{r})$ and $\underline{n}^{*}(\underline{r})$ represent, respectively, the real and adjoint neutron densities of the fundamental mode, the following perturbation expressions are derived from Eqs. (IA) and (2A) (supposing all neutrons are prompt and that the criticality is not affected):

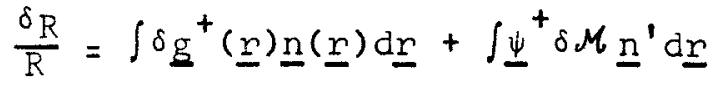

$$
\begin{aligned}
& \left.\frac{\delta R^{*}}{R^{*}}=\int \underline{\underline{n}} * \underline{r}\right) \delta \underline{g}(\underline{r}) d \underline{\underline{r}}+\int \underline{\underline{n}} * \delta \mu \underline{\mu d \underline{r}}
\end{aligned}
$$

where $\Psi^{*}$ and $\Psi$ satisfy the differential equations:

$$
\begin{aligned}
& -\mu^{*} \underline{\Psi}^{+}=\underline{g}^{+} \\
& -\mu \Psi=\underline{g}
\end{aligned}
$$

and where:

$$
\begin{aligned}
& \underline{\mathrm{g}}^{+}=\frac{\underline{h}_{1}^{+}}{\mathrm{Q}_{1}}-\frac{\mathrm{h}_{2}}{\mathrm{Q}_{2}} \\
& \underline{\underline{g}}=\frac{\frac{h_{1}}{Q_{I}^{*}}}{\mathrm{Q}_{1}}-\frac{\underline{h}_{2}}{\mathrm{Q}_{2}^{*}}
\end{aligned}
$$

Each of Eqs. ( $5 \mathrm{~A}$ ) and ( $6 \mathrm{~A}$ ) may be solved by iterative methods.

It may be shown ${ }^{3}$ that the right hand side of $\mathrm{Eq}$. (3A) may be substituted by the expansion:

$$
\frac{\delta R}{R}=\int \delta \underline{g}^{+}(\underline{r}) \underline{n}(\underline{r}) d \underline{r}+\sum_{i=1}^{\infty} \int \Psi(i)(\delta \mu)^{i} \underline{n} d \underline{r}
$$

where functions $\Psi_{(i)}^{\#}$ satisfy the recurrent system:

$$
\left\{\begin{array}{l}
-\mu^{*} \underline{\Psi}_{(1)}^{+}=\underline{\mathrm{g}}^{+} \\
-\mu^{*} \underline{\Psi}_{(i)}^{+}=\underline{\Psi}_{(i-1)}^{+} \quad(i=2,3, \ldots \ldots)
\end{array}\right.
$$

and are shown to vanish for $i \rightarrow \infty$.

In a similar way we may derive an expansion of the right hand side of $\mathrm{Eq}$. (4A):

$$
\frac{\delta \mathrm{R}^{*}}{\mathrm{R}^{*}}=\int \underline{\mathrm{n}}^{*}(\underline{r}) \delta \underline{g}(\underline{r}) \mathrm{d} \underline{\underline{r}}+\sum_{i=1}^{\infty} \int_{\underline{n}}^{*}(\delta \mathcal{M})^{i} \underline{\Psi}(i) \underline{\mathrm{r}}
$$


where functions $\underline{\psi}(i)$ satisfy the recurrent system:

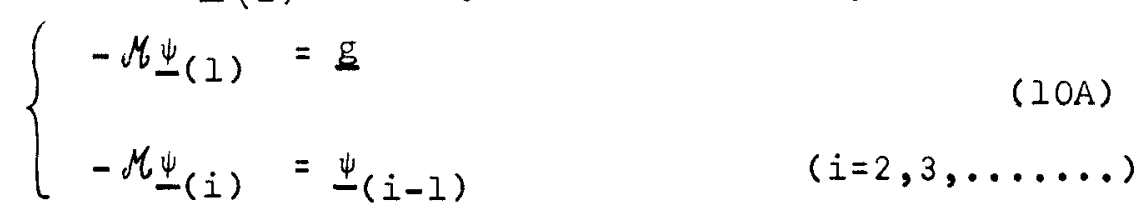

and are also shown to vanish for $i \rightarrow \infty$.

In case of a ratio $\rho$ bil of two quantities $T_{1}$ and $T_{2}$, bilinear with the real and adjoint densities:

$$
\rho_{\text {bil }}=\frac{T_{1}}{T_{2}}=\frac{\int \underline{\underline{n}} * \epsilon_{1} \underline{n d \underline{n}}}{\int \underline{n}^{*}+\epsilon_{2} \underline{n d \underline{n}}}
$$

the following perturbation expression is derived:

where

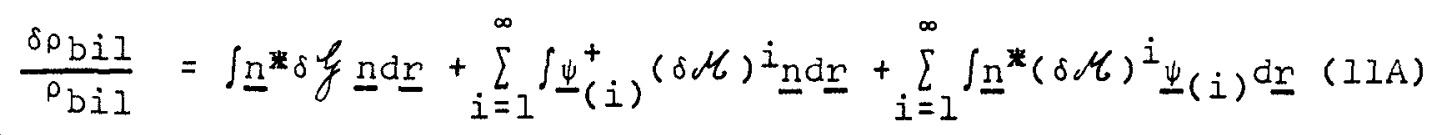

$$
y=\frac{t_{1}}{T_{1}}-\frac{t_{2}}{T_{2}}
$$

and where functions $\Psi_{(i)}^{\#}$ and $\Psi_{(i)}$ satisfy systems (8A) (IOA) with the adjoint and real sources $g^{*}$ and $g$ replaced, respectively, by functions $y^{*} \underline{n}^{*}$ and $\xi^{n}, y^{*}$ being the adjoint operator of $\xi$.

\section{ACKNOWLEDGMENT}

This work has been made under the auspicies of the Fast Reactor Program of CNEN. 


\section{REFERENCES}

1 - P. Aiello et al., Reattore veloce PEC, an Internal CNEN Report, $\operatorname{PRV}(66) 6,(1966)$.

2 - A. Gandini, "Perturbation Methods in Nuclear Reactors from the Importance Conservation Principle", Nucl.Sci.Eng., 35, 141 (1969).

3 - A. Gandini, to be published on Nucl.Sci. Eng.

4 - I.P. Abagjan et al., Gruppovie Konstanty dlia Rasceta Jadernyk Reactorov, M. Go satomizdat (Moscow, 1964).

5 - I. Dal Bono, TAIM - a Multigroup One Dimension Diffusion Code for IBM 7094, an Internal CNEN Report, CEC(66)12, (1966).

6 - I. DaI Bono et al., THE CIAP-ID CODE, a CNEN Report, RT/FI (68) 9 , (1968).

7 - I. DaI Bono et al., THE GIOBPERT-ID CODE, a CNEN Report, $\mathrm{RT} / \mathrm{FI}(68) 10$, (1968).

8 - R.E. Griffith and R.A. Stewards, "A NON-LINEAR PROGRAMMING TECHNIQUE FOR THE OPTIMIZATION OF CONTINUOUS PROCESSING SYSTEMS", Management Sci., 7, 379 (I96I).

9 - A.V. Fiacco and G.P. McCormick, "The Slacked Unconstrained Minimization Technique for Convex Programming", SIAM J.Appl. Math., 15(3), 505 (1967).

10 - A. Gandini, M. Salvatores, G. Sena, Studio di ottimizzazione della distribuzione del berillio nel reattore PEC, an Internal CNEN Report, PRV-r(68)12, (May, 1968). 
SESSION IV

REACTOR DESIGN SYSTEMS

Chairman: C. J. Pfeifer Bettis Atomic Power Laboratory 


\section{BLANK}




\section{VARIATIONAL METHODS AND NEUTRON FLUX SYNTHESIS}

\section{E. L. Wachspress}

\section{Knolls Atomic Power Laboratory*}

This is a review of synthesis techniques for computing neutron fluxes in a reactor. The term synthesis is ambiguous, and this review will be concerned specifically with the construction of approximations in $n$ dimensions using known "base functions" of $r$ dimensions where $r$ is less than $n$. Thus, a Fourier analysis is a synthesis computation. The known base functions are sin $\mathrm{mx}$ and $\cos \mathrm{mx}$, and the approximation might be of the form

$$
f(x, y) \sim \sum_{m=0}^{M}\left[a_{m}(y) \sin m x+b_{m}(y) \cos m x\right] .
$$

Here $n=2, r=1$, and the construction of $f$ involves finding the coefficients $a_{m}(y)$ and $b_{m}(y)$, this being the central problem in the synthesis computation.

We could choose some norm, denoted by $\|\cdot\|$, and choose the combining coefficients in an attempt to minimize

$$
\left\|f(x, y)-\sum_{m=0}^{M}\left[a_{m}(y) \sin m x+b_{m}(y) \cos m x\right]\right\| \text {. }
$$

Questions of existence and uniqueness of "best" solutions to synthesis problems arise, and for Fourier expansions extensive theoretical analysis is available. In most reactor applications, the known base functions are not elementary functions, but rather they are numerical results of auxiliary computations.

A common characteristic of all synthesis methods is the combining of given base functions to approximate a solution to a problem. As the number of linearly independent base functions is increased, higher accuracy is usually obtained. One may avoid difficulties associated with near linear dependence by orthogonalizing base functions. In reactor synthesis studies, one should consider the following: 
1. Given a set of base functions, how does one determine the combining coefficients?

2. Having constructed a synthesis approximation, how does one estimate the error?

3. How many base functions are needed for prescribed accuracy? and

4. How should one determine base functions so that the prescribed accuracy is attained with least total computer time?

Each of these questions cannot be answered without considerable analysis of each specific problem, and even then only partial answers may be found. We shall attempt to offer a few guidelines for such studies, and in particular to demonstrate how variational methodology is particularly suited for analysis of synthesis approximation.

The variational formulation of a synthesis computation consists in defining a functional and choosing the combining coefficients to render the functional stationary. If the functional is a norm, perhaps of the difference between the exact solution and the approximation, then it is nonnegative and the stationary point is a minimum. The functional is often an approximation to some quantity of physical significance. Stationarity then means that first order errors in the synthesis approximation result in second order errors in the value of the functional.

The variational formalism leads to a derived problem in which the cambining coefficients are the unknowns, and the stationarity equations provide a recipe for determining these unknowns. However, there are a number of variational formulations, and these may yield significantly different results with the same base functions. One cannot say that a variational basis for a result makes the result a "good" one. There are "good" and "bad" functionals just as there are good and bad base functions. The approximation with the combining coefficients as variables is know as a "trial function". Thus, in synthesis approximations 
the trial function is a linear combination of base functions in our terminology.

Some of the early synthesis techniques, which were not based on variational techniques, were so simple that they were no doubt used by nuclear reactor analysts at many installations. For example, two space dimension problems were solved approximately by iterating to consistency a sequence of one space dimension problems, alternating between the two space variables and choosing perpendicular bucklings from the previous calculation. These studies predated the first two space dimension multigroup program.

Three space dimension synthesis started along similar lines, although two space dimension calculations from which bucklings were obtained for I-D calculations in the perpendicular direction were not often iterated upon. Local radial bucklings were used for computation of axial flux shapes at a seecific radial location. A forerunner of variational synthesis was John Meyer's'(BAPL) region synthesis where region bucklings and radial-flux-weighted cross-sections were used to compute axial distributions which were then multiplied by the radial shapes to yield 3-D approximations.

The KAPL multichannel flux synthesis scheme ${ }^{2}$ was an attempt at 3-D calculations with few radial nodes. Flux averaged group constants were used for all but the radial diffusion, which was accounted for by interchannel coupling coefficients obtained from partial currents at channel interfaces.

Kaplan (BAPL) was the first to apply variational techniques to 3-D synthesis 3,4 of reactor fluxes, and many programs have been developed along lines proposed by Kaplan. This same variational principle had been applied previously by Calame and Federighi to the thermal spectrum problem. The base functions are known hard and soft energy-spectra and the combining coefficients give the linear combinations of these spectra as a function of position.

The latest major innovation was introduced by selengut who suggested a functional admitting discontinuous trial functions.7 Although the usefulness of discontinuous trial functions in the approximation of continuous solutions may be somewhat obscure, major improvements are possible by this device? $6,9,10$ 
For example, radial base functions obtained from 2-D calculations may be swltched at various reactor elevations. Only flux shapes pertinent to a particular axial zone need be used as base functions in that zone. This enables computation with fewer base functions in each zone than were required in the continuous framework. Likewise, radial discontinuities being permitted, one may now place multichannel synthesis on a variational footing.

Having recognized the applicability of variational methods to flux synthesis computations, various analysts developed schemes for Pn and Sn calculations, for space-angle synthesis,; and for a variety of calculations. These schemes have been reported over the past few years in N.S. and E. and at A.N.S. meetings.

Spatial synthesis mockups provide a flexible tool for kinetics studies in which space and time variation is not separable. This was recognized many years 14 ago but hot been exploited until quite recently $15,16,17,18,19,20,21,10,22,23,24,25$

\section{The Variational Formalism}

A boundary-value-problem is described by a partial differential equatior satisfied over some domain coupled with conditions imposed on the solution over the boundary of the domain and with continuity conditions imposed within the domain. An adjoint B.V.P. may be derived from a given B.V.P. For a "linear" variational formulation one first selects a bilinear functional $F$ which maps each pair of direct and adjoint functions $\psi$ and $\psi^{+}$lying within specified function spaces into a real number, $F\left[\psi, \psi^{+}\right]$. The functional is chosen so that $F$ is stationary about the direct and adjoint functions which solve the direct and adjoint problems. This means that first order perturbations from these solutions leads to only second order perturbations in F. Thus, if $\Phi$ and $\Phi^{+}$ are the solutions and the trial functions are $\Psi=\Phi+a \eta$ and $\Psi^{+}=\Phi^{+}+b \eta^{+}$where $\eta$ and $\eta^{+}$are in the proper function spaces and where $a$ and $b$ are scalars, then

$$
\left.\frac{\partial F}{\partial a}\right|_{a=0}=0 \text { and }\left.\frac{\partial F}{\partial b}\right|_{b=0}=0 \text {. }
$$


In the Kantorovich-Krylov procedure, we let the trial functions be arbitrary linear combinations of know base functions:

$$
\Psi=\sum_{m=1}^{M} a_{m}{ }_{m} \Psi^{+}=\sum_{m=1}^{M} b_{m} \eta_{m}^{+} .
$$

The furcticnal is lirear in $\Psi$ and $\Psi^{+}$. Therefore, the equations of stationarity are two systems of linear equations in $M$ unknowns each, one for the direct and the other for the adjoint combining coefficients:

$$
\begin{aligned}
& \frac{\partial F}{\partial b_{m}}-2, m=1,2, \ldots, M \text { is the Iinear system in the } a_{m} \\
& \frac{\partial F}{\partial a_{m}}=0, m=1,2, \ldots, M \text { is the linear system in the } b_{m} \cdot
\end{aligned}
$$

An additional condition is often required of the functional, and that is that the determinants of these linear systems not vanish for any linearly independent sets of base functions. This assures a unique solution for the combining coefficients. Thus, if any member of the trial function subspace solves the problem, then there will be a stationary point at the solution and the stationarIty equations will pluck out the appropriate coefficients. If the subspace of the trial function does not include the solution, as is more of ten the actual situation, then the bone is that the values obtained from the stationarity equations are such

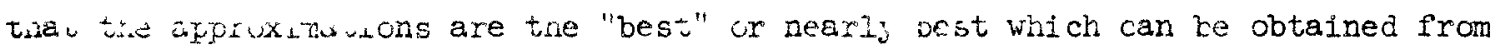
the trzel furctucr subspace. We must of course define "best" in some mathematical sense, perinars in terms of some exror norm.

The varationa! equations may be developed for the discrete multigroup alfin is $e: a t^{\circ-m}$ ifthout great complexity. One neej only be familiar with some

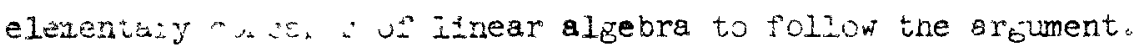


The equations to be solved may be expressed in the matrix form $A \Phi=1 / k B \Phi$, where $A$ includes diffusion, absorption and scattering terms and where $B$ is a fission neutron production matrix. We seek the eigensolution $k_{0}$ and $\Phi_{0}$ with $k_{0}$ the largest (in modulus) eigenvalue of $A^{-1} B$. It is known that $k_{0}$ exists, is real and is a simple eigenvalue, and that the associated eigenvector $\Phi_{0}$ is positive. The adjoint problem $\Phi^{+} A=1 / k \Phi^{+} B$ has a corresponding eigensolution $\mathrm{k}_{\mathrm{O}}$, $\Phi^{+}{ }_{0}$. Let $\underline{\Psi}=\sum_{m=1}^{M} a_{m} \Phi_{m}$ and $\Psi^{+}=\sum_{m=1}^{M} a_{m}^{+} \Phi_{m}^{+}$be trial functions, where the base functions are obtained by some preliminary computation.

The eigenvalue $k$ may be chosen as the functional, which could be the Rayleigh quotient:

$$
\frac{\underline{\Psi}^{+} B \underline{\Psi}}{\underline{\Psi}^{+} \text {A } \underline{\Psi}}=F\left[\underline{\Psi}, \underline{\Psi}^{+}\right]
$$

This functional is not bilinear in $\Psi$ and $\Psi^{+}$. However, the stationarity equations can be expressed as an eigenvalue problem with the combining coefficients as an eigenvector. We define the matrices

$$
\begin{aligned}
& c=\left(c_{m^{\prime}}\right) \text { where } c_{m^{\prime}}=\Phi_{m}^{+} A \Phi_{m^{\prime}} \\
& D=\left(d_{m^{\prime}}\right) \text { where } d_{m^{\prime}}=\Phi_{m}^{+}{ }^{+} \Phi_{m^{\prime}}
\end{aligned}
$$

We define the vectors $\underline{a}=\left[\begin{array}{c}a_{1} \\ a_{2} \\ \vdots \\ a_{M}\end{array}\right]$ and $\underline{a}^{+}=\left[a_{1}+\ldots, a_{M}^{+}\right]$.

The Rayleigh quotient is stationary about solutions to the eigenvalue problems:

$$
C \underline{a}=1 / k \text { D } \underline{a} \text { and } \underline{a}^{+} \mathrm{C}=1 / \mathrm{k} \underline{a}^{+} \mathrm{D}
$$


The eigenvalue $k$ is the value of the functional at the stationary point. The order of each of these matrix equations is equal to $M$.

If there is any set of coefficients for which the true solution is obtained, then this solution will satisfy the above matrix equations. In general, the trial function subspace does not include the exact solution, and the significance of the stationary point may be obscure.

A common procedure for the non-self-adjoint neutron diffusion equations is to choose the same base functions for the direct and adjoint trial function expansions. It is thus assumed that both the direct and adjoint fluxes may be approximated well by linear combinations of these base functions, although the coefficients of combination are not identical for both the direct and adjoint approximations. This is called Galerkin's method. Some insight concerning the nature of these approximations may be gained from a more detailed study of a self-adjoint problem.

Let $Q$ be a symmetric linear partial differential operator with real coefficients and suppose we are to find the minimum eigenvalue $\lambda_{0}$ and associated normalized eigenfunction $\Phi_{0}$ satisfying $Q_{\Phi_{0}}=\lambda_{0} \Phi_{0}$ where $\Phi_{0}$ is in some class $C$ of suitably differentiable functions defined on the domain $D$ with appropriate conditions on the boundary $\Gamma$ of $\mathrm{D}$. These boundary conditions are assumed to be such that the boundary-value-problem is self adjoint. We define the inner product $(a, b)=\int_{D} a b d r$.

Let $\Psi ; m=l, 2, \ldots, M$ be an orthonormal set of base functions capable of ylelding a close approximation to $\Phi_{0}$, and define the normalized function $\eta$ by

$$
\Phi_{0}-\sum_{m=1}^{M} a_{m} \psi_{m}=a_{m}, \quad\left(\psi_{m}, \eta\right)=0
$$

where the $a_{m}$ are the combining coefficients which yield the least possible value for $(\alpha)$. We note that $\left\|\Phi_{0}\right\|^{2}=\sum_{m=1}^{M} a_{m}^{2}+\alpha^{2}=1$ so $|\alpha| / \|$ al is also minimized. 
$\sum^{\mathrm{M}}$

We wish to determine the error associated with approximating $\Phi_{0}$ by

$b_{m} \Phi_{m}$, where the $b_{m}$ are obtained from the Ritz variational principle. We assume $\mathrm{m}=1$

that $0<\alpha<<1$. We define the following quantities:

$$
\begin{array}{lll}
g_{m m}, \equiv\left(\Phi_{m}, q I_{m},\right) & c_{m} \equiv\left(\Psi_{m}, a_{n}\right) & a \equiv\left(\eta, a_{\eta}\right) \\
A \equiv\left(g_{m m}\right) & c^{T} \equiv\left(c_{1}, c_{2}, \ldots, c_{M}\right) & \underline{a}^{T} \equiv\left(a_{1}, a_{2}, \ldots, a_{M}\right)
\end{array}
$$

Then $B\left[\frac{a}{\alpha}\right]=\lambda_{0}\left[\frac{a}{\alpha}\right]$, where

$$
B \equiv\left[\begin{array}{l}
A \stackrel{c}{T} \\
c^{T} d
\end{array}\right]
$$

$$
\begin{aligned}
& \lambda_{0}=\min _{\underline{x}, \beta}\left[\underline{x}^{T_{\beta}}\right] \text { B }\left[\frac{x}{\beta}\right]<\left[\underline{O}^{T}\right] \text { B }\left[\frac{0}{I}\right]=d \\
& \|\underline{\underline{x}}\|^{2}+\beta^{2}=1
\end{aligned}
$$

Strict inequality above follows from the assumption that $\alpha$ is not equal to unity。

Realistic bounds on a are not easily found. Frequently, $a$ has an eigenvalue accumulation point at infinity and $\left(\eta, a_{\eta}\right)$ can be unbounded. For practical purposes, however, the base functions are sufficiently smooth that they may be represented well by finite eigenfunction expansions. If we select $k$ as the number of eigenfunctions which are to be considered in these expansions, where $K>M$, then

$$
\Psi_{m}=\sum_{k=0}^{K} t_{m k} \Phi_{k} \text { and } \eta=\sum_{k=1}^{K} h_{k} \Phi_{k} \text { 。 }
$$

An upper bound on $d$ is then given by $\lambda_{K}$. 
Variational methods are sometimes applied to discrete B.V.P. where $G$ is a matrix of order $N$ and $N>>M$. (This is the case in flux synthesis computation.) Then $\left(\eta, a_{\eta}\right)=\eta^{T} a_{\eta} \leq \lambda_{\max }(a)$ and $p$ is the p-condition number of $a$. We thus reduce the infinite-dimensional space of $a$ to a finite-dimensional subspace in which we bound $\left(\eta, a_{\eta}\right)$, and we consider the error of our approximate solution in the reduced subspace.

The variational approximation yields $d_{0} \sim \sum_{m=1}^{M}\left(a_{m}+\delta a_{m}\right) \Psi_{m}$ where

$$
A(\underline{a}+\delta \mathbf{a})=\left(\lambda_{0}+\delta \lambda\right)(\underline{a}+\underline{\delta} \mathbf{a}),
$$

and where $\underline{\delta a}$ is made unique by normalizing so that $\underline{a}^{\mathrm{T}} \underline{\delta a}=0$.

We must relate $\|\delta a\|$ and $|\delta \lambda|$ to $\mid \alpha$.

First, we recall that

$$
\lambda_{0}+\delta \lambda=\min _{\mathrm{b} \neq 0} \frac{\underline{b}^{\mathrm{T}} \mathrm{Ab}}{\underline{\mathrm{b}}^{\mathrm{T}} \underline{\mathrm{b}}}>\lambda_{0}
$$

From (2), we have

$$
\underline{c}^{\mathrm{T}} \underline{a}+\mathrm{d} \alpha=\lambda_{0} \alpha \text { or } \underline{c}^{\mathrm{T}} \underline{a}=\alpha\left(\lambda_{0}-d\right) \text {. }
$$

Multiplying (2) on the left by $\left(\mathrm{a}^{\mathrm{T}}\right)$, we have

$$
\begin{aligned}
& \underline{a}^{\mathrm{T}} \underline{\mathrm{a}}+\alpha \underline{a}^{\mathrm{T}} \underline{c}=\lambda_{0} \underline{a}^{\mathrm{T}} \underline{a} \text {, or } \\
& \frac{\underline{a}^{\mathrm{T}} \mathrm{A} a}{\underline{\underline{a}}^{\mathrm{T}} \underline{a}}=\lambda_{0}+\frac{\alpha^{2}\left(a-\lambda_{0}\right)}{\underline{\underline{a}}^{\mathrm{T}} \underline{a}}=\lambda_{0}+\frac{d^{2}\left(d-\lambda_{0}\right)}{1-\alpha^{2}} \text {. }
\end{aligned}
$$

Let $p=d / \lambda_{0}$. Then

$$
\begin{aligned}
& \left.\frac{a^{T} A a}{=}=\lambda_{c} \underline{a^{T}}=\frac{a^{2}(p-1)}{1 \cdot \alpha^{2}}\right) \text {, and since } \min _{b \neq 0} \frac{b^{T} A b}{\underline{b}^{T} \underline{b}}<\frac{a^{T} A a}{=} \text { : } \\
& 0<\delta \lambda<\lambda_{0} \frac{\alpha^{2}(p-1)}{1-\alpha^{2}}=\lambda_{0}-0\left(p \alpha^{2}\right) \text {. }
\end{aligned}
$$


We have thus proved the following:

1. The error in estimating $\lambda_{0}$ by the variational method is $\left.\lambda_{0} \cdot 0, p \alpha^{2}\right)$.

2. The eigenvalue obtained by the Ritz variational principle is closer to the true value than the estimate obtained by substituting into the Raylelgh quotient the closest subspace approximation in a least square sense to the true eigenfunction.

These are well known results, but they have been developed here from a different viewpoint than is normally taken.

We now consider $\|\underline{a}\|$. For the symetric matrix $B$ in $(2),(\underline{a}, \alpha)$ is a left eigenvector with eigenvalue $\lambda_{0}$ so that $\underline{a}^{T} A+\alpha \underline{c}^{T}=\lambda_{0} a^{T}$ or $\underline{a}^{T} A=\lambda_{0} \underline{a}-\alpha c^{T}$. Premultiplying (4) by $\underline{a}^{T}$ and using the above, we have $\left(\lambda \underline{a}^{\mathrm{T}}-\alpha \underline{c}^{\mathrm{T}}\right)(\underline{a}+\underline{\delta a})=\left(\lambda_{0}+\delta \lambda\right)\left(\underline{a}^{\mathrm{T}} \underline{a}+\underline{a}^{\mathrm{T}} \delta \underline{a}\right)$, or $-\alpha \underline{c}^{\mathrm{T}} \underline{a}-\alpha \underline{c}^{\mathrm{T}} \delta \underline{a}=\delta \lambda \underline{a}^{\mathrm{T}} \underline{a}$ Therefore,

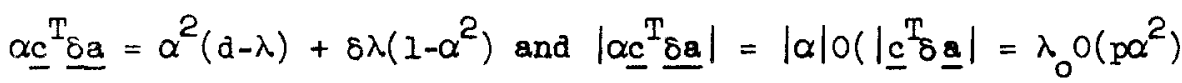
Thus, $\left.\left|\underline{e^{\mathrm{p}}} \underline{\underline{a}}\right|=\lambda_{0} 0, p|\alpha|\right)$ Having shown that $\left|c^{\mathrm{T}} \delta \mathrm{g}\right|=\lambda_{0} O^{\prime}(\mathrm{p}|\alpha|)$, we must still demonstrate that $\|\delta \underline{b}\|$ is itself sma11. We note that $\left(\Phi_{\circ}, \sum_{m} \delta a_{m} w_{m}\right)=a^{T} \underline{\delta a}+\alpha \sum_{m} \delta a_{m}(\eta, \psi m)=0$ This means that $\sum \delta a_{m} \psi_{m}$ is orthogonal to the eigenfunction of $a_{\text {with }}$ eigenvalue $\lambda_{0}$. Therefore

$$
\delta a^{T} a_{\delta} \underline{a}=\left(\sum_{m} \delta a_{m} \psi_{m}, \widehat{a} \sum_{m} \delta a_{m} \psi_{m}\right) \geq \lambda_{1}\|\underline{a}\|^{2}
$$

where $\lambda_{1}$ is the next smallest eigenvalue of $Q$. We now premultiply (4) by $\delta a^{T}$ and use the identity $\underline{A} \underline{a}=\lambda_{0} a-\alpha_{c}$ to get $-\alpha \underline{\delta a^{T}} \underline{c}+\underline{\delta a}{ }^{T} \underline{A} \underline{a}=\left(\lambda_{0}+\delta \lambda\right) \| \underline{\delta a} \mid f$, and since $\left|\underline{\delta a}{ }^{T} \underline{c}\right|=o\left(\lambda_{o} p|\alpha|\right)$ and $\delta \lambda=o\left(p \alpha^{2}\right)$, we have $\left|\delta a^{T} A \delta a-\lambda_{0}\|\delta a\|^{2}\right|=o\left(\lambda_{0} p \alpha^{2}\right)$. 


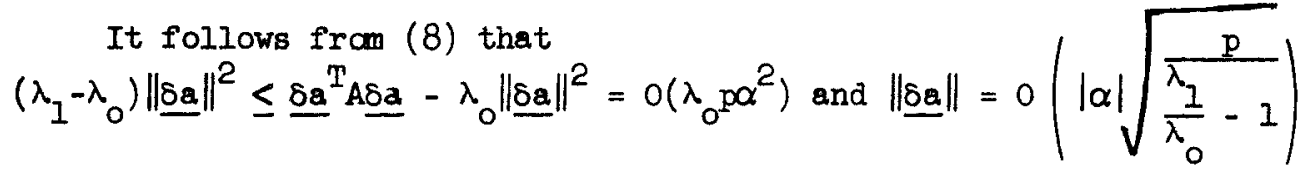

If the base function subspace is such that

$$
\left\|\Phi-\sum a_{m} \Phi_{m}\right\|=|\alpha|
$$

then the eigenfunction approximation obtained from the Ritz variational procedure is such that

$$
\left\|\delta a_{m} \psi_{m}\right\|=0\left(|\alpha| \sqrt{\frac{\overline{\lambda_{1}}}{\lambda_{0}}-1}\right)
$$

This analysis does not apply to the non-self-adjoint neutron flux synthesis computations for which the stationary point is not a minimum. We must seek an alternative justification.

First, we choose a functional of physical significance: the multiplication constant of the reactor. This constant has overshadowed all others in interpretation of multigroup calculations, and is a most logical choice. Second, we use very good trial functions. Continuity arguments can be developed to prove that if the trial function subspace has a point sufficiently close to the solution then there will be a stationary point near the solution. Quantitative results relating errors to eigenvalue separation and best least square fits are of little practical value.

The main justification is numerical results. Comparisons of approximations with known solutions have repeatedly supported the validity of variational synthesis computations.

Having discussed the variational formalism, we now direct our attention to choice of base functions and to the "synthesis" part of variational synthesis. 
Suppose we seek values of a function $f$ of several variables which satisfies $Q_{f}=\lambda_{0} f$, and that we have decided upon a functional $F[g]$ which is stationary about $g=f$. The independent variables are subdivided into two sets which we shall designate by $x$ and $y$. Base functions are determined first by preliminary computations. They depend on only the $y$ variables: $\Phi_{k}(y), k=1,2, \ldots, K$.

The trial function $g$ is chosen as

$$
g(x, y)=\sum_{k=1}^{K} \psi_{k}(x) \Phi_{k}(y)
$$

The free parameters to be found from the variational principle are the combining coefficients which depend upon $x$ only. If $y$ contains $m$ variables and $x$ contains $n$ variables, the initial problem in $n+m$ dimensions is reduced to a similar problem in $n$ dimensions.

For application to reactor design studies, the $\Phi_{k}(y)$ may be asymptotic energy spectra, or neutron flux distributions computed for lower dimensional configurations than the actual reactor, or any of a number of functions chosen for a specific application.

The $\psi_{k}(x)$ may be computed by a technique appropriate for an $n$-dimensional problem in the $x$ variables. A few concrete applications will now be described in an attempt to clarify these concepts.

The Thermal Spectrum Problem

The first application of variational methods to spatial synthesis in neutron diffusion computations was by Calame and Federighi to the thermal spectrum problem. The thermal spectrum may change significantly with position in a reactor. It is relatively hard in and near poison regions and soft in and near water. One could attempt to approximate region-dependent spectra for computation of thermal groupconstants. However, these spectra are sensitive to adjacent region composition and to local flux gradients. This approach has been found to be inadequate for many studies. One may subdivide the thermal energy range into a number of groups to yield required precision. This approach with several coupled thermal groups is valid, but the variational technique has been found to be more efficient. 
Let $\psi_{S}(E)$ be a soft and $\psi_{H}(E)$ a hard spectrum. Both of these may be determined in advance from infinite-medium or some other more sophisticated computations. The thermal neutron flux is approximated by

$$
\Phi(r, E)=\Phi_{S}(r) \psi_{S}(E)+\Phi_{H}(r) \psi_{H}(E)
$$

The coefficients of combination, $\Phi_{S}$ and $\Phi_{H}$, are the free parameters in the trial function. The Rayleigh-Ritz principle has been applied to yield a functional stationary about the desired solution. If this approximation is not adequate for a specific problem, we may add more base spectra characteristic of the problem. Experience has shown that the soft and hard spectra are quite good for a wide range of problems. Accuracy comparable with that attained with several thermal groups has been observed.

There has been some discussion of relative merits of different choices of the adjoint spectra. One school uses the Galerkin approach and another uses adjoint functions of unity and $E$ with the justification that the true adjoint spectrum is more nearly represented by this choice. Galerkin's method may be better suited for numerical calculation, but the difference in computation time between the two methods is not great.

\section{$26,27,28,29,30,31$}

This overlapping group technique has been examined for fast reactors. Here, there is no energy group subdivision as in the multigroup treatment, but a set of base spectra which cover the entire energy range. A thorough description is given by Stacey, and some recent studies were reported by Murley and Williamson. 26

\section{Spatial Synthesis}

Syntnesis of the spatial dependence of neutron filux has its major application to approximation in tiree space dimensions. Relatively efficient two-space-dimension few-group computer programs are currently in use. Some configurations may be analyzed in three dimensions by techniques comparable to those used in two dimensions. We consider here configurations for which three-dimensional computation by conventional finite-difference techniques is impractical for design studies with the present generation of computers. The prevalent synthesis technique involves preliminary generation of a set of flux distributions in two of the three dimensions, which e shall designate as $x-y$ planes. 
Thus, the base functions are:

$$
\Phi_{m g}(x, y), m=1,2, \ldots, M ; g=1,2, \ldots, G
$$

where $g$ is an energy group index and $M$ is the number of 2-D calculations run in advance. The variational synthesis equations developed by Kaplan 4 utilized the trial function:

$$
\Phi_{g}(x, y, z)=\sum_{m=1}^{M} a_{m g}(z) \Phi_{m g}(x, y)
$$

The $z$ variation was discretized by conventional finite-difference techniques with the assumption of a linear flux variation between elevation $z_{i}$ and $z_{i+l}=z_{i}+h_{i}$.

The free parameters in the variational equations then become the values of the combining coefficients at the $z_{i}: a_{m g}\left(z_{i}\right)=a_{m g i}$. Adjoint $2-D$ results have been used, but Galerkin's method is more often applied. Significant time is spent in the preliminary planar computations, and this time is doubled when adjoint fluxes are used.

Kaplan chose the Rayleigh-Ritz functional, and this reduced the three dimensional multigroup problem to a one-dimensional problem. The scalars in the one-dimensional diffusion equation are replaced by matrices of order M. Block methods used in the multichannel synthesis model were applied to solve this variational problem. Some of the mathematical properties of the multichannel synthesis model were lost. The existence of a unique positive eigenfunction could no longer be proved. However, one could invoke the fundamental variational argument that if the base functions provided a close approximation with same set of combining coefficients, then the stationarity equations would yield values reasonably close to this approximation.

Kaplan's model worked well. Realistic 3-D flux approximations were obtained when a sufficient number of pertinent base functions were used. As many as 25 functions were permitted in some studies, but this large number of base functions was not often needed. 
Attempts have been made to use a "collapsed" energy group structure where all groups have identical combining coefficients at an elevation of the reactor. This is a means for reducing the order of the matrices of coefficients in the onedimensional problem. Difficulties were encountered with this approach. The trial function may be so far removed from the solution that meaningless results with extraneous eicenvaiues are obtained. The situation has been improved by more sophisticated adjoirt weignting and by partial group collapsing. ${ }^{24}$ Advantages of collapsing are reduced computation time of the $I-D$ problem and reduced coefficient storage requirements. This is particularly important in transient analysis.

The Rayleigh-Ritz functional for the neutron diffusion operator has diffusion integrals of the type

$$
\text { I. } \int D(r) \nabla_{r} \Phi^{+} \cdot \nabla_{r} \Phi d r
$$

and non-diffusion integrals of the type

$$
\text { II. } \int A(r) \Phi^{+}(r) \Phi(r) d r
$$

If we assume linear variation of flux between axial mesh points to obtain stationarity equations for a discrete set of combining coefficients, then the type I integrals yield the usual type of diffusion coupling between adjacent planes with the scalar diffusion coefficient replaced by a matrix of order M. The non-diffusicn integrais of type II, on the other hand, lead to off diagonal as well as diagonal removal, slowing down, and fission terms. These same terms would be obtained for a true 1-D prohlem and the same assumption of linear flux variation between points. However, wh starcicu (urvugh wiaespreau use) and more convenient formulation involves $m-\gamma$ izaconal ron-diffusion terms, and is derived by the "box integration" method. We cai jotin this form of equacion by wising different quadrature formulas for the tyce I nd a II integrals, this keing equivaient to assuming a step function variatior. of flux for the latter. This is not, however, a consistent variational forma: $=$ s. 
We now turn to a comparatively recent development in reactor computations which provides an alternative variational principle. This principle has many advantages, broadening the scope of synthesis computations. It also provides a functional which resolves the aforementioned inconsistency.

\section{Discontinuous Trial-Functions}

One of the more recent innovations is the use of discontinuous trial functions? The multichannel synthesis formulation has some desireable features, especially in analysis of assemblies for which channels may have same physical significance. More degrees of freedom are introduced when the coefficients of combination of the base functions are permitted to vary from channel to channel. The multichannel flux synthesis scheme was, however, not developed from a variational principle. The functional used initially by Kaplan did not admit discontinuities in trial functions. Hence, even after the introduction of variational methods into synthesis calculations, a somewhat heuristic interchannel coupling was retained in the non-variational multichannel synthesis model.

To place multichannel synthesis on a variational footing, selengut proposed use of a functional which admits discontinuous trial functions. This functional requires both neutron flux and neutron current base functions. Even though flux and current satisfying the diffusion equations are related by $\underline{j}=-D \vee \Phi$, the base functions $\underline{j}$ and $\Phi$ are allowed independent variations. The second order neutron diffusion differential equation is replaced by an equivalent system of two coupled first order equations. The functional is stationary about fluxes and currents which satisfy both equations and which are also continuous.

Selengut was aware of alternative functionals which would accomplish the objective of admitting discontinuities, and some of these have been suggested by others subsequentiy. A multichannel variational synthesis formalism based on Selengut's functional has been devised, but thus far only a few numerical studies have been conducted. 6 
Another advantage to admitting discontinuities is that radial base functions may be changed as a function of height in a 3-D synthesis computation. This was always permitted in the non-variational multichannel synthesis where there is only one base function in each axial zone. Kaplan added this capability to his synthesis mockup and reported significant improvement over the old method. 8

Axial discontinuities are not essential for the introduction of zone-dependent base functions. One can set combining coefficients to zero at elevations where a particular base function is believed to have little influence. 35,36

The difficulty mentioned earlier in connection with non-diagonal coupling from removal, scattering, and fission terms is now resolved by choosing step variations in the flux and axial current combining - coefficients as a function of height. The jumps are taken midway between axial mesh points for the fluxes and at the points for the currents. The diffuston coupling is identical to that obtained by assuming linear variation of fluxes between points. The non-diffusion terms are now ldent1cal with those obtained by the box integration method. We have thus eliminated some arm waving.

We mentioned that synthesis methods usually do not employ analytic base functions, but that they make use of base functions especially tailored to a particular problem. This is a reasonable distinguishing characteristic of synthesis methods. The overlapping group scheme with analytic hard and soft spectra was not called synthesis by Calame and Federighi, but it is so similar in nature to spatial synthesis computation that we have chosen to include this scheme in our synthesis family.

Similarly, discontinuous trial functions lead to nodal mockups of reactors which are strictly speaking synthesis computations when the base functions are obtained from lower dimensional computations. However, Hansen, Johnson and storat expand the flux as a product of cubics in the three dimensions with different coefficients in each box of their nodal mockup, and refer to the variational computation of these coefficients as a synthesis computation. They impose continuity of partial currents on box surfaces while minimizing the square integrals of flux discontinuities at these surfaces. 
Synthesis techniques have been applied to determine the angular dependence of the neutron flux as a function of position in two-space-dimension geometry. 9 Instead of expanding the angular dependence in terms of Legendre polynomials as In the Pn approximations, one may expand in terms of angle base functions chosen for a specific configuration. Natelson, Kaplan and Davis used eccentric circles and ellipses as their base functions in the first such studies. The base functions appropriate for one region may differ appreciably fram those of another region so that many base functions would often be required for a complex configuration. This corresponds to the need for many planar base functions for a 3-D synthesis computation of a many-zoned reactor.

The situation is improved considerably by allowing trial function discontinuities, for we need only use the base functions appropriate for a region in that region. In fact, this space-angle synthesis was made feasible by this device, and the early studies were performed with angle functions tailored to each region. Limited work has thus far been performed in this direction, but one may expect renewed activity when efficient means become avallable for generating realistic angle base functions with the aid of auxiliary transport calculations.

Another variant of space-angle synthesis is where one chooses spatial base functions and computes combining coefficients which depend upon angle. This was investigated by $Z$ wibel and Bowes, who observed that one may sametimes have reasonable spatial base functions instead of angle base functions. Slab absorption probabilities were computed with the trial function:

$$
f(x, u)=(1-c)^{-1}+g_{1}(u) \cosh k x+g_{2}(u) \sinh k x
$$

where $c$ and $k$ were estimated from material properties and $h_{1}$ and $g_{2}$ were computed from the stationarity equations. Excellent agreement with known values was obtained for a wide range of parameters. Results were far more accurate than P-I theory, having errors of about 1-2\% for slabs thin enough to yield P-1 errors of about $40 \%$.

One scheme for generating base functions is to solve a set of inhomogeneous problems with different forcing functions and for various anticipated conditions. These have been called Green's function modes, although they are Green's functions for distributed rather than point sources. These base functions have been used, 
for example, by Dougherty and Shen "in the "semidirect variational" approach, which is especially well suited for studying transients. Studies by Rohr and Becker 39 Indictite that Selengut's runctional with Green's function modes results in an japroved treatment of neutron currents over standard least squares and RayleighPitz functionals. Discontinuities arise when one cuts off base-function "shoulder. ontat de the nonzero source region.

Ancmalies occur in same attempts to use discontinuous trial functions. The additional degrees of freedom are not always beneficial, and more insight is required than for the more conventional variational principles. Kellogg cited a case where a seemingly reasonable choice of base functions yields absurd difference equations. On the other hand, there is a set of base functions which duplicates the box integration method difference equations for all geometries of concern, and this variational principle is to my knowledge the only one which gives the box integration discretization.

Buslik 33 showed how an overdetermined system of interface conditions is obtained from the stationarity equations of Selengut's functional when applied to 3-D synthesis with continuous rather than discrete dependence of the combining coefficients as a function of height. However, other functionals may be used here, as pointea out by Buslik, and this is discussed by several authors. $40,41,42$

Flux discontinuities at surfaces where the base functions for the neutron current vanish lead to decoupled systems with Selengut's functional, and the stationarity equations do not yield the desired stationary point. There are sxtanoous statimary points when the neutron current trial function cannot vary frum zero at a rlux discontinuty. This is remedied by modifying the functional

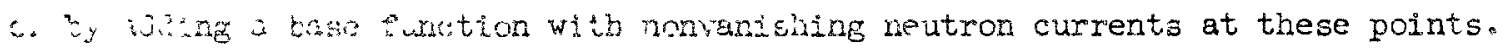

Many wechniques for computing neutron fiuxes during a transient for a point

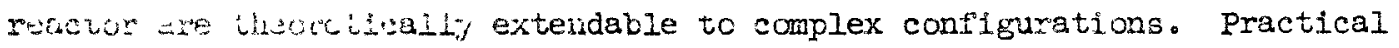

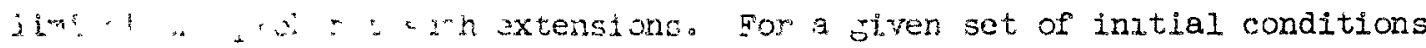
(delayed neutron source, temperature, xenon, rod locations, etc.) one can compute few-group îuxes th diffusion theory to reasonable accuracy in a matter of stconds $f(C)$ 1-D probiems, minutes for 2-D and hours for detailed 3-D mockups. 
This process may have to be repeated many times during a transient study. Even in two-space-dimensions, detailed space-time calculations are too costly for design studies with the current gens ation of computers.

Synthesis methods reduce the orjer of corplexity from $3-D$ to $1-D$, and thus provide a spatial model for kinetics studies of complex configurations." It is not some sophisticated rariaional time-synthesis that one applies. It is just a simplified spatial mockup with well-known time behavior analysis.

The spatial representation is enhanced by use of crial functions characteristic

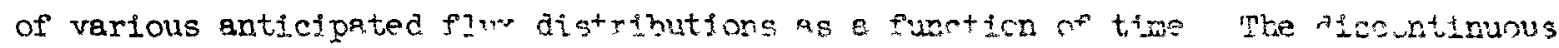
trial function formulation enables switching to new base functions as they beccne more appropriate during the transient. For example, we may compute the coefficients $a_{m}(z, t)$ for

$$
\begin{aligned}
& f(x, y, z, t)=\sum a_{m}(z, t) f_{m(v)}(x, y) \\
& m(t)
\end{aligned}
$$

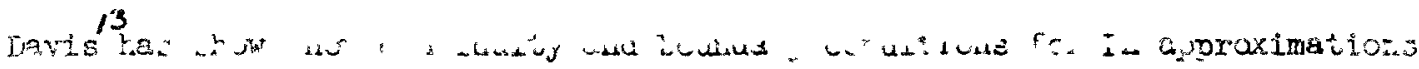

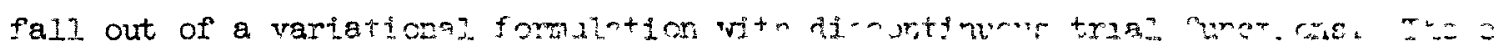
had been considerable controveisy over just which equations weje appropr iete, EIa this variational development helps clerify the sigajficance of dufferent apr reacrsu.

A rather general description of variational synthesis was given by jerrys who

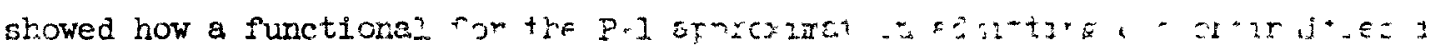
space and energy yields diverse approximations, scre alreac. "

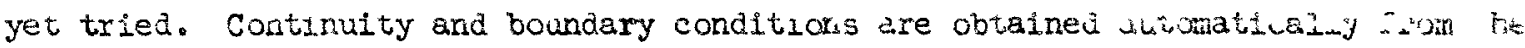
stationarity equations. Although Henry considered time dependence, he did rot choose to consider discontinuities of trial functions with respect to time. 


\section{Linked Calculations}

All the contributed papers in this session are on linked reactor design systems. I should like to call to your attention Session III of the first topical conference sponsored by the Mathematics and Computation Division. This was held at Argonne in May of 1965, and was devoted to the same topic as this session. A paper was presented by $\mathrm{W}$. H. Turner on automated reactor design at KAPL, and in this paper Mr. Turner described some of the thoughts in planning a large modular system of reactor programs. Nick Turner played a key role in the development of the system described therein. His work on datapool and modular programming provided the foundation for our current NOVA system. Many of the Iinked calculations described in today's session employ cancepts which Nick sought to implement. His untimely death, besides being a personal blow to his co-workers, was a great technical loss in this field. It is good to see at this time that some of his dreams regarding linked calculations have now become a reality. 


\section{BIBLIOGRAPHY}

1. J.E. Meyer, "Synthesis of Three-Dimensional Power Shapes", Proceedings of International Conference on Peaceful Uses of Atomic Energy, Geneva, p/636, Volume 11, (1958), Pages 519-522.

2. E. L. Wachspress, R. D. Burgess, S. Baron, "Multichannel Flux Synthesis", Nuclear Science and Engineering, Volume 12, No. 3, (1962), Pages 381-389.

3. S. Kaplan, "Some New Methods of Flux Synthesis", Nuclear Science and Engineer= ing, Volume 13, No. 1, (1962), Pages 22-31.

4. S. Kaplan, "Synthesis Methods in Reactor Analysis", Advance in Nuclear Sclence Volume III (P.R. Greebler, editor), Academic Press, N.Y., (1966).

5. G. P. Calame, F. D. Federight, "A Variational Procedure for Determining Spatially Dependent Thermal Spectra", Nuclear Science and Engineering, Volume 10, No. 2, (1961), Pages 190-201.

6. E. L. Wachspress, "Numerical Studies of Multichannel Variaticnal synthesis", Nuclear Science and Engineering, Volume 26, No.3, (1966), Pages 373-377.

7. E. L. Wachspress, M. Becker, "Variational Synthesis with Discontinuous Trial Functions", Proceedings Conf. Appl • Comp. Methods to Reactor Problems, ANL7050, (1965)。

8. J. B. Yasinsky, S. Kaplan, "Synthesis of Three-Dimensional Flux Shapes Using Discontinuous Sets of Trial Functions", Nuclear Science and Engineering, Volume 28, No. 3, (1967), Pages 426-437.

9. S. Kaplan, J. A. Davis, M. Natelson, "Angle-Space Synthesis - an Approach to Transport Approximations", Nuclear Science and Engineering, Volume 28, No.3, (1967), Pages 364-375.

10. J. B. Yasinsky, "The Solution of the Space-Time Neutron Group Diffusion Equations by a Time-Discontinuous Synthesis Method", Nuclear Science and Engineering, Volume 29, No. 3, (1967), Pages 381-391. 
11. M. Natelson, "A Strategy for the Application of Space-Angle Synthesis to Practical Problems in Neutron Transport", Nuclear Science and Engineering, Volume 31, No. 2, (1968), Pages 325-336.

12. H. S. Zwibel, B. Bowes, "Space-Angle Synthesis", Transactions of the American Nuclear Society, Volume 11, No. 1, (1966), Page 174.

13. J. A. Davis, "Continulty Conditions for a Homogeneous or Heterogeneous Pn Approximation", Nuclear Science and Engineering, Volume 27, No. 3, (1967), Pages $542-548$.

14. E. L. Wachspress, "Digital Computation of Space-Time Variations of Neutron Fluxes in a Complex Reactor Configuration", Codes for Reactor Computations, International Atomic Energy Agency, January 1961, Pages 171-177.

15. D. C. Wade, "Nodal Space-Time Kinetics Models Interpreted in Terms of Modal Expansions", Transactions of the American Nuclear Society, Volume 11, No. 1, (1966), Page 168.

16. J. B. Yasinsky, L。 L. Lynn, S. Kaplan, T. A. Porsching, "A Combined SpaceTime Synthesis Model-Numerical Comparisons with Exact Two-Group Two-Dimensional Transient Solutions", Transactions of the American Nuclear Society, Volume Il, No. 1, (1966), Page 172。

17. J.B. Y६sinsky, "Numerical Studies of Combined Space-Time Synthesis", Nuclear Science and Engineering, Volume 34, No.2, (1968), Pages 158-168.

18. D. E. Dougherty, C. N. Shen, "The Space-Time Neutron Kinetic Equations Obtained by the Semidirect Variational Method", Nuclear Science and Engineering, Volume 13, (1962), Pages 141-148.

19. W. M. Stacey, Jr。, "Variational Functions for Space-Time Neutronics", Nuclear Science and Engineering, Technical Notes, Volume 30, No. 3, (1967), Pages 448451 .

20. W. M. Stacey, Jr., "A Variational Multichannel Space-Time Synthesis Method for Nonseparable Reactor Transients", Nuclear Science and Engineering, Volume 34, No. 1, (1968), Pages 45-56. 
21. J. B. Yasinsky, A. F. Henry, "Some Numerical Experiments Concerning SpaceTime Reactor Kinetics Behavior", Nuclear Science and Engineering, Volume 22, No. 2, (1965), Pages 171-181.

22. S. Kaplan, 0. J. Marlowe, J. Bewick, "Application of Synthesis Techniques to Problems Involving Time Dependence", Nuclear Science and Engineering, Volume 18 No. 2, (1964), Pages 163-176.

23. A. F. Henry, N. J. Curlee, "Verification of a Method for Treating Neutron Space-Time Problems", Nuclear Science and Engineering, Volume 4, No. 6, (1958), Pages 727-744.

24. R. A. Rydin, "Time Synthesis - A Study of Synthesis Modes and Weighting Functions", Transactions of the American Nuclear Society, Volume 10, (1967), Page 559.

25. L. R. Foulke, E. P. Gyltopoulos, "Application of the Natural Mode Approximation to Space-Time Reactor Problems", Nuclear Science and Engineering, Volume 30 , No. 3, (1967), Pages 419-435.

26. T. E. Murley, J.W. Williamson, "Space-Energy Synthesis Techniques for Fast Reactor Calculations", Transactions of the American Nuclear Society, Volume 11, No. 1, (1966), Page 174 。

27. W. M. Stacey, Jr., "Fast-Reactor Calculational Models", Nuclear Science and Engineering, Volume 28, No. 3, (1967), Pages 443-449.

28. A. Travelli, F. Helm, "Calculation of the Sodium Void Effect by Flux Synthesis', Transactions of the American Nuclear Society, Volume 10, No. 1, (1967).

29. B. A. Hutchins, M. D. Kelly, G. L. Gyorey, "Multigroup Two-Dimensional Synthesis Calculations for Fast Power Reactors", Transactions of the American Nuclear Society, Volume 10, No. I, (1967).

30. W. M. Stacey, Jr., "Modal Approximations: Theory and an Application to Reactor Physics", The MIT Press, Cambridge, Massachusetts, (1967)。 
BIBLIOGRAPHY (continued)

31. F. D. Federighi, P.A. Ombrellaro, "Spatially Dependent Spectra at Energies Above Thermal", Transactions of the American Nuclear Society, Volume 5, No. 1, (1962), Page 40.

32. J. B. Yasinsky, S. Kaplan, R. H. Jason, "Initial Studies of Collapsed GroupSpace Synthesis: Anomalies Arising from the Use of Adjoint Weighting", Transactions of the American Nuclear Society, Volume 10, (1967), Page 172.

33. A. J. Buslik, "Interface Conditions for Few-Group Neutron Diffusion Equations with Flux Adjoint Weighted Constants", Nuclear Science and Engineering, Volume 32, No. 2, (1968), Pages 233-240.

34. J. B. Yasinsky, S. Kaplan, "On the Use of Dual Variational Principles for the Estimation of Error in Approximate Solutions of Diffusion Problems", Nuclear Science and Engineering, Volume 31, No. 1, (1968), Pages 80-90.

35. M. Becker, "Overlapping Group Methods with Discontinuous Trial Functions", Nuclear Science and Engineering, Volume 34, No. 3, (1968), Page 339.

36. E. L. Wachspress, "On the Use of Different Radial Trial Functions in Different Axial zones of a Neutron Flux Synthesis Computation", Nuclear Science and Engineering, Volume 34, No. 3, (1968), Page 342.

37. K. F。 Hansen, S. R。 Johnson, R。E. Storat, "A Numerical Method for the ThreeDimensional Multigroup Equations", Transactions of the American Nuclear Society, Volume 10, No. I, (1967), Page 174 .

38. R。 J。Hooper, M. Becker, "Flux Synthesis Using Modified Green's Function Modes", Transactions of the American Nuclear Society, Volume 9, No. 2, (1966), Page 471.

39. P. C. Rohr, M. Becker, "Modal Analysis of Power Tilting", Transactions of the American Nuclear Society, Volume 11, No. 1, (1966), Page 169.

40. A. F. Henry, "Few-Group Approximations Based on a Variational Principle", Nuclear Science and Engineering, Volume 27, No. 3, (1967), Pages 493-510. 
BIBLIOGRAPHY (continued)

41. R. Bobone, "The Method of Solution Functions: A Computer-Oriented Solution of Boundary Value Problems as Applied to Nuclear Reactors - Part I, Cylindrical Reactors in $\rho-\theta$ Geometry", Nuclear Science and Engineering, Volume 29, No. 3, (1967), Pages 337-353.

42. M. Becker, "Assymetric Discontinuities in Synthesis Techniques for Initial value Problems", Nuclear Science and Engineering, Volume 34, No. 3, (1968), Page 343. 


\author{
CARONTE - The EURATOM System for \\ Automatic Control of Linked Calculations \\ G. Buccari - G.Fattori - C.Mongini-Tamagnini \\ EURATOM JOINT NUCLEAR RESEARCH CENTER \\ ISPRA, ITALY
}

\title{
INTRODUCTION
}

Given a group of programs, all concerned with a same field of science (Nuclear Reactor Programs, Civil Engineering Programs, Health and Safety Programs, ...) it happens quite frequently that the solution of a certain problem requires the run of a sequence of programs chosen in a given group, with mutual exchange of data among them.

The procedure followed in the past, when the available computers were the so called "first generation computers", was a very cumbersome one since manual interventions were necessary between nearly each program being run, in order to complete the input data of the program to be executed, starting from the results just obtained. In such a procedure the possibility of errors, besides, was quite remarquable.

Since the advent of the "second generation computers", being in respect to the previous ones much faster and bigger, the interest in the preparation of compilers adapted to the automation of the run of sequences of programs, has increased rapidly in all the world. An example in this direction was studied early in 1962 at the Martin Marietta Company where the APWRC (Army Pressurized Water Reactor Code) system was set up, consisting of four basic programs which were arbitrarily used individually or in a prescribed automatic combination.

However it was only with the advent of the "third generation computers" that actual detailed systems for the automation of sequences were studied throughout the world. The more advanced systems of the kind are specifically constructed for the field of nuclear reactor research and they are being developed in the United States (e.g. ARC $(1)$ and NOVA $(2)$ systems).

Generally such systems control the run of sequences of programs specially written to solve separate reactor problems (Cross section averaging, diffusion and transport calculation, burnup determination, ...). The programs inserted in the system are all written in such a way that the input data and the output results (classified in cross sections, fluxes, isotope concentrations, ...) are standardized, and stocked in a fixed format in an auxiliary memory so as to allow any program of the sequence to directly read the results of any pertinent program previovsly run.

The CARONTE system strongly differs from the existing ones in its main principle, that for the class of programs to be inserted in the CARONTE "PROGRAM LIBRARY", the data to be transferred between the programs need not be classified in a fixed format. Consequently, a program needs no previous significant modifications since its input data and output results do not have to be standardized in any way. They have only to be grouped in numbered sets called DATA SETS, to be transferred, under CARONTE control, to and/or from a DATAPOOL 
stored in auxiliary memory.

Due to this, the DATA SETS stored in the DATAPOOL by the executed programs may have to be mixed and elaborated to form an input DATA SET to a next program. Such an operation is performed, according to the wish of the user, by suitable TRANSFER PROGRAMS running under CARONTE control, and set up for each field of application. The possibility of relying on TRANSFER PROGRAMS for the elaboration of DATA SETS, has been shown to be extremely advantageous since it permits the handling of programs of any class, without previously studying a classification of data transfers in the class, and with a minimum of modification.

THE DATA SETS

More in particular the logical organization of CARONTE is the following. Each program of the CARONTE "PROGRAM LIBRARY" is identified by a name, consisting of up to 8 alphanumeric characters, followed by an ordering number whenever the same program appears more than one time in the considered sequence of programs. Each program contains numbered groups of DATA SETS each of which can be read directly from the DATAPOOL and/or written on it, following indications transmitted by the CARONTE system (in reactor field DATA SETS can be, as an examp.e, energy groups fluxes, self shielding factors, cross sections...).

Whenever a group of data is written from a program on the DATAPOOL, it is automatically labelled by the name of the program itself and the number of the considered DATA SET. It must be noted besides that whenever a program searches a group of data on the DATAPOOL, the label consisting in the name of the program itself and the number of the considered DATA SET, is checked out. As a consequence whenever DATA SET $N$ of program ALFA is directly required by program BETA for its DATA SET $M$, the CARONTE system must automatically change the label "ALFA-SET $N$ " written by program ALFA to the label "BETA-SET M" checked out by program BETA. The INPUT CONTROL cards to be prepared Dy the user must just state:

BETA-SET M FROM ALFA-SET N .

However, as already stated, since input data and output results of the programs are not standardized in any way, it happens very frequently that a DATA SET required by a program of the sequence must be obtained by elaboration of one or more DATA SETS stored on the DATAPOOL. Such an elaboration is performed by TRANSFER PROGRAMS, written for every field of application of CARONTE, running automatically under $\mathcal{E}$ iRONTE control.

\section{THE TRANSFER PROGRAM}

In order to avoid that the user has to trouble himself with the specifications of the TRANSFER PROGRAMS to be used for the elaboration of the DATA SETS, the following procedure has been established.

First of all the DATA SETS contained in the ensemble of the programs of the "PROGRAM LIBRARY" are ordered in numbered "models", such that two DATA SETS belonging to different programs are said to have the same "model number" when they contain the same kind of data, written in the same way. In the case of reactor programs field, a "model" can represent, as an example, a vector containing energy group fluxes: $\phi\left(E_{1}\right), \phi\left(E_{2}\right) \ldots \ldots \phi\left(E_{N}\right)$ in ascending energies order.

The TRANSFER PROGRAMS operate on one or more "models" to produce another "model". In particular the TRANSFER PROGRAM being able to produce "model" number 
$\mathrm{N}$, is identified by the number $\mathrm{N}$. As an example, taken from reactor field, a TRANSFER PROGRAM can be the one that starting from microscopic thermal cross sections, from microscopic fast cross sections, and from combining specifications, prepares a multigroup cross section library.

In any case the user does not deal with the TRANSFER PROGRAMS, since they are automatically controlled by CARONTE. In fact an indication of the type:

"ALFA-SET N FROM BETA-SET M AND GAMMA-SET L"

causes the system CARONTE first to search for the numbers of the models corresponding to ALFA-SET $N$, BETA-SET $M$ and GAMMA-SET L. Supposing to be $K_{1}, K_{2}, K_{3}$, the system will automatically call for execution of the TRANSFER PROGRAM corresponding to $K_{1}$, being able of operate on models $K_{2}$ and $K_{3}$ to produce model $K_{1}$. The TRANSFER PROGRAM will automatically search on the DATAPOOL the data labelled BETA-SET $M$ and GAMMA-SET $L$, and will write its results as labelled ALFA-SET $N$.

It must be noted that in order to give more flexibility to the system it has been taken into account the possibility that more than one transfer program exists, capable to operate on the same data set models, but following different "laws". In such a case the user of the system must add to the pertinent INPUT CONTROL instructions an indication specifying the law he wants. An example taken as before from reactor field can be the preparation of a macroscopic library containing the diffusion coefficient $D$, starting from a microscopic library and from the isotope concentrations. As known the $D$ term can be calculated in many different ways.

\section{THE TEST PROGRAMS}

A run of CARONTE consists in the execution of a sequence of programs from the "PROGRAM LIBRARY". Sequences may be either specified by the user or be self-developing at execution time. The conditions determing the choice of the next program are tested, according to the wish of the user, by TEST PROGRAMS controlled by CARONTE, and set up for every field of application. Loops nested to a depth of four may be set up and executed until certain conditions indicated by the user are satisfied. Such conditions will also be examined by TEST PROGRAMS. An example taken from nuclear reactors can be an iteration between a space independent thermal cross sections averaging program and a space dependent flux calculation program, until self shielding factors are stabilized.

The DATA SETS stored in the DATAPOOL by a program can be utilized by any program following in the same run, as well as by programs executed (even months later) in successive runs of CARONTE. This permits the formation of complex ramified paths, which can be extended indefinitely.

In the case of a prefixed sequence of programs, the names of the programs to be executed must appear in the INPUT CONTROL instructions: ALFA, BETA, GAMMA.

When the sequence is self developing the names of the programs at the branch must be limited by two slashes. The INPUT CONTROL instruction:

$$
\text { ALFA, BETA, /GAMMA , DELTA, EPSILON/,ETA }
$$

indicates that after the execution of program BETA the pertinent TEST PROGRAM will be called by CARONTE, to decide whether the following program to be executed is to be GAMMA, or DELTA, or EPSILON. The INPUT CONTROL instructions must also specify the TEST PROGRAM to be utilized.

Finally in the presence of a loop, the programs contained in the nest are closed in brackets in the INPUT control instruction; an indication of the following type:

$$
\text { ALFA, (BETA, GAMMA, DELTA), EPSILON }
$$

means that a loop between the programs BETA,GAMMA, DELTA, will be executed until certain conditions specified by the user in the INPUT CONTROL are found to be 
verified by the pertinent TEST PROGRAM.

THE INPUT PROGRAMS

Before the execution of a CARONTE run, the user has of course to prepare for each program of the sequence the input data which cannot be derived from previously executed programs, that is from the DATAPOOL, but which derive from the description of the basic data concerning the problem in consideration (as an example, from the given geometrical and physical configuration of a reactor). Such input preparation is always very cumbersome; anyway when the sequence of the programs to be executed is self developing, such a procedure becomes also absurd, since the user is preparing input data for programs which will be never executed.

As a consequence it is of greater interest to set up a group of programs (called INPUT PROGRAMS) which are capable of deriving the pertinent input data of each program inserted in the system, from the given problem description. The INPUT PROGRAMS, like the TRANSFER and TEST PROGRAMS, ran automatically under the direct CARONTE control, and have to be set up for every field of application of CARONTE itself.

\section{THE INPUT CONTROL}

As has been already mentioned the -nput control consists of:

a) the sequence of the programs to be executed. The sequence may contain slashes or brackets indicating respectively the presence of choices between programs at specific points of the sequence, or loops up to fourth order;

b) in the case of self developing sequence or loops, the indications concerning the TEST programs to be utilized to choose the next program to be executed;

c) the indication specifying, for each program of the sequence, the DATA SETS to be obtained by the DATAPOOL. The TRANSFER programs actually necessary will be automatically called by CARONTE;

d) the indications specifying, for each program of the sequence, the INPUT programs to be utilized.

\section{THE SYSTEM ORGANIZATION}

The CARONTE system has been written for IBM $380 / 85$, and runs under the standard IBM O.S. control.

The ensemble of the programs inserted in the CARONTE system (that is the CARONTE PROGRAM LIBRARY), the TRANSFER PROGRAMS, THE TEST PROGRAMS, the INPUT PROGRAMS, and finally the DATAPOOL are all stored on direct access auxiliary storage. The PROGRAM LIBRARY, the ensemble of the TRANSFER, TEST, INPUT PROGRAMS, and the DATAPOOL are open ended.

The CARONTE system itself is organized in two parts, called NUCLEUS and PROCESSOR. The NUCLEUS, always resident in fast memory ( 2000 bytes), alternatively loads the PROCESSOR and the next program to be execated (see Fig. $N^{\circ} 1$ ).

The PROCESSOR, according to the CARONTE INPUT CONTROL instructions, loads the TEST PROGRAMS, if required, to select the next program of the sequence. Besides, the PROCESSOR loads the TRANSFER and INPUT PROGRAMS related to it, in order to prepare the input DATA SETS, which can be derived respectively from the DATAPOOL and from the initial problem description. Finally the PROCESSOR transmits information to the NUCLEUS concerning the flow of DATA SETS of the next program from and/or the DATAPOOL. 


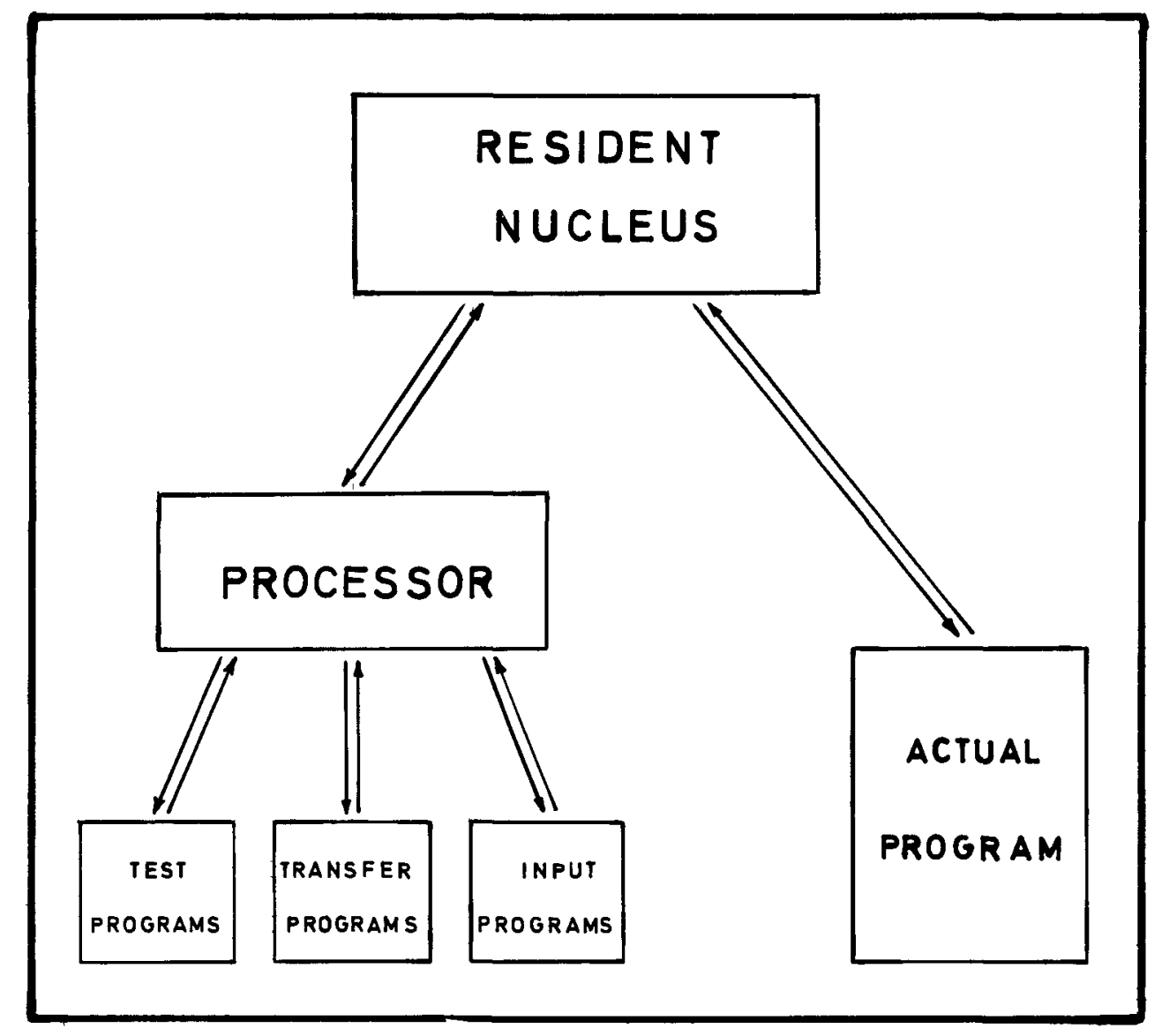

Fig. Nr. 1 - System CARONTE control flow

\section{APPLICATION TO NUCLEAR FIELD}

A certain number of nuclear programs have been chosen from those most utilized and have been adapted to CARONTE. For each of them an appropriate group of data has been selected to be transferred to and/or from the DATAPOOL. They are schematized in Table $\mathrm{N}_{1}$.

Many TRANSFER and TEST programs dealing with elaboration or comparison of DATA SETS have been written.

Finally, in order to avoid to the user the cumbersome numerical INPUT CONTROL instructions accepted by CARONTE, an alphabetic language related to the reactor field, has been developed. One can give instructions, for example, specifying that GAM 22 FAST LIBRARY + GATHER THERMAL LIBRARY + COMBINE SPECIFICATIONS, must be mixed to form the library of DTF4.

A decoding system has been set up, capable of interpreting the alphabetical language and of converting it to the normal numerical control instructions to be supplied to CARONTE. Here again the decoding system is independent from the particular case of reactor programs, since only the "keyword table" is related to the reactor field.

CARONTE has been utilized for various linked calculations concerning 


\begin{tabular}{|c|c|c|}
\hline NUCLEAR PROGRAMS AND SELECTED DATA SETS & $\begin{array}{l}\text { PROGRAM } \\
\text { to } \\
\text { DATAPOOL }\end{array}$ & $\begin{array}{l}\text { DATAPOOL } \\
\text { to } \\
\text { PROGRAM } \\
\end{array}$ \\
\hline $\begin{array}{l}\text { GAM } 22 \text { (Fast cross sections determination) } \\
\text { 1. Source to thermal range } \\
\text { 2. Microscopic fast library } \\
\text { 3. Isotopes concentration }\end{array}$ & $\begin{array}{l}x \\
x \\
x\end{array}$ & \\
\hline $\begin{array}{l}\text { GATHER } 2 \text { (Thermal cross sections determination) } \\
\text { 1. Source to thermal range } \\
\text { 2. Self shielding } \\
\text { 3. Microscopic thermal library } \\
\text { 4. Isotopes concentrations }\end{array}$ & $\begin{array}{l}x \\
x\end{array}$ & $\begin{array}{l}x \\
x\end{array}$ \\
\hline $\begin{array}{l}\text { COMBINE (Complete cross sections library specifications) } \\
\text { 1. Combining specifications }\end{array}$ & $x$ & \\
\hline $\begin{array}{l}\text { DTF } 4 \text { (One dimensional transport criticality) } \\
\text { 1. Microscopic library } \\
\text { 2. Material and fluxes per zone } \\
\text { 3. Fluxes per space interval }\end{array}$ & $\begin{array}{l}x \\
x\end{array}$ & $\begin{array}{l}x \\
x\end{array}$ \\
\hline $\begin{array}{l}\text { 2DF (Two dimensional transport criticality) } \\
\text { 1. Microscopic Library }\end{array}$ & & $x$ \\
\hline $\begin{array}{l}\text { THERMOS (Cell transport) } \\
\text { 1. Microscopic thermal library, isotopes concentrations }\end{array}$ & $x$ & \\
\hline $\begin{array}{l}\text { THERINT (Thermos library specifications) } \\
\text { 1. Thermos library interpreting }\end{array}$ & $x$ & \\
\hline $\begin{array}{l}\text { EQUIPOISE } 3 \text { (One dimensional diffusion criticality) } \\
\text { 1. Macroscopic library for composition } x \\
\text { 2. Macroscopic library }\end{array}$ & & $\begin{array}{l}x \\
x\end{array}$ \\
\hline $\begin{array}{l}\text { GAZE } 2 \text { (Two dimension diffusion criticality) } \\
\text { 1. Microscopic library } \\
\text { 2. Fluxes per space interval }\end{array}$ & $x$ & $\begin{array}{l}x \\
x\end{array}$ \\
\hline $\begin{array}{l}\text { GAD (Zero dimensional burnup) } \\
\text { 1. Microscopic library }\end{array}$ & & $x$ \\
\hline $\begin{array}{l}\text { GAMBLE (Two dimensional diffusion criticality) } \\
\text { 1. Microscopic library }\end{array}$ & & $x$ \\
\hline
\end{tabular}

Table No. 1

The Data SET flow of the nuclear programs inserted in CARONTE 


\section{APPLICATION TO OTHER FIELDS}

The CARONTE system can be applied to any ensemble of scientific or commercial programs, with minor modifications to the existing programs, by the setting up of convenient TRANSFER, TEST, INPUT PROGRAMS.

One can imagine many fields of applicability (for instance the field of heat transfer and engineering problems). However we will mention here a field in which CARONTE is being utilized at the moment at Ispra, that is the field of theoretical design of ion accelerators.

Let's imagine to possess a collection of programs for the potential calculation in various geometrical and physical configurations of the ion source, of the lenses, of the magnet, of the focalising system. A combination of these programs according to the actual characteristics of the machine to be designed, followed by a ray tracing program, would permit an automatic calculation of a given accelerator.

\section{BIBLIOGRAPHY}

1 - C.N. Kelber, G. Jensen, L. Just, J.B. Toppel - "THE ARGONNE REACTOR COMPUTATION SYSTEM, ARC" - Conferencia International Sobre Utilizacion de Reactores de Investigacion y Computo y Matematicas de Reactores - Mexico, May 1967

2 - E.D. Reilly, Jr., W.H. Tumer - "THE AUTOMATION OF REACTOR DESIGN CALCULATIONS AT THE KNOLL ATOMIC POWER LABORATORY" - Conference on the APplication of Computing Methods to Reactor Problems - Argonne, May 1965 


\section{THE CODHOC SYSTEM}

A Modular System of Subroutines for Reactor Calculation

$$
\text { Jean-Paul BAYARD - Roger BOUDET }
$$

Commissariat à l'Energie Atomique - FRANCE 


\section{I - INTRODUCTION}

The codes traditionally used for reactor calculation have two major drawbacks :

1) The user can not modify a code in order to adapt it to a particular problem.

2) Even for the author of the code seemingly small modifications can necessitate an almost complete change in the logical structure of the program (e.g. reorganization of the Input-output).

For these reasons, it was decided at the Computing Departement of the "Commissariat à l'Energie Atomique" to design a new system for reactor calculation based on two concepts : a subroutine library and standardisation of data.

Systems written in other centers, e.g. the ARC System of A.N.L. bear resemblances to our system with regard to overall philosophy.

The CODNUC System has been written for the IBM 360 series (models 65 and 75 ). However we have attempted to have programs as independant as possible of machine or operating system constraints. Many functions specially those concerning the Auxilliary system (see paragraphe III) could have been performed by operating system resources; we have purposely limited ourselves to the possibilities offered by FORTRAN IV, in order to facilitate the adaptation of our system to other machines.

In addition the system was conceived in order to

1) Have a large flexibility enabling users to perform all kinds of reactor calculation.

2) Be able to treat date transmission between two jobs and data transmission between two modules of a program, in a uniform way. of data.

3) Have the fewest possible restriction on the quantity

4) Be able to write subroutines capable of doing calculation for almost any number of points, energy groups, materials, etc...

The flexibility is obtained by separating functions (e.g. 2D. diffusion calculation) from the data (e.g.cross-sections). We find therefore in CODNUC:

- Data modules defined by certain physical characteristics

- A library of specialized subprograms

- An auxilliary data management system

The independance vis-à-vis the operating System is obtained in the following way:

- The internal data are placed on zones of volumes, chosen by the user. The physical nature of these volumes can be magnetic tape or magnetic disk, (the rersion of IBCOM used at our center even allows one to use a part of main storage for this purpose). 


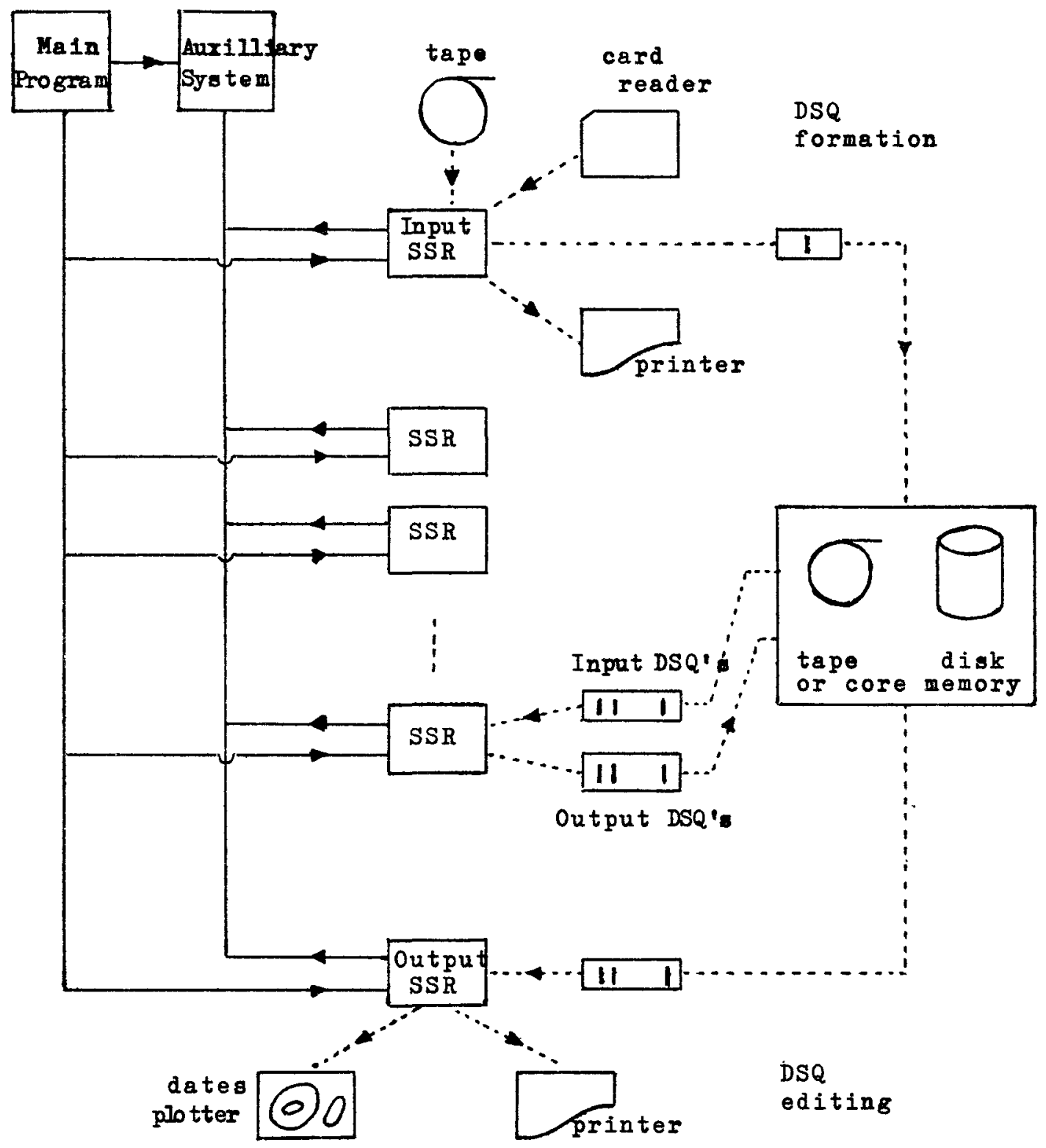

$\begin{array}{ll} & \text { program condections } \\ \ldots . . . . . & \text { trangfer of data }\end{array}$ 
- The Standard Subroutines (SSR) constituting the library and the Auxilliary System (AS) can be in the CODNUC library, in a private library, or read from cards. A calculation can use subroutines from all three of these sources.

- The SSR's are adjustable to storage resources.

The user's principal task is to write a main program which, in the simpler cases (e.g. Flux Calculation) is limited to a series of SSR calls preceeded by a few initializing instructions (see an$n \in x$ ) and which, in more complicated cases (Burn Up, criticality search) may need a good part of FORTRAN possibilities.

In addition an effort has been made in writing the input SSR's in order that the data on cards can be presented simply in a quasi-symbolic language.

\section{II - DATA (Data Sequences)}

The internal data, i.e., data transmitted from one SSR to another, have been divided into a certain number of modules called Data Sequences.

A Data Sequence (DSQ) can be considered as a series of logical FORTRAN records having a well defined structure.

The DSQ's are divided into categories, each corresponding to a type of quantity (Geometrical description of a reactor, cross sections, Flux, etc...). Each category is given a DSQ number.

In order to distinguish DSQ's having the same DSQ number we use an inder whose value is defined by the user.

During the execution of a problem, the DSQ's are placed in the zones associated with the FORTRAN logical numbers. The users are completely free in the choice of zones. The only information needed by the AS in order to exploit a DSQ is the FORTRAN logical number of the zone containing, or to contain, the DSQ. The correspondance between this number and the name of the data set associated with the zone is established by the usual means employed by the operating system. For example, for the 360 operating System this correspondance is established by DD cards written by the user who chooses also the names of his data sets.

It results from the preceeding that the exploitation of a DSQ by a SSR is the same whether the DSQ has been written on tape during an earlier job or written on a disk by another SSR during the same job.

The three pieces of information needed to exploit a DSQ in the course of a problem : DSQ number, index, FORTRAN logical number, are associated during the problem with a single number, the DSQ reference number which characterizes the DSQ during the problem.

\section{III - THE AUXILLIARY SYSTEM (AS)}

The AS is a set of subroutines, written in FORTRAN IV, which enable one to be positionned at the beginning of a logical record belonging to a DSQ. It achieves thusly the simulation of direct access to DSQ's. 
This possibility is achieved by the following means. Each record of a DSQ is preceded by a control record witten by the AS and used only by it to search for records. To enable the AS to do this the control record contains the DSQ number, the index and another number, the record type number. Records containing different kinds of information and belonging to the same DSQ will have different type number. The reading and writting of control records is done by subroutines belonging to the AS. The data records are read and written by the ordinary READ and WRITE instructions.

For example, in order to read the next record of type 4 belonging to a DSQ whose reference number is IREF one would use the following two instructions

$$
\begin{aligned}
& \text { CALI WLECED (IREF,4,\$10) } \\
& \text { READ (NLF) A,B,C, ... }
\end{aligned}
$$

$\$ 10$ corresponds to a return desired in the case where there are no more records of type 4 in the DSQ. The value of NLF is automaticalIy defined by the subroutine WLECED and transferred by a COMMON block - NLF is the FORTRAN logical number given when defining IREF.

Schematically the subroutine WLECED works in the following way. The AS reads the control records one by one, starting with the control record following the last control record read, until a record of type 4 is found, belonging to the DSQ whose reference number is IREF. All the data records met, which belong to other DSQ's or have other types, are ignored.

This organization permits the user if he wishes, to place several DSQ's in the same Data Set, each DSQ being managed by the AS as if it were alone in the Data set.

Another interesting possibility is the following. It is possible to add, in order to satisfy new needs, new types of records to the structure a DSQ. The SSR's using the DSQ with its former structure can still be used, since the new types of records will simply be ignored by these SSR's.

The detailed list of all the instructions relative to the AS is described in a manual distributed to programmers who write SSR's. It is not necessary for the user to be acquainted with all these instructions. The only ones that he needs are the following two which must appear in the main program.

Before using a DSQ for the first time in a problem, the user must use the initializing instruction

CALL WINIST (IREF, NDSQ, IND, NLF)

where IREF is the reference number (positive integer $\leqslant 20$ ), NDSQ the DSQ number, IND the index, an arbitrary positive number, and NLF the FORTRAN logical number associated with the data set containing the DSQ.

Before placing a DSQ on a zone which does not yet contain a $D S Q$, the user must use the following initializing instruction

CALL SRV6 (NLF)

Where NLF is the FORTRAN logical number of the zone. If there are already DSQ's in the zone, they will be unreadable after the call. 
IV - THE STANDARD SUBROUTINES (SSR)

The CODNUC SSR's must obey the following rules

- Every SSR is written in FORTRAN IV

- All of its internal data are in the form of DSQ's and are read and witten with the help of the AS.

- The user has to sypply the storage needed by the SSR. The SSR will have an array whose dimension is adjustable.

- The list of arguments has the following form $N I 1, N I 2, \ldots, N \phi 1, N \phi 2, \ldots, A, I A$

where $N I 1, N I 2, \ldots, N \phi_{1}, N \phi 2, \ldots$ are $D S Q$ reference numbers for input data and for output data, $A$ is the adress of the work zone applied to the subroutine, $L A$ is its length.

- In principle almost all data needed by a SSR is located in DSQ's. However, if some necessary information, such as convergence tests, is not, it will be transmitted by a labeled common appearing in the SSR.

In order to insure a compatibility between different SSR's and between SSR's and other subroutines it has been necessary to impose rules regarding the names of the SSR's and the names of labeled commons.

The SSR's can be divided into four distinct classes according to the nature of their functions: Input, Computation, Edition Utilities. An SSR may divide his task into several parts and have each part taken care of by a (FORTRAN) Subroutine. These subroutines will not be known to the user.

\section{Input SSR's}

This type of SSR transforms non-standard data into standard data (i.e. a DSQ) or a DSQ into another DSQ. For each DSQ used as input data for a computational SSR there are one or several input SSR's capable of creating the DSQ.

In regard to data presentation on cards, we have very flexible rules thanks to a special subroutine that reads and decode the data. This subroutine allows one to use keywords in order to characterize the numerical data which follows, and frees one to a large extent from format considerations.

\section{Computational SSR's}

With the aid of DSQ's, an SSR of this type modifies certain DSQ's and/or creates new ones. The more important SSR's in this category replace a physical problem by a numerical model. (e.g. Flux calculation by $\mathrm{Sn}$ method). These SSR's never use data from cards.

\section{Edition SSR's :}

An SSR of this type exploits one or several DSQ's in order to furnish results that are printed, plotted, or projected on a screen. (e.g. fluxes, map of a reactor, contour lines, ...).

\section{Utilities :}

It is important to be able to manipulate DSQ's independantly of their structure. This type of SSR is used in order to 
transfer a DSQ from one data set to another, to update data sets by removing certain DSQ's, to print the content of a series of DSQ's, etc...

$V-$ ANNEX

\section{1 - Example of a main program ( 1 dimension diffusion)}

We suppose that the user wants to effectuate a 1 dimensional diffusion calculation by using the SSR DF1X. DF1X needs the following DSQ's.

\begin{tabular}{|c|c|c|c|}
\hline \multirow{4}{*}{\multicolumn{2}{|c|}{ Input }} & DSQ Number & 100 \\
\hline & & End point conditions & 200 \\
\hline & & Macroscopic cross-sections & 300 \\
\hline & & Initial Flux & 400 \\
\hline & Output & Final Flux & 400 \\
\hline $\begin{array}{l}\text { tions, and } \\
\text { flux a DSQ } \\
\text { flux on the } \\
\text { tions, a wo } \\
\text { DF X, in ad } \\
\text { gence param }\end{array}$ & $\begin{array}{l}\text { He wants } \\
\text { cross-secti } \\
400 \text { from an } \\
\text { same volum } \\
\text { ork zone of } \\
\text { dition, nee } \\
\text { neter EPS wh }\end{array}$ & $\begin{array}{l}\text { to create the DSQ's geometry, end point } \\
\text { ions, from data on cards, use for the ir } \\
\text { earlier calculation, and place the fir } \\
\text { ne. In order to effectuate the desired } \\
40 \text { ooo words is sufficient. The subrout } \\
\text { eds an estimate VPA for the Beff and } \\
\text { hich will be transmitted to it by the }\end{array}$ & $\begin{array}{l}\text { at condi- } \\
\text { initial } \\
\text { inal } \\
\text { calcula- } \\
\text { utine } \\
\text { conver- } \\
\text { labeled }\end{array}$ \\
\hline
\end{tabular}

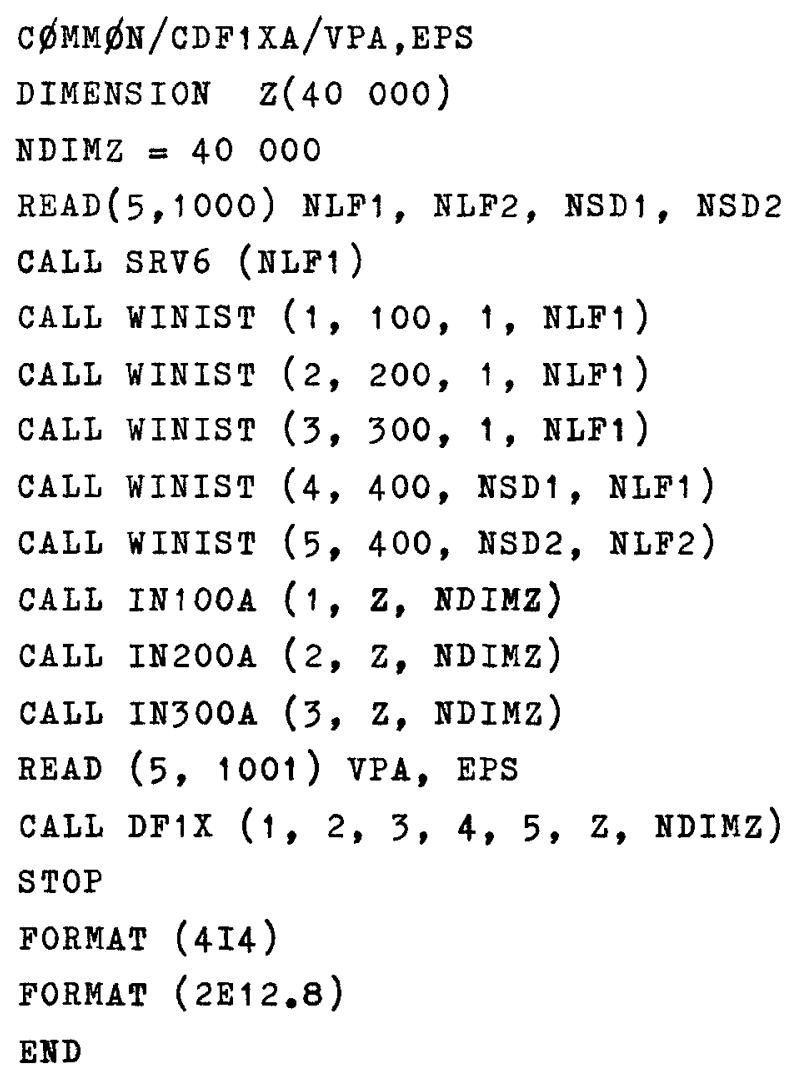


is the three digit number following IN in the name of the SSR unless it is stated otherwise).

\begin{tabular}{|c|c|c|c|c|}
\hline & \multicolumn{3}{|c|}{ Name } & Nature of DSQ created \\
\hline & IN & 100 & A & Geometry for 10 calculation \\
\hline & $\begin{array}{l}\text { IN } \\
\text { IN }\end{array}$ & $\begin{array}{l}110 \\
120\end{array}$ & $\left.\begin{array}{l}A \\
A\end{array}\right\}$ & Geometry for $2 D$ calculation \\
\hline & IN & 151 & A & $\begin{array}{l}\text { Criticality search by macroscopic cross-sections } \\
\text { adjustment }\end{array}$ \\
\hline & IN & 200 & A & Boundary conditions for $1 D$ diffusion calculation \\
\hline & IN & 220 & A & n $2 \mathrm{D}$ \\
\hline & IN & 230 & A & " 2D transport calculation \\
\hline & IN & 300 & A & $\begin{array}{l}\text { Macroscopic (or microscopic) cross-sections ar- } \\
\text { ranged by materials ; for each material, all e- } \\
\text { nergy-groups. Data given by cards. }\end{array}$ \\
\hline & IN & 300 & B & The same as IN $300 \mathrm{~A}$. Data given by tape. \\
\hline & IN & 300 & C & $\begin{array}{l}\text { DSQ } 300 \text { obtained from another DSQ } 300 \text { by elemina- } \\
\text { tion of certain materials. }\end{array}$ \\
\hline & IN & 3001 & $D$ & $\begin{array}{l}\text { DSQ } 300 \text { obtained by selecting certain materials } \\
\text { from a group of DSQ } 300 / \mathrm{s}\end{array}$ \\
\hline IN & $300 \mathrm{E}$ & $-I N$ & N $302 \mathrm{E}$ & $\begin{array}{l}\text { DSQ } 300 \text { formed from a DSQ } 302 \text { (cross sections ar- } \\
\text { ranged by energy-groups ; for each energy group, } \\
\text { all the materials) and conversely }\end{array}$ \\
\hline & IN & 300 & $F$ & $\begin{array}{l}\text { Macroscopic cross-sections calculation from mi- } \\
\text { croscopic cross-sections and materials composi- } \\
\text { tion. }\end{array}$ \\
\hline & IN & 301 & $\mathrm{C}$ & Materials composition \\
\hline & IN & 400 & A & Flux \\
\hline & IN & 401 & $\mathrm{~B}$ & Flux. DSQ 400 formed from a DSQ 401 and conversey \\
\hline & IN & 510 & A & External sources \\
\hline & IN & 920 & $\mathrm{C}$ & Depletion chains \\
\hline
\end{tabular}

3 - Computational SSR's

\begin{tabular}{|c|c|c|}
\hline $\mathrm{Name}$ & Purpose & DSQ's used \\
\hline $\mathrm{DF} 1 \mathrm{~A}$ & 1 D diffusion, multigroup, homogeneous & $100,200,302,400$ \\
\hline $\mathrm{DF} 1 \mathrm{~B}$ & $\begin{array}{l}1 \text { D diffusion, multigroup, homogeneous } \\
\text { and inhomogeneous }\end{array}$ & $100,200,300,510,400$ \\
\hline $\mathrm{DF} 2 \mathrm{~A}$ & $\begin{array}{l}2 \text { D diffusion, multigroup, homogeneous } \\
\text { and inhomogeneous }\end{array}$ & $120,220,302,401$ \\
\hline$D F 2 W$ & 2 D diffusion multigroup, homogeneous & $110,220,300,400$ \\
\hline CRT 1 & Composition criticality search & 151 \\
\hline TR $1 \mathrm{~A}$ & 1 D transport (adaptation of DTF4) & $100,300,400$ \\
\hline TR2A & $\begin{array}{l}2 \text { D transport multigroup, homogeneous } \\
\text { and inhomogeneous }\end{array}$ & $120,230,302,400,510$ \\
\hline
\end{tabular}




\begin{tabular}{|c|c|c|}
\hline ADJ1 & $\begin{array}{l}\text { transforms DSQ's } 200 \text { (end point condi- } \\
\text { tions) and } 300 \text { (cross sections) for an } \\
\text { adjoint flux computation }\end{array}$ & \\
\hline $\operatorname{ADJ} 2$ & $\begin{array}{l}\text { arranges the flux in the correct order } \\
\text { after an adjoint flux computation }\end{array}$ & 400 \\
\hline COLI & boundary conditions & \\
\hline CAIN & $\begin{array}{l}\text { integrals of product of pairs of fluxes } \\
\text { (for synthesis calculation) }\end{array}$ & \\
\hline EVLA & burn up & \\
\hline FNRA & flux normalization & \\
\hline
\end{tabular}

\section{4 - Editing SSR's}

\begin{tabular}{|l|c|}
\hline Name & Purpose \\
\hline EDTA & edition of a DSQ 400 : fluxos, sources, Keff printed \\
EDTB & prints a reactor map \\
\hline
\end{tabular}

\section{5 - Utilities}

\begin{tabular}{|l|l|}
\hline Name & \multicolumn{1}{|c|}{ Purpose } \\
\hline SRVA & selects groups of records on a DSQ \\
SRV1 & copies a DSQ \\
SRV2 & keeps or eliminates certain DSQ's belonging to a data set. \\
SRV4 & $\begin{array}{l}\text { transmits the contents of all the DSQ's belonging to a dat } \\
\text { set : gives the number of records of a DSQ belonging to } \\
\text { each type. } \\
\text { SRV6 }\end{array}$ \\
\hline
\end{tabular}




\section{HITACHI NUCLEAR CODES CONTROL SYSTEM, NCCS}

T. Watanabe, K. Arai and T. Noda

Nuclear Energy Division, Central Research Laboratory, Hitachi, Ltd.

\section{INTRODUCTION}

In the field of nuclear industry, a large number of computer programs have recently been developed utilizing various kinds of nuclear data sets. At present, the demand for precise computations has increasingly caused the complexity of programs and scarcely any problem can be solved without the successive use of different codes. Since most of these codes have been made independently, it is difficult to connect and control each individual nuclear code in order to execute, for instance, two dimensional multi-group burn up calculations. There is a big demand to eliminate difficulties associated with this kind of problem, which has been discussed to some extent in several previously reported articles [1], [2], [3].

The Hitachi Nuclear Codes Control System (NCCS) is one of the solutions to this problem. It is a comprehensive nucear information system with extensive capabilities of data and program management. It unfies and controls different kinds of nuclear data and codes in the use of a highly modulated system.

Firstly it is required, in this system, to establish a data base in which an enormous volume of data necessary for reactor calculations is stored in a form suitable for the efficient data management. To meet this requirement, NCCS contains Sigma Converter I (SC/I).

Secondly, each code in NCCS should be in a modular form for the easy linking and modification. NCCS handles not only newly programmed modules but also those nuclear codes which have previously been programmed for different computers. The Code Translator I ( CT/I) in NCCS translates those codes written for different computers into a form acceptable to the code library of NCCS with the help of hand manipulations.

Thirdly, scheduling and execution of modular computations are controlled by the Code System I (CS/I), the heart of NCCS. As a medium of data communication between modules, a common data strage is provided and is conventionally called Datapool. The structure of the Datapool, however, is automatically constructed and changes during execution depending on module combination and data usage. Since the basic concept of $C S / I$ is not limited to reactor computations, the nucleus of CS/I has been designed so that it may be applicable to many other fields. Computation schedules are described in a special language suitable for the representation of the module linking. 
NCCS is, as a subsystem of the standard operating system, implemented on HITAC 5020F with $131 \mathrm{~K}$ high speed memory [4], an equivalence of CDC 3600 . The conceptual diagram of NCCS is shown in Figure 1. It consists of three parts as mentioned in Section 1:
(1) Code System I
(2) Code (CS/I),
(3) Sigma Converter I (SC/I).

The system components contained in $\mathrm{CS} / \mathrm{I}$ are shown within a square marked by a dotted line in Figure 1. In the case of programming a new module, the main concern is to solve the problem in concern and to maintain the modularity. when integrating an unrelated nuclear code, which will be changed into a modular form, the main concern is the data communication between the code and other modules. The method of data communication between modules is the key to the efficient management of data.

In each module of $\mathrm{CS} / \mathrm{I}$, all I/O operations, except printing and punching, transfer data to or from the Datapool. The Datapool I/O operations are performed by several I/O routines which are common throughout the NCCS system. Input data are once placed into the Datapool. Then, each module gets necessary data from the Datapool and stores intermediate results in the Datapool, where the format of each kind of data is fixed. In some cases, data conversions may be necessary before and after the execution of the calculation in a module in order to adjust the aifference of data formats between the Datapool and the module.

A calculation route between modules is called an inter-module program and an example is shown in Figure 7 of Section 4. In such a program, the Datapool is treated as an extension of the main memory. Main computations are carried out whithin modules, so that the operations necessary in an inter-module program are limited to simple arithmetic operations, branch operations, module callings and $I / O$ operations.

The functions of CS/I are briefly described below. The control tables of the Datapool are built by the Data Editor, and the Code Library is editted by the Code Editor. Under the control of the Nuclear Executor, calculations proceed as follows (See Figure 2):

(1) A given inter-module program is compiled and translated into a pseudo-instruction sequence.

(2) The structure of the Datapool is constructed according to the control tables of the Datapool and the combination of modules.

(3) The pseudo-instructions of the inter-module program are interpreted.

(4) If the current pseudo-instruction specifies to read data, only those input data necessary for the case in concern are placed into the Datapool.

(5) If the current pseudo-instruction specifies to call a module, an $I / O$ control table of the module is constructed and then the module calculation is executed.

The steps from (3) to (5) are repeated as shown in Figure 2 until the end of the programmed procedure.

$\mathrm{CT} / \mathrm{I}$ is a program translator. HITAC 5020F uses a modified FORTRAN language called HARP which covers most functions of IBM FORTRAN 4 . In order to have codes built in the Code Library, they must be changed, if necessary, ina modular form and language translations must be done if they are not written in MARP. Figure 3 indicates these processes. During the process of the program translation, CT/I tabulates various informations useful for 


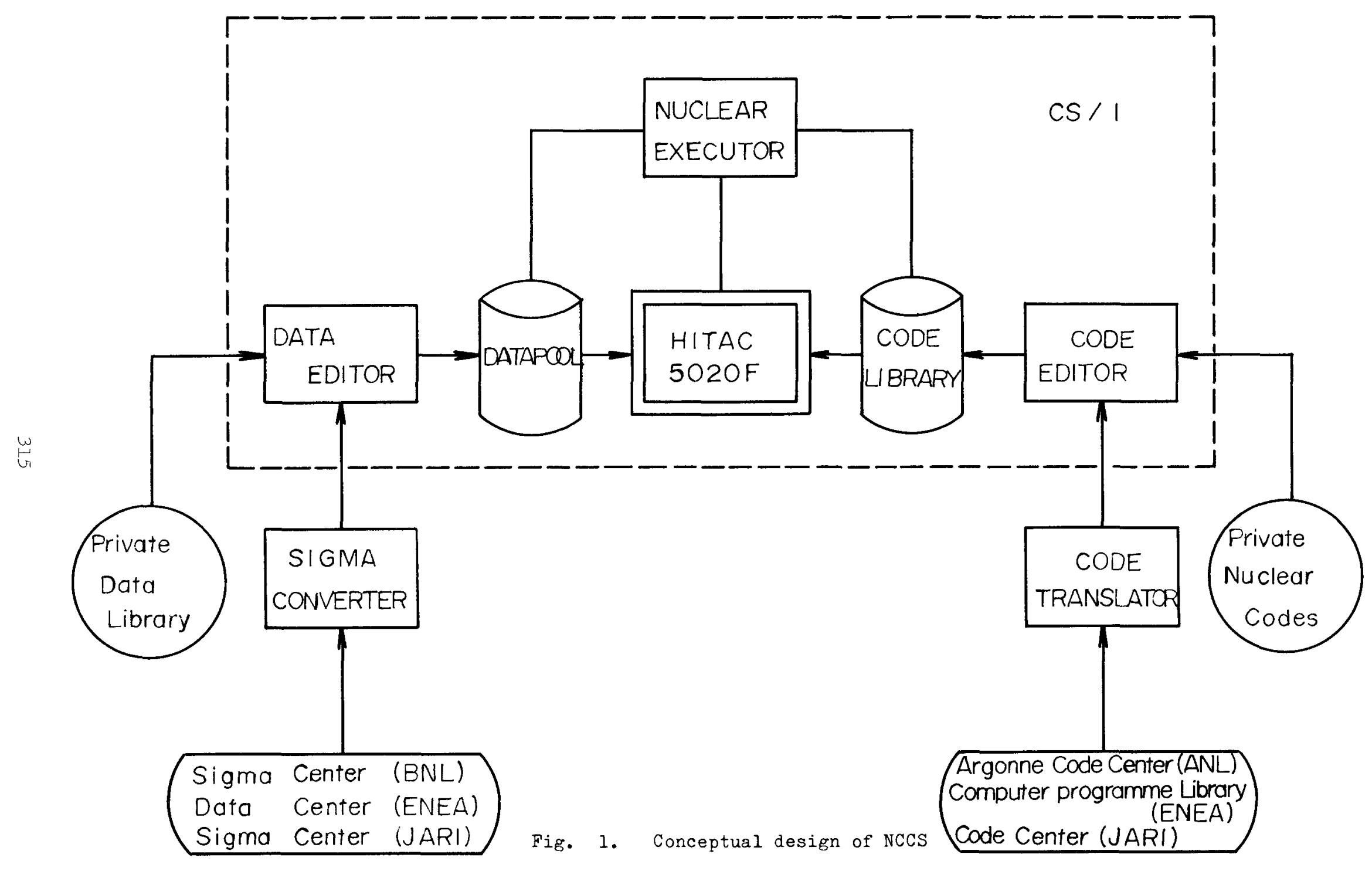




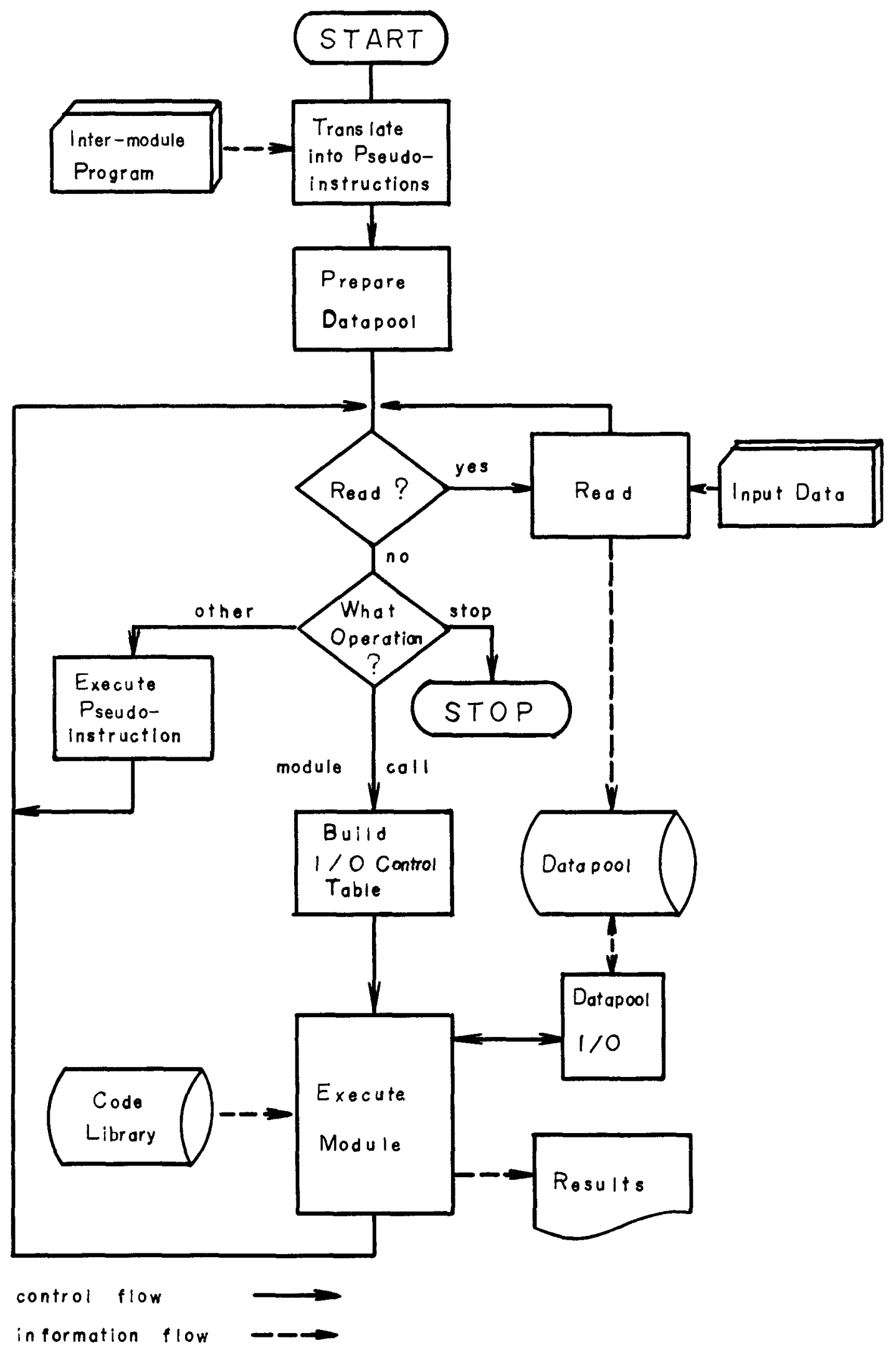

Fig. 2. Operation flow of $\mathrm{CS} / \mathrm{I}$ 
further program modifications as well as tables showing the relation between subprograms within a module.

$\mathrm{SC} / \mathrm{I}$ is a data converter. Since nuclear data are obtained and evaluated by many different institutions, SC/I adjusts the formats of these data to the standard forms of the Data sets in NCCS. Nuclear cross-sections, for example, are processed by SC/I as shown in Figure 4. The nuclear codes such as $M C^{2}$, MUFT and diffusion codes can be used as a part of $\mathrm{SC} / \mathrm{I}$.

As an application of the NCCS system, a reactor computation system for fast breeder reactors (FBRCS/I) is now in the process of testing. Figure 5 shows those codes which have been or will be included in this FBRCS/I.

\section{DATA MANAGEMENT}

In the Datapool, a collection of the same kind of quantities is called a data set (e.g., flux set, diffusion equation coefficient set), and a collection of correlated data sets is called a data block (e.g., geometry block, material block, cross section block). Each data set is treated as one logical unit in all I/O operations. Each data block is assigned a proper block name. Some data sets, however, may have the same set name if they do not belong to the same data block. The Data Editor assigns a data number to each data set so that the number is suitable for quick data reference.

In input data and inter-module programs, the set name is used as the indentifier of the data set. In module programs, a data set corresponds to a variable containing the data number as its value. As the length of a set name is up to 4 characters, the set name can be embedded into the name of this variable, so that both man and machine are able to easily identify the data set.

The actual devices constituting the Datapool are magnetic drums, magnetic disks and magnetic tapes, but the user of NCCS may consider the Datapool to be composed of a lot of "pseudo-tapes", each of which corresponds to one data set. When the Datapool is empty, there is one vacant strage pool in the Datapool and all the length of the pseudo-tapes are zero. When some data are added to a data set, an appropriate amount of the storage is removed from the vacant storage pool and is given to the data set automatically, that is, the length of the pseudo-tape increases according to the amount of data. When the data become unnecessary, the whole or a part of the occupied storage can be returned to the vacant storage pool.

To make the above-mentioned operation possible, drums and disks are divided into the pages of some fixed sizes. When some pages are assigned to a data set, they are formed into a doubly linked list, that is, each non-terminal page has pointers to the next and the previous pages. If a data set is on a magnetic tape, the unused part of the magnetic tape is allotted to this sot during the time when some other data are possibly added to this data set. When a signal is given to notify the end of data addition, the remainder of the magnetic tape becomes a part of the vacant strage pool, so that one magnetic tape is able to contain several data sets. There may exist as many data sets as desired if the total amount of the data contained in these sets does not exceed the capacity of the Datapool.

The operations of pseudo-tapes are performed sequentially by such commands as READ, WRITE, back SPACE, fore SPACE, REWIND, ENDFILE and FREE. 


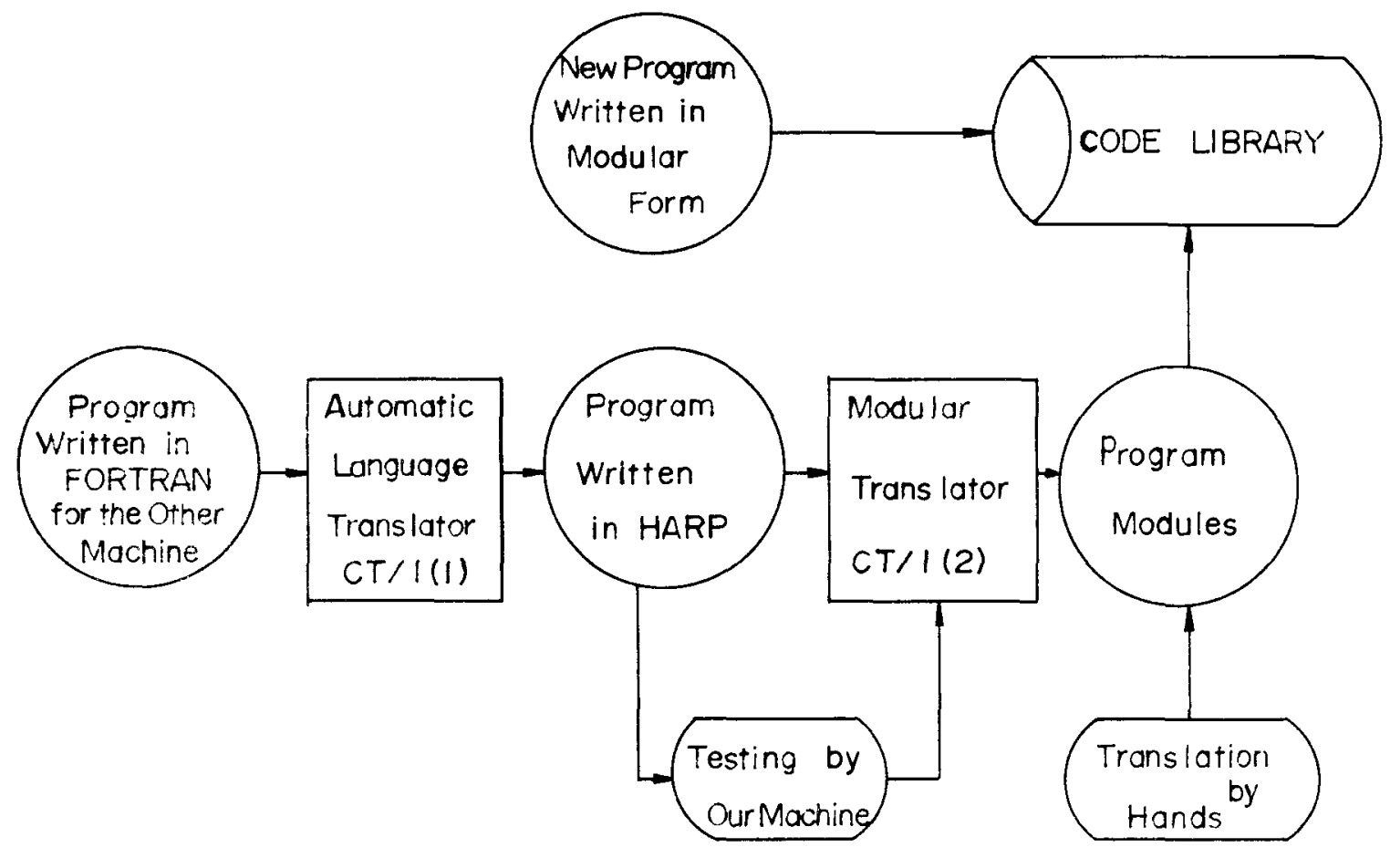

Fig. 3. Conceptual diagram of $\mathrm{CT} / \mathrm{I}$

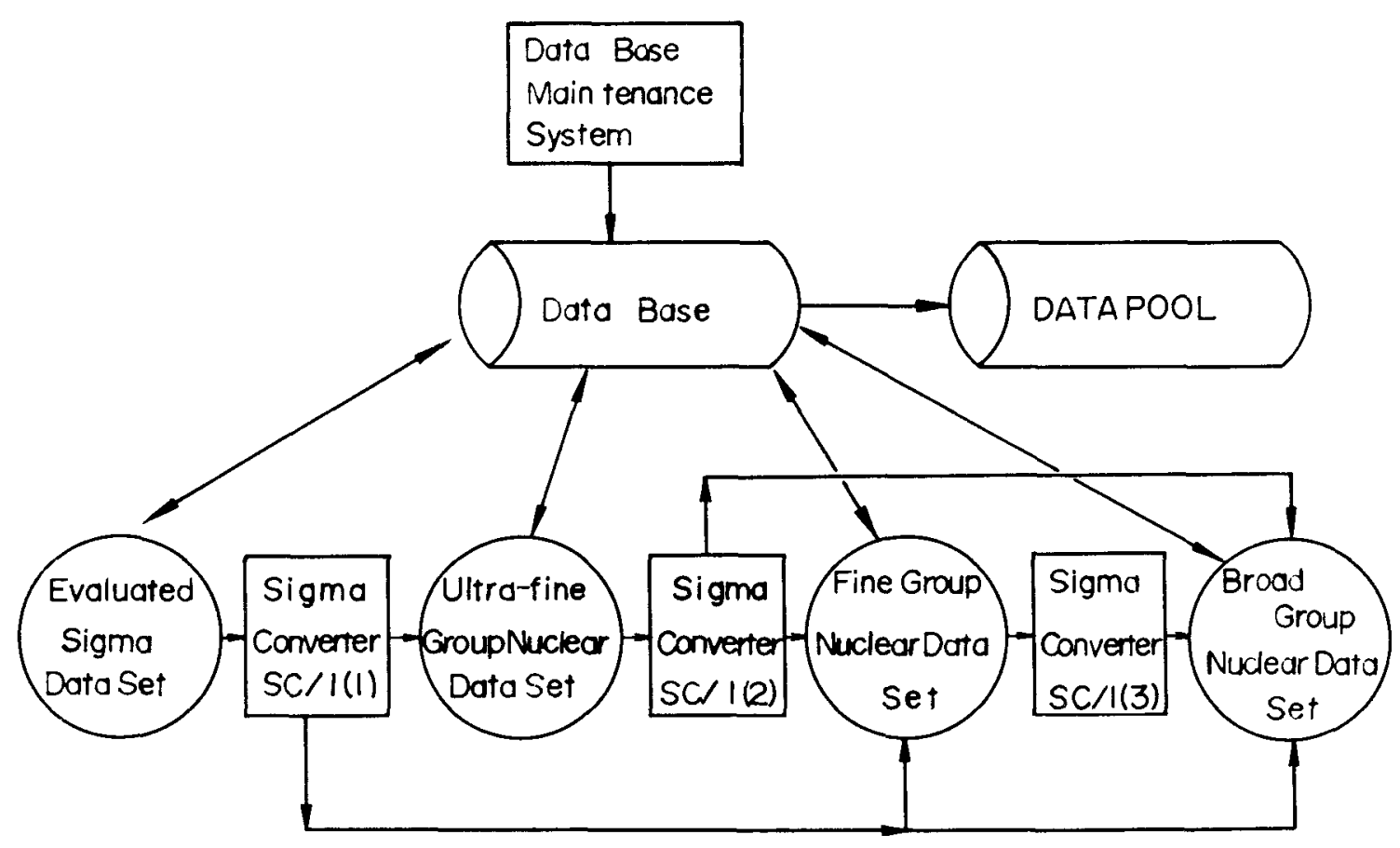

Fig. 4. Process of cross-section degeneration by SC/I 


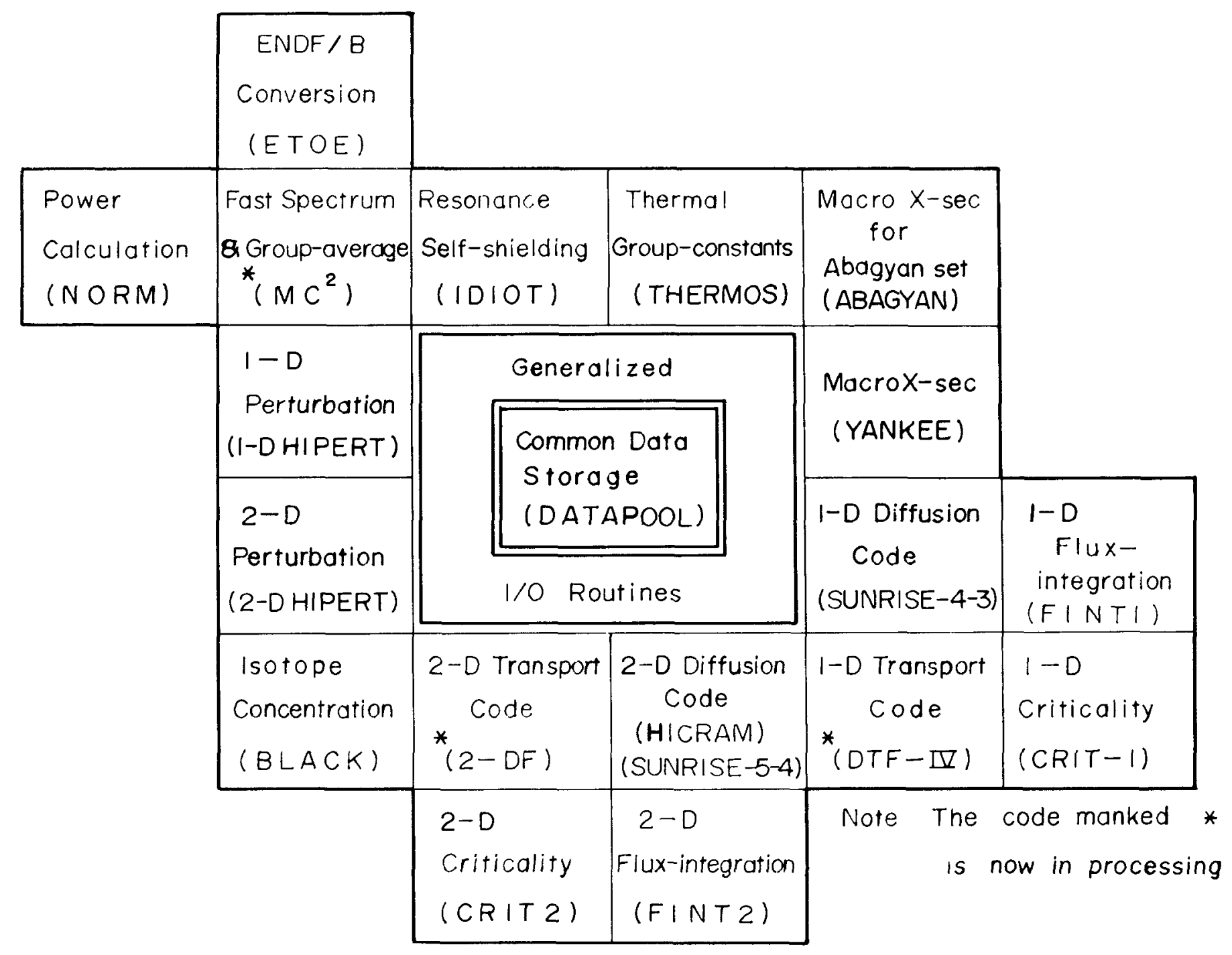

Fig. 5. FBRCS/I 
By the introduction of this pseudo-tape concept, the efficient use of storage spaces can be achieved automatically and all I/0 operations whithin modules can be performed uniformly and machine-independently. This makes it possible to eliminate machine-dependent portions from modules.

Informations of each data set are registered in the Datapool. These informations are of desirable device-type, permissible I/O operation, record size, dimension, data type, initial value, etc. When input data is read, operations such as data-type conversion, data embedding, initial-value filling and apparent-error checking are executed. Some input data such as geometry or material data are reorganized from a form suitable for problem descriptions into a form suitable for module calculations. During this process, insufficiencies of data and inconsistencies between data values are checked. It is remained for each module to verify the applicability of the input data to the module.

The input data format of NCCS is FORMAT-free as shown in Figure 6. It is simple, flexible and easy to write compared with the NAME LIST of FORTRAN and helps visualize the problem to be solved.

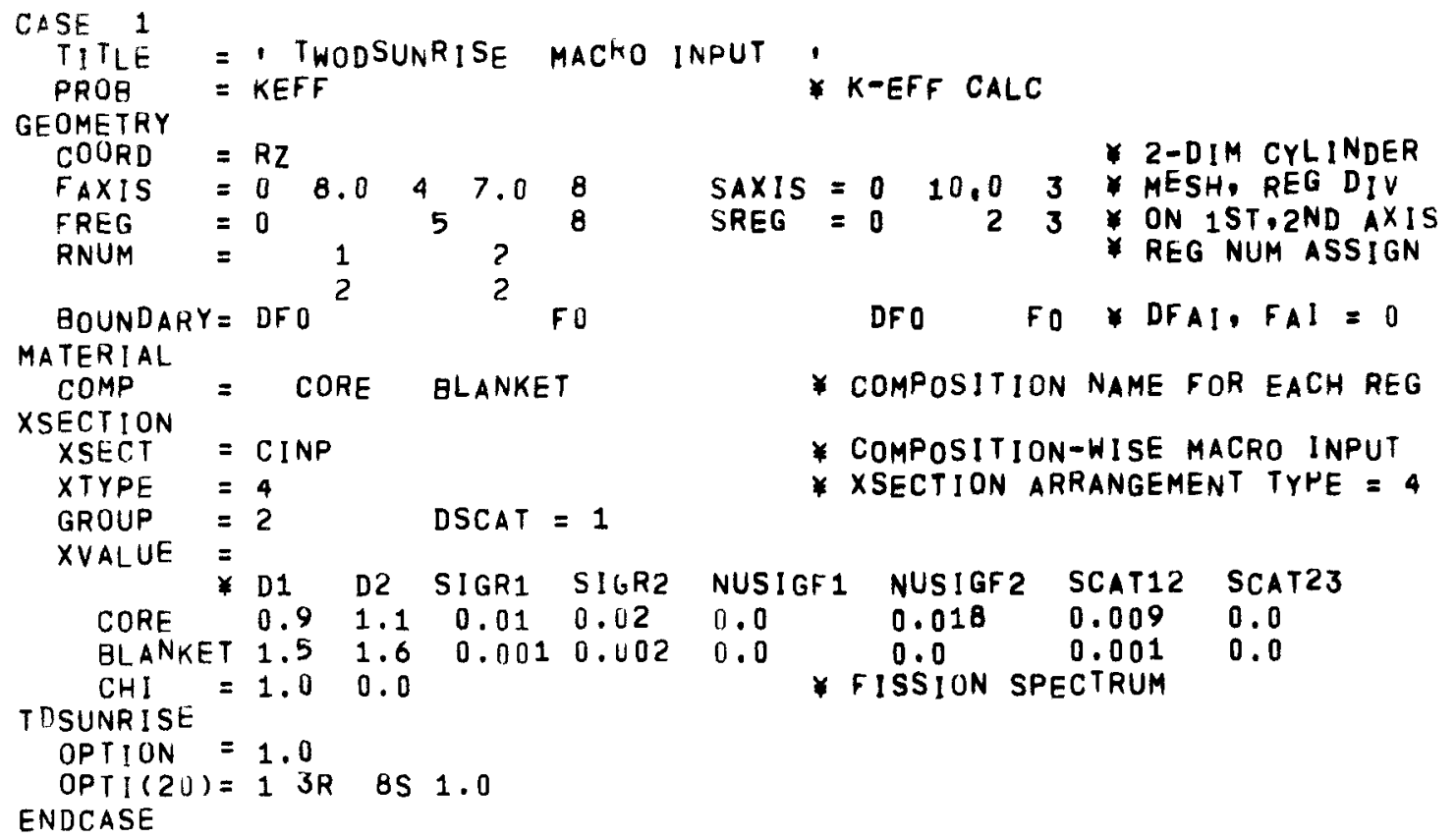

Fig. 6. Sample input data

\section{PROGRAM MANAGEMENT}

The inter-module program language is a small subset of $\mathrm{PL} / \mathrm{I}$ modified with some new data handing features.

In Figure 7, IX, ID, etc., are the Datapool variables. The EXTERNAL declarations mean that the external storages named SYSUT1 and SYSUT2 are allocated to the data sets ABAGYAN and YOM so that the storage locations of these data sets are defined not in the automatic way but in accordance with 


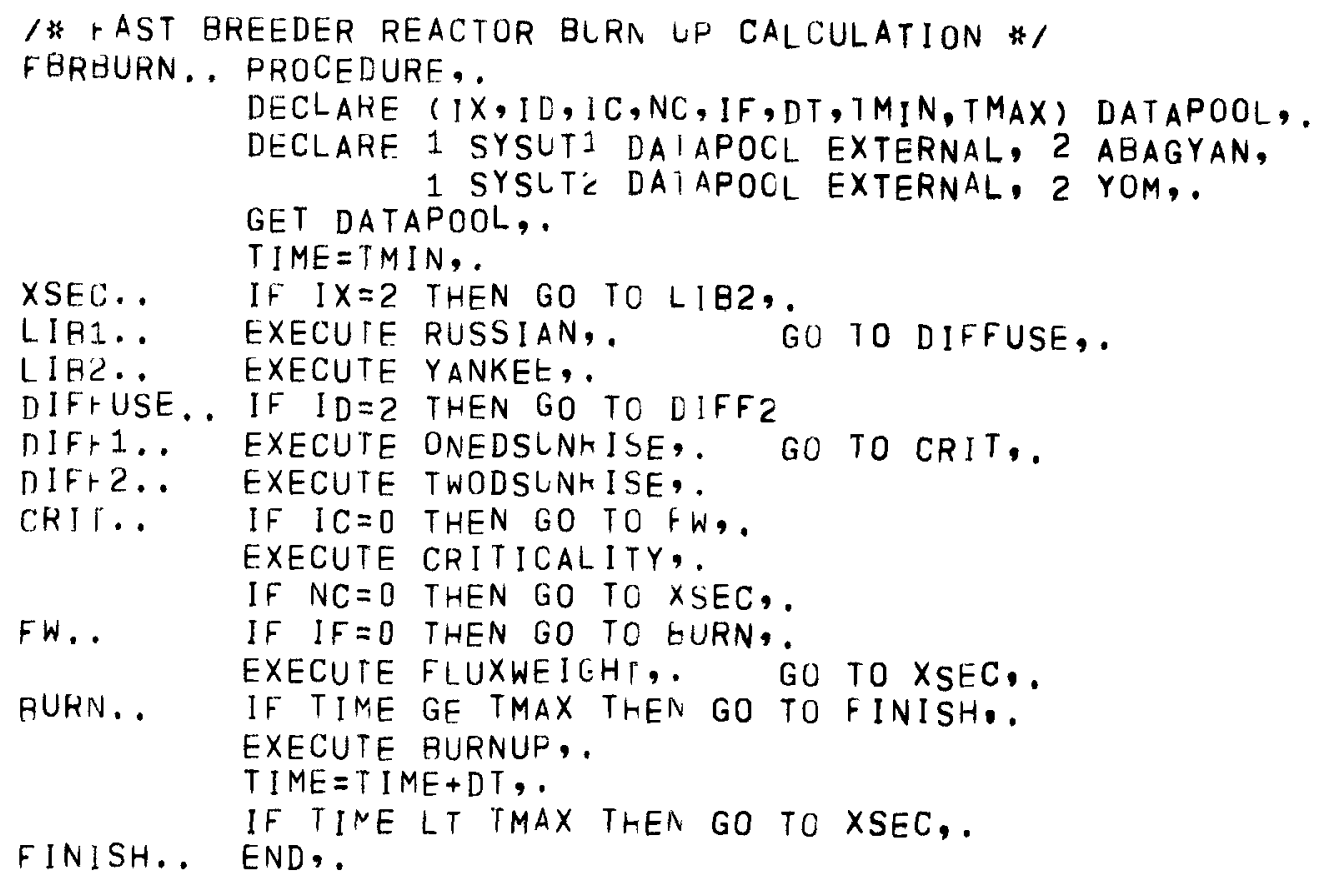

Fig. 7. Example of inter-module program

the user's specification. The declarations of dimension, data type and so on are not necessary for the Datapool variables because they are registered in the Datapool.

Typical inter-module programs are catalogued as standard procedures. The name of a catalogued program is treated in other inter-module programs as if it were a module name.

Each module has a data set list which shows the correspondence of data sets between the module and the Datapool. It is used as an argument list of the module. The data set list is treated in the same manner as an usual data set and its contents can freely be changed if the change causes no inconsistency. If the contents of this list are changed, the module calculation will be performed by using some other data sets according to the specifications given by the new list.

Since modules are not called so frequently as subprograms, the total excution time is not affected by the insertion of table handing operations between module calculations. The amount of the control tables of the Datapool are fairly large, but during the execution of one module, there are not so many tables which are frequently used. When a module is called, the contents of the tables that will be frequently used by this module are collected to construct a resident table typical to that module. At the excution of the module calculation, only this resident table is stored in the main memory and other tables are brought into the main memory only when they become necessary.

In the Code Library, all the programs are stored in the form of machine instructions. When an independent code is built into the Code Library, the 
Code Translator produces such informations as follows:

(I) List of $I / 0$ devices used in the program.

(2) Alphabetically-ordered variable-list describing the usage and attributes of the variables.

(3) Common storage map.

(4) Range of unused statement-numbers.

(5) Subprogram declaration table.

(6) Subprogram reference and argument correspondence table.

(7) Missing subprogram list.

These informations are of great use for modifying and adding new facilities and also for changing the method to solve the problem in concern.

\section{CONCLUSION}

In the process of developing FBRCS/I, it has been realized that the method of data management is the intrinsic problem in linking different programs. To do those data conversions is of ten felt tedious which adjust the difference of data expressions between modules. It is, therefore, necessary to pay much more considerations to the structure of the Datapool, the method of data conversions and the technique of Datapool I/O operations.

If a language has the modularity between subprograms as well as codes, the applicability of NCCS will increase to the great extent. From this point of view, an experimental problem-oriented language has been designed independently of current NCCS. Two outstanding features of this experimental language are of multi-level modularity and of open-ended design. When this new language turns out to be applicable to NCCS, the currently used language will be replaced by this new one. 
6. REFERENCES

(1) E. D. Reilly, Jr. and W. H. Turner, "The Automation of Reactor Design Calculations at the Knolls Atomic Power Laboratory", proceedings of the Conference on the Application of Computing Methods to Reactor problems, 251-260, (1965)

(2) E. Morenoff and J. B. McLean, "Inter-program Communications, Program String Structures and Buffer Files", AFIPS Conference Proceedings Volume $30,175-184$ (1967)

(3) C. N. Kelber, G. Jensen, L. Just and B. J. Toppel, "The Argonne Reactor Computation System, ARC", The paper presented at the Internatioal Conference on Reactor Mathematics Computation, Mexico city, (May 1967)

(4) T. Murata and K. Nakazawa, "Very High Speed Serial and Parallel Computer, HITAC 5020 and 5020E", AFIPS Conference proceedings Volume 26 part I, 187-203 (1964) 


\author{
H. C. Honeck, J. E. Suich, \\ J. C. Jensen, C. E. Bailey, and J.W. Stewart
}

Savannah River Laboratory

E. I. du Pont de Nemours \& Company

Aiken, South Carolina 29801

\title{
1. INTRODUCTION
}

The third generation of computers at most nuclear reactor design installations offers new possibilities to the developers and users of reactor computing codes. In particular, high-speed central processing units are commonplace; almost all systems have large quantities of direct access storage, and many new devices are avaliable to improve the accessibility of the computing system to the reactor designer - such as alphameric display terminals and cathode ray tubes with full graphic capability. The trend in reactor computing systems ${ }^{1, z}$ has been toward utilization of these features of third-generation computers. At the Savannah River Laboratory, the JOSHUA system is being developed to capitalIze on the experience gained with earlier systems, and to include a remote terminal capability.

This paper is a brief introduction to the JOSHUA system. The remainder of this section describes the major considerations in designing such a system and the organization needed to create the system. The operating system is described in the following section, and the last section outines the scientific areas where the system will be first applied.

\subsection{Elements of the System}

JOSHUA is an operating system which provides the data handling services to accomplish extensive, 1terative scientific calculations on a large semipermanent data base. JOSHUA provides standard, "cataloged procedures" for solving routine problems, and allows easy redefinition of these procedures for experimental computation. The user is permitted to intervene between steps of a computation, display results, and possibly redirect the procedure. Individual display terminals for problem setup and output display are central to the design philosophy of JOSHUA, although duplicate facilities are provided for batch mode use where practicable.

The computational system is based on a library of "computational modules" arranged in a varlety of ways to sult the application, and a base of "named data records" of significant computed results and measured values created and displayed by elther the user or modules.

A "named data record" is the smallest collection of logically related information that the user considers a coherent whole. The group of data elements is assigned a unique, qualified name in the natural language of the user's discipline. The logical relationship between records, suggested by the common name qualifiers, is accounted for in the system by cataloging the records as a tree structure. A limited, generally useful set of records is maintained as a protected, read-only "standard" data base. Data created by each active user are cataloged separately. A user may duplicate standard names without overwriting the standard data for other users. Also included in the system is a description of record types so that, upon reference to a record by the name, the data elements can be identified in the display. 
A "computational module" is a logically complete, dynamically executable computer procedure, which receives input from named data records, and places results in other named data records. Since named data records are stored in the system until deleted by the user, they represent information of lasting value (significance) - they are not "scratch," or intermediate computed results. Thus the concept of a logically complete module is equivalent to the concept of a procedure which produces new information of lasting value from old information also of lasting value. Modularity in the conventional sense is achieved by restricting data references to previously defined named data record types.

A "cataloged procedure" is an automated sequence of user data manipulation and module execution that satisfies a particular application. In the simplest case, this will be no more than the creation of input named data records, execution of a single module, and display of output records. In more complicated cases, however, procedures may involve execution of several modules, perhaps even

iteratively. Thus, a procedure is equivalent to a program written in a problemorlented language defined by the functions of the module and terminal commands.

\subsection{Organization}

The JOSHUA Operating System could be used in many areas of scientific computing at Savannah River. Extensive planning and organization is necessary before embarking on a large program to develop applications modules. This section describes this organization and the plans for the first two years of the program.

If the modules and data sets (subsystem) for a specific area of sclentific computing (e.g., reactor physics) do not interact with those of other subsystems, they can be developed independently of the other subsystems. If, on the other hand, several subsystems interact, they must be designed to be compatible. Thus, it is necessary to plan the overall system at an early stage and then to develop individual subsystems. Further, it is essential to organize the personnel involved both in the planning activities and in the code development work to establish lines of communications and areas of responsiblity. This organization is 1liustrated in F1g. 1.

The JOSHUA Task Force, organized in September 1968, consists of representatives from each Division at Savannah River which will utilize the JOSHUA system. Their inftial assignment was to:

- survey all sclent1fic computing at Savannah RIver

- project future computing requirements

- Identily all subsystems and the extent of their interaction

- assign priorities to subsystems and individual modules of subsystems

- develop a schedule to implement each subsystem and the overall system.

This phase of the planning was completed in November 1968.

One of the first results of the Task Force activities was the identification of the following major subsystems which had a high priority for inclusion in the first phase of JOSHUA applications development.

- Reactor Physics 


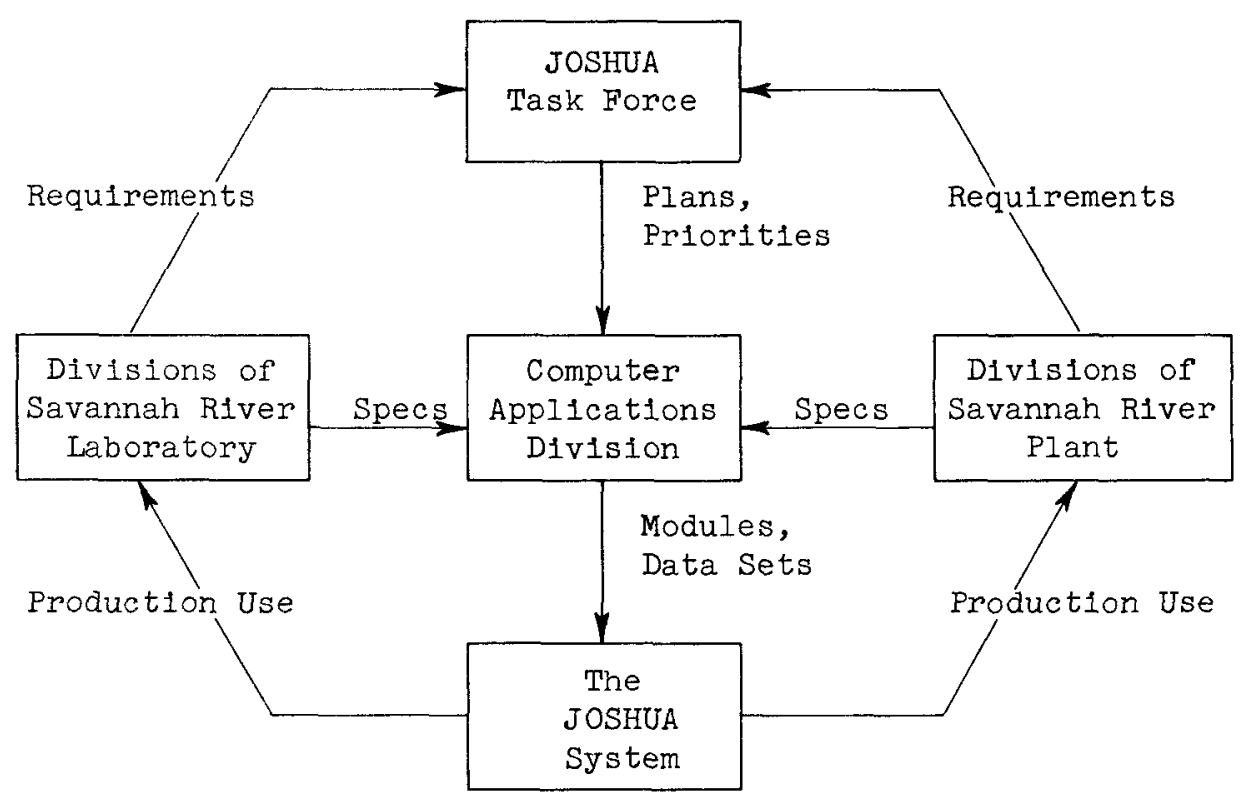

Fig. 1 Organization of the JOSHUA Project

- Reactor Engineering

- Reactor Kinetics

- Plant Simulation and Forecasting

It was evident that the Reactor Physics, Reactor Fingineering, and Reactor Kinetics subsystems interact so strongly that they must be designed concurrently. The Plant Simulation and Forecasting subsystem interacts with the Reactor Physics subsystem, but the interaction is weak enough that it can be neglected at the present time. The two subsystems will be tied together later. other areas such as Chemistry, Chemical Separations, Materials, and Business Data Processing interact only weakly with each other and with the four major subsystems. These were assigned low priorities for inclusion in the first phase of JOSHUA and will not be discussed further here. Areas of highest priority within each subsystem were also identified, and a development schedule was laid out.

The actual implementation of several subsystems began in December 1968 as the joint responsibility of the sponsoring Division (e.g., Theoretical Physics Division, Reactor Engineering Division, etc.) and the Computer Applications Division. The sponsoring Division provides complete specifications for the subsystem; the Computer Applications Division develops the modules and data sets from these specifications and ensures that data sets common to several subsystems are compatible.

\section{OPERATING SYSTEM}

A brief description of the JOSHUA Operating System (JOS) is given in this section. The emphasis is on facilities provided to the user by JoS and how these facilities are used. How the JOS actually provides these facilities is described in a paper ${ }^{3}$ to be given at this conference. 
A simplified diagram of a computing system is shown in Fig. 2. The large block represents the central processing units and fast core storage. " $\varnothing \mathrm{S}$ " indicates that a portion of the core storage must be devoted to an operating system which controls the computer system for the individual tasks above it. "Batch" indicates the possibility of multiprogramming (or multiprocessing) a background stream of jobs outside the reactor computing system (for example, FORTRAN complies, nonreactor computations, etc.). Programming a1ds or control programs that must be provided to interface the reactor computing system with the operating system are subdivided by dashed lines. Separate systems are provided for terminal operation and job execution. "Path" indicates that a program may reside in core to control the flow of computations between the various possible modules. Finally, the modules themselves must be brought into core for execution.

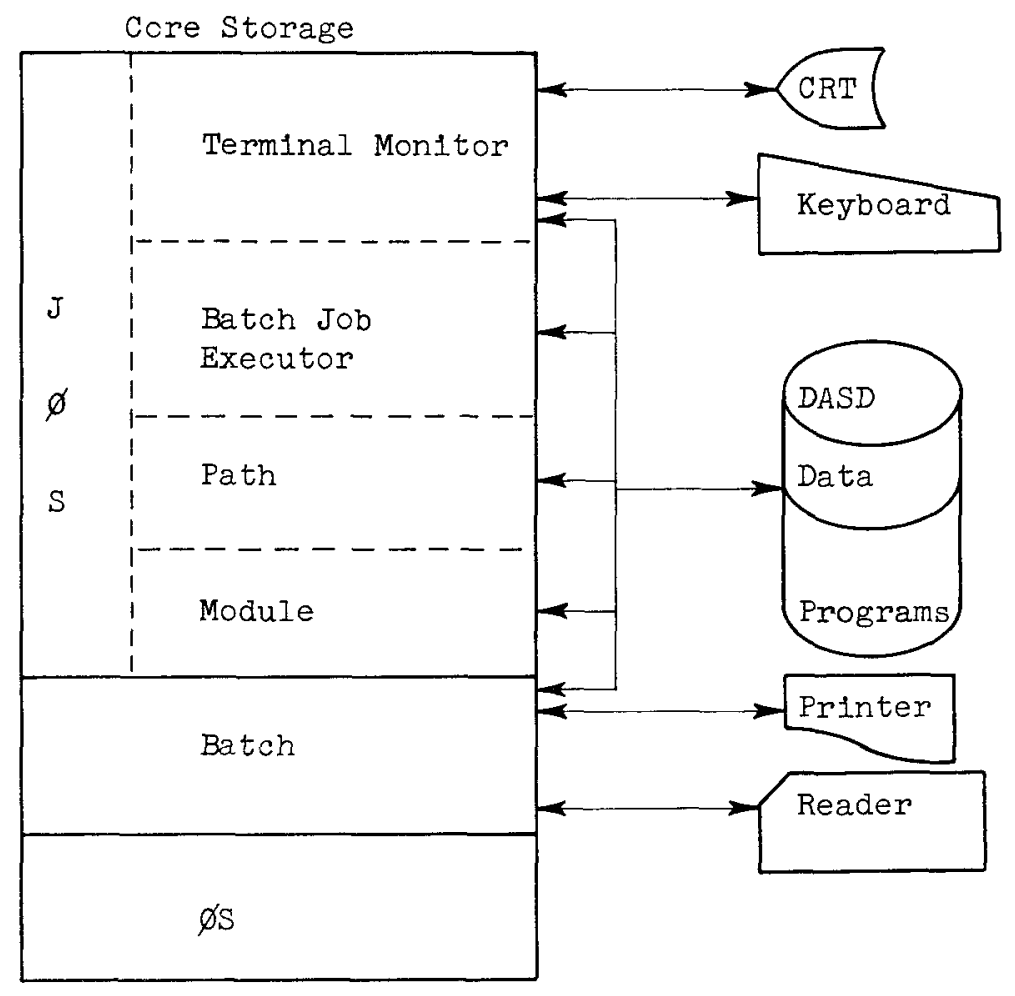

Fig. 2 Simplified Computing System

To the right of the diagram are the various devices involved in the flow of data in and out of the computer and between the varlous segments of core. The two diagrams at the bottom Indicate that the batch mode area utilizes the usual offline printer and card reader of a stacked job processor. The display terminals and remote keyboards show the possibility of allowing a reactor designer to gain easy on-Iine access to the reactor computing system, and to make use of the graphic capabilities of the new terminals in setting up complex input data. All communication between the job control path and module is via data and programs which reside on a direct access storage device (DASD). 
Before discussing how these devices are used, it is necessary to describe how data are handled by the JOS.

\subsection{Named Data}

Fig. 3 1llustrates the problem of handling large quantities of data in a natural way. The flgure shows a typical multigroup data set structured (as in the user's mind) in a hierarchical fashion, that is, as a tree structure. For example, the user may indicate his interest in a data record containing values of the fission cross section for ${ }^{23} 8_{\mathrm{U}}$ in the HAMMER multigroup data set. He cannot, without considerable research, refer to this data record as, for example, record 53 on track 108 of the disk. He can, however, eas1ly refer to this data record with the name

\section{MULTIGRP . HAMMER . U238.XSECTIØN. FISSIØN}

if facilities are provided for using names of this kind in FORTRAN programs, and relating the names with locations on a direct access device. These facilities are provided in JOS by a precompiler and a data manager. Similar facilities are provided in the NOVA system. ${ }^{4}$

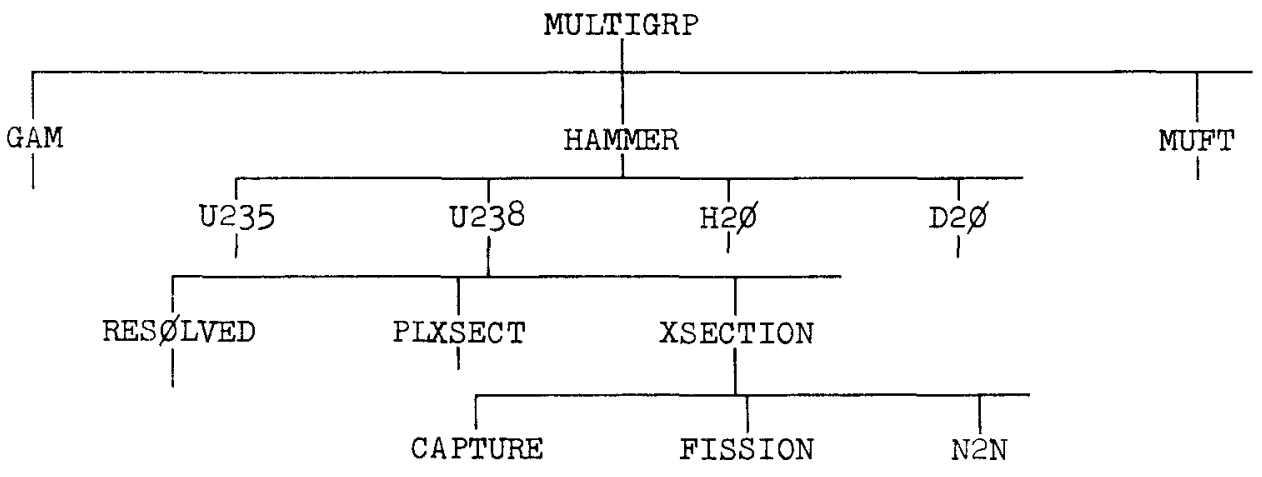

F1g. 3 Typical Multigroup Data Set Structure

A data record referred to by name is called a named data record and is defined by a record name and a list. The list defines the structure of a record and is similar to the usual FORTRAN I/ $\varnothing$ list, except that all indexing must be shown explicltiy and all nonconstant index limits must appear at the beginning of the list. For example, if the user wants to write the record named above from the array, $\mathrm{XF}(\mathrm{N}), \mathrm{N}=1, \mathrm{NEG}$, a sultable list would be:

$$
\mathrm{NEG},(\mathrm{XF}(\mathrm{N}), \mathrm{N}=1, \mathrm{NEG})
$$

The FORTRAN IV language does not provide any direct facility for handling named data records. It is possible to provide FORTRAN-callable subroutines with the record name and list as arguments. This becomes awkward, however, if arbitrary length names and lists are to be allowed. Instead it was declded to extend the FORTRAN language and use a precompiler to interpret the new statements. The basic extension is the addition of a new read/write statement. The normal unformatted read/write statement has the form:

$$
\operatorname{READ}(n, E R R=m) \text { list }
$$


where $n$ is a unit number and $m$ is an error return statement number. The new read statement replaces the unit number with the record name and signals that it is a special JOSHUA statement with a $J$ in column 1:

$$
\text { J } \quad R E A D \text { ( record name, } E R R=m \text { ) list }
$$

For the example above the read statement would be:

$$
\begin{aligned}
& \text { J READ('MULTIGRP'. 'HAMMER' . 'U238' . 'XSECTI } \varnothing N \text { '. 'FISSI ØN', } \\
& 1 \quad E R R=m) \quad N E G,(X F(N), N=1, N E G)
\end{aligned}
$$

The use of ERR is optional. The manner in which the precompiler handles these statements and how the data manager relates names to direct access storage locations is discussed later. Before doing that a more complete description of record name construction is required.

The elements of the record name (e.g., MULTIGRP,FISSIØN) are called qualifiers. A qualifier may be:

- a literal string of 8 or less characters

- an integer

- a FORTRAN variable whose value is either an inter or an 8-character literal string.

For example, the name used in a JOSHUA statement might be:

$$
\text { 'MULTIGRP' - 3726.DIS } \varnothing(\mathrm{N}) \text {. 'XSECTI } \varnothing \mathrm{N} \text { ' . RTYPE }
$$

The quotes around MULTIGRP and XSECTI $\varnothing_{N}$ mean "the literal value of." The integer 3726 refers to a particular version ( 3726 might be the user's badge number) of the multigroup data set. DIS $\varnothing(N)$ is the $N^{\text {th }}$ location in the REAL* 8 array named DIS $\varnothing$ and contains an 8 -character isotope name. RTYPE is a REAL*8 variable containing the reaction type name (e.g., FISSION,CAPTURE).

It is clear that the same record name and list structure can be used for all isotopes and all cross section reaction types. Thus, we can write a general name:

\section{'MULTIGRP' . ?VERSN? . ?DIS $\varnothing$ ? ' XSECTI $\varnothing N$ ' . ?RTYPE?}

Qualifiers enclosed in question marks are called variable qualifiers and a record name containing variable qualifiers is called a record name type. The combination of a record name type and a list defines a named data record type. The example above indicates that the same name and list structure are used for all reaction types, all isotopes, and all versions of the multigroup data set. Obviously, at execution time the variable qualifiers must be replaced with literals, integers, or variables having specific values which refer to a unique named data record.

The first qualifier in a record name is called the data set name (e.g., 'MULTIGRP'). The data set name must not be a variable qualifier and must start with an alphabetic character. The data set name provides the user with a natural major subdivision of the data base. The data set name is used to allocate direct access storage space. 
The hierarchical tree structure of data record names 1llustrated in Fig. 3 provides a convenient and powerful method for maintaining catalogs of record names and pointers. A FORTRAN IV subroutine provides the facility for maintaining and searching catalogs of record names and associated data pointers. These catalogs are themselves part of the data base and are stored on disk. The catalog search subroutine is called from the user module each time a record is to be read or written. The appropriate statements are automatically inserted into the program by the precompiler.

\subsection{Precompiler}

Let us assume that a module has been programmed using JOSHUA read/write statements. The source deck is precomplied to replace JOSHUA statements with a set of FORTRAN IV statements. The resulting source deck can then be compiled with the system's FORTRAN IV complier. The intermediate deck is not punched and the programmer always works with the original source deck.

The precompiler is written in FORTRAN IV and will process four types of JOSHUA statements (each with a $J$ in column 1 ).

- module statement

- read/write statements

- shorthand statements

- variable typing statements

The module statement arranges for communication of control parameters between the module and the batch executor. The read/write statements have been previously defined. The shorthand statements allow the programmer to predefine a frequently used string of qualifiers and use an abbreviated notation thereafter. In the previous example, if many references are made to the same version of the multigroup data set, it would be convenient to include the following statement near the start of the program.

$$
\text { J 'MULTIGRP'. 'HAMMER' } \rightarrow \mathrm{V}
$$

(The arrow is punched as a combination of the - and $>$ characters). A read statement given later in the program could then be written:

$$
\begin{aligned}
& \text { J READ(V.'U238'.'XSECTI ØN'.'FISSI ØN') } \\
& 1 \quad N E G,(X F(N), N=1, N E G)
\end{aligned}
$$

The action of the precompiler is to move the character string defined on the left of the arrow into the portion of the name given by $V$ so that the qualifiers used in the shorthand statement need not be fully defined at the time the shorthand statement is given. The shorthand statement is used only to abbreviate the first part of the name. The number of qualifiers is arbitrary. The shorthand symbol (e.g., V) may be any 1-6 character alphameric string and will not conflict with any FORTRAN variable. Another example is:

J 'MULTIGRP' ' 'HAMMER' .DIS $\varnothing(I) \cdot$ 'XSECTI $\varnothing \mathrm{N}^{\prime} \rightarrow \mathrm{XF}$

$$
D \varnothing 10 \quad I=1, I S \varnothing
$$


The typing statement defines the type (e.g. REAL*8, etc.) of each non-4-byte FORTRAN variable used in a read write statement. Indexing of arrays is not required. For example:

$$
\begin{array}{ll}
\text { J } & R E A L * 8 \text { A, B, C } \\
\text { J } & \text { INTEGER*2 I,J }
\end{array}
$$

These statements are used in addition to the usual FORTRAN typing statements. A later version of the precompiler will use only the usual FORTRAN typing statements and the special typing statements will not be required.

The shorthand and type statements are used by the precompiler to interpret the read/write statements and will not be present in the intermediate source deck. The precompiler replaces each read/write statement with a group of FORTRAN IV statements. These statements:

- define qualifiers as 8-character strings

- compute the length of the list

- call the data management subroutine which locates the record name in its catalogs and passes back the associated pointers to the data

- issue a direct access read/write statement using the pointers and list (This statement is a non-ASA standard FORTRAN statement but is a standard IBM 360 FORTRAN IV statement.).

- transfer to error statements if required.

\subsection{Data Management}

The last two sections have outlined how data records are constructed and named, and how a module can read or write records from direct access storage. If the computer were dedicated to a single user, little more data management capability would be required. However, the computer will be used concurrently for many jobs to achieve reasonable efficiency. At any one time, one or all of the following jobs may be in process:

- manipulation (displaying, modifying, creating) of the data sets from several terminals

- execution of modules inftiated from terminals

- execution of modules initiated from the batch

- execution of non-Joshua batch jobs.

A multilevel, multiuser access method is used to maintain the integrity of the data base during these concurrent operations. Briefly, three levels of data sets are used:

Job - temporary data produced as output from the execution of a module. These data can be read or written by all modules comprising the user's job. At the completion of a job, these data can (at the user's option) be selectively merged with the user data sets. 


User
which can be read by the user's modules.
These data can be written only from a terminal
on the batch input.
Standard - permanently stored data which can be shared (read)
by all users. These data can be written only by
Jos programs.

Any read operation searches all three levels in the above order, and transmits the data found at the lowest level. For example, a module might read data from a standara data set (because no data with the same name were found in the job or user data sets), modify the data, and write the new data with the same name. The new data are written on the job data set, and all future references to that name will retrieve the data from the job data set (since it is searched first). The original data still remain in the standard data set, but it appears to the module that the data have been overwritten. If the module deletes the data, those data in the job data set are deleted, and the standard data are again avaliable to the module.

\subsection{Terminal Facilities}

The JOS provides several facilities for manipuiation of the data base and exeutsu cl mr: $\therefore$ ss, for both terminal and batch operation. Because the facilities for batch operation simulate those for terminal operation, only the terminal facilities Templating, Data Access, Module Execution, and Cataloged Procedure will be discussed here.

The data stored on a DASD are unformatted, that is, in binary form. Templates format the data so they may be displayed (or printed) in an easily readable form. A template is analogous to a FORTRAN FORMAT statement. A template is defined for each named data record type, and the information contained in the template is stored as a data set. Because there will be many data records of the same type, little additional storage is required for temolates.

Six instructions allow the user to access his data from a terminal: IIST, DISPLAY, CØPY, CREATE, MØDIFY, and DELETE. IIST allows the user to look at the catalogs to determine what recoras exist in each data set. In using these commands the user gives a record name to be read, written, or deleted. Normally, reading follows the same hierarchical search through the job, user, and standard data sets, as if the request came from a module. The user can force a search only of his user lata set by preflxing the record name with his user number. Simllarly, the search of the standard data sets can be forced by prefixing the record name with the qualifier 'STD'. All writes (or deletes) refer to the user data sets.

After the input data are set up, the JOS can be commanded to execute a given module. The Jos enters the module into the os batch queue for execution. Following execution the user can display the output records produced by the module. These records will be in the job data set.

Associated with each module is a series of defined input records and output records. A cataloged procedure assists the user in executing a module. The cataloged procedure is stored as a systems data set and contains the names of the input records, the module name, and the names of the output records. When the user executes a procedure, the JOS automatically displays the proper blank records to be created (or existing records to be modified), assists the user in setting up varlable qualifiers in the names, and executes the module. A second procedure automatically displays the output records. 


\section{APPLICATIONS}

The schedule for the development of major application subsystems was given in the Introduction, Section 1. A brief discussion of each subsystem is given here.

In developing each subsystem, the decision to convert an existing program or develop a new program depends on the following factors.

- Is an existing code available?

- Is adequate documentation avaliable?

- Will the theory and techniques in the existing code be adequate or will improvements be required during the next few years?

- Are better techniques (theoretical, numerical, or programing) now avallable than are used in an existing code?

- Would an existing code become a module, part of a module, or several modules?

- How much recoding is required to convert an existing code to run under the JOSHUA system?

- If future improvements to an existing code are anticipated, will they be easier to make if a new code is written now?

- Will a complete recoding yield substantial reductions in computer time?

In nearly every case considered, the decision has been made to develop a new module and utilize the latest theoretical, numerical, and programming techniques.

\subsection{Reactor Physics}

The minimum reactor physics subsystem at Savannah River requires four groups of modules and data sets:

- Cross section data sets and manipulative modules.

- Lattice physics modules.

- Reactor modules.

- Burnup modules.

Several cross section data sets are planned. The first, ENDF/B, contains point cross section data. The second, a fine-group data set, is derived from ENDF/B by simple weighted averaging and is designed to efficiently contain 100 to 1000 group sets. The third data set, a multigroup data set, is derived from the fine-group set by averaging with computed flux distributions. The multigroup data set is designed to efficiently contain 20 to 100 group sets and will be the primary input for lattice physics modules. Finally, a few-group ( 1 to 20 groups) data set will contain the cross sections produced by the lattice physics modules and supply the reactor, burnup, and kinetics modules. 
The lattice physics modules will be based on collision probability methods (e.g., HAMMER) and the Monte Carlo method. A completely new HAMMER system being developed will feature full two-dimensional geometry (clusters, mixed lattices, exact cell boundaries), flexible energy group structures ( 1 to 100 groups), and improved leakage calculations. An equivalent multigroup Monte Carlo module is als be bing developed.

The flrst reactor modules will be two-dimensional diffusion theory modules based on PDQ ard/cr GAUGE. The source-sink code, HERESY-II, will also be used. Two- and three-dimensional synthesis modules will be included later.

Burnup modules are being developed for both simple lattice burnup and for complete reactor life history calculations.

\subsection{Reactor Engineering}

The kasic requirement of the reactor engineering subsystem is to provide for rapid and efficient design of fuel assemblies and reactor charges and specification of their operating characteristics and limits. Three groups of modules and data sets, closely paralleling the reactor physics modules, are required:

- Basic engineering data sets (e.g., defining thermal (1+ uutivities, specific heats) and manipulative modules.

- Pus element design modules.

- Feciztor cnarge design modules.

The basic engineering data sets will relieve the user from providing information repetitively, and ensure that all calculations are made on a consistent basis.

The fuel element design and reactor charge design modules will be used to calculate the temperature distributions in the various components of the reactor system. UItimateiy, modules will be created to provide solutions to the heat diffusion and heat transfer equations in one, two, and three dimensions.

The input information for the engineering modules in almost every application will include results from the reactor physics modules or the kinetics modules, or both. For example, results from the physics modules are necessary to calculate the steady-ztate temperature distributions, and from the kinetics modules to calculate operating limits.

Because of the stron Iopendence of the engineering modules on the physics and kinetics modules, the problems of fuel element and reactor charge design are inherentiy 1terative, and sultable for modular computational techniques.

\subsection{Reactor Kinetics}

The ultimate objective of the kinetic subsystem effort is the capability to analyze the course and consequences of a variety of hypothetical reactor incidents. The kinetic subsystem interacts strongly with both the static reactor physics and static engineering subsystems for the definition of initial reactor conditions. The kinetic neutronics models will include multigroup and delayed neutron effects in multidimensional geometries. The kinetic engineering module will determine all pertinent state variables (e.g., temperature and density of coolant, moderator, and fuel; flow velocity of coolant and moderator; and coolant and moderator vold fraction) as functions of space and time. Feedback to the neutronics module will be included via the updating of nuclear parameters (e.g., neutron cross sections and neutronic diffusion 
coefficients) as functions of the engineering state varlables. The neutronics module will, in turn, provide thermal power feedback information to the engineering module.

The kinetic neutronias module will provide several calculational models including a one-dimensional, finite-difference model similar to existing codes, and synthesis models utilizing elther continuous or discontinuous spatial trial functions in multidimensional geometries.

The inftial kinetic development effort will emphasize the generation of spatial trial functions and the numerical solution of the resulting set of ordinary coupled, linear differential equations for the time-varying model coefficlents. Concurrentiy, an existing, one-dimensional, finite difference code will be modified to provide a standard of comparison for synthesized solutions. These neutronics models will be tested using simple feedback models (e.g., power feedback) but they w1ll also provide for a more detalled feedback treatment.

A parallel effort will be made to develop a kinetic engineering model which will later be coupled with the neutronics model.

Special problems, such as the calculation of spatial xenon oscillations will be treated as separate, specialized subsystems but will utilize existing, applicable program blocks ( $1 . e .$, subroutines, modules, etc.) to treat feedback mechanisms, to define initial conditions, etc.

\subsection{Plant Simulation and Forecasting}

The Plant simulation and forecasting subsystem will be used to develop accurate time schedules for the use of the Plant facilities, record the impact of scheduled processes on inventories, and calculate the costs assoclated with plant operation and the costs of programs and products.

The facility scheduling and inventory managing modules are being developed first; the cost modules will then be developed.

The Plant simulation and forecasting subsystem must be flexible because the users require a wide range of detail. The requirements include:

- Studies where only a facility schedule, with any necessaiy overiap in facility use, is desired.

- Lor-innge studies (10 - 20 years), where schedules for major facilities, inventory accounting for major Itern, and the associated costs are desired.

- Shorter-term studies (6 montns - 2 years), where schedules fù in foulizties, inventory ávounting for all items, and one associated costs are destred.

TC Froide the "lexibility, the data base will include several descriptions if a siven "carnaizn," where a "campaign" encompasses all Plant operations and inventories tc produce a product or carry cut a program.

These descrintiors will permit any desired degree of detail to be specified and will permit cre gereral set of zcheduling, inventory managing, and costing modules $t=$ be used for all types of problems. 


\section{ACKNOWLEDGMENT}

The JOSHUA capabilities now available have been programmed by $R$. L. Boyce

and J. H. Maddox, R. . Priest, W. H. Hightower, C. E. Parker, and A. H. Dobyns.

The information contaired in this article was developed during the course of work under Contract $A T(07-2)-1$ with the U. S. Atomic Energy Commission.

\section{REFERENCES}

1. The Argonne Reactor Computation (ARC) System, USAEC Research and Development Report , ANL-332), Argonne National Laboratory, November 1967.

2. E. E. Re1Ily, Jr. and W. H. Turner, "The Automation of Reactor Design Calculations at the Knolls Atomic Power Laboratory," Proceedings of the Conference or the Application of Computing Methods to Reactor Problems, ANL-7050, 1965.

3. J. E. Sulch, J. C. Jensen, and H. C. Honeck, "Data Management for Multiaccess Computatioril Syrtems," Proceedings of the Conference on the Effective Use of Compitors in tho Nuclear Industry, Knoxville, Tenn., April 21-23, 1969.

4. H, J. Kopp and F, 3. Selergut, "DATATRAN - A Data-Handling Computer Language fur a Lares, Vi:ir Reactor Design System," Proceedings of the International Conference on Tre Utilization of Research Reactors and Reactor Mathematics and Computation, Mexico, D.F., May 2-4, 1967, Vol. 3, pp. 1460-1472, Report CNM-R-2. 
RECENT DEVELOPMENTS AND CAPABILITIES IN THE ARC SYSTEM

\author{
L. C. Just, P. Walker, and B. J. Toppel \\ Argonne National Laboratory \\ Argonne, Illinois
}

The ARC (Argonne Reactor Computation) System is a coordinated system of programs and data that will ultimately encompass the field of reactor computations. The design philosophy and early implementation have been previously reported; $(1,2,3,4)$ the purpose of this paper is to report the present status of the system.

The programs in the ARC system are in the form of modularized computational units rather than stand-alone computer codes. Three types of modules have been developed: computational, system and directive. The computational modules are essentially "black boxes" that are defined in terms of upper and lower interfaces and internal algorithms. Directive modules, called paths, serve to implement a computation by executing computational modules in the proper sequence and by making logical decisions based on results. System modules perform functions of intermodule communication and communication with the operating system. Computational modules and directive modules are restricted to FORTRAN IV while system modules are written in FORTRAN IV and assembly language.

ARC is currently implemented on an IBM 360 model 50-75 combination using OS/360 and ASP and can run on any IBM 360 using Operating System 360 . Because of the nature of reactor problems, ARC requires a fast CPU and large amounts of core and auxiliary storage.

There have been three major approximations to ARC in the last two years, starting with a simple approach to get the project moving and culminating in a system that satisfies the basic ARC requirements.

The first approach was in the form of a large overlay program. The root segment contained the ARC system routines and the computational functions were the segments of the overlay. This system had to be discarded for several reasons: duplicate names for subroutines or common blocks were not allowed and any change in any subroutine necessitated reconstruction of the entire overlay. Most of the programming that was done under this approach was able to be used in later approaches with little change.

These difficulties were overcome by writing subroutines to allow the FORTRAN programmer to interface with the OS/360 MACRO instructions LINK and LOAD.

LINK is an assembly-language subprogram called by

CALL LINK (name, parameter list),

where "name" is the name of an ARC module stored in a library. This call causes "name" to be brought into low core and control to be passed to the entry point of "name." When "name" exits, control passes to the calling subprogram at the statement following the call to LINK, and the space used for "name" is 
released. Computational modules are LINKed.

LOAD is another assembly-language subprogram called by

CALL LOAD (name).

A library is searched for "name," and "name" is brought into high core. All modules LOADed remain in core for the entire ARC run.

Only certain modules are LOADed. These are the ARC system modules and they perform the following functions:

1) System initialization. The first directive module (path) that gains control LOADs the system modules and executes a one-time system initialization. A path can call a path, but only the first can initialize.

2) Process input data.

3) Modify data sets constructed of input data.

4) Produce and maintain tables needed during the run.

According to this description, an ARC run for this second approach should use core as shown in Figure 1 .

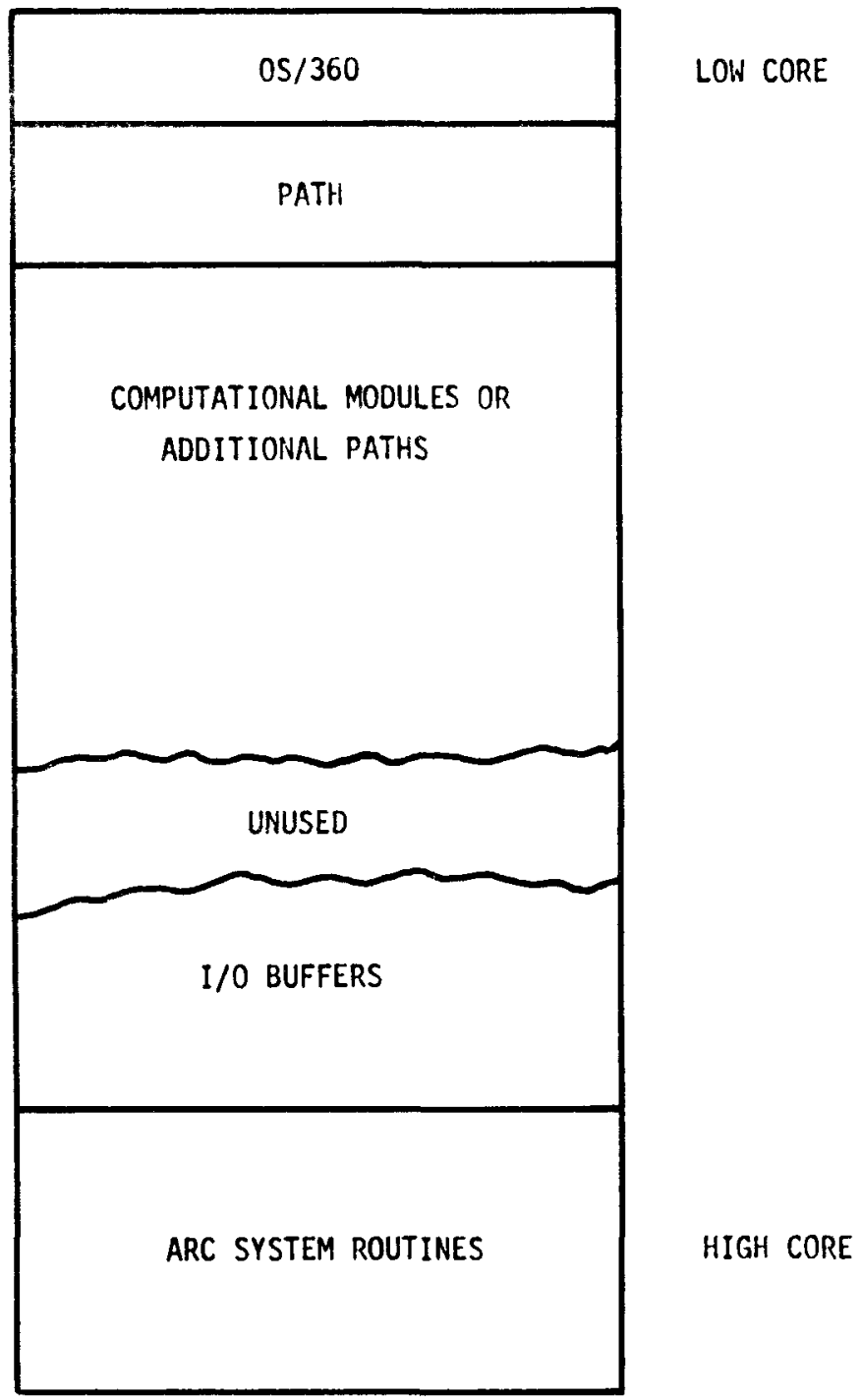

FIG. 1. ARC CORE-USAGE IN NON-MVT ENVIRONMENT WITH SIMPLE LINK. 
The use of LINK and LOAD are completely general; any program in any library can be brought into core and executed. For example, the statement CALL LINK ('FORTRAN4', list)

will cause the FORTRAN compiler to be executed. This could be followed by CALL LINK ('IEWLZWBW', list) to produce a load module. The FORTRAN programmer can thus create executable programs from source libraries or input data as needed during a computation.

The third version of ARC will feature a resident I/O module and will operate under OS/360 with BVT (Multiprogramming with a Variable number of Tasks). While most ARC runs are so large that the computer cannot be shared, MVT overcomes certain shortcomings in PCP (Primary Control Program) and MFT (Multiprogramming with a Fixed number of Tasks). Specifically MFT and PCP reference a half word to tell how large a module has been brought into core by the LINK macro. Interpretation of this half word indicates that a maximum of 512 bk bytes is possible. When a module that is larger than 512 bk bytes is brought into core and executed, trouble will occur because only 512 bk bytes will be released. The $f i x$ for this problem involves using a LOAD-LINK-DELETE sequence for the LINK macro. In this way modules are executed in high core and everything is fine unless a resident $I / O$ routine is LOADed.

With a resident I/O routine LOADed, core can get fragmented by the I/O buffers. Fortunately MVT uses a full word to store module size and allows the normal LINK to be used; computation modules use low core and system modules and $I / O$ can use high core. They absorb core from either end so that the only trouble is a combination of core and $I / O$ requirements that overlap.

A core diagram for MVT is shown in Figure 2. Note that, due to the nature of reactor problems, large production ARC runs will be under MOT (Multiprogramming with One Task).

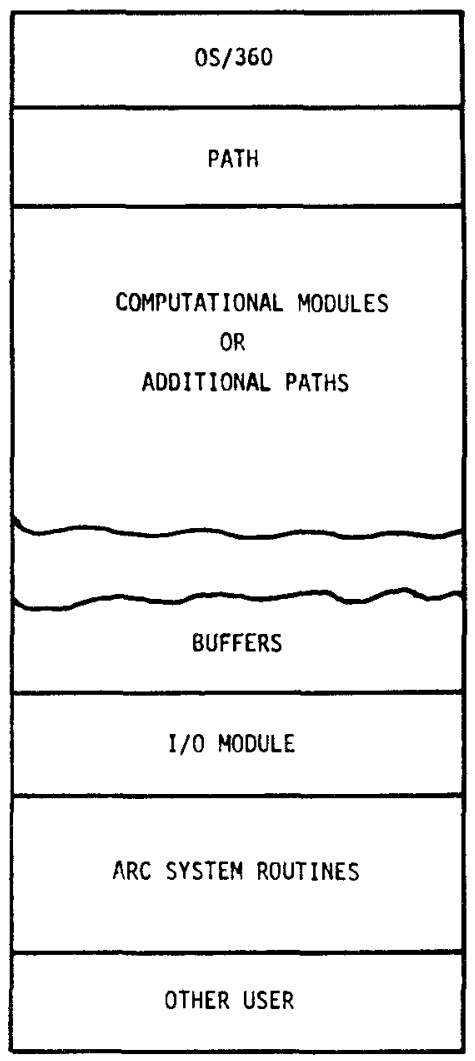

FIG. 2. ARC CORE-USAGE IN MVT ENVIRONMENT. 
Many modules of the ARC system are presently ready for production. With a few exceptions the modules listed in the ARC specification document, ANL-7332, are in final testing states. The exceptions are in the areas of thermal crosssection generation, bivariate cross-section interpolation, transport perturbation, 2D transport theory, and fuel cycle economics. The modules for safety and accident calculations (5) have been programmed on the CDC 3600 and are not yet contained in the ARC system.

The current system has the following capabilities: cross-section generation, one- and two-dimensional diffusion, one-dimensional transport, fuel cycle, and two-dimensional spatial synthesis. The cross-section generation capability is based on $\mathrm{MC} 2$ and includes modules for resonance integral calculations, smooth cross-sections and fundamental mode calculation, broad group collapse, and an input processor. The one- and two-dimensional diffusion capabilities include modules for general neutronics input processing (GNIP), cross-section homogenization (HOMOG), ID diffusion including searches (DARCID), 2D diffusion including searches (DARC2D), ID and $2 D$ diffusion perturbation (PERTID and 2D) and $I D$ and $2 D$ neutron inventory (INVENTID and 2D). There are also group collapse and mesh refinement modules available. The one-dimensional transport capability includes the same general neutronics input processing and homogenization modules and $1 D$ transport modules (SNARCLD), an output manipulation module (OUTMAN) and the ID neutron inventory module. The burnup capability includes modules for fuel cycle calculations and fuel cycle input processing and modules necessary for $I D$ and $2 D$ diffusion calculations. The ARC system enables burnup to also use fluxes generated by a transport neutronics calculation but this has not been tried yet. A 2D spatial synthesis (SYNID) capability is also available; it uses the same neutronics input processing and homogenization modules.

Figure 3 shows the status of some of the modules which have recently been analyzed. A concentrated testing program has been set up for these modules and the status at the end of this testing is reflected in the third bar for each module. The projected time on this testing program is about 15 weeks. Except for the burnup module those modules which are not shown to be fully tested after the testing program include options which are quite obscure and are not considered important at this time.

The $2 D$ transport module is being programmed and will not be available for some time.

In a system of such complexity, organization of the source cards and of the programs on disks is very important. Until recently two system libraries have been kept, one containing each module in load module form and the other containing object modules of any segments the programmers wishes to save. The source cards were kept by the individual programmers. Recent attempts to get listings of the whole system showed the need for more system libraries to enable requests from those wanting to use the ARC system to be fulfilled.

The current system organization includes four libraries.

1. A source library (SOURCE) made up of source segments of reasonable size (less than 500 cards).

2. A partially linked library (SEGLIB) of the same source segments after they have gone through the linkage editor. This library is probably not useful to a requestor.

3. A library (OVERLAY) where organization information for the modules is kept. 
MODULE NAME

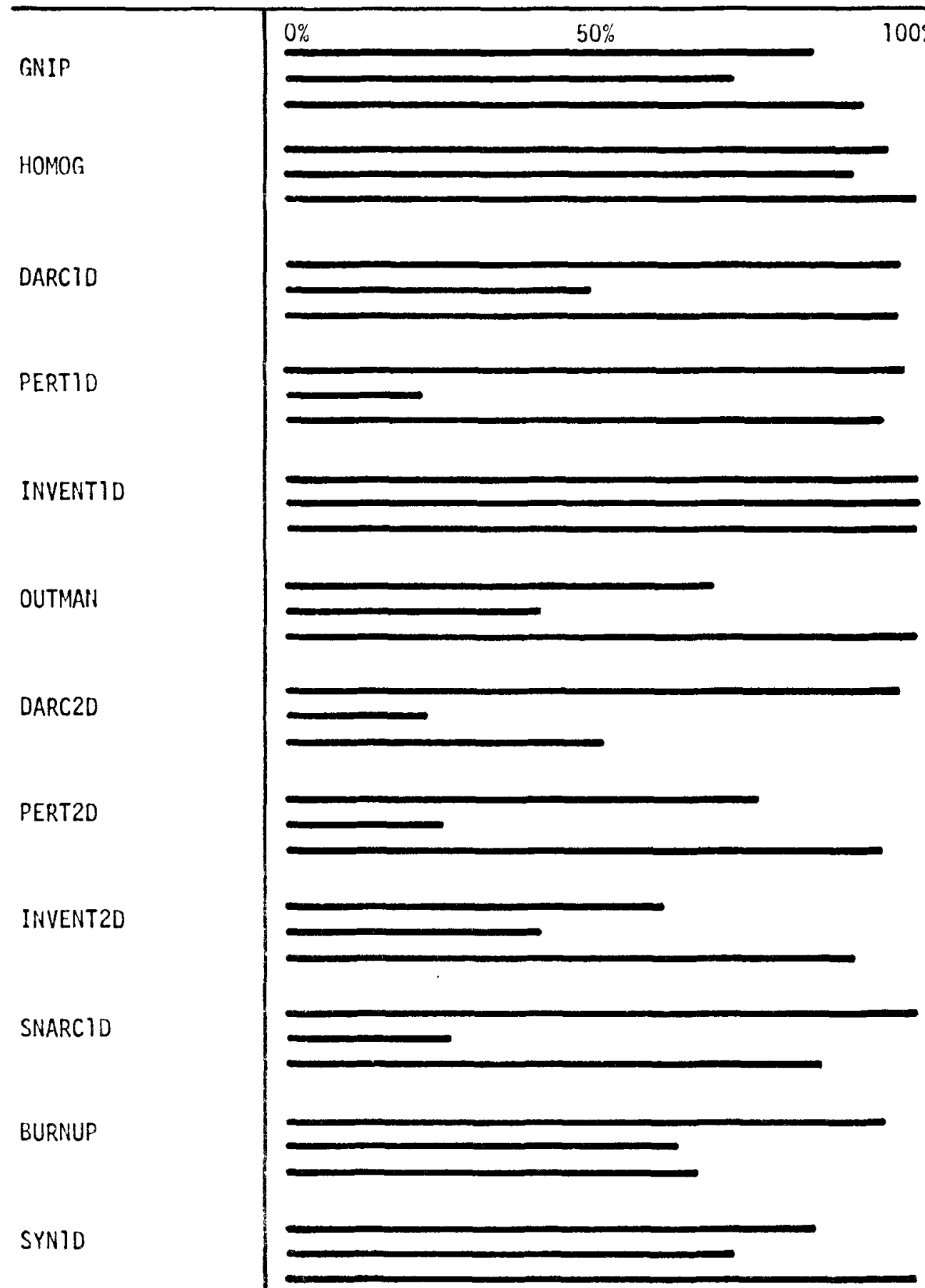

First Bar: Implementation as of March 1, 1969

Second Bar: Tested as of March 1, 1969

Third Bar: Tested after proposed testing program

FIG. 3. STATUS OF ARC MODULES 
4. A Load module library (MODLIB) containing the executable load modules made up from the material in SEGLIB and OVERLAY.

A listing of the SOURCE library and the OVERLAY Iibrary and of the directory of the MODLIB Iibrary gives a complete picture of what the ARC system contains. Copies of the SOURCE library, the OVERLAY library and the MODLIB library with the appropriate data should enable someone at another installation to run ARC joDs with the possibility of making changes.

The ARC system is not yet available through the Argonne Code Center. Current plans call for both individual ARC modules, and collections of standard paths, system modules, and computational modules corresponding to a stand-alone code capability to be available through the Argonne Code Center.

Much effort has been expended in analysis and improvement of the efficiency of the modules. Data set blocking and channel and volume separation are very important. Storing temporary data on the drum has also produced significant time reductions. However, the drum space in Argonne's computer configuration is imited so this advantage has not been fully exploited as yet. Direct access I/O has also been used to advantage by some of the modules.

Argonne has a Large Core Storage unit with 2 million bytes of storage at ache $\vec{*}$ to the $360 / 75$. The common variable dimensioning routine in the ARC

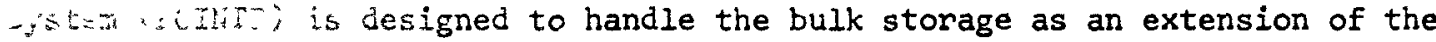

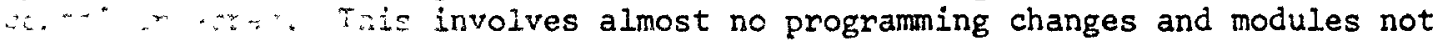
is irg the inj storage are available for use at installations not having bulk stonge croacity. The bulk storage enables large problems to be done faster. iiowever, the modules have all been designed to handle these large problems witnout the bulk storage. Some of the modules contain several algorithms, one of which is designed for large problems and it usually requires more I/O than the others.

A recent experiment with a hardware monitor showed some of the long ARC jobs to be much more efficient than the normal daily job stream (70-95\%) for long ARC jobs as compared to $50 \%$ for daily job stream. This was contradictory to our long-held idea that our long jobs were badly I/O bound. A complete analysis of the data produced by this experiment has not been made but we hope to leam more from this data and from other similar experiments of this type. This monitor was available for only two days so the data gathered is not sufficient to investigate the efficiency completely.

Throughout this project, efforts have been made to have an ARC run look like an ordinary job to the computer. These have been successful if by ordinary jos one implies ondinary large job. As the previous discussion indicates, future development will be on the system to run under MVT. After Argonne converts to MVT efforts will be directed to improving the I/O situation. It is expected that the current rigid interfaces will be retained but that provision will be made for some of the data sets to be broken into elements that can be referenced directly. One simplicication will be to require Data Definition Cards for only the data sets that exist at the start of a run, and those that will be saved. All other data sets will be stored as a member of a run-time scratch library. 


\section{REFERENCES}

1. C. N. Kelber, G. Jensen, L. Just, and B. J. Toppel, "The Argonne Reactor Computation System, ARC," Proceedings of the International Conference on the Utilization of Research Reactors and Reactor Mathematics and Computation, Mexico City, (1967) pp. 1428-1445.

2. B. J. Toppel, The Argonne Reactor Computation System, ARC, ANL-7332 $(1968)$.

3. B. J. Toppel, The Argonne Reactor Computation (ARC) System, Reactor Physics Division Annual Report, July 1, 1966 to June 30, 1967, ANL-7310, pp. 433-436.

4. L. C. Just, and S. G. Sparck, "The ARC System," Applied Mathematics Division Internal Technical Memorandum No. 157 (1968) (unpublished).

5. G. J. Fischer, et al., "Fast Reactor Accident Study Code, SASIA," Conference on the Effective Use of Computers in the Nuclear Industry. 


\title{
Three Dimensional BWR Simulation
}

\author{
by
}

R. L. Crowther, W. P. Petrick and A. Weitzberg

GE-NED

\section{Introduction}

Simulation of the operating characteristics of large Boiling Water Reactor cores requires integration of several areas of technology as illustrated by Fus : The neutron $f 1$ tux and fission power, moderator enthalpy and densitr; $c 0,1 a s i n$ inlet temperature and flow, xenon, and fuel element tempera-

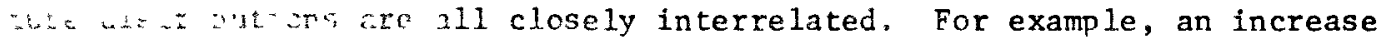
$\therefore$ powt in a local area initiated by withdrawal of a control rod, tends to incroast frel clement tamperature and moderator boiling, both of which result in negative feedtack which tends to reduce the local power. The increase in steam voids increases local pressure drop, which produces a small decrease in flow that feeds back to affect the local steam content and power. The change in flow and heat flux affect the steam content, the local distribution of steam bubbles, and the relationship between the vapor and liquid velocities. The increase in total power increases $s$ team flow to the turbine which decreases the reactor core inlet temperature with a resultant delayed effect on moderator distribution and power. The longer term xenon concentration changes affect the shape of the power and moderator distribution and introduce a gradual perturbation of the other interrelated variables.

The trends in a transient, such as the previous example, are a function of the specific operating state of the reactor and the fission product and isotopic distributions as affected by past history. In a large core, the power distribution is loosely coupled and is reasonably sensitive to local perturbations. Therefore, a reliable simulation model must approximate the preceding trends and effects in three dimensions.

During reactor operation, sufficient information is available from the numerous incore ion chambers so that the reactor operator can continuously observe important reactor operating variables and maintain margin relative to thermal limits without extensive calculations. A three-dimensional simulation model is useful to predict long term future effects of operating variables for purposes of operations optimization. Because of the interrelationship between variables, there is considerable incentive to optimize coolant, control absorber, power and fuel management(1) so as to minimize operating costs. For example, plutonium production can be maximized in a BWR by operating at the minimum flow allowable from the standpoint of thermal limits, followed by maximization of flow to gain reactivity when the control rods are withdrawn. Other alternates such as power coast down, 
FIGLRE I

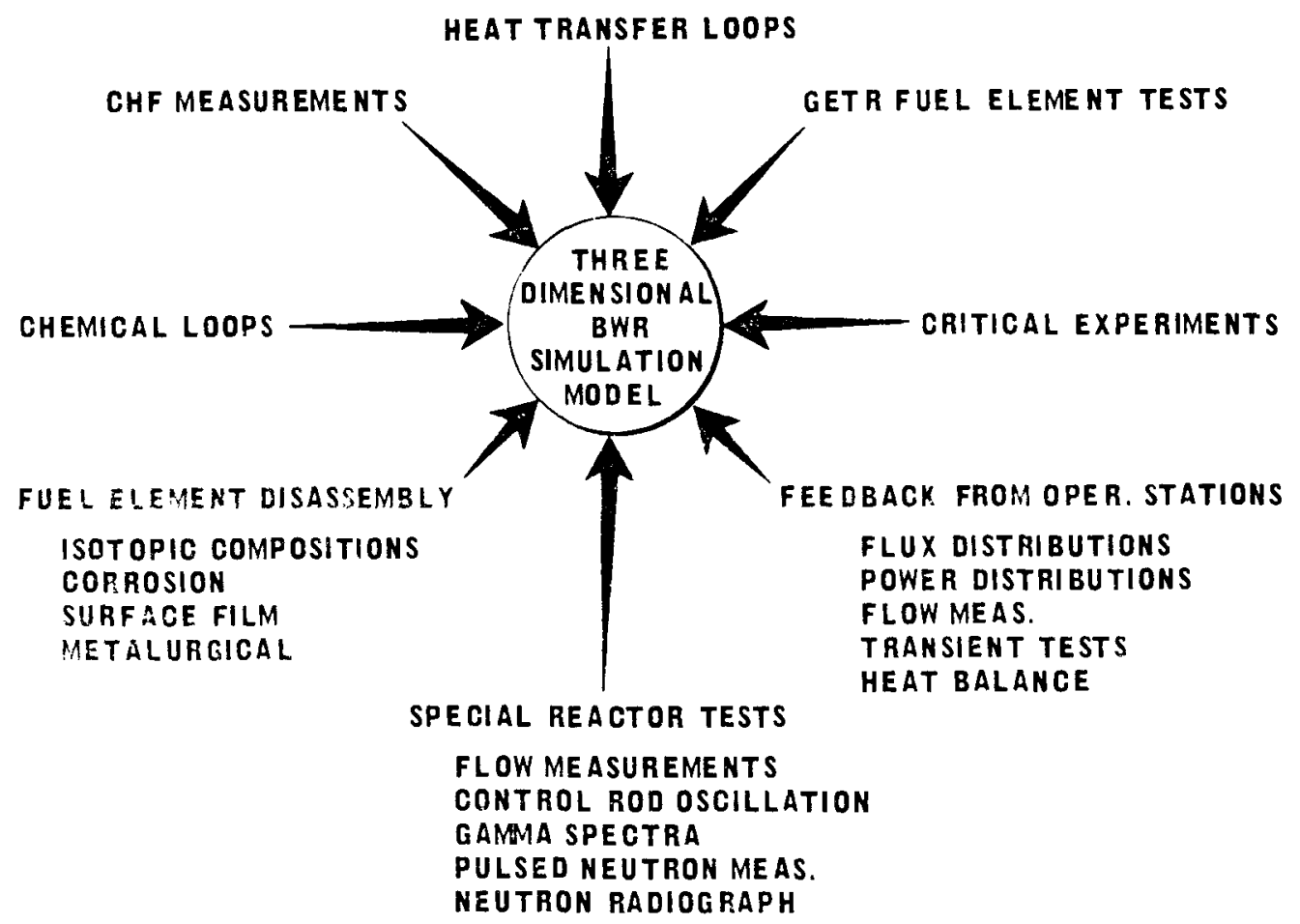


feedwater heater cutout, and special power distribution control to maximize excess reactivity are also available. The degree to which these can be accomplished is a function of the degree to which the three dimensional power distribution is controlled by distribution of fuel exposure, absorber, and steam voids. Thus, the three dimensional simulation program is not essential for plant operation and is used for design and operations optimization.

FLARE, (2) QUAD, (3) TREDA (4) and other three dimensional programs are predecessors of the BWR program. BWR has profited from difficulties experienced in use of its predecessors and includes numerous important improvements. These improvements have resulted in better-agreement with available operating data.

\section{System Features}

The BWR three dimensional simulation and optimization program was developed within the framework of the preceding considerations. The BWR program consists of a large modular system of nuclear-thermal-hydraulic-fuel cycle packages that are coupled to a common data base with general purpose data communication software. The amount of input required is quite large and, therefore, the more difficult areas have been automated. Input can also be obtained through a special interface program coupled with a large tree structured nuclear plant data bank.

The modular $B W R$ program has been structured with future improvement and extensinu in mind. For example, improved modular neutron flux solution packages are plamied such that during the course of a single run, it will be possible to utilize two nr three different three dimensional neutron flux solution modules.

The subroutines utilized for data transfer provide flexible blocking and buffering so as to make most efficient use of disc, drum, or tape devices. Dynamic allocation of data transfer is utilized so as to maximize efficiency, independent of problem size. For example, the maximum number of mesh planes are brought into core on a data load. As a result of these factors, program efficiency has been outstanding.

The program is run on a multi-processor, multi-programming GE-635. The computing facility has 2 processors; 3 disc devices, including a large single head per track unit; 2 fast $786 \mathrm{~K}$ drums; 22 tape drives; and $192 \mathrm{~K}$ of core memory. The BWR program is presently restricted to a maximum of $49 \mathrm{~K}$ of core in order to permit as many as 8 other simultaneous computing tasks within the GE-635 multiprogramming environment. The GECOS-III operator system also permits simultaneous batch processing, time sharing, and remote batch processing. Thus it is possible to access data files from a remote teletype terminal or to initiate or edit BWR output from a remote GE-115 or GE-130 terminal.

A flexible comma loader is utilized for input. A three dimensional array can be loaded by giving the array name followed by the starting subscripts (in parentheses) and the array values. Data repeat also is provided. The output is controlled by edit frequency input controls and some data is edited on a CALCOMP digital plotter.

Future telephone line links with the reactor site are being considered, as shown in Figure 2. A direct telephone data link is possible for coupling the off-site scientific computer with the GEPAC -4020 on-1ine computers that are now standard equipment at BWR nuclear power stations. This is being considered for ease of comparison with the in-core ion-chamber and traversing 
FIGURE 2

INTEGRATED FUTURE OPERATIONS

REACTOR SITE

ANALYSIS AND OPTIMIZATION SYSTEM TELETYPE OR CRT TERMINAL

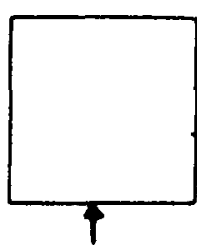

DATA FROM

ON-LINE COMPUTER

TELEPHONE OR OTHER

SERVICE BUREAU, ETC. OFF-SITE SCIENTIFIC COMPUTER

ON-LINE

COMPUTER OPTIONAL DIRECT LINK

UTILITY ENGINEERING OFFICE

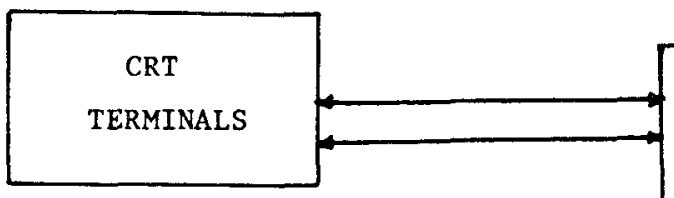

REMOTE

TERMINAL COMPUTER

TELETYPE

TERMINALS 
in-core ion-chamber probe data, for data collection and storage, and for diagnostic analyses.

Nuclear Mode1

The current nuclear model in the BWR program is based on a coarse mesh 1.5 group model. The mesh points are distributed within one fast neutron mean free path. Because of the short thermal neutron mean free path in a water reactor, most neutrons diffuse within the energy span of the fission spectrum. Therefore, the model is oriented toward accurate representation of fast neutron diffusion. Previous experience $(5,6)$ has indicated that the 1.5 group coarse mesh representation is capable of reasonably accurate prediction of global power shapes.

It is important to account for lattice fine structure in derivation of constants for the coarse mesh global model. Separability is assumed for lattice cells consisting of one or more fuel assemblies. Ninety-eight group calculations are carried out parametrically for the lattice cells. These two dimensional calculations approximate the geometric effects in fast fission and include two dimensional Dancoff interaction between fuel rods. Resolved and unresolved resonance Doppler broadening and spatial variations in the thermal neutron spectrum are accounted for by use of transport theory approximations. The latter is particularily important because of the lattice heterogenity introduced by the water spaces between fuel assemblies.

Fuel burnup calculations are carried out for the two dimensional fuel assembly cells by use of 4 th order Runga-Kutta-Gill integration. Twenty heavy nuclides, 29 discrete fission products, one pseudo fission product and 10 flexible nuclides which can be either burnable poisons or heavy nuclides coupled to other chains are provided for in these analyses.

The steam voids introduce special complexity because the nuclear behavior at a point in the reactor is a function of both the instantaneous void content and the previous void history. These effects are accounted for by multivariable nuclear correlations which utilize a combination of spline and rational polynomial correlation techniques.

Doppler broadening is included in the global analysis by use of a simple model which has been derived from physical principles. This correlates well with the detailed multigroup lattice cell calculations. The effective average fuel temperature used in the Doppler representation is obtained by correlation of the results of more detailed numerical calculations.

Xenon is calculated for either equilibrium conditions or for transients that are short compared to the Xe-135 and I-135 destruction and production rates. A transient xenon model is planned and involves implementation of second or fourth order numerical techniques to reduce costs.

Boundary conditions are applied by analytical solution of the reflector equations. This approach has a significant effect on convergence and cost.

Quarter core or half core symmetry is accommodated. Either mirror or periodic symmetry boundary conditions can be applied.

The neutron diffusion equations are solved by use of Gauss-Seidel inner iterations with Chebyshev acceleration of the source. Special power-exposure $(5)$ iteration options are available to solve for the fuel cycle length and the fuel exposure distribution which provide greatest thermal margin 
with all control rods withdrawn.

Development of flexible refueling and fuel cycle software is underway to permit long term fuel cycle studies. Provision for relnsert of fuel assemblies af ter discharge and sharing of fuel between nuclear reactor sites is $\mathbf{l}_{i .}$ sluded. Isotopic compositions are calculated by use of multivariable correlations, the form of which has been derived from phystcal principles. (7)

Direct data couple with fuel cycle economic programs is planned to permit ease of evaluation of the effects of reactor operating variables on power $\cos t$.

Calculations are performed to edit neutron monitor readings for direct comparison with power reactor measurements. Consistency with the on-line computer model $(8)$ is important and has received attention in development and checkout of the nuclear model.

Thermal Hydraulic Model

The loose coupling, which is characteristic of large reactor cores and the important effect of the BWR's large negative power coefficient in providing power distribution stability, make the thermal hydraulic representation of considerable importance in the nuclear analysis. An accurate calculation of the steam void distribution is as important as the accuracy of the nuclear constants.

The thermal-hydraulic model includes a detalled physically oriented representation of subcooled, transition and bulk bolling which is related to heat transfer loop experiments through a few empirical constants that describe the local flow structure. The effects of pressure, flow, and heat flux on local voids are included in the representations. The model agrees well with available experimental data, as summarized in the following section.

The water spaces between fuel channels are considered in the thermal-hydraulic analyses. Direct heating of the bypass region is calculated from $\gamma, n-\gamma$, $n-\alpha$, and neutron slowing down. Indirect heating is calculated from heat transfer across the fuel channel wall. The flow through the bypass region is a complicated function of several leakage paths. In some designs, a stress analysis is required to calculate the leakage flow component.

The fluid properties are calculated internally by use of subroutines that are based on the 1967 ASME International Standards. (9) The critical heat flux ratios and fuel element temperatures can be calculated with several local power peaking and power state options. For example, it is possible to specify that the local power peaking in each channel will be calculated as a function of the position of the nine neighboring control rods and that the temperatures and critical heat flux ratios will be computed for each fuel rod segment for as many as three power states. A "power state" is assumed to be a condition with a different power level but the same power distribution. These states apporximate conditions in a fast transient where the power shape does not change because of the long fuel time constant. 
The comprehensive local power distribution calculation is lengthy and therefore, several alternate less accurate approximations are available on option. The effects of local voids and fuel exposure on the local power distribution are included with approximate correlations. One local power distribution option results in calculations of the local power distribution with methods that are consistent with techniques used in the online computer.

Several optional critical heat flux (heat flux which would cause transition boiling) correlations are included. These include the effects of flow, steam quality, pressure and geometry. The critical heat flux ratio (CHFR) can be edited in three dimensions on option. This option is important because the reactor operator must use the three dimensional shape of the CHFR to make decisions on optimal control rod patterns which optimize fuel cycle or thermal performance. The trends are non-linear. For example, maximum thermal output from a BWR occurs when the power is peaked toward the bottom of the core. Thus minimum peak-to-average is not necessarily the optimum state, and the CHFR distribution is required in order to make good operating decisions.

Other edits include a top view of the core which $11 \mathrm{sts}$ the minimum CHFR and its position in each channel and the overall core MCHFR. In addition, a CHFR histogram plot option is avallable. The results of one case are shown in Figure 3. The left hand scale gives the number of mesh points or fuel rod segments with CHFR between $X$ and $X+\Delta X$ as a function of CHFR $=X$. The right hand scale provides the integral percentage of the mesh points or fuel segments with a CHFR $<$ as a function of CHFR. In this particular example, the CHFR distribution is peaked at a CHFR of 6 and there are no fuel assemblies with a CHFR less than 3.2. This edit provides valuable information on margin relative to the CHF limits. Future improvements will include capability to include CHFR distributions from many reactor operating states in these statistics. This will be valuable in making decisions on several different operating strategies.

Fuel element cladding and $\mathrm{UO}_{2}$ temperatures are also calculated. The effects of variable pellet-to-clad gap conductance and temperature dependence of the $\mathrm{UO}_{2}$ thermal conductivity are included in the model. Temperatures are also calculated for severe states when the CHFR is less than 1.0 and transition boiling is taking place. Surface film deposits and cladding corrosion also are included. Several alternate two and three dimensional temperature edits can be selected. One option includes provision to plot the axial distribution of temperatures and CHFR in the hottest channel. The temperature histogram plot shown in Figure 4 is another edit option.

The detalled temperature calculations are relatively expensive. The local $\mathrm{Kw} / \mathrm{ft}$ is a good approximate measure of $\mathrm{UO}_{2}$ central temperature and, therefore, $\mathrm{Kw} / \mathrm{ft}$ calculations and histogram edits can be selected on option. A $\mathrm{Kw} / \mathrm{ft}$ histogram is shown in Figure 5 .

\section{Experimental Verification}

Feedback from many different reactor sites is aiding in verification of the models in the BWR, three dimensional simulation, program. The accuracy of the simulation also is dependent on the accuracy of the input quantities. Sources of power reactor data which provide a basis for the simulation model include: 
FICURE 3

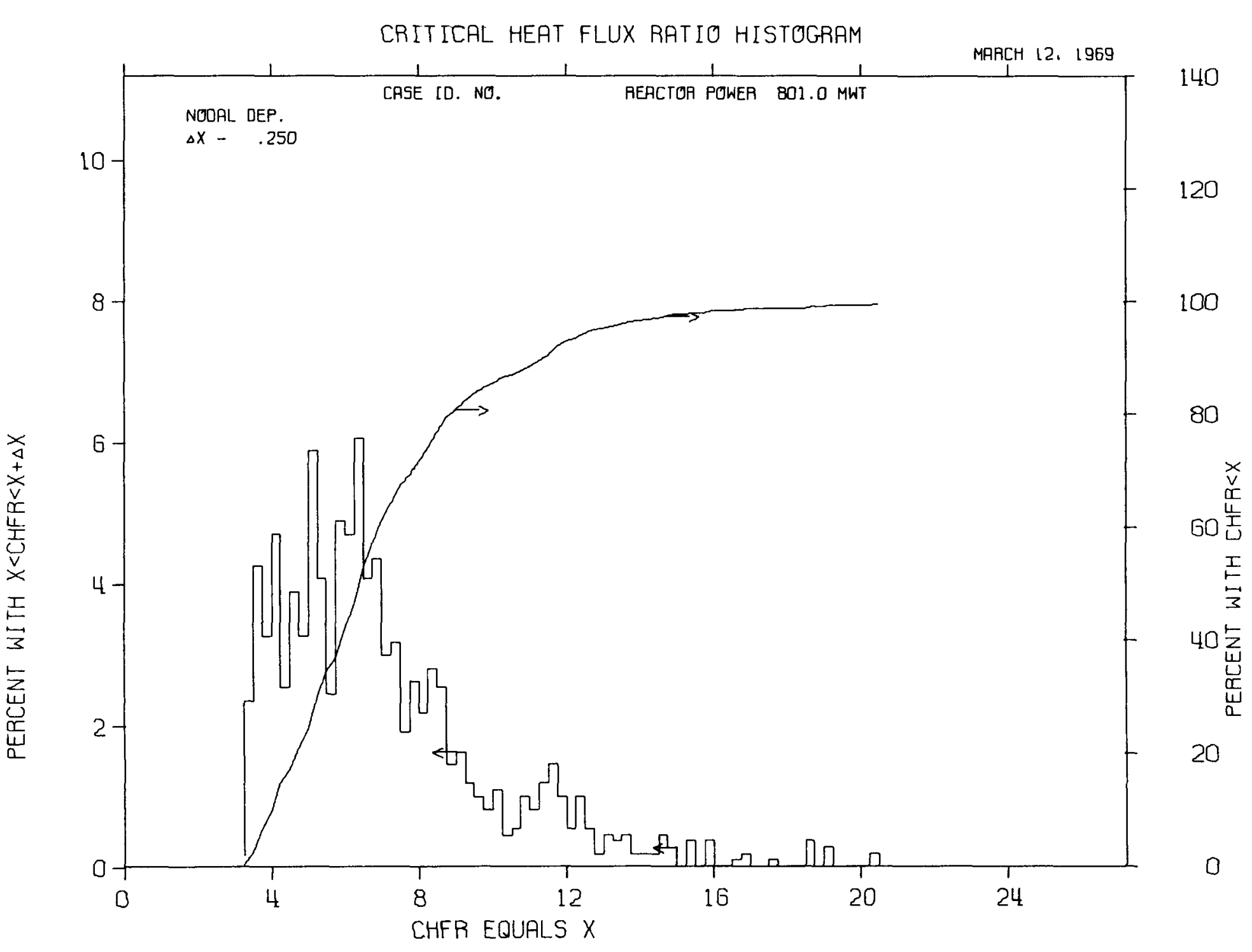


FIGURE 4

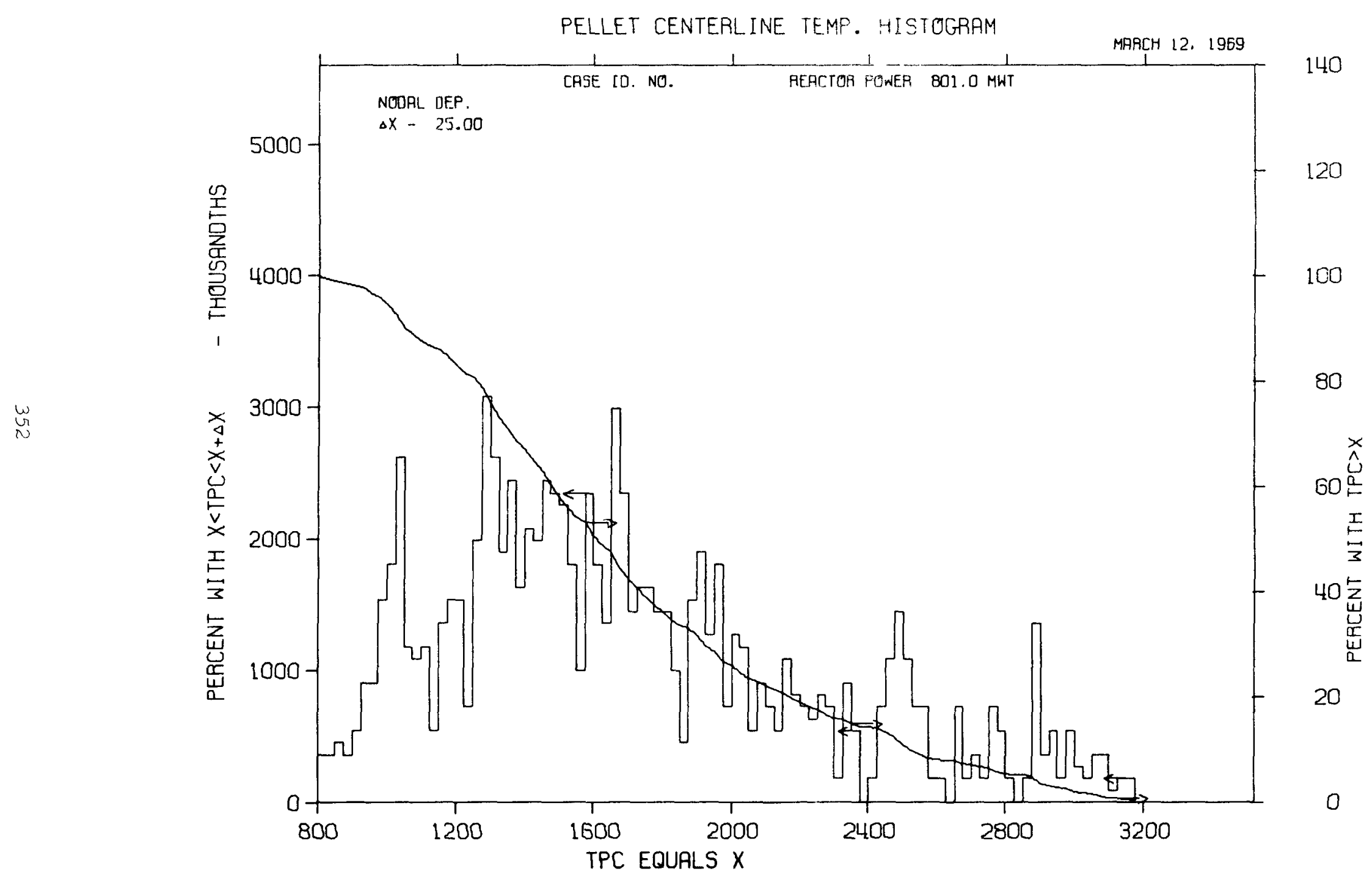


FIGURE 5

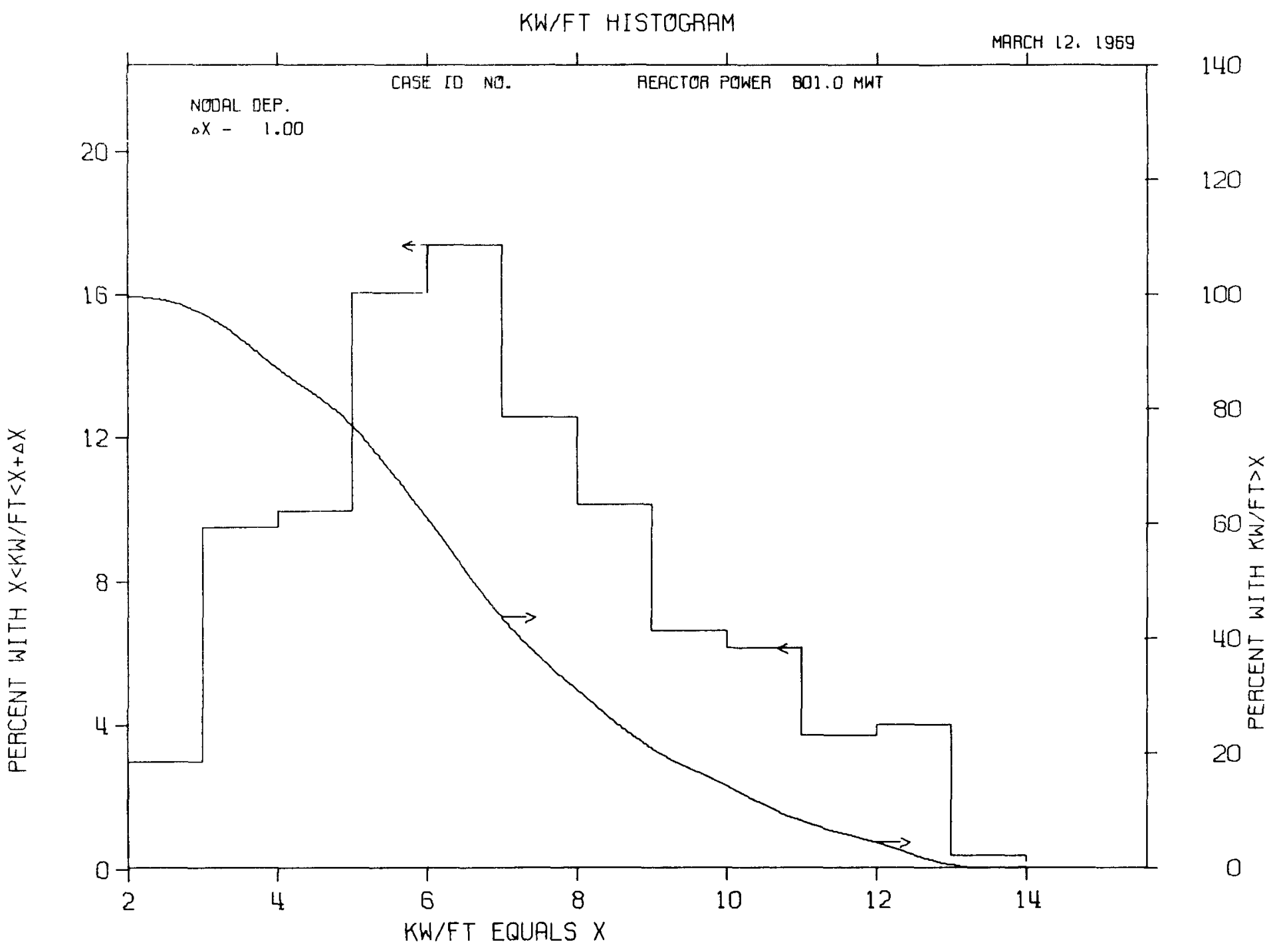


1. Coolant flow, temperature, and pressure drop measurements.

2. Heat balance measurements.

3. Continuous measurements from fixed in-core ion chambers.

4. Periodic detailed axial and radial data from wire scans and traversing incore probes.

5. Special fuel assembly gamma scans to measure global power distribution.

6. Special individual fuel rod gamma scans to measure local power distributions.

7. Detailed isotopic composition measurements at selected positions in individual fuel rods and fuel pellets.

8. Fuel reprocessing plant data from dissolver batch samples.

9. Special flow meter measurements of flow in individual fuel assemblies.

10. Water quali:y measurements.

11. Corrosion and fim deposit measurements from individual fuel rods.

12. Control rod position and worth measurements.

13. Fuel assembly substitution measurements.

14. Cold and hot criticality measurements for many different control rod, fuel assembly and burnable polson configurations.

15. Criticality measurements as a function of reactor power, flow, inlet temperature, fuel exposure, and load following state.

16. Spatial xenon damping measurements for different load following and spatial perturbations.

17. Period measurements.

18. Neutron radiographs of fuel rods and absorbers.

19. Initial conversion ratio measurements by fuel element disassembly and isotopic analysis. 
20. Contro1 rod absorber burnup measurements.

21. Steam carryunder measurements.

This data provides an integral check on the overall coupled nucleartherma1-hydraulic mode1. Collection of some of the power reactor data has required considerable lapsed time. For example, some of the isotopic composition and corrosion measurements from the zircalloy fuel which was inserted in the Dresden reactor in 1960, have required 6 to 8 years. The long interval for the accumulation of fuel cycle data places considerable emphasis on development of reliable analytical models which can predict design and operating variable trends in areas in which there is no data.

Power reactor measurements are made difficult by high temperatures, large radiation levels and inaccessibility. More accurate measurements from operating power reactors is a difficult challenge.

In addition to the power reactor measurements, there is extensive data from critical facilities and flow, heat transfer, and corrosion loops. Some of the data include:

1. Single and multirod steady state and transient critical heat flux measurements.

2. Transition boiling measurements.

3. Steady state and transient void distribution measurements.

4. Criticality measurements from fuel assembly proof tests and from numerous experiments on uniform and clumped fuel rod arrays.

5. Space dependent clumped lattice spectral measurements by $\mathrm{Pu}-239, \mathrm{U}-235$ and other foil activations.

6. Space dependent, clumped lattice, activation measurements of U-238 resonance capture by use of folls and dissolved pellets and by use of $1 / v$ subtraction and cadmium ratios.

7. $M_{\mathrm{n}}$ bath thermal utilization measurements.

8. Criticality for different control rod, temporary poison, and burnable poison arrays.

9. Pulsed neutron measurements.

10. Void simulation measurements.

11. Global wire activations.

12. Temperature coefficient measurements.

13. Hot exponential measurements for plutonium lattices. 
14. Steam separator and jet pump parametric performance measurements.

15. Pressure drop measurements.

Some of the preceding measurements have been completed for a wide variety of BWR lattices including variations in fuel rod diameter and spacing; $\mathrm{H} / \mathrm{U}$ ratio; control blade materials and designs; fuel enrichments and cladding materials; mixed lattices of $\mathrm{UO}_{2}$ and $\mathrm{ThO}_{2}$ fuel rods; different temporary, soluble, and burnable poison designs and concentrations; stainless steel and zircalloy channel materials; local fuel enrichment spatial distributions; and fuel assembly nose piece and fuel rod spacer designs. Recent review papers were published in the reactor physics area. $(6,10)$ It is impractical to review all the comparisons and backup information, and therefore, a small sample has been chosen.

The KRB or Grundremmigen reactor provides a good overall integral test of the simulation model because of the spatial complexity introduced by the use of spatially varying burnable poisons in temporary poison curtains. The Grundremmigen reactor also has traversing incore ion chamber probes (TIP) which can be compared directly with the BWR computer program TIP edits. In addition, local fuel assembly gamma scans have been completed at KRB by disassembling fuel assemblies and scanning individual fuel rods.

A top view of the KRB core is shown in Figure 6 . The core consists of 368 fuel assemblies with 36 zircalloy clad fuel rods per assembly. The active fuel rod length is 130 inches and, thus, from a neutron's point of view, the reactor is large and loosely coupled.

Each fuel assembly is approximately 5 inches across and there are water spaces between assemblies. The design incorporates 89 cruciform control blades that are constructed of $\mathrm{B}_{4} \mathrm{C}$ contained in stainless steel tubes. There are no control rod followers.

One hundred fifty six temporary poison sheets are located in the water spaces at the tips of the control rods. These sheets contain burnable boron poison which is initially distributed in three axial zones.

Seventeen in-core monitor strings are located at the circular positions opposite the cruciform control rods. There are 4 fixed ton chambers uniformly spaced in each strong. Two traversing in-core ion chambers can traverse nine ion chamber strings, including a common position at the center of the core that is used for intercalibration of the TIP chambers. In addition, there are chambers outside of the reactor core which provide little information of value in accessing power distributions and spatial effects.

Local power distribution comparisons with improved nuclear models have indicated errors of 1 to 2 percent. However, greater inaccuracy has been experienced for cases involving inserted control rods. Figure 7 summarizes the results of the ratio of calculations-to-measurements for a central fuel assembly in the KRB reactor with an adjacent inserted control rod. This fuel assembly was taken apart and each fuel rod was individually scanned. 


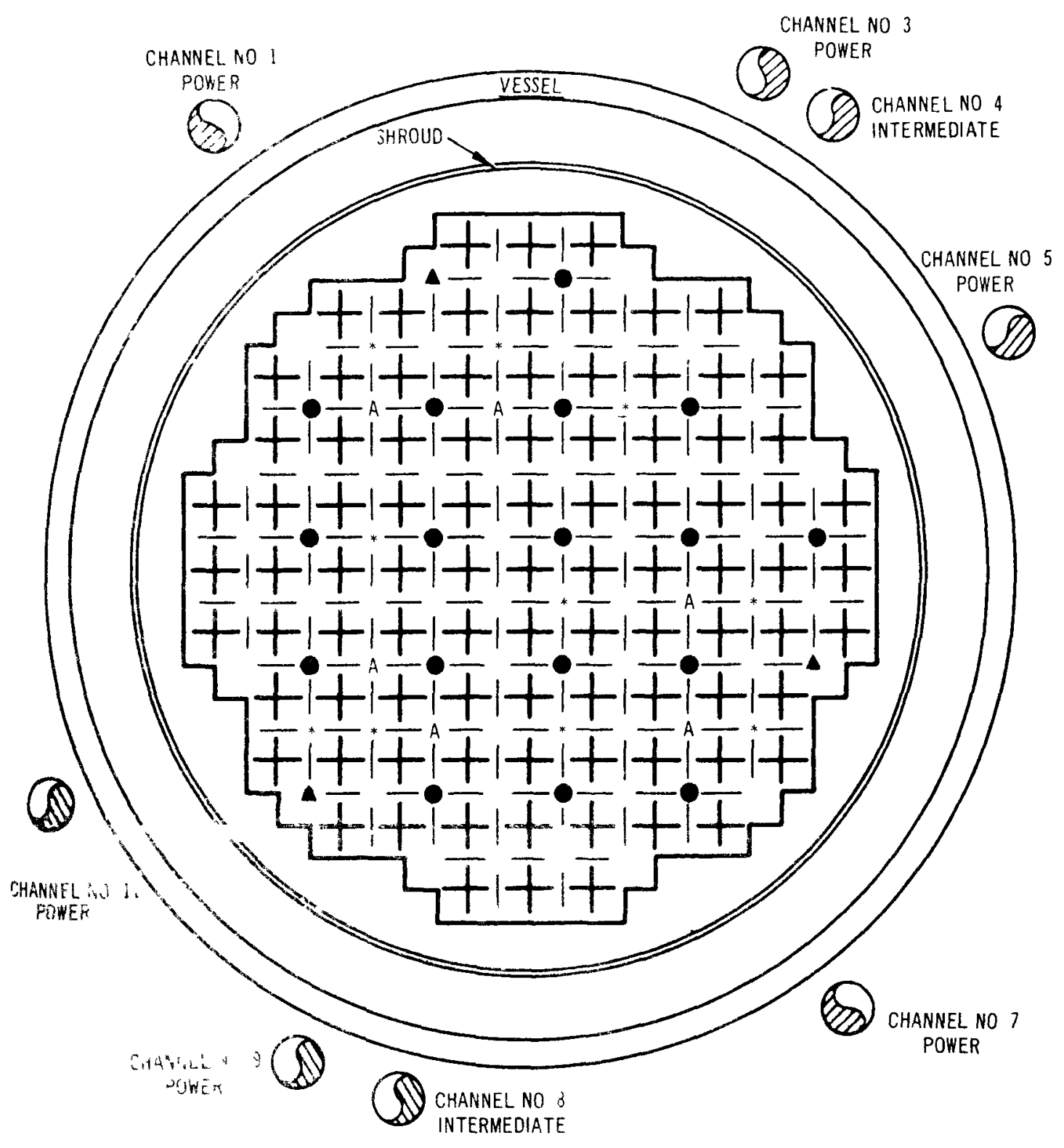

NO OF IN-CORE ASSEMBLIES $-17(0 ;$
NO OF STARTUP CHAMBERS $-3(\mathbf{A})$
ALTERNATE SOURCE LOCATIONS - $6(\mathbf{A})$
SOUPCES $\left.-100^{*}\right)$

FIGURE 6. REACTOR CORF SCHEMATIC 
KRB $\gamma$ SCAN - $6 \times 6$ - HOT - NO VOIDS-

$2000 \mathrm{MWD} / \mathrm{T}$

CURTAIN AND BLADE

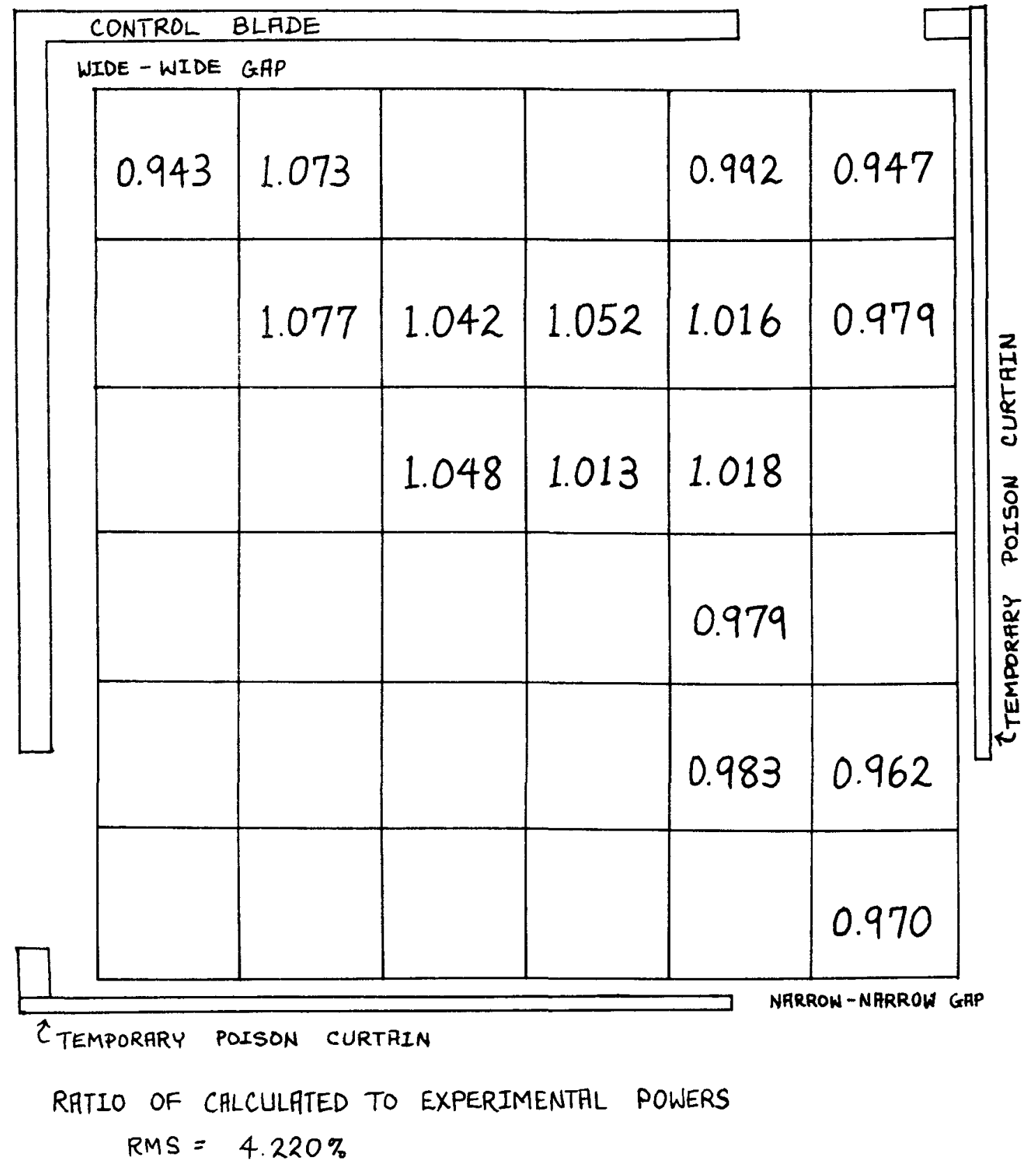

FIGURE 7 
The accuracy of the measurement is approximately $\pm 2-5$ percent. Calculational differences of 7 to 8 percent occur for $a$ few of the fuel rods that are near the control blade. These comparisons are indicative of needed improvement in the nuclear model for cases in which control blades are inserted. A new two dimensional transport theory model(11) has considerable promise for improved accuracy.

The ratio of calculated to measured channel integrated TIP readings is shown in Figure 8 for an early KRB operating condition. The largest difference of about 3 percent occurs near the reflector. In this case, the core was symmetric and all the TIP readings were rotated into one quadrant. The RMS error is $2 \%$.

An axial comparison for one TIP position is shown in Figure 9. This data was taken for the same KRB operating state shown in Figure 8 . The positions of the stainless steel fuel rod spacers are apparent from the dips in TIP trace. The effects of variable axial boron concentration in the temporary sheets also are apparent. The axial agreement is not as good as the radial for this configuration of burnable poisons and control rods. However, the general agreement tends to confirm the coupled nuclear-hydraulic model.

A comparison with measured fissile plutonium isotopic compositions from the Commonwealth Edison, Dresden I reactor is shown in Figure 10. Previous comparisons had indicated a consistent underprediction of fissile plutonium content; (11) however, model improvements have removed most of this discrepancy.

Figures 11 and 12 present a few of the comparisons of predicted voids with available experiments. Figure 11 represents a comparison where bulk boiling is the important phenomena. The comparison in Figure 12 is for subcooled and transition bolling conditions. The thermal-hydraulic model that is used in BWR does a good job of predicting most of the data over a wide range of heat flux, flow, and pressure.

The BWR program will be continually evolved and improved. A long list of improvements has been compiled for future implementation. The general purpose data communication software and the modular structure of the program greatly aid in simplifying the incorporation of major improvements. 


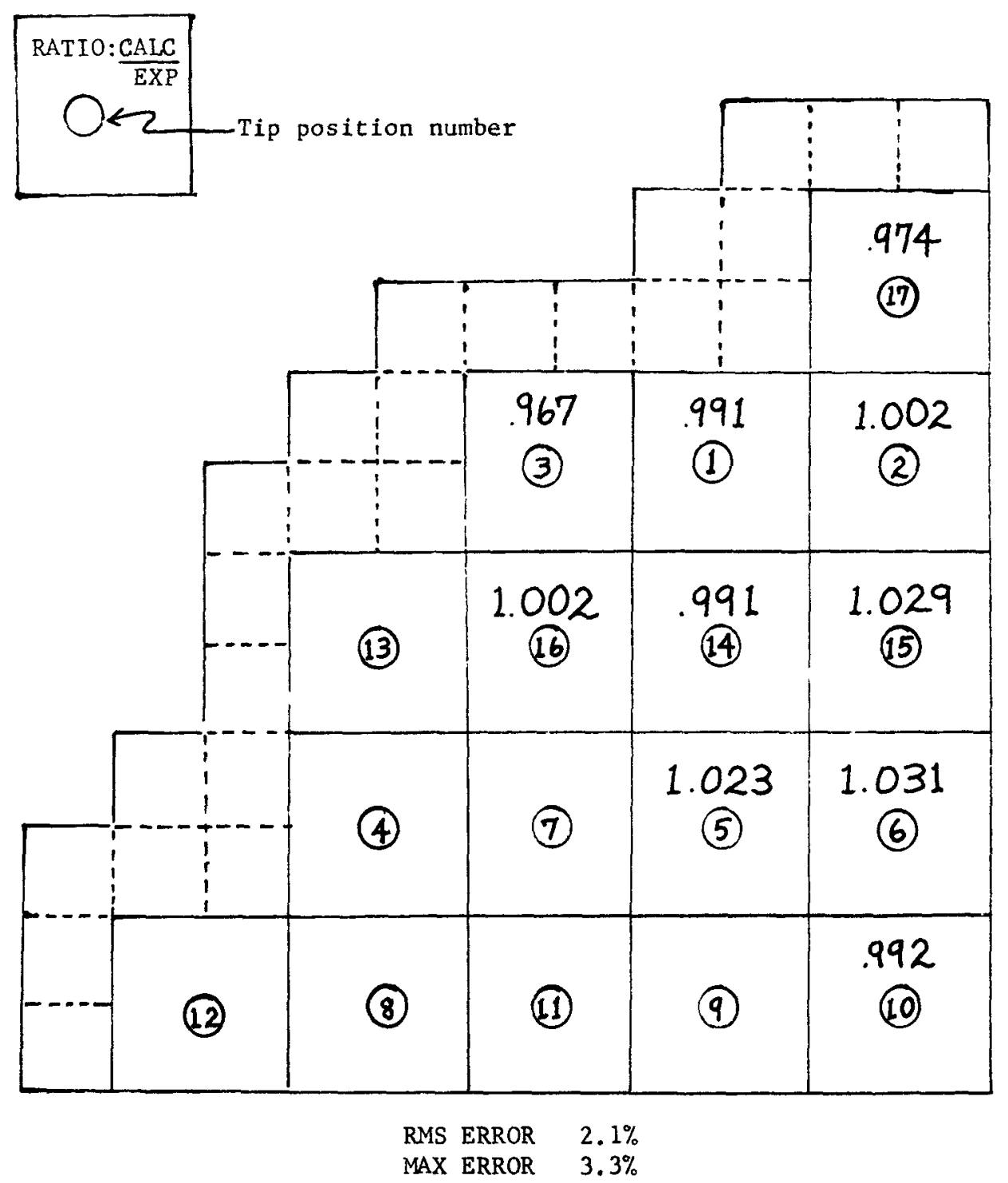

CALC: Average of integrated axial power of surrounding four bundles EXPT: Average of trace (planimeter)

NORMALIZATION: $\quad \sum_{17}$ TIPS $=\sum_{17} \operatorname{TIP}_{i, j}=17.00$

FIGURE 8 


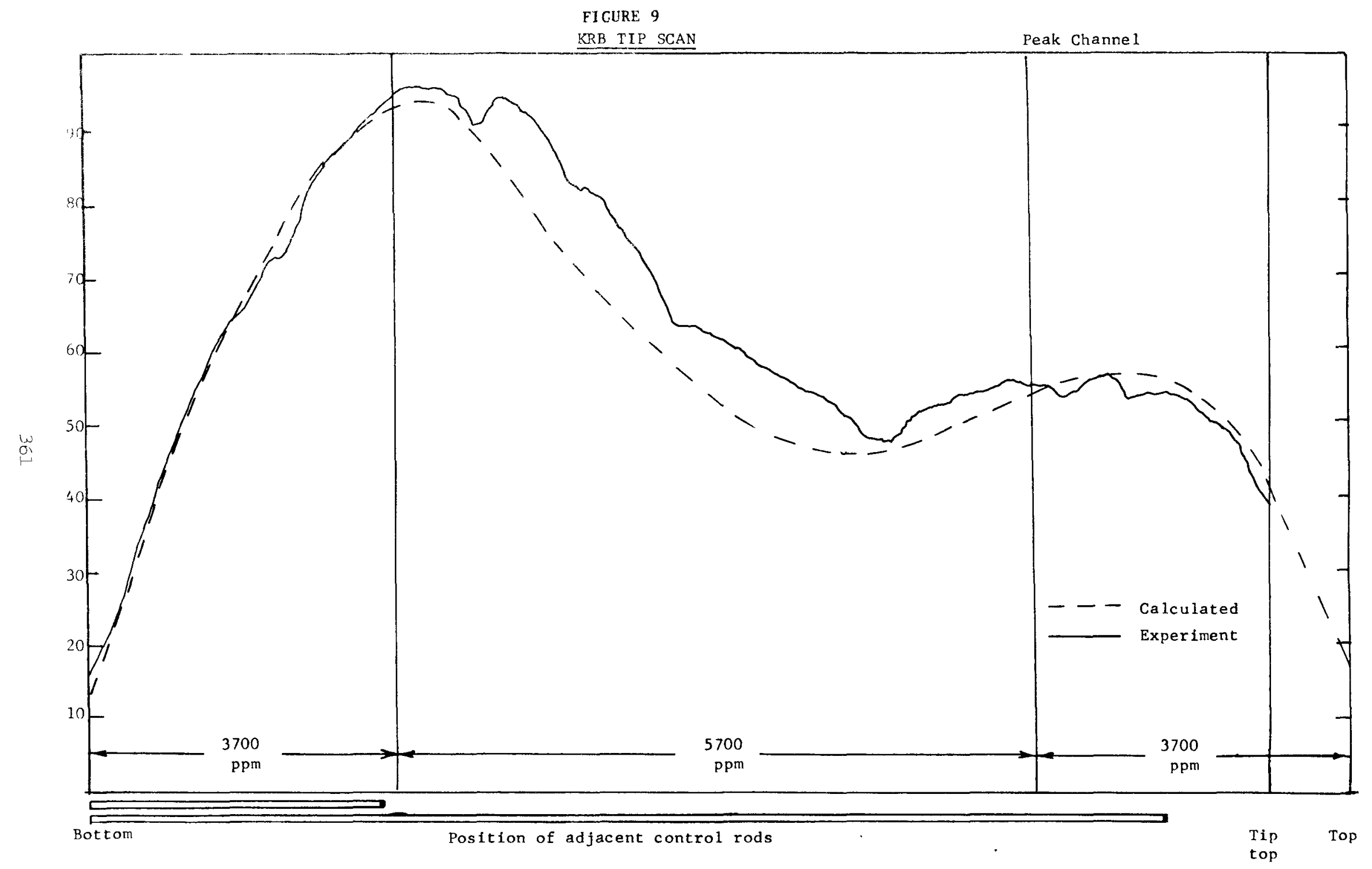




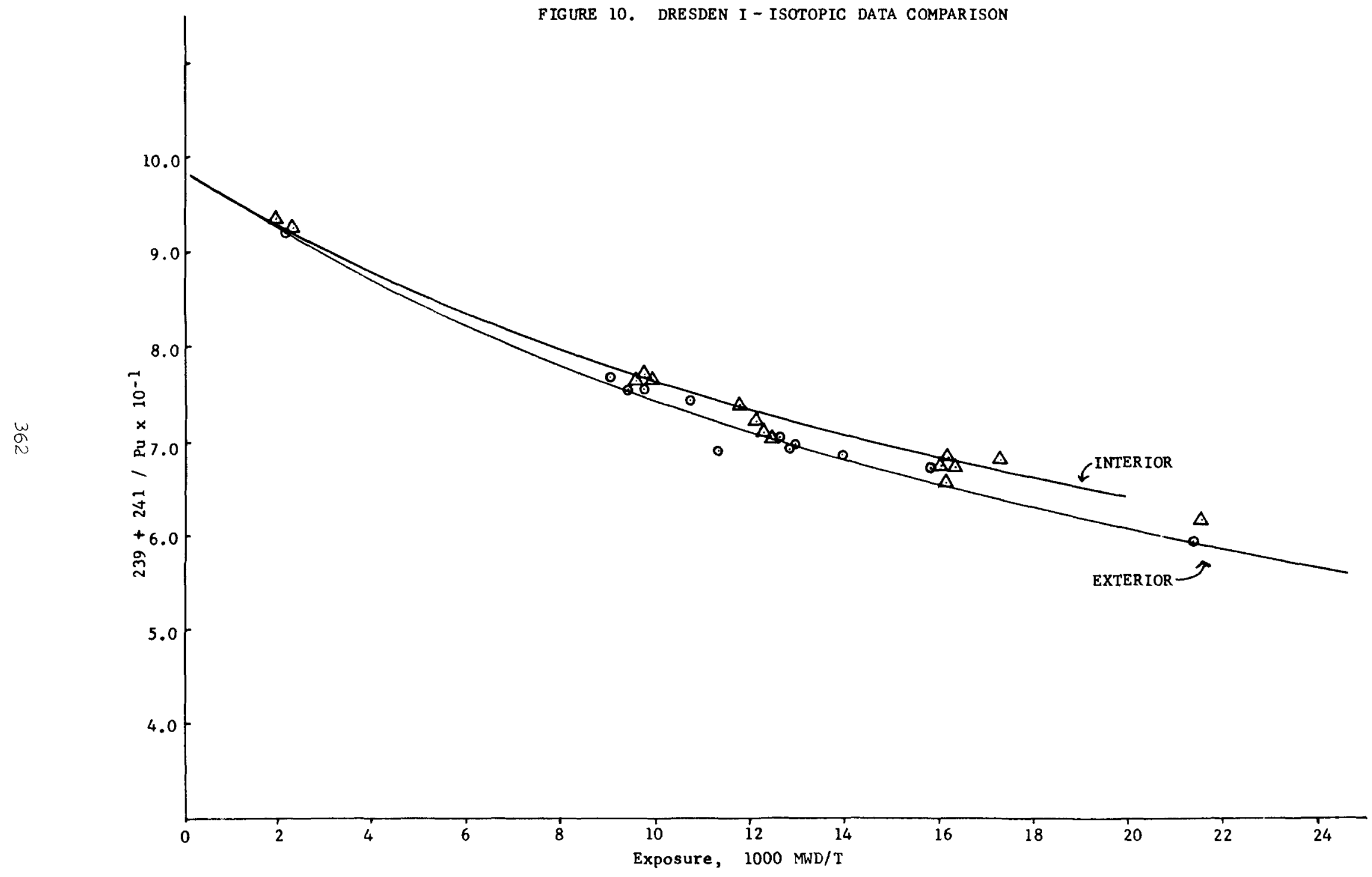


FIGURE 11

COMPARISON WITH MARCHATERRE DATA, 814 psIa

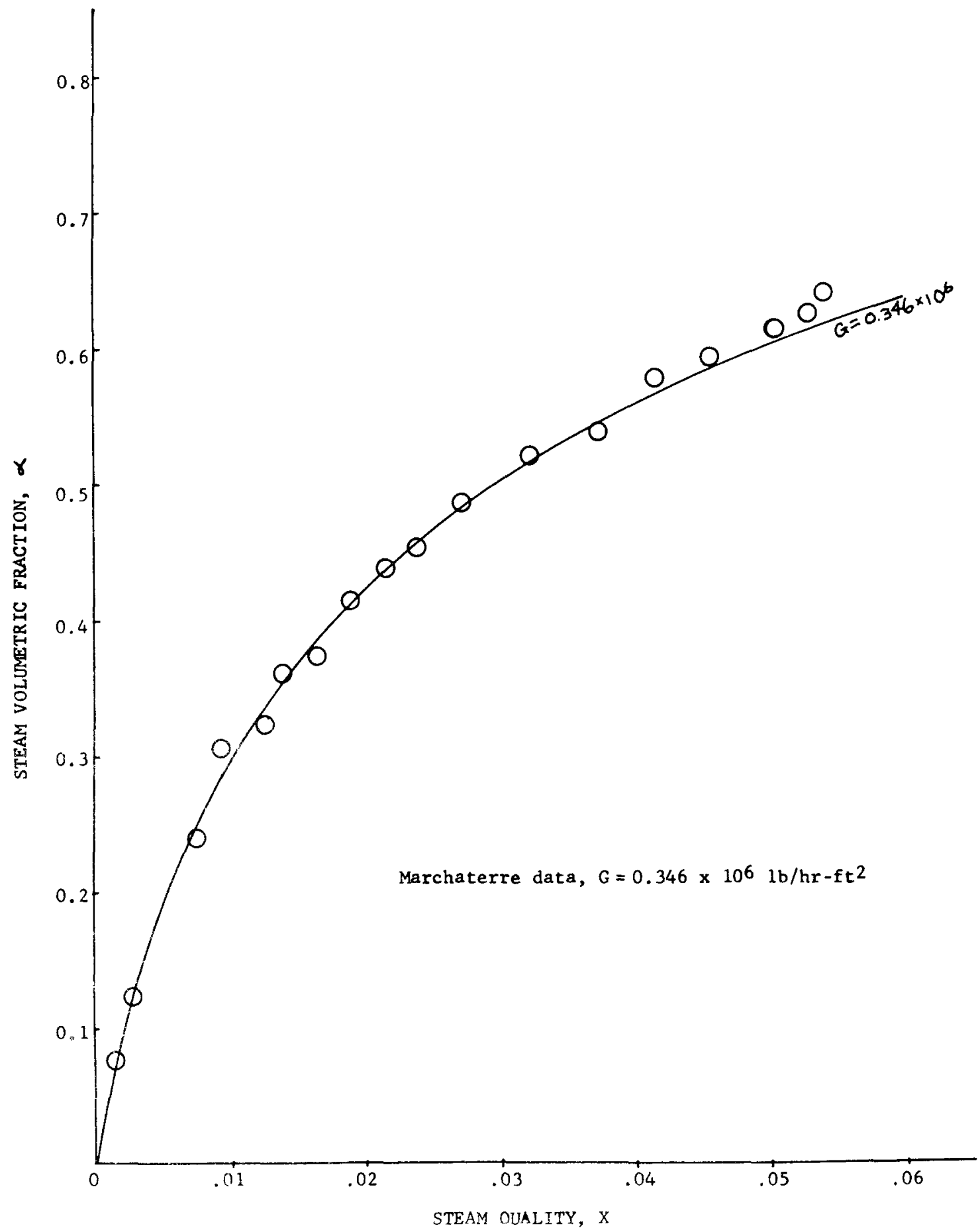


FIGURE 12

COMPARISON WITH CHRISTENSEN DATA, 1000 psia

PRESSURE $=1000 \mathrm{ps} 1 \mathrm{a}$

HEAT FLUX $=0.157 \times 10^{6} \mathrm{BTU} / \mathrm{hr}-\mathrm{ft}^{2}$

MASS FLOW $=0.63 \times 10^{6} \mathrm{lb} / \mathrm{hr}-\mathrm{ft}^{2}$

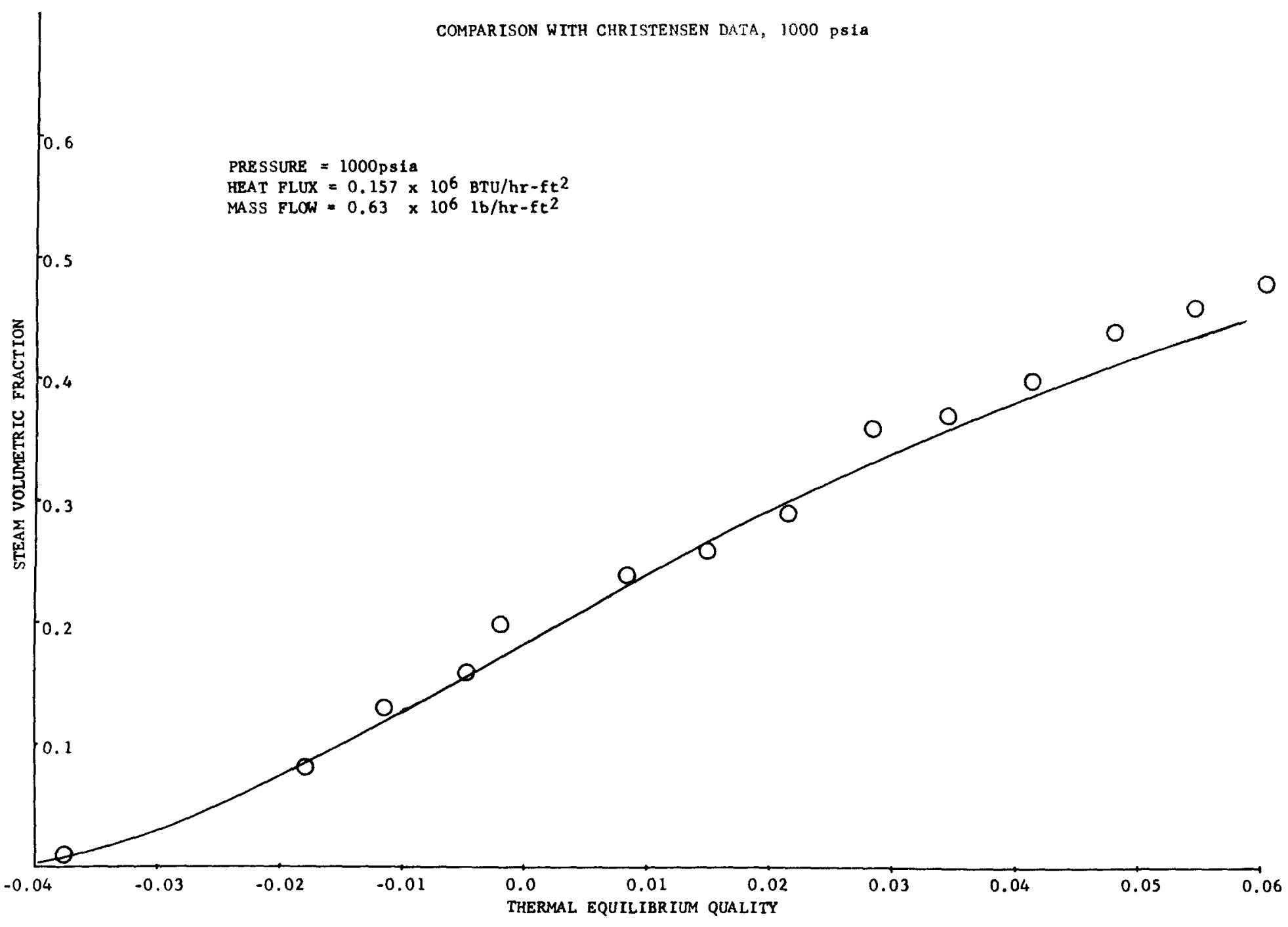




\section{References}

1. R. L. Crowther, "Optimization of Fuel Cycle Performance in Boiling Water Reactors." University of Wisconsin Extension on Fuel Management in Nuclear Power Plants, Dec. 1968. (To be published).

2. D. L. Fischer, J, M. Harriman and M. J. Stedwe11. "FLARE, A ThreeDimensional Boiling Water Reactor Simulator." GEAP-4598. (July 1964)

3. W. V. MacNabb and D. L. Fischer. "A Method For Solving Directly For The Exposure Distribution at the End of a Power Reactor Fue1 Cycle." International Conference on Reactor Mathematics and Computation, Mexico City (May 1967).

4. N. H. Barth. "Physics Specifications for SENN On-Line Computer." GEAP-4346. (July 1964).

5. R. L. Crowther. "Burn-Up Analysis of Large Boiling-Water Reactors." Proceedings of a Panel in Vienna, Apri1 1967 on Fuel Burnup Predictions in Thermal Reactors. IAEA, Vienna (1968).

6. P. G. Aline, R. O. Brugge, W. R. Clancey, R. L. Crowther, D. L. Fischer, C. P. Ruiz, and D. L. West. "Fuel Management and Isotopic Composition Prediction and Experiment in Light Water Power Reactors." Proceedings of International Conf. on the Physics Problems in Thermal Reactor Design. British Nuclear Energy Society. (June 1967).

7. R. L. Crowther. "Extensions of the Power Control Method for Solution of Large Inhomogeneous Reactor Problems." ANL-7050. (1965).

8. R. L. Crowther and L. K. Holland. "Nuclear Applications of On-Line Process Computers." Nuclear News (May 1968).

9. C. A. Meyer, et.al. "Thermodynamic and Transport Properties of Steam." ASME (1967).

10. P. G. Aline, N. H. Barth, W. R. Clancey, R. L. Crowther, D. L. Fischer, J. W. Hallam, A. Weitzberg, F. G. Warzek and I. B. Wall. "The Physics of Non-Uniform BWR Lattices." Proc. of International Conf. on the Physics Problems in Thermal Reactor Design. British Nuclear Energy Society. (June 1967).

11. A. Leonard and C. T. McDanfel. "Transport Solutions For Two-Dimensional Mu1tigroup Thermal-Neutron Distributions in Nonuniform Lattices." Trans. ANS. V11, $\mathrm{n} 2,556 .(1968)$. 


\section{BLANK}


BESSION

HYBRID COMPJTATIONS

chairman: s. I. Juny

University of Tennessee 


\section{BLANK}




\title{
HYBRID SOLUTION OF PARTIAL DIFTERENTIAL EQUATIONS IN NUCLEAR PLANT SIMULATIONS: A REVIEW
}

\author{
R. Vichnevetsky \\ Electronic Associates, Inc. \\ and Princeton University \\ Princeton, New Jersey
}

\section{INTRODUCTION}

The transient simulation of overall nuclear power plants is a task which is extremely demanding in terms of computer time. The presence of partial differential equations in the corresponding mathematical models is probably the most important single factor contributing to this fact. With the rapid increase of the number of plants being constructed, the need for developing economical methods for these kinds of problems has arisen. Hybrid computers, with their analog capability to integrate effectively and at high speed sets of ordinary differential equations, and their digital capability for function generation, function memory and the implementation of complex sequential algorithms, have proven to provide a tool around which such methods could be developed.

The development of an adequate mathematical background of theory in the hybrid solution of the rype of partial differential equations found in the nuclear power industry has taken place urer the rast decade or so. Aithough closely related to the simultaneous development of analog as well as numerical stechods, the unique combination of continuous and discrete alnewatical operators within a single computing system has generated possibilities, as well as problems, which are tiuly "hybrid" in nature. In the present state of the art, many of these problems have been resolved in a satisfactory manner, and many of them remain open to further investigation.

It seems usetui to identify the type of problems for which hybrid computers are being used in nuclear reactors and plant simulations. These problems generally have the following parameters.

(3) Concomitant simulation of more than one dynamic stibsytein, where emphasis is placed upon the translent interaction of the suisystems rather than on the detailed internal behavior of each subsystem.

(b) Overall plant simulations which are used for operation evaluation purposes. That is, many computer runs are requized either to optimize unspecified design paramecors, or to assess the effect of predictable malfunctions.

(c) Detailsd nulti-dimensional transient simulation of reactor cores, with simultaneous consideration of neutrons, thermal and fluia arnamı phenomena. 
(d) Detailed transient simulation of steam generating units, with consideration of all nonlinear phenomena.

Studies as (c) and (d) above are usually performed as a preliminary scep to integrating the resultant programs into larger simulations having the characteristics of (a) and (b).

In summary, the requirement placed upon the computing algorithms and computer systems needed to solve these problems are:

(a) Speed in the computation, to bring these largescale studies into the realm of practicality.

(b) Simplification of the mathematical models to a degree compatible with the representation of the elements of a nuclear plant, in a systems sense, not in a detailed internal sense.

For the kind of studies cited above, i.e., for nuclear plant simulatıon proilems involving significant portions represented by partial differential equations, hybrid computer speed advantages (over numerical simulation) of one to two orders of magnitude have been reported 4,51,5\}.

Functionj11y, the most important characteristic of hybrid con uteng .... ch rakes them suitable for solving partial dif-

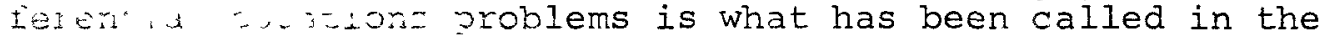
texmincicgy of that discipline the "function storage and

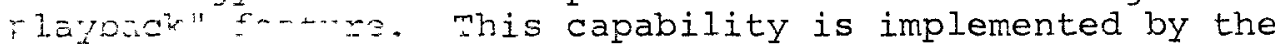
combination of fast analog-to-digital converters by which functions of time (solutions) generated by the analog computer are sampled and converted to digital words, storage of these functions in the form of memory arrays in the digital computer, and digital-to-analog conversers, which reconstitute (or playback) these functions into the analog computer with suitable smoothing. (Some methods require these functions to be played back in the reverse direction of that in which they were generated.)

\section{EQUATIONS AND ASSUMPTIONS}

From a mathematical standpoint, transient field problems found in nuclear power plants generally fall into one of the following categories:

(1) Parabolic partial differential equations describing heat or neluton flux diffusion phenomena

(2) First-order partial differential equations of the material transport type, describing fluid flows, usually with heat exchange

(3) Any combination of the two types above. In particular, coupled first-order partial differential equations in countercurrent heat exchangers lead to problems of the hyperbolic type.

There are some examples of the use of analog/hybrid computers for the solution of elliptic problems for reactor steady-state design, but these seem to be the exception rather than the rule; references 13 and 31 describe such analog/hybrid computer applications.

We shall review in some detail the classes of problems at hand. 


\subsection{The Homogeneous-Reactor Assumption}

Nuclear reactor cores are essentially heterogeneous systems, consisting of an assembly of fuel, moderator, structural eiements, and cooling media. For overall plant studies, the assumption is generally made that a homogeneous mathematical model, where all materials are present in all points in space, is adequate for the neutron flux equations. This assumption makes it possible to represent nuclear reactor core dynamics by partial differential equations where most terms are given artificial continuity properties. This procedure is not dissimilar to statistical mechanics, where macroscopic properties are described by equations obtained by averaging over many microscopic processes.

\subsection{Cocling-Channel, Boilers and Heat Exchangers}

The thermal model of nuclear plants hinges on reasonable modelling of the thermal behavior of cooling channels and heat exchangers. Reduction of the partial differential equations to a suitable form took place in the mid $1950^{\circ} \mathrm{s}$. A very good paper describing simple mathematical models for channel thermal behavior was presented by O'Neill in 195835 ; it is an example of the type of mathematical modeliing that was going on at that time in Great Britain. Similar studies were taking place in the U.S. and in France at about the

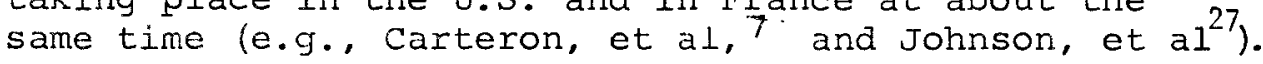

Boilers and heat exchangers lead to partial differential equations of the coupled-first-order type. Generalized use of once-through boilers has resulted in much more complex mathematical models than those of conventional voilers. mest models could not easily be represented cn analo; computers, but are easily implemented by hyorld mec.ods.

Recently, the large-scale hybrid simulation of a complete nuclear power plant, including a detailed nonLien nustation of the once-through boiler, was performed by the British Central Electricity Generating Board. ncoount of this work is to be found in Blare, et a 1,4 . Fiermelink 52 also describes a hybrid program tor the simidision of a once-through steam generator.

\subsection{Reaial Fizimuthal Studies}

Kad d 12 imuthal representations of reactor cores are inosicy of interest for spatial instability problems, suci as:

(a) riose introduced by the interaction between suependently actuated control rods, and

(i) Neutron flux instabilities induced by xenon poisoning.

The concerr ove spatial instability has arisen mostly in :.e mid-to-late $1950^{\prime} \mathrm{s}$, with the construction of iarge nower reactors. 


\subsection{Axial Studies}

By contrast with radial/azimuthal models, axial representation of reactor cores is of more concern in the analysis $n i$ temperature peaks during induced transients. It is generally included in larger simulations involving more or less complete power plants and their control systems.

3. DISCRETE SPACE CONTINUOUS TIME (DSCT) METHOD OF LINES

The hybrid methods of solution of partial differential equations were developed to alleviate some of the limitations of the analog DSCT method of lines (also called parallel-finitedifferences method). The DSCT method is obtained when finite differences approximations are used to replace all partial differential operators involving spatial derivatives, thus resulting in sets of initial valued ordinary differential equations. These can be solved with ease by conventional analog computer methods. This general approach was almost universally adopted in nuclear reactors simulation in the $1950^{\prime}$ 's, when the scope of computer simulation problems was relatively limited and many users still had to establish confidence in simulation results versus experiment. But a general property of the parallel finite differences method is that the erizenont complement needed grows in proportion to the accuracy desired. Very large analog computer systems were elaborated. An extreme, although by no means unreasonable, example is that of the 1500 amplifier Saturn computer manufactured and operated by the English Electric Co. in Great Britain.

The high cost paid for accuracy in the application of the DSCT has fostered the development of hybrid methods of solution of partial differential equations which preserve the speed advantage of analog computers but save on equipment requirements.

Two such methods are:

(a) the CSDT method of lines (or serial-finitedifferences method), and

(b) the multiplexing methods.

4. THE CONTINUOUS-SPACE-DISCRETE-TIME (CSDT) METHOD OF LINES Continuous-space-discrete-time (CSDT) methods of lines (also called "serial", by contrast to the parallel methods previously discussed) allow higher resolution and accuracy to be obtained without the burden of increasing hardware requirements. As potad in the preceding section, the amount of hardware needed to solve partial differential equations by parallel methods grows in direct proportion to the desired accuracy. But if the solution contains low frequency terms only (as is often the case), then the dynamic capabilities of analog hardware are not utilized. The potential speed performance of the latter remains untapped.

The serial methods of solution which we are about to describe require the capability to store and playback functions; i.e., hybrid computing capability. In contrast to parallel modal or finite difference methods, serial methods allow use of the maximum speed capability of the analog computer and hybrid 
interface system, and therefore greater accuracy can be obtained with relatively little equipment.

With the availability of hybrid computers, considerable interest has developed towards serial methods in recent years. Many applications of this method have been reported, especially for the representation of boilers and heat exchangers. Successful applications to core neutronic equations simulation have also been reported, and further development of related methods and algorithms in this area is going on.

\section{1 Serial Method for Cooling Channels and Heat Exchangers}

When applied to first-order partial differential equations (cooling channels and heat exchangers), the serial method results in a sequence of stable, initial-valued ordinary differential equation problems.

Consider the one-fluid equation, where $\boldsymbol{V}$ represents the velocity of the fluid, $T_{w}$ and $T_{f}$ are the wall and fluid temperatures, respectively, and $C$ the ratio of fluid/wall thermal conductivity to fluid specific heat per unit length:

$$
\frac{\partial T_{f}}{\partial t}=-V \frac{\partial T_{f}}{\partial z}+C\left(T_{w}-T_{f}\right) \quad ; z \in[0, L]
$$

becomes, by application of the backward finite differences approximations in time:

$$
\begin{aligned}
& \frac{d T_{f}{ }^{i+1}}{d z}+\left(\frac{1}{V \Delta t}-\frac{c}{V}\right) T_{f}^{i+1}=T_{f}^{i} \frac{1}{V \Delta t}+\frac{c T_{w}^{i+1}}{V} \\
& T^{i}(z)=T\left(z, t^{i}\right) ; t^{i}=i \Delta t ; i=0,1,2 \ldots .
\end{aligned}
$$

This equation can also be rewritten as:

$$
\frac{d T_{f}^{i+1}}{d z}=\frac{1}{V \Delta t}\left(T_{f}^{i}-T_{f}^{i+1}\right)+\frac{C}{V}\left(T_{w}^{i+1}-T_{f}^{i+1}\right)
$$

A hyorid circuit diagram corresponding to the integration of that equation is shown in figure 1 .

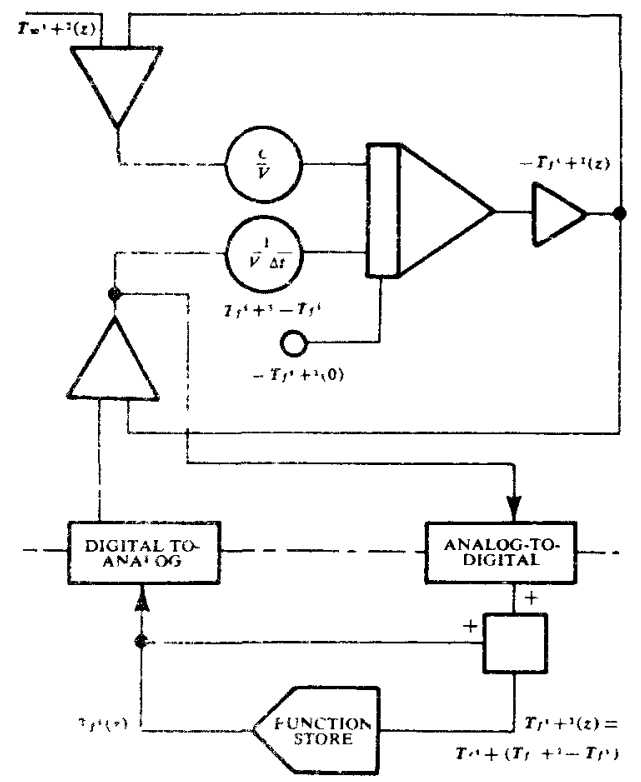

Figure 1

Hybrid Computer Circuit Diagram for the CSDT Integration of a One-Fluid Heat Exchanger 
Note that, in this implementation, the analog to digital conversion is performed on the variable $\left(T_{f}^{i+1}-T_{f}^{i}\right)$

which is of the order $\Delta t$. The scaling factor used for the analog representation of this quantity can be chosen so that the round-off error in the conversion is negligible.

When applying this method to countercurrent heat exchangers, one is faced with the problem of integrating in space two ordinary differential equations for which initial conditions are given at opposite ends of the domain of integration. This is illustrated by the following example:

4.2 Consider the countercurrent heat exchanger of figure 2 , which is described by the equations - (the thermal capacity of the wall is assumed to be negligible):
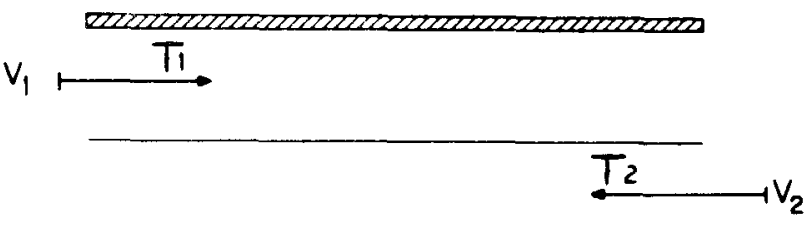

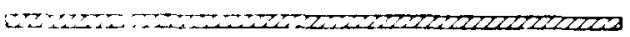

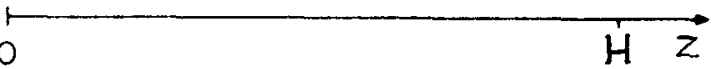

$$
\begin{aligned}
& \frac{\partial T_{1}}{\partial t}=-V_{1} \frac{\partial T_{1}}{\partial z}+k_{1}\left(T_{2}-T_{1}\right) \\
& \frac{\partial T_{2}}{\partial t}=V_{2} \frac{\partial T_{2}}{\partial z}+k_{2}\left(T_{1}-T_{2}\right)
\end{aligned}
$$

where $\sqrt{1}$ and $\sqrt{2}$ are respective velocities of the primary and secondary fluid.

The CSDT method can be applied to this (hyperbolic) problem by integrating each equation at time intervals of $\Delta t / 2$ This is in effect an interlaced (in time) method. The resulting equations are:

$$
\left.\begin{array}{l}
\frac{d T_{1}^{i}}{d z}+\left(\frac{1}{\sqrt{1} \Delta t}+\frac{k_{1}}{V_{1}}\right) T_{1}^{i}=\frac{T_{1}^{i-1}}{\sqrt{1} \Delta t}+\frac{k_{1}}{\sqrt{1}} T_{2}^{i *} \\
T_{2}^{i *}=3 / 2 T_{2}^{i-1 / 2}-1 / 2 T_{2}^{i-3 / 2}
\end{array}\right\}
$$


The * values are seen to be extrapolated values of $T_{1}$ and

$T_{2}$, respectively.

Equation (6) is integrated in the forward direction, and (7) in the backward direction. Initial conditions for these integrations are the input temperatures $T_{1}(t, 0)$ and $T_{2}(t, L)$, respectively.

A resulting simplified hybrid computer block diagram is that of figure 3 .

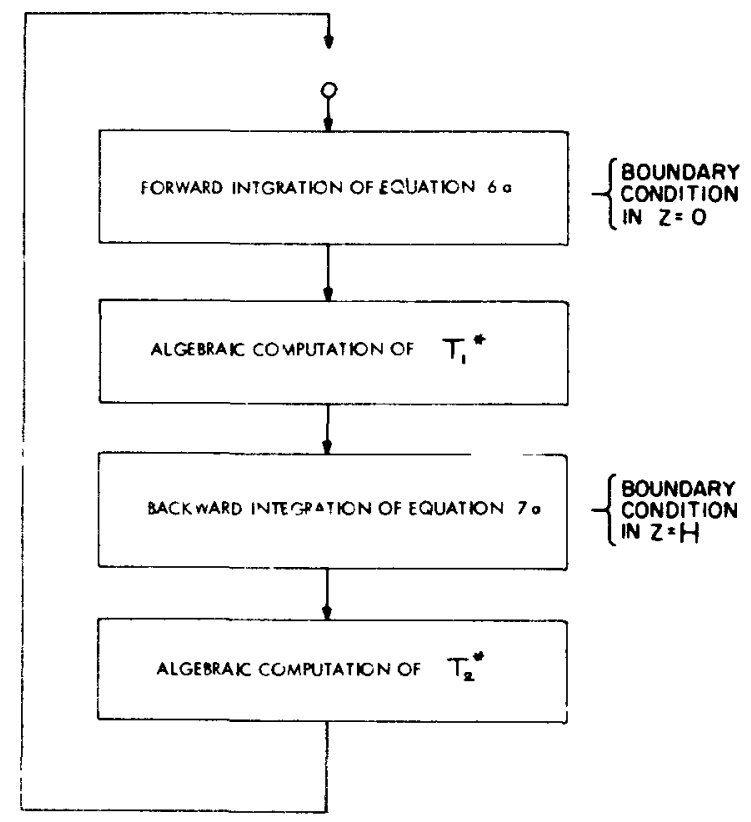

Figure 3

Sequence of Operations in the Hybrid Computer CSDT Integration of the Counter Current

Heat Exchanger

\subsection{Serial Simulation of Once-Through Boilers}

The dynamics of the once-through boilers used in most modern nuclear power stations represent an important phenomenon in the overall transient behavior of the plant. Their accurate representation results in nonlinear-hyperbolic partial differential equations which can easily represent a major obstacle towards computing speed when handled improperly.

the serial method, which is typically hybrid in its approach, lias been applied to this problem. Most of this work to date has taken place in Europe 10, 52, because large hybrid computer systems for nuclear applications were installed there earlier than in the U.S.

whe approach is essentially that described in the preceding section with the addition of appropriate equations describing boiling phenomena and conservation of momentum. 


\subsection{CSDT Method Applied to Parabolic Diffusion Equations}

For parabolic diffusion equations, the application of the serial method results in difference-differential equations with mixed boundary valles and unstable error propagation properties. These apparent difficulties can, however, be overcome if handled correctly. Te shall lliustrate this by referring to the axial neutron diffusion equation.

Let $\phi$ be a cre-group neutron flux density, $l$ the neutron lifetime, $M^{2}$ the migration area and $k$ the effective multiplication factor. A simplified form of the axial neutron kinetics equation is:

$$
\begin{aligned}
& l \frac{\partial \phi}{\partial t}=M^{2} \frac{\partial^{2}}{\partial z^{2}} \phi+(k-1) \phi ; \\
& z \in[0, H] ; \phi(0)=\phi(H)=0
\end{aligned}
$$

Assuming fixed time intervals $t^{i}=i \Delta t$, an approximation of that equation may be written:

$$
\begin{gathered}
\frac{\phi^{i+1}-\Phi^{i}=}{3=}\left[M^{2} \frac{d^{2}}{d z^{2}}+k-1\right] \phi^{i+1}+(1-\theta)\left[M^{2} \frac{d^{2}}{d z^{2}}+k-1\right] \phi^{i} \\
\phi^{i+1}(0)=\phi^{i+1}(H)=0
\end{gathered}
$$

$$
(\theta \text { is here an arbitrary scalar } \leqslant 1 \text { ). }
$$

or:

$$
\frac{d^{2} \phi^{i+1}}{d z^{2}}-\left[\frac{l}{M^{2} \theta \Delta t}-\frac{k-1}{M^{2}}\right] \phi^{i+1}=-\frac{\ell}{M^{2} \theta \Delta t} \cdot \tilde{\phi}^{i}
$$

where

$$
\widetilde{\phi}^{l}=\phi^{i}+(1-\theta) \frac{\Delta t}{l}\left(M^{2} \frac{d^{2}}{d z^{2}}+k-1\right) \phi^{i} \simeq \phi^{i+1-\theta}
$$

represents past information which is stored and played back at the time $t=t^{i+1}$ when equation $(10)$ is
integrated to yield $\phi^{i+1}$. oblajnea recursively by the algebraic expression (taking (10) and (II) into account) :

$$
\begin{aligned}
\widetilde{\phi}^{i+1} & =\phi^{i+1}+(1-\theta) \frac{\Delta t}{l}\left(M^{2} \frac{d^{2}}{d z^{2}}+k-1\right) \phi^{i+1} \\
& =\tilde{\phi}^{i}+\frac{1}{\theta}\left(\phi^{i+1}-\tilde{\phi}^{i}\right)
\end{aligned}
$$

Since, according to (11), $\tilde{\phi}^{i}$ represents an approximation of $\phi$ at time $t=(i+1-\theta) \Delta t$, an initial value for $\widetilde{\phi}$ is obtained simply by equating it with the initial neutron flux $\phi(z, 0)$, and shifting the origin of time consistently. 
A general block diagram of the hybrid function storage and playback involved in equations (10), (12) is shown in figure

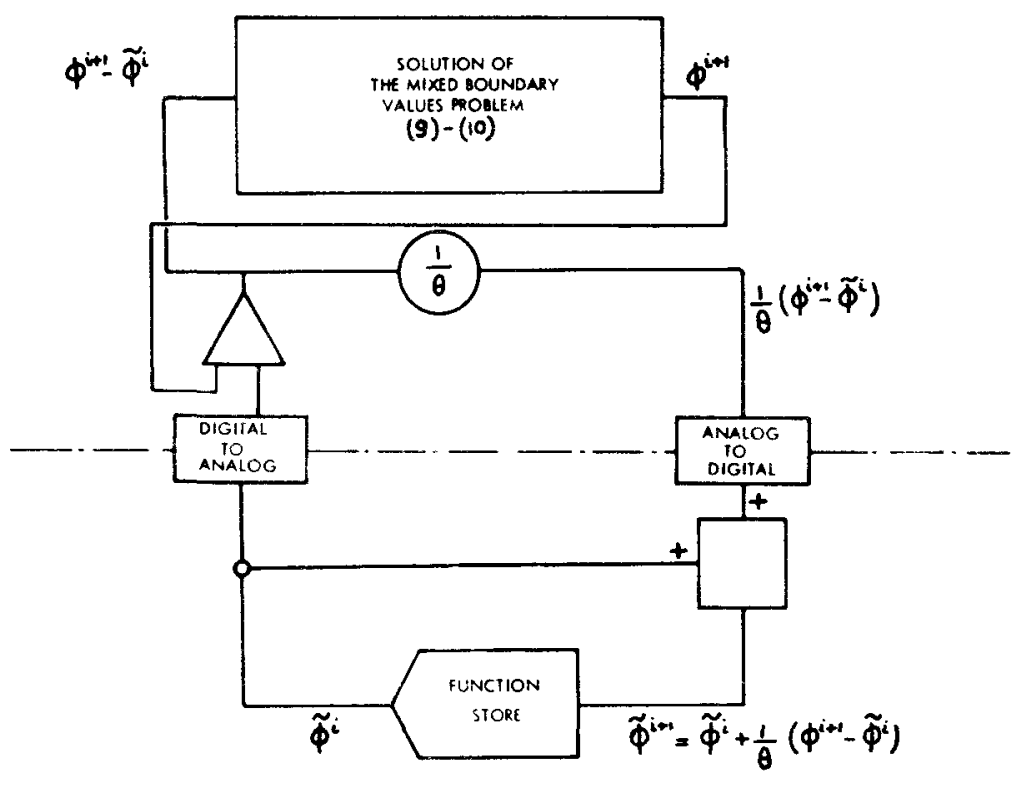

Figure 4

CSDT Hybrid

Integration

of the

Diffusion

Equation

\section{SOLUTION OF THE ASSOCIATED BOUNDARY VALUES PROBLEM}

The reduced problem in ordinary differential equations is of the mixed boundary value type. We also note that the characteristic equation of (10), namely:

$$
\lambda^{2}-\left[\frac{l}{M^{2} \theta \Delta t}-\frac{k-1}{M^{2}}\right]=0
$$

has generally two real roots of opposite sign, which has the result that any direct integration of that equation in the direction of $z$ (or $-z$ ) will have unstable error propagation properties. Accordingly, attempting to solve this problem as an initial value problem (the target shooting method) will only be possible if great care is taken to preserve as much accuracy as possible in the computation. But this, in addition to requiring great care in the implementation, would also require small increments to be taken in the function storage and playback routines, thereby defeating the objective of speed and economy.

What is needed here is an efficient and fast way to solve the boundary values problem (10), since this operation must take place at every time step.

Methods which have been proposed to this effect are:

1. Superposition Method (Calza-Bini, et al, ${ }^{23}$ )

As in the target shooting method, the integration is started at $z=0$ with any value for $\partial \phi /\left.\partial z\right|_{0}$

A solution of the homogeneous equation with boundary value $y=0$ at $z=0$ and $y \neq 0$ at $z=H$ is added to the computed solution so that the boundary condition $\phi(H)=0$ is satisfied. 
Since iteration is avoided, the iteration difficulty is avoided, although the problem of instability still exists. (The major effect of the unstable error propagation property is thet, exror bounds are high.)

2. Green's Functions Method (Witsenhausen 46 )

Green's furctions of the differential equation (10) associated with the boundary conditions (9) are precomputed and stored ir. the digital portion of the hybrid system. The solution of (10), using those Green's functions, is expressed by a functional or definite integral for each value of $z$

3. Decomposition Method (Vichnevetsky ${ }^{55}$ )

The problem is decomposed into two first-order stable problems which can be computed independently. Satisfaction of the boundary conditions is obtained then, either by

superposition ías in (1) above), or by iteration (as in the shooting rethod).

We will analyze in some more detail the two last methods cited. For simplicity, we rewrite equation (10) in the form:

$$
\frac{d^{2} \phi}{d x^{2}}-\gamma \phi=R(z)
$$

Sreen's Finatones Method

To apply the Cras's Functions method, the solution of equation -s exrreses by the integral:

$$
\phi(z)=\int_{0}^{H} G\left(z, z_{1}\right) \cdot R\left(z_{1}\right) d z_{1}
$$

where $G\left(z, z_{1}\right)$ is the Green's function of the problem. For constant $\gamma$ ( 1. e.. independent of $z$ ), an analytic expression of $G\left(z, z_{1}\right)$ is easily obtained.

$$
G\left(z, z_{1}\right)=\frac{1}{\sqrt{\gamma} \sinh \sqrt{\gamma} \cdot H} \begin{cases}\sinh \sqrt{\gamma} z_{1} \cdot \sinh \sqrt{\gamma}\left(1-\frac{z}{H}\right) & \left(z_{1} \leqslant z\right) \\ \sinh \sqrt{\gamma} z \cdot \sinh \sqrt{\gamma}\left(1-\frac{z_{1}}{H}\right) & \left(z_{1} \geqslant z\right)\end{cases}
$$

Several drawioacks of the Green's function method are easily identified. The most important one is that the calculation is lengthy, since $N$ complete integrals ( 14 ) must be computed for each time step if $N$ is the number of points used to store $\phi(z)$. Moreover, for more complex problems, e.g., when $\gamma$ depends on $z$ ), it becomes a relatively complex task to derive the Green's functions themselves.

A variation of the Green's function method is known under the name of the Integral Equation method 46,54 : Rewrite equation in the form:

$$
\frac{d^{2} \phi}{d z^{2}}=\gamma \phi(z)+R(z)
$$

Treating the righthand side as a known "forcing function", the Green's functions of the operator $d^{2} / d z^{2}$ are used to write the solution of (13) in the form: 


$$
\phi(z)=\int_{0}^{H} K\left(z, z_{1}\right)\left[\gamma \phi\left(z_{1}\right)-R\left(z_{1}\right)\right] d z_{1}
$$

where the Green's function $K\left(z_{1} z_{1}\right)$ is now:

$$
K\left(z, z_{1}\right)= \begin{cases}\frac{z}{H}\left(1-\frac{z_{1}}{H}\right) & \left(z \leqslant z_{1}\right) \\ \frac{z_{1}}{H}\left(1-\frac{z}{H}\right) & \left(z \geqslant z_{1}\right)\end{cases}
$$

The problem has been simplified in that the Green's functions

(17) are significantly more simple than (15). But at the same time, we are faced with an integral equation (the unknown

$\phi(z)$ appears in both sides of (16) , which must be solved by some form of iterative scheme, thus increasing the computation time essentially by a number equal to the number of iterations needed at each time step.

For the reasons cited above, both aspects of the application of the Green's function method to problems in nuclear reactor simulation have been little used, and abandoned as a general means of solution.

(Further analysis of these methods can be found in ref. 54 .)

The Method of Decomposition

The method of decomposition applied to (13) consists essentially in replacing this second order problem by two first order-initial value problems. This is achieved by observing that, if $y_{1}(z)$ is a solution of:

$$
\frac{d y_{1}}{d z}+\sqrt{\gamma} y_{1}=R(z)
$$

and $y_{2}(z)$ is a solution of:

$$
\frac{d y_{2}}{d z}-\sqrt{\gamma} y_{2}=y_{1}
$$

then it foilows that $y_{2}$ is a solution of (18), i.e.:

$$
\begin{aligned}
\frac{d^{2} y_{2}}{d z^{2}}-\gamma y_{2} & =\left(\frac{d}{d z}+\sqrt{\gamma}\right)\left(\frac{d}{d z}-\sqrt{\gamma}\right) y_{2} \\
& =\left(\frac{d}{d z}+\sqrt{\gamma}\right) y_{1}=R(z)
\end{aligned}
$$

q.e.d. 
It then becomes a simple matter (because of linearity) to satisfy the boundary conditions of the problem by adding to $y_{2}$ two elementary solutions of the corresponding homogeneous equation; i.e.:

$$
\phi(z)=y_{2}+a_{1} y_{3}+a_{2} y_{4}
$$

where $y_{3}$ and $y_{4}$ satisfy $\left(\frac{d^{2}}{d z^{2}}-\gamma\right) y_{3,4}=0$

and $a_{1}$ and $a_{2}$ afe defined algebraically by the conditions:

$$
\phi(0)=\phi(H)=0
$$

This method has the advantage of economy in computing time as well as a reduction in error bounds, when compared to the other methods.

\section{Space Dependence}

When $\gamma$ is a sunction of $\boldsymbol{z}$ the method of decomposition must oe extended to aliow for this dependence.

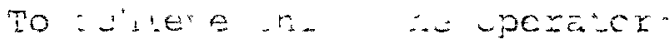

$$
: 1=\frac{d^{2}}{d z^{2}}-\gamma(z)
$$

is decomposed into two operators, respectively stable in the forward and backward directions of integration (meaning $z=0$ to $z=H$ and $z=H$ to $z=0$, respectively):

$$
L(\cdot)=L_{8} \cdot L_{F}
$$

Then, as previously, a particular solution $y_{2}(z)$ of $(13)$ is obtained by solving the two stable problems:

$$
\begin{aligned}
L_{B}\left(y_{1}\right) & =R(z) \\
L_{F}\left(y_{2}\right) & =y_{1} \\
\therefore \quad L\left(y_{2}\right) & =L_{B} \cdot L_{F}\left(y_{2}\right)=R(z)
\end{aligned}
$$

and the solution satisfying the appropriate boundary conditions is obtained by adding to $y_{2}(z)$ solutions of the
homogeneous problem $L(\cdot)=0$

A decomposition such as (24) is provided by writing, formally:

$$
\left.\begin{array}{l}
L_{F}=\frac{d}{d z}-\lambda_{F}(z) \\
L_{B}=\frac{d}{d z}-\lambda_{B}(z)
\end{array}\right\}
$$


The condition of stability requires that $\lambda_{F} \leqslant 0$ and $\lambda_{B} \geqslant 0$ Identifying (13) and (26) yields the conditions on $\lambda_{f}$

and $\lambda_{B}$ :

$$
\begin{aligned}
L(\cdot)=\frac{d^{2}}{d z^{2}}-\gamma(z) & =\left(\frac{d}{d z}-\lambda_{B}\right)\left(\frac{d}{d z}-\lambda_{F}\right) \\
& =\frac{d^{2}}{d z^{2}}-\left(\lambda_{B}+\lambda_{F}\right) \frac{d}{d z}-\frac{d \lambda_{F}}{d z}+\lambda_{F} \lambda_{B}
\end{aligned}
$$

i.e. :

$$
\begin{aligned}
& \lambda_{B}+\lambda_{F}=0 \\
& \frac{d \lambda_{F}}{d z}-\lambda_{F} \lambda_{B}=\gamma(z)
\end{aligned}
$$

which become:

$$
\begin{aligned}
& \lambda_{F}=-\lambda_{B} \\
& \frac{d \lambda_{F}}{d z}+\lambda_{F}{ }^{2}=\gamma(z)
\end{aligned}
$$

This latter equation is stable when integrated in the backward $(z=H$ to $z=0)$ direction, with any initial condition satisfying:

$$
\lambda_{F}(H) \leqslant 0
$$

The condition $\lambda_{F}(z) \leqslant 0$ is then always satisfied. (Note that when $\gamma$ is a constant, and the integration of

(29) is started off with the condition $\lambda_{F}(H)=\sqrt{\gamma}$

then $d \lambda_{F} / d z$ is identically zero and $\lambda_{F} \equiv-\sqrt{\gamma}$ which is an icenuical result to the decomposition method used in the preceding section.

The intergrition of (29) needs to be done only once. However, if $\gamma=\gamma(z, t)$ then (29) must be integrated at each time increment.

In particular, this method is extremely useful for the simulation of the axial response of reactor kinetics with detailed representation of control rods reactivity.

\section{REMARKS ON THE APPLICATION OF THE CSDT METHOD OF LINES}

\subsection{Time Stabs Iity}

In the preceaing sections, only those aspects pertaining to the spatial integration problem have been analyzed. An analysis must be conducted for the stability and convergence of the approximation process in the direction of time. In this direction, we have discrete transition operators and stability aralysis is that of a discrete-time process. 
For linear problems, such a stability analysis can be performed by applying to the solution Fourier transforms in space and Laplace transforms in time. Since trigonometric functions are eigenfunctio: of 1 inear differential operators, the analysis can be performed independently for each harmonic. Stability then requires that all modes-in-time corresponding to trigonometric functions-In-space be stable.

For instance, it is easily shown that the serial integration using backward differences (Section 4.1 ) is always stable, and that the method used in section 4.4 requires that $\theta \geqslant 1 / 2$ for stability.

\subsection{Speed}

Hybrid implementation of the CSDT method of integration hinges on the capability to store and playback functions. This mode of operation heavily taxes the hybrid interface hardware, and high speed of analog integration is on iy possible to the degree allowed by the time taken to read information in and out of the digital computer.

The practical result of this is that the hybrid CSDT method of integration becomes of interest principally for relatively complex pat:aid differential equations. This condition is ful-

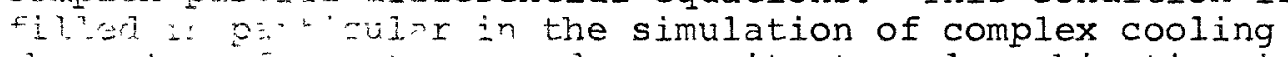
chaune: cor iguratuons and concomitant nuclear kinetics in reactor cores. and even more so in the simulation of oncetnrough boilers.

\section{Stalling}

In the application of the CSDT method, one has in essence the choice of exchanging speed for accuracy by reducing the discrete time step $\Delta t$. The accuracy which can be so achieved is, however, bound by limitations introduced by roundoff error accumulation effects.

This phenomenon, which we may call "stalling" was pointed out by Frank and Lapidus 19. - It is a direct parallel to the roundoff error problem associated with fixed-point numerical integration of ordinary differential equations. In order to reduce the importance of this problem, it is advisable to transfer only increments from the analog to the digital computer. For instance, the diagrams of figures 1 and 4 are crgar: $x_{2} \quad n$ a fashion where only quantities of order $\Delta t$ are transferred. The accuracy of the digital function storage routine car be chosen to produce roundoff errors only at the same point as those introduced by the analog-to-digital converters.

7. APPLICATION OF THE CSDT METHOD OF LINES TO THREE-DIMENS IONAL SIMLLATION OF NUCLEAR REACTORS

When the CSDT method of lines is applied to three-dimensional simulation of nuclear reactors, it is natural to choose the axial direction as that along which the continuous integration takes place. If the neutron flux partial derivatives in the radiai and azimuthal directions were approximated by finitedifferences, this would result in a set of coupled ordinary differential equations. The Green's functions method or the decomposition method are then difficult to apply. By contrast, disposing of the radial-azimuthal problem by applica- 
tion of a modal method results in a set of almost separated ordinary differential equations, for which the Green's function method and the decomposition method apply easily.

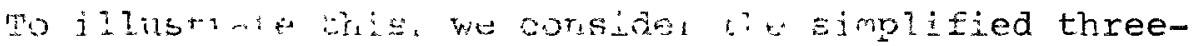

dimensiona syations of a cylindracal gas-cooled reactor:

$$
\begin{aligned}
& l \frac{\partial \phi}{\partial t}=\left(M^{2} \nabla^{2}+k-1\right) \phi \\
& \frac{\partial T_{f}}{\partial t}=-v \frac{\partial T_{f}}{\partial z}+C_{1}\left(T_{m}-T_{f}\right) \\
& \frac{\partial T_{m}}{\partial t}=b \phi+C_{2}\left(T_{f}-T_{m}\right)
\end{aligned}
$$

where:

$$
\begin{aligned}
& T_{m}(r, z \alpha, t)=\text { Finir innperatures } \\
& T_{m}(r, z, \alpha, t)=\text { solva meterual temperatures }
\end{aligned}
$$

Application of the CSDT method of integration in the $z$ directior leads to (using backward differences in time):

$$
\begin{aligned}
& \ell\left(\frac{\phi^{i+1}-\phi^{i}}{\Delta t}\right)=\left[M^{2}\left(\frac{\partial^{2}}{\partial z^{2}}+\frac{\partial^{2}}{\partial r^{2}}+\frac{1}{r} \frac{\partial}{\partial r}+\frac{1}{r^{2}} \frac{\partial^{2}}{\partial \alpha^{2}}\right)+k-1\right] \phi^{i+1} \\
& \frac{T_{f}^{i+1}-T_{f}^{i}}{\Delta i}=-v \frac{d T_{f}^{i+1}}{d z}+C_{i}\left(T_{m}^{i+1}-T_{f}^{i+1}\right) \\
& \frac{T_{m}^{i+1}-T_{m}^{i}}{\Delta t}=b \phi^{i+1}+C_{2}\left(T_{f}^{i+1}-T_{m}^{i+1}\right)
\end{aligned}
$$

Decompose $\Phi^{\prime}(r, z, \alpha)$ into radial azımuthal modes (figure 5 ):

$$
\begin{aligned}
\phi^{i}(r, z ; \alpha) & =\sum_{m=1}^{N} \varphi_{m}^{i}(z) f_{m}(r, \alpha) \\
& \left(\frac{z^{2}}{\partial r^{2}}+\frac{1}{r} \frac{\partial}{\partial r}-\frac{1}{r^{2}} \frac{\partial^{2}}{\partial \alpha^{2}}\right) f_{m}=\lambda_{m} \cdot f_{m}
\end{aligned}
$$
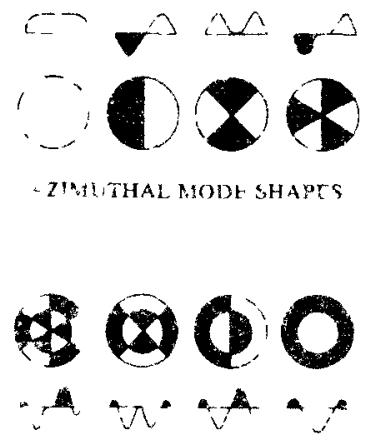

RALIAL VIMTIUAL MODE SHAPIS
Figure 5

Normal RadialAzimuthal Mode Shapes ot a Cylindrical Reactor 


$$
\ell \sum_{m=1}^{N}\left(\frac{\varphi_{m}^{i+1}-\varphi_{m}^{i}}{\Delta t}\right) f_{m}=\sum_{m=1}^{N}\left[M^{2}\left(\frac{d^{2}}{d z^{2}}+\lambda_{m}\right)+k-1\right] \varphi_{m}^{i+1} \cdot f_{m}
$$

This expression can be decomposed into $N$ independent equations by invoking the orthonormality property of the eigenfunctions $f_{m}(r, \alpha)$ :

$$
\begin{gathered}
l\left(\frac{f_{m}^{i+1}-f_{m}^{i}}{\Delta t}\right)=\left[M^{2}\left(\frac{d^{2}}{d z^{2}}+\lambda_{m}\right)+k-1\right] \rho_{m}^{i+1} \\
m=1,2, \ldots . . M
\end{gathered}
$$

When, as is the case in practice, the effective multiplication factor $k$ is a function of $r, \alpha$ and $t$ (through the effect of control rods and temperatures), then one may still decompose (16) into $N$ independent equations by treating the variable part of $k$ as a forcing function at time $t^{i+l}$ in the following manner:

$$
\begin{aligned}
& k=k_{0}+\delta k \\
& \sum_{m=1}^{N}\left(\frac{-f_{m}^{i}}{\Delta t}\right) f_{m}=\sum_{m=1}^{N}\left[M^{2}\left(\frac{d^{2}}{d z^{2}}+\lambda_{m}\right)+k_{0}-1\right] P_{m}^{i+1} f_{m} \\
& \quad P_{m}^{i+1}=2 P_{m}^{i}-P_{m}^{i-1} \delta k \cdot P_{m}^{i+1} \cdot f_{m}
\end{aligned}
$$

wisere

and

This procedure of extrapolation in the * variables is somewhat similar to that used in Section 4.2 .

This method has been applied successfully to startup transient hybrid simulations of large reactor cores.

In such s.miztionj. it is not necessary to apply the modal decomposition method to the radial/azimuthal representation of the temperatures $T_{m}$ and $T_{f} \dot{\phi}$ Indeed, transformation from the modal form (32) in which $\phi$ is expressed to local values in the $(r, \alpha)$ plane is given by:

$$
\phi\left(r_{j}, \alpha_{j}, z, t\right)=\sum_{m=1}^{N} P_{m}(z, t) f_{m}\left(r_{j}, \alpha_{j}\right)
$$

Thus, discrete-point representation of temperatures in the $(r, \alpha)$ plane is handled without difficulty.

But the most convenient method to represent temperatures in

$(r, \alpha)$ may be influenced by specific aonsiderations.

For instance, in the analog simulation of such a problem reported by Carstairs and Taylor 11 , the temperatures as well as the neutron flux are expanded in normal modes of the operator. $\nabla_{r \alpha}^{2}$ 
The hyorid mitzplexing methods vse the fact that the analog implementa"i ir $c$ " the parallel fincte differences method results in misy celis of computing elements naving identical or similar structures.

The major aspects of the application to reactor core problems have been roortid in the literature, and more work on these methods - both practical and theoretical - is in progress.

8.1 Component Multiplexing

Finite difference techniques, when applied to nonlinear partial differential equations, generally result in the need for a number of nonlirear elements such as multipliers and/or function asckacors in each firte difference cell. A useful metisd conos ots ar time-multipiexing only these nonlinear elements wilct, as a rule, are more expensive than linear components. The remaining parallel-finite-difference circuit is then programmed to be exactly the linearized version of the same problem, thus providing a meaningful transient behavior in between updating by the multiplexing routine.

8.2 Finite-Difference cell Multiplexing

When the imount of multiplexed eruioment becomes important, it may oecome easier to multiplex a complete finite-difference cell, including the integrators. In this case, the equations solved are actually discrete both in space and time, even though the transition operator from one time itation to the next involves integration of ordinary differential equations.

In reference 38 , Sanathanan, et aI, apply this method of

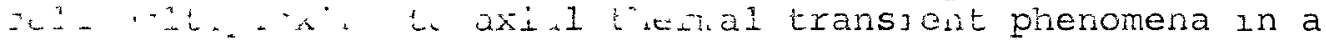
re "los core. A unicue ladial fluile-difrerence cell is inolemenzaj on the aralog computer and multiplexed sequen-

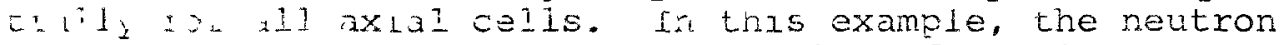
kinetics are conputed on the assumption of a point reactor. Itaration L's used to obtair tat simultaneous solution of the couplea thermal and neutronic equations.

A othur pacar tosarwing solnewhat experimental work in hytord onuter all multiplexing applied to parabolic particl drierentiul equations is reported by Howe and Hsi 16 . In this work, multiplexing is also combined with itcralior $t=$ obtaln the solution of the one-dimensional

into 0 . Uiation.

8.: Moad Militeiexing

Similatude berween cells in aralog crreuit diagrams is what makes wultiplexing applicable to palallei-finite-difference solutsor of cartial differertial equations. But this similitbie suist in the mcarl methods (e.g. Section 7 ) with the aditi crverience that trere is less interaction between nodey $t$ ations tha- interaction betweer. Iinite differences colls. Thersfore, the application of multiplexing to the paraliel recial solution of particl differential equations appears attrarti to No implementation of this approach has been re-

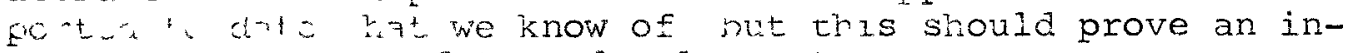
tercbir. arcu .ur future developmicnt. 


\section{CONCLUSIONS}

The recent development of adequate mathematical tools has made it possible to apply hybrid computers to the solution of partial differential equations in nuclear power plants simulations. Although hybrid computers provide an extremely flexible and powerful computing capability, fundamental problems which are very mathematical in their nature had to be resolved before this computing capability could be utilized. This fact is very common in the use of computers for scientific applications. As a corollary, it is to be expected that future developments in the application of hybrid computers to partial differential equations problems in the nuclear power industry will depend on theoretical work as well as on improved hardware capabilities. We are aware of the recent installation of hybrid computers in nuclear research laboratories in this country, in France, Great Britain, Italy and Japan. The work performed in these laboratories will undoubtedly result in new and improved methods of simulation. 


\section{REFERENCES}

1 Albrecht, R.F", "Approximation to the Solution of Partial Differential Equations by the Solution of Ordinary Differential Equations", Numerische Mathematik, Vo1.2, pp 245-262,July 1960.

2 Allen, R.J. et al, "Nuclear Reactor Simulation by Analog Compu"ter - A Literature Survey", Atomic International NAA-SR-3708, p.56, December 1959.

3 Bayly, J.G. and Fearce, R.M., "Method of Studying Multi-Region Reactors with an Analog Computer", Nucl.Sci.Eng., Vo1.2,pp 352$362,1957$.

4 Blake, R., Piggott, M., Smale, R. and Lotito, L., "Hybrid Computer Optimization of a Nuclear Power Station Control System". Int. Symposium on Analog/Hybrid Computation applied to Nuclear Energy, Frocecaings Versailles 1968.

5 Bona, C., "A Mathematical Model for the Simulation of a Heat Exchanger with a Natural Circulation Drum Boiler", 5th AICA Int. Congress 1967 Proceedings, European Academic Press, Brussels,

6 Budak, B.M., "The Method of Straight Lines for Certain Boundary Value Problems of the Parabolic Type", z Vycisl.Mat. i Mat.Fiz 2, 1961,pp 1284-1291, Trans. as USSR Comp. Math. and Math. Phys. 2 (1963) pp 1284-1291.

7 Carteron, I. and Deloux, G., "Representation Analogique des Circuits d'Echange de Chaleur". Application aux Echangeurs de Centrales Nucleaires, L'Onde Electrique pp.37,369 December 1957.

8 Caligiuri, G.P., Claes, W., d'Hoop, H., and Vichnevetsky, R., "Research cn Three Dimensional Simulation of Nuclear Reactors" 3 rd AICA Int. meeting Opatija,1961, Proceedings, European Academic Press, Brussels, 1962.

9 Cerling, I.T. "On the Solution of Nuclear Reactor and Related Problems by Mans of the Hybrid computer", Report \#CD/ASG/N31, Centia: Llecticity Generating Board, London,s.E., 1 JulY 1962.

10 Carling, 1...". "Hybrid Computer Solution of Heat Exchanger Partial Lifferential Equations", Proc. for the Int. Assoc. for Analog Computation, Vol.X, No.1, January 1968.

11 Carstarss, R.L. and Taylor, R.W.. "The Occurrence and control of Spatial Instabilities in Large Reactors", G.E.C.Atomic Energy Review, vol.2,No.2, september 1959.

12 Carstalrs, r.L. and Taylor,R.W., "Reactor Radial Spatial Behavior", Nuclear Power, June 1960 .

13 Chua:g, ..., kazāa, L.F., Winderknecht, T.,"A Stochastic Method of Solvirg Partial Differential Equations Using an Electronic Analog comyter". 1st IFAC Congress, Moscow, 1960.

14 Davis, D. Snd Darker, H.A., "A Hybrid Computer Study of Direct Digital Control of a Nuclear Power station", Int. Symp. on Analog, Hyorid Comp. applied to Nuclear Energy, Versailles, 1968.

15 Dougias, J., "on the Errors in Analog Solutions of Heat Conduction Prublens". Quarterly Applied Math., i4, 1956 pp.333-335.

16 Eyeions,D.A.. Seyfferth,L., Spurgin, A.J., "Analogue Computer Studies of Hear Exchangers", 3rd AICA Int. Meeting, Opatija 1961, Proceedings, European Acad. Press, Brussels 1962. 
17 Fillmore, R.L. " "Two Group Calculation of the Critical core Size of the S.R.E. Reactor", NAA-SR-1517, July 1956

18 Fisher, M.E., "Limitations Due to Noise Stability and Component Tolerance on the Solution of Partial Differential Equations by Differential Analysers", J. of Elec. and Control, Vol.8,No. 2, p.113, February 1960.

19 Frank, A. and Lapidus, L., "The Effect of Error Propagation on Hybrid Computation Application to Chemical Engineering Problems" Proceedings of IFIP Congress 1965, Vol.2, Spartan Books.

20 Friedmann, N.E.."The Truncation Error in the Semi-Discrete Analog of the Heat Equation", J. of Math. Physics, 35 p. 299, 1956.

21 Glasstone, E., The Elements of Nuclear Reactor Theory, Van Nostrand Co., 1956.

22 Godts, J.E.,"Analog Analysis of Transient Neutron Flux by Discontinuous Synthesis", PhD Thesis-Carnegie Inst. of Tech. 1965.

23 Calza-Bini, A. and Turrini, E. "A New Method for the Solution of Partial Differential Equations with Boundary Value Problems", Proceedings of the 5th Int. AICA Congress, European Academic Press, Brussels, 1967.

24 Gyftopoulos, E. and Smets, H.,"Transfer Functions of Distributed Parameter Nuclear Reactor System", Nucl.Sci. and Eng.Vol.5,No. 6, June 1959.

25 Henry, A.F., and German, J.D., "Oscillations in the Power Distribution Within a Reactor". Nuc.Sci and Eng.,Vol2,No.4, 1956.

26 Howe, R.M. and Hsu, S.K.,"Preliminary Investigation of a Hybrid Method of Solving Partial Differential Equations", Proceedings 1968 Fall Joint Computer Conference, AFIPS, Vol. 33.

27 Johnson, S.O., Curlee, N.J. and Reibing, J.V., "Simulation of Hot Channel Boiling in Water-Cooled Reactors", IRE Trans.on Nuc.Sci., Vol NS-5, No. 1, June 1958.

28 Kaczmarec, "Critical Comparison of Nuclear Reactor with Various Types of Controllers", Proceedings - lst IFAC Congress, Moscow, 1960, Pub. by Butterworth Co., London.

29 Iandau, H. G.. "The Accuracy of the Analog Solution of Heat Conduction Problems", J. of Soc. for Industrial and Applied Math., Vol. 11, p. 564, (1963).

30 Leonard, R.R., "Analog Computer Solutions for Xenon Concentration in a Reactor", U.S.Gov. Res. Reports p.500, November 14, 1958.

31 Little, W.D., "Hybrid Computer'Solutions of Partial Differential Equations by Monte Carlo Methods", Proc. of Fall Joint Computer Conference, 1966, AFIPS, Vol. 29.

32 Mitchell, E.E.L.,"Hybrid Techniques for Solution of Partial Differential Equations", SAG Report \#19,1963, Electronic Associates, Inc., Princeton, New Jersey.

33 Miura, I. and Iwata, J., "Study on Time Shared Analog Computation Techniques", SIMULATION pp 318-328, November 1965.

34 Nemehinov, S.V., "The Solution of Boundary Problems for Partial Differential Equations of Second order of Elliptic Type using the Network Method", Original: z Vyeisl.Mat. i Mat. Fiz. 2 (1962) pp 418-436: Trans. USSR Comp. Math. and Math. Phys. Z (1963),pp. 441. 
35 O'Neill,T.J., "The Derivation of Reactor Heat Transfer Transient Equations for Gas Cooled Graphite Moderated Thermal Reactors", 2nd United Nations Int. Conf. on the Peaceful Uses of Atomic Energy, Geneva, 1958.

36 Peden, J.C., "The Study of Multi-Region Reactions with an Analog Computer", Hanford Atomic Prod. Operation H.W.65578, July 1960, p. 50.

37 Petrarca, S., "An Analog Iterative Model for the Calculation of Coolant Density in Pressure-Tube Nuclear Boiler Channel", Proceedings - 5th AICA Congress, European Acad.Press, Brussels 1968.

38 Sanathanan, C.K., Carter, J.C., Bryant, L.T., and Amiot, L.W., "The Application of a Hybrid Computer to the Analysis of Transient Phenomena in a Fast Reactor Core", Nuc.Sci.\& Eng. Vol. 28, pp. 82-92, 1967 .

39 Stacey, W.M., "Modal Approximations", Research Monograph No. 41, The Mass. Institlite of Tech. Press, Cambridge, Mass.

40 Tyror, J. G., "Controlling Instabilities in Graphite Power Reactors", Nuclear Power. June 1959.

41 Vaughan, R.I., "Control Considerations for High Burn-Up", Nuclear Engineering, January 1960.

42 Vichnevetsky, R., "Computing Methods in Nuclear Reactors Kinetic and Thermal Behavior", Int. Seminar on Analog Computation Applied to Nuclear Problems, Proceedings published by European Academic Press, Brussels, 1962.

43 Vichnevetsky, R., "A New Method of Analyzing the Truncation Error in the Finite Difference Representation of Partial Differential Operators", Proceedings of 3rd Int. Analog Computation Meeting, Pub. by European Academic Press, Brussels, 1962.

44 Vichnevetsky, R., "Method of Characteristics in the Hybrid Solution of First order Partial Differential Equations", ECC Report \#60, 1962, Electronic Associates, Inc., Princeton, N. J.

45 Vichnevetsky, R.. "Application of Hybrid Computers to the Integration of Partial Differential Equations of the First and Second Order", Proceedings of IFIP Congress 1968, Edinburgh,Scotland

46 Witsenhausen, H. S., "Hybrid Solution of Initial Value Problems for Partial Differential Equations", Mass. Institute of Tech., Electronjc Systems Lab Memorandum No. 8, 1964.

47 Witsenhausen, H. S., "On the Hybrid Solution of Partial Differential Equations". Proc. of IFIP Congress, 1965, Vol. 2,p.42543). Spartan Books.

48 Caillet, C. and Deat, M., "Possibilites et Limitations du Calcul Analogique pour les Etudes Dynamiques de Centrales Nucleaires" 2nd United Nations Conf. on the Peaceful Uses of Atomic Energy, Geneva, 1958.

49 Schmidt, J.R. and Clark, D.R.,"Analog Simulation Technique for Modeling Parallel Flow Heat Exchangers", IEEE Winter Power Meeting, New York, 1967.

50 Mackay, D. and Fisher, M.E., Analogue Computing at Untra-High Speed, Publishers, John Wiley \& Sons, New York, 1962. 
51 Carlson, A.M., "Hybrid Simulation of an Exchanger-Reactor Control System", Technical Conference on Process Control

(Sponsored by National Research Council), Edmonton, Alberta, Canada, May 1968.

52 Hermelink, M. J. M., "Hybrid Simulation of a Sodium Heated Once-Through Steam Generator", Proceedings of International Symposium on Analogue and Hybrid Computation Applied to Nuclear Energy, Versailles, September 1968, Published by European Academic Press, Brussels.

53 Vichnevetsky, R., "Analog/Hybrid Solution of Partial Differential Equations in the Nuclear Industry", Proceedings of Int. Symposium on Analogue and Hybrid Computation Applied to Nuclear Energy, Versailles, Sept. 1968, Pub. by European Academic Press, Brussels, Belgium. Reprinted in SIMULATION, Vo1.11, No. 6, December 1968.

54 Shu-Kwan Chan. "The Serial Solution of the Diffusion Equation Using Non-Standard Hybrid Techniques", ESL Technical Memorandum \#329, Electronic Systems Lab., Mass. Institute of Tech., October 1967.

55 Vichnevetsky, R. "A New Stable Computing Method for the Serial Hybrid Computer Integration of Partial Differential Equations", Proc. of Spring Joint Computer Conference 1968, AFIPS Vol. 32, Thompson Book Co., Washington, D. C.

56 Vichnevetsky, R., "Serial Solution of Parabolic Pariial Differential Equations: The Decomposition Method for Non-Linear and Space Dependent Problems". Scheduled for publication in SIMULATION, June 1969.

57 DiBartolomeo, M., Mathis, A., Melucci, G., and Turrini, E., "Time Discretization Hybrid Models for Nuclear Reactor Cores", Proceedings of Int. Symposium on Analogue and Hybrid Computation Applied to Nuclear Energy, Versailles, Septembar 1908. Published by European Academic Press, Brussels. 
THE USE OF HYBRID COLPUTERS IN THE ITALIAN CNEN NUCLEAR PROGRAMME

MATHIS A., Prof. Eng.

Comitato Nazionale Fnergia Nucleare

Centro Studi Nucleari della Casaccia - 00060 - Roma (Italy)

\section{The potentialities of hybrid computers}

It is interesting to follow the evolution of the computing equipment for scientific applications in the last years.

For the digital computers, it may be assumed that, in the last five years, the computing power has keen increased by a factor of ten, remaining the cost of the same order.

For example, an IB:-70 4 (first installation in Sep. 1052) with a typical fast memory of $64 \mathrm{~K}$ words, carried out about $10^{5}$ operations per second (a weighted average between sums and multiplications); now, an IBII-360/75, with a typical fast memory of $132 \mathrm{~K}$ words, may perform $4 \div 5.10^{5}$ operations per second.

If the computing power of a digital computer is defined as a quantity proportional to the product of the number of operations per second, by the fast memory dimension, we obtain the above mentioned improvement in computing power.

In the field of analog computation, which practically is equivalent to the field of simulation of dynamic systems, a far-reaching evolution intervened during the last years.

The computing needs, arised by the simulation of engineering systems of higher and higher complexity (e.g. large industrial processes and aerospace systems), have outlined several heavy limitations of classical analog computers, in particular:

- Iimited accuracy in quantity representation (related to the accuracy of electrical components);

- difficulties in the simulation of distributed systems (the feasikility of accurate space-cells models was directly related to the size of the computer); 
- difficulty in the generation of arbitrary functions of sever al variakle. + $r c \cdot x=n+l y$ requested in the simulation of complex physisal prenomena);

- impossibility $=:$ a direct simulation of control systems based on discrete lagiss and on digital computers.

Clearly, the most part of the preceeding requests correspond to typical performances of digital computers, and consequently a growing interest arose for "hybrid" (analog-digital) computing equifment. In this direction, many trials were done, not always successful, based either on the connection of conventio nal analug and dieital computers, or on the combination of digital and anilog principles in the same computing element.

Now a tycical configuration for hybrid computers is establisheo. It is based on a fast iterative analog computer, which, st the same cost, reaches a computing power between two and trres lecades nigher than classical analog computers: such a

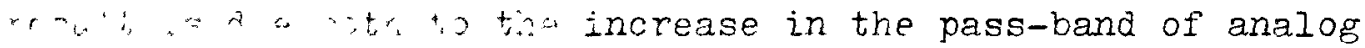
urplitier, and, particularly, to the introduction of solid state jectruica, instead of electromechanical devices, in mode control circuits and in nonlinear computing elements (the minimum operation cycle, comprising the "Initial Condition", "Operation", "Hold" phases, may be reduced from 1 sec to $1 \mathrm{msec}$ ).

The full utilization of a system based on such fast znalog elements reauires by itself the availability at least of a set of fast digital circuits, and possibly of a complete digital computer, designed for on-line operation with the analog section: to these alternatives correspond the two typical configurations of modern "fast iterative analog" and "hybrid" computers. Of course, the truly digital tasks above mentioned can be performec only with the complete configuration, compris ing a stored-orograr digltal computer; in this case the digital computer may be user. with advantage also for the automation of problem setting and checking, and of the operation of the analog computer.

The potentialities of this new kind of computer are not merely due to the addition of a logic and digital capability, but, as the practical experience of these last years as shown, are mainly related to the much higher computation speed of modern iterative analog elements, with respect to classic analog computers.

Consequently, the most effective utilization of hybrid computers has to be expected for those problems which allow 
the full use of the pass-band of new analog elements; in these cases, the competitivity of hybrid computers vs. purely digital ones may be higher than for analog computers in the past. Often this point is not sufficiently taken into account, and the evaluation of hybrid computers is done for applications concerning the solution of sets of slow ordinary differential equations, leading to poor comparison with full digital simulation ${ }^{1}$.

An attempt to compare quantitatively the performances of hybrid and digital computers, in the field of fast iterative integration, may be carried out on the base of a work of W. Giloi2.

Giloi considers a typical average digital computer for scientific computation, with 24 bit word, and $2 \mu s e c$ memory cycle. For a 24 bit word the round-off error, corresponding to the static error in analog computers, is $1 / 2^{24} \cong 10^{-7}$. However the dynamic error in the digital integration of a dynamic model is mainly due to the truncation error related to numerical integration, which depends on the integration formula used in the program, and on the time discretization interval: in fact, this interval cannot be less than the "frame time" of the program, i.e. the time requested by the digital computer for the execution of one step for all the functional relations constituting the model.

Giloi then carried out a comparison of the error vs. frequency plots for the above digital computer and a modern analog computer (with $0.01 \%$ static accuracy and a $300 \mathrm{KHz}$, $3 \mathrm{dR}$ pass-band for amplifiers) (fig. 1). The comparison is made for a typical dynamic model, of a size corresponding to a 100 amplifiers analog computer (with a typical implementation of integrators, summers, multipliers and function generators); the execution on the digital computer of one time step for this model, with least-time programming (in machine language), reauires about 4000 $\div 5000$ memory cycles, depending on the integration formula used (frame-time of $8+10 \mathrm{msec}$, for a memory cycle of $2 \mu \mathrm{sec})$. The error plots for digital computations have been computed by means of the z-transform, and for three different integration formulas (reported on the plots).

For different model sizes, in the case of the digital computer the frame time changes proportionally, and the pass-band for a given error, inversely; in the case of analog or hybrid computer, the pass-band remains the same, and conversely the needs of computing equipment change in the same sense. For different memory cycle times of the digital, the frame time 


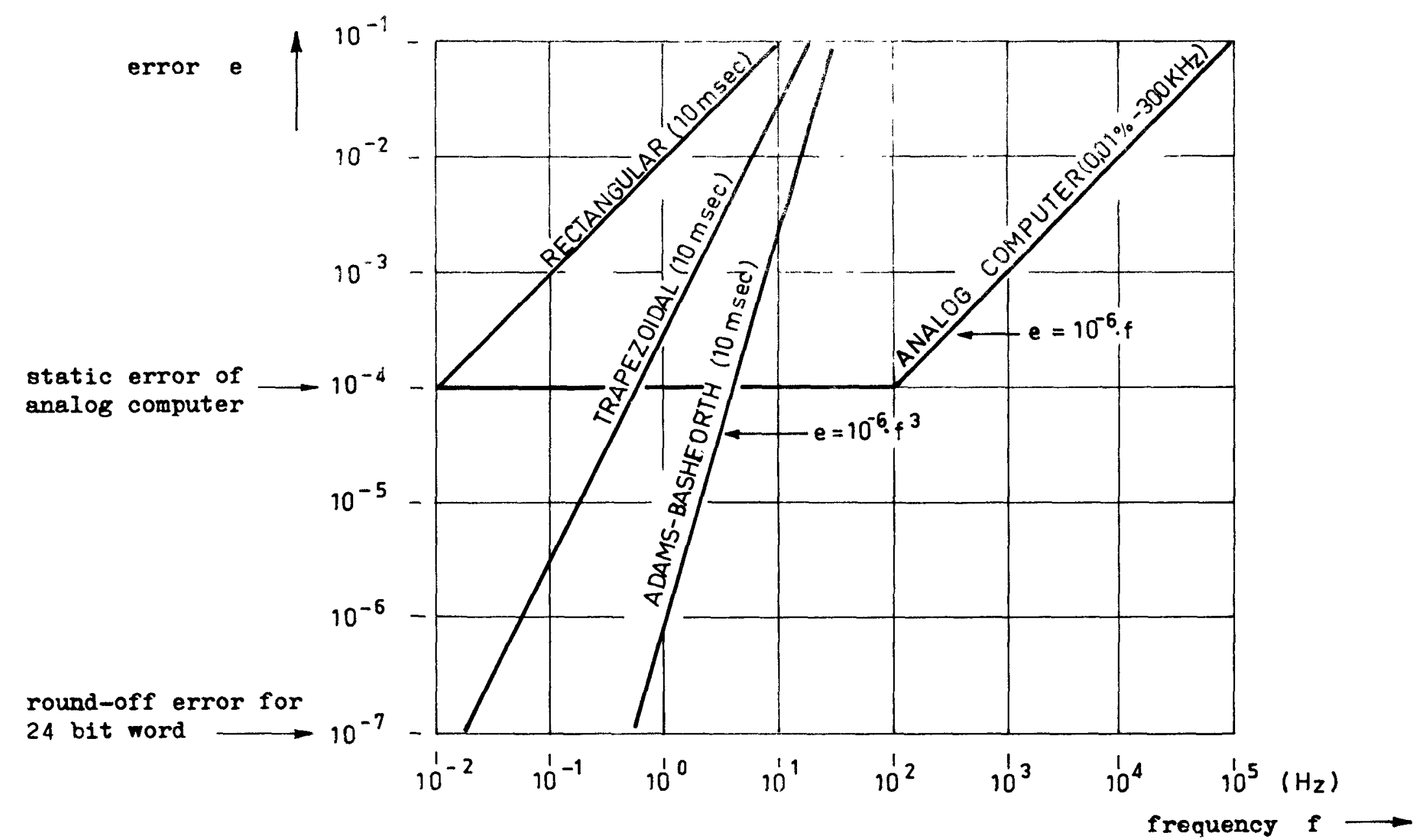

Fig. 1: Error v8. frequency for analog and digital integration (for digital integration, three different formulas are considered, and the frame time is $10 \mathrm{mgec}$ ) (from Giloi, ref.2, with added analytical expressions). 
changes of course proportionally.

For dynamic model integration, the computing power for a given accuracy may be defined as the product of the number of integrationscomprised in the model, by the maximum frequency allowed in the solution for that accuracy. Considering the typical integration run, which computes the behaviour of the model quantities for a given range of the indipendent variable, normally the maximum freauency for the given accuracy should correspond to ten times the frequency related to a period equal to the integration run length. So, the computer power may be directly related to the number of integrations per unit time, allowed for a given accuracy.

From fig. 1 it is clear that for the case above considered (digital computer with 24 bit word and $2 \mu s e c$ memory cycle; iterative analog computer with $300 \mathrm{KHz}$ pass-band and a size of 100 amplifiers), che computing power for fast integrations with an error $\leqslant 10^{-3}$ is about two decades higher for the analog with respect to digital computer.

The mere performance comparison may be of significance only in special cases ( $\mathrm{e} . \mathrm{g}$. when the possibility or not of real-time simulations is a discriminant criterium). But in general the right comparison criterium is the performance vs.cost ratio.

With the above assumption, being $f$ the maximum freauency allowed for a given error e, the integration run length may be written:

$$
R=10 / \mathrm{f}
$$

In the case of the digital computer, with the best integration formula and for the typical 100 amplifiers problem, it is (fig. 1):

$$
e=10^{-6} \cdot f^{3} \quad \text { for } e>10^{-7}
$$

In the case of the analog computer, it is (fig. 1):

$$
e=10^{-6} \cdot f \text { for } e>10^{-4}
$$

Supposing 30 the number of integrations in the typical 100 amplifiers problem and being $H$ the cost per hour of the computer (either digital or analog), the cost per one integration results:

$$
C=\frac{H}{3600} \cdot \frac{10}{f} \cdot \frac{1}{30}
$$

Owing to expression (1) and (2), it is, respectively for digital (subscript d) and analog (subscript a):

$$
c_{d} \cong 10^{-6} \frac{\mathrm{Hd}}{\sqrt[3]{\mathrm{e}}} ; \quad c_{a} \cong 10^{-10} \frac{\mathrm{Ha}}{\mathrm{e}}
$$


E.g., for an error $e=10^{-3}$, wich is practically acceptable in most engineering simulations, it is:

$$
\mathrm{c}_{\mathrm{d}} \cong 10^{-\overline{7}} \cdot \mathrm{H}_{\mathrm{d}} \quad \mathrm{c}_{\mathrm{a}} \cong 10^{-7} \cdot \mathrm{H}_{\mathrm{a}}
$$

and the ratio of integration costs for the two computers results:

$$
\frac{r^{d}}{r_{a}}=100 \cdot \frac{H_{d}}{H_{a}}
$$

Tynical costs per hour for analog and digital computers of the above mentioned features are of the same order; so, the advantace in the nerformace/cost ratio of the modern analog vs.difgtal, in the field of fast integration, remains of the order of two decades, as for the computing power.

For nroblems of larger size, the pass-band of the digital comouter, as said, decreases inversely; while the cost of the analog and hybrid increases less than its size; then it may be as $s$ thet for very large system simulations the advantage of rain and rumil is still increased.

$\therefore$ fact, it should be pointed out that such advantage of chalne and hyrid computers is fully exploited only for problems vich can engage the full pass-band of all analog elements; obviously, the size of the computer cannot be alnay tailored to the requirements of each problem and not all parts of a nroblem normally have the same pass-band requirements. On the other hand, digital computers may be fully utilized also by jobs of kinds far different from dr"namic computations. These are clearly the reasons "hy large analog and hybrid computers, though so advantageous in dynamic simulation, are practically used only bv those organzations, which present heavy computing requests for this kind of activity.

2. Ises of hybrid computers in the nuclear activities

Owing to tre peculiar characteristics of hybrid computers, the most promising applications in the nuclear industry concern particularly the process engineering; in this field, it is to be noted that the results obtained for nuclear processes are almost completely transferable to any other process industry.

Two applications look very promising, with respect to the potentialities of either the classical analog computers and of the digital ones:

a) the dynamic simulation of distributed systems, based mainly on the serial solution of partial differential equations $(\mathrm{PDE})$;

b) the analysis of continuous experimental records (e.g., noise and vibration statistical analysis). 
For the first point, an excellent survey paper was presented recently by $R$. Vichnevetsky 3 .

On the base of experience developed in CNEN organization, some typical applications to nuclear plants simulation will be discussed, and the possibility of using Monte Carlo techniques for hybrid solution of $\mathrm{PDE}$ is outlined.

For the statistical analysis of continuous records (computa tion of amplitude distributions, variance, power density autoand cross-spectra, etc.), hybrid computers look very suitable, and competitive vs. the present day digital computers (mainly because of large fast memory requirements for such kind of applications). In the future, owing to reduced memory and time requirements due to advanced programming techniques (e.g. fast Fourier transform), and to the easier computation of correlation functions on digital computers, it is possible that most part of statistical analysis will be done digitally; anyway, hybrid computer will be conveniently used for analog conditioning and analog-digital conversion of signals coming from magnetic tape analog recorders.

Other applications of hybrid computers, which are common to every field of engineering, concern the development of control systems.

The automatic optimization of dynamic systems is in fact a job which may lead to full utilization of hybrid computers: the dynamic model (process and control systems) is implemented on the analog section, and operated at the maximum speed allowed by the pass-band of the computer; the optimization routine, on the contrary, is implemented on the digital section, which computes the figures of merit, gives the increments to the parameters according to a stated policy, and coordinates the operation of the full model.

The development of control systems based on on-line digital computers is another obvious field of application for hybrid computers; however it should be pointed out that not always this application leads to full utilization of the analog section (e.g. when, for simulation requirements, a process model is operated in real time). In the nuclear field, this application is justified when the potentialities of hybrid computer are utilized for the simulation of complex distributed systems (e.g. space-time reactor core model with two-phase flow feedbacks, once-through steam-generator continuous axial models, etc.), while the simpler part of the plant may be simulated on normal slow analog computer (connected to the hybrid), and eventually the control computer is an autonomous digital process computer (possibly the same that will be actually used on the plant). 
3. Hybrid simulation of reactor cores and heat exchangers

Reactor cores and heat exchangers (in particular steam gene rators) are the components of a nuclear plant which principally require a detailed dynamic analysis.

As it is known, the phenomena involved are the transport of coolant (eventually with change of phase), and the heat transfer, in fuel and cladding, or tube walls. In general the fluid transport (described by 1st order PDE) is characterized by one predominant direction, and the heat transfer (described by 2 nd order PDE) may be considered only in the orthogonal direction.

The possible approaches to the hybrid solution of PDE may be suitably exposed with reference to a simple example: the transport of a liquid in a channel with heat injection (fig.2). The normalized equation for the liquid temperature $T$ (supposed uniform in any channel cross-section) is:

$$
\frac{\partial T(z, t)}{\partial t}+v(z, t) \frac{\partial T(z, t)}{\partial z}=f(T, z, t)
$$

being:

$z$ axial coordinate of the channel;

$t$ time;

$v \quad l i q u i d$ velocity;

$f$ function describing the heat injection.

For the solution on analog (hybrid) computers, only one indipendent variable shall be discretized: space or time.

There are so two possibilities, which lead respectively to the following sets of ordinary differential equations (ODE) (for 1st order backward differences):

Space Discretization ( $k$ indicates discrete $\frac{d T_{k}(t)}{d t}=\frac{v_{k}(t)}{\Delta z}\left[T_{k-1}(t)-T_{k}(t)\right]+f_{k}(t)$ space points)

Time Discretization (i indicates discrete time instants)

$$
\begin{aligned}
\frac{d T_{i}(z)}{d z}= & \frac{1}{v_{i}(z) \cdot \Delta t}\left[T_{i-1}(z)-T_{i}(z)\right]+ \\
& +\frac{1}{v_{i}(z)} f_{i}(z)
\end{aligned}
$$

To these equations correspond the circuits and the forms of solutions indicated in fig. 2, a) and b) respectively.

If the fuel element or tube wall are to be considered, some $O D E$, in the case of space discretization, or same algebrical 

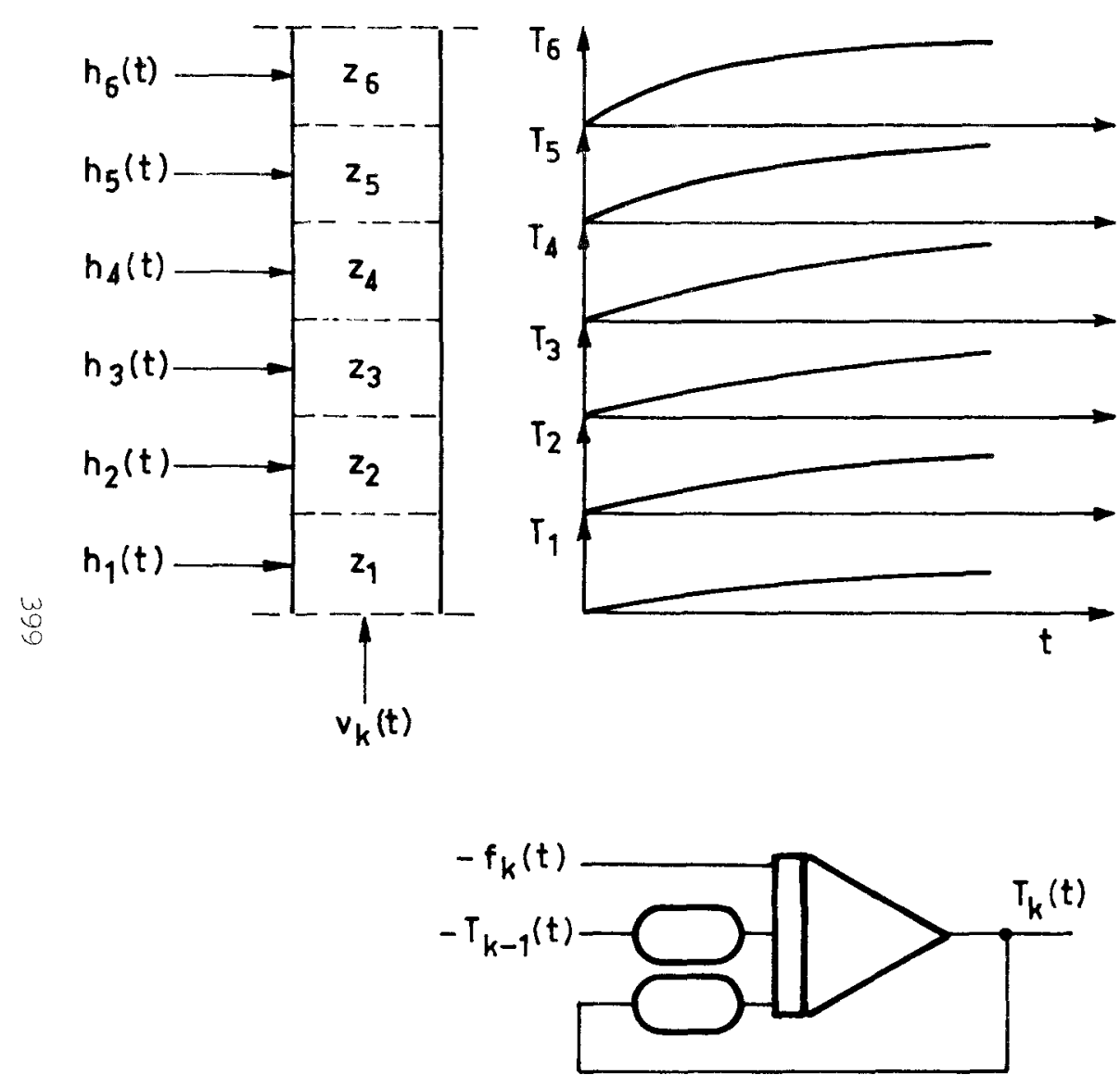

a)
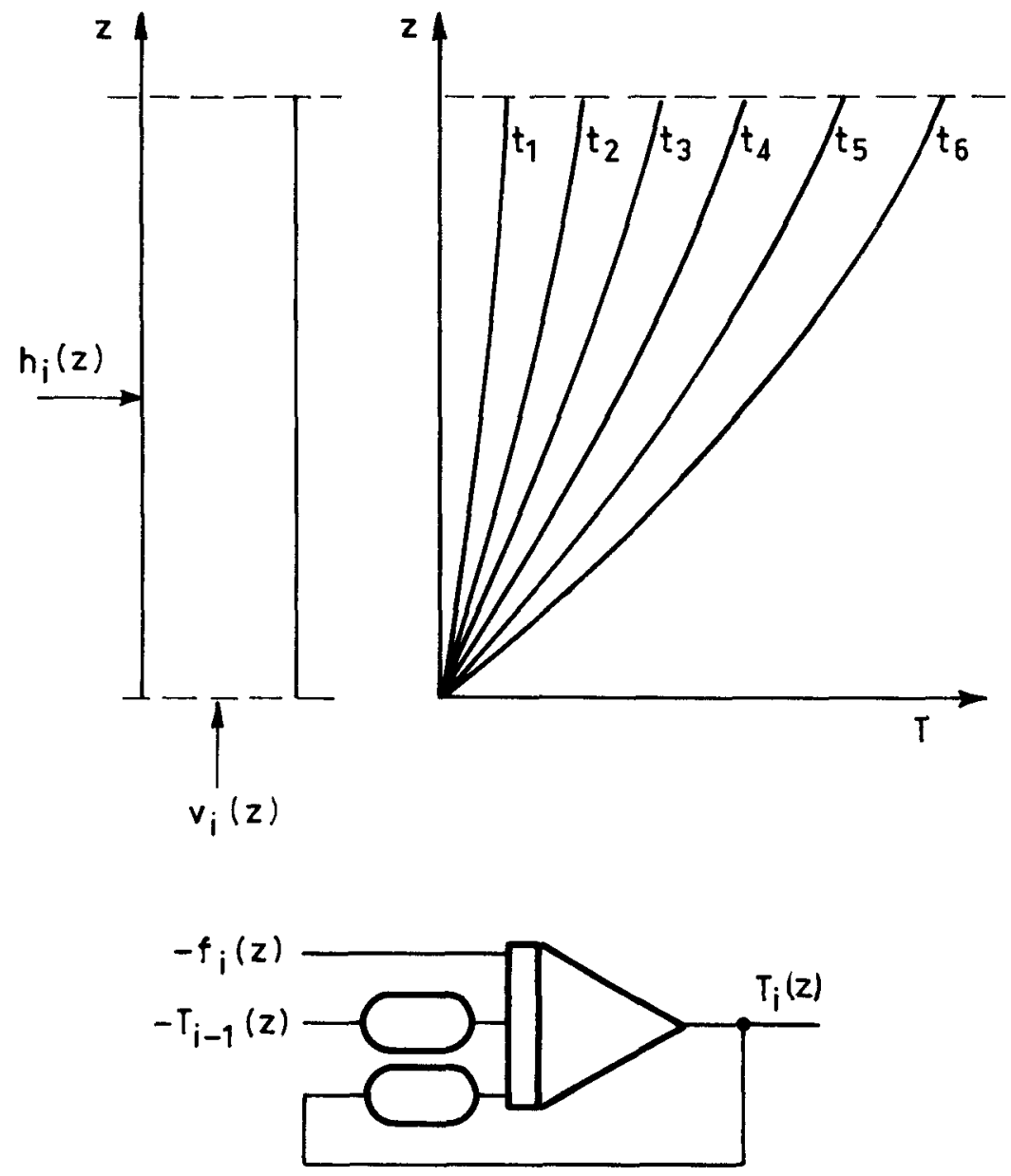

b)

Fig. 2: Space and time-discretization models (a and b respectively)for liquid transport with heat injection in a channel. 
equations, in the case of time discretization, should be added, giving average shell temperatures.

On classical analog computers a circuit for every cell had to be implemented, and the family of solutions came out in paral lel; the requirement of computing equipment was directly proportional with the desired accuracy; eventual space-boundary conditions (e.g. pressure drop in the case of two-phase flow) and feedback effects were easily imposed on the space-discretiza tion model (and not on the time-discretization model, which, having no other advantage, was practically never used).

On a hybrid computer, the ODE's for the various cells may be computed serially by the same analog circuit, if all the input functions are available: initial conditions and external excitations from normal analog models; input functions, which are the solutions for the previous cell, from a function memory (generally these functions are stored in sampled form on the digital section of the hybrid computer). The accuracy is no more related to the equipment used, but to the computation time.

With the serial approach, space and time discretizations lead to far different implications about boundary conditions and feedbacks, in a sense which is the contrary with respect to the parallel approach.

In fact the space-discretization serial model computes successively the whole transient for every cell, receiving from the function memory the preceeding transient (fig. 3). Evidently its application is restricted to the cases where the behaviour of a space cell does not depend on the behaviour of cells ahead, which is not yet known. Therefore the serial method with space differences can be applied only when the iterative model can be kept out from feedback loops (fig. 3). This fact indeed constitutes an heavy restriction for the method. Practically, in CNEN programs, this method has been applied for the dynamic analysis of the boiling hot channel in a liquid cooled core: the hot channel model was open-ended, while the feedbacks were computed by a lumped analog model of the whole core (considering negligible on the whole the feedbacks from the hot channel) 4 .

In the case of time-discretization, there are no limitations related to feedback loops provided that the iteration cycle is sufficiently fast with respect to the system time constants. The unique analog circuit integrates iteratively with respect to the space variable in the sense of fluid motion (only in this sense the solution is stable); it can supply in sampled form the value of variables, at particular points (e.g. the outlet temperature) or averaged and eventually 


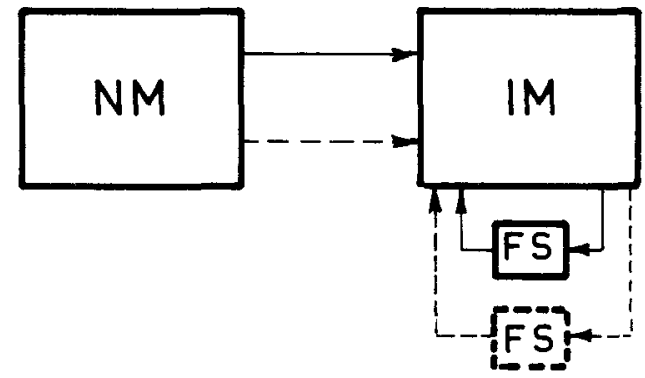

Fig. 3: General block diagram for iterative computation with space discretization:

NM: normal model, in the main computer time, with internal feedbacks;

IM: iterative model;

FS: function storage.

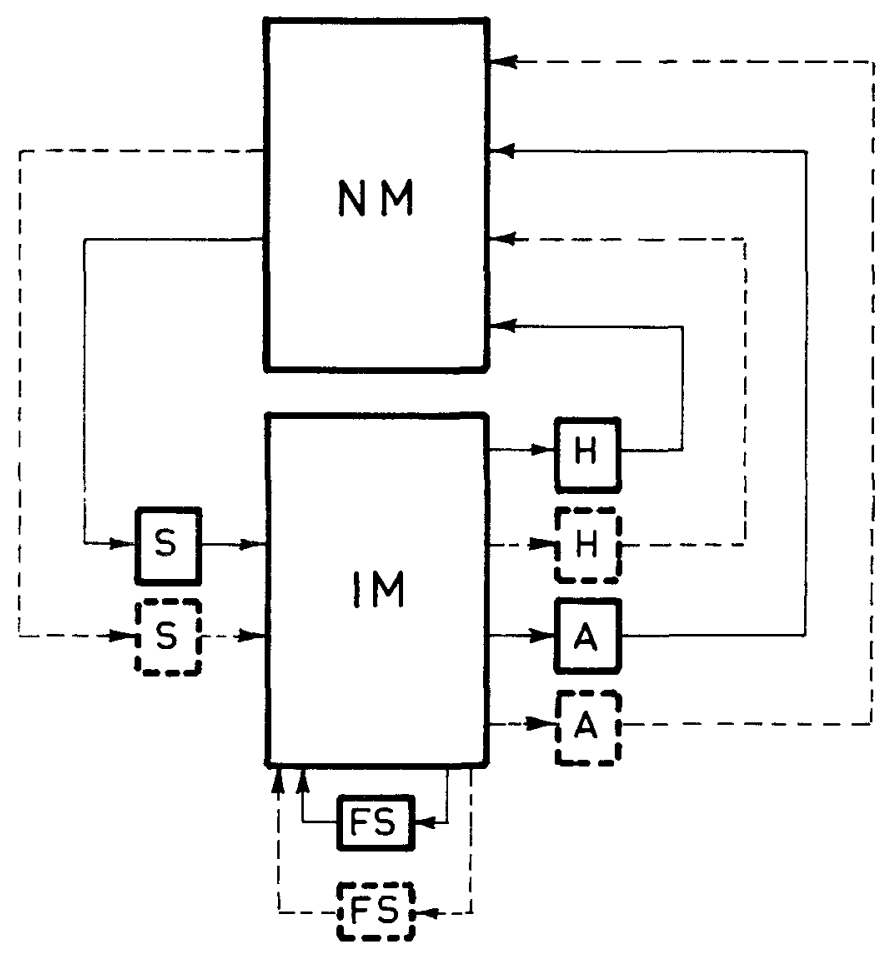

Fig. 4: General block diagram for iterative computation with time discretization:

$\mathrm{NM}$ : normal model, in the main computer time

IN: iterative model;

FS: function storage;

H: holding circuit;

A: averaging circuit;

$S:$ sampling circuit. 
weighted along the channel (e.g. flux weighted averages for temperature and densities) (fig. 4).

These values are supplied with a delay corresponding to the iteretive circuit computation time, and sampled at intervals corresponding to the iteration cycle (which contains the computation time). If the iteration cycle is hort, compared with the significant time constants of the remaining parts of the system, the sampled values can be introduced directly in the analog circuits simulating such remaining parts, which in particular may constitute feedback loops. These external parts may be simulated by normal analog circuits operated at much slower speed, with time as integration variable (fig. 4).

$\Lambda$ said the input functions are supplied by a function memory; tre initial and external conditions may be directly supplied from the external analog circuits, without sampling (in the foresaid hypothesis, the changes in these variables uaring ar zeration cycle are negligible).

in the case of boundary condition at the outlet of the ciarnel (e.g. pressure drop in two-phase-flow), the solution at ever, time instant may be found through a series of automatic cubiterations until the condition is met; if the model is to be timed with external simulation models, the time allowed for subiterations snould be constant 5 .

In the case of countercurrent heat exchangers, the inlet temperatures are given at opposite ends for primary and secondary fluids: solving in parallel the two transport equations, an unstable solution has to be faced. This difficulty may be avoid ed by solving successively each side according to the respective fluid flow: for the other side, the previously computed distribu tion may be taken and the final result may be obtained through a certain number of relaxing subiterations for each time instant; in practise, It can be shown that, if the time discretization interval is sufficiently reduced, these subiterations (and the related logic) are not necessary 6 .

Continuous space-discrete time models, as the above described ones, are obviously particularly suitable for a detailed dynamic analysis of channels, in which the fluid changes phase, flow patterns, and heat transfer rates: typically, channels of boiling reactors, and of once-through steam generators. In these cases, it is easy to implement several thermohydraulic models, to be integrated along the space variable, which may be switched automatically at any channel section, according to the value of correlations computed from the channel local quantities 5,6 . 


\section{Time-discretization models for reactor cores: design criteria}

A quantitative evaluation of hybrid computer potentialities has been made for the simulation of nuclear reactor cores by means of time discretization models 7 .

For a typical hybrid computer, as the EAI-8945, the limit to computational speed of this kind of models is given by the function storage digital routine, whose operation takes a time which is practically proportional to the number of sampled values.

Being $\tau_{T}$ the transit time of the coolant in the core channels, the time available for one computer iteration is $\beta T_{T} / N_{i}(\beta$ is the time scale factor for the whole simulation model, and $N_{i}$ is the number of iterations per coolant transit time).

If $\tau_{\mathrm{s}}$ is the time requested by the routine to store and playback one sampled value, the ratio $\frac{\beta \tau_{T}}{\tau_{s} N_{i}}$ is the maximum number of sampled values, which are available to store the functions of the model.

This number of values may be used in many different ways, according to the aims of the model, but in general it should be:

$$
\frac{\beta \cdot \tau_{T}}{\tau_{S}} \geqslant N_{c} \cdot N_{f} \cdot N_{i} \cdot N_{S}
$$

where:

$N$ is the number of core channels to be simulated;

$\mathrm{N}_{f}^{\mathrm{C}}$ is the number of space-functions per channel to be stored;

$\mathrm{N}_{\mathrm{s}}^{f}$ is the number of sampled values for each function.

In the formula (3) there is an ineauality (greater or equal) for two reasons: first there is a fixed time interval that must be added to $\tau_{s}$ in order to take into account a waiting interval before any memorization; in the second place, there are two time intervals during each computer iteration (Initial Condition and Hold modes) in which only one point per function is memorized.

It is interesting to note that the product $N_{f} \cdot N_{i}$ gives a general idea of the accuracy of the mathematical model; in fact, $N_{f}$ is just the number of radial cells which describe the channel, and $I_{i}$ corresponds to the number of axial cells of an axially discretized model of similar accuracy 8 ; practically, it is suitable that $N_{f}$ and $N_{i}$ be not too different.

The number of sampled values for each function, $N$, is related to the shape of the function (for instance, In the case 
of hoiling coolant, the density pattern may be very irregular, and reauire a very fine sampling) and on the availability of an interpletis: rancut at the output.

It is to no noted that the period of the iterative analog circuit $=\beta \cdot \tau_{T}{ }^{\prime}{ }_{C} \cdot N_{i}$; from expression (3), it should be:

$$
\frac{\beta \cdot \tau_{T}}{N_{c} \cdot N_{i}} \geqslant N_{f} \cdot N_{s} \cdot \tau_{s}
$$

It should be checked that this period is always above the limz: at ted $r_{y}$ tre pass-band of the analog componerts.

Wit. the ahove mentioned computer system, the time $\tau_{s}$, for a routire witten in assembler language, may be about $34 \mu \mathrm{sec}$.

$\tau^{\prime} \tau_{-}=.2 \mathrm{sec}$ (as in a liquid metal cooled fast reactor vole, ard for $\beta=1$ (real time simulation), it is:

$$
\frac{\tau_{\mathrm{T}}}{\tau_{\mathrm{s}}} \cong \operatorname{son}
$$

If is poes-ile to cuild a model with:

- core arcinnels;

- 7 functions per channel;

- 10 iterations per transit time;

- 40 anmpled ralidar per function.

In this sase the iteration period results 9,5 mses, nit Is stlil an admissible value for the analog section of the computer. For one core channel, and 80 sampled values per function, tre iteration period will be $10 \mathrm{msec}$.

An Iterative model operating at these frequencies may be obviously connected without difficulty to real-time analog nial moinols (whose time constants range in tre nrder of many seor. $\therefore$.

A pure digital model of the reactor core of comparable accuracy ( 7 radial cells, 5 axial cells), suitable for two core channel, implemented on IBN-7094 digital computer, shows for the same numerical example and for intermediate transients, a computing time which is $\sim 30$ times the real time duration of the computed transient; besides, this model can not be directly connected to a whole plant simulation. 
5. Hybrid simulation of diffusion in solids

Diffusion (of heat, neutrons, etc.) in solid domains is described by second order PDE.

Two completely different methods may be followed for the solution of this kind of equations on hybrid computers: the first one is based on serial solution after time discretization, as above mentioned for transport phenomena; another method is based on Monte Carlo techniques, and is suitable for point by point solution.

\subsection{Time discretization methods}

In the case of transient diffusion in a domain described by one space coordinate (indefinite slab, symmetric and indefinite cylinder, etc.) a parabolic PDE in space and time has to be considered.

Serial solution of this kind of equations, owing to the two-points boundary conditions, requires time discretization and continuous space integration; with this technique, as seen, it is also easy to take into account variable properties in the space domain (eventually depending on the solution itself), and to comprise the PDE model in a whole system closed-loop simulation model.

However, in this case, difficulties arise in the continuous solution of space equation: in fact, it is a second order ODT, with conditions to be met at both integration boundaries, and its solution show an hypersensitivity to parameter variations (the general solution contains diverging exponential functions); therefore the trial-and-error search for boundary conditions may became very difficult. This instability is peculiar to the type of equation and the way of solution, and appears also in problems which are physically stable 9 .

Many attempts were made to overcome these difficulties; one possibility is the transformation of ODF in an integral equation, and the computation of the required solution satisfying boundary conditions through successive approximations 10. The method is suitable also for non-linear cases, but requires a high and indefinite number of iterations; consequently, it cannot be used in closed loop systems.

Another method has been developed at CNEN laboratories, which avoid the above difficulties, being able to obtain the exact solution in two iterations 11 . It is based on the definition of general integral for linear ODF and is valid for any order of equation; however its application will be 
shown for the simpler 2nd order ODE, as arising from diffusion problem. in heterogeneous domain:

$p(x) \cdot \frac{d^{2} F(x)}{d(x)^{2}}+q(x) \cdot \frac{d F(x)}{d(x)}+r(x) \cdot F(x)=S(x)$

where $F$ is the quantity to be computed; $x$ the space coordinate; $p, q, r$ spcice dependent coefficients and $s$ a source term.

The bcundary conditions are of the type:

$\begin{array}{ll}\varnothing[\dot{F}(0), & F(0)]=0 \\ \psi[\dot{E}(I), & F(I)]=0\end{array}$

beir $x_{3}$ I the far-end coordinate. The above boundary conditions on be wried at each discrete time during the transient.

The method is based on the implementation of three simple anclog aircuits: one for the solution of the comrlete equatru, $\therefore$ r $r_{-} ;$particular solution $\bar{F}(x)$, and the others two

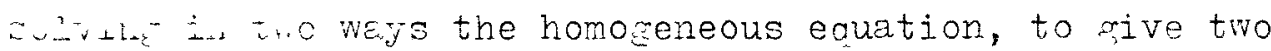
lirearly iroependent particular solutions $\mathrm{p}_{1}(\mathrm{x})$ and $\mathrm{F}_{2}(\mathrm{x})$. ate intili coniitions of these circuits are largely aruitrary and may be choosen so as to have solutions within computer limits.

The Eeneral integral is obtained ky superposition:

$E(x)=\bar{F}(x)+C_{1} F_{1}(x)+C_{2} F_{2}(x)$

where $C_{1}$ and $C_{2}$ are coefficients depending on boundary conditions. In particular, initial conditions (5) are to be met; introducing the expression (7) in (5), a relation $c_{1}=f\left(\mathrm{C}_{2}\right)$ may be derived, and implemented on the computer:

$F(x)=\bar{T}(x)+f\left(C_{2}\right) F_{1}(x)+C_{2} F_{2}(x)$

isfter a trial run of the above circuits from $x=0$ to $x=I$, the analog section is put in Hold mode, and $\mathrm{C}_{2}$ is varied continuously until the far-end boundary condition (6) is satisfied. The following run, with the so found values of $\mathrm{C}_{1}$ and $\mathrm{C}_{2}$, will supply the required solution which satisfies the boundary conditions. The block diagram of the method is given in fig. 5 .

In the case of non-linear problems (e.g. nuclear fuel ther mal properties depending on fuel temperature), the above procedure should be iterated until relaxation at every discrete time: the computation time may become indefinite, 


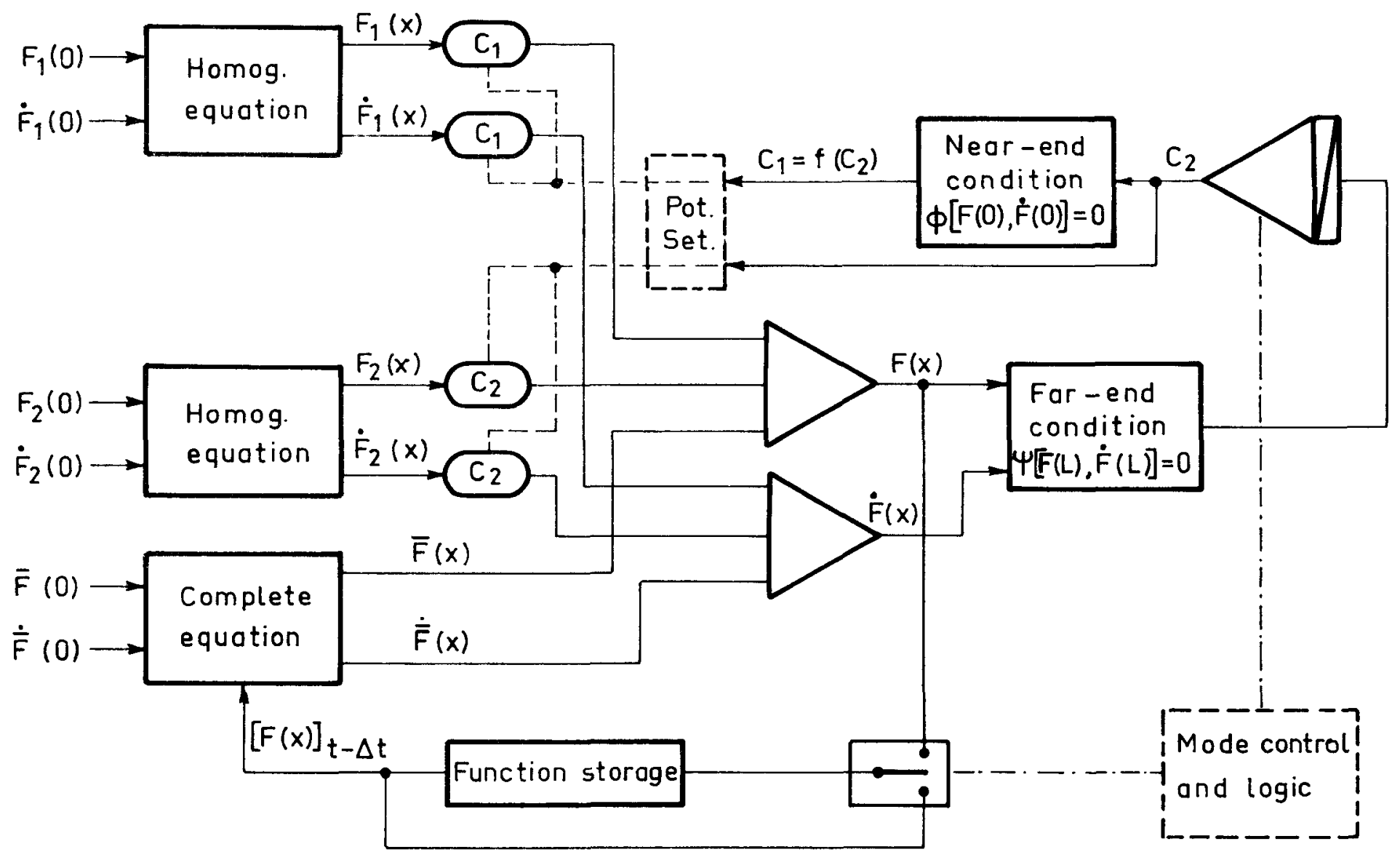

Fig. 5: Block diagram of the superposition method for 2nd order two-point boundary value problem solution. 
and a direct connection to whole system simulation models gets impossible. Froper operators may be introduced to obtain fast convergence (which may allow a fixed number of iterations); however, if the time interval is sufficiently short with respect to system time-constants, it may be shown that the non-linear terms can be conveniently computed with the solution values of the preceeding time interval, so avoiding any subiteration 11 .

Another method of solution by superposition of eq. (4) with constant coefficients has been developed by $R$. Vichnevetsky $;^{12}$ it is based on the precalculation and storage of the stable solution of two first order equations, in which was decomposed the given equation; at each computation time, two other first order equations have to be integrated.

With respect to the preceeding one, this method requires simpler circuits, but more memory and is limited to Iinear nap with constant coefficients.

\subsection{Mite-varlo methods}

The above methods require always simple integration domains, from the points of view of geometry. In fact often nuclear engineering requires detailed distributions, both in steady state and transient, in geometrically complex structures. E.E., in sodium heated or cooled components, temperature gradients in particular cross-sections, following coolant temperature transients, may generate dangerous thermal stresses.

Monte-Carlo methods, originally developed for problems of statistical mechanics, particle diffusion, etc., where classical analytical methods are practically unemployable, can be used also to approximate solution of functional equations of various types, without reference to underlying physical system.

In particular, it can be shown that conditional probability density functions of a Míarkov process satisfies second order elliptical or parabolic PDE, defined in a bounded region, with known boundary values: $i . e$. the same mathematical models which describe steady and transient diffusion phenomena $13,14,15,16$.

Hybrid computers are particularly suitable for the implementation of such probability models: Markov processes are simulated on analog section, exciting simple first-order, linear or non-linear, analog circuits with zero-average white noise (e.g. pseudo-random bynary sequences, from logic section or from digital section of the computer); random walks start from the point of the bounded region, in which the solution is desired; the simulation of boundary and the detection of 
boundary crossings may be done with analog and hybrid components; the result (solution value in the starting point) is obtained by the totalization of values pertaining to each walk (only boundary values at crossing points for steady state cases; a suitable combination of initial values in starting point and boundary values in crossing points, for transient cases): this totalization, with accuracy evaluations, may be easily done by the digital section of hybrid computer 16 .

Fractical experience has shown that these hybrid MonteCarlo models are quite faster than whole-digital computation of the same models, and are advantageous vs. classical hybrid FDE solutions, when only particular variable patterns in complicated boundaries are desired. Besides, it should be noted that probability functions so obtained, with reference to a given geometrical domain, are the resolving functions for the whole class of problems referring to that same geometrical domain: their computation may be done once for always 16 .

\section{Statistical analysis of continuous data}

Noise analysis is getting a growing importance in nuclear field, not only as a theoretical tool, but also as a practical diagnostic aid in component development and plant operation 17,18.

In particular, vibration studies of mechanical components requires the reduction of large lots of continuous records, on the base of statistical analysis: signal coming from accelerometers and/or strain gages may be processed to obtain power density auto-spectra (for the search of preferential vibration fields), and cross-spectra (to evaluate the couplings among structure elements).

A spectrum analyser suitable for this kind of data processing has been implemented on the EAI-8945 hybrid computer ${ }^{19}$. The signals are transferred from the plant to the computer by means of a magnetic analog recorder.

The general block diagram of the hybrid circuit (fig. 6) comprises four main blocks: input conditioning band-pass filters, sin-cos modulators, low-pass filters, output circuits (squarer, resolver); the channels are two, for cross-spectra computation. The spectrum is computed point by point, on the signals plaied back every time by the recorder (in the case of auto-spectra, the two channels, modulated by the two different oscillators, may compute two spectrum points at the same time). 


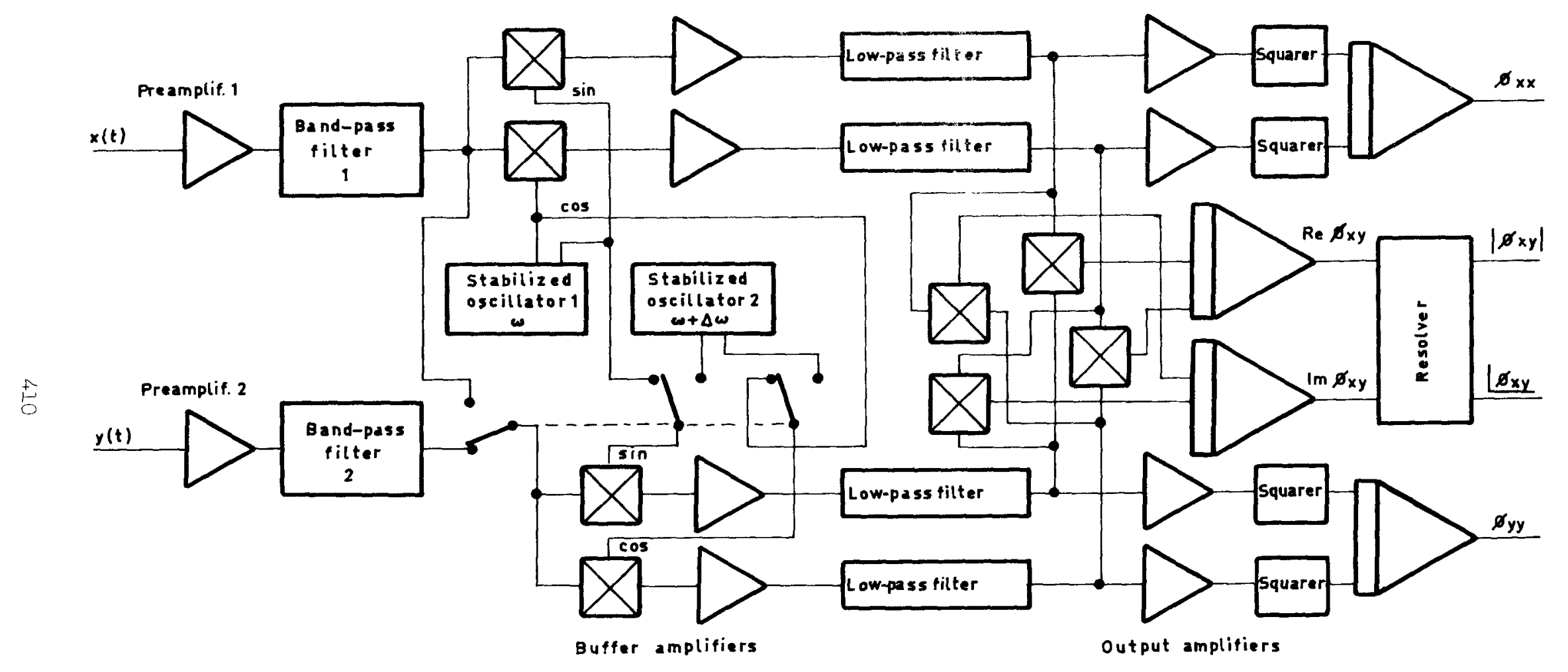

Fig. 6: Block diagram of hybrid circuit for spectral analysis. 
The logic and digital sections of the computer control the operation of the whole system, included the magnetic recorder; store and print the results and set the oscillators at new frequencies, after the computation of each point.

Practical experience has shown that acceptable accuracy may still be obtained for modulation frequencies up to $2 \mathrm{KHz}$, thanks to features of modern analog components. Normally, to fully use this pass-band, the play-back of recorded signals has to be greatly accelerated with respect to recording: this need has to be remembered in the choice of the recorder and recording speeds.

In fig. 7 is given an example of power density spectrum obtained from a fuel assembly vibration record. The spectral resolution reaches $0,5 \mathrm{~Hz}$ (on a full scale of $56 \mathrm{~Hz}$ ), value which is requested for a detailed evaluation of the sharp spectrum peaks (typical of mechanical vibration). The computation time was 2 minutes for run (two spectrum points in the case of auto-spectrum), corresponding to 16 minutes of plant record (play-back/record speed ratio equal to 8 ).

By now the attainment of such performances in other ways does not seem so easy: with digital computers, for memory and computation time requirements; with special purpose equipment, for the very high cost (owing to the unique kind of utilization).

Acknowledrments

The author is grateful to many people of CNEN Reactor Dynamics Group, whose works, mentioned as references, constitute the base for a large part of this paper. 


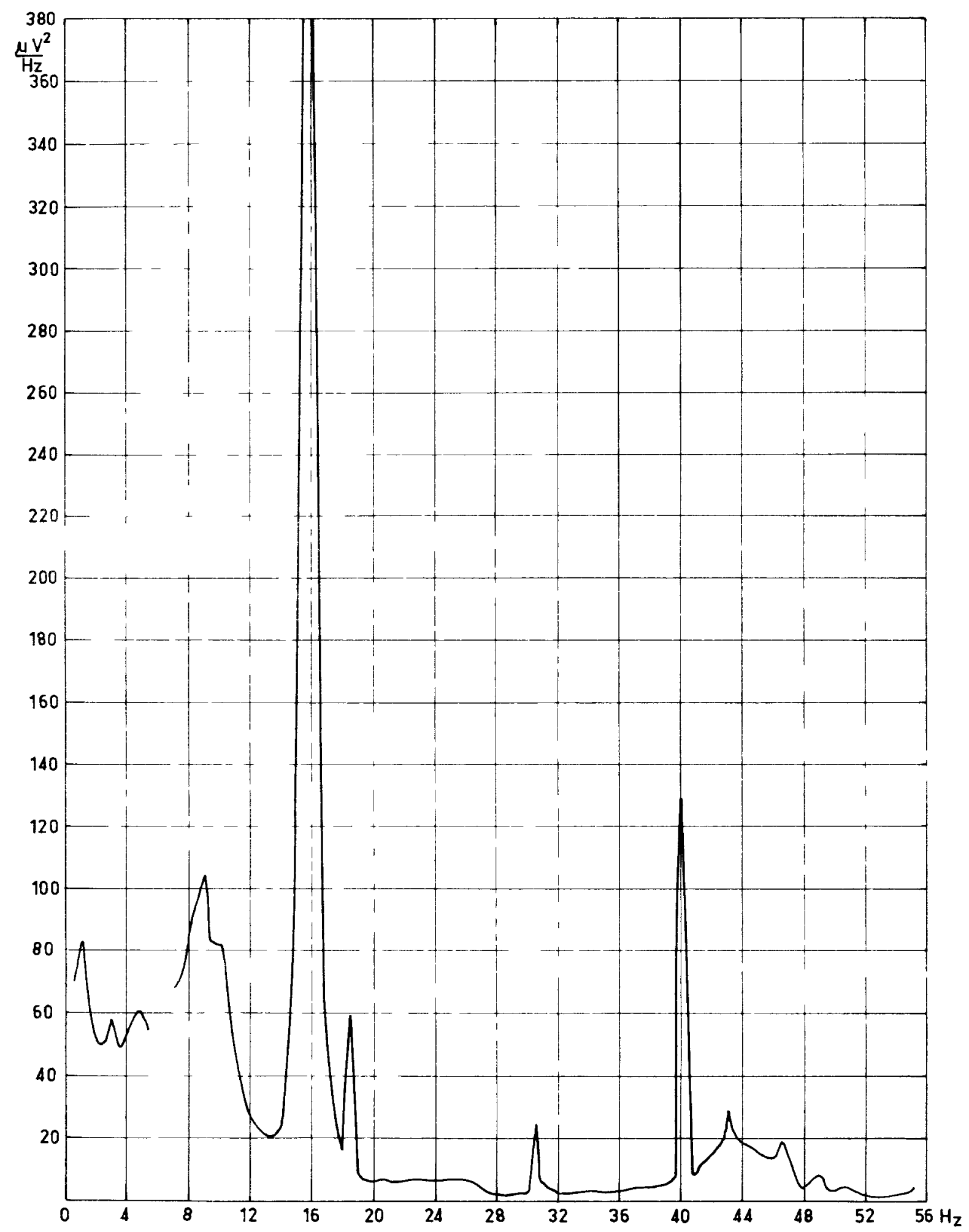

Fig. 7: Power density spectrum of a strain-gage record from a vibratine nuclear fuel assembly. 
1. Ake Rullgâra, "Some Aspects of Hybrid Computers", Simulation, $11(4), 161-164$ (Oct. 1968).

2. W. Giloi, "Future Development in Hybrid Computation", Proc. of 5 th AICA Weetings, Lausanne (1967).

3. R. Vichnevetsky, "Analog/Hybrid Solution of Partial Differential Equations in the Nuclear Industry", Int. Symp. on Analog and Hybrid Computation Applied to Nuclear Energy, Versailles (France) (1968).

4. A. Nathis and G. Valli, "Iterative Analog Models for the Dynamic Study of the Nuclear Power Reactor Cores", Proc. of 4th AICA Neetings, 344-352, Brighton (UK), (1964).

5. M. Di Bartolomeo and A. Mathis, Metodo ibrido per la simulazione delle instabilità di portata in un canale percorso da miscela bifase", Calcolo, 3, Suppl. N.2, 169-194 (1966).

6. S. Petrarca, "Modello ibrido di un generatore di vapore sodio-acqua in controcorrente", 2nd Congress of Associazione Italiana per il Calcolo Automatico, Napoli (1968).

7. M. Di Bartolomeo, A. Nathis, G. Melucci and E. Turrini, "Time Discretization Hybrid Models for Nuclear Reactor Cores", Int. Symp. on Analog and Hybrid Computation Applied to Nuclear Energy, Versailles (France) (1968).

8. A. Mathis, P. Giordano and G. Gatto, "Iterative Methods for the Analog Simulation of Physical Systems Containing Flowing Fluids", Proc. of IFIP Congress 1965, 2, 335-336.

9. D. NacKay and M.E. Fisher, Analogue Computing at Very High Speed, (John Wiley \& Sons, New York, 1962).

10. M.S. Elzas, "An Iterative Hybrid Computing Method for the Solution of Boundary Value Problems", Proc. of 4th AICA Meetings, 166-170, Brighton (UK) (1954).

11. A. Calza Bini and E. Turrini, "A New Hybrid Method for the Solution of Partial Differential Equations with Boundary Value Problems", Proc. of 5th AICA Meetings, Lausanne (1967).

12. R. Vichnevetsky, "A New Stable Computing Method for the Serial Hybrid Computer Integration of Partial Differential Equations", Proc. of 1968 Spring Joint Computer Conference, AFIPS, 32, 143-150. 
13. A.T. Bharucha-Reid, Elements of the Theory of Markov Processes and Their Application, (McGraw-Hill, New York, 1960).

14. K. Chuang, I.F. Kazda and T. Windeknecht, "A Stochastic rethod of Solving Partial Differential Equations Using an Flectronic Analor Computer", Proc. of 1st IFAC Congress, Moscow (1960) .

15. W.I. Little, "Hybrid Computer Solution of Partial Differential Equation by Monte Carlo Methods", Proc. of 1966 Fall Joint Computer Conference, AFIPS, 29.

15. I. Cimorelli, K. Caporaso and A. Federico, "Hybrid Stochastic Vonte Carlo liethods for Solving Heat Transfer Problems in Nuclear Reactors", Int. Symp. on Analog and Hybrid Computation tipplied to Nuclear Energy, Versailles (France) (1968).

17. R. E. Thrig (Coordinator), Noise Analysis in Nuclear Systems, IID-7579, USAEC Symposium Series 4 (June 1964).

1:. D.I. "iper and D.P. Roux (Coordinators), Incipient Failure E. Iurer Flants, Jolv-571011 (Jan. 1968).

1S. I. Cimorelli and A. Federico, "Realizzazione di un analizzatore spettrale con elementi di una calcolatrice ibrida", 2nd Congress of Associazione Italiana per il Calcolo Automatico, Napoli (1968). 


\section{HYBRID SIMULATION IN FFTF SYSTEM CONCEPTUAL DESIGN*}

A. L. Gunby and Grant A. Worth

Battelle Northwest

INTRODUCTION

The Fast Flux Test Facility (FFTF) will be the primary test facility for fast reactor fuels in the USAEC Iiquid Metal Fast Breeder Reactor (LMFBR) development program. As such, the FFMF must provide high plant availability for fuel and material tests in high energy neutron fluxes and high sodium temperatures typical of future LMFBRs.

Battelle Northwest (BNW) has been pursuing conceptual design of the FFTF with the broad objectives of (1) defining the overall configuration of the plant, such as the reactor type, the number of heat transport loops, and the types of components in each loop, and (2) defining those problem areas which require further study as the project moves into preliminary design, such as thermal transients in the heat transport loops resulting from equipment failure. Early in conceptual design, the need for simulation of the reactor and heat transport systems was recognized for both the preceding broad objectives. Hybrid simulation was to be used for fast, inexpensive scoping work for FFTF components and for long-term studies of process control, while all-digital simulation was to be used for detailed calculations and for checking the hybrid.

This paper presents the development of useful models for hybrid simulation of the FFIF reactor and process system and discusses the use of the models throughout conceptual design. Simulation details are discussed where appropriate and plans for future work are outlined.

\section{SUMMARY}

At the start of FFrF conceptual design, hybrid simulation was chosen as the most promising method of performing time-consuming control studies and real-time simulation for plant checkout and operator training. A hybrid computer was purchased for eventual installation in the FFTF plant, and simulation models of the reactor and process systems were developed for the equipment. During the conceptual design phase, the hybrid simulation proved useful at several points during its development, notably ( 1 ) in providing scoping information for early component conceptual studies, (2) in providing conservative, "worst-case" values for thermal transients, and (3) in providing preliminary evaluation of plant control schemes. Model simplifications have been made to fit the simulation on the equipment, with each model portion checked against a detailed all-digital simulation.

* Work performed for the United States Atomic Energy Commission 
Model development will continue as simulation techniques allow expansion and sophistication of models. An approach now in use on the FFTF hybrid (modular programing) allows greater model detail where it is needed by programming system components ( $(. g$. ., vessel, heat exchanger) and system functions (e.g., plant protection system) as separate entities on the digital side. Detail in one part of the simulation is provided by sacrificing detail in another, less-neecied part.

\section{SYSTEM DESCRIPTION}

The FFTF reactor and heat transport system for the current conceptual design is shown schematically in Figure 1. Several basic features have changed during conceptual design, notably a change from 4 loops to 3 , and a change of cold leg primary pumps to the hot leg. The overall layout remains the same, however, with the reactor cooled by three primary sodium loops, each primary loop transporting heat through an intermediate heat exchanger (IHX) to a secondary sodium loop which, in turn, loses its heat in an air-cooled dump heat exchanger (DHX). Throughout conceptual design, component designs have changed, with the hybrid simulation easily accommodating these changes. Current conceptual design of components favors the following: (a) 10-pass crossflow DHX, (b) 1660-tube, counterflow, Fermi-type IHX, (c) Fermi-type sodium pumps. Pump drives will be either the wound-rotor motor type or the electro-magnetic clutch type with a constant speed motor.

T.a reactor core consists of 70 to 80 driver fuel elements in a vertical array, each contairing 2l7-pin subassemblies of $\mathrm{UO}_{2}-\mathrm{PuO}_{2}$ fuel and cooled by sodium. Sodium also passes around the tubes, having leaked past tube-grid plate seal for the purpose of hydraulic balance. Around the core region is the radial reflector, which generates about $5 \%$ of the reactor heat and is cooled by an equivalent sodium flow. Finally, inlet and outlet plena accept and mix their respective input flows to provide core inlet and vessel outlet sodium, respectively.

\section{MODEL DEVELOPMEITT AND USE}

\section{MODEL DEVELOPMEIT}

Hybrid computer models of the FFTF reactor and heat transport system were developed in relation to the need for transient results and also to available equipment. Several distinct phases of development are evident, though they overlapped considerably during design. During the first phase of this development, utilization of the hybrid had to be minimal, as the machine was new and softwere was still being developed. Conceptual design during this period required "scopirg" information for thermal transients, i.e., data on how thermal transients at various points in the proposed FFTF system would be affected by changes in design data or operating conditions. For example, the effect of outlet plenum mixing volume on thermal transients at the vessel outlet nozzles is one input (among many) needed to estimate the desired vessel size. Throughout this phase, models remained fairly simple, with simplifications made in the conservative direction. Only one coolant circuit was modeled.

The second phase of modeling increased the utilization of the hybrid capability. A second coolant circuit was added and portions of the system model were made more sophisticated. During this period of conceptual design, the simulation was used for defining "worst-case" thermal transients (those transients resulting from a set of extreme circumstances) at key points in the process system. Worst-case transients were then used to develop testing objectives in the component development program. For example, thermal shock tests for the primary pumps (in the hot leg) must consider the worst-case conditions of full core $\Delta T$, high--performance fuel, and imperfect outlet plenum mixing. 


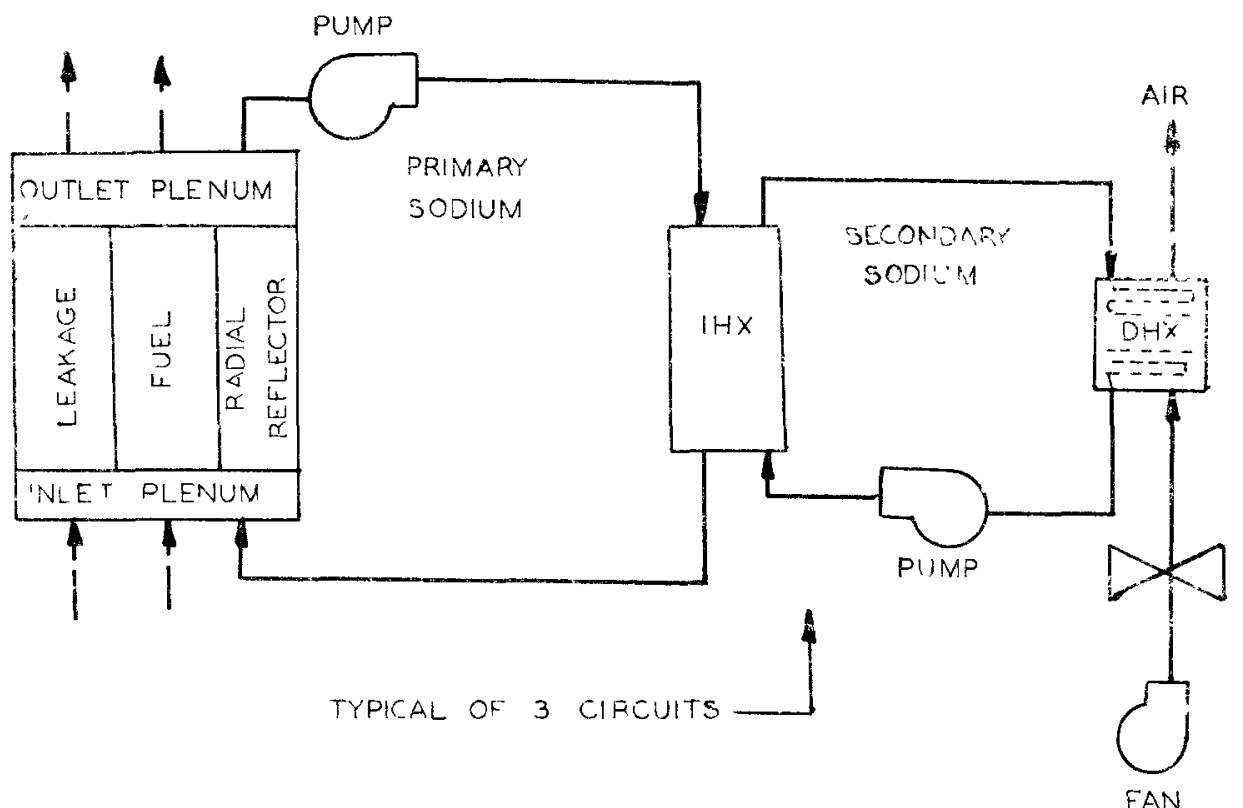

EWURE - SCHEMATIC DIAGRAM OF FFTF REACTOR AND HEAT TRANSPISTT SYSTEM 
The third phase considered control aspects. Process control simulators were added to the overall simulation and a plant protection system was simulated. These improvements allowed the study of control configurations for the reactor and process system, in particular, system responses to reactor power perturbations. Models for each phase are discussed in the following sections.

\section{PHASE 1 MODEL}

\section{Overall Description}

During this period, the analog portion of the hybria simulation was emphasized since the analog-digital interfacing was being completed and familiar analog techniques were dependable. The only portions of the simulation designated for digital simulation were the coolant transport delays and some selected function generators. A single coolant circuit was modeled; thus, transient behavior of all circuits was assumed to be identical. Figure 2 shows the simulated system with the division between analog and digital simulation.

\section{Component Modei Descriptions}

Equipment limitations on the analog side forced an early evaluation of desired model complexity. Two basic considerations were kept in view during this effort: (1) sodium outlet temperature from the modeled component is the parameter of interest; internal temperatures (e.g., nodal temperatures) are not of primary concern, and (2) due to the preliminary nature of conceptual design, accuracies on the order of $\pm 5 \%$ for thermal transients are acceptable. Models were made as complex as necessary with the understanding that as design progresses and greater detail is needed, the models would be made more sophisticated. A complete description of the Phase 1 models may be found in Reference $1 .(1)^{-}$

Reactor Vessel: Neutron kinetics were simulated with three delayed groups, as in the method of Albrecht and Metelmann. (2) An algebraic expression for neutron dynamics was used, to eliminate the need for extremely high-gain analog integration. Heat generation due to fission product decays was separated from the total power generation and also represented by three decay terms. Reactivity feedbacks included linear terms for sodium and core expansion (based on average sodium temperature), and for core bowing (based on core $\Delta T$ ), as well as a logarithmic term for Doppler (based on average fuel temperature). Inlet and outlet plenum models assumed perfect mixing throughout the plenum volume, with heat transfer to steel structures in the region being represented by a single node for each pienum.

Heat transfer within the core was divided in three parts by three coolant streams: fuel coolant, leakage, and radial reflector coolant. The radial reflector region was modeled by a single node as though heat were generated directly in the coolant. Simulation parameters were chosen to account for the stored heat in the reflector and to approximate the time constant of coolant temperature response to power changes. Leakage coolant acquires heat from the fuel coolant through the reactor tubes, and was also simulated by a single axial node. The core itself was simulated by two lumped nodes representing fuel and coolant. This simplification was acceptable based on comparisons with a detailed digital FFrF core model ( 4 axial nodes, 3 fuel nodes plus clad and coolant radially) as shown in Figure 3. For a core scram with full flow, the agreement in tube and vessel outlet temperature transients was good. For a combined power and flow scram, the tube outlet temperatures deviated somewhat but the damping of the outlet plenum provided good agreement at the vessel outlet.

Sodium Systems: Sodium hydraulics were simulated by assuming the DarcyWeisbach flow vs head loss relation, with an exponent of 1.8 . Pump and motor 


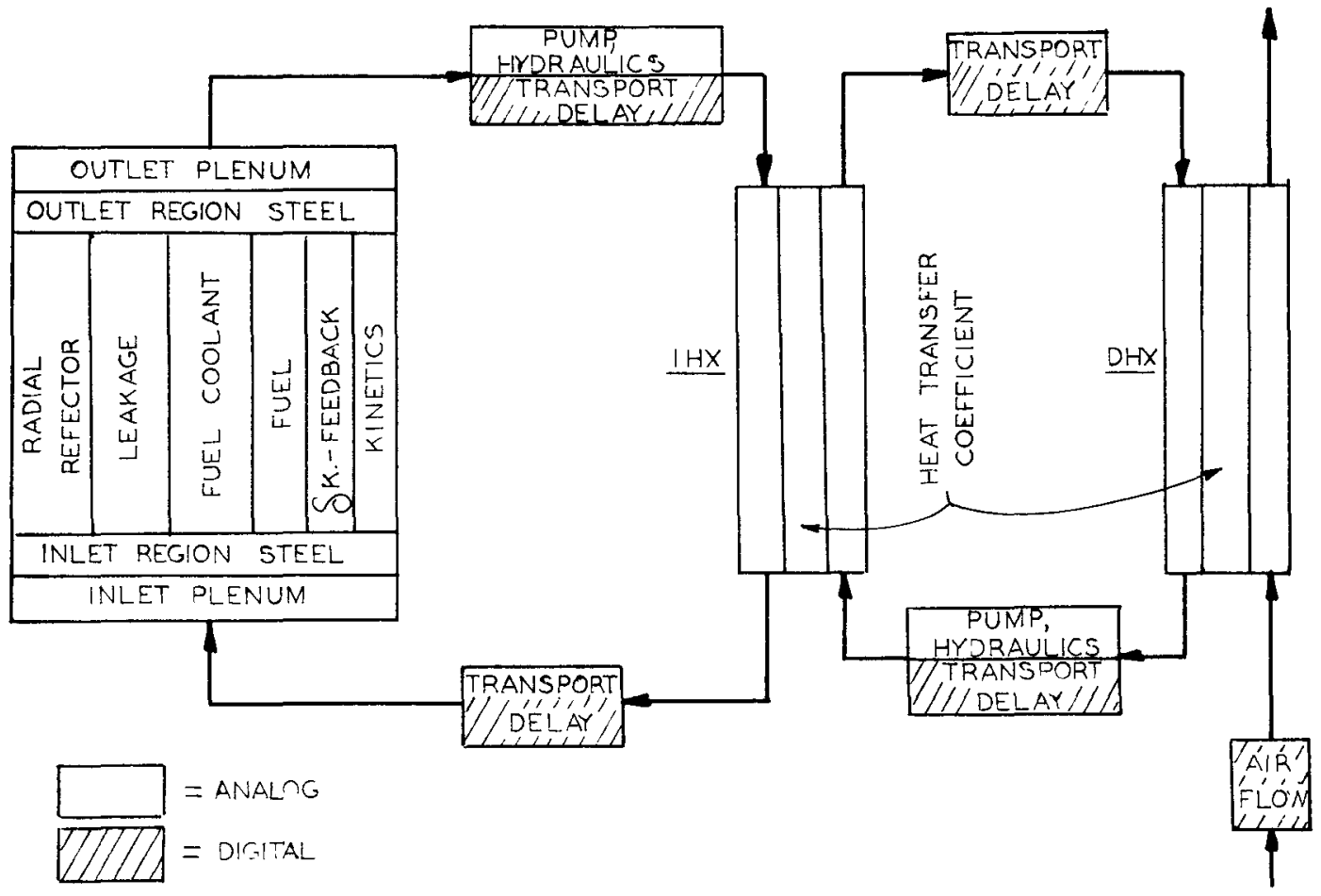

FIGURE 2. SIMULATED FFTF REACTOR AND HEAT TRANSPORT SYSTEM, PHASE ONE. 


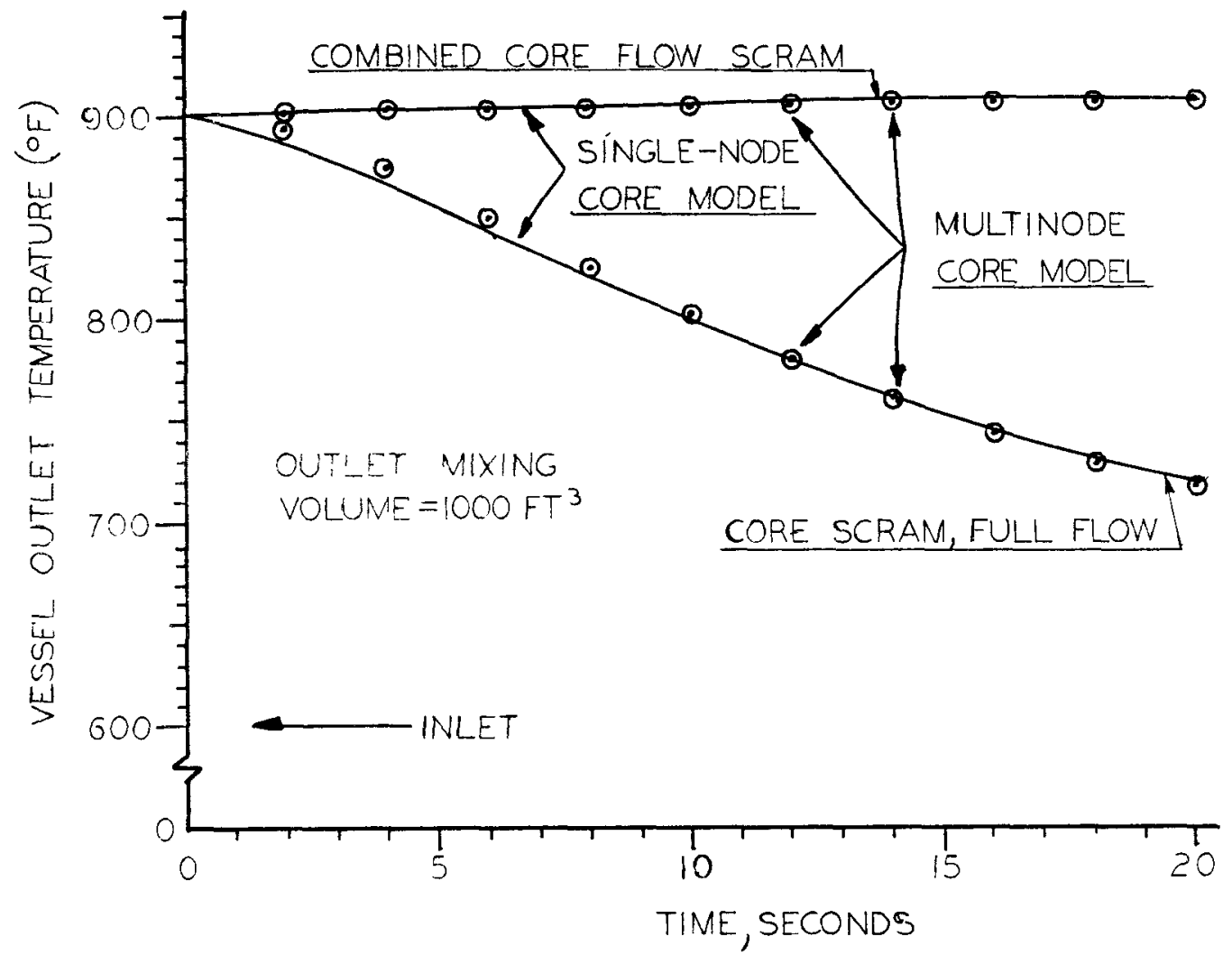

FIGURE 3. VESSEL MODEL COMPARISON, OUTLET TEMPERATURE. TRANSIENT. 
dynamics were simulated with speed control by an eddy-current clutch. Although pipe bends do not follow the above relationship and the reactor is hydraulically complex, comparison to a more detailed hydraulic model (accounting for bends, entrance and exit losses) for a typical primary flow coastdown showed good agreement (Figure 4 ).

Transport delays were the main contribution of the digital capability to the hybrid simulation. Pure delays were assumed as a conservative approach, since stored heat in the piping will tend to dampen thermal transients moving down the pipe.

IHX: The IHX was simulated as a counterflow exchanger divided into 4 nodal segments. Comparisons to models with $1,2,3,6$, and 8 nodes were performed which demonstrated the adequacy of the 4-node approach. Finite difference approximations for the partial differential equations were used, using central difference expressions at the first computed temperature of each coolant stream, and three-point backward expressions elsewhere (i.e., backward with respect to flow direction). The heat transfer coefficient was analogsimulated as being flow and temperature dependent. Stored heat in the tube metal was equally divided between tube and shell sodium. Transient studies showed less than $1 \%$ deviation from models using a separate metal node.

DHX: In spite of its crossflow design, the DHX was simulated as a counterflow device, an assumption which improves with the number of tube passes across the air flow direction. Preliminary model investigations indicated that three nodes would be sufficient axial complexity. Figure 5 shows the relative responses to loss of air flow for the 3-node hybrid model compared to an 18-node crossflow DHX model. Heat storage in the finned tubing was lumped with that for the sodium. This practice assumes that the metal temperature behaves substantially like the sodium temperature. The assumption is still under study at this time. Again, finite difference approximations for the standard partial differential equations were employed, with central difference expressions at the first computed temperature of each coolant stream, and three-point backward expressions elsewhere. The heat transfer coefficient was simulated on the analog side as a function of both coolant flows and temperature.

\section{Use of the Model}

The single coolant circuit model was used for "scoping" conceptual design studies for system components. Thermal transients as functions of operating parameters (power, $\Delta T$ ) and of design data were determined for use as inputs to thermal stress studies of vessel, pump, IHX and DHX. With the expected scope of thermal transients defined, preliminary analysis of specific component concepts could proceed for the purpose of determining concepts most likely to succeed.

For example, Figure 6 shows vessel outlet temperature transient rates as a function of vessel outlet plenum volume for a power scram with full flow. A representative mixed oxide core was assumed. These results were used as inputs to vessel design studies which examined such problems as nozzle spacing, and needs for thermal barriers. The hybrid was able to generate this information quickly and inexpensively.

\section{PHASE 2 MODEL}

The single coolant circuit simulation was expanded during this phase to include a second coolant circuit, with each circuit capable of simulating one, two, or three of the actual circuits. As shown in Figure 7, one circuit is primarily analog-simulated, while the other is digital. The component models used in each coolant circuit (analog or digital) are identical. Heat transfer 


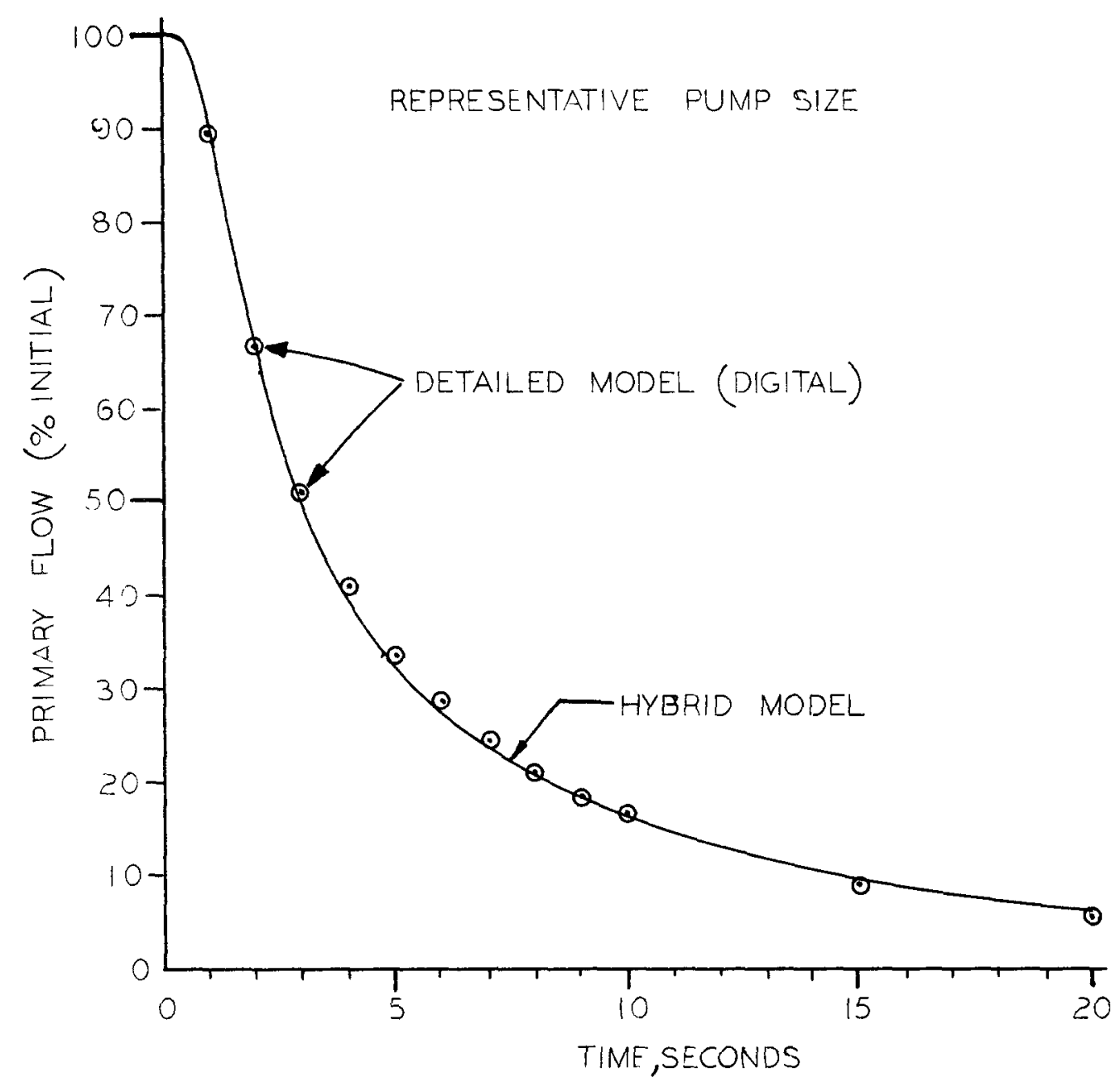

FIGURE 4. FLOW MODEL COMPARISON: PRIMARY FLOW DECAY FOR LOSS OF PUMP MOTOR 


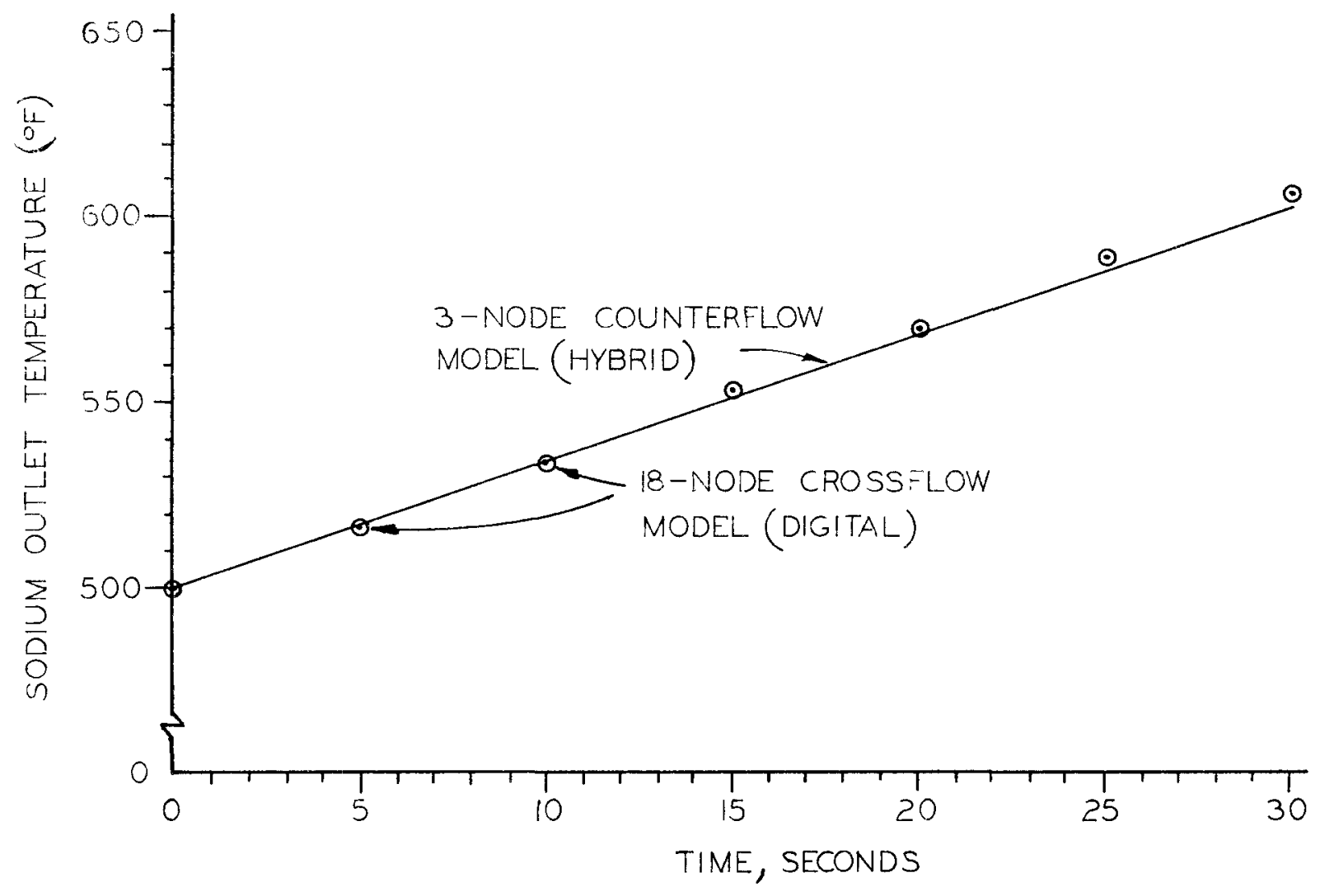

FIGURE 5. DHX SODIUM OUTLET RESPONSE TO LOSS OF AIR FLOW, FULL SODIUM FLOW. 


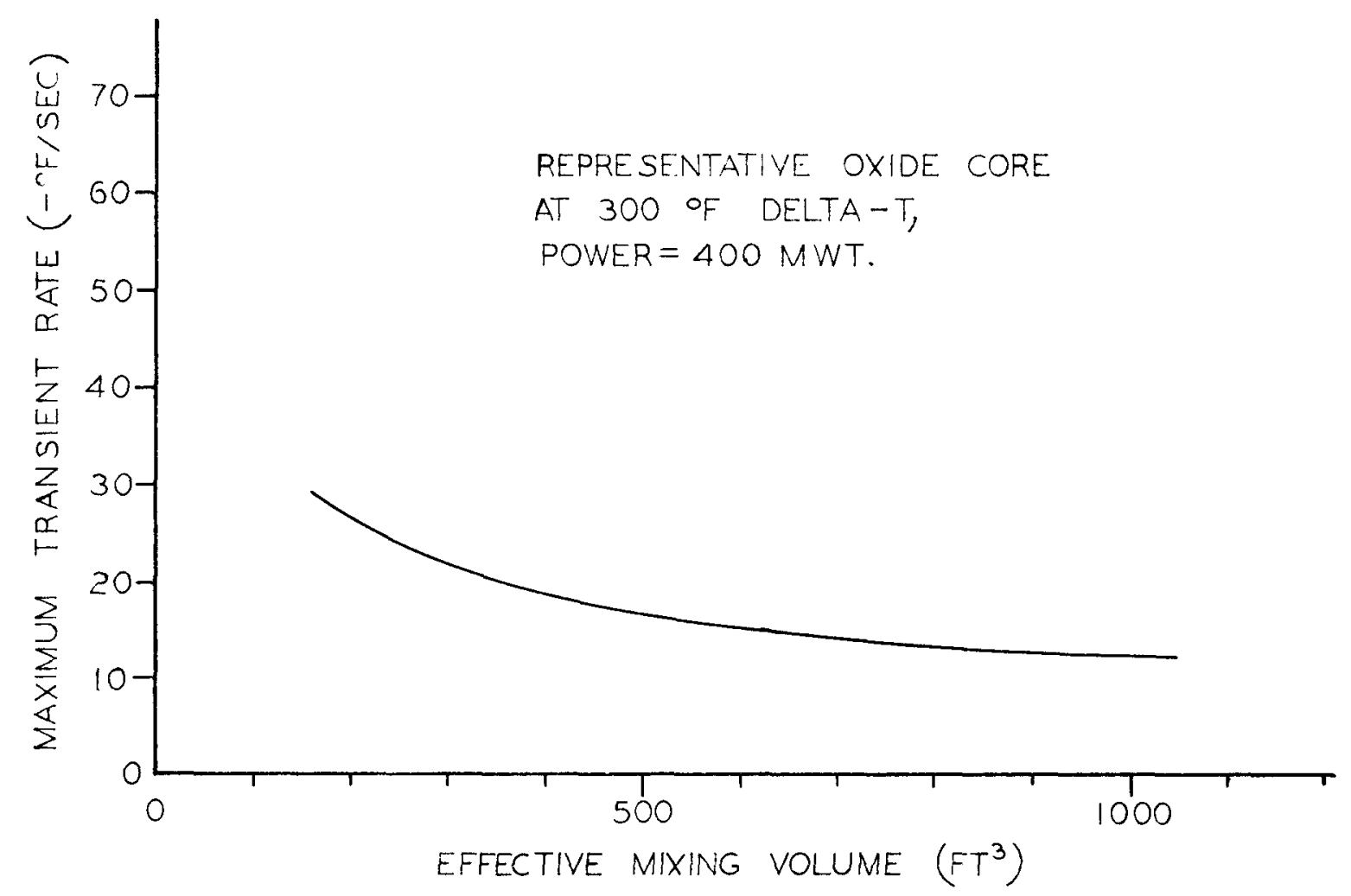

FIGURE 6. EFFECT OF OUTLET PLENUM MIXING ON VESSEL OUTLET THERMAL TRANSIENT DUE TO POWER SCRAM, FULL FLOW. 


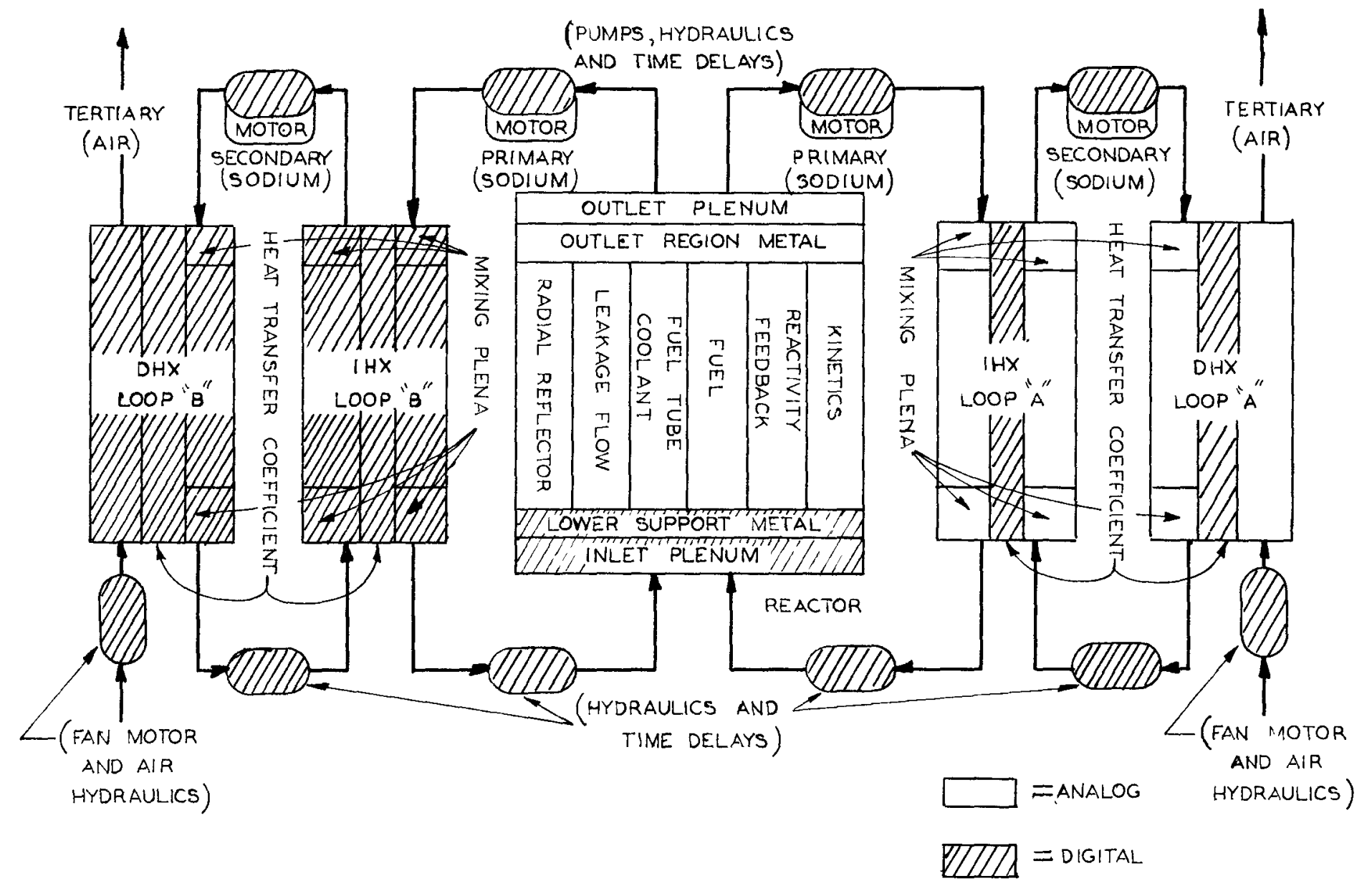

FIGURE 7 FFTF SYSTEM HYBRID SIMULATION, PHASE TWO 
coefficients for the IHXs and DHXs were shifted to the digital side as functions of the appropriate temperatures and flows.

Expansion of the model to the multicircuit status was accomplished without simplifications in model complexity. Indeed, several additions to the models were made. In the reactor model, the fuel was divided into three radial nodes of equal volume, resulting in a better match to the detailed core model for reactor tube outlet temperature responses to combined power and flow scrams. Further, flow-dependent mixing plena were added at the inlet and outlet of each IHX and DHX (total of twelve).

\section{Use of the Model}

This stage of model development was an interim between the single-circuit model and the goal of a multicircuit model with control simulation. During this time, the need arose for defining conservative, "worst-case" transients for component development and testing. A combination of circumstances designed to produce the most severe thermal transients was selected, including highperformance fuel, poor plenum mixing, high power and core $\Delta \mathrm{T}$, and low pump inertias. The hybrid simulation easily assimilated the changes and produced the required thermal transients. An example is shown in Figure 8 , comparing the worst-case primary hot leg transient to the transient resulting from exoected initial design data.

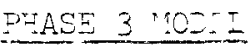

\section{Model Modifications}

During this phase of model development, control loops and a protection system were added to the simulation. Analog simulators of proportional-plusreset controllers were used, separate from the analog console of the hybrid. Switching arrangements allowed a wide variety of process control loops to be formed. Reactor control simulation was based on a reactivity-neutron flux loop which moved a simulated control rod when the flux signal moved outside a preset deadband. A plant protection system was simulated on the digital portion with the capability of looking at any computed variable, comparing it to a setpoint, and initiating control or protective action in any desirea sequence, i.e., power setback, controlled shutdown, and reactor scram.

\section{Use of the Model}

Several plant control systems were studied to examine concept feasibility. Response of the total system to anticipated perturbations (power, flow) was measured, with the process control system programmed to maintain a particular test objective. For example, Figure 9 shows the results of a reactor setback from $400 \mathrm{MWt}_{t}$ to $300 \mathrm{MW}_{t}$ with constant sodium flows. Airflow at the DHX is controlled by a proportional-plus-reset controller with a setpoint adjustment as a function of power level, in order to maintain reactor inlet temperature constant. As power decreases, airflow drops to raise secondary cold leg temperature and hold primary cold leg temperature within $\pm 10^{\circ} \mathrm{F}$. Work of this type is just beginning and will continue through the next design phases. Further model improvements will be made enabling more detailed control studies.

\section{SIMULATION FEATURES}

\section{COMPUTER DESCRIPTION}

The hybrid computer system used in the reactor simulation was developed by interfacing a Beckman Model 2133 analog computer (about 170 amplifiers) with a Digital Equipment Corporation (DEC) PDP-7 digital computer (8K core memory). The interface consists of a 24-channel multiplexer, an analog-to-digital 


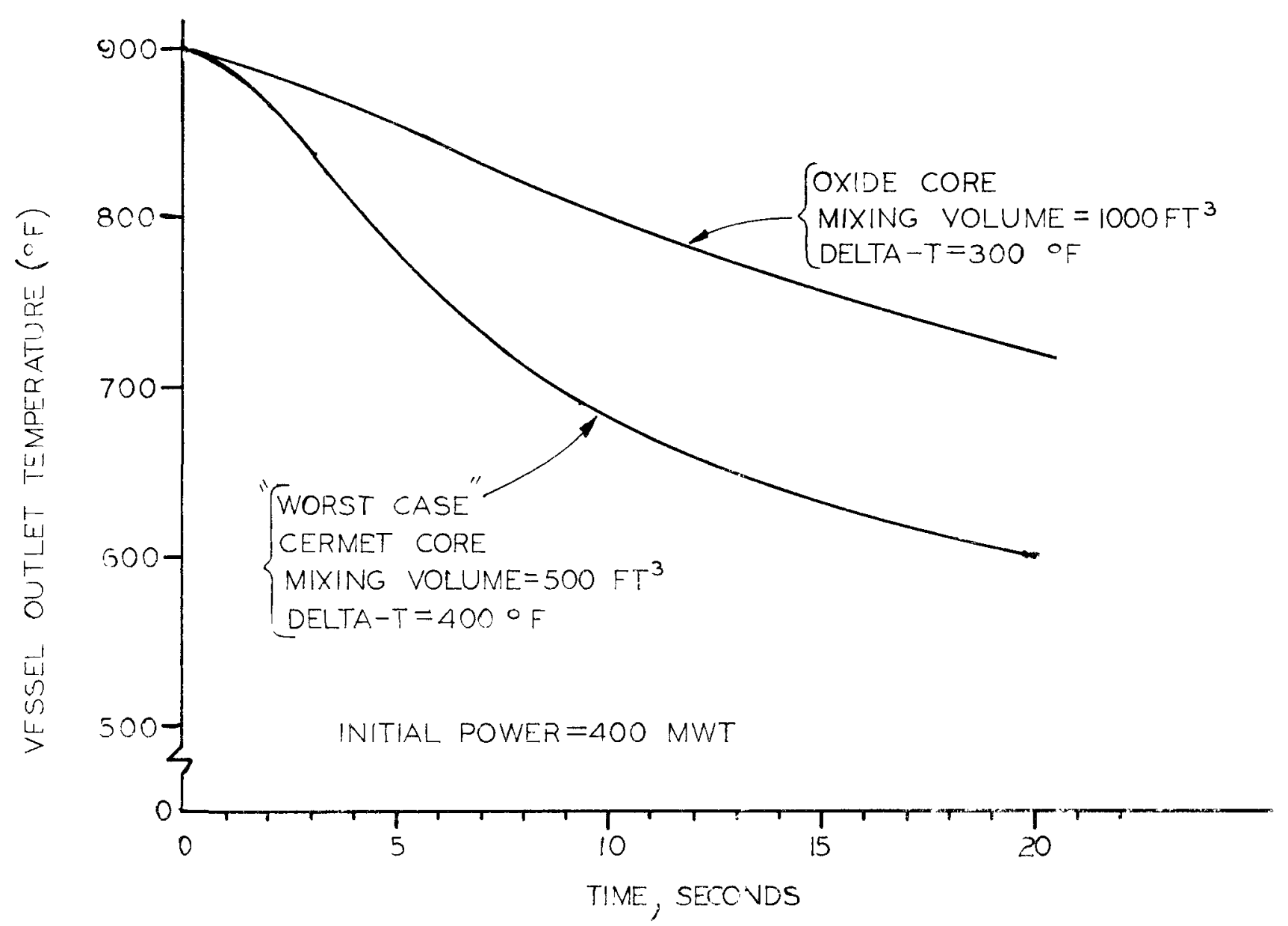

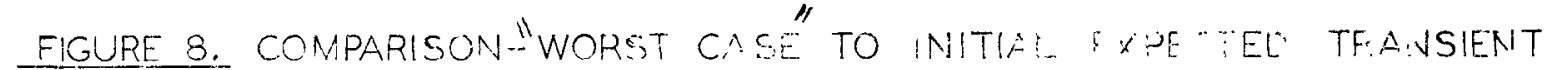
AT VESSEL OUTLET FOR POWER SCFLIA WITW FULL TLOW 

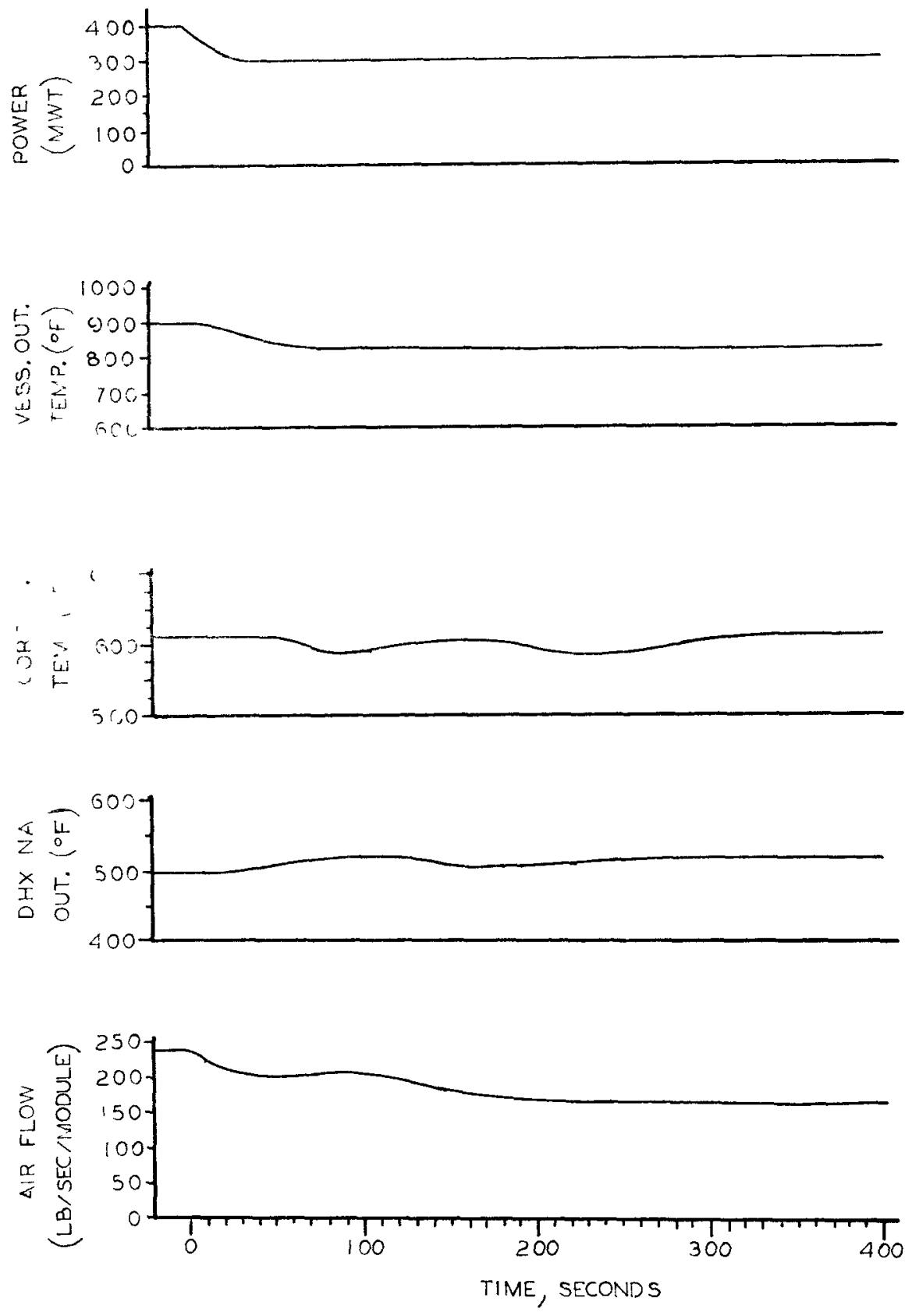

FIGURE 9. PRELIMINARY CONTROL STUDY: SYSTEM RESPONSE TO POWER SETBACK, CONSTANT SODIUM FLOWS. 
converter and 24 digital-to-analog converters. (3) Digital control of analog functions is provided through control interfacing. (3)

The software system used in digital programing is a combination of greatly modified DEC Basic Software System programs and several programs developed independently at BNW. DEC's basic assembler and debugging program (DDT) were modified to make them compatible with a hybrid computer system. BNW software includes magnetic tape editor and loading programs and provides selective core dump capabilities (to magnetic tape).

The actual language used in programming is a "dialect" of DEC's assembly language and is called SIMPL-1 (Simulation Implementing Machine Programming Language - Version 1). (4) The technique is essentially "subroutine programming" in that a subroutine library is available for integration, transport time delays, function generation, and all basic mathematical operations such as multiplication, division, and taking of logarithms. The language provides the capability of doing digital programming with an analog approach, i.e., direct conversion from block diagrams with "black boxes" for integration and other mathematical operations.

Fixed-point arithmetic is used along with analog scaling techniques within the digital program. This was easily done since the entire simulation had originally been scaled for an analog computer. The efficiency of assembly language programming combined with the speed of fixed point arithmetic operations provides the capability of operating the entire simulation in ten times real-time, i.e., ten times as fast as the actual process would operate. Ten times real-time will be referred to as problem-time in this paper. The high speed operation has proven beneficial in performing long-term control studies.

\section{SIMULATION TECHNIQUES}

The division of labor between the analog and digital portions of the hybrid computer has been established primarily on the basis of high gain operations functioning better on the analog computer. If the entire simulation (Phese 3 Model) were placed on an analog computer, 320 amplifiers, 60 integrators, 55 multipliers, 25 function generators, over 250 potentiometers, and at least 20 differential relays would be needed, as well as 8 variable time delays. The hybrid simulation of Phase 3 uses 120 amplifiers, 36 integrators, 30 multipliers, and 130 potentiometers. The digital computer provides the rest of the capability.

\section{Main Simulation Program}

The digital program is broken up into about 20 subprograms, each of which can be entered at a varying rate. The overall frame-time of the digital computer program is about 16.5 milliseconds which corresponds to 0.165 seconds problem time; however, many variables are updated three, four, or even five times during this single cycle. The frequency of iteration can be varied for each subprogram individually to provide stable output signals from each. For example, high gain integrations, such as pump motor speed, require a higher iteration rate than flow integrations to maintain stability.

The simulation itself occupies about 5,000 of the available 8,000 core memory locations on the PDP-7. Of the remaining 3,000 memory locations, about 1,000 are used by an on-line monitoring program, Dynamic Keyboard Monitor (DKM). This program provides the capability of making on-line decisions to initiate control actions or transients, or to alter controller setpoints or safety trip points. This is done by way of the teletype while the computer is in compute mode without returning to initial conditions, and will provide the basis for future operator training. In addition, DKM makes possible the 
changing of instructions during operation of the simulation, providing a powerful debugging tool.

DKM also allows commands to be given to selectively load any of several support programs from magnetic tape into the remaining portion of memory (2,000 locations). These programs (discussed below) can be loaded relocatably and are loaded on an interrupt level so as to not disturb the operation of the simulation. Once they are loaded, DKM can be used to transfer control and initiate program action by these programs.

\section{Simulation Support Programs}

Several support programs share a buffer zone in memory. They are loaded when needed and replaced by other programs upon completing their functions. The first of these automatically sets potentiometers on the analog computer. This provides for easily changing parameter values or initial conditions when a new study is to be performed.

A separate program is also used for monitoring steady-state conditions. It stores the steady-state values of all integrators for use as initial condition settings at a later time. This includes digital and analog variables.

To assist in determining whether the simulation is operating correctly, several programs can be loaded to monitor steady-state conditions and calculate energy balances throughout the simulated system. Heat balances are calculated for the vessel, IHX, and DHX including percent error deviation for each component.

When the simulation is operating, the buffer zone of memory is used for a simulated protection system replacing the preoperational support programs mentioned above. This program monitors any number of variables comparing their values to pre-set trip points. If a trip point is reached, action is automatically taken as specified by the user. This program is used not only for protective functions but also provides the user with the capability of initiating accident conditions. This prevents the necessity of patching special relays and integrators on the analog patch board merely to initiate a particular transient.

During a transient condition a user-specified number of variables can be monitored and their values can be stored in preselected time increments. These values cen be automatically printed out together with a heading describing the transient.

\section{Special Simulation Techniques}

The digital programing in the FFTF Simulation emphasizes special techniques to maximize computer efficiency. As an example, function generation (table lookup) methods developed for multiple input functions are readily available but are time-consuming (e.g., 290 microseconds for two input functions). A time savings of 30 to $65 \%$ has been achieved by breaking these functions into single-variable functions with their outputs being handled algebraically to produce the desired function. Having the programmer spend more time in developing an efficient program is desirable since the plant simulation provides faster-than-real-time operation and since it is to be used for several years.

Other special techniques developed by BNW include several special purpose subroutines. The digital portion of the simulation performs about thirty lowto-medium gain integrations. These integrations are performed by a subroutine which utilizes asynchronous rectangular integration techniques. Normalized mathematical operations are used within the subroutine to provide a maximum 
degree of accuracy. Truncation errors are greatly decreased by accumulating an eighteen bit remainder term from one sample time to another and using it to update the output. The subroutine provides the capability of using an input multiplier with the integration, much the same as gains are used on analog integrators. The amount of computer time needed for one sample time for a single integration is about 65 microseconds.

It is felt that the use of rectangular integration is justified in that all FFTF system models using digital integrations had previously been programmed on the analog computer. These include models of the pump, hydraulics, the IHX, and the DHX. No significant differences were detected in the results of the two approaches.

Another special subroutine being used in the simulation is one which simulates variable flow transport time delays. Coolant pipes can be divided into a large number (several hundred if desired) of nodes and the rate at which temperature transients pass from one node to the next is determined as a function of flow rate. The subroutine requires between 70 and 90 microseconds for each entry regardless of how many nodes the pipe is divided into. In the simulation these time delays have been updated ten times per second, problem time (100 times per second real-time).

\section{RECENT DEVELOPMENTS}

\section{COMPUTING EQUIPMENT}

The FFTF Simulation has recently been transferred to a new hybrid computer facility consisting of an AD-4 (Applied Dynamics) analog computer and a DEC PDP-9 digital computer. The analog computer is solid-state and is equipped with a digital logic patchboard while the PDP-9 has two $8 \mathrm{~K}$ memory banks with the capability of easily expanding to three or four. Cycle time for the PDP-9 is 1.0 microseconds compared to 1.75 microseconds for the PDP-7; however, because of slower hardware arithmetic operations (multiply and divide) there is little speed advantage with the new computer. An interface similar to the one already described is used in linking the two computers together.

A modified version of DEC's Advanced Software System is used for all digital programming. The language used is a version of DEC's assembly language with many BNW-developed macros which have been included as a aprt of DEC's Macro Assembler. This approach has been used in place of many subroutines used in programing on the original simulation because of the extra operating speed gained and because the larger core memory is available.

\section{SIMULATION TECHNIQUES}

As the design of the FFTF progresses beyond the conceptual stages, the need for more complexity in the hybrid model arises. A solution to this problem would be a programming technique which would allow adding complexity to portions of the system which are of major interest and simplifications to other, less important portions. Ideally, the programming would be such that changes could be easily made and any portion of the system could be expanded.

This capability was provided by a "modular" programming approach. The digital program was separated into several programs or modules which can be loaded and operated independently of one another. They communicate only with a master program which defines all system variables that are used by more than one program module. Each program module represents a separate component of the plant simulation, such as an IHX, a DHX, coolant pipes, hydraulics, and reactor kinetics. If a study of only one component is needed then only that one component module reeds to be loaded into memory. 
Modulizing of the Phase 3 model is now complete and it is possible to make studies of more complex system components without having to edit, assemble, load, and debug a single overall program describing the entire simulation. A single module can be modified and combined with the rest of the simulation during the loading process. Detailed models of particular components can be developed separately and studied at the expense of less important, simplified models elsewhere in the system. In this approach, the analog computer serves as a separate module.

The utility of the modular programming will be evident in continuing plant control studies (Phase 3 Model) and in performing pipe rupture studies which will require a widely different model. The pipe rupture studies will be pursued with a complex reactor model and a complex primary coolant loop and pump model. Moedling of secondary loops and DHXs will be rudimentary or nonexistent. These studies, with an all-digital approach, are now in progress and consume from fifty to several hundred times the problem time in the computing process. Hybrid studies will yield great computing cost savings.

\section{CONCLUSIONS}

Experience with the FFTF hybrid simulation leads to the following basic conclusion: using a structured assembly language which gives the programmer the capability of applying well-known analog computer techniques (i.e., "black boxes" for mutiplication, division, and integration) to a digital program, a relatively small computer system can be used efficiently in large scale studies. This can provide the following advantages:

1) Large systems can be simulated, often in faster-thanreal-time operation.

2) Powerful man-machine interaction capabilities can be provided in a manner not at all possible on large, expensive computer systems.

3) Large cost savings can be achieved. The same overall FFTF simulation, if simulated with conventional digital programing techniques, would cost at least 100 times as much to run as it does on the hybrid computer.

\section{ACKNOWLEDGMENTS}

The authors gratefully acknowledge the efforts of H. G. Johnson, I. H. Gerhardstein and M. H. Deardorff in the development of models and techniques in hybrid simulation.

\section{REFERENCES}

1. C. D. Flowers and L. H. Gerhardstein. Analog-Hybrid Dynamic Simulation of the FFTF Reactor and Heat Transport System, BNWL-707, (April, 1968).

2. R. W. Albrecht and C. Metelmann. The Use of Reduced Delayed Neutron Group Representations in Nuclear Reactor Simulations, HW-81076, (March, 1964).

3. M. D. Erickson. The Battelle-Northwest Hybrid Computer System, BNWL-538, (October, 1967).

4. R. D. Benham. SIMPL-1: Simulation Implementing Machine Programming Language - Version 1, BNWL-878, (October, 1968). 
SESSION VI

COMPUTATIONAL TECHNIQUES

Chairman: K. D. Iathrof

Los Alamos Scjentifin Labnratory 


\section{BLANK}




\section{THE EFFECTIVE USE OF THE COMPUTER STORAGE HIERARCHY* \\ by \\ Thomas L. Jordan and William J. Worlton \\ Los Alamos Scientific Laboratory \\ Los Alamos, New Mexico}

\section{INTRODUCTION}

The need for a hierarchy rather than a single level of storage is dictated on both technical and economic grounds. Timing (and hence distance) limitations require the close integration of the main memory with the central processor, and it is therefore possible to incorporate only a limited capacity of main memory without violating these restrictions. Thus, even if main memory costs posed no problem it would not be technically feasible to include unlimited main memory in computers. The dual of this statement is that even though it were technically feasible to do so, the high cost of main memory would impose an economic limit on the capacity that is feasible for users to acquire. Many large capacity requirements must therefore be met with other devices with lower cost and less desirable performance characteristics than those of main memory.

The storage hierarchy poses important problems in the design, selection, and use of computers. Computers are usually designed to achieve the speed potential of their central processor through use of the main memory, and the problem of how to avoid system degradation when other levels of storage are used is left as an exercise for the programer. The design goals for a properly balanced storage hierarchy include minimum access and transmission delay, minimum cost, ease of programming, and maximum reliability. These goals are properly addressed in the design of the computer, in the selection of a properly balanced hierarchy of equipment, and in the use of appropriate programming techniques. Although certain design implications will be noted, this paper will be devoted largely to guidelines for the selection and proper use of the storage hierarchy.

\section{THE STORAGE HIERARCHY: PAST AND PRESENT}

\subsection{History.}

Figure 1 shows the various storage levels which are used in modern computers, together with order of magnitude figures for capacity, access ratio, and cost. Access ratio is defined as the ratio of the access time for any storage level to the access time of main memory. The first electronic computer (ENIAC, 1946) had only two levels of storage--twenty adding and storage registers augmented by three portable function-table units, each of which held 104 pre-set values which could be read in about four add-times. 1 In modern terminology, ENIAC had 20 registers and 312 words of read-only storage. Experience with ENIAC showed that achieving the speed potential of a computer required a larger high-speed memory for both programs and data, so ENIAC's successor,

*Work performed under the auspices of the U. S. Atomic Energy Commission. 


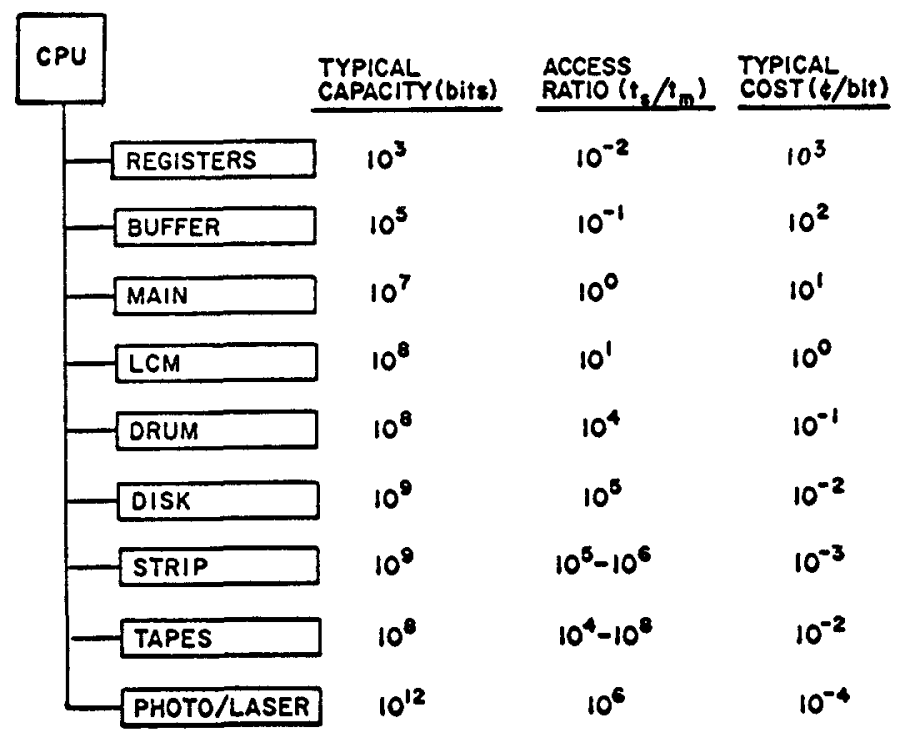

Fig. 1. The storage hierarchy.

EDVAC, was designed with 1024 words of main memory and 4,608 words of magnetic drum storage. 2 Tapes were introduced into the storage hierarchy in the SEAC (1950). 3 The first magnetic disk unit was demonstrated in 1952 by the Bureau of Standards, and the first commercially available disk was the RAMAC, introduced by IBM in 1956. 4 Magnetic card and strip devices offering capacities on the order of $10^{9}$ bits were developed in the early 1960's; these devices are now offered by Honeywell, IBM, NCR and RCA.5, 6 Storage capacities of 1012 bits have been developed using photographic storage on film chips, ${ }^{7}$ and laser storage technology will soon offer about the same capacity. ${ }^{8}$

The gap between main memory and moving-media storage has recently been partly filled by the development of large capacity memory (LCM), which offers relatively low-cost fixed-medium storage which is some three orders of magnitude faster in access time than any moving-medium storage device. ${ }^{9}$ The importance of this new storage level is discussed from the point of view of experience at Los Alamos Scientific Laboratory in Section 4.

The need to provide instructions and operands to the central processor (CPU) at extremely high speeds has traditionally required the use of a rather large amount of high speed, high cost, main memory. The inclusion of a level in the storage hierarchy between the registers and the main memory offers the possibility of using a somewhat lower speed, lower cost, main memory. In this design, an example of which is the IBM-360/85, fetching and storing requirements of the CPU are met by references to a high-speed buffer, which is automatically filled and emptied from main memory as required by the addresses generated by the CPU. The effective cycle time of the system approaches the cycle time of the buffer store, and the effective capacity of the system equals that of the main memory. Figure 2 shows the mean access time of a combination of high-speed buffer and main memory, with access times of 0.1 and 1.0 , respectively, as a function of the fraction of the access requests which are satisfied by the buffer. This fraction is determined by the capacity, sector size, and replacement algorithm of the buffer. The IBM-360/85 uses a buffer of $16 \mathrm{~K}$ $(\mathrm{K}=10248$-bit bytes $)$ with sectors of $1 \mathrm{~K}$; performance studies showed an average value of $f=0.968$ for 19 real programs, but $f<0.01$ for random addressing. 10 This design is a blend of M. V. Wilkes' ideas on slave memories 11 with T. Kilburn's ideas on a one-level storage system.12 It is only recently that a technology appropriate for the inclusion of this new level has become avail- 


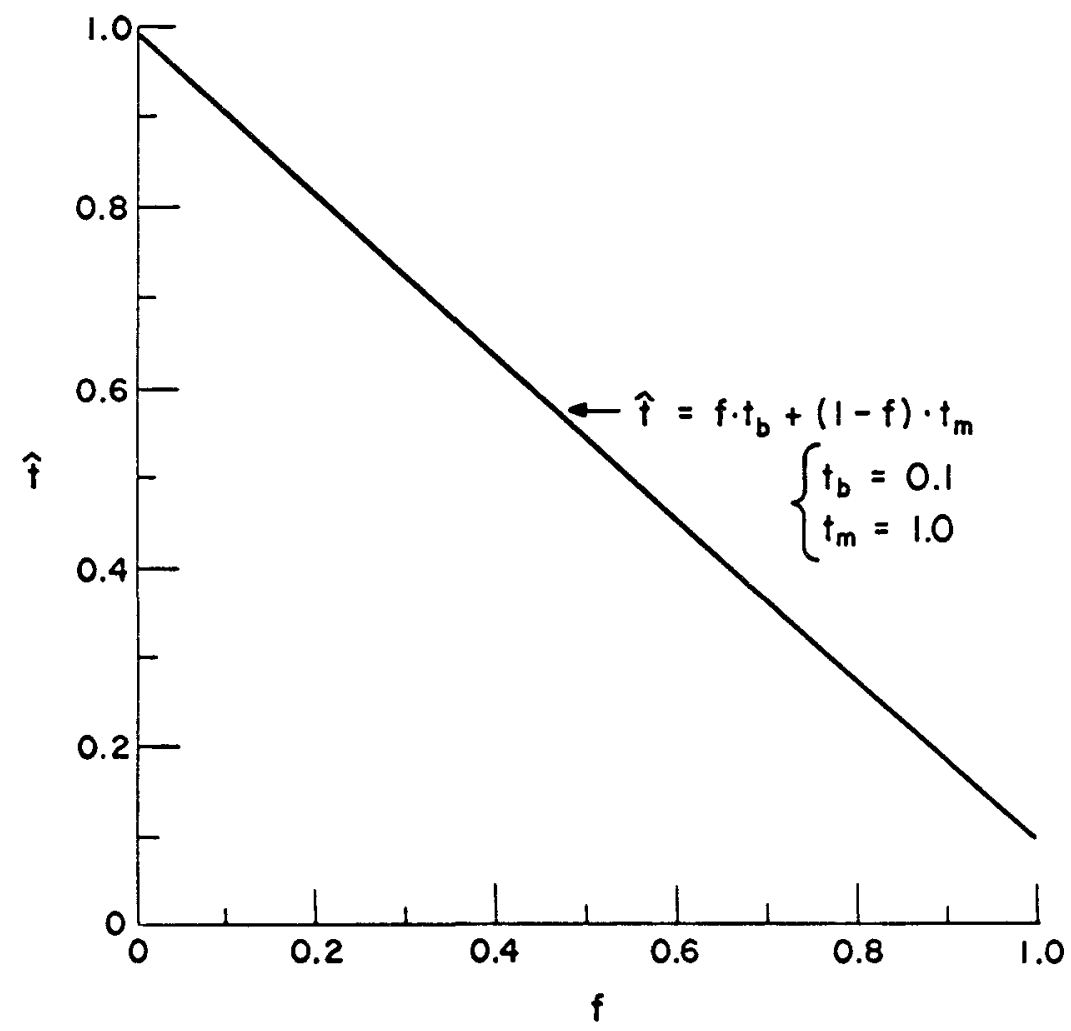

Fig. 2. Mean access time for high-speed buffer and main memory.

able, and the high-speed buffer is expected to become a standard part of the storage hierarchy of future computers.

In summary, then, the history of the computer storage hierarchy is characterized by an increase in the number of storage levels, a decrease in the gaps in access ratio between the levels, and for each level, a decrease in the cost per bit of storage, a decrease in the access time, and an increase in available capacity.

\subsection{State-of-the-art.}

Figure 3 is a state-of-the-art diagram showing access time and capacity for the various technologies which are used in the storage hierarchy, and Fig. 4 is a similar diagram for access time and cost. The orientation of the ellipses in Fig. 3 which define the areas of application implies that within any one of these technologies it is generally true that the use of larger capacities requires longer access times. The superconductive technology is not one that is currently available in the storage hierarchy, but may someday fill the price-performance gap between the static and moving magnetic media.13 If the price reductions which have been projected for semiconductor memories are achieved, this technology may challenge the magnetic media in the lower capacities of main memory applications. 14 The areas covered by these technologies are not static, but tend to migrate toward the upper left, i.e., toward a higher capacity and faster access time.

The orientation of the ellipses in Fig. 4 implies that faster access time within a technology generally requires higher costs. There is room in the hierarchy for storage which falls below and to the left of the positions shown in this diagram, i.e., storage which is either less expensive at the same access 


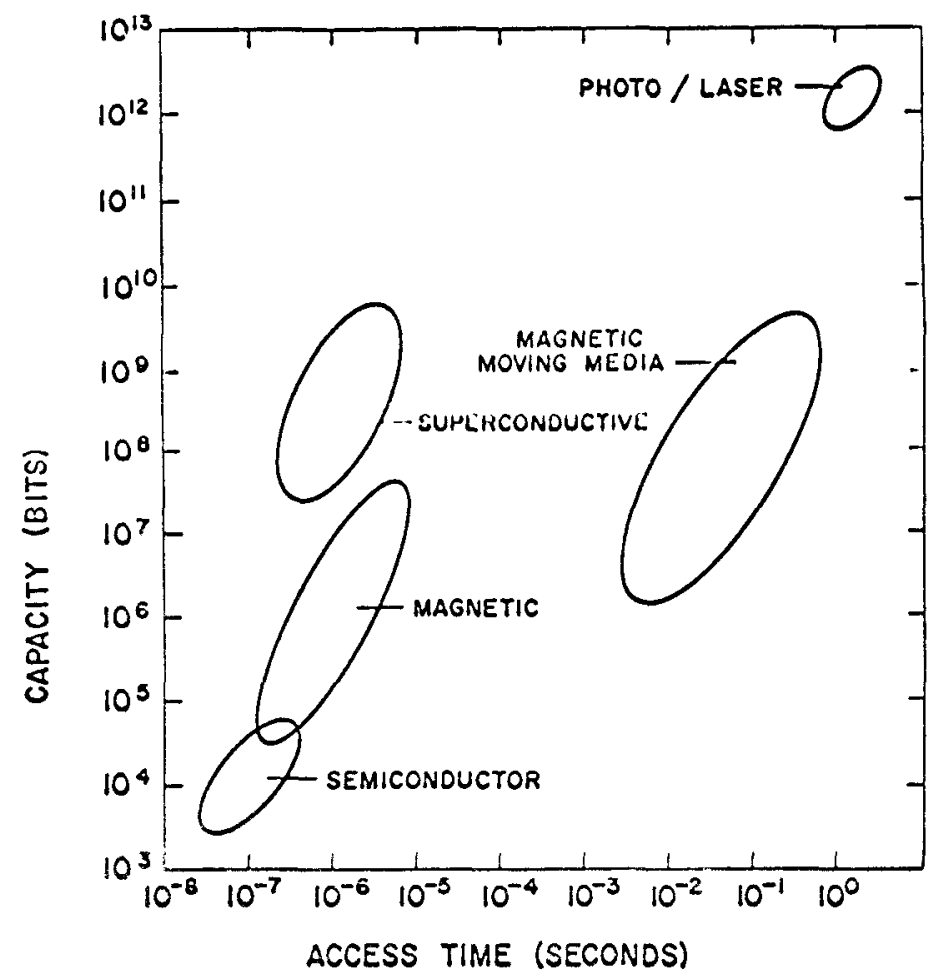

Fig. 3 State-of-the-art: capacity vs. access time.

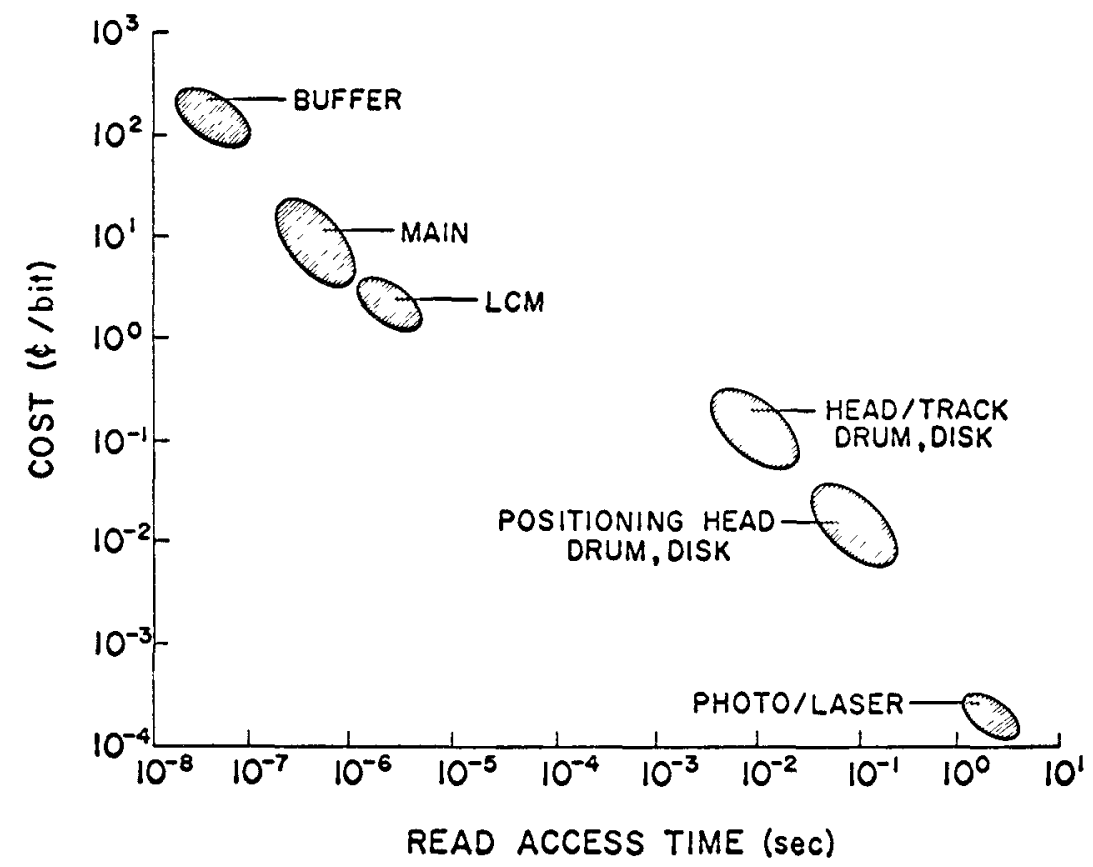

Fig. 4. State-of-the-art: cost vs. access time. 
time, faster at the same cost, or both faster and less expensive. Storage devices which fall below a line connecting any of the areas shown would also likely find a ready market. For example, a device which had an access time of about $100 \mu \mathrm{sec}$ which cost less than LCM but more than drums would find ready acceptance in the capacity range of $10^{8}$ to $10^{9}$ bits. The areas shown in this diagram will migrate in time to positions of faster access time and lower cost.

\section{CYCLIC BUFFERING}

The large access ratios of secondary storage levels require either that the use of these levels must be relatively infrequent or that their use must be overlapped with CPU activity in order to avoid system degradation. "Cyclic buffering" (also called the "three-ring circus") is a method of using secondary storage as an extension of main memory which systematically overlaps secondary storage data flow with calculation. When properly used, this method reduces the secondary storage overhead to a few percent of the time required for the same calculation when only main memory is used, but when improperly used, the overhead can be as much as several hundred percent. Hence the importance of the following analysis.

In this method, three buffers are used for reading, computing, and writing; although the buffers normally occur in main memory, certain designs (e. g., the CDC-7600) allow the use of LCM. Independent devices and channels are needed for reading and writing secondary storage. A block of data is read into the first buffer, and this data is then used for computing, in parallel with the reading of data into the second buffer. The data in the second buffer is next used for computing, in parallel with reading more data into the third buffer and writing the data out of the first buffer. This process continues, with each buffer being used in cycles for reading, computing, and writing, as illustrated in Fig. 5 and Fig. 6.

The parameters of interest here are:

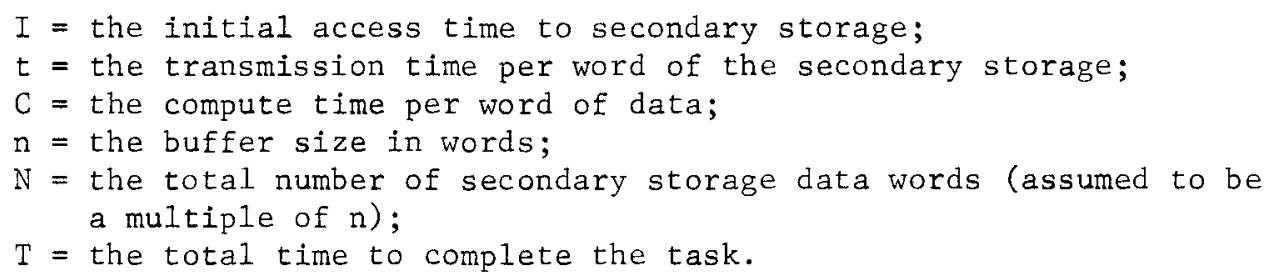

The time to complete the task using main memory for data storage is $\mathrm{T}_{\mathrm{O}}=\mathrm{C} * \mathrm{~N}$. For $\mathrm{I}+\mathrm{t} *_{\mathrm{n}} \leq \mathrm{C} *_{\mathrm{n}}$, i.e., when the read/write time for a buffer of size $\mathrm{n}$ is less than or equal to the compute time for the buffer, the total time is given by

$$
\mathrm{T}_{1}=\mathrm{C} * \mathrm{~N}+2 *(I+t * \mathrm{n})
$$

The buffer size for which $I+t *_{n}=C *_{n}$ is given by

$$
\mathrm{n}_{\mathrm{o}}=\mathrm{I} /(\mathrm{C}-\mathrm{t}) \text {. }
$$

Several important facts can be observed from this simple relation. First, the buffer size necessary to achieve balance in cyclic buffering is directly proportional to the initial access time of the secondary storage being used. Thus, a disk with initial access time of 100 milliseconds requires ten times as much storage per buffer as a drum with initial access time of 10 milliseconds. Second, this balance point cannot be reached at all unless $C>t$. The value of $C$ is a function of the magnitude of the computing task and the speed of the computer; the value of $t$ depends upon the transmission rate of the secondary 


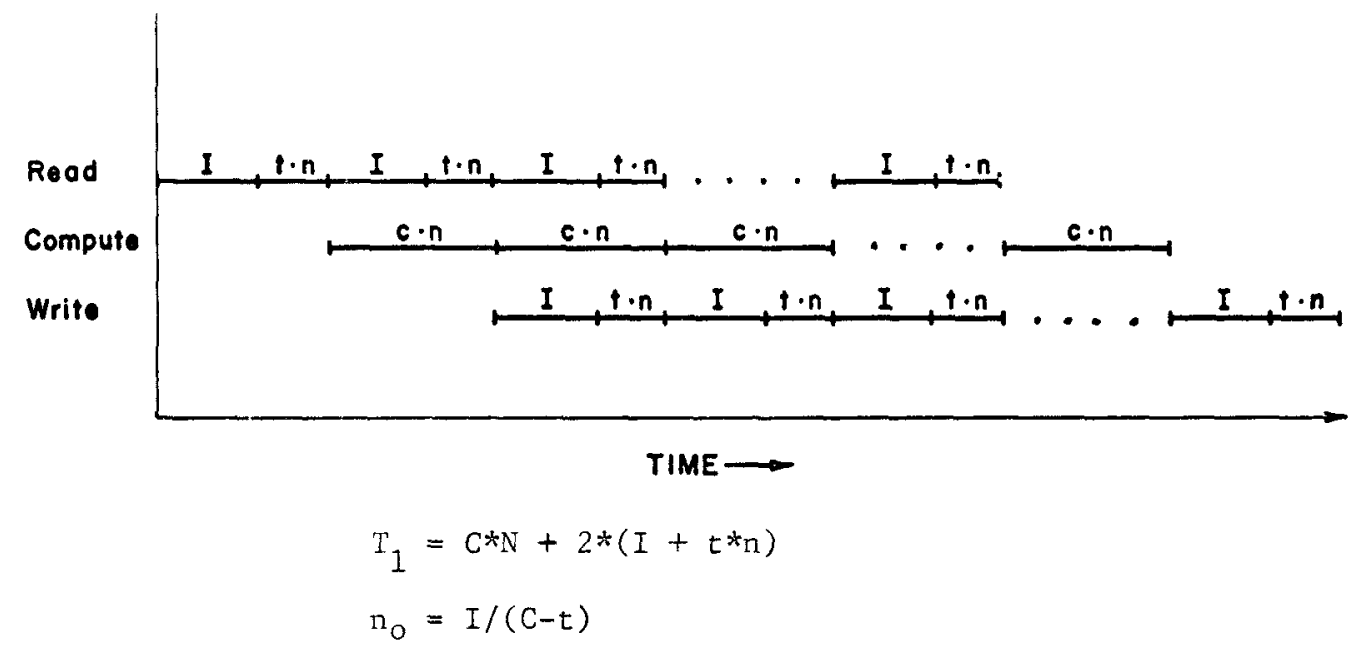

Fig. 5. Cyclic buffer timing for $I+t^{*} n \leq C * n$.

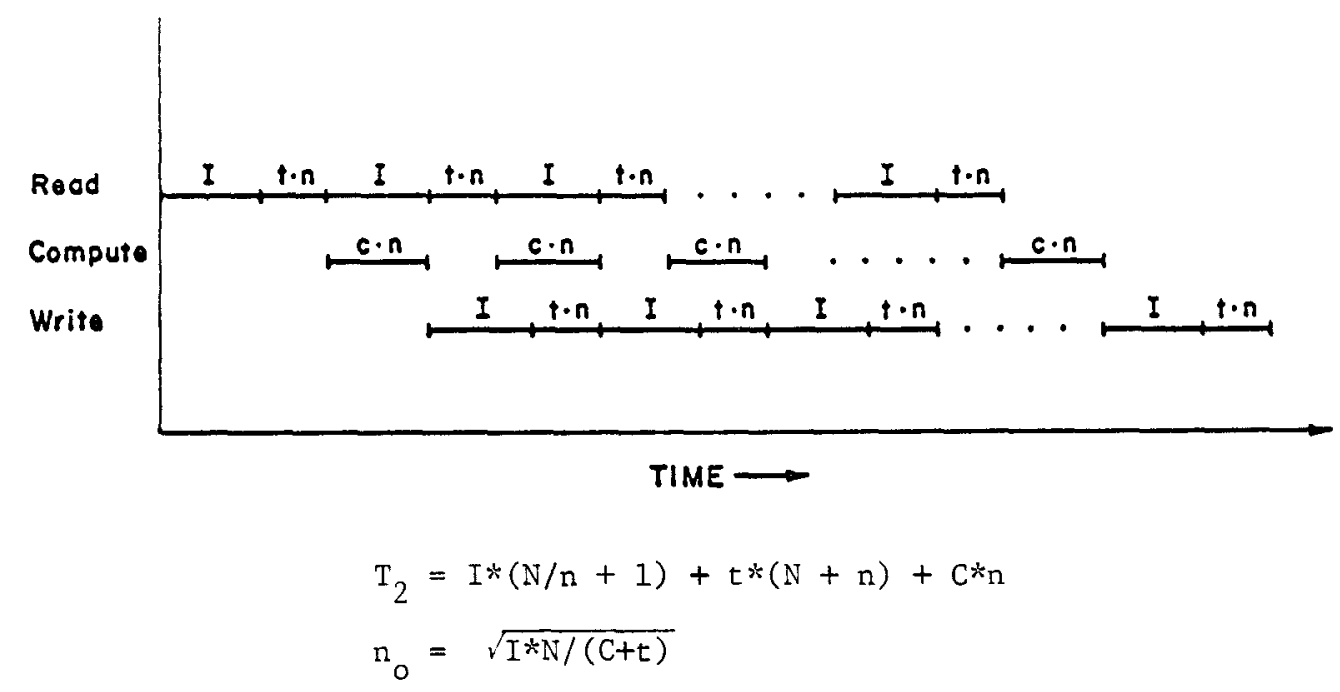

Fig. 6. Cyclic buffer timing for $I+t^{*} n \geq C^{*} n$.

storage. For a given value of $t$ only those tasks for which $C>t$ can use cyclic buffering without unnecessary system degradation. Alternately, for a given value of $C$, only those devices with $t<C$ should be used for secondary storage for cyclic buffering.

When I $+t^{*} n>C^{*} n$, the time to complete a cyclic buffered task is given by

$$
T_{2}=I *(N / n+1)+t *(N+n)+C * n \text {. }
$$

This situation will occur when the buffer is too small, i.e., when $n<n$, or when $t>c$. This function has a minimum at

$$
\mathrm{n}=\sqrt{\mathrm{I} * \mathrm{~N} /(\mathrm{C}+\mathrm{t})}
$$

and this is the optimum buffer size which should be used if $t>C$. Figure 7 illustrates the timing for a cyclic buffered task on the CDC-6600. It is 


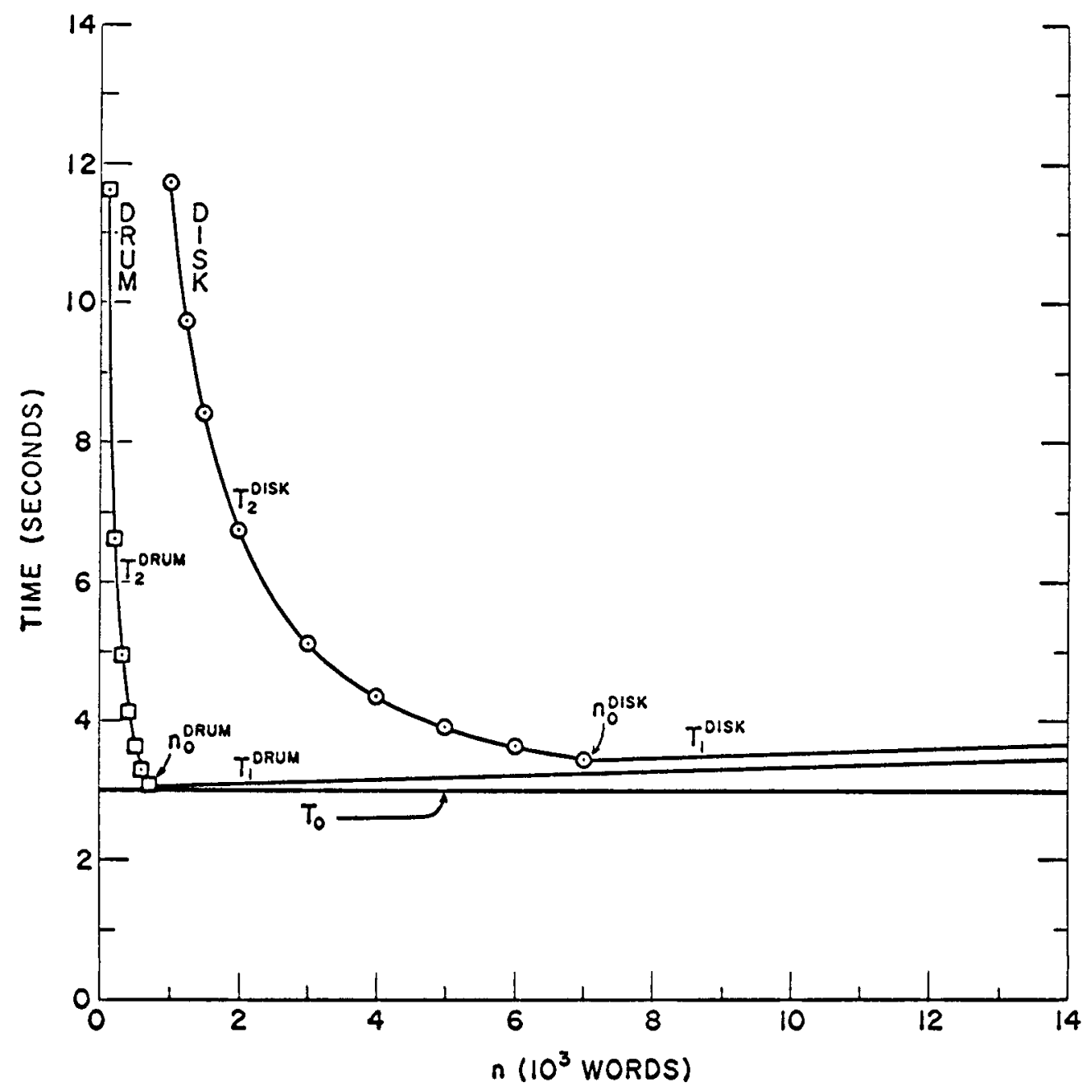

Fig. 7. Cyclic buffer timing comparison for disk and drum.

important to note the extremely large penalty in running time when the buffer size is too small.

This discussion can be made more meaningful by relating it to computing tasks on current computers, and this is done in Table 1 . The value of $\mathrm{C}$ used here is taken from a magnetohydrodynamics code programmed in assembly language on the 6600; the values of $\mathrm{C}$ for the IBM-360/85 and the CDC-7600 are derived from the assumption that the $360 / 85$ and the 7600 are two and four times as fast as the 6600, respectively. A nominal value of 100 milliseconds is used for the access time to disks and 20 milliseconds for drums, rather than measured values. The execution time for this code when the data is memory contained is $\mathrm{T}_{\mathrm{O}}=\mathrm{C}^{*} \mathrm{~N}$, or $3.0,1.5$, and 0.75 seconds, respectively, for the 6600, 360/85, and 7600 . When the buffer size is too small, the penalty in running time is very high, and would make any computer appear to be running many times slower than its CPU potential. However, when the optimum buffer size is used, the penalty for using disks or drums rather than main memory is only a few percent, and the use of cyclic buffering has effectively extended the main memory capacity by $10^{5}$ words in this example. Further, the fraction of overhead time decreases as $N$ 


\begin{tabular}{|c|c|c|c|c|c|c|c|c|}
\hline System & $\begin{array}{l}\text { Secondary } \\
\text { Storage } \\
\end{array}$ & $\begin{array}{c}I \\
(\mu s e c)\end{array}$ & $\begin{array}{c}t \\
(\mu s e c)\end{array}$ & $\begin{array}{c}c \\
(\mu s e c)\end{array}$ & $\begin{array}{c}N \\
\text { (words) } \\
\end{array}$ & $\begin{array}{c}\mathrm{n}_{\mathrm{o}} \\
\text { (words) }\end{array}$ & $\begin{array}{c}\mathbf{n} \\
\text { (words) }\end{array}$ & $\begin{array}{c}\mathrm{T} \\
(\mathrm{Sec}) \\
\end{array}$ \\
\hline CDC- 6600 & 6638-Disk & $10^{5}$ & 16 & 30 & $10^{5}$ & 7142 & $10^{3}$ & 11.75 \\
\hline$"$ & " & $10^{5}$ & 16 & 30 & $10^{5}$ & 7142 & $5 \times 10^{3}$ & 3.93 \\
\hline$"$ & 865-Drum & $2 \times 10^{4}$ & 16 & 30 & $10^{5}$ & 1428 & $10^{3}$ & 3.66 \\
\hline IBM- $360 / 85$ & 2314-Disk & $10^{5}$ & 25 & 15 & $10^{5}$ & 15811 & $10^{3}$ & 12.64 \\
\hline " & " & $10^{5}$ & 25 & 15 & $10^{5}$ & 15811 & $10^{4}$ & 4.00 \\
\hline$"$ & 2301-Drum & $2 \times 10^{4}$ & 6 & 15 & $10^{5}$ & 2222 & $2 \times 10^{3}$ & 1.66 \\
\hline CDC-7600 & 7638-Disk & $10^{5}$ & 1.5 & 7.5 & $10^{5}$ & 16667 & $10^{3}$ & 10.26 \\
\hline$"$ & $"$ & $10^{5}$ & 1.5 & 7.5 & $10^{5}$ & 16667 & $2 \times 10^{4}$ & 1.01 \\
\hline$"$ & $\begin{array}{c}7600 / 150 \\
\text { Drum }\end{array}$ & $2 \times 10^{4}$ & 1.5 & 7.5 & $10^{5}$ & 3333 & $5 \times 10^{3}$ & 0.80 \\
\hline
\end{tabular}

Table 1. Cyclic buffer timing comparisons.

increases, since

$$
\begin{aligned}
\text { Overhead } & =T_{1} / T_{0}-1 \\
& =(C * N+2 *(I+t * n)) /(C * N)-1 \\
& \left.=2 * C+t * *_{n}\right) !(C * N) .
\end{aligned}
$$

The other cost for cyclic buffering is the space in the main memory which is used for the buffers, equal to $3^{*} n$ words. When a computer system is selected, typical values of $n_{0}$ should be calculated, and sufficient space allowed in main memory for these buffers. This space can be reduced by the use of fast access mechanisms such as head-per-track disks or drums, but any such trade-off should include the higher cost per bit and the capacity limitations of these devices as compared to positioning-head devices.

Note that the use of the IBM-2314 on the IBL-360/85 leads to an example where $t>C$; this device cannot provide the necessary transmission rates to sustain cyclic buffering when $C<25 \mathrm{\mu sec} /$ word without system degradation (assuming only one channel each for reading or writing). If its capacity is adequate, the IBM-2301 drum can be used for cyclic buffering in these cases.

In summary, when cyclic buffering is properly used it allows the capacity of secondary storage to be used as though this capacity were part of main memory, with only a small overhead in running time. Proper use of this method requires (1) that the optimum buffer size, $\mathrm{n}_{0}$, be determined, (2) that the main memory be large enough to accommodate three buffers of size $n_{0}$, and (3) that two independent channels and storage devices be used for reading and writing.

\section{LASL EXPERIENCE WITH EXTENDED CORE STORAGE}

\subsection{Introduction.}

The Los Alamos Scientific Laboratory (LASL) accepted the first Control Data Extended Core Storage (ECS) in June 1968. The ECS, compared to disks and tapes, is a secondary storage unit with very high speed and low capacity. The cycle time of ECS is only a few times slower than the 6600 Central Memory (CM). A secondary device of this speed is relatively new to the industry. Hence, the importance of evaluating it as another device in the scheme of computer storage hierarchies is substantial.

A 500K ECS was selected in lieu of $64 \mathrm{~K}$ of additional CM on the basis of the enlarged class of problems that could be computed, the number of 
computers that could access the device, and the approximate 10 to 1 cost ratio per bit of storage. A more complicated programming logic and some slow down in processing time was recognized for those problems which could operate in CM with an additional $64 \mathrm{~K}$ of $\mathrm{CM}$.

Acquisition and use of the ECS storage has raised a number of questions to be answered, including the following:

1) How many machines could a single stack of ECS support? Since LASL was getting another bay of ECS and the bay we had was connected to two CDC$6600^{\prime} s$, it was important to know if it could be added to the stack and also serve three or four computers without degrading significantly either the ECS or the computers. We shall refer to this as the "multiple computer interaction" problem.

2) What effect does introducing ECS into the operating system have upon performance of the computer system and the ECS? We shall refer to this as the "ECS-computer interaction" problem.

3) How effective is ECS as: a) an extension of CM; and b) a replacement for disk for certain critical disk functions?

\subsection{ECS Characteristics.}

The ECS memory is organized with a word size of 488 bits ( 8 CM words plus 8 parity bits), banks of $125,952 \mathrm{CM}$ words, and a total of $1,2,4,8$, or 16 banks interlaced on the low order address bits. The memory cycle time is $3.2 \mu \mathrm{sec}$ with a maximum transfer rate between CM and ECS of .1 usec per CM word. At least $64 \mathrm{~K}$ of $\mathrm{CM}$ and 4 banks of ECS are needed to obtain this maximum transfer rate.

Other characteristics or features of the ECS include: four access channels, allowing service to as many as four computers; sequential service to computers requesting simultaneous service; flag-register interrogation to permit other than sequential service through programming and provide for intercomputer communication; testable bits for checking parity on reading; and interruption of ECS transfers to allow transfers between Peripheral Processors $\left(\mathrm{PP}^{\prime} \mathrm{s}\right)$ and $\mathrm{CM}$.

As a consequence of the ECS organization, characteristics, and features described above, there are several ways in which the ECS and/or the computer can be degraded. To initiate a transfer to or from ECS, various Central Processor ( $C P$ ) and PP functions must be satisfied first. This, plus initiating ECS controls, causes the first CM word of a transfer to occur in $5.4 \mu \mathrm{sec}$ for a read and $3.6 \mu \mathrm{sec}$ for a write. These times are also referred to as access times. Secondly, if a PP references CM during an ECS transfer, the 480-bit ECS word in transfer at the time the PP request is made is completed, and the ECS is delayed 2.7 to $3.3 \mu \mathrm{sec}$ if the transfer is a read and 1.4 to $2.0 \mathrm{\mu sec}$ if the transfer is a write. Thirdly, if two or more computers are connected to a single ECS and simultaneous service is requested, the maximum rate at which ECS can transfer words is degraded to various amounts ranging from $25 \%$ to $100 \%$ of the nominal rate depending upon the banks requested, the number of banks in the ECS configuration, and the number of computers requesting simultaneous service. ECS specifications have been reported elsewhere in more detail. 9

\subsection{LASL Experience.}

4.3.1 Multiple computer interaction. When more than one computer is serviced by a single ECS, there are two degrading effects on the total system. First, during the periods of time more than one computer are simultaneously 
requesting service, central processors of all but one computer are in a wait state. At best, these computers could only be proceeding with unfinished $\mathrm{I} / 0$ requests. Next, the ECS performance is in general degraded because the method of sequentially servicing computers usually interrupts the bank phasing that permits the very high streaming rates between ECS and CM.

The first problem was attacked by obtaining hardware measurements of the number of conflicts that occurred when two CDC-6600's were connected to $500 \mathrm{~K}$ of ECS. Two counters were attached to the ECS controller, one to count the total number of ECS words referenced, the second to count the number of concurrent requests to the same ECS banks. The code selected and run on both computers was a production code that is used extensively at LASL and is typical of several other heavily used production codes. This test was performed in an environment where the ECS usage was as heavy as one could realistically expect and was undoubtedly much heavier than one would encounter on the average. The number of conflicts that occurred was $6.5 \%$ of the total number of ECS words referenced by the two computers. Now if one takes the total number of ECS words addressed and translates these words accessed into minutes by an empirically established formula $(t=14+.15 n$, $t$ in microseconds, for $n$ word transfers), determined by software measurements, one notes that the ECS is busy about 5.1 minutes of the 22 minutes spanning the ECS experiment or less than $25 \%$ of the time. The above formula does not take into consideration the degraded ECS performance that is present when conflicts for service occur. During the time confiicts occur, ECS is performing, on the average, at $60 \%$ of its nominal rate. However. the $25 \%$ figure is about right th allow for this degraded performance. Of the time ECS is active, the duration of conflict is like $10 \%$ of its active period $(6.5 \%$ activity $\div 60 \%$ effectiveness). Hence the total effect of conflicts is no more than $3 \%$ of a system.

The second problem, that of determining the amount of degradation that ECS experiences when sustained simultaneous request to ECS are made, is resolved by an analysis of the behavior of ECS. To illustrate, if one considers two computers and four banks of ECS, and asks what the performance of ECS is when sustained strings of simultaneous requests are made, one can do the following sort of analysis. Let 1, 2, 3, 4 represent the ECS memory banks and A and B the computers. Then by looking at the possible banks requested and requiring four periods of $.8 \mathrm{\mu sec}$ for each bank to cycle, Table 2. illustrates the density of ECS activity for extended concurrent requests.

This analysis leads to the conclusion that the ECS performance is $60 \%$ of its rated capacity. If this analysis is performed for $1,2,4,8$, and 16 banks and $1,2,3$, or 4 computers, and for some configurations of ECS banks and computers, the average ECS performance during periods of multi-computer competition for service, will be as indicated in Table 3 . The computers are assumed to have memories of size $64 \mathrm{~K}$ or greater so that the computer acceptance rate is not a limiting factor.

4.3.2 ECS - Computer interaction problem. As part of the predelivery test, the ECS was tested to determine that it could perform at its advertised rate of 10 words per usec. To achieve this nominal ECS transfer rate, it was necessary to eliminate interference from PP's. What then is the effective transfer rate of ECS when the PP's are allowed to function at their usual rate? Software tests indicate that ECS is transferring at an approximate average rate of $.15 \mu \mathrm{sec}$ per $\mathrm{CM}$ word for equal numbers of reads and writes, in contrast to its nominal rate of .1 usec per $C M$ word. The degradation is roughly twice as great for a read as for a write. This degradation was an anticipated effect, although not to the degree experienced. Just why the degradation is so large is not well understood. 
Banks Initially Requested Simultaneously

\begin{tabular}{|c|c|c|c|c|c|c|c|}
\hline & $(1-1)$ & & $(1-2)$ & & $(1-3)$ & & $(1-4)$ \\
\hline & $\begin{array}{l}A \quad B \\
1\end{array}$ & & $\begin{array}{l}A B \\
I\end{array}$ & & $\begin{array}{l}A \quad B \\
1 .\end{array}$ & & $\begin{array}{ll}A & B \\
1\end{array}$ \\
\hline & - & & 2 & & 3 & & 4 \\
\hline & - & & - & & 2 & & 2 \\
\hline & - & & - & & 4 & & - \\
\hline & 1 & & - & & - & & 1 \\
\hline $40 \%$ & 2 & $40 \%$ & 2 & $80 \%$ & 3 & $80 \%$ & 3 \\
\hline Activity & - & Activity & 3 & Activity & 1 & Activity & 2 \\
\hline & - & & - & & 4 & & 4 \\
\hline & - & & - & & 2 & & - \\
\hline & 2 & & - & & - & & 3 \\
\hline & 3 & & 3 & & 1 & & 1 \\
\hline & - & & 4 & & 3 & & 4 \\
\hline & - & & - & & 2 & & 2 \\
\hline & - & & - & & 4 & & \\
\hline & etc. & & etc. & & etc. & & et \\
\hline
\end{tabular}

Note: Each line of the table represents an $.8 \mu \mathrm{sec}$ time interval.

Table 2. Density of ECS activity for concurrent requests.

\begin{tabular}{rrrll}
\multicolumn{5}{c}{ Computers } \\
Banks & 1 & 2 & 3 & 4 \\
\hline 1 & 2.5 & 2.5 & 2.5 & 2.5 \\
2 & 5 & 4 & 3.75 & 3.76 \\
4 & 10 & 6 & 5.375 & 5.216 \\
8 & 10 & 8 & 7.156 & \\
16 & 10 & 9 & 8.445 & \\
\hline
\end{tabular}

Table 3. Number of CM words transferred/usec.

An unanticipated degradation, more serious than the aforementioned, is something of a converse effect--that of ECS degrading disk performance. In this case, a PP request to access a word from CM is held up until an ECS word is transferred, and possibly longer if the PP request is for a word in one of the banks being serviced by the ECS request. A series of such delays caused by moving a string of words to or from a PP is sufficient to cause the disk to take at least one extra revolution to perform a task. A task can normally be performed in one or two disk revolutions. Software experiments show that it is virtually impossible to overlap ECS and disk activity without causing disk delay. Table 4 contains results of an experiment that demonstrates the capability of the 6600 system to overlap disk activity with 1) CP activity and 2) ECS activity. Times for the isolated tasks and the combined tasks are given.

\begin{tabular}{lcc} 
Activity & Time (sec.) & Range (sec.) \\
\hline Disk only & 4.2 & $3.9-4.6$ \\
CP only & 4.5 & \\
ECS only & 3.7 & \\
Disk \& ECS & 7.6 & $6.4-8.6$ \\
Disk \& CP & 5.0 & N.A. \\
\hline
\end{tabular}

Table 4. Multiple activity data. 
The latter of the two effects described above has a very serious consequence on some of our large production codes that are disk limited. Previous$1 y$, for such problems one had hoped to be able to absorb ECS activity in this I/O limited portion of the code. Unfortunately, there seems to be no simple cure to this problem.

\subsubsection{ECS effectiveness. It is convenient to think of ECS as per-} forming two functions: 1) replacing certain disk functions; 2) serving as an extension of CM. We make this distinction because existing I/O devices are not fast enough to serve as an extension of $\mathrm{CM}$ for a large class of problems.

By transferring certain critical disk functions to ECS, large amounts of computer time have been saved. These functions generally deal with files that are small enough to fit in ECS and cause the computer to be I/O limited when operated from a conventional I/O device. This has been the case in a number of codes that consume large mounts of computer time. Savings ranging from $25 \%$ to $50 \%$ have been characteristic of codes that run for long periods of time, such as two-dimensional neutronics and hydrodynamics codes.

For problems that are using ECS as an extension of CM, more realistically as an extension of CM for data, the cost in additional CP time typically ranges from $10 \%$ to $30 \%$ over that which would be required if the data were completely contained in CM. For some important problems, it was expected that this time would be overlapped with I/O activity. However, this did not occur because of the aforementioned problem of cverlapping ECS activity and disk activity. As a consequence these problems are seeing ECS use as additional overhead.

\subsection{Design implication.}

The ECS is most effective for relatively small well organized data files where the compute time per word of data is also small. This normally implies that the data can be completely contained in ECS and that the data is referenced in a well ordered fashion. This is characteristic of many higher dimensional scientific calculations. If the references to the data are more random in nature, then the access time for a single word is very large compared to such references from central memory. Consequently, the ECS does not provide a good capability for directly addressing single word data items.

To date we have placed very little emphasis on utilizing this device to support software systems. This is in large measure due to competition with applications programs for core, and in some measure due to the scope of programming effort necessary to obtain a system that would take full advantage of the ECS. Other installations, e.g., Brookhaven, are using ECS much more heavily to support software systems.

The Large Core Memory (LCM) of the CDC-7600 is similar in many respects to the ECS with development occurring on a time scale that is not too different from that of the ECS development. Yet one finds therein many important improvements, primarily because the LCM functions have been integrated into the complete hardware design. First, a PP request from central memory is honored sooner during LCM transfers by allowing service at the end of a transfer of a single CM word to or from LCM with the LCM being held up until this service is complete. This kind of priority should avoid the problem of missed disk revolutions that exists on the 6600 with ECS. Second, the delay for accessing the first word of a transfer is relatively shorter, thereby improving the capability for randomly addressing the LCM. This capability is further enhanced by a vocabulary that effects transfers from the LCM to the arithmetic registers of the CP thereby avoiding CM traffic. A basic hardware feature essential to the implementation of the aforementioned features is a buffer of one LCM word 
( 8 CM words) for each of the eight banks. Also, this feature eliminates LCM memory references when additional words referenced are already in the buffer.

\section{THE COMPUTER STORAGE HIERARCHY: THE FUTURE}

It is expected that several changes will occur in the storage hierarchies used by computers in the early 1970's. There will be a wider use of the highspeed buffer to mediate the speed requirements of the CPU with the larger capacity of the main memory. Semiconductor technology will be used for this buffer, with capacities on the order of $10^{5}$ bits and cycle times less than 100 nanoseconds. Although planar film and plated wire technologies are being pursued, magnetic cores will continue to be the dominant storage technology for both main memory and LCM well into the 1970's.

The design parameters for moving-medium storage are importantly influenced by recording density; this includes capacity, access time to a given capacity, transmission rate, and cost per bit. Recording density for magnetic media has been increasing by about an order of magnitude every five years, as indicated in Fig. 8, and this trend is expected to continue; the above mentioned par-

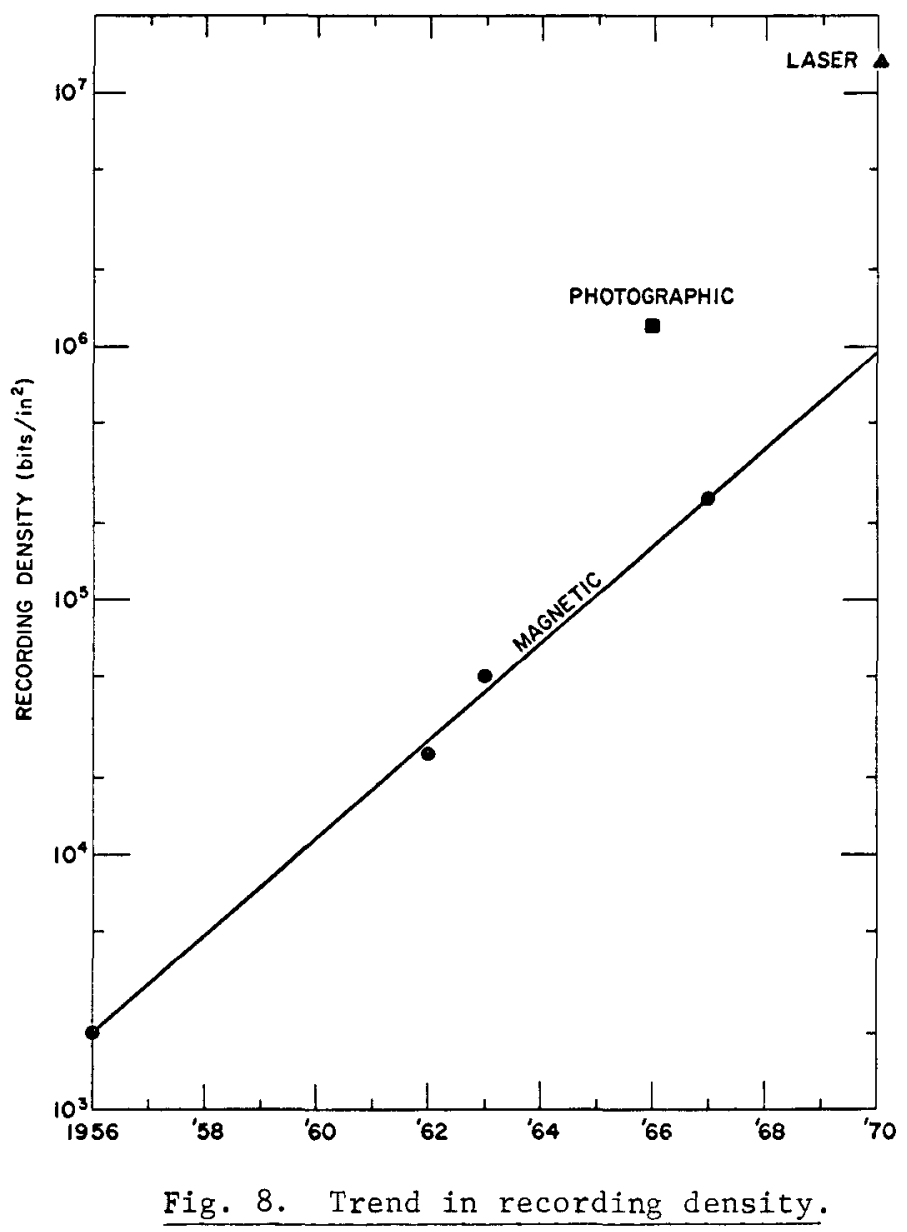

amaters will be favorably influenced thereby. Higher recording density allows more capacity to be included in a disk pack, and removable disk packs will replace more and more functions which have been previously performed by magnetic tapes. The future of magnetic strip devices is dubious. When they were first developed, they filled a need at the high end of the capacity scale which was 
not met by disks. However, high-density disks now offer as much or more capacity as magnetic strips, disk access time is significantly faster, and disks are more reliable, so strip devices may disappear from the hierarchy.

Although there is some expectation expressed in the literature that magnetic recording will offer capacities at the $10^{12}$ bit level in the mid-1970's, it is more likely that requirements for such large capacities will be met by photographic and laser techniques. Both photographic and laser technologies are capable of recording densities of about $10^{8}$ bits per square inch, and devices using these technologies should offer capacities beyond 1012 bits in the 1970 's.

In conclusion, multilevel storage will continue in the forseeable future to pose important and difficult problems which must be dealt with if the user is to achieve the potential of the computer for solving the increasingly complex problems of scientific research. 


\section{REFERENCES}

1. Goldstine, H. H., and Goldstine, A., "The Electronic Numerical Integrator and Computer (ENIAC)," Math Tables and Other Aids to Computation, 2(15), 79-110 (July 1946).

2. Gluck, S. E., "The Electronic Discrete Variable Computer," Electrical Engineering, 72, 159-162 (Feb. 1953).

3. Greenwald, S., et al., "SEAC," Proc. IRE, 41(10), 1300-1313 (Oct. 1953).

4. Hobbs, L. C., "Review and Survey of Mass Memories," 1963 Proc. FJCC, 295-310.

5. Bloom, L., et al., "Card Random Access Memory (CRAM): Functions and Use," 1961 Proc. SJCC, 147-157.

6. Shugart, A. F., and Yang-Hu Tong, "IBM 2321 Data Cell Drive," 1966 Proc. SJCC, $335-345$.

7. Kuehler, J. D., and Kerby, H. R., "A Photo-Digital Mass Storage System," 1966 Proc. FJCC, 735-742.

8. Mc Farland, K., and Hashiguchi, M., "Laser Recording Unit for High Density Permanent Digital Data Storage," 1968 Proc. FJCC, 1369-1380.

9. Jallen, G. A., "Extended Core Storage for the Control Data 64-6600 Systems," 1967 Proc. SJCC, 729-734.

10. Liptay, J. S., "Structural Aspects of the System/360 Mode1 85: The Cache," IBM Systems Journal, 7(1), 15-21 (1968).

11. Wilkes, M. V., "Slave Memories and Dynamic Storage Allocation," IEEE Trans. Elec. Comp., 14(2), 270-271 (April 1965).

12. Kilburn, T., et al., "One Level Storage System," IRE Trans. Elec. Comp., 11(2), 223-235 (April 1962).

13. Burns, L. L., "Cryoelectric Memories," Proc. IEEE, 52(10), 1164-1176 (Oct. 1964).

14. Brown, D. W., and Burkhardt, J. L., "The Computer Memory Market," Computers and Automation, 18(1), 17-26 (Jan. 1969). 


\section{BIBLIOGRAPHY}

1. Anacker, W., and Chu Ping Wang, "Performance Evaluation of Computing Systems with Memory Hierarchies," IEEE Trans. Elec. Comp., 16(6), 764773 (Dec. 1967).

2. Opler, A., "Dynamic Flow of Programs and Data Through Hierarchical Storage," 1965 Proc. IFIP Congress, 273-276.

3. Scarrott, G. G., "The Efficient Use of Multilevel Storage," 1965 Proc. IFIP Congress, $137-141$.

4. Fuchel, K., and Heller, S., "Considerations in the Design of a Multicomputer System with Extended Core Storage," Comm. ACM, 11(5), 334-340 (May 1968).

5. Humphrey, T. A., "Large Core Storage Utilization in Theory and Practice," 1967 Proc. SJCC, 719

6. Hoagland, A. A., "Mass Storage Revisited, 1967 Proc. FJCC, 255-260.

7. Risko, F. D., "New Horizons for Magnetic Bulk Storage Devices," 1968 Proc. FJCC, $1361-1367$.

8. Hobbs, L. C., "Present and Future State-of-the-Art in Computer Memories," IEEE Trans. Elec. Comp., 15(4), 534-550 (Aug. 1966).

9. Bonn, T. H., "Mass Storage: A Broad Review," Proc. IEEE, 54 (12), 1861-1870 (Dec. 1966).

10. Craver, J. S., "A Review of Electromechanical Mass Storage," Datamation, $12(7), 22-28$ (July 1966).

11. Hodges, D. A., "Large-Capacity Semiconductor Memory," Proc. IEEE, 56(7), 1148-1162 (July 1968).

12. Sander, W. B., "Semiconductor Memory Circuits and Technology," 1968 Proc. FJCC, 1205-1211.

13. Nisenoff, N., "Hardware for Information Processing Systems: Today and in the Future," Proc. IEEE, 54(12), 1820-1835 (Dec. 1966).

14. Rajchman, J. A., "Integrated Computer Memories," Scientific American, 217(1), 18-31 (July 1967). 
COMPUTER INDEPENDENCE OF LARGE REACTOR PHYSICS CODES WITH REFERENCE

TO WELL BALANCED COMPUTER CONFIGURATIONS

\author{
by \\ Reimar Froehlich \\ March 3, 1969 \\ Gulf General Atomic Incorporated \\ P. 0. Box 608, San Diego, California 92112
}

\title{
I. INTRODUCTION
}

The use of a universal programming language, e.g. FORTRAN-4, is an important and generally accepted requirement for computer independence of codes. For small core contained codes, which do not utilize specific features of a specific compiler, computer independence is almost guaranteed by writing the code in a universal programming language. For large codes, which make extensive use of scratch data handling to and from secondary storage, a universal programming language might guarantee a computer independent code but due to delays in data handling, not necessarily a very efficient one. As a matter of fact, a severe imbalance between central processor speed and data transfer capabilities of a computer configuration can make the solution of certain large reactor physics problems unfeasible.

The storage requirements of reactor physics and engineering codes frequently exceed the capacity of the main core storage and it is necessary to address further levels of storage without a large time delay caused by long access times or by unsatisfactory transmission rates. The most crucial requirements occur when very little computing is done between accesses to auxiliary storage devices. Multi dimensional diffusion or transport theory calculations are very demanding in this respect.

New developments in computer storage technology have provided equipment and systems which make delays in data transfer within the storage hierarchy almost unnecessary. These developments include extended core storage (also called bulk storage), fast rotating drums and disks with relatively small access times and high transmission rates, high density magnetic tapes, and multi channel data transfer parallel to computing.

In spite of these developments, the data flow within the multilevel storage hierarchy must be programmed by the user for most of the present day computers. This is so because different addressing techniques are used, and because the access times and transmission rates are different for various storage devices, thereby requiring single channel or multichannel data transfer and also specific data blocking and buffering techniques. For large multi dimensional diffusion and transport theory codes, which are in routine production use throughout the 
world, very sophisticated programming techniques have been employed for opt1mizing the performance of these codes on specific computer configurations. For example, extensive assembler language programming efforts were undertaken to avoid the rotational delay for drums or disks in case of sequential data transfer. It is a fact that the so optimized large computer programs are strongly computer dependent; they require excessive reprogramming efforts when another computer type is used or even when hardware modifications are made for the original computer.

Efficient automatic handling of a multilevel storage system was pioneered for the ATLAS computer ${ }^{1}$ and was later adapted to other computers, ${ }^{2}$ especially in connection with multiprogramming systems. In this concept the user addresses his information on a single level and he does not need to know that multi level storage devices are actually involved. In spite of this breakthrough the costeffectiveness of all these single level storage concepts, especially for sequentially oriented data transfer problems, seems to be unclear.

The purpose of the present paper is to analyze the data handling problem from a general point of view by considering its relation to well balanced computer configurations. A simple but typical data transfer model is discussed, and based on this analysis it will be shown how new procedures together with some old ideas can be useful in solving the data handling problem for an important class of reactor physics codes. In this sense it is a contribution to the development of computer independent large reactor physics codes.

The material is presented in the following order: In Section II the concept of a well balanced computer configuration with respect to a specific computer code is discussed. In Section III conventional ways of achieving a well balanced computer configuration are analyzed. Storage device and computer characteristics are presented for clarification of the data transfer problem for present day computers. In Section IV the efficient use of a multilevel storage hierarchy is discussed. In addition an investigation is made to see if an automatic handling procedure for multilevel storage systems can overcome the present computer dependence of large reactor physirs rodes. In Section $\mathrm{V}$ the data handling problem for multi dimensional diffusion theory and transport tieory calculations is investigated. It is shown how new numerical techniques and several programming tricks can be employed to solve the data handling problem for most problems of practical interest. In Section VI the efficiency of rebalancing methods is evaluated in detail for a difficult TRIGA reactor problem and a typical HTGR power reactor problem in two dimensions. The application of rebalancing methods in other diffusion theory and transport theory codes is also mentioned. Finally, in Section VII the major results are summarized.

\section{WELL-BALANCED COMPUTER CONFIGURATIONS}

The specification of a well-balanced computer configuration is a very difficult task even for a well defined class of mathematical problems. One reason is that the programming strategies and numerical methods are not necessarily known, and if known, are still subject to development and change. In this paper, a simplified but, nevertheless, typical situation is considered.

It is assumed, by generalizing earlier work, ${ }^{3}$ a so-called "three ring circus" is used via a number of data channels. That is, three storage buffers in main memory are used cyclically for reading from secondary storage, computing, and writing into secondary storage. Furthermore, it is assumed that there is no (severe) limitation for the physical block size in data transfer, and that reading and writing of data from and into secondary storage can be done in parallel 
via a specified number of channels and also in parallel to computing. A specific computer program is considered and the following notations are used.

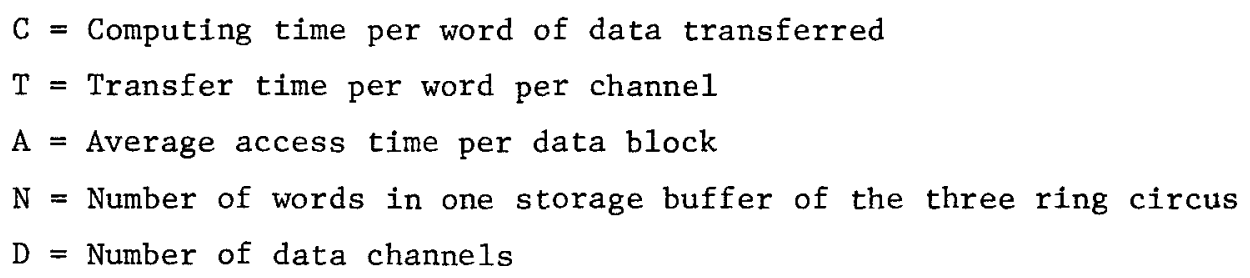

The notation implies that there is only one type of secondary storage device available. If it is further assumed that access times to different storage devices via different data channels overlap and that access is gained parallel to computing, then the following formula holds for a well-balanced computer configuration.

$$
\mathrm{A}+\frac{\mathrm{N}}{\mathrm{D}} * \mathrm{~T}=\mathrm{N} * \mathrm{C}
$$

The left side in Equation 1 is greater than the right side whenever data handling causes delay. For computing bounded problems the right side is greater than the left side. Equation 1 can also be written in the following form

$$
N=\frac{A}{C-\frac{T}{D}},
$$

and the denominator must be positive.

This model is not very sophisticated but the principle of this concept is representative for many large reactor physics codes. A detailed discussion of Eq. 2 will be presented in the next section.

\section{CONVENTIONAL WAYS OF ACHIEVING A WELL-BALANCED COMPUTER CONFIGURATION}

Before a detailed discussion of Eq. 2 is attempted, a familiarization with typical access times and transfer rates for storage devices, and with timing characteristics for arithmetical operations is necessary.

Table 1 summarizes the approximate access times and data transfer times for the most important storage devices. The high speed buffers of present day computers are usually not addressable by the user and are presently economical only in small sizes ( $<256$ words). The transmission rate for drum storage is comparable to the transmission rate of magnetic bulk storage, but the access time for drum storage is greater by a factor of 1000. Also, main magnetic core storage is very expensive 3,4 and is used only in medium capacities $\left(5 * 10^{4}-\right.$ $5 * 10^{5}$ words).

Table 2 presents some typical execution times for floating point operations on present day computers. Manufacturers already offer computers (e.g. CDC 7600 , IBM 360/92) which are faster by more than a factor of 3 compared to the CDC 6600 . 


\begin{tabular}{|l|c|c|}
\hline $\begin{array}{c}\text { Storage } \\
\text { System }\end{array}$ & $\begin{array}{c}\text { Approximate } \\
\text { Access Time }\end{array}$ & $\begin{array}{c}\text { Approximate Data } \\
\text { Transfer Time } \\
\text { Per Word }\end{array}$ \\
\hline $\begin{array}{l}\text { High Speed Buffer } \\
\text { Main Magnetic Core } \\
\text { Bulk Magnetic Core }\end{array}$ & $0.1-1.0 \mu \mathrm{sec}$ & $<.1 \mu \mathrm{sec}$ \\
$\begin{array}{l}\text { Magnetic Drum } \\
\text { Magnetic Disk }\end{array}$ & $\begin{array}{c}<-1.0-10.0 \mu \mathrm{sec} \\
\text { Mass Storage Disks } \\
\text { or Drums }\end{array}$ & $1.0-10.0 \mu \mathrm{sec}$ \\
& $5-100 \mathrm{msec}$ & $4 \mathrm{sec}$ \\
Magnetic Tapes & $5-200 \mathrm{msec}$ & $40 \mu \mathrm{sec}$ \\
\hline
\end{tabular}

Table 1. Storage System Characteristics

\begin{tabular}{|l|c|c|c|}
\hline \multirow{2}{*}{\multicolumn{1}{|c|}{}} & \multicolumn{3}{|c|}{ Execution Times in $\mu s e c$} \\
\cline { 2 - 4 } Operation & UNIVAC 1108 & CDC 6600 & IBM 360/75 \\
(36 bit word $)$ & $(60$ bit word $)$ & $(32$ bit word $)$ \\
\hline Addition/Subtraction & 1.75 & .4 & .85 \\
Multiplication & 2.625 & 1.0 & 2.1 \\
Division & 8.25 & 2.9 & 3.9 \\
\hline
\end{tabular}

Table 2. Execution Times for Floating Point Operations

In order to get a feeling for numbers with respect to a we11-balanced computer configuration an example is given which is not completely realistic due to several simplifications.

EXAMPLE. It is assumed that a CDC 6600 is used with auxiliary disk storage $(A=30,000 \mathrm{usec}, \mathrm{T}=25 \mathrm{\mu sec})$. Also it is assumed that two additions and two multiplications must be performed per word of data transferred. With an overhead factor of 3 it can easily be seen that $C=8.4 \mathrm{\mu sec}$. For a we11-balanced computer configuration one requirement is

$$
C-\frac{T}{D}=8.4-\frac{25}{D}>0
$$

that is, the number of data channels must be greater than two. By using Eq. 2 the following results are obtained:

$$
\begin{array}{llll}
\mathrm{D}=3 & \mathrm{D}=4 & \mathrm{D}=6 & \mathrm{D}=10 \\
\mathrm{~N}=450,000 & \mathrm{~N} \approx 13,950 & \mathrm{~N} \approx 7,080 & \mathrm{~N} \approx 5,080 .
\end{array}
$$


These results show that an excessive amount of buffer storage must be provided if the number of data channels is insufficient.

The example is representative for the situation encountered in many large reactor physics codes. The achievement of a well-balanced computer configuration requires an excessive amount of buffer storage in main memory. The main memory of present-day computers is restricted in size (mainly due to high cost!) and as a consequence $\mathrm{N}$, the number of words in one storage buffer of the three ring circus, is severely limited in size. If data handling causes delay for a specific code (characterized by $C$ ) the question is: What can be done to reduce the right side of $\mathrm{Eq} .2$ to a reasonable magnitude?

1. Decrease the access time A.

This can be done by use of bulk storage, drums instead of disks, faster rotating drums, etc. For sequential data transfer the access time of magnetic tapes is relatively short, but unfortunately the transmission rate is considerably smaller than for most other devices. It should be mentioned that some software packages have a severe limitation for the physical data block size allowed; in this situation, tapes can indeed be advantageous compared to slow rotating disks. For sequential data transfer another sophisticated method has been used with drum storage. 5 The data blocks are adequately located on the drum so that rotational delay times are minimal.

2. Decrease the transfer time $T$.

This can be done by faster rotating drums and disks, higher density of information on magnetic tapes, optimum arrangement of the word-bits on drums or disks with respect to read and write heads, etc.

3. Increase the number of data channels $D$.

The increase of the number of data channels (as the decrease of T) cannot always accomplish a well balanced computer configuration (it can be done if and only if $\mathrm{N}<\mathrm{A} / \mathrm{C}$ ).

For present-day computers the efficient use of either of these three possibilities involves an extensive programming effort or, even worse, makes the code strongly computer and hardware dependent. Frequently, the adjustment of a computer code to one computer configuration by sophisticated assembler programming techniques for data transfer optimization requires many man-months of highly trained personnel. Unfortunately, such an effort does not solve the data transfer problem forever; on the contrary, it is the hour of birth for more difficulties in the future. Whenever the hardware of the utilized computer is modified or the type of computer is changed (which happens too frequently!) extensive new optimization or conversion efforts are required. A cost benefit analysis would be desirable before this type of work is initiated. Important input parameters are: How often will the computer configuration be changed? How much computing cost does the optimization save per month? What is the cost of the optimization and of later conversions? Does one need the highly trained personnel for more important tasks?

The ideal solution for the data transfer problem (or more general, for a wel1-balanced computer configuration), would be one which takes the burden away from the computer user and is, at the same time, open for technical advancement, i.e., increased computer speed, shorter access time and increased transmission rates for storage devices. It is obvious that a satisfactory solution of this very complex and difficult problem is not readily at hand. 


\section{THE EFFICIENT USE OF A MULTILEVEL STORAGE HIERARCHY}

Multilevel stcrage systems have the advantage of greater capacity and/or greater flexibility. Lnfortunately, their efficient use has the disadvantage of an excessive programming burden for the user on present day computers.

An automatic way of handling a multilevel storage system was pioneered for the ATLAS computer.1,6 In later publications $2,7,8$ the method was slightly modified, but the basic concept stayed essentially the same. A strict distinction is made between memory units, called "pages" (core pages, drum pages, disk pages, etc.), and corresponding information units, called "blocks". A block just fits in a page. Each block contains an identification number and the number of possible identifiers is much larger than the total number of pages at al1 storage levels. The block identifier gives fast access to a so-called "block variable" in core storage whose value denotes whether the block is still empty, and if not empty, in which page (or pages) it can be found.

This concept has several advantages: 1) The user addresses his information by block identifiers and a position number within the block. He addresses his information on a single level and he does not need to know that multilevel storage devices are actually involved. 2) If a block of information, residing, for example, in a main core page has to be dumped onto the drum in order to make the core page availit, f r cther use (e.g. one can select the core page at the

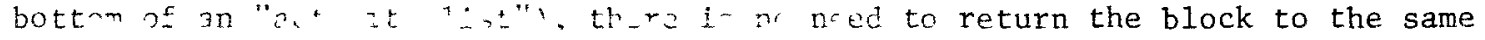
page from which ' with the minimum iatericy an be sulected. 3) codes and data can be relocated quite edsily.

An efficient autonatic way of handling a multilevel storage system takes the programming burden away from the user. He does not need to know which storage devices are involved or how many data channels are used. He simply addresses his information on a single level.

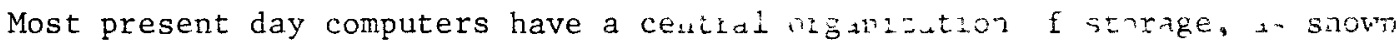
in Fig. 1. This organization appears to be most suitable for applications in which there is a one to one correspondence between a variety of data requirements and a variety of storage characteristics. The hierarchical organization of storage, shown in F1g, 2 , is the other obvious possibility. 7 Here, the different storage devices, ordered in an hierarchical multilevel structure (determined by access time, transfer rate, etc.), permit data transfer between hierarchical neighbors without going through main core storage. The data transfer via the interdevice data channel = is controlled (selection, initiation, addressing, error detection, etc.) by a contiol program resiling, in the main core storage, tut the data transfer itself is perforned indepridentiy. The hierarchical structure is conceptually better suited for an automatzo handling procedure of its multilevel structure, but very little experience (simulation) is presently available for this concept. The realızation of an efficient hierarchical storage system permitting a single level addressing concept would indeed require new hardware and software developments.

Automatic handling of centralized storage systems has been developed in connection with multiprograming systems development. These systems worked successfully on large computer configurations, such as the ATLAS. Recently, IBM developed a similar automatic handling concept for a two level storage system of the IBM $360 / 85$ computer. ${ }^{9}, 10$ This concept was intended as an effective way of providing large main storage capacity while retaining high internal performance. A detailed analysis of the efficiency was performed. 9,10 
FIG. I CENTRAL ORGANIZATION OF STORAGE

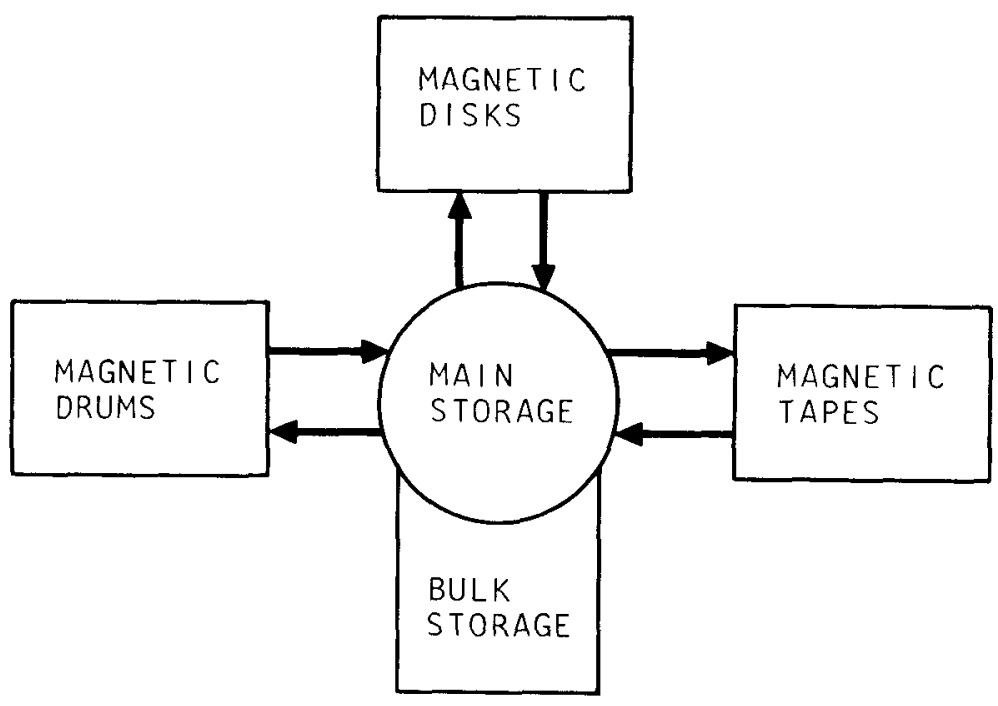

FIG. 2 HIERARCHICAL ORGANIZATION OF STORAGE

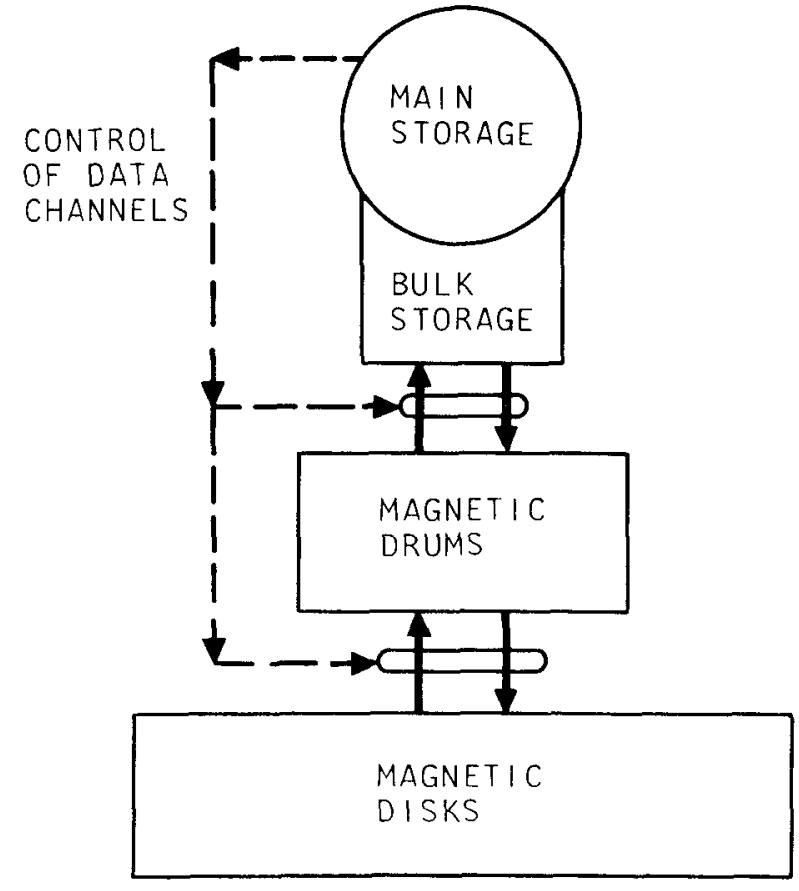


Large reactor physics codes usually require as much core storage as is available on present-day computers. Consequently, batch processing is presently preferred for these codes. Furthermore, the data transfer problem is almost always concerned with sequentially ordered data, which must be channeled through main core storage repeatedly. At present there is little experience available with an automatic multilevel storage handling system in connection with large reactor physics codes. The potential of such systems could be very beneficial, but it is not likely that they will be efficiently operational (if developed at all) within the near future.

\section{IMPROVED METHODS OF ACHIEVING A WELL-BALANCED COMPUTER CONFIGURATION}

As long as computers are not equipped with an efficient automatic handling procedure of their storage hierarchies, there seems to be only one paradoxical way out of the data transfer dilemma for large reactor physics codes: Increase of $\mathrm{C}$, the computing time per word of data transferred. This is indeed a method for increasing the right side in Eq. 2 if the number of words in one storage buffer of the three ring circus is severely limited. It should be remembered that $C$ is not only a computer hardware dependent value but it is also strongly dependent on the nature of the problem to be solved, on the programming strategy used, and on the numerical procedure chosen. On the other hand, an increase of $C$ should be exercised with extreme care; for example, a bad choice of a numerical procedure can lead to an inefficient computer program.

For two- and three-dimensional transport and diffusion theory codes the following procedures can be applied to increase $C$ :

1. Regionwise uniform material properties and meshspacings, and also symmetry properties can be used to reduce drastically the number of difference equation coefficients which must be transferred for each iteration.

2. Simultaneous performance of several fine mesh iterations (e.g. single line successive over-relaxation) as described by W. R. Cadwell.11 The idea is to transfer the necessary information (fluxes and difference equation coefficients) only once and to proceed with a set of $n$ ( 5 to 10 ) iterations concurrently in the following manner: line one is inverted for the first iteration, line two is then inverted for the first iteration, line one is then inverted for the second iteration, line three is then inverted for the first iteration followed by line two for the second iteration followed by line one for the third iteration, and so forth. Performing $n$ iterations concurrently in this manner has exactly the same effect as performing $n$ successive passes through the mesh grid. The advantage is that $C$ is increased by the factor $n$.

3. Coarse mesh variational methods ${ }^{12}, 13,14$ can be applied to accelerate fine mesh iterative procedures by periodically interrupting the latter. Then the variational coarse mesh equations are solved, the fluxes (and if applicable the eigenvalue) are updated, and the fine mesh iterations can continue. The coarse mesh variational methods are very powerful numerical techniques as will be demonstrated for the coarse mesh rebalancing technique in the next section. The coarse mesh variational equations (sometimes called "contracted equations") can be solved by a main memory contained subprogram, thereby avoiding data transfer problems. The coarse mesh equation coefficients can be calculated during the last fine mesh iteration before the interruption takes 
place. All this contributes to an increase of C. A combination of the coarse mesh variational scheme (interrupting every $n$ iteration) with the concurrent performance of $n$ iterations described under 2 looks very attractive. If every fine mesh iteration is followed by a solution of the coarse mesh equations the increase of $\mathrm{C}$ appears to be most consistent.

All these procedures for increasing $C$ and/or the efficiency of the numerical solution method have the following advantages: a) The programming effort can be accomplished once and for all; specifically, no new extensive effort is necessary for converting the code to another computer configuration. b) The procedures are obviously not computer hardware dependent. c) The concepts permit technical advancement (increase of computing speed and/or decrease of access times, etc.) without any difficulty.

\section{EVALUATION OF THE COARSE MESH REBALANCING METHOD FOR REALISTIC REACTOR PROBLEMS}

Coarse mesh variational techniques were discussed by E. L. Wachspress ${ }^{14}$ from a general standpoint and a theoretical foundation for coarse mesh variational techniques was presented by the author ${ }^{12}$ at a similar meeting in Mexico City two years ago. The so-called coarse mesh rebalancing techniques, a special form of the coarse mesh variational methods, have gained widespread usage in two dimensional diffusion theory and transport theory codes.15,16,17 The following numerical evaluation of coarse mesh rebalancing techniques was performed with several of Gulf General Atomic's large diffusion theory production codes.15,18 Interesting results for realistic reactor problems were obtained.

It seems appropriate to start with a summary of the ideas and equations for coarse mesh rebalancing techniques, because this will be helpful for an accurate description of the numerical evaluation.

The discrete form of the steady state multigroup diffusion equations can be written in the following matrix form,

$$
\mathrm{A} \varphi=\frac{1}{\lambda} \mathrm{B} \varphi \text {. }
$$

The order $I$ of the square matrices $A$ and $B$ and the flux vector $\varphi$ is equal to the total number of (fine) energy-space meshpoints. The matrix A includes the diffusion, removal, and scattering processes, while the matrix $B$ represents the neutron fission processes. The $\lambda$ in Eq. 4 is the eigenvalue (k-effective) of the problem.

The I energy-space meshpoints are partitioned in $\mathrm{R}$ disjunctive subsets (coarse meshes) $s_{r}, r=1,2, \ldots, R$. For the GAMBLE- 5 code ${ }^{15}$ the $s_{r}$ are threedimensional parallelepipeds in the space-energy coordinate system. This subdivision is obtained by specifying coarse mesh intervals along each coordinate axis $x, y$ (or $r, z$ ) and for the energy group coordinate. If $\varphi_{a}$ is an approximate solution of Eq. 4 and the components of the column vectors $\varphi_{r}$ and $\delta_{r}$ are defined as

$$
\begin{aligned}
& \varphi_{r}^{(i)}=\varphi_{a}^{(i)}, \delta_{r}^{(i)}=1 \text { for } i \text { an element of } S_{r} \\
& \varphi_{r}^{(i)}=\delta_{r}^{(i)}=0 \text { for all other } i,
\end{aligned}
$$


then the coarse mesh rebalancing equations can be written as

$$
\mathrm{Gd}=\frac{1}{\lambda} \mathrm{Hd}, \mathrm{G}=\left(\delta_{\mathrm{r}}^{*} \mathrm{~A} \varphi_{\mathrm{S}}\right), \mathrm{H}=\left(\delta_{\mathrm{r}}^{*} \mathrm{~B} \varphi_{\mathrm{s}}\right)
$$
The row vector $\delta_{r}^{*}$ is the transposed column vector $\delta_{r}$, and the column vector d is
of order R. The rebalanced flux is

$$
\varphi=\sum_{r=1}^{R} \mathrm{~d}^{(\mathrm{r})} \varphi_{\mathrm{r}}
$$

It was proved ${ }^{12}$ that Eq. 6 has a largest positive eigenvalue $\lambda$ and a corresponding positive eigenvector d whenever $\varphi_{\mathrm{a}}$ is positive.

In the following numerical evaluation of the coarse mesh rebalancing techniques two examples, a TRIGA reactor and an HTGR power reactor, are considered.

\section{TRIGA Reactor with a Centra1 Hole}

A two dimensional $(r, z)$ geometry TRIGA reactor mockup with a central hole is shown in Fig. 3. The central hole, an irradiation region, is surrounded by a stainless steel tube with iron plugs at the top and the bottom. The core (region 2) is imbedded in water and at the top and bottom of the core there are so-called grey regions. The material content of each region is indicated.

The criticality and flux calculations are performed for a 5 energy group model and the macroscopic cross sections (at a temperature of $23^{\circ} \mathrm{C}$ ) are displayed for each region in Table 3 . The following fission spectrum was utilized:

$$
x_{1}=0.86866, x_{2}=0.13134, x_{3}=x_{4}=x_{5}=0
$$

The mesh grid (61* 43 meshpoints) is also indicated in Fig. 3. The mesh size is assumed to be constant between the meshpoints designated with numbers in Fig. 3 .

The central hole makes this problem a very difficult one for most diffusion theory codes which are based on iterative techniques. Four different methods were used to contribute to a solution of this problem:

1. An alternating direction implicit (ADI) iteration method as utilized in GAMBLE-1, a two dimensional multigroup diffusion theory code. 18

2. The GAMBLE-5 method without rebalancing. GAMBLE-5 is a multigroup diffusion theory code based on a successive over-relaxation method.15

3. The GAMBLE-5 method with column/row rebalancing. 15 The fine mesh successive over-relaxation is interrupted every ten iterations and column or row rebalancings are performed alternatively. Each $S_{r}$ for a column rebalancing consists of all the meshpoints in a specific column and group; $R$ is equal to the number of columns times the number of groups. For row rebalancing the situation is similar.

4. The GAMBLE-5 method with coarse mesh rebalancing. 15 The fine mesh successive over-relaxation is interrupted every ten iterations and coarse mesh rebalancings for two different coarse meshes are performed alternatively. For the first coarse mesh each $S_{r}$ consists of column pieces (row numbers 
FIG. 3 TWO DIMENSIONAL R-Z GEOMETRY TRIGA REACTOR MOCKUP WITH CENTRAL HOLE

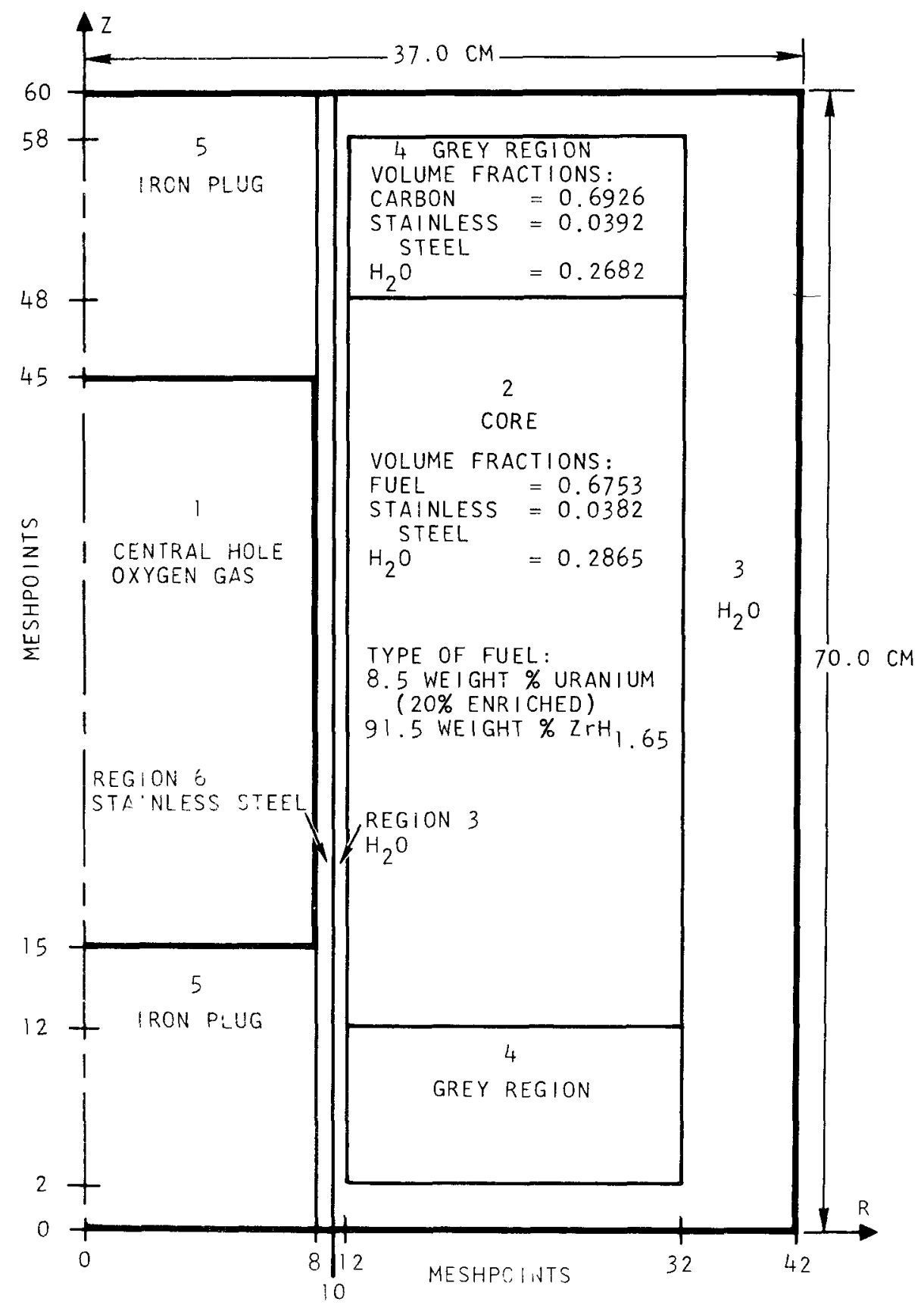


TABLE 3: MACROSCOPIC CROSS SECTIONS FOR A TRIGA REACTOR WITH CENTRAL HOLE

\begin{tabular}{|c|c|c|c|c|c|c|c|c|c|c|}
\hline \multirow{2}{*}{$\begin{array}{c}\text { GROUP } \\
G \\
\end{array}$} & \multirow{2}{*}{$\begin{array}{l}\text { REGION } \\
-R\end{array}$} & \multirow{2}{*}{$\begin{array}{l}\text { DIFFUSION } \\
\text { CONSTANT }\end{array}$} & \multirow{2}{*}{$\begin{array}{c}\text { FISSION } \\
\text { CROSS SECTION } \\
\end{array}$} & \multirow{2}{*}{$\begin{array}{c}\text { REMOVAL } \\
\text { CROSS SECTION } \\
\end{array}$} & \multirow{2}{*}{$\begin{array}{r}\text { PRODUCTION } \\
\text { CROSS SECTION } \\
\end{array}$} & \multicolumn{5}{|c|}{ SCATTERING IMTO GROUP C FROM GROUP: } \\
\hline & & & & & & 1 & 2 & 3 & 4 & 5 \\
\hline \multirow[t]{6}{*}{1} & 1 & $3.204+03$ & 0.0 & $6.186-06$ & 0.0 & 0.0 & 0.0 & 0.0 & 0.0 & 0.0 \\
\hline & 2 & $1.495+00$ & $3.795-04$ & $1.029-01$ & $1.037-03$ & 0.0 & 0.0 & 0.0 & 0.0 & 0.0 \\
\hline & 3 & $1.970+00$ & 0.0 & $1.023-01$ & 0.0 & 0.0 & 0.0 & 0.0 & 0.0 & 0.0 \\
\hline & 4 & $1.982+00$ & 0.0 & $4.415-02$ & 0.0 & 0.0 & 0.0 & 0.0 & 0.0 & 0.0 \\
\hline & 5 & $1.800+00$ & 0.0 & $1.266-02$ & 0.0 & 0.0 & 0.0 & 0.0 & 0.0 & 0.0 \\
\hline & 6 & $1.754+00$ & 0.0 & $1.303-02$ & 0.0 & 0.0 & 0.0 & 0.0 & 0.0 & 0.0 \\
\hline \multirow[t]{6}{*}{2} & 1 & $1.477+03$ & 0.0 & $3.635-06$ & 0.0 & $5.52-06$ & 0.0 & 0.0 & 0.0 & 0.0 \\
\hline & 2 & $7.758-01$ & $2.959-04$ & $1.696-01$ & $7.225-04$ & $1.00-01$ & 0.0 & 0.0 & 0.0 & 0.0 \\
\hline & 3 & $9.909-01$ & 0.0 & $2.016-01$ & 0.0 & $1.00-01$ & 0.0 & 0.0 & 0.0 & 0.0 \\
\hline & 4 & $9.614-01$ & 0.0 & $6.288-02$ & 0.0 & $4.35-02$ & 0.0 & 0.0 & 0.0 & 0.0 \\
\hline & 5 & $8.177-01$ & 0.0 & $4.134-03$ & 0.0 & $1.23-02$ & 0.0 & 0.0 & 0.0 & 0.0 \\
\hline & 6 & $7.788-01$ & 0.0 & $5.046-03$ & 0.0 & $1.20-02$ & 0.0 & 0.0 & 0.0 & 0.0 \\
\hline \multirow[t]{6}{*}{3} & 1 & $1.745+03$ & 0.0 & $2.551-06$ & 0.0 & $1.62-11$ & $3.64-06$ & 0.0 & 0.0 & 0.0 \\
\hline & 2 & $5.316-01$ & $3.398-03$ & $1.348-01$ & $8.258-03$ & $1.73-03$ & $1.69-01$ & 0.0 & 0.0 & 0.0 \\
\hline & 3 & $6.114-01$ & 0.0 & $1.517-01$ & 0.0 & $1.80-03$ & $2.02-01$ & 0.0 & 0.0 & 0.0 \\
\hline & 4 & $7.429-01$ & 0.0 & $4.580-02$ & 0.0 & $5.66-04$ & $6.28-02$ & 0.0 & 0.0 & 0.0 \\
\hline & 5 & $3.919-01$ & 0.0 & $1.094-02$ & 0.0 & $5.70-05$ & $3.50-03$ & 0.0 & 0.0 & 0.0 \\
\hline & 6 & $3.681-01$ & 0.0 & $1.339-02$ & 0.0 & $4.01-05$ & $4.23-03$ & 0.0 & 0.0 & 0.0 \\
\hline \multirow[t]{6}{*}{4} & 1 & $1.754+03$ & 0.0 & $3.764-05$ & 0.0 & 0.0 & 0.0 & $2.55-06$ & 0.0 & $2.15-06$ \\
\hline & 2 & $3.245-01$ & $2.169-02$ & $4.656-01$ & $5.270-02$ & $1.85-07$ & $1.81-05$ & $1.04-01$ & 0.0 & $2.46-02$ \\
\hline & 3 & $3.424-01$ & 0.0 & $8.146-01$ & 0.0 & $2.00-07$ & $2.15-05$ & $1.32-01$ & 0.0 & $3.53-02$ \\
\hline & 4 & $5.998-01$ & 0.0 & $2.288-01$ & 0.0 & $6.09-08$ & $5.90-06$ & $4.02-02$ & 0.0 & 1. 39-02 \\
\hline & 5 & $3.368-01$ & 0.0 & $7.354-02$ & 0.0 & 0.0 & 0.0 & $3.65-03$ & 0.0 & 0.0 \\
\hline & 6 & $3.652-01$ & 0.0 & $8.637-02$ & 0.0 & 0.0 & 0.0 & $3.28-03$ & 0.0 & 0.0 \\
\hline \multirow[t]{6}{*}{5} & 1 & $1.723+03$ & 0.0 & $2.145-06$ & 0.0 & 0.0 & 0.0 & 0.0 & $3.76-05$ & 0.0 \\
\hline & 2 & $1.766-02$ & $6.586-02$ & $1.302-01$ & $1.600-01$ & $2.89-08$ & $2.57-06$ & $1.46-02$ & $4.29-01$ & 0.0 \\
\hline & 3 & $1.497-01$ & 0.0 & $5.486-02$ & 0.0 & $2.77-08$ & $3.10-06$ & $1.86-02$ & $8.07-01$ & 0.0 \\
\hline & 4 & $3.603-01$ & 0.0 & $2.863-02$ & 0.0 & $8.66-09$ & $8.00-07$ & $4.98-03$ & $2.24-01$ & 0.0 \\
\hline & 5 & $3.034-01$ & 0.0 & $2.021-01$ & 0.0 & 0.0 & 0.0 & 0.0 & 0.0 & 0.0 \\
\hline & 6 & $3.202-01$ & 0.0 & $2.374-01$ & 0.0 & 0.0 & 0.0 & 0.0 & 0.0 & 0.0 \\
\hline
\end{tabular}


0 - 14 or $15-45$ or $46-60$ ) in one group, and $R$ is equal to 3 * number of groups * number of columns. For the second coarse mesh each $\mathrm{S}_{\mathrm{r}}$ consists of row pieces (column numbers $0-8$ or $9-42$ ) in one group, and $R$ is equal to $2 *$ number of groups * number of rows.

The numerical results are displayed in Fig. 4 and in Table 4 . They need some detailed explanation:

Figure 4 shows that the alternating direction method and the GAMBLE-5 method without rebalancing have an unsatisfactory convergence behavior. After 150 iterations the discrepancy from the reference k-effective value is still more than one percent. On the other hand, both rebalancing methods show a very satisfactory convergence behavior. The coarse mesh rebalancing method is somewhat better than the column/row rebalancing method. The step changes of k-effective, which can be observed at multipla of ten iterations for both rebalancing methods, demonstrate the effect of rebalancing procedures in an impressive way. The step changes for k-effective in case of "no rebalancing" are caused by an asymptotic convergence acceleration procedure, which has been described in the GAMBLE-5 report. 15

Table 4 presents some selected results of the error in k-effective and the corresponding execution time in minutes. These results are very instructive: 100 iterations for the two different methods with rebalancing take 1.8 and 4.0 minutes longer than the iteration method without rebalancing ( 7.7 minutes), but the error in k-effective shows clearly the superiority of the rebalancing methods. GAMBLE-5 is a highly optimized code with respect to data transfer, whereas GAMBLE- 1 is not optimized and 100 iterations (50 ADI double iterations) in GAMBLE-1 take about 2.3 times longer than 100 GAMBLE-5 iterations. The reference case was checked very carefully to assure a correct result (e.g., the residuals were shown to be extremely small throughout the mesh). The numerical results for this difficult TRIGA reactor problem demonstrate clearly that a small maximum relative flux change for successive iterations does not necessarily guarantee an accurate result. The averaged group-region fluxes obtained with the GAMBLE-5 method without rebalancing were completely inaccurate (in regions 1 and 4 by more than 200 percent!). The alternating direction method gave somewhat better averaged fluxes (with errors in regions 1 and 4 of somewhat less than 50 percent!).

It should be mentioned that the coarse mesh rebalancing method works extremely well for problems with highly nonuniform mesh spacings, for extremely heterogeneous assemblies, and also for cell problems with a very fine mesh grid.

\section{HTGR Power Reactor Problem}

The rebalancing method of the two dimensional multigroup diffusion code GAMBLE-5 is also evaluated for a large HTGR power reactor problem. A seven energy group model in two dimensional $(x, y)$ geometry with approximately 2,600 meshpoints is utilized and the standard column/row rebalancing method is applied. The results are summarized in Table 5 . The most interesting numbers for the reactor designer are the eigenvalue k-effective and the patch power (averaged power values for characteristic regions of an HTGR). The improvement due to the rebalancing method is remarkable. For other codes, which are less optimized with respect to data transfer, the gain would be even greater.

\section{$\underline{\text { Remarks }}$}

A rebalancing method has also been used with great success in the two dimensional few group diffusion theory depletion program GAUGE, 16 and computer time savings of more than 50 percent have been achieved.

The two dimensional transport theory code TWOTRAN ${ }^{17}$ was also equipped with a coarse mesh rebalancing technique, which resulted in remarkable convergence improvements. 
FIG. 4 CONVERGENCE BEHAVIOR OF VARIOUS METHODS FOR A TRIGA REACTOR WITH A CENTRAL HOLE

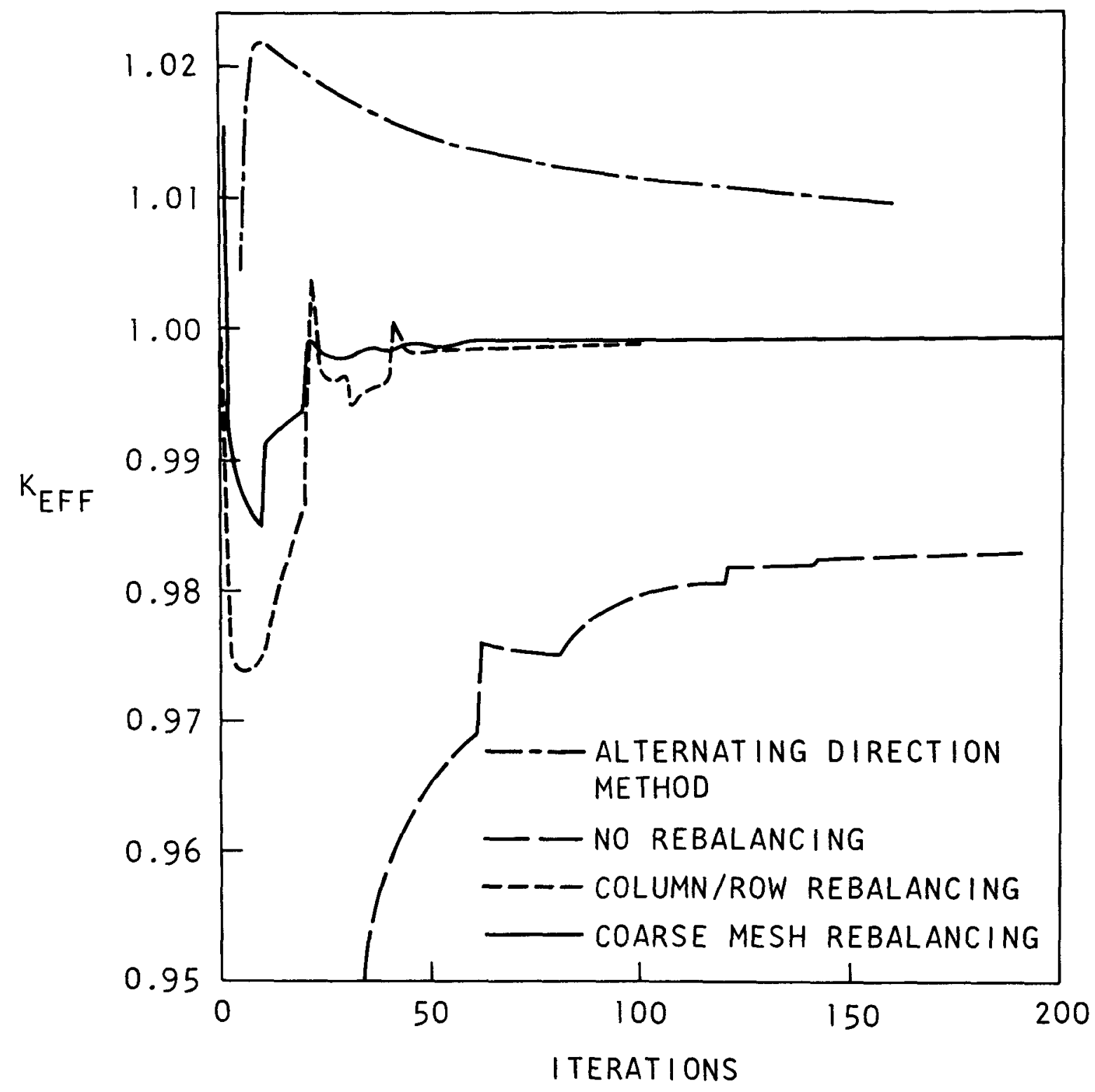


TABLE 4: EVALUATION OF REBALANCING FOR A TRIGA REACTOR WITH CENTRAL HOLE

\begin{tabular}{|c|c|c|c|c|c|c|}
\hline CODE & $\begin{array}{c}\text { TYPE } \\
\text { OF } \\
\text { REBALANC ING } \\
\end{array}$ & $\begin{array}{c}\text { NUMBER } \\
\text { OF } \\
\text { ITERATIONS } \\
\end{array}$ & $\begin{array}{c}\text { MAXIMUM } \\
\text { RELATIVE } \\
\text { FLUX CHANGE } \\
\end{array}$ & K-EFFECTIVE & $\begin{array}{c}\text { ERROR } \\
\text { IN } \\
\text { K-EFFECTIVE } \\
\end{array}$ & $\begin{array}{c}\text { EXECUTJON } \\
\text { TIME IN } \\
\text { MINUTES } \\
\text { (UNIVAC 1108) } \\
\end{array}$ \\
\hline GAMBLE- 5 & NONE & 100 & 0.00231 & 0.97941 & $-1.97 \%$ & 7.7 \\
\hline GAMBLE-5 & NONE & 191 & 0.00016 & 0.98274 & $-1.64 \%$ & 14.0 \\
\hline GAMBLE-5 & COLUMN / ROW & 100 & 0.00030 & 0.99900 & $-0.01 \%$ & 9.5 \\
\hline GAMBLE-5 & COARSE MESH & 100 & 0.00007 & 0.99902 & $-0.01 \%$ & 11.7 \\
\hline GAMBLE- 5 & COARSE MESH & 300 & 0.00000 & 0.99914 & $\begin{array}{c}\text { REFERENCE } \\
\text { CASE }\end{array}$ & 28.0 \\
\hline $\begin{array}{c}\text { GAMBLE-1 } \\
(\mathrm{ADI})\end{array}$ & NONE & 100 & 0.0007 & 1.01141 & $1.23 \%$ & 18.1 \\
\hline $\begin{array}{c}\text { GAMBLE-1 } \\
\text { (ADI) }\end{array}$ & NONE & 160 & 0.0004 & 1.00926 & $1.01 \%$ & 28.6 \\
\hline
\end{tabular}


TABLE 5: EFFICIENCY OF THE GAMBLE-5 REBALANCING METHOD FOR A TYPICAL HTGR (FORT ST. VRAIN) POWER REACTOR PROBLEM

\begin{tabular}{|c|c|c|c|c|c|}
\hline & REBALANCING & $\begin{array}{c}\text { MAXIMUM } \\
\text { RELATIVE } \\
\text { FLUX CHANGE }\end{array}$ & $\begin{array}{c}\text { E-EFFECTIVE } \\
\text { ERROR }\end{array}$ & $\begin{array}{c}\text { PATCH } \\
\text { POWER } \\
\text { ERROR }\end{array}$ & $\begin{array}{c}\text { TIME IN } \\
\text { MINUTES } \\
\text { (UNIVAC 1108) }\end{array}$ \\
\hline NO & 70 & 0.01 & $0.02 \%$ & $84 \%$ & 6.0 \\
NO & 140 & 0.003 & $0.0004 \%$ & $2.2 \%$ & 12.0 \\
YES & 70 & 0.005 & $0.0004 \%$ & $2.0 \%$ & 8.2 \\
YES & 140 & 0.0003 & $0.00001 \%$ & $0.06 \%$ & 14.7 \\
NO & 300 & 0.00003 & REFERENCE CASE & 26.6 \\
\hline
\end{tabular}


Presently, coarse mesh rebalancing methods are being incorporated in a three dimensional diffusion theory code for HTGR reactor applications.

VII . CONCLUSIONS

The use of a universal programming language, e.g. FORTRAN-4, is one important and generally accepted requirement for computer independence of large reactor physics codes. From a practical point of view, computer independence of a code should also imply that the same code runs efficiently on different computer types and configurations. For codes making extensive use of scratch data handling to and from secondary storage, a universal programming language might guarantee that the code runs on different computers, but very often these codes run inefficiently and the solution of practical problems is unfeasible. From a practical point of view, computer independence of a code should also imply that the same code reproduces accurate results on different computers. Different word lengths might jeopardize this requirement. The latter difficulty has not been discussed in this paper. Indeed, one could argue that a code can always be compiled so that double precision arithmetic is utilized. The computing time will usually not increase very much, but the increased storage requirements could be disastrous. Fortunately, the successful solution of the data transfer problem will also contribute to a solution of this storage problem. Therefore, all attention in this paper was focused on the data transfer problem.

The problem of computer independence (with efficiency) of large reactor physics codes is due to the data transfer problem being very closely related to the problem of a well balanced computer configuration. It was shown that the optimization of data transfer, by using expensive fast storage equipment or multichannel data transfer concepts and also by expending sophisticated programming efforts for minimizing access times etc., very often has serious disadvantages. In this manner a strongly computer dependent code is produced which will cause many difficulties in conversion to another computer or even to other hardware equipment.

In principle, an efficient automatic handling concept for a multilevel storage system appears to be possible. Some attractive ideas were described. Such a concept would take the programming burden away from the user and contribute to a computer independent solution of the data transfer problem. The user could address all of his information on a single level and would not need to know that a complicated multilevel storage system is involved. Unfortunately, this would involve a great software and hardware development effort and a useful solution cannot be expected in the near future. On the other hand, the potential benefit appears to warrant great efforts. Computer manufacturers and software development groups should be encouraged to proceed towards a solution.

Meanwhile, other approaches that would contribute to a solution of the data transfer problem should be vigorously pursued. New programming strategies and new numerical techniques should be developed to increase the number of arithmetical operations per word of data transferred. Careful consideration must be given to such methods, because they should not only increase the number of arithmetical operations per word of data transferred but should also improve the overall efficiency. Three successful methods for multidimensional diffusion and transport theory codes were discussed. Detailed numerical results were presented for one of the methods, the coarse mesh rebalancing technique. These results have demonstrated that coarse mesh rebalancing techniques contribute considerably to a solution of the data transfer problem and that they make the numerical solution of extremely difficult problems feasible. 
The computer independence of codes and the analysis of a well balanced computer configuration are two very difficult and complex problems. This paper should be considered as a contribution to a somewhat better understanding of the problems involved; it certainly does not attempt a rigorous solution, and many problems involved were simplified and require further clarification. If the author has succeeded in shedding some light on some prospective solution methods his goal has been reached.

\section{ACKNOWLEDGEMENTS}

The author is grateful to John Dorsey for discussions of programming analysis problems and to Gordon West for his comments and support during the numerical evaluation of coarse mesh rebalancing techniques for TRIGA reactor problems. 
1. T. Kilburn, et al., "One-Level Storage System," IRE Transactions of Electronic Computers, 11 (2), 223-235 (Apri1 1962).

2. E. W. Dijkstra, "The Structure of the "THE" - Multiprogramming System," Commun. ACM (Ass. Comput. Mach.), 11 (5), 341-346 (May 1968).

3. W. J. Worlton, "Computers and the Nuclear Industry, A Look Into the Future," Nucl. News, 11 (4), 42-45 (April 1968).

4. W. J. Worlton and E. A. Voorhees, "Recent Developments in Computers and Their Implications for Reactor Calculations," Proceedings of the Conference on the Application of Computing Methods to Reactor Problems, ANL-7050, 15-32, (1965).

5. J. P. Dorsey, personal communication (August 1968).

6. W. J. Worlton, "The Use of Auxiliary Storage in Nuclear Codes," Trans. Am. Nucl. Soc., 9 (1), 200-201 (June 1966).

7. Ascher Opler, "Dynamic Flow of Programs and Data Through Hierarchica1 Storage," Proceedings of IFIP Congress 65, 1, 273-276 (May 1965).

8. G. G. Scarrott, "The Efficient Use of Multilevel Storage," Proceedings of IFIP Congress 65, 1, 137-141 (May 1965).

9. C. J. Conti, D. H. Gibson and S. H. Pitkowsky, Structural Aspects of the System/360 Mode1 85, IBM TR 00.1688 (January 3, 1968).

10. J. S. Liptay, The Model 85 Buffer Storage, IBM TR 00.1688 (January 3, 1968).

11. W. R. Cadwe11, "The Input-Output Problem in Two-Dimensional Neutron Diffusion Programs with Large Meshes," Trans. Am. Nuc1. Soc., 7 (1), 23-24 (June 1964).

12. R. Froehlich, "A Theoretical Foundation for Coarse Mesh Variational Techniques," Proceedings of the International Conference on Research Reactor Utilization and Reactor Mathematics, Mexico DF, 1, 219-237 (May 1967).

13. E. L. Wachspress, Some New Nonlinear Iteration Techniques for Solving Neutron-Diffusion Problems, ANL-7050, (1965).

14. E. L. Wachspress, Iterative Solution of Elliptic Systems, 270-287, (Prentice Hall Inc., Englewood Cliffs, New Jersey, 1966).

15. J. P. Dorsey and R. Froehlich, GAMBLE-5, A Program for the Solution of the Multigroup Diffusion Equations in Two Dimensions with Arbitrary Scattering for the UNIVAC 1108 Computer, GA-8188 (February 1968).

16. M. Wagner, GAUGE - A Two Dimensional Few Group Neutron Diffusion Depletion Program for a Uniform Triangular Mesh, GA-8307 (March 1968).

17. K. D. Lathrop, TWOTRAN - A Fortran Program for Two Dimensional Transport, GA-8747 (Ju1y 1968).

18. J. P. Dorsey, GAMBLE - A Program for the Solution of the Multigroup NeutronDiffusion Equations in Two Dimensions, with Arbitrary Group Scattering, for the IBM-7090 FORTRAN II System, GA-4246 (June, 1963). 


\section{LIST OF FIGURES}

Figure 1 - Central Organization of Storage

Figure 2 - Hierarchical Organization of Storage

Figure 3 - Two Dimensional R-Z Geometry TRIGA Reactor Mockup with Central Hole

Figure 4 - Convergence Behavior of Varfous Methods for a TRIGA REactor with a Central Hole. 
DATA MANAGEMENT FOR MULTIACCESS COMPUTATIONAL SYSTEMS

J. E. Suich, J. C. Jensen, and H. C. Honeck

Savannah River Laboratory

E. I. du Pont de Nemours and Company

Aiken, South Carolina 29801

\section{THE JOSHUA CONCEPT}

A companion paper describes the development of the JOSHUA system at Savannah River Laboratory. Although the system is intended for application to problems in the management of a nuclear reactor facility, the system is quite general. Generality has been lost only in the sense that the design of the system was tailored to the requirements of the application area.

The JOSHUA system provides data and job management facilities for applications having the following characteristics:

- A large set of dynamically executable modules interfaced through direct access storage.

- An extensive data base of hierarchically ordered, unformatted records.

- A high ratio of records to record-types.

- A substantial amount of "standard," read-only, multiuser data.

- Significant quantities of semipermanent, read/write, single-user data.

- A minor amount of unchecked, temporary, job output data.

- A need for individual display terminal assistance in creating, modifying, and viewing data.

- A fairly long "think time" per display.

- A very long computer time per module execution request.

The machine system for which JOSHUA was designed has the following salient characteristics:

- (Conceptually) different processors for terminal and module execution.

- Attention-driven (interrupt) terminal control processor.

- Multiprogrammed, noninteractive, module execution.

- Processor to processor communication via shared direct access storage.

Such a system seems necessary to efficiently handle the assumed "think" and "compute" times postulated above. A degree of man-module interaction can be provided, when appropriate, by reducing a job to small, independent modules, and permitting terminal display and modification of results between each module. However, the addition of interactive modules, as required in graphics applications, will undoubtedly require a dedicated (multiprogrammed) attention-driven terminal processor (partition). 
A large data base with many individually addressable records requires a cataloging scheme, which is conveniently referenced both by the user at a terminal, and by computational modules during execution. The user must be allowed a natural language naming scheme; the modules require a reference (record) numbering scheme for $I / \varnothing$ access methods.

The heart of the JOSHUA system is the "named access method" which establishes, dynamically, the correspondence between record name and reference number(s).

A convenient way of representing a hierarchically organized data base is by concatenation of qualifying names, ${ }^{2}$ e.g.,

\section{MULTIGROUP. STANDARD. U238. XSECTION. FISSI $\varnothing N$}

The collection of all such names into a general "tree" structure ${ }^{3}$ expresses quite nicely the logical order of the names (and data):

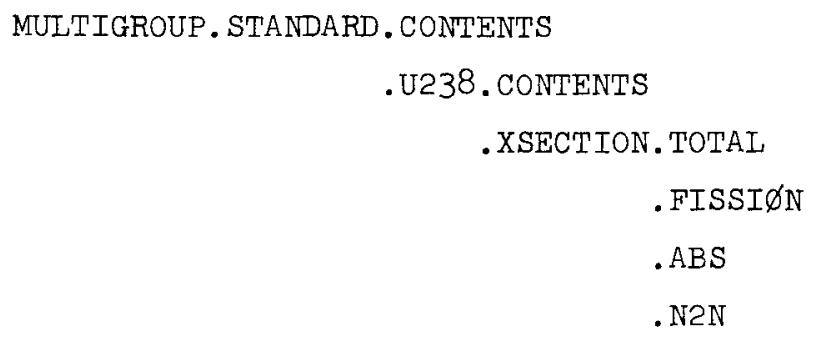

The relationships between names in this tree reflect the most probable order of use of the data; that is, when the user or module refers to some given piece of data, he will very likely refer next to data named nearby in the tree.

Further, reference to a partial name string expresses interest in the associated subtree, and is a very convenient reference for terminal users in requesting multiple displays, and for modules to retrieve whatever data are available.

The data records themselves could be stored either in unformatted form (appropriate for FORTRAN list directed, direct access $I / \varnothing$ ), or formatted as appropriate for terminal display. Considering the fewer data accesses generated by terminal requests, the system is designed for unformatted storage. Displays for terminal users are formatted by maintaining a set of list descriptor records to control the conversion of each list type. Thus, computational modules, for the sake of efficiency, utilize normal $I / \varnothing$ for data retrieval, and are simply provided cataloging services to replace the use of conventional record numbers.

Finally, the distinctions mentioned earlier between standard, multiuser data; semipermanent, single-user data; and temporary job results are accounted for by different procedures for allocation and management of direct access space for each data type. 


\section{DATA STRUCTURES}

The management of data structures of the type described is not a new problem. The use of "list processors" to handle such structure has a venerable history; ${ }^{4}$ and more recently computer graphics systems ${ }^{5}$ have extended the simpler list processing techniques.

Consider the following simple tree:

$$
\begin{aligned}
& \text { A.B.D. } \\
& \text {.C.E.H } \\
& \text {.F.J } \\
& . K \\
& \text {.G }
\end{aligned}
$$

We may also represent this tree graphically:

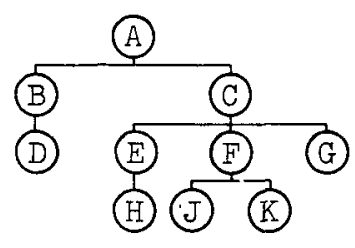

Such a form is inconvenient in that any "node" (circle in the diagram) may have $0,1,2, \ldots, n$ "sons" (lower level connected nodes). Since the tree must be capable of dynamic modification, keeping track of a variable number of sons will be wasteful of space (allowing for a very large number of sons) or of time (reordering a packed tree to admit or remove an additional son).

An alternative form of the general tree is the "binary tree," In which each node has only one son, and one "brother" (node to the right at the same level).

\begin{tabular}{|c|c|}
\hline $\begin{array}{r}1 \\
\text { na }\end{array}$ & 2 \\
\hline brother ${ }^{3}$ & son \\
\hline
\end{tabular}
In our example:

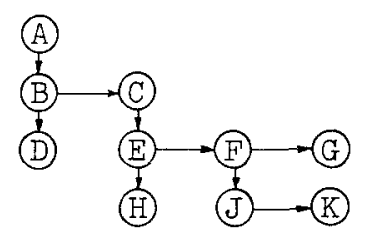

Here, a "father" points only to his first son and to his next brother. Thus the simplest computer structure of a node is:

In which we have allowed two full words for the name of a node, and one word each for pointers to the nodes representing next brothers and first son. The "elements" of node $P$ will be referred to as name $(P)$, brother $(P)$, son $(P)$. The "null" pointer, indicating termination of a branch, is designated $\varnothing$. Thus, e.g., $\operatorname{son}(P)=\varnothing$ states that node $P$ is a terminal node; i.e., that name $(P)$ is the last qualifier of some name string. 
In internal computer form, the "tree" becomes a Iist of chained nodes:

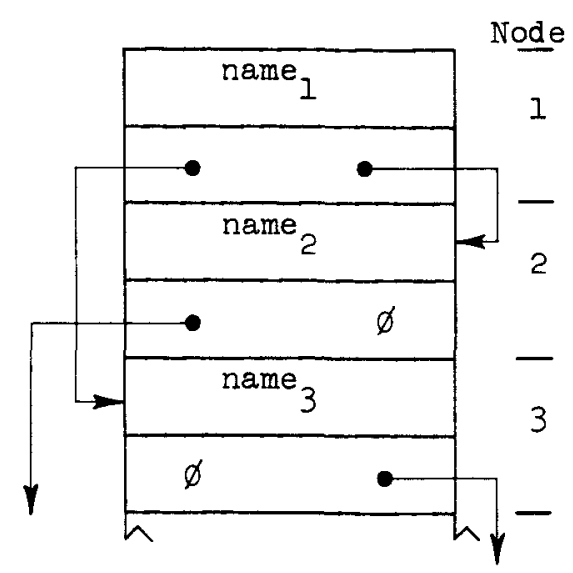

More complex structures have been developed to allow multiple associations between elements of the structure. For our purposes, however, the simple structure is quite sufficient, and can be handled efficiently without a generalized list processor. A useful feature of some list structures are "backward" pointers, in which each node contains pointers to the "father" node and older brother (node to the left at the same level). Such pointers allow one to traverse the tree in any direction, starting from an arbitrary node. For data cataloging, however, we expect only to traverse the tree in natural onder. The only advantage of backward pointers is to avold retracing the entire tree if a reference is similar to the prior reference. That is, a high proportion of the time, it should be possible to retrace the tree from the last node visited to a common node in the old and new name strings, and proceed forward from there. Keeping backward pointers, however, increases storage requirements by 50\% (an additional two full words).

The same retracing advantages can be gained by maintaining a "trace" table of the nodes visited on each reference in an auxiliary stack - a much more economical solution.

In fact, the auxiliary stack is preferable because the complete tree structure cannot be core contained. Thus, all pointers in pages of the tree whlch have been overlaid would be lost without the auxiliary stack.

Another interesting feature of some list structures is a "circular Iist" or "ring" in which the last brother of a family points to the first brother, instead of utilizing the null pointer. This feature (which requires no additional storage) allows consequentive references to proceed without researching a set of brothers, by eliminating the right/left orientation of the simple binary tree. (one can search to the right without having to always locate the "first" son.) We have adopted this convention because of its utility.

Finally, a terminal node (one having no sons) must point to the data record number associated with the name string it terminates. This is done without use of additional storage by restricting node pointers to positive values, and utilizing a negative number as the null pointer. AII pointers are relative to a base; the full word is interpreted as two half words in the form:

\begin{tabular}{|l|l|}
\hline base & displacement \\
\hline
\end{tabular}


For node pointers, the base is the tree page number (equivalent to a physical record number), and the displacement is the relative location of the node within that page. A base of zero refers to the current tree page.

For data pointers, the base is the negative of the data record word count (hence interpreted as a null pointer), while the displacement is the data reference number (equivalent to the first physical record number). Thus, In final form, our sample tree might be (for tree pages of 5 nodes, with pointers expressed in full words):

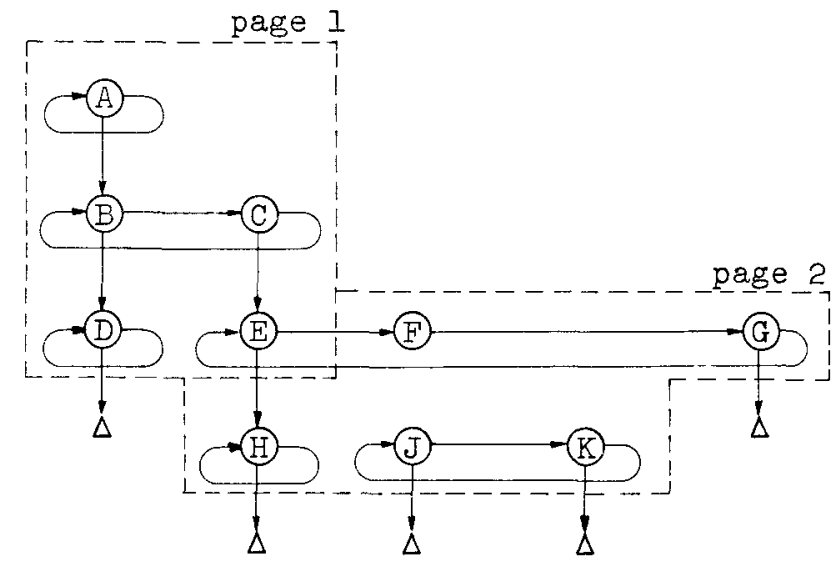

here, the symbol $\Delta$ denotes a data record.

Finally, the name of the first node (tree root) is interpreted as a data set name, allowing the entire data base to be segmented for allocation to direct access devices, reduction in arm contention, maintenance, file protection, etc.

\section{EFFICIENCY OF BASIC AIGORITHMS}

Trees are maintained as direct access data sets with 50 nodes per page (record). Up to 8 pages are in core at any time; each time a pointer is encountered with a nonzero base, a search of pages currently in-core is made. If it is necessary to bring in another page, that page which was least recently visited by a search is selected to be overlaid. If that page had any additions or deletions made to it, the copy on direct access space is rewritten before bringing in the new page. The statistjcs necessary for core management (counts of number of entries, number of additions, deletions, new page accesses, etc.) are available to the user, and can be used to determine the optimum use of in-core space (number and size of pages).

Searches through the trees are quite efficient because of the simple tree structure. The most recent 8 references are traced in the auxiliary stack. Two "modify" operations from a terminal (1nvolving the old record name, the new record name, and record type descriptor) can be completelv traced.

The search routine returns the data pointer associated with the name, or if a subtree is referenced, the data pointer for the leftmost branch. Suisequent searches return data pointers for the remainder of the subtree in natura order. Computational modules are currently restricted to using only complete names. 
Consider a "complete t-ary tree" (each father has $t$ sons, all terminal nodes are at level $\mathrm{k}$ ). The total number of terminal nodes is $t^{\mathrm{k}} \equiv \mathrm{m}$. A search of such a tree would require, on the average, $t / 2$ comparisons at each of $k$ levels, or $\frac{\mathrm{kt}}{2}=\frac{\mathrm{km}^{1 / k}}{2}$ comparicons per searoh. Thus, a tree with $\mathrm{m}=32,768$ terminal nodes and $k=5$ levels (typlcal of a large JOSHUA reactor physics data set) would require an average of 20 comparisons per retrieval for randomly selected names. Because of the large number of roces, as many as 13 pages of the tree may need to be retrleved. However, retrieval of names in their logical (tree) order, should permit 25 to 50 searches without requiring a new page to be brought into rore, at our present page size. Thus we estimate about $10 \%$ of direct access space is devoted to tree storage, 90\% to data; about 95\% of ordered searches will not requine access for new tree pages, while random searches may require $u 15$ accesses for tree pages.

\section{DATA MANTPUIATIJN FACII ITIES}

Computational modules are written in FORTRAN (modified by a precompiler phase to provide read/write facilities). System modifications and improvements have been made transparent to the applications programmers (through the use of a precompler). The operating group mairtains a complete flle of JOSHUA producticn appl- an ior mou'es whech car be recompiled as necessary.

Manipuiation of dara at dispiay tuminals zequires formatting of the FORTRAN (binary) data records for visua iispiay. Tor each type of record defined by an $I / \phi$ list and name sting structire, the applications programmer supplies to the system a "template" to control iormatting.

1) Record Neme: name name $_{2} . .-$. name ${ }_{n}$ where name, is either a literal name (if uniqueiy defined for this record type), or a name embedded in "?" to stgnlfy a raniable name (either computed or user supplied).

2) List: the I $\not x$ list used in FoRTRAN read/write statements.

3) Variable Narle/Gye: for each variakle in the list, a name (to be used later for tutorlal purposes) and type specification (real, integer, character plus length, in bytes) is given. This relieves the restriction to full word variables.

4) Page Type Specification: f'c each fnolied Ir loop, a page type is specifled on which rariales ascociated with that loop will be displayed.

5) Page Descriptions: for each page type mertioned in 4), a picture is created of the desired output format.

In the page descriptions, fleld specifications are given by inserting the symbol " $>$ ", followed by a string of A,I,E,F, or Z's to denote the field count (the $>$ is not included in the count). A blank terminates the field description; decimal points are embedded in $E$ and $F$ strings where desired.

The page type images are analyzed for field locations corresponding to each loop initial variable; fields are analyzed for type and packed into two bytes in the form $\left[t,(w-1)+d^{*} 16\right]$ where $t$ is a code corresponding to $A, I$, $E, F$, or $Z$, and $w, d$ are the conventional integers in a FORTRAN format tw.d. 
Finaliy all strings of more than 2 repeated characters in the page image are packed into two bytes in the form $[(n-2), c]$ where $n$ is the count $(\leq 63)$ of characters $C$ in the string. This page description appears to be at least as compact as standard FORTRAN formats for templates devised so far.

The display package moves data from the input stream as encountered into the next avallable field in an in-core 1mage of the page type specified for 1ts corresponding loop. If page overflow occurs, the page image is forced out of core, and the field into which data from that loop were first moved becomes the next available field. When the limit is exhausted for the innermost loop on a given page which has variable loop limits, the remainder of the page is blanked and forced out of core. Thus, for example, a simple nest with two loops on the same page might behave as follows:

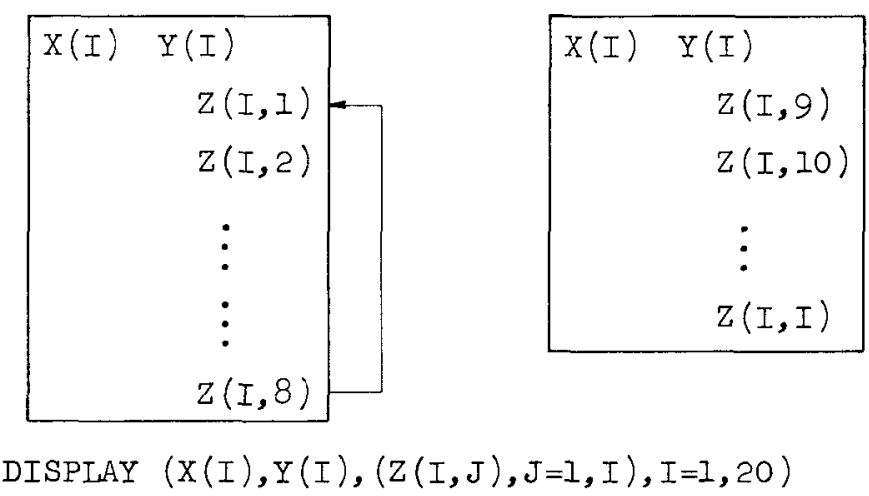

More complex displays are, of course, possible. All pages of a given type are presented in the order they were created before display of the next page type occurs. This is accomplished by developing a list of one-byte page record numbers by type (each page is a direct access record) in the following form:

\begin{tabular}{|l|}
\hline number of page types \\
count of type 1 records \\
record number, first type I record \\
$\vdots$ \\
\hline record number, last type I record \\
\hline count of type a records \\
\hline etc. \\
\hline
\end{tabular}

The one-byte word length restricts the total number of pages to 255, which is a reasonable limit for the present application. 
The overall operation of the display and modify function is illustrated below:

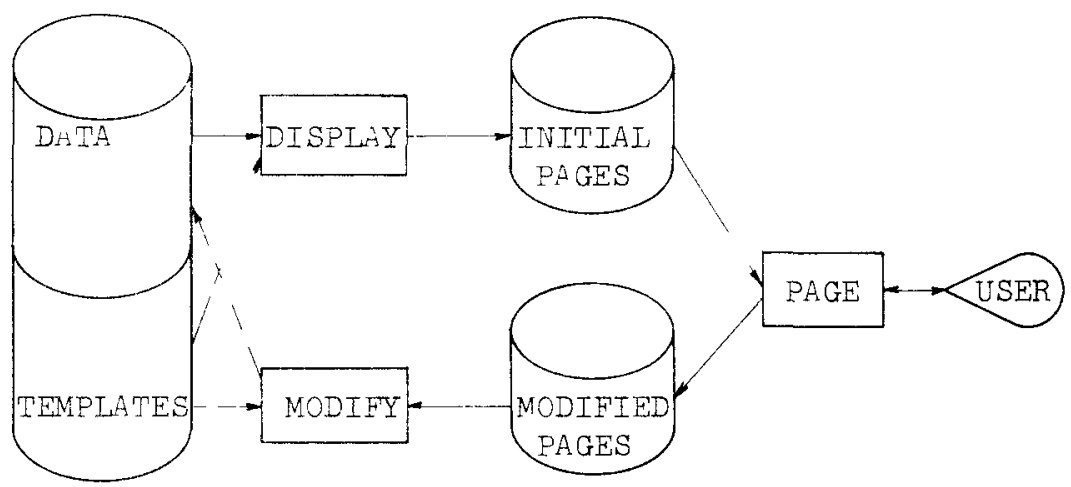

Formatted DISPLAY and MODIFY

The display/modify scheme using "templates" obviates the need for a large library of FORTRAN subroutines to interpret individual record types. We have in essence replaced such a library by a generalized interpretive program to replace FORTRAN $I / \varnothing$.

\section{DATA MANAGEMEIJT}

While the above described data handing system represents an extensive amount of design and programming, the analysis is fairly straightforward. Consideration of the multiaccess features of the JOSHUA system poses a more formidable design problem.

Considering the data base as a whole, the following concurrent activities can be foreseen:

- A significant number $(\geq 8)$ of active terminal requests to either DISPLAY, MODIFY, COPY, Or CREATE records.

- A background batch activity by computational modules reading/writing records.

- System activity in updating standard records, allocating work space, deleting work space, etc.

Furthermore, the system design calls for at least conceptually different machines for terminal and batch activity. Consideration was given to use of $O S / 360$ ENQ-DEQ macro's to ensure the integrity of shared files. How this facility could be implemented on independent processors was not known; furthermore, its use might be system and version dependent. Finally, it was considered extremely difficult to predict the result of the use of such a scheme on system response time. Thus, it was decided to look for a conceptually different scheme for controlling multiple accesses to the shared data base.

A scheme which allows for all concurrent activities except update of standard records is the following:

- Include all data of general utility which is fairly static in "standard," read-only data sets. 
- Provide each active user with an individually maintained, "user" data set. This data set is read only to all users, and can be written into onIy from the terminal by 1 ts owner.

- Dynamically manage "job" data set space to allocate to each batch Job a temporary data set assigned to the user submitting the job. On completion of the job, this data set becomes avallable for interrogation at the terminal, and can be selectively copied into a user data set and/or deleted in toto by the user.

- Restrict updating of standard records to the systems group, who will accomplish this during controlled periods.

These file protection schemes are accomplished by the search and retrieval routines, in a way which also provides a very nice "overwrite" capability for standard record modification. All write operations from a terminal (including copies from other data sets) automatically append the user ID number as name ${ }_{1}$ of the record name. All writes from computational modules automatically append the Job name as name ${ }_{1}$ of the record name. This guarantees the integrity of all data sets. Reads, however, are now ambiguous if the initial data set name is not specified. The system removes this ambiguity by defining a hierarchy of data set names as follows:

$$
\begin{aligned}
& \text { 1. job.name.--- } \\
& \text { 2. user.name.--- } \\
& \text { 3. [standard].name.--- }
\end{aligned}
$$

Any read operation then searches for the named record first appending the "Job" name, then the "user" name, and finally the name as given. A user at a terminal can override this hierarchy by simply giving a first name (data set name) equal to the current job name (a command, "set job," establishes this at any time), his user ID, or the name "STD" for standard. It is not expected that computational modules will alter the established hierarchy.

Thus if a user (or module) writes a record with the same name as a standard record, subsequent references by that user to that name automatically retrieve his "user" record - although the standard record has not been changed for other users of the system. A "delete" of this "standard" record by the user simply restores the existence of the original (true) standard record. Thus comparative studies can easily be accomplished without special concern for the integrity of the standard data set.

The management of "job" or work space is accomplished via a combination of batch job executor and the terminal monitor which provides data and job management services. The batch executor maintains a directory of work space. Each batch job specifies the module name(s) to be executed, and an estimate of job data set size required. If at least the estimated space is available in some contiguous block of free space, the job is assigned the first such block encountered. (The largest available block is not selected because this may compound the space "fragmentation" problem, and is less efficient. ${ }^{6}$ )

Upon termination of the job, the batch executor marks the actual extent of the space used, and updates a catalog on the processor-to-processor shared direct access space, inserting the new job name as a data set name. Thus, the existence of the job data set is unknown to the user at the terminal until 
the job has completed execution. At that polnt, the user is free to display, copy, modify, or delete records (or the entire data set). A delete of the data set returns the work sjace to the free list of the batch executor (simultan-

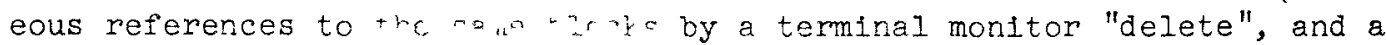
batch executor "alloce: = ciuse, at worst, a lack of recognition by the batch executor of the avajabilit. of space!.

Thus, the space management scheme prevents simultaneous read/write operations to any data set, obviates trs need for system dependent code, and places the primary burden of space maintenance on the user to whom the space is assigned. A method of automatically aeleting old "job" data will also be obviously necessary. The apparently awknari situation of not being free to update standard records will probally prove to be a positive feature. The hierarchy of data sets allows any individual to effectively update standard records; when he has verified the validity of the new record, the systems group can actually change the stardanj record (after warning 911 users of the impending change, so that they may copy the old standard record into their user data set if they wish, thus insulatirle themselves!).

The efficiency of the t-ary tree search technique was mentioned earlier, and applies directly to batch computational module execution because only one task is utilizing the search routine. However, for the case of multiple terminal referenses th $\mathrm{n}^{r}$ the same search routine, traces would be lost from

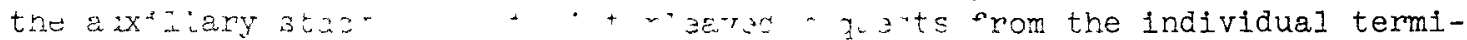
nals. Tubviate thi fr. " an, three traca jer umal al an be maintained in in-sore work space associated witr each terminal. The 8 in-core tree pages are managed as das bed previously to reduce to al space requirements. At worst, for logically orderea searches, each terminal reference, utilizing the saved trace, will generate ore tree page access to restore an overlaid page. Experience witn rultipie terminal activity will be required to examine the net efficiency of dynamic page pool management versus buffer and trace space allocated per terminal.

The Savannah River computing system currentiy nas two IB $B_{1}<3+4$ a rect is cass storage facilities avaliable (with a third probably to be installed in the near future), and some 50-100 potential users of the JosHUA system. For the immediate future, therefore, it seems protatie that the system can operate as though all data sets were always on lire. Data set maintenance is easy a "copy" instruction snecifying only the data set name produces a clean copy (deleted data records mitted with all space recovered) so that users can easily maintain their own space with stardard utilities. It seems likely,

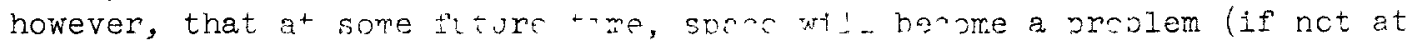

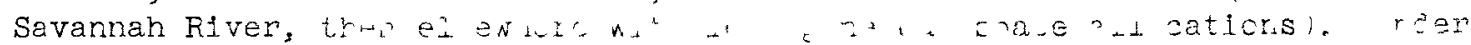

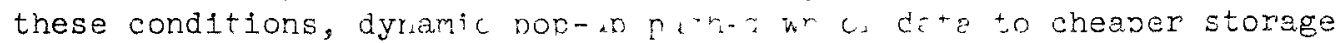
(tape?) will be necessary. Batch compulsuror il raule execution does not seem a problem, In that if the user data set is not on-line, a simple pre/post-job step to readiwrite tre lape car bc int土laved.

For terminal operations, however, pop-up cannot occur before user sign-on, and this will entall a delay of several minutes while the tape is located and mounted. Either time scheduled terminal sessions will be required, faster devices (data cells?) will be required, or the delay must be accepted. The user can, of course, pe interrogating standard files, job files, etc., during this setup period. These problems will be tackled after sufficient experience with the on-iine system has been gatned to permit a guess at the correct answers. 
The JOSHUA capabilities now available have been programmed by R. L. Boyce and J. H. Maddox.

The information contained in this article was developed during the course of work under Contract AT(07-2)-I with the U. S. Atomic Energy Commission.

\section{REFERENCES}

1. H. C. Honeck, J. E. Suich, J. C. Jensen, C. E. Bailey, and J. W. Stewart, "JOSHUA - A Reactor Physics Computational System," proceedings of the Conference on The Effective Use of Computers in the Nuclear Industry, Knoxville, Tenn., April 21-23, 1969.

2. H. J. Kopp and D. S. Selengut, "DATATRAN - A DATA-HANDLING COMPUTER LANGUAGE FOR A LARGE MODUTAR REACTOR DESIGN SYSTEM," Proceedings of the International Conference on The Ut1lization of Research Reactors and Reactor Mathematics and Computation, Mexico City, D. F., May 2-4, 1967, Vo1. 3, pp. 1460-1472, Report CNM-R-2.

3. D. E. Knuth, "The Art of Computer Programming, "Fundamental Algorithms, Vol. 1, Chap. 2.3. (Addison Wesley Publishing Company, Reading, Mass., 1968.)

4. Ibid. Chap. 2.6.

5. J. C. Gray, "Compound Data Structure for Computer Aided Design; A Survey," Proceedings of 22nd National Conference, p 355-365, Thompson Book Company, Washington, D. C., 1967.

6. D. E. Knuth, op. c1t., p 437. 
A BURNUP SYS TEM CONTROL AND CALCULATION METHOD*

E. R. Volk, J. S. Gubkin, D. B. Wehmeyer, C. S. Yeh

(Atomic Power Development Associates, Inc.)

\section{INTRODUCTION}

A one- or two-dimensional burnup system has been designed for use with the multigroup diffusion code SCRAMBLE IIIl. Although the burnup system is specific for use with SCRAMBIE III, it does contain several areas of general interest particularly in the control method used within the calculation and the mothod by which the burnup equations are solved. To better describe the control system, a brief summary of the SCRAMBLE III free-format input metrod, control by directives, and output edits are given.

\section{SCRAMBLE III SUMMARY}

The SCRAMBLE III free-format input allows any alphabetic or numeric data to be read, free of column control, with the primary restriction that alphabetic data are terminated by blank or numeric data, while numeric data are terminated by blank or alphabetic data. The use of fixed or floating point numbers is selected by the programmer; the use of a decimal point is optional in either case.

The directive control system in SCRAMBLE III is based upon a subroutine which compares an alphabetic field to a string of alphabetic fields then returns, as an argument, the integer that corresponds to the location of the equivalent element. For example, in a system using FORTRAN and A4 format,

CALL CRAMIN (AA, NN, WW)

would read the first four characters of the next alphabetic word from the input data set into $W W$ in free-format. Then using

CAIL SCANN(28HONE TWO THREFOURFIVESIX ****,WW,J),

the value of $\mathrm{J}$ will be given a value from 1 to 6 based on the input word being ONE, TWO, THREE, FOUR, FIVE, or SIX. A value of 7 will be assigned to $J$ if no match is found. Thus, $J$ can then be used in a "calculated go to" statement to obtain the desired result.

\footnotetext{
FThis work was supported by the Edison Electric Institute and Central Research Institute of the Electric Power Industry of Japan.
} 
The many output edits within SCRAMBLE III make use of the TAPE (data sets on disk or tape) directive for problem data and fluxes. Thus, to execute the neutron balance edit routine, a directive such as

TAPE 10 NBAL 11

would restore the problem data from information contained on data set 10 and execute the NBAL (neutron balance) edit routine using fluxes from data set 11.

It should also be pointed out that SCRAMBLE III utilizes variables for all input and output logical unit assignments.

With this brief summary of parts of the SCRAMBLE III system, the problem of control with the BURNUP edit routine will be reviewed.

\section{BURNUP CONTROL CRITERIA}

In outlining the requirements for the SCRAMBLE III burnup system, it was desired to specify both major and minor time steps. The major time steps were defined as those times at which flux recalculations, alterations of data, execution of routines, or any other normal SCRAMBIE III operation would be performed. The minor time steps were defined as those at which the isotopic burnup would be recalculated and fluxes renormalized. Thus for major time steps, it was necessary for the burnup system to return control to the SCRAMBLE III control system yet be prepared for a re-entry for continuing the calculation. For the minor time step, all action is performed within the burnup system.

The basic material composition region within SCRAMBIE III during a flux solution is the "zone"; therefore, although isotopic concentrations are used to accumulate burnup, it is the zone region over which control is desired. Thus for each zone, the burnup must be monitored to determine when and if it exceeds the user defined limits. Once the limits have been reached for a particular zone, the action desired by reaching that limit can be initiated. After this action, the problem can then be continued until other burnup limits have been met, and the subsequent required action has been taken in each case. The problem will continue in this same manner until all criteria have been satisfied and the termination requirements have been met. The action which may be specified covers a very wide range of items such as fuel exchanges, fuel replacement, changes in burnup criteria, execution of any edit routine, or change in type of solution, i.e., change from a dimensional search to a material search or to a $k$ effective type of calculation. In addition, the desired action to be specified cannot be assumed to be the same for different zones or the same for the same zone at different burnup limits. It was also desired to operate within the basic framework of the SCRAMBLE III program and to utilize the existing routines to a large extent.

\section{BURNUP CONTROL NETHOD}

The selected solution to the control problem satisfies all the above conditions, with few changes to the basic SCRAMBIE III system. Figure 1 outlines the burnup system control flow logic. The details of the method selected are as follows. 


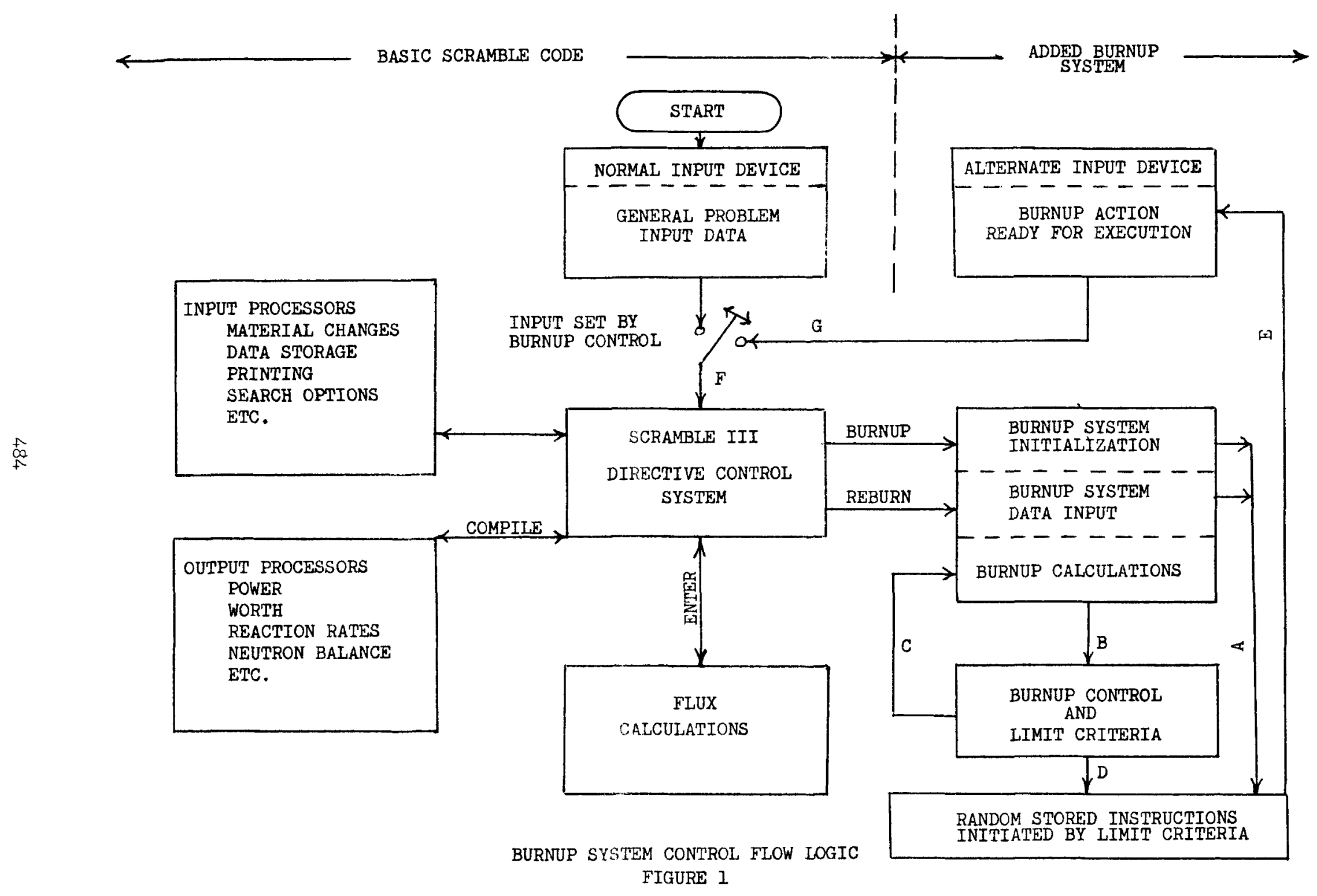


Input to the burnup system includes cards for control criteria, both of major and minor time steps and of burnup limits by zone. These cards are read in by SCRAMBIE III in free-format and are easily recognized by a directive "T" for time steps and a directive "B" for zone burnup. The time control cards have the additional data of "number of days" covered during each minor time step, "reactor power" used to normalize the fluxes, "number of minor time steps" or the number of repetitions of the "number of days" at the "reactor power" given for burnup calculations, and "number of cards" required to contain the special action to be taken on completion of these time steps. If the "number of cards" is 0 , this is not the end of a major time step, and the program continues with the next "T" card. Before discussing the use of this action information, the form of the burnup criteria card will be outlined.

In addition to the "B" specifying that the data following is zone burnup control, there is the "zone number" that will be monitored and to which the limit will apply, "zone burnup limit" that is to be monitored for this zone, and "number of cards" that are necessary to specify the action to be taken in the event the burnup criterion is satisfied.

When a "T" or "B" directive is encountered during the burnup input data, entries are made in the particular table (time limits or zone burnup limits) to which it applies. In addition, a temporary random access data set location is inserted in the table as a pointer to where the applicable action directive cards are to be stored. Since the number of cards are known, the information can then be read as 80 column alphabetic card images, transferred to the location on the random access data set desired, and the pointer updated for the next input information. This movement of data is indicated on Figure $I$ by Path A .

When the input data is complete the time limit conditions will be contained in a table with the information as mentioned previously and in addition, will contain the pointer to the random access disk area where the directives specifying the action to be taken on major time steps are located. A corresponding table is also prepared containing an entry for each zone in the problem. For zones that have no burnup control limit specified, an excessively large value is used to nullify any subsequent action.

The burnup problem will be initiated on detection of the directive card "END". At this time, all tables have been prepared and any following data will be normal SCRAMBLE III input data to be executed only after completing the burnup calculation through all time steps specified.

The burnup system will recalculate the number densities at each minor time step and then check (Path B Figure 1) to see whether a major time step or a zone burnup limit has been reached. If a limit has been reached, a check will be made to determine what action is to be taken at this time. When no limit is indicated, Path C Figure 1 will be followed; however, if action is required by the presence of one or more directive cards in the table, Path D of Figure 1 will be followed.

To prepare the desired action directive data for execution, an auxiliary sequential input/output device (usually disk) is rewound. The number of directive cards and their location on the random access device is obtained from the limit table and then used to transfer the data to the 
sequential device, Path $\mathbf{E}$ Figure 1 . This sequential derice is then rewound and assigned as the normal input unit to SCRAMBLE III. Control is then returned to the normal directive input section of SCRAMBLE III, Path $F$ of Figure 1.

At this point, all isotopic number densities have been updated in the basic SCRAMBIE III storage area, and all accumulated burnup numbers valid only to the burnup system are contained in labeled common.

The directives specified in the input data when either the major time limit or the zone burnup limit is reached will now be executed as shown by Path $G$ of Figure 1. These directives may include such itens as

\begin{tabular}{|c|c|}
\hline ALTER C & Used to shuffle fuel \\
\hline ALTER CON & $\begin{array}{l}\text { Used to change type of problem i.e., a search } \\
\text { problem to } k \text { effective calculation }\end{array}$ \\
\hline ENTER & Resolve the flux solution \\
\hline DUMP & Save problem and fluxes for edit processing \\
\hline COMPIIE & $\begin{array}{l}\text { Tiansfer to edit processing routines. This } \\
\text { directive would be followed by the edit } \\
\text { directives such as }\end{array}$ \\
\hline & RATES - reaction rates \\
\hline & NBAI - neutron balance \\
\hline & PERT - perturbation calculation. \\
\hline
\end{tabular}

To return to the burnup system to continue processing requires the directive REBURN to be read and executed by the SCRAMBLE III control system. After this is done, the user may elect to alter any of the burnup system input data, except the major time steps. The burnup criteria can now be altered to adjust for any fuel changes made by the directives given to the basic SCRAMBLE III control system.

A brief partial example is given to clarify some of these areas. If, for example, channel 6 of a reactor core under consideration contains zone 3 material in the first 10 points and zone 4 material in the last 15 points, the channel 6 specification as normal input to SCRAMBLE III is

\section{$660 Z 3 \quad 10 \quad 2425$}

After entering the burnup system input routine, the following directives are typical of the entries which could control the solution, based on the burnup in a single zone. 


\section{$\underline{\text { CARD }}$}

1

2

3

4

5

6

7

8

9

CONTENTS

B 23.1257

ALTER C6 $0 \quad Z 10 \quad 10 \quad Z 4 \quad 25$

ENTER TAPE 10 DUMP REBURN 10

B 210.253

ALTER C6 0 Zl1 $10 \quad 24 \quad 25$

TAPE 10 DUMP COMPILE NBAL 10

REBURN 10 END

END

END

This set of cards would cause the following sequence of events to

occur.

1. Zone 3 burnup criteria would be set to .125 , and 7 directive cards would be indicated in the burnup limit table for zone 3 .

2. Cards 2 through 8 would be stored on a random access device. The disk pointer location and number of cards stored would be entered in the burnup limit table for zone 3 .

3. Card 9 directive "END" would terminate the burnup system input and start the burnup calculations.

4. When zone 3 reaches a burnup of .125 , cards 2 through 8 would be retrieved from the disk and stored on an auxiliary input device which would be designated the SCRAMBLE III normal input.

5. SCRAMBLE III would read and execute the ALTER card, causing zone 3 to be replaced by zone 10 in channel 6 .

6. A new flux solution would be obtained after reading the directive "ENTER" on card 3. The "TAPE 10 DUMP" directive would store the problem and fluxes on data set 10 for future use.

7. After reading the directive "REBURN 10" (also on card 3), SCRAMBLE III would transfer control back to the burnup system using the revised fluxes and problem data available on data set 10 .

8. The burnup system input would continue to read the auxiliary input device, card 4 would be read, and the burnup value of .25 for zone 10 stored in the burnup limit table. 
9. The burnup system would then read and store the next 3 cards (cards 5 through 7) on the random access device with the number of cards and disk pointer included in the burnup limit table for zone 10.

10. The END directive (card 8) would terminate the burnup system input and processing would continue.

11. When zone 10 reaches a burnup of .25 , the process of retrieving cards 5,6 , and 7 would be repeated; control would then be transferred to the SCRAMBIE III control system where these cards would be read and executed.

12. Card 5, similar to card 2, would alter the material in channel 6 from zone 10 to zone 11 .

13. Card 6 would execute the neutron balance output edit then return control to the burnup system by the directive "REBURN 10" on card 7 as previously indicated.

14. The "END" directive (card 7) would allow the burnup system to continue processing until the next major time limit or zone burnup limit is reached.

Of course, all zones could have limits and directives which would be checked, together with the major time steps at every minor time step. If a major time limit and a burnup limit are reached at the same time, the burnup directive is executed first, followed by execution of the major time limit directives. If two zone burnup limits are reached at the same time, they are executed in the order of zone number.

\section{BURNUP CALCULATIONAL METHOD}

The method used for solution of the burmup ecuations involves a series expansion relation that is quite fast and accurate for both long and short time increments. The programming of this thod is quite simple because a recursion relation for successive terms is easily evaluated.

The method is probably easiest explained using the U-238 chain and following the development of the equations to their final form. The five differential equations for the number densities are

$$
\begin{aligned}
& \frac{d N_{8}}{d t}=-Q_{8} \phi N_{8} \\
& \frac{d N_{9}}{d t}=s_{8} \phi N_{8}-Q_{9} \phi N_{9} \\
& \frac{d N_{10}}{d t}=s_{9} \phi N_{9}-Q_{10} \phi N_{10} \\
& \frac{d N_{11}}{d t}=s_{10} \phi N_{10}-Q_{11} \phi N_{11}
\end{aligned}
$$




$$
\frac{d N_{12}}{d t}=s_{11} \varnothing N_{11}-Q_{12} \varnothing N_{12}
$$

$N=$ number density

$S=$ captur cross section

$\varnothing=\mathrm{flux}$

$Q=$ absorption cross section, including decay

$t=$ time

Using the matrix notation

$N=\left|\begin{array}{c}\mathrm{N}_{8} \\ \mathrm{~N}_{9} \\ \mathrm{~N}_{10} \\ \mathrm{~N}_{11} \\ \mathrm{~N}_{12}\end{array}\right|$

$M=\left|\begin{array}{ccccc}-Q_{8} \varnothing & & & & \\ s_{8} & -Q_{9} \varnothing & & & \\ & s_{9} \varnothing & -Q_{10} \varnothing & & \\ & & s_{10} \varnothing & -Q_{11} \varnothing & \\ & & & s_{11} \varnothing & -Q_{12} \varnothing\end{array}\right|$,

Equations (1) through (5) can be expressed as

$$
\frac{d N}{d t}=M N
$$

Starting with Equation (8) which can be rearranged as

$$
\mathrm{dN}=\mathrm{MNdt},
$$

and letting $N=N(0)$, the initial number densities at time zero, an expression for $N(T)$ where $t=T$ can be derived. Dividing $T$ into $n$ equal parts of $\Delta T$ each, the approximate expression for $N$ at each time step can be written as follows:: 


$$
\begin{aligned}
& N(\Delta T)=N(0)+M N(0) \Delta T \\
& N(2 \Delta T)=N(\Delta T)+M N(\Delta T) \Delta T \\
& N(3 \Delta T)=N(2 \Delta T)+M N(2 \Delta T) \Delta T \\
& \vdots \\
& N(n \Delta T)=N(T-\Delta T)+M N(T-\Delta T) \Delta T
\end{aligned}
$$

The Equation for $N(T)$ can then be written as

$$
N(T)=\left[I+M \Delta T T^{n} N(0)\right.
$$

where $I$ is the unit matrix.

Taking the limit

or

$$
\begin{aligned}
N(T) & \left.=\operatorname{limitr}_{n \rightarrow \infty} I I+\frac{1}{n} M T\right]^{n} N(0) \\
N(T) & =\left[I+\sum_{p=1}^{\infty} \frac{1}{p} M^{p} T^{p}\right]^{N} N(0),
\end{aligned}
$$

which can also be expressed as

$$
N(T)=N(0) e^{M T}
$$

and expanding the first three terms of Equation (16), Equation (18) is obtained.

$$
N(T)=\left[I+M T+M^{2} T^{2}+M^{3} T^{3}+0000\right] N(0)
$$

Defining a recursion relation

$$
\Delta_{\mathrm{p}}=\frac{1}{\mathrm{p}} \operatorname{MT} \Delta_{\mathrm{p}-1}
$$

Equation (18) can be written as

$$
N(T)=N(0)+\Delta_{1}+\Delta_{2}+\Delta_{3}+0 \circ 0
$$

where $\Delta_{0}=\mathrm{N}(0)$

The time dependence of the number density can be expressed in terms of general time $t$ as

$$
N(t)=N(0)+\frac{t}{T} \Delta_{1}+\frac{t^{2}}{T^{2}} \Delta_{2}+\frac{t^{3}}{T^{3}} \Delta_{3}+000
$$

The burnup can then be calculated using $F$ as the fission cross section from

$$
B(T)-B(0)=F \varnothing \int_{0}^{T} N d t \text {. }
$$

Substituting, integrating, and rearranging terms results in the final expression:

$$
B(T)-B(0)+F \varnothing T\left[N(0)+\frac{\Delta_{1}}{2}+\frac{\Delta_{2}}{3}+\frac{\Delta_{3}}{4}+\cdots 0\right]
$$


Equations (20) and (23) are programmed and in use for SCRAMBLE III. Since the series expansion becomes alternating after several terms, it is quite easy to predict and obtain the accuracy desired. The programming is also quite simple since the same loop can determine not only the new number density, but also the new value of burnup.

1. Scramble Users' Manual, Digital Computer Group, Central Theory and Computing, AEE Winfrith, Dorchester, Dorset, England. 
NUCLEAR MODEL PROGRAMMING FOR DATA GENERATION

K. J. Yost

Oak Ridge National Laboratory

Oak Ridge, Tennessee

March 1969 


\section{NUCLEAR MODEL PROGRAMMING FOR DATA GENERATION}

The development of nuclear technology over the past twenty-five years has been accompanied by an extensive effort devoted to the development of particletransport techniques specifically for the description of neutron and, to a lesser extent, gamma-ray transport. As a result, there are now in being complex computer codes which deal with particle transport by way of diffusion theory, polynomial expansion methods, Monte Carlo, discrete ordinates, invariant embedding, etc. The sum total of these efforts over the years at various laboratories and universities represents, among other things, a substantial expenditure of funds. Transport methods research has been justified by the contention that there was no reasonable alternative to developing a calculational capability. As a practical matter, it makes little sense to construct prototypes of promising reactor systems before at least a preliminary check of the design with a series of reliable calculations. In the same spirit, it is impractical technically and politically to detonate a bomb each time a new question regarding weapons effects presents itself.

As we are all aware, transport techniques represent only a part of the problem associated with meaningful transport calculations. Another aspect of the problem is, of course, reliable data on which to base a calculation. Data in this context generally has to do with the description of the various neutron and gamma-ray interaction processes. Relatively much less emphasis has been placed on the development of means for the calculation of nuclear data despite the fact that the motivation is essentially the same. Just as exclusive reliance on experimental methods for the evaluation of reactor performance characteristics is impractical for reasons of time and money, so is the measurement of all basic data required for the calculation of these characteristics. Data measurement tends to be expensive and time consuming, particularly as the data requirements of an ever-expanding nuclear technology become more and more sophisticated. We must, for the foreseeable future, rely on methods for filling in data gaps and, where necessary, generating data to an accuracy which is consistent with that of the transport methods for which they serve as input.

One of the data problems which exemplifies the foregoing observations has to do with secondary gamma-ray production cross sections as employed in shielding and gamma heating salculations. The primary function of a shield is the attenuation of neutron and gamma radiation. Unfortunately, the two reaction mechanisms which are primarily responsible for neutron attenuation, capture and inelastic scattering, themselves constitute sources of gamma radiation. The shape of gamma-ray spectra emitted in both reactions tends to change as a function of incident neutron energy. This change of shape constitutes, as a function of the dependence of gamma-ray attenuation coefficients on gamma-ray energy, a change in the penetrability of the emitted gammas. Thus, for computing secondary gamma-ray dose, one is faced with the necessity of employing a two-dimensional cross-section grid which strictly speaking couples a set of gamma-ray yields to each reported capture or inelastic cross-section value.

A code system for the calculation of gamma-ray production cross sections is shown in block diagram form in Figure 1. The first block represents a nuclear model code which generates the eigenvalues and eigenvectors for the Schroedinger 


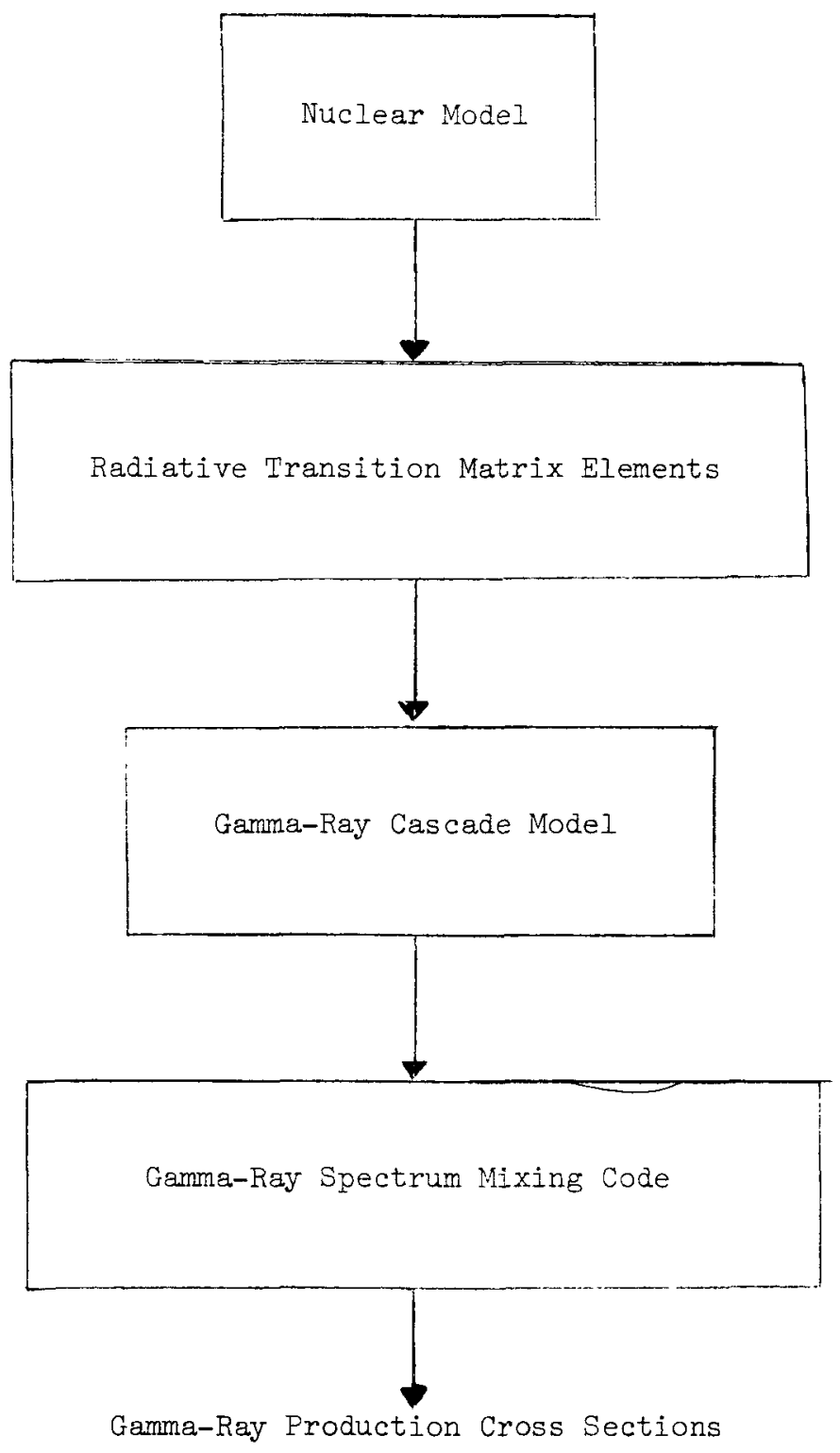

Figure 1. The Calculation of Gamma-Ray Production Cross Sections 
equation

$$
\mathrm{H} \Psi=\mathrm{E} \Psi
$$

for a single particle in a deformed potential well with the nuclear "core" subject to rotational and vibrational collective excitations. In practice the Hamiltonian, $\mathrm{H}$, is made up of a number of terms, each corresponding to a particular problem variable or mode of nuclear excitation. Energy levels are typically assumed to be farticle or collective in nature, or some combination of the two. For ease of computation, particle wave functions are based upon a harmonic oscillator potential. They have the form

$$
{ }_{l} F_{l}\left(-n, l+3 / 2, r^{2}\right) \Psi_{\ell m}(\theta, \varphi) \text {, }
$$

i.e., the product of a confluent hypergeometric function and a spherical harmonic, where $\mathrm{n}$ is given by

$$
\mathrm{n}=\frac{\mathrm{N}-\ell}{2}
$$

IN being the nuclear "shell" index, and $\ell$ is the unpaired particle (nucleon) angular momentum.

Cóllective motion of the nucleus is assumed to take two forms: rotation of the body and vibration of its surface. The rotational wave functions are the Wigner or D-functions, $D_{M K}^{I}\left(\Omega_{\mathbb{E}}\right)$, where $\Omega_{E}$ represents the Euler angles relating the orientation of a set of coordinate axes fixed in the nucleus to space-fixed axes. The symbols I, M, K denote the total nuclear angular momentum, the projection of $I$ on the (space fixed) $z$ axis, and the projection of $I$ on the (nuclear fixed) 3 axis. Quadrupole vibrational coordinates in the nuclear-fixed coordinate system, $a_{0}$ and $a_{2}$, are derived from an expression defining the nuclear surface of the form

$$
r(\theta, \phi)=r_{0}\left[1+\sum_{\mu, m} a_{\mu, m} Y_{\ell m}(\theta, \phi)\right]
$$

where the sum over $\mu$ is restricted to $\mu=2$. Eigenfunctions of the purely vibrational part of the nuclear Hamiltonian have the form

$$
{ }_{1} \mathrm{~F}_{1}\left(-\mathrm{n}_{2}, \mathrm{R}_{3}+1 / 2 ; \mathrm{a}_{2}^{2}\right) \mathrm{H}_{\mathrm{n}_{0}}\left(\mathrm{a}_{0}\right)
$$

where $H_{n_{0}}\left(a_{0}\right)$ is the Hermite polynomial, $n_{2}$ and $n_{0}$ denote the number of energy quanta associated with the $\mathrm{a}_{0}$ and $\mathrm{a}_{2}$ vibrational modes, and $\mathrm{R}_{3}$ is the 3-component of the collective angular momentum.

In summary, the precise form of the nuclear Hamiltonian employed in the model is given by

$$
\begin{aligned}
& H=-\frac{\xi^{2}}{2 M} \nabla^{2}+\frac{M}{2}\left(w_{x}^{2} x^{2}+w_{y}^{2} y^{2}+w_{z}^{2} z^{2}\right)+c \ell \cdot s+D \ell^{2}+\frac{-k^{2}}{2 B} \int \frac{\partial^{2}}{\partial a_{0}^{\prime 2}} \\
& \left.+\frac{1}{2} \frac{\partial^{2}}{\partial a_{2}^{\prime 2}}\right)+\frac{I^{2}-K^{2}}{2 I_{0}}+\frac{K^{2}-h^{2}}{16 B a_{2}^{\prime 2}}+\frac{L^{2}-K^{2}}{2 I_{0}} \frac{1}{\beta_{0}}\left[-2 a_{0}^{\prime}+\frac{1}{\beta_{0}}\left(3 a_{0}^{\prime 2}+2 a_{2}^{\prime 2}\right)\right] \\
& -\frac{I_{+}^{2}+L_{-}^{2}}{2 I_{0} \beta_{0}}\left(\frac{\sqrt{6}}{3} a_{2}^{\prime}-\sqrt{6} \frac{a_{2}^{\prime} a_{0}^{\prime}}{\beta_{0}}\right)+\frac{1}{2} C_{0} a_{0}^{\prime 2}+c_{2} a_{2}^{\prime 2},
\end{aligned}
$$


where $I_{0}$ is the moment of inertia along the nuclear fixed 1 - and 2-coordinate axes, $B$ is a quantity related to the nuclear mass density, I represents the total angular momentum of the nucleus, and $C_{0}$ and $C_{2}$ are constants relating vibrational potential energy to the squares of variables representing the two vibrational modes. The wave function corresponding to the above Hamiltonian derived from the solving of equation (1) is expressed in terms of sums of the products of the D-function and the partial wave functions given in equations (2) and (4). The expansion coefficients evolve from a series of matrix diagonalizations, each one corresponding to the inclusion of successively more terms of the foregoing Hamiltonian.

The second block of Figure 1 represents a routine which calculates radiative transition matrix elements based upon the wave functions generated in step 1. One generally considers electric and magnetic dipole (El and $M l$ ) and electric quadrupole (E2) transitions in defining a gamma-ray cascade. The operator corresponding to these types of radiation for single particle transitions are (in the laboratory reference frame),

$$
\begin{aligned}
& \operatorname{MEI}(\mu)=e\left(I-\frac{Z}{A}\right) r Y_{I \mu}(\theta,) \\
& \operatorname{ME} 2(\mu)=e\left(1+\frac{Z}{A^{2}}\right) r^{2} Y_{2 \mu}(\theta, \varphi)+\frac{3}{4 \pi} Z_{e} R_{0}^{2} \alpha
\end{aligned}
$$

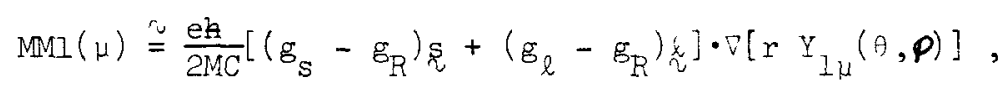

where $\alpha_{2 \mu}$ represents the quadrupole vibrational coordinates relative to the laboratory fixed coordinate axes, $\mathbb{2}$ and $\ell$ denote the spin and orbital angular momentum operators, $M$ and $e$ are the nucleon mass and charge, $R_{0}$ is the nuclear radius, and $g_{\ell}, g_{S}$, and $g_{r}$ are the spin, orbital, and collective angular momentum gyromagnetic ratios, respectively. The matrix elements, which define reduced radiative transition "partial widths," are proportional to the quantity

$$
\left.\int \Psi_{f^{*}{ }^{*}{ }^{*} \Psi_{i}} d_{i}^{i}\right|^{2}
$$

where $\Psi_{1}$ and $\Psi_{f}$ refer to pre- and post-transition states of the nucleus.

Ratios of the products of reduced partial widths and factors of the form $\left(E_{i}-E_{f}\right)^{2 l+1}, \ell=1$ for aipole transitions, $\ell=2$ for quadrupole, define probabilities for particular modes of radiative decay, $P_{\text {if }}$ by way of the expression,

where,

$$
P_{i f}=\frac{P_{i f}}{\sum_{f^{\prime}} P_{i f^{\prime}}}
$$

$$
P_{i f} \approx\left|\int \Psi_{f}^{*} M_{\ell} \Psi_{i}{ }_{i}\right|^{2}\left(E_{i}-E_{f}\right)^{2 \ell+1}
$$

Recall that $\Psi_{i}$ and $\Psi_{f}$ are actually sums over appropriate basis functions. Each matrix element is thus made up of the sum of as many pairs of initial and final state wave function components as are coupled by the operator $M_{\ell}$. In order to save computing time the radial portion of the above integral is parameterized, calculated, and stored to be called from memory as each matrix element component is evaluated. 
Nuclear energy levels, level spins, and parity, and radiative transition probabilities calculated in the preceding two phases of the calculation are then input to the gamma-ray cascade model for the calculation of a gamma-ray spectrum. The capture of a neutron excites the compound nucleus to an energy equal to the binding and kinetic energies of the neutron. The conpound nucleus then may de-excite by emitting gamma rays. In medium and heavy nuclei the evel spectrum has typically been measured (resolved) to an energy, $E_{C}$, of from $1 / 2$ to $3 \mathrm{MeV}$ above the ground state. The situation is pictured in Figure 2. For $E>E_{C}$, one defines a spin and parity dependent level density function, $\rho_{\mathrm{J} \pi}(E)$. The partial width, $\Gamma_{\gamma \ell}^{J \pi}$, corresponding to the excitation of a spin $J$, parity $\pi$ state given the emission of a gamma ray of angular momentum $l$ from a state in the continuum has the form

$$
\begin{aligned}
\Gamma_{\gamma \ell}^{J \pi}(E) & =\int_{E_{C}}^{E}\left(E-E^{\prime}\right)^{2 \ell+1} \rho_{J \pi}\left(E^{\prime}\right) d E^{\prime}+\sum_{k=1}^{N}\left(E-E_{k}\right)^{2 \ell+1} \delta[\pi-\pi(k)] \\
& x \delta[J-J(k)],
\end{aligned}
$$

where $\mathrm{N}$ denotes the number of energy levels in the resolved region $\left(\mathrm{E}_{<}<\mathrm{E}_{\mathrm{c}}\right)$ whose energies are denoted by $E_{k}$. The programming of the cascade model is essentially the same as that involved in certain resonance integral formulations. The output of the program consists of gamma-ray energy group intensities. The gamma groups are arbitrary in magnitude.

The fourth and final stage in the calculation of gamma-ray production cross sections consists of averaging gamma-ray spectra corresponding to possible neutron capture states, generally restricted to those consistent with s- (zero orbital angular momentum) and $p$-( 1 unit of orbital angular momentum) wave neutron capture. Let $i$ and $k$ denote gamma and neutron energy groups, respectively. The cross section for the production of ith group gamma rays corresponding to the capture of a kth group neutron, $\sigma_{p}(i, \vec{k})$, is given by

$$
\sigma_{p}(i, k)=\sum_{\ell} \sum\left\langle\sigma_{J \ell}>I\left(i, k, J, \pi_{\ell}\right)\right.
$$

where $\ell$ indexes neutron angular momentum, $\pi_{2}$ denotes capture state parity, $J$ is the spin of the capture state, $\left\langle\sigma_{\mathrm{J} l}\right\rangle$ is the average capture cross section for spin $J$ states and l-wave neutrons, and I denotes the gamma-ray intensity corresponding to the indicated variables. The $\sigma(i, k)$ are thus the cross sections required for the definition of secondary gamma-ray production cross sections in gamma heating and dose calculations.

Figure 2 exhibits a comparison of measured and calculated level schemes for $239 \mathrm{U}$. The theoretical basis for the nuclear model used for the calculation was developed in the $1950^{\prime} \mathrm{s}$ by the Copenhagen school. ${ }^{-4}$ The treatment of collective excitations is similar to that expounded by Faessler, et. al. 5 The model is discussed in detail in a recent paper. ${ }^{6}$ The gamma-ray cascade model ${ }^{7}$ and the method for calculating secondary gamma-ray production cross sections ${ }^{8}$ also appear in the literature. 
ORNL-DWG 69-2533

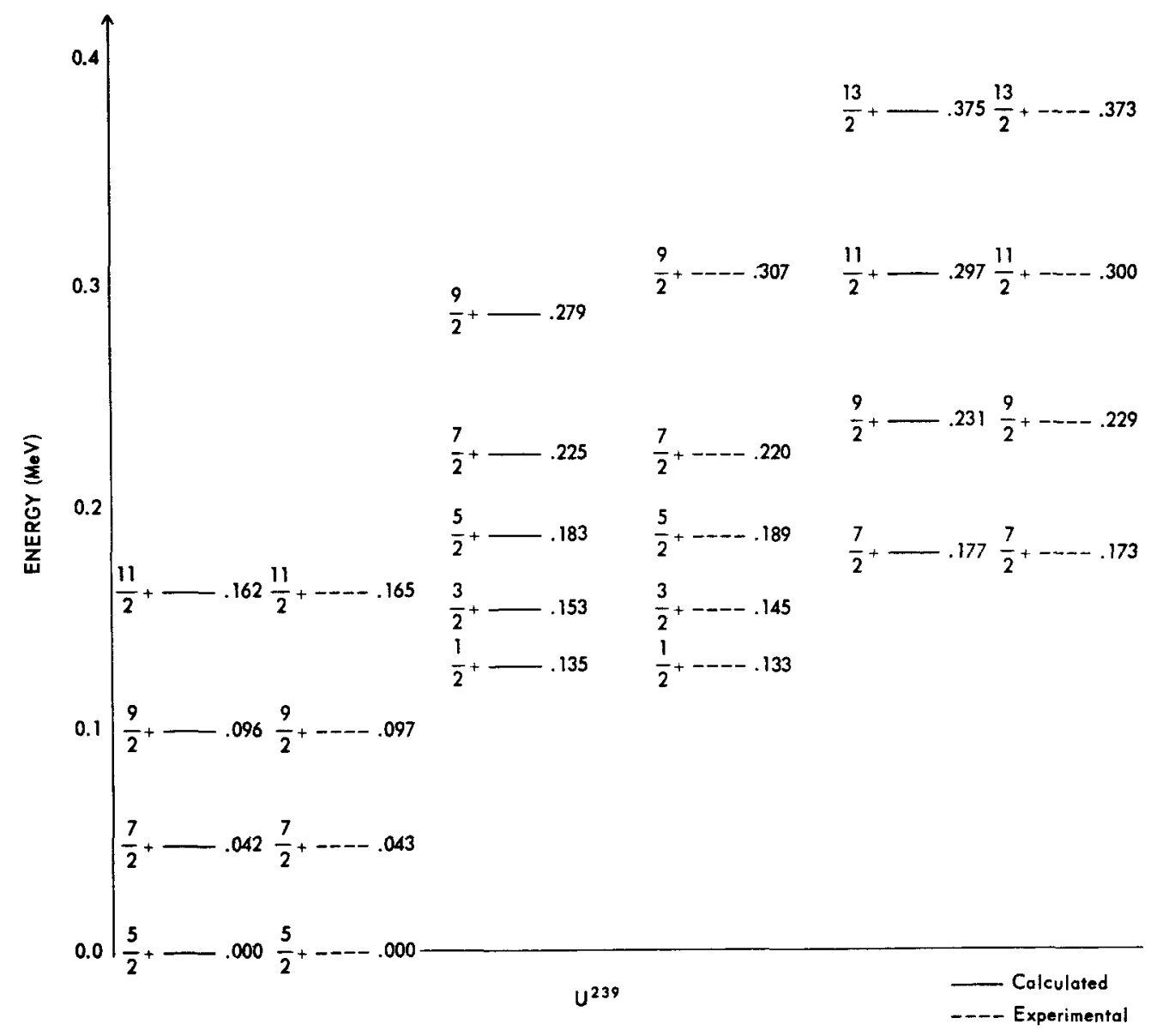

Figure 2. Comparison of Measured with Calculated Energy Level Spectra for ${ }^{239} \mathrm{U}$. 
1. A. Bohr, Kgl. Danske Videnskab. Selskab, Mat. Phys. Medd. 26, 14 (1952).

2. A. Bohr and B. R. Mottelson, Kgl. Danske Videnskab. Selskab, Mat. Phys. Medd. Medd. 27, 16 (1953).

3. S. G. Nilsson, Kgl. Danske Videnskab. Selskab, Mat. Phys. Medd. 29, 16 (1955).

4. B. R. Mottelson and S. G. Nilsson, Kgl. Denske Videnskab. Selskab, Mat. Fys. Skrifter 1, 8 (1959).

5. A. Faessler, W. Greiner, and R. Sheline, "Rotation Vibration Interaction in Deformed Nuclei," Nucl. Phys. 70, 33 (1965).

6. K. J. Yost, P. H. Pitkanen, and C. Y. Fu, "A Unified Nuclear Model for the Generation of Nuclear Data," Nucl. Sci. Eng. (in press).

7. K. J. Yost, "A Method for the Calculation of Neutron-Capture Gamma-Ray Spectra," Nucl. Sci. Eng. 32, 62 (1968).

8. K. J. Yost and S. M. Kremer, "The Application of a Gamma-Ray Cascade Model to the Calculation of Neutron Energy-Dependent Capture Gamma-Ray Production Cross Sections," Nucl. Sci. Eng. (in press). 
BLANK 
SESSION VII

ENGINEERING ANALYSIS

Chairman: J. E. Olhoeft

Westinghouse Corporation, Atomic Power Division 
BLANK 


\title{
ON COMPUTATIONAL METHODS IN SOLID MECHANICS \\ AND STRESS ANALYSIS
}

\author{
Y. R. Rashid \\ Gulf General Atomic Incorporated \\ San Diego, California
}

\section{INTRODUCTION}

Computational problems in solid mechanics generally begin with identification of a structure's configuration and its environment. The former defines the geometry of the structure, which may be one of many types, such as a beam, plate, shell, or general three-dimensional solid. The latter, the environment, imposes on the geometry a set of governing equations which consist of strain-displacement relations, constitutive equations, compatibility relations, boundary conditions, and equilibrium equations. Both the geometry and the environment strongly influence the selection of the computational procedure appropriate to the problem.

The development of computational methods in solid mechanics has emphasized variational procedures in recent years. One method in particular, the finite element method, evolved as an extension of the Rayleigh-Ritz procedure and has become the most extensively used numerical technique in computational mechanics. With the aid of the digital computer, the development of this method has progressed at a phenomenal rate, and the method has come to represent the accepted state of the art in most areas of stress analysis. The major factor responsible for this rapid growth is the method's versatility in treating arbitrary geometries, boundary conditions, and material inhomogeneities.

Despite the significant contributions made by many workers in the general area of finite element analysis, many important problems in stress analysis have received little attention. However, these problems are not unique to the finite element method but are of a fundamental nature and deal primarily with the characterization of the environment. Unfortunately, in many instances the development of the finite element method bypassed this fundamental part of computational problems, and emphasis on expediency rather than rigor has marred many of the method's applications.

In the present paper, we discuss the progress made in the various areas of finite element application. Since a complete survey is virtually impossible because of space limitations, the present survey is, by necessity, arbitrarily selective. The examples given represent cases of recent developments. 


\section{BASIC CONCEPTS}

In the classical Ritz variational procedure, the solution of a boundary value problem is obtained by minimizing a functional that characterizes the problem over the entire domain of interest. The solution is approximated by relatively complete sequences of functions which satisfy appropriate smoothness requirements in the interior of the domain and geometric boundary conditions on the surface. The main difficulty encountered in applying this procedure is finding the appropriate functions which satisfy the completeness and smoothness requirements over the entire domain.

The finite element method generalizes the Ritz concept in a manner which removes this difficulty. We consider a three-dimensional continuum occupying a closed region $\mathrm{R}$ with boundary $\Sigma$. Let the vector field $\{\mathrm{u}(\mathrm{x})\}$, defined in a cartezian reference frame, be continuous in $R$. We assume that $\{u(x)\}$ can be locally expressed as

$$
\left\{u^{m}(x)\right\}=[\phi(x)]\left\{q^{m}\right\}
$$

in a closed subregion $R_{m}$ of $R$. The matrix "shape" function $[\phi(x)]$ is smooth in $R_{m}$ and the vector $\left\{q^{m}\right\}$ is unknown. If $[\phi(x)]$ preserves the continuity of $\{u(x)\}$ across the boundaries of $R_{m}$, then Eq. (1) can be generalized to all similar subregions $R_{1}, R_{2}, \ldots R_{M}$ of $R$.

If the generalized stresses $\left\{\sigma^{\mathrm{m}}\right\}$ and the generalized strains $\left\{\epsilon^{\mathrm{m}}\right\}$ satisfy the relations

$$
\begin{aligned}
& \left\{\sigma^{\mathbf{m}}\right\}=\left[H^{\mathbf{m}}\right]\left\{\varepsilon^{\mathbf{m}}-\theta^{\mathbf{m}}\right\} \\
& \left\{\varepsilon^{\mathbf{m}}\right\}=\left\{\mathrm{Lu}^{\mathbf{m}}\right\}
\end{aligned}
$$

in $R_{m}$, and $\left\{\sigma^{m}\right\}$ satisfy the specified tractions $\left\{S^{m}\right\}$ on $\Sigma_{m}$, then the potential energy functional which characterizes the problem is

$$
\begin{aligned}
& U=\sum_{m=1}^{M} \frac{1}{2} \int_{R_{m}}\left\{L u^{m^{m}}\right\}^{T}\left[H^{m}\right]\left\{L u^{m}\right\} d R_{m}-\int_{R_{m}}\left\{L u^{m_{m}}\right\}\left\{\theta^{T}\right\} d R_{m} \\
& -\int_{R_{m}}\left\{u^{m_{m}}\right\}^{T}\left\{g^{m^{m}}\right\} d R_{m}-\int_{\Sigma_{m}}\left\{u^{m^{T}}\left\{s^{m}\right\} d \Sigma_{m}\right.
\end{aligned}
$$

Equations (2) and (3) are the generalized stress-strain and generalized strain-displacement relations, respectively. The matrix $\left[\mathrm{H}^{\mathrm{m}}\right]$ contains the material constants, $\left\{\theta^{\mathrm{m}}\right\}$ are the temperature strains, $\left\{\mathrm{g}^{\mathrm{m}}\right\}$ are the body forces, and $\mathrm{L}$ is a matrix differential operator defined by the strain-displacement relations appropriate to the problem. If $U$ is stationary in $R$, i.e., 
then $\left\{\mathrm{u}^{\mathrm{m}}(\mathrm{x})\right\}$, and consequently $\{\mathrm{u}(\mathrm{x})\}$, represent the variational solution of the problem.

To make further use of Eq. (4), we require the existence of a one-to-one correspondence between the unknown constants $\left\{q^{m}\right\}$ and the values of the vector field $\{u(x)\}$ at a finite number of points (nodes) located on the surfaces of $\mathrm{R}_{\mathrm{m}}$. We further require that the nodes located on internal surfaces common to adjacent subregions coincide. Denoting these values by the vector $\mathrm{V}$, we have from Eq. (1)

$$
\left\{u^{m}(x)\right\}=[\phi(x)]\left[\phi_{0}\right]^{-1}\left\{v^{m}\right\}
$$

provided $\left[\phi_{0}\right]^{-1}$ exists; $\left[\phi_{o}\right]$ is obtained by evaluating Eq. (1) at the nodes of $R_{m}$, and $\left\{v^{m}\right\}$ is a subvector of $\{V\}$. Substituting Eq. (6) in Eq. (4) and carrying out the variation with respect to $\{\mathrm{V}\}$, we obtain the following system of equations:

$$
\left\{\mathrm{f}^{\mathrm{m}}\right\}=\left[\mathrm{k}^{\mathrm{m}}\right]\left\{\mathrm{v}^{\mathrm{m}}\right\}
$$

where

$$
\begin{aligned}
\left\{f^{\mathrm{m}}\right\}= & {\left[\phi_{0}^{-1}\right]^{\mathrm{T}}\left\{\int_{\mathrm{R}_{\mathrm{m}}}\left([\mathrm{L} \phi]^{\mathrm{T}}\left\{\theta^{\mathrm{m}}\right\}+[\phi]^{\mathrm{T}}\left\{\mathrm{g}^{\mathrm{m}}\right\}\right) d \mathrm{R}_{\mathrm{m}}\right.} \\
& \left.+\int_{\Sigma_{\mathrm{m}}}[\phi]^{\mathrm{T}}\left\{\mathrm{S}^{\mathrm{m}}\right\} \mathrm{d} \Sigma_{\mathrm{m}}\right\}
\end{aligned}
$$

and

$$
\left[k^{m}\right]=\left[\phi_{0}^{-1}\right]^{T}\left(\int_{R_{m}}[L \phi]^{T}[H][L \phi] d R\right) ~\left[\phi_{0}^{-1}\right]
$$

Equations of type (7) are derived for all $R_{i}, i=1,2, \ldots M$, and then merged to obtain the equilibrium equations of the entire solid $\mathrm{R}$ in the form

$$
\{\mathrm{F}\}=[\mathrm{K}]\{\mathrm{V}\}
$$

The matrix $[K]$ is symmetric and generally sparse. 
These derivations have been presented in a generalized sense. Specific forms of the various relations, e.g., stress-strain, strain-displacement, boundary conditions, are considered problen, details and can be found in the referenced literature. In the following sections, we discu application of this general procedure to various problems in solid mechanics.

\section{TWO-DIMENSIONAL ELASTIC SOLIDS}

This method of analysis was applied first to problems in plane elastostatics by Turner $\epsilon$ al. ${ }^{1}$ and, subsequently, by Clough ${ }^{2}$ and then to axisymmetric solids by Clough and Rashid These two types of problems belong to the general class of two-dimensional continuou structures. The displacement field $\{u(x)\}$ consists of two components $u_{1}(x)$ and $u_{2}(x)$ define in the cartesian coordinate system and the cylindrical coordinate system for plane an:' axisymmetric problems, respectively. Various element shapes and the associated approximati. can be found in the referenced literature. The stress-strain and strain-displacement relaticor required for the previous derivations are the classical elasticity equations. 4

The state of the art of two-dimensional elastic analysis is well developed. ${ }^{5-7} ;$ * computational problems associated with two-dimensional problems present no particu! difficulties, and many computer program, for soiving then lave been ir. "sistence for a numis of years.

\section{THREE-DIMENSIONAL ELASTIC SOLIDS}

Because of the many computational difficulties associated with three-dimensional probley. the development of three-dimensional analysis has not progressed verv rapidly $F_{?}$ :

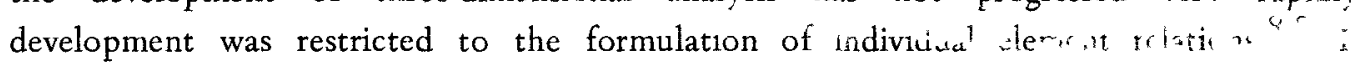
number of equations which nceded to be solved discouraged any practical appucui: Although equation solvers for large systems based on Gaussian elimination had been develop they were restricted to bandwidths of order 400 for a $32 \mathrm{~K}$ computer, ${ }^{10}$ which are consider inadequate for three-dimensional problems. Despite this limitation, results of three-dimension calculations were presented ${ }^{11}$ based on the standard Gaussian elimination. However, experien' indicates that Gaussian elimination for general three-dimensional problems is net economically feasible technique. A special class of thref d noms, nal at $n$, axisymmetric geometry but nonsymmetric loading was treat d by whon. 12

A general solution method, called the alternating component iteration method, has hav developed for problems involving three or more dependent variables. Systems of order $15,1 \mathrm{n}$. and of bandwidth exceeding 1,100 can be economically solved. The details of the method ?* discussed in Refs. 13 and 14. A brief outline of the essential steps is given below.

If $P$ and $Q$ are lists of field and source points, respectively (any set of nodal points $P{ }^{*}$. $Q$ can be nondistinct), then equilibrium equations (10) can be written in the follow partitioned form: 


$$
F_{i}(P)=\sum_{j=1}^{m} k_{i j}(P, Q) v_{j}(Q) ; \quad i=1,2, \ldots, m
$$

where $\mathrm{m}$ is the number of components (dependent variables) that is equal to the number of unknowns at each node, $F_{i}(P)$ and $V_{j}(Q)$ are the force and displacement vectors of the $i^{\text {th }}$ component, and $K_{i j}(P, Q)$ are the stiffness submatrices that couple the $i^{\text {th }}$ and $j^{\text {th }}$ components and nodal point lists $P$ and $Q$. An iterative solution of Eq. (11) can be obtained by successively applying the following equations:

$B_{i}(P){ }^{(s)}=F_{i}(P)-\sum_{j=1}^{i-1} k_{i j}(P, Q) v_{j}(Q)(s)-\sum_{j=i}^{m} k_{i j}(P, Q) v_{j}(Q)(s-1)$

and

$$
\Delta V_{i}(P)^{(s)}=K_{1 i}(P, Q)^{-1} B_{i}(Q)
$$

$$
\begin{gathered}
\mathrm{V}_{i}(\mathrm{P})^{(\mathrm{s})}=\mathrm{V}_{i}(\mathrm{P})^{(\mathrm{s}-1)}+\lambda \Delta \mathrm{V}_{i}(\mathrm{P}) \\
(\mathrm{s}) \\
i=1,2, \ldots, \mathrm{m}
\end{gathered}
$$

where $\lambda$ is an overrelaxation factor in the range $1<\lambda<2$ and $s$ is the iteration cycle; $\mathrm{K}_{\mathrm{ii}}(\mathrm{P}, \mathrm{Q})$ are block-tridiagonal symmetric matrices. The solution for the incremental displacement $\Delta \mathrm{V}_{\mathrm{i}}(\mathrm{p})^{(\mathrm{s})}$ is performed by Gaussian elimination and not by matrix inversion as implied in Eq. (13). This solution reduces to the successive application of the following recursion formulas:

$$
\begin{aligned}
& \mathrm{K}^{*}{ }_{i i}\left(\mathrm{P}_{\alpha}, \mathrm{Q}_{\alpha}\right)=\mathrm{K}_{i i}\left(\mathrm{P}_{\alpha}, \mathrm{Q}_{\alpha}\right)-\mathrm{K}_{i i}\left(\mathrm{P}_{\alpha-1}, \mathrm{Q}_{\alpha}\right)^{\mathrm{T}} \text {. } \\
& \mathrm{K}_{i i}^{*}\left(\mathrm{P}_{\alpha-1}, \mathrm{Q}_{\alpha-1}\right)^{-1} \mathrm{~K}_{i i}\left(\mathrm{P}_{\alpha-1}, \mathrm{Q}_{\alpha}\right) \\
& \mathrm{B}_{i}^{*}\left(\mathrm{P}_{\alpha}\right)^{(s+1)}=\mathrm{B}_{i}\left(\mathrm{P}_{\alpha}\right)^{(s+1)}-\mathrm{K}_{i \mathrm{i}}\left(\mathrm{P}_{\alpha-1}, \mathrm{Q}_{\alpha}\right)^{\mathrm{T}} \\
& K_{i i}^{*}\left(P_{\alpha-1}, Q_{\alpha-1}\right)^{-1} \cdot B_{i}^{*}\left(P_{\alpha-1}\right)^{(s+1)}
\end{aligned}
$$




$$
\begin{aligned}
\Delta V_{i}\left(Q_{\alpha}\right)^{(s+1)}=K_{i i}^{*}\left(P_{\alpha}, Q_{\alpha}\right)^{-1} \cdot B_{i}^{*}\left(P_{\alpha}\right)^{(s+1)}-K_{i i}\left(P_{\alpha}, Q_{\alpha+1}\right) \cdot V_{i}\left(Q_{\alpha+1}\right)^{(s+1)} \\
1 \leq \alpha \leq M
\end{aligned}
$$

where $M$ is the number of blocks of $\mathrm{K}_{\mathrm{ii}}$.

To speed the convergence of the iterative process, the following formula is applied in place of Eq. (14):

$$
\begin{aligned}
v_{j}(n+s)=v_{j}(s)+\lambda \Delta v_{j} & (s) \sum_{i=2}^{n}\left(\frac{\Delta v_{j}^{(s)}}{\Delta v_{j}^{(s-1)}}\right)^{i} \\
j & =1,2, \ldots, N ; n=2,3, \ldots
\end{aligned}
$$

where $v_{j}$ is the $j^{\text {th }}$ displacement unknown and $N$ is the total number of unknowns. Formula (18) predicts, approximately, the $(n+s)^{\text {th }}$ iterate from the displacement increments obtained at cycles $\mathrm{s}-1$ and $\mathrm{s}$.

Convergence of the method is monitored by the criterion

$$
\lim _{\substack{s \rightarrow \infty \\ 1 \leq i \leq N}}\left|\Delta v_{i}(s)\right| \underset{\max }{\rightarrow} \delta
$$

where $\delta$ is a specified small number.

Results of three-dimensional calculations based on this method are given in Fig. 1.

\section{PLATES AND SHELLS}

Problems of the plate and shell type present a fundamental difficulty in selecting the displacement approximations, Eq. (1), which satisfy the appropriate smoothness requirements. Unlike the three-dimensional problem in which the displacement field $\{u(x)\}$ is required to be in class $c^{1}$, here $\{u(x)\}$ must be in $c^{2}$; i.e., the function and its derivatives must be continuous. This was a major source of difficulty in shell analysis, particularly in triangular element formulation. The problem has been solved for plate bending by Clough and Toucher, ${ }^{15}$ who further generalized the concept of discretization to the subregions $R_{m}$ where in each $R_{m}$ the local displacement $\left\{\mathrm{u}^{\mathrm{m}}(\mathrm{x})\right\}$ consisted of three displacement patterns, each of which is defined over a subregion of $R_{m}$. To satisfy smoothness and continuity requirements across the boundaries of $R_{m}$, they introduced homogeneous compatibility relations on the subvector functions of $\left\{u^{\mathrm{m}}(\mathrm{x})\right\}$. 


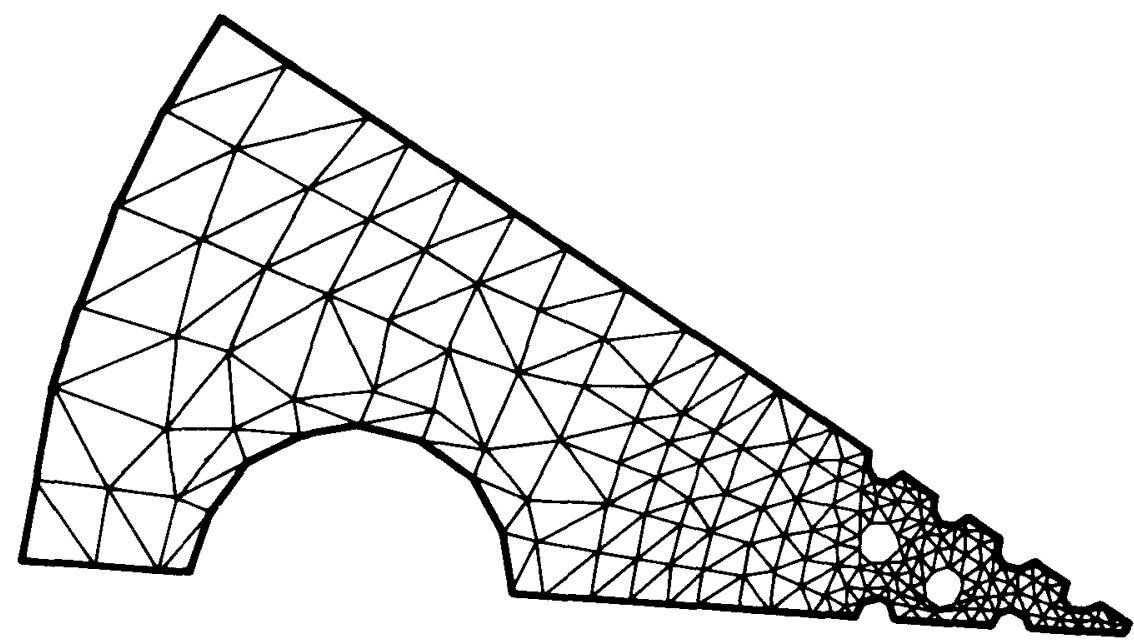

PLAN VIEW OF THE THREE-DIMENSIONAL GRID

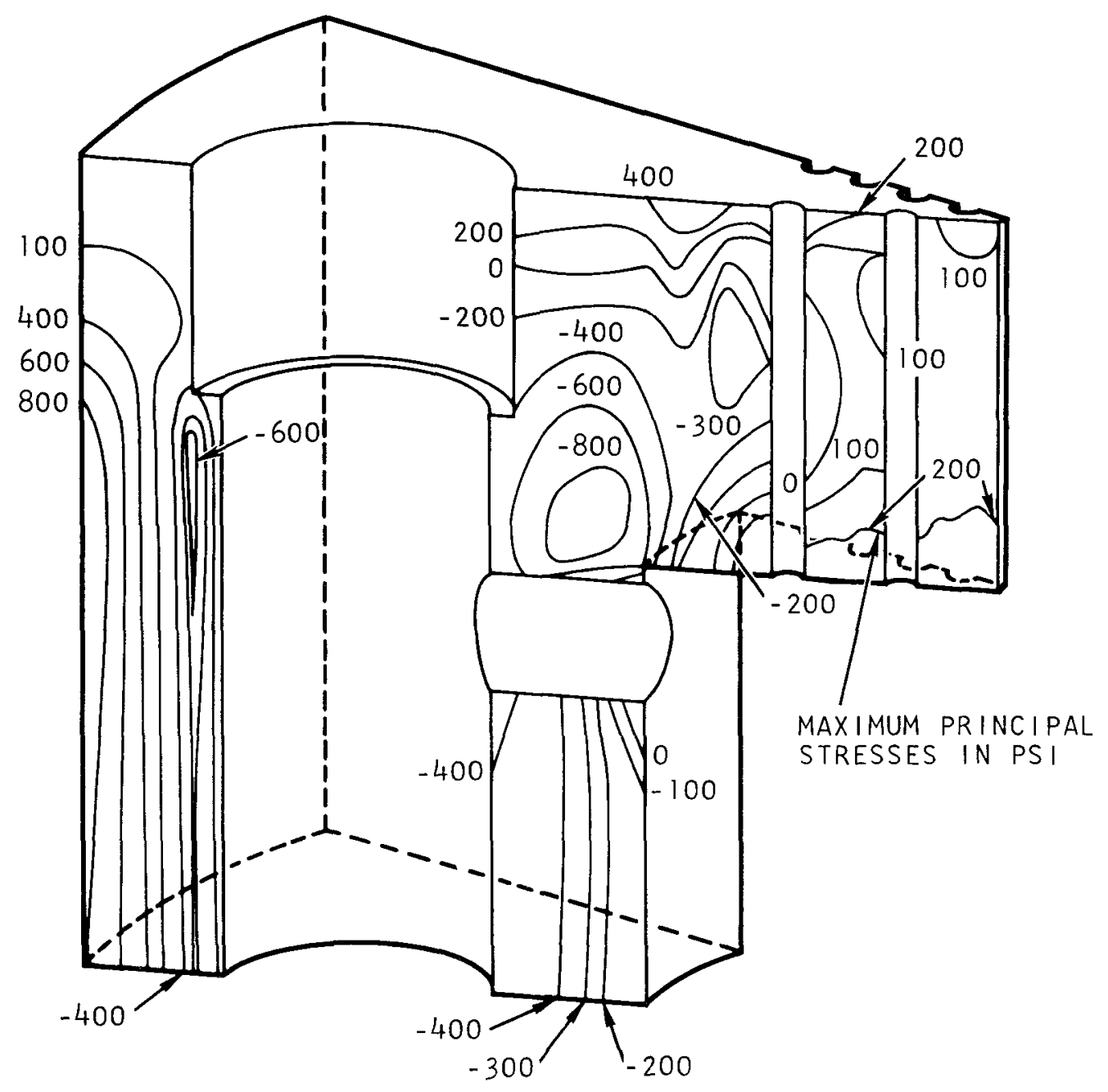

Fig. 1. Example of three-dimensional analysis - prestressed concrete reactor vessel 
Generalization of this type of plate analysis to shell problems was given by Clough and Johnson ${ }^{16}$ and by Prince and Rashid. ${ }^{17}$ Some numerical results are given in Fig. 2.

A different treatment of this problem was given by Herrmann, ${ }^{18}$ who based his formulation on the Reissner variational principle ${ }^{19}$ in which the displacement field as well as the stress field was varied. Numerical results based on this formulation were given by Herrmann and Campbell. ${ }^{20}$

\section{VISCOELASTICITY}

Quasi-static problems are treated by first extending the functional which characterizes the problem to include time. This extended functional is given in the expression

$$
\begin{aligned}
U= & \sum_{m=1}^{M}\left(\frac{1}{2} \int_{R_{m}}\left\{L u^{m}\right\}^{T} *\left[H^{m}\right] *\left\{I u^{m}\right\} d R_{m}\right. \\
& \int_{R_{m}}\left\{L u^{m}\right\}^{T} *\left\{\theta^{m^{m}}\right\} d R_{m} \\
& \left.\int_{R_{m}}\left\{u^{m_{m}}\right\}^{T} *\left\{g^{m_{m}}\right\} d R_{m}-\int_{\Sigma} t *\left\{u^{m^{T}}{ }^{T} *\left\{s^{m}\right\} d \Sigma_{m}\right]\right)
\end{aligned}
$$

in which the convolution form of two functions in space and time is defined by

$$
w_{1} * w_{2}=\int_{0}^{\infty} w_{1}(x, t-\tau) w_{2}(x ; \tau) d \tau
$$

The forms of the displacement approximations, Eq. (1), and the strain-displacement relations, Eq. (3), remain unchanged except for the fact that $\left\{q^{m}\right\}$ is a function of time. The stress-strain relations, Eq. (2), become

$$
\left\{\sigma^{m}(x, t)\right\}=\int_{0}^{t}\left[H^{m}(t-\tau)\right]\left\{\frac{\partial \varepsilon^{m}(x, \tau)}{\partial \tau}-\frac{\partial \theta^{m}(x, \tau)}{\partial \tau}\right\} d \tau
$$

Numerical calculations based on this formulation are given in Refs. 21 and 22. 

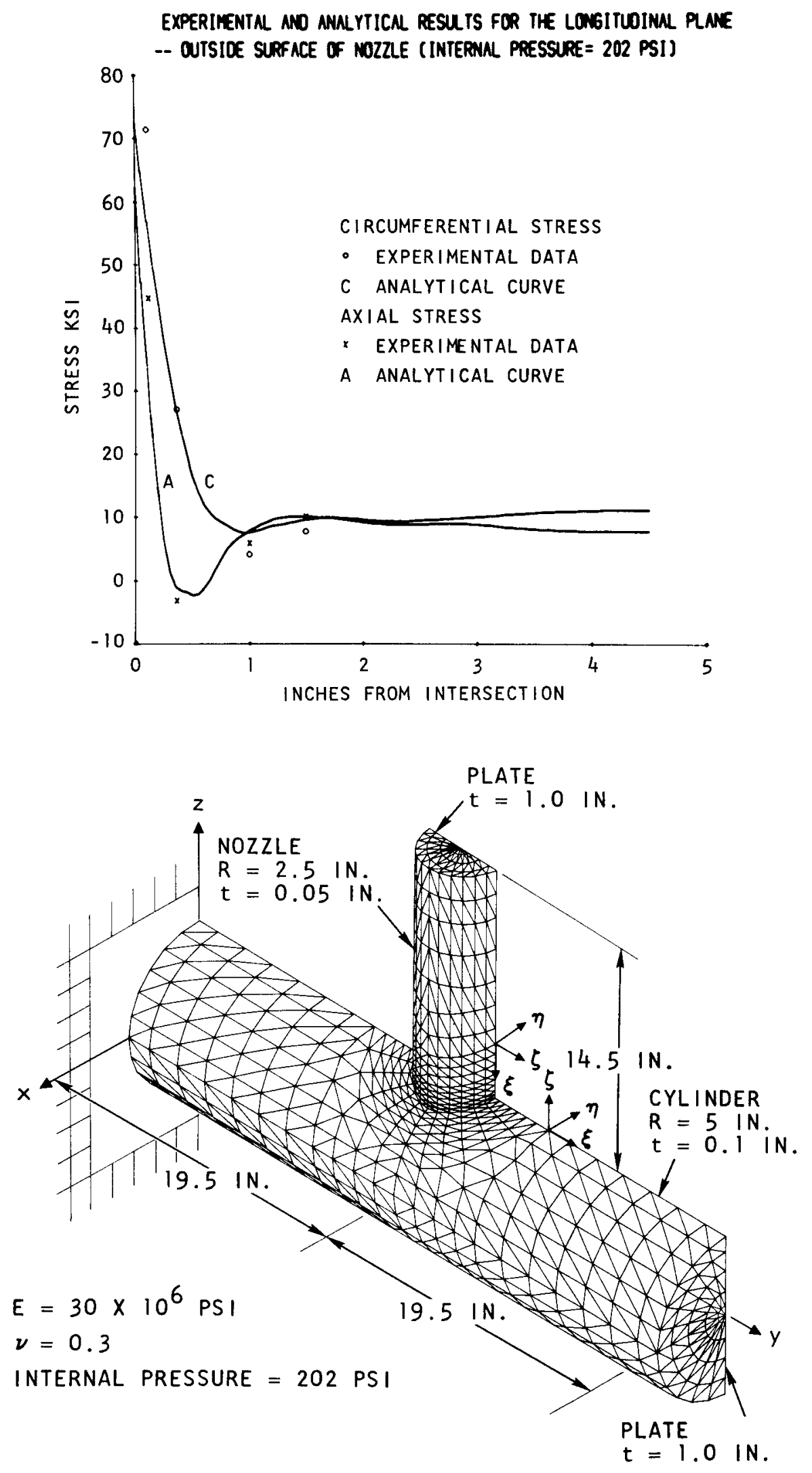

Fig. 2. Example of three-dimensional thin shell analysis 
Some of the materials exhibit this type of behavior only when they are subjected to certain environments, e.g., graphite structures under irradiation. Generalizations of viscoelastic formulation to irradiated materials are given by Rashid and Chang, ${ }^{23}$ and results of some calculations are shown in Fig. 3.

\section{INELASTIC ANALYSIS}

Some of the more important structures which have received considerable attention in recent years, especially in the nuclear industry, are prestressed concrete pressure vessels. These are composite three-dimensional structures in which various steel elements, e.g., prestressing cables, reinforcement bars, and liner, interact with concrete. Because concrete creeps, especially under high temperatures, these structures exhibit a time-dependent response of the type discussed earlier. Furthermore, the assessment of their safety factors is an important consideration in the design of these structures. This involves an elastoplastic analysis in which the internal pressure is varied statically or over a period of time. Nonlinear analysis of this type has been developed for two-dimensional structures; some numerical results are given in Ref. 24 .

\section{HEAT CONDUCTION}

Application of the finite element variational procedure to heat conduction and diffusion problems was given by Zienkiewicz and Cheung for the steady state problem ${ }^{25}$ and Wilson and Nickell for the transient problem. ${ }^{26}$ The variational formulation follows parallel steps as before, except that expressions associated with the displacement field are replaced by the appropriate temperature variables. This formulation is summarized as follows.

The scalar temperature field $Q(x, t)$ is approximated in each subregion $R_{m}$ by the expression

$$
Q^{m}(x, t)=\left\langle\phi(x)>\left\{q^{m}(t)\right\}\right.
$$

The temperature gradients are obtained by differentiating Eq. (23):

$$
\left\{L Q^{m}(x, t)\right\}=[L \phi(x)]\left\{q^{m}(t)\right\}
$$

where $\mathrm{L}$ is the appropriate matrix differential operator. In terms of the convolution notation introduced in Eq. (21), the functional which characterizes the heat conduction problem is

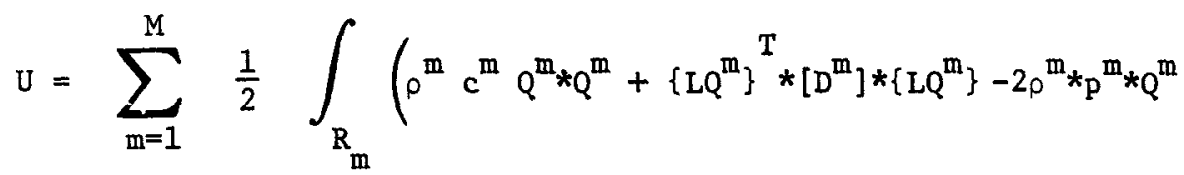

$$
\begin{aligned}
& \left.-2 \rho^{m} c^{m} Q_{0}^{m_{*}} Q^{m}\right) d R_{m}-\int_{\Sigma_{m}} f_{i}^{m} n_{i} * Q^{m} d \Sigma_{m}-\int_{\Sigma} h^{m} *\left(Q_{e}^{m}-\frac{1}{2} Q^{m}\right) * Q^{m} d \Sigma_{m}
\end{aligned}
$$




\section{AXIAL STRESSES IN PS I}

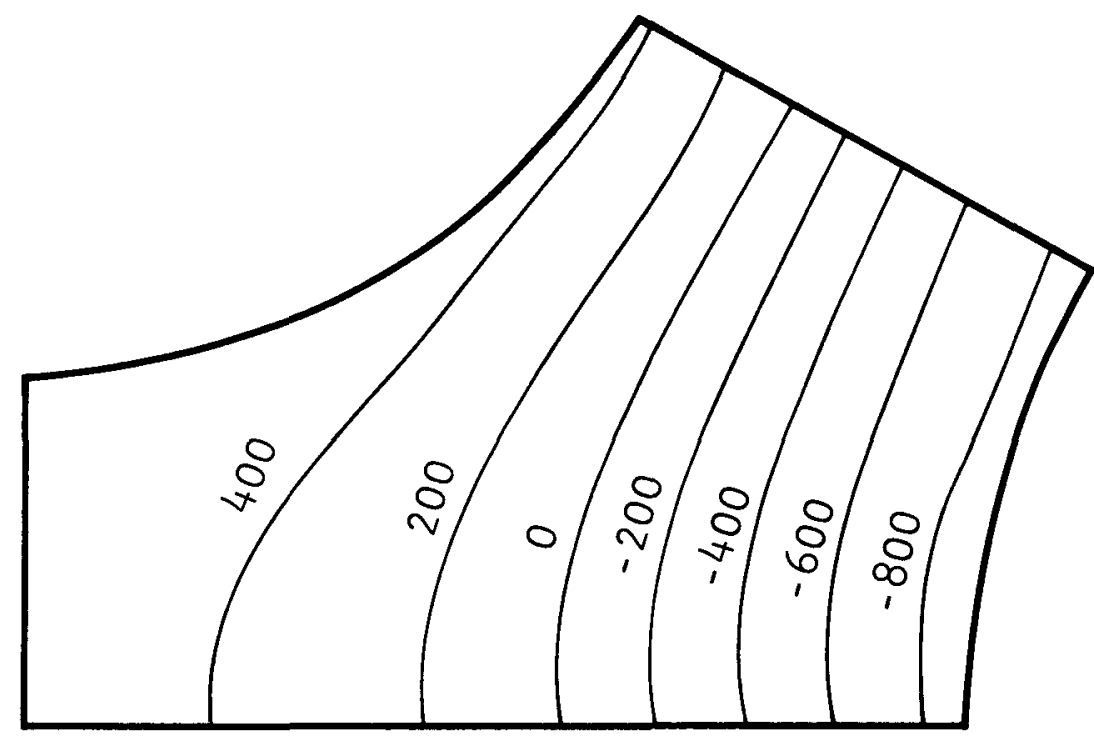

NEUTRON DOSE $=2.4 \times 10^{21}$ NVT

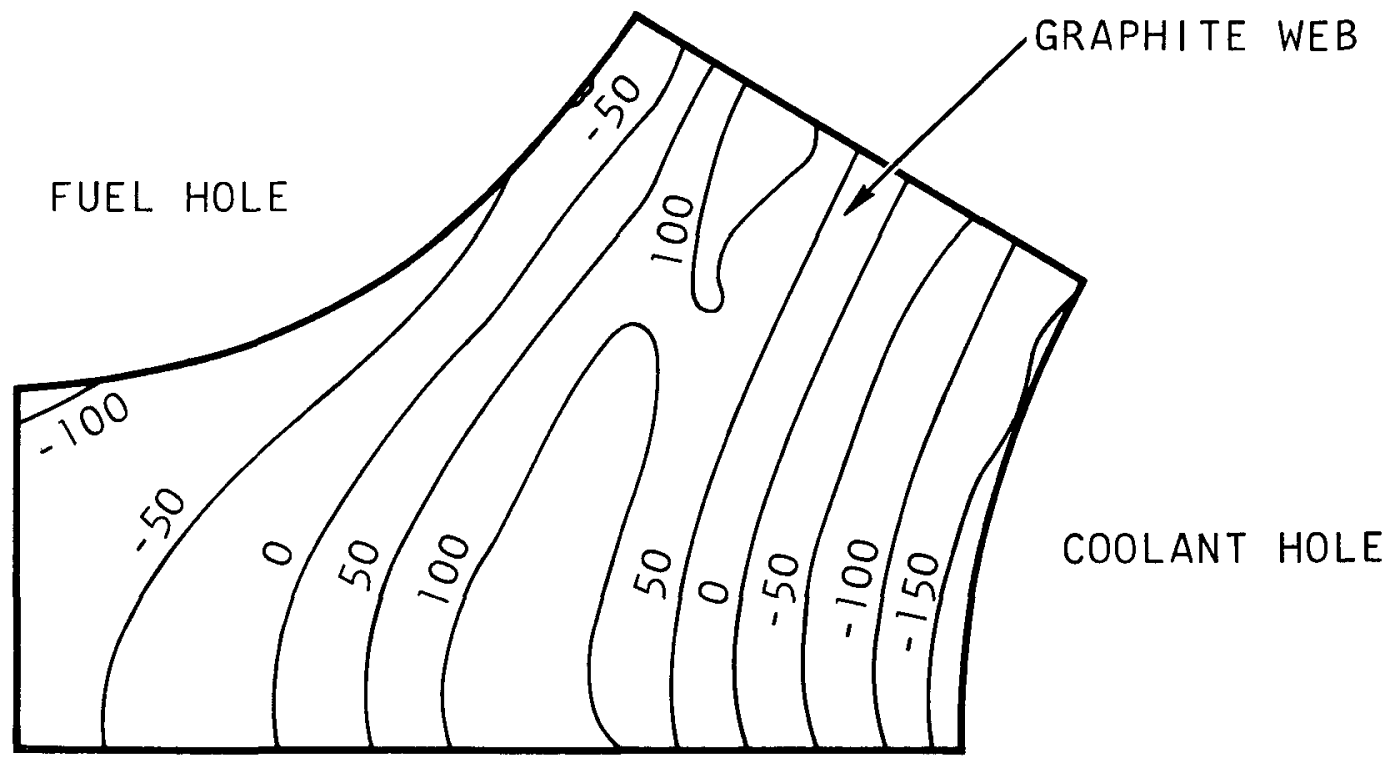

NEUTRON DOSE $=5.0 \times 10^{21} \mathrm{NVT}$

Fig. 3. Stresses in graphite fuel block due to temperature and irradiation 
where $\rho^{\mathrm{m}}=$ the mass density, $c^{\mathrm{m}}=$ the heat capacity per unit mass, $\mathrm{D}^{\mathrm{m}}=$ the conductivity tensor, $Q_{0}^{m}=$ the initial temperature, $f_{i}=$ the prescribed heat flux at the boundary, $n_{i}=$ the outward unit normal to $\Sigma_{\mathrm{m}}, \mathrm{h}^{\mathrm{m}}=$ the heat transfer coefficient for the boundary layer, and $\mathrm{Q}_{\mathrm{e}}^{\mathrm{m}}=$ the known temperature outside the boundary layer.

The above quantities are defined for the subregion $R_{m}$. If $\gamma(t)$ represent the vector of nodal temperature in $\mathrm{R}$, from $\mathrm{Eq}$. (23)

$$
Q^{\mathfrak{m}}(x, t)=\left\langle\phi(x)>\left[\phi_{0}^{\mathbb{m}}\right]^{-1}\left\{\gamma^{\mathbb{m}}(t)\right\}\right.
$$

Substituting Eq. (26) in (25) and carrying out the variation of $U$ with respect to $\gamma(t)$ yields the following equations for the entire region $\mathrm{R}$ :

$$
[C]\{\gamma(t)\}+[K]\{\gamma(t)\}=[C]\{\gamma(0)\}+\{E(t)\}
$$

where

$$
\begin{aligned}
& {[C]=\sum_{m=1}^{M} \int_{R_{m}} \rho^{m_{c} m}\langle\phi\rangle^{T}\langle\phi\rangle \mathrm{dR}_{\mathrm{m}}} \\
& {[K]=\sum_{m=1}^{M} \int_{R_{m}}[L \phi]^{T}\left[D^{m}\right][L \phi] d R_{m}+\int_{\Sigma_{m}} h^{m}\left\langle\phi^{T}\right\rangle\langle\phi\rangle d \Sigma_{m}}
\end{aligned}
$$

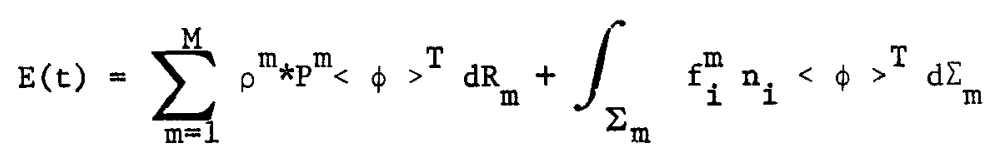

$$
\begin{aligned}
& +\int_{\Sigma_{m}} Q_{e}^{m^{*} h^{m}<\phi>T} d \Sigma_{m}
\end{aligned}
$$

This derivation can be applied to two- and three-dimensional problems, provided that in each problem the appropriate element shape is considered; the shape function $\langle\phi(x)\rangle$ can be of the same form as the displacement shapes.

\section{DYNAMICS}

Dynamic analysis of lumped-parameter and continuous systems based on the classical mode superposition approach is well known. Application of this approach to the finite element method is an obvious extension. The elastostatic procedure described earlier can be modified to include a velocity field consistent with the displacement approximations. Such modification was 
given by Archer. ${ }^{27} \mathrm{~A}$ general analysis procedure as it applies to the particular problem of earthquake response was given by Clough and Chopra. ${ }^{28}$

COUPLED INITIAL BOUNDARY VALUE PROBLEMS

We have already discussed three types of initial boundary value problems, namely, viscoelasticity, transient heat conduction, and vibration problems. Given the right environment, coupling between the thermal and mechanical states due to heat generation caused by dilatational deformations may become significant. This problem was treated by Nickell and Sackman, ${ }^{29}$ who supported their results with several numerical examples.

Another important type of initial boundary value problems is wave propagation. Traditionally, numerical as well as closed form solutions of the problem have been limited by many analytical and computational difficulties. Recently, Becker and Nickell ${ }^{30}$ presented a variational solution of the problem of stress wave propagation thermoviscoelastic material. Numerical results were given for one-dimensional problems.

\section{ACKNOWLEDGMENT}

This work was supported in part by Union Carbide Corporation, Nuclear Division, under Subcontract 2848 (Prime Contract W-7405-eng-26). 


\section{REFERENCES}

1. M. J. Turner, R. W. Clough, H. C. Martin, and L. J. Topp, "Stiffness and Deflection Analysis of Complex Structures," J. Aeron. Sci., 23, 805-823 (1956).

2. R. W. Clough, "The Finite Element Method in Plane Stress Analysis," in Proceedings, American Society of Civil Engineers, Second Conference on Electronic Computations (held in Pittsburgh, September 1960).

3. R. W. Clough and Y. R. Rashid, "Finite Element Analysis of Axisymmetric Solids," J. Eng. Mech. Div. Am. Soc. Civil Engrs., 91, 71-85 (1965).

4. I. S. Sokolnikoff, Mathematical Theory of Elasticity, 2d ed. (McGraw-Hill, New York, 1956).

5. E. L. Wilson, Finite Element Analysis of Two-dimensional Structures, Report 63-2, Structural Engineering Laboratory, University of California, Berkeley (June 1963).

6. E. B. Becker and J. J. Brisbane, Application of the Finite Element Method to Stress Analysis of Solid Propellant Rocket Grains, Vol. 1, Special Report S-76, Rohm and Haas Company, Redstone Arsenal Research Division (November 1965).

7. Y. R. Rashid, "Analysis of Axisymmetric Composite Structures by the Finite Element Method," Nucl. Eng. Design, 3, 163-182 (1966).

8. R. H. Galagher, J. Padlog, and P. P. Bijlaard, "Stress Analysis of Heated Complex Shapes." Am. Rocket Soc. J., 32, 700-707 (1962).

9. R. J. Melosh, "Structural Analysis of Solids," J. Struct. Div. Am. Soc. Civil Engrs., 89 (ST4), 205-223 (1963).

10. C. W. McCormick and K. J. Hebert, Solution of Linear Equations with Digital Computers, California Institute of Technology, Pasadena (September 1965).

11. J. H. Argyris, "Matrix Analysis of Three-Dimensional Elastic Media, Small and Large Displacements," Am. Inst. Aeron. Astronaut. J., 3, 45-51 (1965).

12. E. L. Wilson, "Structural Analysis of Axisymmetric Solids," Am. Inst. Aeron. Astronaut. J., 3, 2269-2274 (1965).

13. Y. R. Rashid, "Three-Dimensional Analysis of Elastic Solids, Part I-Analysis Procedure" (to be published in Int. J. Solids Structures).

14. Y. R. Rashid, "Three-Dimensional Analysis of Elastic Solids, Part II-The Computational Problem" (to be published in Int. J. Solids Structures). 
15. R. W. Clough and J. L. Tocher, "Finite Element Stiffness Matrices for the Analysis of Plate Bending," Proceedings of the Conference on Matrix Methods in Structural Mechanics, AFFDL-TR-NNK80, Air Force Institute of Technology, Wright-Patterson Air Force Base (October 1965).

16. R. W. Clough and C. P. Johnson, "A Finite Element Approximation for the Analysis of Thin Shells," Int. J. Solids Structures, 4, 43-60 (1968).

17. N. Prince and Y. R. Rashid, "Structural Analysis of Shell Intersections" (submitted to International Conference on Pressure Vessels and Piping, September 29-October 2, 1969, Delft, Holland).

18. L. R. Herrmann, "Finite Element Bending Analysis for Plates," J. Engr. Mech. Div. Am. Soc. Civil Engrs., 93 (EM5), 13-26 (1967).

19. E. Reissner, "On a Variational Theorem in Elasticity," J. Math. Phys., 29, $90-95$ (1950).

20. L. R. Herrmann and D. M. Campbell, "A Finite-Element Analysis for Thin Shells," Am. Inst. Aeron. Astron. J., 6, 1842-1947 (1968).

21. Y. R. Rashid and W. Rockenhauser, "Pressure Vessel Analysis by Finite Element Techniques," Conference on Prestressed Concrete Pressure Vessels at Church House, Westminster, S.W. 1, 13-17 March 1967, p. 375 (The Institution of Civil Engineers, London, 1968).

22. Y. R. Rashid, "Analysis of Prestressed Concrete Nuclear Reactor Structures" (short course on Prestressed Concrete Nuclear Reactor Structures, March 18-23, 1968, University of California, Berkeley).

23. Y. R. Rashid and T. Y. Chang, "Stress Analysis of Reactor Graphite Structures" (submitted to Fifteenth Annual Meeting of the American Nuclear Society, June 15-19, 1969, Seattle).

24. Y. R. Rashid, "Ultimate Strength Analysis of Prestressed Concrete Pressure Vessels," Nucl. Eng. Design, 7, 334-344 (1968).

25. O. C. Zienkiewicz and Y. K. Cheung, The Finite Element Method in Structural and Continuum Mechanics (McGraw-Hill, New York, 1967).

26. Edward L. Wilson and Robert E. Nickell, "Application of the Finite Element Method to Heat Conduction Analysis," Nucl. Eng. Design, 4, 276-286 (1966).

27. J. S. Archer, "Consistent Mass Matrix for Distributed Mass Systems," J. Struct. Div. Am. Soc. Civil. Engrs., 89 (ST4), 161-178 (1963). 
28. Ray W. Clough and A. K. Chopra, "Earthquake Stress Analysis in Earth Dams," J. Eng. Mech. Div. Am. Soc. Civil Engrs., 92, 197-211 (1966).

29. R. E. Nickell and J. L. Sackman, "Approximate Solutions in Linear Coupled Thermoelasticity," J. Appl. Mech., 35, 225-266 (June 1968).

30. Eric Becker and R. E. Nickell, "Stress Wave Propagation Using the Extended Ritz Method" (to be presented at ASME/AIAA 10th Structures, Structural Dynamics and Materials Conference, April 14-16, 1969, New Orleans). 


\section{AUTOMATED THERMAL ANALYSIS OF NUCLEAR}

REACTOR COMPONENTS

A. Y. Lee and K. J. Wells

Westinghouse Electric Corporation

Astronuclear Laboratory

Pittsburgh, Pennsylvania 


\title{
AUTOMATED THERMAL ANALYSIS OF NUCLEAR REACTOR COMPONENTS
}

\begin{abstract}
This paper presents the description of the computer code AUTOTEM (AUTOmatic TEMperature calculations). It was developed for automating the procedure of modeling and obtaining temperature distributions for a given two-dimensional irregular body with a geometry description such as engineering drawing information as input. The code meshes the solid into a nodal system as required by the heat transfer calculation codes. The nodal system, as generated, is plotted on the SC 4020 data plotter for visual inspection. Temperature distributions are determined using a finite difference numerical solution. From these calculated nodal temperatures isotherms can be plotted for specified temperature values.

The AUTOTEM code can handle any two-dimensional or axial symmetrical body consisting of a single material. Time dependent internal heat generation, temperature dependent material thermal properties, and time dependent boundary conditions can be considered.
\end{abstract}

\section{INTRODUCTION}

Thermal analysis of a nuclear reactor or other apparatus component from engines: ing drawing information to temperature distribution patterns requires several independent calculations, such as geometry meshing, internal heat generation rate distributions, fluid flow and cycle state point conditions determination, and detailed temperature distribution calculations for the given component. These calculations can be readily automated using a large scale digital computer. This paper describes a computer program called AUTOTEM (AUTOmatic TEMperature calculation) for geometry meshing from drawing data and detailed temperature distribution calculations.

To determine the temperature distribution for an irregular body by finite difference numerical techniques, the body must first be divided into a finite number of lumped masses or nodal points. The majority of the input data required to specify the physical nodal system are the volumes of the nodes, connector lengths, and the interface areas between nodes for evaluating the conductances. The process of preparing this input data is time consuming and error prone. AUTOTEM was developed to eliminate this manual process of input preparation. This program automatically generates the input data described above and calculates the temperature distribution for a two-dimensional plane in $(x, y)$ coordinates or axially symmetrical irregular body in $(r, z)$ coordinates. Any two-dimensional closed geometry which can be described by straight lines and/or circular arcs can be meshed. Information and dimensions shown on engineering drawings of a given component can be utilized readily as input. By using this program, the designer can obtain a quick and accurate appraisal from a thermal standpoint for the design or alternate designs which are still in the preliminary design stage. 
The AUTOTEM includes the MESHER ${ }^{1}$ and TOSS $^{2}$ among its several major sub-programs. The former program was originally developed by Los Alamos Scientific Laboratory and the latter by Oak Ridge National Laboratory. They were extensively modified to become parts of AUTOTEM. The peripheral nodes around the specified geometry are generated by the modified MESHER and the regular interior nodes, and the other data required for temperature distribution calculations are generated by the other sub-programs. To calculate temperature distributions, the user has an option of using the modified TOSS code or another heat conduction analysis code, called TAP-A (Transient Analysis Program, Version A) ${ }^{3}$. TAP-A is a heat conduction code developed by Westinghouse Astronuclear Laboratory for steady-state and transient analysis. These two heat conduction codes can calculate the temperature distribution in an irregular 2 - or 3 - dimensional body. Because MESHER is restricted to mesh a 2-dimensional figure which consists of only one single material, the problems which AUTOTEM can handle will, therefore, be restricted to such 2-dimensional types. However, temperature dependent material thermal properties and the time-dependent internal heat generation rates and convective boundary conditions can be considered in the temperature calculation.

Internal heat generations and fluid boundary conditions are input quantities to AUTOTEM. The internal heat generation rates of the material can be input abtained from cards or from a tape which is previously generated for fission, neutrons, and gamma ray heating rate distributions in the material. The boundary conditions for the heat conduction analysis of a component can also be input obtained from cards or from a tape which is also previously generated. For NERVA nuclear reactor component thermal analysis applications, the convective boundary conditions can be abtained from reactor cycle state point calculations by a thermal and nuclear transient code.

The input data to TOSS or TAP-A are punches out once they are generated by the AUTOTEM, and in this way a problem can be rerun subsequently without requiring a remeshing of the geometry. In addition, AUTOTEM has the capability of plotting the generated nodal network and the desired isothermal temperature lines on the SC-4020 data plotter.

The AUTOTEM is written in Fortran IV language for the 64K CDC-6600 computer. The following sections present a description of the program and a sample problem.

\section{PROGRAM ORGANIZATION}

The AUTOTEM computer code consists of four major sections: 1) meshing the peripheral nodes by MESHER, 2) generating the regular interior nodes and input data required by TOSS or TAP-A, 3) calculating the temperature distributions, and 4) plotting the generated nodal network and isotherms. The overall organization of AUTOTEM is shown schematically in Figure 1. The data generated from one section are stored on tape and are punched out on cards. In this way, the execution of the problem can be stopped at the end of any one section for examination. The punched cards are used as input to continue the execution of the problem. The datastored on tape are used when the problem is executed continuously from one section to the next. For a problem having a complex geometry, it is desirable to stop the execution at the end of the meshing operation and to examine the nodal network until a satisfactory network is obtained.

The AUTOTEM is organized in terms of the overlay setup for the CDC-6600 computer as shown in Figure 2. The first level overlay $(0,0)$ is the control program. It requires only one input card for controlling the execution of the sub-programs which make up the 


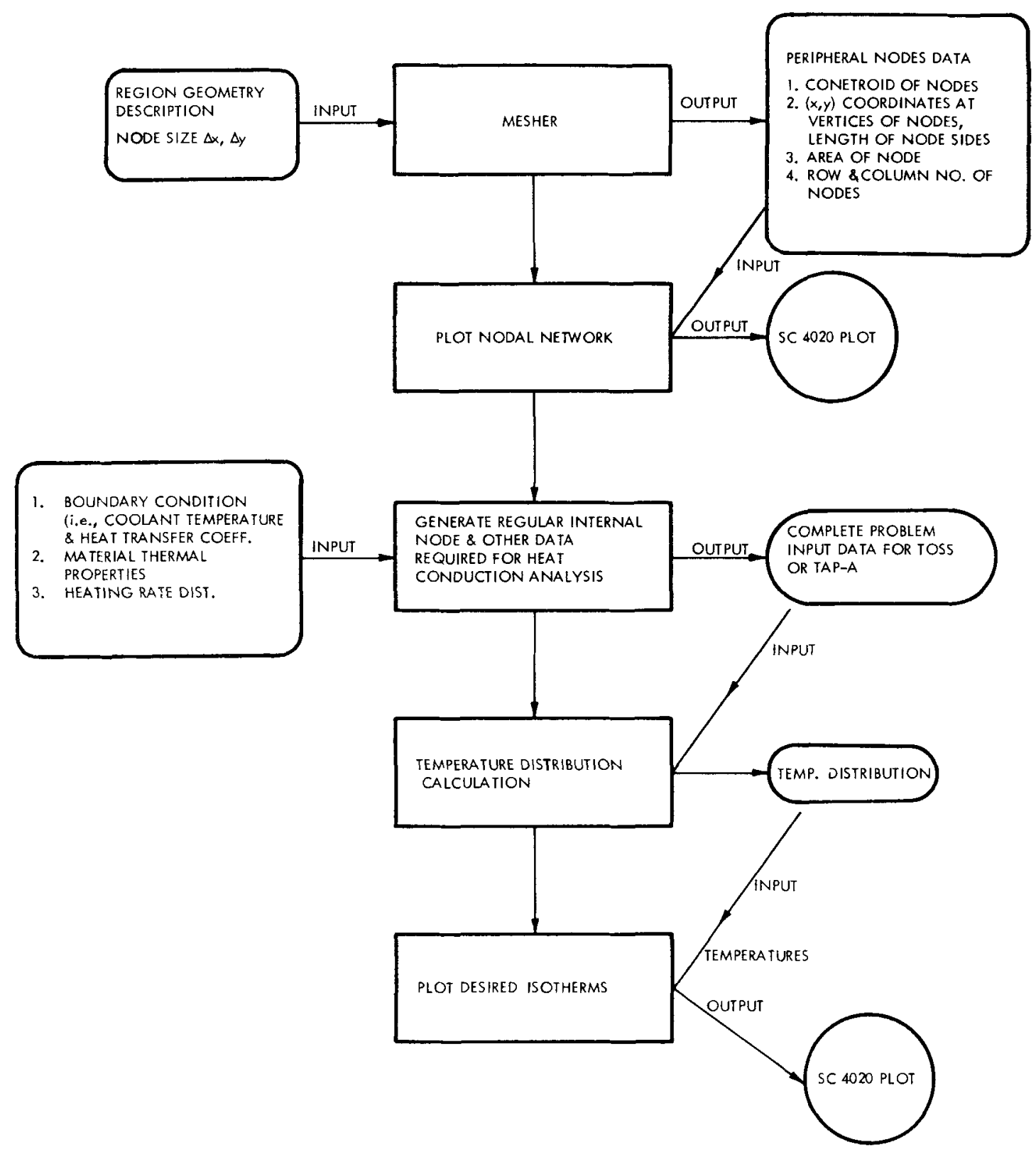

Figure 1. Overall Organization of AUTOTEM 


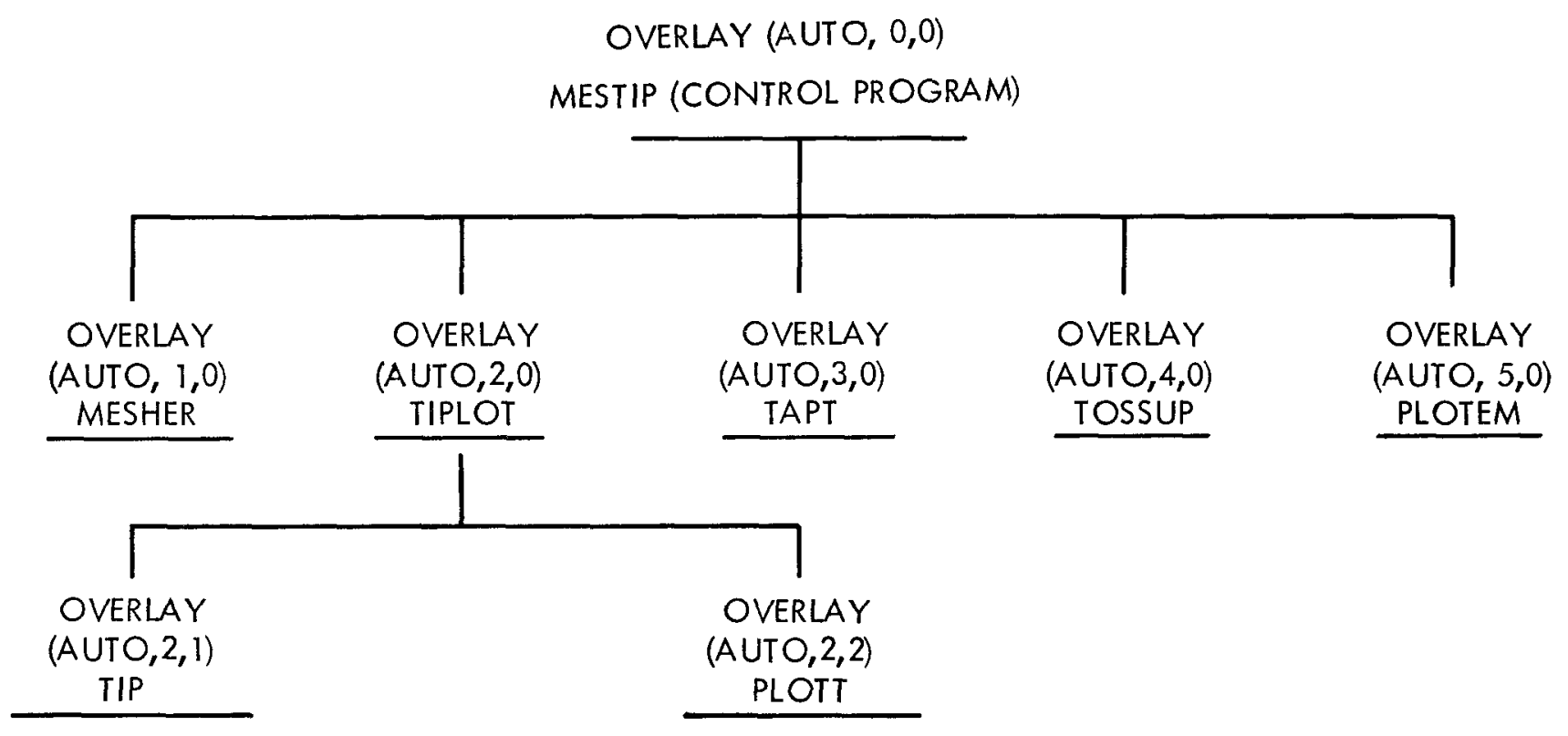

Figure 2. AUTOTEM Computer Program Overlay Organization 
AUTOTEM. These sub-programs in turn require their own input cards.

\section{PROGRAM CAPACITY}

The AUTOTEM program's overall limitations are:

1) The code can handle a 2-dimensional plane geometry in $(x, y)$ coordinates or axial-symmetrical region in $(r, z)$ coordinates.

2) The geometry consists of only one single homogeneous material.

3) Internal void areas are allowed, but the computer running time increases considerably with an increased number of internal void areas.

4) The $\Delta x$ and $\Delta y$ node size must be uniform for the entire figure.

5) The size of the mesh should be chosen such that there are at least two nodes (irregular or regular) between two boundary lines.

6) If the side of an internal polygon is too close to a grid line, overlapping may occur. In this case MESHER would fail. Changing the size of the node would alleviate. this situation.

7) The heat transfer codes have the following capacity restrictions:

TOSS: up to 700 internal nodes, with 8 connectors per node. up to 300 surface nodes, with 3 connectors per node, up to 160 boundary nodes.

TAP-A: up to 900 internal nodes, with 9 connectors per node, up to 400 surface nodes with 4 connectors per node, up to 500 boundary nodes.

\section{PROGRAMS DESCRIPTION}

\section{PROGRAM MESHER TO GENERATE PERIPHERAL NODES}

Any two-dimensional closed configuration which can be specified in terms of si...'. lines and circles, or circular arcs, can be meshed by MESHER. The configuration geren.:" consists of a number of connected segments, each of which is described by some numbcis punched on two or three input cards. These segments must be arranged in sequential ordir so that the area to be meshed will be on the left as one travels along the boundary. A in: or void area that does not intersect another boundary is input as a separate boundary.

To set up a problem, the entire configuration which is to be meshed must lie in the first quadrant of a coordinate system, so that all the coordinates describing the figure are positive or zero. Three types of line segments are accepted as input: 1) straight line, 2) circular arc or closed circle, and 3) closed regular polygon. The desired mesh size L. and $\Delta y$ is also specified in the input. 
The dimensions and orientations of the segments may be partially or completely obtained directly from engineering drawings. For example, for an axially-symmerirical apparatus such as the NERVA nuclear rocket reactor, the reactor centerline may be considered as the $Z$-axis. Any transverse section maybe described in the $(x, y)$ plane (after transformation from the $(r, \theta)$ coordinates, if necessary) and any longitudinal section on the $(r, z)$ plane. If the dimensions on the drawings are based on these coordinates, the input to the MESHER could be directly transcribed from the drawings.

Peripheral nodes (meshing) for a given geometrical configuration utilized by MESHER are generated in the following manner. Initially the first $N$ segments are examined to find a closed curve. When a closed configuration is found, mesh nodes are drawn at the corners of the figure (points of intersection of boundary segments). Subsequently, peripheral nodes along a segment are drawn in between the corner nodes and along the segments until the boundary has been lined with peripheral nodes which are generated by drawing normals from the nearest intersections of the grid lines to the boundary line as shown in Figure 3. This figure shows a rectangle with one corner removed and with an internal circular hole. The boundary of this configuration consists of the straight line segments $A B, C D, D E$ and $E A$. circular arc $B C$ and the internal hole whose center is at point $E$. If the segment is parallel to one of the coordinate axes, all of the peripheral nodes along this boundary line will be rectangular (or square) nodes except at the corners. After the $\mathrm{N}$ segments are examined, the next $M$ segments are examined for another closed curve. The procedure continues, until no segments remain to be meshed. For this example as shown in Figure 3, the first $N$ segments will be the boundary lines $A B, B C, C D, D E$, and $E A$. The next $M$ segments will be the interior circle alone. The mesh will be drawn either on the inside or the outside of each closed curve according to the input instructions. To prevent overlap of corner nodes, the node size $\Delta x$ and $\Delta y$ should be chosen so that there will be room for two nodes in the narrowest space between two boundary lines to be meshed. The output of MESHER is a complete geometrical description of the generated peripheral nodes and consists of the following data:

1) The coordinates of the centroids of each node.

2) The area of each node,

3) the coordinates of the comers of the node

4) the length of each side of the node,

5) location indices $(I, J)$ for each node which represent the node column (I) number and the node row (J) number on a rectangular grid system. A peripheral node carries the same $I$ and $J$ as the rectangular node from which it is derived as shown in Figure 3.

The process of preparing MESHER input data is illustrated by considering the same Figure 3. The specification of the boundary segments starts at one point, say A, and proceeds clockwise or counterclockwise along the entire boundary in such a way that the region to be meshed is at the left-hand side when one circumvents the boundary of the configuration. In this example, the specification of input starts from point $A$ and proceeds counterclockwise. A straight line is specified by the two end points. For the straight line $A B$, the end points from $A\left(x_{1}, y_{1}\right)$ to $B\left(x_{2}, y_{2}\right)$ are specified. A circular arc is specified by the coordinates of the center of the arc, the radius, and the two end points of the arc. For the arc $B C$ in Figure 3, the coordinates of the center $F\left(x_{c}, y_{c}\right)$, the radius $R$, and the arc end 


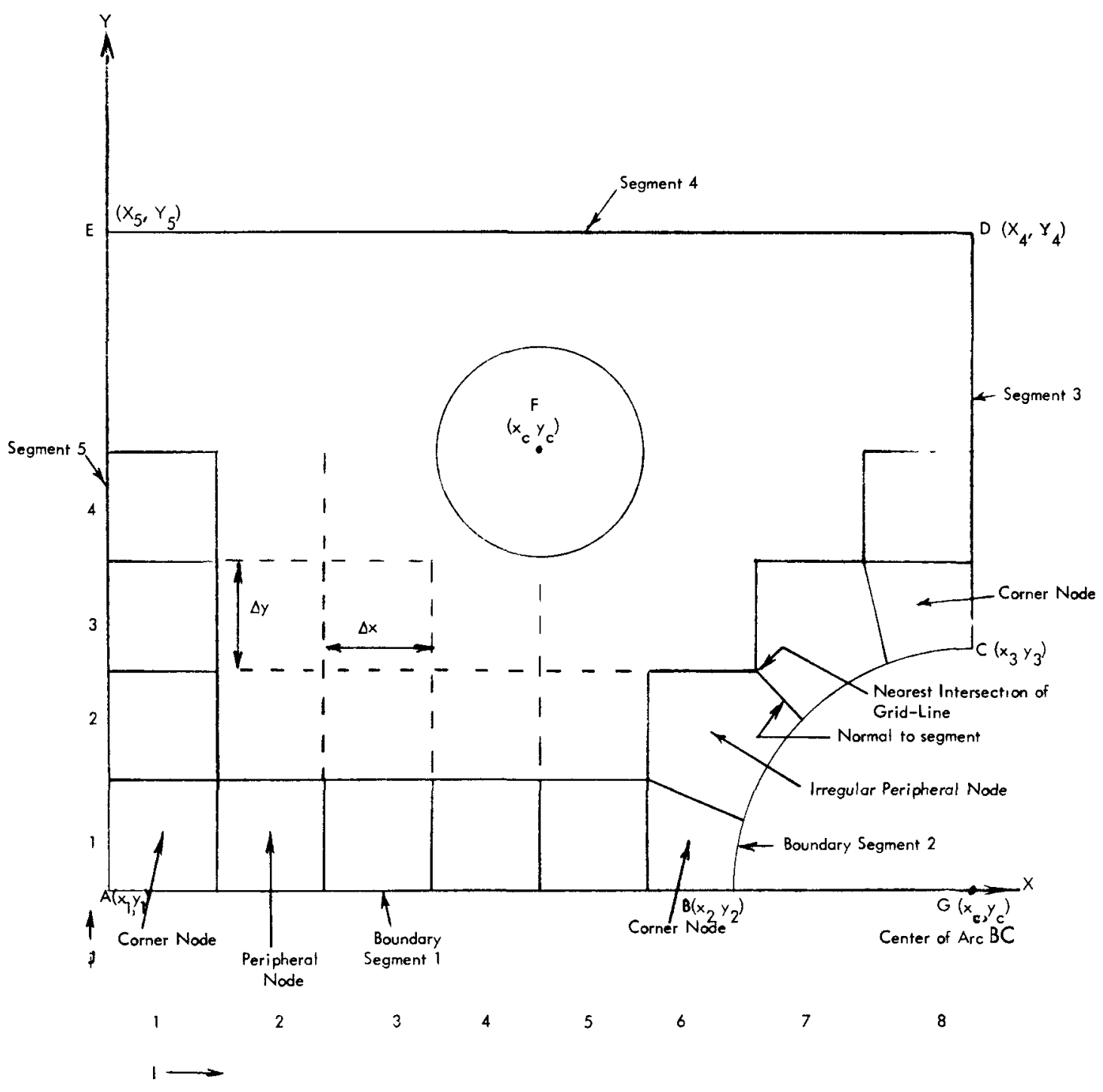

Figure 3. Peripheral Node Generation 
points from $B\left(x_{2}, y_{2}\right)$ to $C\left(x_{3} y_{3}\right)$ are specified. A positive sign for the radius indicates that the region to be meshed is on the convex sie of the arc and a negative sign indicates that the region to be meshed is on the concave side of the arc. The radii for both the arc $B C$ and the internal circle are, therefore, negative. For a complete circle, the two arc end points are omitted or set to zero.

In addition to straight line segments and circular arcs, a regular polygon can be accepted as input. The input specification includes the coordinates of the center of the polygon and the coordinates of the end points of any one side of the polygon. The complete polygon will be generated from these data. The restriction is that the coordinates of the endpoints of the given side must be stated in such order that going from $\left(x_{s_{7}}, y_{s_{7}}\right)$ to $\left(x_{s_{2}}\right.$, $y_{s_{2}}$ ) keeps the interior to be meshed on the left. If only a portion of the polygon is to be included in the figure, the endpoints must also be specified, as in the case of a circular arc. Note in this case that interior referes to the interior of the figure, which may or may not be the interior of the polgon if only a portion is included in the complete figure.

Although the specifications for the geometry to be meshed are comparatively simple, they are not very flexible. In a configuration made up of $N$ segments, the coordinates of each vertex of the configuration are actually input twice, once in the $x_{1}, y_{1}$ fields and once in the $x_{2}, y_{2}$ fields. The MESHER is not equipped at present to sort segments into correct order.' Polygons may close upon themselves and the endpoints of circular arcs must, of course, obey the equation of the circle. There is a numerical tolerance of one-tenth of the mesh spacing built into the program to allow for small numerical inaccuracies in the input and for truncation and rounding errors.

\section{PROGRAM TIP TO GENERATE INTERIOR NODES AND DATA FOR HEAT CONDUCTION ANALYSIS}

The sub-program TIP accepts as input the data stored on tape or from punched cards produced by MESHER, and completes the meshing by generating the regular internal nodes inside the configuration between the peripheral nodes. The generation of regular internal nodes is done in a row-wise order as shown by the dashed lines in figure 3 . The number of regular nodes required to fill a row is determined by the number of increments $(\Delta x)$ between the two peripheral nodes in that row.

Because the geometric description data generated are for the TOSS or TAP-A heat conduction computer code, the assignment of node numbers and connections between nodes will follow the conventions of these two codes. All nodes (peripheral as well as regular interior nodes) generated inside the specified geometric configuration are now called internal nodes. The numbering sequence for the internal nodes ranges from 1 to 1000 . The node number starts from the lowest column and row indexes $\mathrm{I}=1$ and $\mathrm{J}=1$, then proceeds from left to right and from bottom to top of the configuration. The center of an internal node is located at the centroid of the node. At the midpoint of an exterior side of a peripheral node a surface node is assigned. The numbering sequence for surface nodes starts from 1001 up to 2000. The node number also starts from the surfaces of the peripheral node located at the lowest column and row indexes. The boundary condition facing each of the boundary segments is assigned a boundary node. The numbering sequence for boundary node starts from 2001 up to 3000 . The boundary node facing the input segment No. 1 is assigned 2001, the boundary node facing the input segment No. 2 is assigned 2002, etc. It is noted that the numbering sequences for these three types of nodes may start from any other number specified in the input to the computer run. In this way more than one geometry can be meshed separately, then combined into one problem without duplicated node numbers. Figure 4 shows the node numbers for the bottom three rows of nodes, using the same configuration. 


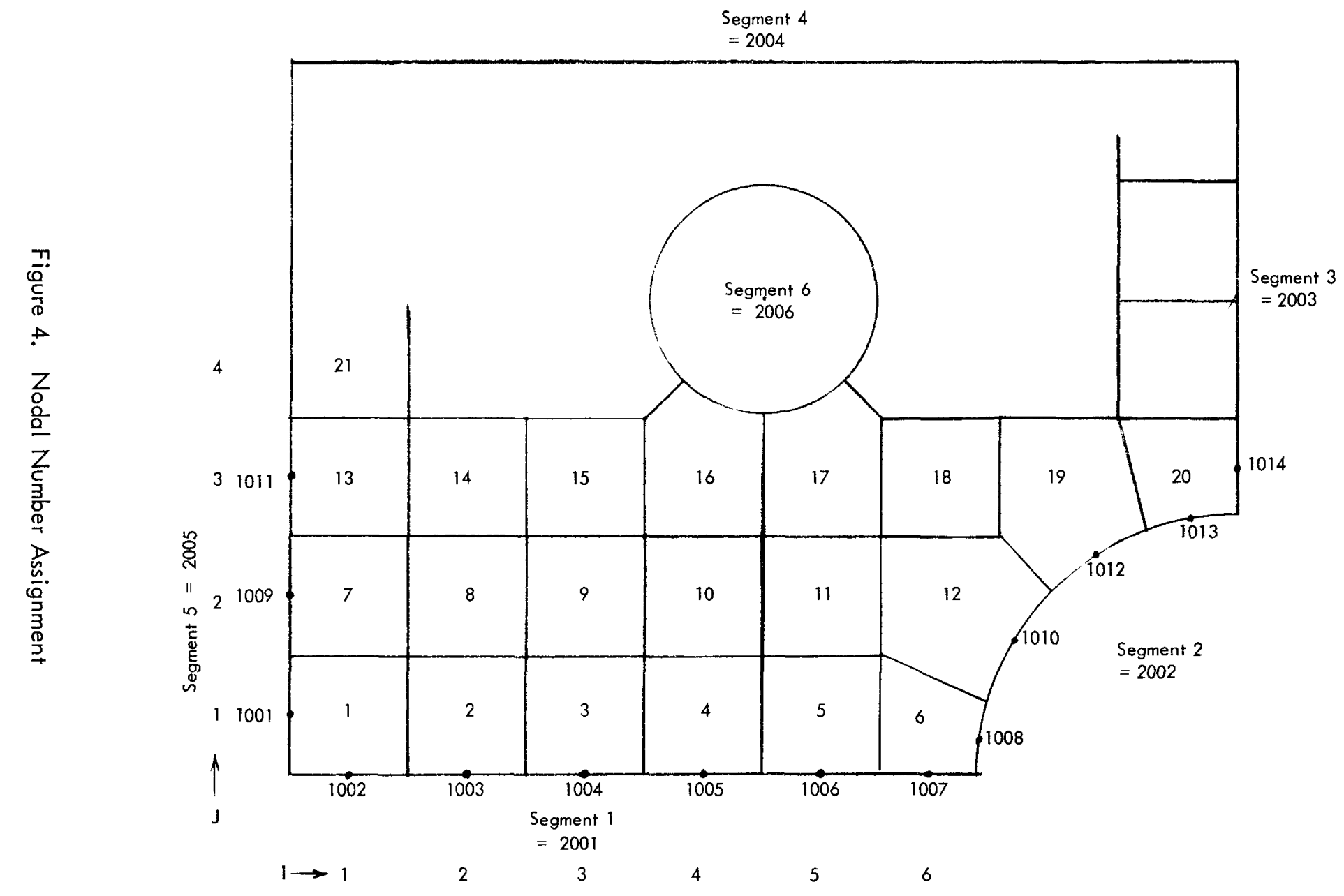


After the node numbers are assigned, the nodes are connected by TIP. The following method is used to find the neighboring nodes. Because the coordinates of the vertices describing a given node are arranged in order when one circumvents it, two pairs of the coordinates are - smpared to two pairs of coordinates on all other nodes (taken two at a time). When two pairs of coordinates on one node are matched to two pairs on another node, a connection is made. If no match is found, the node is connected to a surface node which is located at the midpoint joining these two pairs of coordinates. For the example shown in Figure 4, the node No. 1 is connected to nodes Nos. 2, 7, 1001, and 1002, and the node No. 11 is connected to nodes Nos. 5, 12, 17 and 10 .

The connector length between two adjacent internal nodes is calculated by different means depending on the coordinate system specified. For an $(x, y)$ coordinate system with a constant third dimension (depth), the connector length is calculated as the normal distance from the centroid to the interface of the two adjacent nodes. The heat transfer area between any two nodes is the length of the common side times the depth of the body.

The calculation of the connector lengths for an axial-symmetrical sector in $(r, z)$ coordinates is done in a different manner. The connections which are made in the $Z$ direction are calculated as the normal distance from the centroid of a node to the interface of the two adjacent nodes (above and below). Connections which are made in the radial direction $(R)$ are based on the following equations:

$$
\begin{aligned}
& I_{i}=r_{m} \log _{e}\left(\frac{r_{m}}{r_{i}}\right) \\
& l_{i}=r_{m} \log _{e}\left(\frac{r_{i}}{r_{m}}\right)
\end{aligned}
$$

where

$$
\begin{aligned}
& r_{i}>r_{i} \\
& r_{i}=\text { radius to centroid of node } i \\
& r_{i}=\text { radius to centroid of node } i \\
& r_{m}=\text { radius to interface between nodes } i \text { and } i \\
& I_{i}=\text { connector length in node } i \\
& I_{i}=\text { connector length in node } i
\end{aligned}
$$

The heat transfer area between nodes in the $Z$ direction, which is a sector of an annulus, is equal to the common interface (radial) length times the arc length at a radius and an angle. The radius is calculated at the midpoint of the common side between the two nodes. The heat transfer area between nodes in the $R$ direction is equal to the common interface (axial) length times the arc length at a radius and an angle. The radius is located at the interface between the nodes. The angle is an input number. For internal to surface 
node connection such as from node 1 to 1001,2 to 1003 , etc., the connector length is the distance from the centroid of the node to the midpoint of the boundary side. Since the irregular side (s) of the peripheral node is normal to the boundary side, this method of determining the connector length introduces little error, unless the node is highly distorted. The convective heat transfer area of the peripheral node is the product of the length of its boundary side and the depth of the body. The total convective heat transfer surface area of the configuration is the same as the total actual exterior surface area within the numerical truncation error.

The nodal system generated by AUTOTEM results in an arrangement such that one side of a node is common only to one adjacent node. There will never be more than one smaller node along one side of a larger node. The result will be a uniform nodal model except the irregular peripheral nodes, suitable to finite difference heat transfer calculations.

The volumetric internal heating rate may be a uniform rate for the entire configuration or it may be calculated as the product of two heating rate distributions at the centroids of the node. For the latter the two distributions are input in a tabular format. If the configuration is a plane cross section in an $(x, y)$ coordinate system, the coordinate system for one of these two distributions must be the same as the one used to describe the configuration to be meshed. The heating rate distribution on this plane cross section is a function of the distance from the origin of the coordinates to the centroid of the node. The other distribution is merely a constant multiplier. If the configuration is a transverse plane of an axialsymmetric sector in $(r, z)$ coordinates, one distribution is a function of the $R$ coordinate and the other distribution is a function of the $z$ coordinate of the centroid of the node.

Presently the internal heat generation rates and the boundary conditions required for the temperature calculations are input by cards to the AUTOTEM code as described above. These data could be easily obtained directly from tapes to further automate the system.

\section{TEMPERATURE DISTRIBUTION CALCULATIONS}

The two heat conduction analysis codes TOSS and TAP-A calculate steady-state or transient temperature distribution for a 2-dimensional or 3-dimensional irregular body (2-dimensional for AUTO TEM generated problems). The heat transfer mechanism of conduction, forced and free convection, and radiation between a boundary to an external body of given temperature are considered. Time-dependent internal heat generation rates and boundary conditions, and temperature-dependent material thermal properties are allowed. TOSS and TAP-A are quite similar in programming logic and input data format. The latter code is, however, more efficient and possesses many additional features.

In these codes, an internal node is a point which is associated with a volume, a heat capacity and a heat generation rate. A surface node is a point associated with an area and a film coefficient. The surface nodes are used to link the internal or capacity-bearing nodes with the boundary or sink-temperature nodes. A connector is a path between two nodes along which heat can flow. The connectors are associated with admittances which, in turn, are functions of the material conductivities, resistor lengths, and areas between nodes. The codes thus do not recognize any real geometry, but only the lengths, areas, and volumes of the nodes and connectors.

Both codes solve the general heat-conduction equation 


$$
\nabla(k \nabla t)+q^{\prime \prime \prime}=C_{p} \rho \frac{\partial t}{\partial \theta}
$$

or in the equivalent implicit finite difference form

$$
Q_{i}+\sum Y_{i j}\left(t_{i}^{*}-t_{i}^{*}\right)=\frac{C_{i}\left(t_{i}^{*}-t_{i}\right)}{\Delta \theta}
$$

or exponential explicit finite difference form

$$
t_{i}^{*}=Z_{i}+(1-Z)\left[\sum_{i=1}^{N} Y_{i j}{ }^{*}{ }^{*}+\sum_{i=N+1}^{M} Y_{i j}{ }^{t_{i}}+Q_{i}\right] / \sum_{i=1}^{M} Y_{i j}
$$

where $i \neq i$, and $t^{*}=$ temperature at future time, and

$$
\begin{aligned}
& Q_{i}=q_{i}^{\prime \prime} V_{i} \\
& C_{i}=C_{p_{i}} \rho_{i} V_{i} \\
& V_{i}=I_{i} w_{i}^{d}
\end{aligned}
$$

$$
Z=\exp \left[\frac{-\Delta \theta}{C_{i}} \quad \sum_{i=1}^{M} Y_{i i}\right]
$$

For conduction from an internal node to another internal node

$$
Y_{i j}=\frac{A_{i i}}{\frac{i_{i j}}{k_{i}}+\frac{T_{i i}}{k_{i}}}
$$

For conduction from an internal node to a surface node

$$
Y_{i j}=\frac{k_{i} A_{i j}}{I_{i j}}
$$


The temperature of a surface node is a weighted average of the temperatures of all nodes connected to the surface node

$$
t_{s}=\frac{\sum Y_{i s} t_{i}}{\sum Y_{i s}}
$$

Each surface node must be connected to one, and only one, internal node and one or more boundary nodes.

If $\mathrm{i}$ represents a boundary node,

$$
Y_{\text {is }}=h_{\text {is }} A_{s}
$$

The film coefficient, $h_{i s}$ depends on the mechanism of heat transfer between $s$ and $i$.

For $N$ number of nodes, a system of $N$ linear equations (4) must be solved. The Gauss-Seidel procedure is used to solve this system of $N$ linear equations. The iteration for the $k+1$ time for the $i$ th temperature, $t_{i} k+1$, is

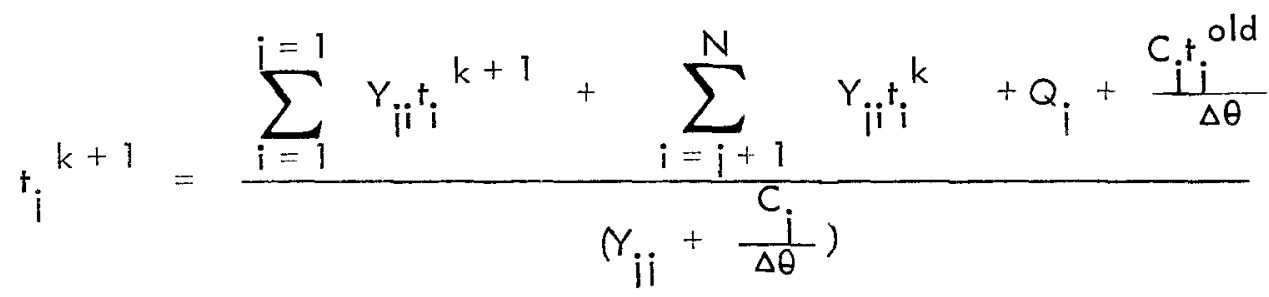

where

$$
Y_{i i}=\sum_{i} Y_{i i}
$$

and $i$ ranges over the neighbors of $i$. This iteration continues until all internal temperatures meet the following criterion:

$$
\left|t_{i}^{k}-t_{i}^{k+1}\right|<\epsilon
$$

where $\epsilon$ is the convergence tolerance. 
The above criterion for convergence has been found necessary but not sufficient for an accurate solution. An additional check is made by performing a heat balance as follows:

A steady state the solution is considered converged, if

$$
|A|>C
$$

where

$$
\begin{aligned}
& A=\frac{\sum_{v} q^{\prime \prime \prime} d v+\sum_{s} \frac{d q}{d n} d s}{B} \\
& B=\text { maximum of } \sum_{V} q^{\prime \prime \prime} d v \text {, or } \sum_{s} \frac{d q}{d n} d s \\
& C=\text { Convergence tolerance on heat balance }
\end{aligned}
$$

If

$$
|A|>C,
$$

a new temperature criterion $\epsilon^{*}$ is calculated

$$
\epsilon^{*}=\frac{\epsilon \times C \times 0.99}{|A|}
$$

For transient problems the solution is considered converged, if

$$
|A|<C
$$

where

$$
\begin{aligned}
& A=\frac{\sum_{v} q^{\prime \prime \prime} d v+\sum_{s} \frac{d q}{d n} d s-\sum_{v} c_{p} d v \int_{0}^{\theta+\Delta \theta} \frac{d t}{d n} d \theta}{B} \\
& B=\text { maximum of } \sum_{v} q^{\prime \prime \prime} d v, \sum_{s} \frac{d q}{d n} \text { ds or } \sum_{v} c_{p} d v \int_{\theta}^{\theta+\Delta \theta} \frac{d T}{d \theta} d \theta
\end{aligned}
$$


If $|A|>C$, the temperature criterion $E$ is modified as in the steady state case.

An accelerating routine is also included in the iteration. This is a second order extrapolation of temperature and is performed after a specified number of iterations. If the following criterion is met:

$$
0<b<1
$$

where

$$
b=\frac{t_{i}^{k}-t_{i}^{k-1}}{t_{i}^{k-1}-t_{i}^{k-2}}
$$

then

$$
t_{i}^{k+1}=\frac{b}{b-1} t_{i}^{k}+\left(1-\frac{b}{b-1}\right) t_{i}^{k-1}
$$

\section{PROGRAM TO PLOT MESHED FIGURE AND ISOTHERMS}

The plot programs used in AUTOTEM are from a standard program package for the SC-4020 CRT data plotter on the CDC- 6600 computer. The user has the option of plotting only the peripheral nodes produced by mesher or the complete nodal network generated by MESHER and TIP. Steady-state isotherms or isotherms at any given time during a transient run can be plotted. In addition, the temperatures of selected nodes or groups of nodes as a function of time can also be plotted for a transient problem.

\section{SAMPLE APPLICATION}

The sample problem presented in this paper is an illustration of the application of AUTOTEM proceeding from engineering drawing information to temperature distribution pattern. A steady state calculation is performed on a carriage clamp which is shown in Figure 5 . It is located in the reactor assembly and is made of stainless steel with a uniform heat generation rate of $30 \mathrm{Btu} / \mathrm{Hr}-\mathrm{in}^{3}$. It is cooled by forced convection along the large circular arc located at the bottom of the part and the internal hole. The other surfaces are assumed to be adiabatic. The dimensions shown on this drawing are sufficient to describe the component completely as required by MESHER. The configuration was transposed to the first quadrant of an $(x, y)$ coordinate system as shown in Figure 6. The $(x, y)$ coordinate of the end points of each of the 14 segments shown are obtained with minor computations from the drawing data. For MESHER input purposes the line $A B$ is considered to be segment No. 1, BC is segment No. 2, etc. The sequence of input for the outer contour is counterclockwise from $A$ to $M$ then back to $A$. The circular hole $N$ is the last input. The circular segment from $E$ to $F$ is the surface which is cooled by a coolant at $600^{\circ} \mathrm{R}$ with a heat transfer coefficient of $1200 \mathrm{Btu} / \mathrm{Hr}-\mathrm{ft}^{2}-{ }^{\circ}$. The internal hole with its center at point $\mathrm{N}$ is cooled by a coolant at $400^{\circ} \mathrm{R}$ with a heat transfer coefficient of $2000 \mathrm{Btu} / \mathrm{Hr}_{-}-\mathrm{ft}^{2}-{ }^{\circ} \mathrm{R}$. The mesh size selected in this case is limited by the size of the internal hole. Because the radius of the hole is 0.5 ", 


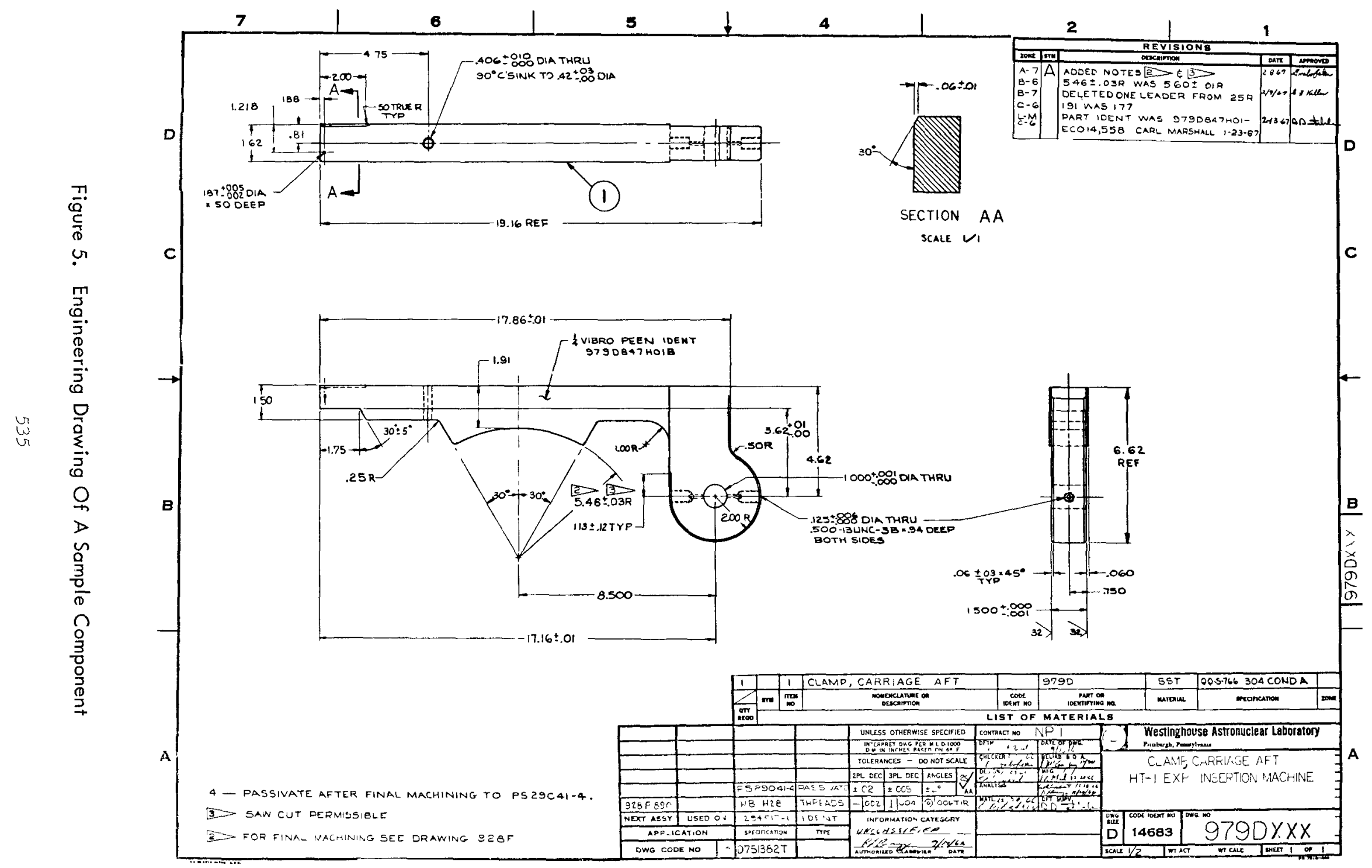




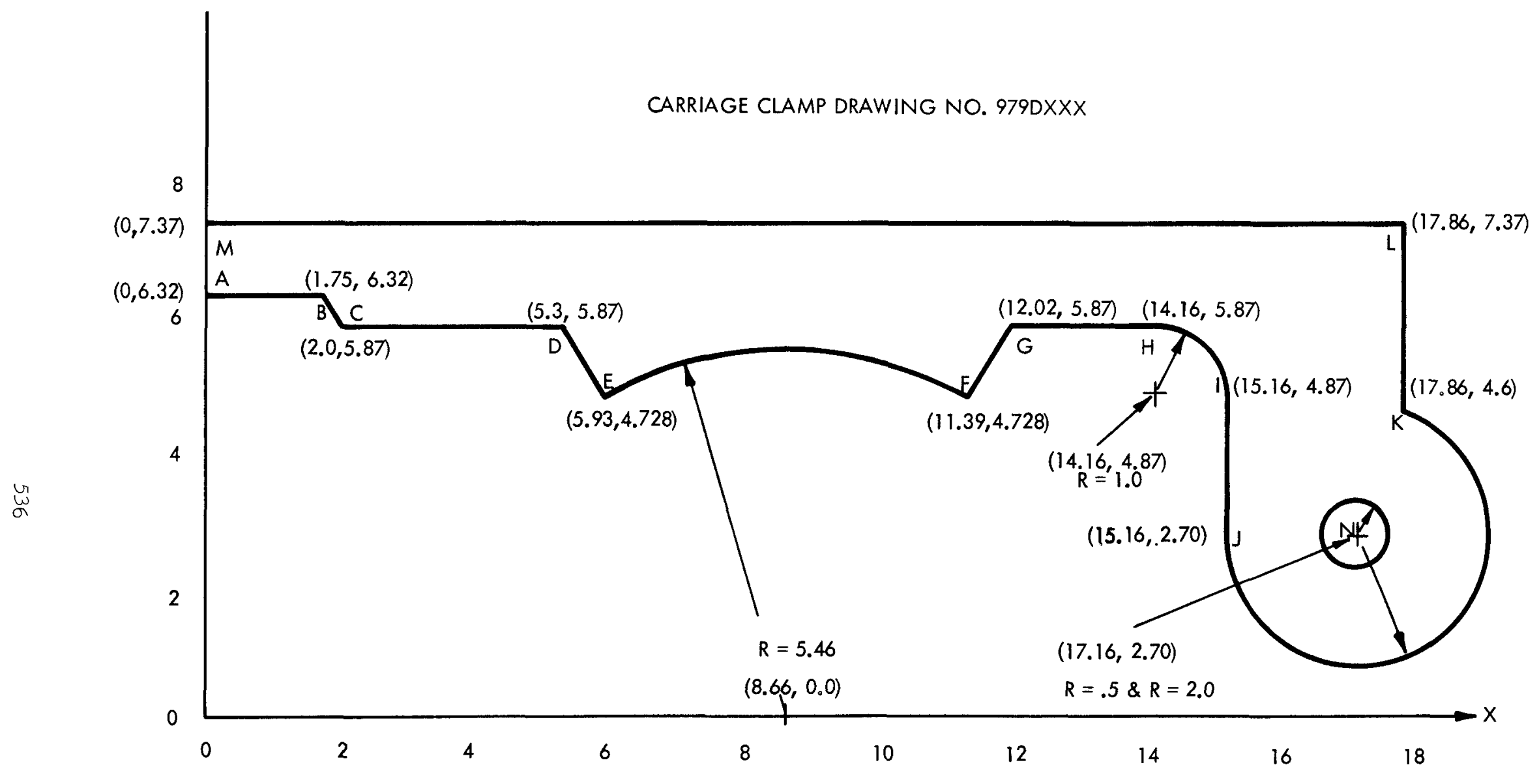

Figure 6. Input Data Layout 
the size of the nodes was selected to be $0.4^{\prime \prime} \times 0.4^{\prime \prime}$ with a tolerance $\epsilon$ of 10 percent. With this tolerance there will not be a node which will have an area less than 0.1 " $\times 0.4$ " $x 0.4^{\prime \prime}(\Delta x \bullet \Delta y \bullet E)$.

The configuration was meshed and the generated peripheral nodes are plotted for visual inspection as shown in Figure 7. Since the nodal system generated was found to be satisfactory, the program was restarted to complete the entire nodal network, which, with its 344 nodes, is also plotted as shown in Figure 8. With the known boundary conditions, the temperature distribution in this component was then computed. The isotherms for the temperature of $500^{\circ} \mathrm{R}$ to $1300^{\circ} \mathrm{R}$ in increments of $50^{\circ} \mathrm{R}$ are plotted and are presented in Figure 9. The temperatures at the nodal points are available in the computer output,

\section{NOMENCLATURE}

C Heat capacity of node, Btu/ $\rho_{R}$

$C_{p}$ Specific heat BTU/LB- ${ }^{\circ}$

$\Delta x \quad$ Node size in the $x$ direction, inches

$\Delta y \quad$ Node size in the $y$ direction, inches

d Depth of a node, inches

$\epsilon \quad$ Convergence tolerance

$\theta \quad$ Angle of a sector, degrees or time, sec

h Film coefficient, BTU/Hr-Ft ${ }^{2}-{ }^{\circ} \mathrm{R}$

I Column index on a rectangular grid system

i Node index

J Row index on a rectangular grid system

i Node index

K Thermal conductivity, BTU $/ \mathrm{Hr}-\mathrm{ft}^{\circ}{ }^{\circ} \mathrm{R}^{\prime}$

I Thermal connector length for a node, inches

$q^{\prime \prime \prime}$ Internal heat generation rate, BTU/ $\mathrm{Hr}-$ in $^{3}$

Q Heat generation, Btu $/ \mathrm{Hr}$

r Radius, inches

o Material density, $\mathrm{lb} / \mathrm{in}^{3}$

$+\quad$ Temperature, ${ }^{\circ} R$ 


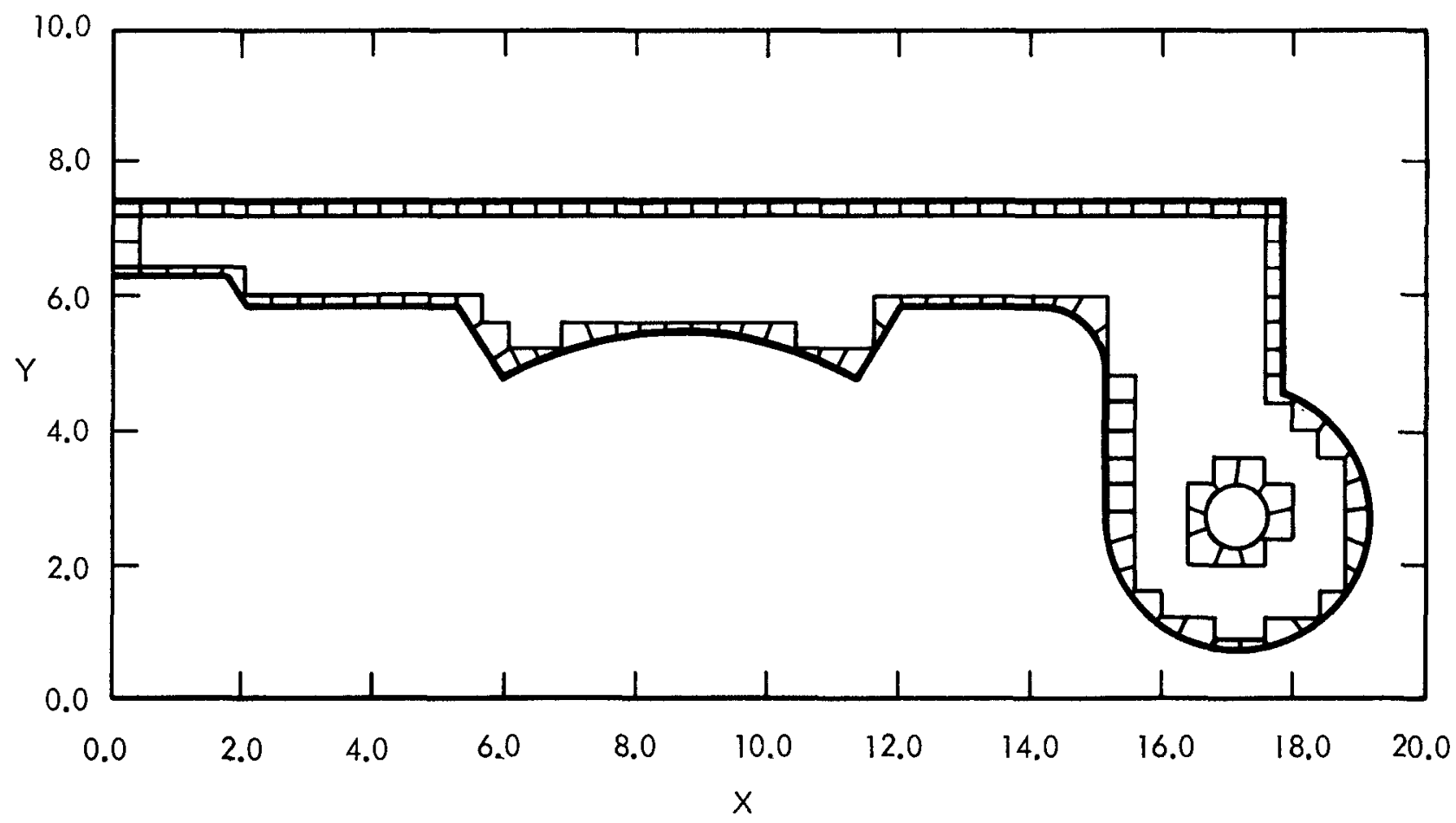

Figure 7. Peripheral Nodal System Generated by Mesher 


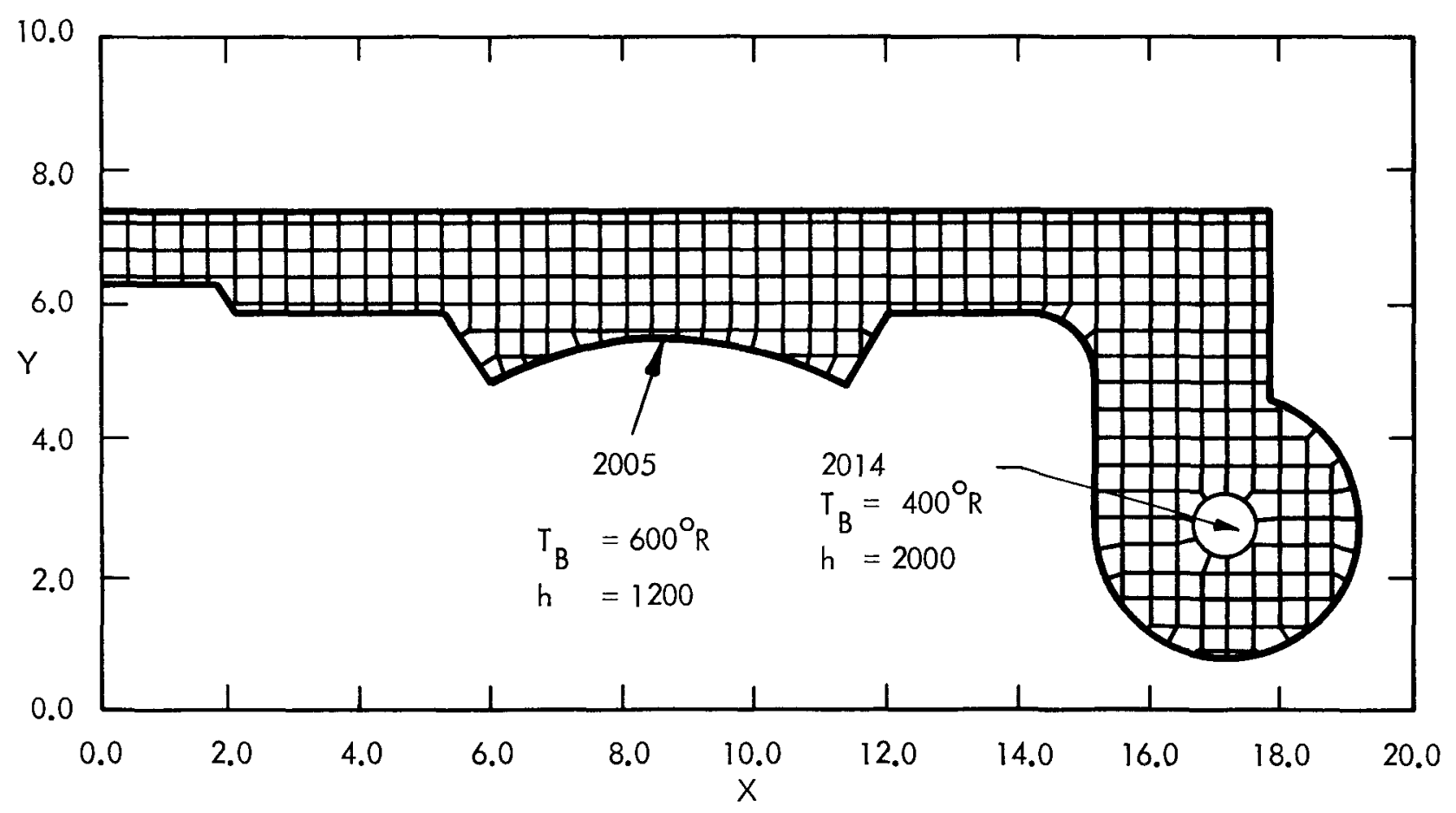

Figure 8. Nodal System Generated By Tip 


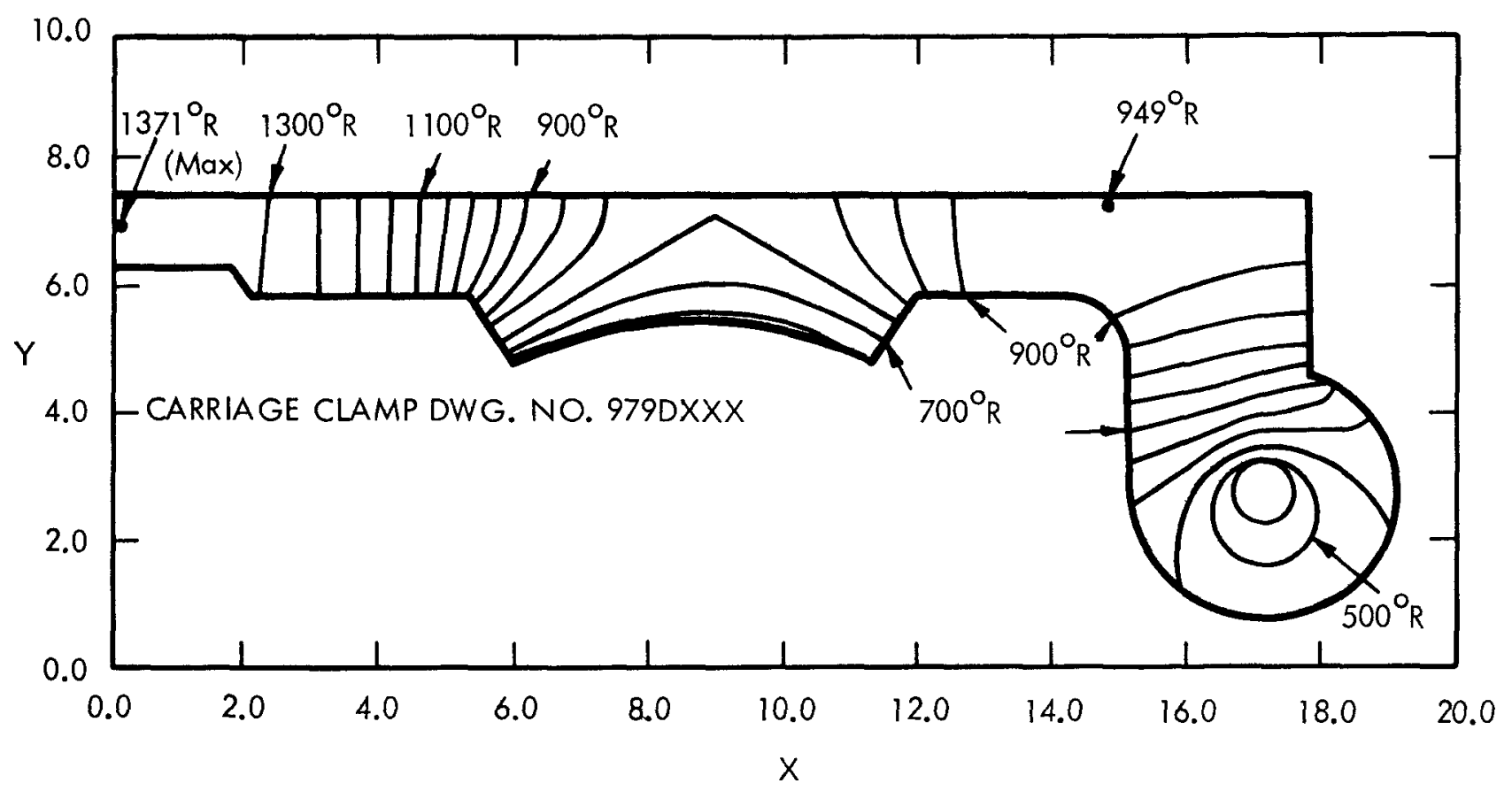

Figure 9. Isothermal Patterns 

$\checkmark \quad$ Nodal volume, in ${ }^{3}$
Width of a node, inches
$x$ Distance from the $y$-axis, in the first quadrant of a Cartesian coordinate system, inches
$y$ Distance from the $x$-axis, in the first quadrant of a Cartesian coordinate system, inches
$z$ Distance from the $x$-axis (axial), inches

\section{ACKNOWLEDGEMENT}

The Nuclear Engine for Rocket Vehicle Application Program (NERVA) is administered by the Space Nuclear Propulsion Office, a joint office of the U. S. Atomic Energy Commission and the National Aeronautics and Space Administration. Aerojet-General Corporation, as prime contractor for the engine system, and Westinghouse Electric Corporation, as subcontractor for the nuclear subsystem, are developing a nuclear propulsion system for space applications.

\section{REFERENCES}

1. R. F. Thomas, Jr., The Rath MESHER a 7090 Program for Preparing Input Data for Heat Conduction Codes, Los Alamos Scientifíc Laboratory, LAMS-2809 (December 1962).

2. B. L. Pierce, Modified Transient and/or Steady-State (TOSS) Digital Heat Transfer Codes, WANL-TME- 1108 (February 1965).

3. B. L. Pierce, TAP-A Program for Computing Transient or Steady State Temperature Distributions, WANL-TME-1872 (December 1968). 
Numerical Analysis of Two-Dimensional, Dynamic, Thermal Stresses in a Hollow Cylinder*

by

J. A. Reuscher and M. R. Scott

Sandia Laboratories

Albuquerque, New Mexico

\section{Introduction}

The operational mode of a fast burst reactor induces severe stress conditions on the fuel material. The energy deposited during the narrow power pulse causes the temperature of the core to rise faster than the fuel material can respond by thermal expansion. A small portion of the tinermal energy is converted into kinetic energy causing the core to vibrate; this, in turn, creates dynamic stresses. The dynamic core expansion exceeds the static expansion which would result if the fuel were heated slowly. The expansion and contraction continues at the natural yibrational frequency of the core until material damping eliminates the oscillations, and the final ragnitude of the expansions are the static values. These dynamic effects disappear within a few nilliseconds after the power pulse. In several instances, the stresses induced by the oscillations of the core have been of sufficient magnitude to cause danage to fuel components and even reactor disassembly, $1,2,3$

Conventionai thermal stress analysis cannot be used to calculate the stresses induced in a fast burst reactor core because the time to achieve the temperature rise is of the same order of magnitude as the natural vibrational period of the core. This means that the effect of mass inertia must be included in the calculations. In addition to the determination of stresses for evaluation of fuel integrity, it is important to calculate the displacements, since the movement of the core surfaces determines the burst characteristics of the reactor.

\section{Theory}

The high-performance fast burst reactors built during the past few years have all had the shape of a hollow right circular cylinder. $4,5,5$ since a onedimensional analysis restricts motion to a single spatial direction, calculated expansions and stresses are overestimated. ${ }^{7}$ In order to properly describe the thermomechanical behavior of the fuel assembly of a fast burst reactor, two spatial directions must be considered, so a computer code, CADETS, has been written to solve the dynamic, thermoelastic, displacement equations in cylindrical geometry with angular symmetry. These equations are

$$
\begin{aligned}
& (2 \mu+\lambda)\left\{\frac{\partial^{2} u}{\partial r^{2}}+\frac{1}{r} \frac{\partial u}{\partial r}-\frac{u}{r^{2}}\right\}+\mu \frac{\partial^{2} u}{\partial z^{2}}+(\lambda+\mu) \frac{\partial^{2} v}{\partial r \partial z} \\
& -(3 \lambda+2 \mu) \alpha \frac{\partial T}{\partial r}=\rho \frac{\partial^{2} u}{\partial t^{2}},
\end{aligned}
$$

*This work was supported by the United States Atomic Energy Commission. 
and

$$
\begin{gathered}
\mu\left\{\frac{\partial^{2} v}{\partial r^{2}}+\frac{1}{r} \frac{\partial v}{\partial r}\right\}+(2 \mu+\lambda) \frac{\partial^{2} v}{\partial z^{2}}+(\lambda+\mu)\left\{\frac{\partial^{2} u}{\partial r \partial z}+\frac{1}{r} \frac{\partial u}{\partial z}\right\} \\
-(3 \lambda+2 \mu) \alpha \frac{\partial T}{\partial z}=\rho \frac{\partial^{2} v}{\partial t^{2}}
\end{gathered}
$$

where

$$
\begin{array}{lll}
\mathrm{u}=\text { radial displacement component } & \mu=\frac{\mathrm{E}}{2(1+\sigma)} \\
\mathrm{v}=\text { axial displacement component } & \lambda=\frac{\sigma \mathrm{E}}{(1+\sigma)(1-2 \sigma)} \\
\mathrm{r}=\text { radial coordinate } & E=\text { Young's modulus } \\
\mathrm{z}=\text { axial coordinate } & \rho=\text { density } \\
\mathrm{t}=\text { time coordinate } & \sigma=\text { Poisson's ratio } \\
\mathrm{T}=\mathrm{T}(\mathrm{r}, \mathrm{z}, \mathrm{t}) \text { = temperature } & \alpha=\text { thermal expansion coefficient. }
\end{array}
$$

These equations describe the radial and axial displacement of the cylinder as a function of space and time for a specified temperature distríbution, and are based on linear thermoelasticity with constant material properties. The temperature function is the driving force in the equations and may be dependent upon both space and time.

After $\mathrm{Eq}$. (1) and (2) are solved for the displacements for a specified time and spatial variation of temperature, the stress components throughout the cylinder are determined from:

$$
\begin{aligned}
& T_{r r}=(\lambda+2 \mu) \frac{\partial u}{\partial r}+\lambda\left(\frac{u}{r}+\frac{\partial v}{\partial z}\right)-(3 \lambda+2 \mu) \alpha \mathrm{T} \\
& T_{\theta \theta}=(\lambda+2 \mu) \frac{u}{r}+\lambda\left(\frac{\partial u}{\partial r}+\frac{\partial v}{\partial z}\right)-(3 \lambda+2 \mu) \alpha T \\
& T_{z z}=(\lambda+2 \mu) \frac{\partial v}{\partial z}+\lambda\left(\frac{\partial u}{\partial r}+\frac{u}{r}\right)-(3 \lambda+2 \mu) \alpha T \\
& T_{r z}=\mu\left(\frac{\partial u}{\partial z}+\frac{\partial v}{\partial r}\right)
\end{aligned}
$$

where these are the radial, tangential, axial, and shear stress components, respectively.

The reactor is initially at rest prior to a burst, so the initial conditions used in solving $\mathrm{Eq}$. (1) and (2) require all displacement components to be zero. That is,

$$
u(r, z, 0)=v(r, z, 0)=0 \text { for all } r \text { and } z \text { at } t=0
$$

Since a fast burst reactor is unrestrained over the majority of its surface, the boundary conditions used in the solution of Eq. (1) and (2) require that there are no normal or shear stresses imposed on the surfaces of the cylinder. These boundary conditions are

$$
T_{r r}\left(R_{i}, z, t\right)=T_{r z}\left(R_{i}, z, t\right)=0 \text { along the inner radial boundary }\left(r=R_{i}\right)
$$




$$
\begin{aligned}
& T_{r r}\left(R_{0}, z, t\right)=T_{r z}\left(R_{0}, z, t\right)=0 \text { along the outer radial boundary }\left(r=R_{0}\right) \\
& T_{z z}(r, 0, t)=T_{r z}(r, 0, t)=0 \text { along the lower axial boundaries }(z=0) \\
& T_{z z}(r, L, t)=T_{r z}(r, L, t)=0 \text { along the upper axial boundaries }(z=L) \text {. }
\end{aligned}
$$

By solving Eq. (1) and (2) along with the initial conditions (7) and boundary conditions (8), (9), (10), and (11), the $\mathbf{u}$ and $\mathbf{v}$ displacement components throughout the cylinder are found for a given temperature function. The dynamic stress components are then calculated from Eq. (3), (4), (5), and (6) using the displacement components and the temperature distribution.

\section{Numerical Solution of the Displacement Equations}

A finite difference scheme similar to that used by Bertholf ${ }^{8}$ is utilized in the solution of the displacement equations. A space grid is superimposed over the hollow cylinder as shown in Fig. 1, and finite difference expressions for Eq. (1) and (2) are written at interior points. For the radial boundaries, fictitious points outside the cylinder are used in order to satisfy the radial boundary conditions (8) and (9). At the ends of the cylinder, boundary values of $\mathrm{u}$ and $\mathrm{v}$ are found from a simultaneous solution of finite difference analogs of the axial boundary conditions (10) and (11).

\section{Interior Difference Equations}

Equations (1) and (2) are written in explicit finite difference form by using central differences for both space and time derivatives. The resulting difference equations for the displacement components are

$$
\begin{aligned}
& u_{i, j, k+1}=u_{i, j, k}\left\{2-\frac{2 c_{1}^{2} \Delta t^{2}}{\Delta r^{2}}-\frac{2 c_{2}^{2} \Delta t^{2}}{\Delta z^{2}}-\frac{c_{1}^{2} \Delta t^{2}}{r_{i}^{2}}\right\} \\
& +u_{i+1, j, k}\left\{\frac{c_{1}^{2} \Delta t^{2}}{\Delta r^{2}}+\frac{c_{1}^{2} \Delta t^{2}}{2 \Delta r r_{i}}\right\}+u_{i-1, j, k}\left\{\frac{c_{1}^{2} \Delta t^{2}}{\Delta r^{2}}-\frac{c_{1}^{2} \Delta t^{2}}{2 \Delta r r_{i}}\right\} \\
& +\frac{c_{2}^{2} \Delta t^{2}}{\Delta z^{2}}\left\{u_{i, j+1, k}+u_{i, j-1, k}\right\} \\
& +\frac{c_{3}^{2} \Delta t^{2}}{4 \Delta r \Delta z}\left\{v_{i+1, j+1, k}-v_{i+1, j-1, k}-v_{i-1, j+1, k}+v_{i-1, j-1, k}\right\} \\
& -\frac{(3 \lambda+2 \mu) \alpha \Delta t^{2}}{\rho 2 \Delta r}\left\{T_{i+1, j, k+1}-T_{i-1, j, k+1}\right\}-u_{i, j, k-1}
\end{aligned}
$$

and

$$
\begin{aligned}
& v_{i, j, k+1}=v_{i, j, k}\left\{2-\frac{2 c_{1}^{2} \Delta t^{2}}{\Delta z^{2}}-\frac{2 c_{2}^{2} \Delta t^{2}}{\Delta x^{2}}\right\}+\frac{c_{1}^{2} \Delta t^{2}}{\Delta z^{2}}\left\{v_{i, j+1, k}+v_{i, j-1, k}\right\} \\
& +v_{i+1, j, k}\left\{\frac{c_{2}^{2} \Delta t^{2}}{\Delta r^{2}}+\frac{c_{2}^{2} \Delta t^{2}}{2 \Delta r r_{i}}\right\}+v_{i-1, j, k}\left\{\frac{c_{2}^{2} \Delta t^{2}}{\Delta r^{2}}-\frac{c_{2}^{2} \Delta t^{2}}{2 \Delta r r_{i}}\right\} \\
& +\frac{c_{3}^{2} \Delta t^{2}}{4 \Delta r \Delta z}\left\{u_{i+1, j+1, k}-u_{i-1, j+1, k}-u_{i+1, j-1, k}+u_{i-1, j-1, k}\right\}
\end{aligned}
$$




$$
\begin{aligned}
& +\frac{c_{3}^{2} \Delta t^{2}}{2 \Delta z r_{i}}\left\{u_{i, j+1, k}-u_{i, j-1, k}\right\}-\frac{(3 \lambda+2 \mu) \alpha \Delta t^{2}}{\rho 2 \Delta z}\left\{T_{i, j+1, k+1}\right. \\
& \left.-T_{i, j-1, k+1}\right\}-v_{i, j, k-1},
\end{aligned}
$$

where $u_{i, j, k} \approx u(r, z, t)$ and $v_{i, j, k} \approx v(r, z, t)$. The subscripts $i, j$, and $k$ refer to the radial, axial, and time directions, respectively. $\Delta r, \Delta z$, and $\Delta t$ are the radial, axial, and time increments where

$$
\Delta \mathrm{r}=\frac{\mathrm{R}_{\mathrm{o}}-\mathrm{R}_{\mathrm{i}}}{\mathrm{N}-2} \text { and } \Delta \mathrm{z}=\frac{\mathrm{L}}{\mathrm{K}-1} \text {. }
$$

$\mathrm{N}+1$ is the total number of radial space points, and $\mathrm{K}$ is the total number of axial space points as indicated in Fig. $1 . R_{0}$ is the outside radius, $R_{i}$ is the inside radius, and $L$ is the length of the cylinder. The symbols $c_{1}, c_{2}$, and $c_{3}$
are wave speeds in the material and are defined as

$$
c_{1}^{2} \equiv \frac{2 \mu+\lambda}{\rho}, \quad c_{2}^{2} \equiv \frac{\mu}{\rho}, \text { and } c_{3}^{2} \equiv \frac{\mu+\lambda}{p} .
$$

The other symbols in Eq. (12) and (13) are as defined previously. These equations are used to calculate displacement components over the portion of the space grid defined by $2 \leq i \leq N$ and $2 \leq j \leq K-1$.

Boundary Difference Equations

The stress free boundary conditions are satisfied along the radial boundaries by requiring that

and

$$
(\lambda+2 \mu) \frac{\partial u}{\partial r}+\lambda\left\{\frac{u}{r}+\frac{\partial v}{\partial z}\right\}-(3 \lambda+2 \mu) \alpha T=0
$$

$$
\frac{\partial u}{\partial z}+\frac{\partial v}{\partial r}=0
$$

at $r=R_{i}$ and $r=R_{0}$. These boundary conditions are written in finite difference form by using central differences in both the radial and axial directions. Since the boundary and interior values of $u$ and $v$ are known from previous calculations, the difference forms of Eq. (14) and (15) are solved for the displacement components at the fictitious points. Hence, at the inside of the cylinder

$$
\begin{aligned}
& u_{1, j, k}=u_{3, j, k}+\frac{\lambda 2 \Delta r}{r_{2}(\lambda+2 \mu)} u_{2, j, k} \\
& +\frac{\lambda \Delta r}{(\lambda+2 \mu) \Delta z}\left\{v_{2, j+1, k}-v_{2, j-1, k}\right\}-\frac{(3 \lambda+2 \mu)}{(\lambda+2 \mu)} 2 \Delta r \alpha T_{2, j, k}
\end{aligned}
$$

and

$$
v_{1, j, k}=\frac{\Delta r}{\Delta z}\left\{u_{2, j+1, k}-u_{2, j-1, k}\right\}+v_{3, j, k},
$$

for $2 \leq j \leq k-1$.

At the outside of the cylinder, the displacement components for the fictitious points are determined from

and

$$
\begin{aligned}
& u_{N+1, j, k}=u_{N-1, j, k}-\frac{\lambda 2 \Delta r}{r_{N}(\lambda+2 \mu)} u_{N, j, k} \\
& -\frac{\lambda \Delta r}{(\lambda+2 \mu) \Delta z}\left[v_{N, j+1, k}-v_{N, i-1, k}\right]+\frac{(3 \lambda+2 \mu)}{(\lambda+2 \mu)} 2 \wedge r \alpha T_{N, j, k}
\end{aligned}
$$

$$
v_{N+1, j, k}=-\frac{\Delta r}{\Delta z}\left[u_{N T, j+1, k}-u_{N, j-1, k}\right]+v_{N-1, j, k}
$$

for $2 \leq \mathrm{j} \leq \mathrm{K}-1$. 


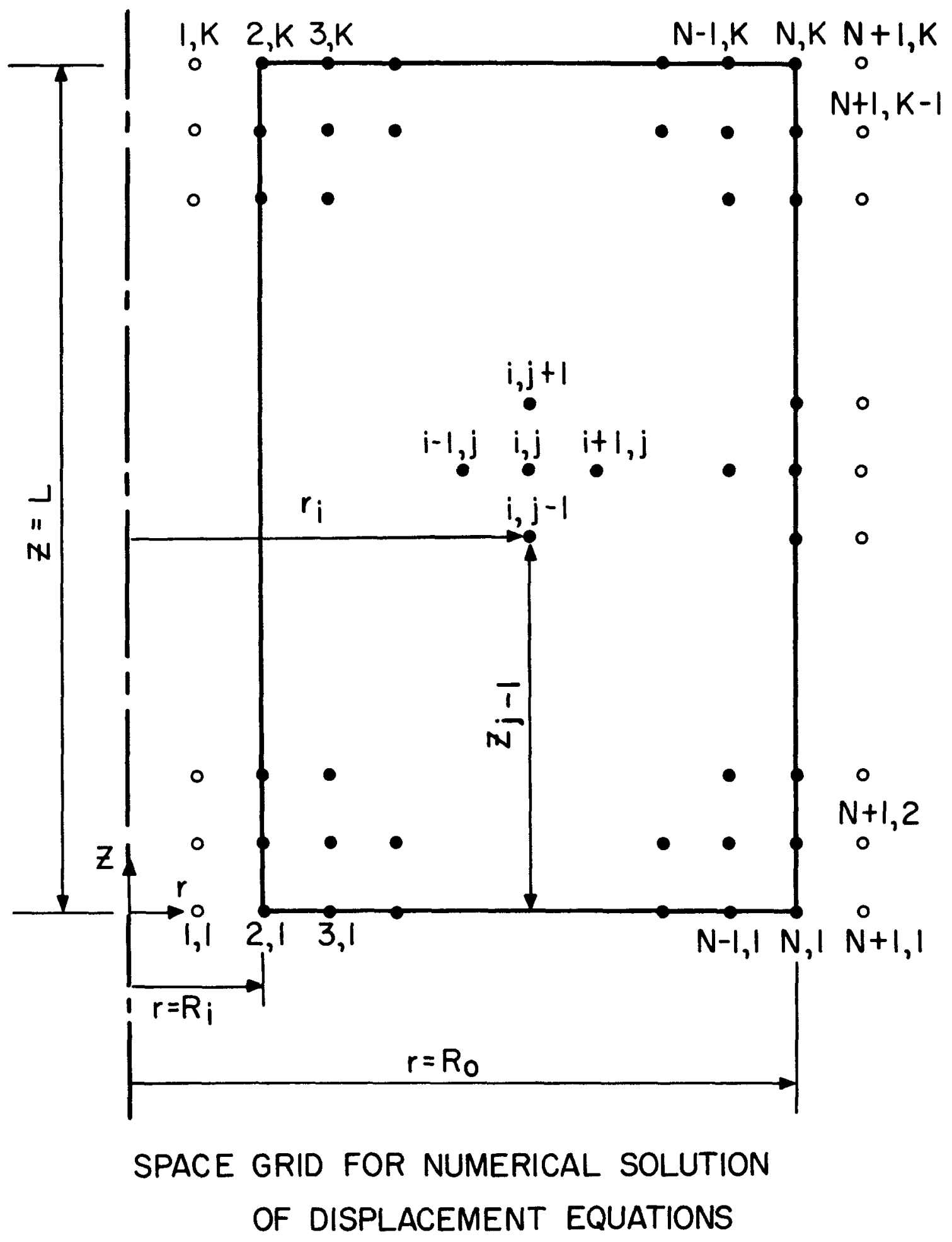

Figure 1. Space Grid for Numerical Solution of Displacement Equations. 
In order to calculate the values of the displacement comnonents along the axial boundaries of the cylinder, a sinultaneous solution of the zero stress boundary conditions is utilized. In general, the following equations are solved at specific space points.

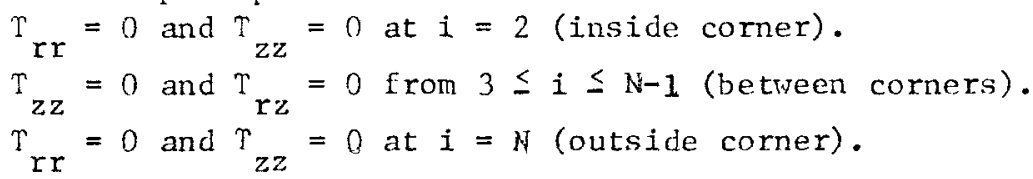

At the lower axial boundary $(z=0)$, axial derivatives are written in forward difference form and radial derivatives are written in fonward, centered, or backward differences, depending upon the location of the derivative across the cylinder. At the lower inside corner $(i=2, j=1)$, the finite difference forms of Eq. (3) and (5) are

$$
\begin{aligned}
\mathrm{T}_{\mathrm{rr}_{2,1}=} & \frac{2 \mu+\lambda}{2 \Delta \mathrm{r}}\left\{-3 \mathrm{u}_{2,1}+4 \mathrm{u}_{3,1}-\mathrm{u}_{4,1}\right\}+\frac{\lambda}{\mathrm{r}_{2}} \mathrm{u}_{2,1} \\
& +\frac{\lambda}{2 \Delta z}\left\{-3 \mathrm{v}_{2,1}+4 \mathrm{v}_{2,2}-\mathrm{v}_{2,3}\right\}-(3 \lambda+2 \mu) \alpha \mathrm{T}_{2,1} \\
\mathrm{~T}_{\mathrm{zz} 2,1}= & \frac{2 \mu+\lambda}{2 \Delta z}\left\{-3 \mathrm{v}_{2,1}+4 \mathrm{v}_{2,2}-\mathrm{v}_{2,3}\right\}+\frac{\lambda}{\mathrm{r}_{2}} \mathrm{u}_{2,1} \\
& +\frac{\lambda}{2 \Delta \mathrm{r}}\left\{-3 \mathrm{u}_{2,1}+4 \mathrm{u}_{3,1}-\mathrm{u}_{4,1}\right\}-(3 \lambda+2 \mu) \alpha \mathrm{T}_{2,1} .
\end{aligned}
$$

and

For simplicity, the time subscript $k$ has been dropped from these equations. The values of the stresses at the corner are zero, and the interior values of $u$ and $v$ are known from previous calculations; therefore, with unknown values on the left-hand side, these equations can be written as

$$
\begin{aligned}
& u_{2,1}\left\{-\frac{3(2 \mu+\lambda)}{2 \Delta r}+\frac{\lambda}{r_{2}}\right\}+\frac{4(2 \mu+\lambda)}{2 \Delta r} u_{3,1}-\frac{(2 \mu+\lambda)}{2 \Delta r} u_{4,1} \\
& -\frac{3 \lambda}{2 \Lambda \mathrm{z}} \mathrm{v}_{2,1}=-\frac{\lambda}{2 \Delta \mathrm{z}} \quad\left\{4 \mathrm{v}_{2,2}-\mathrm{v}_{2,3}\right\}+(3 \lambda+2 \mu) \alpha \mathrm{T}_{2,1}
\end{aligned}
$$

and

$$
\begin{gathered}
u_{2,1}\left\{-\frac{3 \lambda}{2 \Delta r}+\frac{\lambda}{r_{2}}\right\}+\frac{4 \lambda}{2 \Delta r} u_{3,1}-\frac{\lambda}{2 \Delta r} u_{4,1}-\frac{3(2 \mu+\lambda)}{2 \Delta z} v_{2,1} \\
=-\frac{(2 \mu+\lambda)}{2 \Delta z}\left\{4 v_{2,2}-v_{2,3}\right\}+(3 \lambda+2 \mu) \alpha^{T} T_{2,1} .
\end{gathered}
$$

Between the corners along the lower boundary $(3 \leq i \leq N-1), \mathrm{Nq}$. (5) and (6) are written in finite difference form as

$$
\begin{aligned}
\mathrm{T}_{z z_{i, 1}}= & \frac{2 \mu+\lambda}{2 \Delta z}\left\{-3 v_{i, 1}+4 v_{i, 2}-v_{i, 3}\right\}+\frac{\lambda}{r_{i}} u_{i, 1} \\
& +\frac{\lambda}{2 \Delta x}\left\{u_{i+1,1}-u_{i-1,1}\right\}-(3 \lambda+2 \mu) \alpha T_{i, 1}
\end{aligned}
$$

and

$$
\mathrm{T}_{\mathrm{rz}}=\frac{1}{2 \Delta \mathrm{z}}\left\{-3 \mathrm{u}_{\mathrm{i}, 1}+4 \mathrm{u}_{i, 2}-\mathrm{u}_{\mathrm{i}, 3}\right\}+\frac{1}{2 \Delta \mathrm{r}}\left\{\mathrm{v}_{\mathrm{i}+1,1}-\mathrm{v}_{\mathrm{i}-1,1}\right\} \text {. }
$$

Since the stresses are zero and the interior values of $u$ and $v$ are known, these equations can be rearranged in the form

$$
\begin{aligned}
& -\frac{\lambda}{2 \Delta r} u_{i-1,1}+\frac{\lambda}{r_{i}} u_{i, 1}+\frac{\lambda}{2 \Delta r} u_{i+1,1}-\frac{3(2 \mu+\lambda)}{2 \Delta z} v_{i, 1} \\
& =-\frac{(2 \mu+\lambda)}{2 \Delta z}\left\{4 v_{i, 2}-v_{i, 3}\right\}+(3 \lambda+2 \mu) \alpha T_{i, 1} \\
& -\frac{3 \Delta r}{\Delta z} u_{i, 1}-v_{i-1,1}+v_{i+1,1}=-\frac{\Delta r}{\Delta z}\left\{4 u_{i, 2}-u_{i, 3}\right\} .
\end{aligned}
$$

and

At the lower outside corner $(i=N, j=1)$, the finite difference forms of Eq. (3) and (5) are 


$$
\begin{gathered}
{ }_{\mathrm{Tr}_{\mathrm{N}, 1}}=\frac{2 \mu+\lambda}{2 \Delta \mathrm{r}}\left\{3 \mathrm{u}_{\mathrm{N}, 1}-4 \mathrm{u}_{\mathrm{N}-1,1}+\mathrm{u}_{\mathrm{N}-2,1}\right\}+\frac{\lambda}{\mathrm{r}_{\mathrm{N}}} \mathrm{u}_{\mathrm{N}, 1} \\
+\frac{\lambda}{2 \Delta \mathrm{z}}\left\{-3 \mathrm{v}_{\mathrm{N}, 1}+4 \mathrm{v}_{\mathrm{N}, 2}-\mathrm{v}_{\mathrm{N}, 3}\right\}-(3 \lambda+2 \mu) \alpha \mathrm{T}_{\mathrm{N}, 1}
\end{gathered}
$$

and

$$
\begin{aligned}
& \mathrm{T}_{\mathrm{zz}, 1}=\frac{2 \mu+\lambda}{2 \Delta \mathrm{z}}\left\{-3 \mathrm{v}_{\mathrm{N}, 1}+4 \mathrm{v}_{\mathrm{N}, 2}-\mathrm{v}_{\mathrm{N}, 3}\right\}+\frac{\lambda}{\mathrm{r}_{\mathrm{N}}} u_{\mathrm{N}, 1} \\
& \quad+\frac{\lambda}{2 \Delta \mathrm{r}}\left\{3 \mathrm{u}_{\mathrm{N}, 1}-4 \mathrm{u}_{\mathrm{N}-1,1}+\mathrm{u}_{\mathrm{N}-2,1}\right\}-(3 \lambda+2 \mu) \alpha \mathrm{T}_{\mathrm{N}, 1}
\end{aligned}
$$

Again, since the corner stresses are zero and interior values of $u$ and $v$ are known, these equations can be written as

$$
\begin{gathered}
\mathrm{u}_{\mathrm{N}, 1}\left\{\frac{\lambda}{\mathrm{r}_{\mathrm{N}}}+\frac{3(2 \mu+\lambda)}{2 \Delta \mathrm{r}}\right\}-\frac{4(2 \mu+\lambda)}{2 \Delta \mathrm{r}} \mathrm{u}_{\mathrm{N}-1,1}+\frac{(2 \mu+\lambda)}{2 \Delta \mathrm{r}} \mathrm{u}_{\mathrm{N}-2,1} \\
-\frac{3 \lambda}{2 \Delta \mathrm{r}} \mathrm{v}_{\mathrm{N}, 1}=-\frac{\lambda}{2 \Delta \mathrm{z}}\left\{4 \mathrm{v}_{\mathrm{N}, 2}-\mathrm{v}_{\mathrm{N}, 3}\right\}+(3 \lambda+2 \mu) \alpha \mathrm{T}_{\mathrm{N}, 1}
\end{gathered}
$$

and

$$
\begin{aligned}
u_{\mathrm{N}, 1} & \left\{\frac{\lambda}{r_{\mathrm{N}}}+\frac{3 \lambda}{2 \Delta r}\right\}-\frac{4 \lambda}{2 \Delta \mathrm{r}} \mathrm{u}_{\mathrm{N}-1,1}+\frac{\lambda}{2 \Delta \mathrm{r}} \mathrm{u}_{\mathrm{N}-2,1} \\
& -\frac{3(2 \mu+\lambda)}{2 \Delta \mathrm{z}} \mathrm{v}_{\mathrm{N}, 1}=-\frac{(2 \mu+\lambda)}{2 \Delta \mathrm{z}}\left\{4 \mathrm{v}_{\mathrm{N}, 2}-\mathrm{v}_{\mathrm{N}, 3}\right\}+(3 \lambda+2 \mu) \alpha \mathrm{T}_{\mathrm{N}, 1}
\end{aligned}
$$

Thus, with Eq. (22), (23), (26), (27), (30), and (31), there is a set of $2(\mathrm{~N}-1)$ equations and $2(\mathrm{~N}-1)$ unknowns for the $\mathrm{u}$ and $\mathrm{v}$ displacement components along the lower axial boundary from $i=2$ to $i=N$.

The values of $\mathbf{u}$ and $\mathbf{v}$ at the fictitious points at the corners are found by writing Eq. (14) and (15) with central radial differences and forward axial differences. At the point $i=1, j=1$, the displacement components are given as

$$
\begin{aligned}
u_{1,1}= & u_{3,1}+\frac{2 \Delta r \lambda}{(2 \mu+\lambda) r_{2}} u_{2,1}+\frac{\Delta r \lambda}{(2 \mu+\lambda) \Delta z}\left\{-3 v_{2,1}+4 v_{2,2}-v_{2,3}\right\} \\
& -\frac{2 \Delta r(3 \lambda+2 \mu) \alpha}{(2 \mu+\lambda)} \mathrm{T}_{2,1}
\end{aligned}
$$

and

$$
\mathrm{v}_{1,1}=\mathrm{v}_{3,1}+\frac{\Delta \mathrm{r}}{\Delta \mathrm{z}}\left\{-3 \mathrm{u}_{2,1}+4 \mathrm{u}_{2,2}-\mathrm{u}_{2,3}\right\} .
$$

For the fictitious point at $i=N+1, j=1$, the displacement components are

$$
\begin{aligned}
u_{N+1,1} & =u_{N-1,1}-\frac{2 \Delta r \lambda}{(2 \mu+\lambda) r_{N}} u_{N, 1}-\frac{\Delta r \lambda}{(2 \mu+\lambda) \Delta z}\left\{-3 v_{N, 1}+4 v_{N, 2}-v_{N, 3}\right\} \\
& +\frac{2 \Delta r(3 \lambda+2 \mu) \alpha}{(2 \mu+\lambda)} T_{N, 1}
\end{aligned}
$$

and

$$
\mathrm{v}_{\mathrm{N}+1,1}=\mathrm{v}_{\mathrm{N}-1,1}-\frac{\Delta \mathrm{r}}{\Delta \mathrm{z}}\left\{-3 \mathrm{u}_{\mathrm{N}, 1}+4 \mathrm{u}_{\mathrm{N}, 2}-\mathrm{u}_{\mathrm{N}, 3}\right\} \text {. }
$$

At the upper axial boundary $(z=L)$, a similar derivation yields the following set of simultaneous equations for $\mathrm{u}_{2, \mathrm{~K}}, \mathrm{u}_{3, \mathrm{~K}^{\prime}} \cdots, \mathrm{u}_{\mathrm{N}, \mathrm{K}}$, and $\mathrm{v}_{2, \mathrm{~K}}, \mathrm{v}_{3, \mathrm{~K}} \ldots, \mathrm{v}_{\mathrm{N}, \mathrm{K}^{*}}$ 
At the inside upper corner $(i=2, j=K)$,

$$
\begin{aligned}
& u_{2, K}\left\{-\frac{3(2 \mu+\lambda)}{2 \Delta r}+\frac{\lambda}{r_{2}}\right\}+\frac{4(2 \mu+\lambda)}{2 \Delta r} u_{3, K}-\frac{(2 \mu+\lambda)}{2 \Delta r} u_{4, K} \\
& +\frac{3 \lambda}{2 \Delta z} v_{2, K}=-\frac{\lambda}{2 \Delta z}\left\{-4 v_{2, K-1}+v_{2, K-2}\right\}+(3 \lambda+2 \mu) \alpha T_{2, K}
\end{aligned}
$$

and

$$
\begin{gathered}
u_{2, k}\left\{-\frac{3 \lambda}{2 \Delta r}+\frac{\lambda}{r_{2}}\right\}+\frac{4 \lambda}{2 \Delta r} u_{3, K}-\frac{\lambda}{2 \Delta r} u_{4, K}+\frac{3(2 \mu+\lambda)}{2 \Delta z} v_{2, K} \\
=-\frac{(2 \mu+\lambda)}{2 \Delta z}\left\{-4 v_{2, K-1}+v_{2, K-2}\right\}+(3 \lambda+2 \mu) \alpha T_{2, K^{\circ}}
\end{gathered}
$$

Along the upper axial boundary between the corners $(3 \leq i \leq N-1, j=k)$,

$$
\begin{aligned}
& -\frac{\lambda}{2 \Delta r} u_{i-1, K}+\frac{\lambda}{r_{i}} u_{i, K}+\frac{\lambda}{2 \Delta r} u_{i+1, K}+\frac{3(2 \mu+\lambda)}{2 \Delta z} v_{i, K}= \\
& =-\frac{(2 \mu+\lambda)}{2 \Delta z}\left\{-4 v_{i, K-1}+v_{i, K-2}\right\}+(3 \lambda+2 \mu) \alpha T_{i, K}
\end{aligned}
$$

and

$$
\frac{3 \Delta \mathbf{r}}{\Delta z} u_{i, K}-v_{i-1, k}+v_{i+1, K}=-\frac{\Delta r}{\Delta z}\left\{-4 u_{i, k-1}+u_{i, K-2}\right\} \text {. }
$$

At the outside upper corner $(i=N, j=k)$,

$$
\begin{aligned}
& \frac{(2 \mu+\lambda)}{2 \Delta \mathrm{r}} u_{\mathrm{N}-2, \mathrm{~K}}-\frac{4(2 \mu+\lambda)}{2 \Delta \mathrm{r}} \mathrm{u}_{\mathrm{N}-1, \mathrm{~K}}+\left\{\frac{3(2 \mu+\lambda)}{2 \Delta \mathrm{r}}+\frac{\lambda}{\mathrm{r}_{\mathrm{N}}}\right\} \mathrm{u}_{\mathrm{N}, \mathrm{K}} \\
& +\frac{3 \lambda}{2 \Delta \mathrm{z}} \mathrm{v}_{\mathrm{N}, \mathrm{K}}=-\frac{\lambda}{2 \Delta \mathrm{z}}\left\{-4 \mathrm{v}_{\mathrm{N}, \mathrm{K}-1}+\mathrm{v}_{\mathrm{N}, \mathrm{K}-2}\right\}+(3 \lambda+2 \mu) \alpha \mathrm{T}_{\mathrm{N}, \mathrm{K}}
\end{aligned}
$$

and

$$
\begin{gathered}
\frac{\lambda}{2 \Delta r} u_{N-2, K^{-}}-\frac{4 \lambda}{2 \Delta r} u_{N-1, K}+\left\{\frac{3 \lambda}{2 \Delta r}+\frac{\lambda}{r_{N N}}\right\} u_{N, K}+\frac{3(2 \mu+\lambda)}{2 \Delta z} v_{N i, K} \\
=-\frac{(2 \mu+\lambda)}{2 \Delta z}\left\{-4 v_{N, K-1}+v_{N, K^{\prime}-2^{2}}\right\}+(3 \lambda+2 \mu) \alpha T_{N, K^{\circ}}
\end{gathered}
$$

Again the time subscript $k$ has been dropped from these equations. The simultaneous solution of Eq. (36), (37), (38), (39), (40), and (41) gives the 2(N-1) values of $u$ and $v$ across the upper axial boundary of the cylinder. After these values of the displacement components are calculated, the fictitious point values are determined from the following equations.

$$
\begin{aligned}
u_{1, K} & =u_{3, K}+\frac{2 \Delta r \lambda}{(2 \mu+\lambda) r_{2}} u_{2, K}+\frac{\Delta r \lambda}{(2 \mu+\lambda) \Delta z}\left\{3 v_{2, K}-4 v_{2, K-1}+v_{2, K-2}\right\} \\
& -\frac{2 \Delta r(3 \lambda+2 \mu) \alpha}{(2 \mu+\lambda)} T_{2, K} \\
v_{1, K} & =v_{3, K}+\frac{\Delta r}{\Delta z}\left\{3 u_{2, K}-4 u_{2, K-1}+u_{2, K-2}\right\}
\end{aligned}
$$




$$
\begin{gathered}
u_{N+1, K}=u_{N-1, K}-\frac{2 \Delta r \lambda}{(2 \mu+\lambda) r_{N}} u_{N, K}-\frac{\Delta r \lambda}{(2 \mu+\lambda) \Delta z}\left\{3 v_{N, K}\right. \\
\left.-4 v_{N, K-1}+v_{N, K-2}\right\}+\frac{2 \Delta r(3 \lambda+2 \mu) \alpha}{(2 \mu+\lambda)} T_{N, K} \\
v_{N+1, K}=v_{N-1, K}-\frac{\Delta r}{\Delta z}\left\{3 u_{N, K}-4 u_{N, K-1}+u_{N, K-2}\right\}
\end{gathered}
$$

Solution Procedure for the Difference Equations

The CADETS computer code solves the difference equations previously described by first setting all values of $u$ and $v$ to zero. The temperature is varied according to a specified function of space and time. For each time increment $\Delta t$, the interior values of $u$ and $v$ are calculated from Eq. (14) and (15) for $2 \leq i \leq N$ and $2 \leq j \leq K$. Then the values of $u$ and $v$ are determined along the axial boundaries from a simultaneous solution of Eq. (22), (23), (26), (27), (30), and (31) for the lower boundary and of Eq. (36), (37), (38), (39), (40), and (41) for the upper houndary. Next, the fictitious point values of $u$ and $v$ at the corners are calculated directly from Eq. (32), (33), (34), (35), (42), (43), (44), and (45). Finally, the values of $u$ and $v$ along the radial fictitious points are obtained with Eq. (16) and (17) for the inside, and Eq. (18) and (19) for the outside of the cylinder. Thus, all values of $u$ and $v$ are determined for the space grid for a single time step. The temperature is increased and the above procedure is repeated again for the next time step.

After all values of the two displacement components are calculated for a given time step, the stress components are determined from finite difference forms of Eq. (3), (4), (5), and (6). In the interior of the cylinder, these difference equations are

$$
\begin{aligned}
& \mathrm{T}_{\mathrm{rr}}=\frac{(\lambda+2 \mu)}{2 \Delta \mathrm{r}}\left\{u_{i+1, j, k}-u_{i-1, j, k}\right\}+\frac{\lambda}{r_{i}} u_{i, j, k} \\
& \quad+\frac{\lambda}{2 \Delta z}\left\{v_{i, j+1, k}-v_{i, j-1, k}\right\}-(3 \lambda+2 \mu) \alpha T_{i, j, k} \\
& T_{\theta \theta_{i, j, k}}=\frac{(\lambda+2 \mu)}{r_{i}} u_{i, j, k}+\frac{\lambda}{2 \Delta r}\left\{u_{i+1, j, k}-u_{i-1, j, k}\right\} \\
& \quad+\frac{\lambda}{2 \Delta z}\left\{v_{i, j+1, k}-v_{i, j-1, k}\right\}-(3 \lambda+2 \mu) \alpha T_{i, j, k} \\
& T_{z z}=\frac{(\lambda+2 \mu)}{2 \Delta z}\left\{v_{i, j}+1, k-v_{i, j-1, k}\right\}+\frac{\lambda}{r_{i}} u_{i, j, k} \\
& \quad+\frac{\lambda}{2 \Delta r}\left\{u_{i+1, j, k}-u_{i-1, j, k}\right\}-(3 \lambda+2 \mu) \alpha T_{i, j, k} \\
& \quad=\frac{\mu}{2 \Delta z}\left\{u_{i, j+1, k}-u_{i, j-1, k}\right\} \\
& T_{r z} \frac{\mu}{2 \Delta r}\left\{v_{i+1, j, k}-v_{i-1, j, k}\right\} .
\end{aligned}
$$

For the stress components along boundaries, equations such as Eq. (20), (21), (24), (25), (28), or (29) are used to determine the stress magnitudes. Backward and forward differences are used to approximate derivatives at boundaries in the appropriate difference expressions for the boundary values of the stress components. 
A rigorous investigation of the stability of the finite difference scheme is virtually impossible, due to the complexity of the boundary conditions and the inhomogeneous (or temperature) term. However, by making several simplifying assumptions, it is possible to use the Fourier series method to obtain an approximate stability criterion. The Fourier series method was developed by Von Neumann; ${ }^{9}$ and the development given here follows the analysis of Bertholf. 8

In order to use Vor Neumann's method, the following assumptions must be made: (1) the boundary conditions do not contribute to the growth of errors; (2) the inhomogeneous term does not affect the growtl of errors; and (3) the dependent variables may be approximated by a finite Fourier series.

The solutions are then of the form

$$
u(r, z, t)=u_{0} e^{i l r} e^{i m z} e^{n t}
$$

where $1, m$, and $n$ are integers, and $i=\sqrt{-1}$. The positions $r, z$, and $t$ may be written as $r=\alpha \Delta r, z=B \Delta z, t=\gamma \Delta t$, where $\alpha, B$, and $\gamma$ are integers. By defining $\zeta, \eta, \xi$ as

$$
\begin{aligned}
& \zeta=e^{i 1 \Delta r} ; n=e^{i m \Delta z} ; \xi=e^{n \Delta t} \text {, Eq. (50) becones } \\
& u(r, z, t)=u_{0} \zeta^{\alpha} \eta^{\beta} \xi^{\gamma} .
\end{aligned}
$$

The error will not increase as $t$ increases provided that $|\xi| \leq 1$.

Since Eq. (12) and (13) are coupled, it is necessary to either eliminate u or $v$ from one of the equations or to estimate their relative magnitudes. An estination of their relative magnitudes should be sufficient, since the entire development has been heuristic. Thus the assumption that $u=v v$, where $v$ is a constant, can be made.

Substitution of Eq. (51) and $u=v v$ into $\mathrm{Eq}$. (12), after some tedious algebraic manipulation, yields

$$
\frac{\Delta t}{\Delta r} \leq\left[\frac{c_{3}^{2} \Delta r}{4 v \Delta z}+\frac{c_{2}^{2} \Delta r^{2}}{\Delta z^{2}}+\frac{5}{4} c_{1}^{2}\right]^{-\frac{1}{2}}
$$

for the stability criterion for $\mathrm{Lq}$. (12). An analogous procedure for Lq. (13) yields the stability criterion

$$
\frac{\Delta t}{\Delta r} \leq\left[\frac{c_{1}^{2} \Delta r^{2}}{\Delta z^{2}}+\frac{c_{3}^{2} \nu \Delta r}{4 \Delta z}+c_{2}^{2}\right]^{-\frac{1}{2}}
$$

The approxinate region of stability is shown in Fig. 2 for $c_{1}=1.17 \times 10^{5}$ $\mathrm{in} / \mathrm{sec}, c_{2}=5.5 \times 10^{4} \mathrm{in} / \mathrm{sec}, c_{3}=1.03 \times 10^{5} \mathrm{in} / \mathrm{sec}$, and $v=1$. Some numerical results are also given in Fig. 2 for the time-dependent displacement for several values of $\Delta z / \Delta r$. The numerical results show that the heuristic stability analysis given above is valid; however, in practice the values of $\Delta z / \Delta r$ and $\Delta t / \Delta r$ necessary for stability of the difference equations must define a point well within the stability region. 


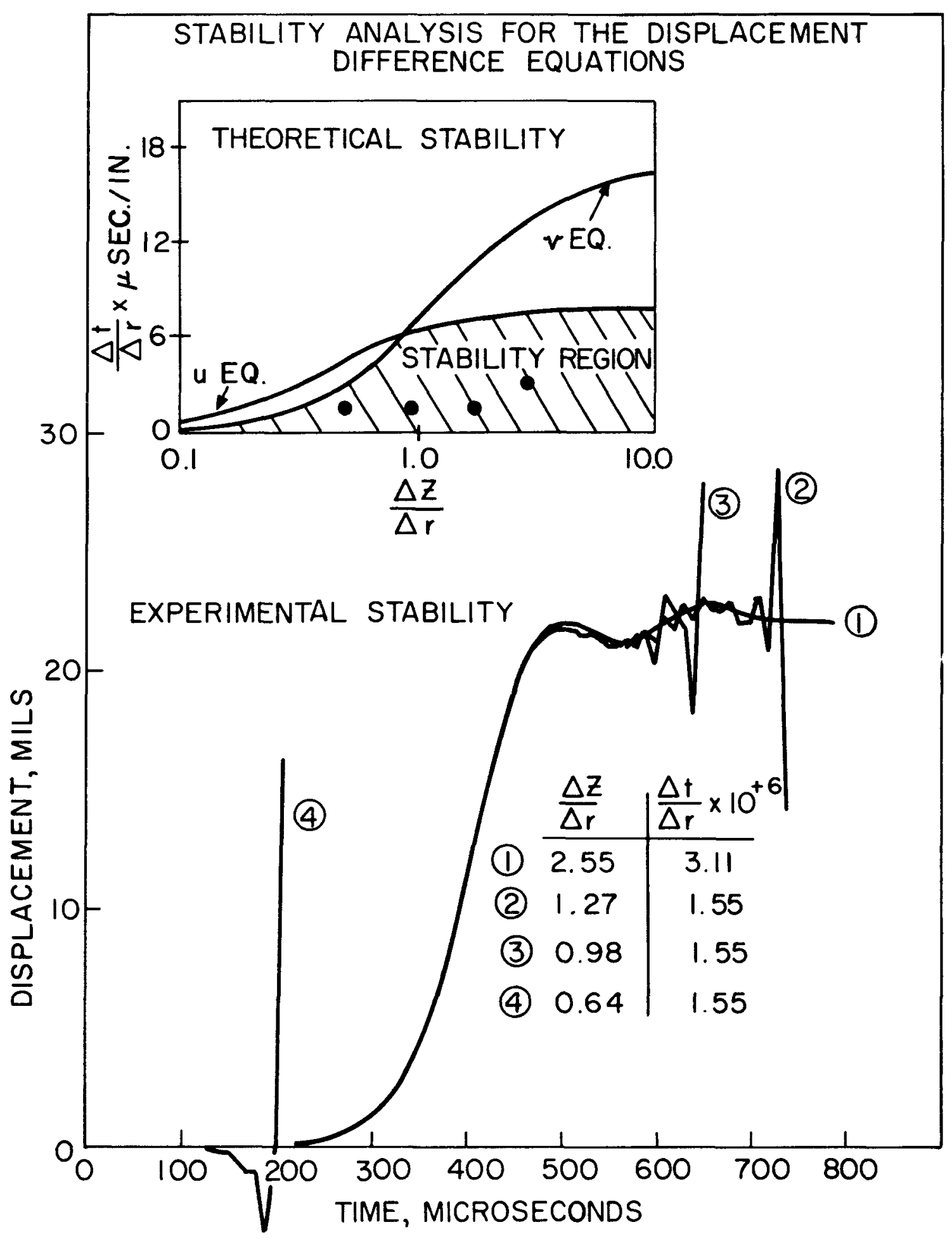

Figure 2. Stability Analysis for the Displacement Difference Equations. 


\section{Results of Calculations}

Calculations have been performed using CADETS for a hollow cylinder with the dimensions, material properties, and temperature conditions appropriate for the Sandia Pulsed Reactor II (SPR II). 5,6 The cylinder has the same dimensions as the upper half of SPR II with an outside radius ( $R_{0}$ ) of 4.039 inches, an inside radius $\left(R_{j}\right)$ of 0.825 inch, and a length (L) of 4.103 inches. The material properties are those of an uranium-10 weight percent molybdenum fuel alloy. $E=13.1 \times 10^{6} \mathrm{psi}, \rho=0.613 \mathrm{lb} / \mathrm{in}^{3}, \sigma=0.358, \alpha=1.225 \times 10^{-5} \mathrm{in} / \mathrm{in}-{ }^{\circ} \mathrm{C}$. The spatial temperature distribution follows the measured neutron flux variation across the core and the temperature rise is calculated from

$$
T(r, z, t)=\frac{T_{\max } F(r, z)}{\exp \left[-\frac{3.52}{b}\left(t-\frac{T}{2}\right)\right]+1}
$$

where

$$
\begin{aligned}
\mathrm{T}_{\max } & =\text { maximum temperature rise } \\
\mathrm{t} & =\text { time } \\
\mathrm{b} & =\text { pulse width at one-half maximur pover } \\
\mathrm{T}_{\mathrm{p}} & =\text { heating time }
\end{aligned}
$$

$F(r, z)=$ normalized spatial distribution of temperature.

This equation is an integration of the symmetric power pulse fiven by Wimett, et al, 10 normalized to the maximum temperature rise. The pulse is symmetric about $\mathrm{T}_{\mathrm{f}} / 2$, the time at which the maximum porer occurs. There is no direct relationship between $\mathrm{b}$ and $\mathrm{T}_{\mathrm{p}}$, but $\mathrm{T}_{\mathrm{p}}=6 \mathrm{~b}$ has heen used for the calculations described in this paper. P'Equation (54) is only an approximation to the actual time variation of temperature in the reactor, but produces results which are in good agreement with experiment. The majority of the temperature rise occurs during a time equal to three pulse widths.

Results are given in Fig. 3 for a maximum temperature rise of $489^{\circ} \mathrm{C}$ and a pulse width of $38 \mathrm{microseconds.} \mathrm{This} \mathrm{figure} \mathrm{shows} \mathrm{the} \mathrm{dynamic} \mathrm{axial} \mathrm{expansion}$ of a point at the average radius on both the top and botton of the cylinder. ilegative expansion is in the negative direction of the coordinate system or downward. The radial expansions of the outside and inside radial surfaces for a point which is located at one-lialf the length are also shown; negative expansions are inward. These curves show that the expansion peaks in the axial direction correspond to expansion valleys in the radial direction. Likewise, peaks in the radial direction correspond to valleys in the axial direction. The predominant vibrational period of $160 \mathrm{microseconds}$ for the radius and $110 \mathrm{microseconds}$ for the axis are shown, as well as higher vibrational periods along the inside radius.

The effect of four different burst conditions on the calculated outside radial expansion is shown in Fig. 4 for a point 0.6 inch from the bottom of the cylinder. The maximun temperature rise and the burst width are given for each curve. As the burst yield diminishes, the magnitude of the dynamic expansion decreases until no oscillations are evident. For the $120^{\circ} \mathrm{C}$ curve, the body is able to respond in unison with the temperature rise so that inertia effects are not important and no oscillations are produced. The vibrational period or the time between oscillation peaks remains the same as burst yield increases. Iowever, higher frequency oscillation modes become apparent at high yields.

If the maximum temperature is held fixed and the burst width is increased, the results shown in Fig. 5 are obtained. The rate of temperature rise is decreased as the burst width increases, which reduces the magnitude of the oscillations. The average expansion remains the same, since the amount of energy being deposited in the cylinder does not change. $\Lambda$ s the heating rate increases, 
CALCULATED DISPLACEMENTS FOR SPR - II UPPER-HALF

$T_{\text {MAX }}=489^{\circ} \mathrm{C}$

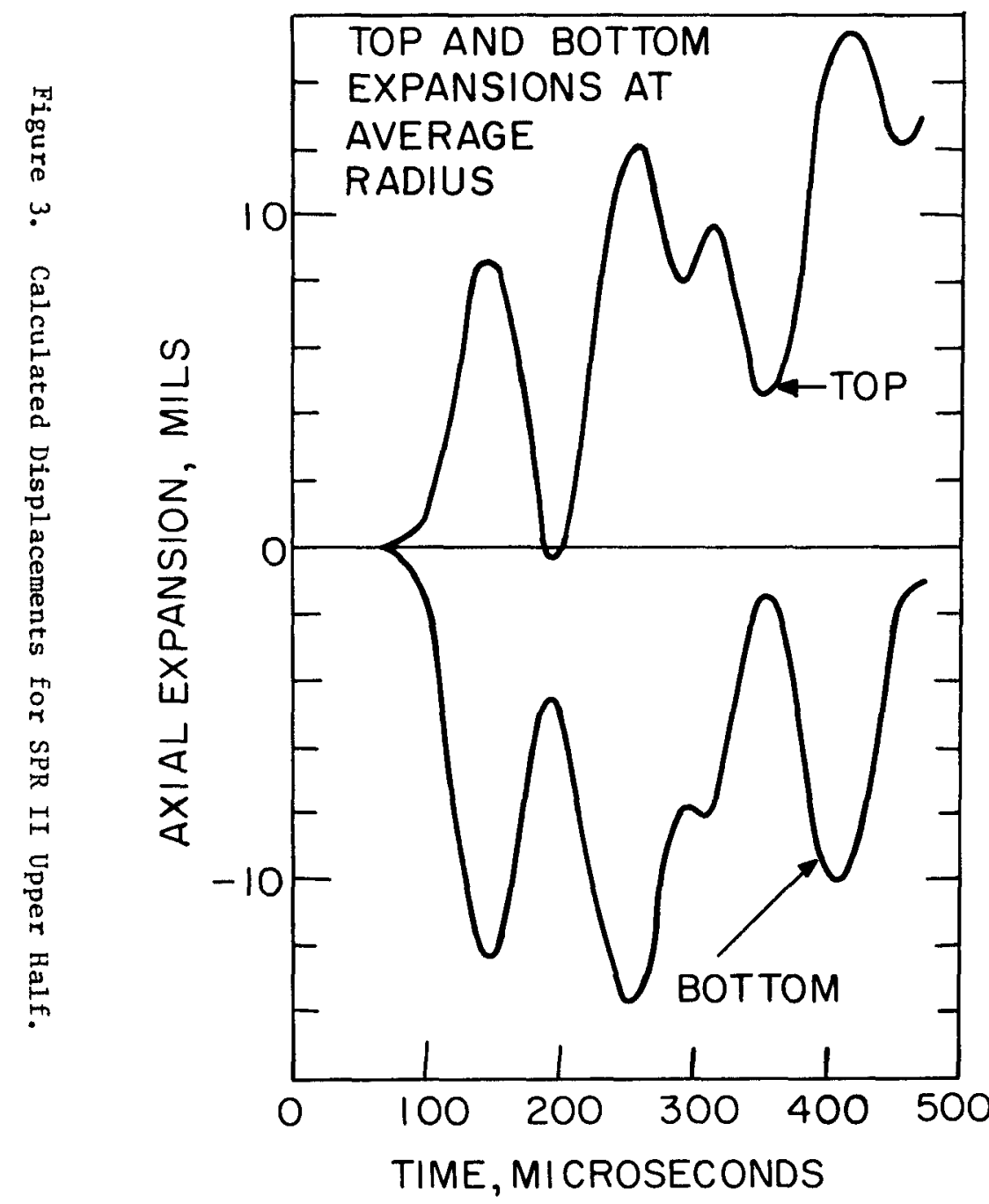

BURST WIDTH $=38 \quad \mu$ SEC

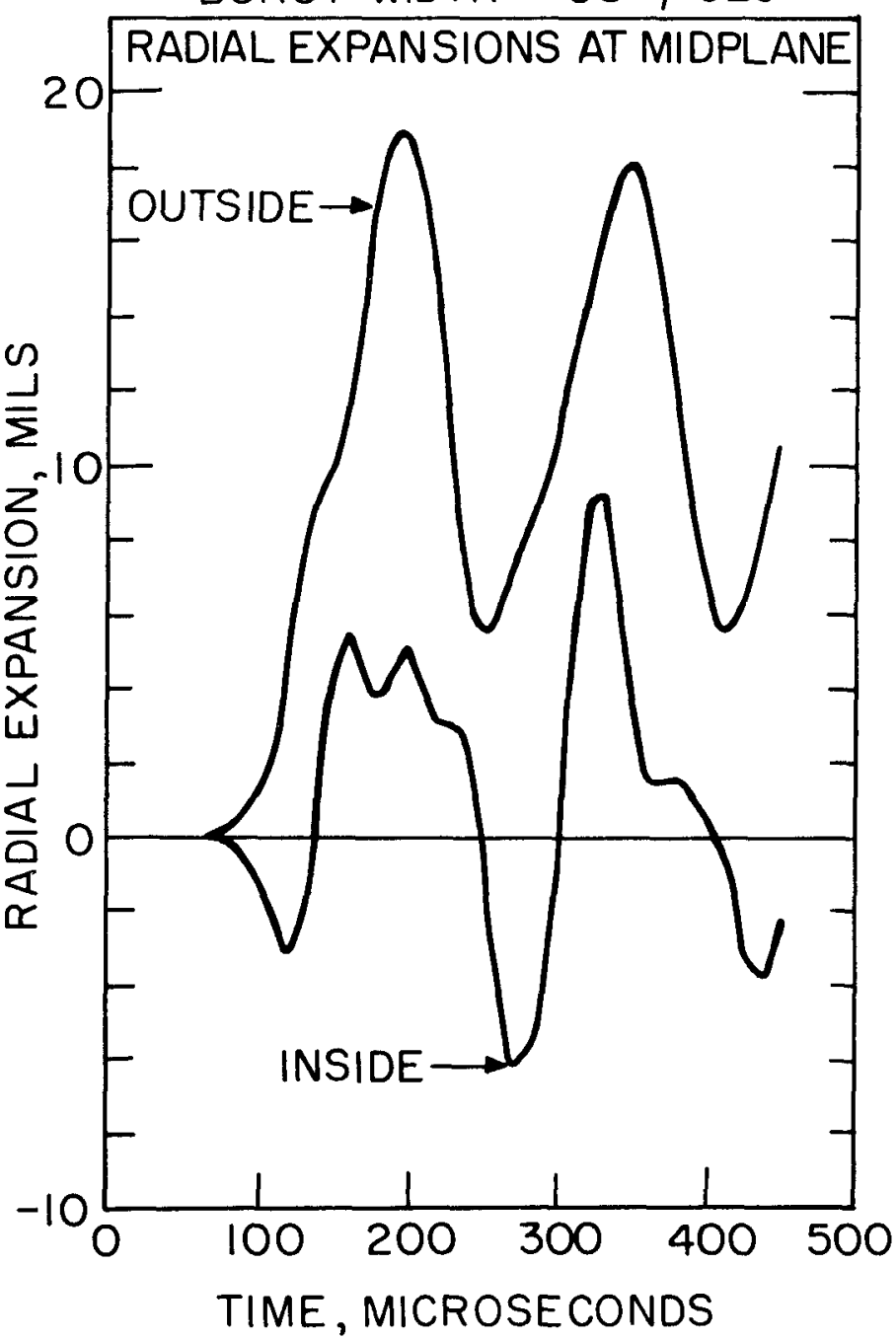




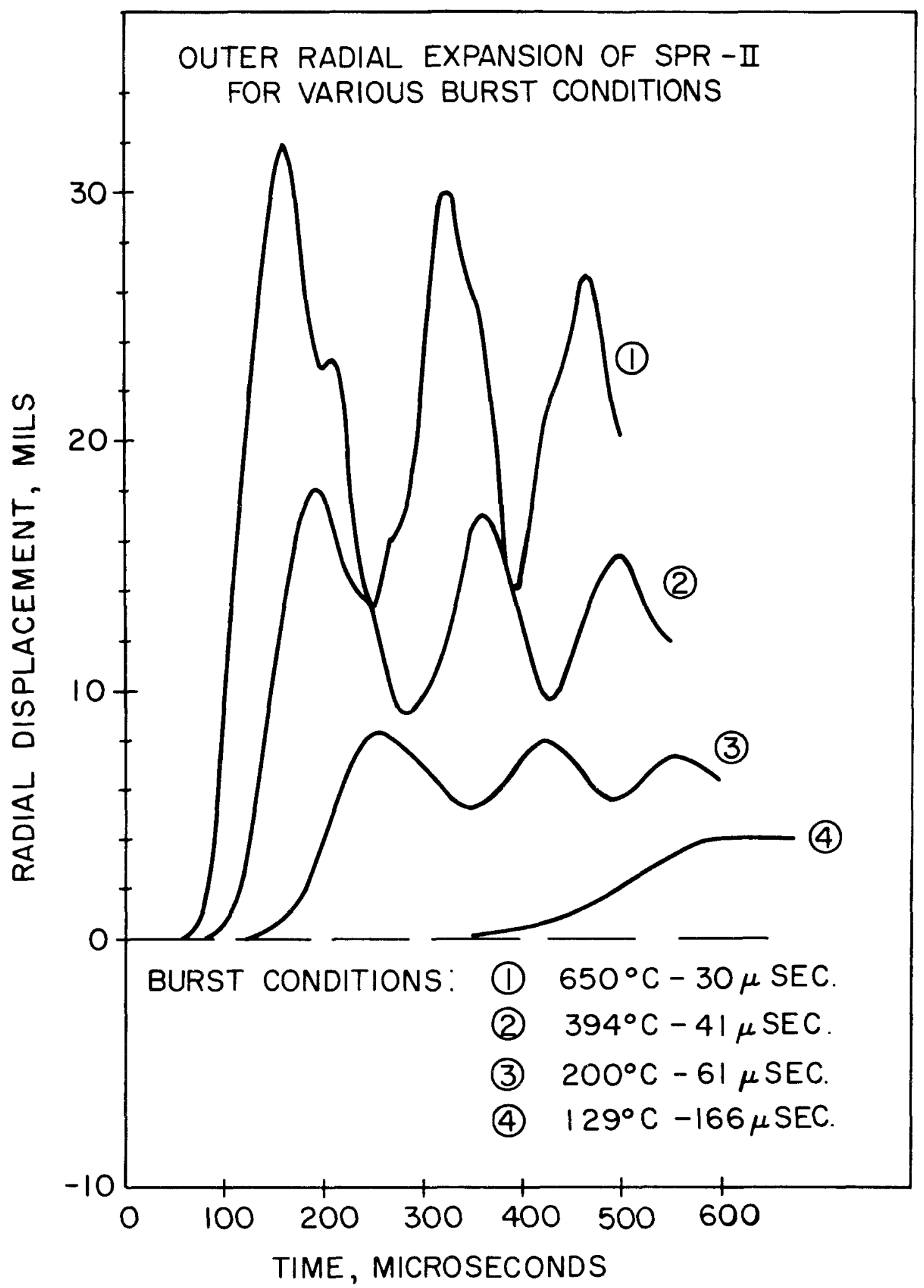

Figure 4. Outer Radial Expansion of SPR II for Various Burst Conditions. 


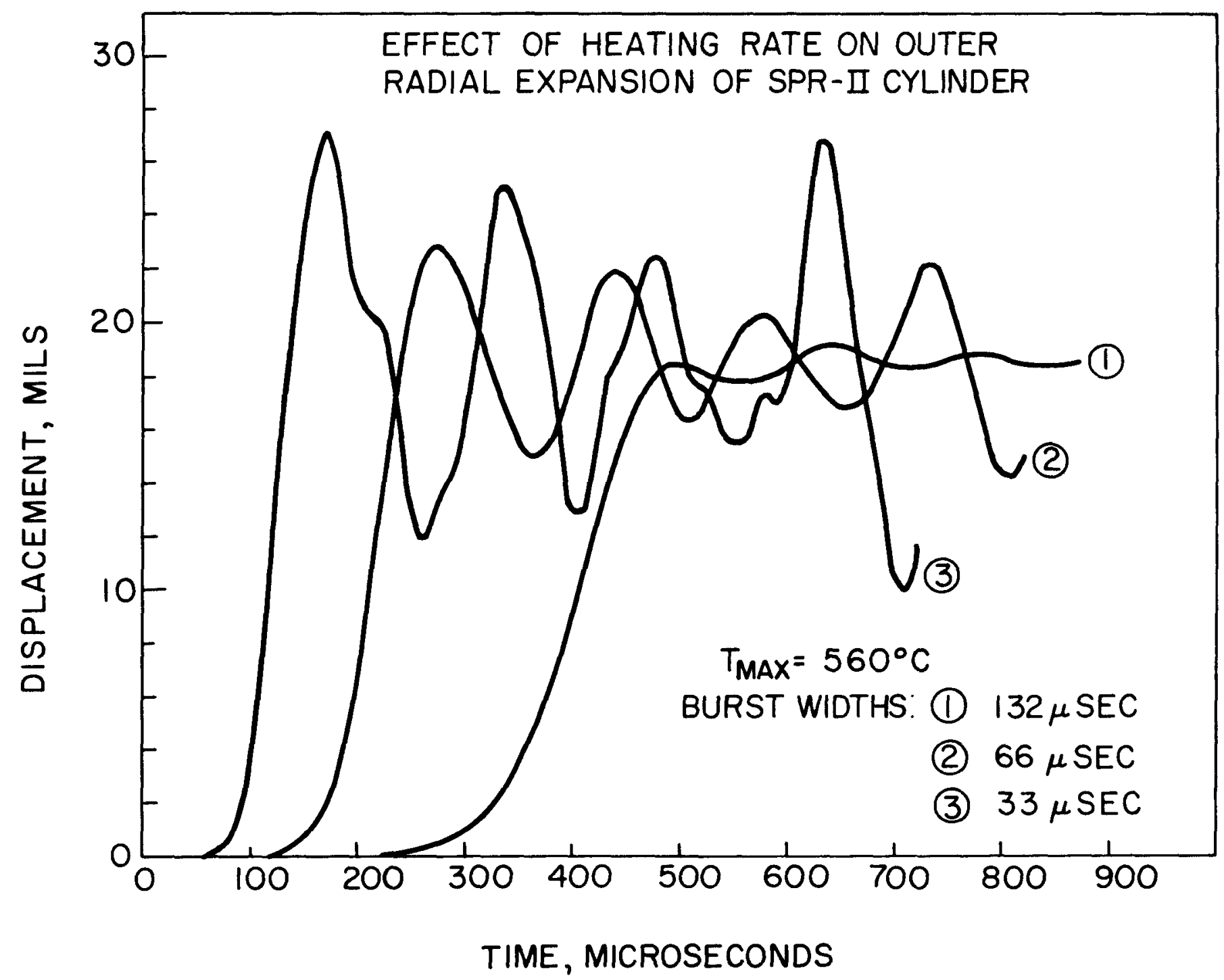


higher frequency oscillations become evident. This figure shows that inertia effects become unimportant as the burst width approaches the vibrational period (160 microseconds) of the cylinder.

\section{Comparison Between Theory and Expcriment}

Some dynamic and static comparisons between experimentally determined displacements and calculated values using CADETS are shown in Figs. 6 and 7 . The measurements were conducted on a central core plate of SPR II using eddy current displacement transducers. 1 Figure 6 shows the dynamic comparison for a burst with a maximum temperature rise of $489^{\circ} \mathrm{C}$ and a width of 38 microseconds. The calculated radial vibrational period of 160 microseconds compares quite favorably with the measured period of 170 microseconds. The outside surface expansions show fairly good agreement between theory and experiment with a difference of about $1.5 \mathrm{mils}$ at the peak expansions. The time scale for the measurements is normalized to the first expansion peak of the calculations. The initial disagreement between theory and experiment up to 120 microseconds is probably due to the sensitivity of the transducers to the transient radiation environment. Higher modes of oscillation are present in the calculations which are not seen in the measurements; this is probably due to several factors. The transducers are unable to resolve the higher frequencies and the data are obtained only for every 20 microseconds, which would tend to smooth out the higher modes. Also, the glory hole surface should behave more like a plate, since there are gaps between adjacent fuel plates. A plate would not exhibit the higher vibrational modes that are seen in a finite cylinder.

The maximum radial expansions are shown in Fig. 7 as a function of peak temperature rise in the fuel plate. The difference between experiment and the two-dimensional calculations is only $2.0 \mathrm{mils}$ at the outsicle surface. The discrepancy between experiment and theory at the glory hole gets larger as the temperature rise increases. The agreement between theory and experiment in Figs. 6 and 7 is excellent, considering that the actual core geometry is considerably more complex than the right circular cylinder used in the calculations. The SPR II core contains gaps, bolt holes, and control rod holes which cannot be included in the calculations.

\section{Conclusion}

The finite difference technique for solving the dynaric themoelastic displacement equations described in this paper provides good agreement between calculated results and measured values. The program CADITS can perform about $3.6 \mathrm{x}$ $10^{5}$ mesh point calculations of $u$ and $v$ per minute of computer time on the CDC6600 . Hollow cylinders with varying lengths and diameters have been successfully run with CADETS as long as stability was maintained by the proper choice of $\Delta t / \Delta r$ and $\Delta z / \Delta r$.

\section{References}

1. J. A. Reuscher, "Dynamic Mechanical Characteristics of the Sandia Pulsed Reactor II," Irans. Am. Nucl. Soc., 11, 220 (1968).

2. R. L. Long, Report of Reactor Excursion During Test of Modified Core, U. S. Army Test and Evaluation Command, White Sands Missile Range, N.M. (July 1965).

3. J. A. Reuscher and J. M. Richter, "Dynamic Mechanical Measurements on the Army Pulsed Radiation Facility Reactor," Trans. Am. Nuc1. Soc., 10, 612 (1967).

4. R. W. Dickinson, et al, Safety Analysis Report for Army Pulse Radiation Facility Fast Pulse Reactor, BRL R 1356 (February 1967). 


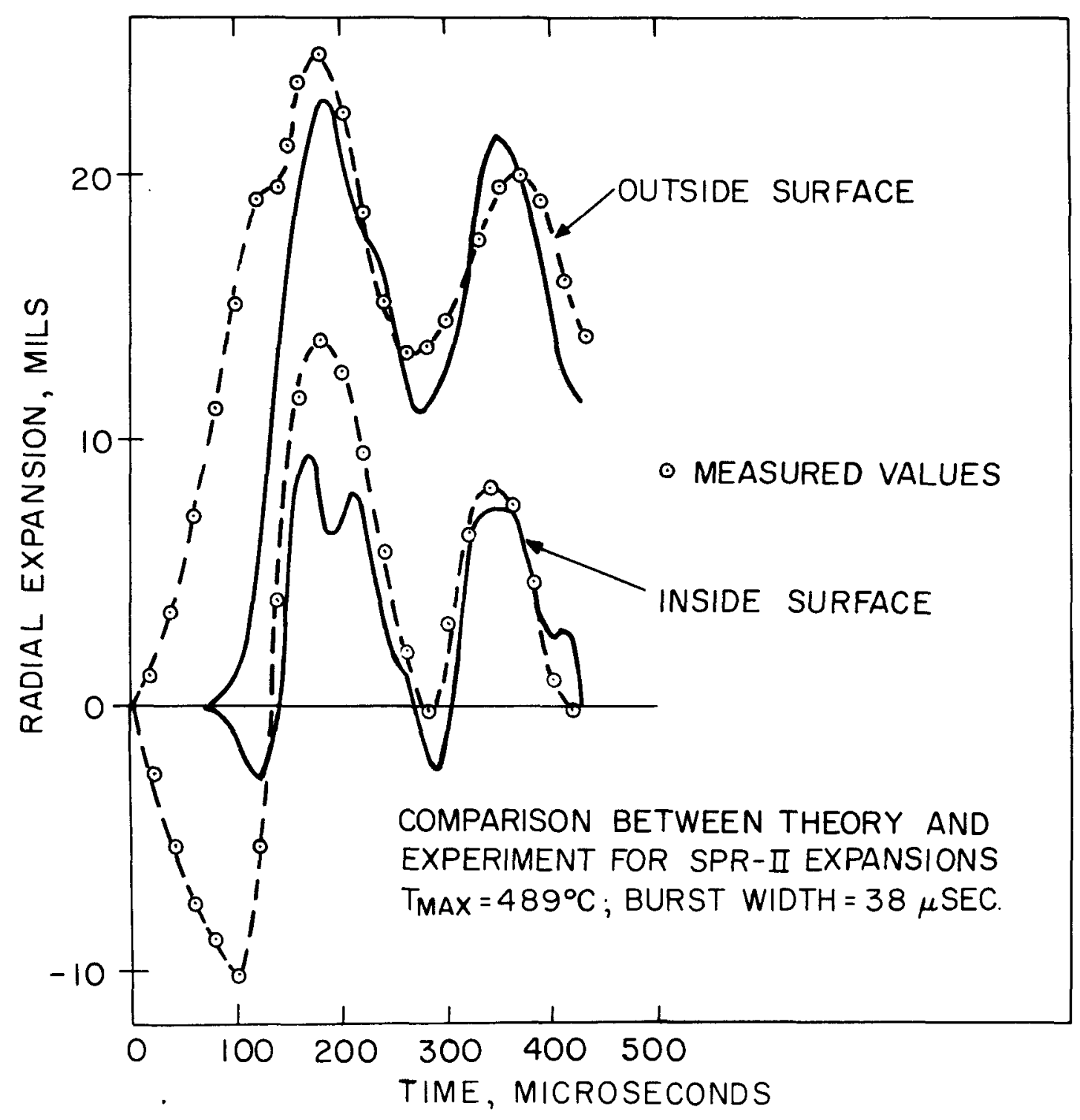

Figure 6. Comparison Between Theory and Experiment for SPR II Expansions. 


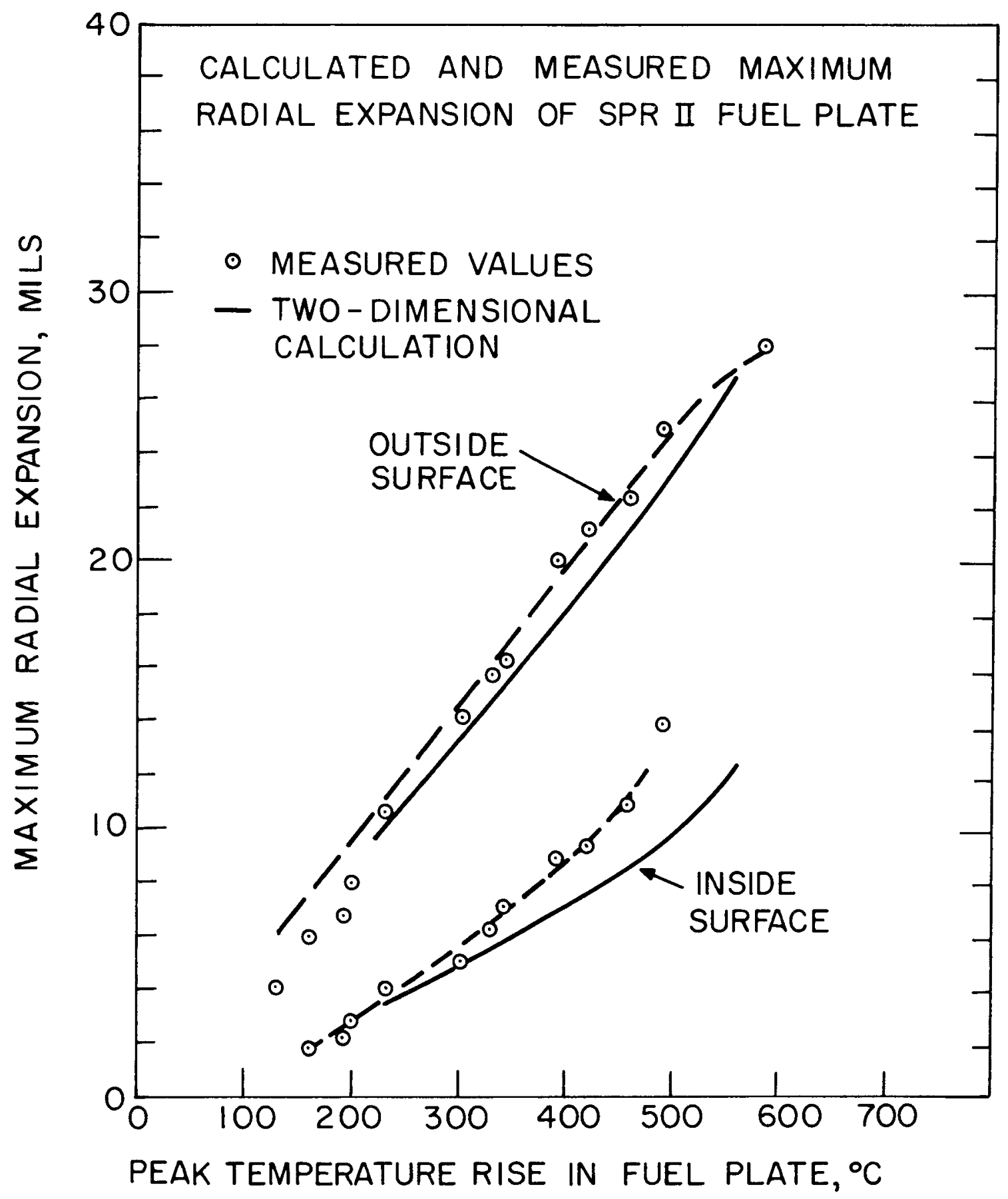

Figure 7. Calculated and Measured Maximum Radial Expansion of SPR II Fuel Plate. 
5. R. L. Coats and P. D. O'Brien, "Pulse Characteristics of the Sandia Pulsed Reactor II," Trans. Am. liuc1. Soc., 11, 219 (1968).

6. R. M. Jefferson, "Sandia Pulsed Reactor II," National Topical Meeting on Fast Burst Reactors, Albuquerque, N.M., (January 28-30, 1969).

7. J. A. Reuscher, "Thermomechanical Analysis of Fast Burst Reactors," National Topical Meeting on Fast Burst Reactors, Albuquerque, N.M., (January 28-30, 1969).

3. L. 1). Bertholf, Longitudinal Elastic Wave Propagation in Finite Cylindrical Bars, WSU SDL 66-03, (July 1966).

9. G. G. O'Brien, M. $\Lambda$. Hyman, and S. Kaplan, "A Study of the Numerical Solution of Partial Differential Equations," J. Math. Phys., 29, 223-250 (1951).

10. T. F. Wimett, R. H. White, W. R. Stratton, and D. P. Wood, "Godiva II-An Unmoderated Pulse-Irradiation Reactor," Nucl. Sci. and Fngr., 8, 691-708 (1960). 
AN EXPLICIT UNLIMITED STABILITY APPROACH

TO THE TRANSIENT CONDUCTION-CONVECTION EQUATIONS

\author{
David J. Bender \\ General Electric Company \\ Breeder Reactor Development Operation
}

\title{
Introduction
}

The transient thermal response of a reactor core to reactivity or flow perturbations is normally analyzed by subdividing the geometry into a nodal structure and maintaining heat balances on each node to determine their temperature history through the transient. Due to the rigorous environment in a core, accuracy in the computation of the nodal temperatures is required to predict when and if the thresholds of material thermal limits are reached.

Since a reactor usually requires quite an extensive nodal structure to describe its complete temperature distribution, and transient analyses involve a great deal of computation, it is of importance to have numerical models available which insure the required accuracy while minimizing computation time.

The nodal structure of the core is constructed by dividing the channels, at different radial locations, into axial sections, with the requirement that the axial power profile is approximately uniform in the node. This results in a model with uniform heat flux from the fuel pin to the coolant along the axial length of the node. This axial section is then subdivided radially, with individual nodes in the coolant, clad and fuel. A typical axial section is shown in Figure 1. This axial section is the basic unit for the thermal analysis, and an examination of the equations for the nodal temperatures within this unit can be generalized to the whole core.*

The computation of the temperatures in the clad and fuel is a straightforward conduction and heat generation problem, and numerical models for this phenomena have been treated extensively. (1) On the other hand, the analysis of the coolant temperature adds another heat transfer mechanism, that of convection. The convection problem requires special consideration because of the transport effects involved. That is, coolant enters the axial section at a given temperature and time, transfers heat by conduction with the fue 1 pin, and exits from the section at another temperature and time. In the analysis, it will be shown that consideration of the transit time of the coolant through the axial section is of kev importance in the correct analys is of the convective

₹ This precludes radial heat transfer between bundles which only becomes important with very large radial temperature gradients across the core, such as in a severe transient causing gross geometrical changes in the core. such a situation is beyond the scope of this analysis. 


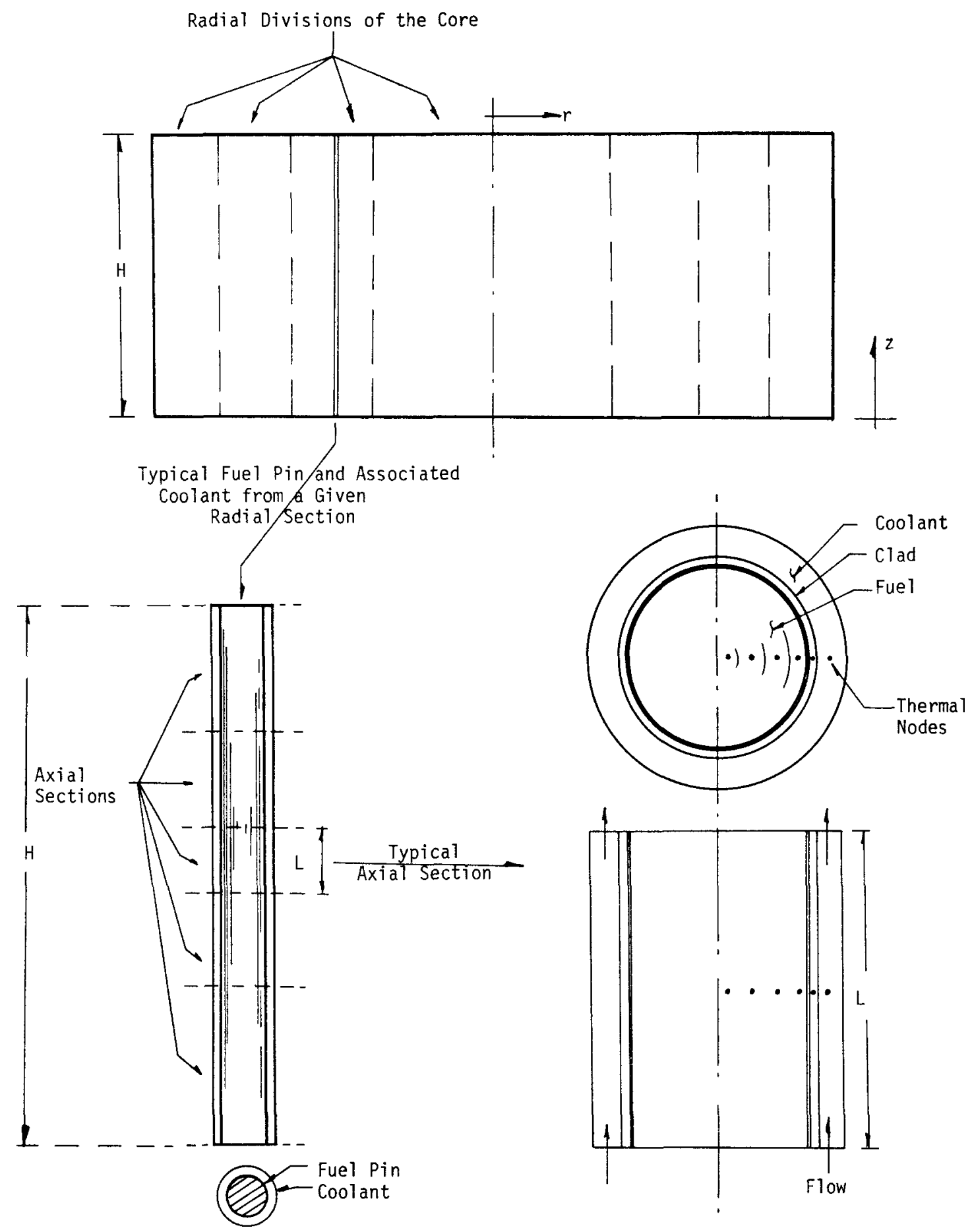

NODALIZATION OF THE REACTOR GEOMETRY FOR THERMAL ANALYSIS

FIGURE 1 
heat transfer. The importance of this transit time through the section had previously been recognized and considered by Calderola, et.al.(2) and Winson. (3)

In keeping with the requirement that the axial section is a basic unit, it is desirable to describe the convective heat transport in terms of the inlet, average and outlet coolant temperatures of the section. Experience with two of the more popular and straight forward methods presently available have shown them to be inaccurate in many of the situations encountered in transient conductive-convective heat transfer analysis. This study was initiated to determine specific shortcomings of present methods and develop a new, more rigorous model.

\section{Review of Existing Convective Finite Difference Techniques}

To demonstrate what is needed in the way of a convective finite difference model, the equation for the temperature of the coolant as a function of time, $t$, and axial position, $z$, is written for one axial section of a reactor channel.

$$
\begin{aligned}
& \rho c A \frac{\partial T_{C}(t, z)}{\partial t}=\frac{1}{R_{W C}}\left[T_{W}(t)-\bar{T}_{C}(t)\right]-\rho c u A \frac{\partial T_{C}(t, z)}{\partial z} \\
& \bar{T}_{C}(t)=\frac{1}{L} \int_{0}^{L} T_{C}(t, z) d z
\end{aligned}
$$

In this formulation it is seen that heat transfer to the coolant is described by the difference between the clad and average coolant temperatures, and is already in finite difference form. Also, axial conduction is neglected, an assumption which is good except at very low flow rates. (4)

The convective term may now be expressed in the form

$$
\frac{\partial T_{C}}{\partial z}=\frac{T_{L}(t)-T_{i n}(t)}{L}
$$

so that the problem now is transferred to determinina a valid expression for the coolant outlet temperature as a function of time. It should be noted here that more complex and exact expressions for $\partial T_{C} / \partial z$ are available (1) than equation (3), but they involve using temperatures outside of the axial section, and thus do not conform to the criteria of the axial section as a basic unit. It is the object of this analysis to determine a valid model for $T_{L}(t)$ so that equation (3) will yield accurate results when used in equation (1). Also, it should be realized that $T_{i n}(t)$ is actually $T_{L}(t)$ from the adjacent upstream axial section, or else the coolant inlet temperature to the core.

The two methods presently in use for evaluating the coolant outlet temperature have been

$$
T_{L}(t+\tau)=T_{L}(t)+\left[\bar{T}_{C}(t+\tau)-\bar{T}_{C}(t)\right]
$$

and

$$
T_{L}(t+\tau)=\bar{T}_{c}(t+\tau)+\left[\bar{T}_{c}(t+\tau)-T_{\text {in }}(t+\tau)\right]
$$


The analysis has shown that the validity of either equations (4) or (5) depends on the relative size of the time increment, $\tau$, and the transit time of the coolant through the node, $\lambda$.

$$
\lambda=\frac{L}{u}
$$

Neither expression (4) or (5) explicitly involve $u$ (or equivalently $\lambda$ ) so that they are only valid when particular relationships exist between $\tau$ and $\lambda$.

Interpreting equations (4) and (5) physically, equation (4) specifies the change in outlet coolant temperature as equal to the change in the average coolant temperature. Equation (5) in essence assumes a linear axial temperature gradient and specifies the change in coolant outlet temperature as twice the change in average coolant temperature. The actual value of the coolant outlet temperature change lies between these two limiting cases depending on the relative size of $\tau$ and $\lambda$, and the nature of the phenomena causing the temperature perturbation.

\section{The Convective Transport Effects}

In order to understand the transient response of the coolant and the transport delay effects involved, a simplified form of the original problem, as stated by equation (1), was selected for study.

The physical model used was simply an axial section of coolant channel with a specified heat input to the coolant. The geometry is shown in Figure 2. By selecting this simplification, the delay time associated with conducting heat to or from the fuel is ignored, but the model is useful in permitting isolation and specific examination of the coolant transport effects. After the solution is found, the heat input can be reassociated with the heat transfer from the fuel pin so that finite difference equations can be formulated.

Initially, it is desirable to find the response of the chosen system to either a step change in coolant flow or a step change in inlet temperature. With the elimination of the time lag between coolant and fuel, it can be shown that a decrease in flow qives an equivalent response to an increase in heat input, and thus all of the major types of perturbations to the coolant temperature can be covered by flow decrease or inlet temperature change. is

The governing equation for the coolant temperature in the selected model

$$
\rho c A \frac{\partial T_{C}(t, z)}{\partial t}+\rho c A u_{F} \frac{\partial T_{C}(t, z)}{\partial z}=\pi D \dot{q}_{F}
$$

The initial condition for the system is that it is in steady state:

$$
T_{C}(0, z)=T_{\text {in }}{ }^{0}+b_{0} z \quad(0 \leq z \leq L)
$$

where $\quad b=\frac{\pi D}{\rho C A}$

$$
\delta_{0}=\frac{\dot{q}_{0}^{\prime \prime}}{u_{0}}
$$


SYSTEM DIAGRAM FOR A SPECIFIED HEAT INPUT

TO THE COOLANT IN AN AXIAL SECTION

FIGURE 2

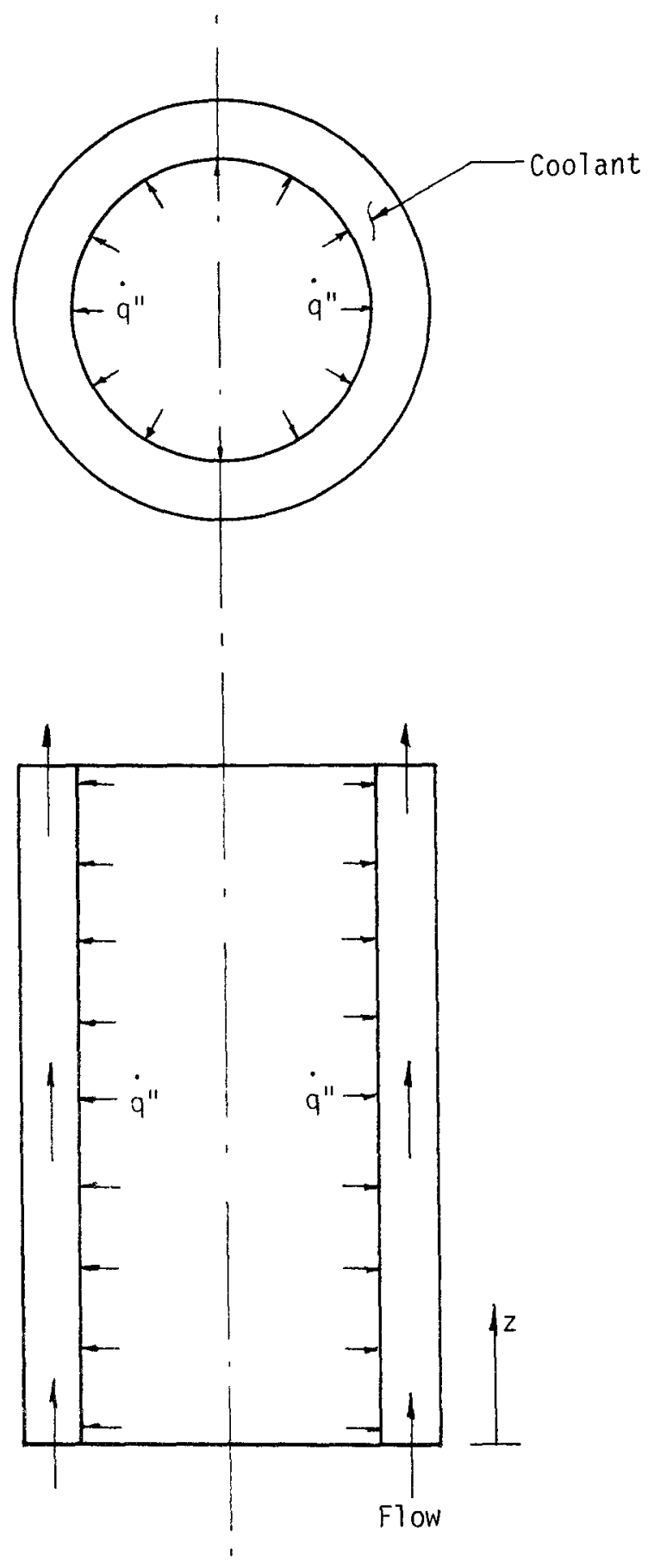


The boundary condition is a step change in coolant inlet temperature

$$
T_{c}(t, 0)=T_{\text {in }} F
$$

Note that the governing equation and boundary condition already incorporate the step changes in flow and heat input.*

Now solving equation (7) by LaPlace transform methods, the solution for the coolant temperature becomes

$$
T_{C}(t, z)= \begin{cases}T_{i n}{ }^{0}+b \delta_{0} z+b u_{F}\left(\delta_{F}-\delta_{0}\right) t & \left(z>u_{F} t\right) \\ T_{i n}{ }^{F}+b \delta_{F} z & \left(z<u_{F} t\right)\end{cases}
$$

and it is noted, in equations (10), that $U_{F} t$ is the distance the perturbation has traveled up the coolant channel from the inlet from time $t=0$.

To further clarify these results, a simple step reduction in flow is examined. Setting

$$
\begin{aligned}
& \dot{q}_{F}^{\prime \prime}=\dot{q}_{0}^{\prime \prime} \\
& T_{\text {in }}=T_{\text {in }}{ }^{\circ}
\end{aligned}
$$

Equations (7) reduce to

$$
T_{c}(t, z)= \begin{cases}T_{C}(0, z)+\dot{b}_{0}^{\prime \prime}\left(1-\frac{u_{F}}{u_{0}}\right) & \left(z>u_{F} t\right) \\ T_{i n}{ }^{0}+\frac{b \dot{q}_{0}^{\prime \prime} z}{u_{F}} & \left(z<u_{F} t\right)\end{cases}
$$

These results are plotted in Figure 3.

Similarly, for a step change in inlet temperature, equations (10) become

$$
T_{c}(t, z)= \begin{cases}T_{i n}{ }^{0}+b \delta_{0} z & \left(z>u_{F} t\right) \\ T_{i n}{ }^{F}+b \delta_{0} z & \left(z<u_{F} t\right)\end{cases}
$$

This is plotted in Figure 4.

By examining Figures 3 and 4 , the transport effects mentioned previously become evident. For the step decrease in flow (or step increase in heat input), the new axial temperature profile in the channel does not fully establish itself unti] $t \geq \lambda$. The effect at the outlet is felt gradually. During the transient, $0 \leq t \leq \lambda$, the temperature for $z>u_{F} t$ maintains its original gradient, the profile being displaced upward, and for $z<u F t$ the new profile is fully established.

* The model has included both step changes in flow and heat input, and thus the verification of the equivalence of $\Delta u$ and $\Delta \dot{q} "$ is possible from the equations. 


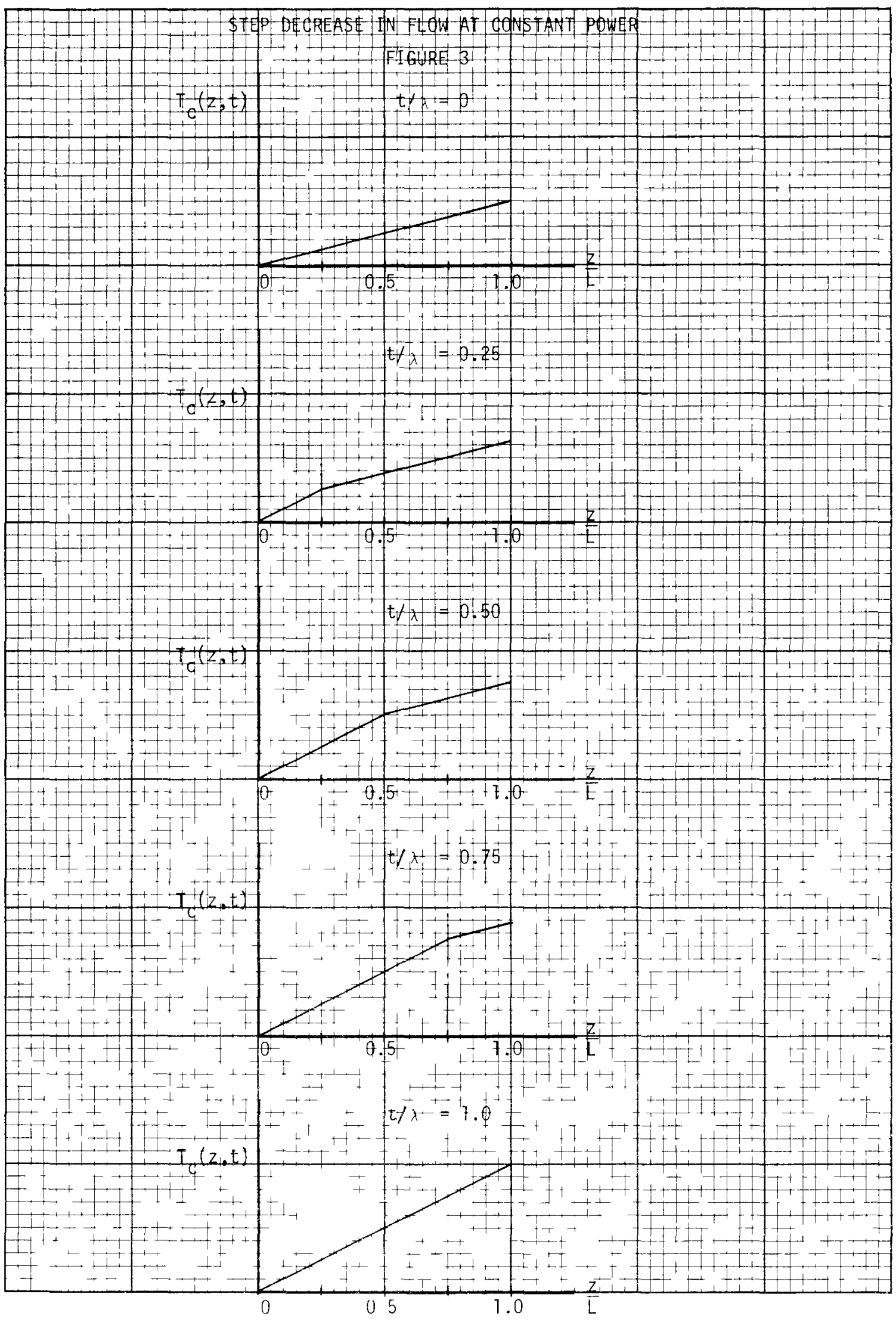




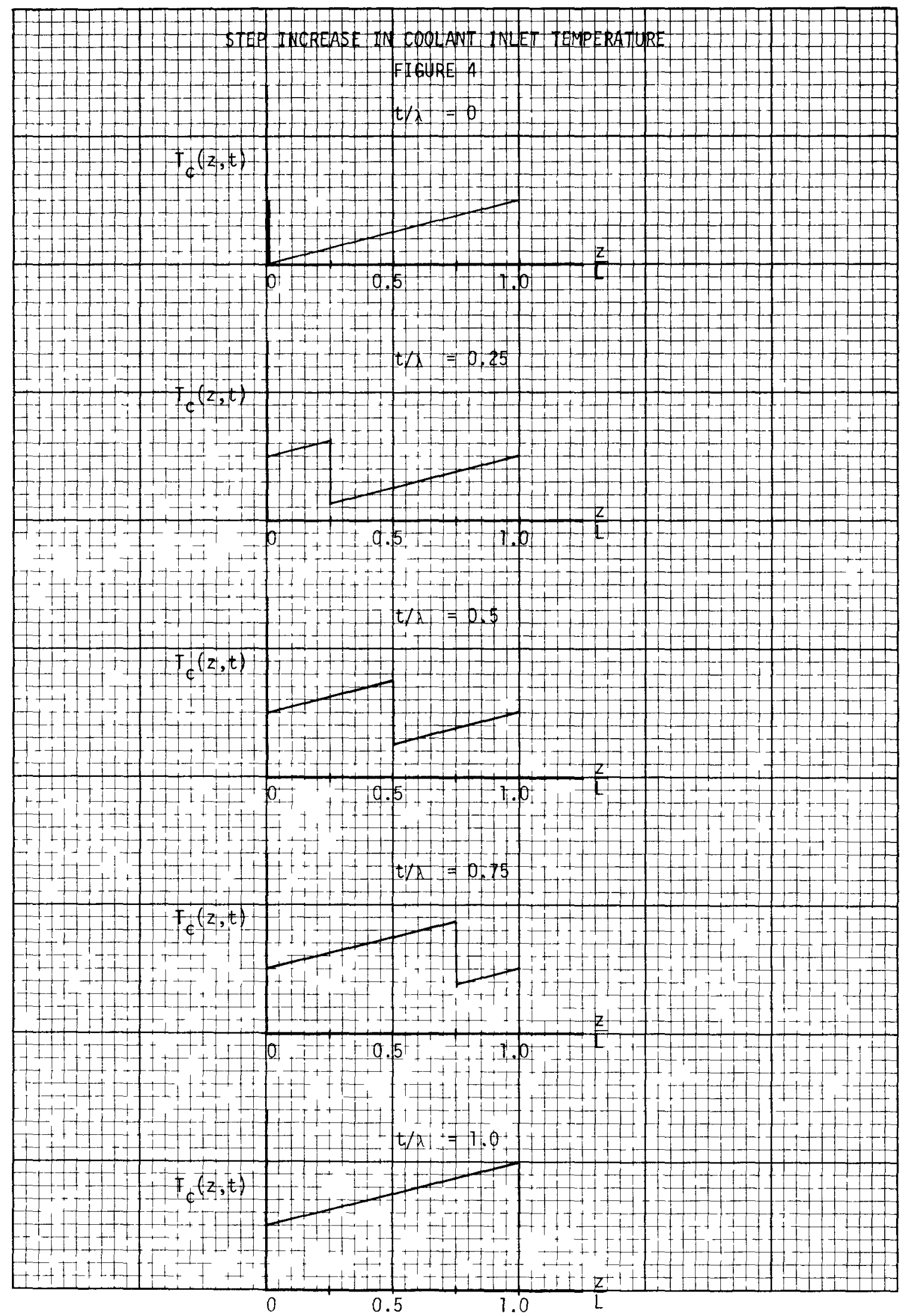


For the step change in inlet temperature, the perturbation propagates up the channel at velocity $u_{F}$ and is not seen at the outlet until $t=\lambda$.

To compare these exact results with the finite difference methods mentioned previously, equations (4) and (5), the average and outlet coolant temperatures are plotted as functions of time in Figure 5 .

For the flow decrease, it can be seen that for $t<<\lambda$, equation (4) is a better finite difference approximation to the actual situation than equation (5). For $t>\lambda$, equation (5) gives a better solution to the coolant response. Thus, neither of equations (4) or (5) are particularly attractive for an arbitrary size time step, for they may or may not yield correct results. That is, accuracy is not insured.

For the step change in coolant inlet temperature, neither of the finite difference formulations, equations (4) and (5), predict the transport delay at a11. In fact, for a large $T_{i n}$ and $t<\lambda$, equation (5) will cause the outlet temperature to drop below its original value.

Formulation and Evaluation of the Corrected Convective Finite Difference Equations

Using solutions (10a) and (10b), a new expression in finite difference form for the coolant temperature can be formulated. The model is slightly more complex than equations (4) or (5), due to the necessity of describing the transport effects, but does give much improved results.

With respect to the coolant outlet temperature, evaluating (10a) at $z=L$;

$$
\begin{aligned}
& T_{L}(t)=\underset{\frac{T_{L}(0)}{\text { Initial }}}{T_{F}}-b u_{F} \frac{\dot{q}_{0}^{\prime \prime}}{u_{0}} t+\underbrace{b \dot{q}_{F}^{\prime \prime} t}_{\text {Heat }} \\
& \text { Outlet Temp Input } \\
& \text { Initial Temp at } \\
& (L-z)=u_{F} t
\end{aligned}
$$

Having identified these terms, it is now possible to set up the equations in terms of nodal temperatures at two consecutive time steps, $j$ and $j+1$. Writina equation (13) in an equivalent finite difference form:

$$
\begin{aligned}
T_{L}^{j+1}=T_{L}^{j}-\left(T_{L}^{j}-T_{i n}^{j}\right) \frac{\tau_{j+1}}{\lambda_{j+1}}+\omega_{C}\left(T_{W}^{j}-\bar{T}_{C}^{j}\right) \tau_{j+1} \quad\left(\tau_{j+1}<\lambda_{j+1}\right) \\
\text { where } \cos _{C}=\frac{1}{\rho c A R_{W C}} \\
{ }^{\tau} j+1=t_{j+1}-t_{j} \\
\lambda_{j+1}=\frac{L}{u_{j+1}}
\end{aligned}
$$

and where the average coolant temperature, $\bar{T}_{C}$, is taken as the "nodel" temperature of the coolant in the axial section. Experience with this equation required that it be modified due to the fact that at any given time step the axial temperature profile in the coolant may not be linear, as assumed in the derivation for equations (10). Thus the final recommended form of the equation is 


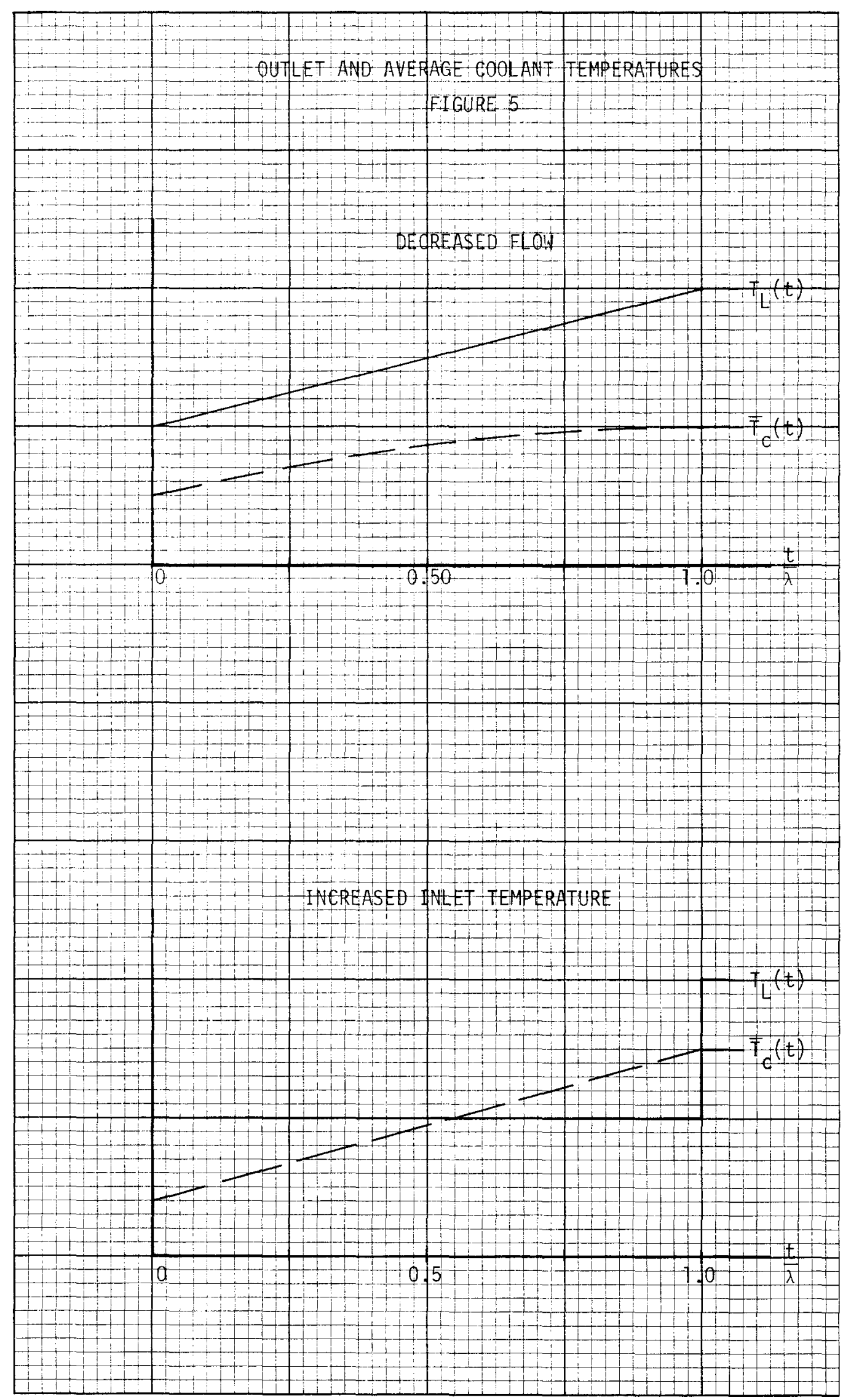




$$
T_{L}^{j+1}=T_{L}^{j}-2\left(T_{L}^{j}-\bar{T}_{C}^{j}\right) \frac{{ }^{j} j+1}{\lambda j+1}+\omega_{C}\left(T_{w}^{j}-\bar{T}_{C}^{j}\right)_{j+1} \quad\left(\tau_{j+1}<\lambda_{j+1}\right)
$$

For $\tau>\lambda$, equation ( $10 \mathrm{~b}$ ) is used and evaluated at $z=L$. Proceeding directly to the finite difference model, it is

$$
T_{L}^{j+1}=T_{i n}^{j+1}+\omega_{c}\left(T_{W}^{j}-\bar{T}^{j}\right) \lambda_{j+1} \quad\left(\tau_{j+1}>\lambda_{j+1}\right)
$$

This expression can be extended to include a linear variation of the inlet temperature*, with the result

$$
\begin{aligned}
& T_{L}^{j+1}=T_{i n}^{j}+\dot{T}_{i n}^{j+1}\left(\tau_{j+1}-\lambda_{j+1}\right)+\omega_{c}\left(T_{w}^{j}-\bar{T}_{c}^{j}\right) \lambda_{j+1} \\
& \text { where } \dot{T}_{i n}^{j+1}=\frac{T_{i n}^{j+1}-T_{i n}^{j}}{\tau_{j+1}}
\end{aligned}
$$

Since a good expression for $T_{L}$ is now available, the average, or nodal, coolant temperature may be evaluated with a simple heat balance.

$$
\bar{T}_{c}^{j+1}=\bar{T}_{c}^{j}+\omega_{c}\left(T_{w}^{j}-\bar{T}_{c}^{j}\right) \tau_{j+1}-\left(T_{L}^{j}-T_{i n}{ }^{j}\right) \frac{{ }^{\tau}}{\lambda+1}
$$

To summarize the computational procedure, the coolant inlet temperature is evaluated as the outlet temperature from the adjacent upstream axial section. Then the outlet temperature is evaluated from either equation (14a) or (14b), and the average coolant temperature from equation (i5). Having found $T_{C}$, it is then possible to evaluate by either an explicit or implicit method the clad and fuel temperatures. If an implicit scheme is used, equation (15) can be reformulated in terms of temperatures at step $j+1$, and included in the implicit solution for the time step.

This is also an appropriate place to point out the fact that equations (14) for $T_{L} j+1$ are not stability limited.

To examine the calculational results of the suggested model, as compared to the other models, a series of transients were run and compared to exact results, as determined from previous considerations.

A computational model was set up which had the capability of calculating a transient by the recommended method and also by two previous methods, equations and $(5)$. The models consisted of one axial section with the coolant coupled by conduction to one fuel node. The three models differed only in their method of calculating the coolant outlet temperature. Specifically, the three methods were

* This would be a typical situation for an axial section downstream from the core inlet during the course of the transient. 
Method 1

$T_{1}$ from Equations (14)

$\overline{\mathrm{T}}_{\mathrm{c}}$ from Equation

Method 2

$T_{L}$ from Equation (4)
$\bar{T}_{C}$ from Equation (15)

Method 3

$T_{L}$ from Equation (5)
$\bar{T}_{C}$ from Equation (15)

Where, in equation (15), $T_{W}$ was replaced by a fuel temperature and $R_{W C}$ was corrected to reflect the correct thermal resistance from fuel to coolant. A straight forward conduction heat-generation finite difference model was used for the fuel temperature.

These three methods were used to calculate the following transients.

\section{(1) Flow Loss}

Figures $6 \mathrm{a}$ and $6 \mathrm{~b}$ show the coolant response to a $50 \%$ step flow loss. It can be seen that overall the new model predicts the best results. Method 2 underpredicts the outlet temperature and thus gives too large a change in the average coolant temperature. Method 3 overpredicts the outlet temperature for $t<\lambda$, but comes into line for $t>\lambda$. It can also be seen that Method 1 does overshoot the temperature by about $70 \%$ but corrects itself. It was found that this problem could be eliminated with a more refined nodal structure in the fuel pin.

On the other hand, when a $95 \%$ flow loss accidient, resulting in very low flow and a large value of $\lambda$, was run, Method 2 yielded better results than Method 3. However, Method I was still superior to either of the other two. In the limit as flow goes to zero, Method 2 becomes a correct solution (as does Method 1). See Figure 6c.

\section{(2) Change in Inlet Temperature}

The step change in inlet temperature is shown in Figures $7 \mathrm{a}$ and $7 \mathrm{~b}$. Since the outlet temperature subsequently undergoes a step change, none of the three methods represents a precise solution. For $t<\lambda$, Method 3 yields very poor results. Method 2 is an improvement but gives ppor results for the average coolant temperature. The best results are again predicted by Method 1, although it smooths out the step in outlet temperature.

However, putting the same inlet temperature change in on a very rapid ramp (Figure 8), Method 1 shows significant improvement, while Method 2 and 3 remain about the same.

(3) Increase in Power

A step increase in power was run with the same qualitative results as the step change in flow problem. When the power change was effected at normal flow 


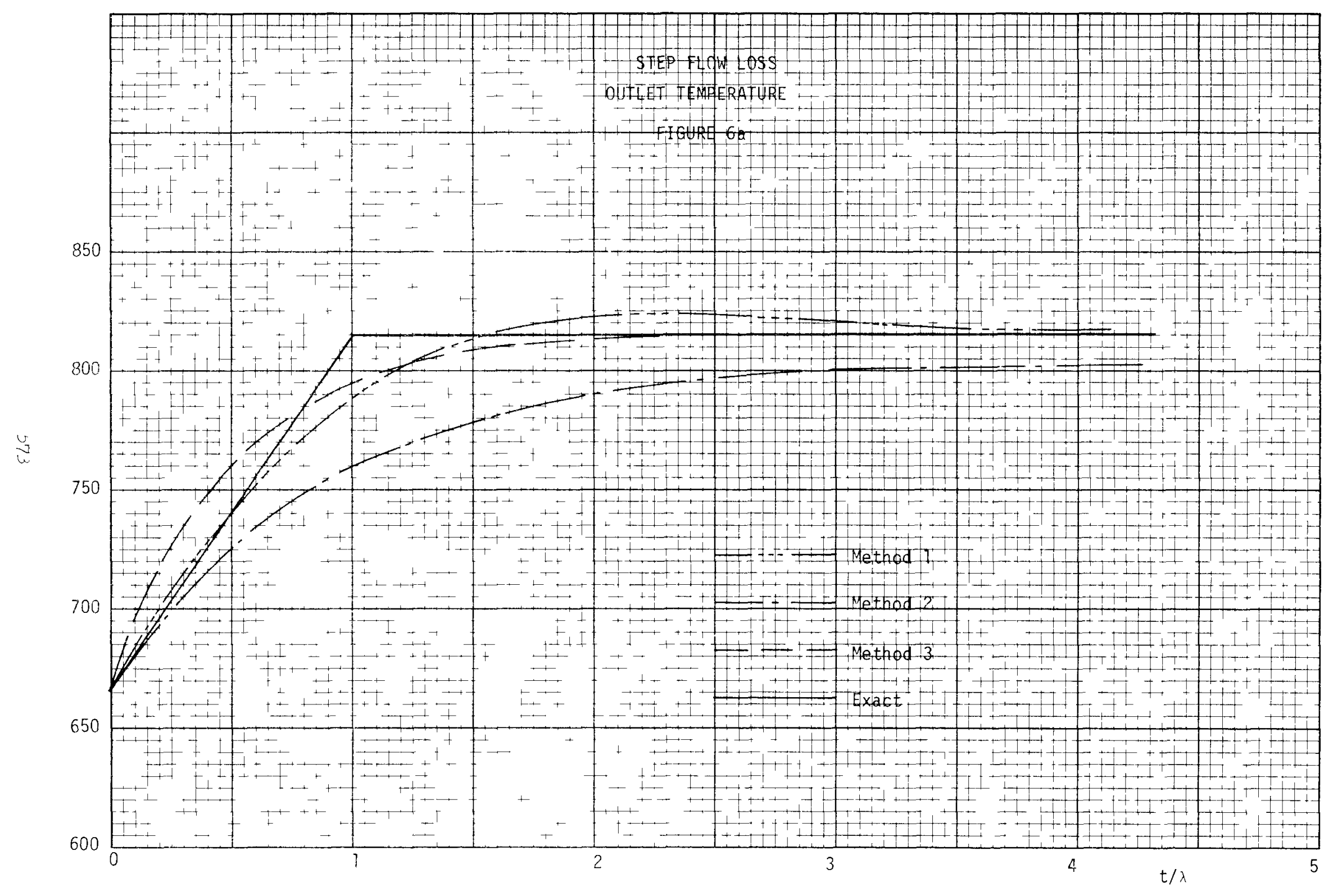




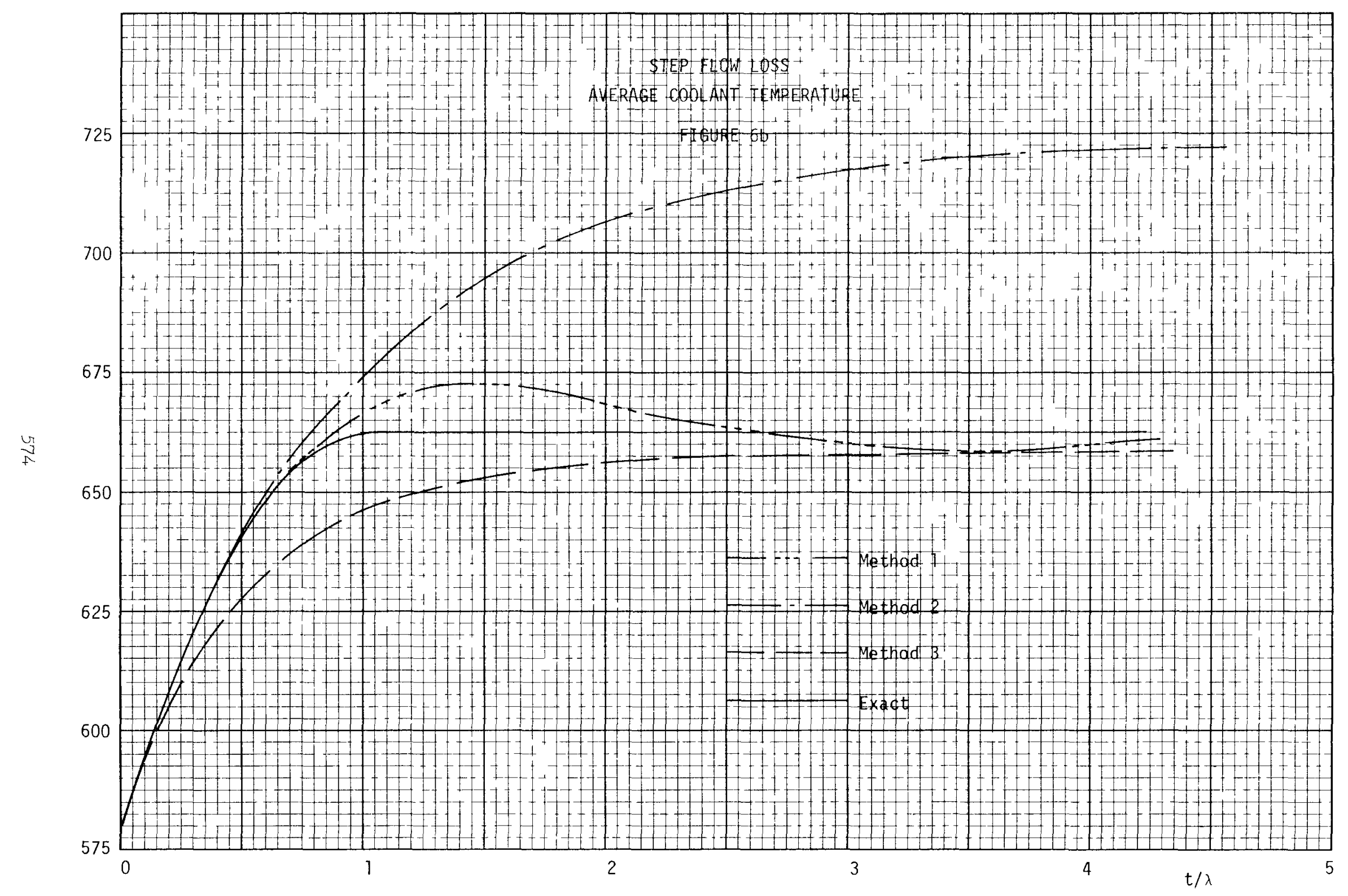




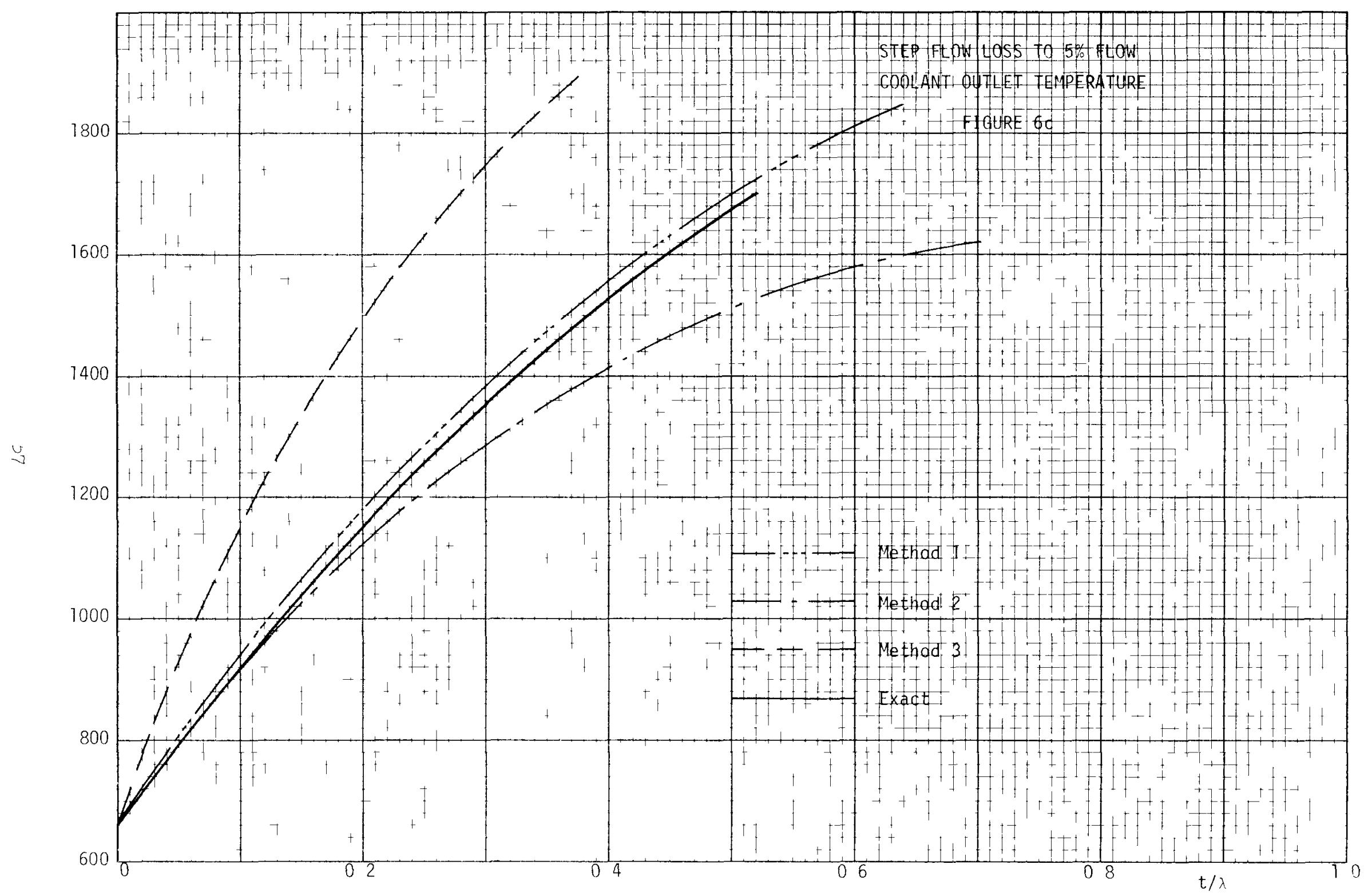




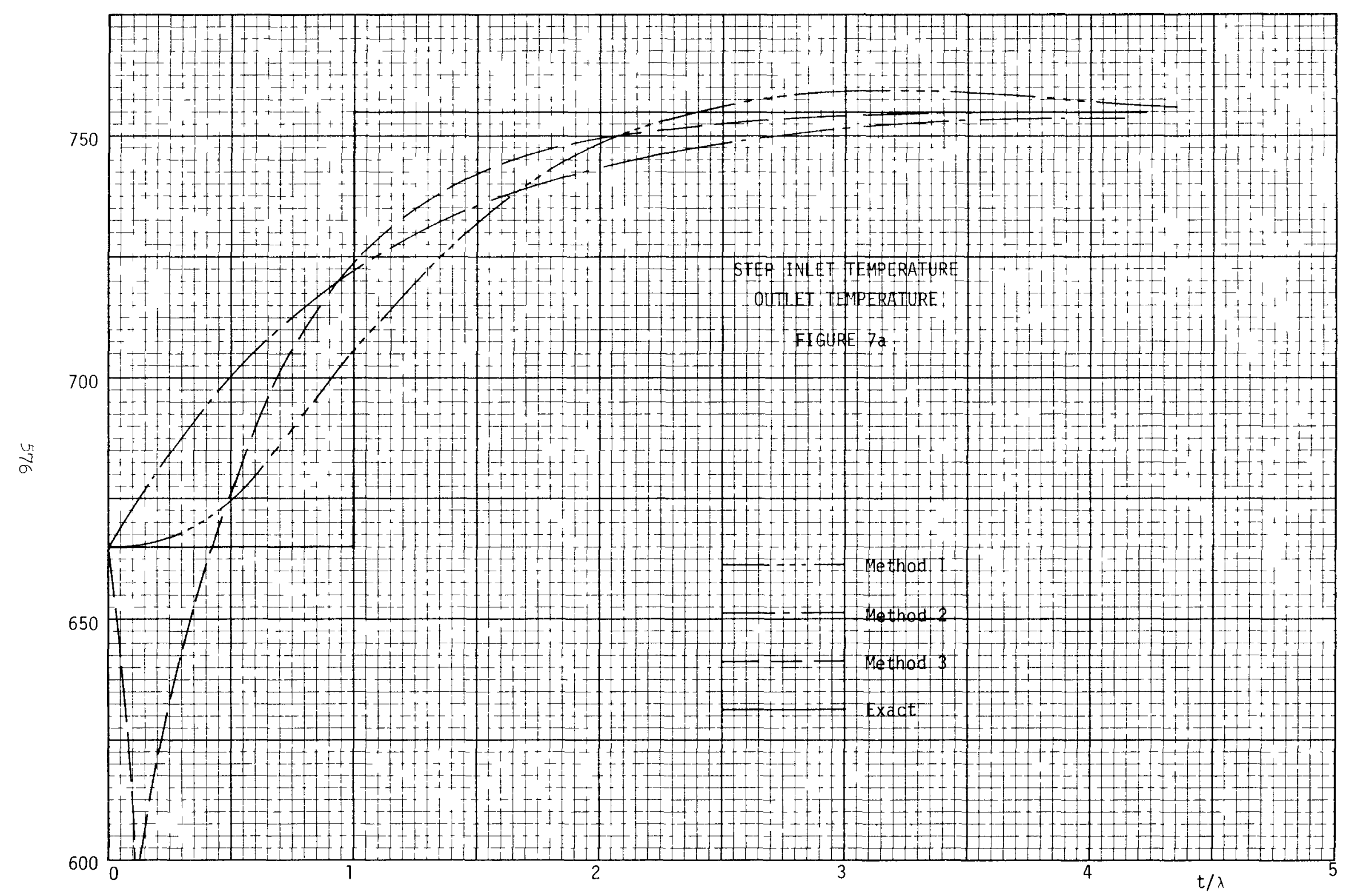




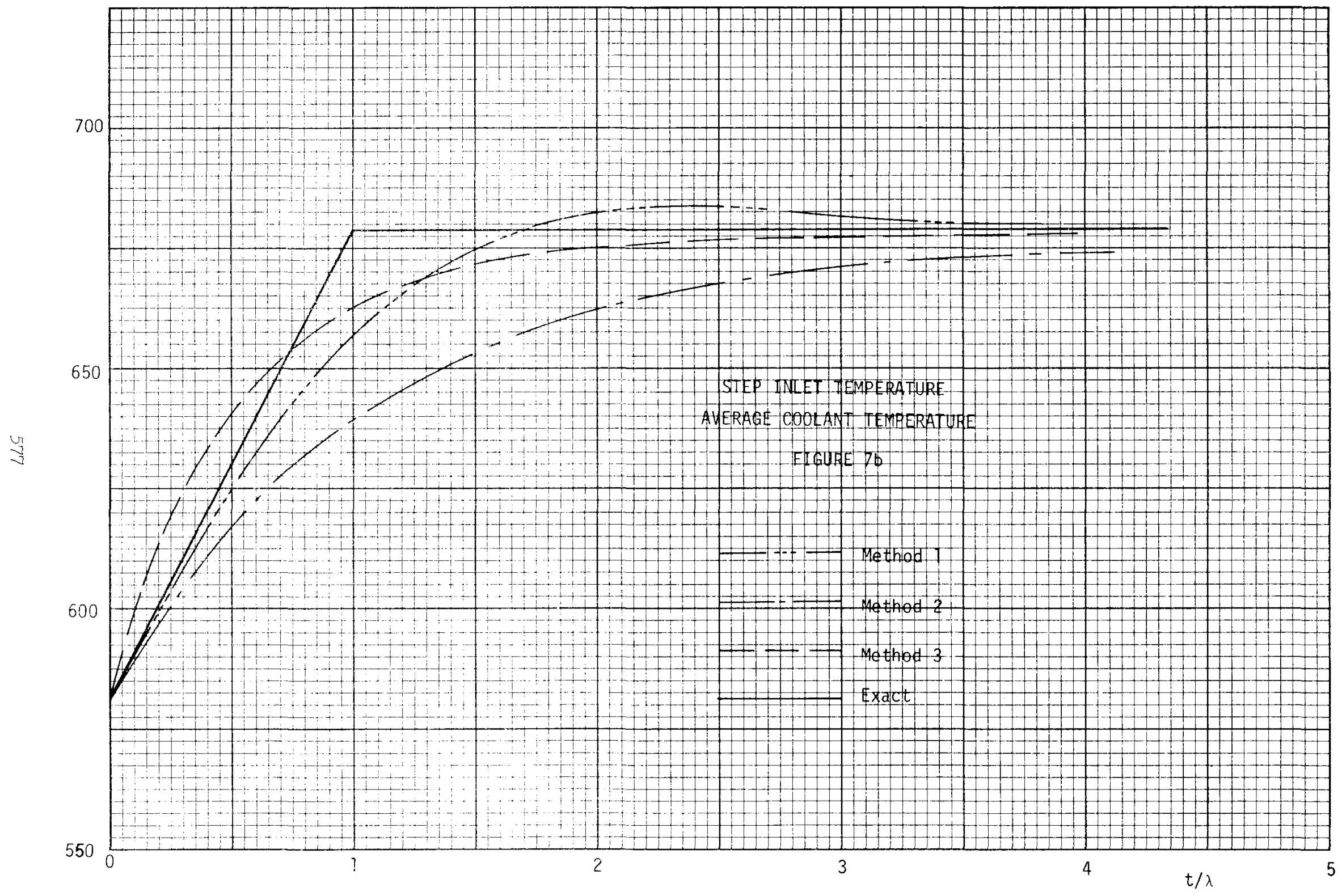




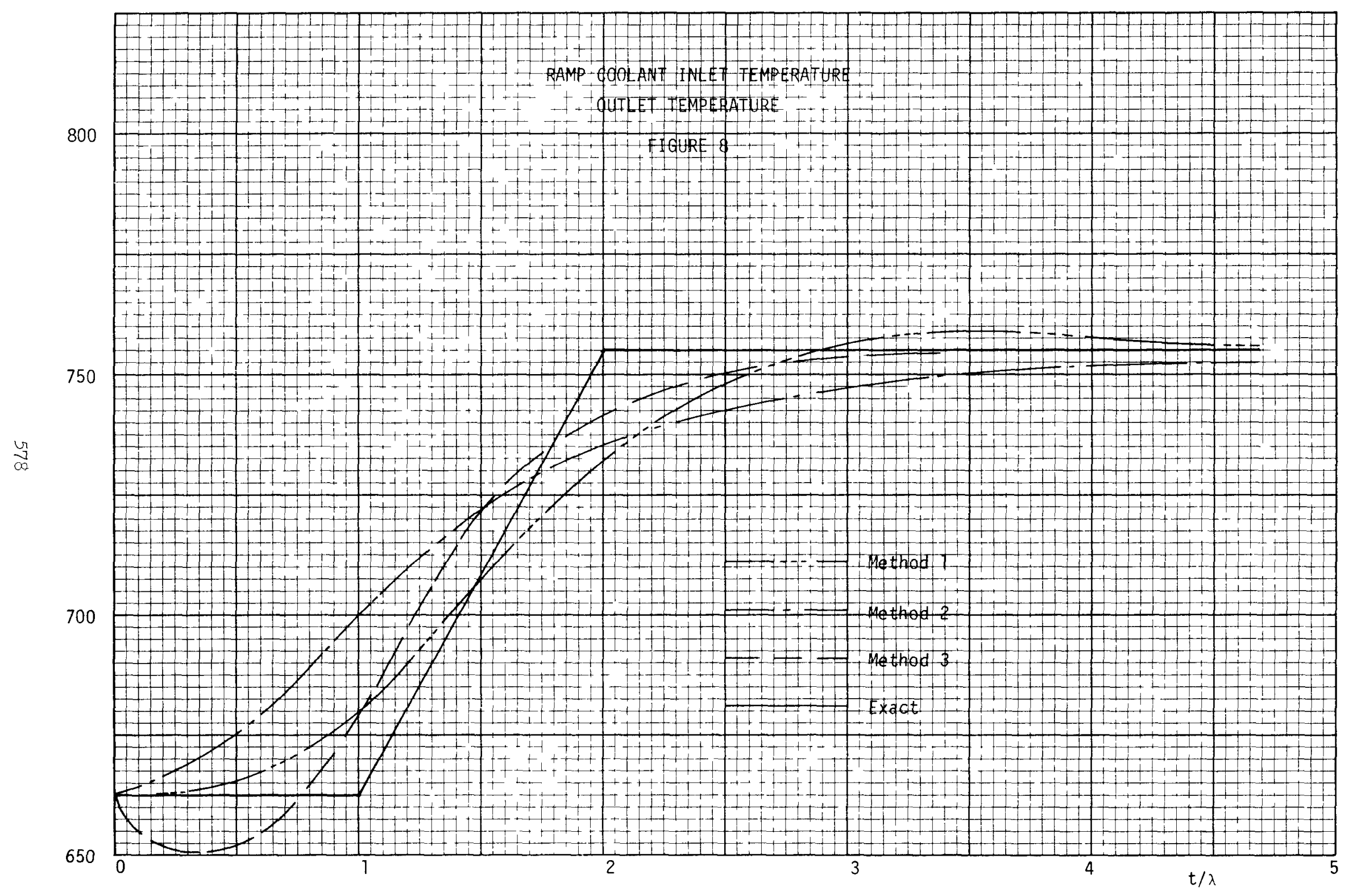


conditions, Method 3 was better than Method 2; the same accidient, at low flow, resulted in Method 2 being better than Method 3. Again, Method 1 gave correct results in both situations.

\section{A Finite Difference Solution to the Axial Section}

Having developed a finite difference model for the coolant, and verified its validity, it was necessary to couple this to a finite difference method to solve for the clad and fuel nodal temperatures. It should be remembered that due to the large temperature gradients in the fuel, the fuel may require as many as ten radial nodes to accurately describe its spatial temperature distribution.

Since the model for the coolant temperature is not stability limited, it was possible to use an unlimited stability solution for the fuel and clad. Several of these methods were examined by using them to calculate typical reactor transients for the basic axial section of nodal temperatures, coupled to the new convective model previously described. They were then evaluated in terms of the computation time required to yield a reasonably accurate solution to the problem.

The first class of unlimited stability solutions are of the implicit type (1), where heat transfer into and out of the node is described by the temperatures at the time step being solved. The resultant equations for the nodal temperatures involve the solution of a set of simultaneous equations. The solution to the simultaneous equations can be obtained quite readily if the coefficient matrix of the simultaneous equations is tridiagonal. (5) Physically, the tridiagonal matrix implies that any given node can transfer heat with only two other nodes. It was felt this was too restrictive a situation, since in many problems commonly considered in accidient analysis, it is necessary to include heat transfer to the coolant not only from the fuel pin, but from core structure and other materials, such as Be0 moderator. Therefore the implicit type methods were ruled out.

There is also an interesting group of unlimited stability, explicit type methods that have been developed by Winson (3)(6). Barakat and Clark (7) and Levy (8). The explicit method calculates the temperatures at the new time step, $. j+1$, in terms of the temperatures at the previous step, $j$, and thus normally results in a very simple computational scheme. Although most explicit methods are stability limited, the three mentioned above have modified the basic explicit scheme to remove the stability problem.

After an examination of these three explicit schemes, it was found that the method of Levy(8) was particularly well suited to the reactor heat transfer problem, and could readily be adapted to the convective heat transfer results. Levy's method was found to give a fast, accurate solution for very general, unrestricted problems.

The following brief description of Levy's method is included in order to illustrate how it is adapted to a very general description of the transient heat transfer in an axial section.

The general equation for the average coolant temperature is written as

$$
\frac{\partial \bar{T}_{c}}{\partial t}=\sum_{i=1}^{N} \omega_{c_{i}}\left[T_{i}-\bar{T}_{c}\right]+\frac{\dot{q}_{c}^{\prime \prime \prime}}{\rho c}-\frac{\left(T_{L}-T_{i n}\right)}{\lambda}
$$


where the heat conduction is included to an from an arbitrary number of adjacent nodes, $N$, and the capability of describing $\gamma$-neutron heating has also been included. Note that the equation has not yet been differenced.

The method of Levy now differences the time derivative in the form

$$
\frac{\partial \bar{T}_{c}}{\partial t}=\left(1+\beta_{c}\right) \frac{\left(\bar{T}_{c}^{j+1}-\bar{T}_{c}^{j}\right)}{\tau_{j+1}}-\beta_{c} \frac{\left(\bar{T}_{c}^{j}-\bar{T}_{c}^{j-1}\right)}{{ }^{j} j+1}
$$

and the right hand side of equation (16) is evaluated at step $j$, resulting in a final form for $\overline{\mathrm{T}}_{\mathrm{C}} \mathrm{j}+1$ of

$$
\begin{aligned}
& \bar{T}_{c}^{j+1}=\bar{T}_{c}^{j}+\frac{{ }^{\tau} j+1}{\left(1+\beta_{c}\right)}\left\{\sum_{i=1}^{N} w_{c_{j}}\left[T_{i}^{j}-\bar{T}_{c}^{j}\right]\right. \\
& \left.+\frac{\dot{q}^{\prime \prime}{ }^{j+1}}{\rho c}-\frac{\left(T_{L}^{j}-T_{i n}^{j}\right)}{\lambda_{j+1}}\right\}+\frac{c}{1+c}\left(\bar{T}_{c}^{j}-T_{c}^{j-1}\right)
\end{aligned}
$$

$$
\text { where } w_{c_{j}}=\frac{1}{\rho C A R{ }_{c i}}
$$

and the $\beta_{C}$ 's are a "stability factor", and are a function of $\tau_{j+1}$ and the $w_{C_{j}}$ 's.

$$
\begin{aligned}
& \beta_{c}= \begin{cases}0 & \Delta_{c}<1 \\
\left(\Delta_{C}-1\right) / 2 & \Delta_{c}>1\end{cases} \\
& \Delta_{c}={ }_{j}+1 \sum_{i=1}^{N}{ }^{w}{ }^{w}{ }_{i}
\end{aligned}
$$

Note that equation (18) is also applicable to the other non-convective nodes in the axial section by dropping out the convective term. 


$$
\begin{aligned}
T_{m}^{j+1} & =T_{m}^{j}+\frac{{ }^{j}+1}{\left(1+\beta_{m}\right)}\left\{\sum_{i=1}^{N_{m}} w_{m_{i}}\left[T_{i}^{j}-T_{m}^{j}\right]\right. \\
& \left.+\frac{\dot{q}^{\prime \prime \prime}+1}{\rho c}\right\}+\frac{\beta m}{\left(1+\beta_{m}\right)}\left[T_{m}^{j}-T_{m}^{j-1}\right]
\end{aligned}
$$

where the subscript " $m$ " was used to denote the particular node in question.

With the generalized heat conduction-generation term that was used for the average coolant in equation (18), the corresponding equations for the outlet coolant temperature become, using equations (14a) and (14b),

$$
\begin{aligned}
& T_{L}^{j+1}=T_{L}^{j}-2\left(T_{L}^{j}-\bar{T}_{c}^{j}\right) \frac{\tau_{j+1}}{\lambda j+1}+\sum_{i=1}^{N} w_{c_{i}}\left(T_{i}^{j}-\bar{T}_{c}^{j}\right)_{j+1} \\
& +\frac{\dot{q}}{\rho \prime \prime}+1_{\rho c}^{\tau}{ }^{\tau+1} \quad\left(\tau_{j+l}<\lambda_{j+1}\right) \\
& T_{L}^{j+1}=T_{i n}^{j}+\dot{T}_{i n}^{j+1}\left(\tau_{j+1}-\lambda_{j+1}\right)+\sum_{j=1}^{N} w_{c_{i}}\left(T_{i}^{j}-\bar{T}_{c}^{j}\right) \lambda_{j+1} \\
& +\frac{\dot{q}^{\prime \prime} \dot{j+1}}{\rho c} \lambda_{j+1} \quad\left(\tau_{j+1}>\lambda_{j+1}\right)
\end{aligned}
$$


To summarize at this point, a set of explicit, unlimited stability equations have been formulated and checked for the nodal temperatures in an axial section of a reactor coolant channel. They include heat transfer by conduction and convection and account for heat generation.

The final step in optimizing the solution is to select an efficient method of time stepping the solution. Since the equations are not stability limited, full advantage should be taken of this characteristic.

It was found that a very satisfactory method of time stepping the solution was to base the time step on an "allowable" temperature change per step, that is specified at the beginning of the solution. At the end of any given step, the new temperatures are checked against the old to insure that they have not exceeded the allowable change. If any have, the time step is halved and the temperatures recalculated. If all the temperatures changes are well below the allowable change (such as by a factor of 2 or 3 ), the size of the time step may be expanded on the next step. In this way, the rate of integration of the soiution is determined by the gradient of the temperature-time relationship. Thus when values are changing slowly, the solution may use large time steps, and when rapid changes are encountered, the size of the step is cut down to maintain accuracy. Typical results with the set of equations specified have indicated that accuracy within $5 \%$ could be obtained with an allowable temperature change per step of $25-50^{\circ} \mathrm{F}$. When specifying the allowable temperature change per step, some consideration must be given to the magnitude of the total temperature variation expect over the entire transient. That is, in a very mild transient, a total temperature change in the coolant on the order of $100^{\circ} \mathrm{F}$ may possibly be expected. In this case, the allowable temperature change would be specified as something typically like $5^{\circ} \mathrm{F}$ per step.

With respect to the programming of the solution at any given axial section and time step, the inlet temperature is evaluated from the outlet of the adjacent upstream section, or else the inlet to the core. Then $T_{L}$ is calculated from equations (21). Next $\bar{T}_{C}$ is determined from (18), and the remaining temperatures of clad, fuel, structure and other materials from equation (20). Note that if $\tau$ and the material properties have not changed from the previous step, the $\beta$ 's do not have to be recalculated. Also, with constant material properties, the $\beta$ 's are the same at any given time step for all the axial sections in a coolant channel. That is, with some consideration to the problem being solved, the number of times the $B$ 's must be calculated can be minimized. Finally, the solution proceeds downstream through the remainder of the axial sections in the coolant channel to the core outlet, and the time step size is then checked for conformance to the specified allowable change. Appropriate action is initiated from this point to either halve $\tau$ and recalculate, or else proceed to the next time step with either the same or an expanded value of $\tau$.

\section{Conclusions}

Postulating an "axial section" of a reactor channel to be the basic nodal unit for transient heat transfer calculations, a finite difference method of calculating the convective heat transfer was developed and checked with sample problems, and found to be superior to existing methods.

As the convective model was explicit and not stability limited, it was coupled to an existing explicit, unlimited stability scheme formulated by Levy (8) for the fuel, clad and structural node temperatures. Finally, determination of an effective time step criteria to take advantage of the unlimited stability nature of the equations has resulted in an efficient and accurate nodal model for the analysis of transient heat transfer in a reactor core. 


\section{Nomenclature}

A Cross sectional area of a node in an axial plane

b See Equation (8b)

c Specific heat

D Inside diameter of the coolant annulus

j Time step index - step most recently solved for

j+1 Time step index - step being solved for

$j-1$ Time step index - step previous to $j$

$L \quad$ Length of the axial section

$\mathrm{N}$ Number of nodes transferring heat to the primary node being considered

q" Heat flux to the coolant

$\dot{q}^{\prime \prime}$ Heat generation rate

$R_{W C}$ Thermal resistance between $c l a d$ and coolant

$R_{c_{i}} \quad$ Thermal resistance between coolant and node $i$

t Time

$T_{c} \quad$ Coolant temperature as a function of $(t, z)$

$\bar{T}_{C} \quad$ Average or nodal coolant temperature

$T_{\text {in }}$ Inlet coolant temperature

$\dot{\mathrm{T}}_{\text {in }}$ Rate of change of inlet coolant temperature

$\mathrm{T}_{i} \quad$ Temperature of a node transferring heat to the primary node being considered

$T_{L} \quad$ Outlet coolant temperature

$T_{W} \quad C l a d$ node temperature 
Nomenclature (Con'd)

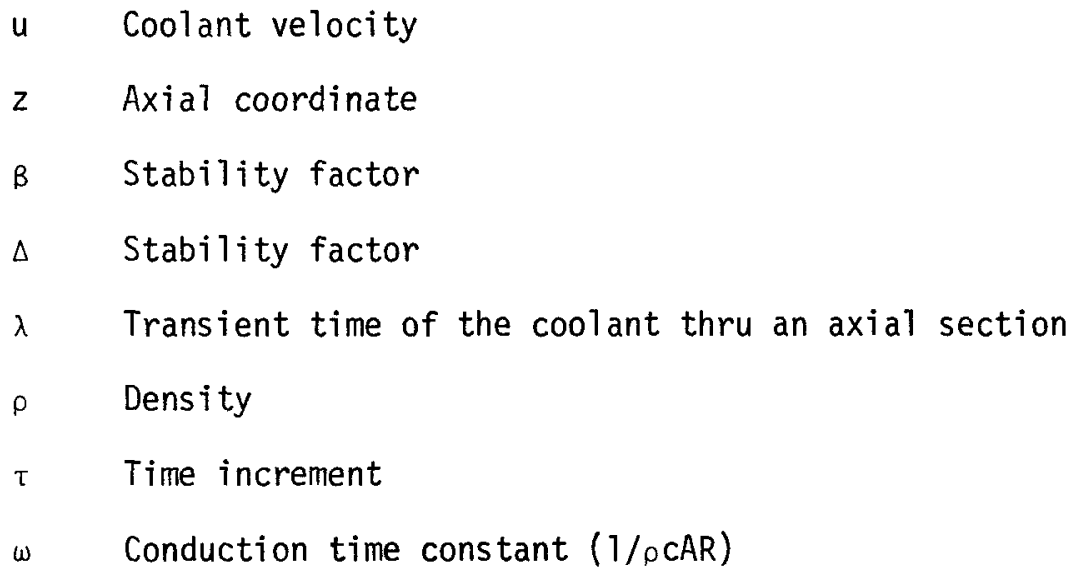


(1) R. D. Richtmyer, Difference Methods for Initial Value Problems, (Interscience, New York, 1957).

(2) Caldarola, Niedermyer, Woit, Reactor Temperature Transients with Spatial Variables, Second Part, Axial Analys is, KFK 618, (1967).

(3) R. W. Winson, Thermal Model for Use in SNAP System Analys is, NAA-SR-10961, (1965).

(4) W. M. Kays, Convective Heat and Mass Transfer, p. 121, (McGraw Hi11, 1966).

(5) F. B. Hildebrande, Methods of Applied Mathematics, p. 503, (Prentice-Ha17, EngTewood Cliffs, N.J., 1961).

(6) R. W. Winson, A Technique for Solving Dynamics Problems by Digital Computation, NAA-SR-10692, (1964).

(7) H. Z. Barakat and J. A. Clark, "On the Solution of the Diffusion Equations by Numerical Methods," J. of Heat Transfer, 88 (4), 421-427 (November 1966).

(8) S. Levy, Use of Explicit Method in Heat Transfer Calculations with an Arbitrary Time step, (General Electric Technical Information Series), 68-C-282, (1968). 


\section{TRACK II, A COMPUTER PROGRAM FOR TRANSIENT THERMAL ANALYSIS OF COMPLEX NUCLEAR REACTOR SYSTEMS WITH MULTIPLE PARALLEL AND SERIES FLOW PATHS}

M. D. Woods and L. D. Hamlin

Westinghouse Electric Corporation

Astronuclear Laboratory Pittsburgh, Pennsylvania 


\title{
TRACK II, A COMPUTER PROGRAM FOR TRANSIENT THERMAL ANALYSIS OF COMPLEX NUCLEAR REACTOR SYSTEMS WITH MULTIPLE PARALLEL AND SERIES FLOW PATHS
}

\begin{abstract}
TRACK $\|$ is a digital computer program for transient and steady state thermal analysis of heat generating solids of arbitrary geometry which are cooled by fluid flowing through multiple parallel or series channels. The hydraulic and heat convection calculations are coupled with finite element heat conduction calculations to determine coolant conditions and material temperature distributions in a single computation. The primary application is to perform detailed thermal analyses of nuclear reactor components such as the reflector, the control mechanism, and fuel cluster assemblies under various operating conditions. A key feature of the program is the versatility of the flow network. Two independent groups of coolant channels are permitted and the channels may be arranged in series and parallel combinations. Additional features include an option for interface conduction between components which are modeled separately and a method to accommodate the effect of fast neutron fluence on component temperature distributions.
\end{abstract}

This paper presents the formulation for the transient thermal calculations and discusses the calculational models with emphasis on the application of the capability of TRACK II for thermal, hydraulic analysis of complex systems. The thermal analysis of the reflector system for the XE-PRIME NERVA reactor is presented as an example of this capability.

\section{INTRODUCTION}

The NERVA program is responsible for the design and development of a nuclear rocket engine for use as a space propulsion system. Nuclear propulsion provides a major advancement in space propulsion capability and gives increased flexibility to spaceflight mission planning. The feasibility and high performance potential of the nuclear rocket propulsion system have been demonstrated by an extensive series of successful reactor and breadboard engine system tests which have been conducted at the Nuclear Rocket Development Station in Nevada.

In the design and development of a nuclear reactor for rocket propulsion application, the reactor components must fulfill the design requirements in a severe thermal, hydraulic and nuclear irradiation environment with a high probability of success. Furthermore, the weight of reactor components must be minimized in order to achieve the performance goals. The dual objectives of high performance with high reliability entail precise determination of stresses and, hence, temperature distributions. Increasingly, this has come to mean computerized finite element solutions which provide a practical approach to analyzing components of complex geometry with non-symmetric boundary conditions. This paper presents a computer solution developed by the Westinghouse Astronuclear Laboratory (WANL) to perform detailed transient and steady state thermal analyses of nuclear reactor components. The 
computations are performed by the TRACK II digital computer program. The temperature distributions are reserved on tape for direct input to stress analysis codes with compatible models. Application of the program to the thermal analysis of the XE-PRIME NERVA reflector system is discussed herein.

\section{SUMMARY OF TRACK II CAPABILITY}

TRACK, ${ }^{1}$ a digital computer program was developed for transient and steady state thermal analysis of heat generating solids of arbitrary geometry which are cooled by fluid flowing through multiple parallel channels. The hydraulic and heat convection calculations are coupled with the heat conduction calculations to determine coolant conditions and material temperature distributions in a single computation. The coolant channels may have individual impedances and be arranged in any non-symmetrical array. The flow in the channels may be either laminar or turbulent. The solid body may be general in shape with temperature distributions determined by a finite difference nodal solution. The primary application is to perform detailed thermal analyses of nuclear reactor components such as the reflector, the control mechanisms, and fuel cluster assemblies under various operating conditions. The computer solution may be applied to other heat transfer systems with or without internal heat generation such as heat exchange apparatus.

TRACK II is an updated version of the basic TRACK program which is capable of performing transient thermal analyses of more complex systems. The program's key features consist of series and parallel combinations of coolant channels that are permitted with two groups of coolant channels to increase the program capacity and flexibility. Figure 1 illustrates the possible combinations of flow systems.
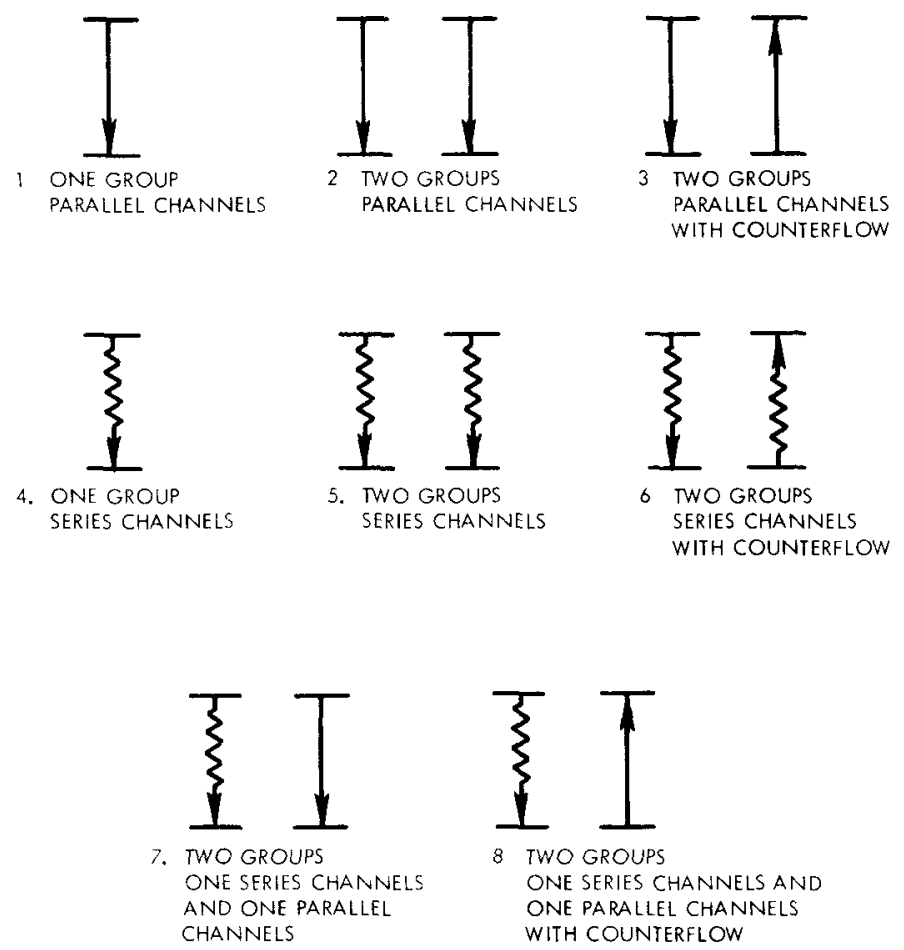

Figure 1. Series and Parallel Channel Combinations 
The TRACK II program includes a technique for simulating interface conduction between adjacent components which are modeled separately. This option permits the subdivision of an assembly of components into smaller units for more convenient modeling and more efficient usage of the nodal capacity of the conduction code. Applicable boundary temperatures are applied through equivalent heat transfer coefficients to the interface surfaces for each of the separate heat conduction models. The boundary conditions are obtained from the most recently calculated temperature distributions in the neighboring component. The equivalent heat transfer coefficients are calculated from the thermal conductivity of the adjacent material and the mean conduction path length $(h=k / x)$. The interface conduction option is properly applied only at low heat flux boundaries such as insulation interfaces.

The effect of fast neutron fluence on component temperature distributions can also be accommodated with the TRACK II code. Many materials exposed for long periods of time to high intensity radiation will have their physical properties altered. A method has been developed for TRACK II which allows for the variation of material thermal conductivity as a function of temperature and fast neutron fluence.

Another option permits intermediate flow input to a series combination of channels. Flow is added or subtracted at specified plena and an enthalpy balance is made between the direct flow and lateral flow streams to determine the mixed mean coolant conditions for downstream hydraulic calculations. Two input options are available: 1) flow rate and specific enthalpy for the lateral flow stream are specified, or 2) the flow rate and specific enthalpy are calculated for interconnection to an external channel through a specified impedance. The coolant pressure and temperature distributions for the external channel may be specified or, where applicable, they may be obtained from hydraulic calculations for a preceding group of channels. Another source of lateral inflow in porous flow through the material boundaries. Porous flow is determined from a correlation of flow rate versus the parameter, $(p \Delta \mathrm{P} / \mu \mathrm{RT})$. The porous flow integrated over a prescribed channel length is lumped and added to specified plena in conjunction with other forms of lateral inflow.

The TRACK II computer program is written in FORTRAN IV language for the CDC 6600 computer. The program is currently dimensioned to require a computer memory bank of 350,000 actual locations. The following is a summary of the capability and capacity of the program.

Hydraulic Models

Transient or Steady State Calculations

Single Phase, One Dimensional Hydrogen, Helium or Nitrogen Flow

Two Groups of Hydraulic Channels

1) 60 channels per group

2) 18 axial increments per channel

3) Turbulent or laminar flow

4) Channels may be series or parallel

5) Plena bleed flow is permitted

Conduction Models

Transient or Steady State Calculations 
Boundary Conditions may be

1) Forced convection

2) Thermal radiation

3) Free convection

4) Adiabatic

Internal Heat Generation

Finite element conduction models

Up to 5 independent conduction models

Up to 11 axial locations

1) Up to 800 nodes per model

2) Up to 400 surface nodes per model

3) Up to 300 boundary nodes per model

Interface conduction between models is permitted

Models may be one, two, or three dimensional

Material properties may vary as a function of temperature and nuclear irradiation fluence.

\section{ANALYSIS FORMULATION}

The system being considered is a heat generating solid body of arbitrary geometry cooled (or heated) by a fluid flowing through multiple parallel or series channels as shown in Figure 2. The channel cross sectional areas are constant over the length, but channels may have different cross sectional areas and impedances. The fluid is considered compressible in a one-dimensional single phase pipe flow with friction and heat transfer across the channel boundary. The flow may be either laminar or turbulent.

The thermodynamic and transport properties of the fluid are temperature and pressure dependent. Hydrogen is the primary coolant considered but other fluids can be used by substituting the appropriate fluid property subroutines. The solid body possesses finite and temperature dependent physical properties. The internal heat generation rate in the solid body may vary longitudinally and transversely.

The hydrodynamic equations in the Eulerian form for the laws of fluid motion are expressed as follows:

$$
\begin{array}{ll}
\text { Continuity: } & \frac{\partial e}{\partial \theta}+\frac{\partial G}{\partial x}=0 \\
\text { Momentum: } & \frac{\partial P}{\partial x}+\frac{1}{g} \frac{\partial G}{\partial \theta}+\frac{1}{g} \frac{\partial\left(G^{2} v\right)}{\partial x}+F=0
\end{array}
$$




$$
\text { Energy: } \quad \frac{\partial(H G)}{\partial x}+\frac{\partial(H e)}{\partial \theta}-\frac{1}{J} \frac{\partial P}{\partial \theta}-Q P=0
$$

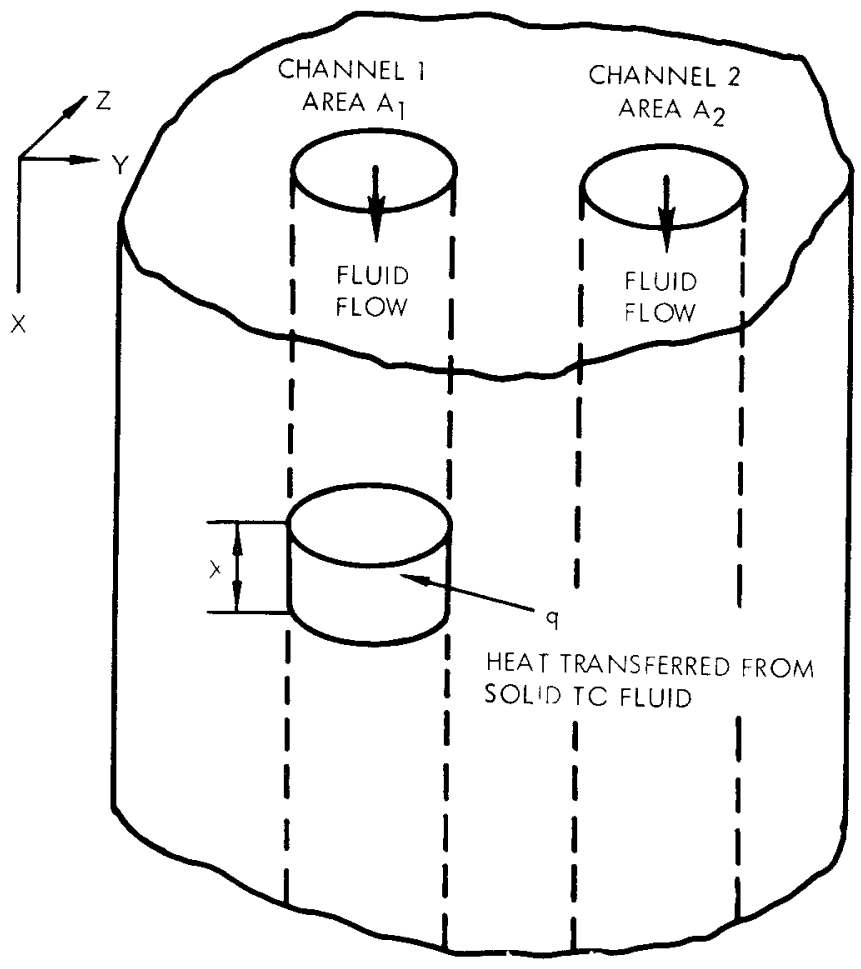

Figure 2. A Heat Generating Solid Cooled by Fluid Flowing in Series or Parallel Channels

The temperature distribution in the solid body is calculated by the Fourier heat conduction equation shown below:

$$
\frac{\partial}{\partial x}\left(k \frac{\partial T}{\partial x}\right)+\frac{\partial}{\partial y}\left(k \frac{\partial T}{\partial y}\right)+\frac{\partial}{\partial z}\left(k \frac{\partial T}{\partial z}\right)+Q^{\prime \prime} \quad(x, y, z, \theta)=e_{p} \frac{\partial T}{\partial \theta}
$$

The convective heat transfer between the fluid and channel wall in the hydraulic calculations is found from the following equation,

$$
q=h A_{s}\left(T_{w}-T_{r}\right)
$$


For small Mach numbers $\left(\langle<1.0)\right.$ the difference between the recovery temperature $\left(T_{r}\right)$ and the fluid free stream temperature $\left(T_{b}\right)$ is not significant so that Equation (5) may be written as

$$
q=h A_{s}\left(T_{w}-T_{b}\right)
$$

The channel wall boundary conditions for the conduction calculation are

$$
-\left.k \frac{\partial T}{\partial n}\right|_{\text {wall }}=h A_{s}\left(T_{b}-T_{w}\right)
$$

From the above formulation, it is seen that the coupling term between the fluid flow and heat conduction calculations is the temperature of the channel walls, Tw. The development of Equations (1) through (7) into finite difference form is given in Appendix A.

The convective heat transfer coefficient, h, used in Equations (6) and (7) is determined by the following type of correlation

$$
N_{u}=f\left(\operatorname{Re}^{n}, \operatorname{Pr}^{m}, \frac{x}{D}, \frac{T_{b}}{T_{w}}\right)
$$

where $\mathrm{Nu}, \mathrm{Re}$, and $\mathrm{Pr}$ are the Nusselt, Reynolds, and Prandtl numbers, respectively. For turbulent flow in smooth tubes, three correlations are available. The first is based on the McCarthy-Wolf ${ }^{2}$ investigation of gaseous hydrogen and helium flow through smooth tubes and is suitable for a wide range of $T_{W} / T_{b}$ ratios, up to 3.5 .

a) McCarthy-Wolf Turbulent Heat Transfer Correlation

$$
h=0.025\left(\frac{k_{b}}{D}\right)\left(\operatorname{Re}_{b}\right)^{0.8}\left(\operatorname{Pr}_{b}\right)^{0.4}\left(\frac{T_{b}}{T_{w}}\right)^{0.55}\left[1+0.3\left(\frac{x}{D}\right)^{0.7}\right]
$$

where the fluid properties are evaluated at fluid bulk temperature.

The second turbulent flow heat transfer correlation is a combination of the Petukhov, ${ }^{3}$ $\mathrm{McC}$ arthy and Wolf correlation which was developed at Westinghouse Astronuclear Laboratory. The Petukhov correlation has the following form:

b) Petukhov Turbulent Heat Transfer Correlation

$$
h=0.021\left(\frac{k_{b}}{D}\right)\left(\operatorname{Re}_{b}\right)^{0.8}\left(\operatorname{Pr}_{b}\right)^{0.4}\left(\frac{T_{b}}{T_{w}}\right)^{P}
$$

where the exponent $P$ is a function of the $x / D$ ratio varying from 0.125 at $x / D=0$ to 0.53 for $x / D \geq 90$. 
The third turbulent flow heat transfer correlation was developed by Los Alamos Scientific Laboratory. 4

c) Los Alamos Scientific Laboratory Correlation

$$
h=0.023\left(\frac{k_{b}}{D}\right)\left(\operatorname{Re}_{b}\right)^{0.8}\left(\operatorname{Pr}_{b}\right)^{0.4} \quad C_{1}\left(\frac{T_{w}}{T_{b}}\right)^{-C_{2}}
$$

In Equation (10) the constants $C_{1}$ and $C_{2}$ are defined as follows:

$$
\text { For } \begin{aligned}
T_{W} / T_{b} & <1.8 \\
C_{1} & =1.0 \\
C_{2} & =0.765
\end{aligned}
$$

For $T_{W} / T_{b} \geq 1.8$

$$
\begin{aligned}
& c_{1}=0.7 \\
& c_{2}=0.20
\end{aligned}
$$

For laminar flow in the gaseous regime, the following relation ${ }^{5}$ is used

$$
h=\left\{4.36+\left(\frac{0.036\left(\frac{D}{x}\right) \operatorname{Re} \operatorname{Pr}}{1+0.001\left(\frac{D}{x}\right) \operatorname{Re} \operatorname{Pr}}\right)\right\}\left(\frac{k}{D}\right)\left(\frac{T_{w}}{T_{b}}\right)^{0.25}
$$

where the properties are evaluated at the coolant bulk temperature.

The friction factor $f$ which is used in determining the term, $F$, in equation (2) is obtained from the following correlations by Taylor 6 for gaseous flow through a smooth tube:

$$
\begin{aligned}
& \text { turbulent flow, } f=\left(0.0014+\frac{0.125}{\left(\operatorname{Re}_{w}\right)^{0.32}}\right)\left(\frac{T_{b}}{T_{w}}\right)^{0.5} \\
& \text { laminar flow, } f=\frac{C}{\operatorname{Re}_{w}}
\end{aligned}
$$

where $\operatorname{Re}_{w}$ is a modified surface Reynolds number in which the gas density is evaluated at the fluid " bulk temperature, but the viscosity is evaluated at channel wall temperature:

$$
\operatorname{Re}_{w}=\left(\frac{G D}{\mu_{w}}\right)\left(\frac{T_{b}}{T_{w}}\right)
$$

The constant, $C$, in Equation (13) depends upon the type of cooling channels used. For example, with cylindrical cooling channels $C=16$ and with annular channels $C=24$. 


\section{MODELING OF THE XE-PRIME REFLECTOR SYSTEM}

The XE-PRIME is a NERVA engine currently being tested at the Nuclear Rocket Development Station in Nevada. A schematic of this engine is shown in Figure 3. The TRACK II digital computer code was used to perform the thermal-hydraulic analysis of the XE-PRIME reflector system. The calculational model included all the major components from the outer diameter of the core to the outer diameter of the pressure vessel. A schematic representation of the system is shown in Figure 4. The system consists of concentric annular regions with a total of 12 control drums. A repeating section representing $1 / 12$ of the total system was chosen for the computational model.

The outer reflector, control drums, tie bolts and inner reflector have cooling passages extending the entire length of the reflector with flow direction as indicated in Figure 4. Flow through the reflector system originates in the reflector inlet plenum at the nozzle end (aft end) of the reactor and exits in to the reflector exit plenum at the forward end. Flow enters the transition region at the forward end and exits into the nozzle chamber while crossflow takes place between these streams.

The flow system was modeled for the TRACK II computer code by using two groups of hydraulic channels. The first group of channels represents a parallel flow region comprising the reflector and control drum coolant passages. The second group of channels represents the series flow through the transition region. A schematic diagram of the hydraulic model is shown in Figure 5. Figure 6 illustrates the hydraulic representation of the transition region. There are three distinct types of coolant flow paths in repeating patterns. As indicated in Figure 6, Types 2 and 3 constitute a parallel flow system in series with Type 1. Each grouping of parallel channels within the series channel structure is called a section which may comprise one or more channels.

The hydraulic diameters, channel lengths, inlet and exit loss coefficients and number of sub channels for each channel were specified as TRACK II input. The number of sub channels is determined by considering the total cross-sectional flow area for the entire system. Boundary conditions to the hydraulic calculation consisted of reflector inlet plenum coolant temperature and pressure, total reflector flow rate, nozzle chamber pressure and coolant channel wall temperature distributions throughout the system.

The heat conduction model was composed of three separate finite element models: 1) the outer reflector, pressure vessel and tie bolts, 2) the control drums and 3) the inner reflector, transition region. Separation of the solid body into independent units allows for more efficient usage of the capacity of the computer program with adequate nodal representation of each component. The conduction models were chosen to take advantage of the symmetry of the system. Two dimensional models with unit depth representing a 30 degree sector of the periphery were made for the outer reflector and control drum. A three dimensional model was constructed for the inner reflector transition region.

In addition to geometry and materials properties, the external boundary conditions to the heat conduction models consisted of heat generation rates and fuel temperatures at the inner diameter of the transition region with coolant temperatures and convective heat transfer coefficients for the internal coolant channels to be supplied from the hydraulic solution.

Using the two group hydraulic model described and the conduction models at seven axial locations, a full power steady state analysis was performed. The problem solution begins with a hydraulic-heat convection calculation for the first group of channels 


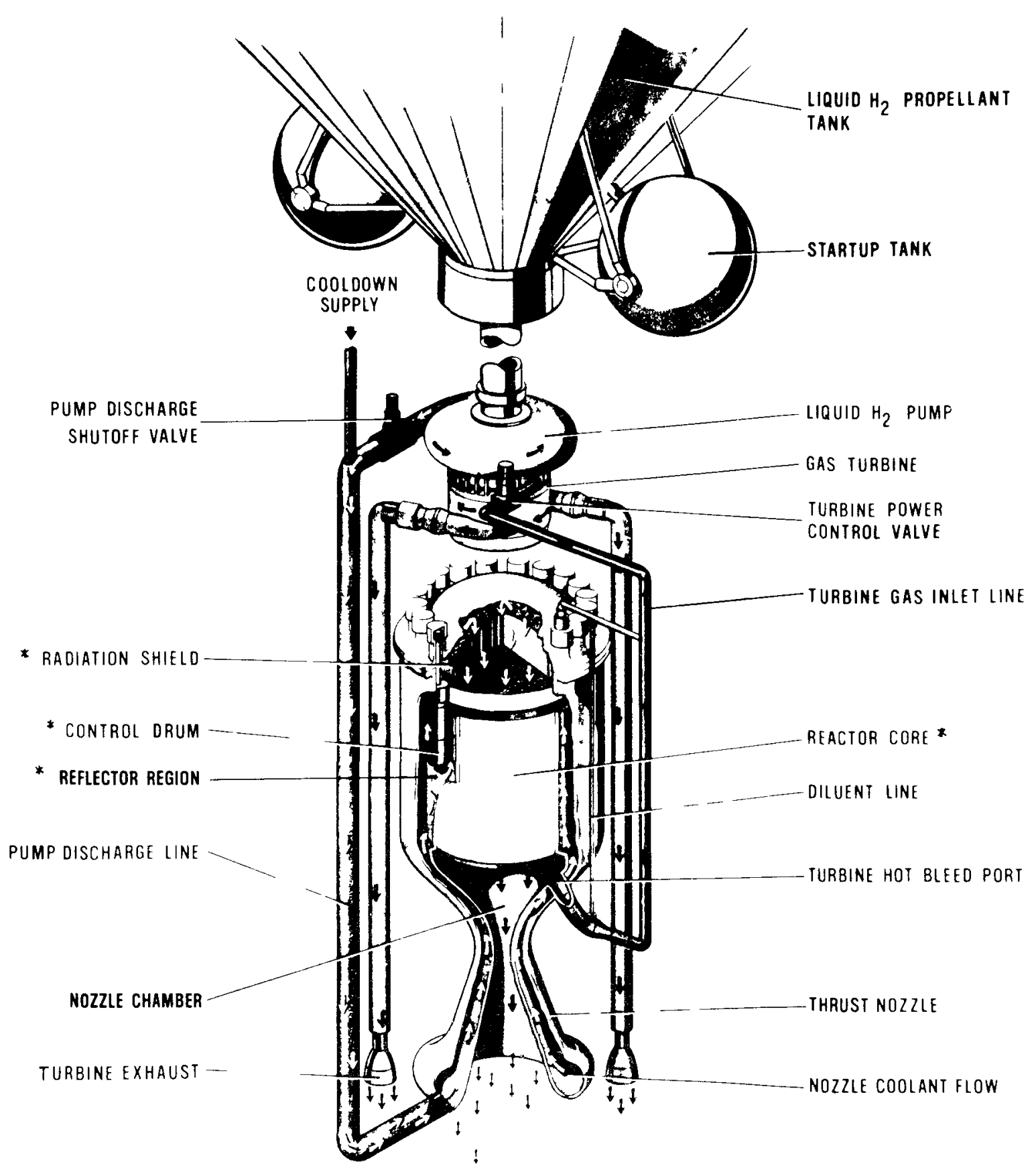

* nuclear subsystem components

Figure 3. XE-PRIME Engine Schematic 

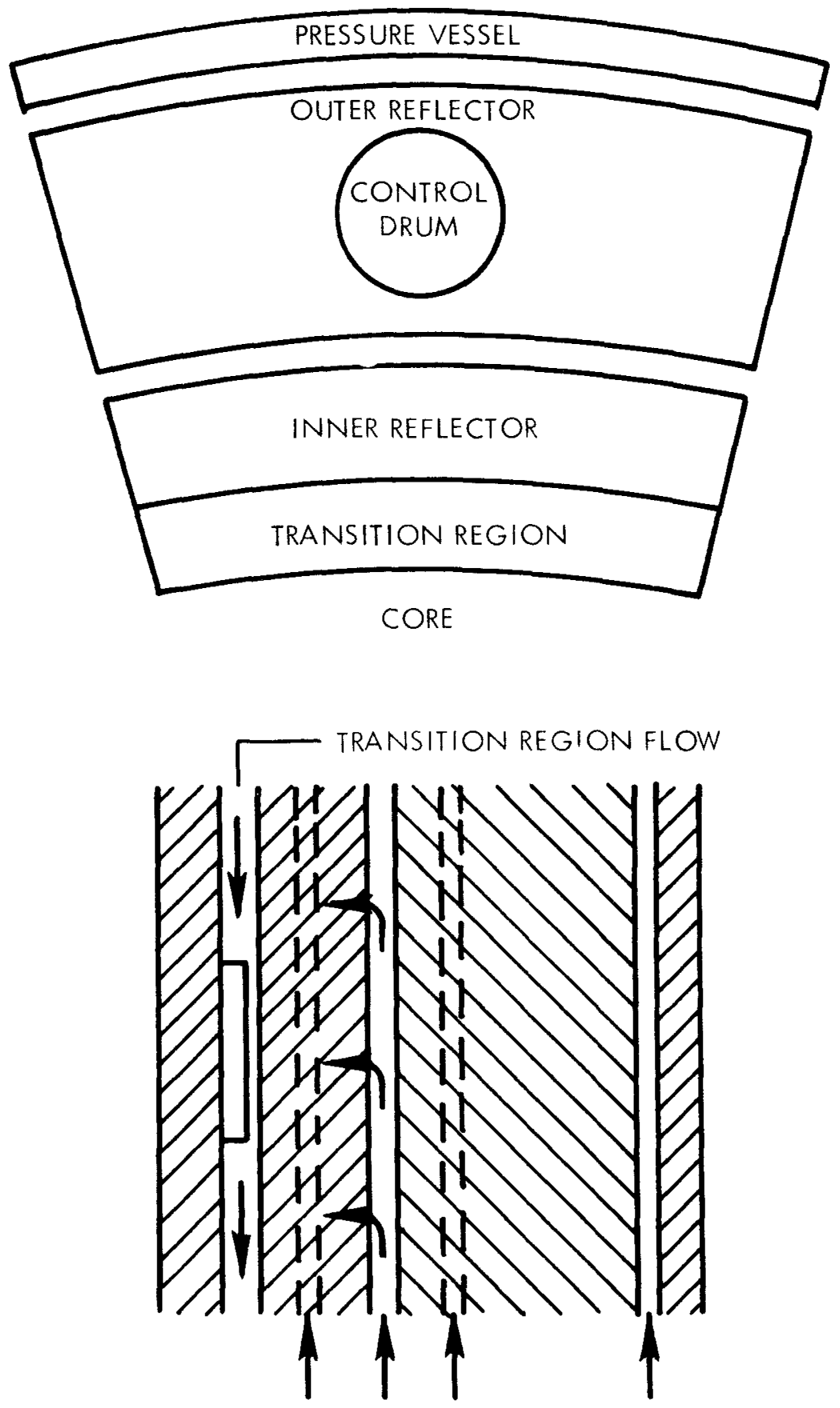

REFLECTOR FLOW

Figure 4. XE-PRIME Reflector System 


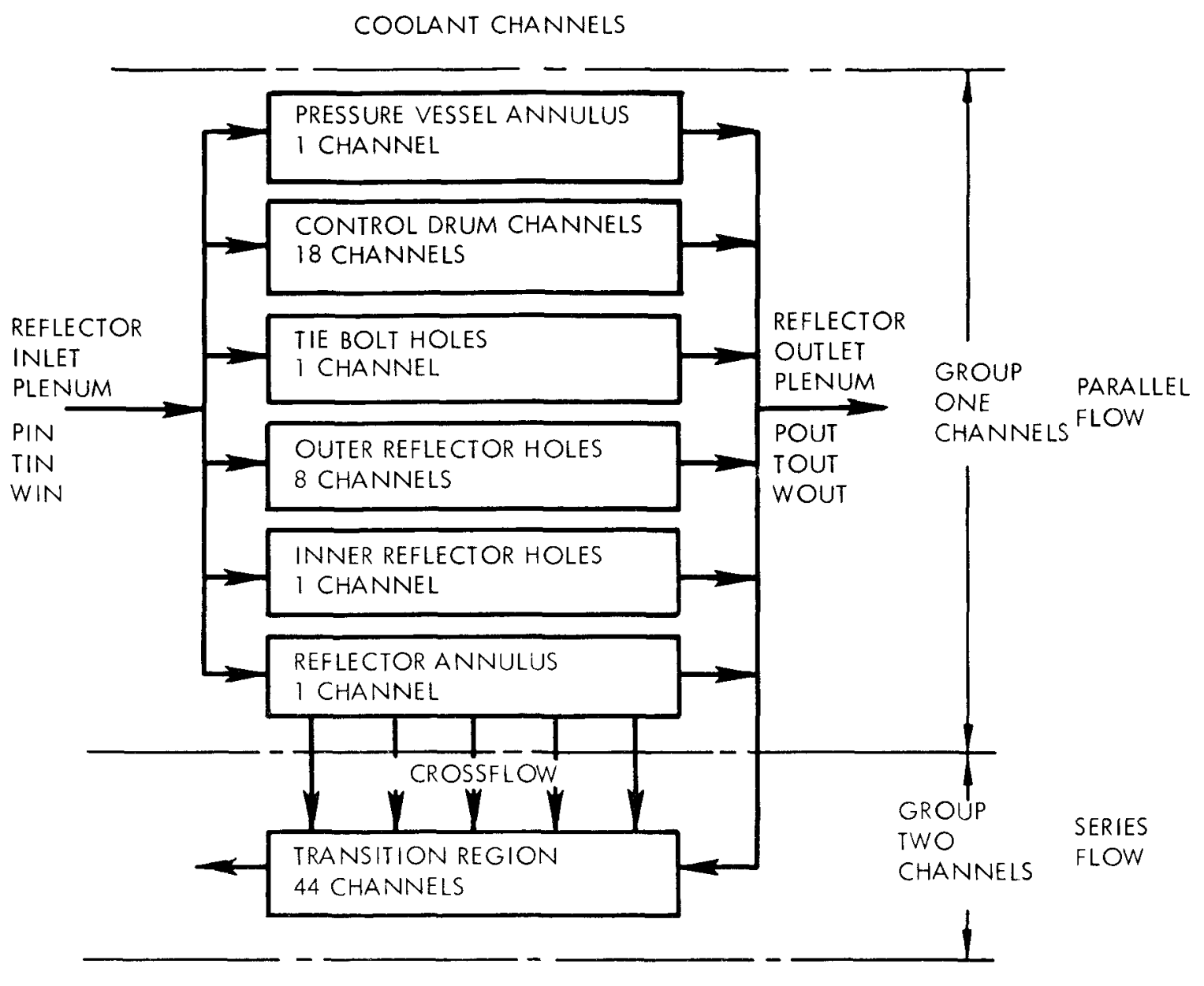

Figure 5. Flow Diagram for the XE-PRIME Reflector System 


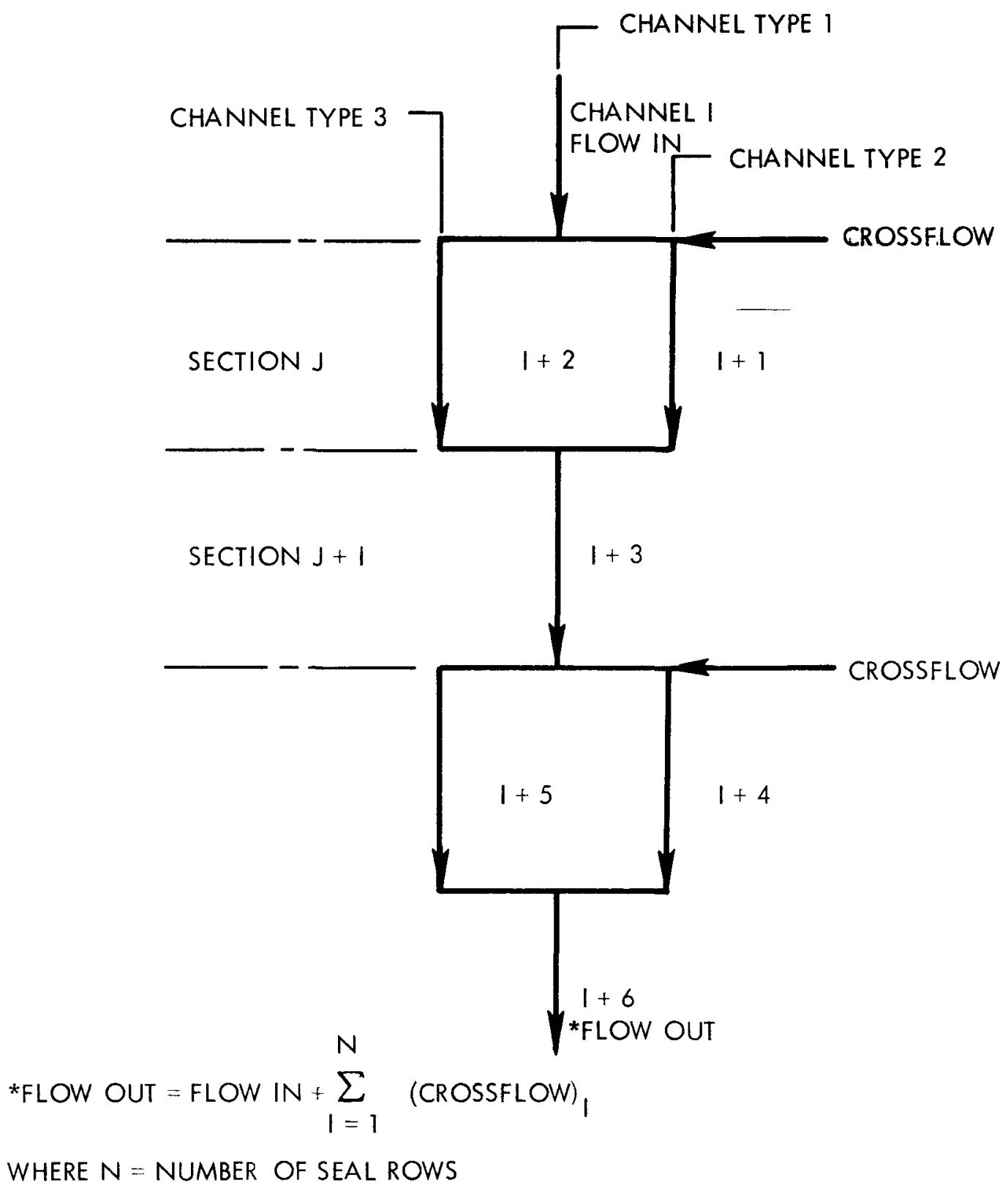

Figure 6. XE-PRIME Transition Region Flow Model 
representing parallel flow through the reflector system. Total flow was specified and a flow balance was performed in such a way that all channels had the same pressure drop within the input tolerance of one percent.

The next step was to compute the coolant conditions in the transition region (Group 2 channels). Calculated coolant temperatures and pressures for Group 1 coolant channels were used as boundary conditions for Group 2 hydraulic calculations. The inlet temperature and pressure were obtained from reflector exit conditions.

Crossflow from the reflector system to the transition region was determined from the coolant pressure and temperature distributions in the reflector annulus, the specified interconnection flow impedance and the plena pressures. By iteration, the inlef flow rate was determined in such a way that the exit pressure agreed with the input specification (nozzle chamber pressure) within one psi.

Upon completion of the hydraulic calculations, boundary conditions for the conduction analysis were prepared from the coolant temperature and convective heat transfer arrays. With the imposed boundary conditions, material temperature distributions were obtained for each component at each of the axial locations. Component surface temperatures were then translated into axial distributions of coolant channel wall temperatures.

The above calculational procedure was repeated until a compatible solution for the hydraulic and conduction models was obtained. The convergence criteria are described in Item 5 of Appendix A. The final temperature distributions were reserved on tape for direct input to structural analysis codes. The printed output included complete display of coolant conditions throughout the system and nodal temperature distributions for the solid body. Typical results for the XE-PRIME full power, steady state analysis are presented in Figures 7-9 (reflector pressure distributions, reflector material temperature distributions and reflector isotherms).

\section{APPENDIX A}

\section{CALCULATIONAL PROCEDURE}

\section{DIFFERENCE EQUATIONS FORMULATION}

The governing differential equations as shown in Equations (1) through ( 3 ) of the text, are expressed in the conservative form as pointed out by Lax. ${ }^{7}$ This philosophy was maintained in the formulation of the finite difference approximation. With the " $i$ " index designating the space variable and the " $i$ " index designating the time variable, in backward difference Equation (1) becomes

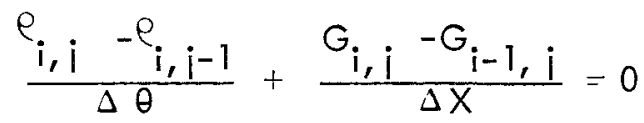

Solving for $G_{i, i}$ one obtains 


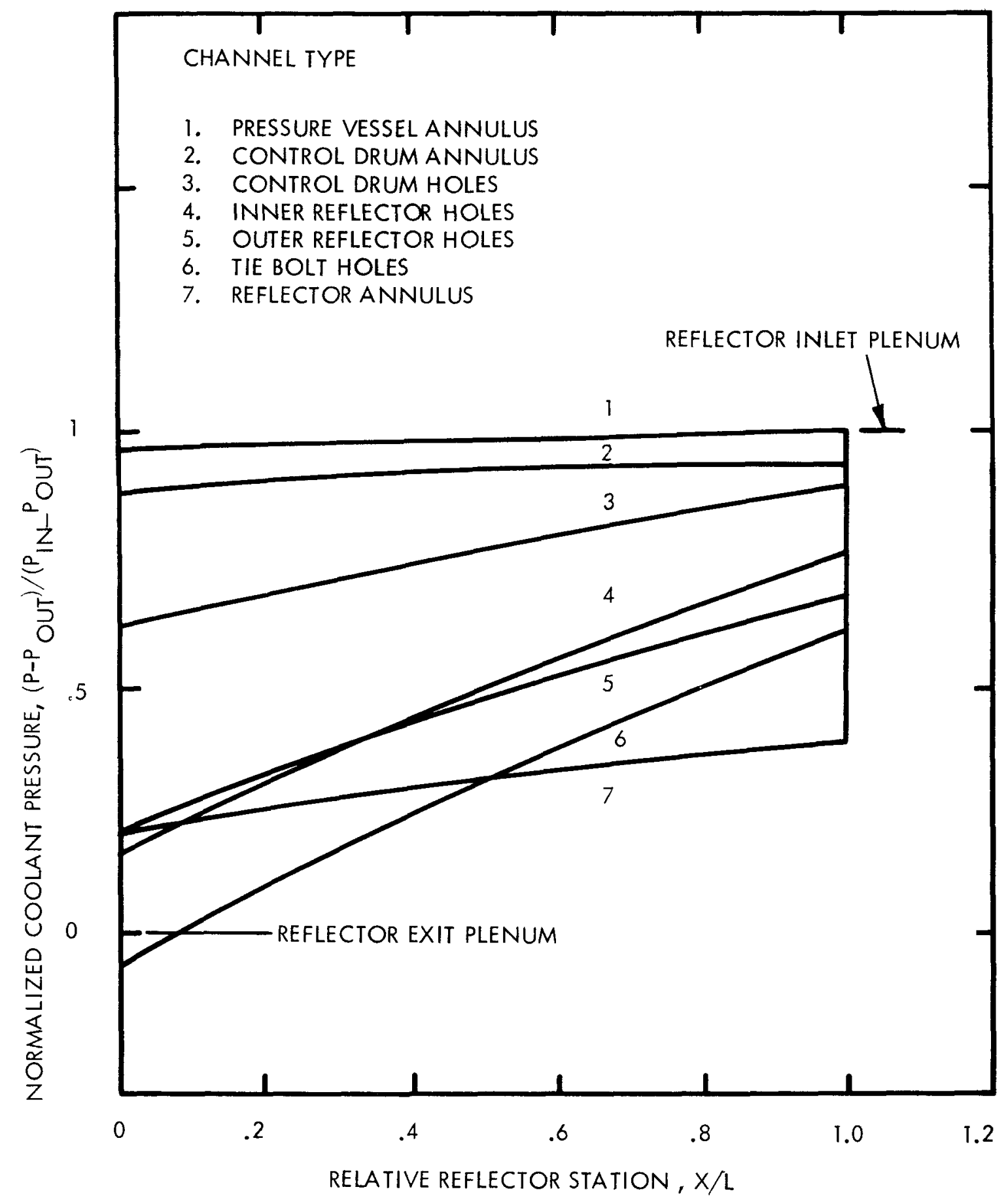

Figure 7. Full Power Steady State Pressure Distributions of the XE-PRIME Reflector System 


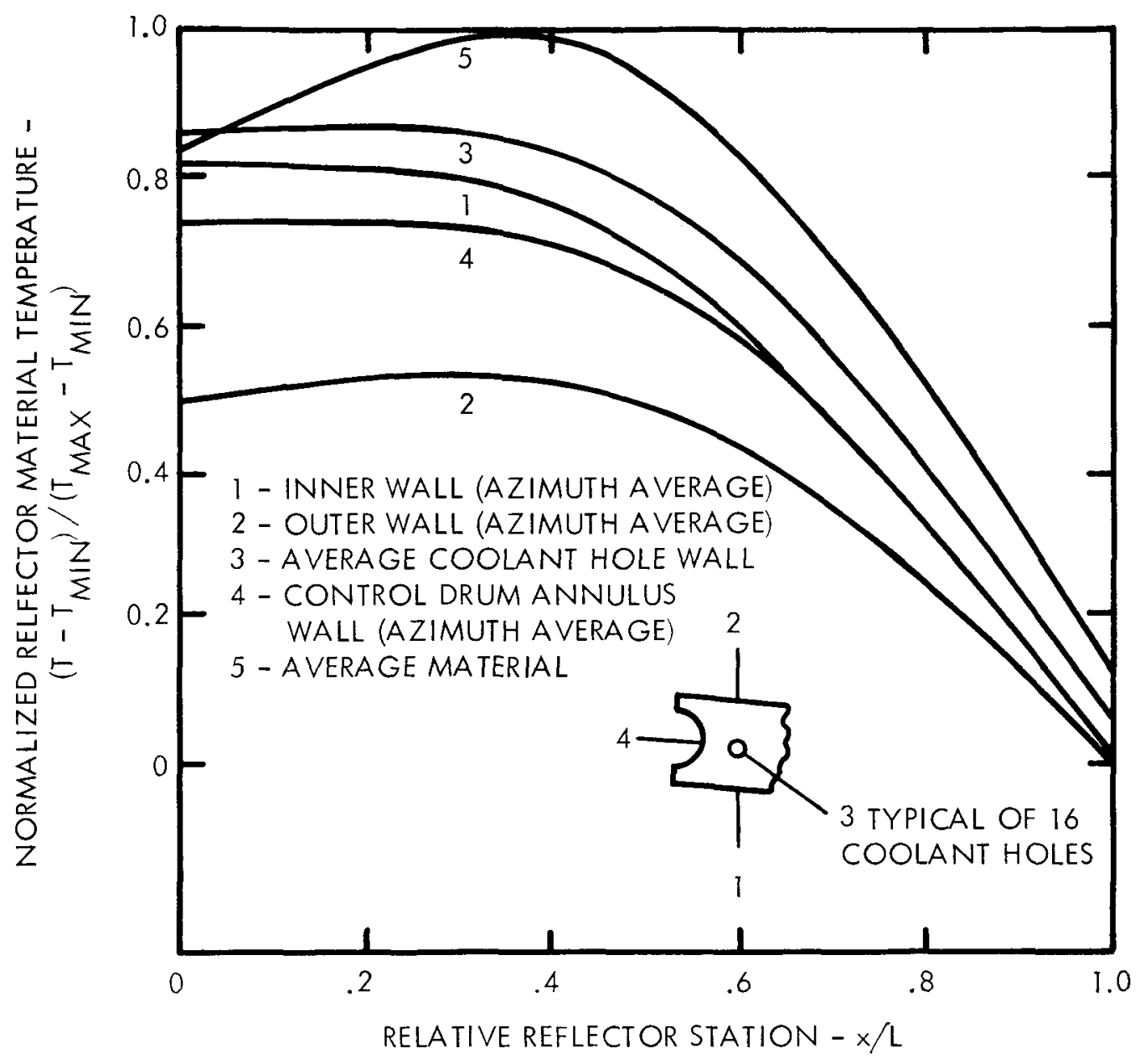

Figure 8. Full Power Steady State Material Temperature Distributions for the XE-PRIME Reflector 


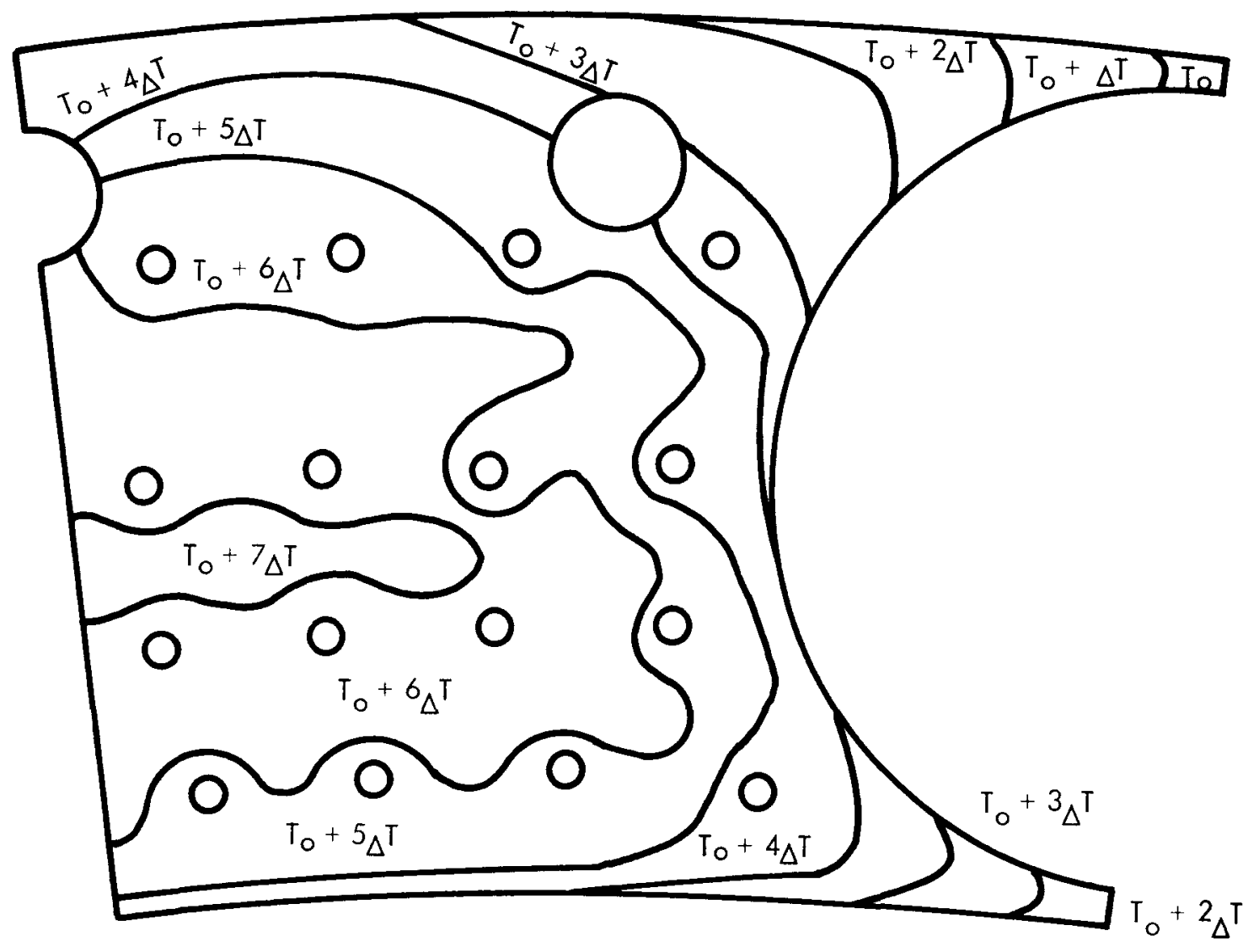

Figure 9. Midplane Isotherms for the XE-PRIME Reflector During Full Power, Steady State Operation 


$$
G_{i, i}=G_{i-1, i}-\frac{\Delta x}{\Delta \theta}\left(e_{i, i}-e_{i, i-1}\right)
$$

Similarly, Equation (2) becomes

$$
\frac{P_{i, i}-P_{i-1, i}}{\Delta X}+\frac{1}{g}\left(\frac{G_{i, i}-G_{i, i-1}}{\Delta \theta}\right)+\frac{1}{g}\left(\frac{G^{2} i, i v_{i, i}-G^{2} i-1, i v_{i}-1, i}{\Delta X}\right)+F_{1, i}=0 \text { (A3) }
$$

The frictional term $\mathrm{F}$ in Equation (A3) is expressed by means of the friction factor " $\mathrm{f}$ " as follows

$$
F=\frac{4 f G^{2}}{2 g \rho D}
$$

Using the average values of mass flow rate and the density along an increment, $i$, the friction ferm is,

$$
\begin{aligned}
& F_{i, i}=\frac{2 f_{i, i} \bar{G}^{2}}{g \bar{\rho} D} \\
& \bar{G}=\frac{G_{i, i}+G_{i-1, i}}{2} \\
& \bar{\rho}=\frac{\rho_{i, i}+\rho_{i-1, i}}{2}
\end{aligned}
$$

The friction factor, $f_{i, j}$, is obtained from empirical correlations for gaseous flow in a smooth tube as shown in $i, i^{\prime}$ Equations (14) and (15). A roughness multiplier can be applied to the friction factor for flow passages which are not smooth. This roughness multiplier may be obtained from experimental data. Substituting Equation (A5) into (A3) and solving for $P_{i, j}$, one obtains

$$
\begin{aligned}
P_{i, i}=P_{i-1, i}-\frac{1}{g}\left[\frac{\Delta X}{\Delta \theta}\left(G_{i, i}-G_{i, i-1}\right)+\left(G_{i-1}^{2} v_{i, i}-G_{i, 1, i}^{2} v_{i-1, i}\right)\right] \\
-\frac{2 f_{i, i} \bar{G} \Delta X}{g \bar{\rho} D}
\end{aligned}
$$


Similarly, in Equation (3) of Section 2, the last term can be expressed as

$$
Q P=\frac{q P}{A}
$$

The energy equation then becomes

$$
\begin{gathered}
\frac{H_{i, j} G_{i, j}-H_{i-1, i} G_{i-1, i}}{\Delta x}+\frac{H_{i, j} e_{i, j}-H_{i, j-1} e_{i, j-1}}{\Delta \theta}- \\
-\frac{1}{J}\left(\frac{P_{i, j}-P_{i, j-1}}{\Delta \theta}\right)-\frac{\bar{q} P}{A}=0,
\end{gathered}
$$

where

$$
\bar{q}=\frac{q_{i, j}+q_{i-1, j}}{2}
$$

Solving for $\mathrm{H}_{i, i}$, one obtains

$$
\begin{gathered}
H_{i, i}=\frac{1}{\left(G_{i, j}+e_{i, j} \frac{\Delta x}{\Delta \theta}\right)}\left\{G_{i-1, i} H_{i-1, i}+\frac{\Delta x}{\Delta \theta}\left(e_{i, j-1} H_{i, j-1}+\frac{1}{J}\left(P_{i, j}-P_{i, j-1}\right)\right)\right. \\
\quad+\frac{\bar{q} p}{A} \Delta x
\end{gathered}
$$

The heat flux, $q$, in Equation (A7) is determined by the convective heat transfer relationship as shown by Equation (6). Equations (A2), (A6), and (A9) are the working finite difference representations for the equations of conservation. These three equations together with the equation of state are sufficient to calculate the fluid conditions $G, Q, P$, and $H$ at any point of the flow system. The calculational procedure is an iteration process as described in the following: For specified values of 1) the channel wall temperature distributions of all channels at time $=\theta_{i}, 2$ ) all the fluid conditions and channel wall temperatures at time $=\theta_{j-1}$ and 3 ) the inlet fluid conditions at time $=\theta_{j}$, the fluid conditions at the end of an Increment at time $=\theta_{i}$ can be calculated. To start the iteration

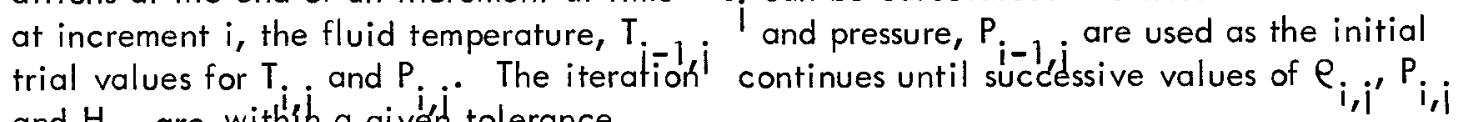
and $\mathrm{H}_{i, i}$ are withíh a givén tolerance.

\section{ENTRANCE AND EXIT PRESSURE LOSSES}

The pressure losses due to frictional force and momentum change inside a channel are calculated by Equation (A6). The pressure loss at the entrance or exit of the channel is computed from the following expression:

$$
\Delta P=C \frac{G^{2} v}{2 g}
$$


The coefficient $C_{L}$ is the inlet or exit loss coefficient of the channel. It can be determined analytically or by experiment.

\section{FLOW RATE ADJUSTMENT}

The total flow of fluid in the system is distributed among the channels in such a way that the overall pressure drop across all channels is the same within a specified tolerance. An estimated flow rate distribution is given as the initial condition.

Following an iteration on flow balance, the flow rate distribution is adjusted according to the following relationship,

$$
\left(G_{k}\right)_{\text {new }}=\left[G_{k}\left(\frac{\Delta P_{k}}{\Delta \bar{P}}-\right)^{\beta}\right] \text { old }
$$

where,

$$
\begin{aligned}
\Delta P_{k}= & \text { overall pressure drop in the } k^{\text {th }} \text { channel } \\
\overline{\Delta P} \quad= & \text { flow weighted average pressure drop of all the channels or specified } \\
& \text { pressure drop of the flow system }
\end{aligned}
$$

The iteration is continued until the flow balance criteria is met or the maximum number of iterations is exceeded.

\section{TEMPERATURE DISTRIBUTION IN THE SOLID BODY}

The TOSS (Transient or Steady State) temperature calculation code was modified and integrated into the TRACK program for solution of the heat conduction model. Equation (4), the heat conduction equation, in finite difference form is:

$V_{q} q^{\prime \prime \prime} q_{, i}+\sum_{p} Y_{p q, i}\left(T_{p, i}-T_{q, i}\right)=\frac{C q}{\Delta \theta}\left(T_{q, i}-T_{q, j-1}\right)$

where the index " $p$ " denotes all neighboring nodes of the internal node " $q$ " and the index " $i$ " denotes the quantities at time $=\theta_{i}$. The term $Y_{p q, ~} i$ is the admittance between adjacent internal nodes or a surface $\mathrm{i}$ and an $\mathrm{pq}, \mathrm{i}$ internal node, $\mathrm{p}$ to $\mathrm{q}$.

For conduction from an internal node to another internal node 


$$
Y_{p q, i}=\frac{A p q}{\frac{l_{p q}}{k_{p, i}}+\frac{q p}{k_{q, i}}}
$$

For conduction from an internal node to a surface node

$$
Y_{p q, i}=\frac{k_{p, i} A_{p q}}{\left.\right|_{p q}}
$$

The boundary heat transmission is calculated by means of the heat transfer coefficient between the coolant and the channel wall surface node. The surface admittance is determined by the mode of heat transfer between the boundary node, $b$, and the channel wall surface node, $w$. The following three modes can be used:

For forced convection

$$
Y_{b w}=h(\theta) A_{W}
$$

For natural convection

$$
Y_{b w}=A_{w} K_{b w}\left|T_{w}-T_{b}\right| E_{b w}
$$

For radiation

$$
Y_{b w}=A_{w} K_{b w} \sigma\left(T_{b}^{2}+T_{w}^{2}\right)\left(T_{b}+T_{w}\right)
$$

where $h(\theta)$ is the heat transfer coefficient which can be a function of time, and $K_{b w^{\prime}}$ and $\mathrm{E}_{\text {bw }}$ are input constants.

To solve the system of linear finite difference equations are shown by Equation (A12), a Gauss-Seidel iteration procedure is used. The $(m+1)^{\text {th }}$ iteration at time $=\theta_{i}$ for the temperature at the $\mathrm{q}^{\text {th }}$ node, $\underset{q, i}{\mathrm{~m}}+\mathrm{i}$, is

$T_{q, i}^{m+1}=\frac{\sum_{p=1}^{q-1} Y_{q p} T_{p, i}^{m+1}+\sum_{p=q+1}^{n} Y_{q p} T_{p, i}^{m}+q_{q, i}^{\prime \prime} V_{o l} v_{q}-C\left(\frac{T_{q, i}^{m}-T_{q, i}-1}{\Delta \theta}\right)}{-Y_{q q}}(A$

where

$$
Y_{q q}=\text { Self admittance }=-\sum_{p}^{n} Y_{q p}
$$


and

$$
T_{q, i-1}=\text { converged temperature for node at time } \theta_{j-1}
$$

and " $p$ " ranges over the " $n$ " neighbors of node " $q$ ". This iteration continues until all internal node temperatures meet the following criteria

$$
\left|T_{q}^{m}-T_{q}^{m+1}\right| \sum \epsilon
$$

where " $\mathrm{m}$ " indexes the number of iterations on the internal node array. The convergence parameter, " $\epsilon "$ is an input quantity with units of ${ }^{O} \mathrm{R} /$ iteration.

\section{COUPLING OF HYDRAULIC AND CONDUCTION CALCULATIONS}

From the above description of the calculational procedure, it is seen that the fluid flow calculation can be completed for one time step when the channel wall temperature distributions through the flow system are known. In the analysis presented herein, the channel wall temperatures are supplied and checked using a temperature distribution calculation of the solid body. Estimates of the initial wall temperature distribution are specified in the input data. The fluid flow calculation for the first time step can then be completed. The resulting fluid bulk temperatures and channel wall heat transfer coefficients are input to the temperature distribution calculations to compute the temperature change of the solid body during this time step at several transverse cross sections of the system (axial heat conduction is neglected). Linear interpolation is used to complete the axial wall temperature profiles. The channel wall temperatures thus obtained are compared to the wall temperatures used in the fluid flow calculation. If they do not agree within a specified tolerance, the entire procedure is repeated using the new channel wall temperatures. This iteration between the fluid flow calculation and the material temperature distribution calculation is continued until the convergence criterion is satisfied. The iteration process is repeated for each succeeding time step. The initial estimates of the wall temperatures at the end of a time step are obtained by linearly extrapolating the wall temperatures from two preceding times. Expressed another way, the channel wall temperatures at $\theta_{i}$ are obtained by extrapolation from the known wall temperatures at $\theta_{i-1}$ and at $\theta_{i-2}$.

\section{NOMENCLATURE}

A Channel cross sectional area, in. ${ }^{2}$

As Channel surface area, in. ${ }^{2}$

C Heat capacity, Btu $/{ }^{\circ} \mathrm{R}$

$C_{L} \quad$ Pressure loss coefficient

$c_{p} \quad$ Specific heat, Btu/ $/ b_{m}-{ }^{o}$ 
D Hydraulic diameter of flow channel, in.

F Frictional force term, $\mathrm{l}_{\mathrm{f}} / \mathrm{in}^{3}$

$f \quad$ Friction factor

$\mathrm{G} \quad$ Mass flow rate, $\mathrm{l} \mathrm{b}_{\mathrm{m}} / \mathrm{sec}-\mathrm{in} .^{2}$

g Gravitational constant, $\left(\mathrm{in} / \mathrm{sec}^{2}\right)\left(\mathrm{l} \mathrm{b}_{\mathrm{m}} / \mathrm{l} \mathrm{b}_{\mathrm{f}}\right)$

H Stagnation specific enthalpy, Btu/ $/ b_{m}$

h Local heat transfer film coefficient, Btu/sec-in. ${ }^{2}-{ }^{o}$

$i, i \quad$ Indices in finite difference equations, $i=$ space variable $; i=$ time variable

J Energy conversion factor, $\mathrm{lb}_{f}-\mathrm{in.} / \mathrm{Btu}$

$\mathrm{K} \quad$ Constant in natural convection and radiation equations

$k \quad$ Thermal conductivity, Btu/sec-in. ${ }^{\circ} R$

I Conduction node length, in.

Nu Nusselt number

P Pressure, psia

Pr Prandtl number

Q Heat transfer per unit time per unit mass of fluid, Btu/sec-lb

q Heat flux, Btu/sec-in. 2

$q^{\prime \prime} \quad$ Internal heat generation rate, Btu/sec-in. 3

Re Reynolds Number

T Fluid bulk or material temperature, ${ }^{\circ} \mathrm{R}$

$T_{B} \quad$ Fluid bulk temperature, ${ }^{\circ} R$

$T_{r} \quad$ Fluid recovery temperature, ${ }^{\circ} R$

$T_{w} \quad$ Material wall temperature, ${ }^{\circ} R$

u Internal energy, $B+u / \mathrm{Ib}_{\mathrm{m}}$

$\checkmark \quad V e l o c i t y$, in/sec

Vol Volume, in. 3 


\begin{tabular}{|c|c|}
\hline$v$ & Specific volume, in ${ }^{3} / \mathrm{lb}_{\mathrm{m}}$ \\
\hline W & Flow rate, $1 b_{m} / \mathrm{Sec}$ \\
\hline$x, y, z$ & Space coordinates \\
\hline Y & Conduction node admittance, $\mathrm{B}+\mathrm{u} / \mathrm{hr}-\mathrm{O}_{\mathrm{R}}$ \\
\hline$\mu$ & Fluid absolute viscosity, $\mathrm{lb}_{\mathrm{m}} / \mathrm{in} .-\mathrm{sec}$ \\
\hline$\theta$ & Time, seconds \\
\hline$\sigma$ & Stefan-Boltzman radiation constant, $0.1713 \times 10^{-8} \mathrm{Btu} / \mathrm{ft}{ }^{2} \mathrm{hr}\left({ }^{\circ} \mathrm{R}\right)^{4}$ \\
\hline $\mathbf{P}$ & Channel perimeter, in. \\
\hline$\rho$ & Mass density, $\mathrm{lb} \mathrm{m}_{\mathrm{in}} \mathrm{in}^{3}$ \\
\hline
\end{tabular}

VII. ACKNOWLEDGEMENT

The Nuclear Engine for Rocket Vehicle Application Program (NERVA) is administered by the Space Nuclear Propulsion Office, a joint office of the U. S. Atomic Energy Commission and the National Aeronautics and Space Administration. AerojetGeneral Corporation, as prime contractor for the engine system, and Westinghouse Electric Corporation, as subcontractor for the nuclear subsystem, are developing a nuclear propulsion system for space applications.

\section{REFERENCES}

1. A. Y. Lee, M. D. Woods, TRACK, A Computer Program for Transient and Steady State Coupled Flow and Heat Conduction Analysis, WANL-TME-1736, (Jan. 1968).

2. J. R. McCarthy and H. Wolf, "Forced Convection Heat Transfer to Gaseous Hydrogen at High Heat Flux and High Pressure in a Smooth, Round, Electrically Heated Tank," ARS Journal 43 (April 1960).

3. B. S. Petukhov, et al., "Heat Transfer Experimental Research for Turbulent Gas Flow in Pipes at High Temperature Difference Between Wall and Bulk Fluid Temperatures". Proc. Intern. Conf. on Heat Transfer, $3^{\text {rd }}$ Conference, (Aug. 7-12, 1966).

4. K. D. Williamson, Jr., et al., "Studies of Forced Convection Heat Transfer to Cryogenic Fluids", C.E.P. Symposium 87(64), (1968).

5. H. L. Langhaar, "Steady State in the Transition of a Straight Tube", Journal of Applied Mechanics, Trans. ASME, 64, (1942). 
6. M. F. Taylor, "A Method of Correlating Local and Average Friction Coefficients for Both Laminar and Turbulent Flow of Gases Through a Smooth Tube with Surface to Fluid Bulk Temperature Ratios from 0.35 to $7.35^{\prime \prime}$, Intern. Conf. on Heat and Mass Transfer Shorter Communications, (Aug. 1967).

7. P. Lax, "Weak Solutions of Nonlinear Hyperbolic Equations and Their Numerical Computation", Communications on Pure and Applied Mathematics, 7 (1954).

8. D. Bagwell, "TOSS - An IBM 7090 Code for Computing Transient or Steady State Temperature Distributions", K-1494, Oak Ridge National Laboratory (Dec. 1961).

\section{REFERENCES FOR ADDITIONAL RESEARCH}

J. E. Mayer, et al. , ART - Program for the Treatment of Reactor Thermal Transients on the IBM-704, WAPD-TM-156 (Nov. 1959)

G. Guderley, Nonstationary Gas Flow in Thin Pipes of Variable Cross-Section, NACA-TM-1196, (Dec. 1948).

R. P. Stein, Transient Heat Transfer in Reactor Coolant Channels, AEC ReportAECU-3600 (Oct. 1957).

E. Jenny, "Unidimensional Transient Flow With Consideration of Friction, Heat Transfer, and Change of Section", The Brown Boveri Review, (Nov. 1950).

W. O. Doggett, R. H. Shultz, Jr., "Transient Heat Transfer in a Convection Cooled Heterogeneous Nuclear Reactor With Axial Power Density", International Developments in Heat Transfer, Part III, pp. 622-633 (ASME, 1961).

L. R. Steele, R. F. Berland, A Transient Multichannel Two-Phase Hydraulic Code NAA-SR-9425 (Dec. 1964).

A. H. Shapiro, The Dynamics and Thermodynamics of Compressible Fluid Flow, Vol. II, (Ronald Press, 1954).

A. Y. Lee, M. D. Woods, A. G. Eggers, "Transient Thermal Analysis of a Heat Generating Solid and Its Application to NERVA Reactor Control Drums", Paper presented at $2^{\text {nd }}$ AIAA Propulsion Joint Specialist Conference, (June 1966).

B. L. Pierce, Modified Transient and/or Steady State (TOSS) Digital Heat Transfer Code, WANL-TME-1108. 
ROBOT - A COMPUTER PROGRAM TO SOLVE THE

BOWING PROBLEM IN ROD-TYPE FUEL ELEMENTS

(LWBR Development Program)

John J. Urbaniak

Westinghouse Electric Corporation

Bettis Atomic Power Laboratory

One of the major problems in the design of civilian nuclear reactors is the accurate prediction of the deflections undergone by rod-type fuel elements. The structural designer, for example, must have a thorough knowledge of the forces and moments present in the various components of his core throughout the life of the reactor. Since the fuel rods are generally supported at intervals by grids, any reaction between the rods and the grids must be predicted so that the grids can be manufactured with the necessary strength and rigidity. These rod-grid reactions are dependent upon both axial and transverse deflections of the fuel rods. From a thermal designer's standpoint, bowing of fuel rods affects the heat transfer capability of the coolant. In particular, if two adjacent rods deflect to within a few thousandths of an inch of each other, the coolant flow is thus restricted and local hot spots may develop on the rod. For these reasons, both axial and transverse deflections of the fuel rods, as well as the axial forces present in the rods must be accurately predictable.

Fuel rods deflect in response to several mechanisms. First of all, reactor operating temperature tends to increase the length of the rod via thermal expansion. However, the supporting grids resist this length increase so that axial forces, called thrusts, arise within the rod. If the rod was manufactured with a slight degree of initial curvature, or if the thermal gradient across the rod induces curvature, the axial forces tend to intensify this curvature leading to transverse deflections or bow of the fuel rod. Also, irradiation, temperature, pressure and stress, as well as fuel-clad interactions affect the bending stiffness of the rod. Thus any externally applied moment, such as that induced by the support grids, will affect the deflected shape of the rod. One of the most interesting factors affecting the fuel rod shape, and the main content of this paper, is the frictional reaction between the rods and the supports. Generally, the support is some type of clamping device which exerts 
a definite frictional resistance to rod motion. It is conceivable, however, that at some times during reactor operation, the net axial force exceeds the support resistance and the rod slips through the support. When this happens, both the axial thrust and the rod's transverse deflection are reduced. Thus we see that in addition to the well known mechanisms such as thermal expansion, curvature, etc., the frictional nature of the supports contributes to the deflected shape of the rod. In order to accurately predict these frictional reactions, the axial thrust and the transverse deflections must be treated as interdependent quantities. To our knowledge, the computer program described in this paper, is the only existing program which contains this interdependence.

References 1 and $\geq$ contain the specifications for ROBOT, a computer program written in FORTRA IV for the CDC-6600 computer, and designed to predict the time history of fuel rod bowing under simulated reactor operating conditions. The fuel rod is idealized as a viscoelastic, multispan beam held in place by elastic supports. The generalized ROBOT support (Figure 1) is flexible with axial spring constant $\mathrm{K}_{\mathrm{m}}$, transverse spring constant $\mathrm{S}_{\mathrm{m}}$, and torsional spring constant $\mathrm{T}_{\mathrm{m}}$. Transverse load $\mathrm{L}_{\mathrm{m}}$, moment $\mathrm{M}_{\mathrm{m}}$, axial load $\mathrm{Z}_{\mathrm{m}}$, and ground motion $W_{m}$ may be applied to the support. Internally generated thrusts $P_{m}, P_{m+1}$ tend to push the rod through the support and friction force $\bar{z}_{m}$ resists this motion. Therefore, when $P_{m}+Z_{m}-P_{m+l}$ exceeds $\bar{Z}_{m}$, the rod will slip at support $m$. Furthermore, the displacement $u$ of the rod may be fixed at $a_{m}$ for support $m$ and the slope of the rod at support $m$ may be fixed at $b_{m}$. In Figure 1 , the rod is positioned so that the z-direction is the direction of increasing core height. We now define "span $n$ " as that portion of the fuel rod lying between supports $n-1$ and $n$, and we wish to compute the history of transverse deflections undergone by the rod in each of its spans.

The deflected shape $u_{n}(z, t)$ of the span lying between supports $n-I$ and $n$ at time $t$ is approximated by a polynomial in the trial functions $f_{i}^{n}(z)$ where:

$$
\begin{aligned}
& f_{1}^{n}(z)=1 \\
& f_{i}^{n}(z)=\left(\frac{z-z_{n-1}}{z_{n}-z_{n-1}}\right)^{i-1} \quad i=2, \ldots, N_{t f}
\end{aligned}
$$

and $\mathrm{N}_{\mathrm{tf}}=$ the number of trial functions to be used. Since displacement $\mathrm{a}_{\mathrm{m}}$ and slope $b_{m}$ may be imposed as boundary conditions at a given support $m$, these trial functions must be combined to form an "admissable" set of trial functions. This new set of trial functions must reflect the imposed deflection and slope 


\section{GENERALIZED ROBOT SUPPORT}

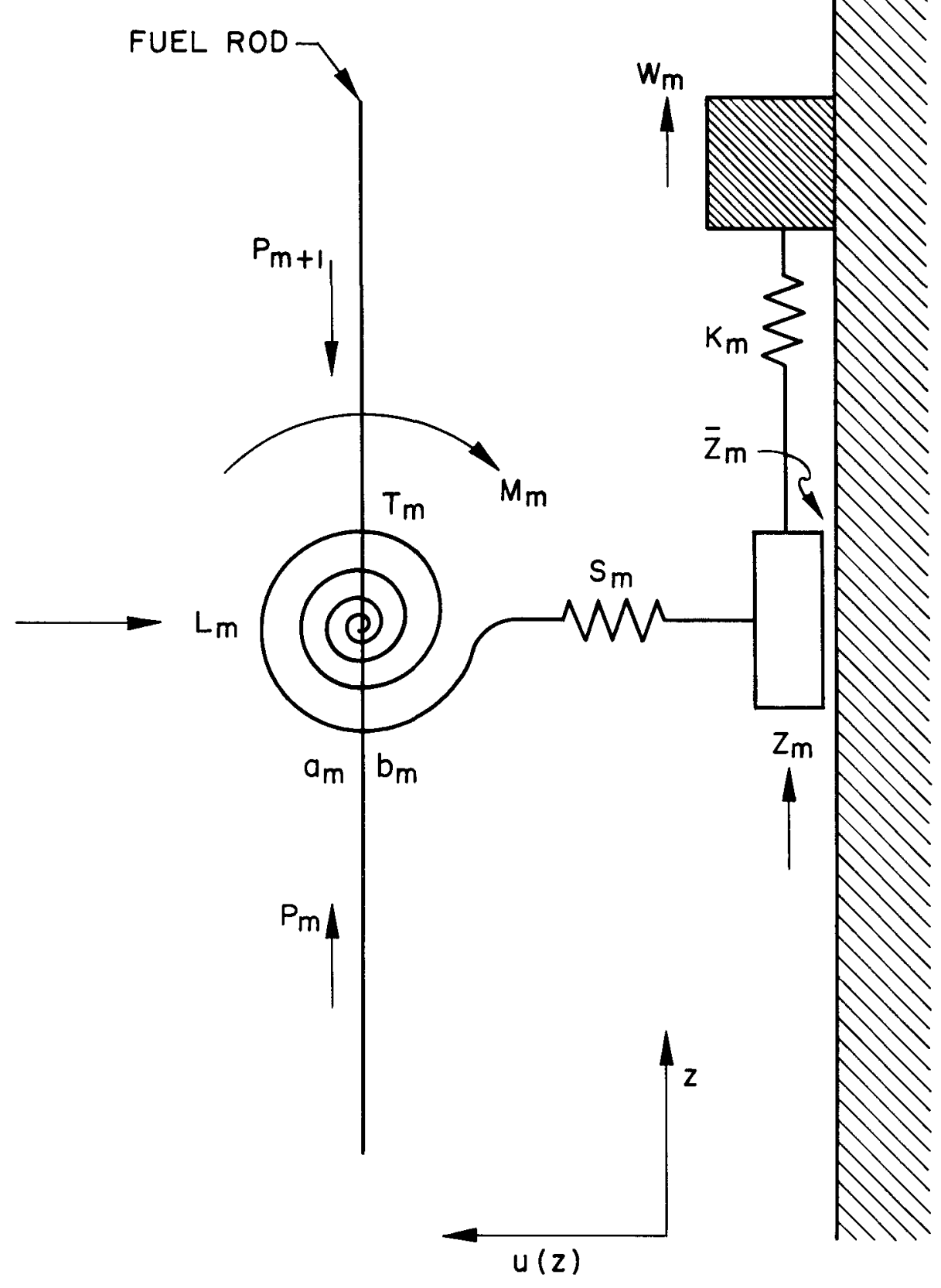

FIGURE I 
at the end supports. Thus we write

$$
u_{n}(z, t) \approx \psi_{n}(z, t)+\sum_{j=1}^{N} d_{n j}(t) \phi_{n}^{n}(z)
$$

where $\mathbb{N}_{b c}$ is the number of imposed boundary conditions, $\psi_{n}(z, t)$ is chosen to satisfy the imposed conditions and $\phi_{j}^{n}(z)$ is a linear combination of the trial functions $f_{i}^{n}(z)$ defined above satisfying the homogeneous version of these conditions. Symbolically we write $\phi_{i}^{n}(z)$ and $\psi_{n}(z, t)$ as the matrix products

and

$$
\phi_{i}^{n}(z)=\left[C_{i j}^{n}\right]\left[f_{j}^{n}(z)\right]
$$

$$
\psi_{n}(z, t)=\left[s_{j}^{n}(t)\right]^{T}\left[f_{j}^{n}(z)\right]
$$

where the matrices $\left[\mathrm{C}_{i j}^{n}\right]$ and $\left[j_{j}^{n}(t)\right]$ are given in Appendix $B$ of reference 1 and $\left[f_{j}^{n}(z)\right]$ is the vector of trial functions described above. Now using the matrix notation

and

$$
\Delta_{n}(t)=\left[j_{n j}(t)\right], D_{n}(t)=\left[d_{n j}(t)\right], c_{n}=\left[C_{i j}^{n}\right]
$$

$$
F_{n}(z)=\left[f_{j}^{n}(z)\right]
$$

equation 1 can be rewritten as

$$
u_{n}(z, t)=\left\{D_{n}(t)^{T} C_{n}+\Delta_{n}(t)^{T}\right\} F_{n}(z) \cdot
$$

By using the principle of virtual work, together with the Newton-Raphson iteration scheme, Newman. (Ref. 2) derives a series of equations relating the axial thrust and transverse displacements to the known forces imposed on the beam at time $t_{k}$. It is assumed that the deflection vector $D_{n, k-1}\left(t_{k-1}\right)$ and the axial thrust $P_{n, k-1}\left(t_{k-1}\right)$ are known at time $t_{k-1}$. The values $D_{n, k}\left(t_{k}\right)$ are then expressed by

and

$$
D_{n, k}\left(t_{k}\right)=D_{n, k-1}+\tilde{D}_{n}
$$

$$
P_{n, k}\left(t_{k}\right)=P_{n, k-1}+\widetilde{P}_{n}
$$

Calculation of $D_{n, k}\left(t_{k}\right)$ and $P_{n, k}\left(t_{k}\right)$ is thus reduced to the calculation of $\tilde{D}_{n}$ and $\widetilde{\mathrm{P}}_{n}$. In the Newton-Raphson iteration, guesses are made for the terms $\widetilde{D}_{n}$ and $\widetilde{P}_{n}$. The equations governing $D_{n, k}$ and $P_{n, k}$ are then approximated by 
linearized Taylor series about the point $\widetilde{D}_{n}$ and $\widetilde{P}_{n}$. This introduces terms of the form $\hat{D}_{n}-\widetilde{D}_{n}$ and $\hat{P}_{n}-\widetilde{P}_{n}$. Solution of these resulting equations for $\hat{D}_{n}$ and $\hat{P}_{n}$ leads to an improved estimate for $\tilde{D}_{n}$ and $\widetilde{P}_{n}$. Eventually the process will converge and the terms $\hat{D}_{n}$ and $\hat{P}_{n}$ will approximately equal $\widetilde{D}_{n}$ and $\widetilde{P}_{n}$.

Thus writing

and

$$
D_{n, k}=\left(D_{n, k-1}+\tilde{D}_{n}\right)+\left(\hat{D}_{n}-\tilde{D}_{n}\right)
$$

$$
P_{n, k}=\left(P_{n, k-1}+\tilde{P}_{n}\right)+\left(\hat{P}_{n}-\tilde{P}_{n}\right)
$$

we arrive at the following fundamental equations, labelled 24 in reference 2 . The $\sigma_{i}, i=1, \ldots, 4$ matrices appearing in these equations are stiffness matrices which can be found in Appendix $C$ of reference 1 . The parameters $\lambda_{n}$ and $\mu_{n}$ express continuity of position and slope between span $n$ and $n+l$. The equations are:

$$
\begin{aligned}
& C_{1}\left[Q_{1}^{1}-\left(P_{1, k-1}+\tilde{P}_{1}\right) Q_{2}^{1}+Q_{3}^{1}+Q_{4}^{1}\right] C_{1}^{T}\left(\hat{D}_{1}-\tilde{D}_{1}\right)+C_{1}\left[\lambda_{1} F_{1}\left(z_{1}\right)+\mu_{1} F_{1}^{\prime}\left(z_{1}\right)\right] \\
& -C_{1} Q_{2}^{1}\left\{C_{1}^{T}\left(D_{1, k-1}+\tilde{D}_{1}\right)+\Delta_{1, k}\right\}\left(\hat{P}_{1}-\tilde{P}_{1}\right) \\
& =C_{1} \int_{0}^{z_{1}} F_{l}^{\prime \prime}(z) M_{n, k} d z+\left(P_{1, k-1}+\widetilde{P}_{1}\right) C_{1} Q_{2}^{1}\left\{C_{1}^{T}\left(D_{1, k-1}+\tilde{D}_{1}\right)+\triangle_{1, k}\right\} \\
& +\left[L_{0}-S_{0, K}\right] C_{1} F_{1}(0)+\left[L_{1}-S_{1, K}\right] C_{1} I_{1}\left(z_{1}\right) \\
& +\left[M_{0}-T_{0, k}\right] C_{1} F_{1}^{\prime}(0)-\left[M_{1}+T_{1, k}\right] C_{1} F_{1}^{\prime}\left(z_{1}\right)
\end{aligned}
$$

for span 1,

$$
\begin{aligned}
C_{n}\left[Q_{1}^{n}\right. & \left.-\left(P_{n, k-1}+\tilde{P}_{n}\right) Q_{2}^{n} P Q_{3}^{n}\right] C_{n}^{T}\left(\hat{D}_{n}-\tilde{D}_{n}\right) \\
& +C_{n}\left[-\lambda_{n-1} F_{n}\left(z_{n-1}\right)-\mu_{n-1} F_{n}^{\prime}\left(z_{n-1}\right)+\lambda_{n} F_{n}\left(z_{n}\right)+\mu_{n} F_{n}^{\prime}\left(z_{n}\right)\right] \\
& -C_{n} Q_{2}^{n}\left\{C_{n}^{T}\left(D_{n, k-1}+\tilde{D}_{n}\right)+\Delta_{n, k}\right\}\left(\hat{P}_{n}-\tilde{P}_{n}\right) \\
& =C_{n} \int_{z}^{z}\left\{F_{n}^{\prime \prime}(z) M_{n, k}\right\} d
\end{aligned}
$$




$$
\begin{aligned}
& +\left(P_{n, k-1}+\tilde{P}_{n}\right) C_{n} Q_{2}^{n}\left\{C_{n}^{T}\left(D_{n, k-1}+\tilde{D}_{n}\right)+\Delta_{n, k}\right\} \\
& +\left[L_{n}-S_{n, k}\right] C_{n} F_{n}\left(z_{n}\right)-\left[M_{n}+T_{n, k}\right] C_{n} F_{n}^{\prime}\left(z_{n}\right)
\end{aligned}
$$

for spans $n=2,3, \ldots, N$ and

$$
\begin{aligned}
F_{n}\left(z_{n}\right)^{T} C_{n}^{T}\left(\hat{D}_{n}-\tilde{D}_{n}\right)-F_{n+1}\left(z_{n}\right)^{T} C_{n+1}^{T}\left(\hat{D}_{n+1}-\tilde{D}_{n+1}\right) \\
=F_{n+1}\left(z_{n}\right)^{T}\left\{C_{n+1}^{T}\left(D_{n+1, k-1}+\tilde{D}_{n+1}\right)+\Delta_{n+1, k}\right\} \\
-F_{n}\left(z_{n}\right)^{T}\left\{C_{n}^{T}\left(D_{n, k-1}+\tilde{D}_{n}\right)+\Delta_{n, k}\right\}
\end{aligned}
$$

and

$$
\begin{aligned}
F_{n}^{\prime}\left(z_{n}\right) & T C_{n}^{T}\left(\hat{D}_{n}-\tilde{D}_{n}\right)-F_{n+1}^{\prime}\left(z_{n}\right)^{T} C_{n+1}^{T}\left(\hat{D}_{n+1}-\tilde{D}_{n+1}\right) \\
= & F_{n+1}^{\prime}\left(z_{n}\right)^{T}\left\{C_{n+1}^{T}\left(D_{n+1, k-1}+\tilde{D}_{n+1}\right)+\Delta_{n+1, k}\right\} \\
& -F_{n}^{\prime}\left(z_{n}-z_{n-1}\right)^{T}\left\{C_{n}^{T}\left(D_{n, k-1}+\tilde{D}_{n}\right)+\Delta_{n, k}\right\}
\end{aligned}
$$

for spans $n=1,2, \ldots, \mathbb{N}-1$.

Thus we have a system of equations relating the transverse deflections and the axial thrust values to the known parameters of the problem.

The unique feature in the ROBOT program is the method used to compute rod slippage at the supports. It is not enough to consider only the maximum axial thrust which will be imposed on a particular span of the beam. Rather, the thrust and displacement must be considered as inter-dependent in order to accurately determine the effects of rod slippage upon the deflected shape of a fuel rod. For example, consider a particular span of a fuel rod in an operating reactor. Thermal expansion tends to increase the length of the span. Since the supports are more or less rigid, some of the force generated by this length increase is absorbed by transverse rod deflections. However, at some time in the history of the rod, the generated thrust may exceed the frictional resistance and the rod slips past the support, thus relieving the force in the span and thereby reducing the rod's transverse deflection. Therefore, rod deflections and axial thrust are intimately connected and must be treated as such for a correct computation of slippage at the supports.

The slippage problem may be described as follows: At a particular time in the history of the reactor, depending upon the thrusts and deflections in each span, a fuel rod may be slipping to the left at some supports, to the 
right at other supports, and at some supports, the rod will not be slipping at all. [In this discussion "left" slippage means the rod is slipping through the support in the direction of decreasing $z$, "right" slippage is the direction of increasing $\mathrm{z} \cdot]$ Thus, at each support $\mathrm{m}$, a slippage value $\mathrm{s}_{\mathrm{m}}$ representing left, right, or no slippage is to be assigned. The set of values $s_{m}$ forms a vector $S$, which we shall call the "slip vector". Physical reasoning suggests that there is a unique configuration for this vector and it is this configuration which must be determined. One approach to solving this problem involves a systematic investigation of all possible configurations. By using equilibrium of forces each incorrect configuration could be eliminated until the true solution was determined. Unfortunately, however, a typical fuel rod may have up to 10 support points. This leads to a total of $3^{10}$ possible configurations for the slip vector. The method employed in the ROBOT program eliminates the need to consider each of the $3^{10}$ possible configurations. It has been proven to be very efficient.

Basically, the ROBOT method of computation of rod slippage at the supports is an iteration. At each new time step, an initial set of values $s_{m}$ is assumed for each support. Let $s_{m}=-1$ to denote left slippage, +1 to denote right slippage and 0 to denote no slippage. At time $t=0$, the guessed values for $s_{m}$ are all zero and at time $t_{k}$, the guessed values for $s_{m}$ are the converged values from $t_{k-1}$. Based on these assumed values, a system of equations is constructed relating the support slippage to the axial forces in each span.

These equations involve calculation of the length of the chord between supports $n$ and $n-1$. Letting $\bar{l}_{n, k}$ be the arc length of span $n$ at time $t_{k}$, the chord length is then given by

$$
\Lambda_{n}(t)=\bar{l}_{n, k}-\frac{1}{2} \int_{z_{n-1}}^{z_{n}} u_{z}^{2}(z, t) d z .
$$

Now when equation (2) is substituted for $u(z, t)$, and the moment curvature relationship applied, $\Lambda_{n}(t)$ becomes

$$
\Lambda_{n}(t)=\bar{l}_{n, k}-\frac{1}{2}\left\{D_{n}^{T}(t) C_{n}+\Delta_{n}^{T}(t)\right\} Q_{2}^{n}\left\{C_{n}^{T} D_{n}(t)+\Delta_{n}(t)\right\}
$$

The term $\bar{l}_{n, k}$ can be divided in two parts, the first of which contains effects of thermal expansion and other axial growth properties and is denoted by $\ell_{n, k}$. The second term of $\bar{l}_{n, k}$ can be expressed as $P_{n}$ k, $l_{p, k}$ where $P_{n}$ is the thrust in span $n$ and $l_{p, n, k}=\frac{\partial l}{\partial P}$. Thus equation (3) becomes 


$$
\Lambda_{n}(t)=l_{n, k}+P_{n} l_{p, n, k}-\frac{l}{2}\left\{D_{n}^{T}(t) C_{n}+\Delta_{n}^{T}(t)\right\} O_{2}^{N}\left\{c_{n}^{T} D_{n}(t)+\Delta_{n}(t)\right\} .
$$

Equations for $\vec{l}_{\mathrm{n}, \mathrm{k}}$ are given in Appendix $\mathrm{A}$ of reference 2 .

Turning now to the equations relating support slippege to axial thrust and transverse displacements in span $n$, we have four possible cases.

CASE 1. Supports $\mathrm{n}$ and $\mathrm{n}-\mathrm{I}$ not slipping (Figure $\varepsilon$ ). Here the chord length is found to be the original span length plus the net motions of the two end supports. Thus

$$
\begin{aligned}
\Lambda_{n}(t)=z_{n}-z_{n-1} & +\left[\frac{P_{n}-P_{n+1}+z_{n}}{K_{n}}-\frac{P_{n-1}-P_{n}+z_{n-1}}{K_{n-1}}\right] \\
& +w_{n}-W_{n-1} \cdot
\end{aligned}
$$

Substituting equation (4) for $\Lambda_{n}(t)$ and replacing $P_{m}$ by $\left(P_{m, k-1}+\tilde{P}_{n}\right)$ $+\left(\hat{P}_{n}-\widetilde{P}_{n}\right)$ and $D_{m}$ by $\left(D_{m, k-1}+\widetilde{D}_{m}\right)+\left(\hat{D}_{m}-\tilde{D}_{m}\right)$ we obtain

$$
\begin{aligned}
{\left[-\frac{1}{K_{n-1}}\right]\left(\hat{P}_{n-1}-\tilde{P}_{n-1}\right)+\left[\frac{1}{K_{n-1}}+\frac{1}{K_{n}}-l_{p, n, k}\right]\left(P_{n}-\tilde{P}_{n}\right)-\left[\frac{1}{K_{n}}\right]\left(\hat{P}_{n+1}-\tilde{P}_{n+1}\right) } \\
+\left[\left\{\left(D_{n, k-1}^{T}+\tilde{D}_{n}\right) C_{n}+\Delta_{n, k}^{T}\right\} Q_{2}^{n}\right] c_{n}^{T}\left(\hat{D}_{n}-\tilde{D}_{n}\right) \\
=l_{n, k}-z_{n}+z_{n-1}-\left[\frac{\left.\left(P_{n, k-1}+\tilde{P}_{n}\right)-\left(P_{n+1, k-1}+\tilde{P}_{n+1}\right)+z_{m}\right]}{K_{n}}\right] \\
+\left[\frac{\left(P_{n-1, k-1}+\tilde{P}_{n-1}\right)-\left(r_{n, k-1}+\tilde{P}_{n}\right)+z_{n-1}}{K_{n-1}}\right]-w_{n}+w_{n-1} \\
-\frac{1}{2}\left\{\left(D_{n, k-1}^{T}+\tilde{D}_{n}^{T}\right) c_{n}+\Delta_{n, k}^{T}\right\} \theta_{2}^{n}\left\{C_{n}^{T}\left(D_{n, k-1}+\tilde{D}_{n}\right)+\Delta_{n, k}\right\}
\end{aligned}
$$

CASE 2. Support $\mathrm{n}$ not slipping, support $\mathrm{n}-1$ slipping and a non-slipping support $m$ to the left of $n$ (Figure 3). Here the chord length is to be computed between supports $m$ and $n$ in the following manner: 


\section{CASE: I. SLIP EQUATION}

CASE I: SUPPORTS $n-1, n$ NOT SLIPPING

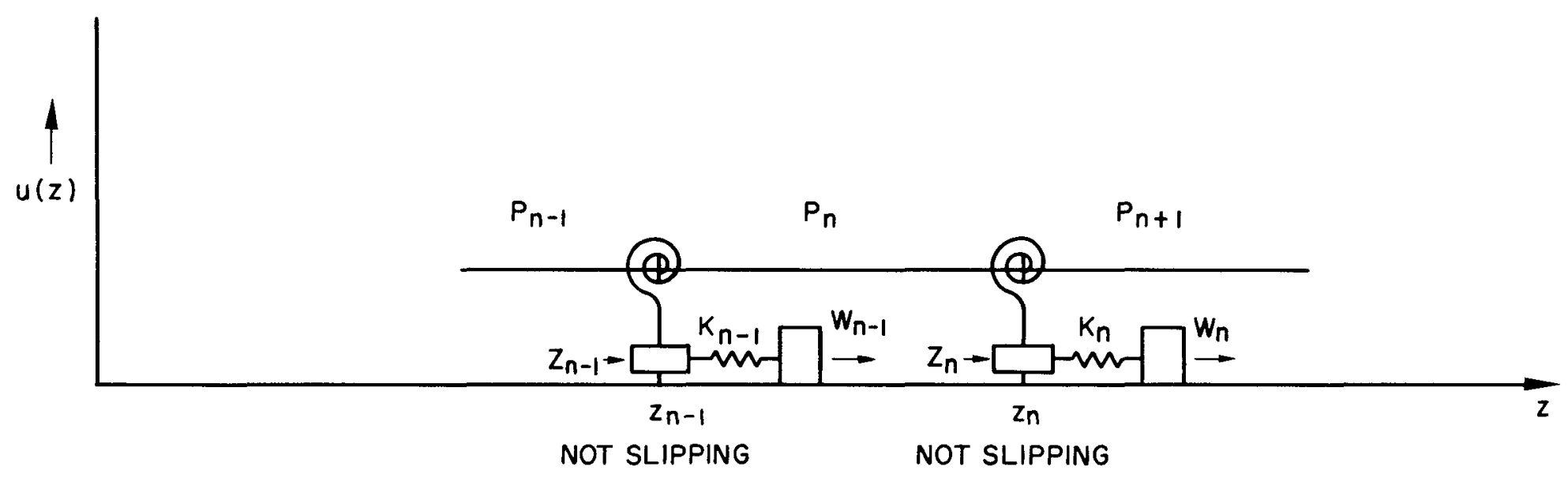

$\Lambda_{n}(\dagger)=z_{n}-z_{n-1}+\left[\frac{P_{n}-P_{n+1}+z_{n}}{K_{n}}-\frac{P_{n-1}-P_{n}+z_{n-1}}{K_{n-1}}\right]+w_{n}-w_{n-1}$

$\Lambda_{n}(t)=$ CHORD LENGTH OF SPAN $n$ AT TIME $\dagger$

FIGURE 2 


\section{CASE: 2 SLIP EQUATION}

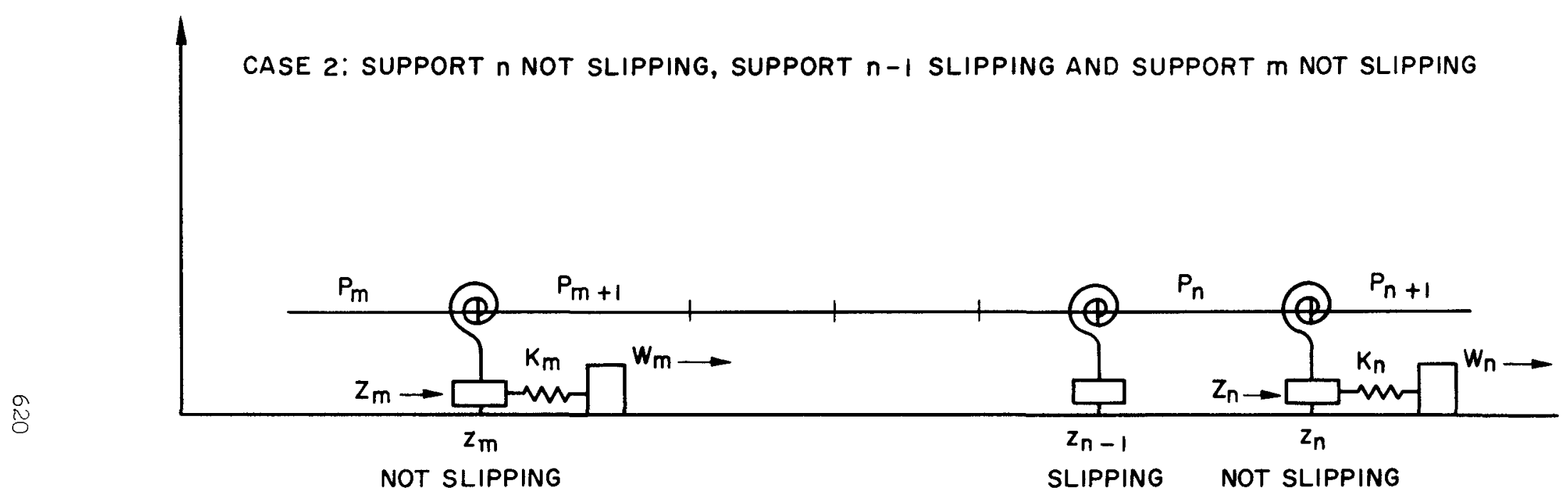

$\Lambda=\sum_{i=m}^{n} \Lambda_{i}(t)=z_{n}-z_{m}+w_{n}-w_{m}-\left[\frac{P_{m}-P_{m+1}+z_{m}}{K_{m}}\right]+\left[\frac{P_{n}-P_{n+1}+z_{n}}{K_{n}}\right]$

$\Lambda_{i}(t)=$ CHORD LENGTH OF SPAN $i$ 


$$
\begin{aligned}
\Lambda(t) & =\sum_{i=m}^{n} \Lambda_{i}(t) \\
& =z_{n}-z_{m}+w_{n}-W_{m} \\
& -\left[\frac{P_{m}-P_{m+1}+z_{m}}{K_{m}}\right]+\left[\frac{P_{n}-P_{n+1}+z_{n}}{K_{n}}\right] .
\end{aligned}
$$

Upon making the same substitutions as in Case 1, we obtain

$$
\begin{aligned}
& {\left[\frac{1}{K_{m}}-\ell_{p, m, k}\right]^{?}\left(\hat{P}_{m}-\tilde{P}_{m}\right)-l_{p, m+1, k}\left(\hat{P}_{m+1}-\tilde{P}_{m+1}\right)-\cdots} \\
& -\ell_{p, n-I k}\left(\hat{P}_{n-1}-\tilde{P}_{n-1}\right)-\left[\ell_{p, n, k}-\frac{I}{K_{n}}\right]\left(\hat{P}_{n}-\tilde{P}_{n}\right) \\
& +\left\{\left[\left(D_{m+1, k-1}^{T}+\tilde{D}_{m+1}^{T}\right) C_{m+1}+\Delta_{m+1, k}^{T}\right] Q_{2}^{m+1} C_{m+1}^{T}\right\}\left(\hat{D}_{m+1}-\tilde{D}_{m+1}\right) \\
& +\cdots+\left\{\left[\left(D_{n, k-1}^{T}+\widetilde{D}_{n}^{T}\right) C_{n}+\Delta_{n, k}^{T}\right] Q_{2}^{n} C_{n}^{T}\right\}\left(\hat{D}_{n}-\tilde{D}_{n}\right) \\
& =-z_{n}+z_{m}-W_{n}+w_{m}+l_{m+1, k}+\cdots+l_{n, k} \\
& -\frac{1}{2}\left\{\left[\left(D_{m+1, k-1}^{T}+\tilde{D}_{m+1}^{T}\right) C_{m+1}+\Delta_{m+1, k}^{T}\right] Q_{2}^{m+1}\left[C_{m+1}^{T}\left(D_{m+1, k-1}+\tilde{D}_{m+1}\right)+\Delta_{m+1, k}\right]\right. \\
& \left.+\cdots+\left[\left(D_{n, k-1}^{T}+\widetilde{D}_{n}^{T}\right) C_{n}+\Delta_{n, k}^{T}\right] Q_{2}^{n}\left[C_{n}^{T}\left(D_{n, k-1}+\tilde{D}_{n}\right)+\Delta_{n, k}\right]\right\} \\
& +\left[\frac{\left(P_{m, k-1}+\tilde{P}_{m}\right)-\left(P_{m+1, k-1}+\tilde{P}_{m+1}\right)-z_{m}}{K_{m}}\right]-\left[\frac{\left(P_{n, k-1}+\widetilde{P}_{n}\right)-\left(P_{n+1, k-1}+\tilde{P}_{n+1}\right)-z_{n}}{K_{n}}\right] \cdot(5 b)
\end{aligned}
$$

CASE 3. Support $n$ not slipping, all supports to the left of $n$ slipping (Fig.4). Here the equations must satisfy equilibrium of forces in the z-direction at support $\mathrm{n}-1$. Thus,

$$
P_{n}-P_{n-1}-z_{n-1}= \pm \bar{z}_{n-1}
$$

where the sign of $\bar{z}$ is chosen so that $\bar{z}$ acts in the opposite directjon of 


\section{CASE: 3 SLIP EQUATION}

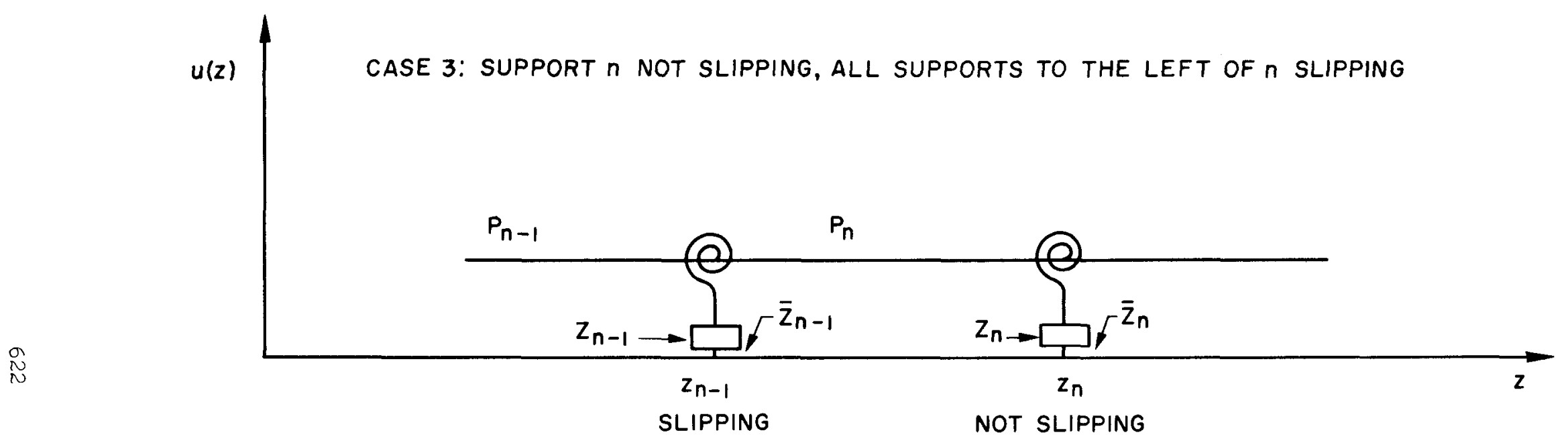

$$
P_{n}-P_{n-1}-z_{n-1}= \pm \bar{z}_{n-1}
$$

FIGURE 4 
slippage. For example, if $\left(P_{n}-P_{n-1}-z_{n-1}\right) \equiv 0$ then the rod will be slipping to the left and $\bar{Z}_{n-1}$ will be positive. Replacing $P_{m}$ by $\left(P_{m, k-I}+\widetilde{P}_{m}\right)+\left(P_{m}-\widetilde{P}_{m}\right)$ we obtain

$$
\begin{aligned}
\left(\hat{P}_{n}-\tilde{P}_{n}\right) & -\left(\hat{P}_{n-1}-\tilde{P}_{n-1}\right)=z_{n-1} \pm \bar{z}_{n-1} \\
& +\left(P_{n-1, k-1}+\widetilde{P}_{n-1}\right)-\left(P_{n, k-1}+\widetilde{P}_{n}\right) .
\end{aligned}
$$

CASE 4. Support $\mathrm{n}$ slipping (Figure 5). Here again equilibrium of z-direction forces must be satisfied. Let the variable $k_{z}=0$ if there is a non-slipping support to the left of $n, k_{z}=I$ if all supports to the left are slipping. Then the relation

$$
P_{n-k_{z}+1}-P_{n-k_{z}}=z_{n-k_{z}} \pm \bar{z}_{n-k_{z}}
$$

must hold. This implies that

$$
\begin{aligned}
&\left(\hat{P}_{n-k_{z}}-\right.\left.\tilde{P}_{n-k_{z}}\right)-\left(\hat{P}_{n-k_{z}+1}-\tilde{P}_{n-k_{z}+1}\right) \\
&=\left(P_{n-k_{z}}+1, k-1+\widetilde{P}_{n-k_{z}+1}\right)-\left(P_{n-k_{z}}, k-1\right. \\
&
\end{aligned}
$$

The equations (5), together with (24), are then solved for the deflections and thrusts. In the overall ROBOT iteration scheme, the slip iteration is the "outer" iteration while the Newton-Raphson iteration forms the "inner" loop. Therefore, for a particular assumed slip vector $S$, the Newton-Raphson solution for the thrust in each span is used to determine whether or not the original assumed $s_{m}$ values are valid. With the converged values for thrust in each span, together with the known friction forces $\bar{z}_{m}$ at each support, a new vector $S^{\prime}$ consisting of values $s_{m}^{\prime}$ is constructed.

The calculation of $s_{m}^{\prime}$ from $S$ and the values of thrust and $\bar{Z}_{m}$ is rather subtle. Remembering that a value of 0 for $s_{m}$ signifies no slip at support $m$, values of $-I$ and +1 represent left and right slippage respectively, we shall use the same notation for the values $s_{m}^{\prime}$. The computation of $s_{m}^{\prime}$ at support $m$ falls into two cases (see Figure 6).

CASE 1. Support $m$ assumed not slipping, that is $s_{m}=0$. Here we must insure that the converged thrusts $P_{m}=\left(P_{m, k-1}+\widetilde{P}_{m}\right)$ and $P_{m+1}=\left(P_{m+l, k-1}+\widetilde{P}_{m+l}\right)$ together with imposed axial force $Z_{m}$ satisfy the relation $\left|P_{m}+Z_{m}-P_{m+l}\right| \leq \bar{Z}_{m}$ where $\bar{z}_{m}$ is the friction force at support $m$. If this is true, set $s_{m}^{\prime}=0$ to 


\section{CASE: 4 SLIP EQUATION}

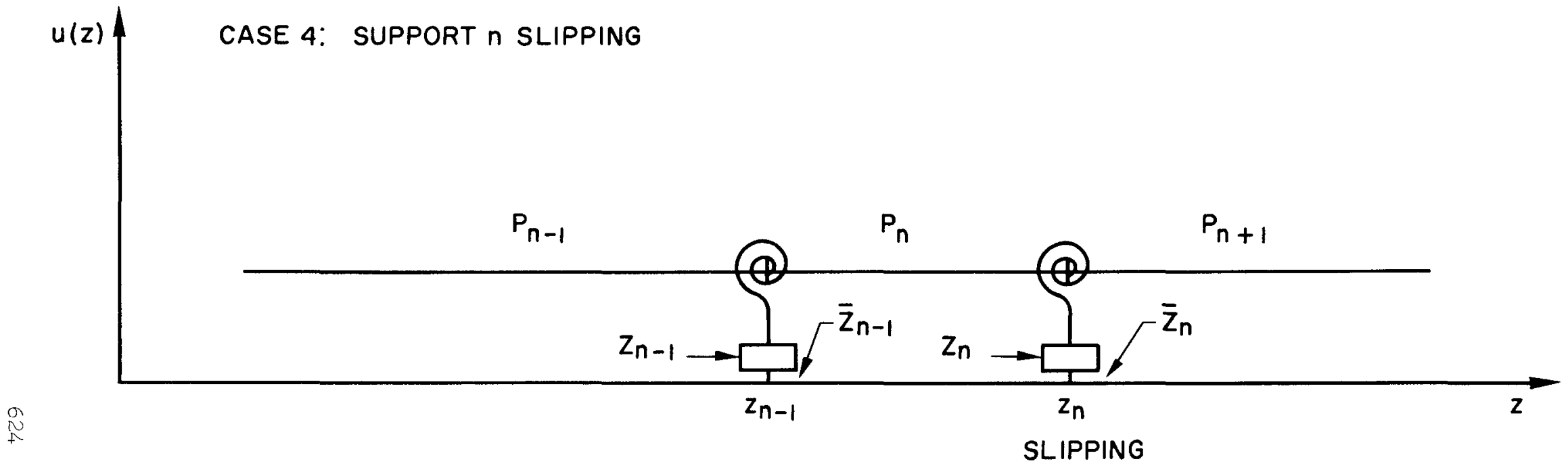

LET $k_{z}=0$ IF THERE IS A NON-SLIPPING SUPPORT TO THE LEFT OF $n, k_{z}=$ I IF ALL SUPPORTS TO THE LEFT ARE SLIPPING. THEN

$$
P_{n-k_{z+1}}-P_{n-k_{z}}=z_{n-k_{z}} \pm \bar{z}_{n-k_{z}}
$$


CALCULATION OF JSLIP $(m)$ FROM ISLIP $(m)$ AND $P_{m}=P_{m} k-1+\tilde{P}_{m}$

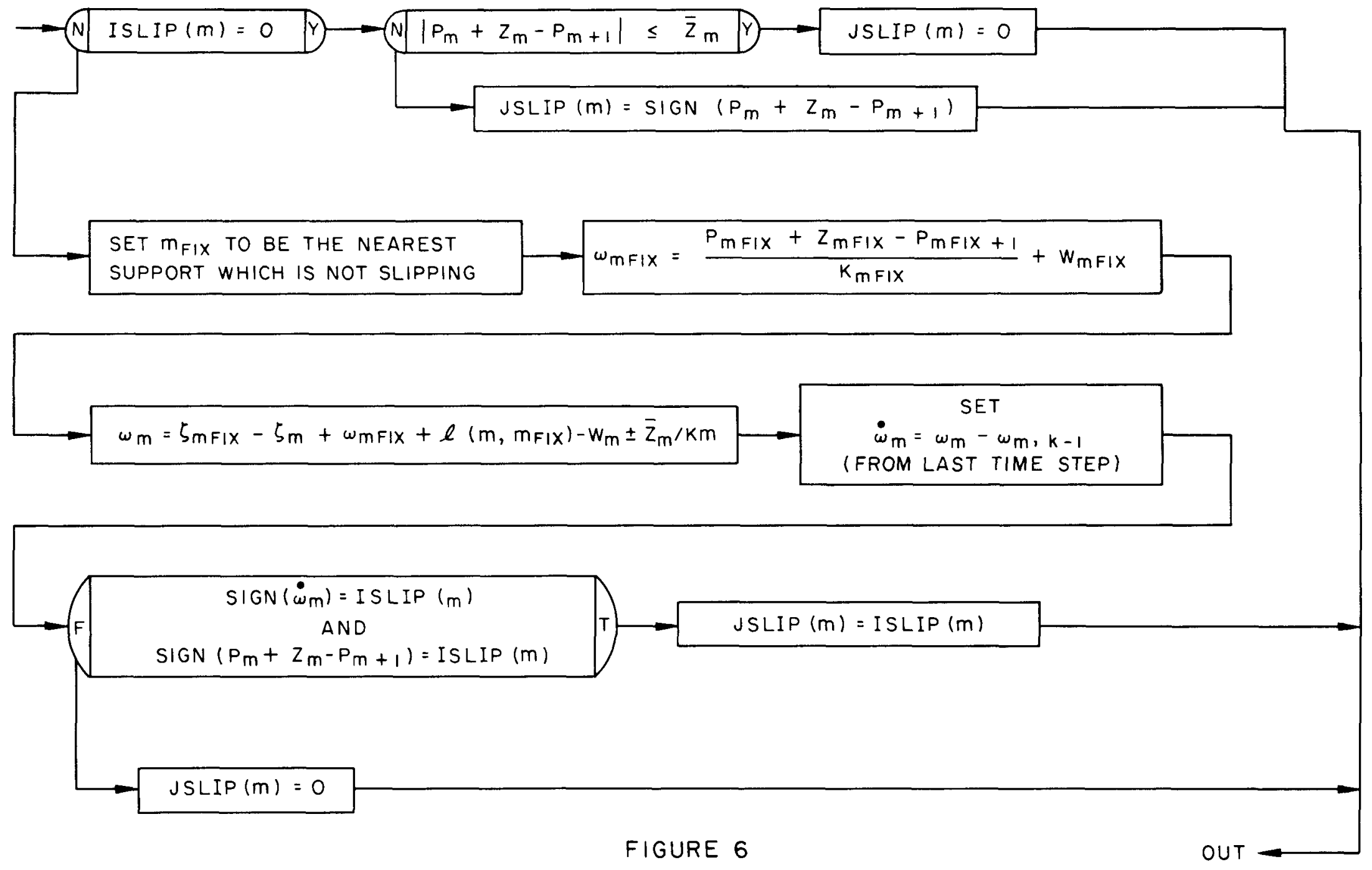


denote no slippage at support $m$. Otherwise, set $s_{m}^{\prime}=\operatorname{sign}\left(P_{m}+z_{m}-P_{m+l}\right)$ to represent either left or right slippage.

CASE 2. Support $m$ assumed to be slipping, that is $s_{m} \neq 0$. In this case the net motion of the support must comply with the value of $\mathrm{s}_{\mathrm{m}}$. For example, if $s_{m}=-l$, the net motion $w_{m}$ of the rod at support $m$ at time $t_{k}$ must be less than the net motion at time $t_{k-1}$. To compute $w_{m}$, we first find the support nearest to $m$ which is not slipping. Denote this support by $m_{f i x}$. Then compute $w_{\text {mfix }}$, the motion of the rod at support $m_{f i x}$ according to the equation

$$
w_{\text {mfix }}=\left[\frac{P_{m f i x}+Z_{m f i x}-P_{m f i x+1}}{K_{m f i x}}\right]+w_{m f i x} .
$$

From this relation we obtain $w_{m}$, the motion of the rod at support $m$ as follows: letting $m_{0}=\min \left(m_{f i x}, m\right), m_{I}=\max \left(m_{f i x}, m\right)$ we obtain

$$
w_{m}=\sum_{m_{0}}^{m_{l}} \bar{l}_{i k}-z_{m_{l}}+z_{m_{0}}+w_{m f i x}-w_{m} \pm \bar{z}_{m} / K_{m}
$$

where the sign of $\bar{z}_{m}$ is opposite to the direction of slippage, i.e., $\operatorname{sign}\left(\bar{z}_{m}\right)$ $=\operatorname{sign}\left(P_{m+1}-P_{m}-Z_{m}\right)$. Now we calculate $\Delta w_{m}$, the change in net motion of the rod at support $m$ from the equation

$$
\Delta \mathrm{w}_{\mathrm{m}}=\mathrm{w}_{\mathrm{m}}-\mathrm{w}_{\mathrm{m}, \mathrm{k}-1}
$$

where $w_{m, k-1}$ is the motion at time $t_{k-1}$. Finally, if the sign of $\Delta w_{m}$ equals $s_{m}$, we set $s_{m}^{\prime}=s_{m}$. If $\Delta w_{m}$ is zero or is opposite to the sign of $s_{m}$, we set $s_{m}^{\prime}=0$. This completes the discussion of the calculation of $s_{m}^{\prime}$, the "improved" guess for the slip vector.

Once the values $s_{m}^{\prime}$ are computed, the slip iteration is assumed to have converged if values $s_{m}^{\prime}$ equal $s_{m}$ for all supports $m$. That is to say, if the assumed values for slippage at the supports lead to values of axial thrust which are consistent with the assumptions, the process has converged and we have found the desired configuration for the slip vector. If this is not the case, that is if $s_{m}^{\prime} \neq s_{m}$ at some support $m$, we use the newly computed values of $s_{m}^{\prime}$ for an improved guess for the slip vector and repeat the process until it does converge.

While support slippage greatly affects the calculation of the axial thrust in each span, it must also be accounted for in the motion imposed on 
the rod. For instance, if $W_{m}$ is the imposed motion of a support which is slipping, it must be adjusted to reflect the total slipped distance if the support stops slipping. In this case, a value $W_{r_{m}}$ called the "reduced" motion of support $m$ is calculated from $w_{m}$ described above. All references to $w_{m}$ in this paper should add the term $W_{r_{m}}$. That is

$$
W_{m}=W_{m}(t) \text { as supplied by the user }+W_{r_{m}}
$$

where $W_{r_{m}}=W_{m}$ from equation (6).

With the equations $(24 a)-(24 d)$ and $(5 a)-(5 d)$ available, we are ready to discuss the overall ROBOT iteration for computing the history of deflections of a fuel rod. Figure 7 is a schematic flow diagram of the iteration.

(1) Set $K T=0$, that is, begin computations at time $=t_{0}$.

(2) Initialize all variables. In particular, set $D_{O_{n}}, P_{\text {on }}=0$ for all spans $n$.

(3) $\mathrm{KT}=\mathrm{KT}+1$, we wish to calculate the deflections and thrusts at time $t_{k}=t_{K T} \cdot$

(4) For each ROBOI support $m$, compute $I_{m}, M_{m}, Z_{m}, \bar{Z}_{m}$, and $W_{m}$. For each span n compute $P_{r_{n}}=$ pressure, $T_{n}=$ temperature, and $\phi_{n}=$ integrated neutron flux. These values are computed from user supplied tables of values versus time.

(5) Begin slip iteration at time $t_{k}$. Set slippage iteration counter KSLIP $=0$.

(6) For each support $m$, guess a slippage value $s_{m}$ for the rod at support $m$. The values $s_{m}$ then form a vector $s$, whose entries will be $0,-1$, or +1 to denote no slippage, slippage to the left, or slippage to the right.

(7) Set KSLIP $=$ KSLIP+1.

(8) Begin Newton-Raphson iteration for deflections $D_{n, k}$ and thrusts $P_{n, k}$. Set KNR $=0$.

(9) Guess values for $\tilde{\mathrm{D}}_{\mathrm{n}}, \tilde{\mathrm{P}}_{\mathrm{n}}$.

(10) Set $\mathrm{KNR}=\mathrm{KNR}+\mathrm{l}$

(11) From the current values of $P_{r_{n}}, T_{n}, \phi_{n}$, together with thrust $\left(P_{n, k-1}+\widetilde{P}_{n}\right)$ compute bending stiffness, axial expansion parameters $\bar{l}_{n, k} l_{p, n, k}$ for each span.

(12) Construct equations $(24 a)-(24 d),(5 a)-(5 b)$ for all spans. This leads to a matrix equation $R x=b$ where $x$ is an unknown vector of the form $(\hat{x}-\tilde{x})$. 
OVERALL FLOW CHART

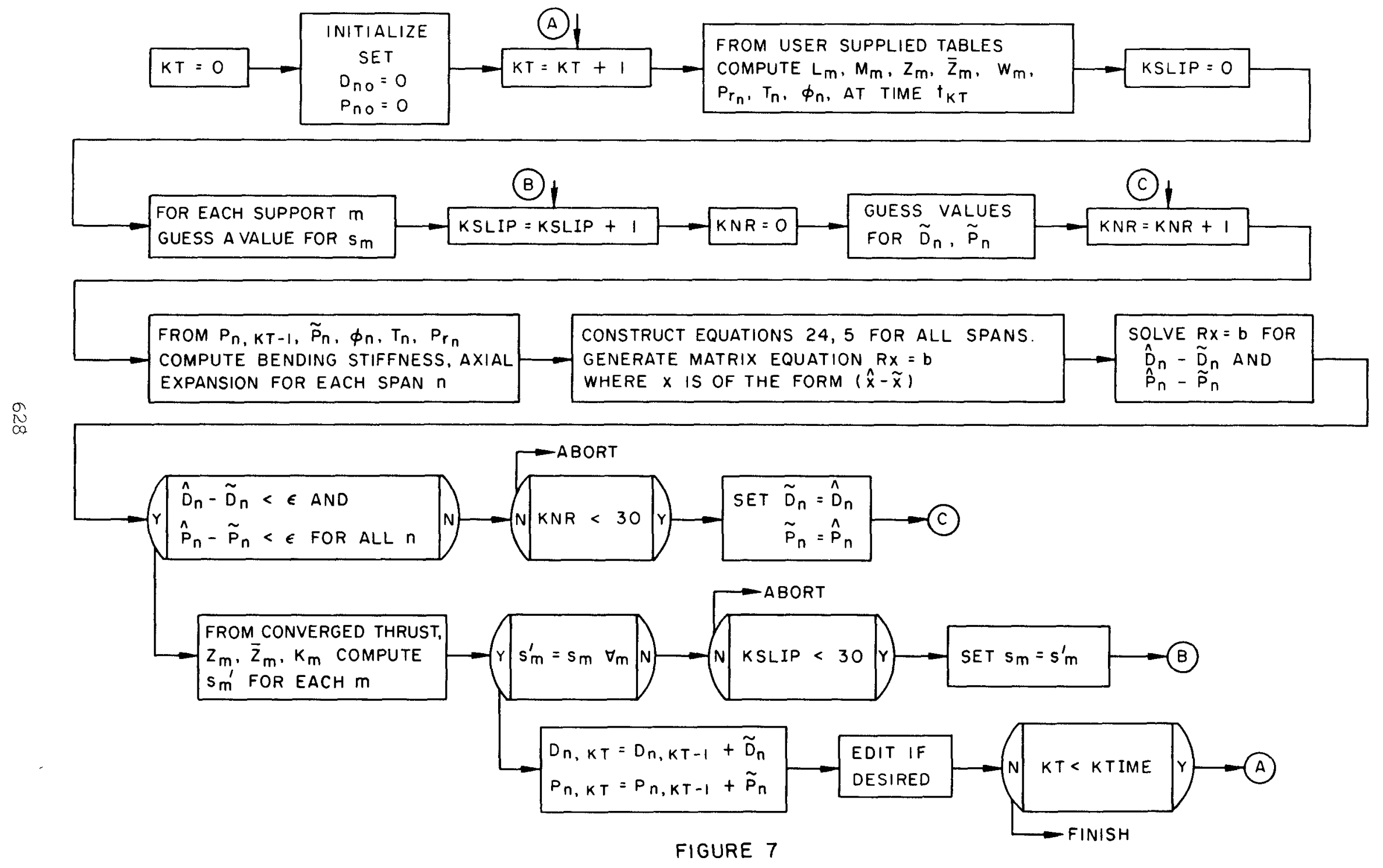


(13) Solve the matrix equation $R x=b$ by Gaussian elimination for $\left(\hat{D}_{n}-\tilde{D}_{n}\right)$, $\left(\hat{\mathrm{P}}_{\mathrm{n}}-\widetilde{\mathrm{P}}_{\mathrm{n}}\right)$.

(14) If $\left(\hat{D}_{n}-\tilde{D}_{n}\right)<\epsilon$ and $\left(\hat{P}_{n}-\widetilde{P}_{n}\right)<\epsilon$ for all spans $n$, proceed to step 16 .

(15) If KNR $<30$ set $\widetilde{D}_{n}=\widehat{D}_{n}, \widetilde{P}_{n}=\hat{P}_{n}$ and go to step 10 . If KNR $\geq 30$ assume no convergence and abort.

(16) Using the converged values for thrust, $\mathrm{P}_{n, k-1}+\widetilde{\mathrm{P}}_{n}$, together with $z_{m}$, $\bar{Z}_{m}$ and $K_{m}$ compute a slippage value $s_{m}^{\prime}$ for each support $m$.

(17) If $s_{m}^{\prime}=s_{m}$ for all $\mathrm{m}$, the iteration is assumed to have converged at time step KT. In this case, proceed to step 19.

(18) If KSLIP $<30$ set $s_{m}=s_{m}^{\prime}$ and go to step 7 . If KSLIP $\geq 30$, assume no convergence on slip vector and abort.

(19) Set $D_{n, k}=\left(D_{n, k-1}+\widetilde{D}_{n}\right), P_{n, k}=\left(P_{n, k-1}+\widetilde{P}_{n}\right)$. Edit the deflections and thrusts if desired and either proceed to step 3 for the next time step or finish the problem.

As mentioned previously, the slip iteration has been proven to be quite efficient. A 10-span sample problem has been run testing the slip convergence algorithm. In this problem, the friction forces $\bar{z}_{m}$ at all supports except support 0 , were set equal to 2.1 pounds. The friction force at support 0 was set to $10^{40}$ pounds to simulate a non-frictional support. In all spans a value of axial expansion was given as an increasing function of time. The problem was run from time $=0$ to time $=16,000$ hours with a $\Delta t$ of 100 hours. The maximum number of slip iterations required at any given time step was 4 and in most cases the procedure converged in 1 slip iteration.

Summarizing this discussion we see that ROBOT is a computer program including the major known mechanisms affecting fuel rod bowing. Effects of rod curvature, externally applied forces and moments, internally generated thrusts, thermal expansion, thermal gradient curvature, fuel-clad interaction as well as slippage at the supports are included. For an in-depth analysis of these mechanisms, one should consult references 1 and 2. The program is written in FORTRAN IV with a few auxiliary CDC-6600 machine language subroutines. All storage allocation is dynamic, and extensive use is made of the routines described in Appendices $A$ and $B$ of reference 3 . 


\section{REFERENCES}

1. J. B. Newman, Elastic Analysis of Thermal Gradient Bowing in Rod-Type Fuel Elements Subjected to Axial Thrust, WAPD-TM-726 (January 1968).

2. J. B. Newman, Inelastic Bowing of Multispan Fuel Rods Subjected to Axial Thrust and Temperature Gradients, WAPD-TM-770 (september 1968).

3. C. J. Pfeifer, CDC-6600 FORTRAN Programming - Bettis Environmental Report, WAPD-TM-668 (January 1967) • 
SESSION VIII

MONTE CARLO

Chairman: F. H. Clark

Oak Ridge National Laboratory 


\section{BLANK}




\title{
A NEW NON-MULTIGROUP ADJOINT MONTE CARLO TECHNIQUE*
}

\author{
by
}

Leo B. Levitt, Atomics International Division of North American Rockwell Corporation

and

Jerome Spanier, Science Center/North American Rockwell Corporation

\section{INTRODUCTION}

A problem of frequent occurrence in nuclear reactor design is the estimation of the probability of an event of low frequency. For example, in the study of radiation shield properties, interest focuses on the occasional particle which succeeds in penetrating the shield. The practical use of Monte Carlo techniques to solve such problems often depends on the application of one form or another of importance sampling. $(1,2,3,4,5)$ However, there is sometimes an alternative to the use of importance sampling as a means of reducing the statistical uncertainty in the estimate of the answer. This alternative consists in transforming the original problem into one in which the event of interest occurs with relatively higher frequency than in the original problem. (One may still, of course, use sophisticated sampling techniques in solving the auxiliary problem, thereby achieving even greater gains in efficiency.)

One way in which such an auxiliary problem arises is through the solution of an adjoint transport equation. $(1,6,7,8,9,10,11)$ The reciprocity relationship shows how "source" to "detector" histories in the original problem may be replaced by "detector" to "source" histories of an adjoint simulation in the estimation of key design parameters. Then in cases where the detector region is small, perhaps even a point, the adjoint simulation might be expected to yield more efficient estimates provided that the source region is "larger" than the detector region. The work "larger" is here used in the sense of probability. The present paper describes a new adjoint technique which appears to be very useful for solving problems in the slowing-down region.

In a sense which will be made more precise later, this technique is closely related to a multigroup adjoint method used by Gelbard and Spanier( $8,9,11)$ to solve problems in the thermal energy range. As in the latter case a connection with dual importance sampling will be demonstrated.

We should point out that adjoint techniques are not the only ones available for transforming a difficult Monte Carlo problem into a more easily solved auxiliary problem. Spanier and Gelbard(11) used the superposition principle

\footnotetext{
*Research supported by USAEC under Contract AT(04-3)-701
} 
to transform certain problems involving the calculation of resonance integrals into one whose solutions were vastly more tractable. In fact, they were able to show infinite gains in efficiency in the limit of resonance escape probabilities near unity. Their method, however, is intended to be used only for fairly simple geometries, such as in calculations involving isolated fuel rods, and for only a portion of the slowing-down region covering the low-lying resolved resonances. Thus, while extremely effective for those problems for which it was designed, the superposition principle does not appear to allow easy generalization to a larger class of problems. The adjoint technique presented in this paper does not suffer from this disadvantage and so might find more general applicability to resonance escape calculations. Furthermore, the ideas of this paper are not really limited to resonance escape problems, although we use such problems to illustrate the method here.

\section{DESCRIPTION OF THE METHOD}

In this section we give both an intuitive and more formal description of the new technique. We begin with the integral equation for the density $\chi(\underline{x}, E, \underline{w})$ of particles emerging from a collision (or the source) ${ }^{*}$

$$
X(\underline{x}, E, \underline{\omega})=\iiint x\left(\underline{x}^{\prime}, E^{\prime}, \underline{\omega}^{\prime}\right) \mathrm{T}\left(\underline{x^{\prime}}, \underline{x} ; E^{\prime}, \underline{\omega}^{\prime}\right) C\left(E^{\prime}, \underline{\omega^{\prime}}, E, \underline{\omega} ; \underline{x}\right) \mathrm{d} x^{\prime} \mathrm{d} E^{\prime} \mathrm{d} \omega^{\prime}+Q(\underline{x}, E, \underline{\omega}) \ldots
$$

In Equation $1, Q(\underline{x}, \mathrm{E}, \underline{\omega})$ is the slowing-down source to the problem, $\mathrm{T}\left(\underline{x^{\prime}}, \underline{x} ; \mathrm{E}^{\prime}, \underline{\omega}^{\prime}\right)$ is the transport kernel

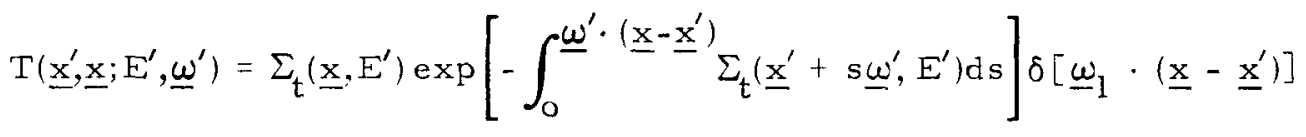

$$
\begin{aligned}
& \delta\left[\underline{\omega}_{2} \cdot\left(\underline{\mathrm{x}}-\underline{\mathrm{x}}^{\prime}\right)\right] \eta\left[\underline{\omega}^{\prime} \cdot\left(\underline{\mathrm{x}}-\underline{\mathrm{x}}^{\prime}\right)\right],
\end{aligned}
$$

where $\Sigma_{t}$ is the total macroscopic cross section, $\underline{\omega}^{\prime}, \underline{\omega}_{1}$, and $\underline{\omega}_{2}$ form an orthonormal triple of vectors and $\eta(t)=1$ if $t>0, \bar{\eta}(t)=0$ if $t \leq 0$, and $\mathrm{C}\left(\mathrm{E}^{\prime}, \underline{\omega}, \mathrm{E}, \underline{\omega} ; \underline{x}\right)$ is the collision kernel

$$
C\left(E^{\prime}, \underline{\omega^{\prime}}, E, \underline{\omega} ; \underline{x}\right)=\frac{\sum_{s}\left(\underline{x}, E^{\prime}\right)}{\sum_{t}\left(\underline{x}, E^{\prime}\right)} P\left(E^{\prime}, \underline{\omega}^{\prime}, E, \underline{\omega} ; \underline{x}\right),
$$

where $\mathrm{P}$ is a properly normalized probability density function for scattering from energy $E^{\prime}$, direction $\underline{\omega}^{\prime}$ into energy $E$ and direction $\underline{\omega}$ at $\underline{x}, \Sigma_{s}$ is the macroscopic scattering cross section. For scattering which is elastic, and isotropic in the CM system,

$$
\begin{aligned}
& P\left(E^{\prime}, \underline{\omega^{\prime}}, E, \underline{\omega} ; \underline{x}\right)= \frac{1}{E^{\prime}(1-\alpha)} \delta\left(\mu_{L}-\frac{A \mu_{c}+1}{\sqrt{A^{2}+2 A \mu_{c}+1}}\right) \\
& \text { for } \alpha E^{\prime} \leq E \leq E^{\prime}, \\
&= 0 \text { otherwise, }
\end{aligned}
$$

*See, for example, Appendix 2 of Reference 12 . 
where $\mu_{\mathrm{L}}=\underline{\omega} \cdot \underline{\omega}^{\prime}$ is the cosine of the scattering angle in the laboratory system, $\mu_{\mathrm{C}}=\left[2\left(\mathrm{E} / \mathrm{E}^{\prime}\right)-(1+\alpha)\right] /(1-\alpha)$ is the cosine of the scattering angle in the $\mathrm{CM}$ system, $\alpha=(A-1)^{2} /(A+1)^{2}$, and $A$ is the mass of the scattering nucleus at $x$. In Equation 4 and all subsequent references to $P$ a factor $1 / 2 \pi$, corresponding to a uniform choice of azimuthal angle, has been omitted for convenience. None of our conclusions is affected by this omission.

To calculate an absorption rate over a region $R$, one writes

$$
\begin{aligned}
I & -\iiint_{R} \frac{\sum_{a}(\underline{x}, E)}{\sum_{t}(\underline{x}, E)} \psi(\underline{x}, E, \underline{\omega}) \mathrm{d} \underline{x} \mathrm{~d} E \mathrm{~d} \underline{\omega} \\
& =\iiint\left[\int_{R} \frac{\sum_{a}(\underline{x}, E)}{\sum_{t}(\underline{x}, E)} X\left(\underline{x^{\prime}}, E, \underline{\omega}\right) \mathrm{T}\left(\underline{x^{\prime}}, x ; E, \underline{\omega}\right) \mathrm{d} \underline{x^{\prime}}\right] \mathrm{d} \underline{x} \mathrm{~d} E \mathrm{~d} \underline{\omega},
\end{aligned}
$$

where $\Sigma_{\mathrm{a}}$ is the macroscopic absorption cross section and $\psi(\underline{\mathrm{x}}, \mathrm{E}, \underline{\omega})=\Sigma_{\mathrm{t}}(\underline{\mathrm{x}}, \mathrm{E}) \mathrm{F}(\underline{\mathrm{x}}, \mathrm{E}, \underline{\omega})$ is the vector collision density, $F$ the vector flux. Then the equation adjoint to the pair of equations ( 1 and 5 ) is

$$
\begin{aligned}
x^{\prime}(\underline{x}, E, \underline{\omega}) & =\iiint \lambda^{\prime \prime}\left(\underline{x}^{\prime}, E^{\prime}, \underline{\omega}^{\prime}\right) \mathrm{T}\left(\underline{\mathrm{x}}, \underline{x^{\prime}} ; E, \underline{\omega}\right) C\left(E, \underline{\omega}, E^{\prime}, \underline{\omega}^{\prime} ; \underline{x^{\prime}}\right) \mathrm{d} \underline{x}^{\prime} \mathrm{d} E^{\prime} \mathrm{d} \underline{\omega}^{\prime} \\
& +\int_{R} \frac{\sum_{\mathrm{a}}\left(\underline{x}^{\prime}, E\right)}{\sum_{\mathrm{t}}\left(\underline{x}^{\prime}, \mathrm{E}\right)} \mathrm{T}\left(\underline{\mathrm{x}}, \underline{x}^{\prime}, \mathrm{E}, \underline{\omega}\right) \mathrm{d} \underline{x}^{\prime},
\end{aligned}
$$

and the integral which corresponds to Equation 5 is

$$
I=\iiint Q(\underline{x}, E, \underline{\omega}) X^{\prime}(\underline{x}, E, \underline{\omega}) \mathrm{d} \underline{x} \mathrm{~d} E \mathrm{~d} \underline{\omega} .
$$

Upon multiplying Equation 1 by $X^{*}(\underline{x}, E, \underline{\omega})$ and Equation 6 by $\chi(\underline{x}, E, \underline{\omega})$ and inte grating these equations, one sees that

$$
I=I " \text {. }
$$

This suggests that the absorption rate I may be estimated in two different ways: one might construct Monte Carlo estimates of the density $X$ through a simulation of Equation $I$ and use any estimator whose mean is the fraction of particles absorbed in R. Alternatively, one might construct Monte Carlo estimates of the density $X^{*}$ through a simulation of Equation 6 and use any estimator whose mean is the weighted fraction which arrives at the source region defined by $Q \neq 0$. Such simulations are, of course, subject to an interpretation of Equations 1 and 6 as proper transport equations, a point which we now discuss more fully.

Estimation of I through a simulation of Equation 1 is, of course, straightforward. Because the functions $Q, T$, and $P$ are properly normalized density functions, one may select a starting position $\underline{x}_{0}$, energy $E_{O}$, and direction $\underline{\omega}_{0}$ from the density $Q$, move the particle along $\underline{\omega}_{0}$ to its first collision at point $\underline{x}_{1}$ chosen from the density $T\left(\underline{x}_{0}, \underline{x}_{1} ; E_{0}, \underline{\omega}\right)$, absorb the particle at $\underline{x}_{1}$ with 
probability $1-\Sigma_{s}\left(\underline{x}_{1}, E_{o}\right) / \Sigma_{t}\left(\underline{x}_{1}, E_{o}\right)$ or scatter it with probability $\Sigma_{s}\left(\underline{x}_{1}, E_{o}\right) /$ $\Sigma_{t}\left(\underline{x}_{1}, E_{0}\right)$, to a new energy $E_{1}$ and direction $\underline{\omega}_{1}$ selected from the density $P$. The simulation continues through alternate selections from $T$ and $P$ until termination occurs in some region. In the particle population so constructed, the density emerging from collisions is an estimate of $\chi(x, E, \omega)$ and a valid estimator of $I$ is the fraction of particles absorbed in $R$ or equivalently, the sum over all collisions of the expected contributions to the absorption rate in $\mathrm{R}$ for a particle which has just emerged from collision.

A proper simulation of Equation 6, however, is more complicated because of the fact that the adjoint source, transport kernel, and collision kernel are not properly normalized. In fact, it is somewhat nontrivial even to sample the adjoint source function

$$
Q *(\underline{x}, E, \underline{\omega})=\int_{R} \frac{\sum_{a}\left(\underline{x^{\prime}}, E\right)}{\Sigma_{t}\left(\underline{x^{\prime}}, E\right)} T\left(\underline{x}, \underline{x^{\prime}} ; E, \underline{\omega}\right) d \underline{x}^{\prime} .
$$

Physically the spatial positions $\mathrm{x}$ in $Q^{*}$ represent scattering points from which particles travel along $\omega$ to an absorbing event in $R$. One might sample for such an $x$ through a rejection technique, but this would be very inefficient if $\mathrm{R}$ were small. A more attractive alternative is to start the particle uniformly in $R$ and trace it back to the point of its previous collision. Thus if $\underline{\omega}_{0}$ is selected from an isotropic distribution, $E_{O}$ from

$$
\frac{\Sigma_{a}\left(\underline{x}_{0}, E\right)}{\sum_{t}\left(\underline{x}_{0}, E\right)} / \int \mathrm{d} E \frac{\Sigma_{a}\left(\underline{x}_{0}, E\right)}{\sum_{t}\left(\underline{x}_{0}, E\right)}, \underline{x}_{0} \in R
$$

where $\underline{x}_{0}$ is selected uniformly in $R$, then $\underline{x}_{1}$ may be chosen from the density

$$
T\left(\underline{x}_{0}, \underline{x}_{1} ; E_{0}, \underline{\omega}_{0}\right)=T\left(\underline{x}_{1}, \underline{x}_{0} ; E_{0},-\underline{\omega}_{0}\right) \frac{\Sigma_{t}\left(\underline{x}_{1}, E_{0}\right)}{\Sigma_{t}\left(\underline{x}_{0}, E_{0}\right)} .
$$

Thus, a particle with coordinates $\left(\underline{x}_{1}, E_{0}, \underline{\omega}_{0}\right)$ selected in this manner will represent a sample drawn from $Q *(\underline{x}, E,-\underline{\omega})$ provided it is assigned a weight

$$
W_{O}=4 \pi V_{R}\left[\int \frac{\Sigma_{a}\left(\underline{x}_{0}, E\right)}{\Sigma_{t}\left(\underline{x}_{0}, E\right)} d E\right] \frac{\Sigma_{t}\left(\underline{x}_{0}, E_{o}\right)}{\Sigma_{t}\left(\underline{x}_{1}, E_{0}\right)},
$$

where $V_{R}=$ volume of $R$.

The reversal of direction in $Q *$ occurs, then, in a very natural way and suggests that we might more easily simulate the density $X^{*}(x, E,-\omega)$ [replacing $\underline{\omega}$ by $-\underline{\omega}$ in Equation 6] than the density $X^{*}(\underline{x}, E, \underline{\omega})$. We will still, of course, obtain $\bar{I} *$ by replacing $\underline{\omega}$ by $-\underline{\omega}$ in Equation 7 as well. The equation satisfied by $X^{*}(\underline{x}, E, \underline{-\omega})$ is 


$$
\begin{aligned}
& x^{*}(\underline{x}, E,-\underline{\omega})=\iiint x^{*}\left(\underline{x}^{\prime}, E^{\prime},-\underline{\omega}^{\prime}\right) \mathrm{T}\left(\underline{x}, \underline{x}^{\prime} ; E,-\underline{\omega}\right) \mathrm{C}\left(\mathrm{E}, \underline{\omega}, \mathrm{E}^{\prime}, \underline{\omega}^{\prime} ; \underline{x}^{\prime}\right) \mathrm{d} \underline{x}^{\prime} \mathrm{d} \mathrm{E}^{\prime} \mathrm{d} \underline{\omega}^{\prime}
\end{aligned}
$$

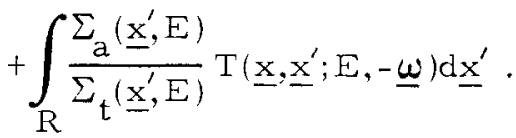

Notice we have made use of the fact that the collision kernel $C$ depends only on $\underline{\omega} \cdot \underline{\omega}^{\prime}=(-\underline{\omega}) \cdot\left(-\underline{\omega}^{\prime}\right)$.

Pursuing this line of thought, upon collision at $x_{l}$ we may choose to absorb the particle with probability $\Sigma_{a}\left(\underline{x}_{1}, E_{o}\right) / \Sigma_{t}\left(\underline{x}_{1}, E_{0}^{\prime}\right)$ and, with probability $\Sigma_{\mathrm{s}}\left(\underline{x}_{1}, E_{0}\right) / \Sigma_{t}\left(\underline{x}_{1}, E_{0}\right)$, to scatter into a new direction $\underline{\omega}_{1}$ and energy $E_{1}$. To perform the transformation in energy and direction we must sample $E_{1}$, $\underline{\omega}_{1}$ from

$$
\begin{aligned}
\frac{1}{W_{c}} C\left(E_{1}, \underline{\omega}_{1}, E_{o}, \underline{\omega}_{o} ; \underline{x}_{1}\right) & =\frac{1}{W_{c}} \frac{\sum_{s}\left(\underline{x}_{1}, E_{1}\right)}{\sum_{t}\left(\underline{x}_{1}, E_{l}\right)} \frac{1}{E_{l}(1-\alpha)} \delta\left(\mu_{L}-\frac{A \mu_{c}+1}{\sqrt{A^{2}+2 A \mu_{c}+1}}\right), \\
\quad \text { for } E_{o} \leq E_{1} \leq E_{o} / \alpha, & \\
& =0 \text { otherwise, }
\end{aligned}
$$

and use a weight

$$
\begin{aligned}
& W_{c}=\frac{\iint C\left(E_{1}, \underline{\omega}_{1}, E_{0}, \underline{\omega}_{0} ; \underline{x}_{1}\right) d E_{1} d \underline{\omega}_{1}}{\sum_{s}\left(\underline{x}_{1}, E_{0}\right) / \Sigma_{t}\left(\underline{x}_{1}, E_{0}\right)} \\
& =\int_{E_{0}}^{E_{0} / \alpha} \frac{\Sigma_{s}\left(\underline{x}_{l}, E_{1}\right) d E_{1}}{\Sigma_{t}\left(\underline{x}_{l}, E_{1}\right) E_{1}(1-\alpha)} / \Sigma_{s}\left(\underline{x}_{1}, E_{0}\right) / \Sigma_{t}\left(\underline{x}_{1}, E_{0}\right) .
\end{aligned}
$$

Having arrived at $\left(\underline{x}_{1}, E_{1}, \underline{\omega}_{1}\right)$ in this fashion, we next sample for $\underline{x}_{2}$ from

$$
T\left(\underline{x}_{1}, \underline{x}_{2} ; E_{1}, \underline{\omega}_{1}\right)=T\left(\underline{x}_{2}, \underline{x}_{1} ; E_{1},-\underline{\omega}_{1}\right) \frac{\Sigma_{t}\left(\underline{x}_{2}, E_{1}\right)}{\Sigma_{t}\left(\underline{x}_{1}, E_{1}\right)},
$$

making use of the weight

$$
W_{T}=\frac{\sum_{t}\left(\underline{x}_{1}, E_{1}\right)}{\sum_{t}\left(\underline{x}_{2}, E_{1}\right)} .
$$


Sampling the adjoint kernels $\mathrm{C}$ and $\mathrm{T}$ alternates until the particle is absorbed, escapes from the system, or is scattered above energies of interest. Then the product of the particle's weight and $\mathrm{Q}(\underline{\mathrm{x}}, \mathrm{E}, \underline{-\underline{\omega})}$ produce an unbiased estimate of $I^{*}=\mathrm{I}$. We emphasize that the density being constructed in this adjoint simulation is $X^{*}(\underline{x}, \mathrm{E}, \underline{\underline{\omega}})$ rather than the solution of Equation 6.

This same difficulty, that of constructing Monte Carlo solutions of equations with unnormalized kernels, becomes even more apparent in working with the integro-differential equation for the vector flux, $F(\underline{x}, E, \underline{w})$. This transport equation may be written

$$
\begin{aligned}
& \underline{\omega} \cdot \underline{\nabla} F(\underline{x}, E, \underline{\omega})+\Sigma_{t}(\underline{x}, E) F(\underline{x}, E, \underline{\omega})=\iint \Sigma_{t}\left(\underline{x}, E^{\prime}\right) C\left(E^{\prime}, \underline{\omega}^{\prime}, E, \underline{\omega} ; \underline{x}\right) F\left(\underline{x}, E^{\prime}, \underline{\omega}^{\prime}\right) d E^{\prime} \mathrm{d} \underline{\omega}^{\prime} \\
&+Q(\underline{x}, E, \underline{\omega}) \\
&= \iint \Sigma_{s}\left(\underline{x}, E^{\prime}\right) P\left(E^{\prime}, \underline{\omega}^{\prime}, E, \underline{\omega} ; \underline{x}\right) F\left(\underline{x}, E^{\prime}, \underline{\omega}^{\prime}\right) d E^{\prime} \mathrm{d} \underline{\omega}^{\prime} \\
&+Q(\underline{x}, E, \underline{\omega}) .
\end{aligned}
$$

The adjoint integro-differential equation is (11)

$$
\begin{aligned}
& \underline{-\underline{\omega}} \cdot \underline{\nabla} F *(\underline{x}, E, \underline{\omega})+\Sigma_{t}(\underline{x}, E) F *(\underline{x}, E, \underline{\omega})=\iint \Sigma_{s}(\underline{x}, E) P\left(E, \underline{\omega}, E^{\prime}, \underline{\omega^{\prime}} ; \underline{x}\right) F *\left(\underline{x}, E^{\prime}, \underline{\omega}^{\prime}\right) d E^{\prime} d \underline{\omega}^{\prime} \\
&+Q^{\prime} * *(\underline{x}, E, \underline{\omega}) .
\end{aligned}
$$

Then with suitable boundary conditions on $F$ and $F^{*}$, we have

$$
\left.\iint Q * * * \underline{x}, E, \underline{\omega}\right) F(\underline{x}, E, \underline{\omega}) d \underline{x} d E d \underline{\omega}=\iint Q(\underline{x}, E, \underline{\omega}) F *(\underline{x}, E, \underline{\omega}) d \underline{x} d E d \underline{\omega} .
$$

For the problem we are studying, Equation 5 implies that

$$
Q * *(\underline{x}, E, \underline{\omega})=\left\{\begin{array}{l}
\Sigma_{\mathrm{a}}(\underline{x}, E), \underline{x} \in R \\
0, \text { otherwise. }
\end{array}\right.
$$

One difficulty with constructing a simulation of Equation 16 arises because of the $-\underline{\omega}$ in the leakage term. To circumvent this difficulty we use the same tatic as before; we define

$$
F^{\dagger}(\underline{x}, E, \underline{\omega})=F *(\underline{x}, E,-\underline{\omega}) .
$$

The replacement of $\underline{\omega}$ by $-\underline{\omega}$ in Equation 16 gives 
$\underline{\omega} \cdot \underline{\nabla} F^{\dagger}(\underline{x}, E, \underline{\omega})+\Sigma_{t}(\underline{x}, E) F^{\dagger}(\underline{x}, E, \underline{\omega})=\iint \Sigma_{s}(\underline{x}, E) P\left(E, \underline{\omega}, E^{\prime}, \underline{\omega}^{\prime} ; \underline{x}\right) F^{\dagger}\left(\underline{x}, E^{\prime}, \underline{\omega}^{\prime}\right) d E^{\prime} d \underline{\omega}^{\prime}$

$$
+Q * *(\underline{x}, E,-\underline{\omega}) \text {. }
$$

an equation which may be readily simulated and which leads to a model different from, but equivalent to, the one already described. We would expect this equivalence since the equality

$$
\iint \mathrm{QF} *=\iint \mathrm{Q} X^{*}
$$

for all source functions $Q$ implies that

$$
F * X^{*} \text {. }
$$

Therefore a simulation of the function $\chi^{*}(\underline{x}, E,-\omega)$ must ultimately be statistically equivalent to a simulation of $F^{*}(\underline{x}, E, \underline{\underline{\omega}})=F^{\dagger}(\underline{x}, E, \underline{\omega})$.

Based on the integro-differential Equation 18, which is now a conventional transport equation, a sampling procedure which suggests itself is the following: Select a starting direction $\omega_{0}$ from an isotropic distribution, $\underline{x}_{0}$ uniformly in $R$, and $E_{o}$ from $\Sigma_{a}\left(\underline{x}_{0}, E\right) / \int \mathrm{d} E \Sigma_{a}\left(\underline{x}_{0}, E\right)$. The source weight is

$$
\mathrm{W}_{\mathrm{o}}^{\prime}=4 \pi \mathrm{V}_{\mathrm{R}} \int \Sigma_{\mathrm{a}}\left(\underline{x}_{\mathrm{o}}, \mathrm{E}\right) \mathrm{d} \mathrm{E} \text {. }
$$

Next, the position $\underline{x}_{1}$ of first collision is chosen from $\mathrm{T}\left(\underline{x}_{0}, \underline{x}_{1} ; E_{0}, \underline{\omega}_{0}\right)$. At $\underline{x}_{1}$, absorption occurs with probability $\Sigma_{a}\left(\underline{x}_{1}, E_{0}\right) / \Sigma_{t}\left(\underline{x}_{1}, E_{0}\right)$ and scattering with probability $\Sigma_{s}\left(\underline{x}_{1}, E_{o}\right) / \Sigma_{t}\left(\underline{x}_{1}, E_{o}\right)$. In the latter event, a new energy $E_{1}$ and direction $\underline{\omega}_{1}$ are selected from

$$
\begin{aligned}
& \frac{1}{W_{c}^{\prime} \cdot \sum_{s}\left(\underline{x}_{1}, E_{0}\right)} \sum_{t}\left(\underline{x}_{1}, E_{1}\right) C\left(E_{1}, \underline{\omega}_{1}, E_{0}, \underline{\omega}_{0} ; \underline{x}_{1}\right) \\
& =\frac{1}{W_{c}^{\prime} \cdot \Sigma_{s}\left(\underline{x}_{l}, E_{0}\right)} \frac{\Sigma_{s}\left(\underline{x}_{l}, E_{l}\right)}{E_{1}(1-\alpha)} \delta\left(\mu_{L}-\frac{A \mu_{c}+1}{\sqrt{A^{2}+2 A \mu_{c}+1}}\right), \\
& \text { for } \mathrm{E}_{\mathrm{O}} \leq \mathrm{E}_{\mathrm{l}} \leq \mathrm{E}_{\mathrm{O}} / \alpha \\
& =0 \text { otherwise, }
\end{aligned}
$$

and a multiplicative weight factor

$$
W_{c}^{\prime}=\int_{E_{0}}^{E_{0} / \alpha} \frac{\Sigma_{s}\left(\underline{x}_{1}, E_{1}\right) d E_{1}}{E_{l}(l-\alpha)} / \Sigma_{s}\left(\underline{x}_{1}, E_{0}\right)
$$


is assigned. This alternation between collision and transport kernels continues until the particle is absorbed, escapes from the system, or is scattered above energies of interest. This sampling procedure may be shown to be satistically equivalent to the one described earlier based on the integral equation for $\chi^{*}$.

While the two "analog" adjoint sampling and weighting procedures just described lead to the same expected values, they do, in general have different variances. Furthermore, the weight factors $W_{c} \cdot W_{t}$ and $W_{c}^{\prime}$ (see Equations 12,14 , and 21) result in significantly larger variances than would arise in direct mode simulation. As is observed in Reference 11 , this appears to be so precisely because the weights seem in no way to reflect the importance of the event being weighted.

One may ask whether the use of appropriately altered collision and transport kernels in an adjoint mode simulation might eliminate some or all of this additional variance. We can get some feeling for the need for such improvement by examining a constant cross section model. Under this as sumption the two analog adjoint sampling schemes become identical, and the weight factor arising from collision at an energy $E_{1}$ (assuming isotropic scattering in the CM system) is

$$
W=\int_{E_{0}}^{E_{0} / \alpha} \frac{d E_{1}}{E_{1}(1-\alpha)}=\frac{\ln \frac{1}{\alpha}}{1-\alpha}
$$

It seems clear that, even in this relatively simple case, the variability of the total weight with the number of collisions, as well as with the mass of the scatterers present, will cause a harmful effect on the variance. This difficulty may be virtually eliminated by a physically intuitive biasing (13) of the adjoint scattering kernel. ing:

To see this, we examine the energy-angle relationship for elastic scatter-

$$
E_{L}=\frac{E_{U}}{2}\left[(1+\alpha)+(1-\alpha) \mu_{c}\right]
$$

where $E_{L}$ and $E_{U}$ designate lower and upper energies, respectively. In direct mode simulation, $\mu_{c}$ is determined through a random sampling of its density function $f$ by setting a random number

$$
r=\int_{-1}^{\mu} \mathrm{f}(\mu) \mathrm{d} \mu
$$

after which, since $E_{U}$ is known, $E_{L}$ is determined through Equation 23. If one could envision a filmed sequence depicting this collision in time, it would seem natural to regard the adjoint collision as what would be seen if the film were run backward. This implies that the scattering angle in the CM system should be preserved, that is, selected by means of Equation 24, as in a direct mode collision. This would suggest the use of a biased scattering kernel equal to $E_{L} / E_{U}$ times the normalized probability of slowing down from $E_{U}$ to $E_{L}$. The factor $E_{L} / E_{U}$ properly normalizes the kernel for adjoint sampling of the upper energy $E_{U}$ and may be applied to any elastic angular distribution $f(\mu)$, as long as $f(\mu)$ is independent of energy. 
It remains to be seen that the use of this altered scattering kernel removes most of the extra variance caused by the factor Equation 21. One also may wonder whether the use of still other kernels might not be equally good or perhaps even better from the standpoint of variance. Both of these equations may be settled by examining transformations of the adjoint transport equation designed to achieve unit weight factors, as we now do.

We begin with the integro-differential Equation 18 for the function $\mathrm{F}^{\dagger}$, which is related to the adjoint flux $F *$ through Equation 17 . We set ourselves the goal of finding a transformation of Equation 18 which will lead to an adjoint equation which can be simulated without the need for weights on every collision. We shall discover a transformation which avoids weights except at boundary crossings between regions of different scattering cross section and which is equivalent to a time reversal of the direct mode collision.

Let $\psi^{\infty}(\mathrm{x}, \mathrm{E}, \underline{\omega)}$ be a prospective transforming function and multiply Equation 18 by $\psi^{\infty}$; further let

$$
\eta(\underline{x}, E, \underline{\omega}) \equiv F^{\dagger}(\underline{x}, E, \underline{\omega}) \psi^{\infty}(\underline{x}, E, \underline{\omega}) .
$$

Then Equation 18 becomes

$$
\begin{aligned}
& \underline{\omega} \cdot \underline{\nabla} \eta(\underline{x}, E, \underline{\omega})+\Sigma_{t}(\underline{x}, E) \eta(\underline{x}, E, \underline{\omega})=\iint_{\psi^{\infty}\left(\underline{x}, E^{\prime}, \underline{\omega}, \underline{\omega}\right)}^{\left.\omega^{\prime}\right)} \Sigma_{s}(\underline{x}, E) P\left(E, \underline{\omega}, E^{\prime}, \underline{\omega^{\prime} ; \underline{x}}\right) \eta\left(\underline{x}, E^{\prime}, \underline{\omega}^{\prime}\right) \mathrm{d} E^{\prime} \mathrm{d} \underline{\omega}^{\prime} \\
& +\psi^{\infty}(\underline{x}, E, \underline{\omega}) Q * *(\underline{x}, E,-\underline{\omega}) .
\end{aligned}
$$

If the integral which appears on the right hand side is to be interpreted as a scattering rate at $\left(\underline{x}, E^{\prime}, \underline{\omega}^{\prime}\right)$ and if unit weights are demanded on every collision (i.e., scattering-in rate = scattering-out rate) then we must have

$$
\Sigma_{s}\left(\underline{x}, E^{\prime}\right)=\iint_{\psi^{\infty}\left(\underline{x}, E^{\prime}, \underline{\omega}^{\prime}\right)} \Sigma_{s}(\underline{x}, E) P\left(E, \underline{\omega}, E^{\prime}, \underline{\omega}^{\prime} ; \underline{x}\right) \mathrm{d} E \mathrm{~d} \underline{\omega} .
$$

In a problem consisting of an infinite lattice of two regions, I and II, then we would want for $\underline{x} \in \mathrm{I}$

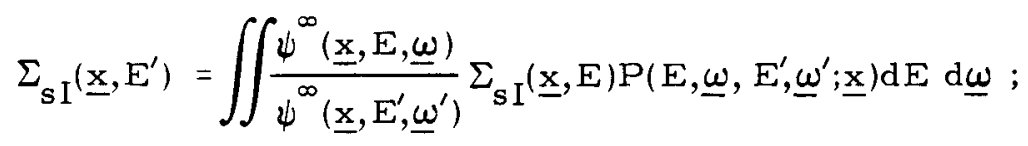

and for $\underline{x} \in I I$,

$$
\Sigma_{S I I}\left(\underline{x}, E^{\prime}\right)=\iint_{\psi^{\infty}(\underline{x}, E, \underline{\omega})}^{\left.\psi^{\infty}, E^{\prime}, \underline{\omega}^{\prime}\right)} \Sigma_{s I I}(\underline{x}, E) P\left(E, \underline{\omega}, E^{\prime}, \underline{\omega}^{\prime} ; \underline{x}\right) \mathrm{d} E d \underline{\omega} .
$$

We notice that the se equations are the transport equations for the flux $\psi^{\infty}(\underline{x}, E, \underline{\omega})$ in a source-free infinite medium of Region I and Region II scatterers, 
respectively. Since such a flux is isotropic and spatially constant we may write

$$
\begin{aligned}
\psi^{\infty}(\underline{x}, E, \underline{\omega}) & =\frac{1}{4 \pi} \psi_{I}^{\infty}(E), \underline{x} \in I \\
& =\frac{1}{4 \pi} \psi_{I I}^{\infty}(E), \underline{x} \in I I .
\end{aligned}
$$

If we assume scattering is elastic and isotropic in the CM system both in $I$ and II, then writing Equation 4 in the CM system gives

$$
\begin{aligned}
P\left(E, \underline{\omega}, E^{\prime}, \underline{\omega}^{\prime}, \underline{x}\right) & =\frac{1}{E\left(1-\alpha_{I}\right)} \delta\left[\mu_{c}-\frac{2 E^{\prime}}{E\left(1-\alpha_{I}\right)}+\frac{1+\alpha_{I}}{1-\alpha_{I}}\right], \underline{x} \in I \\
& =\frac{1}{E\left(1-\alpha_{I I}\right)} \delta\left[\mu_{c}-\frac{2 E^{\prime}}{E\left(1-\alpha_{I I}\right)}+\frac{1+\alpha_{I I}}{1-\alpha_{I I}}\right], \quad \underline{x} \in I I .
\end{aligned}
$$

Then Equation 28 becomes

$$
\Sigma_{S I}\left(E^{\prime}\right) \psi_{I}^{\infty}\left(E^{\prime}\right)=\int_{E^{\prime}}^{E^{\prime} / \alpha_{I}} \frac{\Sigma_{S I}(E) \psi_{I}^{\infty}(E)}{E\left(1-\alpha_{I}\right)} d E,
$$

and Equation 29 becomes

$$
\Sigma_{S I I}\left(E^{\prime}\right) \psi_{I I}^{\infty}\left(E^{\prime}\right)=\int_{E^{\prime}}^{E^{\prime} / \alpha_{I I}} \frac{\Sigma_{S I I}(E) \psi_{I I}^{\infty}(E)}{E\left(1-\alpha_{I I}\right)} d E
$$

From this it easily follows that

$$
\psi_{I}^{\infty}\left(E^{\prime}\right)-\frac{k}{\sum_{s I}\left(E^{\prime}\right) E^{\prime}}
$$

and

$$
\psi_{I I}^{\infty}\left(E^{\prime}\right)=\frac{k}{\sum_{S I I}\left(E^{\prime}\right) E^{\prime}},
$$

where $k$ is any constant. If we choose $k=1$ and substitute into Equation 26 we arrive at 
$\underline{\omega} \underline{\nabla} \eta(\underline{x}, E, \underline{\omega})+\Sigma_{t}(\underline{x}, E) \eta(\underline{x}, E, \underline{\omega})=\iint \Sigma_{s}\left(\underline{x}, E^{\prime}\right) \frac{E^{\prime}}{E} P\left(E, \underline{\omega}, E^{\prime}, \underline{\omega}^{\prime}, \underline{x}\right) \eta\left(\underline{x}, E^{\prime}, \underline{\omega}^{\prime}\right) d E^{\prime} d \underline{\omega}^{\prime}$

$$
+\frac{Q(\underline{x}, E,-\underline{\omega})}{\sum_{S}(\underline{x}, E) E}
$$

where

$$
\eta(\underline{\mathrm{x}}, \mathrm{E}, \underline{\omega})=\frac{F^{\dagger}(\underline{\mathrm{x}}, \mathrm{E}, \underline{\underline{\omega}})}{\sum_{\mathrm{s}}(\underline{\mathrm{x}}, \mathrm{E}) \mathrm{E}} .
$$

Equation 35 for the transformed adjoint flux $\eta$ is now in a form suitable for simulation by Monte Carlo without the use of weights at every collision. Indeed, precisely because $\left(E^{\prime} / E\right) P\left(E, \underline{\omega}, E^{\prime}, \omega^{\prime}, x\right)$ is a properly normalized scattering kernel from energy $E^{\prime}$ to energy $E$, the integral term in Equation 35 is the scattering rate at $\left(\underline{x}, E^{\prime}, \underline{\omega}^{\prime}\right)$ in the flux $\eta$. While we have assumed that scattering is isotropic in the $\mathrm{CM}$ system, our conclusions remain valid as long as the scattering is elastic. This follows from the relationship $\partial \mu_{C} / \partial E_{U}=-\left(E_{L} / E_{U}\right) \partial \mu_{c} / \partial E_{I}$ which depends only on Equation 23. Notice that we have derived the physically intuitive altered kernel $\left(E^{\prime} / E\right) P$ based only on the requirement of unit weights at everycollision. We conclude that no other transformation will accomplish this.

The only non-standard feature of Equation 35 is that the flux $\eta$ is discontinuous at the interface between Regions I and II, owing to the possible discontinuity in $\Sigma_{S}(x, E)$ there. However,

$$
\Sigma_{s}(\underline{x}, E) \eta(\underline{x}, E, \underline{\omega}) \quad \frac{F^{\dagger}(\underline{x}, E, \underline{\underline{\omega}})}{E}
$$

is continuous everywhere, so that the appropriate discontinuity in $\eta$ may be achieved by multiplying the weight of each particle by $\Sigma_{S I}(E) / \Sigma_{S I I}(E)$ when the particle passes from Region I into Region II and by the inverse factor upon crossing from II into I.

The adjoint sampling procedures which result from this transformation may be described as follows a starting direction $\omega_{0}$ is chosen from an isotropic distribution, $\underline{x}_{O}$ is selected uniformly in $R$, and $E_{O}$ is chosen from

$$
\frac{Q\left(\underline{x}_{O}, E,-\underline{\omega}_{O}\right)}{\Sigma_{s}\left(\underline{x}_{0}, E\right) E} / \int \frac{Q\left(\underline{x}_{O}, E,-\underline{\omega}_{O}\right)}{\Sigma_{s}\left(\underline{x}_{0}, E\right) E} d E=\frac{\Sigma_{a R}(E)}{\Sigma_{s R}(E) E} / \int_{0}^{E} \max \frac{\Sigma_{a R}(E)}{\Sigma_{s R}(E) E} d E .
$$

A starting weight factor

$$
4 \pi v_{R} \int_{0}^{E} \frac{\Sigma_{a R}(E)}{\sum_{s R}(E) E} d E
$$


is assigned each particle. First collision points are selected from $\mathrm{T}\left(\mathrm{x}_{\mathrm{O}}, \mathrm{x}_{1} ; \mathrm{E}_{\mathrm{O}}, \underline{\omega}_{\mathrm{O}}\right)$ and the history terminates with probability $\Sigma_{\mathrm{a}}\left(\underline{x}_{1}, \mathrm{E}_{\mathrm{o}}\right) / \Sigma_{\mathrm{t}}\left(\underline{x}_{1}, \mathrm{E}_{\mathrm{o}}\right)$. With probability $\Sigma_{\mathrm{s}}\left(\underline{x}_{l}, E_{\mathrm{o}}\right) / \Sigma_{\mathrm{t}}\left(\underline{x}_{1}, E_{\mathrm{o}}\right)$, a new energy $E_{1}$ and direction $\underline{\omega}_{1}$ are chosen from $\left(E_{0} / E_{1}\right) P\left(E_{1}, \underline{\omega}_{1}, E_{0}, \underline{\omega}_{0} ; x_{1}\right)$. The only weight factors are the ones occurring upon passing from one region into another having a different scattering cross section. In this fashion the transformed adjoint flux $\eta$ is constructed. Then one uses the relationships

$$
\begin{aligned}
\iint Q * * F & =\iint \Sigma_{a R^{*}}(E) F(\underline{x}, E, \underline{\omega}) \\
& =\iint Q F^{\dagger} \\
& =\iint Q \eta / \iota^{\infty} \\
& =\iint Q \eta \Sigma_{S} E
\end{aligned}
$$

to convert $\eta$ tallies into estimates of the absorption rate. That is, estimators of $\eta$ must be multiplied by the factor $\Sigma_{\mathrm{S}} \mathrm{E}$ in order to yield estimators of the absorption rate in $R$. It is evident that the scheme described above may be immediately generalized to any number of regions containing elastic scatterers.

It seems apparent that most of the deleterious effects of the adverse weight factors (Equation $12 \times$ Equation 14 and Equation 21) have been eliminated by the above transformation. Numerical evidence in support of this will be offered in the next section. However, one should expect such a reduction in variance through an interpretation involving dual importance sampling.( 8,11$)$ That is, the function $\psi^{\circ}(\underline{x}, E, \underline{\omega})$ of Equation 30 which accomplishes the transformation may be viewed as a region-dependent dual importance function, since the flux $F$ is an appropriate importance function for solution of adjoint equations and since $\psi^{\infty}$ is an approximation to $F$. Despite the discontinuity in $\psi^{\infty}$ (which, of course, is not present in $F$ ), the flux $\psi^{\infty}$ will be of a good approximation to $F$ deep in the interior of any region. To this extent one might anticipate variance reduction to be accomplished through the use of the transformation via $\eta$. This was the same philosophy Spanier and Gelbard successfully applied in solving multigroup thermal problems through solutions of transformed adjoint equations.

\section{NUMERICAL RESULTS}

A computer program, STRIFE, was written to compare the performance of the transformed adjoint method with that of an analog adjoint method, as well as to explore the range of problems in which an adjoint method is preferable to a direct method.

A constant cross section case was examined first in order to easily compare direct, analog adjoint, and transformed ajoint methods. A two-region repetitive slab lattice consisting of a graphite moderating region and a highly absorbing region containing a single isotope of large, but finite mass was selected. By varying the thickness of the absorbing region several problems with a range of "resonance" escape probabilities were studied. These results are shown in Figure 1, (based on Table 1) in which the relative standard deviation per history (in percent) is plotted against $1-p$, the "resonance" absorption probability. As can be seen, when $1-p$ is very small the advantages of adjoint 
TABLE 1

CONSTANT CROSS-SECTION RESULTS

\begin{tabular}{|c|c|c|c|c|c|}
\hline \multicolumn{2}{|c|}{ Direct } & \multicolumn{2}{|c|}{$\begin{array}{c}\text { Untransformed } \\
\text { Adjoint }\end{array}$} & \multicolumn{2}{|c|}{$\begin{array}{c}\text { Transformed } \\
\text { Adjoint }\end{array}$} \\
\hline $1-p$ & $\sigma_{\mathrm{R}}(\%)$ & $1-p$ & $\sigma_{\mathrm{R}}(\%)$ & $l-p$ & $\sigma_{\mathrm{R}}(\%)$ \\
\hline $\begin{array}{l}0.2955 \\
0.4031 \\
0.4978 \\
0.6336 \\
0.72904 \\
0.76468 \\
0.7988\end{array}$ & $\begin{array}{l}0.697 \\
0.590 \\
0.453 \\
0.334 \\
0.278 \\
0.240 \\
0.203\end{array}$ & $\begin{array}{l}0.06889 \\
0.1010 \\
0.1323 \\
0.1916 \\
0.2435 \\
0.2951 \\
0.3640 \\
0.4952 \\
0.6402 \\
0.73335 \\
0.76820 \\
0.80510\end{array}$ & $\begin{array}{l}0.4118 \\
0.3946 \\
0.4208 \\
0.4204 \\
0.4424 \\
0.4241 \\
0.4063 \\
0.4024 \\
0.4488 \\
0.4958 \\
0.5501 \\
0.65256 \\
0.7321 \\
0.7423\end{array}$ & $\begin{array}{l}0.004473 \\
0.01779 \\
0.06893 \\
0.10126 \\
0.1322 \\
0.19106 \\
0.2946 \\
0.4933 \\
0.5692 \\
0.6327 \\
0.73175 \\
0.76829 \\
0.8036\end{array}$ & $\begin{array}{l}0.2315 \\
0.3495 \\
0.4284 \\
0.4742 \\
0.5580 \\
0.6635 \\
0.6537\end{array}$ \\
\hline
\end{tabular}

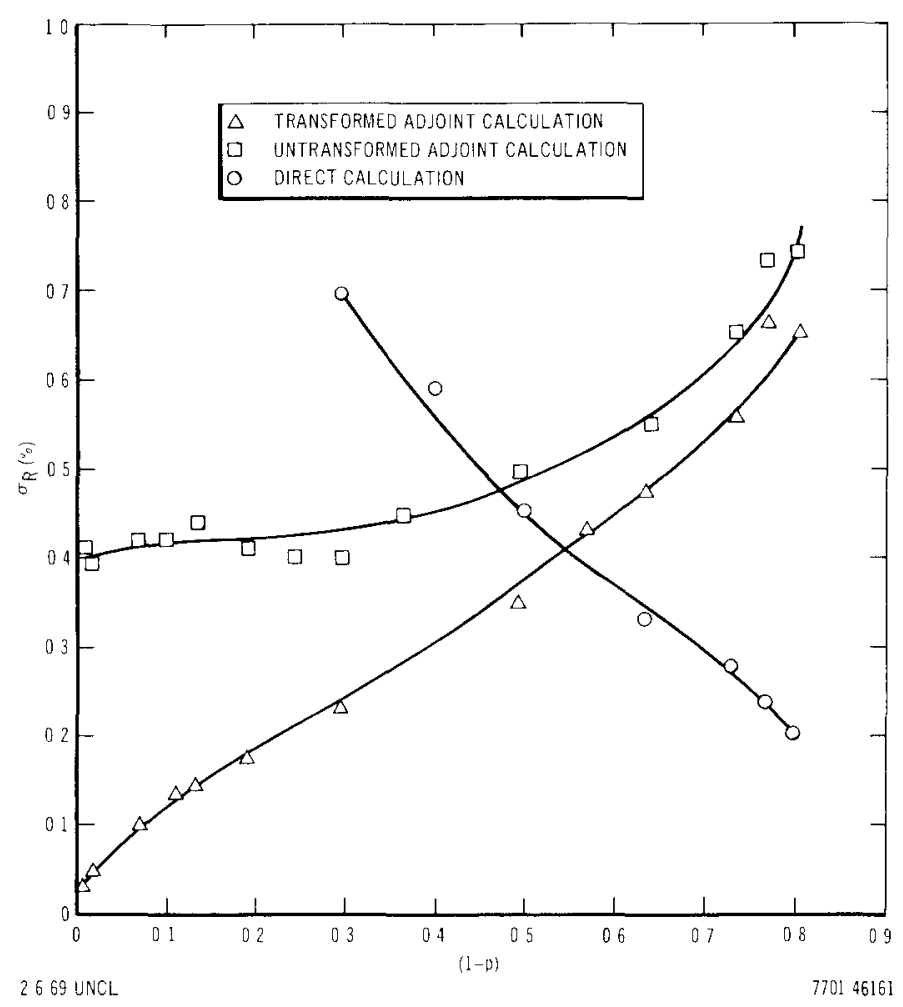

Figure 1. Comparison of Relative Errors, Constant-Cross-Section Case 
over direct methods are apparent. Of even greater significance is the fact that the advantage of the transformation increases with decreasing $1-p$ to such an extent that accurate estimates of $1-p$ in this range may be obtained very cheaply. The running times obtained reflect additional advantage of adjoint over direct methods, namely, the shorter time per history due primarily to the fact that adjoint particles are born with a distribution of energies rather than at one end of the energy range. For the problems studied this additional advantage was worth a factor of about 1.7 (not shown in Figure 1 or Table 1). The transformed adjoint method is 200 times as efficient as the analog adjoint method for small $1-p$ and still 3 times as efficient for $1-p=0.3$. Figure 2 shows the advantage (in time) resulting from the transformation for a wide range of $1-p$. Notice that while the advantage diminishes considerably as 1 - p increases, so does the need to perform the calculation using adjoint techniques.

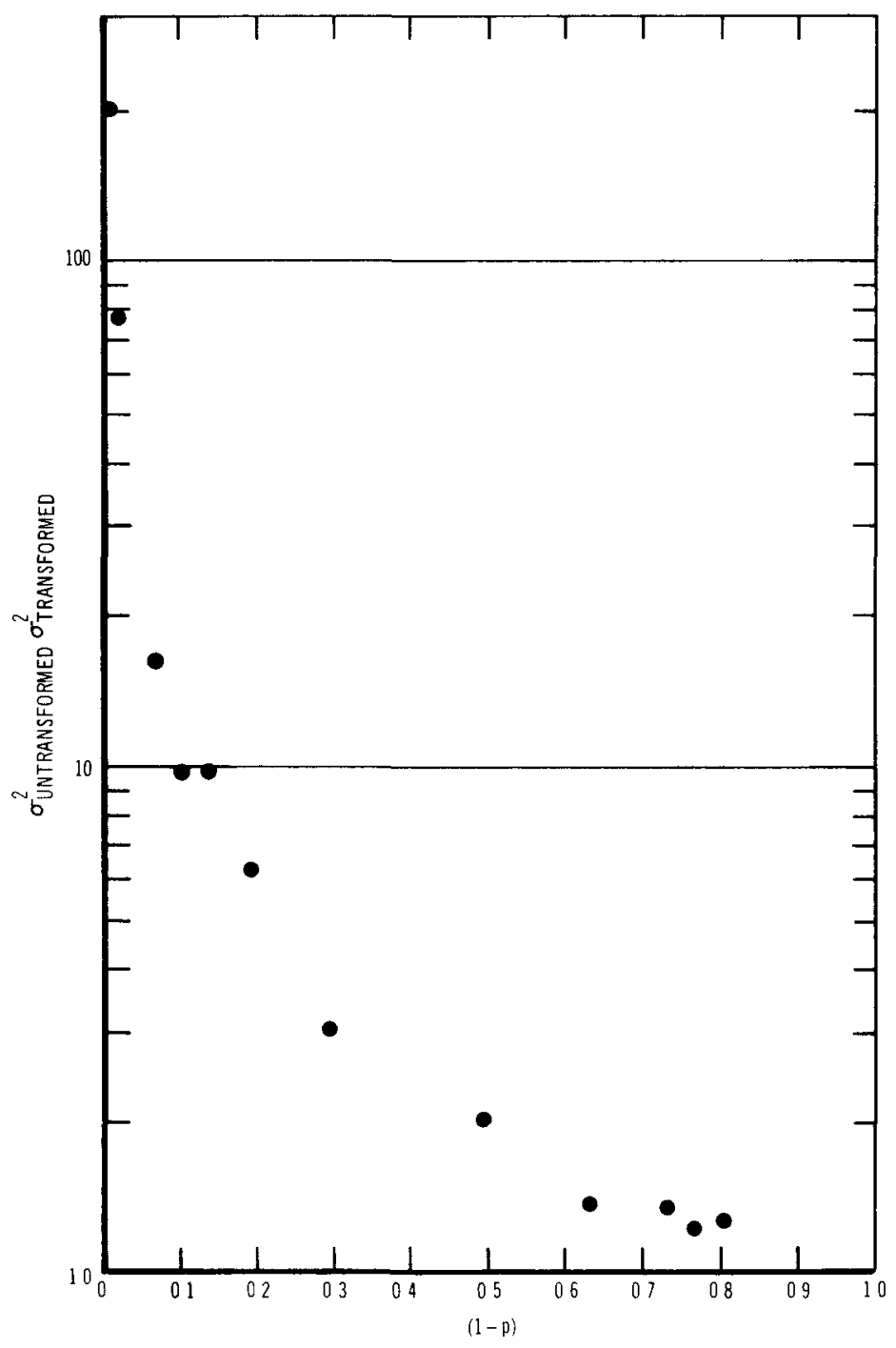

Figure 2. Computational Advantage of Adjoint Transformation. 
Figure 3 and Table 2 show a comparison of direct and transformed adjoint results for a practical two-region problem with a graphite moderator and a gold resonance absorber. In such a problem, the energy-dependence of the resonance absorber cross section gives rise to considerable latitude in the choice of sampling procedure for adjoint source energies. This energy-dependence also tends to reduce the relative efficiency of adjoint versus direct procedures. The results shown in Figure 3 and Table 2 were obtained by forcing half the adjoint particles to start inside an energy interval slightly larger than an interval containing the dominant gold resonance. Even with such a relatively simple procedure, the advantage of transformed adjoint to direct method for small $\mathrm{l}-\mathrm{p}$ is impressive.

TABLE 2

ENERGY-DEPENDENT CROSS-SECTION RESU LTS

\begin{tabular}{l|r|l|l}
\hline \hline \multicolumn{2}{c|}{ Direct } & \multicolumn{2}{c}{$\begin{array}{c}\text { Transformed } \\
\text { Adjoint }\end{array}$} \\
\hline \multicolumn{1}{c|}{$1-\mathrm{p}$} & $\sigma_{\mathrm{R}}{ }^{(\sigma)}$ & \multicolumn{1}{c}{$1-\mathrm{p}$} & $\sigma_{\mathrm{R}}(\%)$ \\
\hline 0.0056926 & 13.23 & 0.006543 & 1.79 \\
0.026082 & 5.62 & 0.02598 & 1.75 \\
0.046006 & 4.06 & 0.04899 & 1.86 \\
0.16254 & 2.29 & 0.1615 & 2.20 \\
0.24405 & 1.73 & 0.2453 & 2.12 \\
0.35103 & 1.28 & 0.32495 & 2.78 \\
\hline
\end{tabular}

Figure 3. Comparison of Relative Errors, Two-Region Gold Resonance Problem

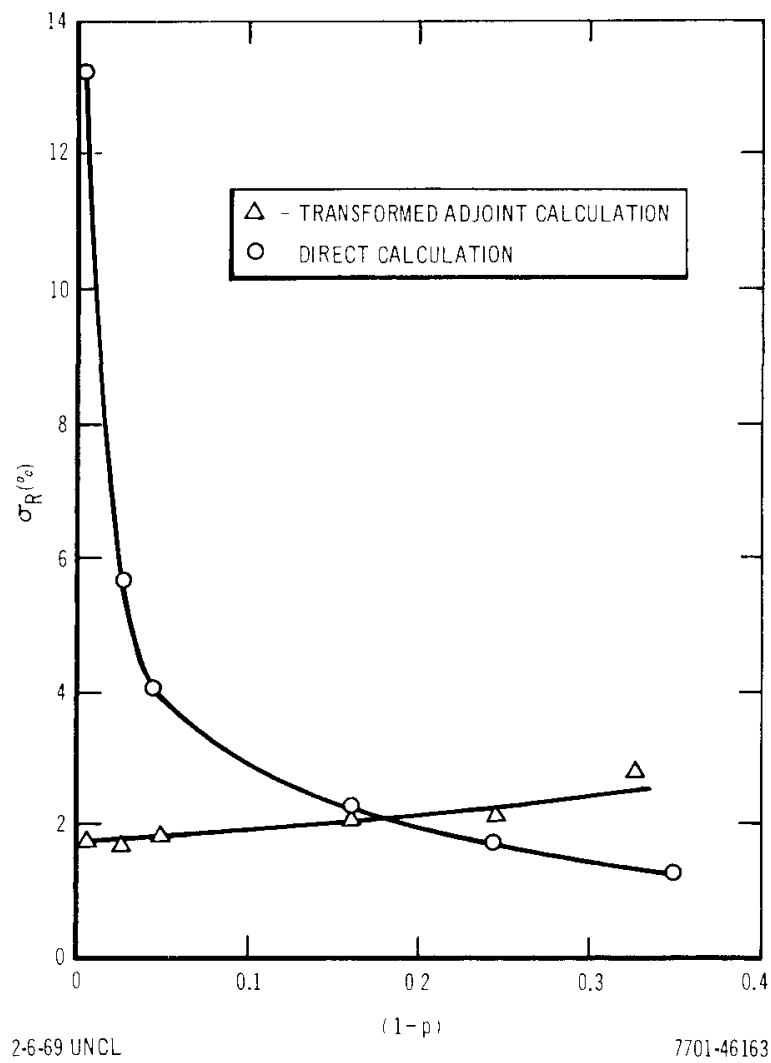




\section{SUMMARY AND CONCLUSIONS}

This paper has described an adjoint technique which appears to be very useful for solving problems in the slowing-down region below the threshhold for inelastic scattering. The physically intuitive basis for this technique is by means of an exact reversal of direct Monte Carlo random walks, including the preservation of scattering angles. This idea was previously used in a combined direct-adjoint Monte Carlo calculation of resonance integrals. (13) We have shown that this reversal may be also accomplished by a transformation of the adjoint transport equation by means of a discontinuous importance function which, in each region of the problem, is the flux in an infinite medium of scatterer appropriate for that region. From the latter point of view this transformation may be seen to be a logical extension of continuous energies of the adjoint multigroup formulation of Spanier and Gelbard.

In addition to the variance reduction it achieves, the principal advantages of the adjoint technique described here stem from its ease of implementation, computational speed, and its ability to utilize the same nuclear data as an equivalent direct Monte Carlo calculation.

This new technique considerably expands the range of problems tractable by Monte Carlo. It seems particularly well-suited, for example, to the analysis of foil experiments and computational times appear to be sufficiently low to permit parametric studies to be performed cheaply.

\section{ACKNOW LEDGMENT}

The authors are indebted to R. C. Lewis of Atomics International for developing the STRIFE Program and for invaluable help in organizing the results presented in this paper.

Typical running times for transformed adjoint problems were 1 minute or less on the CDC -6600 . 


\section{REEER ENCES}

1. R. R. Coveyou, V. R. Cain, and K. J. Yost, "Adjoint and Importance in Monte Carlo Application," Nu. Sci. and Eng., 27, 219-234 (1967).

2. L. Levitt, "The Use of Self-Optimized Exponential Biasing in Obtaining Monte Carlo Estimates of Transmission Probabilities," Nu. Sci. and Eng., 31, 500-504 (1968).

3. J. Spanier, "An Analytic Approach to Variance Reduction," submitted to SIAM J. Appl. Math.

4. V. R. Cain, "Application of $\mathrm{S}_{\mathrm{N}}$ Adjoint Flux Calculation to Monte Carlo Biasing," Trans.A.N.S., 10, 399 (1967).

5. M. Leimdörfer, "On the Transformation of the Transport Equation for Solving Deep Penetration Problems by the Monte Carlo Method," Trans. Chalmers Univ. of Tech., Gothenberg, Sweden, No. 286 (1964).

6. H. Kahn, Applications of Monte Carlo, AECU-3259, Rand Corporation (1954).

7. C. W. Maynard, "An Application of the Reciprocity Theorem to the Acceleration of Monte Carlo Calculations," Nu. Sci. and Eng., 10, 97, (1961).

8. J. Spanier, "A Unified Approach to Monte Carlo Methods and as Application to a Multigroup Calculation of Absorption Rates," SIAM Review, $\underline{4}$, $115-134$ (1962).

9. E. M. Gelbard, H. R. Ondis and J. Spanier, MARC-A Multigroup Monte Carlo Program for the Calculation of Capture Probabilities, WAPD-TM$273(1962)$.

10. M. H. Kalos, "Monte Carlo Integration of the Adjoint Gamma-Ray Transport Equation," Nu. Sci. and Eng., 33, 284-290 (1968).

11. J. Spanier and E. M. Gelbard, Monte Carlo Principles and Neutron Transport Problems, Addison-Wesley Publishing Co. (London and Reading, Mass., 1969).

12. G. Goertzel and M. H. Kalos, Progress in Nuclear Energy, D. J. Hughes, Ed., Series I, 2, 315-369, Pergamon Press (New York, 1958).

13. L. B. Levitt, A New Use of Adjoints in the Monte Carlo Estimation of Resonance Integrals, AEC Research and Development Report NAA-SR12494 (September 5, 1967). 
TIIE USE OF THIRD GENERATION COMPUTERS IN MONTE CARLO CRITICALITY CALCULATIONS*

by

\author{
N. F. Cross \\ Computing Technology Center \\ E. C. Crume \\ Y-12 Plant
}

March 1969
Union Carbide Corporation
Nuclear Division
Oak Ridge, Tennessee

*This document is based on work performed at the Computing Technology Center operated by Union Carbide Corporation for U. S. Atomic Energy Commission. 
The advent of third generation computers such as the IBM 360 series has made possible the economic application of Monte Carlo techniques to solve many practical nuclear criticality problems without resorting to significant geometrical simplifications. The characteristics of these computers that contribute most to this application are: (1) the decreased characteristic operation time, (2) the increased fast memory capacity, and (3) the decreased input/output device access time. These represent significant improvements over the previous generation computers.

The decreased operation time is important because the Monte Carlo technique requires many program statements to be executed thousands to millions of times in the solution of a single problem, and each statement consists of several operations. The IBM 360 series computers have operation times of the order of microseconds. The operation times of the IBM 360 Model 65 are from 3 to 5 times shorter than that of the 7090 series computers of the previous generation. The IBM 360 Mode 175 has operation times 5 to 8 times faster than the IBM 7090 series. Thus, problems which previously would have demanded inordinate amounts of computing time may now be attacked.

The increased fast memory capacity is important because it allows the detailed description necessary to solve geometrically complicated problems with numerous different material constituents without requiring simplifications to fit more limited fast memory capacity. Up to a factor of 100 times the fast memory capacity of the 7090 series is possible; in general, however, economics would dictate a smaller increase. For example, the IBM 360 Model 65 at the Oak Ridge Computing Technology Center has a factor of 4 greater capacity than an IBM 7090.

The decreased access time of input/output devices now available such as disc packs and data cells reduces time spent on the computer. llowever, this is only a second order benefit.

An example of a computer program which makes effective use of these characteristics in KENO. $1,2,3$ KENO is a Monte Carlo nuclear criticality code which uses multi-energy-group neutron interaction cross sections. It may be used to calculate the criticality condition of individual systems containing fissile material and finite or infinite spatial arrays of such systems. Is mentioned above, KENO makes use of the large fast memory to store a truly three-dimensional geometrical description of a system and, if required, the description of a three-dimensional spatial lattice of individual systems, either finite or infinite. Such descriptions are more accurate than the usual one- or two-dimensional approximations that are necessary to prevent exceeding the fast memory capacity of earlier computers.

$\Lambda$ modification of KENO which requires a very large fast memory capacity: is one in which the neutron interaction cross sections are not averaged over energy ranges into a so-called group structure but are entered as individual points. This type of cross section representation is the most suitable for a nuclide which exhibits resonance behavior. A second modification which uses large amounts of fast memory is the addition of the 05R geometry package, GEOM. 4 This package allows the geometrical description of irregular shaped systems such as the one shown in figure 1.

As in other Monte Carlo criticality programs, the behavior of individual neutrons is tracked from birth in the system to death or escape from the system. This information is used to determine the criticality condition of the system. A track consists of the numerous interactions a neutron undergoes with the materials in the system during its life. To generate these tracks, it is necessary that major Fortran loops be executed a very large number of times during a calculation. The compilers available for the third generation of computers provide a two-pronged attack on the problem of increasing the efficiency of these major Fortran loops. First, these new compilers allow larger programs to be written, making it possible to save computing time by 


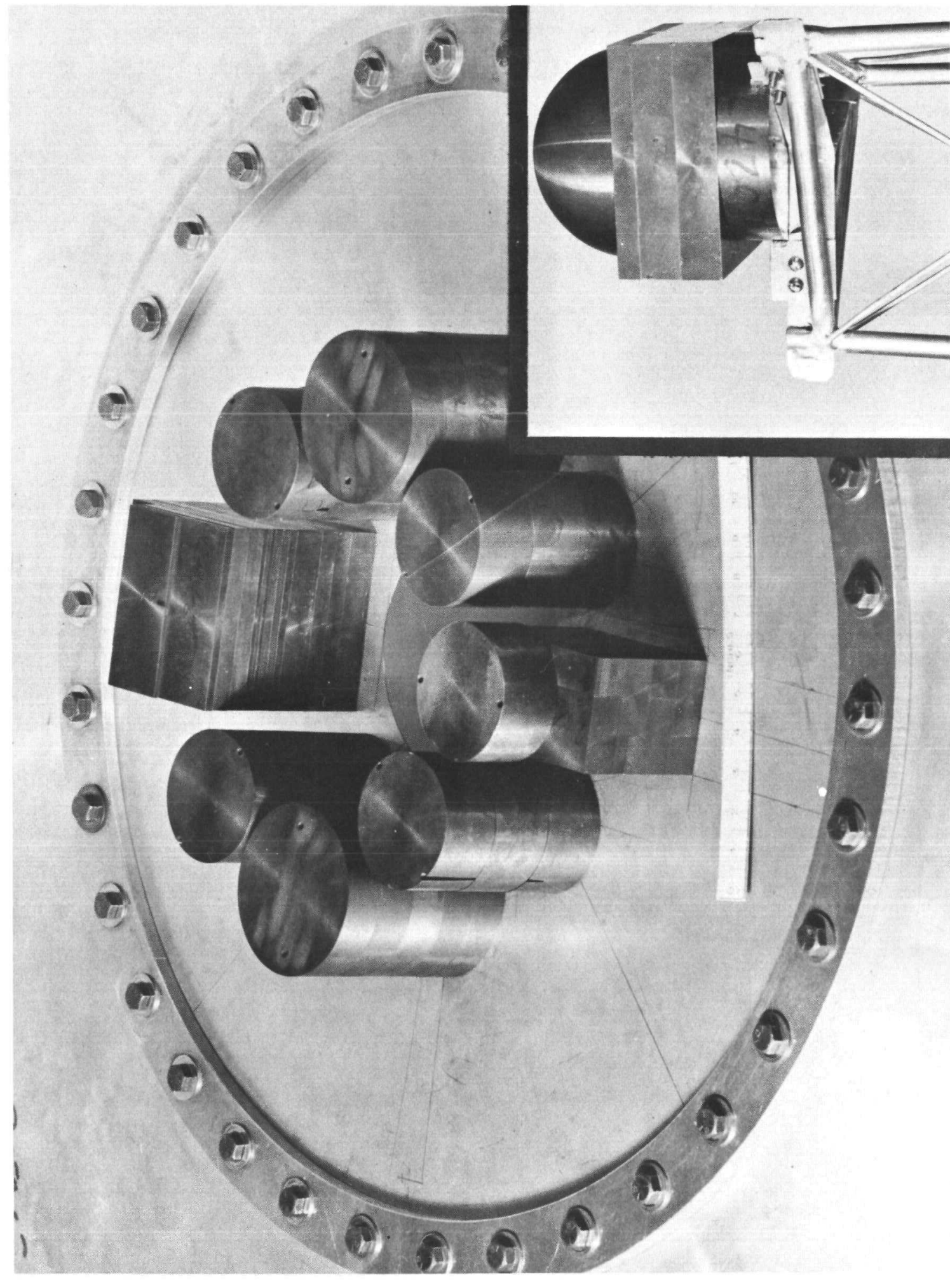

Figure 1. A complex critical uranium metal system requiring the use of the GEOM package to adequately describe the geometry. 
incorporating the Fortran statements equivalent to a number of external subroutines directly in the major loops. Secondly, computing time was saved by using the program compilation optimization options of these new compilers. In some instances a factor of two or more was gained by using the highest level of optimization. Because some programs are not amenable to optimization, it is necessary to subject calculational results to an independent check when using the higher levels of optimization. Once the validity of the results has been established the program may be assumed to be amenable to optimization. However, this procedure must be repeated each time a program change is made and the optimization options are employed.

In the most recent versions, typical KENO execution times, for results at a very acceptable level of statistical uncertainty, range from less than 2 minutes for geometrically simple solid metal systems to several hours for geometrically complicated systems with low atomic number neutron moderators and reflectors. The mean execution time for routine problems falls in the 10 to 30 minute range. The index of criticality used is the k-effective of a system and is defined to be exactly unity for a just critical system. The acceptable level of uncertainty referred to above corresponds to $0.5 \%$ in k-effective. It is to be noted that much greater uncertainty in the results is often allowable for systems where k-effective is $10 \%$ or more from just critical. In such cases an acceptable answer to a practical problem can be obtained in a few minutes computing time whereas it might take an hour or more to reduce the statistical uncertainty to the $0.5 \%$ level.

Some examples of experimentally critical systems that have been calculated successfully using KENO are shown in figures $1,2,3$, and 4 .

Figure 1 illustrates a critical assembly ${ }^{5,6}$ of uranium metal enriched to 93.2\% in the 235 isotope, consisting of eight units sitting on a diaphragm with an irregularly shaped centerpiece positioned in the center hole of the diaphragm. This centerpiece is shown in the insert of figure 1 . The eight units consist of an approximate parallelepiped with an irregular top, a parallelepiped, and six cylinders of assorted sizes. The centerpiece, which penetrated the hole in the support diaphragm consists of a cylinder topped by a parallelepiped topped by a hemisphere. The KENO calculation for this system yielded a $k$-effective of $1.002 \pm .005$ in 10 minutes.

Figure 2 illustrates a hydrggeneously moderated array of eight $20.9 \mathrm{Kg}$ units of $93.2 \%$ enriched uranium. Each unit consists of a cylindrical metal unit contained in a plexiglas parallelepiped having a wall thickness of 1.27 $\mathrm{cm}$. KENO calculated a k-effective of $0.995 \pm .008$ for this system in 15 minutes.

Figure 3 consists of a composite array ${ }^{7}$ of four $20.9 \mathrm{Kg}$ of $93.2 \%$ enriched uranium metal units and four $2.1 \mathrm{Kg}$ of $92.6 \%$ enriched uranyl nitrate solution contained in a plexiglas bottle. KENO calculated a k-effective of $1.005 \pm$ .008 for this array in 9 minutes.

Figure 4 illustrates a critical system of 125 units, each consişting of a cylindrical plexiglas container filled with uranyl nitrate solution. Each unit contains $1.92 \mathrm{Kg}$ of U-235. A k-effective of $0.994 \pm .009$ was calculated by KENO in 18 minutes.

One of the most recent additions to the capabilities of the KENO program is that of varying certain parameters of a system to satisfy given criticality conditions. Such techniques are usually referred to as criticality searches. These searches have been used primarily in solving nuclear criticality safety problems such as finding the maximum safe sizes of storage and shipping arrays and the maximum safe sizes of fissile units in fixed arrays. The main advantage of using KENO for such searches is that it can treat three-dimensional arrays that are finite in all dimensions without using empirical corrections to infinite array results. lowever, criticality searches using Monte Carlo techniques have been prohibitively expensive in the past because of the large 


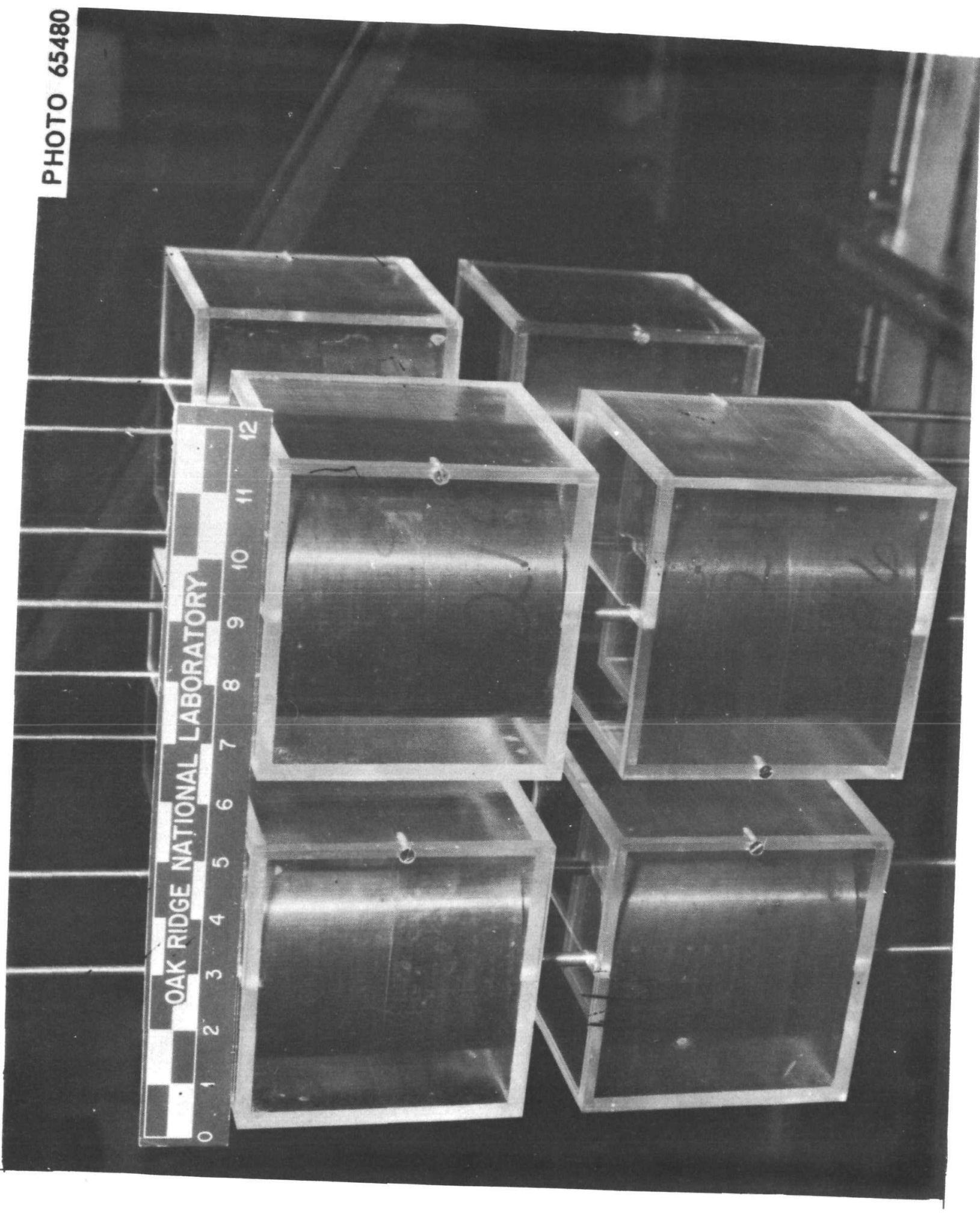

Figure 2. A critical moderated array of eight metal units. 


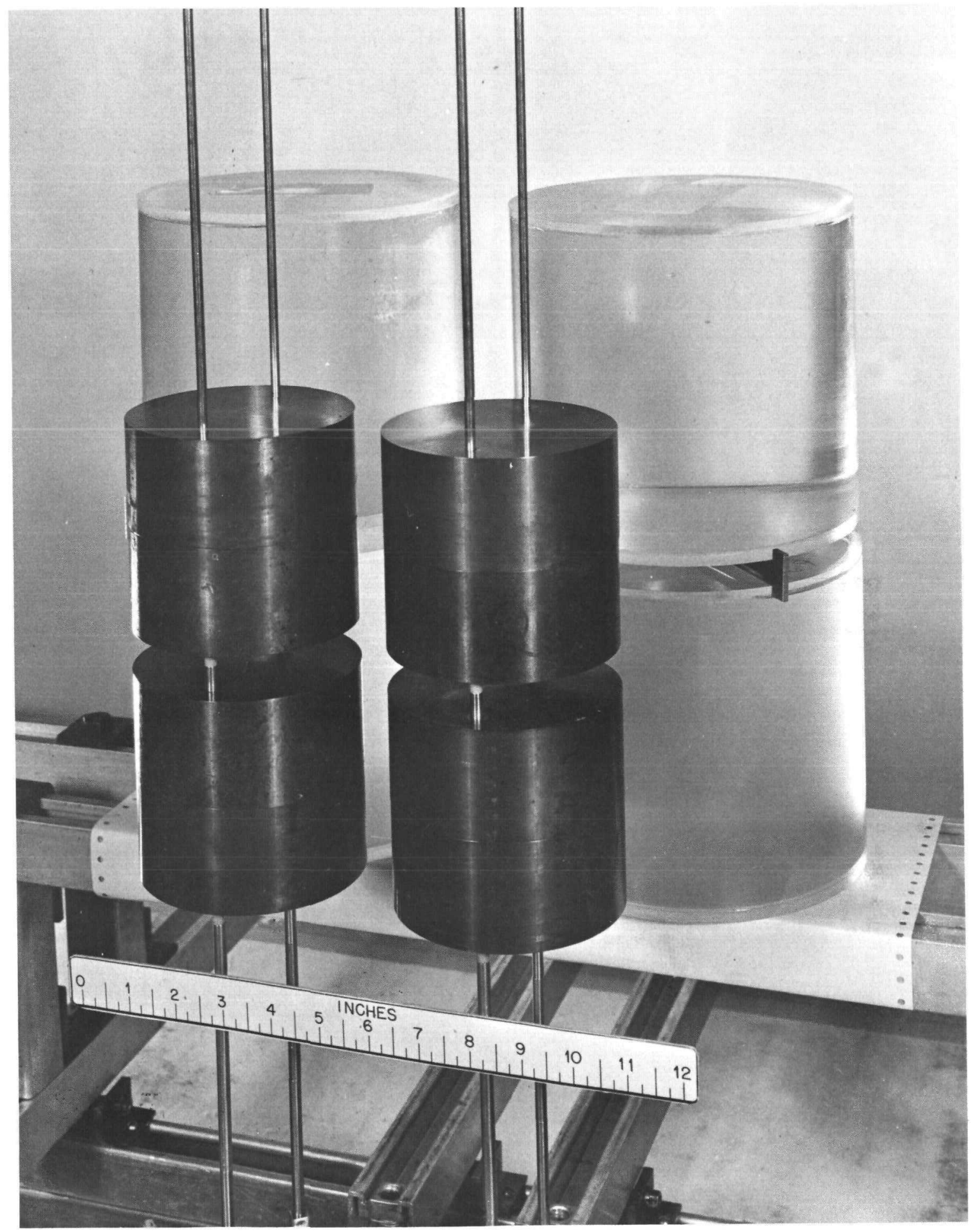

Figure 3. A critical system composed of four uranium metal units and four units of uranyl nitrate solution in plexiglas containers. 


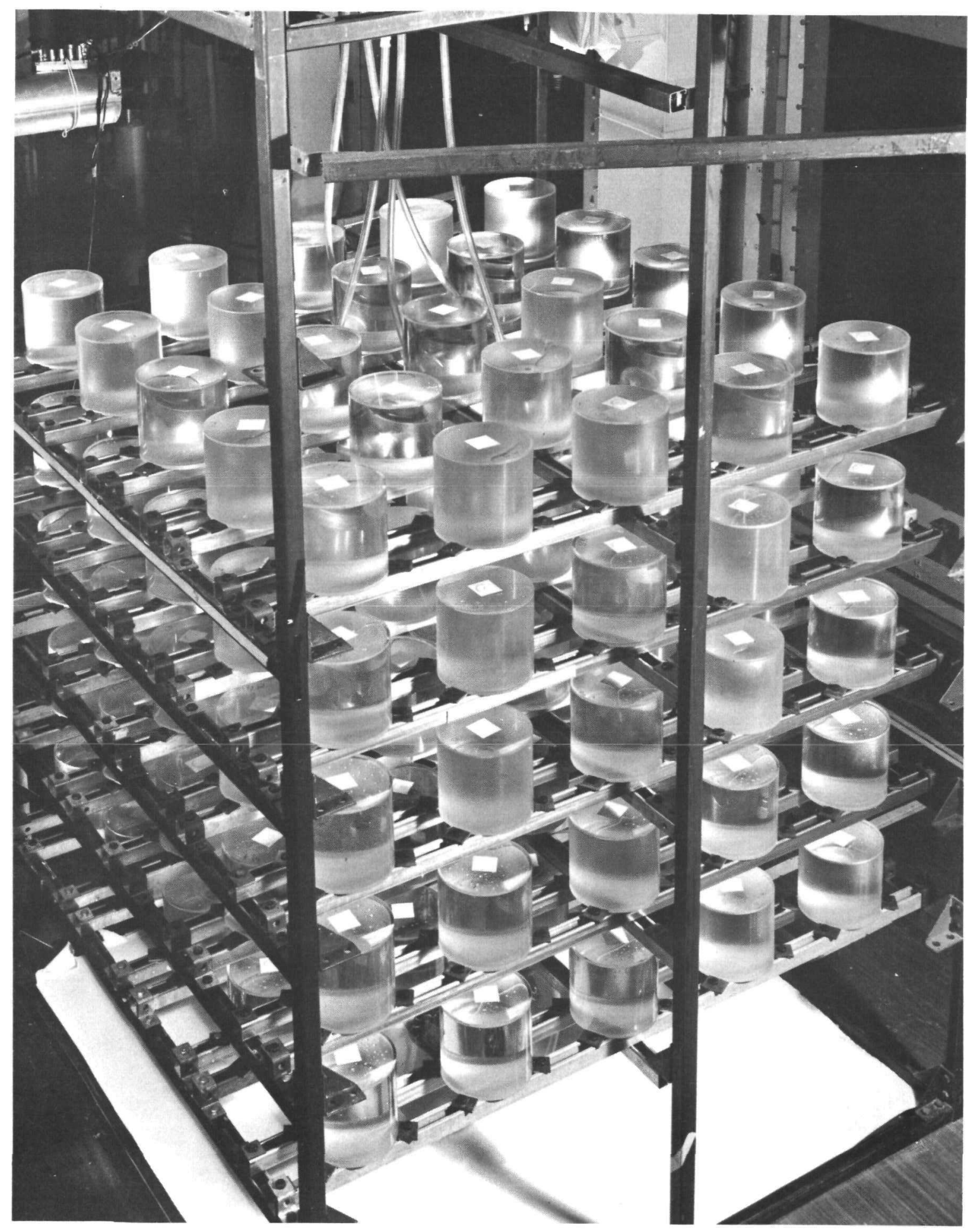

Figure 4. A critical system composed of 125 units of uranyl nitrate in plexiglas containers. 
amounts of computer time required for the solution of a single problem. Because KENO executes so rapidly, it is now practical to conduct such searches. Several search procedures have been implemented; however, because there are so many possible variations within the program's framework, it is doubtful that one generalized search package can be written. Following is a brief description of some of the search packages.

Center-to-center spacinf search. This search package is extremely useful in the determination of the minimum safe spacing of a predetermined number of fissile units. It's most important application is in the shipping and storage of nuclear materials. The center-to-center spacing between fissile units in a rectangular lattice configuration may be determined for any desired value of k-effective. The user may specify the desired value of k-effective, the desired level of uncertainty in k-effective, and the maximum number of iterations to be allowed. The search is terminated when (1) k-effective is within the prescribed limits, (2) the maximum number of iterations have been executed, or (3) the fissile units touch (i.e. the spacing can never get small enough to achieve the desired value of $k$-effective.)

Dimension search. The size of any one or more regions within a unit can be varied to obtain the desired value of k-effective. This search is limited to the alteration of the size of spheres, cylinders, cubes, and rectangular parallelepipeds. The search is terminated when (1) k-effective is within the prescribed limits, (2) the maximum number of iterations have been executed, (3) the region to be altered becomes so small it no longer exists or becomes so large it comes into contact with the next larger region. It should be noted that the size of one region may be altered while holding all other regions constant or several concentric regions may be altered simultaneously.

Search on the number of units in an array. Work is currently underway to develop a technique to search on the number of units in an array. This search package will be useful in determining the maximum number of specified units with a specified spacing that can safcly be stored or shipped.

All of these search packages utilize linear extrapolation techniques in the attempt to achieve the desired criticality condition.

The third generation computers constitute a significant contribution to the development of Monte Carlo criticality codes such as KENO. Without their increased fast memory capacity it would be difficult to adequately describe both the geometry and the properties of the constituents of the system under investigation. The decreased operation time has made possible the economical calculation of the criticality condition of simple and complicated units of fissile materials and spatial arrays of such units. It also enables KENO to conduct criticality searches. Thus problems involving nuclear criticality safety may be attacked in a more accurate and economical manner than before. 
1. G. E. Whitesides, G. W. Morrison, and E. C. Crume, "Few Group Monte Carlo Criticality Calculations," Trans. American Nuc1. Soc. 9, 133, (June 1966).

2. G. E. Whitesides and N. F. Cross, "On the Treatment of Hydrogen Scattering in Multigroup Monte Carlo Criticality Calculations," Trans. American Nucl. Soc. 10, 239, (1967).

3. G. E. Whitesides and N. F. Cross, KENO-A Multigroup Monte Carlo Criticality Program, CTC-5, (to be published).

4. D. C. Irving, R. M. Freestone, Jr., and F. B. K. Kam, 05R, A GeneralPurpose Monte Carlo Neutron Transport Code, ORNL-3622, or TID-4500, (Feb. 1965).

5. D. C. Irving and J. T. Mihalczo, Monte Carlo Calculations for Enriched Uranium Metal Assemblies," Trans. American Nucl. Soc. 7, 284, (Nov. 1964).

6. J. T. Mihalczo, Multiplication Factor of Uranium Metal by One-Velocity Monte Carlo Calculations, ORNL-TM-1220 (August 19, 1965).

7. J. T. Thomas, Critical Three-Dimensional Arrays of Neutron-Interacting Units, Part II, ORNL-TM-868, (July 1964).

8. J. T. Thomas, Critical Three-Dimensional Arrays of Neutron-Interacting Units, ORNL-TM-719 (1963). 


\title{
AN EXPERIMENTAL MONTE CARLO PROGRAM FOR THREE DTMENSIONAL NUCLEAR SYSTEIMS
}

\author{
Charles S. Shapiro* \\ IBM Scientific Center \\ Palo Alto, California
}

\section{INTRODUCTION}

The introduction of larger, more powerful computers requires a re-examination of how one constmets algorithms to solve a problem. The most efficient method of solving a large scientific problem with a computer can be a very sensitive function of the speed of the central processing unit (CPU), the size and speed of fast and auxiliary memory devices, the size and speed of I/O devices, etc. An algorithm efficient for one computer may be completely inefficient for a computer of a different architecture. The goal of this project was to investigate the feasibility of utilizing the Monte Carlo technique for solving a large threedimensional nuclear reactor design problem. It was simulated by the advent of the large and fast digital computers available today. A more complete and detailed report on this work appears in reference 1.

Finite Difference Approaches: The two most popular approaches for solving the neutron transport equation for reactor design involve the discrete ordinate method $\left(D_{n}\right)^{(2)}$, and the diffusion approximation. Both of these approaches utilize various approximations that reduce the transport equation to systems of finite difference equations. Because of the size of these problems and the available computing power, most of these codes are one-dimensional codes and a few are twodimensional. There are also some three-dimensional codes, but these can handle only very small problems. The realization of a practical three-dimensional code is much sought after by the nuclear reactor industry as its use could result in greater understanding and economies in reactor design and operation.

When one examines the new computers available today, the big advance vis-a-vis these reactor problems is the large increase in computational speed of CPU's. There are also increases in the size and speed of fast memory, and some improvement in I/O abilities, however, the increase in CPU speed outpaces the other improvements. In examining how one would solve a three-dimensional DS problem or a three-dimensional diffusion theory problem of realistic size on the large computers available today, one can quickly recognize that these problems are severely I/O bound and still cannot be handled economically.

The Monte Carlo Approach: It is for the above reasons that the Monte Carlo approach was investigated, as it appeared possible to keep most of the computation core-contained using this approach. Hence, the large increase in CPU speed

$\begin{aligned} \text { *Present Address: } & \text { Department of Physics } \\ & \text { San Francisco State College } \\ & \text { San Francisco, California }\end{aligned}$


could be effectively utilized. Therefore, of the many Monte Carlo formulations available, we have chosen an approach which allows all of the data required to solve for the fission source to be stored in fast memory. As the number of spatial zones in a realistic problem can easily number in the hundreds of thousands, we limit ourselves to storing only three-dimensional arrays in fast memory. The scalar neutron flux, which is of four dimensions (three space and one energy) is not determined until after the fission source (three dimensions) has converged to a desired accuracy.

Another important reason why a Monte Carlo approach can be advantageous is due to the well known fact that in numerical integration (essentially we are numerically integrating the transport equation) the efficiency of Monte Carlo improves vis-a-vis a finite difference approach as the number of dimensions in the problem increases. One should observe in the description of the Monte Carlo program that appears later in this report, the amount of work performed per neutron history is very insensitive to the number of dimensions.

In light of the wide interest today in fast reactors, another motivation behind a Monte Carlo program for reactor design is the advantages of this method for fast reactors vis-a-vis thermal reactors.

The Transport Equation: We seek the solution for the neutron flux $\phi(E, \vec{r}$, $\Omega)$ to the time independent neutron transport equation that is everywhere real and positive, and we also seek its associated eigenvalue $\mathrm{k}$. The value of $\mathrm{k}$ yields the effective multiplication constant of the assembly. A significant factor in our approach is that the fission source can be written $\frac{\chi(E)}{k} s(\vec{r})$, where $X(E)$ is the fission spectrum. We write it in this manner to explicitly show that since we know $\chi(E)$, the unknown part is just a function of the three spatial coordinates, This fact is of central importance in our method which solves first for $S(\stackrel{3}{r})$, a three-dimensional array. Our approach will be to assume an initial $S(\hat{r})$, and use it to generate one generation of neutron histories. This in turn yields a new fission source. This new source is used to create another generation of neutron histories and another new fission source. The process is repeated for as many iterations as necessary for the source $S(\vec{r})$ to converge to the correct solution. Knowledge of $S(\vec{r})$ yields directly the power distribution in the reactor which is of prime interest in reactor design. These iterations on the source, when compared to $\mathrm{DS}_{\mathrm{n}}$ and diffusion theory, are essentially the "outer iterations".

For the special case of an infinite homogeneous medium, we can easily solve for $\mathrm{k}$ in a multi-group approximation, and hence we use this as a test case in checking out the program.

\section{PHYSICAL ASSUMPTIONS}

In an investigative program of this type, it was felt that it would be best to keep all physical assumptions as simple as possible while at the same time not detracting from the purpose of the investigation. The purpose here was to examine the feasibility of the Monte Carlo approach for reactor design. Using simple geometry and elementary physics models yields conclusions about the Monte Carlo approach while avoiding the burden of extraneous details. At worst this approach would shed some doubt on the relationship of our numerical results to a real reactor, but this is not our primary purpose here. Also, Monte Carlo programs are generally built up in modules (for example, a geometry subroutine, a collision subroutine, etc.), and it is an easy matter to exchange these modules with more sophisticated ones. 
The physical assumptions we have used in the program are:

1. Energy Dependence: We assume discrete energy groups.

2. Geometry: The geometry is determined by intersecting planes that are parallel to the cartesian coordinate planes. Provision is made for specularly reflecting planes to take advantage of symetric reactors.

3. Material Composition: We assume the material in each spatial zone is homogeneous throughout the zone. Each material has its own cross-section set which is referred to by the integer stored in $\operatorname{MR}(i, j, k)$, where $i, j, k$ denotes the zone.

4. Neglect of Neutron Flux: Although we are actually considering the full angular dependent neutron flux when we follow each history through its life in the reactor, we shall record only the fission source and discard the information generated about the energy dependent neutron flux. Otherwise we would have to store a four-dimensional array which would not fit into fast memory. It is only after the fission source has converged that we calculate the scalar neutron flux. To accomplish this we must output intermediate results about the neutron histories onto external storage devices, and then process these records to determine the flux.

5. Isotropic Scattering: We assume scattering events are isotropic in the laboratory frame of reference.

6. Transport Approximation: To counteract the inaccuracy resulting from the use of the isotropic scattering approximation, use is made of the transport approximation. Here, the total cross-section $\Sigma_{\mathrm{T}}$ is replaced by the transport cross-section $\Sigma_{T R}$ where $\Sigma_{T R} \equiv \Sigma_{T}-\bar{\mu} \Sigma_{S}$ and $\bar{\mu}$ is the average cosine of the scattering angle. To preserve neutrons, the ingroup scattering cross-section $\Sigma_{S i} \rightarrow i$ and the total scattering cross-section $\Sigma_{S i}$ are adjusted so that $\Sigma_{S i}=\Sigma_{j} \Sigma_{S i j}$ and $\Sigma_{T_{i}}=\Sigma_{S_{i}}+\Sigma_{C_{i}}+\Sigma_{F_{i}}$. Here $\Sigma_{C_{i}}$ is the capture cross-section in group i.

7. Source Generation: Neutron histories from the source in each zone are generated spatially uniformly throughout the zone.

8. Cross-Sections: Macroscopic cross-sections are generated for each material. These are calculated in a separate computer program from the microscopic cross-sections and the isotopic concentrations of the elements that make up each different material.

9. Track Length Method: The track length method of generating fissions is used. In this method one generates fissions from each track length the neutron undergoes by multiplying the neutron track length in a zone (the neutron flux) by the fission cross-section. In a fast reactor like the ZPR-III, a neutron will typically undergo 20 collisions before it is absorbed. If one uses the collision method then all information generated by the 20 previous track lengths are lost. This method is also superior when a zone is very thin.

\section{DESCRTPTION OF THE PROGRAM}

A flow chart of the program appears in Figure 1. The input data includes specification of the geometry, the identification of reflection planes (if any), the specification of which material is in which geometrical zone, the number of energy groups, the velocity mesh, the initial source, various convergence criteria, and whether we want to converge on the total $k$, the regional $k$, or whether it is a fixed source problem (for shielding calculations). The cross-sections are read in for each material type. These include total, scattering, transport, $\cup \Sigma_{F}$ (fission), the fission spectrum and the group to group scattering matrix.

\section{Preparation of Source}

The initial source can be read in via cards, or generated by the program. 
FIGURE 1

Monte Carlo Flow Chart

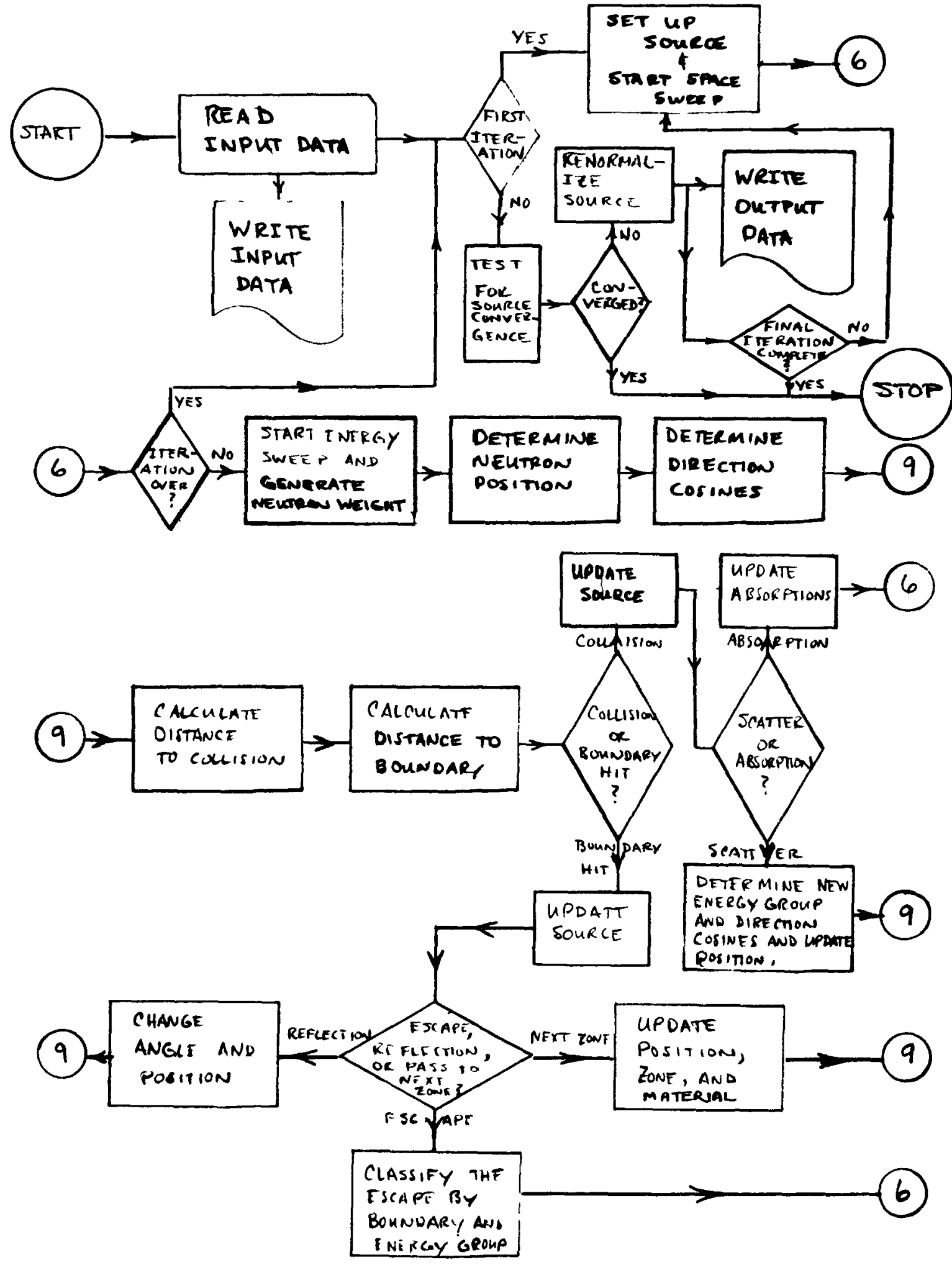


Experience has shown that a good source to start with is a flat distribution for the zones which contain fissionable material. This insures that there is some contribution to all of the energy-space harmonics. Then as the iterations take place, the transients should die away leaving only those modes that represent the steady-state source distribution.

Calculation of the Fission Source: The initial source before an iteration is called SOLD $(i, j, k)$. As each track length d occurs in any zone $i, j, k$, the quantity du $\Sigma_{F} W$ is accumulated in the array $\operatorname{SNEW}(i, j, k)$, i.e., the new source. Here $\mathrm{d}$ is essentially the neutron flux, $\mathrm{d} \Sigma_{\mathrm{F}}$ is the density of fissions in the zone, $\mathrm{d} \cup \Sigma_{\mathrm{F}}$ is the number of neutrons emitted from these fissions, and $\mathrm{W}$ is the weight of the neutron. When all of the neutron histories have depleted SOLD $(i, j, k)$, we sum up all the elements of SNEW, and the ratio of the total number of neutrons in SNEW to the total number in SOLD yields $k$, the multiplication constant of the reactor. The ratio of SNEW and SOLD for each zone yields a $k$ for each zone, which we call the regional multiplication constants. These are also calculated but only the maximum and minimum ( $k_{\text {MAX }}$ and $k_{M T N}$ ) of these are printed. We also calculate the quantity DLTASR which $1 \mathrm{~s}$ defined as

$$
\begin{array}{r|c|}
\operatorname{DLTASR}=\operatorname{MAX} & \operatorname{SNEW}(i, j, k)-\operatorname{SOLD}(i, j, k) * \mathrm{k} \\
\cline { 2 - 2 } & \operatorname{SNEW}(i, j, k)
\end{array}
$$

and reflects the maximum relative change of the regional sources from one iteration to the next. This quantity is used to determine convergence, as it is compared after each iteration to an input error criteria epsilon. Quantities also tabulated during each iteration include number of histories, number of absorptions, number of escapes, and number of times CUBE1 and CUBE2 (the geometry subroutines) are entered. For the last iteration, the distribution by boundary of escapes is also tabulated.

Source Weighting: After the source settles down to the "statistical region" (i.e., after a sufficient number of iterations when the source has converged to the point where its fluctuations in each zone are statistical in nature and cannot be reduced further) a provision was added to the program to weight the source after each iteration so that all the information from the iterations in the statistical region can be utilized. This effectively increases the accuracy of the source as the weighted source is based on all the histories followed once convergence is established into the statistical region. Experience with ZPR-III and the fictitious reactor indicates that starting with a flat initial source requires about four iterations to enter the statistical region.

Calculation of the Generation Time: Here we define the generation time as the average lifetime of fission neutrons from birth until death by absorption or escape from the system. This quantity is rather difficult to obtain using nonstochastic approaches, but is extremely easy to obtain using Monte Carlo. We define $D(i, j, g)$ as the $j^{\text {th }}$ track length in the life of the $i$ th history of neutrons with energy group $g ; W(i, j, g)$ as its weight for that track length; and $\mathrm{v}(i, j, g)$ as the velocity for that track length. Then the generation time $\bar{g}$ is given by the average over all the histories of the lifetime $t_{i}$ of the $i^{\text {th }}$ history.

$$
t_{i}=\sum_{j} \frac{D(i, j, g) W(i, j, g)}{v(i, j, g)}
$$

and

$$
\bar{g}=\frac{1}{W} \sum_{i} t_{i}
$$


where $\mathrm{W}$ is the total weight of all the neutron histories followed.

One problem in calculating $\bar{g}$ is that the answer is sensitive to the choice of velocity mesh, and is particularly sensitive to the velocity choice in the lowest energy group. This sensitivity can be alleviated somewhat by taking many velocity groups, or by modifying the code so that velocity is a continuous variable.

The array TRKTTM $(g)=D(i, j, g) W(i, j, g)$ summed over $i$ and $j$ is in itself a useful quantity in that it yields the energy dependence of the neutron flux averaged over the space for the entire reactor. For example, it is useful in calculating effective cross-sections. Hence this array is printed out after each generation (iteration).

Program Output Format: In the printout for each iteration, the data for the unweighted source is printed out first, followed by a listing of the unweighted source for that iteration; then the data for the weighted source is printed out, followed by a listing of the weighted source (used as the source for the successive iteration). In the data summary printout at the end of the program output, XKAYEF, XMMAXKR, etc., refer to the values for the weighted source, where as XKAY1, XMAXK1, etc., refer to the values for the unweighted source. For example, a typical data sumary is reproduced in Table 1.

Calculation of the Scalar Neutron Flux: The scalar neutron flux is a fourdimensional array and in general would be too large to store in fast memory. For this reason the Monte Carlo program is divided into two sections. The first is concerned with obtaining a converged fission source distribution. Once this is obtained, we proceed to calculate the flux as follows. Using the converged. source, we go through one final iteration with a very large number of histories. However, for this iteration, each time we generate a track length we record in a buffer array ARR1 values of the zone $i, j, k$ in which the track length occurs, the energy group $g$, the neutron weight $W$, and the track length $d$. If the track length ends in an absorption, we indicate this by a negative sign in front of $g$. When the buffer array is filled, these records are outputted to an auxiliary storage device (e.g., tape). After the completion of this last large iteration, another program, TDC, takes the tapes and sifts and sorts the information on them to obtain values of the neutron flux and absorption distributions.

\section{DESCRIPTION OF PROBLEIMS TREATED}

We have treated in detail two kinds of reactors. The first is a fictitious reactor with simple properties and fictitious cross-sections which was utilized fully to check out the program and to investigate some of the properties of the method, the accuracy of the answers, the dependence of the answers on number of histories, spatial mesh spacing, etc. The second reactor treated in detail was the ZPR-III (Zero Power Reactor-III) assembly number 1A, which was constructed at Argonne National Iaboratory in 1955. This critical assembly is a fast breeder type of reactor and is typical of the kind of reactors for which the Monte Carlo program would be most suitable.

Fictitious Reactor: This reactor is a homogeneous cube $3 \mathrm{cms}$ on a side. Most of the test runs used two energy groups with $\Sigma_{\mathrm{T}}=2.0 / \mathrm{cm} ; \Sigma_{\mathrm{S}}=1.0 / \mathrm{cm} ; \Sigma_{\mathrm{F}}=1.0 / \mathrm{cm}$; $\Sigma_{\mathrm{C}}=0.0, \Sigma_{\mathrm{S} 11}=0.5, \Sigma_{\mathrm{S} 12}=0.5 ; \Sigma_{\mathrm{S} 21}=0.5 ; \Sigma_{\mathrm{S} 22}=0.5$, and $\chi=0.5$ for both energy groups. Of the many runs on this reactor, the parameters that were varied included spatial mesh size, different initial sources, different reflection planes that created essentially different reactors (e.g., all reflection plans $\rightarrow$ infinite homogeneous medium, a slab reactor is created by four plane parallel reflection planes, a two-dimensional infinite rectangle is created by two plane parallel 
TABLE 1

Typical Data Summary Output

\begin{tabular}{|c|c|c|c|c|}
\hline $\begin{array}{l}\text { XATION NUMBER OF } \\
\text { MEER SOURCE NEUTRONS }\end{array}$ & $\begin{array}{rl}X & T \\
\text { EFFECT } & -1\end{array}$ & $\begin{array}{l}\text { KREGIONAL } \\
\text { MAXIMUM }\end{array}$ & $\begin{array}{l}\text { K REGIONAL } \\
\text { MINIMUM }\end{array}$ & $\begin{array}{l}\text { NUMBER OF NUMBER OF NUMBER OF NUMBER OF } \\
\text { HISTORIES COLLISIONS ABSORPTNS ESCAPES }\end{array}$ \\
\hline
\end{tabular}

DLTASR

$17.029650 E$ O2 $6.508977 E-01 \quad 2.345065 E$ OO $\quad 3.717525 E-01$

$\begin{array}{llll}1296 & 75197 & 7.4803 E & 02 \\ 1425 & 23906 & 8.4773 E & 02 \\ 1476 & 31334 & 8.7659 E & 02 \\ 1488 & 29328 & 8.9859 E & 02 \\ 1507 & 31257 & 9.2771 E & 02 \\ 1526 & 32424 & 9.3135 E & 02 \\ 1518 & 31504 & 9.1569 E & 02 \\ 1508 & 30960 & 9.1617 E & 02 \\ 1512 & 30032 & 9.1599 E & 02 \\ 1513 & 31534 & 9.3114 E & 02\end{array}$

$3.3191 \mathrm{~F} \quad 02$

$7.5089 E-01$

$29.459153 \mathrm{E}$ O2 $8.758475, \mathrm{E}-01$ 1.66 $6645 \mathrm{E}$ 00 $6.543648 \mathrm{E}-J 1$

$3 \quad 2.010213 E$ 03 $9.353821 E-01$ 1..7U1709E $00 \quad 7.714223 E-01$

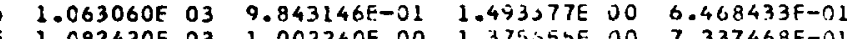

$1.082420 \mathrm{E}$ O3 $1.002240 \mathrm{EE}$ 00 $1.37555 \mathrm{SE}$ 00 $7.337468 \mathrm{E}-01$

$1.165026 \mathrm{E}$ O3 $1.072728 \mathrm{E}$ ON $1.102624 \mathrm{E}$ 00 $9.047053 \mathrm{EE}-\mathrm{O}$

$8 \quad 1.112263 E$ O3 $1.029873 E$ DO $1.103375 E$ DO $9.695753 E-01$

10 1.087716E 03 $1.007144 E 00 \quad 1.114460 \mathrm{E} 00 \quad 9.600026 \mathrm{E}-01$

9.159 GE 02

$2.0333 E 02 \quad 4.5033 E-01$

$1.5132 E$ O2 $5.2172 E-01$

1.5271 . 02 $3.6592 E-01$

1.48562 O?

$1.6421 E$ O?

. $2283 \mathrm{E}-01$

WEICHTS D

$\begin{array}{lll}1.6392 E & 02 & 9.6299 \mathrm{E}-02 \\ 1.4877 E & 02 & 6.596 \mathrm{AF}-02\end{array}$

XKAYEF, XMAXKR, XKINKR, DLTASR AND GENTHA

$0.1016332 E$ 01 $0.1098124 E-02$ C.3190605t-01

$0.1160653 E$ Ol $0.1029873 E-01$ 0.1014826t 00

$0.9009263 E$ OO $0.6625872 E-020.8139444 E-O 1$

. lslbszbe co 0.1100109E-0l 0.1040861e 00

0.879005 CE-07 $0.4170157 E-17 \quad 0.2042096 r-U$

$7.508378 E-01$
$4.732208 \mathrm{E}-01$

$4.732208 \mathrm{E}=01$
$4.503281 \mathrm{E}-0$

$5.217203 \mathrm{E}-0$

$3.659209 E-n 1$

$4.763213 E-01$

$4.178569 E-01$
$3.058176 E-0$

$3.475999 E-31$
$3.815590 E-01$

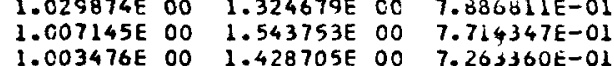
ITERATICNS 5 THROUGH 10

XKAYEF, XMAXKR, XMINKR, DLTASR ANO GENTIM

MEAN ON VARIANCE AND GENTIM DEVIATION.

$0.1016333 E$ O1 0.1018

$0.1391006 E$ Ol $0.6721731 E$ E 02 0.3190670E-01

$0.7349371 E$ CO $0.1049657 E-02$ 0.3239613E-OL

$0.8790 C 5 O E-07 \quad 0.4170157 E-17 \quad 0.2042096 E-C \theta$

XKAYEF $=$ XKAYEI $=$ Total effective multiplication factor

XMAXKR = Maximum Regional Multiplication Factor for the Core Only, Weighted Source

XMAXK $="$ " " " " " " " " " " " " " " " " " " " " XMINKR = Minimum " " " " " " " " " " " " " " " " " " " " " " " " XMINKI = " " " " " " " " " " " " " " " " " " " " " "

DLTASR = Maximum (Regional) Fractional Change in the Source in the Core Only, Weighted Source DLTASI $=$

GENTIM = Generation Time (Seconds)
UNWEIGHTED

SOURCE 
reflection planes, etc.). Because of the symmetry of this reactor, runs were made treating the whole reactor, half, one-quarter, and one-eighth of the reactor. The number of energy groups was also varied between one and eight. For the calculation of generation time, the velocities chosen for the two groups were $1.4 \times 10^{9} \mathrm{cms} / \mathrm{sec}$ ( $1 \mathrm{MEV}$ neutrons) and $2.2 \times 10^{5} \mathrm{cms} / \mathrm{sec}$ (thermal neutrons).

ZPR-III: A complete physical description of this reactor appears in reference 3. The ten energy group microscopic cross-section set used was taken from Table 14 of that reference. The three energy group microscopic cross-section set used was taken from reference 4, Table 7-5. This reactor is rectangular in shape and consists of a critical core of one composition surrounded by a blanket with a different material composition. It is fairly typical in its nuclear properties and should be representative of the fast power reactors that are being actively planned today. The dimensions of the core (in centimeters) are $30.7 \mathrm{x}$ $38.73 \times 45.4$ and the blanket extends $23 \mathrm{cms}$ beyond each core face.

\section{RESULTS}

In this section we shall discuss some of our results regarding various questions of interest. These include running times for realistic problems, rates of convergence, accuracy of answers, and effects of spatial mesh size and energy group structure on muning time.

In general, we have found that starting with a flat initial source leads to "convergence" of the fission source in four or five iterations. This compares favorably with $S_{N}$ calculations where typically $100(5)$ outer iterations are necessary. The number of iterations to "convergence" will vary somewhat depending on the problem, the space-energy mesh, the choice of initial source, and the definition of convergence. In Figure 2 we have plotted $k, k_{\text {MAX }}$, and $k_{\text {MTN }}$ as a function of iteration number for a ZPR-III run. This run had TOOO zones and an initial flat source of 25 particles per zone. We also include in this figure $k_{\text {MAX }}$ and $k_{\text {MTN }}$ for just the core region of the reactor. The fission source resulting Irom this run is too voluminous to present here (1000 regions). The distribution of escapes by boundary was as follows: $1.656 \times 10^{3}$ neutrons leaked from the $\mathrm{x}$ boundary, $1.332 \times 10^{3}$ from the $\mathrm{y}$ boundary, and $1.098 \times 10^{3}$ from the $\mathrm{z}$ boundary. As the initial source had 25,000 neutrons, this means $16.3 \%$ of the neutrons escaped from this reactor. This particular mun took a few more iterations to converge than usual because the reactor was divided into a $10 \times 10 \times 10$ mesh (equivalent to a $20 \times 20 \times 20$ mesh by utilizing the symmetry of the ZPR-III) with the core represented by a $6 \times 6 \times 6$ mesh. Hence the core contains 216 regions and the blanket 784 . As the fission source in the core regions are two orders of magnitude larger than in the blanket, there is a mismatch in the relative importance of the regions and the source takes longer to converge from the initially flat source used. Some test muns indicate a more efficient procedure is to begin with a small number of histories for a few iterations, and then multiply the resulting source everywhere by a constant factor for use as the source for a larger run. For the same ZPR-III run, we have listed in Table 2 the group dependent track length which is sumed over the entire reactor. This array is used in the calculation of the generation time and, as described earlier, is essentially the group dependent scalar neutron flux averaged over the entire reactor.

In Table 3 we list the results of a ZPR-III man. One can see that all of the quantities listed take about four or five iterations to converge. The standard deviations listed are based on iterations five through ten; the first four iterations are ignored. The $\mathrm{k}_{\mathrm{MAX}}, \mathrm{k}_{\mathrm{M} \amalg N}$, and DLTASR are listed both for the core and for the entire reactor. 
ZPR III PROBLEM - FIGURE 2

10-Group, $10 \times 10 \times 10$ Geometry, 25 Neutrons/Zone Initially Runs $652,654,655, \& 656$

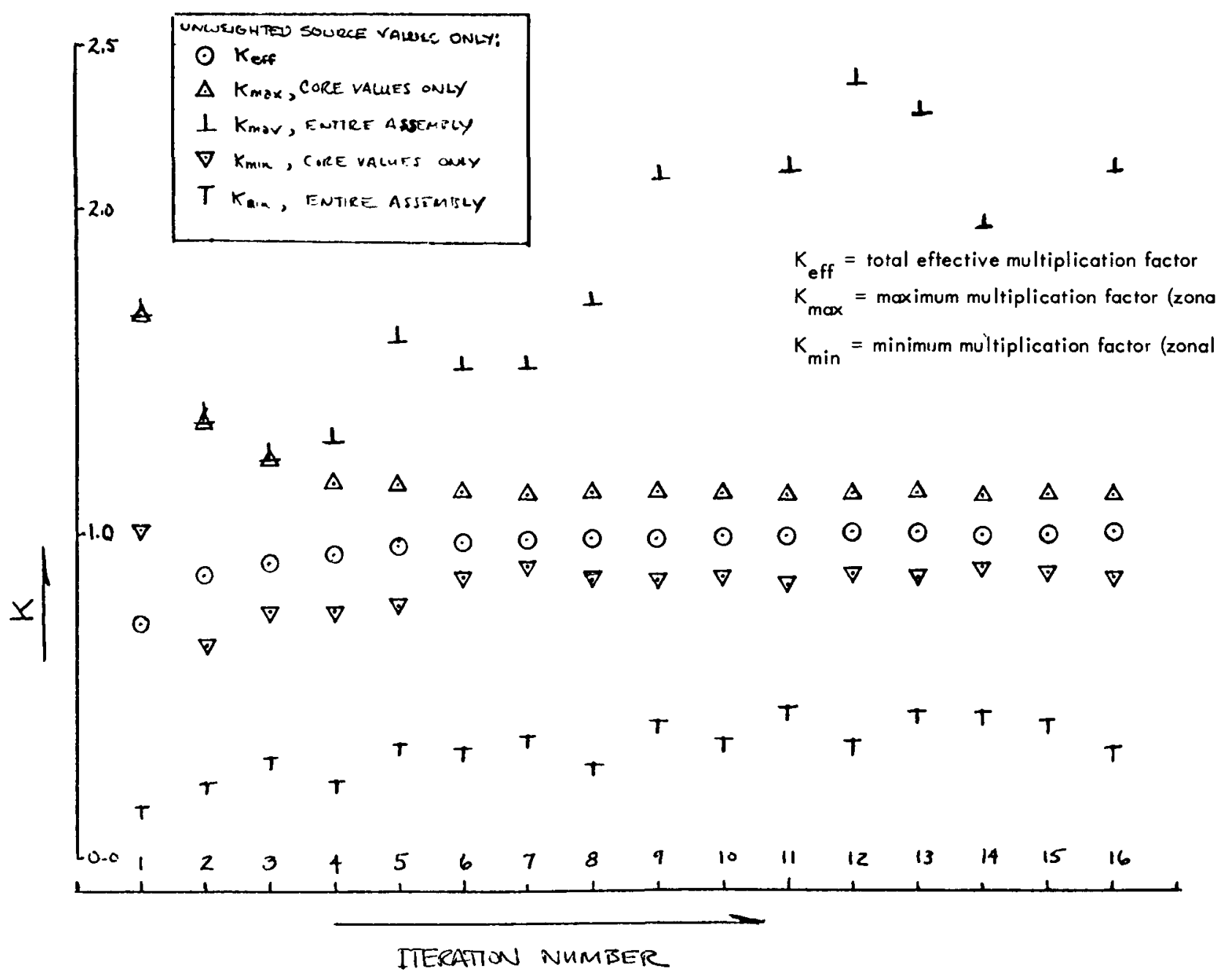


TABLE 3

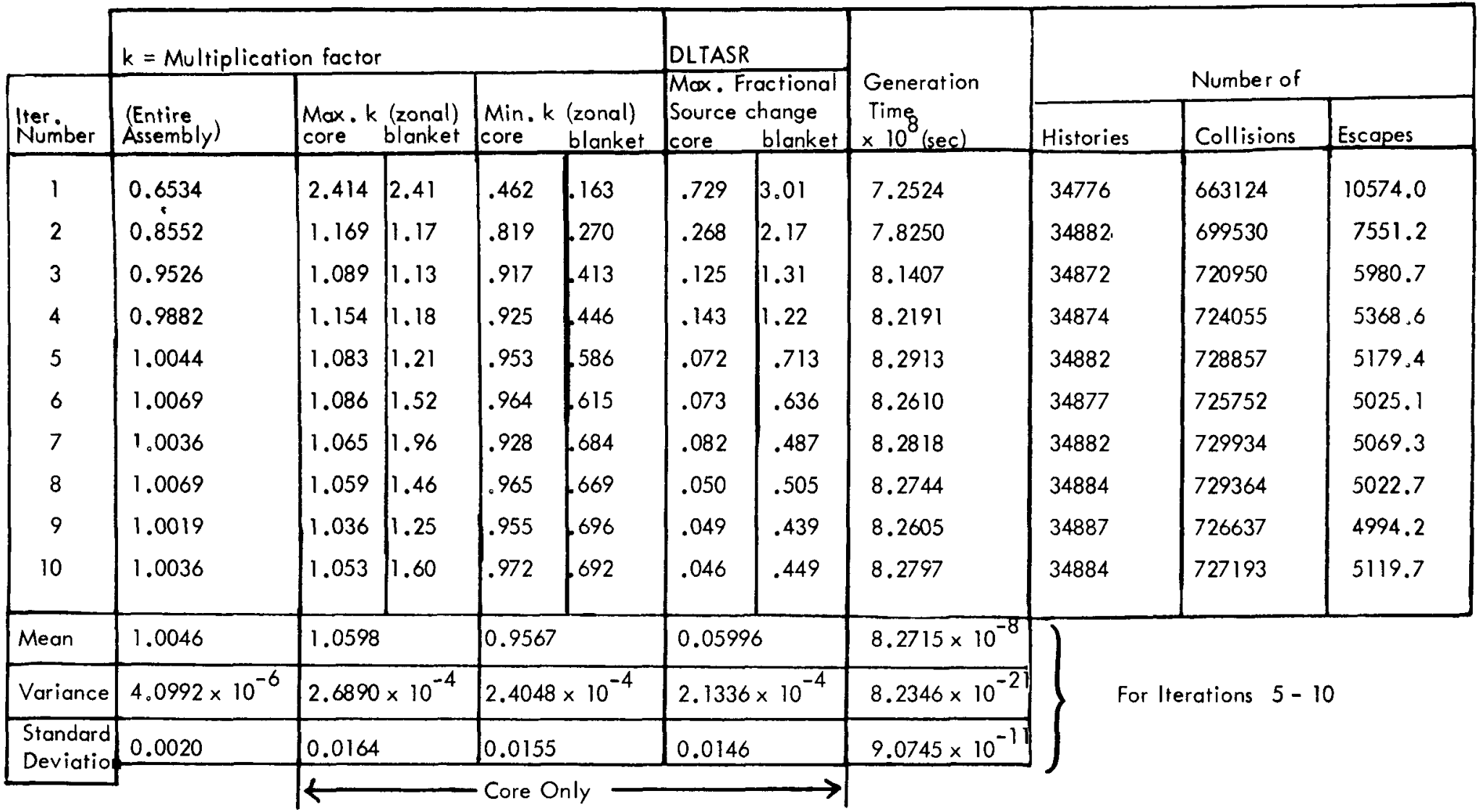

Initial Total Number of Neutrons $=34560$ (160 neutrons per zone)

Problem Description: ZPR III as described in Table 7-16 (Assembly 1A), ANL 5800, using three-group microscopic cross-section set of Table 7-5, ANL 5800. The geometry is $6 \times 6 \times 6$ (216 zones) with three reflecting planes ( $1 / 8$ of a $12 \times 12 \times 12$ rectangular assembly). Core is $4 \times 4 \times 4$.

Number of Absorptions $=34560-$ (number of escapes)

Runs "601\& 602 
TABLE 2

TOTAL NEUTRON TRACK LENGTH BY ENERGY GROUP

\begin{tabular}{ccc}
$\begin{array}{l}\text { Energy } \\
\text { Group }\end{array}$ & $\begin{array}{c}\text { Lowest Energy Bound } \\
\text { of the Group }\end{array}$ & $\begin{array}{c}\text { Total Neutron Track Length } \\
\text { (space averaged neutron flux) }\end{array}$ \\
\cline { 2 - 4 } 1 & 1.35 & $1.94485 \times 10^{5}$ \\
2 & 0.825 & $1.70244 \times 10^{5}$ \\
3 & 0.5 & $3.68280 \times 10^{5}$ \\
4 & 0.3 & $3.74189 \times 10^{5}$ \\
5 & 0.18 & $3.17896 \times 10^{5}$ \\
6 & 0.11 & $2.09187 \times 10^{5}$ \\
7 & 0.0674 & $1.55207 \times 10^{5}$ \\
9 & 0.025 & $1.10010 \times 10^{5}$ \\
10 & 0.0091 & $2.93189 \times 10^{4}$ \\
9 & 0.0 & $4.76518 \times 10^{3}$
\end{tabular}

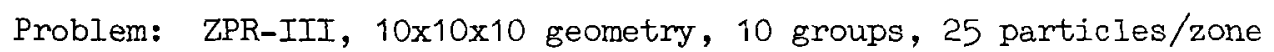

Run \#656, 16th iteration

Those external quantities referring to the entire reactor invariably occur in the blanket as the neutron population in these areas is much lower than in the core, resulting in much larger statistical variations in the blanket. For example in the run referred to in Figure 2, DLTASR in the core is a factor of ten smaller than is DLTASR in the blanket. This can be seen in Fig. 3.

One should note that these quantities (DLTASR, $k_{\text {MAX }}$, and $k_{M T N}$ ) are extremums of a large number of quantities, each of which has statistical variations. The average value of the regional $\mathrm{k}$ 's of course have a much smaller variation. Another reason these variations are large for the first few iterations is that the initial flat source is a very poor approximation for the outer zones.

The accuracy of the total $k$ varies as the inverse square root of the number of histories. For the mun with 35,000 histories per iteration, $(\sqrt{35,000})^{-1} \simeq .005$ and the relative standard deviation was .002. For a run with 1,500 histories per iteration, $(\sqrt{1,500})^{-1} \simeq .025$ and the relative standard deviation was .02 . This is typical of the many mus executed. The variation in the regional $\mathrm{k}^{\prime} \mathrm{s}$ goes roughly as the inverse square root of the source in each zone. The ratio of the number of histories in the two above runs was $35,000 / 1,500 \simeq 23, \sqrt{23} \simeq 5$, and so we expect roughly a factor of five improvement in accuracy in one case over the other. This is what is observed. The generation time, like the total k, is an integral quantity over the entire reactor, and therefore its accuracy also behaves as the inverse of the square root of the number of histories.

Table 4 has some interesting results using the fictitious reactor. Here we have varied spatial mesh size as well as initial number of particles. If one looks at the three cases with 5,400 particles with the three-dimensional cube, the error in the total $k$ is about the same. If one compares the $3 \times 3 \times 3$ mun with 27,000 particles to the $2 \times 2 \times 2 \mathrm{mu}$ (identical to the previous reactor except the symmetry of the reactor was used to treat only one-eighth of the reactor), the accuracy of the total $\mathrm{k}$ 's are roughly proportional to the square root of the ratio of particles as expected. In both these problems, however, the initial source per zone was 1,000, and so we see the $k_{\text {MAX }}$ and $k_{\text {MIN }}$ are about the same. 


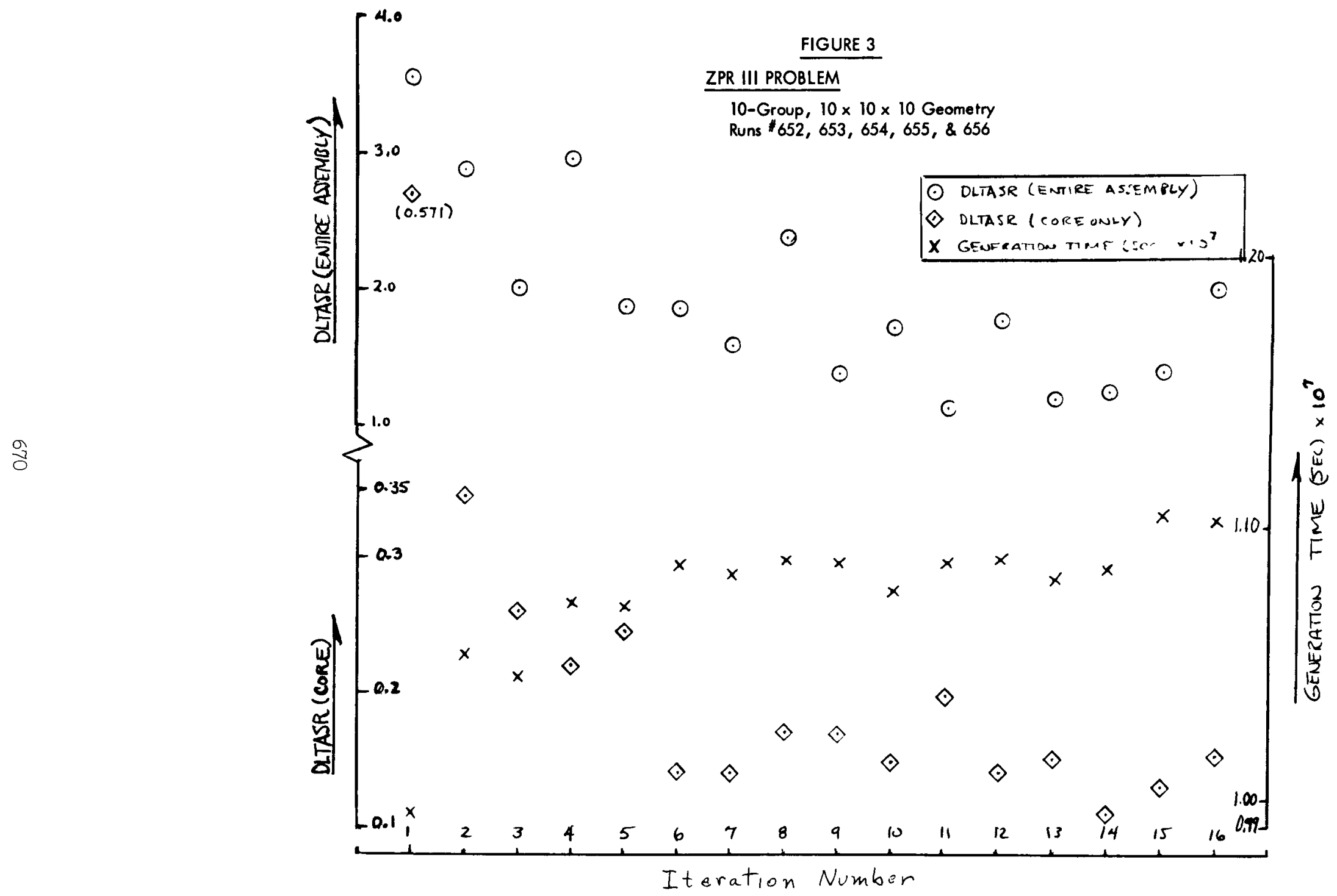




\section{FICTITIOUS REACTOR; CUBE (each side is $3 \mathrm{cms}$ )}

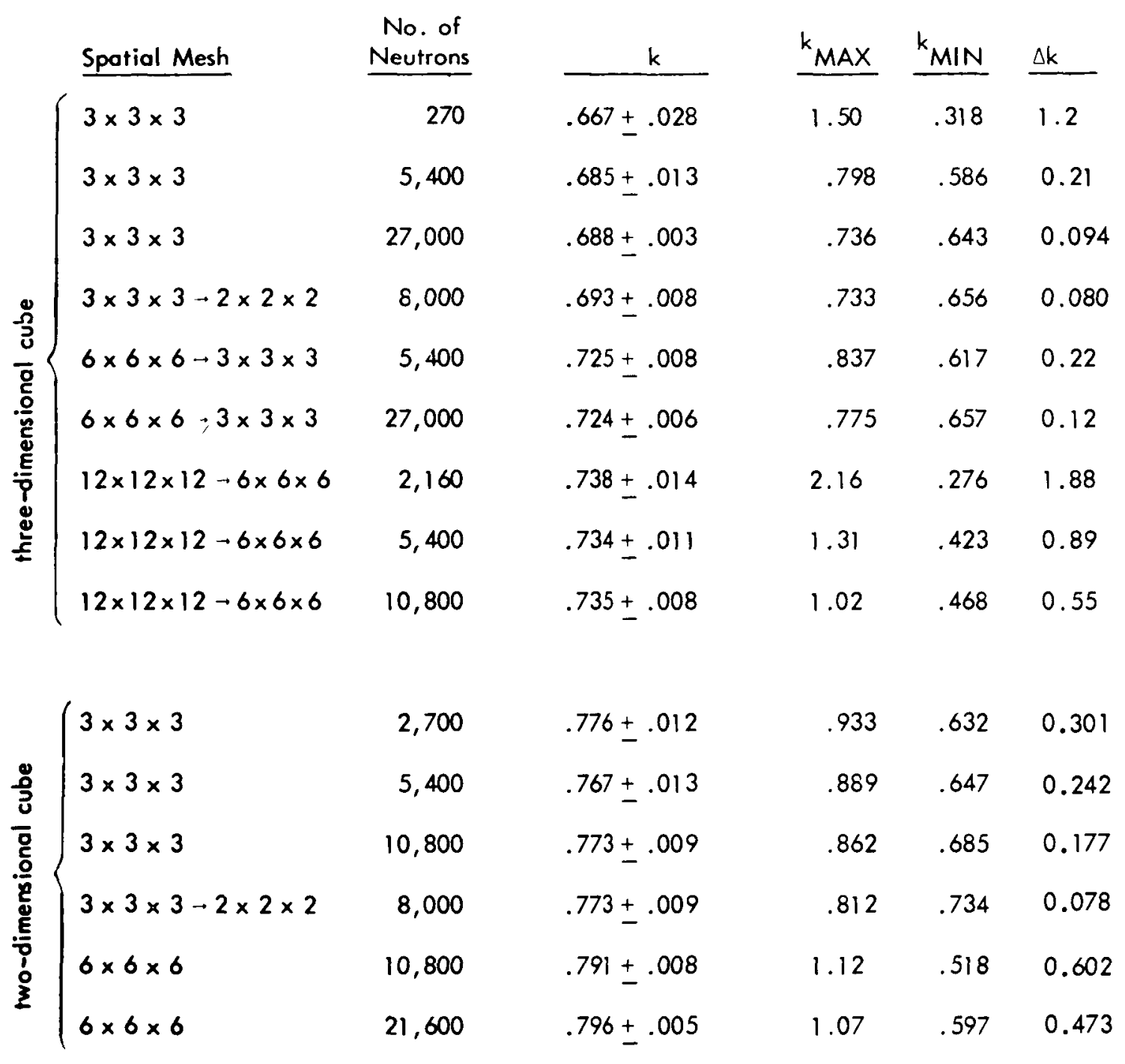

$\cdot k=k_{\text {MAX }}-k_{\text {MIN }}$ 
The spread in $k_{\text {MAX }}$ and $k_{\text {MMN }}\left(k=k_{\text {MAX }}-k_{\text {MmN }}\right)$ depends on the regional source intensity as expected.

The variation of $k$ with mesh size can be seen by comparing the $3 \times 3 \times 3,6 \times 6 \times 6$, and $12 \times 12 \times 12$ runs. The true fission source for this problem has a cosine distribution which we are approximating by a piecewise flat source. As the number of zones increases, the approximation to the true solution improves and the eigenvalue changes accordingly. The change in $\mathrm{k}$ with decreasing mesh size gets smaller and smaller, and the appropriate mesh to use would be when the change in $k$ is obscured in the statistical uncertainty. Perhaps some kind of extrapolation formula could be used to estimate $\mathrm{k}$ with an infinitely small mesh size. Also, performing a linear approximation to the source distribution within each spatial zone (as opposed to the flat distribution used here) should improve the accuracy obtained. Examination of the results for the two-dimensional cube also presented in Table 9 shows the same behavior as above.

The value of the generation time obtained for the run in Figure 2 is $10.9 \times 10^{-8}$ sec. This run used the ten group cross-section set. The generation time obtained with the three group cross-section set is $8.3 \times 10^{-8} \mathrm{sec}$. These values compare favorably with experimental measurements 2 of the generation times on similar ZPR-III assemblies which range from $6 \times 10^{-8}$ to $20 \times 10^{-8} \mathrm{sec}$.

Timing Studies: Most of the execution time is consumed in the history following section of the program. The convergence tests, source updating, and other auxiliary computations that are performed after each iteration consume only a small percentage of time. The most important section time-wise in the history following are the geometry subroutines (called CUBE 1 and CUBE 2) which occupy about $35 \%$ of the time.

In Table 5 are listed some of the timing data obtained. Listed are time per history, time per collision, time per entry into CUBE 1, number of entries into CUBE 1 per history, and number of nuclear collisions per history.

\section{TABLE 5 - RESULTS OF TIMING STUDIES}

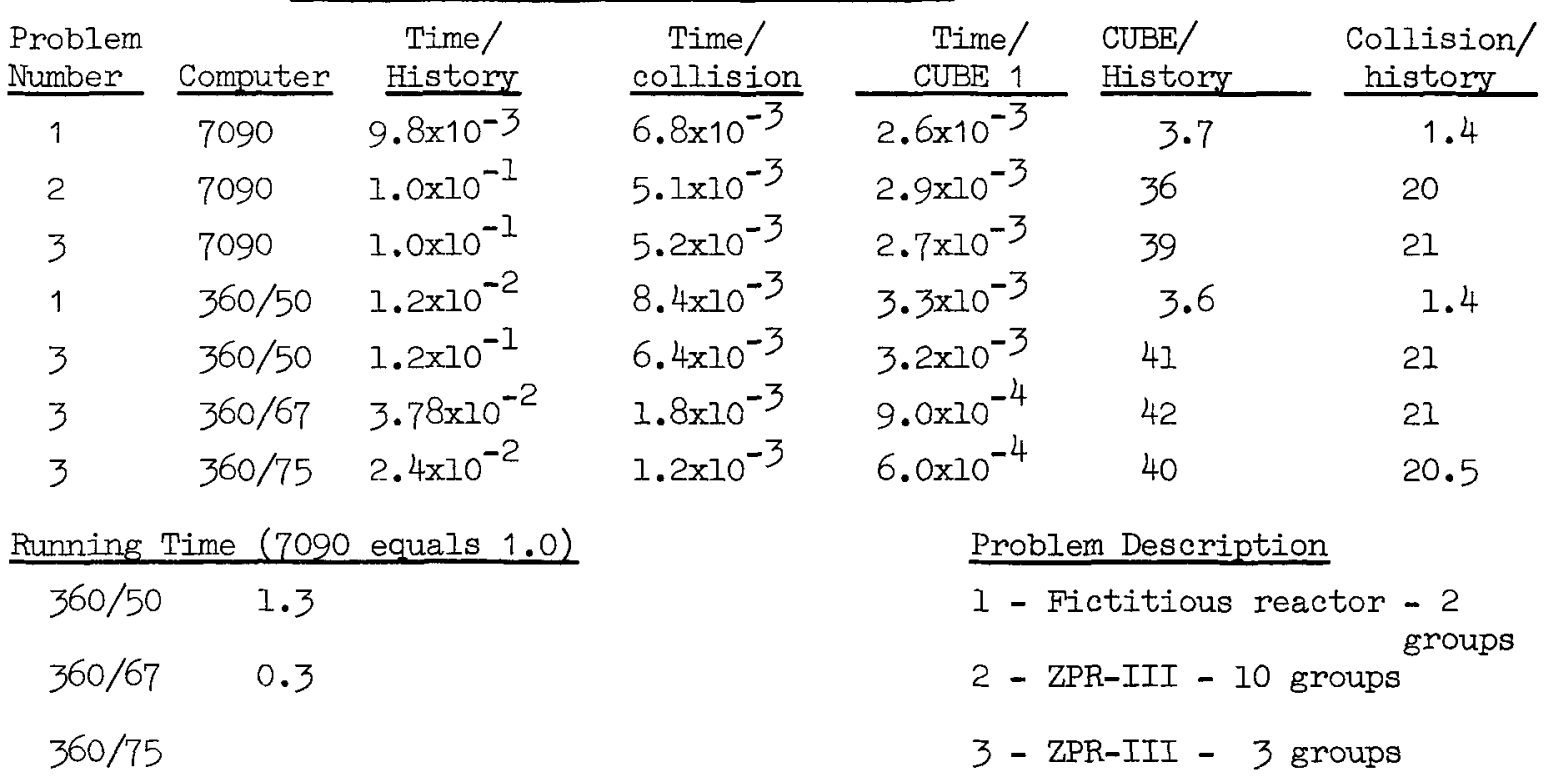

Times are compared for a number of different IBM computer models for three different problem types. The various quantities listed are useful in estimating 
execution time. The running time for a given problem is directly proportional to the initial source size (this determines the number of histories per iteration), and the number of entries into CUBE 1. The latter is a function of the average number of nuclear collisions, and the number of boundary hits, which depends on the choice of spatial mesh size. The number of histories followed depends on the number of energy groups and the initial source size.

In order to predict the computer time required for a large run, it is necessary to have a small preliminary run in order to determine the various quantities like number of collisions per history.

The choice of the initial source intensity depends on the accuracy that is required for various quantities of interest. On the basis of discussion presented earlier, one can roughly predict the source intensity needed to obtain confidence levels for quantities like $\mathrm{k}$ or generation time. However, quantities like $k_{\text {MLN }}$, $k_{\text {MAX, }}$, and DLTASR depend very much on the spatial mesh size. It is important to realize that the accuracy requirements one puts on a Monte Carlo problem are of a different nature than that of a finite difference approach like $\mathrm{S}_{\mathrm{N}}$. Also, the running time for a problem is very sensitive to the kind of convergence criteria that the user supplies. He must determine his accuracy requirements very carefully and then pose the appropriate convergence criteria to the code.

Table 6 lists the time per iteration as a function of the number of energy groups for the $3 \times 3 \times 3$ fictitious reactor. It is clear that the computation time is rather insensitive to the number of energy groups, this being an advantage of the Monte Carlo approach. Of course, for a given number of histories, the accuracy of a group dependent quantity like the neutron flux would decrease with an increase in the number of groups. However, group-averaged quantities would improve in accuracy. This is an example of the different questions that should be asked of a Monte Carlo program as opposed to a finite difference approach.

Various attempts at variance reduction are discussed in reference 1.

TABLE 6 - DEPENDENCE OF RUNNING TIME ON THE NUMBER OF GROUPS

\begin{tabular}{cc}
$\begin{array}{c}\text { Number of } \\
\text { Energy Groups }\end{array}$ & $\begin{array}{c}\text { Average Time } \\
\text { per iteration }\end{array}$ \\
\hline 1 & 12.958 \\
2 & 13.098 \\
3 & 13.119 \\
4 & 13.392 \\
8 & 14.174
\end{tabular}

Fictitious Reactor - Run \#70

$3 \times 3 \times 3$ geometry with 3 reflecting planes, initially

40 particles/zone

\section{CONCLUSIONS}

We have arrived at the conclusion that for a large class of realistic reactor problems, the Monte Carlo method is both feasible and economical on computers available today. The particular approach we used was designed to core contain much of the calculations, and hence efficiently utilize the fast CPU speeds and large fast memories of these computers. Integral quantities like the multiplication constant and the generation time can easily be obtained. 
We have demonstrated here the accuracy and running time obtainable for the fission source distribution of a ZPR-III fast reactor. One can estimate the accuracy and running time for obtaining the fission source for other reactors from the results presented in this report and a few small experimental computer muns. Further work is required to determine the obtainable precision of neutron flux distributions.

It is the author's opinion that the Monte Carlo method can and will be extensively used for nuclear reactor design studies in the future. The Monte Carlo method is capable of handling very sophisticated physics models and complicated geometries, and its strength as a tool ffor computation will increase still further as CPU speeds improve. Kolsky $(6)$ projects that within 5 years, computers of 500 million instructions per second will be in operation. Additionally, there is a considerable amount of work in progress today by a number of competent investigators on the subject of variance reduction techniques for this type of problem. These efforts should result in further improvement in the economics of this approach. We believe Monte Carlo has a bright future for these large problems.

\section{ACKNOWLEDGENMENTS}

The author expresses his appreciation to Mr. George Joanou for contributing many of the ideas behind this project and for many fruitful discussions. Appreciation is also due $\mathrm{Mr}$. Garrett Garrettson for his aid in the programing and running of the code, and for his help in the writing of this report. 
1. C. S. Shapiro, A Three-Dimensional Monte Carlo Neutron Transport Code for Nuclear Reactor, IBM Scientific Center Report \#320-3233 (March 1969) Palo Alto.

2. Bengt Carlson, "Numerical Solution of Neutron Transport Problems", Proc. of Symposia in Applied Mathematics, Vol. II (1960).

3. J. K. Long et al. "Fast Neutron Power Studies with ZPR-III," P/598, Proc. Geneva Conf. Peaceful Uses of Atomic Energy, (1958).

4. "Reactor Physics Constants," Argonne National Lab (A.N.L. -5800), 2nd ed., (1963).

5. Personal Communication, George Joanou (1968).

6. H. G. Kolsky, "Some Computer Aspects of Meteorology" IBM Journal of Research \& Development (Nov. 1967). 
COHORT-II - A MONTE CARLO GENERAL PURPOSE SHIELDING COMOUTER CODE

bý Leonard Soffer and Lester C. Clemons, Jr.

Lewis Research Center

National Aeronautics and Space Administration

Cleveland, Ohio

\section{INTRODUCTION}

An efficient Monte Carlo shielding code should have reasonable running times, flexible geometry routines, and flexibility in biasing. It should be able to generate and track secondary radiation and employ good coding nractices to speed input and to clarify output.

COHORT-II is a Monte Carlo general purpose shielding code which has been written with the intention of satisfying these criteria. In its oresent form, it represents a major revision and modermization of the original COHORT code. 1,2 Much effort has been spent on integrating the formerly seoarate segments into one package and extending its geometry and biasing capability. The code can track either neutrons or gamma rays born in a spherical, cylindrical or rectangular paralleleniped source regions propagating through shield geometries describable by general quadratic surfaces. In addition, the code can generate secondary particles to be used as innut for a subsequent run. Biasing features include preferential source varticle selection and the exponential transform. Number fluxes in energy bins averaged over regions and at arbitrarily located point detectors are determined along with an estimate of the statistical precision. Energy integrated quantities such as dose rates and heating rates are also obtained.

This paper describes the general features of the code, results of some checkout calculations and its present status.

\section{CODE DESCRIPTION}

General - The code is written completely in Fortran-IV for the IBM7094 computer. The overlay feature of the compiler has been used to allow sufficient storage for large problems; variable dimensioning has been incorporated. The program consists of a main link and 5 dependent links. At any given time, the main link and only one of the dependent links are stored in core; the remaining links are stored on the overlay tape.

The main link acts as an executive routine, calling in the other links as required to manipulate data or perform calculations. The first link reads in and prints out input data and cross-sections and computes the storage requirements of the problem. 
The second link then calculates the parameters (spatial coordinates, direction cosines, energy, particle weight and region of birth) of primary source neutrons or photons from spatial, angular and energy distributions innut by the user. These parameters are written on an internal scratch tape and used by the particle tracking routines. If secondary gammas or leakage particles are being tracked from a previously run problem, then a physical source tape will be available from this run. In this case link 2 is bypassed with control proceeding directly from link 1 to link 3.

The great majority of computing time is spent in the third link which performs the bulk of the Monte Carlo calculations. Each particle is treated individually. The uncollided flux is first calculated for any ooint detectors that may be present. Since the direction cosines of the particle are known, the estimated track length of the particle in all regions of the system along its projected path is then computed. The distance to the next collision is then computed for the actual particle and it is tracked to its collision point. The type of interaction, energy and direction cosines after scattering are then determined. If any point detectors are present, an estimate of the uncollided flux from the collision point to every detector is made. Track lengths are estimated through the system along its new direction (after scattering) and the cycle repeats. All scoring, both track lengths in regions and at point detectors, is performed by estimation. If secondary gammas are to be generated, neutron collision data are written on an intermal tane and stored. Histories are terminated when a particle escapes from the geometry, when its energy or weight falls below a pre-assigned cut-off value, or when it has undergone more than a pre-specified number of collisions. When this occurs, an appropriate counter is incremented and a new particle started.

After the desired number of particles has been followed, control passes to the lth link. This link prints out the results of the problem. The output lists the reasons for termination of the histories and energy deposition in the regions. The number fluxes sorted according to energy bins at point detectors and/or estimated in every region are then printed out. Energy integrated quantities such as total dose rates and heating rates are next obtained from the computed filuxes using input resmonse functions. Statistics on fluxes are computed as in reference 3. The total number of histories $\mathrm{N}$, is divided into $\mathrm{G}$ groups of $\mathrm{n}$ histories per group. Group scores $\mathrm{S}_{\mathrm{g}}$ are accumulated. The standard deviation per history is

$$
\sigma=\frac{1}{n} \frac{1}{\sqrt{G-1}} \sqrt{\overline{s g}^{2}-(\overline{S g})^{2}}
$$

Both absolute and percent deviations are printed.

If no secondary gamma source is to be written the problem terminates at this point; otherwise control proceeds to the 5th link. This link generates a secondary gamma source tape identical in format to a primary particle source tape. Neutron collision data are read in from the tape oreviously orepared in link 3. Probability tables giving the energy and number of secondary gammas emitted per capture or inelastic event are required as innut. When this link is completed the problem ends. 
The program is designed to require a minjmum number of passes on the computer and with no physical. tase randlinf br the user excent when a leakage tape or a secondary gamma tape has been requested. A complete analysis of a reactor shield including primary neutrons and primary and secondary photons, for example, would require three mactine rus.

Geometry - The shield reometry is flexibie with space divided into homogeneous regions bounded by surfaces describable by a general quadratic equation.

This equation is of the form

$$
\mathrm{Ax}^{2}+\mathrm{By}^{2}+\mathrm{Cz}^{2}+\mathrm{Dxy}+\mathrm{Exz}+\mathrm{Fyz}+\mathrm{Cx}+\mathrm{Hy}+\mathrm{Jz}+\mathrm{K}=\mathrm{O}
$$

and allows a description of such surfaces as spheres, cylinders, cones, ellipsoids, hyperboloids and planes at arbitrary location and orientation to the coordinate axes.

Special forms of the general equation are also incoroorated into the geometry routine for convenience. These are:

$$
\begin{gathered}
A\left(x-x_{0}\right)^{2}+B\left(y-y_{0}\right)^{2}+C\left(z-z_{0}\right)^{2}+D=0 \\
x-x_{0}=K \\
y-y_{0}=K \\
z-z_{0}=r
\end{gathered}
$$

Equation (3) can be used for specifying spheres, cylinders, ellinsoids and hyperboloids with axes parallel to the coordinate axes; while equations (4) to $(6)$ allow description of planes parallel to the $x, y$, and $z$ axes, respectively. Use of these specialized equations when possible not only requires less input, but also uses less computer time than use of equation (2).

The shield geometry, using equations (2) to (6), should be distinguished from the source geometry. The shape that the source regions can have is more restricted. Only spheres, cylinders and rectangular parallelenipeds whose axes are parallel to the coordinate axes are permitted as source volumes.

The geometry calculations parormed by the code have two basic functions. Given the spatial coordinates and direction cosines of a particle these are: (1) to determine the region the particle is in; and (2) to comute the distance from the particle to the nearest boundary in its direction of travel. The method used is the same as that of reference 4 .

The numerous and time-consuming geometry calculations required of all general Monte Carlo codes has been reduced by performing some of the geometry calculations for particle tracking and track length estimation simultaneously. In tracking a particle from one collision point to the next, the information obtained from the geometry subroutine (region of location, distance to nearest 
boundary) is available to both the particle tracking and track length estimation computations which proceed essentially simultaneously until the collision point is reached.

Additional time savings can be achieved by terminating the estimation calculation for either point detectors or track length regions when the detector or region is more than a pre-assigned number of mean free paths from the collision point. When this occurs, the contribution from that collision ooint is presumed to be negligible compared to other collisions much closer. These estimation cutoff parameters are at the complete control of the user.

Biasing - Two standard biasing features are available to the user. These are (1) selection of source particles with respect to preferred locations, energies and directions, and (2) use of the exponential transform during tracking. Where $\mathrm{bi}^{\mathrm{i}}$ ing is used the weight of a particle is changed to preserve the original ...tribution.

The selection of the parameters needed to describe a source oarticle, namely: the snatial coordinates, direction cosines and energy, are indenendent of each other. thus, biasing in their selection may also be nerformed independently.

Flexibility in source selection biasing is valuable. It allows the user to make use of any initial knowledge he has about the importance of particles born at certain locations or emitted in certain directions or energies upon the final result.

The exponential transform is also available during particle tracking. The code uses this by defining a fictitious cross-section $\sum$ as follows:

$$
\sum^{*}=\sum(1-K)
$$

where $\sum$ is the actual cross-section and $K$ is a biasing parameter that can be varied for each region and for particles traveling in certain directions within a given region. The distance to the next collision $d$, is chosen as

$$
d=-\frac{I}{\Sigma} * \ln \xi
$$

where $\xi$ is a random number. When $\sum^{*}<\sum$ the distance to the next collision is increased over the unbiased distance. This serves to stretch the path length, and is the usual technique used to enhance deep penetration in shields. Then $\sum>\sum(k<0)$ the path length is compressed.

The biasing features are optional and a completely unbiased nroblem can be mun, if desired.

Cross-sections - Cross-sections are entered on cards in a prescribed format. A table of cross-sections vs. energy is always required. For ohotons the total, Compton scatter plus pair production, and Compton scatter crosssections are required. For neutrons a similar table listing the total, 
elastic plus inelastic scattering and elastic scattering cross-sections is entered. Linear interpolation is used in the above table to determine crosssections at intermediate energies. In addition to the above table neutron problems require other tables. The angular distribution of elastically scattered neutrons is determined by entering a table listing the cumulative probability distribution of a neutron scattering through a given angle vs. incident neutron energy for each nuclide. The energy of the neutron after inelastic scattering is determined from tables listing the energy levels of each nuclide and the probability of exciting these levels vs. incident neutron energy for each element. Finally, problems where secondary gammas are to be generated require tables giving the probability of emitting secondary photons of various energies vs, incident neutron energy for each element.

Since cross-sections may be required many times in tracking the varticle, interpolation to find the proper value can become time-consuming. This has been reduced in the code by use of an efficient interpolation routine. Instead of searching sequentially through the energies to find the bounding values, the routine first identifies which half of the energy range the particle lies in, then which quarter of the range, and so on, oermitting the rapid identification of its bounding energy values. Cross-section retrieval time is further reduced by having the energy spacing identical for all elements. In this way, when a particle crosses a boundary from one material to another, its location in the cross-section table has not changed. In addition, further savings are effected by using the cross-sections to compute the probability of a given type of interaction. These include the probability of a total scattering occurring and probabilities of an elastic scatter for neutrons, or a Compton scatter for gammas. The interaction probabilities are stored in the cross-section tables, thereby taking no additional storage. Computation time is reduced as they are calculated in the imput link, rather than in the history tracking link.

\section{CODE CHECKOUT}

At present the code has been completed, its basic mechanics have been checked out and it is running on the IBM-7094. A user's report ${ }^{5}$ is in preparation. Several auxiliary programs have been written to assist in vreparation of neutron cross-section data. A recent compilation 6 lists data on inelastic neutron scattering and on capture gammas in a format suitable for the code. Plans are also being made to obtain cross-sections in a suitable format directly from the ENDF tape.

Several types of checkout calculations were performed to assure that the basic code mechanics of tracking and handling particles was working properly. At the present time, methods for selecting proper biasing for a given nroblem. have not been investigated. The checkout calculations included a point isotropic fission neutron source in polyethylene, a point isotropic monoenergetic ( $3 \mathrm{Mev)} \mathrm{gamma} \mathrm{source} \mathrm{in} \mathrm{aluminum,} \mathrm{and} \mathrm{two} \mathrm{problems} \mathrm{involving} \mathrm{transmission} \mathrm{of}$ particles through ducts. The fission neutron problem was set up to check out fluxes obtained from the track lengths in regions, while the gamma oroblem was intended to check out the fluxes at point detectors. Both problems were run with and without biasing to ensure that no errors were present in the biasing 
coding, but without any attempt to obtain optimum biasing. The two duct problems were run to ensure the proper functioning of the geometry routine under a variety of geometries.

Point fission neutron source in polyethylene - A spherical shell was set up for the neutron point source problem and energy fluxes in 14 bins from 0.1 to $18 \mathrm{Mev}$ were obtained by the track length method. An energy cutoff of 0.1 Mev was used. 20,000 histories were run in 45 and 30 minutes, respectively, for the biased and unbiased problems on the 7094 .

For the biased problem a source energy biasing scheme was arbitrarily set up as shown in the table below. The exponential transform was also employed using an arbitrary value of $\mathrm{K}=0.4$ for each region.

TABLE 1 - Energy Biasing for Neutron Problem

\begin{tabular}{|c|c|l|l|}
\hline Group & Energy bounds, Mer & $\begin{array}{c}\text { Fission Spectrum } \\
\text { Fraction }\end{array}$ & $\begin{array}{c}\text { Biased Spectrum } \\
\text { Fraction }\end{array}$ \\
\hline & & & \\
1 & $0.1-1$ & 0.310 & 0.15 \\
2 & $1-2$ & 0.296 & 0.15 \\
3 & $2-3$ & 0.185 & 0.15 \\
4 & $3-4$ & 0.1032 & 0.10 \\
5 & $4-6$ & 0.0806 & 0.15 \\
6 & $6-8$ & 0.0192 & 0.10 \\
7 & $8-10$ & 0.00416 & 0.08 \\
8 & $10-12$ & 0.000854 & 0.07 \\
9 & $12-15$ & 0.00019 & 0.04 \\
10 & $15-18$ & 0.0000154 & 0.01 \\
\hline
\end{tabular}

Spectra for the unbiased neutron problem at 30 and $60 \mathrm{gm} / \mathrm{cm}^{2}$ are shown on figure 1 and 2 respectively, along with the spectra calculated from the Moments method. 7 Spectra for the biased fission neutron source at the same respective depths are shown on figures 3 and 4 . Moments method soectra are also shown on these figures. From the figures it can be seen that there is fairly good agreement between the Monte Carlo and Moments calculations, for both the unbiased and biased problems, at the shallower penetration. At the deeper penetration, the unbiased spectrum is not in good agreement with the Moments method, while the biased results are notably better. Thus, figure 1 shows that the code mechanics are working properly in performing an unbiased calculation of track lengths in regions. The fact that the biased calculation checks the Moments spectra at the shallow penetration and improves the results at the deeper penetration, as shown in the remaining figures, indicates that the biasing coding is also working properly. 


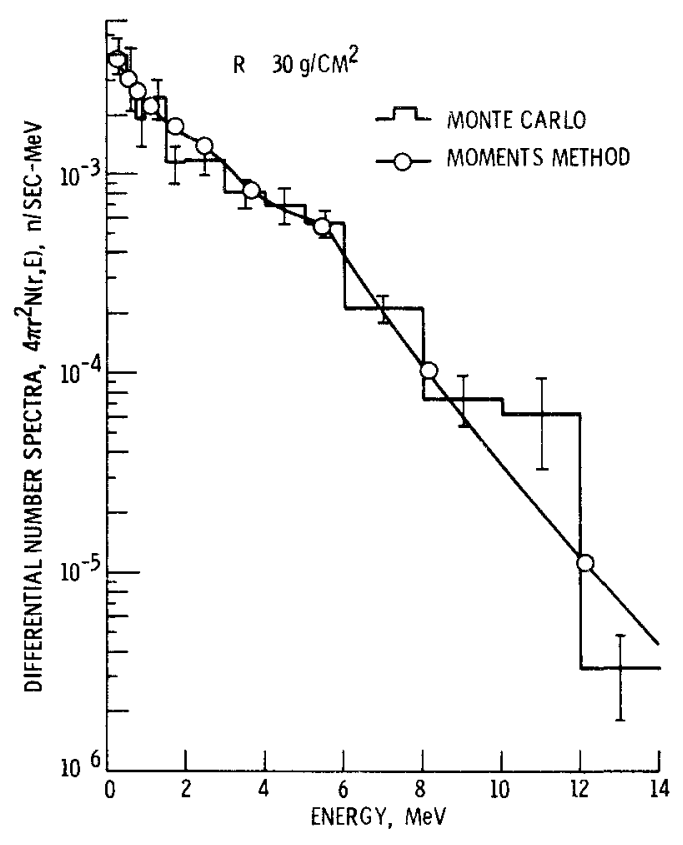

Figure l - Fast neutron spectra in polyethylen Pt isotropic fission source Depth $=30 \mathrm{~g} / \mathrm{cm}^{2}$ Unbiased calculation

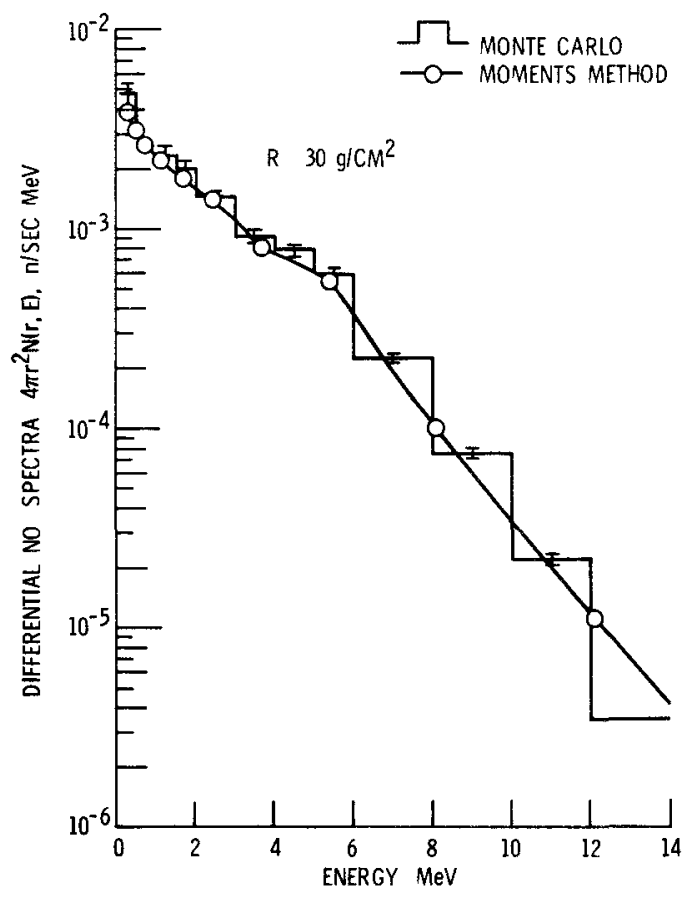

Figure 3 - Fast neutron spectra in polyethylene Pt isotropic fission source Depth $=30 \mathrm{~g} / \mathrm{cm}^{2}$ Biased calculation.

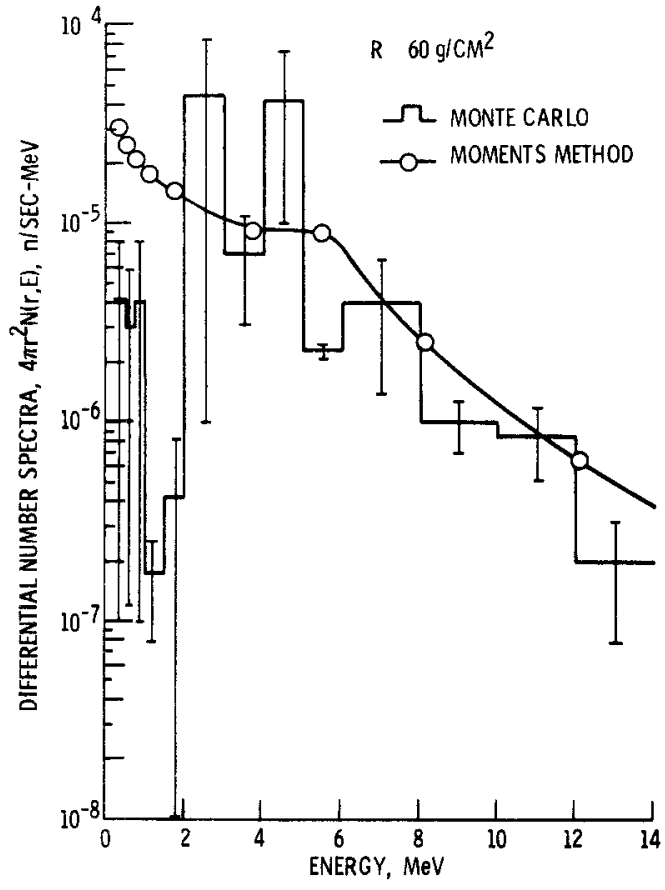

Figure 2 - Fast neutron spectra in polyethylene. Pt. Isotropic fission source. Depth $=60 \mathrm{~g} / \mathrm{cm}^{2}$. Unbiased calculation.

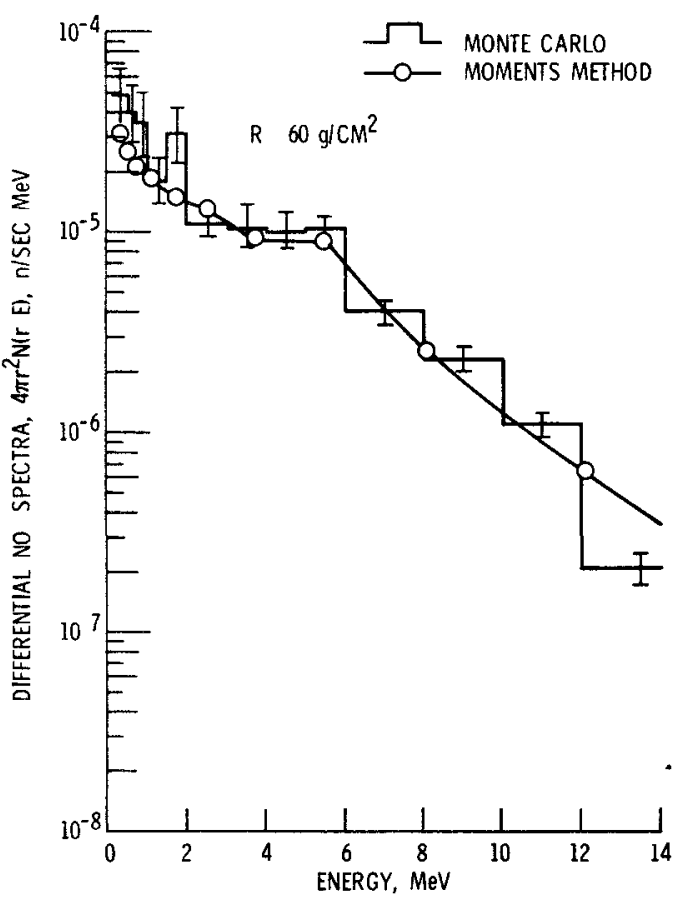

Figure 4. - Fast neutron spectra in polyethylene. Pt isotropic fission source. Depth $=60 \mathrm{~g} / \mathrm{cm}^{2}$. Biased calculation 


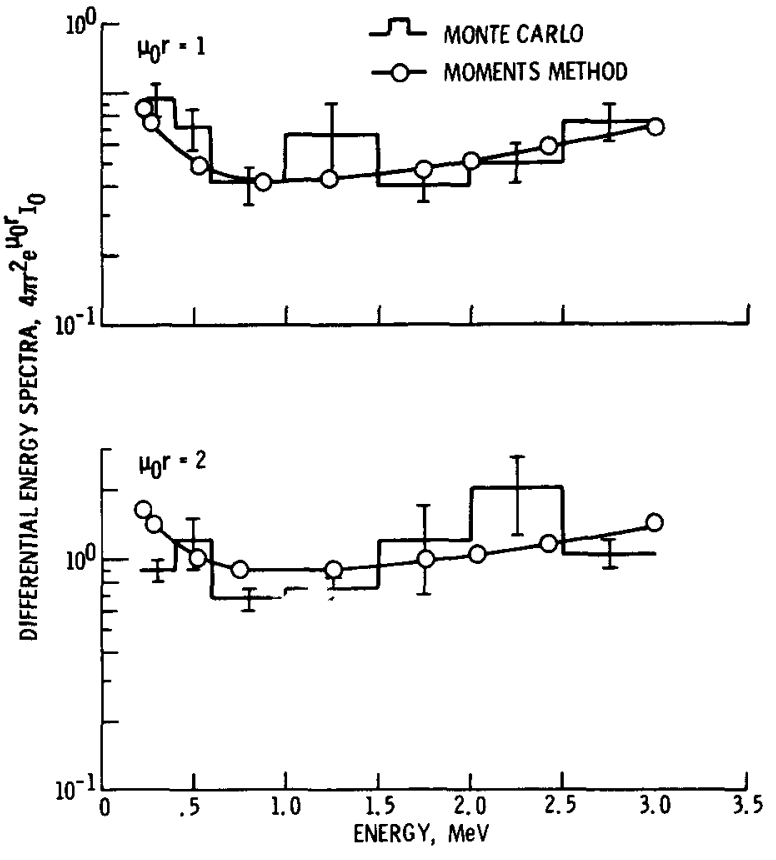

Figure 5. - Gamma energy spectra in aluminum.

Pt. isotropic $3 \mathrm{MeV}$ source. Unbiased calculation.

Depth -1 and 2 mean free paths.

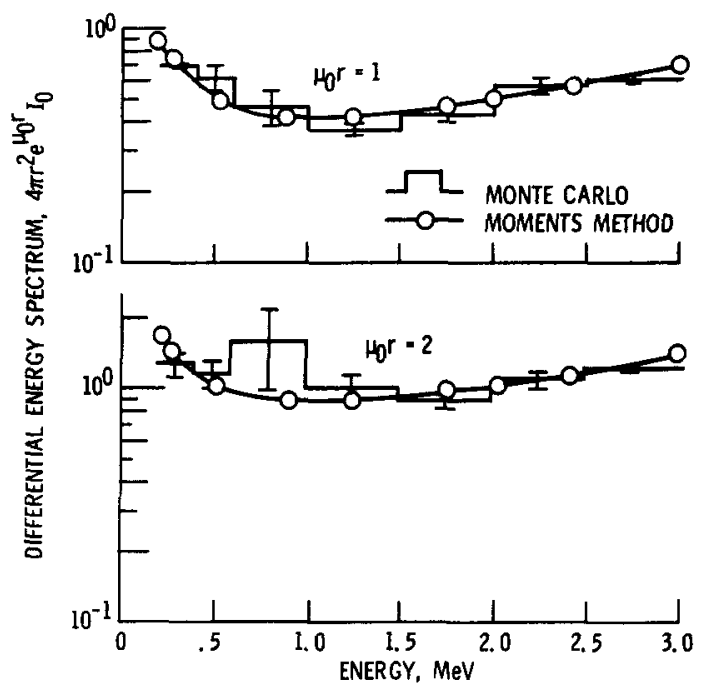

Figure 6. - Gamma energy spectra in aluminum. Pt. isotropic $3 \mathrm{MeV}$ source. Depth -1 and 2 mean free paths. Biased calculation.

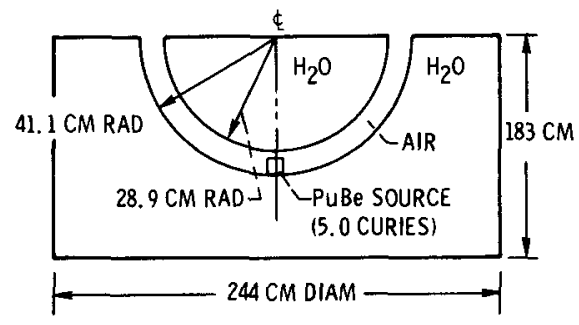

Figure 7. - Hemispherical duct configuration in water.

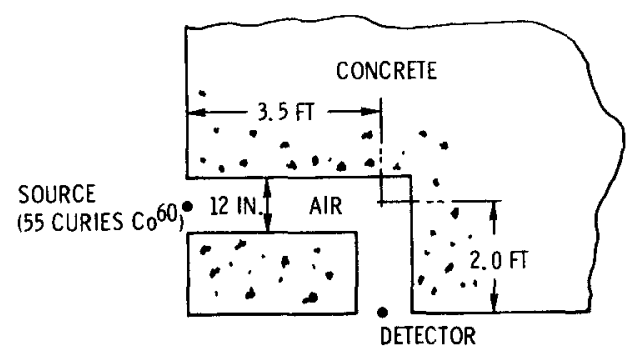

Figure 8. - Rectangular L-shaped duct configuration. 
Point 3 Mev gamma source in aluminum - For the gamma point isotronic problem a large aluminum sphere was calculated with detector points located along the $\mathrm{z}$ axis at 1 and 2 mean free paths from the source at the center. An energy cutoff of $0.2 \mathrm{Mev}$ was used. Again 20,000 histories were run in about $30 \mathrm{~min}-$ utes for each problem.

In the biased problem, the photons were biased arbitrarily in the polar angle as shown in the table below. As in the neutron problem, an exponential biasing factor of $\mathrm{K}=0.4$ was uniformly applied.

TABLE 2 - Polar Angle Biasing for Gamma Problem

\begin{tabular}{|c|c|}
\hline $\cos \theta i-\cos \theta i+1$ & $\begin{array}{c}\text { Fraction in } \\
\text { Angular Interval }\end{array}$ \\
\hline $1.0-0.9985$ & 0.10 \\
$0.9985-0.9962$ & 0.35 \\
$0.9962-0.966$ & 0.25 \\
$0.966-0.866$ & 0.15 \\
$0.866-0.707$ & 0.10 \\
$0.707-(-1.0)$ & 0.05 \\
\hline
\end{tabular}

The gamma spectra at 1 \& 2 mean free paths for the unbiased oroblem are shown in figure 5, while the corresponding spectra for the biased problem are shown in figure 6. Spectra from the Moments method are also shown in both figures. Observation of figures 5 and 6 lead to the same general conclusions already observed in the previous discussion on the neutron problem. The gamna problem indicates that fluxes at point detectors are being computed properly.

Neutron and gamma duct problems - Additional checkout was performed by calculating two fairly complex duct geometries. A sketch of the first duct, a hemispherical duct in water, is shown in figure 7. The duct contains a point neutron source. Measured \& calculated spectra and integrated neutron fluxes were obtained at the tank center and duct centerline. ${ }^{9}$ Considerable neutron streaming in the duct was observed. The measured and calculated fluxes above $0.5 \mathrm{Mev}$ agreed within $50 \%$, while the fluxes above $2 \mathrm{Mev}$ were within 25\%.

The configuration for the second duct calculated, an L-shaped rectangular cross-section duct in concrete, is shown in figure 8 . The dose rate 10 was measured at a detector placed at one end of the duct due to a 55 curie co-60 source located at the other end. The same configuration was calculated by COHORT. The measured value of $852 \mathrm{mr} / \mathrm{hr}$ and the calculated value of $895 \pm$ $60 \mathrm{mr} / \mathrm{hr}$ were in good agreement. The duct problems indicate the COHORT geometry routines are whrking properly for different geometries. In addition, they represent additional checkout of fluxes at point detectors. 
The COHORT code has been greatly reworked and modernized. It has been integrated into a single package with more flexible geometry and biasing. The basic mechanics of the code have been checked out for several problems, and it is presently operable on the IBM-7094.

\section{REFERENCES}

1. D. G. COLLINS and T. W. DEVRIES, "Monte Carlo Calculations of Energy Deposition and Radiation Transport," Vol. I - Validation of COHORT Codes, Vol. II - Utilization of COHORT Codes. FZK-176-I and 2, General Dynamics Corp. (Dec. 1963).

2. D. G. COLLINS and M B. WELLS, "COHORT - A Monte Carlo Program for Calculation of Radiation Heating Transport," RRA-T62, Vols. I to IV, Radiation Research Assoc. (Sept. 1966).

3. D. SPIELBERG, "THENA - A System of Fortran Programs for Radiation Transport and Heating Calculations in Complex Reactor Geometries," UNC-5148, United Nuclear Corp. (March 1966).

4. R. E. MALENFANT, "QAD - A Series of Point Kernel General Purpose Shielding Programs," LA-3573, Los Alamos Scientific Lab. (April 1967).

5. L. CLEMONS, JR. and L. SOFFER, "A Users Report for the COHORT-II Monte Carlo Shielding Code," (To be published).

6. J. D. MARSHALL, "Inelastic Neutron and Capture-Gamma Ray Cross-Section Libraries for the COHORT Monte Carlo Procedure," RRA-T83, Radiation Research Assoc. (Feb. 1968).

7. H. GOLDSTEIN, Fundamental Aspects of Reactor Shielding, p. 384, AddisonWesley Pub. Co., Reading, Mass. (1959).

8. H. GOLDSTEIN and J. E. WILKINS, JR., "Calculations of the Penetrations of Gamma Rays," NYO-3075, Nuclear Development Assoc., Inc. (June 1954).

9. L. CLEMONS, G. N. WRIGHTS and D. SHOOK, "Monte Carlo Calculations of a Hemispherical Duct Neutron Streaming Experiment," paper to be presented at the 15th Annual Meeting of the American Nuclear Society, (June 1969).

10. C. W. TERRELI, et al., "Radiation Streaming in Ducts and Shelter Entranceways," ARF-1158-AO2-7, Illinois Inst. Tech. (April 1962). 


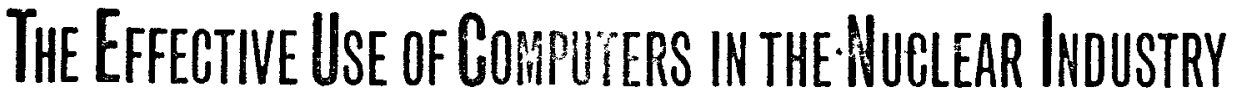 \\ A CONFERENCE AT KNOXVILLE, TENN. A APRIL 21-23, 1969 sponsored by}

THE MATHEMATICS AND COMPUTATION DIVISION OF THE AMERICAN NUCLEAR SOCIETY THE OAK RIDGE NATIONAL LABORATORY AND THE UNIVERSITY OF TENNESSEE

April 8, 1969

Mr. R. C. Dreyer

USAEC - DTIE

Oak Ridge, Tenn.

Dear Mr. Dreyer:

This is in confirmation of the transmittal of the papers for the conference on "The Effective Use of Computers in the Nuclear Industry," on April 3, 1969.

The enclosed notebook contains a publication release signed for each paper that will be published in the Proceedings of the conference.

There will be a special distribution made:

(a) I free copy to each author of a conference paper

(b) I copy to each of the other attendees at the conference

Money to cover all attendees other than authors will be forwarded to you in one check at the time of the conference.

In addition to the above special distribution which we will furnish to you in the near future, we understand that a TID-4500 Mathematics and computers distribution will be made and that the Proceedings will also be placed in the Federal Clearinghouse (CFSTI) for sale.

We have received a packet of labels to be used for the special distribution. We will type these labels and return them to you before the time that you will need them for the distribution.

Need I request that the abstracts be included in NSA?

If you need any additional information or if $I$ can expedite the publication of these papers in any way, please do not hesitate to call me.

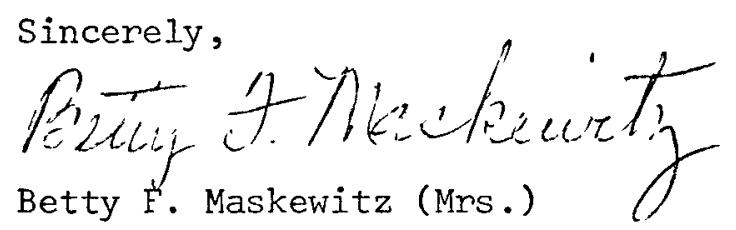

$\mathrm{BFM} / \mathrm{mw} I$

Enc. 
SESSION I. KINETICS

"Review of Computational Methods for Space-Time Kinetics", A. F. Henry (W-BAPL), Invited.

1. "TART: An LMFBR Transient Analysis Project", M. G. Stevenson, B. E. Bingham (BळW)

2. "Coupling of Dynamics Calculations in the FREADM Code", D. D. Freeman (GE-APO, Sunnyvale)

3. "Fast Reactor Accident Study Code, SASIA", G. J. Fischer, J. C. Carter, T. J. Heames, D. R. MacFarlane, N. A. McNeal, C. K. Sanathanan, W. T. Sha, C. K. Youngdahl (ANL)

4. "On the Solution of the Time-Dependent Group Diffusion Equations by an Implicit Time Differenced Iterative Method", J. B. Yasinsky, L. A. Hageman (W-BAPL)

5. "Numerical Solution of the Two-Dimensional TimeDependent Multigroup Equations", W. T. McCormick, $J r ., K . F$. Hansen (MIT)

6. "Nuclear Reactor Spatial Kinetics Calculations", G. Buffoni, A. Galati, P. Loizzo, S. Lopez, F. Norelli, A. Pepino (CNEN, Casaccia, Italy)

LUNCHEON

Speaker: W. H. Hannum (USAEC) "Some Thoughts on AEC Funded Coding" 


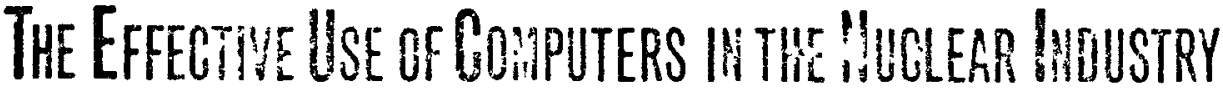 \\ A CONFERENCE AT KNOXVILLE, TENN. APRIL 21-23, 1969 sponsored by}

THE MATHEMATICS ANO COMPUTATION DIVISION OF THE AMERICAN NUCLEAR SOCIETY

THE OAK RIDGE NATIONAL LABORATORY AND THE UNIVERSITY OF TENNESSEE

\section{PUBLICATION RELEASE FORM}

This certifies that the paper

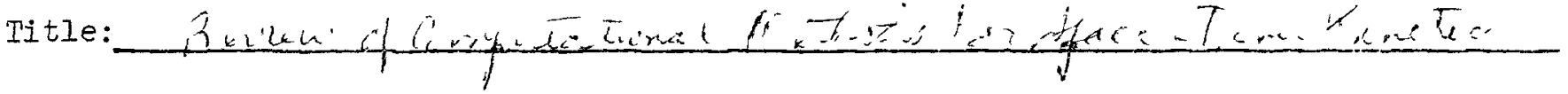

Authors:

CIItenis

Organization:

$11 . T$

is cleared for publication. It is intended for cral Presentation at the above meeting. The paper will be published in the proceedings of the mecting by the USAEC Division of Technical Information Extension, Oak Ridge, Tennessee. DTI nay reprunce and make TID-4500 standard distribution, including copies to Federal Clearinghou, $s$, depository libraries and announce in NSA.

The paper contains no information that need be withneld from publicatiun becus? of national security interests, patent considerations, or proprietary information interests. Permission is hereby granted to publish it in the proceeairgs of the meeting which $I$ understand will receive the above standard distribution.

This release is submitted by:

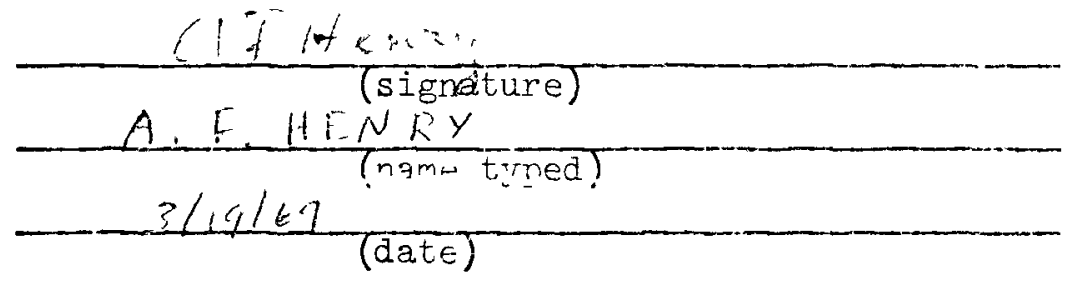

The Author's copy of the proceedings should be mailed to the following complete address:

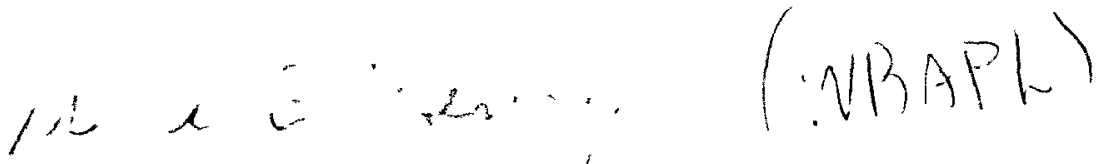

$$
\begin{aligned}
& \text { n) } 1 \text { U }
\end{aligned}
$$

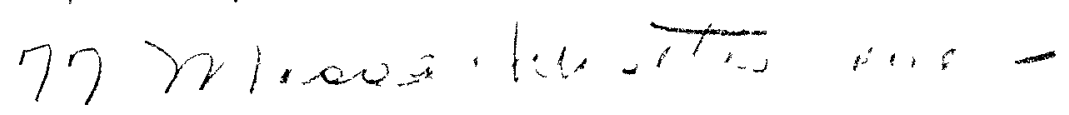

$$
\begin{aligned}
& \text { Ler.in.... }
\end{aligned}
$$




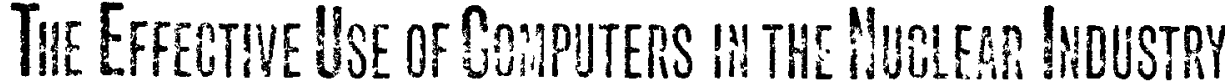

A CONUFERENCE AT KNOXVILLE, TENN. A APRIL 21-23, 1969 sponsored by

THE MATHEMATICS ANO COMPUTATION DIVISION OF THE AMERICAN NUCLEAP SOCIETY THE OAK RIDGE NAIIONAL LAEORATORY AND THE UNIVERSITY OF TENINESSEE

\section{PUBLICATION RELEASE FORM}

This certifies that the paper

Title: _ - TART - An LMFBR Transient Analysis Project

Authors: M. G. Stevenson, B. E. Bingham

Organization: Babcock and Wilcox Company

is cleared for publication. It is intended for Oral Presentation at the above meeting. The paper will be published in the proceedings of the meeting by the US.IFC Division of Technical Information Extension, Oak Ridge, Ternessee. DII may reprcduce and make TID-4500 standard distribution, including copies to Federal Clesringhouse, depository libraries and announce in NSA.

The paper contains no information that need be withheld from publication because of national security interests, patent considerations, or proprietary informstion interests. Permission is hereby granted to publish it in the proceedings of the meeting which I understand will receive the above standard distribution.

This release is submitted by:

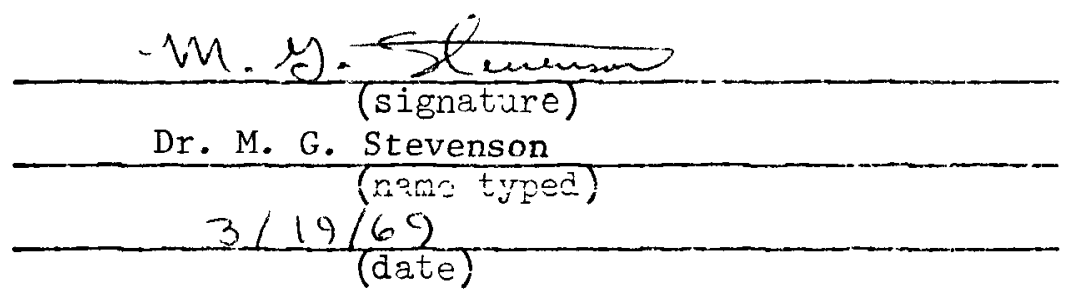

The Author's copy of the proceedings should be mailed to the following complete address:

Dr. M. G. Stevenson

BABCOCK \& WILCOX COMPANY

P. O. Box 1260

Lynchburg, Virginia 24505 


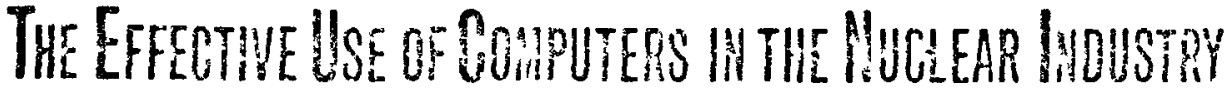

A CONFERENCE AT KNOXVILLE, TENN. APRIL 21-23,1969 sponsored by

THE MATHEMATICS AND COMPUTATIOIN DIVISION OF THE AMERICAN NUCLEAR SOCIETY THE OAK RIDGE NATIONAL LABORATORY AND THE UNIVERSITY OF TENNESSEE

\section{PUBLICATION RELEASE TORM}

This certifies that the paper

Title: "COUPLING OF DYNAMICS CALCULATIONS IN THE FREADM CODE"

Authors: D. D. Freeman

Organization: General Electric Company, Breeder Reactor Development Operation, 310 DeGuigne Drive, Sunnyvale, California 94086

is cleared for publication. It is intended for Oral Presentation a the above meeiing. The paper will be publishes in the proceedings of the meeting by the USAIC Division of Technical Information Extension, Oak Ridge, Tennessee. DTI may reprojue and make TD-4500 standard distrihution, includirg copies to Federal Clearinghous, depository Iibraries and announce in NSA.

The paper contains no information that need be withheld from publication because of national security interests, patent considerations, or proprietary informatior. interests. Permission is hereby granted to publish it in the proceedings of the ineeting which I understand will receive the above standard distribution.

This release is submitted by:

$\frac{\frac{\text { Ax }}{\text { D.D. Freeman }} \text { (signature) }}{\text { March 5, 1969 (narié typed) }}$

The Author's copy of the proceedings should be mailed to the following complete address:

D.D. Freeman

General Electric Company

Breeder Reactor Development Operation

310 DeGuigne Drive

Sunnyvale, California

$$
94086
$$




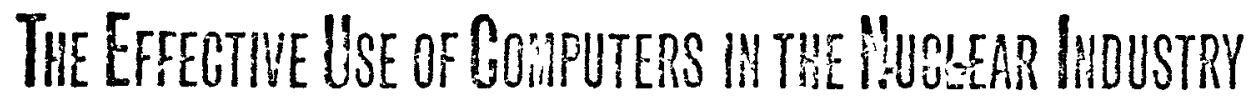

$I-3$

A CONFERENCE AT KNOXVILLE, TENN. P APRIL 21-23, 1969 sponsored by

THE MATHEMATICS AND COMPUTATION DIVISION OF THE AMERICAN NUCLEAR SOCIETY

THE OAK RIDGE NATIONAL LABORATORY AND THE UNIVERSITY OF TENNESSEE

PUBLICATION RELEASE FORM

This certifies that the paper

Title: Fast Reactor Accident Study ConE

$S A S I A$

Authors: G.J. Fischer, J.C. Carter, T.J. Hames, D.R.

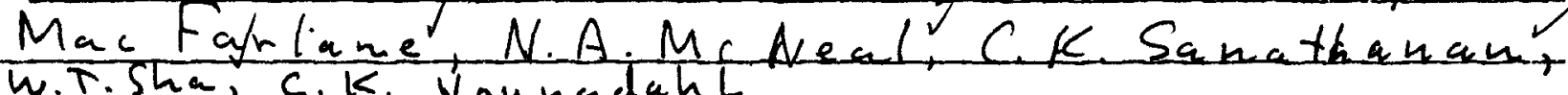
W.T. Sha, C.K. YoungdahL

Organization:

A NL

is cleared for publication. It is intended for Oral Presentation at the above meet-

ing. The paper will be published in the proceedings of the meeting by the USAEC

Division of Technical Information Extension, Oak Ridge, Tennessee. DTI may reproduce

and make TID-4500 standard distribution, including copies to Federal Clearinghouse,

depository libraries and announce in NSA.

The paper contains no information that need be withheld from publication because of national security interests, patent considerations, or proprietary information interests. Permission is hereby granted to publish it in the proceedings of the meeting which I understand will receive the above standard distribution.

This release is submitted by:

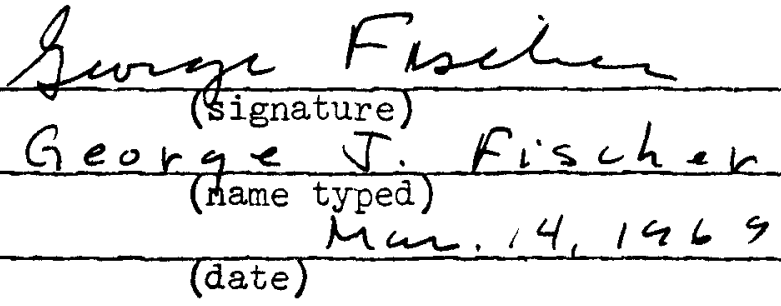

The Author's copy of the proceedings should be mailed to the following complete address:

Dr. Gunge Frith

$A N L, D-209$

Q200 So Cuss Are.

Argonne, \&ll.

$$
\text { cónsa }
$$

MRS BETTY MASKEWITZ. ChaIrman, ORNL. PO BOX X, OAK RIDGE, TENNESSEE, 37830 


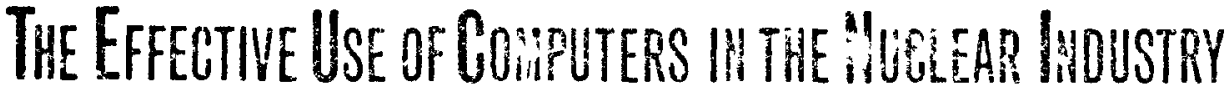

A CONFERENCE AT KNOXVILLE, TENN. APRIL 21-23, 1969 spor.sored by

THE MATHEMATICS ANJ COMPUTATION DIVISION OF THE AMERICAN NUCLEAR SOCIETY

THE OAK RIOGE NATIONAL LAEORATORY ANO THE UNIVERS'TY OF TENNESSEE

\section{PUBIICATION RELEASE FORI}

This certifies that the paper

Title: On the Solution of the Time-Dependent Group Diffusion Equations

By an Implicit Time Differenced Iterative Method

Authors:

J. B. Yasinsky and I. A. Hageman

Organization:

Westinghouse Electric Corporation

Bettis Atomic Power Laboratory

is cleared for publication. It is intended for $\mathrm{Cr} \equiv \mathrm{l}$ Fresentation at the above meet.ing. The paper will be published in the proceedings of the meeting by the USAEC Jivision of Technical Information Extension, Oak Ridge, Tennessee. DII may reproüuce and make TID-4500 standard distribution, including copies io Federal Clearinglouse, depository libraries and announce in INSA.

The paper contains no information that need be withheld from publisation becruse of nationa: security interests, patent considerations, or proprietary insormation interestis. Permission is hereby granted to publish it in the procsedings cf the meeting which $I$ understand will receive the above standard distribution.

This release is submitted by:

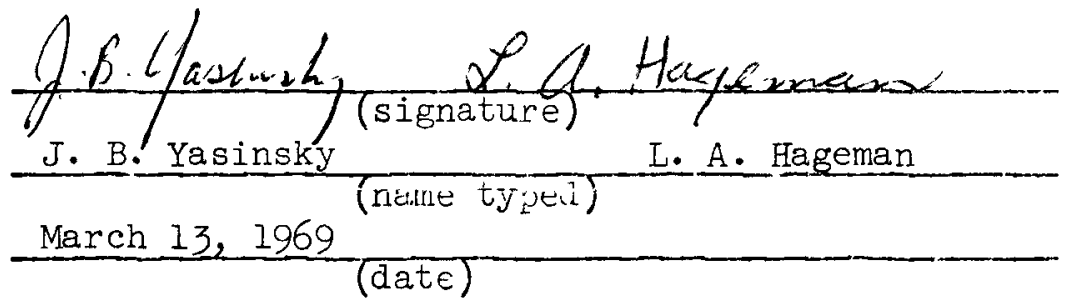

The Author's copy of the proceedings should be mailed to the following comolete address:

J. B. Yasinsky

L. A. Hageman

Westinghouse Electric Corporation

Bettis Atomic Power Laboratory

P. O. Box 79

West Mifflin, Pennsylvania 15122 
A CONFERENCE AT KNOX'VILLE, TENN. APRIL 21-23, 1969 sponsored by

THE MATHEMATICS AND COMPUTATION DIVISICN OF THE AMERICAN NUCLEAR SOCIETY

THE OAK RIDGE NATIONAL LABCRATORY AND THE UNIVERSITY OF TENNESSEE

\section{PUBLICATION RELEASE FORM}

This certifies that the paper

Title: Numerical Solution of the Two-Dimensional Time Dependent

Multigroup Equations

Authors: W.T. McCormick, Jr.

K. F. Hansen

Organization: Department of Nuclear Engineering, Massachusetts Institute. of Technology,

is cleared for publication. It is intended for Oral Presentation at the aoove mesting. The paper will be published in the proceedings of the mecting by the USAEc

Division of Technical Information Extension, Oak Ridge, Tennessee. DTI nay reprojucc and make TID-4500 standard distribution, including copies to Federal Clearingrous 2 depository libraries and announce in NSA.

The paper contains no information that need be withheld from pubIication keciuse of national security interests, patent considerations, or proprietary information interests. Permission is hereby granted to publish it in the proceedings of tre meeting which I understand will receive the above standard disjribution.

This release is submitted by:

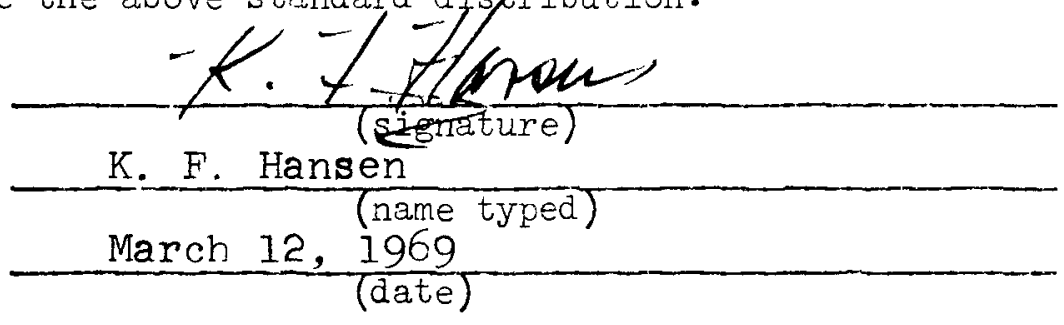

The Author's copy of the proceedings should be mailed to the following complete address:

Dr. K. F. Hansen

Room 24-109

Massachusetts Institute of Technology

Cambridge, Massachusetts 02139 


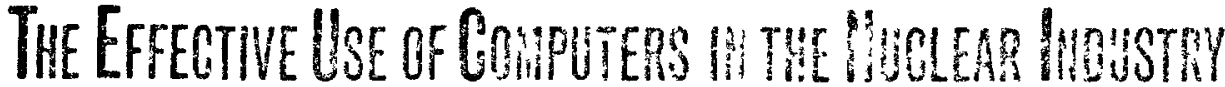

A CONFERENCE AT KNOXVILLE, TENN -APR!L : :-23, 1969 sponsored by

THE MATHEMATICS AND COMPUTAIION DIVISION OE THE AMERICAN NUCLEAR SOCIETYY THE OAK RIDGE NATIONAL I-ABORATORY AND THE UNIVERSITY OF TENNESSEE

\section{PUBIICATION RETAEASE: FORM}

This certifies that the paper Title: NUCLEAR REACTOR SPATIAL RINETICS CALCULATIONS

Autinors: G.BUFFONI, A.GALATI, P.LOIZZO, S.LOPEZ, F.NORFILI, A.PEPINO

Organization: CONITATO NAZIONALE PER L'ENERGIA NUCLEART

L.F.C.R. - C.S.N. CASACCIA - 00060 S.MARIA DI GALERIA (ROME, ITALY)

is cleared for publication. It is intenajed for Oral Presentation at the above meeting. The paper will be published in the yroceedings of the meetire by the USt.Ts Division of Technical Information Lxtensiun, nak Ridge, Tennessee. DTI may reproduce and make TID-4500 standard distribution, inciuding copies to Federaj. Cleasingho, is, depository libraries ard announce in NSA.

The paper contains no information that reed be withheld from publication because of national security interests, patent considerations, or proprietary irformatior interests. Permission is hereby granted to publish it in the proceedings of the meating which I understand will receive the above standard djstribution.

This release is submitted by:

$\frac{\frac{(\text { signature })}{\text { P.ToTzzo }}}{\text { (name typed) }}$

The Author's copy of the proceedings should be aliled to the following complete address:

LABORATCRIO FISICA I CALCOLO REATTORI

C.S.N. CASACCIA

O0060 S.MARIA DI GALERIA

(RONE - ITALY) 


\section{in

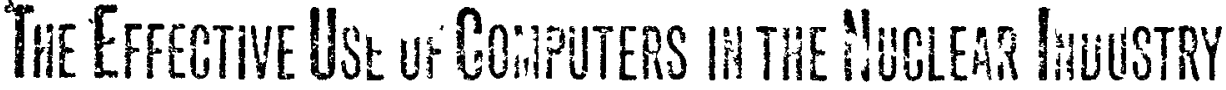 \\ A CONFERENCE AT KNICXVILLE, TENN. APRIL 21-23, 1959 sponsored by}

THE MATHEMATICS AND COMPUTATION DIVISION OF THE AMERICAN NUCLEAF SOCIETY

THE OAK RIOGE NATIONAL LABORATORY AND THE UNIVERSITY OE TENNESSEE

\section{PUBLICATIOIN RELEASE FORM}

This certifies that the paper

Title:__ Some Thoughts on AEC Funded Coding

Authors: W. H. Hannum and J. W. Lewellen

Organization: U. S. Atomic Energy Commission

is cleared for publication. It is interded for Oral Presertation at the above meeving. The paper will be published in the proceedings of the meetirg by the USAFC

Division of Technical Information Extension, Oak Ridge, Tennessee. DTI may seproduce and make TID-4500 stardard distribution, including copies to Federal Clearinghona. depository Iibraries and announce in NSA.

The paper contains no information that need be withheld from publication becalse of national security interests, patent considerations, or proprietary information interests. Permission is hereby granted to publish it in the proceedings of the meeting which I understand will receive the above stendard distribution.

This release is submitted by:

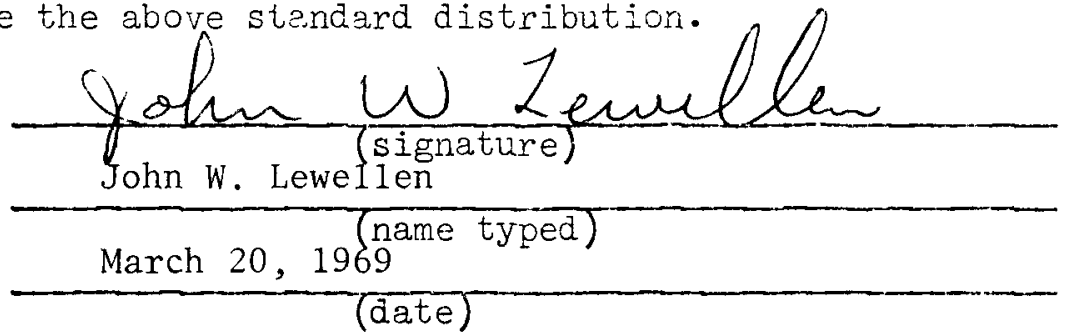

The Author's copy of the proceedings should be mailed to the following complete address:

Dr. W. H. Hannum, Chief

Reactor Physics Branch

Division of Reactor Development and Technology

U. S. Atomic Energy Commission

Washington, D. C. 20545
Mr. J. W. Lewe1len

Reactor Physics Branch

Division of Reactor Development and Technology

U. S. Atomic Energy Commission

Washington, D. C. 20545 


\section{SESSION II. DATA PROCESSING}

1. "Third Generation Computer Used to Control Fuel Element Assembly", G. E. Cort, R. J. Agosti (WANL)

\& "Cophpulerized Dafa Processing of Nerva Naciers Subsystem Test Datax, W. L. Knecht- (WANt)

3. "Use of a Process Control Computer System in Analysis of Neutron Cross Section Data and the Control of Neutron Data Acquisition Facilities Around a Van-deGraaff and an Electron Linear Accelerator", H. Horstmann, A. DeKeyser, H. Schmid (EURATOM, Geel, Belgium)

4. "Design of a Nuclear Data Acquisition System", R. N. Macdonald (IBM, Washington, D.C.)

5. "Application of an Image Processing System to Fragmentation Studies", M. K. Butler (ANL)

6. NOISY 1-A Program for Calculation of Space Dependent Auto and Cross Spectral Densities in Reactors", J. R. Sheff (BNWL) 


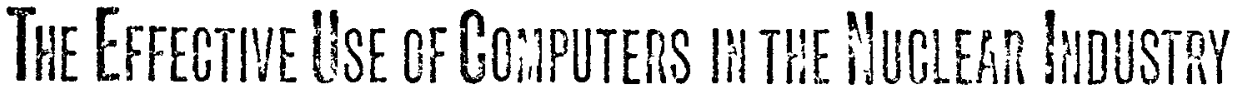 \\ A CONFERENCE AT KNOXVILLE, TENN. APRIL '1-23,1959 sponsored by}

THE MATHEMATICS AND COM. 'UTATION DIVISION OF THE AMERICAN NUCLEAR SOCIETY THE OAK RIDGE NATIONAL LABORATORY AND THE UNIVERSITY OF TENNESSEE

PUBLICATION RELEASE FORM

This certifies that the paper Titie: _. Third Generation Computer Used To Control Fuel Element Assembly

Authors: G. E. Cort and R. J. Agosti

Organization: Westinghouse Electric Corporation, Astronuclear Laboratory, Pittsburgh, Pennsylvania

is cleared for publication. It is intended for Oral Presentation at the above meeting. The paper will be published in the frocesdings of the meeting by the USAEC Division of Technical Information Extension, Oak Ridge, Tennessee. DTI may reprojuce and make TID-4500 standard distribution, including copies to Federal Clearinghouse, depository libraries and announce in NSA.

The paper contains no information that need be withheld from publication because. of national security interests, patent considerations, or proprietary information interests. Permission is hereby granted to publish it in the proceedings of the meeting which I understand will receive the above standard distribution.

This release is submitted by:

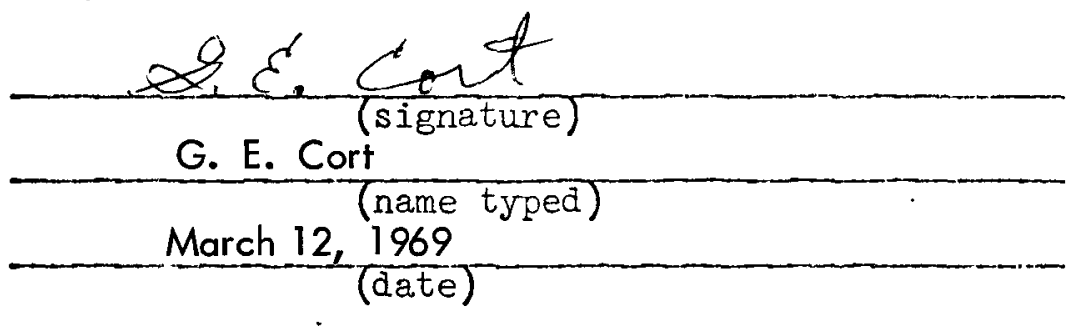

The Author's copy of the proceedings should be mailed to the following complete address:

$$
\text { Rt 51, Bx } 10864
$$


A CONFERENCE AT KNOXVILLE, TENN. APRIL 21-23,1969 sponsored by

THE MATHEMATICS AND COMPUTATION DIVISION OF THE AMERICAN NUCLEAR SOCIETY

THE OAK RIDGE NATIONAL LABORATORY AND THE UNIVERSITY OF TENNESSEE

$33+5=9$

\section{PUBLICATION RELFASE FORM}

This certifies tinat the paper

Titie: Use of a Process Control Computer System in Analysis of Neutron Cross Section Data and the Control of Neutron Data Acquisition Facilities around a Van de Graaff and an Electron Linear Accelerator

Authors: H. Horstmann, A. De Keyser, and H. Schmid

Organization: Central Bureau for Nuclear Measurements, EURATOM, Geel, Belgium

is cleared for publication. It is intended for Cral Presentation at the abore meeting. The paper will be published in the proceedings of the meeting by the UsAEC Division of Technical Information Extension, Oak Ridge, Tennessee. DTT may reproduze and make TID-4500 stardard distribution, including copies to Federal Clearinghouse, depository libraries and announce in NSA.

The paper contains no information that need be withheld from publication because of national security interests, patent considerations, or proprietary information interests. Permission is hereby granted to publish it in the proceedings of the meeting which I understand will receive the above standard distribution.

This release is submitted by:

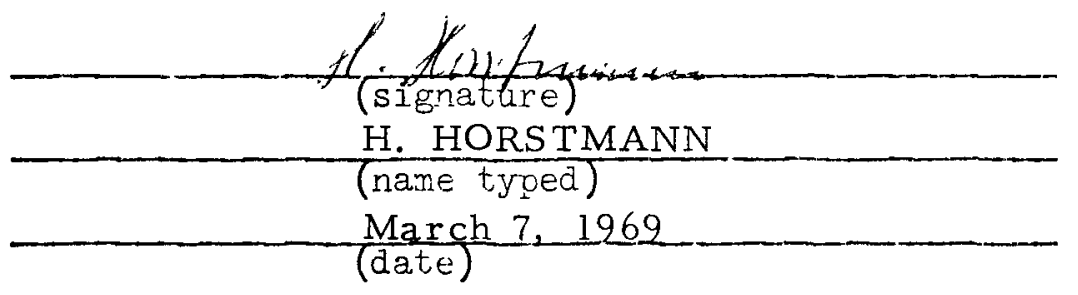

The Author's copy of the proceedings should be mailed to the following complete address:

H. Horstmann

Central Bureau for Nuclear Measurements EURATOM

Steenweg naar Retie

Geel, Belgium 
A CONFERENCE AT KNOXVILLE, TENN. APRIL 2i-23,1909 sponsored by

THE MATHEMATICS AND COMPUTATION DIVISION OF THE AMERICAN NUCLEAR SOCIETY

THE OAK RIDGE NATIONAL LABORATORY AND THE UNIVERSITY OF TENNESSEE

PUBLICATION RELEASE FORM

This certifies that the paper

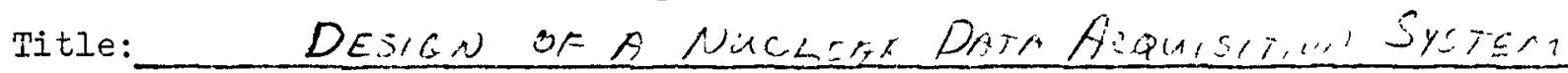

Authors:

Rogen M MAONONAD

Organization:____ Is:1

is cleared for publication. It is intended for Oral Presentation at the above neeting. The paper will be published in the proceedings of the meeting by the uSAFC

Division of T'echnical Infornation Extensicn, Oak Ridge, Tennessee. DTI may reprouuce and make TI-4500 stanaard distribution, including copies to Federal Clearingincuse,

depository libraries and announce in $\mathbb{N S A}$.

The paper contains no information that need be withheld from publication because of national security interests, patent considerations, or proprietary information

interests. Permission is hereby granted to publish it in the proceedings of the meeting which I understand will receive the above standard distribution.

This release is submitted by:

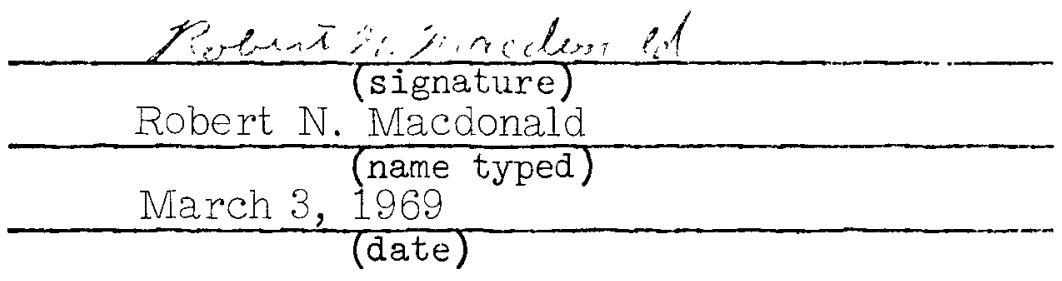

The Author's copy of the proceedings should be mailed to the following complete address:

Dr. Robert N. Macdonald

IBM DACS Center, \#41G

7 th Floor

1900 L Street, N. W.

Washington, D C. 20036 


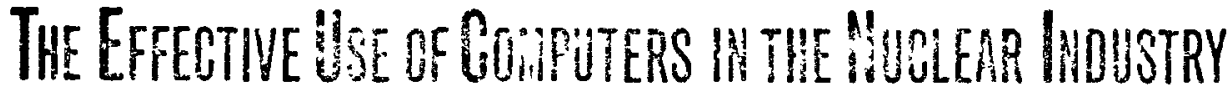 \\ A CONFERENCE AT KNONOXILLE, TENN. APRIL 21-23,1969 sponsored by}

THE MATHEMATICS AND COMPUTATION OIVISION OF THE AMERICAN NUCLEAR SOCIETY THE OAK RIDGE NATIONAL LABORATORY AND THE UNIVERSITY OF TENNESSEć

\section{PUBLICATION REIEASE FCRM}

This certifies that the paper

Title: APPLICATION OF AN IMAGE PROCESSING SYSTEM TO FRAGMENTATION STUDIES

Authors: Margaret K. Butler

Organization: Argonne National Laboratory

is cleared for publication. It is intended for Oral Presentation at the above meeting. The paper will be published in the proceedings of the meeting by the USAIC Division of Technical Information Extension, Oak Ridge, Tennessee. DTI may revroaices and make TID-4500 standard distribution, including copies to Fedoral Clearing-ouse, depository libraries and arrounce in IISA.

The paper contains no information that need be withheld from publication becase of national security interests, patent considerations, or proprietary infcrmation interests. Permission is hereby granted to publish it in the proceedings of the mesting which I understand will receive the above standard distribution.

This release is submitted by:

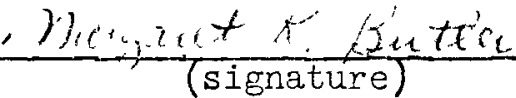
Margaret $K$. Butler

$-\frac{(\text { name typed })}{(\text { date })}$

The Author's copy of the proceedings should be mailed to the following complete address:

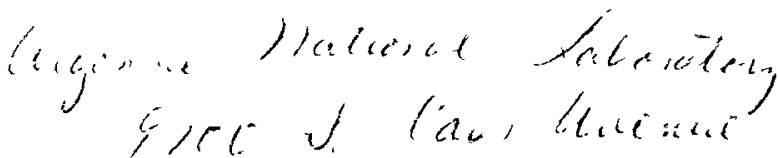

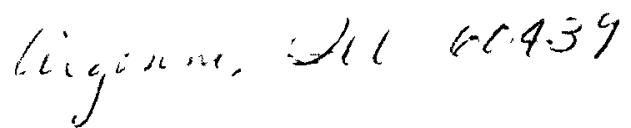


A CONFERENCE AT KNOXVILLE, TENN. AFRIL 21-23, 1969 sponsored by

THE MATHEMATICS AND COMPUTATION DIVISION OF THE AMERICAN NUCLEAR SOCIETY

THE GAY. RIDGE NATIONAL LABORATORY AND THE UNIVERSITY OF TENNESSEE

\section{FUEIICATION REIEASE FORM}

This certifies that the paper

Title: NOISY1 - A Program for Calculation of Space Dependent Auto and Cross Spectral Densities in Reactors

Authors:_James R. Sheff

Organization: Battelle-Northwest, P. O. Box 999, Richland, Washington 99352

is clearea for publication. It is intended for Oral Presentation at the above me zting. The paper will be published in the proceedings of the meeting cy the USAEC Division of Technical Information Extension, Oak Ridge, Tennessee. DTI may reproduce and make TID-4500 standard distribution, including copies to Federal Clearinghouse, depository libraries and announce in NSA.

The paper contains no information that need be withield from publication because of national. security interests, patent corsiderations, or proprietary information interests. Permission is hereby granted to publish it in the proceedings of the weeting which $I$ understand will receive the above standard distribution.

This release is submitted by:

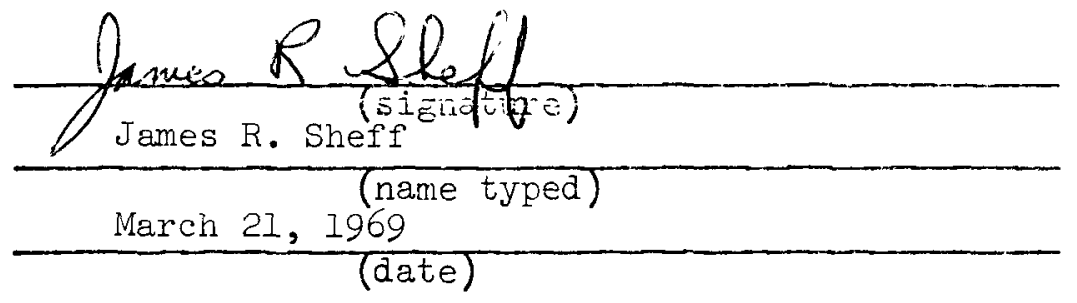

The Author's copy of the proceedings should be mailed to the following complete address: 
SESSION III. MULTIGROUP CALCULATIONS

1. "The Application of the Block Inversion Technique to Multi-Dimensional Reactor Calculations", M. R. Wagner (GGA-Europe)

4 "A New F/st Plongram for Thr le Dimensionaly FawGrou plu Cald hlations in Heterogeneous Beactors", A. Fengusen (CBGB, Landod, England)

3. "Reduction of Machine Time in Discrete Ordinates Calculations", R. E. Sullivan, L. K. Albers (NASA$L R C$ ), and D. Yu (Univ. of Okla.)

4. "A Two-Dimensional Transport Theory Program in a Modular System Environment", J. P. Friedman, B. W. Crawford (GE-KAPL)

5. "Optimization of the Doppler Effect in a Fast Material Test Reactor by Linear Programming Techniques", A. Gandini, M. Salvatores, G. Sena (CNEN, Casaccia, Italy ) 


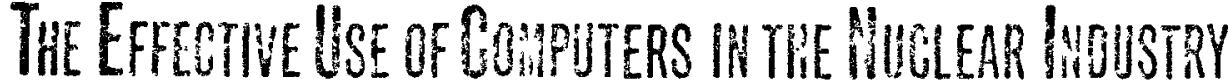 \\ A CONFERENCE AT KNOXVILLE, TENN. APRIL. 21-23.1969 sponsored by}

THE MATHEMATICS AND COMPUTATION DIVISION OF THE AMERICAN NUCLEAR SOCIETY

THE OAK RIDGE NATIONAL LA.BORATORY AND THE UNIVERSITY OF TENNESSEE

\section{PUBLICATION REIEASE FORM}

This certifies that the paper

Title: The Application of the Biock Inversion Technique to Multi-Dimensional Reactor Calculations

Authors: M. R. Wagner

Organization: Gulf General Atomic Europe, Zurich, Switzerland

is cleared for publication. It is intended for Oral Fresentation at the above meeting. The paper will be published in the proceodings of the meeting by tre USAJC Division of Technical Information Extension, Oak Ridge, Tennessee. DTI mey reproduce and make TID-14500 siandard distribution, including copies to Federal Clearinghous , depository libraries and announce in NSA.

The paper contains no information that need be withheld from publication bevalse of national security interests, patent considerations, or proprietary informatior: interests. Permission is hereby granted to publish it in the proceedings of the meeiing which I understand will receive the above siknday aistribution.

This release is subritted by:

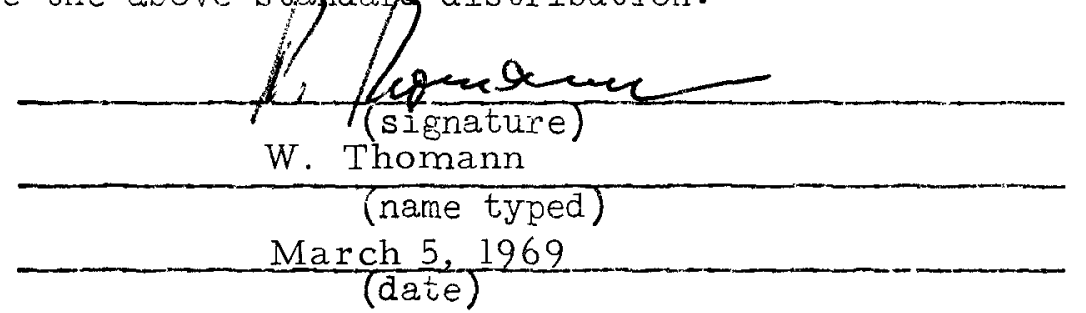

The Author's copy of the proceedings should be mailed to the following complete address:

Mr. M. R. Wagner

Gulf General Atomic Europe

Weinbergstrasse 109

8006 Zurich, Switzerland 


\section{A CONFERENCE AT KNOXVILLE, TENN. APRIL 21-23, 1969 sponsored by}

THE MATHEMATICS AND COMPUTATION DIVISION OF THE AMERICAN NUCLEAR SOCIETY

THE OAK RIDGE NATIONAL LABORATORY AND THE UNIVERSITY OF TENNESSEE

\section{PUBLICATION RELEASE FORM}

This certifies that the paper

Title: Reduction of Machine Time in Discrete Ordinates Calculations

Authors: Robert E. Sullivan, Lahsoong $\mathrm{Yu}$, and Lynn K. Albers

Organization:

IJASA-Lewis Research Center

is cleared for publication. It is intended for Oral Presentation at the above meeting. The paper will be published in the proceedings of the meeting by the USAIC Division of Technical Information Extension, Oak Ridge, Tennessee. DTI may reproduce and make TID-4500 standard distribution, including copies to Federal Clearinghouse, depository libraries and announce in NSA.

The paper contains no information that need be withrield from publication because of national security interests, patent considerations, or proprietary infornation interests. Fermission is hereby granted to publish it in the proceedings of the ineeting which I understand will receive the above standard distribution.

This release is submitted by:

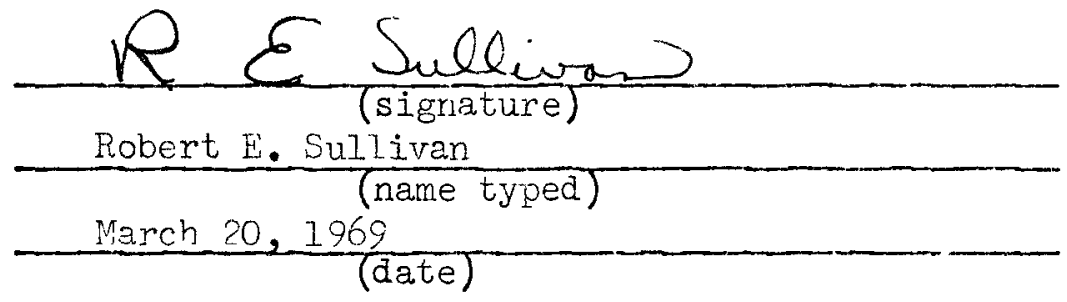

The Author's copy of the proceedings should be mailed to the following complete adaress:

Mr. Robert E. Sullivan

NASA-Lewis Fesearch Center

21000 Brooknark Road

Cleveland, Ohio 44135

Mail Stop: 49-2 


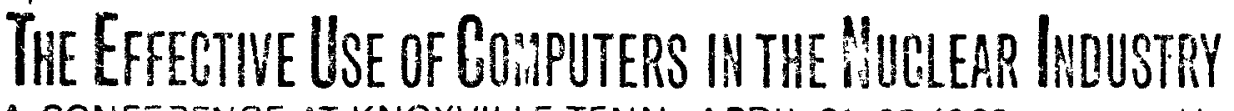 \\ A CONFERENCE AT K.NOXVILLE, TENN. •APRIL 21-23, 1969 sponsored by}

THE MATHEMATICS AND COMPUTATION DIVISION OF THE AMERICAN NUCLEAR SOCIETY

THE OAK RIDGE NATIONAL LABORATORY AND THE UNIVERSITY OF TENNESSEE

\section{PRLICATION RELEASE FORM}

This certifies that the paper

Title: A TWO-DIMENSIONAL TRANSPORT THEORY PROGRAM IN A MODULAR SYSTEM ENVIRONMENT

Authors:__. P. Friedman and Bmice W. Crawford

Organization:

Knolls Atomic Power Laboratory

is cleared for publication. It is intended for Oral Presentation at the above mes:ing. The paper will be published in the proceedings of the meeting by the ISAEC Division of Technical Information Extension, Oak Ridge, Iennessee. DTI may reuroduse and make TID-4500 standard distribution, including copies to Federal Clearinghcuse, depository libraries and announce in NSA.

The paper contains no information that need be withheld from publication because of national security interests, patent considerations, or proprietary information interests. Permission is hereby granted to publish it in the proceedings of the meeting which I understand will receive the above standard distribution.

This release is submitted by:

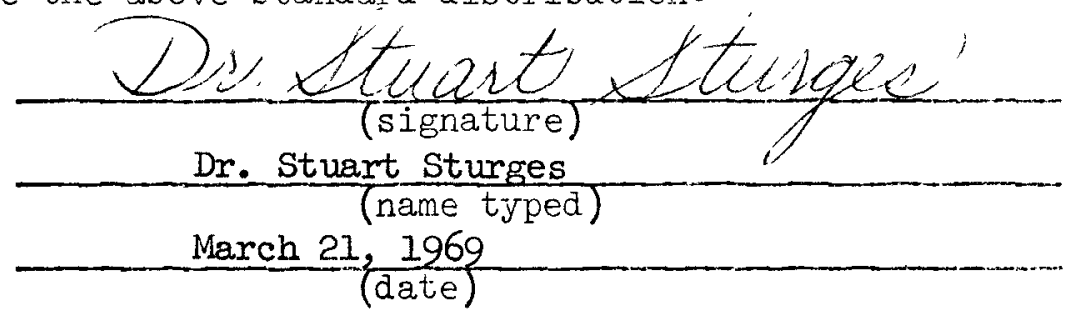

The Author's copy of the proceedings should be mailed to the following complete address:

Jack P. Friedman and

Bruce W. Crawford

Building G-2, Room 152

Knolls Atomic Power Laboratory

Box 1072

Schenectady, New York 12301 


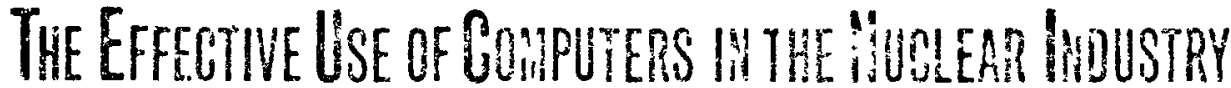 \\ A CONFERENCE AT KNOXVILLE, TENN. APRIL 21-23, 1969 sponsored by}

THE MATHEMATICS AND COMPUTATION DIVISION OF THE AMERICAN NUCLEAR SOCIETY

THE OAK RIDGE NATIONAL LAEORATOFY ANO THE UNIVERSITY OF TENNESSEE

\section{PUBLICATION RELEASE FORIA}

This certifies that the paper Title: OPTIIIZATION OF TIE DOPPLER LFTECT IN A FAST IATLRIAL TEST REACTOR BY PROGRAIIIIHG TECINIRUES

Authors: A.Gandini, M.Salvatores, G.Sena

Organization:_C.M.E.H. - L.E.C.R.C.S.N. CASACCIA

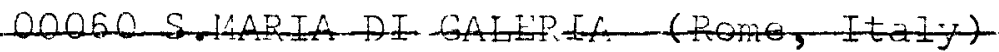

is cleared for publication. It is intended for Oral Presentation at the above reeting. The paper will be published in the proceedings of the meeting by tile USALC Division of Technical Infornation Extension, Dak Ridge, Tennessee. DTI nay reproducs and make TID-4500 standard distribution, including copies to Federal Clearinghouse, depository libraries and annource in INSA.

The paper contains no information that need be withheld from publication because of national security interests, patent considerations, or proprietary information interests. Permission is hereby granted to publish it in the proceedings of tre meeting which I understand will receive the above standard distribution.

This release is submitted by:

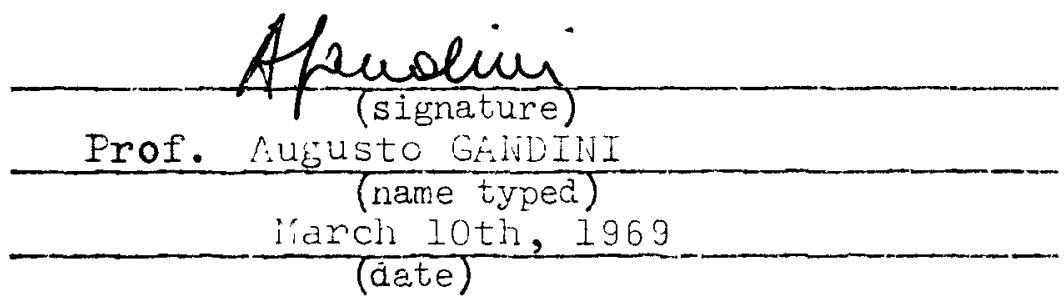

The Author's copy of the proceedings should be mailed to the following complete address:

LABCRATRRIO PISICA E CALCOLO REATTORI

C.S.IV. CASACCIA

OOO6O S.IARIA DI GALEPIA

(Rome, ITALY) 
SESSION IV. REACTOR DESIGN SYSTEMS 9:00 AM

"Variational Foundations of Flux Synthesis", E. L. Wachspress (GE-KAPL), Invited.

1. "CARONTE-The EURATOM System for Automatic Control of Linked Calculations", G. Buccari, G. Fattori, C. Mongini-Tamagnini (EURATOM-CCR, Ispra, Italy)

2. "The CODNUC System-A Modular System of SubRoutines for Reactor Calculation", J. P. Bayard, R. Boudet (CEN, Saclay, France)

3. "The Hitachi Nuclear Codes Control System, NCCS", T. Watanabe, K. Arai, T. Noda (Hitachi, Ltd., Tokyo, Japan)

4. "JOSHUA-An Operating System for Reactor Physics

Computations", J. E. Suich, J. C. Jensen, H. C. Honeck (SRL)

5. "Recent Developments and Capabilities in the ARC System", L. C. Just, P. Walker, B. J. Toppel (ANL)

6. "Three Dimensional BWR Simulation", R. L. Crowther, W. P. Petrick, A. Weitzberg (GE-NED, San Jose) 


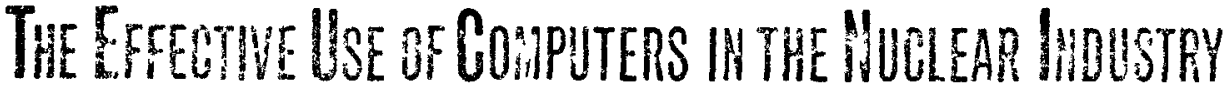

A CONFERENCE AT KNOXVILLE, TENN. APRIL 21-23, 1969 sponsored by

THE MATHEMATICS AND COMPUTATION DIVISION OF THE AMERICAN NUCLEAR SCCIETY THE OAK RIDGE NATIONAL LABORATORY AND THE UNIVERSITY OF TENNESSEE

\section{PUBIICATION RELEASE FORM}

This certifies that the paper

Title: VARIATIONAL METHODS AND NEUTRON FLUX SYNTHESIS

Authors:

E. L. Wachspress

Organization: Knolls Atomic Power Laboratory, General Electric Company

is cleared for publication. It is intended for Oral Presentation at the above meeting. The paper will be published in the proceedings of the metting by tile USAHC Division oi Technical Information Extension, Oak Ridge, Tennessee. DTI nay reprodics and make IID-4500 standard distribution, including copies to Federal Clearinghorse: depository libraries and annource in NSA.

The paper contains ro information that need be withheid from pubiication becalse of national security interests, patent considerations, or proprietary irformaion interesls. Permission is hereby granted to publish it in the proceedings of the meeting which I understand will receive the above standard distribution.

This release is submitted by:

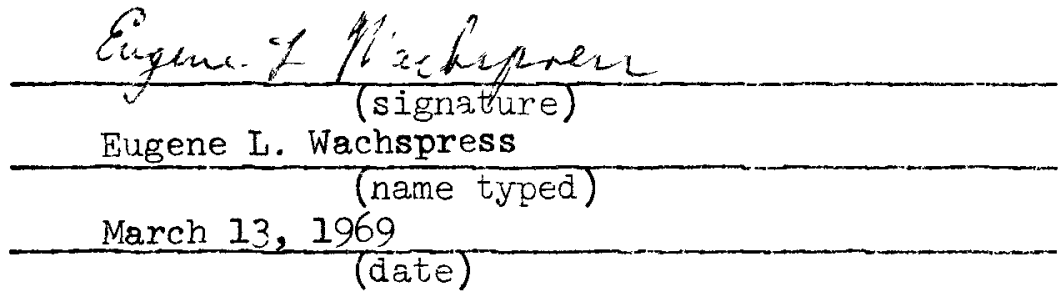

The Author's copy of the proceedings should be mailed to the following conplete ad ress: 


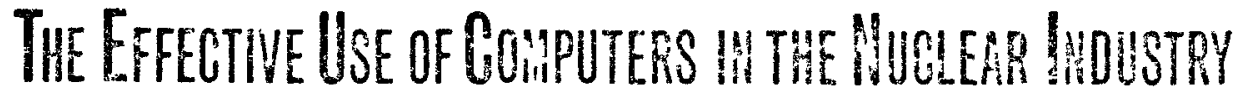

A CONFERENCE AT KNOXVILLE, TENN - APRIL 21-23.1959 suonsored by

THE MATHEMATICS AND COMPUTATION DIVISION OF THE AMERICAN NUCLEAR SOCIETY THE OAK RIDGE NATIONAL LABORATORY AND THE UNIVERSITY OF TENNESSEE

\section{PUBLICATION RELTASE FORM}

This certifies that the paper

Title: CARONTE - The EURATOM System for Automatic Control of Linked Calculations

Authors: G. Buccari, G. Fattori, C. Mongini Tamagnini

Organization:__EURATOM

is cleared for publication. It is intended for Oral Presentation at the above moeting. The paper will be published in the proceedings of the meeting by the USAEC Division of Technical Information Extension, Oak Ridge, Tennessee. DTI ray reproduce and make TID-4500 standard distribution, including copies to Federal Clearinghcus $\triangleq$, depository libraries and announce in NSA.

The paper contains no information that need be withheld from publication because of national security interests, patent considerations, or proprietary information interests. Permission is hereby granted to publish it in the proceedings of the meating which I understand will receive the above standard distribution.

This release is submitted by:

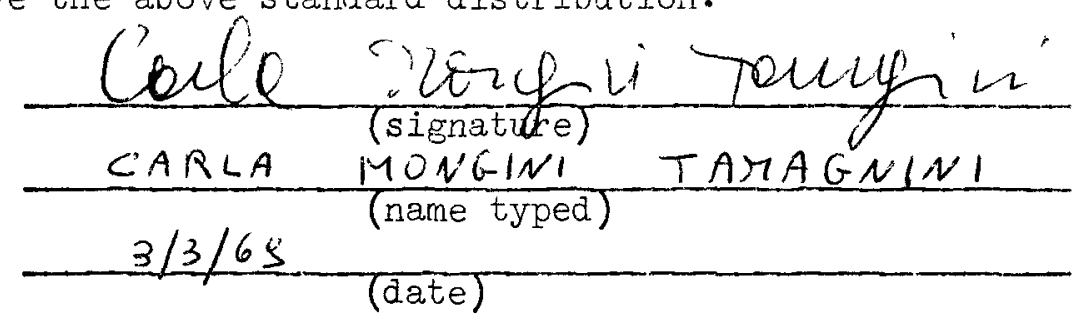

The Author's copy of the proceedings should be mailed to the following complete address:

G. Buccari

G. Fattori

C. Mongini Tamagnini

C.E.T.I.S.

EURATOM CCR

21020 Ispra

Italy 


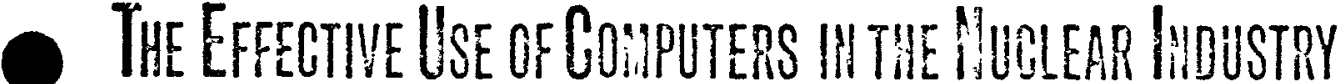

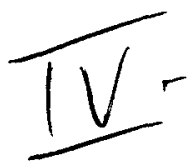

A CONFERENCE AT KNOXVILLE, TENN. APPRIL 21-23, 1969 sponsored by

THE MATHEMATICS AND COMPUTATION DIVISION OF THE AMERICAN NUCLEAR SOCIETY

THE OAK RIDGE NATIONAL LABORATORY AND THE UNIVERSITY OF TENNESSEE

PUBLICATION RELEASE FORM

This certifies that the paper

TitIE: THE CODNUC SYSTEM - A MODULAR SYSTEM OF SUBROUTINES FOR REACTOR CALCULATION

Authors: Jean-Panl BAYARD Roger BOVDET

Organization: COMMISSARIAT à l'ENERCiE ATOMIQUE - ERANCE

is cleared for publication. It is intended for Oral Presentation at the above meeting. The paper will be published in the proceedings of the meeting by the USAEC Division of Technical Information Extension, Oak Ridge, Tennessee. DTI may reproduce and make TID-4500 standard distribution, including copies to Federal Clearinghouse, depository libraries and announce in ISSA.

The paper contains no information that need be withheld from publication because of national security interests, patent considerations, or proprietary information interests. Permission is hereby granted to publish it in the proceedings of the meeting which I understand will receive the above standard distribution.

This release is submitted by:

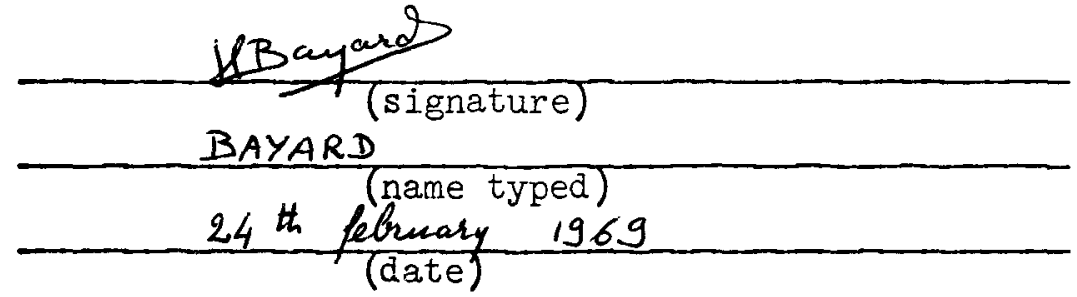

The Author's copy of the proceedings should be mailed to the following complete address:

J.P. BAYARD

D. C.E. - C.E.N. SACLAY

91 Gif SUR YVETTE

FRANCE 


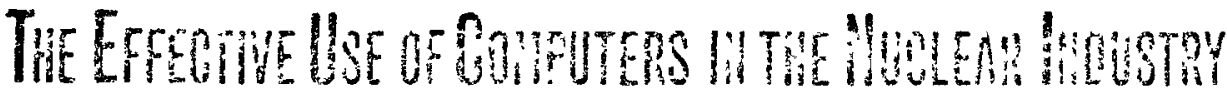

A CONFERENCE AT KNOXVILLE. TENN. APRIL 21-23, 1969 sponsored by

THE NIATHEIAATICS AND COMPIJTATION D: "SION OF THE AME?IZAN NUCLEAR SOCIETY THE OAK RILIGE NAIICNAL LaEORATORY AND THE UNIVERSITY OF TENINESSEE

\section{PUBLICATION RELEASE FORM}

This certifies that the paper

Mitle: Hitachi Nuclear Codes Control System, NCCS

Authors: T. Watanabe, K. Arai and T. Noda

Organization: Nuclear Energy Division, Central Research. Laboratory, Hitachi, Ltd.

is clearea for publication. It is intended for Oral Presentation at the abrva meeting. The paper will be publisned in the proneerings of the mestine hy the LE IE: Divisior of Technical Intormation Extension, Oak Ridge, Tennessee. DII may feprodure and make PDV-4500 standara distribution, in=luding copies to Federal Clearinghouse, depository librarjes and aunounce in NSA.

The paper contains no information that, need be withheld from publication because of national security interests, patent considerations, or proprietary information interests. Permission is hereby granted to publish it in the oroceedings of the leeting which I understand will receive the above standard distribution.

This release is submitted by:

$\frac{\text { Tam detatallah- }}{\text { Tan watanabe }}$

The Author's copy of the proceedings should be mailed to the following complete zadress:

Tan Watanabe

Nuclear Energy Division, Central Research Laboratory

Hitachi, Ltd. Ozenji, Kawas aki, Kanagawa, Japan 


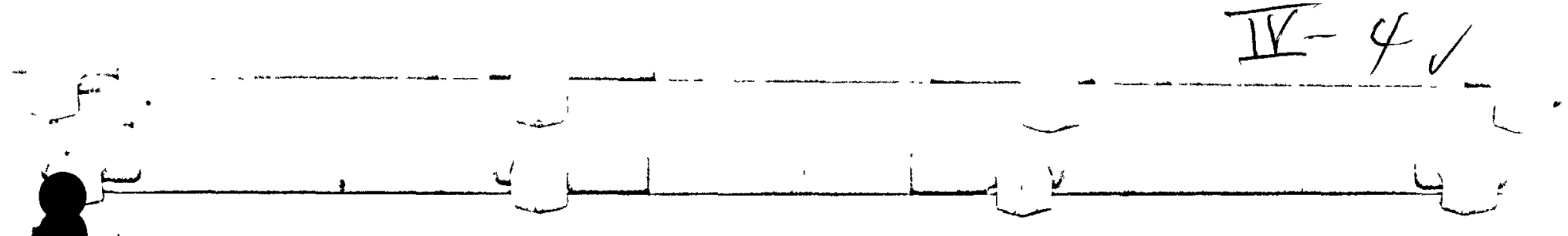

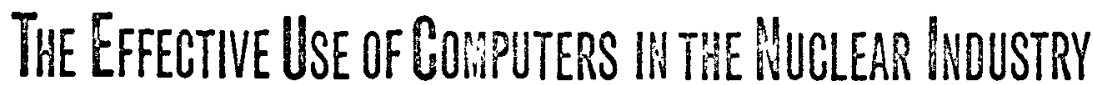

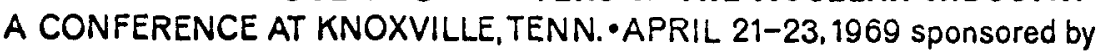

THE MATHEMATICS AND COMPUTATION DIVISION OF THE AMERICAN NUCLEAR SOCIETY

- THE OAK RIDGE NATIONAL LABORATORY AND THE UNIVERSITY OF TENNESSEE

\section{FUBLICATION REIEASE FORM}

This certifies that the paper

Title: JOSHUA - A Reactor Physjes Computational System

Authors: H. C. Honeck, et al

Organization: Savannah River Laboratory, E. I. du Pont de Nemours and Co., Aiken, S, C. 29801

is cleared for publication. It is intended for Oral Presentation at the above meeting. The paper will be published in the proceedings of the meeting by the USAEC Division of Technical Information Extension, Oak Ridge, Tennessee. DII may reproduce and make TID-4500 standard distribution, including copies to Federal Clearinghouse, depository libraries and announce in NSA.

The paper contains no information that need be withheld from publication because of national security interests, patent considerations, or proprietary information interests. Permission is hereby granted to publish it ip the proceedings of the meeting which $I$ understand will receive the above standard of stribution.

This release is submitted by:

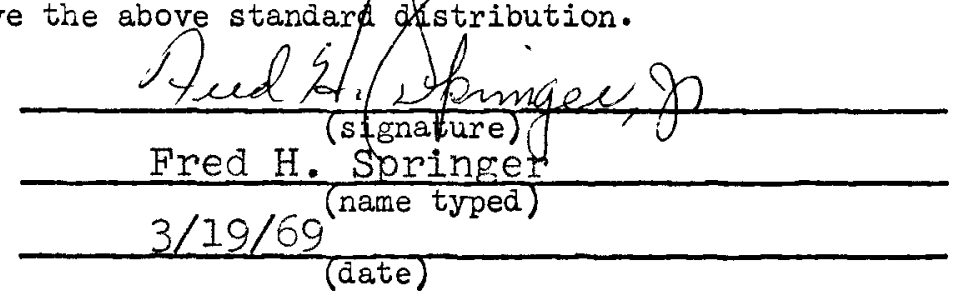

The Author's copy of the proceedings should be mailed to the following complete address:

J. I. OswaId

Savannah River Laboratory

E. I. du Pont de Nemours and Co.

Aiken, South Carolina 29801 


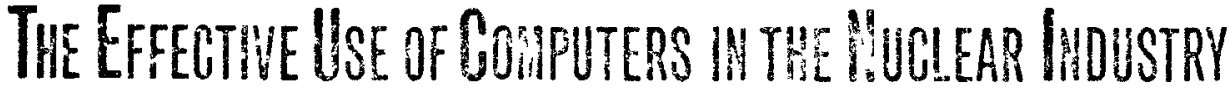 \\ A CONFERENCE AT KNOXYILLE, TENN. APRIL 21-23,1969 sponsored by}

THE MATHEMATICS AND COMPUTATION DIVISION OF THE AMERICAN NUCLEAR SOCIETY

THE OAK RIDGE NAIIONAL LABORATORY AND THE UNIVERSITY OF TENNESSEE

\section{PUBLICATION RELEASE FORM}

This certifies that the paper TitIe: RECENT DEVELOPMENTS AND CAPABILITIES IN THE ARC SYSTEM

Authors: L. C. Just, P. Walker, and B. J. Toppel

Organization: Argonne National Laboratory, Argonne, Illinois

is cleared for publication. It is intended for Oral Presentation at the above meeting. The paper will be published in the proceedings of the meeting by the USAFC Division of Technical. Information Extension, Oak Ridge, Tennessee. DTI may feproduce and make TID-4500 standard distribution, including copies to Federal Clearinghouse;

depository libraries and announce in NSA.

The paper contains no information that need be withheld from publication because of national security interests, patent considerations, or proprietary information interests. Permission is hereby granted to publish it in the proceedings of the mesting which I understand will receive the above standard distribution.

This release is submitted by:

(sigrature)
Mrs. Phyllis Walker
(name typed)
March 19. 1969
(date)

The Author's copy of the proceedings should be mailed to the following conplete address:

Applied Mathematics Division

Building, 221 - Room C216

Argonne National Laboratory

9700 South Cass Avenue

Argonne, Illinois 60439 


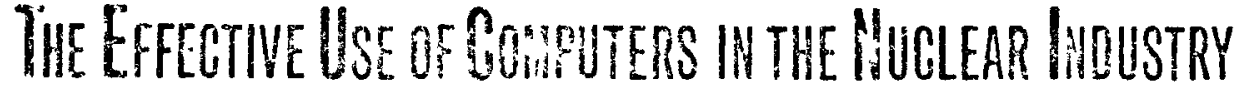 \\ A CONFERENCE AT KNOX̌VILIE, TENN - APFIL 21-23,1969 sponsored by}

THE MATHEMATICS AND COMPUTATION DIVISION OF THE AMERICAN NUCLEAR SOCIETY

THE OAK RIDGE NATIONAL LABORATORY AND THE UNIVERSITY OF TFNNESSEE

\section{PUBLICATION RELEAST FORM}

This certifies that the paper

Title: Three Dimensional BWR Simulation

Authors: R. L. Crowther, W. P. Petrick, and A. Weitzberg

Organization:___ General Electric Company

is cleared for publication. Il is intended for Oral Presentation at the above reer,ing. The paper will be published in the proceedings of the meeting by the USAEC Division of Technical Information Extension, Oak Ridge, Tennessee. DTI may reproduce and make TD-4500 standard distribution, including copies to Federal clearinghouse, depository libraries and announce in IVSA.

The paper contains no information that need be withneld from publication becuse of national security interests, patent considerations, or proprietary information interests. Permission is hereby granted to publish it in the proceedings of tre leeting which I understand will receive the above standard distribution.

This release is submitted by:

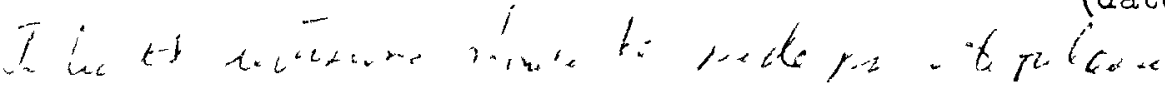

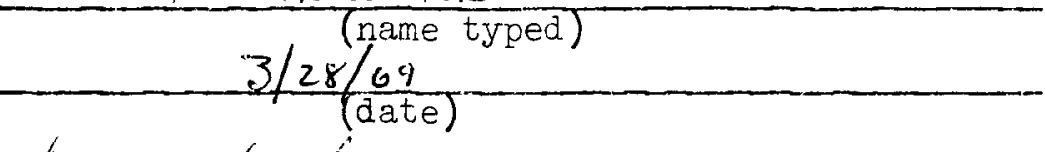

The Author's copy of the proceedings should be mailed to the following complete address:

General Electric Company

Mail Code 523

175 Curtner Avenue

San Jose, California 95125 
SESSION V. HYBRID COMPUTATIONS 1:20 PM

"Hybrid Solution of Partial Differential Equations in

Nuclear Plants Simulation: A Review", R. Vichnevetsky ( $E A I)$, Invited.

1. "The Use of Hybrid Computers in the Italian CNEN Nuclear Program", A. C. Mathis (CNEN, Casaccia, Italy)

E. "T. ansients in an One-Through Steam Generator" C. K. Shananan A. A sandbetg (Unjo. of (II.) and F. Al Chark, O A-Burke R. S. Stone (ORNL)

3. "Hybrid Computations in FFTF System Conceptual Design", A. L. Gunby, G. A. Worth (BNWL)

4. "Use of An Analog Compurer in the Design of a Cotitrol System for a Nuclear Reactor Operating Near Pyonmpt Griticat, O.W. Burke, L. C. Odkes, S? F. Dito, TI Anderson (ORNLF) 


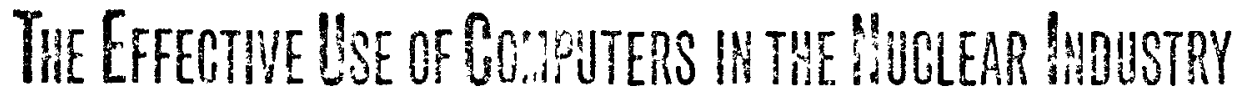 \\ A CONFERENCE AT KNOXVILLE, TENN. APRIL 2:-23,1969 sponsored DY}

THE MATHEMATICS AND COMPUTATION DIVISION OÉ THE AMERICAN NUCLEAR SOCIETY

THE OAK RIDGE NATICNAL LABORATORY AND THE UNIVERSITY OF TENNESSEE

PUBLICATION RELFASE FORM

This certifies that the paper

Title: HYBRID SOLUTION OF PARTIAL DIFFERENTIAI, EOUATTONS

IN NUCLEAR PIANT SIMULATIONS. A REVIEW

Authors: Robert Vichnevetsky

Organization: Electronic Associates, Inc.

P. O. Box 582 e Princeton, N. I.

is cleared for puolication. It is intended for Orai Presentation at tre abova neeting. The paper will be pablished in the proceedings of the meeting by tne TSAEC Division of Technical Information Extension, vak Ridge, Tennessee. DTI may reproduce and make TID-4500 standard distribution, including copies to Federa: Clearinghouse, depository libraries and announce in INSÁ.

The paper contains no information that need be witheld from publication because of national security interests, patent considerations, or proprietary informajion interests. Permission is hereby granted to publish it in the proceedings of the mest... ing which I understand will receive the abové standara distribution.

This release is submitted by:

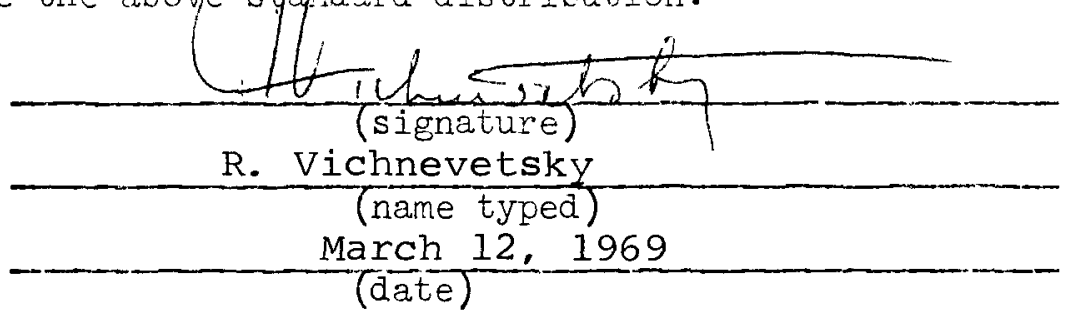

The Author's copy of the proceedings should be wailed to the following complete jedreas:

Dr. R. Vichnevetsky

P. O. Box. 582

Electronic Associates, Inc.

Princeton, New Jersey 08540 


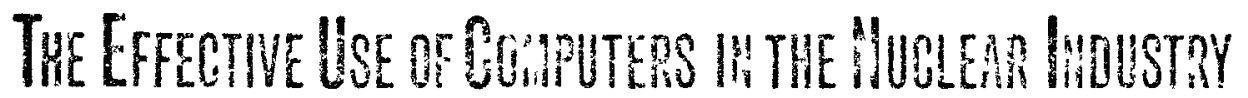 \\ A CONFERENCE AT KNOXVILLE, TENN - AFRIL 21-23, 1950 sponsored by}

THE MATHEMATICS AND COMPLTATION D.VISION OF THE ANIERICAN NUCLEAR SOCIETY

THE OAK RIDGE NATIONAL LABORATOFY AND THE UNIVERSITY OF TENNESSEE

\section{PUBLICAMION RELFASE FORM}

This certifies that the paper

Title: THE USE OF HYRRID COIFUPEKS IN THE ITALIAN CHEN NUCLEAK EROTEATE

Authors: "ATHIS A., Frof. Fing.

Organization: Comitato irazionale per 1'Energia Nucleare - Jentro Studi

Nucleari della Casaccia - $00050 \mathrm{ROAA}$ (Italy)

is cleared for publication. It is intended for Oral Presentation at the abure meeting. The paper will be published in the proceedings of the meeting by the UJAFC Division of Technical Information Extension, Oak Ridge, Tennessee. DTI may recroduce and make IID-4500 standard distribution, including copies to F'ederal slearinghowe, deposjtory libraries and announce in INSA.

The paper contains no information that need be withheld from publication bectuse of national security interests, patent considerations, or proprietary informetion interests. Permiscion is hereby granted to publish it in the proceedings of the reeting which I understand will receive the above standerd distribution.

This releasc is submitted by:

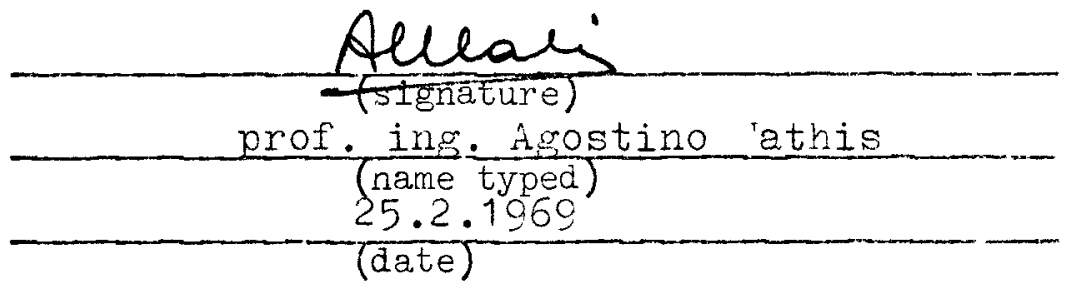

The Author's copy of the proceedings should be mailed to the following complete address:

Prof. A. Matnis

Comitato Nazionale Energia Nucleare

Centro Studi Nucleari della Casaccia

00060 Casaccia - Roma (ITALY) 
A CONFERENCE AT KNOXVILLE, TENN. APRIL 21-23, 1969 sponsored by

THE MATHEMATICS AND COMPUTATION DIVISION OF THE AMERICAN NUCLEAR SOCIETY THE OAK RIDGE NATIONAL LABORATORY AND THE UNIVERSITY OF TENNESSEE

\section{PUBLICATION REIEASE FORM}

This certifjes that the naper Title: HYBRID SIMULATION IN FFTF SYSTEM CONCEPTUAL DESIGN

Authors: A. L. Gunby and G. A. Worth ${ }^{2}$

Organization:

Battelle Northwest: (1) FFTF Project, (2) Applied Physics

and Electronics Department.

is cleared for publication. It is intended for Oral Presentation at the above reeting. The paper will be published in the proceedings of the meeting by the USAEC Division of Technical Information Extension, Oak Ridge, Tennessee. DTI may reprozucs and make TID-4500 standard distribution, including copies to Federal Clearindhouse, depository libraries and anrounce in NSA.

The paper contains no information that need be withheld from publication becuuse of national security interest,s, patent considerations, or proprietary information interests. Permission is hereby granted to publish it in the proceedings of the ineting which I understand will receive the above standard distribution.

This release is submitteri by:

\begin{tabular}{|c|}
\hline A. L. Gunby (signature) \\
\hline March 12, (name typed) \\
\hline
\end{tabular}

The Author's copy of the proceedings should be mailed to the following complete zadress:

Albert L. Gunby 1009 Torbett St. Richland, Washinaton 99352
Grant A. Worth 2403 Torbett St. Richland, Washington 99352 
SESSION VI. COMPUTATIONAL TECHNIQUES

1. "The Effective Use of the Computer Storage Hierarchy", T. L. Jordan, W. J. Worlton (LASL)

2. "Computer Independence of Large Reactor Physics Codes With Reference to Well Balanced Computer Configurations", R. Froehlich (GGA)

(3. Ttorge Alocation and Data Xansfer in the pro-z Peosram", W. R. Gashet (W-BADL)

4. "Data Management for Multiaccess Computational Systems", J. E. Suich, J. C. Jensen, C. E. Bailey, H. C. Honeck, R. A. Priest (SRL)

5. "A Burnup System Control and Calculation Method", E. R. Volk, J. S. Gubkin, D. B. Wehmeyer, C. S. Yeh (APDA)

6. "Some Aspects of Nuclear Model Programming", K. J. Yost (ORNL) 


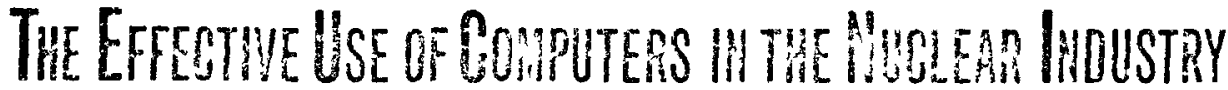

A CONFEREATE AT KNOXVILLE, TENN - APRIL 21-23,1969 sponsored by

THE MATHEMATICS AND COMPUTATION DIVISION OF THE ANIERICAN NUCLEAR SOCIETY

THE OAK RIDGE NATICNAL LABORATORY AND THE UNIVERSITY OF TENNESSEE

\section{PUBLICATION RELEASE FORM}

This certifies that the paper

Title: THE EFFECTIVE USE OF THE COMPUTER STORAGE HIERARCHY

Authors: Thomas L. Jordan and William J. Worlton

Organization: Los Alamos Scientific Laboratory

is cleared for publication. It is intended for Oral Presentation at the ac e nesring. The paper will be published in the proceedings of the meeting by the UiAEC Division of Technical Information Extension, Oak Rilge, Tennessee. DII may reprnicke and make TID-4500 standard distribution, including copies to Federal Cledrinchnuse, depository libraries and announce in IISA.

The paper contains no information that need be withheld from publication becanse of national security interests, patent considerations, or proprietary information interests. Permission is hereby granted to publish it in the proceedings cf the lusting which $I$ understand will receive the above standard distribution.

This release is submitted by:

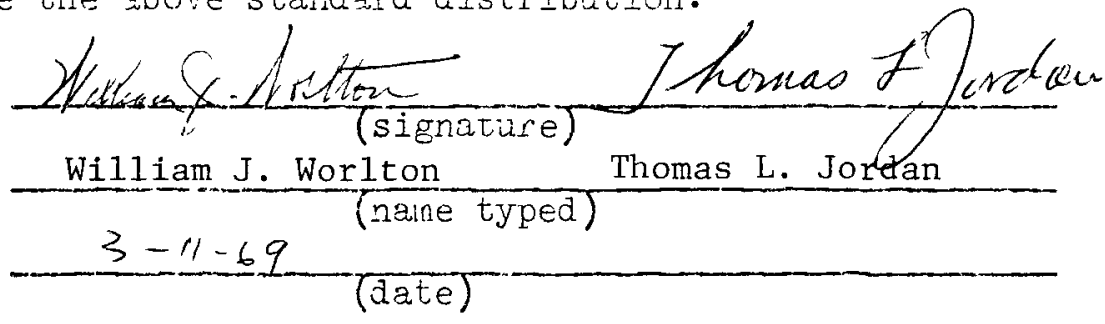

The Author's copy of the propediros at puid te mided to the following complete address:

William J. Worlton, CADP

Los Alamos Scientific Laboratory

P. O. Box 1663

Los Alamos, New Mexico 87544
Thomas L. Jordan, C-DO

Los Alamos Scientific Laboratory

P. O. Box 1663

Los Alamos, New Mexico 87544 


\section{The EFfective Ulse of CoMputers in the Nuclear lidustrit \\ A CONFERENCE AT KNOXVILLE, TENN. APRIL 21-23,1969 sponsored by}

THE MATHEMATICS AND COMPUTATION DIVISION OF THE AMERICAN NUCLEAR SOCIETY

THE OAK RIDGE NATIONAL LABORATORY AND THE UNIVERSITY OF TENNESSEE

\section{PUBLICATION RELEASE FORM}

This certifies that the paper

Title:___ Computer Independence of Large Reactor Physics Codes with Reference to

Well Balanced Computer Configurations

Authors: Reimar Froehlich

Organization: Gulf General Atomic Incorporated

P. O. Box 608, San Diego, California 92112

is cleared for publication. It is intended for Oral Presentation at the above mest-

ing. The paper will be published in the proceedings of the meeting by the USAEC

Division of Technical Information Extension, Oak Ridge, Tennesset. DTI day ceproike and make TID-4500 standard distribution, including copies to Federal clearinghouse, depository libraries and announce in NSA.

The paper contains no information that need be withheld from publication becoxse of national security interests, patent considerations, or proprietary information interests. Permission is hereby granted to publish it in the proceedirgs of the reeting which I understand will receive the above standard distribution.

This release is submitted by:

Reime triklich (signature)

Reimar Froehlich

$\frac{\text { Reimar Froeh1ich }}{\text { (name typed) }}$

(date)

The Author's copy of the proceedings should be mailed to the following complete adaress:

Dr. Reimar Froehlich

Gulf General Atomic Incorporated

P. O. Box 608

San Diego, California 92112 


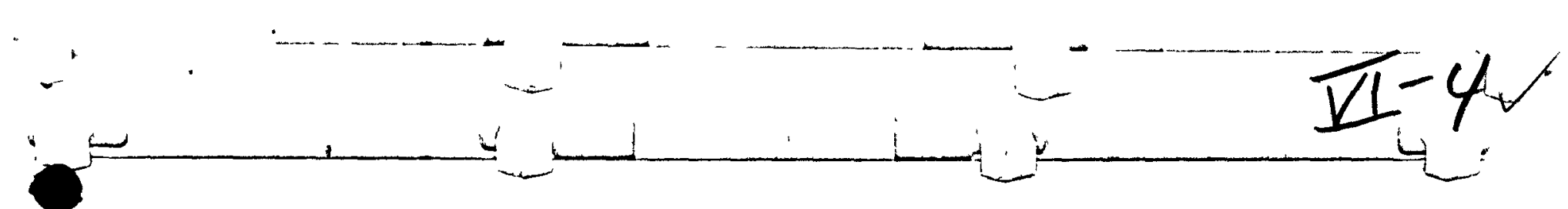

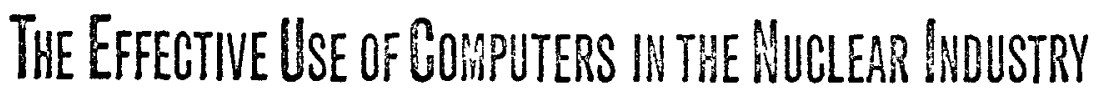 \\ A CONFERENCE AT KNOXVILLE, TENN. A APRIL 21-23, 1969 sponsored by}

THE MATHEMATICS AND COMPUTATION DIVISION OF THE AMERICAN NUCLEAR SOCIETY

, THE OAK RIDGE NATIONAL LABORATORY AND THE UNIVERSITY OF TENNESSEE

\section{PUBLICATION RELEASE FORM}

This certifies that the paper

Title: Data Management for Multiaccess Computational Systems

Authors: J.E. Suich, et aI.

Organization: Savannah River Laboratory, E. I. du Pont

de Nemours and Co., Aiken, S. C. 29801

is cleared for publication. It is intended for Oral Presentation at the above meeting. The paper will be published in the proceedings of the meeting by the USAEC

Division of Technical Information Extension, Oak Ridge, Tennessee. DTI may reproduce and make TID-4500 standard distribution, including copies to Federal Clearinghouse, depository Iibraries and announce in NSA.

The paper contains no information that need be withheld from publication because of national security interests, patent considerations, or proprietary information interests. Permission is hereby granted to publish it in the proceedings of the meeting which I understand will receive the above standafd distribution.

This release is submitted by:

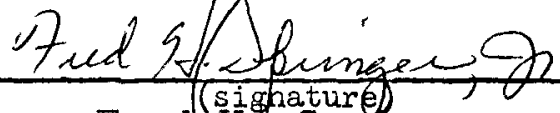

$$
\begin{aligned}
& \text { Fred H. Springer } \\
& \text { 3/19/6ge typed) } \\
& \text { (date) }
\end{aligned}
$$

The Author's copy of the proceedings should be mailea to the following complete adaress:

J. I. OswaId

Savannah River Laboratory

E. I. du Pont de Nemours and Co.

Aiken, South Carolina 29801 


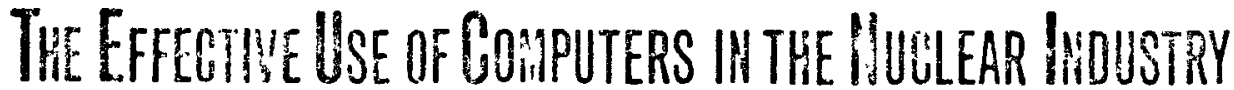 \\ A CONFERENCE AT KNOXVILLE, TENN. APRIL 21-23,1969 sponsorad by}

THE MATHEMATICS AND COMPUTATION DIVISION OF THE AMERICAN NUCLEAR SOCIETY

THE OAK RIDGE NATIONAL LABORATORY AND THE UNIVERSITY OF TENNESSEE

\section{PUBLICATION RELEASE FORM}

This certifies that the paper

Title:

A BURNUP SYSTEM CONTROL AND CALCULATION METHOD

Authors: E. R. Volk, J. S. Gubkin, D. B. Wehmeyer, C. S. Yeh

Organization: ATOMIC POWER DEVELOPMENT ASSOCIATAS, INC.

is cleared for publication. It is intended for oral Presentation at the above neeting. The paper will be published in the proceedings of the meeting by the USAEC Division of Technical Information Extension, Oak Ridge, Tennessee. DTI may reproduce and make TID-4500 standard distribution, including copies to Federal Clearinghouse, depository libraries and announce in NSA.

The paper contains no information that need be withheld from publication because of national security interests, patent considerations, or proprietary information interests. Permission is hereby granted to publish it in the proceedings of the meeting which $I$ understand will receive
This release is submitted by:

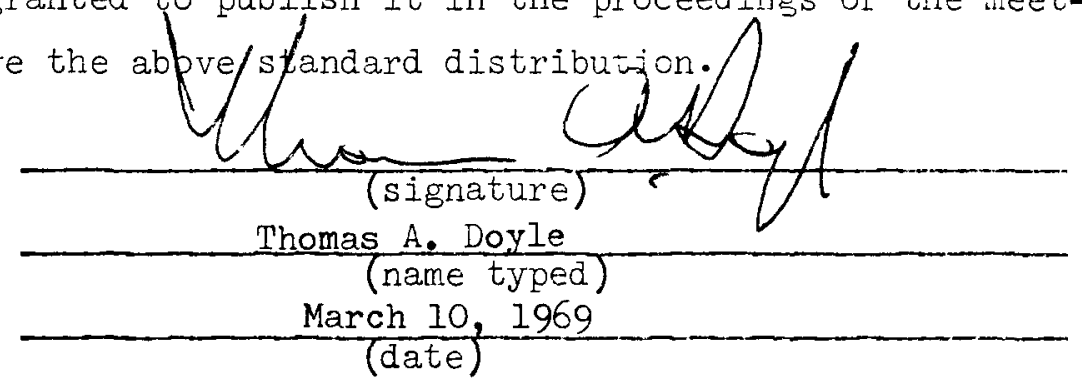

The Author's copy of the proceedings should be mailed to the following complete address:

Eugene R. Volk, Head

Mathematical Analysis Section

Atomic Power Development Associates, Inc.

1911 First Street

Detroit, Michigan 48226 


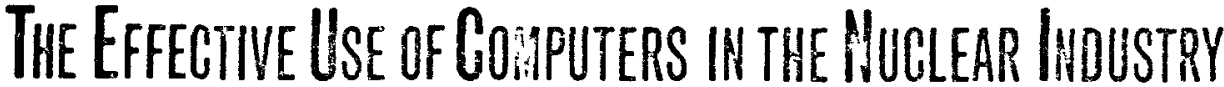 \\ A CONFERENCE AT KNOXVILLE, TENN. APRIL 21-23, 1969 sponsored by}

THE MATHEMATICS AND COMPUTATION DIVISION OF THE AMERICAN NUCLEAR SOCIETY THE OAK RIDGE NATIONAL LABORATORY AND THE UNIVERSITY OF TENNESSEE

\section{PUBLICATION RELEASE FORM}

This certifies that the paper

Title: Nuclear Model Programming for Data Generation

Authors: K. J. Yost

Organization: Oak Ridge National Laboratory

is cleared for publication. It is intended for Oral Presentation at the above meeting. The paper will be published in the proceedings of the meeting by the USAEC Division of Technical Information Extension, Oak Ridge, Tennessee. DTr alay reprcaixce and make TID-4500 standard distribution, including copies to Federal Clearinghouse, depository libraries and announce in NSA.

The paper contains no information that need be withheld from publicaticn because of national security interests, patent considerations, or proprietary information interests. Permission is hereby granted to publish it in the proceedings of the meeiing which I understand will receive the above standard distribution.

This release is submitted by:

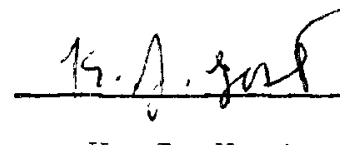

$\frac{\text { K. J. Yost }(\text { signature) }}{\text { March 11, 1969 (name typed) }}$

The Author's copy of the proceedings should be mailed to the following complete address: Neutron Physics Division Oak Ridge National Laboratory P. O. Box X Oak Ridge, Tennessee 37830 


\section{WEDNESDAY, APRIL 23, 1969}

SESSION VII. ENGINEERING ANALYSIS 9:00 AM "Review of Computational Methods in Solid Mechanics and Stress Analysis", Y. R. Rashid (GGA), Invited.

1. "Automated Thermal Analysis of Nuclear Reactor Components", A. Y. Lee, K. J. Wells (WANL)

2. "Numerical Analysis of Two-Dimensional, Dynamic, Thermal Stresses in a Hollow Cylinder", J. A. Reuscher, M. R. Scott (Sandia)

3. "An Explicit Unlimited Stability Approach to the Transient Conduction Convection Equations", D. J. Bender (GE-APO, Sunnyvale)

4. "TRACK II, A Computer Program for Transient Thermal Analysis of Complex Nuclear Reactor Systems With Multiple Parallel and Series Flow Paths", M. D. Woods, L. D. Hamlin (WANL)

5. "ROBOT-A Computer Program to Solve the Bowing Problem in Rod-Type Fuel Elements", J. J. Urbaniak (W-BAPL) 


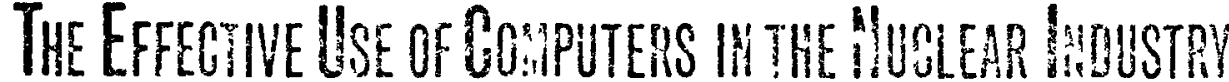

A CONFERENCE AT KNOXVILLE, TENN. APRIL 2:-23,1969 sponsored by

THE MATHEMATICS AND COMPUTATION DIVISION OF THE AMERICAN NUCLEAR SOCIETY THE OAK RIDGE NATIONAL LABOPATORY AIUD THE UNIVERSITY OF TENNESSEE

\section{PUBL.TCATION RTLEASE FORM}

This certifies that the paper

Title: "On Computational Methods In Solid Mechanics and Stress Analysis"

Authort: Y. R. Rashid

Organization: Gulf General Atomic

is cleared for pliblication. It is intended for Cral Presentaticr at the above me ing. The paper will be published in the proceedings of the meetirg by the USAEC Divizion of Technical Information Extension, Oak Ridge, Tennessee. DII may reproduce and make TID-4500 standard distribution, including copies to Federal Clearinghous depository libraties and announce in ITSA.

The paper contains no information that need be withheld from rublicatiun beziuse of national security interests, patent considerations, or proprietary informationinterests. Permission is hereby granted to publish it in the proceedings of the 'wseting which I understard will receive the above standard distripution.

This release is submitted by:

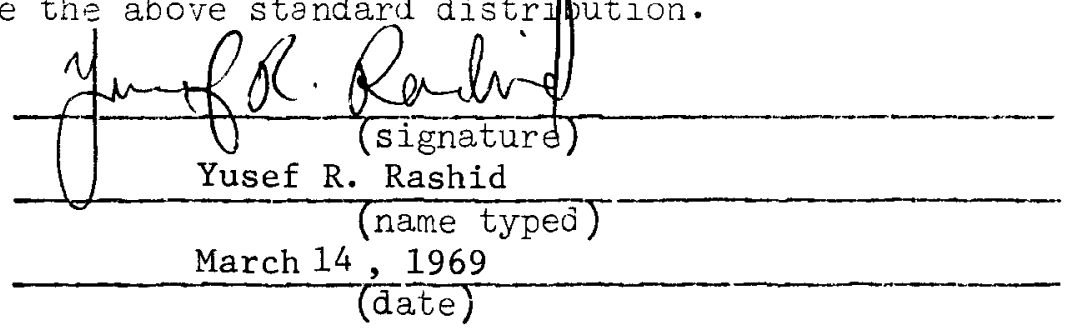

The Author's copy of the proceedings should be mailed to the following comelete address:

Dr. Yusef R. Rashid

Gulf General Atomic

P. 0. Box 608

San Diego, California 92112 


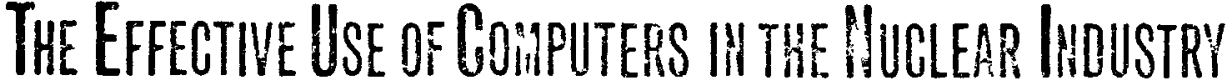

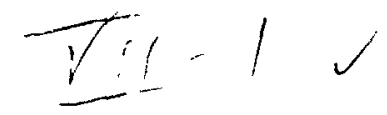

A CONFERENCE AT KNOXVILLE, TENN. APRIL 21-23,1969 sponsored by

THE MATHEMATICS AND COMPUTATION DIVISION OF THE AMERICAN NUCLEAR SOCIETY THE OAK RIDGE NATIONAL LABORATORY AND THE UNIVERSITY OF TENNESSEE

\section{PUBIICATION RETEASE FORM}

This certifies that the paper Title: Automated Thermal Analysis of Nuclear Reactor Components

Authors:

A. Y. Lee and K. J. Wells

Organization:

Westinghouse Electric Corporation, Astronuclear Laboratory,

Pittsburgh, Pennsylvania

is cleared for publication. It is intended for Oral Presentation at the above meeting. The paper will be published in the proceedings of the meeting by the USAEC Division of Technical Information Extension, Oak Ridge, Tennessee. DTI may revroduce and make TID-4500 standard distribution, including copies to Federal Clearinghouse:

depository libraries and announce in NSA.

The paper contains no information that need be withhela from publication becast. of national security interests, patent considerations, or proprietary information interests. Permission is hereby granted to publish it in the proceedings $0:-$ the neeting which I understand will receive the abovg standard distribution.

This release is submitted by:

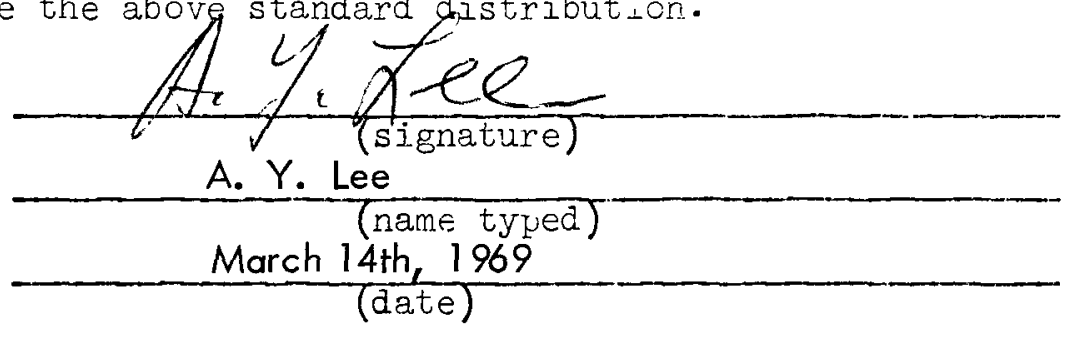

The Author's copy of the proceedings should be mailed to the following complete address:

Westinghouse Electric Corporation

Astronuclear Laboratory

Box 10864

Pittsburgh, Pennsylvania 15236 


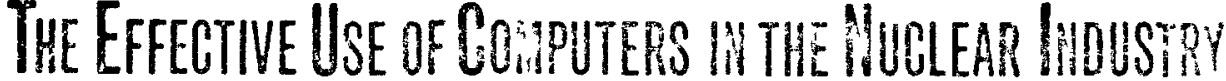

A CONFERENCE AT KNOXVILLE,TENN •APRIL 21-23,1969 sponsored

THE MATHEMATICS AND COMPUTATION DIVISION OF THE AMEPICAN NUCLEAR SOCIETY THE OAK RIDGE NATIONAL LABORATORY AND THE UNIVERSITY OF TENNESSEE

\section{PUBIICATION REIEASE FORM}

This certifies that the paper Title:

Numerical Analysis of Iwo-Dimensional, - Themal-stresses in a Hollow Cylinder

Authors: J. A. Reuscher and M. R. Scott

Organization: Sandia Laboratories; Albuquerque, New Mexico

is cleared for publication. It is intended for Oral Presentation at the above meeting. The paper will be published in the proceedings of the meeting by the USALC Division of Technical Information Extension, Oak Ridge, Tennessee. DTI may reproduce and mike TID-4500 standard distribution, including copies to Federal Clearinghous =, depository Iibraries and announce in NSA.

The paper contains no information that need be withheld from publication because of national security interests, patent considerations, or proprietary information interests. Permission is hereby granted to publish it in the proceedings of the meeting which I understand will receive the above standard distribution.

This release is submitted by:

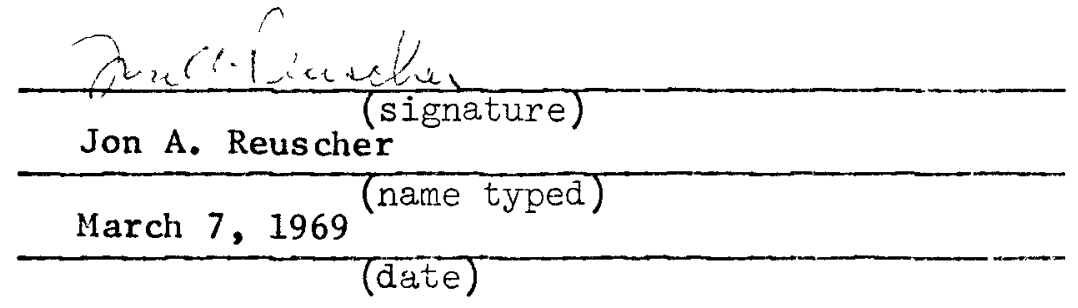

The Author's copy of the proceedings should be mailed to the following complete address:

J. A. Reuscher Division 5222

Sandia Laboratories

P. O. Box 5800

Albuquerque, N. M. 87115
M. R. $S \cot t$

Division 5222

Sandia Laboratories

P. O. Box 5800

Albuquerque, N. M.

87115 
A CONFERENCE AT KNOXVILLE, TENN - APRIL 21-23, 1969 sponsored by

THE MATHEMATICS AND COMPUTATION DIVISION OF THE AMERICAN NUCLEAR SOCIETY THE OAK RIDGE NATIONAL LABORATORY AND THE UNIVERSITY OF TENNESSEE

\section{PUBI ICATION RETEASE FORN}

This certifies that the paper

Title: An Explicit Unlimited Stability Approach to the Transient ConductionConvection Equations

Authors: David J. Bender

Organization: General Electric Company, Breeder Reactor Development Organization

is cleared for publication. It is intended for oral Presentation at the above meeting. The paper will be published in the proceedings of the meetir.g by the USAES Division of Technical Information Extension, Oak Ridge, Tennessee. DTI may sanrcauce and make TID-4500 standard distribution, including copies to Feceral Clearinghouse, devository libraries and announce in NSA.

The paper contains no information that need be withheld from publication beause of national security interests, patent considerations, or proprietary information

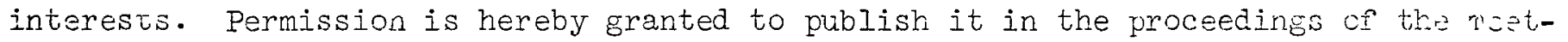
ing which I understand will receive the abowe standard distribuvion.

This release is submitted by:

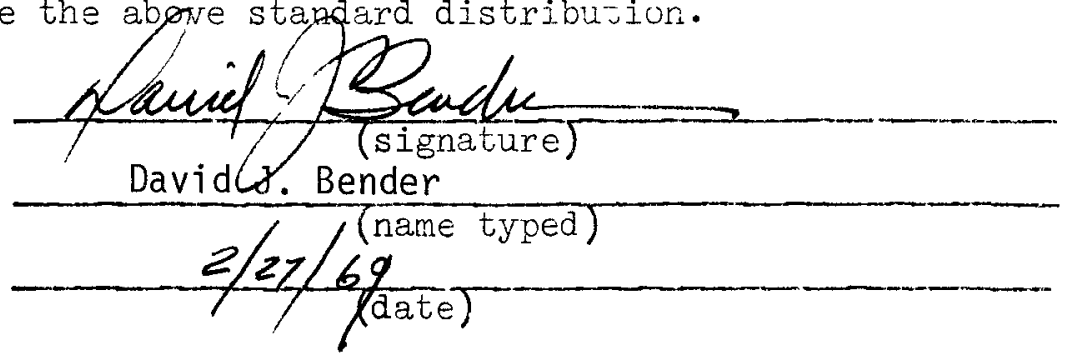

The Author's copy of the proceedings should be mailed to the following complete address:

Mr. David J. Bender

General Electric Company

Breeder Reactor Development Operation

310 DeGuigne Drive

Sunnyvale, Cal ifornia

94086 


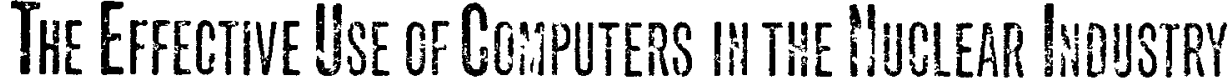 \\ A CONFERENCE AT KNOXVILLE, TENN. A APRIL 21-23,1969 sponsored by
}

THE MATHEMATICS AND COMPUTATION DIVISION OF THE AMERICAN NUCLEAR SCCIETY THE OAK RIDGE NATIONAL LABORATORY AND THE UNIVERSITY OF TENNESSEE

\section{PUBLICATION RELEASE FORM}

This certifies that the paper

Title: TRACK II, A Computer Program for Transient Thermal Analysis of Complex Nuclear Reactor Systems with Multiple Parallel and Series Flow Paths

Authors: M. D. Woods and L. D. Hamlin

Organization: Westinghouse Electric Corporation, Astronuclear Laboratory

is cleared for publication. It is intended for Oral Fresentation at the above meting. The paper will be published in the proceedings of the meeting by tre USAPC Division of Technical Information Extension, Oak Riage, Tennessee. DII may reproiluee and make TID-4500 standard distribution, including copies to Federal Clearinghous?, depository libraries and announce in NSA.

The paper contains no information that need be withheld from publication becalase of national security interests, patent considerations, or proprietary information interests. Permission is hereby granted to publish it in the proceedings of the weeting which I understand will receive the above standard distribution.

This release is submitted by:

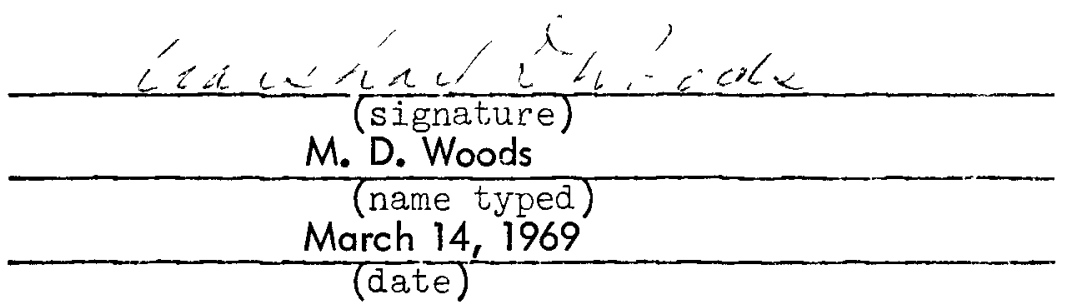

The Author's copy of the proceedings should be mailed to the following complete address:

\author{
Westinghouse Electric Corporation \\ Astronuclear Laboratory \\ Box 10864 \\ Pittsburgh, Pennsylvania \\ 15236
}


A CONFERENCE AT KNOXVILLE, TENN. APRIL 21-23, 1969 sponsored by

THE MATHEMATICS AND COMPUTATION DIVISION OF THE AMERICAN NUCLEAR SOCIETY

THE OAK RIDGE NATIONAL LABORATORY AND THE UNIVERSITY OF TENNESSEE

\section{PUBLICATION RELEASE FORM}

This certifies that the paper

Title:__ROBOT - A Computer Program to Solve the Bowing Problem in

Rod-Type Fuel Elements (LWBR Development Program)

Authors: John J. Urbaniak

Organization:

Westinghouse Electric Corporation

Bettis Atomic Power Laboratory

is cleared for publication. It is intended for Oral Presentation at the above neeting. The paper will be published in the proceedings of the meeting by the UCAEC

Division of Technical Information Extension, Oak Ridge, Iennessee. DTI may reproduce and make $T D-4500$ standard distribution, including copies to Federal Clearinghouse,

depository libraries and announce in NSA.

The paper contains no information that need be withheld from publication because of national security interests, patent considerations, or proprietary information interests. Permission is hereby granted to publish it in the proceedings of the meeting which I understand will receive the above standard distribution.

This release is submitted by:

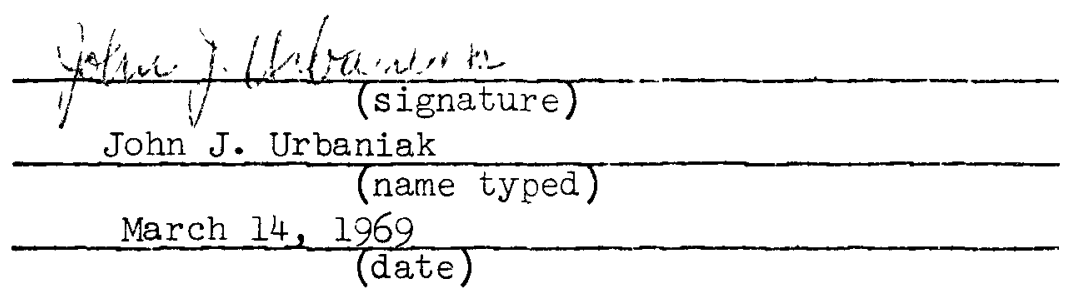

The Author's copy of the proceedings should be mailed to the following complete address:

John J. Urbaniak Westinghouse Electric Corporation Bettis Atomic Power Laboratory P.0. Box 79 West Mifflin, Pennsylvania 15122 
SESSION VHI. MONTE CARLO

1:00 PM

1. “A New Non-Multigroup Adjoint Monte Carlo Technique", L. B. Levitt (AI) and J. Spanier (NAR)

2. "The Use of Third Generation Computers in Monte Carlo Criticality Calculations", N. F. Cross (UCND)

3. "An Experimental Monte Carlo Program for Three Dimensional Nuclear Systems", C. S. Shapiro (San Francisco State College)

4. COHORT II-A Monte Carlo General Purpose Shielding Computer Code", L. C. Clemons, Jr., L. Soffer (NASA LRC) 


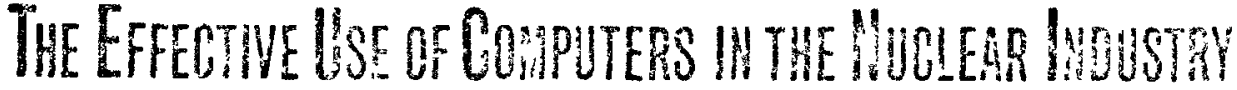 \\ A CONFERENCE AT KNOXVILIE, TEINN. APRIL 21-23, 1969 sponsored by}

THE MATHEMATICS AND COMPUTATION DIVISION OF THE AIVERICAN NUCLEAR SOCIETY THE OAK RIDGE NATIONAL LABORATORY AND THE UNIVERSITY OF TENNESSEE

\section{PUBLICATION RELEASE FORM}

This certifjes that the paper

Titbe:

A New Non-Mult igroup Adjoint Monte Carlo Technique

Authors:__Leo B. Levitt and Jerome Spanier

Organization: htomics International Division and Science Center of North American Rockwell Corporation

is cleared for publication. It is intended for oral Presertation at the abcre reeting. The paper will be published in the proceedings of the meeting by the USAEC Division of Technical Information Extension, Oak Ridge, Tennessee. DTI may seproiuce and rake TID-4500 standard distribution, including copies to Federal clearirghcase,

a depository libraries and announce in NSA.

The paper contains no information that need be withheld from publication vecause of national security interests, patent considerations, or proprietary information interests. Permission is hereby granted to publish it in the proceedings of tre meeting which I understand will receive the above standard distribytion.

This release is submitted by:

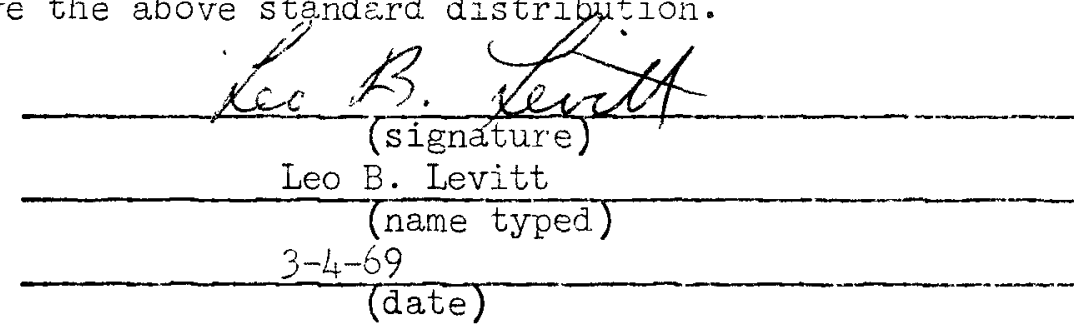

The Author's copy of the proceedirgs should be mailed to the following complete aduress:

Ir. Leo B. Levitt

Atomics International

P. O. Eox 309

Canoga Park, Calif. 91304 


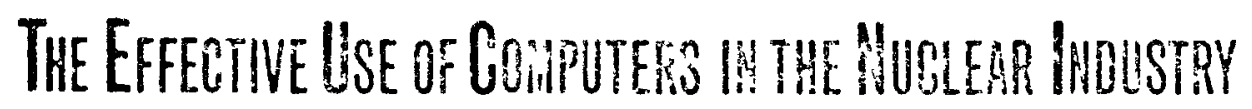

VIII- 3

A CONFERENCE AT KNOXV!LLE, TEI!N. APR!! 21-23, 1969 sponsored by

THE MATHEMATICS AND COMPUTATION DIVISION CF THE AMERICAN NUCLEAR SOCIETY

THE OAK RIDGE NATIONAL LABORATORY AND THE UNIVERSITY OF TENNESSEE

PUBLICATION RELEASE FORM

This certifies that the paper

Titi: AN EXPEFIMENAL HONTE CARLO FFOGEAM FOR THEE E DIMENSIONAL NUCLEAR SYSTEMS

Authors: CHARLFS S. SHAPIRO

organization: Sen Trivisesce State College

is cleared for publication. It is intended for Oral Presentation at the above meeting. The paper will be published in the proceedings of the meeting by the USAEC Division of Technical Information Extension, ak Ridge, Tennessee. DTI may reproduce and make TID-4500 standard distribution, including copies to Federal Clearinghouse, depository libraries and announce in NSA.

The paper contains no information that need be withheld from publication because of national security interests, patent considerations, or proprietary information interests. Permission is hereby granted to publish it in the proceedings of the meeting which $I$ understand will receive the above standard distribution.

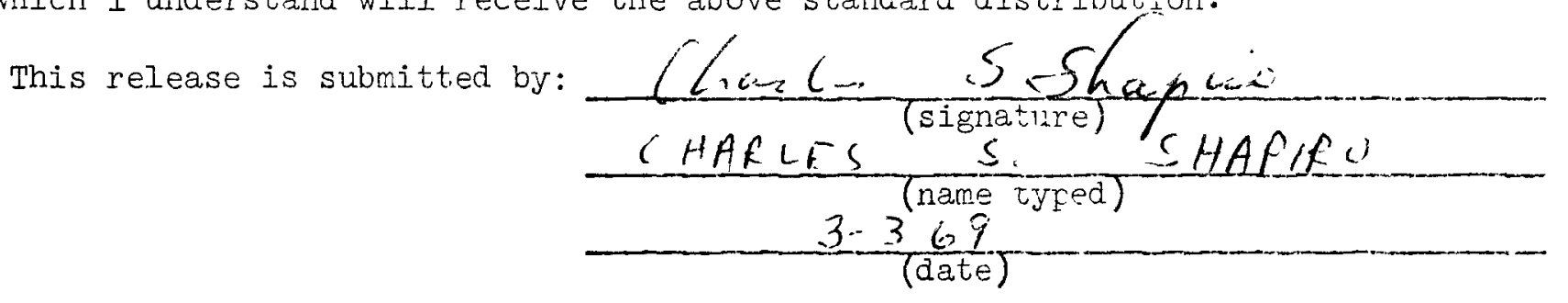

The Author's copy of the proceedings should be mailed to the following complete address: fret Chaeta: S. Shapici

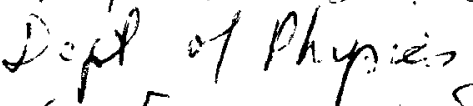

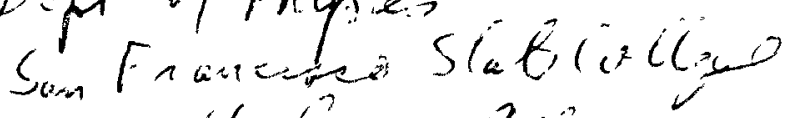

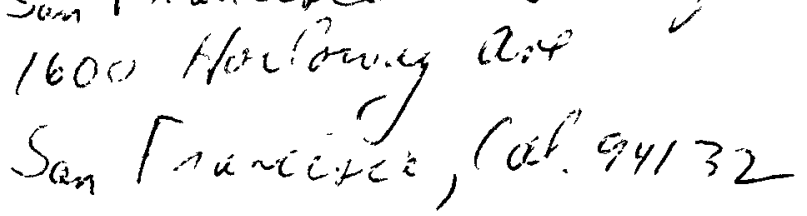

MRS. BETTY MASKEWITZ, ChaIrman, ORNL, PO BO: X OAK RIDGE. TENNESSEE, 37830 


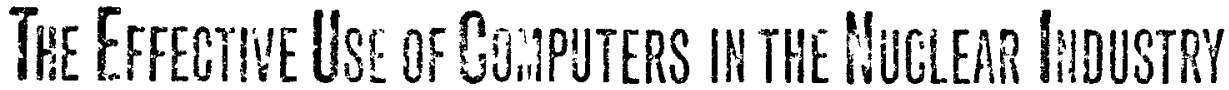 \\ A CONFERENCE AT KNOXVILLE, TENN.・APRIL 21-23,1969 sponsored by}

THE MATHEMATICS AND COMPUTATION DIVISION OF THE AMERICAN NUCLEAR SOCIETY

THE OAK RIDGE NATIONAL LABORATORY AND THE UNIVERSITY OF TENNESSEE

\section{PUBLICATION RELEASE FORM}

This certifies that the paper

Title: COHORI-II - A Ionte Carlo General Puroose Shielding Comnuter Code

Authors: Leonard Soffer and Lester C. Clemons, Ir.

Organization: IIASA-Lewis Research Center, Cleveland, Ohio

is cleared for publication. It is intended ior Oral Presentation at the above meei,-

ing. The paper will be published in the proceedings of the meeting by the USAIC

Division of Technical Information Extension, Oak Ridge, Tennessee. DTI may reproduce and make TID-4500 standard distribution, including copies to Federal Clearinghouse,

depository libraries and announce in NSA.

The paper contains no information that need be withheld from publication because of national security interests, patent considerations, or proprietary iniormation interests. Permission is hereby granted to publish it in the proceedings of the neeting which I understand will receive the above standard distribution.

This release is submitted by:

$\frac{\frac{i}{\text { Lemard soffer }} \text { (signature) }}{\text { (name typed) }}$

The Author's copy of the proceedings should be mailed to the following complete 2ddress:

Leonarl Soffer

IJASA-Lew is Research Center

21000 Frookpark Road

Cleveland, Chio 44135

IS: $49-2$ 